\title{
Schwingungsspektroskopische Untersuchungen zur Chiralitätserkennung und Torsionsdynamik bei Alkoholen
}

\author{
DisserTATION \\ zur Erlangung des mathematisch-naturwissenschaftlichen \\ Doktorgrades "Doctor rerum naturalium“ \\ der Georg-August-Universität Göttingen \\ im Promotionsprogramm Chemie \\ der Georg-August University School of Science (GAUSS)
}

vorgelegt von

Robert Medel

aus Leipzig

Göttingen, 2020 


\section{Betreuungsausschuss}

Prof. Dr. Martin A. Suhm, Institut für Physikalische Chemie, Georg-August-Universität Göttingen

Prof. Dr. Ricardo A. Mata, Institut für Physikalische Chemie, Georg-August-Universität Göttingen

Mitglieder der Prüfungskommission

Referent: Prof. Dr. Martin A. Suhm, Institut für Physikalische Chemie, Georg-August-Universität Göttingen

Korreferent: Prof. Dr. Ricardo A. Mata, Institut für Physikalische Chemie, Georg-August-Universität Göttingen

Weitere Mitglieder der Prüfungskommission:

Prof. Dr. Konrad Koszinowski, Institut für Organische und Biomolekulare Chemie, Georg-August-Universität Göttingen

Dr. Sebastian Kruss, Institut für Physikalische Chemie, Georg-August-Universität Göttingen

Dr. Oliver Bünermann, Institut für Physikalische Chemie, Georg-August-Universität Göttingen

Dr. Tim Schäfer, Institut für Physikalische Chemie, Georg-August-Universität Göttingen

Tag der mündlichen Prüfung: 09.06.2020 


\section{Inhaltsverzeichnis}

English Summary $\quad$ V

1 Einleitung 1

2 Grundlagen 5

2.1 Überschallexpansionen . . . . . . . . . . . . . . . . . . . 5

2.2 Chiralität .......................... 6

2.3 Chiralitätserkennung . . . . . . . . . . . . . 8

2.4 Implikationen von Chiralitätsinduzierter Spinselektivität . . . . . . . . . 11

2.5 Schwingungsspektroskopie . . . . . . . . . . . . . . . 13

2.6 Benchmarking und Fehlerkompensation . . . . . . . . . . . . 16

3 Experimentelle und quantenchemische Methoden 19

3.1 Filet-Jet . . . . . . . . . . . . . . . . . . . 19

3.1.1 Einstellung von Konzentrationen . . . . . . . . . . . . 20

3.1.2 Nachbearbeitung von Filet-Jet-Spektren . . . . . . . . . . . . 23

3.2 Ragout-, Popcorn- und Falafel-Jet . . . . . . . . . . . . . . . . 25

3.3 RESS- und Risotto-Jet . . . . . . . . . . . . . . . . 26

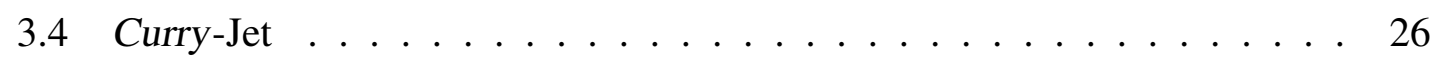

3.5 Verwendete Substanzen . . . . . . . . . . . . . . . . . . 28

3.6 Quantenchemische Rechnungen . . . . . . . . . . . . . . . . 29

3.7 Modellierung von theoretischen Spektren . . . . . . . . . . . . 31

4 Chiralitätssynchronisation in Ethanol-Aggregaten 35

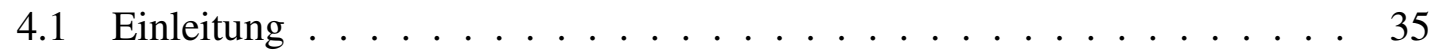

4.2 Ethanol-Monomere . . . . . . . . . . . . . . . 35

4.3 Ethanol-Dimere . . . . . . . . . . . . . . . 40

4.3.1 Einleitung: Adaptive Aggregation . . . . . . . . . . . . . . 40

4.3 .2 Motivation . . . . . . . . . . . . . . . 40

4.3 .3 Konformationsisomerie . . . . . . . . . . . . . . . . . . . . . 41

4.3.4 Schwingungsspektren: Überblick . . . . . . . . . . . . . . . . . . . . 43 45

4.3.5 Quantenchemische Vorhersagen . . . . . . . . . . . 4 45

4.3.6 Modellierung von Schwingungsspektren . . . . . . . . . . . . . . 48

4.3.7 Energetisch höher liegende Konformere . . . . . . . . . . . . . . . 58

4.3.8 Bedeutung von $\mathrm{C}-\mathrm{H}$. . O-Kontakten . . . . . . . . . . . 61

4.3.9 Zusammenfassung, Schlussfolgerungen und Ausblick . . . . . . . 63

4.4 Ethanol- und Methanol-Trimere . . . . . . . . . . . . . . . 66

4.4.1 Konformationsisomerie . . . . . . . . . . . . . . 66

4.4.2 Schwingungsspektren ................ 72 
4.5 Ethanol- und Methanol-Tetramere . . . . . . . . . . . . . . 80

4.5.1 Konformationsisomerie . . . . . . . . . . . . . . . 80

4.6 Ethanol- und Methanol-Pentamere . . . . . . . . . . . . . . . . . . 85

4.6.1 Konformationsisomerie . . . . . . . . . . . . . 85

4.7 Ethanol- und Methanol-Hexamere . . . . . . . . . . . . . . 86

4.7.1 Konformationsisomerie . . . . . . . . . . . . . 86

4.8 Schwingungsspektren von Ethanol- und Methanol-Tetrameren bis -Hexameren . . . . . . . . . . . . . . . . . . 90 90

4.8.1 Methanol-Schwingungsspektren . . . . . . . . . . . . . 90

4.8.2 Ethanol-Schwingungsspektren . . . . . . . . . . . . . . 92

4.9 Vergleichende Betrachtungen und Ausblick . . . . . . . . . . . . . . . 94

4.9.1 Chiralitätssynchronisation in Abhängigkeit der Clustergröße . . . 94

4.9.2 Relative Stabilität der cyclischen Bindungsmotive . . . . . . . . . 96

4.9.3 Schrittweise Dissoziationsenergien . . . . . . . . . . . . . 98

4.9.4 Große Cluster und Kristallisation . . . . . . . . . . . . . . 100

5 Torsionsisomerie von Alkoholen $\quad 103$

5.1 Einleitung . . . . . . . . . . . . . . . . 103

5.2 OH-Streckschwingung als Sonde . . . . . . . . . . . . . 105

5.3 Existierende Korrelationsmodelle . . . . . . . . . . . . . . . . 106

5.4 Neues Korrelationsmodell . . . . . . . . . . . . . . . . . . . . 108

$5.5 n$-Octanol . . . . . . . . . . . . . . . . . . 118

5.6 Isopinocampheol . . . . . . . . . . . . . . . . . . . . . . 119

5.6.1 Konformationsisomerie . . . . . . . . . . . . . . . . . . . 119

5.6.2 Schwingungsspektren . . . . . . . . . . . . 121

5.7 Borneol . . . . . . . . . . . . . . . . . . . 123

5.7.1 Konformationsisomerie . . . . . . . . . . . . . 123

5.7 .2 Schwingungsspektren . . . . . . . . . . . . . . . 124

$5.8 \alpha$-Fenchol - Teil $1 \ldots \ldots \ldots$

5.8.1 Konformationsisomerie . . . . . . . . . . . . . . 127

5.8.2 Schwingungsspektren . . . . . . . . . . . . . . 127

5.9 Barrierenhöhemodell für Tunnelaufspaltungen in Alkoholen . . . . . . . 138

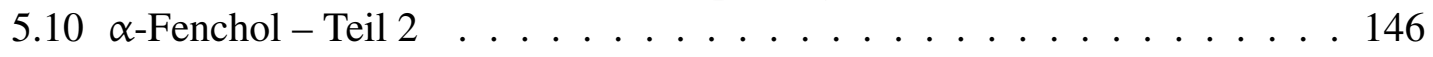

5.11 Pinakolylalkohol . . . . . . . . . . . . . . . . . 151

5.12 tert-Butyldimethylcarbinol . . . . . . . . . . . . . 156

5.13 tert-Butylcarbinol . . . . . . . . . . . . . . . . . 158

5.14 Tri-tert-butylcarbinol . . . . . . . . . . . . . . . . 160

5.15 2-Butanol . . . . . . . . . . . . . . . . . . 162

5.15 .1 Konformationsisomerie . . . . . . . . . . . . . . . 162

5.15 .2 Schwingungsspektren . . . . . . . . . . . . . 168

5.16 Benzylalkohol . . . . . . . . . . . . . . . . . . . 172

5.16.1 Konformationsisomerie . . . . . . . . . . . . . . 172

5.16 .2 Schwingungsspektren . . . . . . . . . . . . . . 178

5.17 1-Phenylethanol . . . . . . . . . . . . . . . . . . 181

5.17 .1 Konformationsisomerie . . . . . . . . . . . . . . 181

5.17 .2 Schwingungsspektren . . . . . . . . . . . . 185 
5.18 Fazit und Aktualisierung des Korrelationsmodells . . . . . . . . . . . . 188

5.19 Anwendungen des Korrelationsmodells . . . . . . . . . . . . . . . . 191

5.20 Ausblick . . . . . . . . . . . . . . . . . . . . . . . 192

6 Chiralitätsdiskriminierung von Alkoholen mit $\alpha$-Pinen 195

6.1 Vorwort . . . . . . . . . . . . . . . . . . . 195

6.2 Einleitung . . . . . . . . . . . . . . . . 195

6.3 Schwingungsspektren . . . . . . . . . . . . . . . . . . 197

6.4 Quantenchemische Rechnungen . . . . . . . . . . . . . . 199

6.5 Diskriminierung mit 2-Butanol . . . . . . . . . . . . . . . . . . 208

6.6 Fazit . . . . . . . . . . . . . . . . . . . . 214

7 Selbstdiskriminierung von $\alpha$-Fenchol $\quad 215$

7.1 Einleitung . . . . . . . . . . . . . . . . 215

7.2 Quantenchemische Rechnungen . . . . . . . . . . . . . 215

7.3 Schwingungsspektren . . . . . . . . . . . . . . . 217

8 Komplementarität in Dimeren von Benzylalkohol und 1-Phenylethanol 219

8.1 Vorwort . . . . . . . . . . . . . . . . . . 219

8.2 Einleitung . . . . . . . . . . . . . . . . . . . 219

8.3 Chiralitätssynchronisation in Benzylalkohol-Dimeren . . . . . . . . 221

8.3 .1 Einleitung . . . . . . . . . . . . . . 221

8.3.2 Quantenchemische Rechnungen ............ . 223

8.3.3 Schwingungsspektren ................. 228

8.4 Chiralitätsdiskriminierung in 1-Phenylethanol-Dimeren . . . . . . . . . 238

8.4 Einleitung . . . . . . . . . . . . . . . . 238

8.4.2 Quantenchemische Rechnungen ... . . . . . . . . . . 238

8.4.3 Schwingungsspektren . . . . . . . . . . . . . 241

8.5 Chiralitätsinduktion in gemischten 1-Phenylethanol-Benzylalkohol-

Dimeren . . . . . . . . . . . . . . . . . 250

8.5.1 Einleitung . . . . . . . . . . . . . . . . . . . . . . 250

8.5.2 Quantenchemische Rechnungen ... . . . . . . . . . 250

8.5.3 Schwingungsspektren . . . . . . . . . . . . . 252

8.6 Substitutionseffekte und chirodiastaltische Energien . . . . . . . . . . . 257

8.7 Ausblick . . . . . . . . . . . . . . . . . . . . . . . 263

9 Umbau des RESS- zum Risotto-Jet 265

9.1 Übersicht . . . . . . . . . . . . . . . . . . . . . . . 265

9.2 Messungen am RESS-Jet . . . . . . . . . . . . . . . . . . . . . . . . . . . 265

9.2.1 Aufbau des RESS-Jets . . . . . . . . . . . . . . . . . . 265

9.2.2 n-Pentan . . . . . . . . . . . . . . . . . 269

9.2.3 $n$-Dodecanol in $n$-Pentan . . . . . . . . . . . . . . 272

9.2 .4 Methanol . . . . . . . . . . . . . . . . . . . . . . . . . . . . . . . . . . . . . . . .

9.3 Umbau zum Risotto-Jet . . . . . . . . . . . . . . . . . . . . . 275

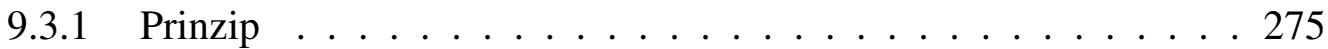

9.3 .2 Vorversuche . . . . . . . . . . . . . . . 277

9.3 .3 Mischkammer . . . . . . . . . . . . . . . . 279 
9.3.4 Zuleitungssystem . . . . . . . . . . . . . . . 281

9.3.5 Synchronisation ................... 283

9.3.6 Heliumverbrauch . . . . . . . . . . . . . . . . 286

9.3.7 Charakterisierung des Konzentrationsverlaufes . . . . . . . . . 286

9.3.8 Optimierung der Aufnahmebedingungen . . . . . . . . . . . . . 289

9.3.9 Variation der Expansionstemperatur am Beispiel Methanol . . . . 292

9.4 Fazit und Ausblick . . . . . . . . . . . . . . . . . . . . . . 294

10 Fazit und Ausblick: Chiralitätserkennung mit prochiralen Molekülen $\quad 297$

$\begin{array}{ll}\text { Danksagung } & 303\end{array}$

$\begin{array}{ll}\text { A Anhang } & 305\end{array}$

Über den Autor $\quad 327$

Persönliche Daten und Ausbildung . . . . . . . . . . . . . . . . . . . 327

Publikationen . . . . . . . . . . . . . . . . . . . 327

Betreute studentische Arbeiten . . . . . . . . . . . . . . . . . 328

$\begin{array}{lr}\text { Spektrenverzeichnis } & 329\end{array}$

$\begin{array}{ll}\text { Literaturverzeichnis } & \mathbf{3 3 3}\end{array}$ 


\section{English Summary}

In the present thesis different implications of molecular chirality are explored by experimental and quantum chemical methods. Discriminating interactions between molecules of different handedness, termed chirality recognition, are one consequence of chirality. Another one is conformational variety in alcohols due to the accompanying low symmetry. In this context special attention is given to conformational interconversion, including quantum tunneling.

The systems are studied in an isolated and cooled condition in supersonic jet expansions, which facilitates the comparison with theory. This is a topic of current interest for chiral systems, since it was recently suggested that an allegedly major contribution to chirality recognition, the chiral-induced spin selectivity (CISS) effect, is either missing $[1,2]$ or strongly underestimated [3] by many popular theoretical methods. One experimental challenge is to generate and analyze appropriate model systems, such as alcohol clusters, in supersonic jet expansions, as they often feature constituents of low volatility. Strategies to optimize existing techniques and to explore new approaches are therefore another topic of this thesis. The $\mathrm{OH}$ stretching vibrations of the molecular systems are probed by linear FTIR and Raman spectroscopy.

The 1st chapter is a brief introduction to the importance of chirality, chirality recognition and conformational isomerism. Alcohols are introduced as a substance class and it is explained why they are appropriate model systems for the investigated phenomena.

In chapter 2 the basics of supersonic jet expansions, chirality, chirality recognition, implications of CISS, vibrational spectroscopy and benchmarking of quantum chemical methods are explained.

The 3rd chapter is an overview of the applied experimental and quantum chemical methods. Multiple apparatuses used to combine jet expansions with vibrational spectroscopy are detailed, as well as the simulation of theoretical spectra.

Chapter 4 covers ethanol, the alcohol prototype for conformational isomerism and chirality synchronization. The subtle conformational balance in the ethanol monomers and dimers is used as a first benchmark for predictions from theoretical methods, mostly popular dispersion corrected density functional methods with various basis sets. Most methods fail at this challenge, while B3LYP-D3(BJ) and B2PLYP-D3(BJ) (when used at least with a triple- $\zeta$ basis set with minimal augmentation) reproduce experimental results for energetic order, geometric parameters (from rotational spectroscopy) and vibrational spectra reasonably well. Some alterations to previous assignments for the vibrational spectra of ethanol dimers are proposed. Larger clusters of ethanol and methanol are explored up to the hexamer. Complementary FTIR and Raman spectra confirm cyclic binding motifs 
and (in the case of ethanol) intramolecular conformational diversity, but due to a lack of conformational resolution no individual structures can be assigned. A switch from a homochiral preference in the dimer to a heterochiral advantage in larger clusters is predicted.

The 5th chapter explores the conformational landscape of larger alcohols as a prerequisite for their application in chirality recognition in subsequent chapters. A model correlating the experimental fundamental transitions of the $\mathrm{OH}$ stretching vibration in jet expansions with the predictions of the vibrational wavenumber in double-harmonic approximation at B3LYP-D3(BJ)/may-cc-pVTZ level is developed. Based on experimental literature data for about 40 alcohol conformers it is found, that a high correlation can be achieved by separating the alcohols into classes by their degree of substitution: tertiary, secondary and primary alcohols as well as methanol. Resulting mean absolute errors and root mean square errors of the correlations are below $2 \mathrm{~cm}^{-1}$ and maximum errors are below $4 \mathrm{~cm}^{-1}$ for all classes. These findings imply an almost constant total $\mathrm{OH}$ stretching anharmonicity within the classes, and a systematic change between them. The predictive quality of the model (including energetic conformational preferences) is tested and confirmed for further alcohols. The conformational landscapes of isopinocampheol, borneol, $\alpha$-fenchol, pinacolyl alcohol, tert-butyldimethylcarbinol, tert-butylcarbinol, tri-tert-butylcarbinol, 2-butanol, benzyl alcohol and 1-phenylethanol are explored in detail.

As expected, transitions for some alcohols cannot be described by this model because changes in the tunneling dynamics upon vibrational excitation lead to band splittings. Therefore a model is adapted and extended to predict ground state splittings. It is assumed that double-minimum potentials with similar barrier widths and comparable effective tunneling masses can be used. Based on the WKB approximation, experimental tunneling splittings can then be correlated with barrier heights from routine calculations in double-harmonic approximation. This highly simplified model leads to surprisingly good correlations for both protiated and deuterated alcohols. Outliers can be explained by a potential form strongly deviating from the double-minimum assumption (for methanol, tert-butyl alcohol and ethanol) or by an anomalously high tunneling mass (for benzyl alcohol). Another relation is presented to describe and predict the isotope effect on tunneling splittings from experimental data of one isotopologue without the need to consult quantum chemical calculations at all. This correlation incorporates ethanol and methanol as well, indicating similar tunneling masses for many alcohols. A number of predictions for yet undetermined splittings are made.

Interestingly, the surprisingly complex vibrational spectrum of the asymmetric molecule $\alpha$-fenchol suggests a major tunneling splitting in the $\mathrm{OH}$ torsional states, even though it is known that the introduction of even slight asymmetry tends to quench delocalization. This might be the result of the combination of an accidental near-degeneracy of two conformers, a low interconversion barrier and a small effective tunneling mass.

In chapter 6 chirality discrimination between the unsaturated monoterpene $\alpha$-pinene and five different alcohols ( $\alpha$-fenchol, borneol, isopinocampheol, 1-phenylethanol and 2-butanol) is explored. For three of the five systems shifts in the $\mathrm{OH}$ stretching frequency of the most stable complex are observed upon change of relative handedness. Four quantum chemical methods are tested for whether they can reproduce these shifts in sign and magnitude. Only B3LYP-D3(BJ) achieves this in a satisfactory manner, while the spec- 
troscopic predictions of $\omega \mathrm{B} 97 \mathrm{X}-\mathrm{D}$ and M06-2X are not better than those of the clearly oversimplified semi-empirical method AM1. On a molecular level these shifts (or their absence) are explained by changes in the torsional preference about the $\mathrm{OH} \cdots \pi$ hydrogen bond. The main results of this chapter are also available in English language in reference [4].

Chapter 7 focuses on the chirality self-discrimination in the dimers of $\alpha$-fenchol. The most stable homochiral conformer is tentatively assigned.

In the 8th chapter the aggregation of benzyl alcohol (phenylmethanol) and 1phenylethanol is investigated. These two compounds form complexes in a very analogous manner, e.g., the dimers differ only in two methyl groups which have no direct steric impact on the structures. However, benzyl alcohol is a transiently chiral molecule, which leads to chirality self-synchronization, while 1-phenylethanol is permanently chiral and exhibits chirality self-discrimination. By comparing the interconversion between homo- and heterochiral dimers of benzyl alcohol with the statistical formation for dimers of 1-phenylethanol, complementary information is obtained regarding relative stability and band positions. The homochiral preference in the dimer of benzyl alcohol is confirmed, while in addition two heterochiral dimers are assigned. Analogous structures (and corresponding deficiencies of theoretical predictions for them) are observed for 1-phenylethanol.

In addition the most stable mixed dimer is tentatively assigned with a homochiral induction and a preference for 1-phenylethanol as the donor of the $\mathrm{OH} \cdots \mathrm{O}$ hydrogen bond and benzyl alcohol as the donor of the $\mathrm{OH} \cdots \pi$ hydrogen bond. Reasons for this allocation of roles are quantum chemically predicted to be based on the influence of the methyl group on intramolecular flexibility, overruling the opposing expectation from its inductive effect.

The topic in chapter 9 is the investigation of two alternative experimental approaches for the generation of small clusters of low volatile compounds in supersonic jet expansions. The first technique is the direct expansion of a compressed and heated liquid into vacuum. After limited results with this approach, the apparatus was subsequently modified to include a heated pre-expansion chamber where the injected liquid is vaporized and mixed with carrier gas. With this second technique spectra comparable with more established techniques used in this thesis are obtained. One possible advantage of this approach might be the generation of a defined gas mixture of two low volatile compounds - independent of the individual vapor pressures - by complete vaporization of a liquid mixture with the same composition.

In the outlook of this thesis (chapter 10) some interesting systems for chirality recognition with prochiral molecules are suggested. Prochiral molecules are remarkable in the fact, that they are achiral but are nevertheless expected to show chirality recognition due to their two enantiotopic sides. This might enable an approach to distinguish between two contributions to chirality recognition: differences in matching of spatial shape and quantum mechanical effects depending on actual chirality of the constituents, such as CISS. 
In conclusion subtle energetic and spectral differences between alcohol monomer conformers and between complexes of different relative handedness were detected. Models for predicting $\mathrm{OH}$ stretching transitions and tunneling splittings of monomeric alcohols were presented. For the investigated model systems these phenomena can be described by the density functional method B3LYP-D3(BJ)/may-cc-pVTZ in double-harmonic approximation in an overall satisfactory and cost-effective manner when combined with empirical corrections. Similar results are obtained with B2PLYP-D3(BJ) (where possible), but this method is at the moment still too cost-prohibitive for a broader application. The obtained results suggest that these quantum chemical methods can describe CISS reasonably well or/and effects of CISS on chirality recognition are not as important as claimed. Observed deviancies between experiment and theory are limited and might be possibly explained by the used double-harmonic approximation and the semi-empirical treatment of exchange and correlation (including dispersion) in the DFT approach, without the need to assume a major (mostly) missing component, such as CISS.

An overview of the investigated systems is given in figure 1 on page IX. 


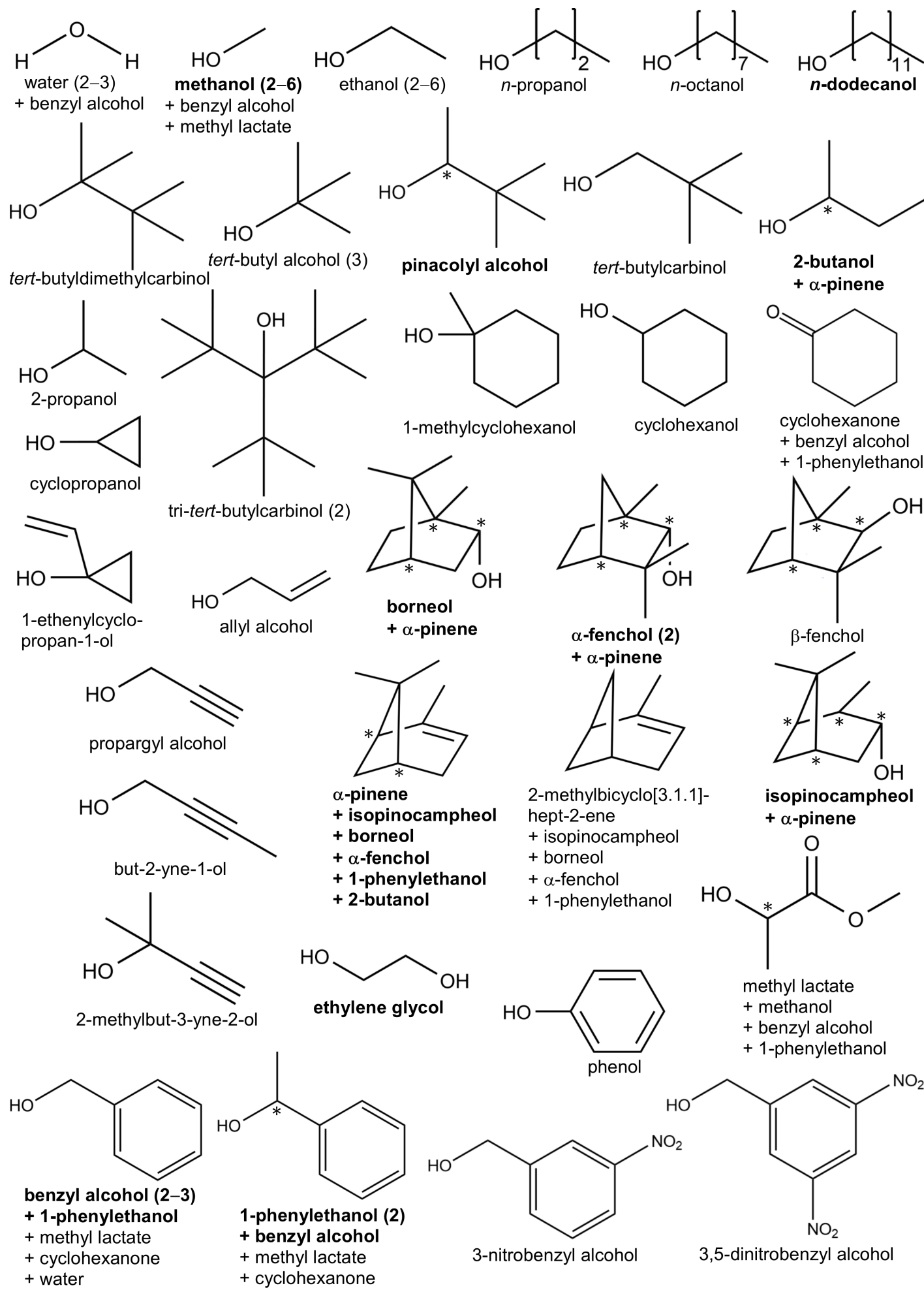

Fig. 1: Overview of systems investigated in this thesis. If self-aggregation was studied, the number of molecules in the clusters is stated in parentheses. For mixed dimers the second constituent is listed below after a plus sign. Names of systems, for which new experimental data is presented, are printed in bold. The systems in plain typeface were studied only by quantum chemical methods, but were compared with experimental data from literature in most cases. Structural formulas are shown with arbitrary absolute configurations and conformations. Stereogenic centers are marked with an asterisk *. 



\section{Einleitung}

Das Phänomenen der Chiralität fasziniert Chemiker schon seit langer Zeit. Bestimmte Moleküle weisen zwei eng verwandte Versionen auf, die sich wie Bild und Spiegelbild verhalten (Enantiomere). Diese zeigen nahezu identische physikalischen Eigenschaften, wechselwirken aber mit anderen chiralen Molekülen teils deutlich unterschiedlich. Dies wird als Chiralitätserkennung bezeichnet.[5] Da auch viele biologisch relevante Moleküle chiral sind, ist dies ein in der Natur fast allgegenwärtiges Phänomen. Vielleicht am intuitivsten äußerst sich dies bei unterschiedlichen Geruchseindrücke einiger Enantiomere, wie bei denen des in dieser Arbeit näher untersuchten $\alpha$-Pinen.[6]

Die Enantiomere von chiralen Substanzen können jedoch auch noch weit gravierendere Unterschiede in ihren biologischen Wirkungen aufweisen. Traurige Bekanntheit hat in diesem Zusammenhang der Wirkstoff Thalidomid im Rahmen des Contergan-Skandals erlangt. Das als Schlaf- und Beruhigungsmittel verabreichte Racemat (1:1-Mischung der Enantiomere) zeigte unerwartete fruchtschädigende Nebenwirkungen. Die beabsichtigte sedierende Wirkung wurde dem $(+)-(R)$-Enantiomer zugeschrieben, die teratogene Wirkung dagegen wesentlich dem (-)-(S)-Enantiomer.[7-9] Doch auch alleinige Verabreichung des (+)-Enantiomers hätte die Katastrophe nicht verhindert, denn Thalidomid racemisiert schnell in vivo.[7-9] Entscheidend ist daher neben der Erkennung der Chiralität auch deren Stabilität. Für Thalidomid und seine Derivate wurde gezeigt, dass die Racemisierung bereits durch eine Substitution des Wasserstoffatoms am stereogenen Zentrum durch ein Deuteriumatom deutlich verlangsamt werden kann. Dies erlaubt es die Wirkungen der beiden Enantiomere besser zu unterscheiden.[7-9] Verantwortlich hierfür ist der Isotopeneffekt auf die Schwingungsnullpunktsenergien der Reaktionsteilnehmer, einschließlich des Übergangszustandes, in der für die Racemisierung verantwortlichen Tautomerisierung der chiralen Keto- in die achirale Enol-Form (Abb. 1.1).

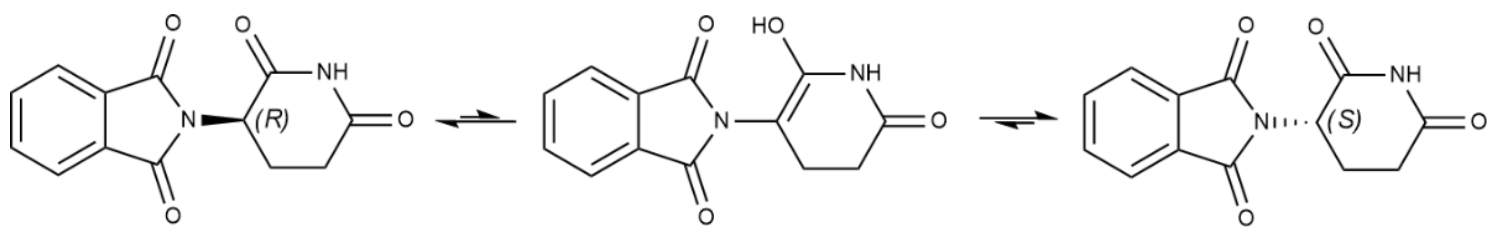

Abb. 1.1: Keto-Enol-Gleichgewicht von Thalidomid, welches die Umwandlung zwischen dem $(+)-(R)$-Enantiomer und dem $(-)-(S)$-Enantiomer ermöglicht.

Der Isotopeneffekt auf die Reaktionsgeschwindigkeit kann besonders ausgeprägt sein wenn Tunnelprozesse zur Reaktion beitragen - auch in biologischen Systemen.[10] Für die Racemisierungsgeschwindigkeit von Thalidomid in Blutplasma zeigen sich nicht nur deutliche Unterschiede zwischen den Isotopologen, sondern ebenso zwischen den Enantiomeren. Dies deutet auch bezüglich dieser Reaktion auf eine Chiralitätserkennung mit einem katalysierenden Plasmaprotein hin.[8] Für die unterschiedlichen biologischen Wirkungen werden verschiedene Bindungsstärken an das Protein Cereblon verantwort- 


\section{Einleitung}

lich gemacht. Dies wird damit begründet, dass das (-)-Enantiomer in weitgehend unveränderter Form (im Vergleich zur Kristallstruktur ohne Protein) bindet, während das (+)-Enantiomer hierfür seine Konformation stärker ändert.[9] Dies wird als adaptive Aggregation bezeichnet.

Als elementares Modellsystem für diese sowohl grundlegenden wie auch biologisch relevanten Phänomene (Chiralitätserkennung, Konformationsvielfalt, adaptive Aggregation, Umwandlung zwischen enantiomeren Strukturen, Tunnelprozesse, Isotopeneffekte) wird in dieser Arbeit die Stoffklasse der Alkohole untersucht. Gemäß IUPAC-Nomenklatur [11] sind Alkohole chemische Verbindungen, bei denen eine Hydroxygruppe (OH) an ein gesättigtes Kohlenstoffatom gebunden ist, welches mit drei weiteren Atomen oder Gruppen $\left(\mathrm{R}^{1}, \mathrm{R}^{2}\right.$ und $\left.\mathrm{R}^{3}\right)$ kovalent verknüpft ist. Abb. 1.2 zeigt die allgemeine Formel eines Alkohols. Durch Torsion um die O-C-Bindung können verschiedene Konformationen angenommen werden, dies ist anhand von Newman-Projektionen illustriert. Die Konformere können sich über niedrige Barrieren und durch isotopenabhängige Tunnelprozesse auch noch bei niedrigen Temperaturen ineinander umwandeln. Daher kann bei starker Kühlung, wie in einer Überschallexpansion, häufig die stabilste Konformation identifiziert werden.

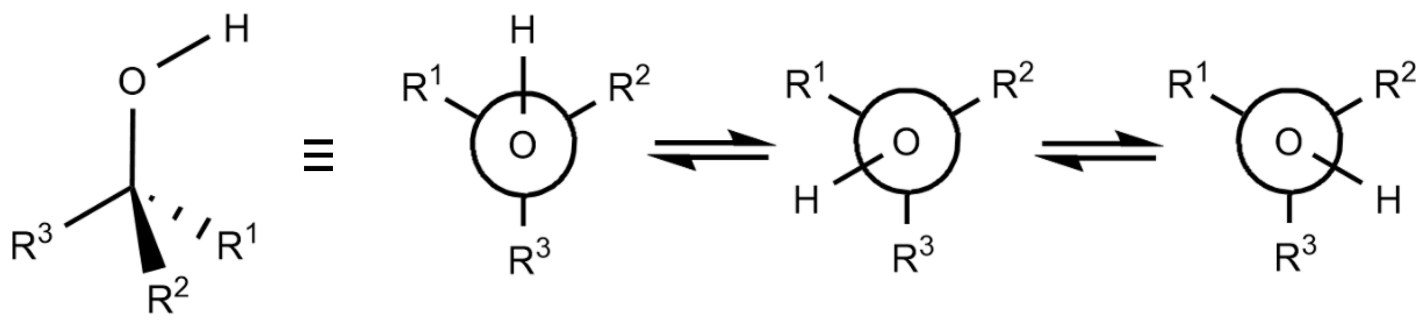

Abb. 1.2: Allgemeine Keilstrichformel eines Alkohols und Konformationen bezüglich der O-CTorsion in Newman-Projektion mit Blickrichtung entlang des $\mathrm{O} \rightarrow \mathrm{C}$-Vektors.

Chemisch besonders interessant sind Alkohole aufgrund ihrer vielfältigen Variationsmöglichkeiten durch die Wahl der Substituenten. Für $\mathrm{R}^{1}=\mathrm{R}^{2}=\mathrm{R}^{3}$ (Beispiel: Methanol) sind die drei möglichen Konformationen in Abb. 1.2 identisch. Chiralität kann bereits für diesen einfachsten Fall über sterisch anspruchsvolle Substituenten eingebracht werden, da diese zu Abweichungen von der gezeigten ideal gestaffelten Anordnung führen können (Beispiel: Tri-tert-butylcarbinol). Bei $\mathrm{R}^{1} \neq \mathrm{R}^{2}=\mathrm{R}^{3}$ ergeben sich drei unterschiedliche Konformationen, wobei sich zwei enantiomer zueinander verhalten (Beispiel: Ethanol). Ethanol ist transient chiral und wechselt durch die Tunneldynamik auch bei sehr tiefen Temperaturen seine Chiralität auf einer Picosekunden-Zeitskala. Dies kann durch Deuterierung fast um einen Faktor sechs verlangsamt werden.[12] Eine Möglichkeit, effektiv permanente Chiralität zu erhalten, ist es, alle drei Substituenten ungleich zu wählen, also $\mathrm{R}^{1} \neq \mathrm{R}^{2} \neq \mathrm{R}^{3}$ (Beispiel: 2-Butanol). In diesem Fall ist das asymmetrisch substituierte Kohlenstoffatom ein stereogenes Zentrum und alle drei Konformere sind zueinander diastereomer. Auch die Substituenten selbst können stereogene Zentren oder weitere Freiheitsgrade enthalten. Die niedrige Symmetrie, die Voraussetzung für Chiralität ist, geht daher in der Regel mit Konformationsvielfalt bei Alkoholen einher. Dies ist daher eines der Hauptthemen dieser Arbeit.

Die OH-Streckschwingung der Hydroxygruppe ist für spektroskopische Untersuchungen aus verschiedenen Gründen gut geeignet. Sie ist besonders hochfrequent und somit 
wenig von der Kopplung oder spektralen Überlagerung mit anderen Schwingungen beeinflusst.[13] Vor allem ist ihre Frequenz empfindlich auf die Konstitution und Konformation des Alkohols, sowie auf die Ausbildung einer Wasserstoffbrücke.[13, 14] Letztere kann durch Koordination von anderen funktionellen Gruppen gebildet werden, wie z. B. mit der Hydroxygruppe eines zweiten Alkohol-Moleküls. Abb. 1.3 zeigt die allgemeine Formel eines solchen Alkohol-Dimers. Ein Molekül nimmt dabei die Donor- und das andere die Akzeptorrolle für die Wasserstoffbrücke an.

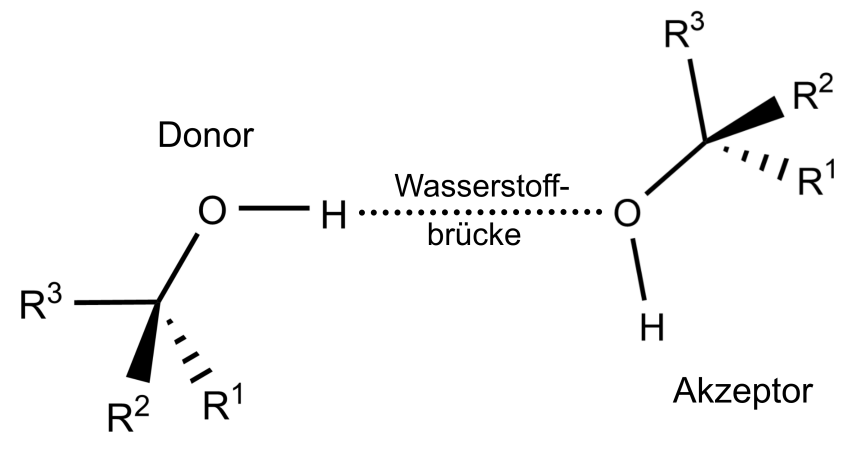

Abb. 1.3: Allgemeine Formel eines Alkohols-Dimers.

Für $\mathrm{R}^{1} \neq \mathrm{R}^{2} \neq \mathrm{R}^{3}$ weisen im gezeigten Fall beide Alkohol-Moleküle die gleiche Konstitution und Konformation, aber unterschiedliche Konfigurationen auf. Wird durch gedachte Vertauschung von $\mathrm{R}^{1}$ und $\mathrm{R}^{2}$ die Konfiguration eines der beiden Moleküle invertiert, so wird ein hierzu diastereomeres Dimer erhalten. Dies ist ein Beispiel für Chiralitätsdiskriminierung zwischen permanent chiralen Substanzen. Sind beide Moleküle dagegen transient chiral, wird dies als Chiralitätssynchronisation bezeichnet. Als dritte Möglichkeit kann zwischen jeweils einer permanent und einer transient chiralen Verbindung Chiralitätsinduktion stattfinden. Chiralitätsdiskriminierung, -induktion und -synchronisation sind die drei Aspekte der Chiralitätserkennung.[5] Die Untersuchung dieses Phänomens ist das zweite Hauptthema dieser Arbeit. Häufig zeigen die Alkohole dabei zudem adaptive Aggregation. Quantenchemische Methoden werden daraufhin evaluiert, ob sie die dabei auftretenden subtilen Einflüsse auf Energie, Struktur und Schwingungsfrequenzen beschreiben können.

Die Erkennung lässt sich durch die Eigenschaften der einzelnen Substituenten beeinflussen. Stärker wechselwirkende Substitutenten können tendenziell zu einer energetisch und spektral ausgeprägteren Chiralitätserkennung führen. Neben der Dispersionswechselwirkung zwischen ausgedehnten aliphatischen Gruppen (Beispiel: $\alpha$-Fenchol) können hierfür auch weitere funktionelle Gruppen eingesetzt werden, wie eine Phenylgruppe (Beispiel: 1-Phenylethanol), oder eine weitere Hydroxygruppe (Beispiel: Ethylenglycol). In den beiden letztgenannten Fällen wird so die Ausbildung weiterer Wasserstoffbrücken möglich und beide Moleküle können sowohl Donor- wie auch Akzeptorrollen annehmen.

Mit Zunahme der Wechselwirkungen sind die Moleküle jedoch auch schwieriger in die Gasphase zu bringen - eine Voraussetzung für die Untersuchung von Monomeren und kleinen Clustern in einer Überschallexpansion. So weisen viele der in dieser Arbeit verwendeten Substanzen Normalsiedetemperaturen oberhalb von $200{ }^{\circ} \mathrm{C}$ auf. Der dritte Fokus dieser Arbeit liegt daher auf der Optimierung und Erweiterung von experimentellen Techniken für die Untersuchung von schwerflüchtigen Substanzen. 



\section{Grundlagen}

\section{1 Überschallexpansionen}

Um grundlegende Eigenschaften von Molekülen, wie Konformationspräferenzen, Tunneldynamiken [15] oder Clusterbildung, zu charakterisieren und auf möglichst einfache Weise mit theoretischen Vorhersagen vergleichen zu können [16], ist es von Vorteil, die Systeme bei tiefer Temperatur und isoliert in der Gasphase zu untersuchen. Eine Möglichkeit, diese beiden scheinbar sich widersprechenden Anforderungen zu erfüllen, bieten Überschallexpansionen. Diese werden erzeugt, indem ein Gasgemisch (seltener auch eine flüssige oder überkritische Lösung [17]) von einer Region hohen Druckes durch eine Düse in eine Umgebung niedrigen Druckes expandiert wird. Die Abmessungen der Düse sind dabei deutlich größer als die mittlere freie Weglänge der Teilchen (in Abgrenzung zur verwandten Effusion). Die hohe Zahl von Stößen im Bereich der Düse führt zu einer adiabatischen und isentropen Umverteilung der inneren Energie von Molekülen in gerichtete Bewegung. Mit wachsendem Abstand von der Düse fallen die Dichte und lokale Temperatur der Expansionen ab, die Teilchen treten in einen nahezu stoßfreien Bereich ein (,Zone of Silence"). Mit abnehmender Temperatur beginnt die Geschwindigkeit der Teilchen formal die Schallgeschwindigkeit zu übersteigen, daher der Begriff „Überschallexpansion“ (im Englischen: „Jet“). Die Kühlung der verschiedenen Freiheitsgrade durch Stöße verläuft unterschiedlich effektiv, sodass sich die Moleküle in einem Nicht-Gleichgewichtszustand befinden. Getrennt für die einzelnen Formen von Freiheitsgraden lassen sich dennoch in günstigen Fällen Gleichgewichte annehmen und Temperaturen ermitteln. Werte, die in der Ruhezone einer Expansion mit Helium als Trägergas erreicht werden können, liegen im Bereich von wenigen Kelvin für Translations- [18] und Rotationsfreiheitsgrade [1921], sowie etwa 30-100 K für Schwingungsfreiheitsgrade [19] (für nicht zu hochfrequente Schwingungen) und 50-70 K für Konformationsfreiheitsgrade [21, 22] (bei niedrigen Barrieren). Die Kühlung lässt sich über die Zusammensetzung der expandierten Gasmischung beeinflussen. Mit massereicheren und besser polarisierbaren Teilchen lassen sich tiefere Temperaturen erreichen und höhere Umwandlungsbarrieren für Konformationsfreiheitsgrade überwinden.[20, 23, 24] Die Temperaturabnahme kann zu einer starken Übersättigung des Gasgemisches führen, sodass kalte Moleküle und kleine Cluster in relativ hoher Konzentration erhalten werden können, während Kondensation zu größeren Aggregaten weitgehend vermieden werden kann.[18] Die freie Expansion wird seitlich und in Expansionsrichtung (Machscheibe) durch die Wechselwirkung mit dem Hintergrundgas begrenzt. Als Einstieg in weiterführende Literatur zu Theorie und technischer Realisierung von Überschallexpansionen seien die Ref. [25] und [18] empfohlen. 


\section{Grundlagen}

\subsection{Chiralität}

Der Begriff „Chiralität“ stammt aus dem Griechischen und bedeutet in etwa „Händigkeit“. Dies ist ein Verweis auf eine besondere Beziehung zwischen unserer linken und rechten Hand: sie sind sich sehr ähnlich, aber nicht identisch, sondern verhalten sich wie Bild und Spiegelbild. Diese Eigenschaft teilen sich unsere Hände mit vielen anderen makroskopischen Objekten, aber auch mit molekularen Systemen. Allen chiralen Anordnungen gemeinsam ist die Abwesenheit von Drehspiegelachsen $\left(S_{n}\right)$ : Symmetrieelemente, an denen sich eine Struktur auf sich selbst abbilden lässt, und zwar über eine Rotation um $360^{\circ} / n$ und eine Spiegelung an einer Ebene senkrecht zu dieser Achse. Dies schließt Spiegelebenen $\left(S_{1}=\sigma\right)$ und Inversionzentren $\left(S_{2}=i\right)$ mit ein.

Im engeren Sinne als chiral werden häufig nur Moleküle bezeichnet, deren spiegelbildlichen Stereoisomere (Enantiomere) bei Zimmertemperatur getrennt werden können. In organischen Molekülen beruht Chiralität in den meisten Fällen auf der Präsenz eines asymmetrisch substituierten Kohlenstoffatoms (stereogenes Zentrum). Doch ist dies weder eine notwendige noch eine ausreichende Bedingung für Chiralität. So existieren auch achirale Moleküle mit mehreren stereogenen Zentren (meso-Verbindungen) und chirale Moleküle ohne diese. Letztere werden stattdessen über Chiralitätsebenen oder -achsen klassifiziert.[26]

Aber auch konstitutionell achirale Moleküle weisen vielfach chirale Konformationen auf. Der Übergang von permanenter zu transienter Chiralität ist fließend und die Einstufung für ein Molekül hängt von den Bedingungen, insbesondere der Temperatur, und der betrachteten Zeitskala ab. So wandelt sich Ethylenglycol bei Zimmertemperatur schnell zwischen zueinander enantiomeren Konformeren um, in einer Überschallexpansion ist dagegen jedes Molekül effektiv in einer bestimmten Händigkeit eingefroren.[27] Für enantiomerenreine helikale „,molekulare Propeller“ konnte gezeigt werden, dass schon durch geringe strukturelle Modifikationen die Lebensdauer einer bestimmten chiralen Konformation bei Zimmertemperatur von Tagen zu Millionen Jahren verändert werden kann.[28]

Der Umwandlungspfad zwischen enantiomeren Anordnungen kann schematisch anhand des Verlaufes der potentiellen Energie $V$ entlang einer Desymmetrierungskoordinate $\pm q$ veranschaulicht werden (Abb. 2.1). Im hypothetischen Grenzfall der perfekt permanenten Chiralität ist die Barriere unendlich hoch und die Enantiomere existieren als zeitlich unbegrenzt stabile Zustände eines Moleküls, die sich nicht gegenseitig beeinflussen. Endlich hohe Barrieren können dagegen potentiell thermisch überwunden oder auch noch bei tiefen Temperaturen quantenchemisch durchtunnelt werden [15]. Der Tunneleffekt führt dazu, dass die Zustände wechselwirken und entsprechend der Symmetrie des Potentials Superpositionen positiver und negativer Parität (Verhalten bei Punktspiegelung) bilden. Die Energiedifferenz zwischen diesen Eigenzuständen des Systems wird als Tunnelaufspaltung $\Delta$ bezeichnet. Bemerkenswert ist, dass diese zeitunabhängigen Lösungen der Schrödinger-Gleichung insgesamt symmetrisch oder antisymmetrisch und damit achiral sind. Da in der Realität jede Barriere endlich ist, wirft die Quantenmechanik die grundlegende Frage auf, warum überhaupt lokalisierte chirale Moleküle vorgefunden werden und nicht deren symmetrische und damit achirale Superpositionen. Mehrere Erklärungsansätze werden hierfür diskutiert.[29, 30]

Wird ein chiraler Ausgangszustand betrachtet, welcher als Linearkombination der Eigenfunktionen konstruiert werden kann, so wird in zeitabhängiger Lösung eine Oszil- 
lation der Aufenthaltswahrscheinlichkeit zwischen den enantiomeren Anordnungen gefunden. Diese Tunnelfrequenz entspricht gerade der Übergangsfrequenz zwischen den Eigenzuständen $\omega=\Delta / \hbar$. Sie kann in einer Überschallexpansion als Abgrenzung zwischen den Grenzfällen permanenter und transienter Chiralität genutzt werden, wenn sie mit der Stoßhäufigkeit verglichen wird. Mit dieser Überlegung wurde eine Tunnelfrequenz von etwa $1 \mathrm{MHz}$ als Grenze vorgeschlagen.[5] Die Tunnelfrequenz sinkt mit zunehmender Barrierenhöhe, Barrierenbreite und effektiver Tunnelmasse. Daneben kann die Barriere ggf. auch thermisch überwunden werden, für die Bedingungen in einer HeliumÜberschallexpansion liegt die Grenze für die Barrierenhöhe in einem Bereich von etwa $5 \mathrm{~kJ} \mathrm{~mol}^{-1}$.[23]

Bei verschwindender Barriere hat die Aufenthaltswahrscheinlichkeit schließlich ihr Maximum in einer symmetrischen Molekülstruktur bei $q=0$. Ist diese Symmetrie auf eine Drehspiegelachse (aber nicht auf eine reine Drehachse) begründet, so kann dieser besondere Fall von Achiralität als Prochiralität bezeichnet werden.[31] Diese Eigenschaft stellt gewissermaßen eine Vorform von Chiralität dar, da letztere durch eine einzelne Modifikation der molekularen Struktur, wie eine Addition oder Substitution, erreicht werden

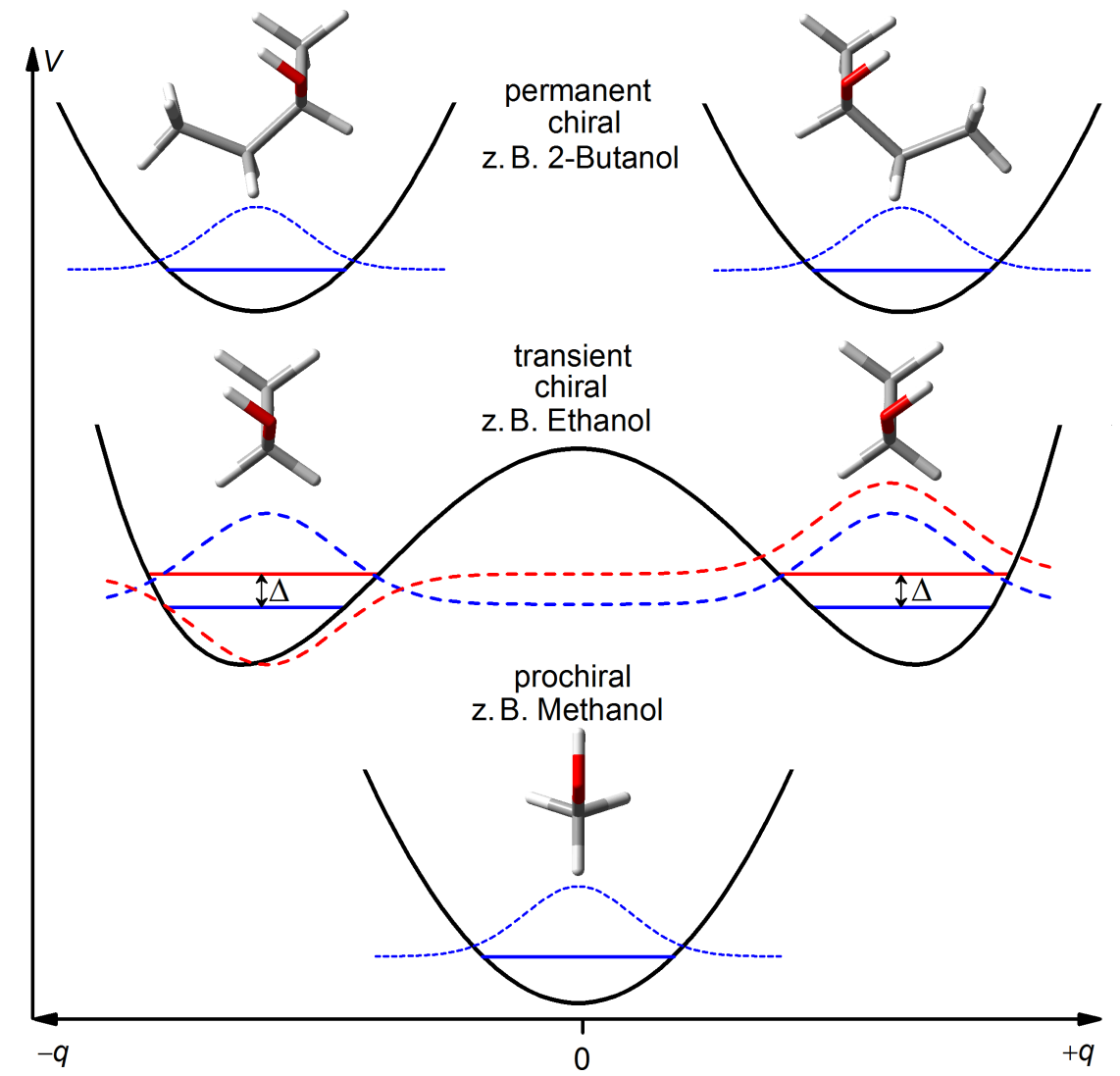

Abb. 2.1: Schematischer Verlauf der potentiellen Energie $V$ entlang einer Desymmetrierungskoordinate $\pm q$ für permanent, transient und prochirale Verbindungen mit in dieser Arbeit näher betrachteten Beispielen. Die jeweiligen Grundzustände sind blau dargestellt. Für den transient chiralen Fall ist zusätzlich der erste angeregte Zustand in rot mit aufgeführt. Die Tunnelaufspaltung $\Delta$ trennt in diesem Fall den symmetrischen Grundzustand und den antisymmetrischen ersten angeregten Zustand. Qualitative Wellenfunktionen der Zustände sind gestrichelt dargestellt. 


\section{Grundlagen}

kann. Das Molekül ist zwar insgesamt achiral, besitzt aber zwei spiegelbildliche (enantiotope) Hälften. ${ }^{a}$

\subsection{Chiralitätserkennung}

Enantiomere besitzen weitgehend gleiche physikalische Eigenschaften. Eine bedeutende Ausnahme hiervon ist, dass von Enantiomeren die Polarisationsebene von linear polarisiertem Licht in gegenläufige Richtungen gedreht wird. Dies ist die Grundlage der gängigsten Bestimmungsmethode für die Höhe der Enantiomerenreinheit einer permanent chiralen Substanz. Hierfür wird das Ausmaß der Drehung einer Probe bestimmt, auf Konzentration und Schichtdicke normiert (spezifischer Drehwinkel) und mit einer enantiomerenreinen Referenz verglichen (bei gleicher Temperatur, Lichtwellenlänge und ggf. Lösungsmittel). Dieses Verhältnis entspricht dem Enantiomerenüberschuss der Probe. In einem racemischen Gemisch kompensieren sich gerade die Beiträge der Enantiomere und es wird keine Drehung beobachtet. Die unterschiedliche Wechselwirkung mit linear polarisiertem Licht wird in dem Modell verständlich, welches elektromagnetische Strahlung als eine Überlagerung aus zirkular polarisierten Anteilen darstellt. In zirkular polarisiertem Licht rotiert der Wellenvektor und beschreibt während der Ausbreitung eine helikale Form. Diese ist ebenfalls chiral, sodass die Anteile von den beiden Enantiomeren in einer Form von Chiralitätserkennung leicht unterschiedlich gebrochen und absorbiert werden. In Folge der hierdurch veränderten Überlagerung führt dies zur Drehung der Polarisationsebene. Dies wird in direkter Weise auch in der Circulardichroismus-Spektroskopie genutzt.

Weitere nachgewiesene enantioselektive Einflüsse sind magnetische Effekte auf die Photochemie [32] und Chiralitätsinduzierte Spinselektivität (Kap. 2.4). Bezüglich weiterer physikalischer Eigenschaften, wie der Energie der beiden Enantiomere, werden auch äußerst subtile Unterschiede erwartet.[33] Dies ist eine Folgerung aus der Paritätsverletzung in der schwachen Kernkraft. Ein experimenteller Nachweis für molekulare Systeme steht allerdings noch aus. Eine ausreichende Asymmetrie durch diesen Einfluss ist aber eine mögliche Erklärung für die im vorherigen Abschnitt angesprochene beobachtete Lokalisierung von permanent chiralen Strukturen. Auswirkungen von Asymmetrie auf Tunneldynamiken werden auch in dieser Arbeit betrachtet, allerdings auf einer völlig anderen Energieskala. Denn berechnete Auswirkungen der Paritätsverletzung [33, 34] sind in jedem Fall um viele Größenordnungen kleiner als in dieser Arbeit behandelte Effekte. Daher werden im Weiteren Enantiomere als effektiv isoenergetisch behandelt.

Auch zwischen chiralen Objekten untereinander finden unterschiedliche Wechselwirkungen statt. Dies kann anschaulich wieder makroskopisch an den menschlichen Händen erklärt werden. Achirale Objekte, wie z. B. ein Zylinder oder ein Würfel, können auf gleiche bzw. spiegelbildliche Weise mit unserer linken und rechten Hand aufgenommen werden. Die Wechselwirkung einer Hand mit anderen chiralen Objekten, wie z. B. einer zweiten, linken oder rechten, Hand, ist dagegen deutlicher unterscheidbar. Das Händeschütteln ist in der westlichen Gesellschaft ein verbreitetes Begrüßungsritual. Dabei ist es Konvention, dass wir uns die jeweils rechte Hand reichen. Wenn wir unserem Gegenüber

${ }^{\text {a}}$ Oder eine kompliziertere Beziehung zwischen Molekülteilen bei einer Drehspiegelachse $S_{n}$ mit $n>1$. 
dagegen unerwartet die linke Hand entgegenstrecken, führt dies zu einem sehr merklich unterschiedlichem Händedruck (dies kann der Leser mit seinen beiden eigenen Händen leicht nachprüfen). Wenn sich beide Parteien dagegen jeweils die linke Hand geben, ergibt sich wieder der gewohnte, wenn auch spiegelbildliche Handschlag. Entscheidend ist also die relative, nicht die absolute Chiralität der Objekte. ${ }^{b}$ Diese Unterschiede in der Wechselwirkung werden als Chiralitätserkennung bezeichnet.

Chiralitätserkennung kann weiter danach unterteilt werden, ob die beteiligten Moleküle permanent chiral oder transient chiral bzw. prochiral sind. Einen Überblick für Beispiele für molekulare Komplexe in Überschallexpansionen bietet Ref. [5].

Die Erkennung zwischen permanent chiralen Konstituenten wird als Chiralitätsdiskriminierung bezeichnet.[5] Da die Chiralität der Moleküle fixiert ist, kann sich in einer Überschallexpansion kein Gleichgewicht zwischen den Komplexen mit gleicher Chiralität der Moleküle (homochiral) und denjenigen unterschiedlicher Chiralität (heterochiral) ausbilden - auch wenn es hierfür durch einen Energieunterschied eine thermodynamische Triebkraft gibt. Dieser Energieunterschied zwischen homo- und heterochiralen Anordnungen wird als chirodiastaltische Energie bezeichnet.[36] Ein negatives Vorzeichen bedeutet dabei, dass der homochirale Komplex energetisch günstiger ist. Da die chirodiastaltische Energie in der Regel nur wenigen Prozent der Bindungsenergie entspricht [5], erfolgt die Bildung von homo- und heterochiralen Clustern in einer Überschallexpansion weitgehend statistisch.

Sind die beteiligten Moleküle identisch bzw. enantiomer, kann dies als Selbstdiskriminierung einer Verbindung bezeichnet werden. In diesem Fall werden in einer racemischen Mischung die gleichen Mengen an homo- und heterochiralen Dimeren gebildet, während größere heterochirale Cluster statistisch wahrscheinlicher sind als homochirale Cluster der gleichen Größe..$^{\mathrm{c}}$ Der Anteil der homochiralen Cluster $x_{\text {hom }}(n)$ der Größe $n$ stehen dabei zu dem der heterochiralen $x_{\text {het }}(n)$ im Verhältnis gemäß Gl. 2.1 (Herleitung in Ref. [37]).

$$
\frac{x_{\text {hom }}(n)}{x_{\text {het }}(n)}=\frac{1}{2^{n-1}-1}
$$

Durch Variation des Enantiomerenüberschusses lassen sich die Anteile von homochiralen und heterochiralen Clustern kontrollieren und so Informationen über die Zuordnung von Banden gewinnen. Während das Spektrum der reinen homochiralen Cluster direkt durch die Expansion von enantiomerenreiner Substanz zugänglich ist, kann ein künstliches Spektrum der heterochiralen Cluster durch passende Differenzbildung erhalten werden. Dabei wird der homochirale Anteil herausgerechnet, wobei für jede Clustergröße die Differenzbildung mit einer unterschiedlichen Gewichtung erfolgen muss. Bei Verwen-

\footnotetext{
${ }^{\mathrm{b}}$ Durch die Paritätsverletzung wäre auch hier ein extrem subtiler Unterschied in Abhängigkeit von der absoluten Chiralität zu erwarten. Studien, die einen solchen Nachweis in Lösung bei Zimmertemperatur behaupten, z. B. Ref. [35], werden vom Autor der vorliegenden Arbeit mit Skepsis betrachtet.

${ }^{c}$ Dies kann leicht an der analogen Binomalverteilung eines wiederholten Münzwurfes nachvollzogen werden. Mit steigender Zahl der Würfe wird es zunehmend unwahrscheinlicher, für alle Würfe einheitlich Kopf oder Zahl zu erhalten.
} 


\section{Grundlagen}

dung von enantiomerenreiner und racemischer Substanz gilt hierfür Gl. 2.2:

$$
A_{\text {het }}(\tilde{v}, n)=\frac{2^{n-1} A_{\mathrm{rac}}(\tilde{v})-A_{\mathrm{er}}(\tilde{v})}{2^{n-1}}
$$

Dabei ist $A_{\text {het }}(\tilde{v}, n)$ das Absorption- oder Ramanspektrum der heterochiralen Cluster der Größe $n, A_{\text {rac }}(\tilde{v})$ das Spektrum der racemischen Substanz und $A_{\text {er }}(\tilde{v})$ das Spektrum der enantiomerenreinen Substanz (letzteres ist gleichbedeutend mit dem der homochiralen Cluster, abgesehen von Monomer-Signalen). In einem Spektrum der heterochiralen Cluster der Größe $n$ erscheinen Banden von größeren heterochiralen Clustern als negative Banden. Ein Beispiel hierfür ist die Differenzbildung für Cluster von 1-Phenylethanol in Abb. 8.8 auf S. 242 in Kap. 8.4. Ein weiteres in dieser Arbeit behandeltes Beispiel ist die Selbstdiskriminierung von $\alpha$-Fenchol (Kap. 7) .

Chiralitätsdiskriminierung zwischen zwei unterschiedlichen Substanzen wird in Kap. 6 zwischen permanent chiralen Alkoholen und $\alpha$-Pinen untersucht. In diesem Fall gilt ähnliches hinsichtlich der statistischen Bildung von gemischten Dimere: Werden beide Substanzen enantiomerenrein eingesetzt, werden nur gemischte Dimere einer bestimmten relativen Chiralität gebildet. Liegt dagegen mindestens eine der Substanzen racemisch vor, werden statistisch die gleichen Mengen an gemischten Dimeren verschiedener relativer Chiralität erhalten.

Sind alle beteiligten Moleküle transient chiral oder prochiral, wird dies als Chiralitätssynchronisation bezeichnet. Sofern die Racemisierungsbarrieren auch im Cluster ausreichend niedrig bleiben, kann sich ein Gleichgewicht zwischen homo- und heterochiralen Strukturen ausbilden. Dagegen kann der Anteil der homo- und heterochiralen Cluster nicht über einen Enantiomerenüberschuss kontrolliert werden. Diese Komplementarität hinsichtlich Gleichgewichtseinstellung und Kontrolle zwischen Chiralitätsdiskriminierung auf der einen Seite, und Chiralitätssynchronisation auf der anderen Seite, wird in Kap. 8 genutzt. In dieser Arbeit untersuchte Systeme mit Selbstsynchronisation sind Cluster von Ethanol und Methanol (Kap. 4) sowie von Benzylalkohol (Kap. 8.3).

In gemischten Clustern aus permanent und transient/prochiralen Verbindungen tritt Chiralitätsinduktion auf. Das permanent chirale Molekül kann dabei seinem flexibleren Wechselwirkungspartner eine bestimmte Chiralität (gleich oder entgegengesetzt) bevorzugt aufprägen. Ein anschauliches makroskopisches Beispiel ist hierfür das Anziehen von Einweg-Laborhandschuhen. Anders als bei den meisten Winterhandschuhen, muss hierbei nicht darauf geachtet werden, dass ein zur jeweiligen Hand passender Handschuh gegriffen wird. Stattdessen passt sich der flexible Handschuh der Chiralität der Hand an. Chiralitätsinduktion hat große Bedeutung in der asymmetrischen Katalyse. Bei der Reaktion eines prochiralen Substrates mit achiralen Reagenzien entstehen die Enantiomere des Produktes in gleichen Mengen. Wird hingegen ein chiraler Katalysator eingesetzt, so sind die Übergangszustände der Reaktion durch Chiralitätsinduktion diastereomer und ein Enantiomer des Produktes wird im Überschuss erhalten. In Bezug auf Gleichgewichtseinstellung und Kontrolle sind Chiralitätsinduktion und -synchronisation in molekularen Komplexen weitgehend analog. Ein hier behandeltes Beispiel für Induktion ist das gemischte Dimer aus 1-Phenylethanol und Benzylalkohol (Kap. 8.5). Im Ausblick dieser 
Arbeit (Kap. 10) werden einige weitere Systeme zur Untersuchung von Chiralitätsinduktion und -synchronisation mit Beteiligung von prochiralen Molekülen vorgeschlagen.

\subsection{Implikationen von Chiralitätsinduzierter Spinselektivität}

Lange Zeit war es Konsens, dass Chiralitätserkennung allein, oder zumindest entscheidend, durch die unterschiedlichen dreidimensionalen Anordnungen der Enantiomere und die daraus resultierenden unterschiedlichen Wechselwirkungen zwischen den Molekülgruppen bestimmt wird. Mit Konzepten wie dem Schlüssel-Schloss-Prinzip [38] oder der Drei-Punkte-Regel [39] könnte Chiralitätserkennung daher grundsätzlich auch klassisch verstanden und - mit geeigneter Parametrisierung - modelliert werden (Molekülmechanik). Zwei Effekte aus der schwachen Kernkraft und der Quantenelektrodynamik gehen jedoch über dieses anschauliche Bild hinaus. Der eine ist die zuvor erwähnte Paritätsverletzung, der zweite sind chiralitätsdiskriminierende Terme in der Dispersions-, Induktions- und Resonanzenergie, die systematisch hetero- gegenüber homochiralen Anordnungen stabilisieren.[40, 41] Allerdings wurden diese beiden Effekte gegenüber dem räumlichen Beitrag zur chirodiastaltischen Energie als sehr geringfügig bewertet, zumindest für kleine molekulare Cluster.[41-43]

Vor wenigen Jahren wurde jedoch ein weiterer, stärker signifikanter Beitrag zur Wechselwirkung vorgeschlagen, der ebenfalls nur zwischen chiralen Molekülen wirksam ist und auf Chiralitätsinduzierter Spinselektivität (CISS) beruht.[1, 2] CISS ist ein vor zwei Jahrzehnten entdeckter [44] dynamischer quantenmechanischer Effekt, der eine bevorzugte Richtung der Elektronenbewegung in einem chiralen Molekül beschreibt, abhängig vom Spin des Elektrons und der absoluten Chiralität des Moleküls.[45] Die bei der Bildung eines molekularen Komplexes stattfindende Ladungspolarisation stellt eine solche Verschiebung von Elektronendichte dar und soll zu unterschiedlichen relativen Spinpolarisationen in homo- und heterochiralen Komplexen führen.[1,2] Bei engem Kontakt der Moleküle führt dies über die Austauschwechselwirkung zu einer verringerten Destabilisierung durch den spin-abhängigen Anteil, wenn Moleküle gleicher Chiralität interagieren.[2] Als Resultat soll der zusätzliche Beitrag von CISS daher systematisch homochirale Anordnungen gegenüber heterochiralen stabilisieren. Dieser Effekt hängt in seiner Größe, aber nicht in seinem Vorzeichen, von der räumlichen Orientierung der Enantiomere zueinander ab. Bei großflächigerem und engerem Kontakt nimmt er zu. Auch wenn ein direkter Kontakt der Moleküle benötigt wird, soll die Chiralität der Moleküle als ganzes relevant sein, sodass auch weiter von der Kontaktstelle entfernte Chiralitätselemente Einfluss nehmen.[2]

Die Größe dieses Effektes wurde in Ref. [1] ohne nähere Begründung für schwache Ladungspolarisationen mit einigen $\mathrm{kJ} \mathrm{mol}^{-1}$ angegeben. Eine konkrete Rechnung wurde in Ref. [2] für die homo- und heterochiralen Dimere von $\mathrm{H}_{3} \mathrm{C}-\mathrm{CFClBr}$, die linear entlang der vier Kohlenstoffatome mit Kontakt über die Methylgruppen ausgerichtet wurden, durchgeführt. Für einen intermolekularen C..C-Abstand von $2.6 \AA$ wurde eine relative Stabilisierung der homochiralen Anordnung von $0.5 \mathrm{kcal} \mathrm{mol}^{-1}\left(\approx 2 \mathrm{~kJ} \mathrm{~mol}^{-1}\right)$ mit symmetrie-adaptierter Störungstheorie angegeben. Dies ist ähnlich groß wie typische chirodiastaltische Energien zwischen kleinen Molekülen.[5] Falls dieser Effekt derartig si- 


\section{Grundlagen}

gnifikant wäre, sollte daher für chirodiastaltische Energien ein merklicher Trend in Richtung homochiraler Präferenz gefunden werden können. Allerdings ist der von den Autoren von Ref. [2] benannte typische intermolekulare Abstand zwischen Kohlenstoffatomen ( $3 \AA ̊$ und weniger) unrealistisch. Die hierfür benannte Quelle (Ref. [46]) gibt stattdessen tatsächlich einen Wert von 3.6 ̊ an. Dies entspricht auch gut dem Gleichgewichtsabstand für den Modell-Komplex gemäß eigener Optimierung auf B3LYP-D3(BJ)/may-cc-pVTZNiveau. Bei dieser Distanz ist die Diskriminierung im Diagramm 4B von Ref. [2] praktisch nicht mehr vorhanden. Unklar ist auch, inwieweit dieser Effekt temporär oder statisch ist. Von den Autoren wird angegeben, dass die Spin-Polarisation auf einer Zeitskala von typischerweise Mikrosekunden nach Bildung des Komplexes wieder randomisiert (mit Spekulation über eine Verlängerung) [1, 2], im Kontrast hierzu wurden die Rechnungen offenbar zeitunabhängig ausgeführt.

Empirische Befunde für Kristalle deuten dagegen eher in Richtung einer heterochiralen Bevorzugung.[43, 47] Diese könnte auf einem (umstrittenen) geometrisch begründeten Trend in der Packungsdichte [43, 48] oder auf höherer Bedeutung des Resonanzterms in makroskopischen Kristallen [40] basieren, oder auch schlicht ein statistisches Artefakt der Datenlage sein [49]. Es ist daher nicht klar, ob bei vernachlässigbarer Bedeutung von CISS eine Präferenz bei Kristallstrukturen zu erwarten wäre. Eine der Verzerrungen bei Vergleich von bekannten Kristallstrukturen ist, dass eine racemische Verbindung (Bezeichnung für einen geordneten Kristall der beiden Enantiomere) experimentell nicht zugänglich ist, wenn das Konglomerat (nebeneinander vorliegende enantiomerenreine Kristalle) merklich stabiler ist. Dies gilt umgedreht nicht, da ein enantiomerenreiner Kristall (mit physikalischen Eigenschaften nahezu identisch zum Konglomerat) stets aus einer ebensolchen Lösung erhalten werden kann.[47, 49] Wegen der statistischen Bildung in Überschallexpansionen kann dagegen erwartet werden, dass stets homo- und heterochirale Cluster von permanent chiralen Verbindungen beobachtbar sind und damit verglichen werden können. Dafür erweist sich mit dieser Methode mangels eines Gleichgewichtseinstellung allerdings die Bestimmung von chirodiastaltischen Energien experimentell als schwierig. Systeme mit Chiralitätsinduktion oder -synchronisation lassen hingegen bei niedrigen Umwandlungsbarrieren zwischen homo- und heterochiralen Clustern prinzipiell eine direkte Abschätzung der chirodiastaltischen Energie zu (oder zumindest einer Untergrenze bei Nicht-Beobachtung einer der Varianten). Dagegen ist in diesen Fällen die korrekte Identifikation der Cluster schwieriger. Dieses Problem wird in Kap. 8 näher diskutiert. Auch falls CISS nur zeitlich begrenzte Auswirkungen auf die chirodiastaltische Energie hat, wäre der Effekt eher auf der Mikrosekundenzeitskala einer Überschallexpansion, denn bei Kristallstrukturen, nachweisbar.

Eine erwähnenswerte weitere Implikation von Effekten, die explizit von der relativen Chiralität abhängen, wie CISS, ist, dass die für Gemische häufig mit weitgehend willkürlichen Kriterien (wie der CIP-Nomenklatur [26]) festgelegten Begriffe „,homochiral“ und „heterochiral“ eine physikalische Bedeutung im Sinne von „durch CISS stabilisiert“ bzw. ,durch CISS destabilisiert“ erhalten. Dies ist von Bedeutung, da auch für permanent chirale Moleküle die Richtung der physikalischen Auswirkungen der Chiralität veränderlich sein kann. So wurde gefunden, dass bereits eine Konformationsänderung durch Torsion um eine C-C-Einfachbindung die optische Aktivität invertieren kann.[50] Dies führt zu Unsicherheit bei der Einstufung von Komplexen mit mehreren beteiligten Konformationen, wie beispielsweise dem in Kap. 7 behandelten Dimer von $\alpha$-Fenchol. Daher 
sind die in dieser Arbeit verwendeten Begriffe homo- und heterochiral unter Vorbehalt zu sehen. In allen Definitionen mit klarer relativer Chiralität ausgestattete Komplexe sind nur solche, in denen die Moleküle symmetrieäquivalent sind, also durch Operationen wie Translation, Drehung oder Drehspiegelung ineinander überführt werden können. In dieser Arbeit behandelte Beispiele hierfür sind bestimmte Tri- und Tetramere von Ethanol und Methanol sowie bestimmte Dimere von Benzylalkohol und 1-Phenylethanol.

In Komplexen aus unterschiedlichen Molekülen ist schließlich völlig unklar, welche Paarung der Enantiomere durch solche Effekte relativ stabilisiert oder destabilisiert wird.[41] Auch die Nutzung der Vorzeichen der experimentell bestimmten spezifischen Drehwinkel der Substanzen ist uneindeutig, da diese nahe Zimmertemperatur und in Lösung oder reiner Flüssigkeit bestimmt werden. Thermische Anregung von Konformationen und Schwingungen [50], sowie Solvatation [51] können zu Inversion führen. Eine mögliche Lösung für dieses Problem könnte in Zukunft ggf. die Berechnung von optischen Aktivitäten für deformierte Cluster-Fragmente darstellen. Allerdings wäre die naheliegend erscheinende Vermutung, dass gleiche Vorzeichen der optischen Aktivität auch relative Stabilisierung durch CISS bedeuten, noch zu beweisen.

Wegen der quantenmechanischen Natur von CISS ist nicht zu erwarten, dass der Effekt von Kraftfeldmethoden erfasst werden kann, einschließlich der neueren Varianten.[2] Methoden, die auf Dichtefunktionaltheorie (DFT) basieren, sollen dagegen grundsätzlich CISS beschreiben können.[3, 52] Dabei hat allerdings das Ausmaß der berücksichtigten Spin-Bahn-Kopplung und das verwendete Austausch-Korrelationsfunktional großen Einfluss.[3] Der CISS-Effekt soll in bisherigen theoretischen Beschreibungen um mehrere Größenordnungen unterschätzt werden.[3]

Eine der Zielsetzungen dieser Arbeit ist es daher für einige molekulare Systeme zu prüfen, inwieweit experimentelle Ergebnisse für Chiralitätserkennung durch populäre DFTMethoden korrekt vorhergesagt werden können. Größere Diskrepanzen bei Strukturen, relative Energien oder Schwingungswellenzahlen können Hinweise auf Schwächen der Theorie liefern. Hierzu könnten auch mögliche nicht oder unzureichend berücksichtige Effekte, wie CISS, beitragen.

\subsection{Schwingungsspektroskopie}

Eine Möglichkeit, Moleküle und Cluster in einer Überschallexpansion zu charakterisieren, ist die Schwingungsspektroskopie. Molekülschwingungen beschreiben die Bewegungen der Atomkerne in der elektronischen Potentialhyperfläche des Systems. In der einfachsten Form können Parabeln als Potentialkurven entlang der Auslenkung der einzelnen Schwingungen angenommen werden, sodass die rücktreibende Kraft proportional zur Auslenkung aus dem Minimum ist (harmonischer Oszillator). Aus der quantenmechanischen Behandlung ergeben sich diskrete, äquidistante Energieniveaus für jede Schwingung. Die Gesamtschwingungsenergie $h c G$ eines polyatomaren Moleküls kann als Funktion der individuellen Schwingungsquantenzahlen $v_{i}$ der $n$ Schwingungsmoden gemäß Gl. 


\section{Grundlagen}

2.3 beschrieben werden:

$$
\begin{aligned}
G\left(v_{1}, v_{2}, \ldots, v_{n}\right) & =\sum_{i}^{n} \omega_{i}\left(v_{i}+\frac{1}{2}\right) \\
\omega_{i} & =\frac{1}{2 \pi c} \sqrt{\frac{k_{i}}{\mu_{i}}}
\end{aligned}
$$

$\omega$ ist die harmonische Schwingungswellenzahl und ergibt sich gemäß Gl. 2.4 aus der Kraftkonstanten $k$ (entspricht der Krümmung der Parabel) und der reduzierten Masse $\mu$ der jeweiligen Schwingung $i$. Es ist zu erkennen, dass die Schwingungen auch im Grundzustand $\left(v_{1}, v_{2}, \ldots, v_{n}=0\right)$ noch Energie besitzen, dies ist die Schwingungsnullpunktsenergie.

Tatsächlich weicht die Potentialform vor allem bei großen Auslenkungen von einer Parabel ab und die Anregung einer Schwingungsmode hat Auswirkungen auf die anderen Moden des Systems. Dies kann allgemein mit Gl. 2.5 [53] beschrieben werden:

$$
G\left(v_{1}, v_{2}, \ldots, v_{n}\right)=\sum_{i}^{n} \omega_{i}\left(v_{i}+\frac{1}{2}\right)+\sum_{i}^{n} \sum_{j \geq i}^{n} x_{i, j}\left(v_{i}+\frac{1}{2}\right)\left(v_{j}+\frac{1}{2}\right)
$$

Gl. 2.3 wird darin mit den zusätzlichen Termen erweitert, die diagonale $x_{i, i}$ und außerdiagonale Anharmonizitätskonstanten $x_{i, j}(j>i)$ enthalten. Die diagonalen Anharmonizitätskonstanten beschreiben die intrinsische Anharmonizität der Schwingungen, während die außerdiagonalen Anharmonizitätskonstanten die Wechselwirkungen zwischen den Schwingungsmoden erfassen. Für eine noch genauere Beschreibung ist es möglich Gl. 2.5 in einer Reihenentwicklung fortzusetzen.

In dieser Arbeit werden Fundamentalübergänge zwischen dem Grundzustand des Systems und dem ersten angeregten Zustand einer bestimmten Schwingung untersucht. Die Übergangswellenzahl ergibt sich aus der Differenz der beiden Zustände gemäß den beiden Modellen zu:

$$
\begin{aligned}
\text { harmonisch: } G\left(v_{i}=1\right)-G\left(v_{i}=0\right)=\omega_{i} \\
\text { anharmonisch: } G\left(v_{i}=1\right)-G\left(v_{i}=0\right)=\omega_{i}+2 x_{i, i}+\frac{1}{2} \sum_{i}^{n} \sum_{j>i}^{n} x_{i, j}=\tilde{v}_{i}
\end{aligned}
$$

Für experimentelle und anharmonische (sowie entsprechend korrigierte harmonische) Wellenzahlen von Fundamentalübergängen wird in dieser Arbeit das Symbol $\tilde{v}$ verwendet. Die diagonalen Anharmonizitätskonstanten sind für OH-Streckschwingungen deutlich negativ.[54] Die außerdiagonalen Anharmonizitätskonstante sind vom Betrag geringer und variieren im Vorzeichen, sodass sie sich in Summe weitgehend kompensieren.[54] Daher sind die harmonischen Übergangswellenzahlen $\omega$ größer als die anharmonischen $\tilde{v}$. Harmonische Wellenzahlen lassen sich, im Gegensatz zu anharmonischen, kostengünstig berechnen. Bei strukturell eng verwandten Systemen kann für analoge Schwingungen angenommen werden, dass die anharmonischen Terme in Summe vergleichbar sind und sich daher bei Differenzbildung weitgehend kompensieren. Solche empirische Korrekturen auf Basis von experimentellen Daten werden in Kap. 5.3 im Detail diskutiert. 
Auch die Schwingungsnullpunktsenergie wird von der Anharmonizität beeinflusst, wie der Vergleich zwischen Gl. 2.8 und 2.9 zeigt:

$$
\begin{aligned}
\text { harmonisch: } G\left(v_{1}, v_{2}, \ldots v_{n}=0\right) & =\frac{1}{2} \sum_{i}^{n} \omega_{i} \\
\text { anharmonisch: } G\left(v_{1}, v_{2}, \ldots v_{n}=0\right) & =\frac{1}{2} \sum_{i}^{n} \omega_{i}+\frac{1}{4} \sum_{i}^{n} \sum_{j \geq i}^{n} x_{i, j}
\end{aligned}
$$

Bei der Berechnung von Energiedifferenzen zwischen sehr ähnlichen Isomeren einer Struktur ist auch hier weitgehende Kompensation für die anharmonischen Terme zu erwarten.

Zur experimentellen Bestimmung der Wellenzahlen und relativen Intensitäten von Fundamentalübergängen werden in dieser Arbeit zwei Spektroskopie-Methoden eingesetzt: Infrarot- und Raman-Spektroskopie.

Bei der Infrarot-Spektroskopie wird elektromagnetische Strahlung absorbiert, die resonant zur Energiedifferenz zwischen den Schwingungsniveaus ist. Die Wahrscheinlichkeit des Überganges hängt in erster Näherung davon ab, wie stark sich das Dipolmoment mit der Schwingungskoordinate ändert. Die Annahme einer linearen Änderung wird als elektrische Harmonizität bezeichnet und bildet zusammen mit der harmonischen Näherung für die Potentialform die sogenannte doppelt-harmonische Näherung. Beide Annahmen werden in den Rechnungen dieser Arbeit stets zusammen verwendet und verkürzt als harmonische Näherung bezeichnet. Die IR-Aktivität von OH-Streckschwingungen nimmt bei der Ausbildung von Wasserstoffbrücken stark zu, weshalb die Untersuchung dieser Schwingungen ausgezeichnet geeignet ist, um auch geringe Konzentrationen von molekularen Komplexen nachzuweisen. Bei Infrarot-Spektroskopie besitzen reine Übergänge zwischen Schwingungsniveaus (Q-Zweig) häufig keine oder nur eine geringe Intensität. Sstattdessen verteilt sich die Intensität stärker auf viele Übergänge, welche eine Änderung des Rotationszustandes beinhalten. Diese kombinierten Rotationsschwingungsübergänge bilden bei Rotationsanregung den R- und bei -abregung den P-Zweig des Spektrums. Da in dieser Arbeit instrumentelle Auflösungen mittlerer Größe $\left(2-5 \mathrm{~cm}^{-1}\right)$ verwendet und Systeme mit relativ kleinen Rotationskonstanten untersucht werden, können weder einzelne Übergänge noch die beiden Zweige aufgelöst werden. Stattdessen werden Banden als Gesamtheit dieser Übergänge beobachtet. Die starke Kühlung in der Überschallexpansion vereinfacht die Spektren allerdings durch weitgehende Entvölkerung von höheren Schwingungs-, Rotations- und Konformationszuständen, wodurch die Zahl und Breite der Banden verringert wird.

In dieser Arbeit wird Infrarot-Spektroskopie in Form der Fourier-Transform-InfrarotSpektroskopie (kurz: FTIR-Spektroskopie) verwendet. Hierbei wird die Intensität der Strahlung aus einer breitbandigen Quelle nach Passieren der Überschallexpansion in Abhängigkeit der Spiegelposition eines Interferometers detektiert. Nach FourierTransformation des Interferogramms wird ein Einkanal-Spektrum $I(\tilde{v})$ erhalten, welches mit einem kurz davor aufgenommenen Hintergrund-Spektrum $I_{0}(\tilde{v})$ zu einem Extinktionsspektrum $E(\tilde{v})=\lg \frac{I_{0}(\tilde{v})}{I(\tilde{v})}$ verrechnet wird. Weitere Details des Messablaufs werden bei der Beschreibung der Apparaturen und im Kontext der Ergebnisse diskutiert. Einen 


\section{Grundlagen}

Überblick über die Kopplung von FTIR-Spektroskopie mit Überschallexpansionen bieten Ref. [18] und [55]. Für eine tiefergehende und umfassendere Beschreibung der FTIRSpektroskopie sei Ref. [56] empfohlen.

Bei der Raman-Spektroskopie wird keine breitbandige, sondern eine hochenergetische monochromatische Strahlungsquelle, wie ein Laser, eingesetzt. Mit zunehmender Wellenzahl des eingestrahlten Lichtes besteht eine steigende Wahrscheinlichkeit, dass ein Teil der Energie des Photons auf Materie übertragen und z. B. eine Schwingung anregt wird (Raman-Streuung). Das detektierte gestreute Licht hat dann eine etwas geringe Energie als das eingestrahlte. Diese Verschiebung heißt Stokes-Shift und entspricht der Wellenzahl des Schwingungsüberganges und wird daher in dieser Arbeit ebenfalls mit $\tilde{v}$ bezeichnet. Auch eine Übertragung von Energie vom Molekül auf die einfallende Strahlung ist möglich (Anti-Stokes). Dies setzt allerdings eine vorherige thermische Anregung des Moleküls voraus und ist für die hier untersuchten hochfrequenten Schwingungsmoden ohne Bedeutung. Der Raman-Streuquerschnitt (und damit die Intensität des detektierten Signals) hängt davon ab, wie sich die Polarisierbarkeit des Moleküls entlang der Schwingungskoordinate ändert. Besonders für (annähernd) symmetrische Systeme können durch die unterschiedlichen Auswahlregeln die relativen IR-Aktivitäten und Raman-Streuquerschnitte der Schwingungsmoden sehr unterschiedlich groß sein. Durch die Kombination dieser beiden Formen von Schwingungsspektroskopie lassen sich daher für geeignete Systeme komplementäre Informationen erhalten. Weiterhin sind durch Raman-Streuung reine Schwingungsübergänge und damit die Beobachtung des schmaleren Q-Zweiges begünstigt.[14] Bei geeigneter instrumenteller Auflösung und geringer Bedeutung von anderen Verbreiterungsmechanismen können daher Banden von verschiedenen Konformeren besser aufgelöst werden, im Vergleich zu den hier eingesetzten FTIR-Methoden. Dies ist in dieser Arbeit vor allem für OH-Streckschwingungsbanden der Konformere von Alkohol-Monomeren von Vorteil, für die sich Raman-Spektroskopie zudem im direkten Vergleich als empfindlicher erweist. Dieser Vorteil geht für Cluster weitgehend verloren, da der Raman-Streuquerschnitt bei der Ausbildung von Wasserstoffbrücken deutlich weniger steigt als die IR-Aktivität. Für weiterführende Informationen zur Raman-Streuung sei auf die Ref. [57, 58] verwiesen.

\subsection{Benchmarking und Fehlerkompensation}

Die Ergebnisse aus quantenchemischen Rechnungen sind unabdingbar für die Interpretation der in dieser Arbeit aufgenommenen Schwingungsspektren. Dabei können die experimentellen Befunde aber auch als Referenzen genutzt werden, um die Vorhersagequalität von quantenchemischen Elektronenstrukturmethoden zu bewerten, das sogenannte Benchmarking [16]. Um die Vergleichbarkeit von Theorie und Experiment zu erhöhen, wird in dieser experimentell fokussierten Arbeit versucht der Theorie möglichst entgegen zu kommen: Es werden Modellsysteme kleiner bis mittlerer Größe, isoliert bei tiefen Temperaturen in einer Überschallexpansion, untersucht. Das offensichtlichste Defizit in der Vergleichbarkeit zwischen den hier verwendeten experimentellen und quantenchemischen Methoden ist die Verwendung der harmonischen Näherung. Um diese Lücke zu verringern wurden zwei Ansätze durch Oswald et al. [59] evaluiert: Zum einen die Verwen- 
dung von anharmonischen Korrekturen, wie VPT2 [60], auf der Theorieseite. Und zum anderen die Konstruktion von ,experimentellen harmonischen Spektren“ durch Nutzung experimenteller Anharmonizitätsparameter. Der erste Ansatz ist allerdings im Fall von VPT2 auf relativ starre, lokalisierte, resonanzfreie und kleine Systeme beschränkt.[59] Der zweite Ansatz setzt dagegen umfangreiche Bestimmungen von Oberton- und Kombinationsbanden voraus, was wegen der geringen Intensitäten gerade für molekulare Cluster sehr herausfordernd ist [61].

Als ein dritter Ansatz wird in dieser Arbeit versucht, durch geeignete Systemwahl bestmöglich Fehlerkompensation bezüglich der Anharmonizität, aber auch anderer molekularer Eigenschaften zu nutzen. Das Phänomenen der Chiralitätserkennung bietet hierfür besonders günstige Voraussetzungen. Denn in den verglichenen homo- und heterochiralen Komplexen ist ein Wechselwirkungspartner in der Regel nur gespiegelt. Geringe Unterschiede in der Stärke von Wasserstoffbrücken, und damit in der OH-Streckschwingung, sind so durch geringfügig unterschiedliche sekundäre Wechselwirkungen bedingt - vorbehaltlich eines unbekannten Einflusses von CISS. Genutzt wird auch der Vergleich mit in verschiedener Hinsicht einfacheren, aber analogen Modellsystemen, wie etwa zwischen Methanol- und Ethanol-Clustern in Kap. 4, oder zwischen dem permanent chiralen 1Phenylethanol und dem transient chiralen Phenylmethanol (Benzylalkohol) in Kap. 8. Mit Serien von chemisch eng verwandten Systemen lassen sich zufällige und systematische Kompensationen unterscheiden. Daher werden in Kap. 6 fünf verschiedene Alkohole mit $\alpha$-Pinen kombiniert. Und in Kap. 5 wird mit Daten von etwa 40 Alkohol-Konformeren ein Modell zur Vorhersage von experimentellen $\mathrm{OH}$-Streckschwingungsübergängen entwickelt - unter Nutzung von systematischer Fehlerkompensation für die Anharmonizität.

Auch auf theoretischer Seite beruht beispielsweise der Status des „Gold-Standards“ von $\operatorname{CCSD}(\mathrm{T}) / \mathrm{CBS}$ auf teilweiser Fehlerkompensation zwischen der störungstheoretischen Behandlung der Triples und der Vernachlässigung höherer Terme [62]. Hierdurch ist $\operatorname{CCSD}(\mathrm{T})$ nicht nur günstiger, sondern in den meisten Fällen sogar genauer als die formal korrektere Variante CCSDT mit iterativer Behandlung der Triples.

Um bei beobachteten Populationsunterschieden mögliche kinetische Einflüsse zu beurteilen, wird in dieser Arbeit ein besonderes Augenmerk auf Umwandlungsbarrieren zwischen Konformeren gelegt. In diesem Kontext wird die Tunneldynamik von Alkoholen relevant. Auch für diesen besonders anharmonischen Effekt lässt sich bei geeigneten Voraussetzungen eine hohe Korrelation zwischen experimentellen Daten und den Ergebnissen von kostengünstigen Rechnungen in harmonischer Näherung herstellen, wie in Kap. 5.9 gezeigt wird. 



\section{Experimentelle und quantenchemische Methoden}

\subsection{Filet-Jet}

Der Filet-Jet ist eine Forschungsapparatur, mit der Moleküle und molekulare Cluster in eine Überschallexpansion mittels FTIR-Spektroskopie charakterisiert werden können. Abb. 3.1 bietet eine Übersicht über den Aufbau.

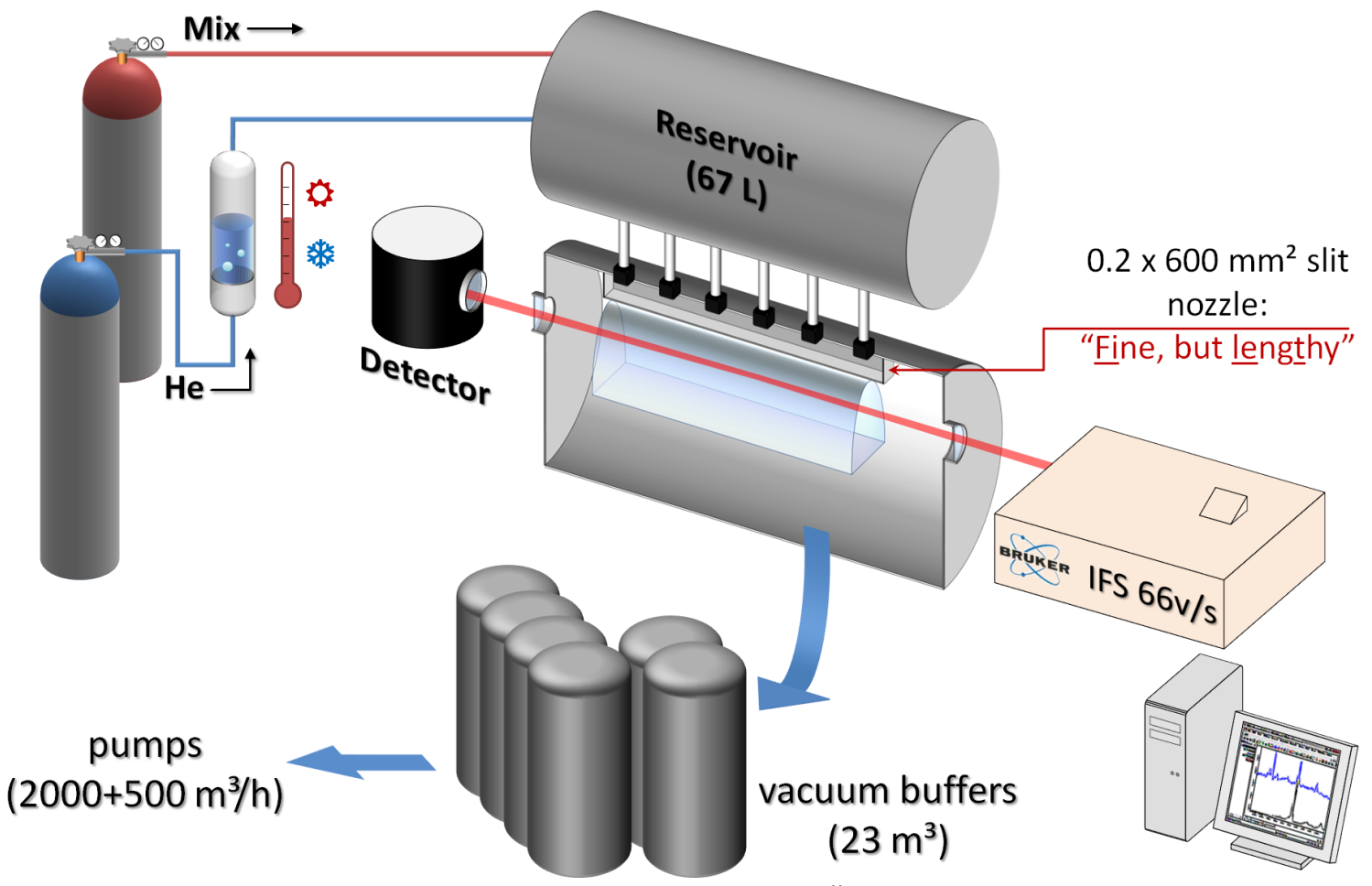

Abb. 3.1: Schematischer Aufbau des Filet-Jets. Übernommen aus Ref. [54].

Die zu untersuchende flüssige oder feste Substanz wird in einem Gasphasen-Sättiger von einem Trägergas (in der Regel 1.6 bar Helium) durchströmt, welches auf diese Weise mit dem Dampf- bzw. Sublimationsdruck der Substanz angereichert wird. Die Konzentration kann über eine Temperierung der Sättiger variiert werden. Dabei ist die Temperatur nach oben durch die Labortemperatur begrenzt, um Kondensation in der übrigen ungeheizten Apparatur zu vermeiden. Mit der Gasmischung aus dem Sättiger wird ein Reservoir mit 67 Liter Volumen bis zu einem gewählten Stagnationsdruck, typischerweise 0.751.25 bar, befüllt. Wahlweise kann über einen weiteren Sättiger oder eine Druckgasflasche eine zweite Substanz zugemischt oder das Gasgemisch weiter verdünnt werden. Das Mischungsverhältnis kann über gepulste Öffnungen von Magnetventilen zwischen Sättigern 
und Reservoir kontrolliert werden. Durch sechs weitere parallel angeordnete Magnetventile gelangt die Gasmischung gepulst in eine Präexpansionskammer, aus der sie über eine $600 \mathrm{~mm}$ lange und $0.2 \mathrm{~mm}$ breite Schlitzdüse in die Jetkammer und die daran anschlieBenden Puffervolumina expandiert. Die besonderen Abmessungen der Schlitzdüse sind namensgebend für das Akronym Filet: Fine but lengthy. Die bis zu $23 \mathrm{~m}^{3}$ großen Puffervolumina begrenzen den Druckanstieg in der Jetkammer während des intensiven Gaspulses auf $<1$ mbar. Das Gas wird über bis zu zwei Pumpstände mit 2000 bzw. $500 \mathrm{~m}^{3} \mathrm{~h}^{-1}$ Pumpleistung in einer typischen Zeitspanne von 30-80 s evakuiert und das Experiment anschließend wiederholt.

Die Expansion wird wenige Zentimeter unterhalb der Schlitzdüse von einem IR-Strahl gekreuzt und FTIR-spektroskopisch untersucht. Als Strahlungsquelle wird eine Wolframlampe mit $150 \mathrm{~W}$ Leistung verwendet. Die Strahlung durchläuft ein Bruker IFS 66v/s Spektrometer mit einem $\mathrm{CaF}_{2}$-Strahlteiler und gelangt über ein $\mathrm{CaF}_{2}$-Fenster und eine $\mathrm{CaF}_{2}$-Sammellinse mit $500 \mathrm{~mm}$ Breitweite in die Jetkammer. Auf der anderen Seite wird die Strahlung über eine $\mathrm{CaF}_{2}$-Linse mit $250 \mathrm{~mm}$ Brennweite wieder parallelisiert (Linsenabstand $776 \mathrm{~mm}$ ) und gelangt über ein $\mathrm{CaF}_{2}$-Fenster in die Detektorkammer. In dieser wird über einen Parabol-Spiegel und durch einen Filter $\left(4100-2500 \mathrm{~cm}^{-1}\right)$ auf einen mit Flüssigstickstoff gekühlten InSb-Detektor fokussiert. Spektrometer, Jetkammer und Detektorkammer werden evakuiert, und die Übergänge zwischen diesen mit von Wasserdampf und Kohlenstoffdioxid befreiter Druckluft gespült. Durch die Verwendung weiterer Strahlungsquellen, Optiken und Detektoren sind Spektralbereiche vom nah- bis zum ferninfraroten zugänglich [55]. In dieser Arbeit wird der oben genannte, vom Filter begrenzte, Spektralbereich im mittleren Infrarot untersucht. Die Messsequenz besteht aus 20 Hintergrundscans und 2 Vorscans vor der Expansion, einem Probescan von 100 ms Dauer während des 150 ms langen Gaspulses und zwei Nachscans. Vor- und Nachscans dienen Kontrollzwecken. Die Synchronisation ist näher in Ref. [37] und [55] beschrieben und wird in Kap. 9 für den Risotto-Jet adaptiert. Verwendet wurde für die hier durchgeführten Messungen am Filet-Jet eine Spiegelgeschwindigkeit von $80 \mathrm{kHz}$, der Aufnahmemodus Single Sided Fast Return, eine Auflösung von $2 \mathrm{~cm}^{-1}$ und eine Phasenauflösung von $16 \mathrm{~cm}^{-1}$. Die Interferogramme werden über eine zwei- bis vierstellige Zahl an Wiederholungen gemittelt, fouriertransformiert (Apodisierungsmethode Norton-Beer Medium, Phasenkorrektur nach Mertz, Zero-Filling Faktor 4) und zu Extinktionsspektren verrechnet.

\subsubsection{Einstellung von Konzentrationen}

Die Clustergrößenverteilung in einer Überschallexpansion (und bei Gemischen auch die Zusammensetzung der Cluster) wird entscheidend von den Konzentrationen der Analyten und des Trägergases im expandierten Gasgemisch beeinflusst. Durch Variation dieser Parameter lassen sich Informationen über die Zuordnung von Banden erhalten, da diese je nach Zusammensetzung und Größe der verursachenden Cluster unterschiedlich mit den Expansionsbedingungen skalieren. Für die Einstellung der Partialdrücke im Reservoir des Filet-Jets stehen die folgenden Stellschrauben zur Verfügung: die Temperatur und der Heliumdruck der beiden Sättiger, die Öffnungs- und Schließzeiten der Magnetventile zwischen den Sättigern und dem Reservoir, sowie der Stagnationsdruck des Reservoirs. 
Durch Veränderung des letztgenannten werden die Konzentrationen aller Substanzen im Reservoir gleichmäßig skaliert. Sowohl die höhere Analytkonzentration, wie auch die tendenzielle stärkere Kühlung durch den höheren Heliumdruck, begünstigen die Bildung größerer Cluster. Deren Signale sind aber häufig eher unerwünscht, während die Signale von Monomeren und Dimeren bei hohen Konzentrationen in eine Sättigung gehen oder sogar abnehmen. Bei schwerflüchtigen Substanzen kann aber bereits die Bildung von Dimeren in ausreichender Menge herausfordernd sein. In diesen Fällen wurde gefunden, dass durch die Verwendung von hohen Stagnationsdrücken ( $>1$ bar) sehr viel schneller ein gutes Signal-zu-Rausch-Verhältnis erreicht werden kann. Dies gilt trotz der geringeren Wiederholrate des Experiments, da sich die Wiederauffüllung des Reservoirs und die Evakuierung der Puffervolumina verlängert.

Durch die Temperaturen der Sättiger können die Dampfdrücke der Substanzen und damit die Konzentrationen im Reservoir gesteuert werden. Zur Abschätzung kann angenommen werden, dass sich im Sättiger ein Phasengleichgewicht einstellt. Der Stoffmengenanteil im Gemisch kann dann aus dem Dampfdruck der Substanz bei der eingestellten Temperatur und dem Druck des Trägergases abgeschätzt werden. Der Dampfdruck ist über Inter- und Extrapolation von Dampfdruckkurven zugänglich, die für viele Substanzen in der Literatur dokumentiert sind, meist in Form der Antoine-Gleichung (Gl. 3.1) mit den Antoine-Parametern $A, B$ und $C$.

$$
p / \mathrm{kPa}=10^{\left(A-\frac{B}{C+T}\right)}
$$

Die Dampfdrücke der Alkohole, die in Kap. 6 mit $\alpha$-Pinen kombiniert werden, variieren über drei Größenordnungen. Die Dampfdruckkurven erlauben es Bedingungen abzuschätzen und einzustellen, bei denen ähnliche Konzentrationsverhältnisse vorliegen. Tab. 3.1 listet die hierfür verwendete Werte sowie die Dampfdrücke bei der am häufigsten verwendeten Sättigertemperatur $24^{\circ} \mathrm{C}$.

Besonders herausfordernd erwiesen sich die Untersuchungen der schwerflüchtigen Monoterpenalkohole Isopinocampheol und Borneol, auch weil sie bei Zimmertemperatur

Tab. 3.1: Antoine-Parameter, Temperaturbereiche der zugrunde liegenden Messwerte und berechnete (meist extrapolierte) Dampfdrücke bei $24{ }^{\circ} \mathrm{C}$ für einige in dieser Arbeit verwendeten Substanzen. Antoine-Parameter für Isopinocampheol wurden gemäß Daten aus Ref. [63] selbstbestimmt. Der Dampfdruck von Borneol wurde abweichend gemäß der Gleichung von Clarke und Glew, wie angegeben in Ref. [64], ermittelt. Mögliche Abweichungen zwischen enantiomerenreinen Substanzen und racemischen Gemischen wurden vernachlässigt.

\begin{tabular}{llcccc}
\hline Substanz & $A$ & $\begin{array}{c}B \\
/ \mathrm{K}\end{array}$ & $\begin{array}{c}C \\
/ \mathrm{K}\end{array}$ & $\begin{array}{c}\theta \text {-Bereich } \\
/{ }^{\circ} \mathrm{C}\end{array}$ & $\begin{array}{c}p\left(24{ }^{\circ} \mathrm{C}\right) \\
/ \mathrm{Pa}\end{array}$ \\
\hline Isopinocampheol [63] & 5.6086 & 1350.539 & -128.635 & $10-40$ & 4 \\
Borneol [64] & - & - & - & $0-35$ & 3 \\
$\alpha$-Fenchol[65] & 5.89569 & 1394.774 & -113.018 & $59-201$ & 21 \\
2-Butanol [65] & 6.59921 & 1314.19 & -86.6 & $22-119$ & 2300 \\
Benzylalkohol [65] & 6.18533 & 1526.52 & -113.214 & $131-234$ & 8 \\
1-Phenylethanol [65] & 7.63184 & 2671.522 & -1.726 & $131-203$ & 40 \\
$\alpha$-Pinen [66] & 5.93206 & 1418.738 & -68.039 & $17-167$ & 550 \\
\hline
\end{tabular}


als Feststoffe vorliegen. Hier konnte der Autor der vorliegenden Arbeit auf Erfahrungen aus seiner Masterarbeit [67] zurückgreifen. Dort wurde festgestellt, dass die erhaltenen Gasphasen-Konzentrationen bei Verwendung von Flüssigkeiten sehr konstant sind, da diese vom durchströmendem Helium aufgewirbelt werden. Dagegen beeinflusst die Oberfläche eines Feststoffes stark die vom Gasstrom aufgenommene Menge, welche sich während der Messung deutlich verringern kann. Neben der sich durch Sublimation allgemein verringernden Substanzmenge ist hierfür die Ausbildung von Kanälen im Feststoff verantwortlich, die die Kontaktfläche mit dem Trägergas weiter verringern. Die ohnehin sehr geringen Sublimationsdrücke von Isopinocampheol und Borneol werden daher nicht erreicht. Mit verschiedenen Gegenmaßnahmen konnte die Konzentration allerdings sowohl in ihrer Höhe, wie auch in ihrer Stabilität, gesteigert werden.

Als erste Möglichkeit erhöht Mörsern der Substanz die Oberfläche und beschleunigt so die Einstellung des Gleichgewichts. Jedoch besteht die Gefahr, dass feines Pulver vom Gasstrom mitgerissen wird und zu Störungen führt (siehe nächster Abschnitt 3.1.2).

Durch eine Verringerung des Heliumdrucks im Sättiger lässt sich prinzipiell der Stoffmengenanteil der Substanz in der Gasphase steigern. Allerdings führt dies zu einer deutlichen Verlangsamung der Befüllung des Reservoirs, insbesondere bei hohen Stagnationsdrücken. Zudem besteht die Gefahr, dass der Druck im Sättiger dabei unter dem Atmosphärendruck fällt, sodass Luft durch Undichtigkeiten eindringen kann. Daher wurde nach einigen Tests der am Filet-Jet etablierte Wert von 1.6 bar beibehalten.

Erfolgreich erwies sich die Nutzung von deutlich kürzeren Öffnungs- und längeren Schließzeiten des gepulst arbeitenden Magnetventils, sodass der Heliumstrom häufiger gestoppt wird, anstatt quasi-kontinuierlich zu fließen. Durch den längeren Kontakt kann sich das Gleichgewicht im Sättiger besser einstellen. Die langsamere Befüllung des Reservoirs muss dabei nicht zwangsläufig zu Einbußen in der Wiederholungsrate des Experiments führen, da gerade bei hohen Stagnationsdrücken (und insbesondere bei Verwendung von nur einem der beiden Pumpstände) der limitierende Faktor häufig die Evakuierung der Puffervolumina ist.

Schließlich ist es vorteilhaft, die Konzentration einer zweiten leichter flüchtigen Substanz, wie $\alpha$-Pinen, im Reservoir weniger durch Kühlung, sondern mehr durch kurze Öffnungs- und lange Schließzeiten des zugehörigen Magnetventils herunterzuregeln. Dies erlaubt es das Reservoir größtenteils mit dem Gasgemisch des ersten Sättigers zu befüllen, sodass die dortige Konzentration der schwerflüchtigen Verbindung möglichst wenig im Reservoir verdünnt wird.

Mit Gl. 3.2 lässt sich das Stoffmengenverhältnis $x_{\mathrm{A}} / x_{\mathrm{B}}$ zweier Substanzen A und B aus den Dampfdrücken $p$ und den Öffnungs- $t_{\mathrm{o}}$ und Schließzeiten $t_{\mathrm{S}}$ der Magnetventile abschätzen. Die Brüche auf der rechten Seite der Gleichung repräsentieren dabei den Zeitanteil, den die beiden Magnetventile jeweils während eines Zykluses geöffnet sind.

$$
\frac{x_{\mathrm{A}}}{x_{\mathrm{B}}}=\frac{p_{\mathrm{A}}}{p_{\mathrm{B}}} \cdot \frac{t_{\mathrm{o}, \mathrm{A}}}{\left(t_{\mathrm{o}, \mathrm{A}}+t_{\mathrm{s}, \mathrm{A}}\right)} \cdot \frac{\left(t_{\mathrm{o}, \mathrm{B}}+t_{\mathrm{s}, \mathrm{B}}\right)}{t_{\mathrm{o}, \mathrm{B}}}
$$

Diese Gleichung enthält als Annahmen die diskutierte Gleichgewichtseinstellung, identische Strömungsgeschwindigkeiten durch beide Magnetventile und schnelle Öffnungsund Schließvorgänge der Magnetventile. 
Der höchste Anteil an gemischten Dimeren ist bei einer stöchiometrischen 1:1Mischung beider Komponenten zu erwarten, sofern diese ähnlich flüchtig sind und damit auch eine ähnliche Aggregationstendenz haben. Jedoch lässt sich die absolute Konzentration des gemischten Dimers in der Expansion noch deutlich durch die Verwendung eines moderaten Überschusses der leichter flüchtigen Komponente steigern. Hierdurch wird auch die Bildung größerer Cluster begünstigt. Diese sind aber tolerabel, sofern deren Banden als solche identifiziert werden und nicht spektral mit den Dimerbanden überlappen. Bei großem Überschuss einer Komponente sinkt die Bandenintensität gemischter Dimere dagegen wieder.

\subsubsection{Nachbearbeitung von Filet-Jet-Spektren}

Die Beobachtung sehr schwacher Signale erhöht die Bedeutung von Störquellen, welche aber durch Nachbearbeitung von Spektren verringert werden können. Drei dieser Störquellen werden im Folgenden betrachtet und Gegenmaßnahmen erläutert.

Die erste Störquelle ist elektronisch begründet und äußert sich darin, dass in einigen Wiederholungen des Experiments die Magnetventile nicht öffnen. Scans werden aber dennoch vom Spektrometer aufgenommen, die in die Mittelung einfließen und das Signal-zuRausch-Verhältnis senken. Der Anteil dieser Leerspektren betrug zeitweise bis zu 30\%, konnte aber durch Austausch von Kabeln und Herabsetzen von elektronischen Schwellenwerten in den unteren einstelligen Prozentbereich gesenkt werden. Diese Leerspektren wurden aussortiert, hierfür wurden alle Interferogramme einzeln (statt gemittelt) fouriertransformiert und zu Extinktionsspektren für jede einzelne Wiederholung verrechnet. Durch die Abwesenheit der intensiven $\mathrm{CH}-$ Streckschwingungsbanden der hier untersuchten Verbindungen sind auch Einzelspektren eindeutig als Leerspektren identifizierbar. Sowohl die drei- bis vierstellige Zahl an Fouriertransformationen für jede Messreihe, wie auch das manuelle Durchsehen aller Einzelspektren ist sehr zeitaufwändig, daher sind Leerspektren tolerabel, solange deren Anteil gering bleibt.

Allerdings wirkte sich eine zweite Störquelle ebenfalls auf einzelne Wiederholungen aus, sodass eine Kontrolle der Einzelspektren ohnehin notwendig wurde. Vom Heliumstrom können kleine Partikel von Feststoffen mitgerissen und vom Sättiger in das Reservoir, und schließlich während der Expansion in den Detektionsbereich gelangen. Dort können sie teils so drastische Streueffekte hervorrufen, dass ein einzelner betroffener Scan eine ganze Messreihe unbrauchbar machen kann. Dies ist in Abb. 3.2 an zwei Einzelspektren der selben Messreihe beispielhaft gezeigt. Während in der Expansion mit nur gasförmigem Borneol die CH-Schwingungsbande im Bereich von $3000-2850 \mathrm{~cm}^{-1}$ nur sehr schwach zu erkennen ist, ist diese im Spektrum einer Expansion mit Borneol-Partikeln etwa 25 fach verstärkt. Zusätzlich tritt dort eine breite $\mathrm{OH}-\mathrm{Streckschwingungsbande} \mathrm{zwi-}$ schen 3400-3100 $\mathrm{cm}^{-1}$ auf, wie sie auch für hochkonzentrierte Expansionen am PopcornJet beobachtet wird (Abb. 5.11 auf S. 125), allerdings anders als dort ohne Hinweise auf kleinere Cluster. Das Spektrum ist von weiteren starken Streuartefakten geprägt, wie einer erhöhten Basislinie. Vergleichbare Effekte werden für fluktuierende Flüssigkeitsexpansionen am RESS-Jet in Kap. 9 näher diskutiert. Dieses Problem trat sehr stark mit Borneol auf, welches auch ohne Mörsern als sehr feines Pulver vorlag, sowie im geringeren Maße auch mit Isopinocampheol. Mit $\alpha$-Fenchol wurden hingegen keine solche Schwierigkei- 


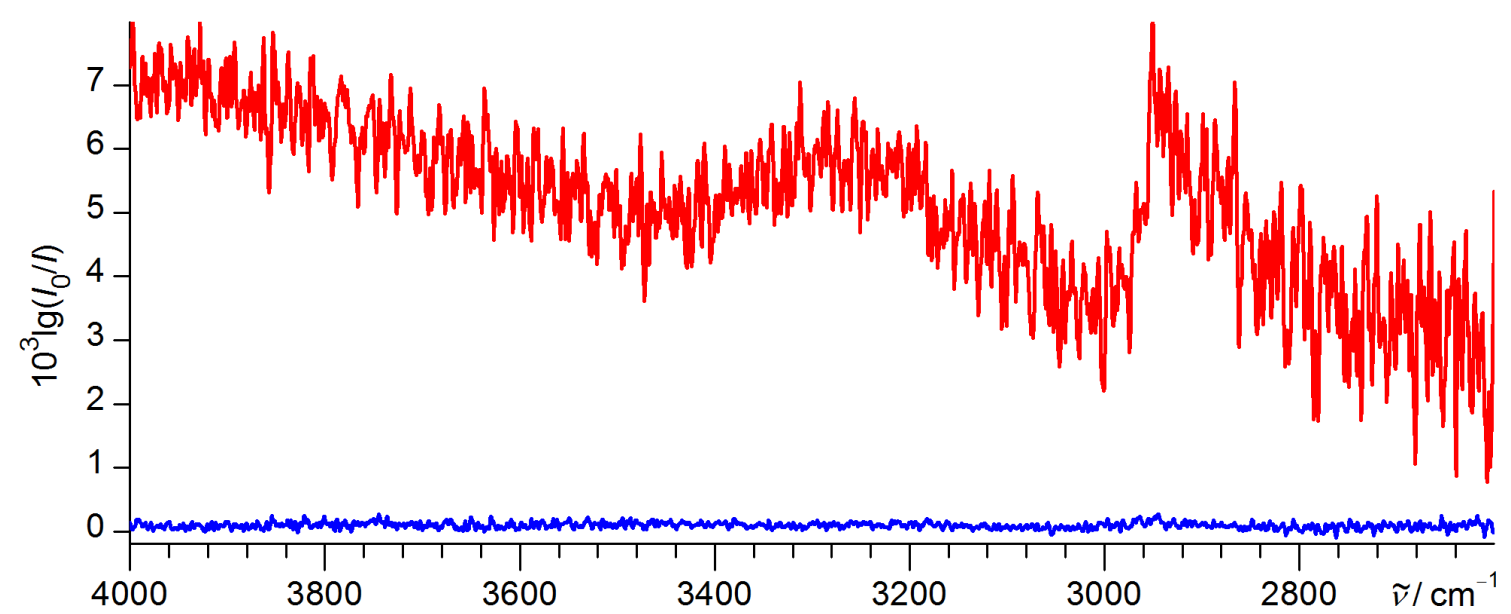

Abb. 3.2: Filet-Jet-Einzelspektren einer Expansion von Borneol mit (rot) und ohne (blau) Feststoff-Partikeln. Die vertikalen Positionen der Spektren sind wie gemessen.

ten festgestellt, vermutlich wegen der weichen und leicht klebrigen Konsistenz dieser Substanz.

Eine dritte Störungsquelle für Messungen im $\mathrm{OH}-$ Streckschwingungsbereich ist die Präsenz von Rotationsschwingungsübergängen von atmosphärischem Wasser. Trotz Evakuierung bzw. Trockenluft-Spülung des Strahlenganges führen Konzentrationsdrifts und/oder -schwankungen zu einer unvollständige Kompensation zwischen Hintergrund- und Probescans. Je nach Richtung der Änderung treten daher positive oder negative Signale auf, die andere Banden überlagern können. Prinzipiell kann dieses Problem ebenfalls durch Aussortieren der am stärksten in diese Richtung betroffenen Einzelspektren behoben werden, sodass sich im Mittel wieder eine Kompensation ergibt. Gegen dieses Verfahren spricht der hohe Aufwand, sowie der Verlust von Einzelspektren, die in von Wasserübergängen nicht betroffenen Bereichen positiv zum Signal-zu-Rausch-Verhältnis beitragen. Daher wurden stattdessen von den Spektren nachträglich ein passend skaliertes Spektrum der Rotationsschwingungsübergänge von Wasser subtrahiert. Dieses Spektrum wurde gewonnen, indem eine Minute lang Hintergrundscans und danach eine Minute lang Probescans gemessen werden. Dies entspricht der täglichen Routine für die Bewertung der spektroskopischen Bedingungen (Noise Test Challenge, NOTCH [54]). Durch den mittleren Abstand von einer Minute zwischen Hintergrund und Probe (statt wenigen Sekunden im Messbetrieb) tritt hier eine stärkere Konzentrationsdrift auf. Für alle Spektren wurde das selbe NOTCH-Spektrum verwendet, welches wegen seiner sehr stabilen Basislinie und der Abwesenheit von Signalen weiterer IR-aktiver Spurengase, wie Kohlenstoffdioxid und Methan, ausgewählt wurde. Durch die lange Aufnahmezeit ist auch das Rauschniveau des NOTCH-Spektrums sehr gering und beeinflusst daher auBerhalb des Bereichs der Wasserübergänge das korrigierte Jet-Spektrum praktisch nicht. Eine solche Korrektur ist exemplarisch in Abb. 3.3 gezeigt. Erst nach Korrektur wird in diesem Spektrum das schwache Signal der freien $\mathrm{OH}-$ Streckschwingung im stabilsten Konformer von Isopinocampheol bei $3648 \mathrm{~cm}^{-1}$ erkennbar. Dass dieses Signal real und kein Artefakt der Korrektur ist, zeigt der Vergleich mit weniger betroffenen Jet-Spektren, dem Gasphasen-Spektrum bei Zimmertemperatur und quantenchemischen Rechnungen in Kap. 5.6. Durch die stabile Basislinie und das niedrige Rauschniveau des NOTCHSpektrums werden Spektralbereiche ohne Wasserübergänge durch die Korrektur praktisch 


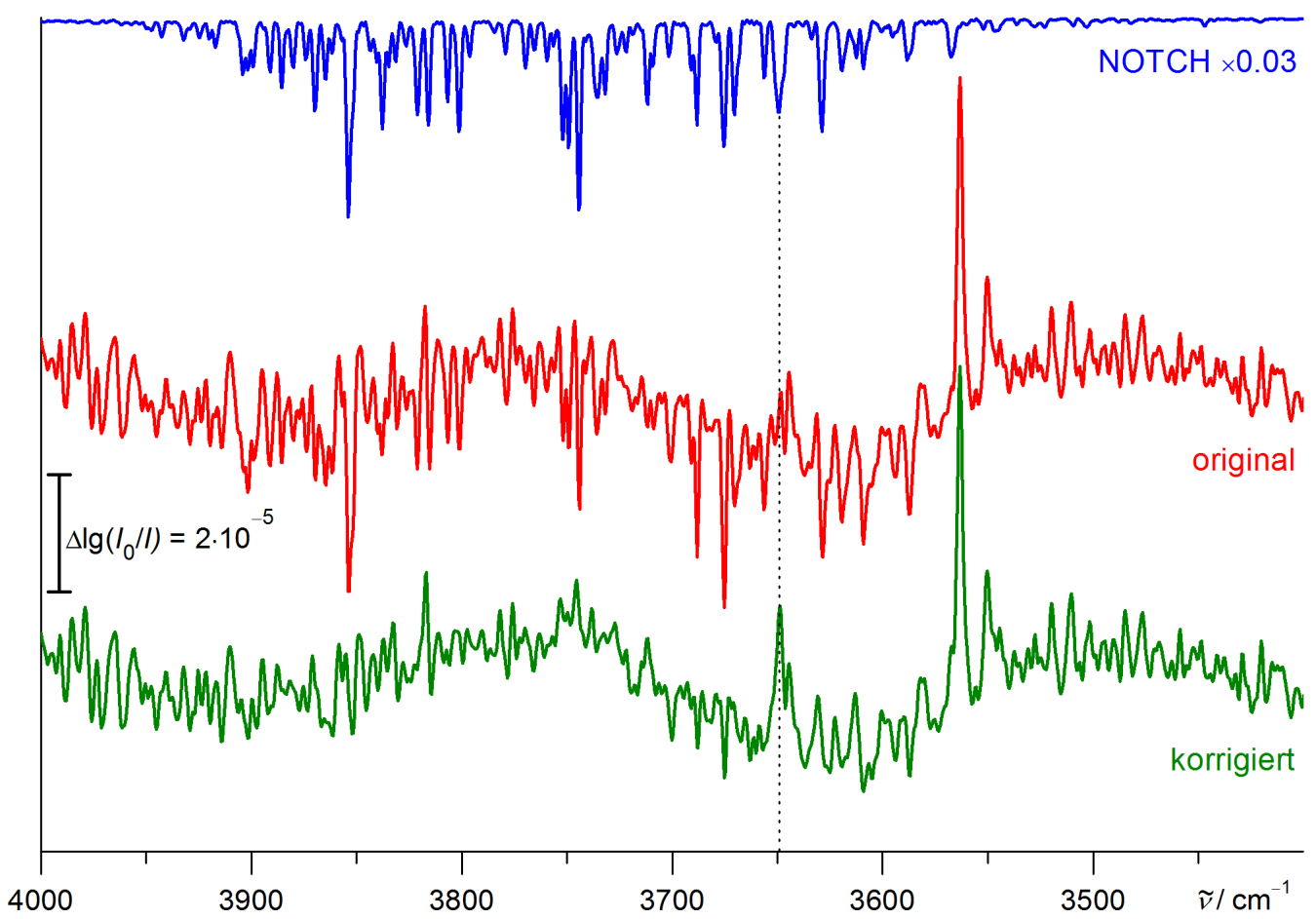

Abb. 3.3: Exemplarische Korrektur eines Filet-Jet-Spektrums (Mischung von (-)Isopinocampheol mit (+)- $\alpha$-Pinen) durch Subtraktion eines skalierten NOTCH-Spektrums. Markiert ist der $\mathrm{OH}-$ Streckschwingungsfundamentalübergang des stabilsten Konformers von Isopinocampheols bei $3648 \mathrm{~cm}^{-1}$, welcher erst nach der Korrektur erkennbar wird.

nicht beeinflusst. Diese Korrektur ist nicht perfekt, da die Formen und relative Intensitäten der Rotationsschwingungsübergänge auch von Temperatur- und Druckänderungen im Strahlengang beeinflusst werden, die aber gering ausfallen. Ungefähr die Hälfte aller in dieser Arbeit gemessenen Filet-Jet-Spektren wurde auf diese Weise korrigiert. Die hierfür verwendeten Skalierungsfaktoren des NOTCH-Spektrums sind im Spektrenverzeichnis (S. 329 ff.) dokumentiert.

\subsection{Ragout-, Popcorn- und Falafel-Jet}

In dieser Arbeit werden verschiedene ältere Messungen am Ragout-, Popcorn- und Falafel-Jet verwendet und teilweise neu ausgewertet. Diese Apparaturen funktionieren nach sehr ähnlichen Grundprinzipien wie der zuvor beschriebene Filet-Jet, sodass hier nur die wichtigsten Unterschiede genannt und auf weiterführende Literatur verwiesen wird.

Der Ragout-Jet (Rapid aquisition giant outlet) kann als direkter Vorläufer des Filet-Jets angesehen werden, durch den er schließlich ersetzt wurde. Neben verschiedenen Detailverbesserungen ist der Hauptfortschritt der Einsatz einer schmaleren, aber wesentlich längeren Schlitzdüse am Filet-Jet, die vor allem den optischen Absorptionsweg vergrößert, aber auch Einfluss auf die Clusterbildung hat.[37] Eine ausführliche Beschreibung des Ragout-Jets findet sich in Ref. [68] und ein Vergleich mit dem Filet-Jet in Ref. [37].

Kernelement des Popcorn-Jets (Poppet controlled resistively heated nozzle) ist ein geheizter Aufbau, welcher höhere Konzentrationen für schwerflüchtige Substanzen ermöglicht. Hierfür wird die Substanz auf Molsiebkügelchen aufgetragen und in einer geheizten 
Probenkammer platziert. Die Probenkammer ist von Überstromventilen begrenzt, sodass sich bei erhöhter Temperatur der Dampfdruck einstellen kann, der schließlich vom Trägergas aufgenommen wird. Dem Trägergas kann durch einen vorgeschalteten Sättiger, analog zum Filet-Jet, auch eine leicht flüchtige Substanz beigemischt werden. Für die Untersuchung von leicht flüchtigen Substanzen ist der Popcorn-Jet aber dem Filet-Jet unterlegen, vor allem aufgrund des deutlich kürzeren Absorptionsweges. Eingesetzt wurde bis vor kurzem eine Doppelschlitzdüse, die jüngst durch eine etwas längere gewinkelte Schlitzdüse ersetzt wurde, inspiriert vom verwendeten Design des im Rahmen dieser Arbeit umgebauten Risotto-Jets (Kap. 9). Mehr Details zum aktuellen Aufbau des PopcornJets finden sich in Ref. [69] und für teils ältere Versionen der Apparatur in den genannten jeweiligen Quellen der Spektren.

Der Falafel-Jet (Fast laser-heated individually fed lump) nutzte weitgehend die Infrastruktur (Spektrometer, Gaszufuhr, Vakuumsystem) des Popcorn-Jets, allerdings wurde dort eine schwerflüchtige Substanz nicht thermisch, sondern durch schnelle Laserverdampfung in den Heliumstrom eingebracht. Der Jet ist in Ref. [70] beschrieben. Da durch diese Verdampfungsform keine ausreichenden Konzentrationen erreicht werden konnten, wurde dieses Prinzip wieder aufgegeben.

\subsection{RESS - und Risotto-Jet}

Da der RESS-Jet und der aus ihm entstandende Risotto-Jet während dieser Arbeit in fortwährender Weiterentwicklung waren, werden diese Apparaturen im Kontext der erzielten Ergebnisse in ihrem eigenen Kapitel 9 (ab S. 265) vorgestellt.

\subsection{Curry-Jet}

Das Akronym Curry steht für „Classical Unrestricted Raman Spectroscopy“. Der Aufbau der Apparatur, die Raman-Spektroskopie mit einer Überschallexpansion koppelt, ist in Abb. 3.4 schematisch dargestellt. Analog zum Filet-Jet kann Trägergas mit dem Dampfdruck von leichtflüchtigen Substanzen über zwei kühlbare Glassättiger angereichert und in einem Reservoir (4.7 Liter) gemischt werden. Im Gegensatz zum Filet-Jet erfolgt die Expansion kontinuierlich, statt gepulst, durch eine heizbare Schlitzdüse mit einer deutlich kleineren Querschnittsfläche von $4 \mathrm{~mm} \times 0.15 \mathrm{~mm}$. Weitere Düsenformen stehen modular zur Verfügung. Die Jetkammer kommt ohne zusätzliche große Puffervolumina aus und wird von zwei Wälzkolbenpumpen (500 und $250 \mathrm{~m}^{3} \mathrm{~h}^{-1}$ ) und einer Drehschieberpumpe $\left(100 \mathrm{~m}^{3} \mathrm{~h}^{-1}\right)$ im Messbetrieb auf einem Hintergrunddruck von etwa 1 mbar gehalten. Alternativ kann Trägergas auch direkt in das Reservoir und durch einen anschließenden geheizten Metallsättiger geleitet werden, wodurch die Untersuchung von schwerflüchtigen Substanzen ermöglicht wird. Die Expansion wird von einem Strahl eines Lasers gekreuzt (Spectra Physics Millenia eV, 532 nm, 25 W, kontinuierliche Arbeitsweise). Durch den im Vergleich zu FTIR-Optiken deutlich kleineren Strahldurchmesser lassen sich gezielt bestimmte Regionen der Expansion untersuchen. Typische Abstände zwischen Düse und Laser betragen 0.5-3.0 mm. Die durch Wechselwirkung mit Molekülen und Clustern entstehende Raman-Streuung wird senkrecht zu Laser und Expan- 


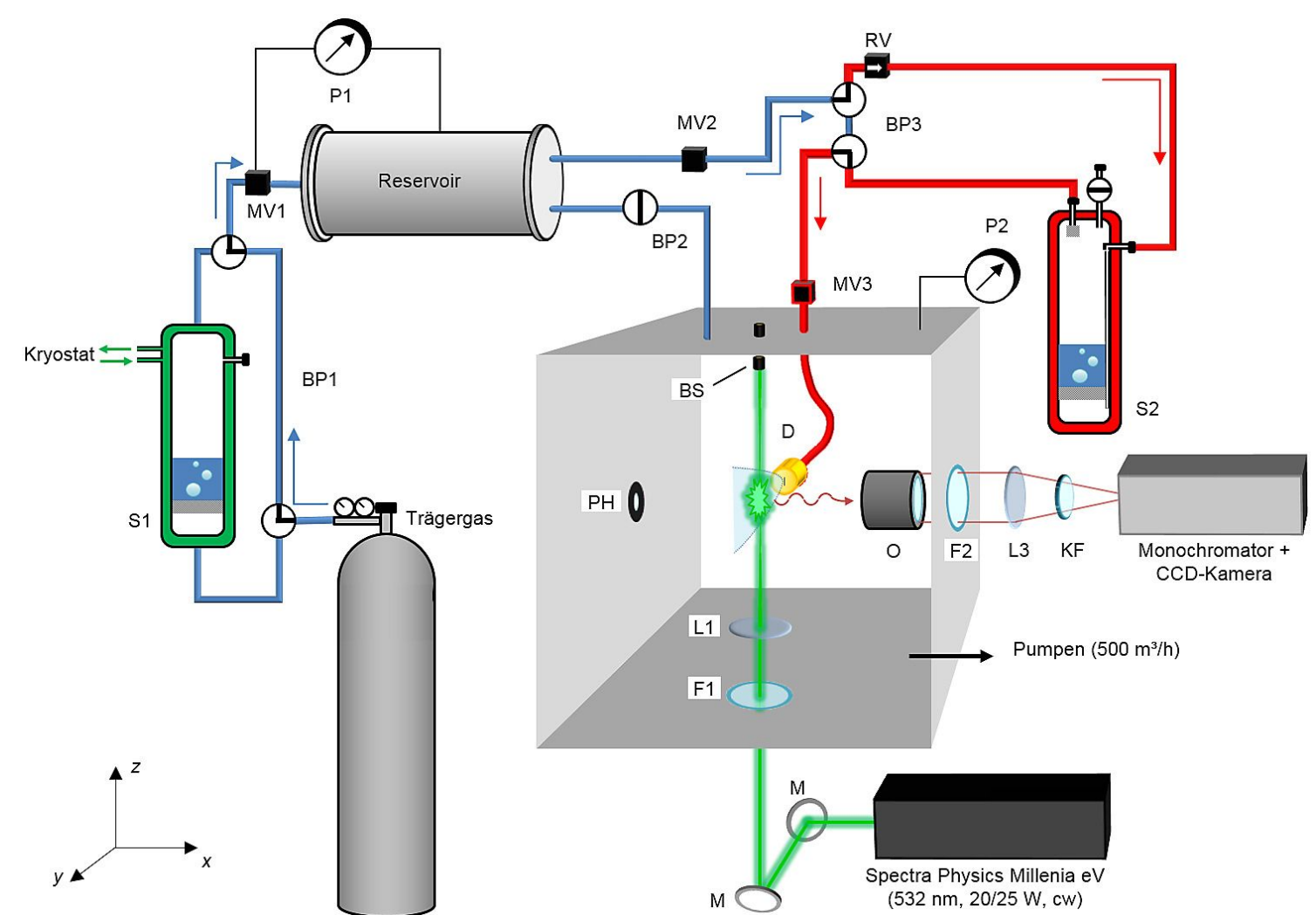

Abb. 3.4: Schematischer Aufbau des Curry-Jets. Übernommen aus Ref. [71], lizenziert nach CC BY-NC-ND 4.0.

sion durch ein Kamera-Objektiv und eine Linse auf einen Monochromator (McPherson Inc. Model 2051, 1200 Linien/mm Gitter, Auflösung ca. $1-2 \mathrm{~cm}^{-1}$ ) fokussiert. Über einen Kantenfilter wird Rayleigh-Streuung geblockt. Die Raman-Streuung wird schließlich über eine mit Flüssigstickstoff gekühlte CCD-Camera (Princeton Instruments PyLoN400B, $1340 \times 400$ Pixel von $20 \mu \mathrm{m} \times 20 \mu \mathrm{m})$ detektiert. Der gleichzeitig untersuchbare Spektralbereich beträgt etwa $\pm 10 \mathrm{~nm}$ um eine Zentralwellenlänge, dies entspricht einem Fenster von etwa $450 \mathrm{~cm}^{-1}$ im OH-Streckschwingungsbereich. Die Kamera wird wiederholt über einige Minuten belichtet. Die einzelnen Messblöcke werden über ein Skript verglichen, um Pixel mit Spikes von kosmischer Strahlung zu identifizieren und deren Wert durch eine Mittelung der nicht betroffenen Blöcke zu ersetzen. Abschließend werden die Spektren aller Messblöcke einer Messreihe gemittelt. Das täglich nachjustierte Setup wird über Vakuum-Emissionslinien einer Neon-Gasentladungsröhre kalibriert, sodass den Pixeln Wellenzahlen zugeordnet werden.

Eine tiefer ins Detail gehende Beschreibung des Curry-Jets findet sich in der Dissertation von S. Bocklitz [72], auch mit Verweisen auf die historische Entwicklung des Setups. 


\subsection{Verwendete Substanzen}

Tab. 3.2 listet die in dieser Arbeit verwendeten Substanzen. Für einige enantiomerenangereicherte Substanzen wurde von den Herstellern kein Enantiomerenüberschuss, sondern nur ein spezifischer Drehwinkel angegeben. Durch Vergleich mit Referenzwerten in der Literatur (Tab. 3.3 und 3.4) wurden die in Tab. 3.2 mit einem Stern ${ }^{*}$ gekennzeichneten Werte für den Enantiomerenüberschuss ee über Gl. 3.3 abgeschätzt.

$$
e e=\left|\frac{[\alpha]_{D}^{20}(\text { Probe })}{[\alpha]_{D}^{20}(\text { Referenz })}\right|
$$

Für die Probe von $(+)-\alpha$-Pinen des Herstellers TCI wurde der gleiche Betrag des spezifischen Drehwinkels $\left(45^{\circ} \mathrm{mlg}^{-1} \mathrm{dm}^{-1}\right.$ ) wie für (-)- $\alpha$-Pinen angegeben, sodass der $e e$-Wert (87\%) übernommen wurde.

Literaturwerte für den spezifischen Drehwinkel von $\alpha$-Fenchol weisen eine nicht unerhebliche Spannweite von -10.35 bis $-13.37^{\circ} \mathrm{mlg}^{-1} \mathrm{dm}^{-1}$ auf (Tab. 3.4). Da diese Werte bereits um die vorletzte Jahrhundertwende ermittelt wurden, ist unklar, inwieweit diese jeweils tatsächlich einer weitgehend diastereo- und enantiomerenreinen Referenz entsprechen. Auf Grundlage dieser Literatur-Referenzen berechnete $e e$-Werte variieren zwischen 77 und 99\% für die verwendete Probe. Sigma Aldrich vertreibt einen Analysestandard, in dessen Spezifikation die Diastereomerenreinheit mit $\geq 99.0 \%$ und der spezifische Dreh-

Tab. 3.2: Verwendete Chemikalien mit CAS-Nr., Diastereomerenreinheit, Enantiomerenüberschuss und Hersteller. Angeben sind Werte aus der Spezifikation des Herstellers und aus dem Analysezertifikat der Charge (letzteres in Klammern). Die mit einem Stern* gekennzeichneten eeWerte wurden über Drehwerte abgeschätzt (s. Text). Zusätzlich wurde racemisches $\alpha$-Pinen durch Mischung der Enantiomere hergestellt.

\begin{tabular}{|c|c|c|c|c|}
\hline Substanz & CAS-Nr. & Reinheit / \% & $e e / \%$ & Hersteller \\
\hline$(+)$ - $\alpha$-Pinen & $7785-70-8$ & $\geq 97.0(98.6)$ & $\geq 81^{*}\left(87^{*}\right)$ & $\mathrm{TCI}$ \\
\hline$(+)-\alpha$-Pinen & $7785-70-8$ & $\geq 97.5(98.5)$ & $\geq 91.0(98.7)$ & Sigma Aldrich \\
\hline$(-)-\alpha$-Pinen & $7785-26-4$ & $\geq 98$ (98.75) & $\geq 87(87)$ & J\& K Scientific \\
\hline$(-)-\alpha$-Pinen & $7785-26-4$ & $\geq 98(98.07)$ & $\geq 87$ (87) & J\& K Scientific \\
\hline (-)-Isopinocampheol & $25465-65-0$ & $\geq 97.5(99.5)$ & $\geq 94.0(99.9)$ & Sigma Aldrich \\
\hline (-)-Borneol & $464-45-9$ & $\geq 96$ (98.9) & $\geq 93.8^{*}\left(96.1^{*}\right)$ & Acros Organics \\
\hline (+)- $\alpha$-Fenchol & $2217-02-9$ & $\geq 95.5(98.5)$ & $\geq(60-77) *(77-99) *$ & Sigma Aldrich \\
\hline (-)-1-Phenylethanol & $1445-91-6$ & $\geq 98.5$ (99.6) & $\geq 94(99.6)$ & Sigma Aldrich \\
\hline$( \pm)$-1-Phenylethanol & $98-85-1$ & $98(98.5)$ & - & Fluka \\
\hline$( \pm)$-1-Phenylethanol & $98-85-1$ & 98 (99.5) & - & Aldrich \\
\hline$( \pm)$-2-Butanol & $78-92-2$ & $\geq 99.45(99.75)$ & - & Sigma Aldrich \\
\hline (+)-2-Butanol & $4221-99-2$ & $98(99.4)$ & k. A. $\left(91.3^{*}\right)$ & abcr GmbH \\
\hline ( \pm )-Pinakolylalkohol & $464-07-3$ & $98(98.8)$ & - & Aldrich \\
\hline Benzylalkohol & $100-51-6$ & 99 & - & Alfa Aesar \\
\hline Helium & $7440-59-7$ & 99.996 & - & Linde AG \\
\hline$n$-Pentan & $109-66-0$ & $>99$ & - & Sigma Aldrich \\
\hline$n$-Pentan & $109-66-0$ & $>99$ & - & Acros \\
\hline$n$-Dodecanol & $112-53-8$ & $\geq 98$ & - & Sigma Aldrich \\
\hline Methanol & $67-56-1$ & $\geq 99.8$ & - & Sigma Aldrich \\
\hline Ethylenglycol & $107-21-1$ & 99 & - & abcr GmbH \\
\hline
\end{tabular}


Tab. 3.3: Literaturwerte für die spezifischen Drehwinkel von (-)-Borneol (in Ethanol) und (+)-2Butanol.

\begin{tabular}{lcc}
\hline Substanz & {$[\alpha]_{D}^{20}($ Referenz $) / \frac{\mathrm{ml}}{\mathrm{gdm}}$} & Quelle \\
\hline (-)-Borneol & $-38^{\circ}$ & {$[73]$} \\
(+)-2-Butanol & $+15^{\circ}$ & {$[74]$} \\
\hline
\end{tabular}

Tab. 3.4: Literaturwerte für den spezifischen Drehwinkel von (-)- $\alpha$-Fenchol (in Ethanol).

\begin{tabular}{crc}
\hline$[\alpha]_{\mathrm{D}} / \frac{\mathrm{ml}}{\mathrm{gdm}}$ & $\theta /{ }^{\circ} \mathrm{C}$ & Quelle \\
\hline$-10.35^{\circ}$ & 19 & {$[75]$} \\
$-10.9^{\circ}$ & 15 & {$[76]$} \\
$-13.37^{\circ}$ & k. A. & {$[77]$} \\
$-12.7^{\circ}$ & k. A. & {$[78]$} \\
\hline
\end{tabular}

winkel mit $11 \pm 0.5^{\circ}$ angegeben ist. Mit dieser aktuellen Referenz wird ein $e e$-Wert von $94 \pm 4 \%$ für die Probe erhalten. In dieser Arbeit vorgestellte Ergebnisse aus der Chiralitätserkennung mit $\alpha$-Pinen bestätigen, dass die Enantiomerenreinheit der verwendeten $\alpha$-Fenchol Probe hoch ist (Diskussion in Kap. 7.3).

Die beiden eingesetzten Proben von ( \pm )-1-Phenylethanol wurden zusätzlich an einem Krüss PT-8000T Polarimeter auf ihren racemischen Charakter überprüft. Die erhaltenen spezifische Drehwerte von jeweils $[\alpha]_{\mathrm{D}}^{22}=-0.3^{\circ} \frac{\mathrm{ml}}{\mathrm{dmg}}$ ergeben im Vergleich zu der Angabe für die verwendete enantiomerenreine Probe von $[\alpha]_{\mathrm{D}}^{20}=-45.7^{\circ} \frac{\mathrm{ml}}{\mathrm{dmg}}$ einen vernachlässigbaren $e e$-Wert von $0.7 \%$.

\subsection{Quantenchemische Rechnungen}

Alle in dieser Arbeit durchgeführten quantenchemischen Berechnungen wurden mit dem Programmpaket Gaussian 09 Rev. E.01 [79] durchgeführt. ${ }^{a}$ Hauptsächlich genutzt wurde Dichtefunktionaltheorie (DFT) mit den Funktionalen B3LYP [80-83], $\omega$ B97X [84], M06-2X [85], B2PLYP [86] und APF [87]. Diese wurden, mit Ausnahme von M062X und in einigen Fällen B3LYP, um teils verschiedene, semi-empirische Zwei-KörperDispersionskorrekturen ergänzt (für einen Überblick hierzu sei Ref. [88] empfohlen). Diese Kombinationen sind B3LYP-D3(BJ), $\omega$ B97X-D, B2PLYP-D3(BJ) und APF-D. M06$2 \mathrm{X}$ berücksichtigt stattdessen nicht-kovalente Wechselwirkungen mit hoher Gewichtung von hierfür relevanten Referenzsystemen in der Parametrisierung. Alle DFT-Methoden wurden mit einem ultrafeinen Integrationsgitter (int=ultrafine) verwendet, sofern nicht explizit anders angegeben. Näherungen für die Berechnung von Integralen gemäß

\footnotetext{
${ }^{a}$ Wegen technischen Limitierungen mussten davon abweichend zwei einzelne Rechnungen mit Gaussian 16 Rev. A.03 durchgeführt werden. Der Erhalt von identischen Ergebnissen mit beiden Programmversionen wurde für die hierbei verwendete Methode B2PLYP-D3(BJ) an einer weiteren Struktur verifiziert.
} 
der Density Fitting Methode (auch bekannt als Resolution of Identity) oder spezielle Berücksichtigungen von Spin-Bahn-Kopplung (wie beispielsweise in Ref. [3] für den CISSEffekt) wurden nicht angewendet. Eingesetzte wellenfunktionsbasierte ab initio Methoden sind Møller-Plesset-Störungstheorie 2. Ordnung (MP2) und Coupled Cluster with Singles, Doubles and perturbative Triples [CCSD(T)]. Ebenfalls zu Vergleichszwecken verwendet wurde die semi-empirische Methode Austin Model 1 (AM1) [89].

An Basissätzen wurden u. a. die Varianten der Karlsruhe-Familie def2-TZVP und def2QZVP [90] genutzt. Augmentierte Versionen dieser Basissatz-Familie mit zusätzlichen diffusen Funktionen wurden veröffentlicht [91], sind aber nicht in Gaussian 09 implementiert. Eine Triple- $\zeta$-Basis mit Augmentierung ist in der Form von aug-cc-pVTZ als Teil der korrelationskonsistenten Dunning-Familie verfügbar, ist aber sehr rechenaufwändig. Als Kompromiss wurden zusätzlich die Kalender-Basissätze von Truhlar [92] erprobt, in denen die Zahl der diffusen Funktionen schrittweise verringert wird. Das Kürzel der vollaugmentierten Variante ,aug“ wurde von Truhlar zu „August“ umgedeutet. Ein Zurückschreiten im Kalendermonat entspricht einer Verringerung der Augmentierung. Minimalaugmentiert sind „may“ für cc-pVTZ und „,apr“ für cc-pVQZ. Die Implementierung in Gaussian 09 weicht allerdings insofern von den publizierten Versionen ab, dass stets diffuse Funktionen vom s- und p-Typ erhalten bleiben.

Für Punktrechnungen mit $\operatorname{CCSD}(\mathrm{T})$ wurde eine Basissatz-Extrapolation gemäß der Methode aus Ref. [93] durchgeführt (Gl. 3.4). Der auf diese Weise extrapolierte Basissatz ist mit einem Sternchen * gekennzeichnet.

$$
\begin{aligned}
& E(\mathrm{CCSD}(\mathrm{T}) / \text { aug-cc-pVQZ } *)=E(\mathrm{CCSD}(\mathrm{T}) / \text { may-cc-pVTZ }) \\
& +E(\mathrm{MP} 2 / \text { aug-cc-pVQZ) }-E(\mathrm{MP} 2 / \text { may-cc-pVTZ })
\end{aligned}
$$

Energetische Minima für Moleküle wurden mit sehr engen Konvergenz-Kriterien (fopt=verytight) und für molekulare Cluster mit engen Konvergenz-Kriterien (fopt=tight) gemäß dem Berny-Algorithmus optimiert. Relevante intra- und intermolekulare Freiheitsgrade wurden in der Struktursuche auf systematische Weise durch manuelle Erstellung von Startstrukturen entlang dieser Koordinaten berücksichtigt. Dies ist am Beispiel der Alkohol/ $\alpha$-Pinen Komplexe in Kap. 6.4 näher erläutert. Zu allen Minima wurden Frequenzrechnungen in doppelt-harmonischer Näherung (freq) durchgeführt, teilweise unter zusätzlicher Berechnung von Raman-Aktivitäten und Depolarisationsverhältnissen (freq=raman). Minima wurden durch die Abwesenheit von imaginären Frequenzen bestätigt und Unterschiede in der Schwingungsnullpunktsenergie in der relativen Energie zwischen Strukturen berücksichtigt.

Das Auffinden von Übergangszuständen ist besonders für flexible molekulare Cluster anspruchsvoll. Am zuverlässigsten erwies sich in dieser Arbeit die Nutzung folgender Parameter: opt $=$ ( $t s, c a l c f c$, noeigen, tight). ts bedeutet hier, dass ein Übergangszustand (transition state) optimiert werden soll, calcfc dass initial die Kraftkonstanten voll berechnet (statt nur abgeschätzt) werden und noeigen dass Tests für die Zahl der Eigenwerte als häufige Ursache für Abbrüche unterdrückt werden. Entscheidend ist allerdings eine Startstruktur, die dem gesuchten Übergangszustand bereits gut entspricht. Entsprechende Strukturen wurden als energetische Maxima von relaxierten Scans (opt=modredundant) einer Koordinate erhalten, welche dem Reaktionspfad möglichst gut entspricht (meist ein Torsionswinkel). Übergangszustände wurden durch das Vorlie- 
gen von genau einer imaginären Koordinate als solche bestätigt. Über Visualisierung der Koordinate mit GaussView 5.0.9 wurde verifiziert, dass diese die angegebenen Minima verbindet.

\subsection{Modellierung von theoretischen Spektren}

Bei Vorhandensein mehrerer Banden in den experimentellen Spektren, die auf Konformationsvielfalt hinweisen, werden diese mit modellierten theoretischen Spektren verglichen. Die explizite Simulation erlaubt es das Zusammenspiel aus Populationsunterschieden, verschiedenen Bandenstärken und spektralen Überlagerungen der Konformere besser zu beurteilen. Die Annahme einer Boltzmann-Verteilung bei einheitlicher Konformationstemperatur ist Grundlage für die Abschätzung von relativen Populationen. Die relative Absorbanz (bzw. Raman-Streuung) $A_{i} / A_{j}$ der Übergänge zweier Konformere $i$ und $j$ kann mit Gl. 3.5 abgeschätzt werden:

$$
\frac{A_{i}}{A_{j}}=\frac{B_{i}}{B_{j}} \cdot \frac{g_{i}}{g_{j}} \cdot \frac{\sigma_{j}}{\sigma_{i}} \cdot \exp \left(-\frac{E_{i}-E_{j}}{R T_{\mathrm{K}}}\right)
$$

Dabei sind $B$ die berechneten Bandenstärken (IR-Aktivitäten oder Raman-Streuquerschnitte), $g$ die Entartungsfaktoren bezüglich der Chiralität (eins für achirale und zwei für chirale Strukturen), $\sigma$ die Rotationssymmetriezahl (diese entspricht der Zahl an Drehoperationen einschließlich der Identität in der Charaktertafel der Punktgruppe), $E_{i}-E_{j}$ der schwingungsnullpunktskorrigierte Energieunterschied bei $T=0, R$ die Gaskonstante und $T_{\mathrm{K}}$ die Konformationstemperatur. Eine in dieser Formel enthaltene Näherung ist, dass Unterschiede in den Schwingungs- und Rotationszustandssummen der Konformere, mit Ausnahme der Rotationssymmetriezahl, als vernachlässigbar angenommen werden. Eine ausführliche Ableitung dieser Beziehung findet sich in Ref. [94].

Die in den Simulationen verwendeten Konformationstemperaturen beruhen auf experimentellen Bestimmungen gemäß der entsprechend umgestellten Form von Gl. 3.5. Diese Bestimmungen wurden bei vergleichbaren Expansionsbedingungen für die Konformere von Ethanol, deren Energiedifferenz experimentell gut bekannt ist, durchgeführt. In Kap. 4 wird dies näher diskutiert und die Übertragbarkeit auf Ethanol-Dimere geprüft.

Bei Vorliegen hoher Barrieren werden getrennte Boltzmann-Verteilungen modelliert, die in passender Weise gewichtet werden. Ein Beispiel ist die Expansion eines racemisches Gemisches einer permanent chiralen Substanz. Wegen der statistischen Bildung von homo- und heterochiralen Clustern werden zwei voneinander unabhängige BoltzmannVerteilungen für homo- und heterochirale Cluster modelliert und mit gleicher Gewichtung überlagert.

Hinsichtlich der Bandenstärken werden berechnete IR-Aktivitäten direkt verwendet, während die aus Gaussian 09 erhaltenen Raman-Aktivitäten und Depolarisationsverhältnisse zunächst in Raman-Streuquerschnitte umgerechnet werden. Dabei wird eine teilweise thermische Schwingungsanregung, die Anregungswellenlänge des Lasers sowie die polarisationsabhängige Detektionsempfindlichkeit des Monochromators und der angeschlossenen CCD-Kamera berücksichtigt. In dieser Arbeit simulierte Raman-Spektren sind daher nicht allgemeingültig, sondern speziell für Vergleiche mit Curry-Jet-Spektren 
angepasst. Aus den Raman-Aktivitäten $A_{\mathrm{R}}$ und den Depolarisationsverhältnissen $P$ werden zunächst gemäß Gl. 3.6 und 3.7 die Ableitungen der isotropen und anisotropen Polarisierbarkeiten $\alpha^{\prime}$ und $\gamma^{\prime}$ berechnet.[94]

$$
\begin{aligned}
\alpha^{\prime 2} & =\frac{A_{\mathrm{R}}}{45}\left(1-\frac{7 P}{3 P+3}\right) \\
\gamma^{\prime 2} & =\frac{A_{\mathrm{R}} P}{3 P+3}
\end{aligned}
$$

Die wellenzahlabhängige Empfindlichkeit des Curry-Jets für die Polarisation des Streulichts wird durch einen Korrekturfaktor $f(\tilde{v})$ über ein Polynom 9. Grades berücksichtigt (Gl. 3.8, bestimmt von Maxim Gawrilow).

$$
\begin{aligned}
f(\tilde{v})=1.48 & +7.2138 \cdot 10^{-10} \tilde{v}^{3} \mathrm{~cm}^{3}-7.9373 \cdot 10^{-13} \tilde{v}^{4} \mathrm{~cm}^{4} \\
& +2.7716 \cdot 10^{-16} \tilde{v}^{5} \mathrm{~cm}^{5}-0.2071 \cdot 10^{-22} \tilde{v}^{7} \mathrm{~cm}^{7} \\
& +0.4414 \cdot 10^{-26} \tilde{v}^{8} \mathrm{~cm}^{8}-2.8952 \cdot 10^{-31} \tilde{v}^{9} \mathrm{~cm}^{9}
\end{aligned}
$$

Die Streuquerschnitte $\sigma(\tilde{v})$ werden schließlich aus Gl. 3.9 unter Verwendung der Laserwellenlänge $\lambda_{\text {Laser }}=532.27 \mathrm{~nm}$ und der Schwingungstemperatur $T_{\mathrm{S}}$ erhalten. $[57,95]$ Für letztere wird hier pauschal ein Wert von $100 \mathrm{~K}$ verwendet. Da in dieser Arbeit ausschließlich Spektren im hochenergetischen OH-Streckschwingungsbereich (mit einer Ausnahme im OD-Streckschwingungsbereich) von Molekülen und Clustern in Überschallexpansionen modelliert werden, ist der Einfluss der thermischen Schwingungsanregung vernachlässigbar.

$$
\sigma(\tilde{v})=\frac{2 \pi^{2} h \lambda_{\text {Laser }}^{-1}}{45 c} \cdot \frac{\left(\lambda_{\text {Laser }}^{-1}-\tilde{v}\right)^{3}}{\tilde{v}\left(1-\exp \left(-\frac{h c \tilde{v}}{k_{\mathrm{B}} T_{\mathrm{S}}}\right)\right)} \cdot\left(45 \alpha^{\prime 2}+4 \gamma^{\prime 2}+\frac{3 \gamma^{\prime 2}}{f(\tilde{v})}\right)
$$

Die für Gl. 3.8 und 3.9 benötigten Wellenzahlen der Fundamentalübergänge $\tilde{v}$ werden durch eine einfache Korrektur von harmonischen Wellenzahlen $\omega$ erhalten. Entgegen der verbreiteten Praxis einer multiplikativen Skalierung, wird in dieser Arbeit stattdessen eine Konstante subtrahiert. Dies entspricht der Annahme einer konstanten Anharmonizität, da nur begrenzte Wellenzahlverschiebungen von Hydroxy-Oszillatoren in ähnlicher chemischer Umgebung betrachtet werden. Dies wird in Kap. 2.5 und 5.4 diskutiert. Die Konstante wird dabei empirisch für eine gute Übereinstimmung zwischen experimentellen und theoretischen Spektrum gewählt, teilweise unter Verwendung einer Position einer sicher zugeordneten Bande.

Die Berücksichtigung von relativen Populationen und Bandenstärken führt zu relativen Bandenintensitäten. Diese bilden ein Strichspektrum mit scharfen Übergängen. Um auch Überlagerungen von Banden modellieren zu können, werden diese entweder über ein normiertes Gauß-Profil gemäß Gl. 3.10 oder über ein normiertes Lorentz-Profil gemäß Gl. 3.11 verbreitert. 


$$
\begin{aligned}
\text { Gauß-Profil: } A(\tilde{v}) & =B \cdot \sqrt{\frac{4 \ln 2}{\pi \mathrm{FWHM}^{2}}} \exp \left(\frac{-4\left(\tilde{v}-\tilde{v}_{0}\right)^{2} \ln 2}{\mathrm{FWHM}^{2}}\right) \\
\text { Lorentz-Profil: } A(\tilde{v}) & =B \cdot \frac{1}{2 \pi} \cdot \frac{\mathrm{FWHM}}{\left(\tilde{v}-\tilde{v}_{0}\right)^{2}+(\mathrm{FWHM} / 2)^{2}}
\end{aligned}
$$

Hierbei sind $\tilde{v}_{0}$ die Zentralwellenzahl und FWHM die volle Halbwertsbreite (Full Width at $\underline{\text { Half }}$ Maximum). Letztere ist definiert über $A\left(\tilde{v}_{0} \pm \mathrm{FHWM} / 2\right)=0.5 A\left(\tilde{v}_{0}\right)$. Durch die Normierung gilt unabhängig von der Halbwertsbreite stets $\int A(\tilde{v}) \mathrm{d} \tilde{v}=B$.

Die Wahl der Profilform (Gauß oder Lorentz) und der Größe der vollen Halbwertsbreite erfolgt empirisch durch Vergleich mit dem Experiment. Dabei werden Banden von Isomeren stets einheitlich modelliert. $A(\tilde{v})$ wird für jede Bande auf einem Wellenzahlgitter von $0.1 \mathrm{~cm}^{-1}$ berechnet und für alle Banden aufsummiert, um das verbreiterte Profilspektrum zu erhalten. Die Strichspektren werden derart skaliert (über Division durch $A\left(\tilde{v}_{0}\right)$ ), dass deren Peakhöhen mit den Maximalintensitäten der unüberlagerten Banden übereinstimmen. ${ }^{\mathrm{b}}$ Durch diese Darstellungsform wird in der Kombination aus Strich- und Profilspektrum leicht ersichtlich, wie viel jedes Signal zu einer Überlagerung beiträgt. Dies ist beispielhaft in Abb. 3.5 dargestellt. Dort sind Signale gleicher Intensität mit Gauß- bzw. Lorentzprofilen von FWHM $=3 \mathrm{~cm}^{-1}$ mit kleiner werdenden Abständen (in Schritten von $1 \mathrm{~cm}^{-1}$ ) überlagert.

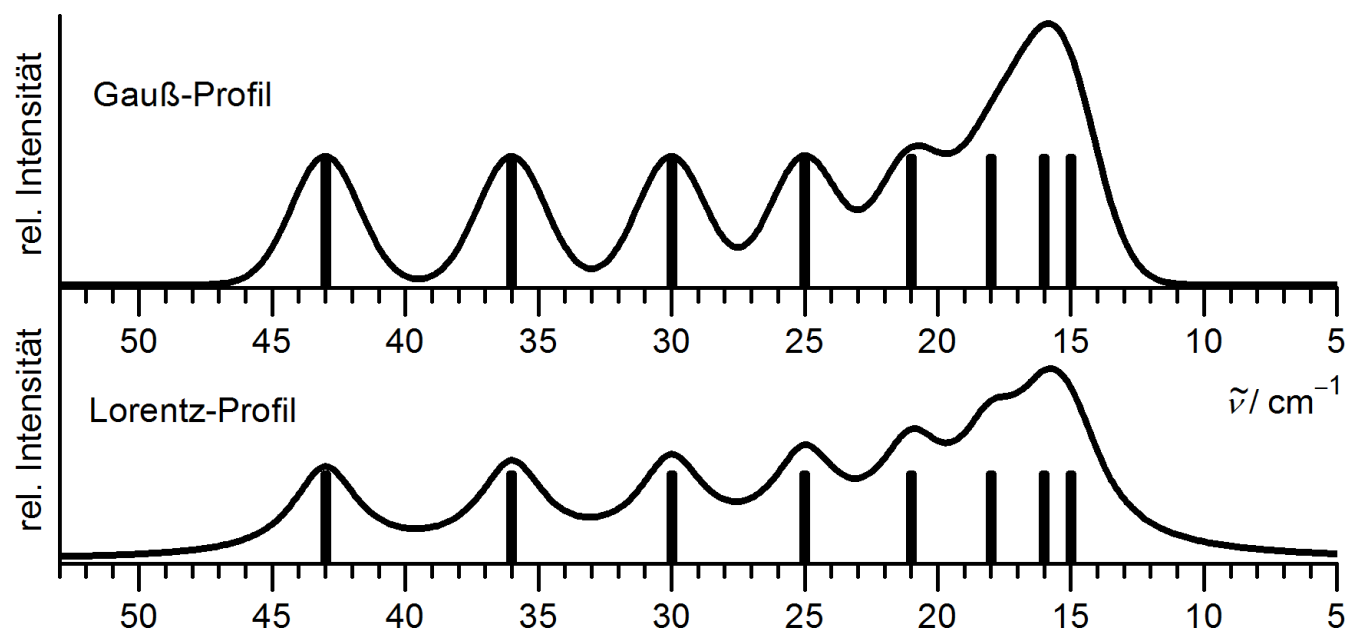

Abb. 3.5: Beispielhafte Überlagerung von Banden gleicher Intensität mit Gauß- bzw. LorentzProfil mit jeweils FWHM $=3 \mathrm{~cm}^{-1}$. Beide Spektren weisen die gleiche Gesamtintensität auf.

Solange die Differenz der Zentralwellenzahlen größer als die volle Halbwertsbreite ist, hat die Überlagerung mit einem Gaußprofil kaum Einfluss auf die Position und Intensität der Bandenmaxima. Dagegen beeinflussen die ausladenden Flanken des Lorentz-Profils noch über deutlich größere spektrale Distanzen benachbarte Banden. Zudem sind die Maximalintensitäten geringer. Da die Konzentrationen von Clustern in einer Überschallexpansion nur über die berechneten Bandenstärken grob abgeschätzt werden können, können nur

\footnotetext{
${ }^{\mathrm{b}}$ Ausnahmen werden für die Abb. 4.19, 4.22 und 4.30 gemacht, da hier eine Vielzahl von Banden hoher Halbwertsbreite überlagert werden, die einzeln kaum erkennbare Maximalintensitäten haben. Diese werden einheitlich hochskaliert, um dennoch relative Intensitäten beurteilen zu können.
} 
3 Experimentelle und quantenchemische Methoden

relative, statt absolute, Intensitäten von Banden zwischen Experiment und Theorie verglichen werden. Hierzu werden die simulierten Spektren auf visuell vergleichbare Intensität zu den experimentellen Spektren skaliert. 


\section{Chiralitätssynchronisation in Ethanol-Aggregaten}

\subsection{Einleitung}

Ethanol ist ein wichtiger Prototyp für die in dieser Arbeit untersuchten intra- und intermolekularen Effekte und Phänomene. Es ist zum einen eines der kleinsten Moleküle mit Isomerie bezüglich der Torsion um eine $\mathrm{C}-\mathrm{O}$-Bindung, zum anderen treten in seinen Clustern adaptive Aggregation und Chiralitätssynchronisation auf. Wegen seiner großen industriellen Bedeutung, u. a. als Lösungsmittel, Biokraftstoff und Genussmittel/Droge, ist es eine intensiv (aber noch lange nicht umfassend) untersuchte Substanz, sodass auf umfangreiche experimentelle Vergleichswerte zurückgegriffen werden kann. Die geringe molekulare Größe von Ethanol erlaubt darüber hinaus quantenchemisch auch den Vergleich mit Ergebnissen aus der Verwendung aufwendiger quantenchemischer Rechenmethoden mit großen Basissätzen und aus wellenfunktionsbasierten Methoden mit hoher Elektronenkorrelation. Aufbauend auf der Konformationsisomerie der EthanolMonomere und -Dimere werden hier verschiedene Dichtefunktionalmethoden für die anschließende Anwendung auf größere Systeme evaluiert. Dazu zählen auch cyclische Ethanol-Cluster mittlerer Größe, denen eine besondere Bedeutung in Flüssigkeit [96] und Lösung [97-99] zugeschrieben wird, deren Isomerie aber bisher nur unzureichend untersucht wurde. Die in dieser Arbeit vorgeschlagenen Gasphasen-Strukturen bei tiefen Temperaturen könnten somit auch künftig als Referenzen für Modelle der kondensierten Phase dienen.

\subsection{Ethanol-Monomere}

Als isoliertes Molekül tritt Ethanol in drei Konformationen auf (Abb. 4.1), die sich im Wesentlichen in Bezug auf den $\mathrm{C}_{\beta} \mathrm{C}_{\alpha} \mathrm{OH}$-Torsionswinkel unterscheiden. Dieser beträgt im trans-Konformer $(t)+180^{\circ}$ (gleichbedeutend mit $-180^{\circ}$ ) und in den gauche-- und gauche+-Konformeren $(g-/ g+)$ etwa $-60^{\circ}$ bzw. $+60^{\circ}$. Während $t$ eine Spiegelebene beinhaltet und somit achiral ist, sind $g-$ und $g+$ transient chiral und verhalten sich enantiomer zueinander.

Darüber hinaus interagieren aufgrund der energetischen Entartung, der niedrigen Barriere und der geringen Masse des Protons die beiden $g$-Wellenfunktionen miteinander. Hierdurch kommt es zu einer Tunnelaufspaltung von $\Delta=h c 3.23 \mathrm{~cm}^{-1}=$ $0.0193 \mathrm{~kJ} \mathrm{~mol}^{-1}[100]$. Experimentell und quantenchemisch gut etabliert ist die etwas niedrigere Energie von $t$ gegenüber $g$. Allerdings hängen Abschätzungen dieser Energiedifferenz stark von der experimentellen Methodik und bei ab initio Methoden vom Basissatz und dem Ausmaß der berücksichtigten Elektronenkorrelation ab. Tab. 4.1 listet 


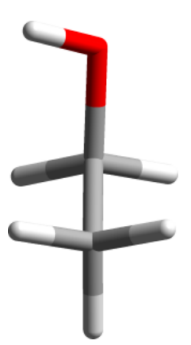

gauche-

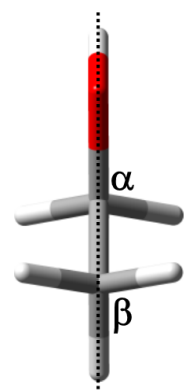

trans

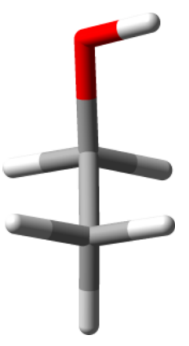

gauche+

Abb. 4.1: Die drei Konformere des Ethanol-Monomers. Die gepunktete Linie deutet die Spiegelebene an, die das trans-Konformer in zwei enantiotope Halbräume teilt und an der sich auch die beiden enantiomeren gauche-Konformere ineinander überführen lassen. Markiert sind zudem die $\alpha$ - und $\beta$-Kohlenstoffatome.

hierzu einige Literaturwerte. Am ehesten verlässlich ist experimentell wohl der rotationsspektroskopische Wert von $0.47 \mathrm{~kJ} \mathrm{~mol}^{-1}$ [100, 104], der auch gut mit dem Ergebnis von $0.51 \mathrm{~kJ} \mathrm{~mol}^{-1}$ auf CCSD(T)/aug-cc-pVTZ//MP2/aug-cc-pVTZ-Niveau (mit harmonischer Schwingungsnullpunktskorrektur auf MP2/6-311++(3df,2p)-Niveau) [106] übereinstimmt. Eine exakte Übereinstimmung ist vor allem aufgrund der Vernachlässigung von anharmonischen Effekten, wie der erwähnten Tunnelaufspaltung, nicht zu erwarten. Dennoch erscheint eine Übereinstimmung zumindest innerhalb einer Spanne von etwa 0 bis $1 \mathrm{~kJ} \mathrm{~mol}^{-1}$ Energievorteil für das $t$-Konformer als Auswahlkriterium für eine theoretische Methode zur Vorhersage der Isomerie von Ethanol-Clustern sinnvoll.

Tab. 4.1: Experimentelle und $a b$ initio Literaturwerte für die Energie- und $\mathrm{OH}-$ Streckschwingungswellenzahl-Differenz zwischen den trans- und gauche-Konformeren von Ethanol. Theoretische Werte sind in doppelt-harmonischer Näherung und mit Schwingungsnullpunktkorrektur berechnet. Experimentelle Ergebnisse sind chronologisch geordnet (alt oben, neu unten). Die experimentellen Abschätzungen der harmonischen Wellenzahldifferenz wurden unter Vernachlässigung außerdiagonaler anharmonischer Beiträge sowie deren Fehler nach der Größtfehlermethode aus den Unsicherheiten der Einzelwerte berechnet.

\begin{tabular}{lcc}
\hline Methode & $\begin{array}{c}E_{0}(g)-E_{0}(t) \\
/ \mathrm{kJ} \mathrm{mol}\end{array}$ & $\begin{array}{c}\omega(t)-\omega(g) \\
/ \mathrm{cm}^{-1}\end{array}$ \\
\hline Ramanspektroskopie [101] & 1.32 & - \\
Rotationsspektroskopie [12] & $0.49(6)$ & - \\
Photoakustische Spektroskopie, Obertöne [102] & $2.9(4)$ & $14(7)$ \\
Ferninfrarotspektroskopie [103] & 0.50 & - \\
Rotationsspektroskopie [100, 104] & 0.47 & - \\
Ramanspektroskopie, Deuterierung [22] & - & $23(16)$ \\
FTIR, Oberton [105] & - & $20.5(13)$ \\
\hline CCSD(T)/aug-cc-pVTZ//MP2/aug-cc-pVTZ [106] & 0.51 & - \\
CCD/6-311++G** [107] & 1.39 & - \\
MP2/aug-cc-pVTZ [106] & 0.89 & 10.0 \\
MP2/6-311++G(2d,p) [108] & 1.27 & 11.4 \\
\hline
\end{tabular}


Das isolierte Ethanol-Monomer liegt somit in einem engen Gleichgewicht von zwei Klassen von Konformeren vor, bei dem $t$ energetisch und $g$ dagegen statistisch, wegen seiner zweifachen Entartung, bevorzugt ist. Je nach Konformationstemperatur sind daher entweder das $t$-Konformer oder die beiden $g$-Konformere stärker populiert.

Die Fundamentalübergänge der OH-Streckschwingungen der beiden Konformere wurden Ramanspektroskopisch in einer Überschallexpansion $\mathrm{zu} 3678 \mathrm{~cm}^{-1}$ für $t$ und $\mathrm{zu}$ $3660 \mathrm{~cm}^{-1}$ für $g$ bestimmt,[22] deren Werte sich aber durch eine nachträgliche Rekalibrierung [109] auf $3677 \mathrm{~cm}^{-1}$ und $3659 \mathrm{~cm}^{-1}$ verschieben. Die experimentelle anharmonische Wellenzahldifferenz beträgt somit etwa $18 \mathrm{~cm}^{-1}$. Die Bande von $g$ erscheint im Vergleich zur Bande von $t$ weder aufgespalten noch verbreitert, da die Tunnelaufspaltung im angeregten Zustand der $\mathrm{OH}-$ Streckschwingung offenbar ähnlich genug zu der des Grundzustandes ist, sodass die Übergänge zwischen den jeweils symmetrischen und antisymmetrischen Kombinationen der $g$-Wellenfunktionen innerhalb der Messgenauigkeit aufeinander fallen.[22] Kollipost et al. [61, 105] lokalisierten FTIRspektroskopisch den $t$-Übergang damit übereinstimmend bei $3676.6(2) \mathrm{cm}^{-1}$ und vermuten das $g$-Bandenzentrum bei $3659.3(4) \mathrm{cm}^{-1}$, sowie eine verringerte Tunnelaufspaltung im angeregten Zustand von $2.8(1) \mathrm{cm}^{-1}$. Allerdings zeigen die zugeordneten Banden in der Gasphase bei Zimmertemperatur starke Überlagerungen und sind auch in der gekühlten Überschallexpansion ungenügend instrumentell aufgelöst, sodass die Werte unsicher bleiben. Experimentelle Abschätzungen der harmonischen Wellenzahldifferenz für den direkteren Vergleich mit der Theorie unter harmonischer Näherung sind schwieriger, da außerdiagonale Beiträge zur Anharmonizität nicht bekannt sind. Tab. 4.1 listet hierzu drei Werte, die aus experimentellen Bandenpositionen unter Vernachlässigung außerdiagonaler Beiträge abgeleitet wurden, sowie ab initio Ergebnisse.

In Tab. 4.2 sind in der vorliegenden Arbeit erhaltene Ergebnisse von Rechnungen in harmonischer Näherung mit verschiedenen Kombinationen aus DFT-Funktionalen und Basissätzen sowie die jeweils benötigte Rechenzeit für eine Punktrechnung eines ButanolTetramers angegeben. Das Butanol-Tetramer wurde gewählt, da es mit 20 Atomen der 2. Periode in etwa den in dieser Arbeit größten intensiver untersuchten Strukturen entspricht. Die Rechenzeiten wurden bei Benutzung von 16 Kernen und 32 GB RAM auf der mpi-Queue des Rechenzentrums der Gesellschaft für wissenschaftliche Datenverarbeitung mbH Göttingen (GWGD) ermittelt. Da hier u. a. nicht durchgehend exakt gleiche Hardware vorausgesetzt werden kann, sind die Werte nur bedingt reproduzierbar und als grobe Orientierung zu verstehen. Die Angabe $>2880$ min $(\hat{=} 2 \mathrm{~d})$ bedeutet, dass die Rechnung nicht in der maximalen Nutzungszeit von zwei Tagen abgeschlossen werden konnte.

B3LYP-D3(BJ) scheint mit zunehmender Göße der korrelationskonsistenten DunningBasissätze auf einen Energieunterschied von etwa $0.25 \mathrm{~kJ} \mathrm{~mol}^{-1}$ (etwas unterhalb des experimentellen Wertes von $0.47 \mathrm{~kJ} \mathrm{~mol}^{-1}$ ) sowie auf eine Differenz in der harmonischen Wellenzahl von $16.5 \mathrm{~cm}^{-1}$ zu konvergieren (experimentell/anharmonisch: $18 \mathrm{~cm}^{-1}$ ). Im Rahmen der Näherungen und experimentellen Unsicherheiten sind harmonische Theorie und Experiment damit in durchaus guter Übereinstimmung. Diese Konvergenz erfolgt aber je nach verwendeter Konstruktion des Bassisatzes unterschiedlich schnell. Mit nichtaugmentierten Basissätzen der Dunning-Familie cc-pVXZ wird eine sehr große Zahl an Basisfunktionen benötigt, dagegen ist die Konvergenz dieser Eigenschaften bereits mit nur minimaler Augmentierung bei Triple- $\zeta$ (may-cc-pVTZ) praktisch erreicht. Bei der Karlsruhe-Familie def2-XZVP wird dagegen noch mit Triple- $\zeta$ eine fast perfekte energeti- 
sche Parität zwischen $t$ und $g$ vorhergesagt. Auch mit der größten verfügbaren Quadrupel$\zeta$-Qualität liegt der Wert etwas niedriger als mit den größeren Dunning-Bassisätzen, möglicherweise aus Mangel an diffusen Funktionen als Pendant der Augmentierung.[91] B3LYP/aug-cc-pVTZ ohne Dispersionskorrektur liegt bezüglich des Energieunterschiedes etwas näher am Experiment, allerdings ist nicht zu erwarten, dass diese Fehlerkompensation auch auf Cluster übertragen werden kann.

B2PYLP-D3(BJ) scheint auf einen Energieunterschied näher am experimentellen Wert $\mathrm{zu}$ konvergieren, allerdings wegen des enthaltenen MP2-Anteils deutlich langsamer als B3LYP-D3(BJ). M06-2X unterschätzt die Energiedifferenz etwas, $\omega$ B97X-D deutlicher bis hin zur Parität. Zusätzlich getestet wurden MP2/aug-cc-pVDZ und APF-D/6$31++\mathrm{G}(\mathrm{d}, \mathrm{p})$, um aktuelle publizierte Ergebnisse $[110,111]$ zu Ethanol in späteren Abschnitten dieser Arbeit besser einordnen zu können. MP2 überschätzt den energetischen Vorteil von $t$ recht deutlich (Tab. 4.1 und 4.2). APF-D/6-31++G(d,p) sagt dagegen qualitativ falsch eine energetische Bevorzugung von $g$ voraus.

Einige hier vorausgewählte Methoden werden im Folgenden für die Beschreibung von Ethanol-Dimeren weiter evaluiert. 
Tab. 4.2: Schwingungsnullpunktskorrigierte Energie- und harmonische OH-Streckschwingungswellenzahl-Differenzen der Ethanol-Konformere mit verschiedenen quantenchemischen Methoden. Die angegebene Rechenzeit bezieht sich auf eine Punktrechnung eines $n$-Butanol-Tetramers.

\begin{tabular}{|c|c|c|c|c|}
\hline Methode & Basissatz & $\begin{array}{c}E_{0}(g)-E_{0}(t) \\
/ \mathrm{kJ} \mathrm{mol}^{-1}\end{array}$ & $\begin{array}{c}\omega(t)-\omega(g) \\
/ \mathrm{cm}^{-1}\end{array}$ & $\begin{array}{c}t_{\mathrm{R}} \\
/ \mathrm{min}\end{array}$ \\
\hline B3LYP-D3(BJ) & def2-TZVP & 0.00 & 16.7 & 3 \\
\hline B3LYP-D3(BJ) & def2-QZVP & 0.16 & 16.6 & 72 \\
\hline B3LYP-D3(BJ) & aug-cc-pVDZ & 0.35 & 20.6 & 6 \\
\hline B3LYP-D3(BJ) & aug-cc-pVTZ & 0.26 & 16.3 & 144 \\
\hline B3LYP-D3(BJ) & aug-cc-pVQZ & 0.24 & 16.6 & $>2880$ \\
\hline B3LYP-D3(BJ) & aug-cc-pV5Z & 0.25 & 16.6 & $>2880$ \\
\hline B3LYP-D3(BJ) & aug-cc-pV6Z & 0.25 & 16.5 & $>2880$ \\
\hline B3LYP-D3(BJ) & cc-pVTZ & -0.28 & 17.4 & 5 \\
\hline B3LYP-D3(BJ) & cc-pVQZ & 0.02 & 16.7 & 49 \\
\hline B3LYP-D3(BJ) & cc-pV5Z & 0.20 & 16.6 & 616 \\
\hline B3LYP-D3(BJ) & cc-pV6Z & 0.24 & 16.6 & $>2880$ \\
\hline B3LYP-D3(BJ) & may-cc-pVTZ & 0.24 & 16.5 & 10 \\
\hline B3LYP-D3(BJ) & jun-cc-pVTZ & 0.22 & 16.5 & 15 \\
\hline B3LYP-D3(BJ) & jul-cc-pVTZ & 0.25 & 16.1 & 22 \\
\hline B3LYP-D3(BJ) & apr-cc-pVQZ & 0.25 & 16.5 & 89 \\
\hline B3LYP & may-cc-pVTZ & 0.57 & 15.5 & 11 \\
\hline B2PLYP-D3(BJ) & def2-TZVP & 0.27 & 14.7 & 19 \\
\hline B2PLYP-D3(BJ) & def2-QZVP & 0.47 & 14.5 & 961 \\
\hline B2PLYP-D3(BJ) & may-cc-pVTZ & 0.82 & 14.2 & 104 \\
\hline B2PLYP-D3(BJ) & jun-cc-pVTZ & 0.82 & 14.1 & 187 \\
\hline B2PLYP-D3(BJ) & jul-cc-pVTZ & 0.67 & 13.5 & 274 \\
\hline B2PLYP-D3(BJ) & aug-cc-pVTZ & 0.60 & 13.3 & 760 \\
\hline B2PLYP-D3(BJ) & aug-cc-pVQZ & 0.54 & 14.4 & $>2880$ \\
\hline M06-2X & def2-TZVP & 0.24 & 19.3 & 4 \\
\hline M06-2X & may-cc-pVTZ & 0.27 & 18.1 & 11 \\
\hline M06-2X & aug-cc-pVTZ & 0.22 & 18.7 & 224 \\
\hline M06-2X & aug-cc-pVQZ & 0.19 & 19.4 & $>2880$ \\
\hline$\omega \mathrm{B} 97 \mathrm{X}-\mathrm{D}$ & def2TZVP & -0.01 & 19.2 & 4 \\
\hline$\omega \mathrm{B} 97 \mathrm{X}-\mathrm{D}$ & may-cc-pVTZ & 0.08 & 17.3 & 13 \\
\hline$\omega \mathrm{B} 97 \mathrm{X}-\mathrm{D}$ & aug-cc-pVTZ & 0.05 & 17.8 & 127 \\
\hline$\omega \mathrm{B} 97 \mathrm{X}-\mathrm{D}$ & aug-cc-pVQZ & 0.00 & 18.3 & $>2880$ \\
\hline APF-D & $6-31++\mathrm{G}(\mathrm{d}, \mathrm{p})$ & -0.17 & 16.4 & 2 \\
\hline MP2 & aug-cc-pVDZ & 0.94 & 12.9 & 24 \\
\hline
\end{tabular}




\subsection{Ethanol-Dimere}

\subsubsection{Einleitung: Adaptive Aggregation}

Bei der Aggregation von Molekülen können die intermolekularen Wechselwirkungen die intramolekulare Konformationspräferenz verändern, es wird von adaptiver Aggregation gesprochen. Bevor dies für Dimere und Cluster näher untersucht wird, lohnt sich ein Blick auf die Gegebenheiten im Festkörper, denn die kristalline Phase kann als Entsprechung eines besonders großen, geordneten Clusters im thermodynamischen Gleichgewicht angesehen werden. Ethanol bildet hier unendliche Ketten aus, innerhalb der die Moleküle über kooperative Wasserstoffbrücken verbunden sind.[112] Die Ketten enthalten dabei abwechselnd ein $t$ - und ein $g$-Konformer. Ein näherer Blick auf die Kristallstruktur (Abb. 4.2) zeigt, dass innerhalb dieser Struktur zusätzlich auch die transiente Chiralität der enantiomeren $g+-$ und $g$--Konformere alterniert (Chiralitätssynchronisation).

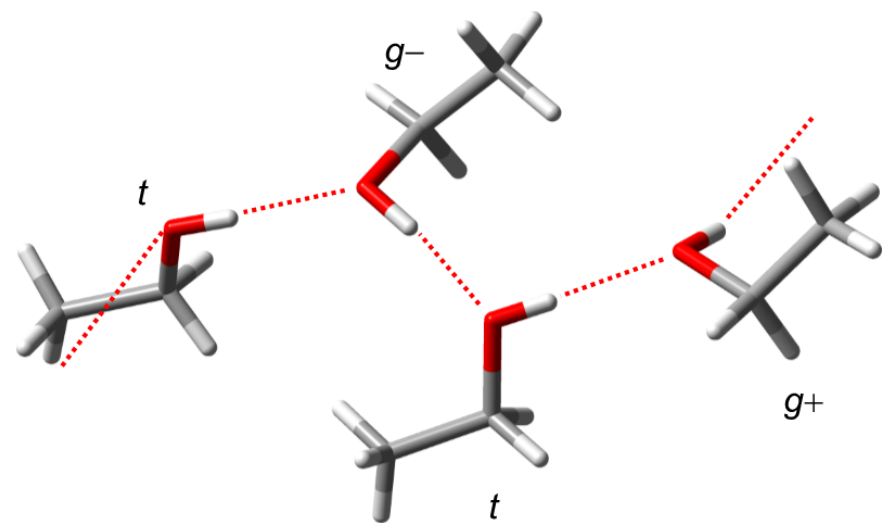

Abb. 4.2: Ausschnitt der Kristallstruktur von Ethanol nach kristallographischen Daten aus Ref. [112]. OH ‥ O-Wasserstoffbrücken sind mit roten gestrichelten Linien hervorgehoben.

Unter hohem Druck findet eine Umwandlung zu einer all-t-Struktur statt [113], in der aber auch Hinweise auf eine Unordnung bezüglich der Hydroxyprotonenposition gefunden wurden, welche zu einer $g$-Population von etwa ein Drittel führt.[114] Dieser Befund wurde aber durch eine jüngste Neutronendiffraktionsstudie an EtOD in Frage gestellt.[115] Es ist daher noch unklar, ob es sich um eine Fehlinterpretation aufgrund der schwierigen Bestimmung von Positionen von Wasserstoffatomen mittels Röntgendiffraktometrie oder um einen tatsächlichen Isotopeneffekt handelt.

Als Fazit lässt sich feststellen, dass auch in der kristallinen Phase das intramolekulare Konformeren-Gleichgewicht sehr fragil ist, ähnlich zu den Monomeren in der Gasphase. Entsprechendes lässt sich daher auch für Aggregate zwischen diesen beiden Extremen erwarten. Den ersten Schritt in der Aggregation stellt die Bildung von Dimeren aus zwei Ethanol-Molekülen dar, welcher in diesem Kapitel näher betrachtet wird.

\subsubsection{Motivation}

Die Konformationsisomerie der Ethanol-Dimere wird seit über 20 Jahren detailliert untersucht,[22, 96, 98, 106, 108, 110, 116-125] innerhalb des Arbeitskreises Suhm stand sie zuletzt vor etwa 10 Jahren im Fokus.[22, 57] Einige Entwicklungen des letzten Jahrzehntes lassen es lohnenswert erscheinen, die bisher nur unvollständig beantworteten 
Fragestellungen, nach der genauen energetischen Abfolge der Ethanol-Dimere sowie der Zuordnung der Schwingungsspektren, erneut zu ergründen. Dies sind auf experimenteller Seite vor allem erweiterte rotationsspektroskopischen Daten von Loru et al. [110] sowie ferner auch bezüglich des Signal-zu-Rausch-Verhältnisses verbesserte FTIR- und Raman-Spektren von Oswald [21, 126]. Auf theoretischer Seite sind es die Entwicklung und Popularisierung von neueren DFT-Methoden, bei denen versucht wird lange bekannte Defizite älterer Methoden in der Berücksichtigung der Dispersionswechselwirkung zu beheben. Der anhaltende Fortschritt in der Computertechnologie erlaubt auch zunehmend Vergleiche mit Ergebnissen aus Rechnungen mit größeren Bassisätzen. Dabei sind nicht nur mögliche Erkenntnisse für Ethanol selbst interessant, sondern auch Rückschlüsse auf die Vorhersagekraft dieser Methoden, um sie für die Beschreibung weniger gut erforschter molekularer Systeme im weiteren Verlauf dieser Arbeit auszuwählen. Aufgrund der geringen Energieunterschiede und den flexiblen Strukturen sind die Ethanol-Dimere ein besonders herausfordernder Testfall sowohl für Experiment wie auch Theorie. So urteilten noch 2015 Vargas-Caamal et al. [123], aufbauend auf ähnlichen Einschätzungen von Provencal et al. [119]:

,$[\ldots .$.$] classifying structures according to their energies in the particular case of$ the ethanol dimer, where the energy differences are too small to be discerned by experimental or theoretical methods, is a futile exercise."

Insbesondere auf theoretischer Seite sind DFT-Methoden sicherlich noch weit davon entfernt im Allgemeinen die benötigte Genauigkeit im Bereich von Zehntel kJ mol${ }^{-1} \mathrm{zu}$ erreichen. Dennoch wird im Folgenden gezeigt, dass sich für geeignete Systeme dennoch gute Vorhersagen unter Ausnutzung von Fehlerkompensation erhalten lassen.

\subsubsection{Konformationsisomerie}

Als primäres Bindungsmotiv der Dimere von Ethanol ist eine lineare Struktur mit einer $\mathrm{OH}$... O-Wasserstoffbrücke sowohl experimentell wie auch theoretisch gut etabliert. Cyclische Dimere ohne Wasserstoffbrücke wurden vereinzelt auch theoretisch analysiert, aber energetisch deutlich höher gefunden.[106, 123] Darüber hinaus würden sie sich wegen der schwachen Wechselwirkung vermutlich schwingungsspektroskopisch nur sehr schwierig vor dem Hintergrund der stets präsenten Ethanol-Monomere nachweisen lassen. Daher werden cyclische Dimere in dieser theoretischen Untersuchung nicht weiter berücksichtigt.

Bei der Ausbildung einer $\mathrm{OH}$...O-Wasserstoffbrücke werden die beiden EthanolMoleküle inäquivalent, da eines die Rolle des Donors (D) und das andere die Rolle des Akzeptors (A) annimmt. Die Dimere werden im Folgenden klassifiziert durch Nennung des Konformers des Donors, gefolgt von der des Akzeptors, beispielsweise $g-g+$. Da zudem eines der beiden freien Elektronenpaare des Akzeptors koordiniert wird, wird die Spiegelsymmetrie eines eventuell beteiligten $t$-Monomer-Konformers gebrochen. Alle so gebildeten Dimer-Konformere sind somit asymmetrisch und bei der Betrachtung der relativen Population müssen daher keine Unterschiede in der Entartung oder Rotationssymmetriezahl berücksichtigt werden.

Die enantiomeren Strukturen lassen sich durch die Koordination des anderen Elektronenpaares erhalten, wenn zusätzlich eventuell beteiligte $g$-Monomere gespiegelt werden. 
So ist beispielsweise die Kombination $g-g+$ bei Koordination des einen Elektronenpaares enantiomer zu $g+g$ - bei Koordination des anderen Elektronenpaares. Um die spektroskopisch unterscheidbaren diastereomeren Strukturen zu erhalten, ist es daher ausreichend nur die Koordination eines festgelegten Elektronenpaares zu betrachten. In dieser Arbeit ist dies stets das rechte, wobei sich dies auf die Blickrichtung entlang des $\mathrm{O} \rightarrow \mathrm{H}$ Vektors bezieht, während der $\mathrm{O} \rightarrow \mathrm{C}$-Vektor nach unten zeigt. Dies entspricht der Darstellung von $t$ in Abb. 4.1 auf S. 36. Dieses Vorgehen folgt der von Ref. [108] eingeführten Konvention. Da Donor und Akzeptor nicht äquivalent sind, ergeben sich somit $3^{2}=9$ Variationen (Klassen) von Ethanol-Dimeren: $g+g+, g+g-, g+t, g-g+, g-g-, g-t, t g+$, $t g-$ und $t t$. Weitere Isomere innerhalb dieser Klassen können sich bezüglich der Torsion der beiden Moleküle um die Wasserstoffbrücke ergeben, die näherungsweise durch den $\mathrm{C}_{\alpha}^{\mathrm{D}} \mathrm{O}^{\mathrm{D}} \mathrm{O}^{\mathrm{A}} \mathrm{C}_{\alpha}^{\mathrm{A}}$-Torsionswinkel beschrieben werden kann. Diese Isomere werden im Folgenden durch eine angehängte Ziffer benannt, z. B. $g+g+2$, in der Energiereihenfolge auf dem Niveau der initialen Struktursuche. Die Nennung keiner Ziffer impliziert hier die erste Struktur, sofern nicht ausdrücklich die gleichnamige übergeordnete Klasse behandelt wird. Dyczmons [106] argumentierte allerdings, dass die energetisch höheren Isomere innerhalb einer Klasse wegen des Mangels an Barrieren bei der Torsion um die Wasserstoffbrücke nicht stabil wären. Dies ist darin begründet, dass die von ihm für zwei Fälle berechneten Übergangszustände nach Schwingungsnullpunktsenergie-Korrektur energetisch tiefer liegen als die Konformere selbst. In einer alternativen Interpretation als angeregte Torsionszustände können diese allerdings zum Spektrum beitragen. Im Folgenden werden diese Strukturen daher in der Boltzmann-Verteilung berücksichtigt und geprüft, ob diese Annahme die Übereinstimmung zwischen Theorie und Experiment verbessert oder verschlechtert.

Die Berücksichtigung aller neun Klassen in der Literatur erfolgte erst ab 2005 durch Emmeluth et al.[108] Davor erschienene Arbeiten,[106, 116-120, 122] aber auch vereinzelt jüngere Untersuchungen [96, 124] betrachteten nur Teilmengen dieses Konformationsraumes, da wichtige Isomerien übersehen wurden, zumeist die Inäquivalenz der beiden $g$-Konformere im Dimer. Die neueren Studien finden fast einheitlich $g+g+$ als globales Minimum, während das globale Minimum der meisten älteren Studien mindestens ein $t$-Konformer enthält. Letzteres ist in einem rückblickend unzureichenden Rechenniveau, z.B. DFT ohne Dispersionskorrektur, begründet, und/oder weil in vielen Fällen $g+g+$ übersehen wurde. Untersuchungen auf Basis von MP2 [106, 108, 110, 121] kommen zu dem globalen Minimum $g+g+$ erst mit größeren nachträglichen Energie-Korrekturen (unter Verwendung der CCSD(T) Monomer-Energiedifferenz), da MP2 gerade mit moderaten Basissätzen die Stabilität des $t$-Monomer-Konformers deutlich überschätzt.

Die Rotationsspektroskopie erlaubt eine direktere experimentelle Zuordnung bestimmter Konformere als die Schwingungsspektroskopie. Die Ethanol-Dimer-Konformere $g+g+, g-t$, und $g+t$ wurden 2005 durch Hearn et al. [121] in einer Helium-Expansion nachgewiesen. Finneran et al. [125] bestätigten 2016 dieses Ergebnis und fanden zusätzlich in einer Argon-Expansion ausschließlich $g+g+.2017$ gelang Loru et al. [110] in einer Helium-Expansion der Nachweis von nunmehr sechs Konformeren und die Bestimmung der Häufigkeitsabfolge (unter Verwendung von theoretischen DipolmomentKomponenten), aus der die qualitative Energieabfolge in Gl. 4.1 abgeleitet wurde (unter 
Annahme einer Gleichgewichtsverteilung):

$$
E(g+g+)<E(g-t) \approx E(t t)<E(g+t) \approx E(t g+)<E(g-g+)
$$

In einer Neon-Expansion blieben davon die drei stabilsten $(g+g+, g-t$ und $t t)$, in einer Argon-Expansion erneut nur die stabilste Konformation $g+g+$ nachweisbar.

\subsubsection{Schwingungsspektren: Überblick}

Schwieriger gestaltet sich dagegen die schwingungsspektroskopische Zuordnung der Konformere. Erste Spektren der Dimere im OH-Streckbereich, gebildet in einer Überschallexpansion mit Helium als Trägergas, wurden 1999 von Häber et al. [127] (FTIR) und 2000 von Provencal et al. [119] (Cavity-ring-down-Spektroskopie) veröffentlicht. Diese zeigen eine Bandenformation mit drei ausgeprägten Maxima bei 3531.2, 3539.5 und $3547.5 \mathrm{~cm}^{-1}$ [119], wobei die größere Halbwertsbreite des mittleren Signals auf die Anwesenheit von insgesamt mindestens vier Konformeren hinweist. Emmeluth et al. $[108,128]$ konnten mit schrittweiser Argon-Beimischung zeigen, dass sowohl die zentrale wie auch die hochfrequente Bande gegenüber der niederfrequenten an Intensität verlieren und damit die niederfrequente Bande dem stabilsten Konformer $g+g+$ zugeordnet werden kann. Der gleiche Befund ergab sich mit Stickstoff-Beimischung. Oswald [126] bestätigte dies auch mit Sauerstoff-Beimischung. Spektren der Dimere in Matrix-Einbettung sind davon deutlich abweichend, mit $t t$ als stabilstes Konformer in einer Argon- und einer tg-Spezies in einer Stickstoff-Matrix.[118] Dieser Wechsel der bevorzugten Konformation in Abhängigkeit der Umgebung unterstreicht die Subtilität der Isomerie. Spektren von Zielke und Suhm [129] sowie Wassermann et al. [14, 22] zeigen bei Detektion der Dimere in einer Helium-Überschallexpansion mittels Raman-Spektroskopie eine zur IRDetektion vergleichbare Bandenformation, die bei Kühlung durch Beimischung von besser polarisierbareren und massereicheren Trägergasen (Ar, $\mathrm{N}_{2}$ ), sowie bei Änderung des Detektionsabstandes von der Expansionsdüse, auch eine ähnliche Temperaturabhängigkeit aufweist. Nur in einer Neon-Expansion behalten, abweichend vom Trend, die hochund die niederfrequente Bande eine vergleichbare Intensität, während die Konformere, die die breitere zentrale Bande verursachen, weitgehend relaxieren. Aus der Korrelation zwischen den Dimer-Donor- und den mittels Raman-Spektroskopie besser beobachtbaren Dimer-Akzeptorbanden wurden weitere Hinweise für die Zuordnung erhalten, wodurch u. a. für das Dimer der hochfrequenten Bande ein $t$-Akzeptor abgeleitet werden konnte. Einen Überblick über die wichtigsten Spektren bietet Abb. 4.3, in der diese von oben nach unten mit tendenziell abnehmender Konformations-Temperatur angeordnet sind. Diese Abfolge wurde aus dem $t / g$-Verhältnis der Monomer-Banden abgeschätzt. Dies erfolgte für die Ramanspektren quantitativ durch Integration der Banden durch die jeweiligen Experimentatoren und für die weniger gut aufgelösten FTIR-Spektren an dieser Stelle nachträglich qualitativ anhand der Maximalintensitäten. Auch wenn die KonformationsTemperatur zwischen so verschiedenen Spezies wie Monomer und Dimer nicht vollständig übertragbar ist, sollte die Tendenz grundsätzlich erhalten bleiben. Anomalien können sich aber durch unterschiedliche Relaxationsmechanismen der Trägergase ergeben wenn hohe Barrieren, relativ zur verbleibenden thermischen Energie, überwunden werden müs- 


\section{Chiralitätssynchronisation in Ethanol-Aggregaten}

sen. Das vom Trend abweichende Raman-Spektrum der Neon-Expansion wird in Hinblick darauf an späterer Stelle diskutiert.

Ein Problem für den direkten Vergleich zwischen den IR- und Raman-Spektren, sowie für deren Modellierung mit theoretischen Daten, ist die unzureichende Kalibrierung der älteren Raman-Daten. So wurden die Daten u. a. mit Luft- statt Vakuum-Emissionslinien von Neon kalibriert (ein Fehler, der erst 2016 behoben wurde).[109] Korrigieren lässt sich dies nachträglich mit einer Verrechnung der Frequenzinformation mit dem Brechungsindex von Luft, aber auch danach blieben deutliche Diskrepanzen von bis zu etwa $1.5 \mathrm{~cm}^{-1}$ für die absoluten Bandenpositionen zwischen verschiedenen Raman-Messungen, teils vom selben Messtag. Daher wurde ein pragmatischer Ansatz gewählt, indem die Frequenzinformation des Raman-Spektrums der Helium-Expansion bei $3 \mathrm{~mm}$ Detektionsabstand multiplikativ auf Übereinstimmung mit der niederfrequenten Dimerbande des

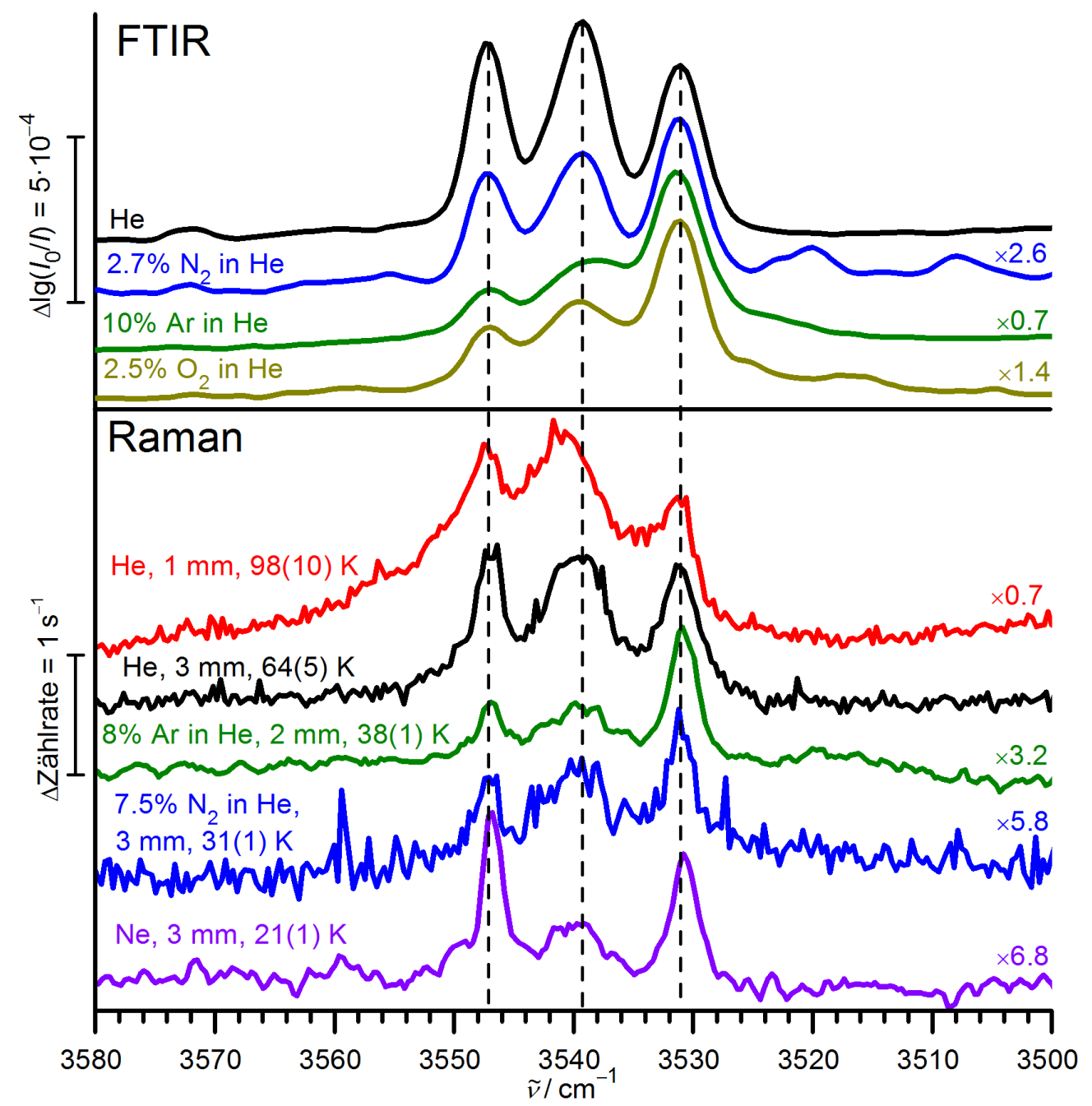

Abb. 4.3: Übersicht von FTIR- und Raman-Spektren im OH-Streckbereich für die Donorbanden von Ethanol-Dimeren mit unterschiedlichen Trägergasmischungen und Düsenabständen, die zu unterschiedlichen Monomer-Konformationstemperaturen führen (jeweils für FTIR und Raman absteigend von oben nach unten sortiert). Die Spektren wurden mit den angegebenen Faktoren auf vergleichbare Maximalintensität der niederfrequenten Bande bei $3531 \mathrm{~cm}^{-1}$ skaliert. Die FTIRSpektren wurden bereits in Ref. [126], die Raman-Spektren (von oben nach unten) in den Ref. [21], [21], [22], [21], [22] veröffentlicht, in Ref. [21] allerdings nicht in diesem Spektralbereich. 
FTIR-Spektrums der Helium-Expansion gebracht wurde. Diese beiden Spektren wurden ausgewählt, da sie die ähnlichsten Expansionsbedingungen aufweisen und zudem das jeweils beste Signal-zu-Rausch-Verhältnis haben. Die anderen Raman-Spektren wurden auf diese Referenz unter Nutzung der in allen Raman-Spektren intensiven und scharfen $t$ Monomerbande skaliert. Die verwendeten Skalierungen sind im Anhang in Tab. A.3 auf S. 307 dokumentiert.

Die Zuordnung der Donor-Banden lässt sich Stand der letzten Analyse aus dem Jahr $2010[22,57]$ wie folgt zusammenfassen. Die niederfrequente Bande wird sehr sicher von dem stabilsten Konformer $g+g+$ verursacht, dies ergibt sich aus der Korrelation mit a) den rotationsspektroskopischen Ergebnissen, b) den Intensitäten der Akzeptorbanden und c) den theoretischen Rechnungen auf verschiedenen Niveaus. Der hochfrequenten Bande wird ein $t$-Akzeptor gemäß Indizien aus b) und c) zugeordnet, allerdings ist die Konformation des Donors unklar. Vermutet wurde hier $g-t$ aufgrund der theoretisch vorhergesagten höheren Stabilität und höheren $\mathrm{OH}-$ Streckfrequenz gegenüber $g+t$. Allerdings basiert diese Vermutung auch auf dem Umstand, dass zu diesem Zeitpunkt $t t$, im Gegensatz zu $g-t$ und $g+t$, noch nicht rotationsspektroskopisch nachgewiesen war. Für die zentrale Bande werden zwei Bandenzentren bei 3541 und $3539 \mathrm{~cm}^{-1}$ (Werte nach Neukalibrierung) vermutet, wobei das für den niederfrequenten Teil verantwortliche Konformer als weniger stabil eingeschätzt wird.

\subsubsection{Quantenchemische Vorhersagen}

Für die initiale systematische Struktursuche wurde auf Basis der für die Monomere gefundenen Ergebnisse B3LYP-D3(BJ)/may-cc-pVTZ als bester Kompromiss aus Genauigkeit und Rechenkosten ausgewählt. Dafür wurden Startstrukturen für alle neun Klassen mit Koordination des rechten freien Elektronenpaares in $30^{\circ}$ Intervallen bezüglich des $\mathrm{C}_{\alpha}^{\mathrm{D}} \mathrm{O}^{\mathrm{D}} \mathrm{O}^{\mathrm{A}} \mathrm{C}_{\alpha}^{\mathrm{A}}$-Torsionswinkels erstellt. Geometrieoptimierung dieser 108 Startstrukturen resultierte in 20 diastereomeren Endstrukturen. Diese Konformere wurden mit den auf MP2/6-311++G(d,p)-Niveau optimierten Strukturen von Loru verglichen, die als Basis der rotationsspektroskopischen Zuordnungen verwendet wurden. Dabei konnte festgestellt werden, dass die sechs energetisch niedrigsten Strukturen qualitativ gut den dort abgebildeten MP2-Strukturen entsprechen (ein detaillierter Vergleich einiger Konformere erfolgt weiter unten in Tab. 4.4). Diese sechs Strukturen sind in Abb. 4.4 dargestellt. Auch die berechnete Energieabfolge entspricht gut, wenn auch nicht perfekt, der von Loru experimentell abgeschätzten (siehe Tab. 4.3), was bezüglich der sehr geringen Energieabstände von wenigen Zehntel $\mathrm{kJ} \mathrm{mol}^{-1}$ und den günstigen Rechenkosten der Methode bemerkenswert ist. Nach diesen sechs Hauptkonformeren innerhalb von nur etwa $0.5 \mathrm{~kJ} \mathrm{~mol}^{-1}$ folgt eine etwas größere energetische Lücke von etwa $0.4 \mathrm{~kJ} \mathrm{~mol}^{-1}$ bis zur siebentstabilsten Struktur $g-g+2$ und dann erneut eine energetische Lücke von etwa $0.5 \mathrm{~kJ} \mathrm{~mol}^{-1} \mathrm{zu}$ den restlichen 13 Konformeren. Die Strukturen und die spektralen Eigenschaften dieser weiteren, bisher nicht rotationsspektroskopisch nachgewiesenen 14 weiteren Konformere sind im Anhang in Abb. A.1 auf S. 305 und Tab. A.1 auf S. 306 dokumentiert. Die Vermutung, dass sechs Konformere energetisch besonders relevant sind, geht auf Dyczmons zurück [106]. Allerdings sei darauf hingewiesen, dass die sechs von ihm benannten Konformere nicht identisch mit den hier berechneten und von Loru et al. nachgewiesenen sind, da Dyczmons $g+t$ fälschlich als enantiomer zu $g-t$ eingeordnet hat. 
Diese sechs Hauptkonformere wurden anschließend mit weiteren DFT-Methoden nachoptimiert. Der Vergleich mit dem wesentlich größeren Basissatz aug-cc-pVQZ bestätigt, dass B3LYP-D3(BJ) praktisch nicht mehr von größeren Basissätzen jenseits von may-cc-pVTZ profitiert, so wie es bereits für die Ethanol-Monomer-Konformere gefunden wurde. Auch Vergleichsrechnungen mit verschärften numerischen Randbedingungen (superfine Integrationsgitter und verytight Optimierungs-Konvergenzkriterien) ergaben vernachlässigbare Unterschiede bezüglich relativer Energien $\left(\leq 0.04 \mathrm{~kJ} \mathrm{~mol}^{-1}\right)$ und relativer OH-Streckschwingungswellenzahlen $\left(\leq 0.3 \mathrm{~cm}^{-1}\right)$. Mit def2-TZVP werden dagegen noch deutlich von den größeren Basisätzen abweichende Ergebnisse erhalten, hier scheint sich die auf diesem Niveau für die Ethanol-Monomere gefundene fälschliche energetische Parität zwischen $t$ und $g$ sich auch negativ auf die Dimer-Energien auszuwirken. Mit der größeren def2-QZVP Basis werden ähnliche Werte wie mit may-cc-pVTZ erhalten, wobei sich die für die Monomere berechnete etwas geringere Stabilität von $t$ tendenziell auch auf die Dimere zu übertragen scheint. B3LYP/may-cc-pVTZ ohne Dispersionskorrektur kann wie erwartet trotz guter Beschreibung der Monomer-Energiedifferenz die Energieabfolge der Dimere in keinster Weise erfassen.

B2PLYP-D3(BJ)/jun-cc-pVTZ liefert ähnlich gute Ergebnisse wie B3LYP-D3(BJ), was insofern überraschend ist, als die Energiedifferenz zwischen $t$ und $g$ im Monomer für diese beiden Methoden deutlich unterschiedlich ist $\left(0.82\right.$ gegenüber $\left.0.24 \mathrm{~kJ} \mathrm{~mol}^{-1}\right)$. Allerdings ist B2PLYP-D3(BJ)/jun-cc-pVTZ bezüglich der Rechenkosten deutlich zu aufwändig für den Einsatz als Routinemethode für größere Systeme. Für B2PLYP müsste hierfür der Basissatz bis auf def2-TZVP verkleinert werden, was aber auch hier

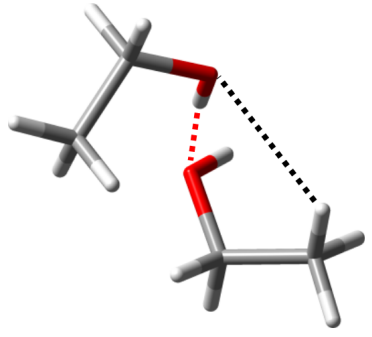

$g+g+0$

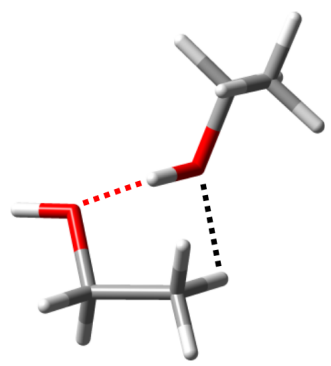

$g+t \quad 0.32$

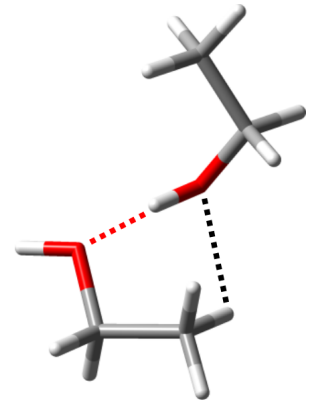

$g-t 0.11$

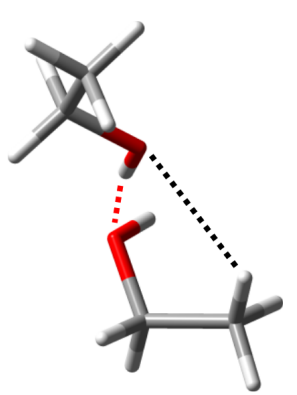

$\operatorname{tg}+0.52$

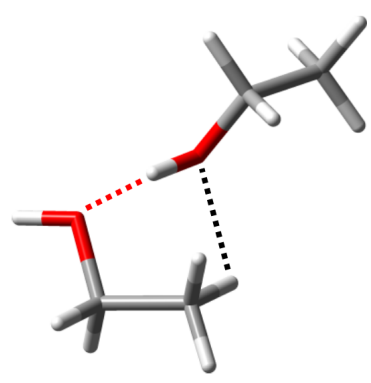

tt 0.31

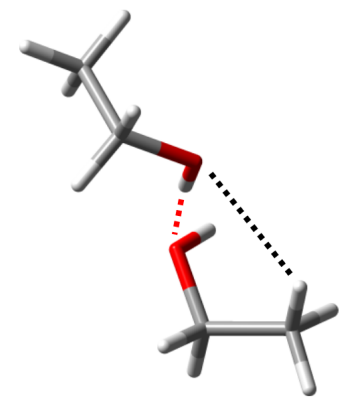

$g-g+0.53$

Abb. 4.4: Strukturen der sechs stabilsten Ethanol-Dimer-Konformere auf B3LYP-D3(BJ)/maycc-pVTZ-Niveau mit nullpunktskorrigierten Energien in $\mathrm{kJ} \mathrm{mol}^{-1}$ relativ zum globalen Minimum $g+g+$. OH $\cdots$ O-Wasserstoffbrücken sind mit roten, $\mathrm{CH} \cdots \mathrm{O}$-Kontakte mit Abständen $<3.0 \AA$ mit schwarzen gestrichelten Linien hervorgehoben. 
Tab. 4.3: Berechnete nullpunktskorrigierte Energien in $\mathrm{kJ} \mathrm{mol}^{-1}$ der sechs Hauptkonformere des Ethanol-Dimers relativ zu $g+g+$ im Vergleich zur qualitativen experimentellen Abfolge aus Ref. [110]. Werte für MP2/6-311++G(d,p) aus Ref. [110] und CCSD(T)/380 cGTOs//MP2/6$311++\mathrm{G}(3 \mathrm{df}, 2 \mathrm{p})$ aus Ref. [108] sind im Gegensatz zu den restlichen Counterpoise-korrigiert. Bis auf die letztgenannten sind alle Werte harmonisch schwingungsnullpunktskorrigiert auf dem Niveau der Strukturoptimierung.

\begin{tabular}{|c|c|c|c|c|c|c|}
\hline & $g+g+$ & $g-t$ & $t t$ & $g+t$ & $\operatorname{tg}+$ & $g-g+$ \\
\hline Experiment [110] & & & $\approx$ & $<$ & $\approx$ & $<$ \\
\hline B3LYP-D3(BJ)/may-cc-pVTZ & 0 & 0.11 & 0.31 & 0.32 & 0.52 & 0.53 \\
\hline B3LYP-D3(BJ)/aug-cc-pVQZ & 0 & 0.14 & 0.32 & 0.33 & 0.50 & 0.54 \\
\hline B3LYP-D3(BJ)/def2-TZVP & 0 & 0.50 & 0.84 & 0.80 & 0.80 & 0.51 \\
\hline B3LYP-D3(BJ)/def2-QZVP & 0 & 0.19 & 0.41 & 0.41 & 0.52 & 0.51 \\
\hline B3LYP/may-cc-pVTZ & 0 & -0.07 & -1.03 & 0.35 & -1.06 & -0.17 \\
\hline B2PLYP-D3(BJ)/jun-cc-pVTZ & 0 & 0.07 & 0.23 & 0.25 & 0.55 & 0.69 \\
\hline B2PLYP-D3(BJ)/def2-TZVP & 0 & 0.40 & 0.69 & 0.66 & 0.67 & 0.54 \\
\hline M06-2X/aug-cc-pVTZ & 0 & 0.72 & 1.50 & 0.98 & 1.40 & 0.76 \\
\hline$\omega$ B97X-D/aug-cc-pVTZ & 0 & 0.40 & 0.42 & 0.21 & 1.31 & 0.46 \\
\hline APF-D/6-31++G(d,p) & 0 & 0.32 & 1.01 & 0.39 & 1.30 & 0.88 \\
\hline MP2/6-311++G(d,p) [110] & 0 & -0.29 & -0.35 & 0.07 & 0.25 & 0.61 \\
\hline MP2/aug-cc-pVDZ & 0 & -0.04 & 0.08 & -0.06 & 1.15 & 0.59 \\
\hline $\begin{array}{l}\text { CCSD(T)/def2-TZVP// } \\
\text { M06/def2-TZVP [123] }\end{array}$ & 0 & 1.09 & 2.76 & 1.26 & 1.21 & 1.17 \\
\hline $\begin{array}{l}\text { CCSD(T)/aug-cc-pVQZ*// } \\
\text { B3LYP-D3(BJ)/aug-cc-pVQZ }\end{array}$ & 0 & -0.05 & 0.03 & 0.22 & 0.33 & 0.59 \\
\hline $\begin{array}{l}\text { CCSD(T)/380 cGTOs// } \\
\text { MP2/6-311++G(3df,2p) [108] } \\
\text { ohne Nullpunktskorrektur }\end{array}$ & 0 & 0.19 & 0.36 & 0.39 & 0.39 & 0.72 \\
\hline
\end{tabular}

mit deutlichen Defiziten für die relativen Energien gegenüber dem größeren Basissatz erkauft werden müsste. Die Ergebnisse aus M06-2X/aug-cc-pVTZ, $\omega$ B97X-D/aug-ccpVTZ, APF-D/6-31++G(d,p) und MP2/aug-cc-pVDZ zeigen ebenfalls deutliche Widersprüche zur experimentellen Abfolge. Bei diesen vier Methoden findet bei der Nachoptimierung von $g-g+$ zudem deutliche Strukturänderungen statt, die in Abschnitt 4.3.7 näher diskutiert werden. Auch die Ergebnisse weiterer Methoden aus der Literatur (MP2/6311++G(d,p) [110] und CCSD(T)/def2-TZVP//M06/def2-TZVP [123]) sind inkompatibel mit der experimentellen Abfolge (Tab. 4.3). Die hochkorrelierte ab initio Methode CCSD(T) kommt hier nicht zur Geltung, vermutlich wegen des zu kleinen Basissatzes und/oder ungeeigneten M06-2X-Geometrien. Unter Verwendung von BasissatzExtrapolation auf aug-cc-pVQZ* und den B3LYP-D3(BJ)/aug-cc-pVQZ-Geometrien und -Schwingungsnullpunktsenergien werden dagegen deutlich kompatiblere Werte erhalten - abgesehen von einer Überschätzung der Energie von $g+g+$. Auch Energien auf CCSD(T)/380 cGTOs//MP2/6-311++G(3df,2p) aus Ref. [108] sind in sehr guter Übereinstimmung, allerdings enthalten diese keine Schwingungsnullpunktskorrektur. Die hier 
durchgeführten Rechnungen und auch solche auf MP2/6-31++(2d,p)-Niveau [106] ergeben, dass die Unterschiede in der Schwingungsnullpunktsenergie ähnlich groß sind wie die in der elektronischen Energie und daher die Abfolge der Gesamtenergie deutlich beeinflussen.

Für $g+g+$, $t t$ und $g-t$ konnten von Loru et al. aus der natürlichen KohlenstoffIsotopenverteilung zudem experimentelle Werte für die $\mathrm{C}_{\alpha}^{\mathrm{A}} \mathrm{C}_{\beta}^{\mathrm{A}} \mathrm{C}_{\alpha}^{\mathrm{D}} \mathrm{C}_{\beta}^{\mathrm{D}}$-Torsionswinkel ermittelt werden. Der Vergleich in Tab. 4.4 zeigt, dass nur B3LYP-D3(BJ) und B2PLYPD3(BJ), mit jeweils ausreichend großer Basis, sowie $\omega$ B97X-D/aug-cc-pVTZ alle drei Werte innerhalb der experimentellen Fehlergrenzen reproduzieren können. Allerdings gilt dies unter dem Vorbehalt, dass quantenchemisch der Einfluss der Nullpunktsschwingungen auf die Geometrien nicht berücksichtigt ist.

Tab. 4.4: Gegenüberstellung zwischen experimentell und quantenchemisch ermittelten $\mathrm{C}_{\alpha}^{\mathrm{A}} \mathrm{C}_{\beta}^{\mathrm{A}} \mathrm{C}_{\alpha}^{\mathrm{D}} \mathrm{C}_{\beta}^{\mathrm{D}}$-Torsionswinkeln $\phi$ für drei Ethanol-Dimer-Konformere. Berechnete Werte außerhalb des experimentellen Fehlerintervalls sind in roter Schriftfarbe hervorgehoben. Die Werte für $\phi(g+g+)$ aus Ref. [110] wurden im Vorzeichen geändert, da mutmaßlich das Enantiomer betrachtet wurde. Der Winkel der in Ref. [110] abgebildeten MP2/6-311++G(d,p)-Struktur für $g+g+$ hat eindeutig das hier angegebene positive Vorzeichen.

\begin{tabular}{llll}
\hline Methode & \multicolumn{1}{c}{$\phi(t t) /^{\circ}$} & $\phi(g+g+) /^{\circ}$ & $\phi(g-t) /^{\circ}$ \\
\hline Experiment [110] & $-138.3(83)$ & $49.3(89)$ & $63.5(41)$ \\
MP2/6-311++G(d,p) [110] & -137.5 & 39.7 & 57.4 \\
MP2/aug-cc-pVDZ & -128.4 & 41.6 & 62.3 \\
B3LYP-D3(BJ)/def2-TZVP & -134.0 & 51.4 & 56.7 \\
B3LYP-D3(BJ)/may-cc-pVTZ & -132.9 & 52.8 & 60.8 \\
B3LYP-D3(BJ)/aug-cc-pVQZ & -132.9 & 53.1 & 61.1 \\
B2PLYP-D3(BJ)/def2-TZVP & -134.9 & 46.7 & 56.3 \\
B2PLYP-D3(BJ)/jun-cc-pVTZ & -133.2 & 47.8 & 60.3 \\
M06-2X/aug-cc-pVTZ & -141.6 & 35.9 & 64.1 \\
$\omega B$ B7X-D/aug-cc-pVTZ & -137.2 & 50.4 & 63.5 \\
APF-D/6-31++G(d,p) & -134.7 & 46.4 & 58.5 \\
\hline
\end{tabular}

\subsubsection{Modellierung von Schwingungsspektren}

Nach Abgleich mit den rotationsspektroskopischen Daten können nun schwingungsspektroskopische Vorhersagen geprüft werden. Aufgrund der zu erwartenden spektralen Überlagerung von mindestens sechs energetisch relevanten Konformeren ist es notwendig das Spektrum explizit zu modellieren, um die Vereinbarkeit von Experiment und Theorie beurteilen zu können. Damit dies mit Theorie in der harmonischen Näherung sinnvoll ist, ist Voraussetzung, dass die Unterschiede bezüglich der Anharmonizität klein sind gegenüber den Unterschieden in der harmonischen Wellenzahl. Ein Deuterierungsexperiment 
[22] und Oberton-Messungen [61, 102] deuten darauf hin, dass dies sowohl für EthanolMonomere wie auch -Dimere eine plausible Annahme ist.

\section{Expansion in Helium bei hohem Düsenabstand}

Der einzige bisherige konkrete Modellierungsversuch des Spektrums unter Berücksichtigung einer Boltzmann-Verteilung und IR-Aktivitäten stammt von Dyczmons [106], der eine zum Experiment ähnliche Dreizack-Formation (mit einigen Defiziten) erhalten konnte. Allerdings wurden die dafür verwendeten MP2-Rechenergebnisse sehr stark nachträglich korrigiert, sowohl bezüglich der relativen Energien, wie auch wenig nachvollziehbar bezüglich der relativen Wellenzahlen. Zudem wurden nur fünf der energetisch niedrigsten Konformere berücksichtigt (ohne $g+t$ ).

Als beste verfügbare Schätzung für die Konformations-Temperatur wird der von Oswald [21] Raman-spektroskopisch aus dem Monomer-Verhältnis bestimmte Wert von 64(5) $\mathrm{K}$ für eine Helium-Expansion bei $3 \mathrm{~mm}$ Detektionsabstand verwendet. Um die Anharmonizität in 1. Ordnung zu korrigieren, wurden die harmonischen Wellenzahlen der Konformere für alle Methoden einheitlich derart verschoben, dass die Position von $g+g+$ mit dem Maximum der entsprechend zuvor zugeordneten niederfrequenten Bande von $3531.2 \mathrm{~cm}^{-1}$ übereinstimmt. Die Halbwertsbreite des Gaußprofils der einzelnen Banden wurde empirisch auf einheitlich $4 \mathrm{~cm}^{-1}$ gesetzt. Eine Gegenüberstellung des experimentellen FTIR-Spektrums von Ethanol in einer Helium-Expansion mit simulierten Spektren auf B3LYP-D3(BJ)-Niveau mit verschiedenen Basissätzen ist in Abb. 4.5 gezeigt. Bereits bei Beschränkung auf die sechs Hauptkonformere liefert B3LYP-D3(BJ)/may-cc-pVTZ eine befriedigende Übereinstimmung mit dem Experiment. Die spektrale Breite der Bandenformation wird gut vorhergesagt, wenn auch scheinbar etwas zu kleineren Wellenzahlen verschoben. Die charakteristische Dreizack-Form deutet sich an, ist aber längst nicht so prägnant wie im Experiment. Das Defizit auf der hochfrequenten Seite kann weitgehend durch die Berücksichtigung der 14 bisher nicht nachgewiesenen Konformere behoben werden, die alle in einem relativ hohen Frequenzbereich absorbieren sollen. Allerdings nicht so hoch, dass eine sehr schwache Bande bei $3572 \mathrm{~cm}^{-1}$ (siehe Abb. 4.5) damit erklärt werden könnte, es sei denn die deutlich schwächere Bindung geht zusätzlich mit einer deutlich geringeren Anharmonizität einher. Dies wäre zumindest nicht völlig überraschend, da die Gesamtanharmonizität des $\mathrm{OH}$-Oszillators bei Ausbildung einer Wasserstoffbrücke in Alkohol-Dimeren wegen eines deutlichen Zunahme der diagonalen Komponente tendenziell steigen sollte.[61] Das Verschwinden der Bande bei Senkung der Konformationstemperatur würde hierzu auch passen, Oswald et al. vermuten hier alternativ als Zuordnung eine Kombinationsbande.[126]

Die nun bereits gute Übereinstimmung lässt sich weiter verbessern, wenn die Anharmonizität in 2. Ordnung korrigiert wird. Dafür wird angenommen, dass die Anharmonizität der OH-Streckschwingung des Donors für alle Dimer-Konformere nicht mehr identisch sei, sondern sich stattdessen bei der Bildung der Wasserstoffbrücke vergleichbar ändert. Dies bedeutet, dass der Unterschied in der Anharmonizität der beiden MonomerKonformere $g$ und $t$ in den Dimeren erhalten bleibt. Der experimentelle Frequenzunterschied beträgt $18 \mathrm{~cm}^{-1}$, der harmonisch berechnete $16.5 \mathrm{~cm}^{-1}$. Wird diese Differenz von $1.5 \mathrm{~cm}^{-1}$ zur Korrektur auf alle Wellenzahlen der Dimer-Konformere mit $t$-Donor addiert, ergibt sich nun eine ausgezeichnete Übereinstimmung mit dem Experiment. Neben der 


\section{Chiralitätssynchronisation in Ethanol-Aggregaten}

fehlenden Anharmonizität werden durch ein solches Verfahren tendenziell auch Fehler in der harmonischen Wellenzahldifferenz mitkorrigiert. Eine vergleichbare Korrektur der relativen Wellenzahlen wurde zuvor auch von Emmeluth et al.[108] verwendet. Das größte verbleibende Defizit ist die etwas zu stark ausladende Flanke auf der niederfrequenten Seite der Simulation, die von $g-g+$ verursacht wird. Die Halbwertsbreite der niederfrequenten experimentellen Bande wirkt gegenüber der hochfrequenten Bande allerdings in der Tat etwas höher (obwohl letztere vermutlich durch energetisch höherliegende Konformere verbreitert wird). Die Vergrößerung des Basissatzes von may-cc-pVTZ auf aug-ccpVQZ hat praktisch keine Auswirkung auf die Simulation (Abb. 4.5). Bei Reduzierung auf def2-TZVP wird dagegen eher eine schmalere Formation mit zwei Maxima und einer Dominanz von $g+g+$ erhalten. Diese ändert sich auch mit nachträglicher Korrektur der Anharmonizität nicht signifikant (nicht abgebildet). Mit def2-QZVP sind die simulierten Spektren sehr ähnlich zu denen mit Dunning-Basissätzen ab may-cc-pVTZ (nicht dargestellt).

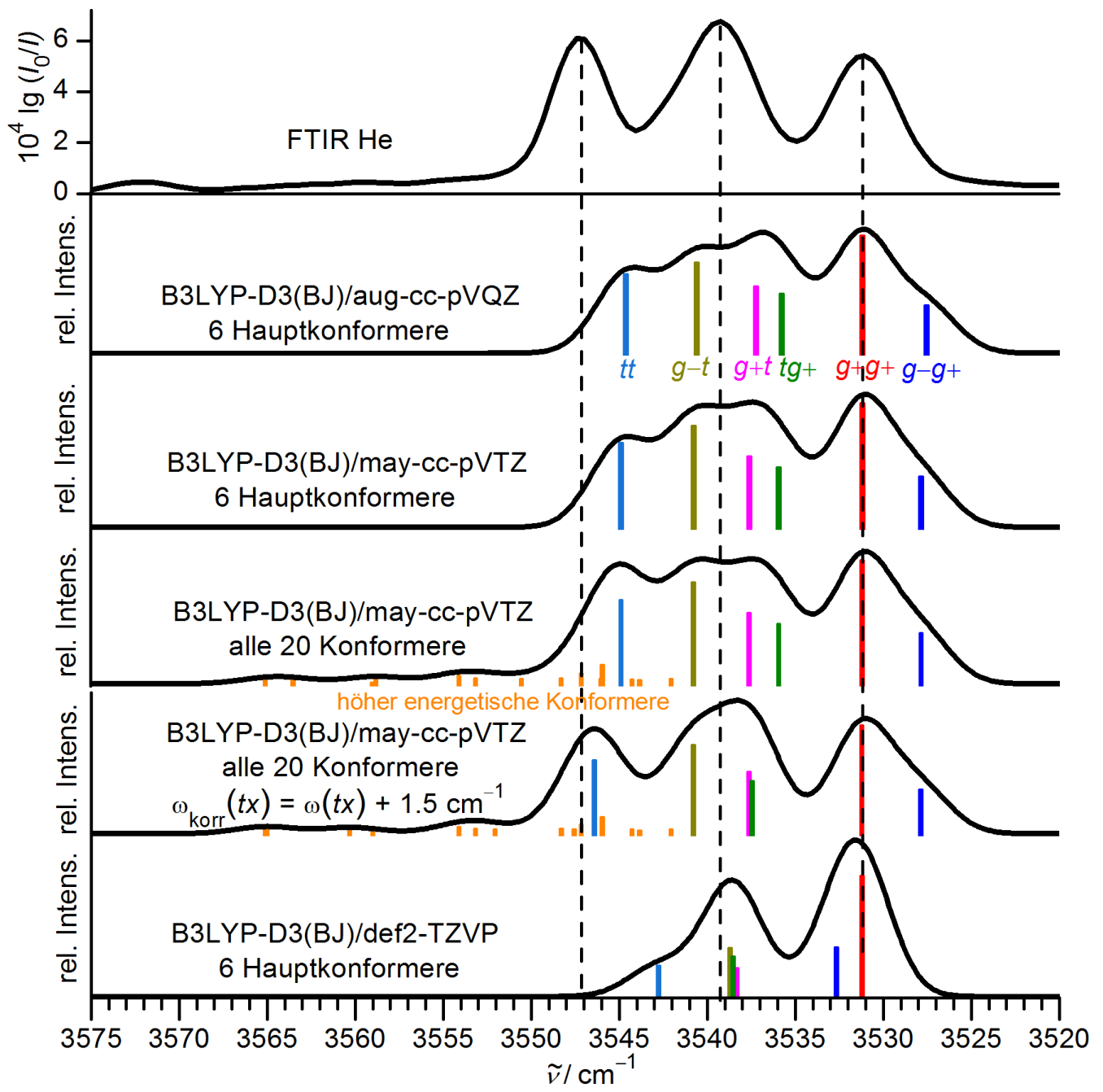

Abb. 4.5: Vergleich des experimentellen FTIR-Spektrums von Ethanol in einer He-Expansion [126] im OH-Streckbereich der Donorbanden mit verschiedenen Simulationen. Simulationsparameter sind Verschiebung der harmonischen Wellenzahlen für $\tilde{v}(g+g+) \stackrel{!}{=} 3531.2 \mathrm{~cm}^{-1}$, Konformationstemperatur $64 \mathrm{~K}$, Gaußprofile mit voller Halbwertsbreite $4 \mathrm{~cm}^{-1}$. 
Abb. 4.6 und 4.7 zeigen die Simulation des selben Spektrums mit weiteren Methoden. B2PLYP-D3(BJ)/jun-cc-pVTZ liefert initial eine mäßige Übereinstimmung, die sich aber mit der aus den Monomeren abgeleiteten zusätzlichen Anharmonizitätskorrektur von $3.9 \mathrm{~cm}^{-1}$ stark verbessert. Die bereits bei B3LYP-D3(BJ) gefundene niederfrequente Flanke durch $g-g+$ verwandelt sich hier in eine ausgeprägte Schulter, die nicht im Experiment vorhanden ist. Mit dem kleinen Basissatz def2-TZVP werden auch hier deutlich weniger kompatible Ergebnisse erhalten, da sich sowohl die relativen Energien wie auch die Frequenzabfolge stark ändern. $\omega$ B97X-D/aug-cc-pVTZ erzielt eine mäßige Übereinstimmung, allerdings mit einer von B3LYP-D3(BJ) und B2PLYP-D3(BJ) stark abweichenden Energie- und Frequenzabfolge der Konformere. $\omega$ B97X-D zeigt auch mit den rotationsspektroskopischen Ergebnissen zumindest für die Strukturen und relative Energien der drei energetisch niedrigsten Konformere gute Übereinstimmung, daher bleibt eine vertauschte Frequenzabfolge von $t t$ und $g-t$ eine nicht völlig abwegige alternative Zuordnung. M062X/aug-cc-pVTZ sagt eine mit dem Experiment inkompatible Doppelpeak-Struktur vorher. Da die harmonische Frequenzdifferenz zwischen $t$ und $g$ für $\omega$ B97X-D und M06-2X

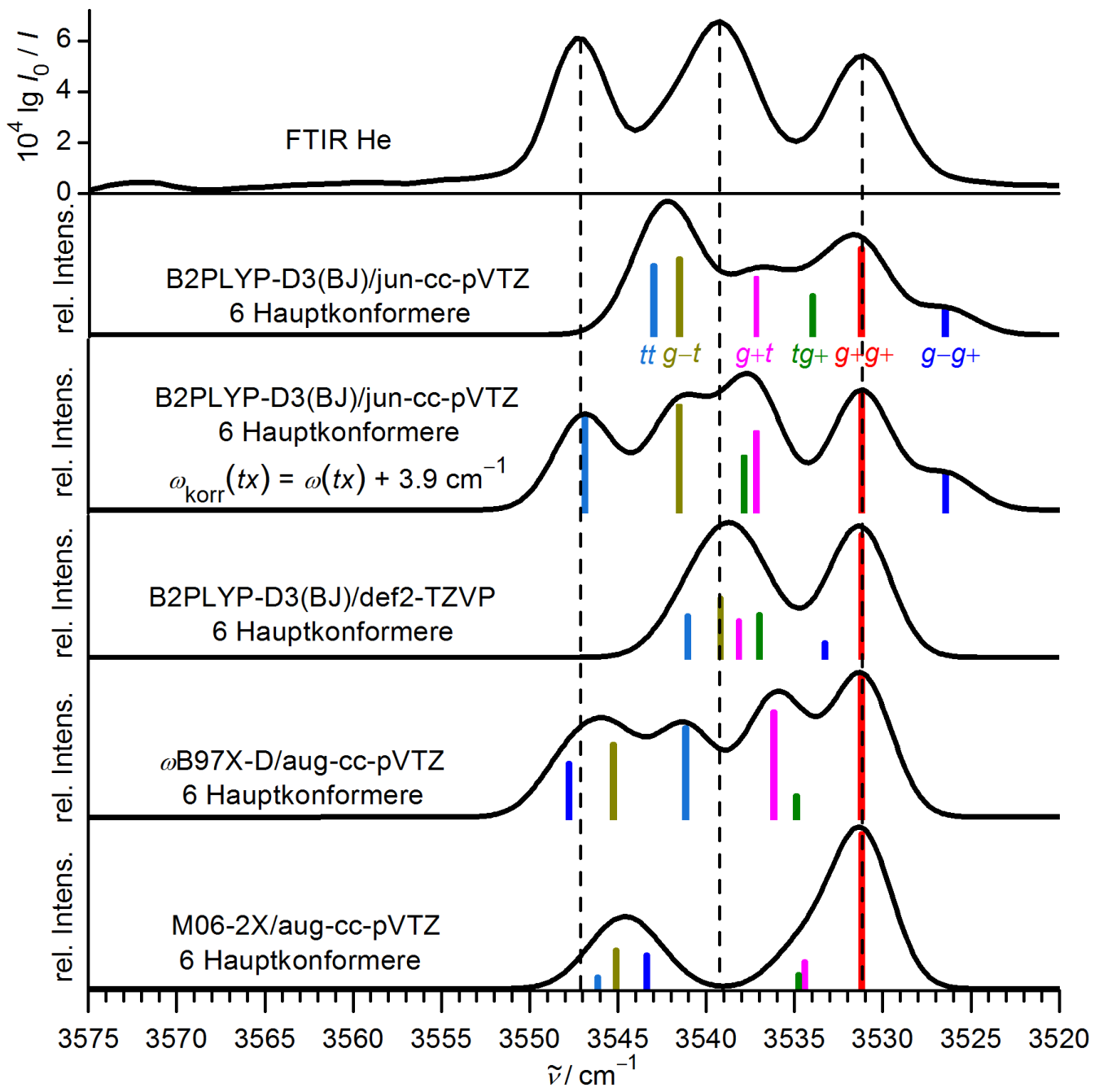

Abb. 4.6: Vergleich des experimentellen FTIR-Spektrums von Ethanol in einer He-Expansion [126] im OH-Streckbereich der Dimer-Donorbanden mit verschiedenen Simulationen. Simulationsparameter sind Verschiebung der harmonischen Wellenzahlen für $\tilde{v}(g+g+) \stackrel{!}{=} 3531.2 \mathrm{~cm}^{-1}$, Konformationstemperatur $64 \mathrm{~K}$ und Gaußprofile mit voller Halbwertsbreite $4 \mathrm{~cm}^{-1}$. 


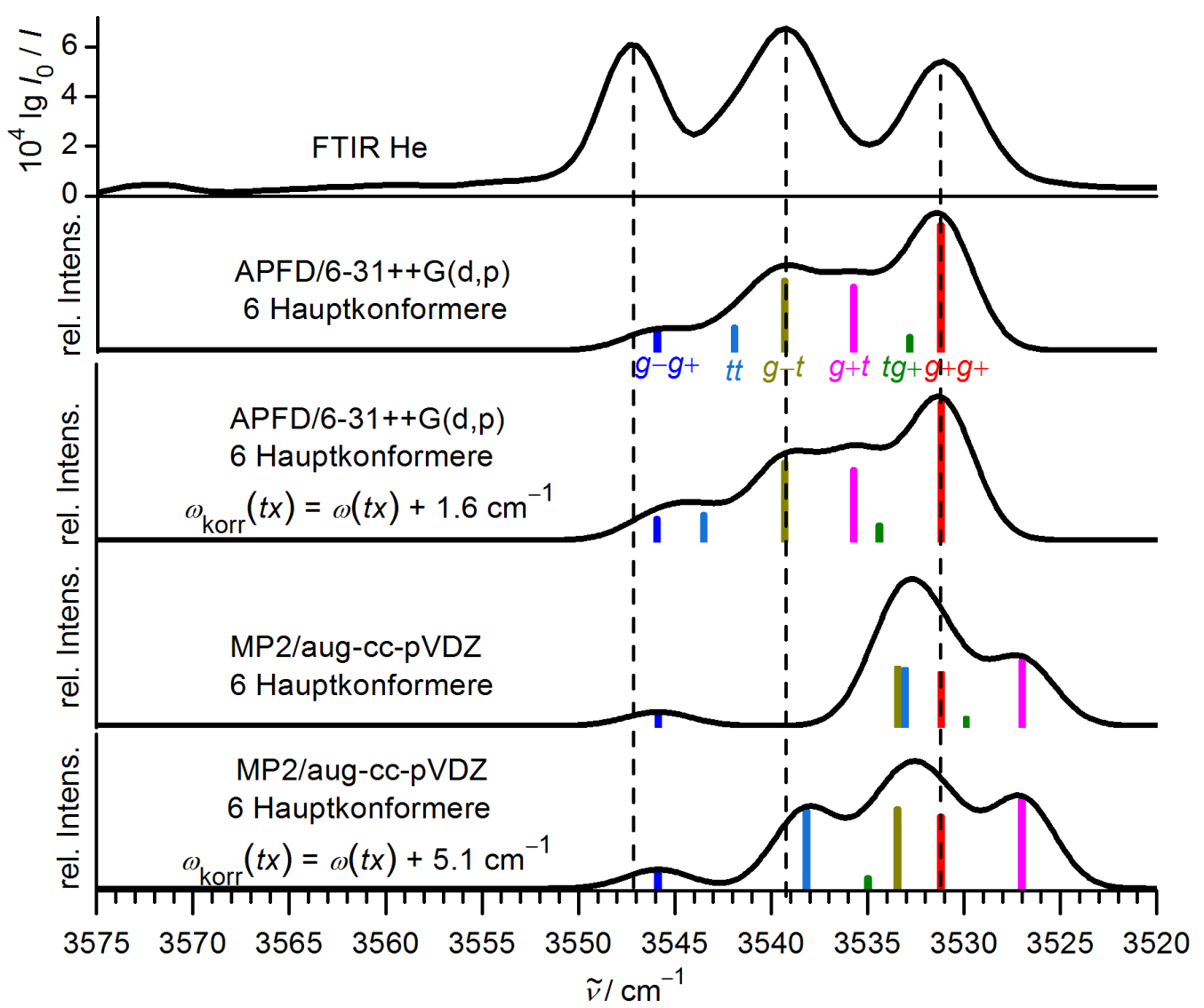

Abb. 4.7: Vergleich des experimentellen FTIR-Spektrums von Ethanol in einer He-Expansion [126] im OH-Streckbereich der Dimer-Donorbanden mit verschiedenen Simulationen. Simulationsparameter sind Verschiebung der harmonischen Wellenzahlen für $\tilde{v}(g+g+) \stackrel{!}{=} 3531.2 \mathrm{~cm}^{-1}$, Konformationstemperatur $64 \mathrm{~K}$ und Gaußprofile mit voller Halbwertsbreite $4 \mathrm{~cm}^{-1}$.

mit 17.8 bzw. $18.7 \mathrm{~cm}^{-1}$ bereits nahe am experimentellen Wert $\left(18 \mathrm{~cm}^{-1}\right)$ liegt, hat hier eine Korrektur kaum Auswirkungen auf die Vorhersage. Auch Simulationen mit APFD/6-31++G(d,p) und MP2/aug-cc-pVDZ zeigen sowohl mit, wie auch ohne, zusätzlicher Korrektur kaum Ähnlichkeit mit dem Experiment (Abb. 4.7).

Als Gesamtergebnis lässt sich festhalten, dass das experimentelle FTIR-Spektrum am besten mit B3LYP-D3(BJ) und einer Basissatzgröße ab may-cc-pVTZ sowie einer überschaubaren anharmonischen Korrektur modelliert werden kann. Auch B2PLYPD3(BJ)/jun-cc-pVTZ liefert eine gute Übereinstimmung, benötigt aber eine größere Korrektur und ist deutlich rechenaufwendiger. Dieser schwingungsspektroskopische Befund steht im Einklang mit der zuvor gefundene gute Kompatibilität mit den rotationsspektroskopischen Daten für diese Methoden. Um zu überprüfen, ob dies nur für den Einzelfall dieser speziellen experimentellen Bedingungen gilt oder die Methode eine breitere Vorhersagekraft bietet, werden im Folgenden auch die Spektren bei anderen Bedingungen (Übersicht in Abb. 4.3) mit B3LYP-D3(BJ)/may-cc-pVTZ simuliert.

Raman-Spektroskopie kann eingesetzt werden, um das FTIR-Spektrum zu bestätigen oder bei Unterschieden komplementäre Informationen zu erhalten. Für den Fall der EthanolDimere wurde gefunden, dass die Bandenformation im Raman-Spektrum eine sehr große Ähnlichkeit zu der im FTIR-Spektrum aufweist. Dies bestätigt die theoretischen Vor- 


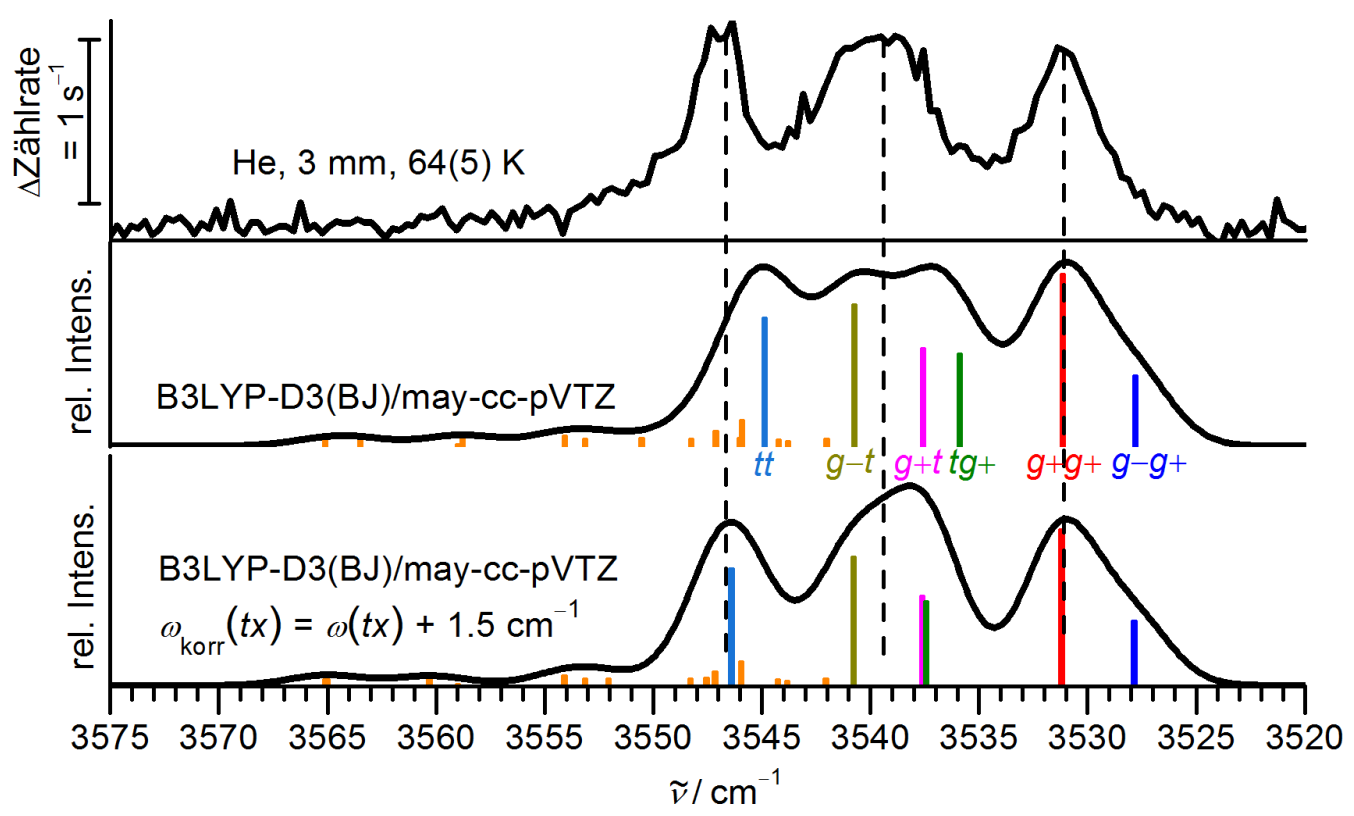

Abb. 4.8: Vergleich des experimentellen Raman-Spektrums von Ethanol in einer He-Expansion bei $3 \mathrm{~mm}$ Detektionsabstand [21] im OH-Streckbereich der Dimer-Donorbanden mit verschiedenen Simulationen. Simulationsparameter sind Verschiebung der harmonischen Wellenzahlen für $\tilde{v}(g+g+) \stackrel{!}{=} 3531.2 \mathrm{~cm}^{-1}$, Konformationstemperatur $64 \mathrm{~K}$ und Gaußprofile mit Halbwertsbreite $4.0 \mathrm{~cm}^{-1}$.

hersagen, dass die in diesem Spektralbereich absorbierenden/streuenden Ethanol-Dimere linear und somit asymmetrisch sind, sowie, dass sich zwischen den Konformeren die Verhältnisse der IR-/Raman-Streuquerschnitte wenig unterscheiden. Entsprechend gut ist auch die Modellierung des Raman-Spektrums mit ansonsten identischen SimulationsParametern in Abb. 4.8.

\section{Expansion in Helium bei geringem Düsenabstand}

Ein Möglichkeit die Konformations-Temperatur zu erhöhen beim Curry-Jet ist die Verringerung des Abstandes zwischen Düse und Detektionsbereich. Beim Übergang von $3 \mathrm{zu}$ $1 \mathrm{~mm}$ steigt die aus den Monomeren abgeschätzte Konformations-Temperatur von 64(5) auf 98(10) K, dieser Wert wurde entsprechend für die Boltzmann-Gewichtung der Dimere verwendet. Zusätzlich wurde die verwendete Halbwertsbreite in der Simulation empirisch auf $5 \mathrm{~cm}^{-1}$ erhöht, um die thermische Verbreiterung der Banden zu berücksichtigen. Es gewinnen die hochfrequente und zentrale Bande an Intensität gegenüber der niederfrequenten, ein Effekt der von der Theorie korrekt beschrieben wird, wie in Abb. 4.9 ersichtlich. Die besonders weit auslaufende Flanke der hochfrequenten Bande kann qualitativ gut durch die Population der energetisch höheren 14 Konformere erklärt werden, was ein Indiz für deren Existenz ist. Dagegen fällt weiterhin die niederfrequente Flanke der Formation steil ab, was gegen eine Streuung von $g-g+$ in diesem Bereich spricht. Insgesamt scheint das gesamte simulierte Spektrum gegenüber dem experimentellen um etwa $1-2 \mathrm{~cm}^{-1} \mathrm{zu}$ tieferen Wellenzahlen verschoben zu sein. Dies könnte auf eine Überlagerung mit heißen Banden hindeuten, die tendenziell bei höheren Wellenzahlen durch eine Schwächung der Wasserstoffbrücke durch thermische Anregung niederfrequenter Moden 


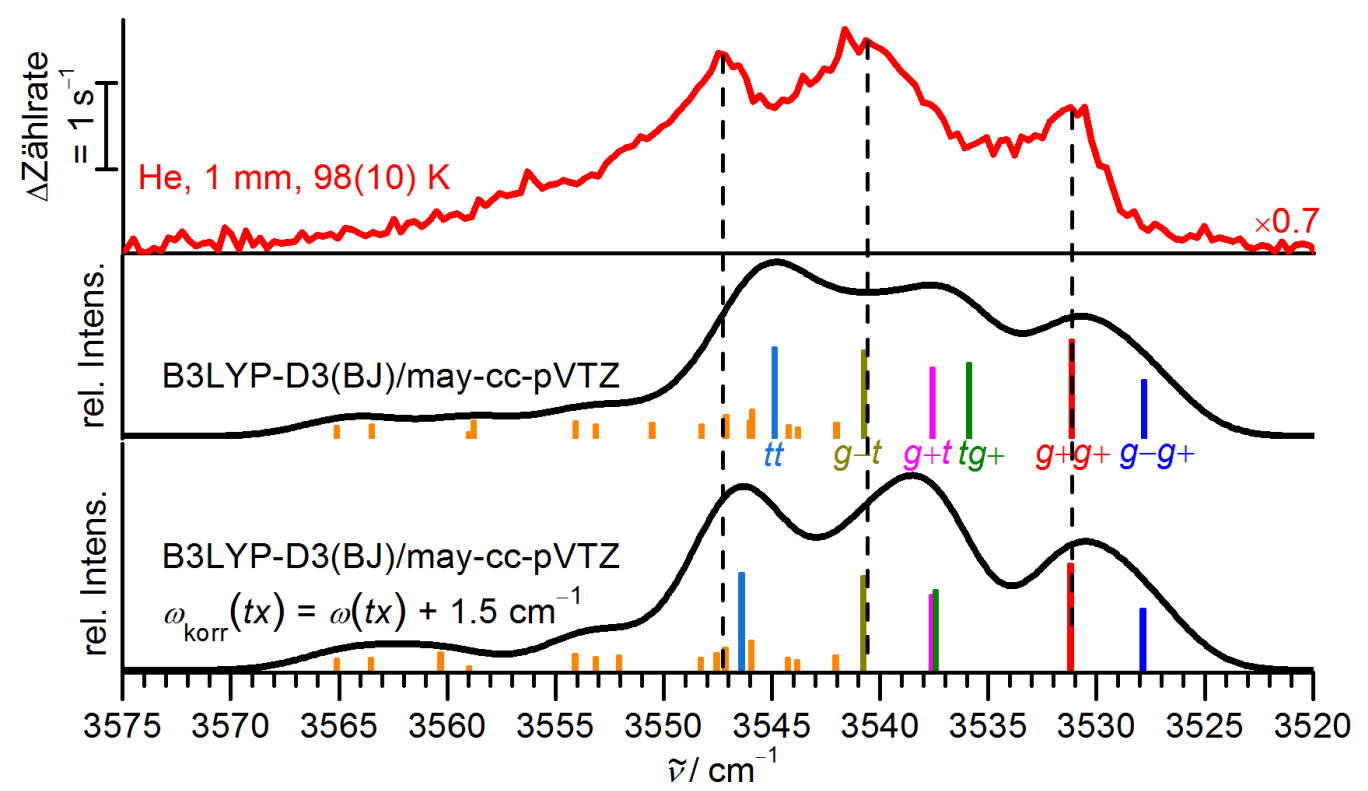

Abb. 4.9: Vergleich des experimentellen Raman-Spektrums von Ethanol in einer He-Expansion bei $1 \mathrm{~mm}$ Detektionsabstand [21] im OH-Streckbereich der Dimer-Donorbanden mit verschiedenen Simulationen. Simulationsparameter sind Verschiebung der harmonischen Wellenzahlen für $\tilde{v}(g+g+) \stackrel{!}{=} 3531.2 \mathrm{~cm}^{-1}$, Konformationstemperatur $98 \mathrm{~K}$ und Gaußprofile mit voller Halbwertsbreite $5 \mathrm{~cm}^{-1}$.

erscheinen.[130] Dieser anharmonische Effekt kann auf dem verwendeten Theorieniveau nicht berücksichtigt werden.

\section{Expansion mit Beimischung von schwereren Trägergasen}

Eine Erhöhung des Abstandes der Detektion über $3 \mathrm{~mm}$ hinaus führt zu keiner weiteren Erniedrigung der Konformationstemperatur.[57] Eine andere Möglichkeit tiefere Temperaturen $\mathrm{zu}$ erreichen, ist die Beimischung von massereicheren und besser polarisierbaren Teilchen in das Trägergas.[23] Dabei sind niedrige Anteile erstrebenswert um die als Nebeneffekt unerwünschte Clusterbildung mit den Teilchen gering zu halten. Diese schrittweise Umhüllung der Dimere ist mit einer zusätzlichen Tiefverschiebung des Absorptions- bzw. Streubereichs verbunden,[126] entsprechende Banden werden in Abb. 4.3, vor allem bei Stickstoff-Beimischung, bereits sichtbar. Der oberere Teil von Abb. 4.10 zeigt die Effekte der Beimischung von $8 \%$ Ar bei $2 \mathrm{~mm}$ und von $7.5 \% \mathrm{~N}_{2}$ bei $3 \mathrm{~mm}$ Düsenabstand im Vergleich mit der Simulation. Die ermittelten Konformations-Temperaturen aus dem Monomer-Verhältnis betragen 38(1) bzw. 31(1) K. Für die Simulation wurde eine Temperatur von $35 \mathrm{~K}$ und eine reduzierte volle Halbwertsbreite von $3 \mathrm{~cm}^{-1}$ verwendet. Wie zu erwarten findet hier, umgekehrt zur zuvor betrachteten Temperaturerhöhung, ein Einbruch der hochfrequenten und der zentralen Bande relativ zur niederfrequenten statt, qualitativ übereinstimmend zwischen Experiment und Theorie. Ein quantitativer Vergleich ist wegen des erniedrigten Signal-zu-Rausch-Verhältnisses schwierig, aber es erscheint, als wäre in der Simulation die zentrale Bande vor allem wegen des Beitrages von $g-t$ etwas zu intensiv. Bei derart niedrigen Temperaturen wirken sich nun auch bereits kleine Fehler in den berechneten Energien signifikant aus. Etwas höhere relativen Energien (im Bereich von etwa $0.1-0.2 \mathrm{~kJ} \mathrm{~mol}^{-1}$ ) für $g-t, g+t$ und $g-g+$ als besserer An- 

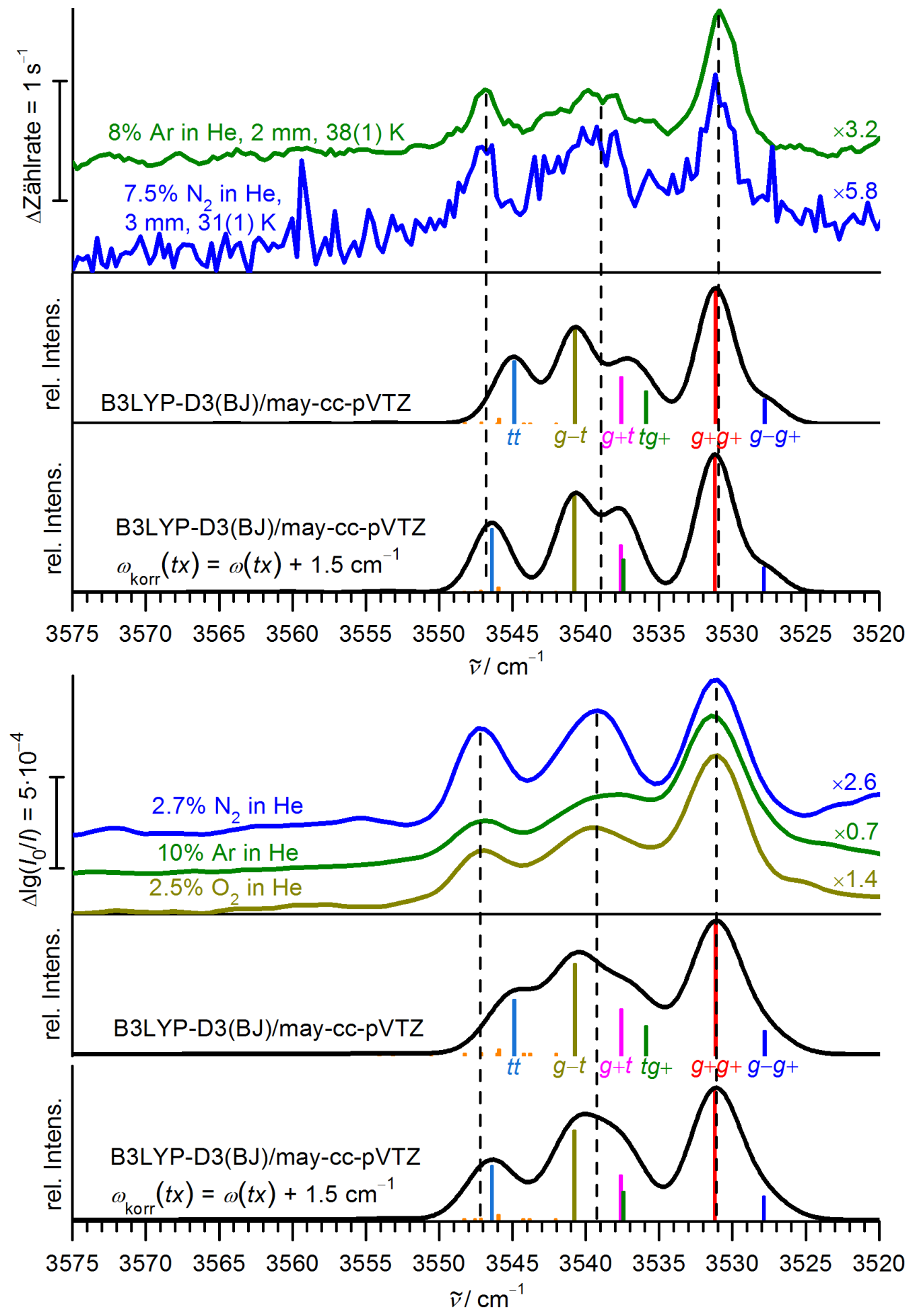

Abb. 4.10: Oben: Vergleich von experimentellen Raman-Spektren von Ethanol in einer HeExpansion mit 8\% Ar [22] bzw. 7.5\% $\mathrm{N}_{2}$ [21] Beimischung bei 2 bzw. $3 \mathrm{~mm}$ Detektionsabstand im OH-Streckbereich der Dimer-Donorbanden mit verschiedenen Simulationen. Unten: Vergleich der experimentellen FTIR-Spektren von Ethanol in einer He-Expansion mit 2.7\% $\mathrm{N}_{2} / 10 \% \mathrm{Ar}$ / 2.5\% $\mathrm{O}_{2}$ Beimischung [126] im OH-Streckbereich der Dimer-Donorbanden mit verschiedenen Simulationen. Simulationsparameter sind Verschiebung der harmonischen Wellenzahlen für $\tilde{v}(g+g+) \stackrel{!}{=} 3531.2 \mathrm{~cm}^{-1}$, Konformationstemperatur $35 \mathrm{~K}$ und Gaußprofile mit voller Halbwertsbreite $3 \mathrm{~cm}^{-1}$ (Raman) bzw. $4 \mathrm{~cm}^{-1}$ FTIR). 


\section{Chiralitätssynchronisation in Ethanol-Aggregaten}

näherung zu der von Loru et al. [110] experimentellen Abfolge in Tab. 4.3 würden die Übereinstimmung mit dem experimentellen Spektrum verbessern.

Entsprechende Relaxationsexperimente mit Beimischung von $\mathrm{Ar}, \mathrm{N}_{2}$ und zusätzlich $\mathrm{O}_{2}$ wurden auch in FTIR-detektierten Expansionen durchgeführt, siehe unterer Teil von Abb. 4.10. Der Vergleich mit der Simulation bestätigt die aus der Ramanspektroskopischen Untersuchung gewonnenen Befunde. Bei weiterer Erhöhung des Anteils dieser schweren Trägergase verbleibt schließlich allein $g+g+$ als einziges nachweisbares Konformer ohne Umhüllung.[22, 108, 110]

\section{Expansion in Neon und Relaxationspfade}

Noch tiefere Temperaturen ohne das Problem der Clusterbildung mit dem Trägergas lassen sich alternativ mit Neon erzielen. Die von Wassermann ermittelte Monomer-Konformationstemperatur sinkt hier bis auf 21(1) K. Der Vergleich mit anderen experimentellen Spektren in Abb. 4.3 und auch der Simulation in Abb. 4.11 zeigt eine deutliche Abweichung vom bisher beobachteten Trend. Während die zentrale Bande wie erwartet schwach ausfällt (insbesondere auf der niederfrequenten Seite), weist die hochfrequente nun wieder eine ähnliche Intensität wie die niederfrequente auf. Dieser Effekt kann nicht mehr durch eine Gleichgewichtsverteilung mit einer einheitlichen Konformationstemperatur erklärt werden, hier erscheint eine kinetische Stabilisierung von $t t$ wahrscheinlich. Gemäß den berechneten Bandenpositionen sind $g+g+, t t$ und $g-t$ noch am stärksten populiert, was der Beobachtung von Loru et al. [110] entspricht, die in einer Neon-Expansion nur noch diese drei Konformere nachweisen konnte. Sinkt die Konformationstemperatur tief genug, können Barrieren oberhalb einer bestimmten Schwelle nicht mehr überwunden werden. $t t$ erscheint aus verschiedenen Gründen prinzipiell hierfür besonders anfällig. So enthält $t t$ zweifach das stabilste Monomer-Konformer $t$. Falls also schon vor der Bil-

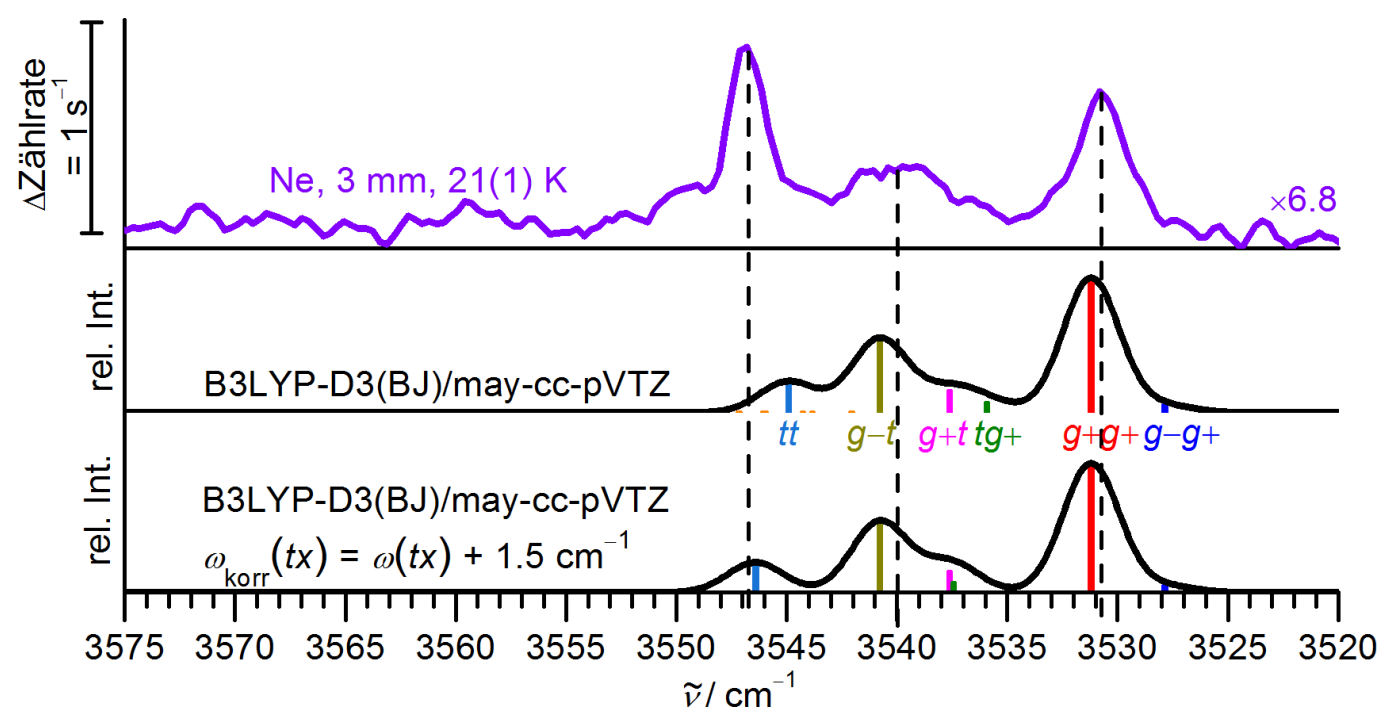

Abb. 4.11: Vergleich des experimentellen Raman-Spektrums von Ethanol in einer Ne-Expansion bei $3 \mathrm{~mm}$ Detektionsabstand [22] im OH-Streckbereich der Dimer-Donorbanden mit verschiedenen Simulationen. Simulationsparameter sind Verschiebung der harmonischen Wellenzahlen für $\tilde{v}(g+g+) \stackrel{!}{=} 3531.2 \mathrm{~cm}^{-1}$, Konformationstemperatur $21 \mathrm{~K}$ und Gaußprofile mit voller Halbwertsbreite $3 \mathrm{~cm}^{-1}$. 
dung der Dimere eine signifikante Relaxation der Monomer-Konformere stattfindet (die errechnete Konformationstemperatur von $21 \mathrm{~K}$ basiert auf einem beobachteten Populationsanteil von $88 \%$ t im Monomer), so erscheint es plausibel, dass initial im hohen Maße $t t$ gebildet wird. Bei anschließender ebenfalls schneller Abkühlung des gebildeten Dimers mit resultierender gehemmter Isomerisierung würde daher eine besonders starke Abweichung von der Gleichgewichtspopulation für $t t$ resultieren. Zusätzlich erfordert $t$, anders als die anderen Konformere, zwei intramolekulare Umwandlungsschritte um zu $g+g+\mathrm{zu}$ relaxieren. Dabei scheint aber die erste Umwandlung eines $t$-Konformers entscheidender, da die als Zwischenprodukt erhaltenen $g / t$-gemischten Dimer-Klassen offenbar weiterhin effektiv relaxieren können. Für die Umwandlung der neun Dimer-Klassen untereinander sind folgende Elementarschritte anzunehmen:

- Typ A) intramolekulare Umwandlungen zwischen $t, g+$ und $g-$

- Typ B) Wechsel des koordinierten Elektronenpaares des Sauerstoffatoms

- Typ C) Tausch von Donor- und Akzeptorrolle zwischen den Molekülen [131]

Alle Klassen von Ethanol-Dimeren (mit Ausnahme von $g-g-$ ) müssen einen oder mehrere Elementarschritte des Typs A durchlaufen um in das globale Minimum $g+g+$ relaxieren zu können. Etwaige klassenspezifisch hohe Barrieren für Typ A könnten aber durch vorangestellte Umlagerungen nach Typ B und/oder C umgangen werden. Könnte beispielsweise hypothetisch $t g+$ nicht direkt in $g+g+$ über Typ A relaxieren, könnte es sich zunächst über Typ B in $t g-$ (bzw. dessen Enantiomer), über Typ C in $g+t$ oder über die Kombination beider Schritte zu $g-t$ umlagern. Eine ausreichend niedrige Barriere für Typ A in nur einer dieser vier Klassen wäre daher ausreichend für eine effektive Relaxation. Für $t t$ führen Umwandlungen nach Typ B und Typ C dagegen nur erneut zu $t t$, sodass weniger Relaxationspfade für A zur Verfügung stehen und eine kinetische Hemmung wahrscheinlicher wird. Dyczmons hatte Umwandlungsschritte nach Typ A und Typ B an je einem Beispiel untersucht und für Typ A eine höhere Barriere gefunden (3.4 gegenüber $1.3 \mathrm{~kJ} \mathrm{~mol}^{-1}$ ),[106] was diese Einschätzung stützt. Barrieren für Umwandlungen nach Typ C für Alkohol-Dimere scheinen tendenziell nochmals leicht höher zu liegen (3.9-4.7 kJ mol ${ }^{-1}$, allerdings berechnet für das Paar Methanol/Ethanol und mit einem anderen Theorie-Niveau).[131] Eine systematische quantenchemische Erforschung dieser Umwandlungsreaktionen könnte in Zukunft tiefere Einsichten in diese Dynamik bieten. Zuvor wäre aber eine Bestätigung des experimentellen Befundes in einer Neon-Expansion mit FTIR-Detektion sinnvoll. Der in Erprobung befindliche Gratin-Jet [132] könnte genutzt werden um die hohen Kosten von Ne durch Gasrecycling zu verringern. Dass dagegen $t t$ bei der ebenfalls starken Kühlung durch Beimischung von elektronenreicheren Trägergasen wie Ar relaxieren kann,[108, 110, 125] lässt einen alternativen assoziativen Relaxationsmechanismus vermuten, bei dem nicht nur Stöße, sondern auch temporär gebildete Cluster mit den besser polarisierbaren Teilchen involviert sind.[23]

\section{Deuteriertes Ethanol}

Ein weiterer Test, ob das experimentelle Spektrum mit harmonischer Theorie adäquat beschrieben werden kann, ist ein Austausch des Protons mit einem Deuteron im HydroxyOszillator. Dabei kann vereinfacht angenommen werden, dass die harmonische Wellen- 


\section{Chiralitätssynchronisation in Ethanol-Aggregaten}

zahl nur durch die Änderung der reduzierten Masse beeinflusst wird, während das Potential unverändert bleibt. Dies wurde von Wassermann et al. genutzt, um die diagonalen Anharmonizitätskonstanten von Ethanol-Monomeren und -Dimeren abzuschätzen.[22] Allerdings stimmen die mit diesem Verfahren ermittelten Werte eher schlecht mit solchen aus späteren Obertonmessungen überein.[105]

Durch Schwingungsnullpunktsenergie-Effekte verändern sich die hier berechneten relativen Energien der Konformere vernachlässigbar, im Bereich von $0.02 \mathrm{~kJ} \mathrm{~mol}^{-1}$. Die Raman-Streuquerschnitte nehmen durch Deuterierung ab, für Konformere mit $t$-Donor etwas stärker (ca. Faktor 0.52) als für solche mit $g$-Donor (ca. Faktor 0.57). Dieser Unterschied ist allerdings zu schwach um bei dem vorliegenden Signal-zu-Rausch-Verhältnis für Zuordnungen ausgenutzt zu werden. Die IR-Aktivitäten nehmen ähnlich stark ab, allerdings ohne klare Diskriminierung zwischen den Konformeren. Für Jet-Messungen von EtOD liegen bisher nur Spektren mit Raman-, aber nicht mit FTIR-Detektion vor. Die für die Simulation berechneten spektroskopischen Daten für EtOD-Dimere sind im Anhang in Tab. A.2 auf S. 307 dokumentiert. Aus dem EtOD-Spektrum wurde keine Konformationstemperatur aus dem Monomer-Verhältnis abgeschätzt, daher wurde die Temperatur $(115 \mathrm{~K})$ aus dem unter gleichen Bedingungen aufgenommenen EtOH-Spektrum von Wassermann [57] übernommen. Bei Vergleich der Spektren von Oswald und Wassermann für EtOH wurde bei kurzen Düsenabständen festgestellt, dass diese hinsichtlich Konformationstemperaturen und Halbwertsbreiten nicht direkt vergleichbar sind, vermutlich wegen baulicher Änderungen des Curry-Jets in den dazwischenliegenden sieben Jahren. Für die Anharmonizitätskorrektur 1. Ordnung für EtOD wurde analog zu EtOH das stabilste Konformer $g+g+$ auf die Position der niederfrequenten Bande von $2607.4 \mathrm{~cm}^{-1}$ verschoben und dieser Wert $\left(-45.0 \mathrm{~cm}^{-1}\right)$ für alle anderen Konformere übernommen. Die experimentelle Differenz der OD-Streckschwingungswellenzahlen zwischen $t$ und $g$ beträgt $14 \mathrm{~cm}^{-1}$, die mit B3LYP-D3(BJ)/may-cc-pVTZ berechnete $13.0 \mathrm{~cm}^{-1}$, daher wurde für die Anharmonizitätskorrektur 2. Ordnung Konformere mit $t$-Donor um $+1 \mathrm{~cm}^{-1} \mathrm{zu}-$ sätzlich verschoben. Der Vergleich zwischen Experiment und Simulation ist in Abb. 4.12 gezeigt. Wie bereits für EtOH ergibt sich für EtOD nach Anharmonizitätskorrektur 1. Ordnung eine gute, nach 2. Ordnung eine ausgezeichnete Übereinstimmung zwischen Experiment und Simulation. Die gemachten Annahmen und die Aussagekraft der Theorie in Form von B3LYP-D3(BJ)/may-cc-pVTZ bestätigen sich hier. Auch bei EtOD zeigt sich bei hoher Konformationstemperatur auf der hochfrequenten Seite der Bandenformation zusätzliche Ramanstreuung, die auf schwächer gebundene Konformere und/oder heiße Banden der Haupkonformere hinweist. Das einzige größere Defizit der Modellierung ist erneut die vorhergesagte niederfrequente Schulter durch $g-g+$, für die es auch bei EtOD keine experimentelle Evidenz gibt.

\subsubsection{Energetisch höher liegende Konformere}

Auf die Frage, an welcher spektralen Postion das sechststabilste Konformer $g-g+$ absorbiert bzw. streut, liefert auch die Messung mit EtOD keine Antwort. Da dieses Konformer rotationsspektroskopisch nachgewiesen wurde,[110] können die Struktur und die ungefähre relative Energie nicht als Fehlvorhersagen der Theorie abgetan werden.

Eine Parallele zeigt sich zu dem zweitstabilsten Dimer von 2,2,2-Trifluorethanol, welches ebenfalls der Klasse $g-g+$ angehört. Dieses enzieht sich bisher ebenso dem 
schwingungsspektroskopischen Nachweis [133, 134], während es rotationsspektroskopisch nachgewiesen werden konnte - wenn auch in deutlich geringerer Konzentration als nach den Berechnungen erwartet.[135] Solche Diskrepanzen zwischen Vorhersagen für homo- und heterochirale Kombinationen können prinzipiell in einen von dem Theorieniveau nicht oder ungenügend berücksichtigten Effekt wie CISS (Kap. 2.4) begründet sein. Zur Bewertung eines solchen systematischen Einflusses ist aber die Betrachtung einer größeren Zahl an Systemen notwendig, für die die vorliegende Arbeit einen Beitrag zu leisten versucht.

Wie zuvor erwähnt, sind die genaue Struktur, die relative Energie und die relative $\mathrm{OH}$ Streckfrequenz für $g-g+$ von Ethanol deutlich stärker abhängig vom verwendeten Theorieniveau als für die anderen Konformere. Diese strukturellen Unterschiede für $g-g+$ sind in Abb. 4.13 exemplarisch anhand der Ergebnisse für B3LYP-D3(BJ)/may-cc-pVTZ und $\omega$ B97X-D/aug-cc-pVTZ visualisiert. Die Struktur für $\omega$ B97X-D wirkt insgesamt kompakter und bildet einen zweiten $\mathrm{C}-\mathrm{H} \cdots \mathrm{O}$-Kontakt $<3.0 \AA$ aus, weist aber auch einen ungewöhnlichen $\mathrm{C}_{\alpha}^{\mathrm{D}} \mathrm{O}^{\mathrm{D}} \mathrm{O}^{\mathrm{A}} \mathrm{C}_{\alpha}^{\mathrm{A}}$-Torsionswinkel auf (siehe Vergleich der Konformere weiter unten). Vermutlich tritt hier eine Konkurrenz zwischen Dispersions- und Dipol-DipolWechselwirkung auf, deren Balance von den Methoden unterschiedlich bewertet wird. Tab. 4.5 listet die Differenz zwischen der $\mathrm{OH}-$ Streckschwingungswellenzahl von $g-g+$ und $g+g+$ in Abhängigkeit des Theorie-Niveaus und die Diederwinkel $\mathrm{C}_{\alpha}^{\mathrm{A}} \mathrm{C}_{\beta}^{\mathrm{A}} \mathrm{C}_{\alpha}^{\mathrm{D}} \mathrm{C}_{\beta}^{\mathrm{D}}$ und $\mathrm{C}_{\alpha}^{\mathrm{D}} \mathrm{O}^{\mathrm{D}} \mathrm{O}^{\mathrm{A}} \mathrm{C}_{\alpha}^{\mathrm{A}}$ für $g-g+$. Es lässt sich deutlich eine positive Korrelation zwischen den Werten der Diederwinkel und der relativen $\mathrm{OH}-$ Streckschwingungswellenzahl erkennen.

Eine nähere Analyse der experimentellen Struktur von $g-g+$ würde daher nicht nur eine Bewertungsmöglichkeit von quantenchemischen Methoden für die Beschreibung solcher flexiblen Anordnungen bieten, sondern auch Anhaltspunkte dafür liefern an welcher

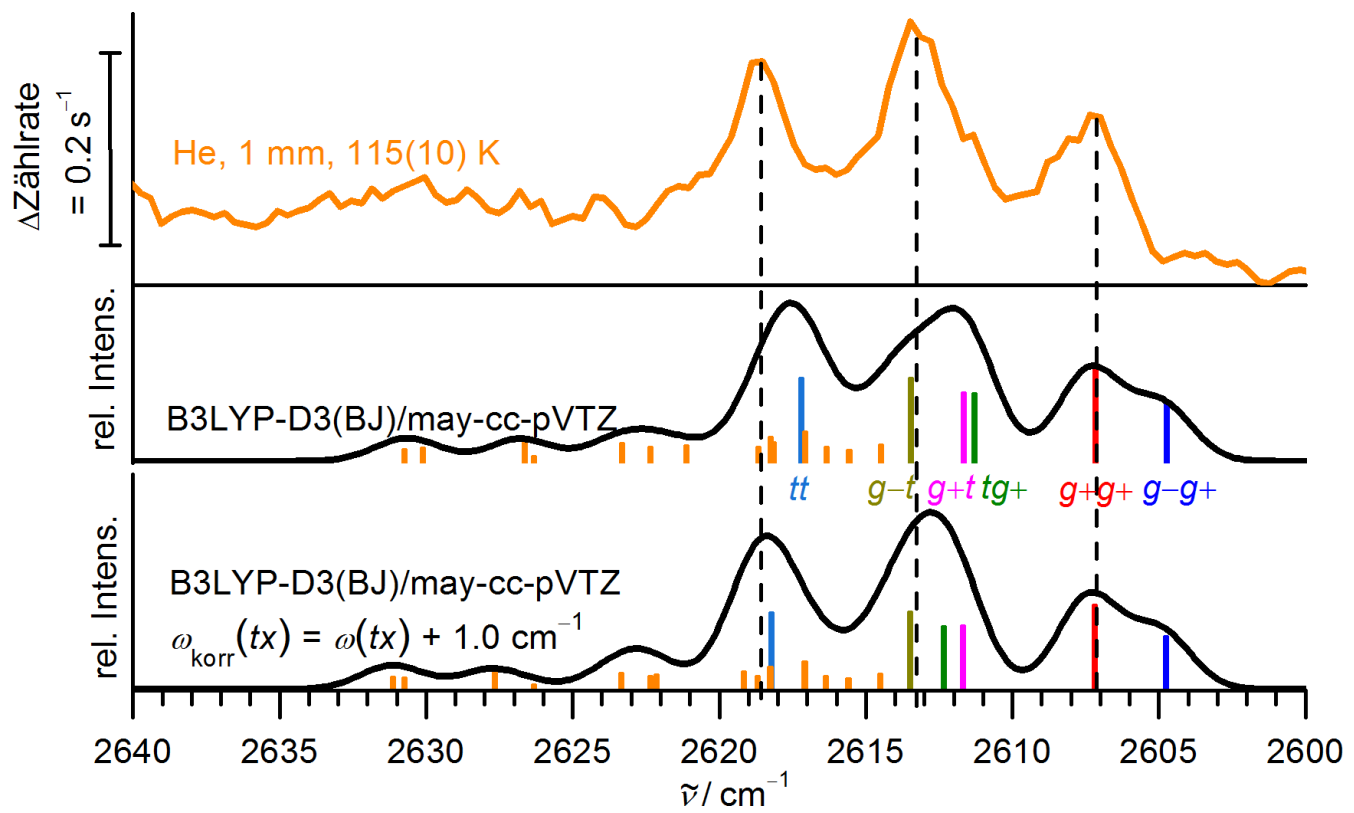

Abb. 4.12: Vergleich des experimentellen Raman-Spektrums von Ethanol-OD in einer HeExpansion bei $1 \mathrm{~mm}$ Detektionsabstand [22] im OD-Streckbereich der Dimer-Donorbanden mit verschiedenen Simulationen. Simulationsparameter sind Verschiebung der harmonischen Wellenzahlen für $\tilde{v}(g+g+) \stackrel{!}{=} 2607.4 \mathrm{~cm}^{-1}$, Konformationstemperatur $115 \mathrm{~K}$ und Gaußprofile mit voller Halbwertsbreite $2.5 \mathrm{~cm}^{-1}$. 


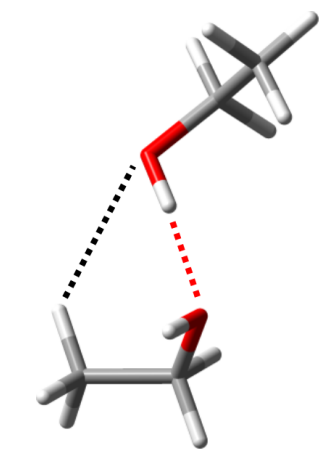

B3LYP-D3(BJ)/

may-cc-pVTZ

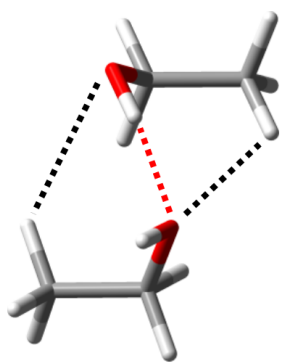

$\omega \mathrm{B} 97 \mathrm{X}-\mathrm{D} /$

aug-cc-pVTZ

Abb. 4.13: Strukturen von $g-g+$ auf B3LYP-D3(BJ)/may-cc-pVTZ und $\omega$ B97X-D/aug-cc-pVTZ-

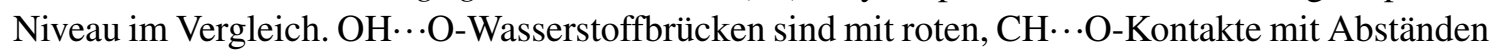
$<3.0 \AA$ mit schwarzen gestrichelten Linien hervorgehoben. Für $\omega$ B97X-D/aug-cc-pVTZ wurde zusätzlich verifiziert, dass es sich um das tiefste Minimum der $g-g+-$ Klasse handelt.

Stelle $g-g+$ im Schwingungsspektrum zu erwarten wäre. Anders als für die drei stabilsten Konformere war eine experimentelle Klärung der genauen Struktur von $g-g+$ wegen der geringen Population in Zusammenhang mit den niedrigen natürlichen ${ }^{13} \mathrm{C}$ - und ${ }^{18} \mathrm{O}$ Isotopenhäufigkeiten bisher nicht möglich.[110] Dies könnte aber durch Anreicherung der ${ }^{13} \mathrm{C}_{\alpha^{-}},{ }^{13} \mathrm{C}_{\beta^{-}}$und optional ${ }^{18} \mathrm{O}$-Isotopologen von Ethanol bewerkstelligt werden diese sind kommerziell verfügbar und für die Rotationsspektroskopie werden nur sehr geringe Mengen benötigt. Die Praktikabilität wurde bereits für gemischte Dimere aus Ethanol und Fenchon demonstriert, bei denen drei Konformere durch den Einsatz der ${ }^{13}$ C-Isotopologen zugeordnet werden konnten.[136] Dabei wurde gefunden, dass experimentelle Geometrien und die Energieabfolge der Konformere gut mit solchen berechnet auf B3LYP-D3(BJ)/6-311++G(d,p)-Niveau übereinstimmen.

Eine denkbare, mit den harmonischen Vorhersagen von B3LYP-D3(BJ) und B2PYLPD3(BJ) mit größeren Basissätzen vereinbare Möglichkeit, wäre eine deutlich geringere

Tab. 4.5: Abhängigkeit der Geometrie von $g-g+$ (repräsentiert durch die $\mathrm{C}_{\alpha}^{\mathrm{A}} \mathrm{C}_{\beta}^{\mathrm{A}} \mathrm{C}_{\alpha}^{\mathrm{D}} \mathrm{C}_{\beta}^{\mathrm{D}}$ - und $\mathrm{C}_{\alpha}^{\mathrm{D}} \mathrm{O}^{\mathrm{D}} \mathrm{O}^{\mathrm{A}} \mathrm{C}_{\alpha}^{\mathrm{A}}$-Torsionswinkel) und der Differenz der OH-Streckschwingungswellenzahl zu $g+g+$, vom Theorieniveau.

\begin{tabular}{|c|c|c|c|}
\hline Methode & $\phi\left(\mathrm{C}_{\alpha}^{\mathrm{A}} \mathrm{C}_{\beta}^{\mathrm{A}} \mathrm{C}_{\alpha}^{\mathrm{D}} \mathrm{C}_{\beta}^{\mathrm{D}}\right)$ & $\begin{array}{c}\phi\left(\mathrm{C}_{\alpha}^{\mathrm{D}} \mathrm{O}^{\mathrm{D}} \mathrm{O}^{\mathrm{A}} \mathrm{C}_{\alpha}^{\mathrm{A}}\right) \\
/^{\circ}\end{array}$ & $\begin{array}{l}\Delta \omega_{\mathrm{OH}} \\
/ \mathrm{cm}^{-1}\end{array}$ \\
\hline B2PLYP-D3(BJ)/jun-cc-pVTZ & -68 & -118 & -5 \\
\hline B3LYP-D3(BJ)/aug-cc-pVQZ & -65 & -117 & -4 \\
\hline B3LYP-D3(BJ)/may-cc-pVTZ & -64 & -116 & -3 \\
\hline $\mathrm{MP} 2 / 6-311++\mathrm{G}(\mathrm{d}, \mathrm{p})$ & -68 & -119 & 0 \\
\hline B3LYP-D3(BJ)/def2-TZVP & -50 & -100 & 1 \\
\hline B2PLYP-D3(BJ)/def2-TZVP & -52 & -99 & 2 \\
\hline M06-2X/aug-cc-pVTZ & -63 & -100 & 12 \\
\hline MP2/aug-cc-pVDZ & 5 & -53 & 15 \\
\hline APF-D/6-31++G(d,p) & 6 & -55 & 15 \\
\hline$\omega \mathrm{B} 97 \mathrm{X}-\mathrm{D} /$ aug-cc-pVTZ & 10 & -49 & 17 \\
\hline
\end{tabular}


Anharmonizität von $g-g+$ gegenüber den anderen Konformeren. Dies würde statt der nicht beobachteten niederfrequenten Schulter zu einer höherfrequenten Position im Bereich der anderen Konformere führen. Dies könnte spekulativ auch eine alternative Erklärung für die laut Simulation zusätzlich zu $t$ benötigte Intensität für die hochfrequente Bande sein.

Diese Frage steht auch im Zusammenhang mit der nach der Bedeutung der anderen energetisch höher liegenden Konformere, die durch Torsion um die Wasserstoffbrücke innerhalb einer Klasse erhalten werden, wie z. B. $g-g+2$. Dyczmons hatte für $g+t 2$ und $g-t 2$ gefunden, dass die Umwandlungsbarriere in $g+t$ bzw. $g-t$ nach Nullpunktskorrektur negativ wird.[106] Dies wurde als eine barrierefreie Relaxation gedeutet, sodass diese Konformere keine Population aufweisen und damit nicht zu den Spektren beitragen. Eine entsprechende Betrachtung wurde hier auch für $g-g+2$ durchgeführt, und ebenfalls gefunden, dass die Umwandlung in $g-g+$ nach Nullpunktskorrektur barrierefrei abläuft. $g-g+2$ wird mit einer Energie von $0.92 \mathrm{~kJ} \mathrm{~mol}^{-1}$ relativ zum globalen Minimum auf B3LYP-D3(BJ)/may-cc-pVTZ-Niveau nur moderat instabiler als die bisher nachgewiesenen Konformere vorhergesagt. Durch den geringen Energieabstand zwischen $g-g+$ und $g-g+2$ von nur $0.4 \mathrm{~kJ}$ mol ohne Barriere kann eine alternative Interpretation auch eine gemittelte Struktur auf der flachen Torsionskoordinate zwischen beiden elektronischen Minima sein (Large Amplitude Motion). Sowohl $g-g+2$, wie auch der Übergangszustand (auf der elektronischen Potentialfläche), weisen eine deutlich höhere $\mathrm{OH}$ Streckschwingungswellenzahl auf als $g-g+$. Ein solcher Beitrag könnte die Abwesenheit von $g-g+$ an der erwarteten spektralen Position erklären.

Die jeweiligen stabilsten Minima der neun Klassen haben (auf B3LYP-D3(BJ)/maycc-pVTZ-Niveau) berechnete $\mathrm{C}_{\alpha}^{\mathrm{D}} \mathrm{O}^{\mathrm{D}} \mathrm{O}^{\mathrm{A}} \mathrm{C}_{\alpha}^{\mathrm{A}}$-Torsionswinkel zwischen $-104^{\circ}$ und $-131^{\circ}$. Diese entsprechen den berechneten Winkeln im Methanol- $\left(-121^{\circ}\right)$ und Wasser-Dimer $\left(-122^{\circ}\right)$, die nur jeweils eine stabile Konformation aufweisen.[137, 138] In diesem Hinsicht erscheinen die auf einigen Niveaus deutlich abweichenden Werte für $g-g+$ (Tab. 4.5) ungewöhnlich. Für die energetisch höher liegenden, bisher nicht nachgewiesenen, Ethanol-Konformere lässt sich dagegen keine klare Präferenz für einen zweiten Winkelbereich erkennen.

Sollten diese höheren Konformere von Ethanol nicht populiert sein, würde in den hier durchgeführten Modellierungen der Spektren im Bereich der hochfrequenten Bande Intensität fehlen, zumindest für die Spektren relativ warmer Expansionen mit Helium als Trägergas. Konformere, die laut Modellierung neben $t t$ zur hochfrequenten Bande beitragen sind nach aufsteigender Energie $g-g+2, g+g-, g+t 2, g+t 3, g+g+3$ und $t g-$, wovon nur $g+g-$ und $t g-$ als stabil verbleiben würden. Eine alternative Erklärung wäre, wie diskutiert, stattdessen die Präsenz von $g-g+$ in diesem Bereich, sei es durch eine falsch vorhergesagte relative harmonische Wellenzahl, eine durch die Theorie nicht erfassbare geringere Anharmonizität oder eine Large Amplitude Motion.

\subsubsection{Bedeutung von $\mathrm{C}-\mathrm{H} \cdots$-O-Kontakten}

Interessant ist ebenfalls die Frage, ob und wie sich die gefundene Energieabfolge der Dimer-Konformere rationalisieren lässt. Der Befund, dass das globale Minimum $g+g+$ gleich doppelt das im Monomer instabilere $g$-Konformer enthält, zeigt, dass die Abfolge maßgeblich von Unterschieden in den intermolekularen Wechselwirkungen bestimmt 
wird. Wassermann et al. haben als wichtigen Beitrag für die unterschiedliche Stabilisierung die Anzahl intermolekularer C-H - . O-Kontakte mit Abständen $<3.4 \AA$ vorgeschlagen [22], hauptsächlich ausgebildet mit einem freien Elektronenpaar des SauerstoffAtoms des Donor-Moleküls. Tab. 4.6 listet solche Kontakte auf B3LYP-D3(BJ)/may-ccpVTZ-Niveau mit Angabe der konkreten Abstände. In der Tat weisen die sieben stabilsten Dimer-Konformere alle einen solchen Kontakt mit einem Abstand von $<2.9 \AA$ auf. Dies gilt allerdings auch für fünf der dreizehn weniger stabilen Konformere bzw. sogar für neun wenn die (willkürliche) Grenze auf den vorgeschlagenen Wert von $<3.4 \AA$ A erweitert wird. Die geringere Stabilität von Konformeren mit $g$--Akzeptor wurde damit begründet, dass diese aus geometrischen Gründen keinen (oder laut der hier geführten Analyse immerhin einen deutlich längeren) Kontakt ausbilden können. Allerdings wird $g+g-$ immerhin als achtstabilstes Konformer vorhergesagt, in dieser Sichtweise aber möglicherweise wegen eines ähnlich kurzen, alternativen Kontaktes mit dem Sauerstoff-Atom des Akzeptors-Moleküls vom 3.0 ̊. Ein zweiter Kontakt mit dem Akzeptor, zusätzlich zu dem Kontakt mit dem Donor, wird von Wassermann et al. auch als Hypothese für die besondere Stabilität von $g+g+$ und $g-t$ angeführt. Tatsächlich wurde aber hier gefunden, dass ein oder sogar zwei solcher Kontakte mit dem Akzeptor-Sauerstoff bei 15 der 20 Dimer-Konformere ausgebildet werden, häufig ebenfalls in Ergänzung zu dem meist kürzeren Kontakt mit dem Donor-Sauerstoff. Das Erklärungsvermögen solcher Merkmale erscheint daher gering, insbesondere in dieser einfachen Betrachtung ohne nähere Analyse in Bezug auf die Linearität des Kontaktes und der tatsächlichen Ausrichtung auf das freie Elektronenpaar. Es erscheint wahrscheinlicher, dass sich zumindest die längeren

Tab. 4.6: Relative nullpunktskorrigierte Energien und Längen der intermolekularen C-H $\cdots \mathrm{O}-$ Kontakte $<3.4 \AA$ (getrennt nach Donor- und Akzeptor-Sauerstoff) der Ethanol-Dimer-Konformere auf B3LYP-D3(BJ)/may-cc-pVTZ-Niveau.

\begin{tabular}{llll}
\hline $\begin{array}{l}\text { Dimer- } \\
\text { Konformer }\end{array}$ & $\begin{array}{c}E_{0}^{\text {rel }} \\
/ \mathrm{kJ} \mathrm{mol}^{-1}\end{array}$ & $\begin{array}{c}d\left(\mathrm{C}-\mathrm{H} \cdots \mathrm{O}^{\mathrm{D}}\right) \\
/ \AA\end{array}$ & $\begin{array}{c}d\left(\mathrm{C}-\mathrm{H} \cdots \mathrm{O}^{\mathrm{A}}\right) \\
/ \AA\end{array}$ \\
\hline$g+g+$ & 0.00 & 2.8 & 3.1 \\
$g-t$ & 0.11 & 2.8 & 3.1 \\
$t t$ & 0.31 & 2.8 & - \\
$g+t$ & 0.32 & 2.9 & 3.2 \\
$t g+$ & 0.52 & $2.8,3.3$ & - \\
$g-g+$ & 0.53 & $2.8,3.3$ & 3.4 \\
$g-g+2$ & 0.92 & 2.9 & 3.0 \\
$g+g-$ & 1.40 & 3.3 & 3.0 \\
$g-t 2$ & 1.46 & 2.9 & 3.1 \\
$t t 2$ & 1.55 & 2.8 & 3.4 \\
$g+t 2$ & 1.60 & 2.9 & 3.0 \\
$g+g+2$ & 1.69 & 3.3 & $3.1,3.4$ \\
$g-g+3$ & 1.70 & - & 3.3 \\
$t g+2$ & 1.77 & $2.9,3.3$ & - \\
$g+t 3$ & 1.81 & - & 3.3 \\
$g-g-$ & 1.87 & - & 3.2 \\
$t g+3$ & 1.89 & 3.4 & - \\
$g+g+3$ & 1.90 & $2.9,3.3$ & 3.4 \\
$t g-$ & 1.95 & 3.4 & - \\
$g+g-2$ & 2.81 & - & $3.0,3.4$ \\
\hline
\end{tabular}


Kontakte als Nebenaspekt bei der Optimierung der Dispersionswechselwirkung ergeben und damit keine eigenen spezifischen Wechselwirkungen darstellen, durch deren Abzählen sich tiefere Einsichten erhalten lassen. In dieser Arbeit werden daher in den Abbildungen der Strukturen nur Kontakte innerhalb einer deutlich engeren Grenze von $<3.0 \AA$ markiert, was auch eher einem üblichen Grenzwert bei der Untersuchung dieser Art von Wechselwirkungen entspricht.[139]

\subsubsection{Zusammenfassung, Schlussfolgerungen und Ausblick}

Aus den in den vorherigen Abschnitten diskutierten Resultaten lassen sich einige vorläufige Schlussfolgerungen ziehen: für zu erwartende Eignung der evaluierten quantenchemischen Methoden für diese Art Systeme, den Rahmenbedingungen für die Modellierung von Spektren von Überschallexpansionen und schließlich konkret für die Interpretation des Schwingungsspektrums der Ethanol-Dimere.

B3LYP-D3(BJ) (mit ggf. geringer Korrektur) liefert eine weitgehend schlüssige und insgesamt die bisher beste bekannte Beschreibung der vorliegenden experimentellen Daten für Ethanol-Dimere betreffend Energieabfolge, einiger geometrischer Parameter und der Schwingungsspektren im OH-Streckschwingungsbereich bei verschiedenen Expansionsbedingungen. Die Größe des Basissatzes lässt sich dabei offenbar ohne Einbußen in der Genauigkeit für die hier spektroskopisch relevanten Größen bis auf Triple- $\zeta$ mit minimaler Augmentierung reduzieren, was gegenüber dem populären aug-cc-pVTZ Basissatz die Rechenzeit für größere Systeme (mit etwa 20 Atomen der zweiten Periode) um etwa 90-95\% reduziert. B3LYP-D3(BJ)/may-cc-pVTZ lässt sich daher in vielen Fällen auch direkt zur Struktursuche einsetzen ohne dafür zunächst auf ein niedrigeres Niveau zurückgreifen zu müssen. Ergebnisse mit dem viel genutzten, noch etwas kleineren def2-TZVP Basissatz sind wegen einigen Abweichungen dagegen mit etwas Vorsicht zu behandeln. B2PLYP-D3(BJ) mit ausreichend großer Basis liefert ähnlich gute Ergebnisse, ist aber sehr viel teurer hinsichtlich der Rechenzeit. Die Ergebnisse von $\omega$ B97X-D und stärker noch M06-2X erscheinen auch in Kombination mit einem relativ großen Basissatz wie aug-cc-pVTZ weniger kompatibel mit dem Experiment. Ähnliches gilt auch für APF-D/6-31++G(d,p) und MP2/aug-cc-pVDZ. Ob diese vorläufigen Einschätzungen auch auf größere System mit ähnlichen Wechselwirkungen übertragen werden können, wird im weiteren Verlauf dieser Arbeit an einigen Beispielen überprüft werden.

Die Modellierung des Schwingungsspektrums mit quantenchemischen Rechnungen einschließlich Frequenzanalyse in harmonischer Näherung und Annahme einer einfachen Boltzmann-Verteilung zwischen den Konformeren erwies sich als sehr erfolgreich, auch für verschiedene Expansionsbedingungen und nach Deuterierung. Die aus den Monomeren abgeleitete Konformationstemperatur lässt sich offenbar gut auf die Dimere übertragen. Eine zukünftige Anwendung dieses Befunds auf die Rotationsspektren von Ethanol in Helium-Expansionen könnte dazu genutzt werden, die experimentelle Energieabfolge der Dimere nicht nur qualitativ, sondern quantitativ zu bestimmen (unter Nutzung berechneter Dipolmoment-Komponenten). Die Modellierung von Schwingungsspektren stößt allerdings an ihre Grenzen, wenn deutlich unterschiedliche Anharmonizitäten oder hohe Umwandlungsbarrieren (relativ zur Konformationstemperatur) im System präsent sind. Hilfreich kann ein gutes Verständnis der Monomere sein, um bereits hier soweit wie möglich Abweichungen zwischen Theorie und Experiment zu charakterisieren, da sie sich zu- 


\section{Chiralitätssynchronisation in Ethanol-Aggregaten}

mindest teilweise auch auf Dimere übertragen. Eine einfache nachträgliche Korrektur des Frequenzunterschiedes zwischen den Monomeren auf die Dimere erwies sich als erfolgreich, eine entsprechende Korrektur der relativen Energien würde dagegen zu einer Verringerung der Übereinstimmung führen. Mit B3LYP-D3(BJ)/may-cc-pVTZ und B2PLYPD3(BJ)/jun-cc-pVTZ wurden recht unterschiedliche Monomer-Energieunterschiede berechnet $\left(0.24\right.$ bzw. $\left.0.82 \mathrm{~kJ} \mathrm{~mol}^{-1}\right)$, dagegen stimmen die relativen Dimer-Energien deutlich besser überein. Diese Befunde deuten darauf hin, dass bestimmte Defizite bei der energetischen Beschreibung der isolierten Monomere nicht zwangsläufig relevant für Aggregate sein müssen.

Bezüglich der Interpretation der Schwingungsspektren der Ethanol-Dimere lassen sich hier einige Revisionen gegenüber dem Stand aus dem Jahr 2010 [22] treffen. Die Zuordnung der niederfrequenten Bande $\mathrm{zu} g+g+$ wurde erneut bestätigt (eventuell mit einen Beitrag von $g-g+$ ), die hochfrequente Bande scheint dagegen, anders als von Wassermann et al. vermutet, maßgeblich von $t t$ verursacht zu werden. In den relativ warmen Expansionen mit Helium als Trägergas spielen aber vermutlich auch ergänzende Beiträge von energetisch höher liegenden Konformeren eine Rolle. Die Vermutung von Wassermann et al. für zwei Bandenzentren in der breiten zentralen Bande wurde erhärtet, allerdings mit Beteiligung von insgesamt drei Konformeren mit $g-t$ im Bereich um $3541 \mathrm{~cm}^{-1}$ sowie $g+t$ und $t g+$ vermutlich nahe beieinander im Bereich von etwa $3537-3538 \mathrm{~cm}^{-1}$. Die von Wassermann et al. gemachte Beobachtung, dass die niederfrequente Seite eher den energetisch etwas höher liegenden Konformeren zugeordnet werden kann, ist mit den berechneten Bandenpositionen im Zusammenhang mit den theoretisch und rotationsspektroskopisch abgeschätzten Energieabfolge kompatibel.

Als Fazit für das Schwingungsspektrum zeigen die fünf robust erscheinenden Zuordnungen der Konformere zu den drei Dimer-Banden eine Parallele zur spektralen Abfolge der Monomere: die Dimere der hochfrequenten Bande enthalten nur $t$-Konformere $(t t)$, die der zentralen Bande Mischungen aus $t$ und $g(t g-, t g+$ und $g+t)$ und die der niederfrequente Bande schließlich nur $g$-Konformere $(g+g+)$ (siehe Abb. 4.14). Zukünfti-

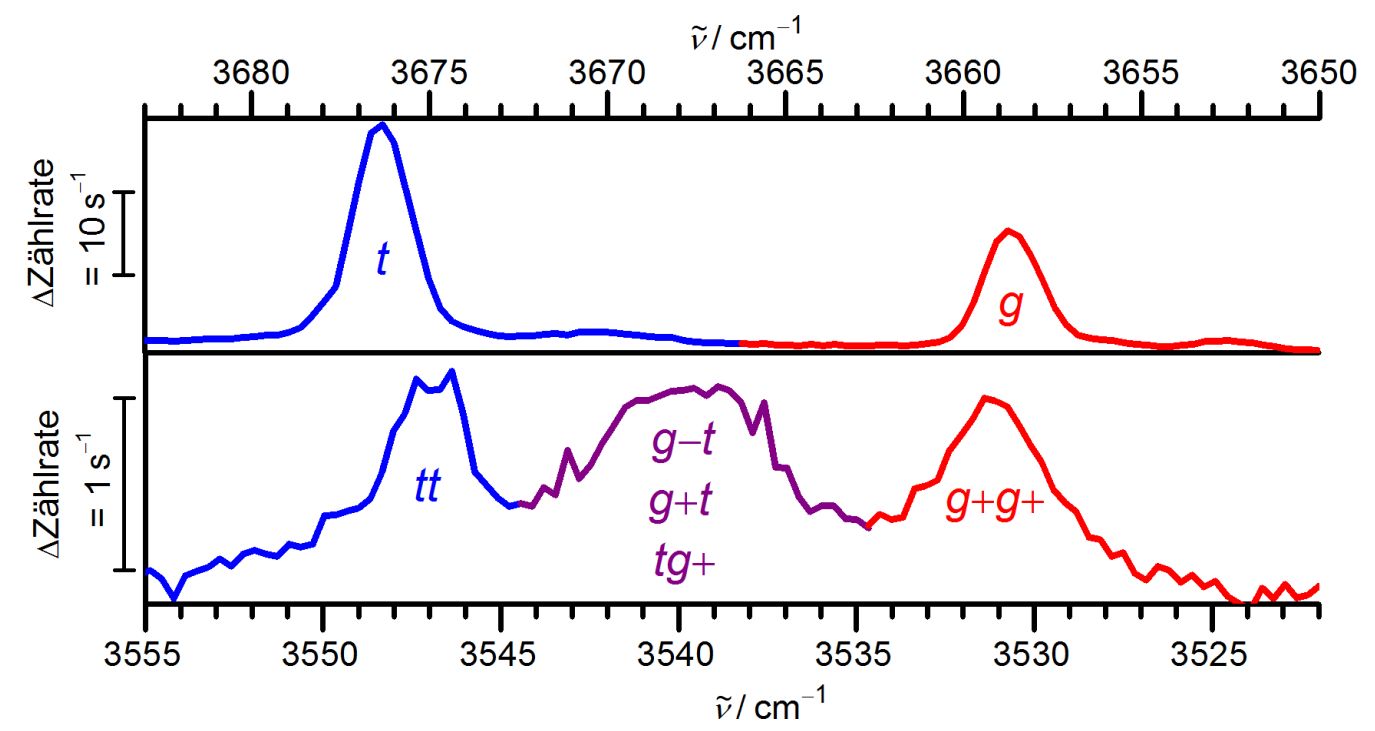

Abb. 4.14: Gegenüberstellung der Monomer-Region (oben, einschließlich schwachen DimerAkzeptor-Banden) mit der Dimer-Donor-Region (unten) im Raman-Spektrum von Ethanol in einer He-Expansion bei 3 mm Detektionsabstand [22] im OH-Streckbereich mit Bandenzuordnungen. 
ge experimentelle Ansatzpunkte sind schwingungsspektroskopisch die Bestätigung der Ergebnisse der Expansion in Neon mit FTIR-Detektion im Gratin-Jet und ferner der Versuch in Helium-Nanotröpfchen hochaufgelöst die relativen Positionen der Bandenzentren besser zu bestimmen. Rotationsspektroskopisch könnte eine quantitative Ermittlung der Energiedifferenzen versucht werden sowie die gezielte Suche nach weiteren höher energetischen Konformeren. Auch auf theoretischer Seite eröffnen die EthanolDimere viele lohnende Möglichkeiten. Tiefere Einblicke in die Dynamik des Systems könnten über eine systematische Untersuchung der Übergangszustände gewonnen werden. Die geringe Größe der Ethanol-Dimere lässt zudem (ggf. lokal oder explizit korrelierte) CCSD(T)-Geometrieoptimierungen mit harmonischen Schwingungsrechnungen sowie anharmonische Schwingungsrechnungen auf mittleren Elektronenstruktur-Niveau zu. Allerdings stellt für letztgenanntes die Präsenz von mehreren Methylgruppen und die Flexibilität der intermolekularen Anordnung besondere Herausforderungen dar. Eigene Versuche im Rahmen dieser Arbeit mit Schwingungsstörungstheorie (VPT2) [60] führten zu keinen verwertbaren Ergebnissen. Auch mit verschärften numerischen Randbedingungen (opt=verytight und grid=superfine) konnte keine Reproduzierbarkeit erhalten werden. Kleinste Strukturabweichungen auch innerhalb der Konvergenzkriterien (resultierend aus unterschiedlichen Startstrukturen vor der Optimierung) führten noch zu deutlich abweichenden Ergebnissen, die teilweise auch imaginäre anharmonische Frequenzen oder unrealistische Infrarot-Aktivitäten beinhalteten. Verantwortlich könnten neben der Flexibilität des Systems auch numerische Instabilitäten im Gaussian Programmpaket sein.[54]

Zusammengefasst sind Ethanol-Dimere ein wichtiger Prototyp für grundlegende intraund intermolekulare Phänomene organischer Moleküle, die dennoch eine besondere Herausforderung sowohl für experimentelle wie auch theoretische Methoden darstellen, die an diesem System erprobt und in Folge verbessert werden können. 


\subsection{Ethanol- und Methanol-Trimere}

\subsubsection{Konformationsisomerie}

Der zweite Schritt in der Aggregation von Ethanol ist die Bildung von Trimeren. Für die Homologen Methanol [130, 140, 141] und Wasser [142], sowie auch Ethanol selbst[141, 143, 144], ist experimentell wie quantenchemisch etabliert, dass beim Übergang vom Dimer zum Trimer ein Wechsel des Bindungsmotivs von linear zu cyclisch stattfindet. Es bildet sich eine kooperative Anordnung von drei Wasserstoffbrücken aus (unter Inkaufnahme nicht unerheblicher Ringspannung [142]), bei der jedes Molekül sowohl als Donor wie auch als Akzeptor jeweils einer Wasserstoffbrücke agiert. In gemischten Trimeren, bei der ein Molekül ein deutlich besserer Akzeptor ist, kann auch eine anti-kooperative Anordnung konkurrenzfähig sein, bei der beide Elektronenpaare des selben Moleküls koordiniert sind. Ein Beispiel hier für ist das Trimer aus einem Methanol- und zwei Chlorwasserstoff-Molekülen.[145]

Die Alkylreste (bzw. bei Wasser die nicht-koordinierenden Wasserstoff-Atome) können in der cyclischen Anordnung entweder jeweils oberhalb ( $\underline{u} p)$ oder unterhalb ( $\underline{\text { down }})$ der Ringebene liegen, die aus den drei Sauerstoffatomen aufgespannt wird. Dies entspricht der Koordination des linken oder rechten freien Elektronenpaares des Akzeptors in der Wasserstoffbrücke. Strukturen, in denen eine oder mehrere Alkylketten in der Ringebene liegen, entsprechen Übergangszuständen sowohl bezüglich des Seitenwechsels der Alkylkette wie auch für den Wechsel des koordinierten Elektronenpaares.[142] Für die relative Ausrichtung der Alkylreste ergeben sich für Trimere prinzipiell zwei Alternativen: entweder sie liegen alle drei auf der selben Seite, oder einer der Reste liegt auf der gegenüberliegenden Seite wie die anderen beiden. In der Literatur haben sich für diese Anordnungen die Bezeichnungen иии und иид etabliert, alternativ auch Wanne und Sessel genannt. Abb. 4.15 zeigt diese beiden Strukturmotive am Beispiel des Methanol-Trimers. Während die Anordnung иии für Moleküle mit identischer intramolekularer Konformation der Punktgruppe $C_{3}$ angehören kann, ist uud inhärent asymmetrisch $\left(C_{1}\right)$. Alle cyclischen Trimer-Strukturen sind damit chiral. Um die Betrachtungen auf zueinander diastereomere Strukturen zu beschränken, wird analog zu den Ethanol-Dimeren hier willkürlich die Koordination bestimmter Elektronenpaare festgelegt. Es wird daher stets das Enantiomer

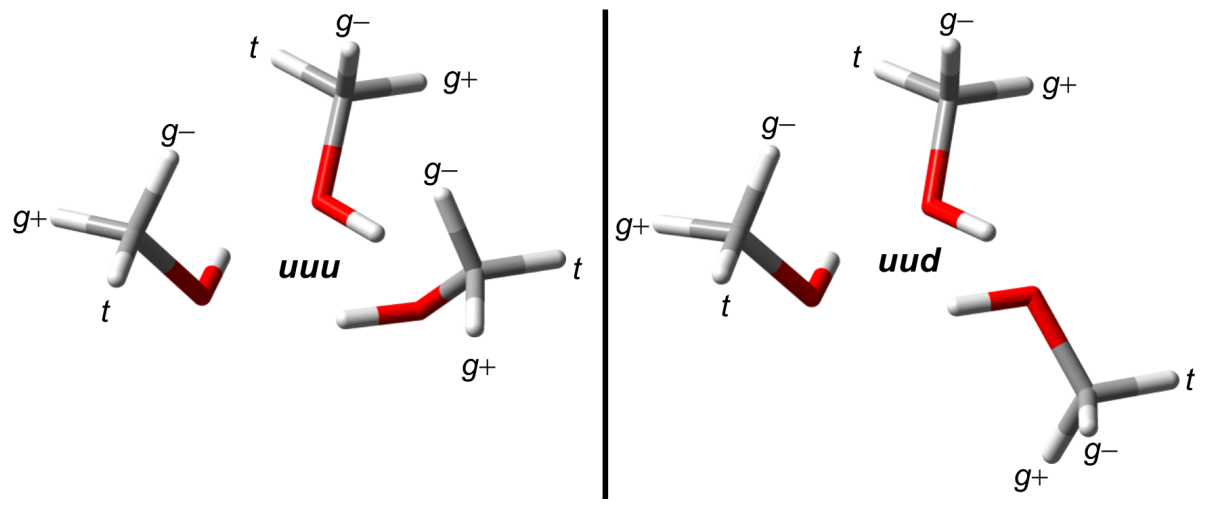

Abb. 4.15: Die Strukturmotive иии und иud des Methanol-Trimers auf B3LYP-D3(BJ)/maycc-pVTZ-Niveau. Die Methyl-Wasserstoffatome sind jeweils mit derjenigen Ethanol-MonomerKonformation beschriftet, die sich ergibt, wenn Methanol an dieser Position formal zu Ethanol erweitert wird. 
betrachtet, bei dem die Wasserstoffbrücken im Uhrzeigersinn verlaufen (in der Richtung von Donor zu Akzeptor $\mathrm{OH}$-..O), wenn der Cluster von der Seite betrachtet wird, auf der sich die Mehrzahl der Alkylreste befindet - die $u$-Seite. Dies entspricht einer Koordination von Molekülen in $u$-Stellung am linken und solchen in $d$-Stellung am rechten Elektronenpaar.

Das uиd-Motiv ist energetisch deutlich gegenüber иии bevorzugt. Dabei spielen sterische Aspekte aber wohl nur eine untergeordnete Rolle, da dieser Energievorteil auch im sterisch wenig anspruchsvollen Wasser besteht $\left(E_{0}^{\mathrm{rel}}=2.4 \mathrm{~kJ} \mathrm{~mol}^{-1}\right)$ auf B3LYP-D3(BJ)/may-cc-pVTZ-Niveau) und über Methanol $\left(2.8 \mathrm{~kJ} \mathrm{~mol}^{-1}\right)$ hin zu tertButylalkohol (3.0 $\mathrm{kJ} \mathrm{mol}^{-1}$ ) nur moderat ansteigt. Als wichtiger können hier Dipol-DipolWechselwirkungen zwischen den unterschiedlich ausgerichteten, polaren $\mathrm{O}-\mathrm{C}-$ (bzw. für Wasser O-H-) Bindungen vermutet werden. Dafür spricht auch eine Analogie zu den Dimeren: Die $\mathrm{C}_{\alpha}^{\mathrm{D}} \mathrm{O}^{\mathrm{D}} \mathrm{O}^{\mathrm{A}} \mathrm{C}_{\alpha}^{\mathrm{A}}$-Torsionswinkel (bzw. HOOH bei Wasser) sind zwischen Molekülen in $u$ - und $d$-Stellung im Bereich zwischen $-114^{\circ}$ und $-135^{\circ}$ in den erwähnten drei Systemen und damit nahe an den bevorzugten Werten in den Dimeren $\left(-121^{\circ}\right.$ bis $\left.-137^{\circ}\right)$. Zwischen zwei Molekülen in $u$-Stellung liegen die Winkel für die drei Systeme dagegen im Bereich zwischen $-16^{\circ}$ und $-23^{\circ}$.

Die Bildung des Methanol-Trimers kann auch als Chiralitätsinduktion des transient chiralen Methanol-Dimers mit dem prochiralen Methanol-Monomer beschrieben werden.[5] Die Koordination an den beiden enantiotopen Elektronenpaaren des Monomers führt dabei zu den diastereomeren uиu- bzw. uиd-Konformationen des Trimers.

Wie die Beispiele des Phenol- [146], 2,2,2-Trifluorethanol- [147] und des homochiralen Methyllactat-Trimers [129, 148] zeigen, kann bei ausreichend starken attraktiven Wechselwirkungen zwischen den Resten auch das $и и и$-Motiv bevorzugt sein. Prinzipiell kommt für einen solchen Wechsel in der Konformationspräferenz auch die Dispersionswechselwirkung zwischen aliphatischen Alkylgruppen in Frage, wie anhand der Isomerisierung für $n$-Alkane von einer gestreckten in eine Haarnadel-Konformation ab einer Kettenlänge von etwa 17 Kohlenstoffatomen gezeigt wurde.[149] Der Vergleich von Methanolund Ethanol-Clustern ist daher auch hinsichtlich des Aspektes interessant, ob sich durch die Verlängerung der Kettenlänge zumindest eine Annäherung der Strukturmotive andeutet, die schließlich zu einem Wechsel der Präferenz führen könnte. Auch durch kovalente Bindungen lässt sich eine entsprechende Struktur erzwingen: die stabilste $C_{3}$ symmetrische Konformation von cis-cis-1,3,5-Cyclohexantriol mit drei intramolekularen Wasserstoffbrücken entspricht einem иии Ethanol-Trimer nach formaler Eliminierung von drei Wasserstoff-Molekülen.[150]

Bei bisherigen quantenchemischen Studien zu Ethanol-Trimeren [96-98, 116, 117, 122, 141, 151-155] lag der Fokus weitgehend auf Isomerie im übergeordneten Wasserstoffbrücken-Motiv, vor allem für die Konkurrenz zwischen linearen und cyclischen Konformeren. Die intramolekulare Isomerie wurde nicht oder nur stark vereinfacht betrachtet, da hieraus resultierende energetische und spektrale Effekte tendenziell kleiner sind. Häufig wurde dabei eine all-t-Struktur angenommen, $[141,151,152]$ da $t$ die stabilere Monomer-Konformation ist. Eine solche Vorannahme führt bereits für das Dimer zum falschen globalen Minimum, kann aber in einem übergeordneten Kontext eine legi-

${ }^{a}$ Die Vorzeichen der Winkel beziehen sich jeweils auf das Enantiomer mit Koordination des rechten Elektronenpaares in der betrachteten Wechselwirkung, in Entsprechung der Konvention für die EthanolDimere. 
time Vereinfachung darstellen. Matisz et al. beschränken sich dagegen auf all- $g$, da die Autoren $g$ als stabiler annehmen.[153] Weitere Studien, die die intramolekulare Isomerie miteinbezogen haben [97, 116, 117, 122, 154], gelangten zur all-t-Struktur dagegen vermutlich aufgrund eines zu niedrigen Rechenniveaus - in den meisten Studien B3LYP ohne Dispersionskorrektur. Bei den wenigen Untersuchungen [96, 98, 155], die gemischte Kombinationen angeben, bleibt dagegen unklar, ob das Ergebnis tatsächlich durch eine systematische Suche des Konformationsraumes erhalten wurde.

Wird Methanol durch Kettenverlängerung formal zu Ethanol erweitert, ergeben sich je nachdem, welches der drei Methyl-Wasserstoffatome substituiert wird, die unterschiedlichen intramolekularen Ethanol-Konformere. Dies kann für Trimere in $3^{3}=27$ Variationen erfolgen. Durch die bei $и и и$ vorhandene lokale Symmetrie gehören allerdings einige Variationen zu der selben Struktur, da sie sich durch zyklische Permutation ineinander überführen lassen. Dies entspricht einer Drehung der Struktur um die (pseudo-)dreizählige Achse und gruppentheoretisch der Anwendung der Symmetrieopertationen $C_{3}$ und $C_{3}^{2} \operatorname{der} C_{3}$-Punktgruppe auf die Variationen. So ist beispielsweise $u u u / g+t t$ identisch zu $u u и / t g+t$ und $и и и / t t g+$. Bei solchen gleichwertigen Bezeichnungen der selben Struktur wird hier stets die nach alphabetischer Sortierung erste verwendet, womit bezüglich der Priorität gilt: $g$ vor $t$ und $g+$ vor $g-.8 \times 3=24$ Variationen gehören zu identischen Strukturen, während die verbleibenden drei Variationen zu intramolekular einheitlichen Strukturen gehören, bei denen die $C_{3}$-Symmetrie erhalten bleibt. Insgesamt sind damit für $и и и$ 11 diastereomere Strukturen zu erwarten.

Die Moleküle in uud sind dagegen grundsätzlich inäquivalent, sodass hier die volle Zahl an 27 Strukturen resultiert. Die Angabe der intramolekularen Konformationen erfolgt hier in der Abfolge uud im Uhrzeigersinn der Donor-Akzeptor-Richtung.

Für die Struktursuche wurden zunächst die beiden Methanol-Trimer-Motive иии und uud auf B3LYP-D3(BJ)/may-cc-pVTZ-Niveau optimiert, zu Ethanol-Trimeren erweitert und reoptimiert. Alle 38 Rechnungen führten ohne Isomerisierungen im Optimierungsverlauf zu den erwarteten Ethanol-Trimer-Strukturen. Zusätzlich wurden für Vergleichszwecke die 27 uud-Strukturen auch ohne Dispersionskorrektur optimiert.

Bei Erweiterung der Methanol-Trimere zu verschiedenen Ethanol-Trimeren fällt auf, dass die endständige Methylgruppe von Ethanol in $\frac{2}{3}$ der Fälle eher nach außen zeigt $(t$ für alle Moleküle, $g+$ für $u$ und $g-$ für $d$ ), in den verbleibenden Fällen dagegen eher in Richtung der (pseudo-)dreizähligen Achse ( $g$ - für $u$ und $g+$ für $d$ ), siehe Abb.4.15. Es zeigt sich, dass die letztgenannte Ausrichtung tendenziell zu einer Stabilisierung der resultierenden kompakteren Struktur, vermutlich durch Dispersionswechselwirkung, führt. Die Ausnahme von dieser Regel ist, wenn mehr als ein Molekül auf der gleichen Seite der Ringebene eine solche Ausrichtung hat, da dann offenbar sterische Abstoßung zwischen den räumlich nahen, endständigen Methylgruppen die Struktur destabilisiert. Für die bessere Verständlichkeit der Struktur-Bezeichnungen wird das betreffende Konformer im erstgenannten stabilisierenden Fall in grüner Schriftfarbe hervorgehoben, im zweiten destabilisierenden Fall die betreffenden Konformere dagegen in roter Schriftfarbe.

Auch wenn die $и и и$-Konformere sich auch für Ethanol energetisch wenig relevant erweisen, wird zunächst dieses Strukturmotiv analysiert, da sich hier die grundsätzlichen Prinzipien leichter nachvollziehen lassen als in der komplexeren Situation bei uud. In Abb. 4.16 ist die relative Energie der $и и u$-Ethanol-Trimere in Abhängigkeit von der Anzahl der nach innen gerichteten $g$-Konformere dargestellt. In der linken äußeren Spalte 
der Abbildung sind die $u и u$-Konformere zu finden, bei denen alle endständigen Methylgruppen nach außen zeigen. Durch die geringe Wechselwirkung der Ethylgruppen untereinander benötigt die schrittweise Umwandlung von $t$ - zu $g$-Konformeren Energiebeträge in ähnlicher Höhe wie beim isolierten Monomer $\left(0.24 \mathrm{~kJ} \mathrm{~mol}^{-1}\right.$ auf diesem Rechenniveau). Die intramolekular neutrale Umwandlung eines $g+$ in ein nach innen gerichtetes $g$ - (linke innere Spalte) bewirkt dagegen einen intermolekularen Energiegewinn von etwa $1.0 \mathrm{~kJ} \mathrm{~mol}^{-1}$, da eine kompaktere Struktur entsteht. Zwischen $g+t g-$ und $g+g-t$ kommt es zu einer energetischen Aufspaltung, die allein aus der veränderten Reihenfolge der drei Konformere resultiert. Die Energieniveaus der Strukturen bezüglich dieser Isomerie werden in Abb. 4.16 und in folgenden Abbildungen farblich einheitlich hervorgehoben. Die Umwandlung eines zweiten $g+$ in $g-$ ist dagegen aus sterischen Gründen mit einem leichten (rechte innere Spalte), die eines dritten (rechte äußere Spalte) mit einem deutlichen Energienachteil verbunden. Die stabilste $u u u$-Struktur stellt $g-t t$ dar, mit einem nach innen zeigenden $g$-Konformer und zwei aus sterischen Gründen nach außen zeigenden Konformeren in der intramolekular stabileren $t$-Konformation.

Für die relative Energie der 27 uud-Konformere, zusammengefasst in Abb. 4.17, gelten die gleichen Prinzipien, allerdings in unterschiedlich starker Ausprägung und überlagert von weiteren subtilen intermolekularen Effekten. Ohne nach innen gerichtete $g$ Konformere ist auch bei uud tendenziell ein hohe Anzahl an $t$-Konformeren energetisch bevorzugt, auch wenn $t t t$ etwas überraschend nicht das Minimum dieser Kategorie ist. Bei einer Umwandlung eines nach außen in ein nach innen gerichtetes $g$-Konformer findet auch bei uud ein Energiegewinn statt, der aber schwächer ausfällt als für иии. Der Grund hierfür könnte sein, dass die nach innen gerichtete Ethylgruppe hier nur mit ma-

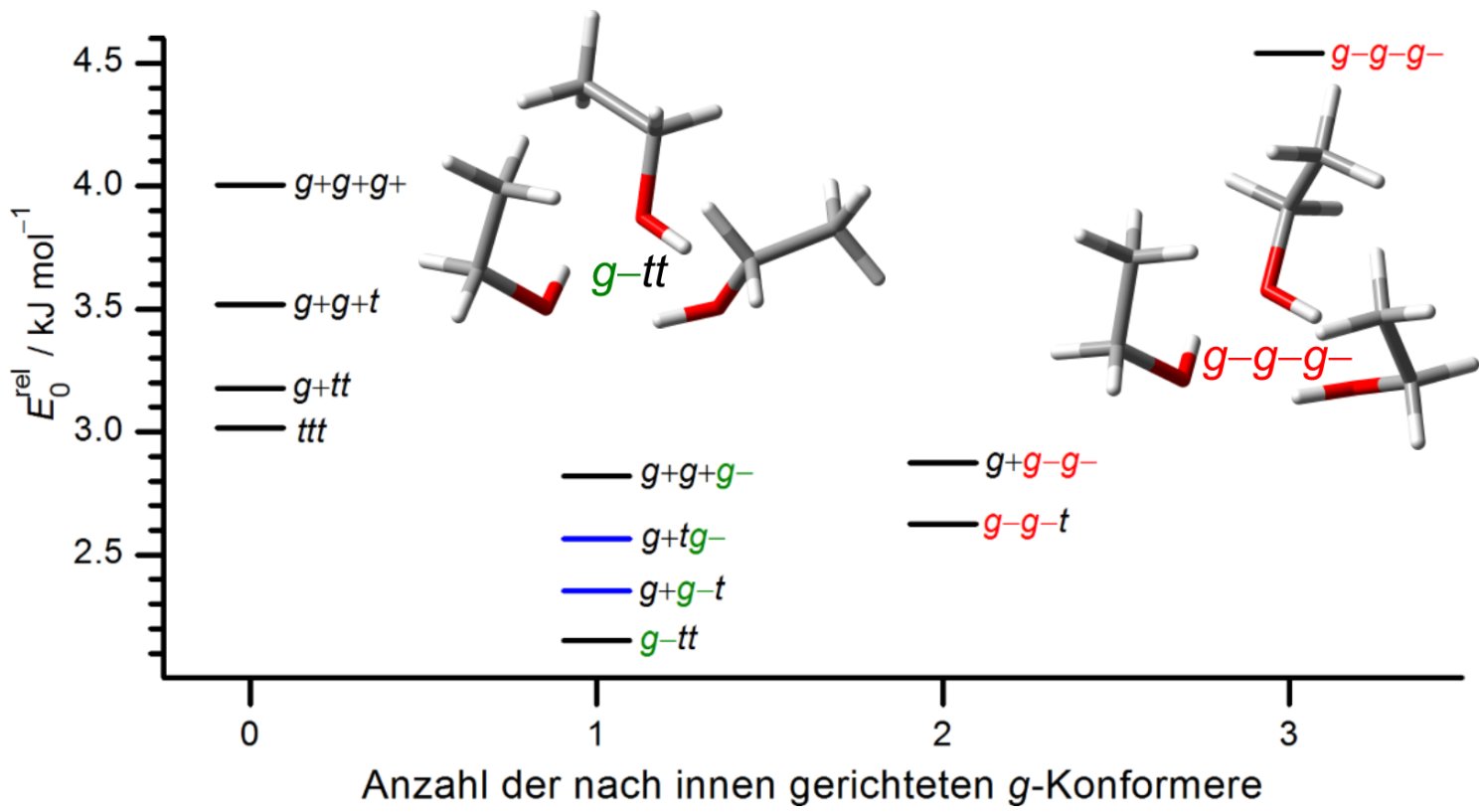

Abb. 4.16: Relative Energie der Isomere des $u и u$-Strukturmotivs des Ethanol-Trimers in Abhängigkeit von der Anzahl der nach innen gerichteten $g$-Konformere auf B3LYP-D3(BJ)/may-ccpVTZ-Niveau. Die angegebenen Werte der relativen Energie beziehen sich auf das globale Minimum mit $u u d$-Strukturmotiv. Die Strukturen des stabilsten und instabilsten $и и u$-Trimers sind als Insert eingefügt. Die Energieniveaus von $g+t g-$ und $g+g-t$, die sich allein durch eine veränderte Reihenfolge von äquivalenten Ethanol-Konformeren unterscheiden, sind blau hervorgehoben. 


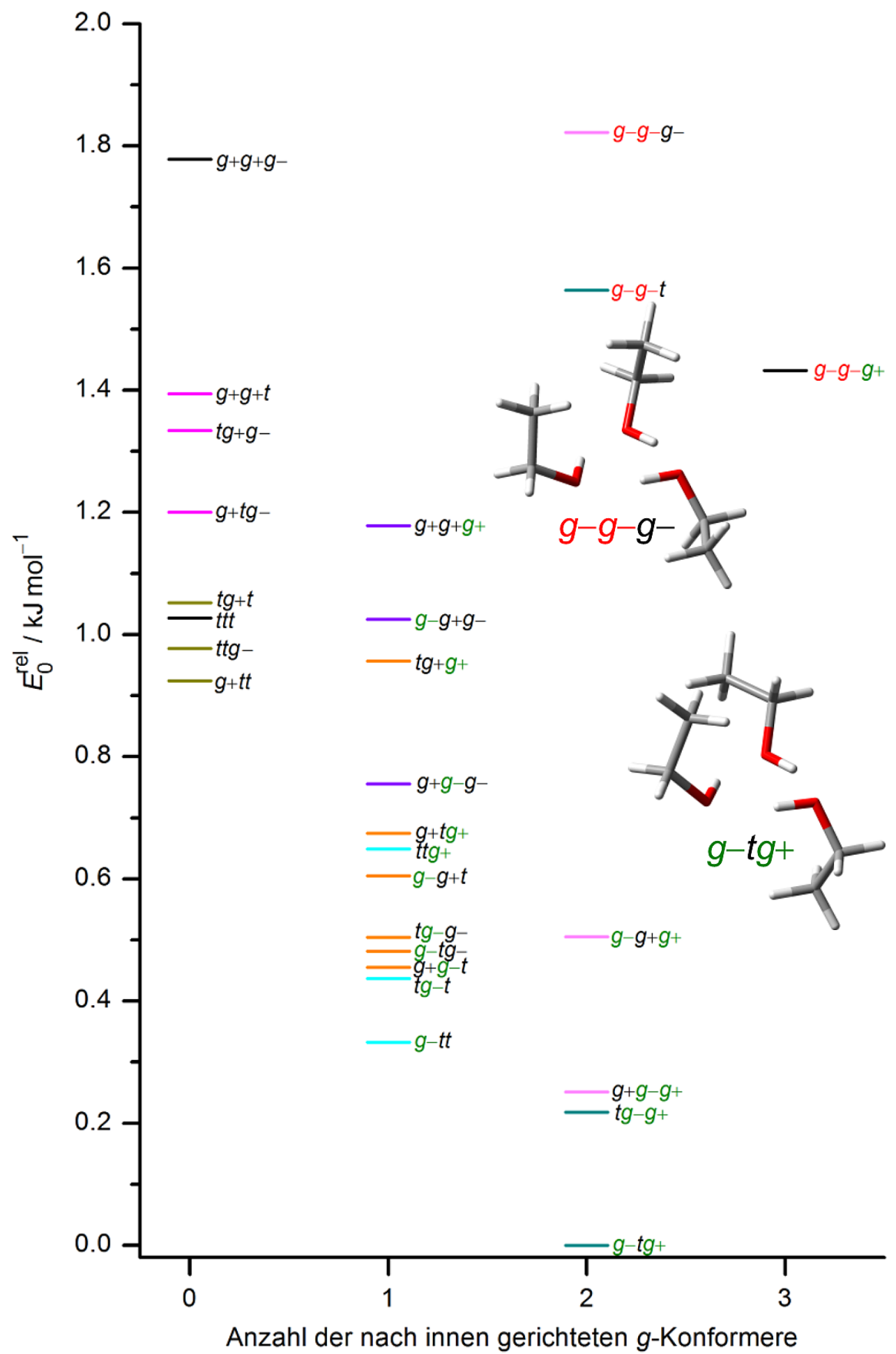

Abb. 4.17: Relative Energie der Isomere des uud-Strukturmotivs des Ethanol-Trimers in Abhängigkeit von der Anzahl der nach innen gerichteten $g$-Konformere. Die Strukturen des stabilsten und instabilsten $u u d$-Trimers sind als Insert eingefügt. Die Energieniveaus von Isomeren, die sich allein durch eine veränderte Reihenfolge von äquivalent ausgerichteten Ethanol-Konformeren unterscheiden, sind jeweils farblich einheitlich markiert. 
ximal einer, statt wie bei иии zwei, anderen Ethylgruppe(n) auf auf der gleichen Seite wechselwirken kann. Dafür spricht, dass der entsprechende Energiegewinn für die Isomerisierung noch kleiner ausfällt, wenn die Ethylgruppe sich ohne direkten Nachbarn in $d$-Position befindet. Mit einem zweiten nach innen gerichteten $g$-Konformer lässt sich noch einmal ein ähnlich großer Energiegewinn erreichen, allerdings nur wenn die beiden Reste auf unterschiedlichen Seiten des Rings platziert sind. Auf gleicher Seite findet dagegen ein deutlicher Energieanstieg statt, der durch ein drittes nach innen gerichtetes $g$-Konformer auf der anderen Seite wieder abgemildert werden kann.

Als globales Minimum wird $u u d / g-t g+$ berechnet, bei dem auf beiden Seiten des Rings jeweils ein $g$-Konformer nach innen gerichtet ist. Das aus sterischen Gründen nach außen gerichtete dritte Ethanol-Molekül nimmt hier die intramolekular stabilere $t$-Konformation an. Diese Struktur ist als Insert in Abb. 4.17 dargestellt. Für das zweitstabilste EthanolTrimer-Konformer $u u d / t g-g+$ sind nur die beiden Konformere in $u$-Position vertauscht. Auch wenn nachträglich die $t / g$-Energiedifferenz auf den experimentellen Wert des Monomers korrigiert wird (was angesichts einer sich deutlich verschlechternden Übereinstimmung mit dem Experiment im Fall der Dimere eher fragwürdig erscheint) bleibt $u u d / g-t g+$ als globales Minimum erhalten. Dies lässt die Vorhersage zumindest hinsichtlich intramolekularer Defizite des Theorieniveaus robust erscheinen lässt. Das globale Minimum des Ethanol-Trimers ist mit drei beteiligten unterschiedlichen Konformeren des gleichen Moleküls maximal diversifiziert, was nach Kenntnisstand des Autors der vorliegenden Arbeit ein Novum für Trimere ist. Im Gegensatz zum stabilsten Ethanol-Dimer $g+g+$ sind die beiden beteiligten $g$-Konformere im Trimer in ihrer transienten Chiralität entgegengesetzt synchronisiert. Als Bestandteile von $u u d / g-t g+$ lassen sich am ehesten die Motive der Dimere $g-g+, t g+$ und $g+t 3$ wiedererkennen, wenn auch deutlich durch die geometrischen Anforderungen des gespannten Ringes verzerrt.

Die einzige andere Studie für Ethanol-Trimere unter Einbeziehung intramolekularer Isomerie und Verwendung einer Dispersionskorrektur [B3LYP-D/6-311++G(2df,p)] von Balanay et al. [96] gibt dagegen $u u d / t g+g+$ als Minimum an, wobei unklar bleibt, wie die Autoren zu dieser Struktur gelangt sind. Dass die Autoren als stabilste Dimer-Struktur $g-t$ angeben und den Widerspruch zu früheren experimentellen und theoretischen Studien (siehe Kap. 4.3) nicht kommentieren, lässt auch das Ergebnis für das Trimer zweifelhaft erscheinen. $u u d / t g+g+$ wird in der vorliegenden Arbeit nur an fünfzehnter Stelle mit einem energetischen Nachteil von $1.0 \mathrm{~kJ} \mathrm{~mol}^{-1}$ gefunden.

Ein völlig anderes Bild ergibt sich dagegen aus den Optimierungen ohne Dispersionskorrektur. Hier wird uud/ttt als globales Minimum gefunden und die relative Energie ist im Wesentlichen eine Funktion der Zahl der $g$-Konformere. Besonders ungünstig ist es, wenn $g$-Konformere nach innen zeigen, unabhängig von der Anzahl und Ringseite. Das globale Minimum mit Dispersionskorrektur $u u d / g-t g+$ mit zwei nach innen zeigenden $g$-Konformeren gehört daher ohne Dispersionskorrektur mit zu den besonders instabilen Strukturen. Die relative Energie von $u u d / t t t$ gegenüber $u u d / g-t g+$ verändert sich durch Verzicht auf die Dispersionskorrektur von 2.5 auf $-1.0 \mathrm{~kJ} \mathrm{~mol}^{-1}$. Von dieser $\mathrm{Ge}-$ samtänderung von $-3.5 \mathrm{~kJ} \mathrm{~mol}^{-1}$ könnte in erster Näherung nur $-0.7 \mathrm{~kJ} \mathrm{~mol}^{-1}$ durch die höhere berechnete intramolekulare Stabilität der $t$-Konformere ohne Dispersionskorrektur erklärt werden. Dies zeigt, dass die Dispersionskorrektur auch für kleine Cluster wie Ethanol-Trimere sehr relevant sein und den Charakter bestimmter geometrischer Aspekte von destabilisierend zu stabilisierend ändern kann. Andere Arbeiten haben dagegen eine 


\section{Chiralitätssynchronisation in Ethanol-Aggregaten}

Dispersionskorrektur für Ethanol-Cluster im Vorfeld als unerheblich eingeschätzt und daher darauf verzichtet,[124, 153] obwohl die zusätzlichen Rechenkosten vernachlässigbar sind.

Das stabilste Trimer der verwandten Verbindung 2,2,2-Trifluorethanol kann als $u u u / g-g-t$ klassifiziert werden.[147] Die Bedeutung der Fluoratome für intermolekulare Wechselwirkungen ist so groß, dass nicht nur die drei Reste auf eine Ringseite gebracht werden, sondern auch in einem besonders ausgeprägten Fall von adaptiver Aggregation die im isolierten Monomer besonders instabile $t$-Konformation von 2,2,2-Trifluorethanol ausgebildet wird.

\subsubsection{Schwingungsspektren}

Bevor Spektren der Ethanol-Trimere diskutiert werden, ist es erneut hilfreich zuerst den einfachen Fall von Methanol ohne intramolekulare Konformationsisomerie zu betrachten.

\section{Schwingungsspektren des Methanol-Trimers}

Spektren des Methanol-Trimers wurden bereits vielfach detailliert analysiert.[127, 129, 130, 156-161] Diese Ergebnisse dienen sowohl als Referenz für die Güte der hier gemachten spektroskopischen Vorhersagen, wie auch als Vergleichsmöglichkeit für die komplizierten Ethanol-Spektren. Für das Methanol-Trimer ist unter Überschallexpansionsbedingungen energetisch nur die Population des $u u d$-Konformers zu erwarten. Wegen der nur schwach verzerrten $C_{3}$-Symmetrie des Ringes sind die O-H-Oszillatoren nahezu äquivalent und koppeln, im Gegensatz zum Dimer, stark miteinander. Es ergeben sich zwei fast entartete phasenverschobene Kombinationen und eine phasengleiche Kombination der Auslenkungen der drei OH-Streckschwingungen. Die beiden phasenverschobenen Kombinationen sind stark IR-, aber nur wenig Raman-aktiv. Die phasengleiche Kombination ist dagegen umgekehrt stark Raman- und schwach IR-aktiv. Der Vergleich von IR- und Raman-Spektren bietet daher für Trimere wichtige ergänzende (komplementäre) Informationen.

Abbildung 4.18 zeigt je ein Filet- und ein Curry-Jet-Spektrum [129] von Methanol im Dimer- und Trimer-Bereich im Vergleich mit Simulationen. Am hochfrequenten Rand des Spektralbereiches ist die Dimer-Donor-Bande von Methanol zu erkennen. Auch nach durchgeführter nachträglicher Korrektur des älteren Raman-Spektrums um den Brechungsindex von Luft verbleibt eine Diskrepanz zum FTIR-Spektrum von etwa $1 \mathrm{~cm}^{-1}$ in der Bandenposition.

Durch Vergleich mit den quantenchemischen Rechnungen lässt sich die Bande mit Doppelmaximum im Zentrum des abgebildeten Spektralbereichs (markiert mit zwei F für Fundamentalschwingung) den phasenverschobenen Kombinationen der OHStreckschwingungen des $u u d$-Trimers zuordnen. Alle berechneten harmonischen Wellenzahlen, sowohl der Dimere wie auch der Trimere, wurden für eine gute Übereinstimmung mit dieser Bande einheitlich um $-92 \mathrm{~cm}^{-1}$ verschoben. Die berechnete Aufspaltung der Bande von $7 \mathrm{~cm}^{-1}$ stimmt gut mit der experimentellen überein. Auch die etwas höhere IR-Aktivität der etwas niederfrequenten Schwingung findet sich im Experiment wieder. Allerdings könnte dieser Unterschied von der Theorie überschätzt werden, denn die Intensitäten gleichen sich in einer Neon-Expansion stärker an, daher wird eine Überlappung 
mit einer heißen Bande vermutet.[129] Das FTIR-Spektrum zeigt weitere schwächere Signale (markiert mit S, S, H, D und R), deren Zuordnung in der Vergangenheit kontrovers diskutiert wurde.[129, 130, 156-161] Das Signal R wurde durch Vergleich mit dem Raman-Spektrum als die Bande der nur sehr schwach IR-aktiven phasengleichen Schwingung des Trimers identifiziert.[130] Die verbleibenden Signale wurden dagegen als Summenbanden (S), einer heißen Bande (H) und einer Differenzbande (D) mit jeweiliger Beteiligung der $\mathrm{OH}-$ Streckschwingungs-Fundamentalen und von niederfrequenten van der Waals-Moden interpretiert.[129] Mit der hier verwendeten harmonischen Theorie lassen sich die Positionen solcher Banden anharmonischen Ursprungs nur sehr grob und die Intensität überhaupt nicht abschätzen. Die stark Raman-aktive Bande weist dagegen keine solche Satelliten auf. Die Davydov-Aufspaltung zwischen den phasenverschobenen und der phasengleichen Schwingung wird von der harmonischen Theorie etwas überschätzt. Die Dimerbande hat im IR- und Raman-Spektrum jeweils eine Halbwertsbreite von etwa $6 \mathrm{~cm}^{-1}$, allerdings mit etwas unterschiedlichen Bandenformen. Die Trimerbanden lassen sich gut mit Lorentzprofilen mit einer etwas höheren vollen Halbwertsbreite von $8 \mathrm{~cm}^{-1}$ modellieren. Als mögliche relevante Mechanismen für die größere Breite der TrimerBanden kommt hier, neben Unterschieden in der Rotationsstruktur, vor allem eine schnel-

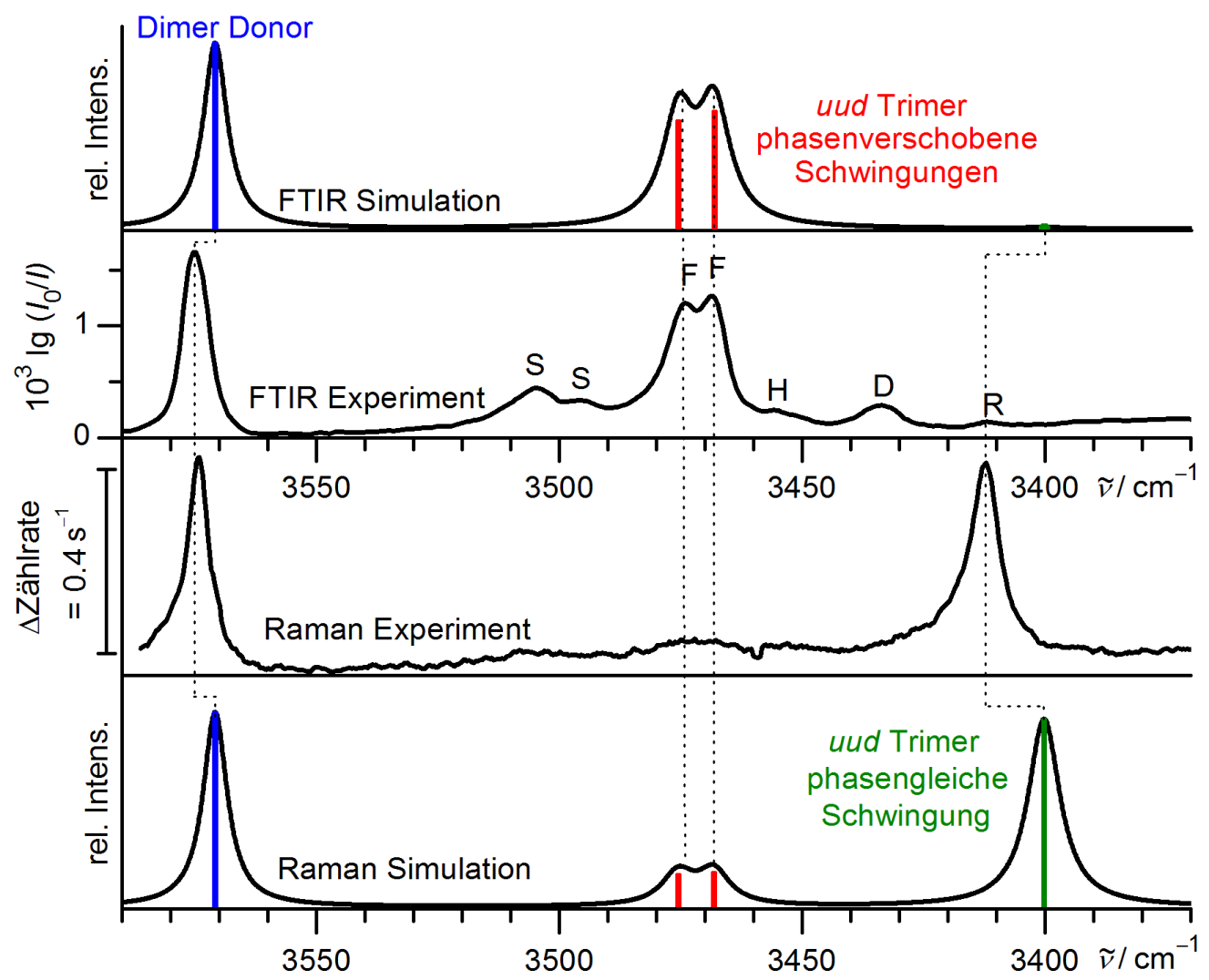

Abb. 4.18: Experimentelle [129] und simulierte IR- und Raman-Spektren von Methanol im Dimerund Trimer-Bereich. Im FTIR Experiment markiert F Fundamentalbanden, S Summenbanden, H eine heiße Bande, D eine Differenzbande und R die vornehmlich Raman-aktive Bande.[130] Simulationsparameter sind einheitliche Verschiebung der harmonischen Wellenzahlen für eine gute Übereinstimmung der phasenverschobenen Schwingungen des Trimers $\left(-92 \mathrm{~cm}^{-1}\right)$, ein Dimer-zuTrimer-Verhältnis von 2.7 (IR) bzw. 2.1 (Raman) und Lorentz-Profile mit Halbwertsbreite $6 \mathrm{~cm}^{-1}$ für die Dimer- und $8 \mathrm{~cm}^{-1}$ für die Trimer-Banden. 


\section{Chiralitätssynchronisation in Ethanol-Aggregaten}

lere Umverteilung der Schwingungsenergie im stark gekoppelten Oszillator-System in Betracht [130, 162], was zu einer homogenen Lebensdauerverbreiterung führt. Auf die ebenfalls denkbare Möglichkeit einer Aufspaltung durch konzertiertes Protonentunneln wird weiter unten beim Vergleich zwischen Methanol- und Ethanol-Trimeren eingegangen.

Bei IR-Detektion in ultrakalten $(0.4 \mathrm{~K})$ Helium-Nanotröpfchen sind Rotationsstruktur und heiße Banden als Verbreiterungsmechanismen weitgehendst eliminiert, auch dort erscheint die Bande des Trimers deutlich breiter als die des Dimers.[163] Auch die Satellitenbanden scheinen sich dort anzudeuten, sind aber aufgrund des schlechteren Signal-zuRausch-Verhältnisses nicht im Detail beurteilbar.

\section{Schwingungsspektren von Ethanol-Trimeren}

Im Filet-Jet-Spektrum von Ethanol mit Helium als Trägergas [126] wurde durch Konzentrationsvariation [119] den Trimeren eine breite, nur wenig strukturierte Bande mit Maximum bei $3447 \mathrm{~cm}^{-1}$ zugeordnet (Abb. 4.19). Eine entsprechend zugeordnete Bande mit größenselektiver NRIDip-IR-Spektroskopie ist dagegen noch deutlich breiter und zu höheren Wellenzahlen verschoben.[144] Im Curry-Jet-Spektrum [95] findet sich eine intensive, stärker verschobene Bande bei $3385 \mathrm{~cm}^{-1}$, die in Analogie zu Methanol den phasengleichen Schwingungen der Trimere zugeordnet werden kann. Die Bande ist breiter als ihre Entsprechung bei Methanol, aber weniger breit als die Bande der phasenverschobenen Schwingungen bei IR-Detektion. Die große Diskrepanz zwischen IR- und RamanAktivitäten und die Analogie zu Methanol beweist, dass die Ethanol-Trimere ebenfalls cyclisch sind, in Übereinstimmung mit den quantenchemischen Vorhersagen. Die naheliegende Erklärung für die Verbreiterung der Banden bei Ethanol gegenüber Methanol ist die vorhergesagte Konformationsvielfalt der Ethanol-Trimere, deren Einfluss auf die $\mathrm{OH}-$ Streckschwingungsmoden im Folgenden näher betrachtet wird.

Kopplungskonstanten wurden für Methanol-Trimere zuvor anhand eines einfachen Modells abgeleitet, welche der bekannten Hückel-Methode für Molekülorbitale ähnelt. Annahmen sind dabei, dass die entkoppelten Schwingungen identische Frequenzen haben und dass mindestens zwei der drei Kopplungen identisch sind.[130] Diese Annahmen sind bereits für Methanol etwas willkürlich und wegen der intramolekularen Konformationsisomerie bei Ethanol-Trimeren höchstens noch für die wenigen intramolekular einheitlichen Strukturen sinnvoll. Zudem sind experimentelle Vergleichswerte wegen der fehlenden Konformationsauflösung bei Ethanol nicht verfügbar. Daher wird hier auf eine analoge Analyse verzichtet und stattdessen ein noch einfacherer phänomenologischer Ansatz verwendet. Eine wichtige Kenngröße hierfür ist die Aufspaltung der beiden phasenverschobenen Schwingungen, die durch den leichten Bruch der $C_{3}$-Symmetrie im uudMethanol-Trimer, sowohl experimentell wie berechnet, etwa $7 \mathrm{~cm}^{-1}$ beträgt. Für die 27 uud-Trimere wurden für diese Aufspaltung Werte zwischen 2 und $23 \mathrm{~cm}^{-1}$ berechnet, welche deutlich mit der angenäherten Symmetrie der Cluster, in Abhängigkeit von der Stellung der Ethylgruppen, korrelieren. Abb. 4.20 zeigt zur Veranschaulichung die Extrema dieser Skala. Für $u u d / g+g+g-$ lassen sich die Projektionen der Ethylgruppen auf die Ringebene gut durch eine Drehung um $120^{\circ}$ überführen, sodass nur eine sehr geringe Aufspaltung von $2 \mathrm{~cm}^{-1}$ resultiert. Erwartungsgemäß hat auch das ebenfalls annähernd symmetrische $u u d / t t t$ Konformer eine geringe Aufspaltung $\left(4 \mathrm{~cm}^{-1}\right) . u u d / g-g-g+$ 
mit drei nach innen zeigenden Ethylgruppen ist dagegen deutlich weniger symmetrisch $\left(16 \mathrm{~cm}^{-1}\right)$, da die Gruppen auf der selben Ringseite wegen des sterischen Konfliktes weniger äquivalent zur alleinigen Gruppe auf der anderen Seite sind. Für das ebenfalls in Abb. 4.20 gezeigte Trimer $u u d / t g-g-$ lassen sich die Ethylgruppen durch Drehung um die $C_{3}$-Achse nur sehr unvollständig ineinander überführen, die spektrale Aufspaltung ist daher besonders groß $\left(23 \mathrm{~cm}^{-1}\right)$.

Neben der Symmetrie und der Stärke der Kopplung zwischen den Oszillatoren wird auch die Stärke der Wasserstoffbrücke und damit die $\mathrm{OH}-$ Streckschwingungswellenzahl von den beteiligten Monomer-Konformeren beeinflusst. Eine entkoppelte Situation lässt sich durch arithmetische Mittelung der drei Schwingungswellenzahlen betrachten. Da die $t$-Konformere eine inhärent höhere Wellenzahl haben, werden im Folgenden nur die all-g-Trimere näher betrachtet. Abb. 4.21 zeigt die Korrelation zwischen der Anzahl der nach innen gerichteten $g$-Konformere und der gemittelten $\mathrm{OH}$ Streckschwingungswellenzahl. Es lässt sich erkennen, dass die Isomerisierung eines nach außen gerichtetes $g$-Konformer in ein nach innen gerichtetes stets mit einer Erhöhung

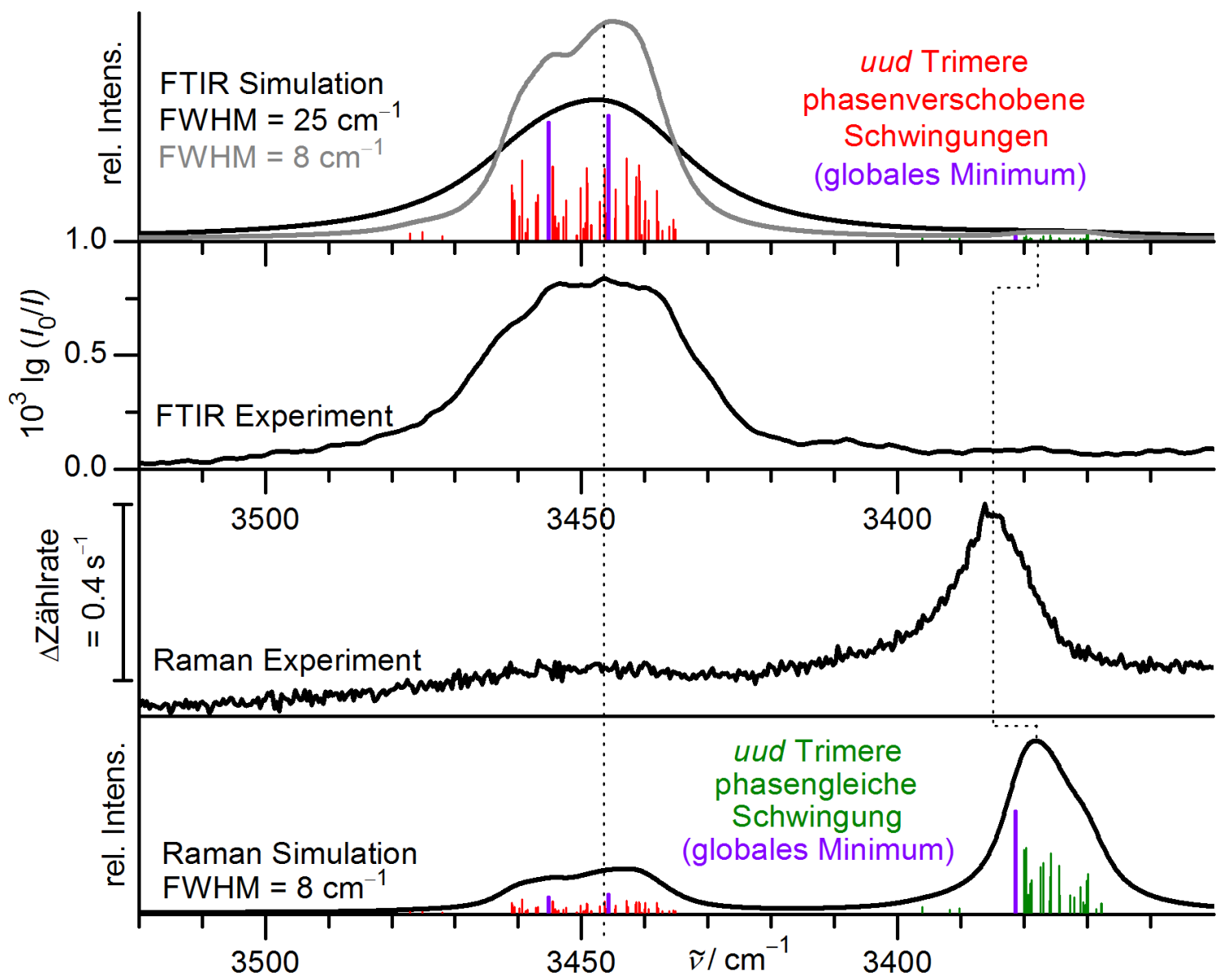

Abb. 4.19: Experimentelle und simulierte IR- [126] und Raman-Spektren [95] von Ethanol im Trimer-Bereich. Simulationsparameter sind einheitliche Verschiebung der harmonischen Wellenzahlen für eine gute Übereinstimmung der IR-Bande $\left(-107 \mathrm{~cm}^{-1}\right)$, eine Konformationstemperatur von $64 \mathrm{~K}$ und Lorentz-Profile mit voller Halbwertsbreite $25 \mathrm{~cm}^{-1}$ für die IR- und $8 \mathrm{~cm}^{-1}$ für die Raman-Banden. Eine alternative Modellierung des IR-Spektrums mit einer vollen Halbwertsbreiten von $8 \mathrm{~cm}^{-1}$ ist grau dargestellt. Die Übergänge des globalen Minimums $u u d / g-t g+$ sind violett hervorgehoben. 
der mittleren $\mathrm{OH}-$ Streckschwingungszahl verbunden ist, was als Schwächung einer oder mehrerer Wasserstoffbrücken interpretiert werden kann. Die Konkurrenz mit den sekundären Wechselwirkungen (Dispersion/sterische Abstoßung) führt in diesen Fällen zu Abweichungen von der bestmöglichen Wasserstoffbrücken-Geometrie für das Trimer. Dieser Effekt ist besonders stark wenn mehrere Ethylgruppen sterisch ungünstig wechselwirken. Die Kombination mit der großen Aufspaltung durch die hohe Asymmetrie der hiervon betroffenen Isomere führt jeweils zu einer besonders hochfrequenten IR-aktiven Schwingung, die in der Simulation Abb. 4.19 deutlich von den anderen Banden abgesetzt im Bereich von 3480-3470 $\mathrm{cm}^{-1}$ erkennbar sind. Allerdings sind deren Boltzmann-gewichteten Intensitäten durch die hohe relative Energie der Cluster gering.

Mit den berechneten spektralen Auswirkungen der Konformationsvielfalt lässt sich die Raman-Bande der phasengleichen Moden in Abb. 4.19 modellieren. Dies gelingt gut, sowohl bezüglich der Breite wie auch der leicht asymmetrischen Form der Bande, wenn die aus den Monomeren abgeleitete Konformationstemperatur von $64 \mathrm{~K}$ und die gleichen volle Halbwertsbreite wie bei Methanol $\left(8 \mathrm{~cm}^{-1}\right)$ verwendet wird. Im Gegensatz zu Methanol werden die nur sehr schwach IR-aktiven, phasengleichen Schwingungen der

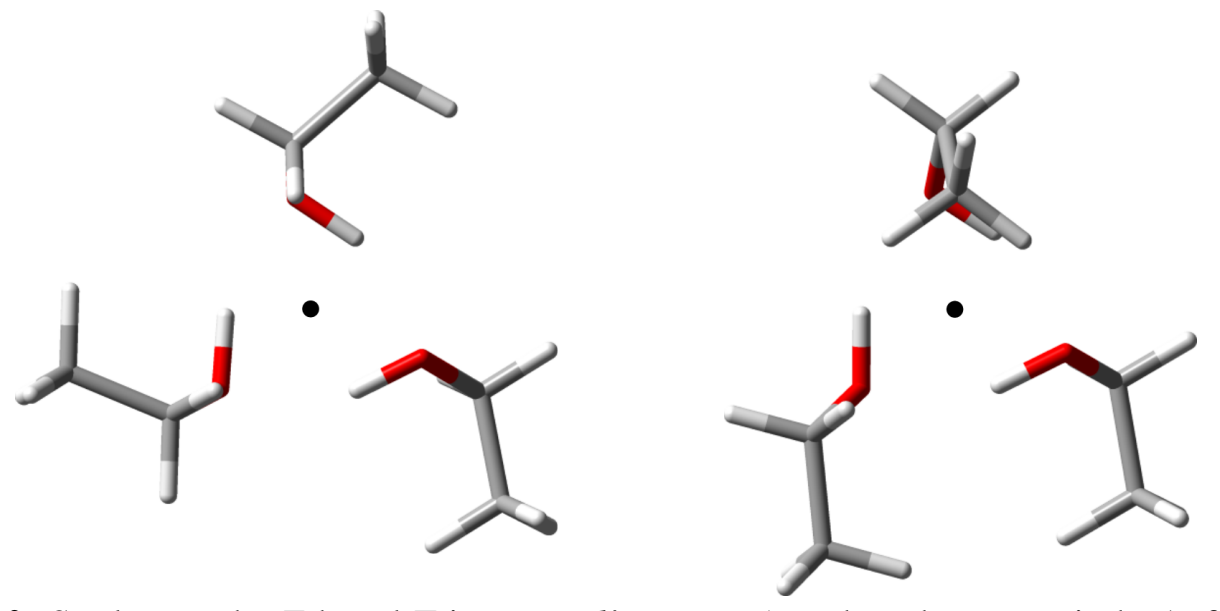

Abb. 4.20: Strukturen der Ethanol-Trimere $u u d / g+g+g-$ (annähernd symmetrisch, Aufspaltung der phasenverschobenen $\mathrm{OH}-$ Streckschwingmoden $2 \mathrm{~cm}^{-1}$ ) und $u u d / t g-g-$ (stärker asymmetrisch, Aufspaltung $23 \mathrm{~cm}^{-1}$ ). Die Blickrichtung auf die Strukturen erfolgt jeweils entlang der Pseudo- $C_{3}$-Drehachsen, deren Positionen durch schwarze Punkte markiert sind.

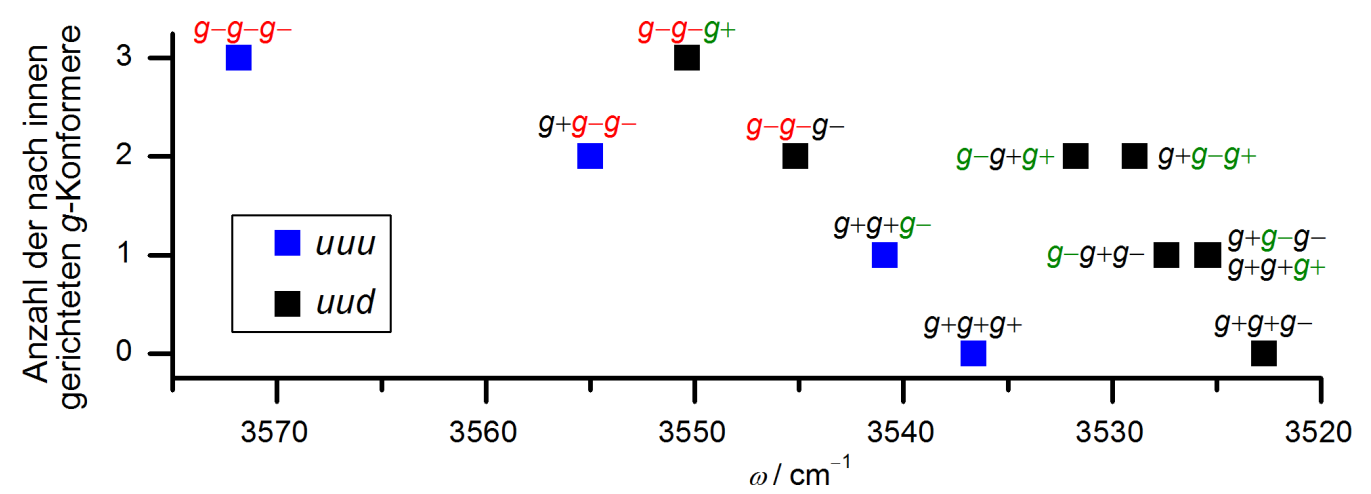

Abb. 4.21: Korrelation zwischen der Anzahl der nach innen gerichteten $g$-Konformere und der gemittelten harmonischen OH-Streckschwingungswellenzahl für all- $g$-Ethanol-Trimere. Die Werte für $u u d / g+g-g-$ und $u u d / g+g+g+$ koinzidieren. 
Ethanol-Trimere nicht im FTIR-Spektrum beobachtet - vermutlich eine Folge der Intensitätsverteilung auf mehrere Konformere.

Für die Modellierung der IR-Bande der phasenverschobenen Schwingungen von Ethanol wird dagegen eine deutlich erhöhte volle Halbwertsbreite von etwa $25 \mathrm{~cm}^{-1}$ benötigt, um die Halbwertsbreite der experimentellen Bande zu erreichen. Eine solch stark Verbreitung zwischen Methanol und Ethanol, sowie zwischen den Raman- und IR-aktiven Moden von Ethanol, ist auffällig. Die berechnete Konformationsvielfalt reicht nicht als (alleinige) Erklärung für die Breite der Bande aus - so wie mit Konformationsvielfalt auch die Bandbreite an Signalen im IR-Spektrum des Methanol-Trimers nicht erklärt werden konnte.[130]

Ein interessanter denkbarer Verbreiterungsmechanismus ist eine spektrale Aufspaltung durch konzertiertes Protonentunneln, bei dem die drei Wasserstoffbrücken effektiv ihre Richtung ändern. Dies wurde bisher vor allem für $S_{4}$-symmetrische AlkoholTetramere und größere symmetrische Cluster erwogen [130, 164], für Wasser- [142, 165] und Alkohol-Trimere [129] dagegen nur sehr eingeschränkt. Analog zum Tetramer erfolgt die konzertierte Tunnelbewegung für das Methanol-Trimer in einem symmetrischen Doppelminimumpotential, allerdings mit dem Unterschied, dass enantiomere statt identische Strukturen ineinander umgewandelt werden. Der Übergangszustand hierfür hat für das $u u d$-Methanol-Trimer $C_{\mathrm{s}}$-Symmetrie. Die Energiebarriere für die globalen Minimumstrukturen der Trimere beträgt ohne (mit) voller Nullpunktskorrektur 100 (77) kJ mol ${ }^{-1}$ für Methanol und 104 (79) $\mathrm{kJ} \mathrm{mol}^{-1}$ für Ethanol (B3LYP-D3(BJ)/may-cc-pVTZ). Dieser Mechanismus kommt aus verschiedenen Gründen nicht für die abnormale Breite der IRBande der Ethanol-Trimere als Erklärung in Frage:

- Die Barrierenhöhe für das stabilste Ethanol-Trimer ist nicht oder zumindest nicht substantiell genug geringer als die berechneten für das Methanol-Trimer, WasserTrimer [165] und Methanol-Tetramer.[164] Für die beiden letztgenannten Cluster wurden Tunnelaufspaltungen von (teils deutlich) kleiner als $1 \mathrm{~cm}^{-1}$ abgeleitet.[164, 165]

- Die Bande der Raman-aktiven phasengleichen Schwingung sollte durch diesen Mechanismus stärker verbreitert sein, da diese Schwingungskoordinate im Wesentlichen der Tunnelbewegung entspricht, sodass im schwingungsangeregten Zustand eine veränderte Tunnelaufspaltung zu erwarten ist.[164] Im Gegensatz zu dieser Erwartung wird beobachtet, dass stattdessen die Bande der stark IR-aktiven Schwingungen wesentlich breiter ist.

- Anders als für Methanol, ist für die meisten uud-Ethanol-Trimere die Symmetrie des Doppelminimumpotentials gebrochen, da die Umkehr der Richtung der Wasserstoffbrücken hier zu einer diastereomeren Struktur führt. Durch die Isomerisierung wandeln sich nach außen zeigende $g$-Konformere in $t$-Konformere um und umgekehrt. So sind beispielsweise über diese Koordinate $g-t g+$ und das um $0.3 \mathrm{~kJ} \mathrm{~mol}^{-1}$ instabilere Enantiomer von $g+g-g+$ verknüpft. Schon ein leichter Bruch der Symmetrie mit aufgehobener Entartung der Energiezustände kann Tunnelaufspaltungen effektiv unterdrücken.[166-168]

Konzertiertes Protonentunneln als relevanter Verbreiterungsmechanismus für die beobachteten Ethanol-Trimer-Banden kann daher ausgeschlossen werden. 


\section{Chiralitätssynchronisation in Ethanol-Aggregaten}

Aus der Analogie zum Methanol-Trimer, bei dem die Bande der phasenverschobenen Schwingungen mehrere Satellitenbanden aufweist, drängt sich eine ähnliche Hypothese für Ethanol auf. Durch die zusätzliche Konformationsvielfalt kann es bei Ethanol zu einer spektralen Überlappung vieler dieser Banden kommen, wodurch die beobachtete breite Bande resultieren könnte. Mit einem einfachen Modell wird daher im Folgenden untersucht, ob die Annahme von zusätzlichen Kombinationsbanden als Verbreiterungsmechanismus für die Ethanol-Bande zumindest in der Größenordnung plausibel ist. Hierzu wird vereinfacht nur die niederfrequenteste Mode des Clusters betrachtet, die einer synchronen Schwingung der Alkyl-Reste relativ zur Ringebene entspricht. An- bzw. Abregung dieser Mode kann zusammen mit der Anregung der OH-Streckschwingung erfolgen, sofern dies im Sinne eines Schwingungs-Franck-Condon-Prinzips [130] begünstigt wird. Für die Beobachtung einer Differenzbande ist allerdings eine thermische Anregung der niederfrequenten Mode Voraussetzung. Für das Methanol-Trimer wird die Wellenzahl dieser van der Waals-Mode zu $31 \mathrm{~cm}^{-1}$ berechnet. Die Positionen der sich ergebenden Kombinationsbanden stimmen grob mit denen der experimentellen Satellitenbanden überein (siehe obere Hälfte von Abb. 4.22). Die relativen Intensitäten dieser Satellitenbanden betragen etwa 30\% (Summebanden) bzw. 20\% (Differenzbanden) der Fundamentalbanden. Für die Übertragung des Modells auf Ethanol wird die Wellenzahl der jeweiligen van der WaalsMode der einzelnen Konformere verwendet. Die berechneten Werte liegen zwischen 13 und $30 \mathrm{~cm}^{-1}$ mit einem Mittelwert von $20 \mathrm{~cm}^{-1}$. Diese Erniedrigung der Frequenz ist im

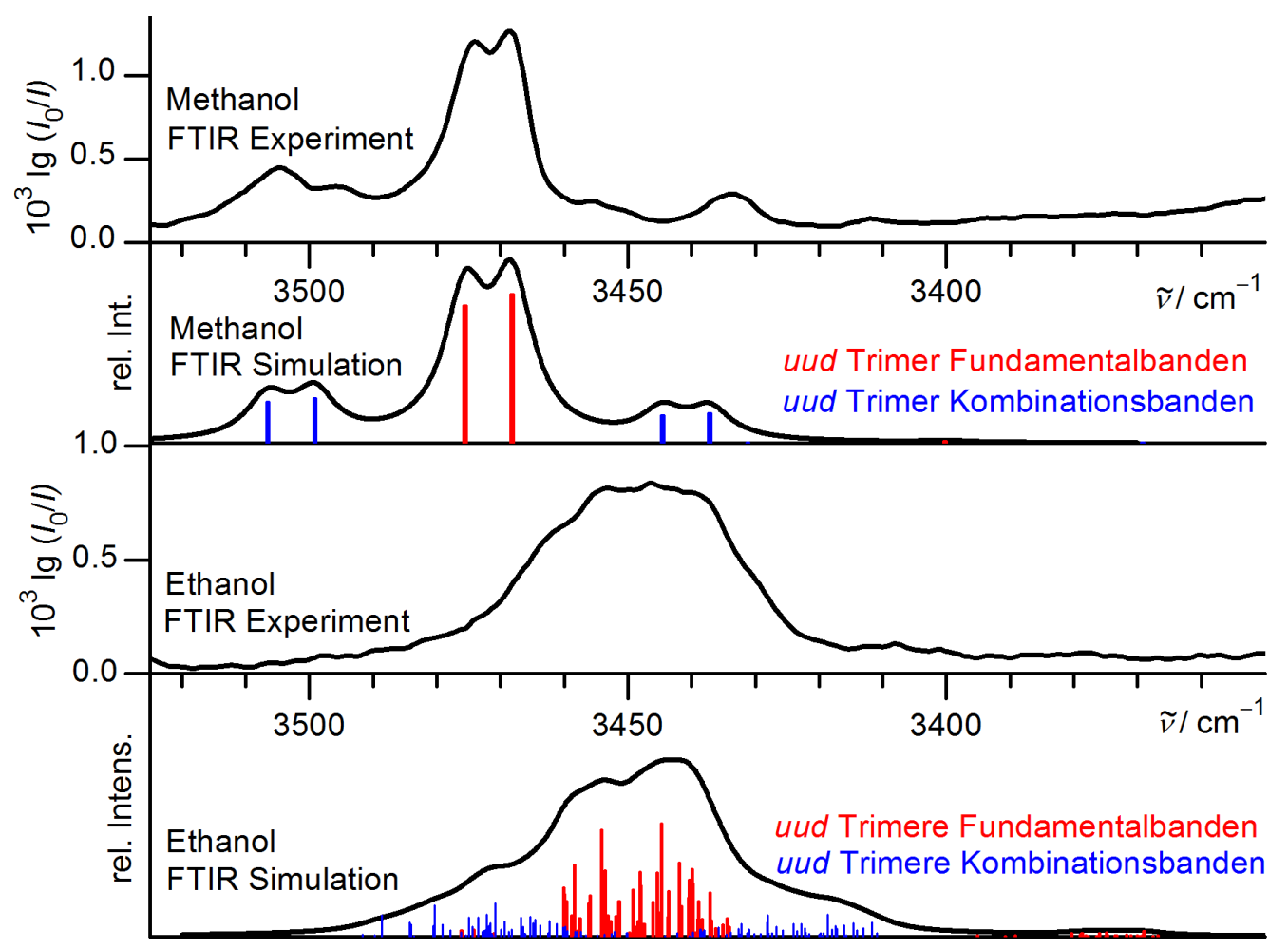

Abb. 4.22: Experimentelle und simulierte IR-Spektren von Methanol [129] und Ethanol [126] im Trimer-Bereich. Simulationsparameter sind Verschiebung der harmonischen Wellenzahlen um $-92 \mathrm{~cm}^{-1}$ (Methanol) und $-108 \mathrm{~cm}^{-1}$ (Ethanol), eine Konformationstemperatur von $64 \mathrm{~K}$, Lorentz-Profile mit Halbwertsbreite $8 \mathrm{~cm}^{-1}$, Kombinationsbanden mit Summe bzw. Differenz der Wellenzahlen der OH-Streckschwingungs-Fundamentalen und der jeweils niederfrequentesten Mode mit jeweils 30\% (Summe) bzw. 20\% (Differenz) Intensität der Fundamentalen. 
Wesentlichen auf die höhere bewegte Masse der Ethyl- gegenüber den Methylgruppen zurückzuführen. Die relativen Intensitäten der Kombinationsbanden werden von Methanol auf Ethanol direkt übertragen. Tendenziell sollten aber die Differenzbanden in Ethanol durch die geringen Frequenzen der van der Waals-Moden eine etwas höhere Intensität haben. Tatsächlich lässt sich somit eine Gesamtbreite der Bande grob übereinstimmend mit dem Experiment erhalten (Abb. 4.22), wenn für die Einzelbanden die geringere Halbwertsbreite von $8 \mathrm{~cm}^{-1}$ angenommen wird, die für die anderen Banden der Trimere von Methanol und Ethanol gefunden wurde. Die Feinstruktur der Bande wird mit diesem krudem Modell erwartungsgemäß nicht wiedergegeben. Die Intensitäten der Kombinationsbanden werden vermutlich deutlich von den anharmonischen Kopplungen im jeweiligen Konformer abhängen, sowie für die Differenzbande vom Ausmaß der thermischen Anregung der niederfrequenten Mode. Letzteres gilt auch für weitere heiße Banden, die im Modell nicht berücksichtigt wurden. Weitere niederfrequente Moden können relevant sein, wie für Methanol vermutet [130], deren spektrale Übergänge im anharmonischen Fall zudem vom Anregungszustand der anderen Moden abhängen. Eine nähere Analyse könnte hier künftig mit hochwertigen anharmonischen Rechnungen zunächst für das Methanol-Trimer ansetzen.

Experimentell bleibt mit den verfügbaren Daten offen, wie viele und welche EthanolTrimer-Konformere tatsächlich populiert werden. Die größeren Breiten der IR- und Raman-Banden im Vergleich zu Methanol geben nur indirekte Hinweise auf die vorhergesagte Konformationsvielfalt, da offenbar auch andere Verbreiterungsmechanismen eine Rolle spielen. Durch die große Zahl an überlagerten Konformeren, die hohen Halbwertsbreiten und die starke Kopplung zwischen den Oszillatoren reagieren die simulierten Spektren wenig empfindlich auf die genaue Konformerenpopulation. Eine Modellierung auf Basis von B3LYP/may-cc-pVTZ ohne Dispersionskorrektur ergibt sehr ähnliche simulierte Spektren, trotz der zuvor diskutierten drastisch unterschiedlichen Energieabfolge der Konformere. Auch der Versuch, die Energie- oder Wellenzahldifferenz nachträglich auf den Wert im Monomer zu korrigieren, hat nur sehr wenig Einfluss auf die simulierten Spektren.

Der berechnete Energievorteil des globalem Minimums gegenüber den energetisch nächsthöheren Konformeren liegt im gleichen Bereich wie für die Ethanol-Dimere, daher könnte Kühlung mit Neon eine weitgehende Relaxation ermöglichen (sofern die Barrieren hierfür niedrig genug sind). In Abb. 4.19 sind die Übergänge des globalen Minimums violett hervorgehoben. Bei einer starken Absenkung der Konformationstemperatur ist tendenziell eine Verschmälerung und möglicherweise eine leichte Aufspaltung der IR-aktiven Bande (wie bei Methanol) zu erwarten. Die Raman-aktive Bande sollte sich ebenfalls verschmälern, während sich ihr Schwerpunkt leicht zu höheren Wellenzahlen verschiebt.

Rotationsspektroskopie verbleibt als vielversprechendster Ansatz um einen direkten Nachweis von einzelnen Konformeren zu erreichen. Die in die Empfindlichkeit quadratisch eingehende größte Dipolmoment-Komponente der Ethanol-Trimere ist mit etwa 0.7 D niedriger als die der bisher nachgewiesenen Ethanol-Dimere mit etwa 2.0 D. Tabelle A.4 im Anhang auf S. 308 listet die rotationsspektroskopischen Kenngrößen für die stabilsten Trimere für eine zukünftige Bestätigung oder Widerlegung der hier vorhergesagten Strukturen. 


\subsection{Ethanol- und Methanol-Tetramere}

\subsubsection{Konformationsisomerie}

Auch für die Tetramere von Ethanol ist in Analogie zu den Homologen Wasser und Methanol die starke Bevorzugung von cyclischen Strukturen gut etabliert.[98, 141, 143, 144, 151, 152, 169] Im Gegensatz zum Trimer können die Alkylgruppen wegen der geraden Zahl an Molekülen im Tetramer eine vollständig alternierende Anordnung udud annehmen, die das bekannte globale Minimum für Methanol [130, 140, 157, 170] und Wasser [171] darstellt. Alternative Anordnungen sind uиdd, иииd und ииии, die in Abb. 4.23 für Methanol mit berechneten relativen Energien und Punktgruppen gezeigt sind. uudd und uииd sind sich energetisch nahe, da beide zwei günstige alternierende $u d$ - und zwei ungünstige $u u$ - bzw. $d d$-Anordnungen zwischen benachbarten Molekülen beinhalten, nur in unterschiedlicher Abfolge. ииии weist vier $и и$-Anordnungen auf und hat daher etwa die doppelte relative Energie. Die Umwandlung von zwei $u d$ - in $u u$-Anordnungen benötigt daher stets etwa $3 \mathrm{~kJ} \mathrm{~mol}^{-1}$. Dieser Wert stimmt auch gut mit dem für die Isomerisierungsenergie des Methanol-Trimers von uud zu uuu überein $\left(2.8 \mathrm{~kJ} \mathrm{~mol}^{-1}\right)$.

Weitere Minima auf der Potentialfläche des cyclischen Methanol-Tetramers konnten nicht gefunden werden, wie etwa diskutierte tetraedrische Anordnungen [172]). Die Bildung des Methanol-Tetramers aus zwei transient chiralen Dimeren kann als Chiralitätssynchronisation beschrieben werden.[5]

Existierende quantenchemische Betrachtungen zu Ethanol-Tetrameren [96, 98, 141, 152, 173] haben ähnliche Einschränkungen oder Defizite wie zuvor für die Trimere diskutiert. Nur in einer einzigen neueren Studie mit Einsatz einer Dispersionskorrektur [96]

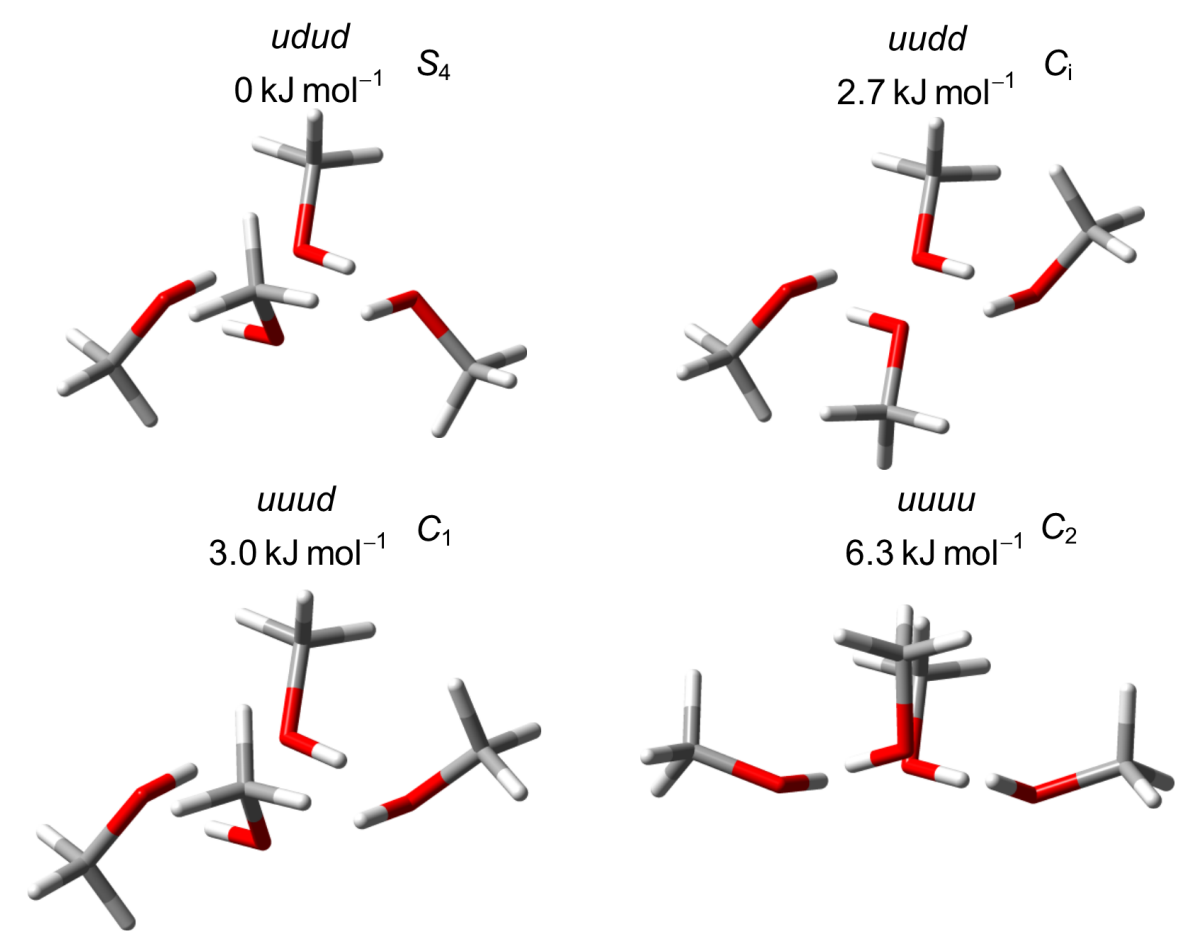

Abb. 4.23: Die Strukturen der vier Isomere des cyclischen Methanol-Tetramers sowie deren relative Energien und Punktgruppen. Das hintere Methanol-Molekül ist jeweils vergleichbar ausgerichtet, womit insbesondere die große Verzerrung der Anordnung im Fall von ииии deutlich wird. 
scheinen keine Vorannahmen für die beteiligten Monomer-Konformere gemacht worden zu sein. Allerdings schienen deren Ergebnisse bereits für das Trimer zweifelhaft, die angegebene Struktur des Tetramers wird weiter unten eingeordnet.

Für die Erweiterung von Methanol- zu Ethanol-Tetrameren durch Verlängerung der Alkylgruppen ergeben sich $3^{4}=81$ Variationen. Durch Anwendung der Gruppentheorie lässt sich zeigen, dass die $S_{4}$-Symmetrie der $u d u d$-Grundstruktur zu 24 diastereomeren $u d u d$-Ethanol-Tetrameren führt. Je vier Variationen gehören zu $18 C_{1}$-symmetrischen Strukturen. So ist beispielsweise die Variation $g+t t t$ identisch zu $t t g+t$ (Anwendung der Symmetrieoperation $C_{2}$ ) und enantiomer zum Paar $t g-t t\left(S_{4}\right) / t t t g-\left(S_{4}^{3}\right)$. Sechs Variationen lassen sich durch die $C_{2}$-Operation (aber nicht durch $S_{4}$ und $S_{4}^{3}$ ) in sich selbst überführen und gehören zu insgesamt drei Strukturen, die durch die Untergruppe $C_{2}$ beschrieben werden. Beispiele hierfür sind $g+t g+t$ und das hierzu enantiomere $t g-t g-$. Die drei verbleibenden Variationen lassen sich durch alle Symmetrieoperationen der Punktgruppe in sich selbst überführen und gehören folglich zu drei Tetrameren mit erhaltener $S_{4}$-Symmetrie, dies sind $t t t t, g+g-g+g-$ und $g-g+g-g+$. Für eine eindeutige Benennung wird die im vorherigen Kapitel eingeführte Nomenklatur der Trimere hier fortgeführt: Aufzählung in der Reihenfolge udud im Uhrzeigersinn der Wasserstoffbrücken und Wahl der alphabetisch niedrigsten Variation als Bezeichnung. Aus den Variationen des oben aufgeführten $C_{1}$-symmetrischen Beispiels wird daher die Bezeichnung $g+t t t$ und für das $C_{2}$-symmetrische Beispiel $g+t g+t$ ausgewählt.

Im Gegensatz zum uud-Trimer können beim udud-Tetramer ohne sterische AbstoBung zwei $g$-Konformere auf der selben Seite nach innen zeigen, da diese nicht direkt benachbart sind. Als globales Minimum ergibt sich gemäß den bereits für die Trimere abgeleiteten Stabilitätsregeln die heterochirale Struktur $u d u d / g-g+g-g+$. Dieser $S_{4-}$ symmetrische-Cluster ist in Abb. 4.24 zusammen mit den jeweils stabilsten Strukturen der anderen Grundmotive des Ethanol-Tetramers gezeigt. Die Anordnung in allen vier Wasserstoffbrücken entspricht der des Dimers $g-g+$. Für die Energieabfolge innerhalb der $u d u d$-Ethanol-Tetramere ergibt sich eine noch deutlichere Hierarchie als für die Trimere hinsichtlich der Anzahl der nach innen gerichteten $g$-Konformere (Abb. 4.25 auf S. 83). Der energetische Vorteil gegenüber der nächst stabileren Struktur $g-g+g-t$ beträgt $0.5 \mathrm{~kJ} \mathrm{~mol}^{-1}$, der auch gegenüber einer denkbaren nachträglichen Korrektur der Monomer- $g / t$-Differenz robust erscheint. Allerdings ist das globale Minimum gleich doppelt statistisch gegenüber den meisten anderen Konformeren benachteiligt. Bedingt durch die $S_{4}$-Symmetrie ist die Struktur zum einen achiral, zum anderen weist sie eine Rotationssymmetriezahl von zwei auf. Dies multipliziert sich zu einer statistischen Benachteiligung um den Faktor vier gegenüber asymmetrischen Konformeren. Analog zum Monomer ergibt sich für die Population der beiden wichtigsten Tetramer-Konformere damit eine Konkurrenz zwischen einer energetisch und einer statistisch bevorteilten Konformation. Bei einer angenommenen Konformationstemperatur von $64 \mathrm{~K}$ für Überschallexpansionen mit Helium als Trägergas wäre daher $g-g+g-t$ als das am höchsten populierte Konformer einzuschätzen. $g-g+g-t$ enthält als Dimer-Motive zweimal $g-g+$ und jeweils einmal $t g+$ und $g-t$. Bei gleicher Anzahl der nach innen gerichteten $g$-Konformere bestimmt sich die energetische Reihenfolge der Konformere, wie bereits beim Trimer festgestellt, im Wesentlichen durch Anzahl der $t$-Konformere mit resultierenden Energieunterschieden in der Nähe der bekannten Monomer- $g / t$-Differenz. Ebenfalls lassen sich auch hier energetische Aufspaltungen erkennen, wenn unterschiedliche Reihenfolgen von in ihrer Ausrich- 
tung äquivalenten intramolekularen Konformere möglich sind, beispielsweise zwischen $g+g-t t$ und $g+t g+t$. Die Energieniveaus solcher Isomere sind wie zuvor bei den Trimeren jeweils in einer gemeinsamen Farbe dargestellt. Eine besonders große Aufspaltung dieser Art tritt für Strukturen mit zwei nach innen gerichteten $g$-Konformeren auf, deren Ethylgruppen entweder beide auf der selben Seite oder alternativ auf den unterschiedlichen Seiten des Wasserstoffbrücken-Ringes liegen können. Dieser Energieunterschied liegt im Bereich von etwa $0.5 \mathrm{~kJ} \mathrm{~mol}^{-1}$ und korreliert mit einem auffälligen strukturellen Effekt, der nur auftritt wenn ein nach innen gerichtetes $g$-Konformer einzeln auf einer Seite liegt. Die innere Struktur der Ethanol-udud-Tetramere ist ohne dieses Merkmal nahezu planar $\left(\mathrm{O}_{u} \mathrm{O}_{d} \mathrm{O}_{u} \mathrm{O}_{d}\right.$-Torsionswinkel von $\left.0-5^{\circ}\right)$, analog zum $u d u d$-Methanol-Tetramer $\left(3^{\circ}\right)$. Mit einem einzelnen nach innen gerichteten $g$-Konformer auf einer Seite erhöht sich dieser Wert auf $9-11^{\circ}$, bei zwei jeweils einzeln nach innen gerichteten $g$-Konfomeren auf jeder Seiten sogar auf $15-16^{\circ}$. Diese strukturelle Änderung entspricht einer dreidimensionalen Verzerrung eines (näherungsweise) planaren Quadrates in einen unregelmäßigen Tetraeder. Der Grenzfall eines regelmäßigen Tetraeders wäre bei einem Winkel von etwa $70.5^{\circ}$ erreicht. Diese Faltung des Ringes führt zu einer etwas kompakteren Struktur und optimiert damit vermutlich die Dispersionswechselwirkung. Liegen zwei nach innen gerichtete $g$-Konformere auf der selben Seite, tritt der Effekt nicht auf, es sei denn auf

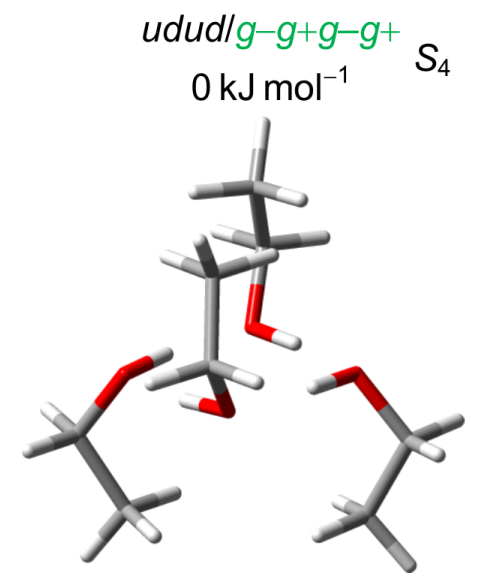

uuudlg-tg-g+

$3.1 \mathrm{~kJ} \mathrm{~mol}^{-1}$

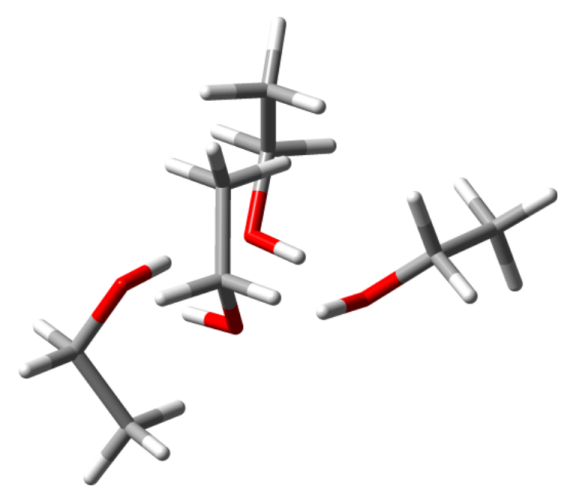

uudd/g-ttg+ $4.0 \mathrm{~kJ} \mathrm{~mol}^{-1}$ $C_{1}$

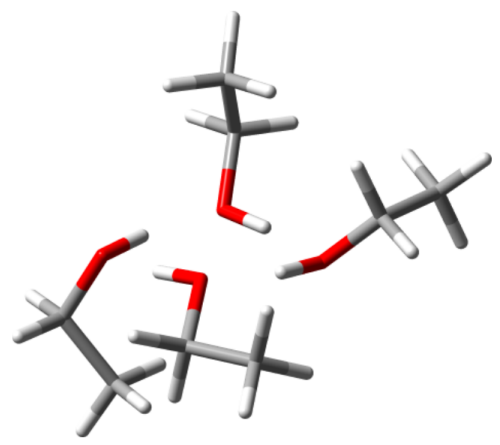

uuuulg-tg-t $6.5 \mathrm{~kJ} \mathrm{~mol}^{-1}$ $C_{2}$

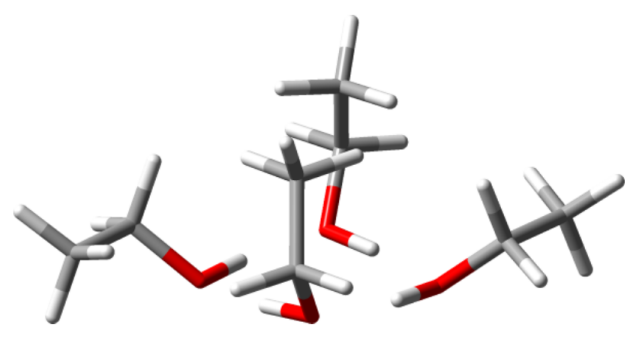

Abb. 4.24: Die jeweils stabilsten Strukturen der vier Grundmotive des Ethanol-Tetramers und deren relative Energien und Punktgruppen. Erkennbar ist die Bevorzugung von nach innen gerichteten $g$-Konformeren sofern sterisch günstig, ansonsten von $t$-Konformeren. 


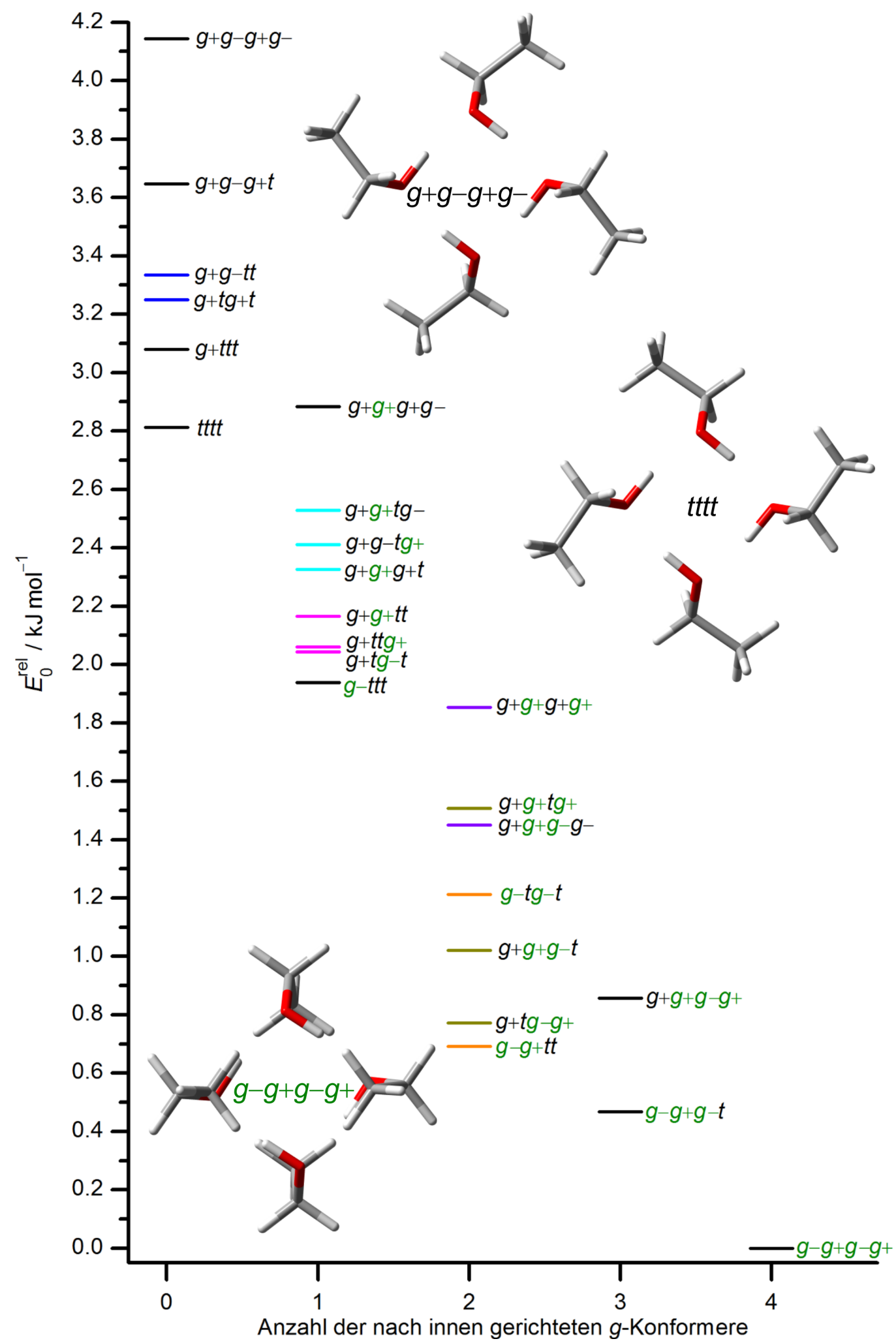

Abb. 4.25: Relative Energie der $u d u d$-Ethanol-Tetramere in Abhängigkeit von der Anzahl der nach innen gerichteten $g$-Konformere auf B3LYP-D3(BJ)/may-cc-pVTZ-Niveau. Die Energieniveaus von Isomeren, die sich allein durch eine veränderte Reihenfolge von äquivalenten Ethanol-Konformeren unterscheiden, sind jeweils farblich markiert. Die Strukturen der drei $S_{4}$ symmetrischen Strukturen sind als Insert mit Blickrichtung entlang der Drehspiegelachse eingefügt. 


\section{Chiralitätssynchronisation in Ethanol-Aggregaten}

der anderen Seite liegt ein drittes, aber kein viertes solches Konformer. Eine weitere Annäherung der auf der gleichen Seite liegenden Alkylgruppen lässt sich durch kovalente Bindungen erzwingen: im ebenfalls heterochiral synchronisierten Ethylenglycol-Dimer $[27,174]$ ist mit $68^{\circ}$ der ideale Tetraeder fast erreicht.

Ohne Dispersionskorrektur ergibt sich, wie bereits für die Trimere, eine sehr stark abweichende Energieabfolge. $g-g+g-g+$ ist nicht länger das globale Minimum, sondern die instabilste (!) aller $u d u d$-Strukturen mit einer relativen Energie von $3.9 \mathrm{~kJ} \mathrm{~mol}^{-1}$ gegenüber dem neuen globalen Minimum tttt. Die Gesamtänderung des Energieunterschiedes zwischen diesen beiden Strukturen beträgt $6.9 \mathrm{~kJ} \mathrm{~mol}^{-1}$ durch Verzicht auf die Dispersionskorrektur. Auch strukturell ergeben sich deutliche Veränderungen. So erhöht sich der C...C-Abstand der diagonal gegenüber liegenden, nach innen gerichteten endständigen Methylgruppen bei $g-g+g-g+$ von 4.0 auf 5.0 ̊. Auch die oben diskutierte deutliche Abweichung von der Planarität für bestimmte Konformere findet ohne Dispersionskorrektur nicht statt.

Das zweitstabilste $u u d d$-Methanol-Tetramer besitzt $C_{\mathrm{i}}$-Symmetrie. Von den daraus abgeleiteten 81 möglichen Variationen für $u u d d$-Ethanol-Tetramere sind neun gegenüber der Operation $C_{\mathrm{i}}$ invariant, sie gehören dementsprechend zu neun $C_{\mathrm{i}}$-symmetrischen Strukturen. Die verbleibenden 72 Variationen bilden 36 Enantiomerenpaare. Hiervon wurden nur die neun symmetrischen Strukturen sowie die asymmetrische $g-t t g+$ Struktur optimiert, denn nach den bisherigen Erfahrungswerten sollte das Minimum dieses Strukturmotivs entweder $t g-t g+, g-t g+t$ oder $g-t t g+$ sein, bei denen auf jeder Ringseite ein nach innen gerichtetes $g$-Konformer ausgebildet wird und die beiden verbleibenden Moleküle aus sterischen Gründen die $t$-Konformation annehmen. Von diesen zehn näher untersuchten Strukturen sind tatsächlich die drei vermuteten am stabilsten mit der asymmetrischen $g-t t g+$ als energetisch niedrigsten. Die Energie relativ zum globalen Minimum $u d u d / g-g+g-g+$ beträgt $4.0 \mathrm{~kJ} \mathrm{~mol}^{-1}$.

Von der weiter oben erwähnten einzigen anderen Studie [96] für Ethanol-Tetramere mit Dispersionskorrektur und gemischten intramolekularen Konformationen wurde $u u d d /$ $t g+t g+$ als Ergebnis der Optimierung angegeben. Allerdings ist, wie bereits beim Trimer, völlig unklar, ob überhaupt andere Isomere evaluiert wurden. Dass die gleichen Konformere als Ergebnisse der Optimierungen mit und ohne Dispersionskorrektur angegeben werden, spricht klar dagegen. $u u d d / t g+t g+$ liegt in der vorliegenden Arbeit mit einer relative Energie von $4.9 \mathrm{~kJ} \mathrm{~mol}^{-1}$ energetisch weit über dem globalen Minimum.

Die einzige bisher nachgewiesene konkrete Ethanol-Tetramer-Struktur lässt sich als uudd/tg+tg- klassifizieren - einem Zufallsfund durch stöchiometrischen Lösungsmitteleinschluss bei Kristallisation eines Silber-Komplexes aus alkoholischer Lösung.[175] Für uudd/tg+tg- wurde dagegen in der vorliegenden Arbeit eine berechnete relative Energie von $5.5 \mathrm{~kJ} \mathrm{~mol}^{-1}$ in der Gasphase gefunden, was auf einen stabilisierenden Einfluss des umgebenden Kristalls hindeutet.

Das uuud-Methanol-Tetramer ist asymmetrisch, alle vier Moleküle sind inäquivalent und es ist damit die volle Zahl an 81 diastereomeren uuud-Ethanol-Tetrameren dieses Strukturmotivs zu erwarten. Von diesen wurde nur eine kleine Auswahl optimiert, als stabilste Struktur hat sich davon wie erwartet $g-t g-g+$ mit drei nach innen gerichteten $g$ Konformeren und einer Energie von $3.1 \mathrm{~kJ} \mathrm{~mol}^{-1}$ relativ zum globalen Minimum ergeben.

Das ииии-Methanol-Tetramer hat $C_{2}$-Symmetrie, bei der die beiden inäquivalenten Paare an Methylgruppen unterschiedlich stark aus der Ringebene ausgelenkt sind. Die 
Struktur kann über einen $C_{4}$-symmetrischen Übergangszustand in eine identische Anordnung umwandeln, wobei die Methylgruppen-Paare ihre Auslenkung vertauschen. Die abgeleiteten 81 Variationen für Ethanol-Tetramere lassen neun $C_{2}$-symmetrische und 72 asymmetrische Strukturen erwarten. Dabei wird in der Regel die Symmetrie des Übergangszustandes und damit des Doppelminimumpotentials gebrochen und die Strukturen lassen sich als 39 zueinander diastereomere Paare hinsichtlich dieser Isomerie klassifizieren. Die Unterscheidung beruht darauf, welches der gegenüberliegenden EthylgruppenPaare stärker aus der Ringebene ausgelenkt ist. Die Ausnahme von der Regel stellen die drei Strukturen mit einheitlicher intramolekularer Konformation dar, bei denen die Symmetrie des Isomerisierungspotentials wie beim Methanol-Tetramer erhalten bleibt. Näher untersucht wurden hier nur die neun $C_{2}$-symmetrischen Strukturen, wobei im Einklang mit der Erwartung $g-t g-t$ als stabilste Struktur mit einer Energie von $6.5 \mathrm{~kJ} \mathrm{~mol}^{-1}$ relativ zum globalen Minimum gefunden wird. Die Ethylgruppen der nach innen gerichteten $g$-Konformere liegen in dieser Struktur näher zusammen (d.h. stärker aus der Ringebene ausgelenkt) als im isomeren $t g-t g-$ mit einer Energie von $7.6 \mathrm{~kJ} \mathrm{~mol}^{-1}$ relativ zum globalen Minimum.

$\mathrm{Da}$ die OH-Streckschwingungsbanden der Tetramere teilweise mit denen der Pentaund Hexamere überlappen, werden im Folgenden zuerst deren Strukturen besprochen bevor eine Modellierung der Schwingungsspektren in diesem Spektralbereich versucht wird.

\subsection{Ethanol- und Methanol-Pentamere}

\subsubsection{Konformationsisomerie}

Nach den Ergebnissen früherer Untersuchungen [140, 157, 176] existieren für das Methanol-Pentamer bis zu vier cyclische Strukturen mit alternativen up/down-Anordnungen der Methylreste: uиdиd, иииdd, ииииd und иииии. Von diesen können nur ииdиd und uuudd als Minima auf B3LYP-D3(BJ)/may-cc-pVTZ-Niveau bestätigt werden. Das globale Minimum ist wie erwartet uudud mit einem Maximum an möglicher alternierender Anordnung der Methylgruppen und einem energetischen Vorteil von $2.5 \mathrm{~kJ} \mathrm{~mol}^{-1}$ gegenüber uuudd. Dieser Energiebetrag ist etwas geringer als der erwartete Wert von etwa $3.0 \mathrm{~kJ} \mathrm{~mol}^{-1}$ für die Umwandlung zweier $u d$ in $u u / d d$-Anordnungen und deutet auf eine höhere Flexibilität des Ringes gegenüber den Tri- und Tetrameren hin.

Die Untersuchung der Gesamtmenge der zu erwartenden, sich aus der asymmetrischen Struktur uudud ableitenden $3^{5}=243$ Ethanol-Pentamer-Isomere übersteigt die Möglichkeiten dieser Arbeit. Aus der Extrapolation der Befunde für Trimere und Tetramere lässt sich jedoch entweder $u u d u d / t g-g+g-g+$ oder $u u d u d / g-t g+g-g+$ als globales Minimum erwarten. Optimierung der beiden Strukturen zeigt, dass die erstgenannte überraschend deutlich $\left(1.4 \mathrm{~kJ} \mathrm{~mol}^{-1}\right)$ stabiler ist als die zweitgenannte. $u u d u d / t g-g+g-g+$ ist in Abb. 4.26 dargestellt. Eine dritte evaluierte Struktur $u u d u d / g-g-g+g-g+$ mit allen Ethylresten nach innen gerichtet hat eine relative Energie von $1.3 \mathrm{~kJ} \mathrm{~mol}^{-1}$. Der erweiterte und damit flexiblere Ring lässt hier offenbar mehr Raum für die Anordnung von benachbarten Ethylgruppen, womit die zuvor abgeleiteten Stabilitätsregeln aufgeweicht werden. Die von Balanay et al. [96] angegebene Struktur kann als $u u d u d / t g+t g-g+$ klassifiziert werden und hat nach Reoptimierung eine relative Energie von $2.5 \mathrm{~kJ} \mathrm{~mol}^{-1}$. Berechne- 
te Schwingungsspektren für Pentamere basieren wegen der großen Energieabstände im Folgenden ausschließlich auf uudud (Methanol) und uudud/tg-g+g-g+ (Ethanol).

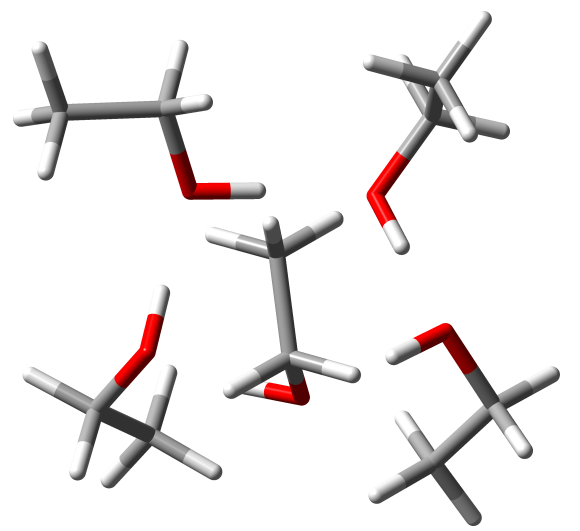

Abb. 4.26: Struktur des vermuteten globalen Minimums der Ethanol-Pentamere $u u d u d / t g-g+g-g+$.

\subsection{Ethanol- und Methanol-Hexamere}

\subsubsection{Konformationsisomerie}

Für Wasser sind die verschiedenen, nahezu isoenergetischen Hexamer-Strukturen die ersten, die eine voll dreidimensionale Anordnung darstellen, bei der Wasser-Moleküle teilweise als Akzeptor und Donor von mehreren Wasserstoffbrücken agieren.[177] Dies beruht aber vor allem darauf, dass Wasser ein zweites acides Wasserstoffatom aufweist. Die bis hierhin beobachtete Homologie zwischen Wasser und kurzkettigen Alkoholen in ihren globalen Minimumstrukturen ist damit gebrochen. Eine stärker zweidimensionale $S_{6}$-symmetrische Struktur von Wasser konnte aber dennoch kinetisch stabilisiert in Helium-Nanotröpfchen beobachtet werden.[178]

Für Methanol-Hexamere wurde zuvor quantenchemisch wie experimentell ein Gleichgewicht zwischen einer $S_{6}$-symmetrischen Sessel- und einer $C_{2}$-symmetrischen Wannenkonformation gefunden.[179] Beide entsprechen Sechsringen, bei denen die Sauerstoffatome nur noch sehr grob in einer Ebene liegen. Mit der Wellung des Ringes bei steigender Ringgröße ohne klare Referenzebene wird die Einstufung von AlkylgruppenAusrichtungen als $u$ oder $d$ in ihrer ursprünglichen Bedeutung daher zunehmend problematisch. Hier erweist sich die bereits eingangs erwähnte Beziehung als hilfreich, nach der Moleküle in $u$-Position stets am linken und solche in $d$-Position am rechten Elektronenpaar koordiniert werden, wenn die Wasserstoffbrücken von der betrachteten Seite im Uhrzeigersinn angeordnet sind. Damit lässt sich die bisherige Nomenklatur weiterhin eindeutig weiterführen. Demnach lässt sich die Sesselkonformation als $u d u d u d$ und die Wannenkonformation als uududd klassifizieren. Für die letztgenannte wurde allerdings hier gefunden, dass diese bei Verwendung der Dispersionskorrektur zu einer zwar weiterhin ähnlichen, aber asymmetrischen und kompakteren Struktur kollabiert, die $1.0 \mathrm{~kJ} \mathrm{~mol}^{-1}$ instabiler als die Sesselkonformation ist. Diese neue chirale Wannenkonformation besitzt allerdings einen statistischen Vorteil von sechs (zweifach durch die Chiralität und dreifach durch die unterschiedliche Rotationssymmetriezahl), sodass zu erwarten ist, dass 
beide Konformere bei einer angenommenen Konformationstemperatur von $64 \mathrm{~K}$ ähnlich populiert sind. Dieser Umschlagpunkt in der relativen Population wurde experimentell bei einer Clustertemperatur von etwa $100 \mathrm{~K}$ bestimmt, allerdings ist unklar inwieweit dieser indirekt abgeleitete Wert tatsächlich der effektiven Konformationstemperatur entspricht.[179]

Weitere Extrapolation der in dieser Arbeit gefundenen Stabilitätsregeln für cyclische Ethanol-Cluster lässt für das stabilste Hexamer das Konformer $u d u d u d / g-g+g-g+g-g+$ mit $S_{6}$-Symmetrie erwarten. Die Struktur ist in Abb. 4.27 dargestellt. Die SauerstoffAtome nehmen hier eine Sesselkonformation ein, die Methylengruppen liegen zwischen einer axialen und äquatorialen Ausrichtung, die endständigen Methylgruppen zeigen nach innen. Durch den hohen statistischen Nachteil dieser Struktur ist aber zu erwarten, dass Strukturen mit leichten Abweichungen stärker populiert sind. So beträgt die berechnete Energie für die Umwandlung eines $g$ - in ein $t$-Konformer nur $0.6 \mathrm{~kJ} \mathrm{~mol}^{-1}$.

Für Ethanol-Hexamere wurde erst kürzlich eine rein theoretische Arbeit von Malloum et al. publiziert,[111] an der sich sowohl die Möglichkeiten wie auch die Fallstricke bei der Untersuchung von Ethanol-Clustern aufzeigen lassen. Die Vorhersagen aus dieser Studie bieten sich als Vergleiche für die hier durchgeführten Rechnungen an. Wie die Autoren treffend anmerken, sind nicht nur Ethanol-Hexamere, sondern auch -Trimere, -Tetramere und -Pentamere bisher nur sehr unzureichend charakterisiert worden - eine Lücke, die die hier vorliegende Arbeit zum Teil zu schließen versucht. Warum die Autoren angesichts dieses Befundes dagegen direkt die extrem komplexe Potentialfläche der Hexamere als Studienobjekt gewählt haben, bleibt etwas unverständlich. Um die hochdimensionale Fragestellung zu bewältigen, wurden Strukturen mit ABCluster erzeugt (einem Algorithmus basierend auf einem Lennard-Jones-Kraftfeld), von denen eine Auswahl mit APFD/6-31++G(d,p) und MP2/aug-cc-pVDZ nachoptimiert wurde. Ein Vortest dieser quantenchemischen Methoden anhand von experimentellen und theoretischen Ergebnissen für Monomere und Dimere erfolgte nicht. Für das Monomer wird eine nullpunktskorrigierte Energiedifferenz von $0.2 \mathrm{kcal} \mathrm{mol}^{-1}$ auf APF-D/6-31++G(d,p)-Niveau zugunsten des $t$-Konformers angeben. Allerdings konnte dieser Wert in der vorliegenden Arbeit nicht reproduziert werden. Persönliche Korrespondenz mit den Autoren ergab, dass hier Er-
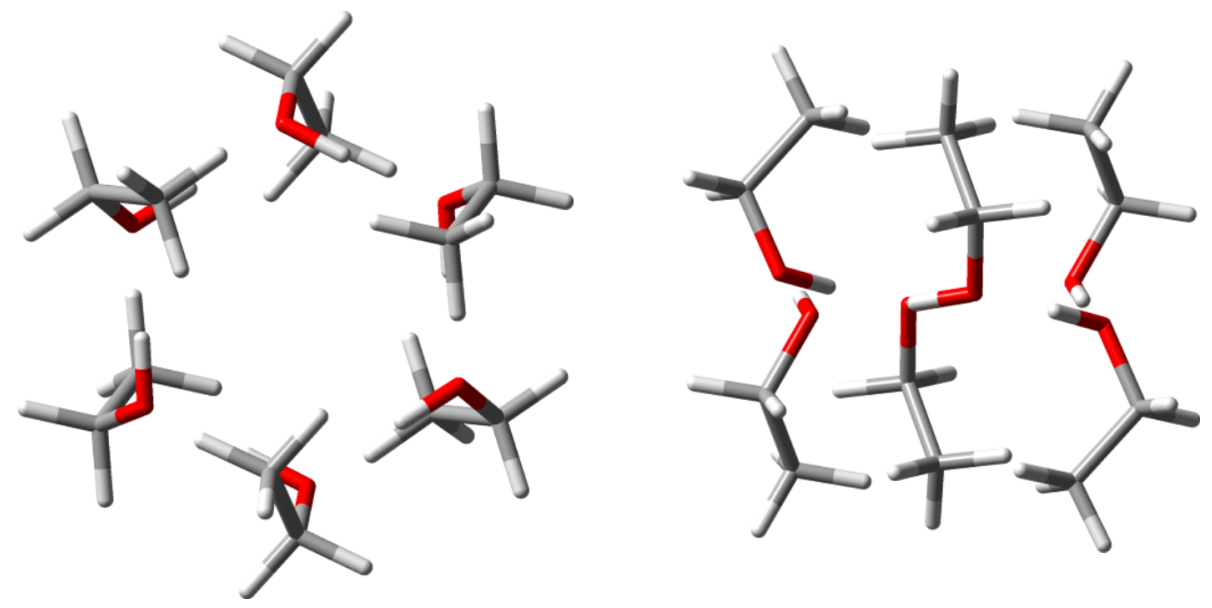

Abb. 4.27: Struktur des vermuteten globalen Minimums der Ethanol-Hexamere $u d u d u d / g-g+g-g+g-g+$ auf B3LYP-D3(BJ)/may-cc-pVTZ-Niveau mit Blickrichtung entlang (links) und senkrecht zur $S_{6}$-Drehspiegelachse. 
gebnisse vertauscht wurden und sich der angegebene Wert tatsächlich auf M06-2X/6$31++\mathrm{G}(\mathrm{d}, \mathrm{p})$ bezieht $\left(0.7 \mathrm{~kJ} \mathrm{~mol}^{-1} \approx 0.2 \mathrm{kcal} \mathrm{mol}^{-1}\right.$ nach eigener Rechnung). Wie bereits in Kap. 4.2 gezeigt wurde, beträgt die tatsächliche $t / g$-Energiedifferenz für APF-D/6$31++\mathrm{G}(\mathrm{d}, \mathrm{p})$ dagegen $-0.2 \mathrm{~kJ} \mathrm{~mol}^{-1}$. Dies entspricht einer qualitativ falschen Vorhersage für eine stabilere $g$-Konformation von Ethanol. Für MP2/aug-cc-pVDZ beträgt die Differenz dagegen $+0.9 \mathrm{~kJ} \mathrm{~mol}^{-1}$ - eine Überschätzung der Stabilität von $t$, wie auch aus früheren MP2-Studien bekannt.[106, 108] Wie in Kap. 4.3 gezeigt wurde, sind auch die Vorhersagen dieser beiden Methoden für Ethanol-Dimere bezüglich Energieabfolge, Geometrien und Schwingungsspektren wenig kompatibel mit experimentellen Ergebnissen.

Bei der Generierung von Hexamer-Startstrukturen wenden die Autoren eine Beschränkung an: nur Kombinationen der drei Ethanol-Konformere werden berücksichtigt, bei denen kein Konformer einzeln vorkommt, d.h. zum Beispiel die Zusammensetzung $4 t 1 g+1 g-$ wird explizit ausgeschlossen. Diese Beschränkung wird doppelt begründet. Die erste Begründung basiert auf der hier bereits mehrfach zitierten Arbeit von Balanay et al. [96], nach der die Bildung größerer Ethanol-Cluster in Lösung auf den Dimeren beruht, da deren Signale bei steigender Konzentration zurückgehen. Dies basiert allerdings auf einer sehr fraglichen und angefochtenen [180] Zuordnung, die auf unskalierten harmonischen Wellenzahlen von Gasphasen-Strukturen basiert. Selbst falls die Bildung von Hexameren aus irgendeinem Grund kinetisch auf die Zusammenlagerung von Dimeren beschränkt sein sollte, bleibt unverständlich warum daraus gefolgert werden könnte, dass keine einzeln vorkommenden Konformere in den Hexameren vorkommen sollten. Wie zuvor gezeigt wurde, gibt es keinerlei Anhaltspunkte dafür, dass die Dimere stets aus zwei identischen Monomer-Konformeren zusammengesetzt wären, noch dass deren nachfolgende Isomerisierung im Hexamer als gehindert zu erwarten wäre.

Die zweite angeführte Begründung besagt, dass die ausgeschlossenen Kombinationen mit einzelnen Monomer-Konformeren keine symmetrischen Cluster bilden könnten und daher per se weniger stabil wären. Dieses Prämisse ist zum einen in dieser Allgemeinheit inhaltlich fragwürdig: Symmetrie mag zwar vom menschlichen Verstand bevorzugt werden, allerdings ist nicht klar warum dies so generell auch für die Natur zutreffen sollte. Zum Beispiel sind speziell die stabilsten Ethanol-Dimer- und Methanol-TrimerStrukturen nachweislich asymmetrisch, obwohl symmetrische cyclische Strukturen möglich wären. Es ist daher stets der Einzelfall zu betrachten. Zum anderen wird dieses Ausschlusskriterium auch falsch angewendet, denn die explizit ausgeschlossene Kombination $4 t 1 g+1 g-$ könnte sehr wohl symmetrische Cluster bilden, wenn $g+$ und $g-$ durch ein Symmetrieelement zweiter Art ineinander überführt werden. Schlussendlich widerspricht auch das Ergebnis der Studie der eigenen Prämisse - das gefundene globale Minimum ist eine asymmetrische Struktur!

Für jede der 13 berücksichtigten Kombinationen aus Monomer-Konformeren haben die Autoren 100 Strukturen mit ABCluster generiert, von denen jeweils 8-15 handverlesen (für möglichst unterschiedliche Strukturen) ausgewählt wurden. Insgesamt wurden 139 Strukturen auf APF-D-Niveau nachoptimiert, von denen anschließend wiederum 68 mit MP2 neu berechnet wurden. Ein näherer Blick auf diese 13 Kombinationen zeigt aber, dass insgesamt 5 Kombinationen sich enantiomer zu jeweils einer anderen verhalten z. B. $4 t 2 g+$ und $4 t 2 g-$. Dies zeigt sich darin, dass immer wieder Strukturen innerhalb dieser enantiomeren Kombinationen-Paare als isoenergetisch gefunden werden. Allerdings bleiben diese Übereinstimmungen sporadisch, was stark darauf hindeutet, dass die Struktursuche 
nicht ausführlich genug war. Nur für zwei der fünf enantiomeren Kombinationen-Paare wurden letztlich auch enantiomere Minimumstrukturen gefunden.

Etwas überraschend ist, dass sowohl mit APF-D wie auch MP2, trotz so deutlich unterschiedlicher Monomer-Präferenz, das gleiche globale Minimum gefunden wurde: eine heterochirale asymmetrische Struktur, die als uududd/tg-tg-g+g+ klassifiziert werden kann und sich von der Wannen-Konformation des MethanolHexamers ableitet. Für B3LYP-D3(BJ)/may-cc-pVTZ kann dies nicht bestätigt werden, hier liegt $u u d u d d / t g-t g-g+g+0.8 \mathrm{~kJ} \mathrm{~mol}^{-1}$ über dem vermuteten globalen Minimum $u d u d u d / g-g+g-g+g-g+$. Dies gilt auch für die weiteren zehn stabilsten APFD- und fünf stabilsten MP2-Strukturen, die ebenfalls für diese Arbeit nachoptimiert wurden. Dies bestärkt die Vermutung, dass $u d u d u d / g-g+g-g+g-g+$ tatsächlich das globale Minimum auf B3LYP-D3(BJ)/may-cc-pVTZ-Niveau darstellt. Umgekehrt hat $u d u d u d / g-g+g-g+g-g+$ auf APF-D-Niveau eine relative Energie von $1.6 \mathrm{~kJ} \mathrm{~mol}^{-1}$ und ist leicht asymmetrisch. ${ }^{\text {b }}$ Auf MP2-Niveau ist die Struktur wieder $S_{6}$-symmetrisch, hat aber eine relative Energie von über $6 \mathrm{~kJ} \mathrm{~mol}^{-1}$ gegenüber dem globalen Minimum. Dies zeigt erneut, dass die quantenchemische Beschreibung von Ethanol-Clustern herausfordernd ist und daher ein schrittweises Vorgehen, beginnend mit kleinen Clustern in der Gasphase und mit wiederholter experimenteller Verifikation der Ergebnisse, dringend angeraten ist.

Nachtrag: Zwischen Einreichung und Veröffentlichung der vorliegenden Arbeit ist ein weiterer Artikel der selben Autoren erschienen [181], der nun auch Ethanol-Dimere bis -Pentamere abdeckt. Auch hierfür wurde erneut MP2/aug-cc-pVDZ als Rechenniveau eingesetzt, welches, wie hier gezeigt, experimentelle Ergebnisse für EthanolMonomere und -Dimere nicht gut beschreibt. Mit zusätzlichen DLPNO-CCSD(T)/CBSPunktrechnungen wurde eine deutlich bessere Übereinstimmung für relative Energien erreicht, allerdings wurde diese Korrektur für die größeren Cluster ab den Trimeren nicht mehr angewendet. Für die Trimere finden Malloum et al. dennoch das selbe globale Minimum wie in der vorliegenden Arbeit. Allerdings ist bei diesem Befund eine gewisse Vorsicht angebracht, denn es gibt Anzeichen dafür, dass die von den Autoren verwendete halb-automatische Konformerensuche auch für kleinere Ethanol-Cluster unzureichend ist. So wurden von den durch intramolekulare sowie $u и d / u u u$-Isomerie zu erwartenden diastereomeren 38 cyclischen Ethanol-Trimeren nur 24 gefunden. Auch bereits für die leichter überschaubaren Ethanol-Dimere wurden nur Strukturen aus 7 der 9 bekannten Klassen [108] erhalten ( $g-g-$ und $t g-$ fehlen) - ein Warnzeichen, welches trotz der Vergleiche mit der Literatur offenbar unbemerkt bliebt. Für Ethanol-Tetramere wurde mit $u d u d / g-g+t t$ ein abweichendes globales Minimum gefunden, welches in der vorliegenden Arbeit an dritter Position mit einer relativen Energie von $0.7 \mathrm{~kJ} \mathrm{~mol}^{-1}$ steht.

\footnotetext{
${ }^{\mathrm{b}}$ Die kartesischen Koordinaten der APF-D-Strukturen sind nicht, wie in der Publikation angegeben, in der Supporting Information verfügbar, sondern müssen beim Erstautor angefragt werden.
} 


\subsection{Schwingungsspektren von Ethanol- und Methanol-Tetrameren bis -Hexameren}

Bevor die Schwingungsspektren der Ethanol-Cluster mittler Größe besprochen werden, wird zunächst wieder der einfachere Fall von Methanol ohne relevante Konformationsvielfalt betrachtet.

\subsubsection{Methanol-Schwingungsspektren}

Wegen der großen Zahl an nahezu äquivalenten Oszillatoren, starker Kopplung und teils hoher Symmetrie ergeben sich komplexe Bandenmuster, die sich deutlich IR- und Ramanspektrokopisch unterscheiden. Diese Bandenmuster sind in Abb. 4.28 dargestellt. Für die $S_{4}$-symmetrischen Tetramere von Methanol und Ethanol wird eine hochfrequente BSchwingung erhalten, die sehr schwach IR-aktiv und mäßig Raman-aktiv ist und bei der Paare an $\mathrm{OH}-$ Oszillatoren phasenverschoben zueinander schwingen. Die mittelfrequente, zweifach entartete E-Schwingung ist sehr stark IR-, aber kaum Raman-aktiv. Die ESchwingungen entsprechen phasenverschobenen Schwingungen jeweils gegenüberliegender OH-Oszillatoren, während die anderen Oszillatoren nicht beteiligt sind. Die niederfrequente phasengleiche A-Schwingung aller $\mathrm{OH}$-Oszillatoren ist schließlich IR-inaktiv, aber sehr stark Raman-aktiv. Für das leicht asymmetrische Pentamer ergibt sich ein sehr ähnliches Bandenmuster, welches im Vergleich zum Tetramer zu niedrigeren Frequenzen verschoben ist und bei der alle Banden zweifach leicht aufgespalten sind, mit Ausnahme der niederfrequenten phasengleichen Schwingung. Auch die Schwingungen der $S_{6^{-}}$ symmetrischen Hexamere zeigen ein vergleichbares, noch etwas stärker tiefverschobenes

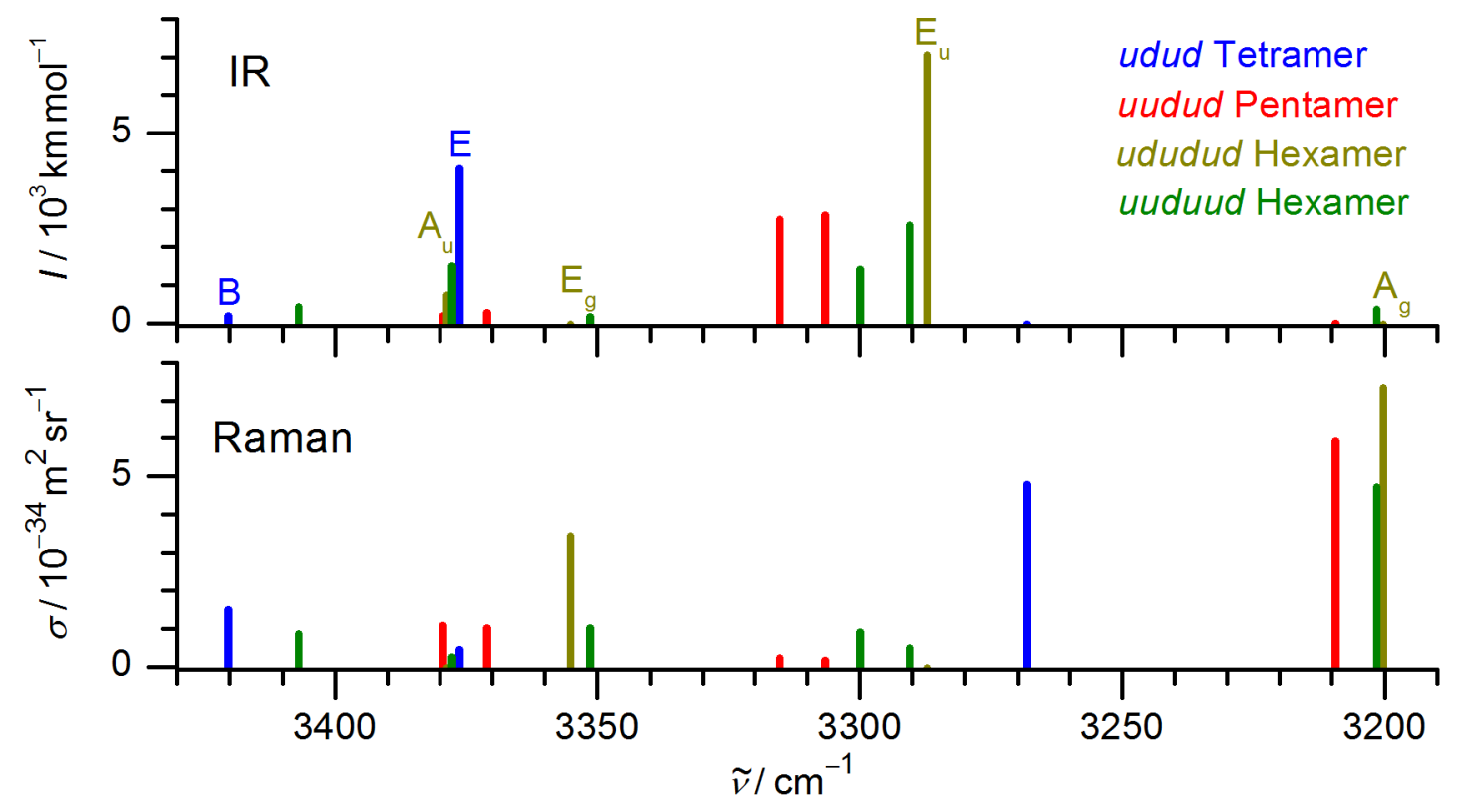

Abb. 4.28: Auf B3LYP-D3(BJ)/may-cc-pVTZ mit doppelt-harmonischer Näherung berechnete Wellenzahlen, IR-Aktivitäten, Raman-Streuquerschnitte und irreduzible Darstellungen für $\mathrm{OH}$ Streckschwingungen von Tetrameren, Pentameren und Hexamere von Methanol. Nicht markierte Schwingungen transformieren nach A. Entartete Schwingungen sind mit kumulierten Aktivitäten bzw. Streuquerschnitten dargestellt. 
Muster mit einer $A_{u^{-}}$, je zweifach entarteten $E_{g}$ - und $E_{u^{-}}$sowie einer $A_{u^{-}}$Schwingung. Die erniedrigte Symmetrie im vermutlich ähnlich stark populierten asymmetrischen uuduudHexamer führt zu weiteren Aufspaltungen und Umverteilung der Intensitäten.

Aus diesen quantenchemischen Vorhersagen lässt sich erwarten, dass die Banden des Tetramers zumindest noch teilweise von denen der größeren Oligomeren separiert sind (insbesondere wenn die Methanol-Konzentration nicht zu hoch gewählt wird, sodass nur wenig Hexamer vorliegt), während eine spektrale Unterscheidung von Penta- und Hexameren sowie noch größeren Clustern [140] ohne zusätzliche Größenselektivität sehr schwierig erscheint. In den experimentellen IR- und Raman-Spektren einer Expansion von Methanol in Helium bestätigt sich die erwartete teilweise Überlappung von Clustern dieser Größe (Abb. 4.29).

Die Zuordnung der breiten, stark rotverschobenen Banden im IR- (Maximum bei $3230 \mathrm{~cm}^{-1}$ ) und Raman-Spektrum (Maximum bei $3172 \mathrm{~cm}^{-1}$ ) zu Clustern größer als Tetramere beruht auf deren Konzentrationsabhängigkeit.[158, 163] Größenselektive VUVIR-Spektren von Methanol- [182-184] und Ethanol- [144] Clustern zeigen deutlich breitere und zu höheren Wellenzahlen verschobene Banden im Vergleich zur direkten FTIRDetektion. Eine Übereinstimmung ist die zunehmend geringere Steigerung der Rotver-

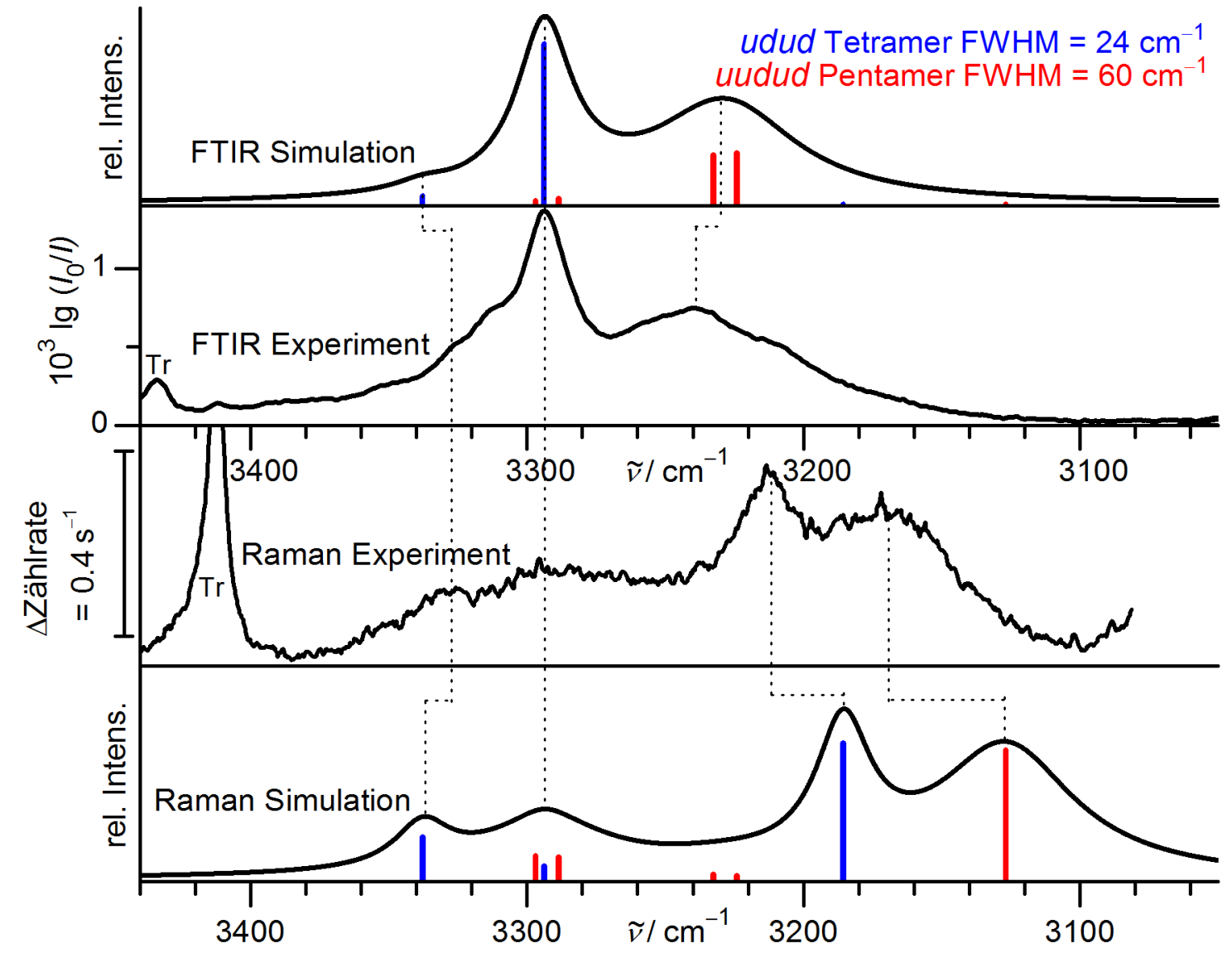

Abb. 4.29: Experimentelle [129] und simulierte IR- und Raman-Spektren von Methanol im Bereich mittelgroßer Cluster. Simulationsparameter sind einheitliche Verschiebung der harmonischen Wellenzahlen $\left(-82.5 \mathrm{~cm}^{-1}\right)$ für eine gute Übereinstimmung der intensivsten IR-Bande des Tetramers, ein Tetramer : Pentamer Verhältnis von 2.2 (IR) bzw. 1.3 (Raman) sowie Lorentz-Profile mit Halbwertsbreite $24 \mathrm{~cm}^{-1}$ (Tetramer) bzw. $60 \mathrm{~cm}^{-1}$ (Pentamer). 


\section{Chiralitätssynchronisation in Ethanol-Aggregaten}

schiebung mit der Clustergröße, die jenseits des Pentamers kaum noch stattfindet.[158, 185]

Die empirische Anharmonizitätskorrektur $\left(-82.5 \mathrm{~cm}^{-1}\right)$ und die Halbwertsbreite $\left(24 \mathrm{~cm}^{-1}\right)$ des Tetramers wurden für die Modellierung anhand der relativ scharf aus der Bandenformation herausstechenden Spitze bei $3294 \mathrm{~cm}^{-1}$ angepasst. Die Zuordnung dieser Bande zum Tetramer wurde experimentell zuvor aus der Konzentrationsabhängigkeit abgeleitet [158] und ist durch die erzielte Größenselektivität bei Detektion in verschiedenen Massenkanälen für Methanol in Helium-Nanotröpfchen bestätigt.[163] Die Anharmonizitätskorrektur wurde für alle Banden, einschließlich denen der Pentamere, übernommen. Die Halbwertsbreite des Pentamers wurde dagegen unabhängig zu $60 \mathrm{~cm}^{-1}$ gewählt. Letztgenannter Wert stellt eine obere Grenze dar, weil auch weitere Isomere des Pentamers sowie Hexamere und noch größere Cluster zur Verbreiterung beitragen können. Aufgrund der hohen spektralen Ähnlichkeit lassen sich diese Cluster ohne Größenselektivität [156, 179] nicht mehr unterscheiden. Im Hinblick auf ein möglichst einfaches Modells mit der geringsten Zahl an notwendigen Parametern wird hier auf eine Postulierung der Anwesenheit von Strukturen jenseits des stabilsten Pentamers verzichtet. Das Verhältnis Tetramer : Pentamer beträgt in der IR-Simulation 2.2:1 und in der Raman-Simulation 1.3: 1. Dies steht in Einklang mit dem Befund für das Dimer: Trimer Verhältnis in Abb. 4.19, welches ebenfalls für die Expansion mit IR-Detektion größer war als für die Raman-detektierte Expansion.

Das Spektrum von Methanol-Clustern in Helium-Nanotröpfchen ist im OH-Streckschwingungsbereich [163] erstaunlich ähnlich zu dem hier gezeigten in einer Überschallexpansion. Dies widerspricht den Ergebnissen einer älteren Studie [186] im COStreckbereich, nach denen die kinetische Barriere für die Ring-Insertion bei Anlagerung eines weiteren Moleküls in einer solchen ultrakalten Umgebung nicht mehr überwunden werden kann und sich für Tetra- und Pentamere sich daher sogenannte Lasso-Strukturen [187] mit Molekülen in exocyclischer Position bilden. Die Insertion würde dagegen auch eher dem Befund für Wasser-Cluster entsprechen.[178]

\subsubsection{Ethanol-Schwingungsspektren}

Die Umwandlung von intramolekularen Konformeren innerhalb der $u d u d$-EthanolTetramere hat ähnliche spektrale Auswirkungen wie beim uud-Trimer. Die $S_{4}$-Symmetrie wird im unterschiedlichen Ausmaße gebrochen, was zu berechneten Aufspaltungen der E-Schwingung zwischen 0 und $30 \mathrm{~cm}^{-1}$ führt. Eine besondere Bedeutung kommt dabei dem zweitstabilsten Tetramer $u d u d / g-g+g-t$ zu, welches wegen seiner Chiralität in der Simulation bei der angenommene Konformationstemperatur von $64 \mathrm{~K}$ fast so stark populiert ist wie die globale Minimumstruktur und dessen (Pseudo-)E-Schwingungen eine relativ große Aufspaltung von $22 \mathrm{~cm}^{-1}$ aufweisen. Für die Tetramere wird analog zum Trimer gefunden, dass $t$ - und nach innen gerichtete $g$-Konformere die mittlere $\mathrm{OH}$ Streckschwingungszahl erhöhen im Vergleich zu nach außen gerichteten $g$-Konformeren, was in der Überlagerung zu der spektralen Verbreiterung beiträgt.

Das experimentelle FTIR-Spektrum einer Expansion von Ethanol in Helium (Abb. 4.30) hat große Ähnlichkeit mit dem von Methanol, ist im Tetramer-Bereich aber verbreitert. Dies kann sehr gut mit der modellierten Konformationsvielfalt der Ethanol- 
Tetramere erklärt werden, wenn eine zu Methanol identische individuelle Halbwertsbreite von $24 \mathrm{~cm}^{-1}$ für die Tetramere und $60 \mathrm{~cm}^{-1}$ für das vermutlich wichtigste PentamerKonformer angenommen wird. Keine definitive Erklärung konnte dagegen gefunden werden für eine Schulter bei $3288 \mathrm{~cm}^{-1}$, die eine Entsprechung bei Methanol bei $3312 \mathrm{~cm}^{-1}$ hat und bei Beimischung von Argon in die Expansion prominenter wird.[126] Möglich wäre hier ein Beitrag von Hexameren gemäß den Bandenmustern in Abb. 4.28 auf S. 90.

Das Raman-Spektrum von Ethanol-Überschallexpansionen ist nur bis zu einer unteren Wellenzahl von $3258 \mathrm{~cm}^{-1}$ bekannt. Die in dem darüber liegenden Bereich von etwa $100 \mathrm{~cm}^{-1}$ beobachtete breite Streuung kann analog zu Methanol den nicht aufgelösten hochfrequenten und mäßig Raman-aktiven Banden der Ethanol-Cluster zugeordnet werden. Für den noch tiefer liegenden, bisher nicht experimentell untersuchten WellenzahlBereich ist eine Vorhersage gezeigt. Für das Verhältnis von Tetramer:Pentamer wurde hier ein Wert von $1.8: 1$ angenommen.

Die deutlich vergrößerte Halbwertsbreite der Methanol- und Ethanol-Tetramerbanden gegenüber denen der Trimere geht vermutlich am ehesten auf eine schnellere Umvertei-

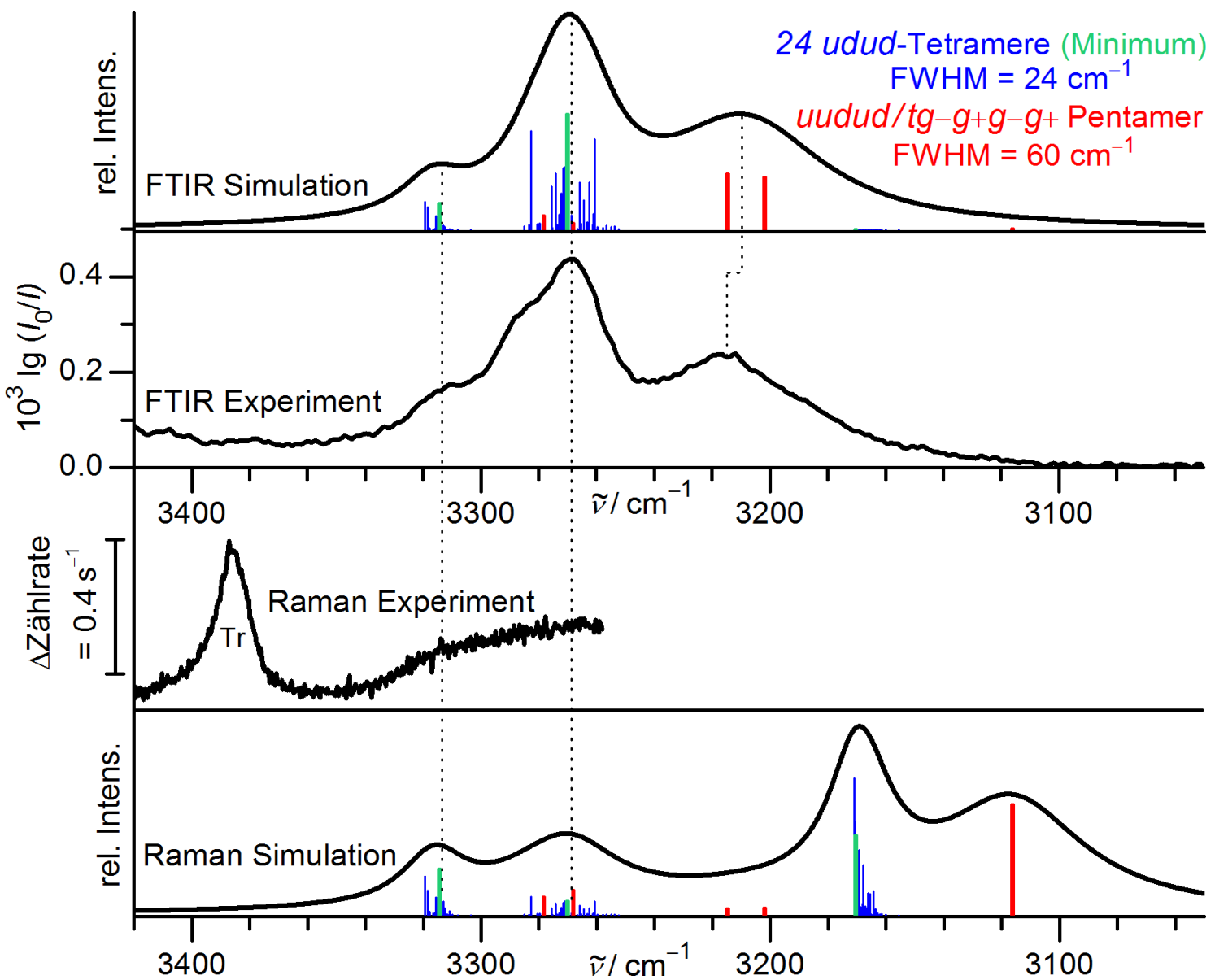

Abb. 4.30: Experimentelle [126, 129] und simulierte IR- und Raman-Spektren von Ethanol im Bereich mittelgroßer Cluster. Simulationsparameter sind einheitliche Verschiebung der harmonischen Wellenzahlen $\left(-103 \mathrm{~cm}^{-1}\right)$ für eine gute Übereinstimmung der intensivsten IR-Bande des Tetramers, ein Tetramer : Pentamer Verhältnis von 2.8 (IR) bzw. 1.8 (Raman), eine Konformationstemperatur von $64 \mathrm{~K}$ sowie Lorentz-Profile mit Halbwertsbreite $24 \mathrm{~cm}^{-1}$ (Tetramer) bzw. $60 \mathrm{~cm}^{-1}$ (Pentamer). Die Übergänge des globalen Minimums der Ethanol-Tetramere ( $u d u d / g-g+g-g+$ ) sind in grün hervorgehoben. 
lung der Schwingungsenergie zurïck. Auch konzertiertes Protonentunneln könnte hierzu einen kleinen Beitrag haben, die Barrierenhöhe sinkt vom Trimer zum Tetramer von etwa 100 (80) auf 85 (50) $\mathrm{kJ} \mathrm{mol}^{-1}$ ohne (mit) voller Schwingungsnullpunktskorrektur, ohne wesentliche Unterschiede zwischen den jeweils stabilsten Methanol- und EthanolStrukturen. Allerdings sind auch für die meisten Ethanol-Tetramere die Potentiale asymmetrisch und Tunnelaufspaltungen damit unwahrscheinlich. Die berechnete Barrierenhöhe sinkt für das Methanol-Pentamers nicht weiter ab, dies entspricht dem Befund für Fluorwasserstoff-Cluster gleicher Molekülzahl.[188] Entgegen der Erwartung ist der Übergangszustand aber nicht wie für das Trimer $C_{\mathrm{s}^{-}}$, sondern deutlich asymmetrisch, was ein Hinweis auf einen komplexeren Umwandlungsmechanismus sein könnte. Für das Methanol-Hexamer blieb die Suche nach dem entsprechenden Übergangszustand aus unklarem Grund ohne Ergebnis.

Schwingungsspektroskopisch ist die hier näher untersuchte Konformationsvielfalt von Ethanol-Tetrameren plausibel, es können aber keine Banden einzelnen Konformeren zugeordnet werden. Für die besser auflösende Rotationsspektroskopie ist das globale Minimum $u d u d / g-g+g-g+$ aufgrund des symmetriebedingt fehlenden Dipolmoments unsichtbar. Die nächsthöheren Konformere erlangen durch ihre Asymmetrie einen statistischen Vorteil in der Population und ein kleines Dipolmoment, was ihren Nachweis möglich aber schwierig erscheinen lässt. Dass sehr kleine bzw. symmetriebedingt sogar fehlende Dipolmomente von Komplexen aber kein zwangsläufiger Ausschlussgrund für den rotationsspektroskopischen Nachweis sein müssen, wurde erst kürzlich anhand des apolaren Ameisensäure-Dimers demonstriert, dessen $(\mathrm{HCOOH})(\mathrm{DCOOH})$-Isotopologes mit einem berechneten Dipolmoment von 0.004 D charakterisiert werden konnte.[189] Allerdings lassen sich Ameisensäure-Dimere in deutlich höherer Konzentration präparieren als Ethanol-Tetramere. Die vorhergesagten rotationsspektroskopischen Kenngrößen der drei stabilsten polaren Ethanol-Tetramere sind im Anhang dieser Arbeit in Tab. A.5 auf S. 308 aufgeführt.

\subsection{Vergleichende Betrachtungen und Ausblick}

Wie in den vorherigen Kapiteln gezeigt wurde, leiten sich die stabilsten Strukturen der Ethanol-Cluster von denen der Methanol-Cluster ab. Zur Einordnung der gewonnenen Erkenntnisse werden nun einige vergleichende Betrachtungen zwischen Clustern mit unterschiedlicher Molekülzahl und Alkylkettenlänge durchgeführt. Zudem wird diskutiert, wie sich weitere Vergrößerung der Cluster, sowohl hinsichtlich Molekülzahl wie auch Kettenlänge, auswirken könnte.

\subsubsection{Chiralitätssynchronisation in Abhängigkeit der Clustergröße}

Das Ethanol-Dimer ist ein Beispiel homochiraler Chiralitätssynchronisation, da das global stabilste Dimer die Anordnung $g+g+$ besitzt. Das stabilste heterochirale Dimer $g-g+$ hat demgegenüber eine relative Energie von $0.5 \mathrm{~kJ}$ mol (B3LYP-D3(BJ)/may-cc-pVTZ).

Durch Erweiterung der stabilsten homo- $(g+g+)$ und heterochiralen $(g-g+)$ Dimere jeweils mit einem $t$-Konformer lassen sich die energetisch niedrigsten Trimere mit homochiraler $(u u d / g-t g-)$ und heterochiraler $(u u d / g-t g+)$ Anordnung von $g$-Konformeren 
erhalten (bzw. deren Enantiomere). Durch die für den Ringschluss notwendige Wasserstoffbrückengeometrie werden die Dimere verzerrt und die mutmaßlich für die höhere Stabilität von $g+g+$ verantwortliche räumliche Nähe der beiden Ethylgruppen wird aufgehoben (siehe Abb. 4.31). Stattdessen erscheint nun die Wechselwirkung der endständigen Methylgruppe mit den Hydroxygruppen des Rings und dem dritten Molekül entscheidend für die Stabilitätsreihenfolge. Die heterochirale Anordnung stellt eine insgesamt kompaktere Anordnung dar, was zu einem Energievorteil von $0.5 \mathrm{~kJ} \mathrm{~mol}^{-1}$ führt. Die chirodiastaltische Energie ist damit für Dimer und Trimer im Vorzeichen umgekehrt. Wird der Begriff Chiralitätssynchronisation in engerem Sinne auf Cluster beschränkt, die ausschließlich aus transient chiralen Monomeren bestehen, so sind die energetisch stabilsten all-g-Trimere homochiral $u u d / g+g+g+$ und heterochiral $u u d / g+g-g+$. Diese lassen sich analog durch Erweiterung der gleichen Dimere um ein $g+$-Konformer erhalten und es ergibt sich mit gleicher Begründung ein berechneter Energievorteil von $0.9 \mathrm{~kJ} \mathrm{~mol}^{-1}$ ebenfalls zugunsten der heterochiralen Kombination.

Allerdings bleibt das $t$-Konformer nur in einer achiralen Umgebung auch tatsächlich perfekt symmetrisch, in den hier betrachteten Trimeren weicht der $\mathrm{C}_{\beta} \mathrm{C}_{\alpha} \mathrm{OH}$ Torsionswinkel von $\pm 180^{\circ}$ ab. Dabei kann für sämtliche cyclischen Trimere festgestellt

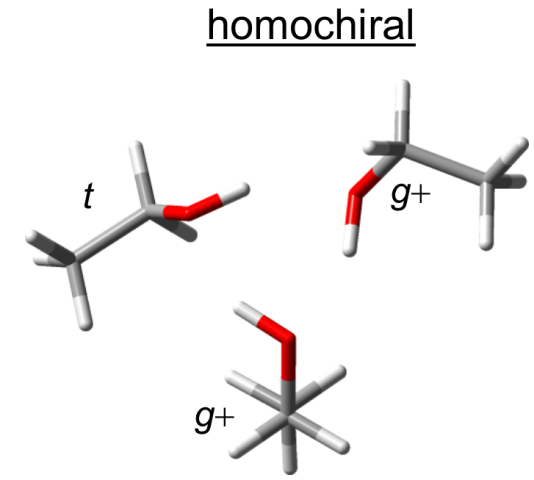

Enantiomer von uud/g-tg$0 \mathrm{~kJ} \mathrm{~mol}^{-1}$ heterochiral

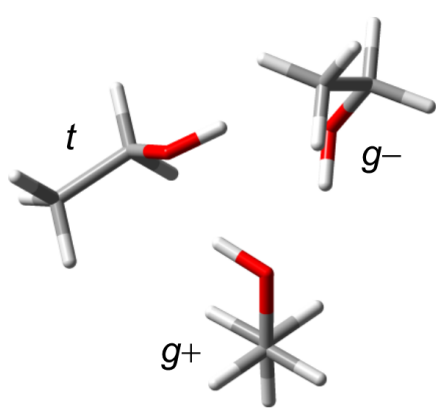

Enantiomer von uudlg-tg+

$-0.5 \mathrm{~kJ} \mathrm{~mol}^{-1}$

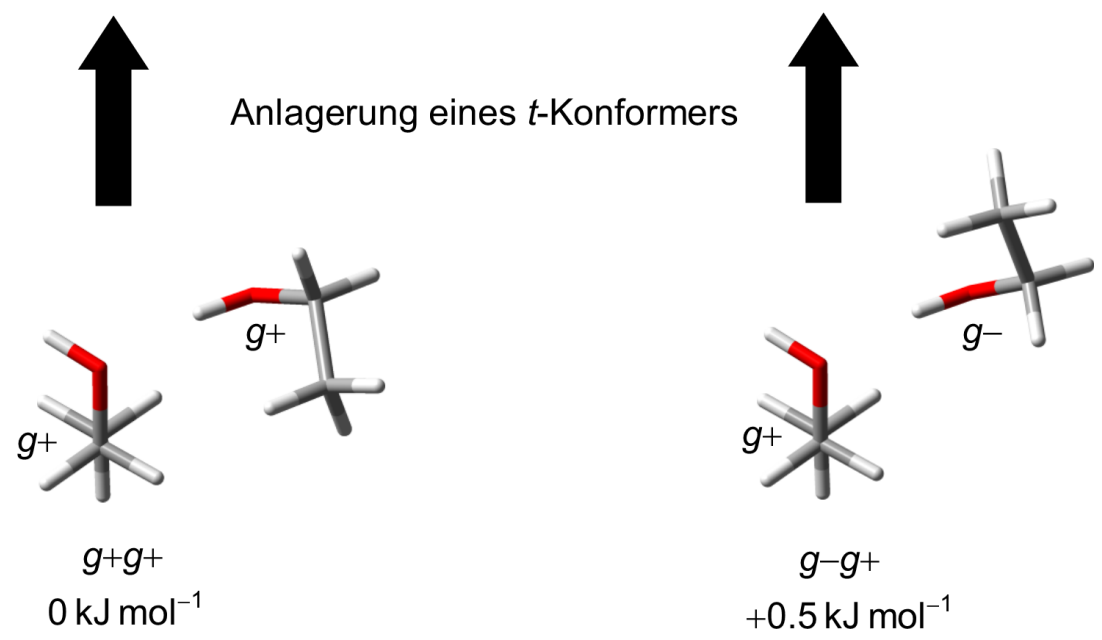

Abb. 4.31: Änderungen in der Struktur und in der relativen Energie bei Erweiterung der stabilsten homo- und heterochiralen Dimere zu den stabilsten homo- und heterochiralen Trimere bei Anlagerung eines $t$-Konformers. 


\section{Chiralitätssynchronisation in Ethanol-Aggregaten}

werden, dass der Winkel für beteiligte $t$-Konformere stets negativ ist (etwa im Bereich $-170 \pm 5^{\circ}$ ) wenn sich der Ethylrest in $u$-Stellung und stets positiv wenn er sich in $d$ Stellung befindet. Damit kann auch im Sinne der Vorzeichen der drei intramolekularen $\mathrm{C}_{\beta} \mathrm{C}_{\alpha} \mathrm{OH}$-Torsionswinkel $u u d / g-t g-$ als das stabilste homochirale Trimer eingestuft werden, welches instabiler ist als das heterochirale $u u d / g-t g+$.

Auch für Tetramere gelten ähnliche Prinzipen. Das heterochirale globale Minimum $u d u d / g-g+g-g+$ ist gegenüber den homochiralen Strukturen $u d u d / g-t g-t$ um

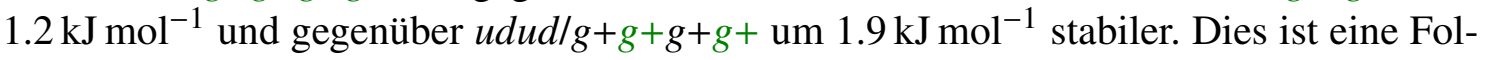
ge der vermutlich elektrostatisch begründeten alternierenden up-down-Anordnung der Alkylketten in cyclischen Clustern. Für eine möglichst günstige Anordnung mit nach innen gerichteten $g$-Konformeren muss auch die Chiralität alternieren und mit der Position im Ring synchronisiert sein. In dieser Betrachtung sind $u d u d / g-g+g-g+$ und $u d u d / g+g-g+g-$ phasenverschoben, was mit einem Energieunterschied von $4.1 \mathrm{~kJ} \mathrm{~mol}^{-1}$ einhergeht. Auch für Pentamere und Hexamere lassen sich nach den vorläufigen Erkenntnissen eine Bevorzugung von heterochiralen Anordnungen vermuten. Für die EthanolKetten im Kristall wird eine ebenfalls heterochirale Anordnung gefunden.[112] Die bevorzugte homochirale Anordnung im Dimer ist daher die Ausnahme und nicht die Regel für Ethanol.

\subsubsection{Relative Stabilität der cyclischen Bindungsmotive}

Eine der Fragestellungen, die als Motivation für Behandlung dieses Themas diente, ist, ob sich durch Wechselwirkung zwischen verlängerten Alkylgruppen die Energieabfolge der cyclischen Strukturmotive von Alkohol-Trimeren und -Tetrameren verändern lässt, analog zu der Faltung von $n$-Alkanen.[149] Abb. 4.32 zeigt die relative Energie verschiedener Strukturmotive von Methanol zu Ethanol. Es lassen sich nur geringe Änderungen in der relativen Energie erkennen. Die einzige Ausnahme stellt das uudd-Motiv für das Tetramer dar, dessen relative Energie relativ deutlich ansteigt. Eine einfache Erklärung hierfür konnte nicht gefunden werden. Die Diskrepanz zum eng verwandten Motiv uииd könnte aber darin begründet liegen, dass uuиd bei Ethanol drei nach innen zeigende $g$ Konformere aufweisen kann, während für uudd eine solche Ausrichtung nur zweifach möglich ist ohne sterische Hinderung. Die übergeordnete Hierarchie der intermolekularen Isomerie bezüglich der Koordination der verschiedenen Elektronenpaare, die zu den $u / d$-Sequenzen führt, wird bei Ethanol teilweise durchbrochen durch die zusätzliche intramolekulare Isomerie. Bei den Ethanol-Trimeren ist die ungünstigste Struktur mit uиd-Motiv nur noch energetisch wenig höher als die stabilste mit uuu-Motiv. Bei den Tetrameren ist die ungünstigste Struktur mit $u d u d$-Motiv sogar instabiler als die jeweils stabilsten mit uuиd- und uudd-Anordnung. Die intramolekulare Isomerie ist daher nicht vernachlässigbar wenn Strukturmotive verglichen werden und es ist zu vermuteten, dass dies mit steigender Kettenlänge noch stärker gilt. Ergebnisse in der Literatur, die z. B. für $n$-Alkanole ab $n$-Butanol vorhersagen, dass lineare Trimere stabiler als cyclische wären,[141] berücksichtigen in aller Regel keine intramolekulare Isomerie. Die hier berechnete Energiespanne innerhalb von cyclischen Ethanol-Trimeren ist aber bereits größer als der dort berechnete Energieunterschied zwischen cyclischen und linearen Struktur- 
motiven für $n$-Butanol-Trimere mit scheinbar willkürlich ausgewählten intramolekularen Konformationen.

Die Alkylkette in Ethanol ist noch zu kurz, um daraus extrapolieren zu können, ob bei weiter steigender Kettenlänge ein Wechsel des Strukturmotivs zu erwarten ist oder nicht. Eine Klärung dieser Frage für $n$-Alkanole mit längerer Alkylkette erscheint aber quantenchemisch und besonders experimentell sehr schwierig. Neben steigenden Kosten pro Rechnung wegen zunehmender Größe des Systems ist das Hauptproblem die exponentiell steigende Konformormationsvielfalt. $n$-Propanol besitzt neun verschiedene MonomerKonformere (acht davon bilden vier Enantiomerenpaare), sodass bereits für uud-Trimere $9^{3}=729$ Konformere zu erwarten sind. Hier müsste demnach sehr selektiv vorgegangen werden um aus repräsentativen Konformeren Stabilitätsregeln, analog zu den hier vorgestellten für Ethanol-Cluster, abzuleiten und so die wahrscheinlichen globalen Minima bestimmen zu können. Experimentell zeigen die IR- und Raman-Spektren von $n$ Propanol im OH-Streckschwingungsbereich größere Komplexität für die Monomer- und Dimer-Banden, für die analog zu Ethanol aber noch teilweise eine Konformationsauflösung erreicht wird.[57, 127] Für größere Cluster werden dagegen noch breitere und weniger strukturierte Banden als für Ethanol beobachtet. Für längere $n$-Alkanole scheint sich der Trend fortzusetzen.[14, 57, 119] Die grundsätzlichen spektralen Muster bleiben dabei aber mindestens bis einschließlich $n$-Propanol erhalten, was zeigt, dass auch die Cluster des $n$-Propanols cyclische Strukturen bevorzugen. Dieser Bereich wurde zuvor von Wassermann [57] hinsichtlich des bevorzugten Strukturmotivs des Trimers analysiert. Die fehlende Aufspaltung der Trimer-Bande im IR-Spektrum wurde dabei als Hinweis darauf interpretiert, dass das verursachende $n$-Propanol-Trimer bereits ein $C_{3}$-symmetrisches иии-Motiv aufweisen könnte. Einer solchen Interpretation der Bande muss angesichts der
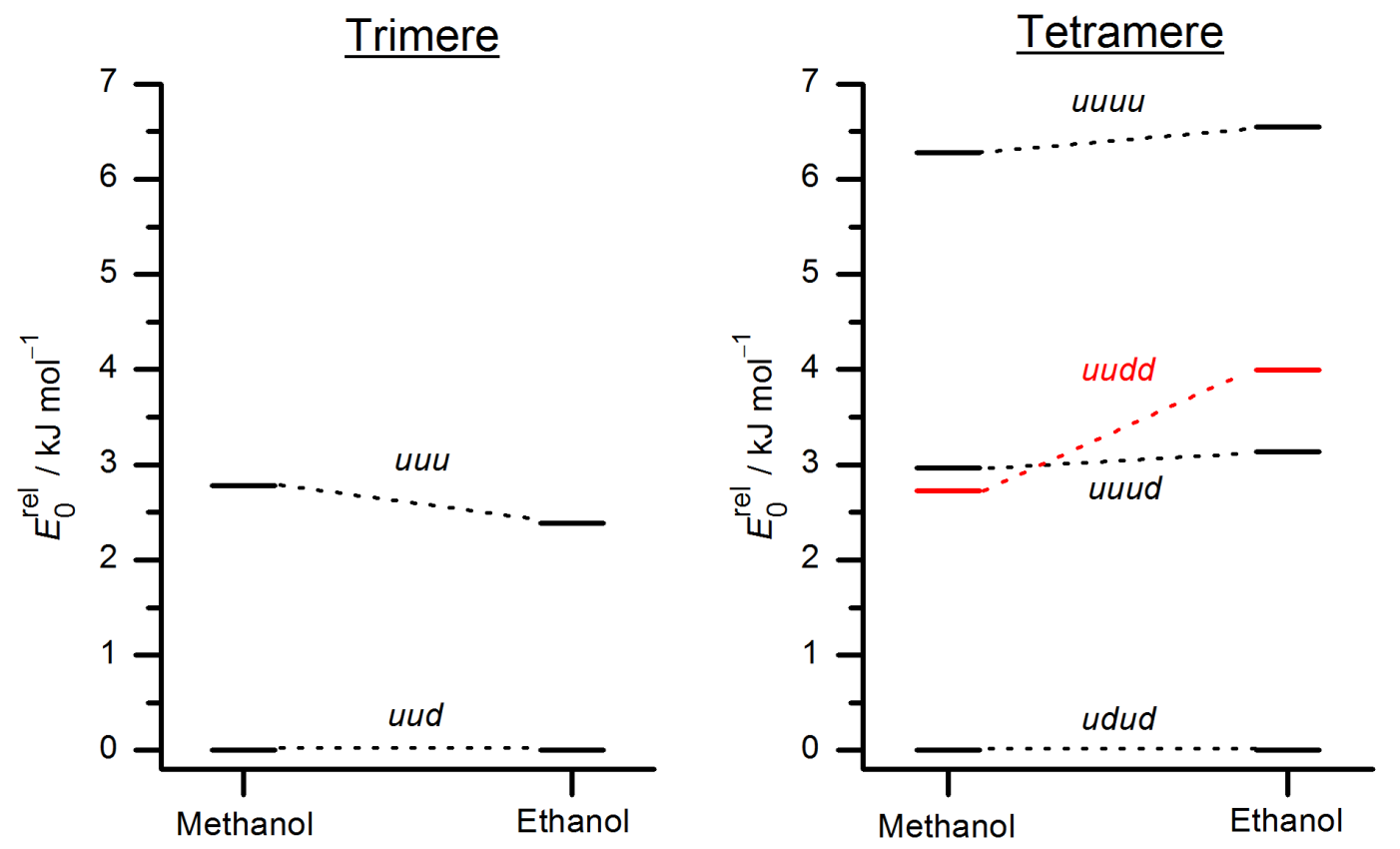

Abb. 4.32: Änderung der relativen nullpunktskorrigierten Energien der Strukturmotive von Alkohol-Trimeren und -Tetrameren beim Übergang von Methanol zu Ethanol auf B3LYPD3(BJ)/may-cc-pVTZ-Niveau. Für Ethanol werden die Strukturmotive durch die jeweils stabilste gefundene Konformation repräsentiert. 
Befunde für Ethanol widersprochen werden. Bereits für das Methanol-Trimer ohne relevante Konformationsvielfalt kann die Aufspaltung gerade noch nachgewiesen werden, da diese eine ähnliche Größe wie die individuelle volle Halbwertsbreite der Übergänge aufweist. Durch Konformationsvielfalt und auch durch Überlagerung mit möglichen Kombinationsbanden, die aufgrund der zunehmend niederfrequenten van der Waals-Moden stärker mit den den Fundamentalbanden überlappen würden, würde eine solche Aufspaltung verdeckt werden. Selbst wenn angenommen wird, dass das $n$-Propanol-Trimer keinerlei relevante Konformationsvielfalt oder Kombinationsbanden aufweisen würde, müsste für die Modellierung der beobachteten breiten Bande eine erhebliche natürliche Linienbreite angenommen werden, die eine Aufspaltung in der Größenordnung wie für das MethanolTrimer komplett überdecken würde. Daher lassen sich aus der fehlenden Aufspaltung keine Schlüsse auf die Symmetrie des stabilsten $n$-Propanol-Trimers ziehen.

Eine weitere experimentelle Komplikation sind die etwa dreifach höheren Barrieren für Isomerisierungen innerhalb der verlängerten Alkylkette für $\mathrm{C}-\mathrm{C}$-Torsion (etwa $15 \mathrm{~kJ} \mathrm{~mol}^{-1}$, gegenüber $5 \mathrm{~kJ} \mathrm{~mol}^{-1}$ für C-O-Torsion [190]), sodass kaum zu erwarten ist, dass die $n$-Propanol-Trimer-Konformere in eine Gleichgewichtsverteilung relaxieren können. Dies erschwert den Vergleich von quantenchemischen und experimentellen Ergebnissen zusätzlich.

Bei der schwingungsspektroskopischen Untersuchung in anderen Spektralbereichen wird neben der Unterscheidung von Konformeren auch die von unterschiedlichen Clustergrößen schwierig, die im OH-Streckschwingungsbereich für Trimere und teilweise noch für Tetramere gegeben ist. Für die Untersuchung von $n$-Alkanen [149] konnten noch relativ einfach Bedingungen ohne Clusterbildung ausgewählt werden, da der Haarnadel„Komplex“ der wechselwirkenden Alkylketten bereits durch kovalente Verknüpfung im Monomer vorgebildet ist. Trimere und Tetramere liegen dagegen stets gemeinsam mit (mindestens) Monomeren und Dimeren vor, die entweder spektral oder räumlich [179] getrennt werden müssen.

Eine experimentelle Antwort auf diese Fragestellung ist daher in näherer Zukunft nicht in Aussicht.

\subsubsection{Schrittweise Dissoziationsenergien}

Während bisher ausschließlich relative Energien innerhalb einer gegebenen Clustergröße diskutiert wurden, ist auch ein Vergleich der absoluten Dissoziationsenergien zwischen verschiedenen Clustergrößen aufschlussreich. Hierfür wird eine Darstellung der Daten in Form der schrittweisen Dissoziationsenergie gewählt. Diese beschreibt, wie viel Energie benötigt wird, um ein einzelnes Alkohol-Molekül aus einer bestimmten Clustergröße zu entfernen, bzw. umgekehrt wie viel Energie frei wird, wenn der Cluster durch Anlagerung eines Moleküls aus dem nächstkleineren Cluster gebildet wird. Dabei werden stets die jeweils stabilsten Konformere betrachtet. Die Gesamtdissoziationsenergie eines Clusters entspricht der Summe der einzelnen Schritte. Abb. 4.33 vergleicht die schrittweisen Dissoziationsenergie für Methanol und Ethanol in Abhängigkeit der Clustergröße.

Als Grenzwert für unendliche Clustergröße wäre im Idealfall eine Annäherung an den experimentellen Wert für die auf 0 K extrapolierte Sublimationsenthalpie [191] zu erwarten. Anders als für Methanol, konnte für Ethanol kein Wert für die Sublimationsenthalpie gefunden werden, daher sind zusätzlich die Verdampfungsenthalpien bei Standardbedin- 
gungen für Methanol und Ethanol [192] mitangegeben, auch wenn die Vergleichbarkeit aufgrund von thermischen Effekten begrenzt ist. Für alle Clustergrößen und auch die flüssige Phase lässt sich erkennen, dass Ethanol-Moleküle stets etwas stärker gebunden sind als Methanol-Moleküle. Neben zusätzlichen dispersiven Wechselwirkungen der verlängerten Alkylgruppe dürfte dies vor allem in verstärkten Wasserstoffbrücken durch den größeren induktiven Effekt der Ethylgruppe begründet sein, die vor allem die Akzeptorstärke von Ethanol steigert. Entsprechend agiert Ethanol im gemischten Dimer mit Methanol als Akzeptor.[131] Dieser Trend setzt sich für die Verdampfungsenthalpie mit steigender Kettenlänge fort.[192]

Die Ähnlichkeit zwischen Methanol und Ethanol äußert sich nicht nur in analogen Strukturen, sondern auch in einer sehr ähnlichen Abhängigkeit der schrittweisen Dissoziationsenergie von der Clustergröße. Das dritte Alkohol-Molekül wird bei der Bildung des Trimers etwa doppelt so stark gebunden wie das zweite bei der Bildung des Dimers. Dies lässt sich anhand der Ausbildung von zwei weiteren Wasserstoffbrücken bei der Bildung des Trimers nachvollziehen. Die Verstärkung der Wasserstoffbrücken durch Kooperativität und die gleichzeitige Schwächung durch Ringspannung halten sich offenbar etwa die Waage. Ein Maximum an Bindungsenergie wird dagegen bei der Anlagerung des vierten Alkohol-Moleküls frei, obwohl hier nur eine weitere Wasserstoffbrücke ausgebildet wird. Offenbar nimmt hier die Ringspannung deutlich ab und die Kooperativität zu. Bei der Bildung des Penta- und Hexamers werden diese Effekte wieder kleiner, die Dissoziationsenergie geteilt durch die Gesamtzahl der Moleküle bzw. der Wasserstoffbrücken nimmt aber weiter zu. Es ist zu vermuten dass die Kooperativität und Ringspannung schließlich für weiter steigende Ringgrößen in eine Sättigung laufen, sodass die schrittweise Dissoziationsenergie etwa konstant wird.[140] Stärker dreidimensionale, dichter gepackte Strukturen können diesen Trend aber beeinflussen und eine zusätzliche Stabilisierung ermöglichen. Schließlich ist für den Übergang zur kristallinen Strukturen mit parallelen

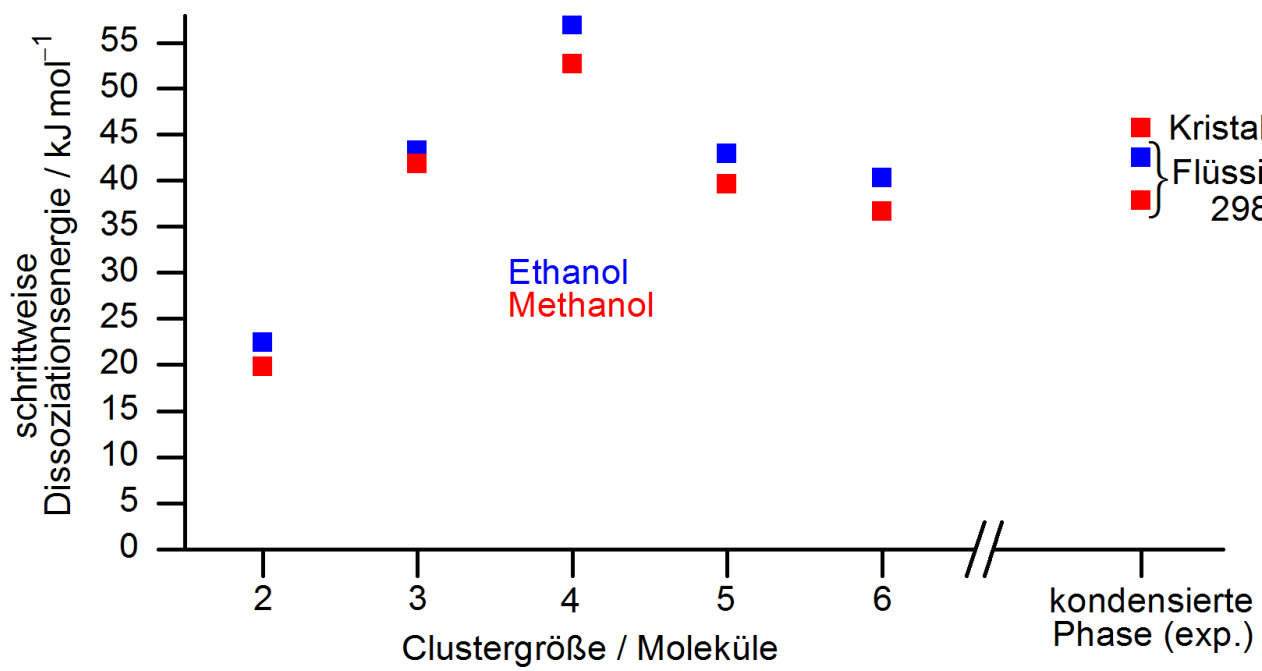

Abb. 4.33: Abhängigkeit der berechneten schrittweisen Dissoziationsenergie von der Clustergröße auf B3LYP-D3(BJ)/may-cc-pVTZ-Niveau. Die Werte für die kondensierte Phase sind experimentell ermittelt worden und entsprechen der auf $0 \mathrm{~K}$ extrapolierten Sublimationsenthalpie von Methanol [191] und der Verdampfungsenthalpie bei Standardbedingungen für Methanol und Ethanol [192]. 
linearen statt cyclischen Anordnungen noch ein Wechsel des Bindungsmotivs zu erwarten mit Konvergenz auf die Sublimationsenthalpie bei $0 \mathrm{~K}$.

\subsubsection{Große Cluster und Kristallisation}

Für Cluster mit drei bis sechs Alkohol-Molekülen wurden in dieser Arbeit nur komplett cyclische Anordnungen erwogen. Dies beruht zum einen auf der guten Übereinstimmung mit den Schwingungsspektren, aber auch auf zahlreichen quantenchemischen Vorarbeiten, die verschiedene Strukturmotive verglichen haben.[96, 140, 141, 155, 157, 187, 193, 194] Nicht-cyclische Strukturmotive sind zumindest für Cluster mittlerer Größe von $n$ Alkanolen aus verschiedenen Gründen energetisch nicht relevant. Lineare Strukturen vermeiden zwar Ringspannung und ggf. ungünstige $u / d$-Ausrichtungen, weisen aber eine Wasserstoffbrücke weniger und geringere Kooperativität auf. Lasso-Strukturen [187], mit einem oder mehreren Molekülen außerhalb des Rings, haben die gleiche Anzahl an Wasserstoffbrücken wie komplett cyclische Anordnungen, dabei sind allerdings die exocyclischen Wasserstoffbrücken anti-kooperativ, da zwei Donoren um die Elektronendichte eines Akzeptors konkurrieren. Zudem kommt es vor allem bei kleineren Clustern durch die Ringverengung zu einer Erhöhung der Ringspannung. Solche höher energetischen Strukturen können aber auch für kleine Cluster in geeigneter Umgebung wie Matrizen [195] und Helium-Nanotröpfchen [186] kinetisch stabilisiert werden. Mit steigender Clustergröße sinkt für zwar solche alternativen Strukturmotive der energetische Nachteil [140, 194], für Methanol wurde aber in einer aktuellen Studie [194] gefunden, dass die größtmögliche Ringstruktur bis einschließlich 13 Moleküle das globale Minimum bleibt. Mit 14 Methanol-Molekülen ist das gefundene globale Minimum dagegen eine Anordnung von zwei Siebenringen, die nur über sekundäre Wechselwirkungen im Kontakt stehen. Der Grund für den Wechsel des Strukturmotivs ist hier offenbar, dass die sekundären Wechselwirkungen zwischen zwei mittelgroßen Ringen stärker sind als innerhalb eines einzelnen großen Ringes. Die Aufspaltung von Vierzehn- in zwei Siebenringe wird energetisch möglich, da bei dieser Größe nur noch geringe Unterschiede in der Ringspannung und Kooperativität zu erwarten sind. Entsprechende größenabhängige Gründe lassen sich dann auch für die Kristallisation vermuteten, bei der für Methanol [196, 197] und Ethanol [112] Ringe durch Ketten ersetzt werden. Pro Kette wird im Vergleich zum Ring eine Wasserstoffbrïcke weniger ausgebildet, zumindest sofern an der Oberfläche des Kristalls keine Absättigung durch Abweichungen von der Ordnung erfolgt, z. B. in Form von Schlaufen. Dies kann durch die offenbar stärkeren sekundären Wechselwirkungen zwischen den Ketten kompensiert werden, da diese mit den Längen der Ketten skalieren. Damit lässt sich verstehen, dass diese Umwandlung erst ab einer gewissen Molekülzahl und einem ausreichenden Verhältnis von Volumen und Oberfläche des Clusters bzw. Kristalls ablaufen kann. Für Wasser, welches wegen seiner stärker dreidimensionalen Aggregation in größeren Clustern mit Alkoholen nur bedingt vergleichbar ist, beginnt die Kristallisation bei etwa 90 Molekülen.[198] Entsprechende experimentelle größenselektive Methoden für große Cluster scheinen auch grundsätzlich geeignet um die Kristallisation in Methanol- [199-201] und Ethanol-Clustern [200] zu verfolgen. Abb. 4.34 stellt Ausschnitte aus den Kristallstrukturen von Ethanol und Methanol gegenüber (letzteres in seiner $\alpha$-Modifikation bei tiefen Temperaturen). Erstmalig wird hier ein Bruch in der Analogie zwischen Ethanol und Methanol bezüglich des $u / d$-Strukturmotivs ge- 
funden. Klassifiziert nach den koordinierten Elektronenpaaren hat Ethanol die Struktur $(u u d d)_{n} /(t g+t g-)_{n}$, während Methanol als $u_{n}$ eingeordnet werden kann. Die Bedeutung von $u$ als $u p$ und $d$ als down relativ zu einer Ringebene verliert bei der Beschreibung von Ketten allerdings ihren ursprünglichen Sinn.
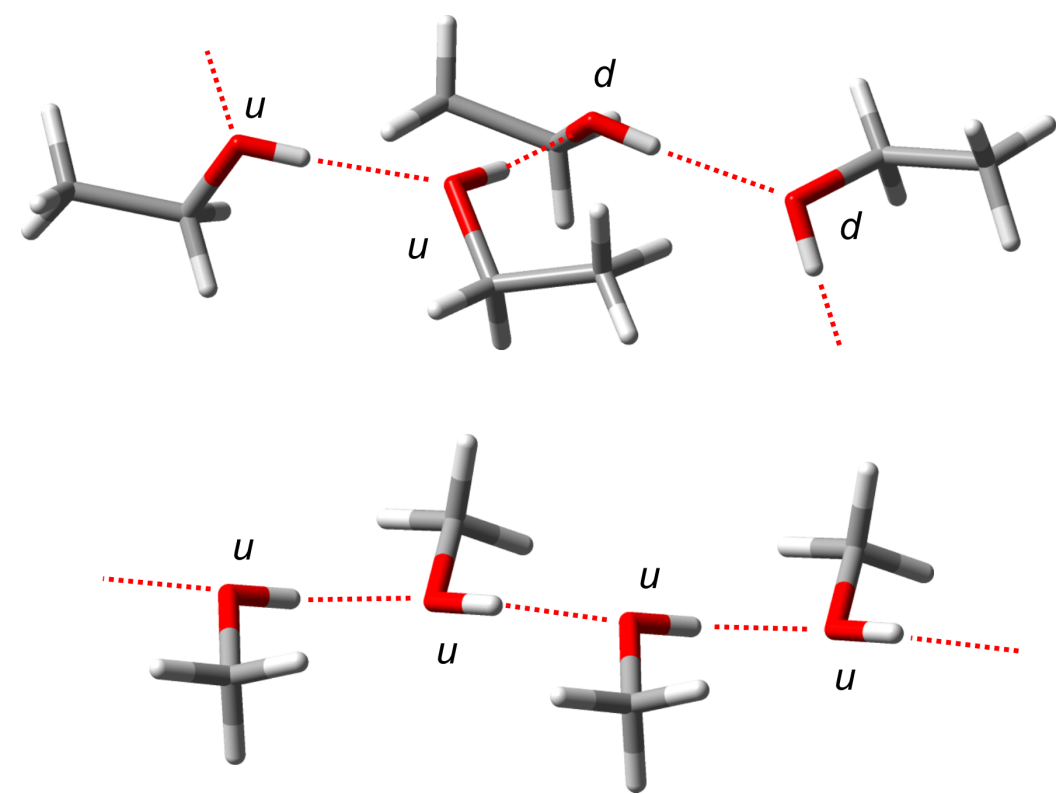

Abb. 4.34: Ausschnitte der Kristallstrukturen von Ethanol und Methanol ( $\alpha$-Modifikation) im Vergleich, nach kristallographischen Daten aus Ref. [112] und [196]. 



\section{Torsionsisomerie von Alkoholen}

\subsection{Einleitung}

Die meisten einwertigen Alkohole besitzen mehrere unterscheidbare Konformere bezüglich der Torsion um die C-O-Bindung. Wie im vorherigen Kapitel am Prototyp Ethanol deutlich wurde, ist ein gutes Verständnis dieser Isomerie wichtig, um auch Aggregate von Alkoholen beschreiben zu können. Auch die Reaktivität verschiedener AlkoholKonformere kann sich deutlich unterscheiden [202]. Eine einfache Möglichkeit, um zu analysieren welche $\mathrm{C}-\mathrm{O}$-Torsionsisomere $\mathrm{zu}$ erwarten sind und in welcher Beziehung sie zueinander stehen, ist, statt der Alkohole selbst, die Alkoholat-Ionen zu betrachten, welche sich durch Deprotonierung der Hydroxygruppe des Alkohols ergeben. Ist das Sauerstoffatom an ein gesättigtes Kohlenstoffatom gebunden, so können diese beiden Atome als $\mathrm{sp}^{3}$-hybridisiert beschrieben werden. Das Alkoholat-Ion weist in diesem Modell drei freie Elektronenpaare auf, durch deren jeweilige Protonierung die verschiedenen Alkohol-Konformere erhalten werden. Die freien Elektronenpaare stehen dabei gestaffelt zu den Substituenten des benachbarten $\alpha$-Kohlenstoffatoms. Die Beziehungen der Alkohol-Konformere zueinander lassen sich durch Analyse der Topizität dieser Elektronenpaare erhalten. Wenn die Elektronenpaare homotop sind, da sie sich über eine Drehung des Moleküls um eine $C_{n}$-Achse ineinander überführen lassen, so sind die entsprechenden Alkohol-Konformere identisch, wie z. B. bei Methanol. Bei enantiotoper Beziehung, d. h. bei Überführung durch eine Spiegelung, nicht aber durch eine Drehoperation, sind die Konformere enantiomer zueinander, wie z. B. die beiden $g$-Konformere von Ethanol. Lassen sich zwei Elektronenpaare des Alkoholat-Ions durch keine Symmetrieoperation ineinander überführen, so sind die Alkohol-Konformere diastereomer, wie z. B. das $t$ und die $g$-Konformere von Ethanol. Dieses Konzept ist am Beispiel des Ethanolat-Ions in Abb. 5.1 illustriert.

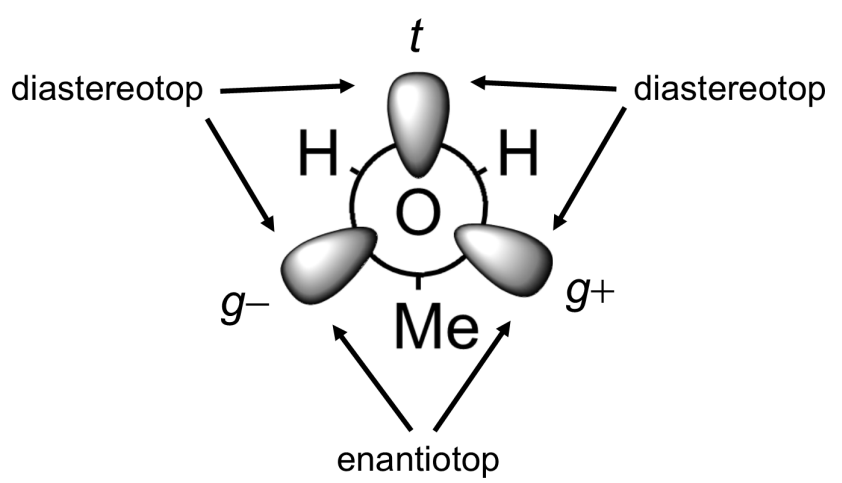

Abb. 5.1: Newman-Projektion des Ethanolat-Ions entlang der O-C-Bindung. Angegeben sind die Topizität der freien Elektronenpaare und die Ethanol-Konformere, die sich jeweils durch Protonierung des Elektronenpaares ergeben. 


\section{Torsionsisomerie von Alkoholen}

Seitdem diese Isomerie von Kolbe 1860 [203] vorhergesagt wurde, werden Alkohole traditionell nach der Anzahl $n$ der weiteren Kohlenstoffatome, die direkt an das $\alpha$ Kohlenstoffatom gebunden sind, in verschiedene Klassen eingeteilt. Bei $n=1$ handelt es sich um primäre, bei $n=2$ um sekundäre und bei $n=3$ um tertiäre Alkohole. Methanol mit $n=0$ gehört gemäß dieser Einteilung einer eigenen Klasse an, wird davon abweichend aber nicht selten dennoch als primärer Alkohol bezeichnet.

Moleküle mit einer Hydroxygruppe, welche an ein ungesättigtes $\alpha$-Kohlenstoffatom gebunden ist, sind in der Regel durch die Keto-Enol-Tautomerisierung instabil. Ausnahmen hiervon treten auf, wenn die Doppelbindung mesomeriestabilisiert ist, insbesondere als Teil eines aromatischen Systems. Letztere Verbindungen werden nach ihrer Stammverbindung als Phenole bezeichnet, diese fallen gemäß IUPAC Nomenklatur allerdings nicht unter die Definition der Alkohole, da diese ein gesättigtes $\alpha$-Kohlenstoffatom voraussetzt.[11] In Phenolen kann das Sauerstoffatom als $\mathrm{sp}^{2}$-hybridisiert angesehen werden, sodass das p-Orbital in Konjugation mit dem aromatischen Ring steht. Wegen dieser Bindungssituation weisen Phenole einige graduelle chemische Unterschiede zu Alkoholen auf, wie z. B. eine erhöhte Acidität. In Phenolen ist nach diesem Modell das Hydroxyproton und das einzelne freie Elektronenpaar in der Ringebene zu erwarten, was mit der experimentell gefundenen Molekülgeometrie [204] im Einklang steht. Phenole werden dennoch nicht selten unter dem Begriff ,,aromatische Alkohole“ eingeordnet, oft zusammen mit bestimmten Alkoholen nach IUPAC-Definition, wie Benzylalkohol.[205] Dies kann durch weitgehende Gemeinsamkeiten, z. B. bei der Ausbildung von Wasserstoffbrücken, begründet werden.

Bei der Erforschung von intermolekularen Wechselwirkungen im Kontext von Wasserstoffbrücken werden bevorzugt solche Hydroxyverbindungen eingesetzt, die keine unterscheidbaren Konformere aufweisen, wie z. B. Methanol, tert-Butylalkohol, Phenol und Wasser.[132, 206-212] Durch die Vermeidung von intramolekularer Konformationsvielfalt können so Spektren und quantenchemische Rechnungen vereinfacht werden. Dies wurde auch im vorherigen Kapitel beim Vergleich der Cluster von Methanol und Ethanol deutlich. Zwar liegt auch in der vorliegenden Arbeit, durch den im Zentrum stehen Begriff der Chiralitätserkennung, der Schwerpunkt eher auf der Untersuchung von intermolekularen Wechselwirkungen. Dabei kann allerdings das Thema der intramolekularen Isomerie nicht auf diese Weise umgangen werden. Denn für die notwendige Chiralität der Alkohole darf das Alkoholat-Ion keine Spiegelebene enthalten, wodurch enantiomere Konformere ausgeschlossen sind. Alternativ sind zwar auch chirale Alkohole mit identischen Konformeren möglich, wenn das Alkoholat-Ion z. B. durch die Punktgruppe $C_{3}$ beschrieben wird, allerdings sind Beispiele hierfür sehr selten. Ein solches wird in Form von Tri-tert-butylalkohol in Kap. 5.14 diskutiert. Alkoholat-Ionen chiraler Alkohole sind in der Praxis fast immer $C_{1}$-symmetrisch mit drei zu erwartenden diastereomeren Konformeren bezüglich der $\mathrm{C}-\mathrm{O}$-Torsion. Bevor Beispiele von Chiralitätserkennung behandelt werden können, ist es daher sinnvoll zunächst die Konformere der dafür eingesetzten Alkohole zu charakterisieren. Hierfür wird in diesem Kapitel die OH-Streckschwingung als Sonde eingesetzt, da die spektralen Positionen und relativen Intensitäten ihrer Übergänge Hinweise auf die im Gleichgewicht vorliegenden Konformeren liefern. Für die Vorhersage der spektralen Positionen wird im Folgenden ein übergeordnetes Modell anhand von Literaturdaten entwickelt, welches mit weiteren Alkoholen geprüft und erweitert wird. 
Weitere Isomerien können auftreten, wenn der Alkohol zusätzliche konformationelle Freiheitsgrade aufweist, z. B. wenn sich auch durch Torsion um C-C-Bindungen unterschiedliche Geometrien ergeben. Da bereits die C-O-Torsionsisomerie eine zusätzliche Komplikation bei der Untersuchung von Chiralitätserkennung darstellt, erscheint es naheliegend die $\mathrm{C}-\mathrm{C}$-Torsionsisomerie durch geeignete Molekülgeometrie möglichst auszuschließen. Dies kann erreicht werden wenn Substitutenten so gewählt werden, dass die $\mathrm{C}-\mathrm{C}$-Torsion nur in weiteren identischen Konformeren resultiert, wie es z. B. bei einer Methyl- oder tert-Butylgruppe der Fall ist. Ein in diesem Kapitel behandeltes chirales Beispiel hierfür ist Pinakolylalkohol (tert-Butylmethylcarbinol). In einem starren bicyclischen Kohlenstoffgerüst kann die $\mathrm{C}-\mathrm{C}$-Torsion auch fast komplett unterbunden werden, behandelte Beispiele hierfür sind Borneol, Isopinocampheol und $\alpha$-Fenchol. Diesen Alkoholen wird 2-Butanol gegenübergestellt, dessen zusätzliche Isomerie bezüglich der Torsion einer Ethylgruppe in der Tat zu besonders komplexen Spektren führt, sowohl für den isolierten Alkohol, wie auch in der Chiralitätserkennung mit anderen Molekülen. Die Spektren von 2-Butanol können dennoch weitgehend analysiert werden. In geeigneter Weise in das Molekül eingebracht, kann zusätzliche Flexibilität in der C-C-Torsion aber auch zu einer Verringerung der Zahl von energetisch relevanten Konformere führen, wie anhand von 1-Phenylethanol und seinem achiralen Derivat Benzylalkohol gezeigt wird.

\subsection{OH-Streckschwingung als Sonde}

Die Wellenzahl der OH-Streckschwingung von Alkoholen ist nicht nur sensitiv für den Akzeptor einer Wasserstoffbrücke, sondern auch im freien Molekül für die Konstitution und Konformation des Alkohols selbst. Letzteres gilt allerdings im geringeren Ausmaße, sodass sich $\mathrm{OH}-$ Streckschwingungswellenzahlen zwischen den verschiedenen Konformeren eines Alkohols für gewöhnlich nur um einstellige bis geringe zweistellige Werte in reziproken Zentimetern unterscheiden. Größere Verschiebungen können allerdings durch die Ausbildung von intramolekularen Wasserstoffbrücken entstehen.[213] Um derart geringe spektrale Unterschiede aufzulösen und mit quantenchemischen Vorhersagen gut vergleichen zu können, ist es hilfreich spektrale Verbreiterungen und Verschiebungen zu minimieren, die durch Rotations- und Schwingungsanregung sowie Umgebungseinflüsse entstehen. Die Untersuchung in Überschallexpansionen stellt daher eine besonders geeignete Präparationsmethode dar, während Untersuchungen in der ZimmertemperaturGasphase, in Flüssigkeit, in Lösung oder in Matrizen nur eingeschränkt geeignet sind (aber dennoch wichtige Vergleichswerte liefern können). Auch Ramanspektroskopie der $\mathrm{CH}$-Streckschwingung zeigt Potential, allerdings ist die Zuordnung gerade bei größeren Alkoholen wegen der Kopplung der meist vielfach vorhandenen Oszillatoren dieses Typs und häufig auftretenden Fermi-Resonanzen herausfordernd.[214]

Eine weitere wichtige Dimension für die Zuordnung von Alkohol-Konformeren sind deren Energieunterschiede. Bezüglich der $\mathrm{C}-\mathrm{O}$-Torsion liegen diese meist in einem $\mathrm{Be}-$ reich von nur etwa $1 \mathrm{~kJ} \mathrm{~mol}^{-1}$. Die Isomerisierung erfolgt in der Regel über recht niedrige Barrieren und wird wegen der geringen Masse des Protons von Tunnelprozessen unterstützt, sodass von einer effektiven Einstellung des Gleichgewichts auch unter Überschallexpansionsbedingungen ausgegangen werden kann. Dieser Umstand wurde im vorherigen Kapitel anhand der Ethanol-Konformere für die Abschätzung einer effektiven Konformat- 


\section{Torsionsisomerie von Alkoholen}

ionstemperatur genutzt. Ein solches Vorgehen setzt allerdings voraus, dass die Energieunterschiede experimentell sehr genau bekannt sind, was bisher nur für sehr wenige Alkohole der Fall ist, wie Ethanol (Kap. 4.2) und $n$-Propanol [215]. Quantenchemische Routinemethoden gelten in diesem engen Energiebereich nur als begrenzt zuverlässig. Daher ist es entscheidend stets beide Dimensionen, Bandenpositionen und -intensitäten, zu berücksichtigen, was hier durch explizite Simulation der Spektren erfolgt, auch um berechnete IR- und Raman-Aktivitäten miteinzubeziehen und um Überlagerungen zu modellieren.

Aus Empfindlichkeitsgründen können aber oft nicht alle Konformere nachgewiesen werden. So wurde beispielsweise für die beiden chiralen Alkohole Menthol und Neomenthol nur jeweils eine Monomerbande beobachtet.[216] Rechnungen auf verschiedenen Niveaus ergaben widersprüchliche Vorhersagen für das stabilste Konformer. Eine isolierte Zuordnung anhand von harmonischen $\mathrm{OH}-$ Streckschwingungswellenzahlen wäre daher nicht möglich gewesen, dies gelang aber durch den Vergleich der beiden Systeme. Denn robuster als die Energieunterschiede wurde vorhergesagt, dass zwei Konformere, jeweils eins von Menthol und Neomenthol, sehr ähnliche OHStreckschwingungswellenzahlen aufweisen sollten - so wie auch die Positionen der Banden beider Systeme im Experiment fast übereinstimmen. Das so zugeordnete Konformer von Menthol wurde später rotationsspektroskopisch bestätigt [217], für Neomenthol steht dies noch aus.

Ohne Vergleich mit anderen Systemen kann es hingegen zu Fehlzuordnungen kommen, auch wenn die Anzahl und Wellenzahldifferenzen von beobachteten Banden mit den Vorhersagen quantenchemischer Rechnungen grundsätzlich kompatibel erscheinen. So wurden für Benzylalkohol zwei Banden bei 3650 und $3585 \mathrm{~cm}^{-1}$ beobachtet und auch zwei Konformere theoretisch vorhergesagt.[218, 219] Auch die berechnete Wellenzahldifferenz der beiden Konformere $\left(\Delta \omega=30 \mathrm{~cm}^{-1}\right.$ auf HF/6-31G-Niveau [218]) entsprach zumindest grob der experimentellen Differenz $\left(\Delta \tilde{v}=65 \mathrm{~cm}^{-1}\right)$. Dementsprechend wurden die Banden zugeordnet. Dies wurde aber durch eine spätere Untersuchung [220] revidiert, die stattdessen die höherfrequente Bande dem niederfrequenten Monomer und die niederfrequente Bande einem Dimer zuordnete, während das höherfrequente Monomer zu hochenergetisch ist um nachgewiesen zu werden (dies wird in Abschnitt 5.16 noch näher betrachtet). Diese neue Zuordnung basierte u. a. auf dem Vergleich mit den strukturell verwandten Alkoholen Ethanol und 2-Phenylethanol, aus dem eine Skalierung der berechneten harmonischen Wellenzahlen abgeleitet und auf Benzylalkohol angewandt wurde.

Um zukünftige Zuordnungen zu erleichtern (und vergangene zu überprüfen) erscheint es daher sinnvoll eine breitere Basis von bekannten Werten für OH-Streckschwingungswellenzahlen zusammenzustellen, sodass ein einfacher Vergleich mit strukturell ähnlichen Alkoholen möglich wird. Auf Grundlage der Korrelation zwischen berechneten harmonischen Wellenzahlen und experimentellen Fundamentalübergängen können Vorhersagen für weitere Systeme getroffen werden. Ein solches Modell, Korrelationsmodell genannt, wird in diesem Kapitel entwickelt.

\subsection{Existierende Korrelationsmodelle}

Die quantenchemische Vorhersage von Schwingungsfrequenzen hat sich nicht nur für schwingungsspektroskopische Zuordnungen, sondern auch für thermodynamische und ki- 
netische Beschreibungen von molekularen Systemen, zu einem unverzichtbaren Hilfsmittel entwickelt. Um experimentelle, d.h. anharmonische Schwingungswellenzahlen über kostengünstige Rechnungen von harmonischen Wellenzahlen abschätzen zu können, wurden bereits vielfach empirische Verknüpfungen dieser beiden Größen abgeleitet. Diese Modelle erfreuen sich großer Beliebtheit, so nähert sich gemäß Google Scholar und Web of Science die Arbeit von Scott und Radom [221] 7000 Zitationen - mit weiterhin mehreren neuen pro Woche, trotz der vermeintlich überholten quantenchemischen Methoden aus dem Jahr 1996.

Der am weitesten verbreitete Ansatz für die Beschreibung der Korrelation beinhaltet eine multiplikative Skalierung der harmonischen Wellenzahl $\omega$ mit einem einzelnen empirischen Parameter $\lambda$ (Gl. 5.1), der meist für die gesamte Breite an Fundamentalübergängen angepasst wird.[222]

$$
\tilde{v}=\lambda \omega
$$

Die einfache mathematische Form und universelle Anwendbarkeit auf beliebige Schwingungen geht allerdings mit Abstrichen in der Genauigkeit einher. Durch die große Bandbreite von Schwingungswellenzahlen (mit intermolekularen Schwingungen im Bereich von wenigen $\mathrm{cm}^{-1}$ am einen Ende und Hydrid-Streckschwingungen von bis zu etwa 4000 $\mathrm{cm}^{-1}$ am anderen Ende) wird ein Defizit der für die Anpassung von $\lambda$ benutzten Methode der kleinsten Fehlerquadrate bedeutsam. Diese minimiert die Summe der Residuen $R$ nach Gl. 5.2, sodass die hochfrequenten Schwingungen effektiv höher gewichtet werden, denn deren prozentuale Abweichungen wirken sich absolut stärker aus.[221]

$$
\min \sum_{i} R_{i}^{2}=\min \sum_{i}\left(\lambda \omega_{i}-\tilde{v}_{i}\right)^{2}
$$

Verfeinerte Verfahren bestimmten daher unterschiedliche Werte für $\lambda$ für die Skalierung von hohen und niedrigen Frequenzen (mit willkürlicher Abgrenzung).[221, 223-225] Eine solche zweite Anpassung nach Gl. 5.3 berücksichtigt die niederfrequenten Schwingungen stärker.

$$
\frac{1}{\tilde{v}}=\frac{1}{\lambda \omega}
$$

Vielfach wurde dabei gefunden [221, 222, 224-227], dass das DFT-Funktional B3YLP, mit ausreichend großem Basissatz,[223, 227] im Vergleich mit anderen Methoden eine besonders hohe Korrelation zwischen experimentellen und in harmonischer Näherung berechneten Wellenzahlen aufweist.

Modelle mit anderen funktionalen Zusammenhängen [228, 229], auch mit mehr als einem Parameter [230, 231], sind weniger verbreitet. Weiterhin gibt es Argumente dafür, dass eine Skalierung bereits auf der Ebene der Kraftkonstanten, und nicht erst auf der der Wellenzahlen, stattfinden sollte.[226, 232, 233] Zudem lassen sich die unterschiedlichen Schwingungsformen (Streck-, Torsions- und Deformationsschwingungen) besser getrennt als gemeinsam skalieren.[226, 227, 232] Für höhere Genauigkeit existieren spezielle Anpassungen für Schwingungen bestimmter funktionaler Gruppen, beispielsweise für Carbonyl-Liganden in Rhodium-Komplexen [234]. Auch für verschiedene Schwingungsformen von Jet-gekühlten Biomolekülen erwiesen sich getrennte Anpassungen als 


\section{Torsionsisomerie von Alkoholen}

erfolgreich, darunter auch für freie OH-Streckschwingungen.[227] Für monomere Alkohole und Phenole wurde eine Korrelation zwischen quantenchemischen Vorhersagen und Messwerten für OH-Streckschwingungswellenzahlen 2011 von Spanget-Larsen et al. untersucht [231], dort allerdings mit Fokus auf Moleküle mit starken bis sehr starken intramolekularen Wasserstoffbrücken bei Zimmertemperatur. Zwei spezielle Modelle in der Literatur basieren auf experimentellen Werten für OH-Streckschwingungsfrequenzen von monomeren Alkoholen in Überschallexpansionen. Das erste ist ein reparametrisiertes AMBER/parm99.dat-Kraftfeld von Cézard et al.[235], welches allerdings auf die Vorhersage von spektralen Verschiebungen bei der Dimerisierung von Alkohole abzielt. Zudem sind zwei der für die Parametrisierung genutzten konformationellen Zuordnungen für Monomere ( $g$-Konformer von $t$-Butyldimethylcarbinol und $T_{6} t$ von $n$-Octanol) wahrscheinlich Fehlzuordnungen (dies wird in Abschnitt 5.5 und 5.12 begründet). Ein zweites solches Modell ist das ebenfalls rein empirische 3-Parameter-Modell, welches von Wassermann $[14,57]$ für $n$-Alkanole entwickelt und von Xue [236] für verzweigte aliphatische Alkohole erweitert wurde. Das 3P-Modell wurde auf Basis von harmonischen B3LYP/6-311+G(d)-Wellenzahlen abgeleitet, für die gefunden wurde, dass deren Differenzen gut experimentelle Verschiebungen zwischen Konformeren entsprechen. Ein interessanter Aspekt des Modells ist neben seiner Einfachheit (eine einzelne mathematische Formel wird auf Basis der Geometrie eines Konformers zur Berechnung benutzt), die Erklärung von Frequenzverschiebungen durch konkrete molekulare Einflüsse auf die Hydroxygruppe. Ein wichtiger Nachteil von solchen rein empirischen Modellen ist allerdings, dass diese keine Vorhersagen für bisher nicht-parametrisierte Einflüsse machen können. Dazu zählen in diesen beiden Beispielen z. B. Ringspannung oder nichtaliphatische Gruppen.

\subsection{Neues Korrelationsmodell}

Auf Basis der Ergebnisse von früheren Korrelationsmodellen werden die folgenden Grundlagen für ein neues Modell festgelegt:

- Die experimentellen Werte werden ausschließlich aus Bestimmungen für Fundamentalübergänge von Alkoholen in Überschallexpansionen ausgewählt.

- Die Auswahl wird auf Alkohole mit vergleichbarer chemischer Struktur zu den hier untersuchten Zielmolekülen beschränkt (Alkohole mit Kohlenwasserstoffresten ohne starke intramolekulare Wasserstoffbrücken).

- Korreliert werden die experimentellen Werte mit Vorhersagen aus B3LYP-D3(BJ)/ may-cc-pVTZ in harmonischer Näherung.

- Der funktionale Zusammenhang zwischen experimentellen und berechneten Daten wird möglichst einfach gewählt, mit einem einzelnen anzupassenden Parameter.

Diese Grundlagen werden im Folgenden etwas näher erläutert. Für experimentelle Werte wurde eine umfangreiche Literaturrecherche durchgeführt, die neben öffentlich zugänglicher Literatur auch Abschlussarbeiten des Arbeitskreises Suhm umfasst. Werte aus letzteren stammen dabei konkret aus der Diplomarbeit von Xue [236], der Bachelorarbeit von Falkowski [237] und der Masterarbeit von Hartwig [150]. 
Für die experimentelle Bestimmung der OH-Streckschwingungswellenzahlen von monomeren Alkoholen in Überschallexpansionen wurden verschiedene Methoden mit unterschiedlichen Vor- und Nachteilen eingesetzt. Ramanstreuung ist universell einsetzbar für alle Alkohole und erlaubt durch die Beobachtung von reinen Schwingungsübergängen (Q-Zweig) auch eine spektrale Auflösung von Banden, die sich in ihrer Position nur um wenige $\mathrm{cm}^{-1}$ von einander unterscheiden. Ein Nachteil ist die Streuung der symmetrischen Streckschwingung der schwierig zu vermeidenden Wasserverunreinigung, die im typischen Monomerbereich von Alkoholen aufritt und dort andere Banden verdecken kann.[238] Ein weiterer Nachteil ist die schwierige Kalibrierung, die für den Curry-Jet über die Jahre verfeinert wurde.

FTIR-Spektroskopie ist dagegen selbst-kalibrierend, allerdings ist die Auflösung limitiert durch die Beobachtung von kombinierten Rotationsschwingungsübergängen ( $\mathrm{P}$ - und R-Zweig) mit begrenzter instrumenteller Auflösung durch die limitierte Expansions- und damit Scandauer bei der Verwendung von gepulsten Überschallexpansionen. Eine Alternative hierzu ist Cavity-Ringdown-Spektroskopie an einer hochfrequent-gepulsten Expansion mit höherer erzielbarer instrumenteller Auflösung.[119, 158] Auch die Kopplung von hochaufgelöster FTIR-Spektroskopie mit kontinuierlichen Expansionen ist möglich [18], wurde aber, soweit dem Autor bekannt, noch nicht für die Untersuchung von Alkoholen eingesetzt.

Auch der IR-Teil von Doppelresonanzmethoden [218-220, 239, 240] ist durch die Bandbreite des Lasers in der Auflösung limitiert, allerdings fällt dies weniger ins Gewicht, da Überlagerungen konformeren- und größenselektiv aufgelöst werden können. Letzteres ist relevant, da auch freie OH-Gruppen von Dimeren in diesen Spektralbereich absorbieren können.[22] Allerdings sind UV-IR-Doppelresonanzmethoden auf Moleküle mit UVChromophor begrenzt (in der Regel aromatische Systeme) und benötigen eine zusätzliche Kalibrierung. Die Erweiterung auf einige aliphatische Alkohole gelang teilweise durch Kopplung mit VUV-Ionisation,[144, 182-184] allerdings erfolgt diese nicht-resonant und daher geht auch der Hauptvorteil der UV-Doppelresonanzmethoden, die Konformerenselektivität, verloren. Zudem sind die bestimmten Bandenpositionen von Ethanol mit dieser Methode gegenüber denen aus direkter Detektion aus unbekanntem Grund signifikant verschoben. Auch die Detektion der IR-Methoden wird durch die Anwesenheit von Wasser beeinträchtigt, allerdings weniger von solchem in der Expansion selbst, sondern eher im Strahlengang des optischen Aufbaus. Wasser reduziert die Strahlungsintensität und damit die Empfindlichkeit im Bereich seiner Rotationsschwingungsübergänge, welche bei Konzentrationsdrifts auch direkt das Differenzspektrum im Monomerbereich überlagern können. Dem wird bei den hier eingesetzten FTIR-Methoden durch Evakuierung, Spülung mit Trockenluft und Bildung von Differenzspektren entgegengewirkt (Details hierzu in Kap. 3.1.2). Ein Vorteil der laserbasierten Methoden ist das enge Strahlprofil, mit dem gezielt bestimmte Abschnitte der Expansion untersucht und damit Informationen über relative Stabilität von Konformeren gewonnen werden können. Dies wurde bereits im vorherigen Kapitel für Ethanol-Dimere genutzt und wird auch hier an einem monomeren System angewendet.

Für die Alkohole Methanol, Ethanol, Benzylalkohol, 1-Phenylethanol, 1-Indanol und Cyclopentanol liegen Bestimmungen von Bandenpositionen aus unterschiedlichen Detektionsmethoden vor (Tab. A.7 und A.8 im Anhang). Diese liegen in guter Übereinstimmung jeweils in einem Wellenzahlbereich von $0-2 \mathrm{~cm}^{-1}$, was auch als eine gute Abschätzung 


\section{Torsionsisomerie von Alkoholen}

für die experimentelle Unsicherheit der hier verwendeten Werte gelten kann. Ausnahmen hiervon sind Werte, die wie erwähnt mit VUV-IR-Methoden bestimmt wurden, sowie für den speziellen Fall von Methanol mit schwieriger Bestimmung des Bandenzentrums. Bei der etwas subjektiven Auswahl aus mehreren leicht abweichenden Bestimmungen werden Werte aus Ramanspektroskopischen (sofern vorhanden) und jüngeren Untersuchungen bevorzugt. Wellenzahlen, die vor 2016 mit dem Curry-Jet bestimmt wurden, wurden hier nachträglich um $-1 \mathrm{~cm}^{-1}$ nachkorrigiert,[109] damit wird in der Tat eine bessere Übereinstimmung mit Werten aus anderen Methoden erreicht. Die ausgewählten Werte sind im Anhang in den Tabellen A.6-A.9 dokumentiert. Der für das Trainingsset des Korrelationsmodells verwendete Wert ist dort jeweils zuerst angegeben, Werte anderer Bestimmungen sind zu Vergleichszwecken dahinter aufgelistet. In Verlauf dieses Kapitels neu bestimmte oder aktualisierte Wellenzahlen sind entsprechend ohne Quellenangabe angegeben. Diese werden für die um den Testdatensatz ergänzte Version des Modells am Ende des Kapitels verwendet.

Für die Auswahl der zu berücksichtigenden Alkohole muss ein Kompromiss aus chemischer Ähnlichkeit und ausreichendem Datenumfang getroffen werden. Bei im Folgenden näher behandelten (transient oder permanent) chiralen Alkoholen handelt es sich allesamt um Alkohole mit Kohlenwasserstoffrest und (weitgehend) unkoordinierter Hydroxygruppe. Nicht berücksichtigt werden daher Alkohole mit weiteren Heteroatomen, da diese zusätzliche Effekte (starke Bindungspolarisierungen, intramolekulare Wasserstoffbrücken), und damit weitere Herausforderungen bei der quantenchemischen Beschreibung, einbringen können. Ebenso nicht berücksichtigt werden Alkohole, die intramolekulare Wasserstoffbrücken mit ungesättigten Kohlenwasserstoffresten eingehen können, z. B. 2-Phenylethanol. Davon ausgenommen werden solche, die eine sehr ungünstige Geometrie für intramolekulare Wasserstoffbrücken aufweisen (z. B. 1-Phenylethanol), auch weil einige dieser Moleküle im Zentrum des Interesses der vorliegenden Arbeit stehen. $\mathrm{Ob}$ die dennoch beobachtete hohe relative Stabilität und vergleichsweise niedrige $\mathrm{OH}$ Streckschwingungsfrequenz bestimmter Konformere dieser Alkohole auf eine schwache intramolekulare Wasserstoffbrücke zurückgeführt werden kann, ist umstritten (siehe Diskussion zu Benzylalkohol in Abschnitt 5.16).

$n$-Alkanole werden nur bis einschließlich $n$-Propanol berücksichtigt, da eine Verlängerung der Alkylkette weit entfernt von der Hydroxygruppe keine signifikanten spektralen Auswirkungen mehr hat: Die Bandenpositionen sind im Rahmen der Messgenauigkeit praktisch identisch mit $n$-Propanol [14], im Einklang mit den Rechnungen. Ein Einbeziehen aller $n$-Alkanole wäre weitgehend redundant und würde das relative Gewicht von relevanteren Alkoholen in der Anpassung verringern. Ähnliches könnte auch für die Erweiterung von Isopropylmethanol zu Cyclohexanol argumentiert werden, allerdings erfolgt die Verlängerung hier doppelt am $\gamma$-Kohlenstoffatom und es besteht die Möglichkeit eines Einflusses auf die Hydroxygruppe durch Ringspannung und Fixierung [13].

Aus verschiedenen Gründen werden Phenole nicht berücksichtigt. Die zuvor diskutierten Unterschiede in den Bindungsverhältnissen der Hydroxygruppe erschweren die Vergleichbarkeit mit Alkoholen. Auch die Behandlung von Phenolen als eigenständige Gruppe ist für ein Korrelationsmodell weniger interessant, da verfügbare Werte andeuten, dass zumindest Kohlenwasserstoffsubstituenten am Ring die OH-Streckschwingungsfrequenz nur wenig beeinflussen. So sind die Werte für Phenol $\left(3656.7 \mathrm{~cm}^{-1}\right.$ [241]), trans-orthoKresol (3656 cm $\mathrm{cm}^{-1}$ [128]) und para-Kresol $\left(3656 \mathrm{~cm}^{-1}\right.$ [128]) im Rahmen der Messge- 
nauigkeit praktisch identisch. Phenole sind auch für die Chiralitätserkennung aus geometrischen Gründen weniger geeignet, da sowohl das $\alpha$ - wie auch die $\beta$-Kohlenstoffatome durch ihre $\mathrm{sp}^{2}$-Hybridisierung als potentielle Stereozentren ausfallen, und mögliche Stereozentren in den anderen Substituenten des Phenylringes relativ weit entfernt von der Hydroxygruppe liegen würden. Zudem ist bereits die Stammverbindung Phenol relativ schwer flüchtig (Normalsiedetemperatur $182^{\circ} \mathrm{C}$ ). Prinzipiell erlauben Phenole alternativ auch helicale Chiralität,[242] allerdings sind entsprechende Moleküle durch das hierfür benötigte ausgedehnte Ringsystem äußerst schwerflüchtig.

Während Tunnelprozesse die Einstellung des Gleichgewichts fördern, können sie die Zuordnung auch erheblich erschweren, wenn sie zu größeren spektralen Tunnelaufspaltungen führen. Die Bandenpositionen betroffener Konformere können durch Theorie in harmonischer Näherung, sowie auch solcher mit lokaler anharmonischer Korrektur, nicht vorhergesagt werden. Systeme, in denen Hinweise darauf vorliegen, werden daher ausgeschlossen - dies betrifft 3-Pentanol [236] und 2,2-Dimethyl-1-propanol [236]. Für letztgenanntes Molekül werden die Schwierigkeiten bei der Zuordnung in Abschnitt 5.13 etwas näher betrachtet. Eine Ausnahme wird für Methanol gemacht, welches ebenfalls u. a. wegen einer Tunneldynamik eine komplexe Bandenstruktur zeigt.[54] Da Methanol aber nur ein Konformer aufweist, reduziert sich das Problem auf eine größere Unsicherheit in der Bestimmung des Bandenzentrums.[54] Die etwas stärker unsichere Position von Methanol hat aber keine Auswirkung auf die Korrelationsfunktionen der anderen Alkoholklassen, da diese besser getrennt betrachtet werden sollten, wie im Folgenden gezeigt wird.

Insgesamt wurden im Trainingsset 37 Konformere von 18 Alkoholen berücksichtigt, davon 7 Konformere von 3 tertiären Alkoholen, 15 Konformere von 9 sekundären Alkoholen, 14 Konformere von 5 primären Alkoholen, sowie Methanol.

Korreliert werden die experimentellen Werte mit berechneten auf B3LYP-D3(BJ)/maycc-pVTZ-Niveau in harmonischer Näherung. Wie im vorherigen Kapitel bei der Beschreibung von Ethanol gefunden wurde, scheint die Methode für die Beschreibung von Alkoholen ein guter Kompromiss aus Rechenkosten und Genauigkeit zu sein, insbesondere wenn auch anschließend Aggregate auf gleichem Niveau behandelt werden sollen. Die in Literatur durchgeführten Zuordnungen wurden auf ihre Plausibilität überprüft, indem stets auch andere Konformere der jeweiligen Alkohole mit in Betracht gezogen wurden sowohl hinsichtlich relativer Energie innerhalb des Systems, wie auch der Einordnung von $\mathrm{OH}-$ Streckschwingungswellenzahlen relativ zu anderen Alkoholen im Rahmen des Korrelationsmodells. Nur für zwei Zuordnungen ( $g$-Konformer von $t$-Butyldimethylcarbinol und $T_{6} t$ von $n$-Octanol) [235] wurden größere Diskrepanzen gefunden, auf die in eigenen Abschnitten zu diesen Alkoholen näher eingegangen wird.

Lange bekannt aus Messungen in verdünnter Lösung und in der Gasphase ist die Tendenz, dass die Wellenzahl des OH-Streckschwingungsfundamentalüberganges mit steigendem Substitutionsgrad am $\alpha$-Kohlenstoffatom sinkt, also von Methanol zu primären, sekundären und tertiären Alkoholen (siehe z. B. Ref. [13, 243] und dahin enthaltene Referenzen). Die analytische Bedeutung dieser Beobachtung hat Eingang in Grundkurse und anwendungsorientierte Lehrbücher zur Schwingungsspektroskopie gefunden.[244] 
Auch für Alkohole in Überschallexpansionen findet sich diese Tendenz wieder, wie die Übersicht der 37 Datenpunkte in Abb. 5.2 zeigt.

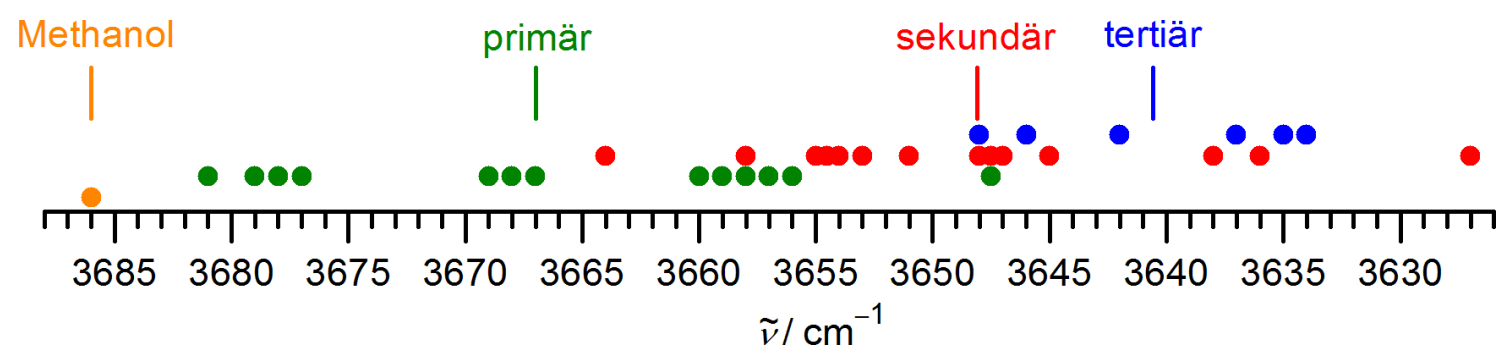

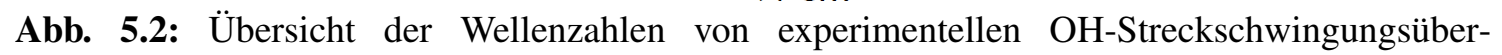
gängen von Alkoholen in Überschallexpansionen, aufgeteilt nach Substitutionsgrad des $\alpha$ Kohlenstoffatoms (Klassen). Die senkrechten Striche repräsentieren die Mittelwerte der jeweiligen Klassen.

Gemessen an den Mittelwerten (markiert durch senkrechte Striche im Diagramm) sinkt die Wellenzahl des Übergangs um $19 \mathrm{~cm}^{-1}$ von Methanol zu primären Alkoholen, um weitere $19 \mathrm{~cm}^{-1}$ von primären zu sekundären Alkoholen und nochmals um $7-8 \mathrm{~cm}^{-1}$ von sekundären zu tertiären Alkoholen. Aufgrund des begrenzten Umfangs des Datensatzes und der großen Streuung der Datenpunkte innerhalb der Klassen, sind dies aber nur grobe Richtwerte. Es existieren Überlappungen zwischen primären und sekundären, sowie noch ausgeprägter zwischen sekundären und tertiären Alkoholen. Diese Streuung besteht nicht nur zwischen den verschiedenen Alkoholen einer Klasse, sondern auch zwischen Konformeren eines Alkohols. Das Extrembeispiel ist hierfür 1-Indanol, dessen Konformere sowohl den hochfrequentesten wie auch den niederfrequentesten Datenpunkt für sekundäre Alkohole stellen, mit einer Differenz von $37 \mathrm{~cm}^{-1}$. Eine Aufschlüsselung der Datenpunkte in einzelne Konformere erfolgt am Ende des Kapitels in Abschnitt 5.18, nachdem in den folgenden Abschnitten noch einige weitere Datenpunkte von bisher nicht untersuchten Alkoholen ergänzt wurden.

Eine Auswertung von Literaturwerten für Messungen von Zimmertemperatur-Gasphasen durch Lutz und van der Maas [13] ergab folgende charakteristische Bereiche: primär $3681-3667 \mathrm{~cm}^{-1}$, sekundär $3670-3637 \mathrm{~cm}^{-1}$ und tertiär $3646-3641 \mathrm{~cm}^{-1}$. Dies lässt sich um einen Wert von $3681 \mathrm{~cm}^{-1}$ für Methanol ergänzen.[245] Diese Bereiche zeigen grobe Übereinstimmung mit ihren Entsprechungen in der Überschallexpansion in Abb. 5.2. Abweichungen sind vermutlich am ehesten auf Unterschiede in den Stichproben zurückzuführen. Daneben können die Gasphasenwerte aber auch durch heiße Banden, Überlappungen zwischen Konformeren und unerkannte spektrale Tunnelaufspaltungen beeinflusst sein. Die Bestimmung von Bandenzentren in der Gasphase ist daher schwierig, so wie es auch für Methanol noch in der Überschallexpansion gilt. Vergleiche mit Werten in Lösung sind nur sehr eingeschränkt möglich, denn der zusätzliche Lösungsmittelshift ist nicht einheitlich, sondern konstitutions- und konformationsabhängig.[13] Für apolare Lösungsmittel werden als charakteristisch primär $3640 \mathrm{~cm}^{-1}$, sekundär $3630 \mathrm{~cm}^{-1}$ und tertiär $3620 \mathrm{~cm}^{-1}$ angegeben [246], dies entspricht Tiefverschiebungen von etwa $20-40 \mathrm{~cm}^{-1}$ relativ zu den Werten der Gasphase.

Auch in den hier berechneten harmonischen Wellenzahlen zeigt sich die Tendenz für eine sinkende OH-Streckschwingungswellenzahl mit steigendem Substitutionsgrad, allerdings in geringerer Ausprägung. Dies führt dazu, dass in einer Auftragung der experimentellen gegen die harmonischen Werte sich die Datenpunkte entsprechend ihrer Alko- 
holklasse weitgehend entlang diagonaler Achsen separieren. Diese Korrelation zwischen den experimentellen und berechneten harmonischen Wellenzahlen ist in Abb. 5.3 dargestellt. Diese Diagonalen sind als Ausgleichsgeraden der Form $\tilde{v}=\omega+\kappa$ angepasst und sind, wegen der unterschiedlichen Werte für $\kappa$, gegeneinander verschoben. Dies lässt vermuten, dass die Verknüpfung zwischen experimentellen und harmonischen Werten besser mit separaten Anpassungen für jede Alkoholklasse statt global für sämtliche Datenpunkte gelingen könnte. Die Verwendung von Ausgleichsgeraden der Form $\tilde{v}=\omega+\kappa$ in Abb. 5.3 bedarf einer näheren Erläuterung. Wie zuvor diskutiert, erfolgt davon abweichend in bestehenden Korrelationsdiagrammen der funktionale Zusammenhang zwischen experimentellen Fundamentalübergängen und berechneten harmonischen Wellenzahlen meist über eine multiplikative Skalierung nach Gl. 5.4.

$$
\tilde{v}=\lambda \omega
$$

Im Kontrast hierzu erfolgt die Verknüpfung beider Größen im gängigsten Modell für die Beschreibung von Schwingungszuständen in polyatomaren Molekülen (siehe Kap. 2.5)

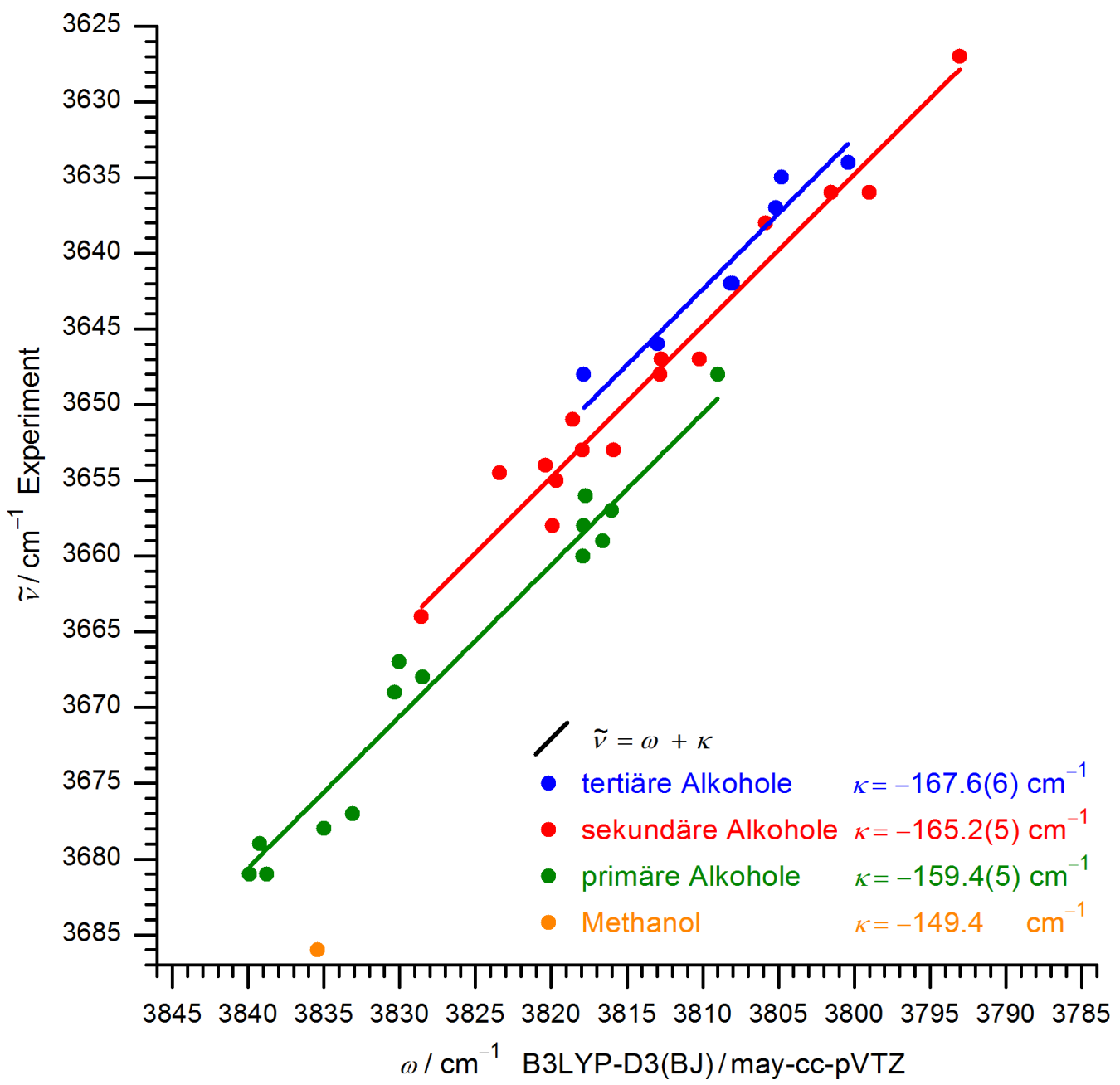

Abb. 5.3: Korrelation von experimentellen OH-Streckschwingungswellenzahlen von Alkoholen mit in harmonischer Näherung mit B3LYP-D3(BJ)/may-cc-pVTZ berechneten. Die Ausgleichsgeraden entsprechen der Gleichung $\tilde{v}=\omega+\kappa$ für die verschiedenen Alkoholklassen. 


\section{Torsionsisomerie von Alkoholen}

additiv gemäß Gl. 5.5 über die diagonalen $x_{i i}$ und außerdiagonalen $x_{i j}$ Anharmonizitätskonstanten.

$$
\tilde{v}_{i}=\omega_{i}+2 x_{i, i}+\frac{1}{2} \sum_{i}^{n} \sum_{j>i}^{n} x_{i, j}
$$

Beide Zusammenhänge lassen sich gemäß Gl. 5.6 verknüpfen. Zur Interpretation kann angenommen werden, dass sich die Anharmonizitätskonstanten $x_{i, i}$ und $x_{i, j}$ ebenfalls proportional zu $\omega_{i}$ nach G1. 5.7 verhalten. Die Abweichung des Parameters $\lambda$ vom Zahlenwert 1 repräsentiert dann nach Gl. 5.9 einen mittleren Faktor für eine mit $\omega_{i}$ skalierende Anharmonizität. Durch die Anpassung sind in $\lambda$ aber auch systematische Fehler aus der Bestimmung von $\tilde{v}_{i}$ und vor allem aus der Berechnung von $\omega_{i}$ enthalten.

$$
\begin{array}{ll}
\lambda \omega_{i}=\omega_{i}+2 x_{i, i}+\frac{1}{2} \sum_{i}^{n} \sum_{j>i}^{n} x_{i, j} & \mid \text { Proportionaliätsannahme } \\
\lambda \omega_{i}=\omega_{i}+\omega_{i} 2 x_{i, i}^{\prime}+\omega_{i} \frac{1}{2} \sum_{i}^{n} \sum_{j>i}^{n} x_{i, j}^{\prime} & \mid \text { Ausklammern von } \omega_{i} \\
\lambda \omega_{i}=\omega_{i}\left(1+2 x_{i, i}^{\prime}+\frac{1}{2} \sum_{i}^{n} \sum_{j>i}^{n} x_{i, j}^{\prime}\right) & \text { | Division durch } \omega_{i} \\
\lambda=1+2 x_{i, i}^{\prime}+\frac{1}{2} \sum_{i}^{n} \sum_{j>i}^{n} x_{i, j}^{\prime} &
\end{array}
$$

Eine solche Abnahme der Anharmonizität mit sinkender Schwingungsfrequenz ist eine sinnvolle Annahme, wenn ein breiter Frequenzbereich betrachtet wird, wie es bei universalen Korrelationsmodellen für Schwingungen der Fall ist. Dies muss aber nicht zwangsläufig auch die beste Annahme für den hier betrachteten engen Frequenzbereich von $\mathrm{OH}-$ Oszillatoren in sehr ähnlicher chemischer Umgebung sein. Eine alternative Annahme ist, dass die Anharmonizität in einem solchen Fall näherungsweise konstant bleibt und die beobachteten Änderungen in den experimentellen Wellenzahlen allein auf Variationen in den harmonischen Wellenzahlen zurückzuführen sind. Dies lässt sich über Gl. 5.10 mit einem Parameter $\kappa$ modellieren, der eine konstante Anharmonizität repräsentiert (wobei auch hier etwaige Fehler in der Bestimmung bzw. Berechnung von $\tilde{v}$ und $\omega$ in $\kappa$ mit eingehen).

$$
\begin{aligned}
\tilde{v}_{i} & =\omega_{i}+\kappa \\
\kappa & =2 x_{i, i}+\frac{1}{2} \sum_{i}^{n} \sum_{j>i}^{n} x_{i, j}=\text { const. }
\end{aligned}
$$

Beide Ansätze wurden in Form von linearen Regressionen nach der Methode der kleinsten Fehlerquadrate an die Daten angepasst, sowohl für die Gesamtheit aller Alkohole, wie auch getrennt für die Alkoholklassen. Die Ergebnisse sind Tab. 5.1 zusammengestellt. Die 
Güte der Anpassungen werden für solche Korrelationsdiagramme in der Literatur in der Regel anhand des RMSE (Root Mean Square Error, Wurzel des mittleren quadrierten Fehlers) nach Gl. 5.12 bewertet. Allerdings ist die Eignung des RMSE durchaus umstritten, da dessen Wert nicht nur mit der mittleren Fehlergröße, sondern auch mit deren Varianz und tendenziell der Anzahl der Datenpunkte skaliert.[247, 248] Daher wird hier ebenfalls die einfacher interpretierbare Kenngröße MAE (Mean Absolute Error, mittlerer absoluter Fehler) nach Gl. 5.13 mitberechnet. Zumindest für die hier diskutieren Daten lassen sich aus den beiden Kenngrößen aber die gleichen qualitativen Schlussfolgerungen ziehen. Zusätzlich wird auch das größte absolute Residuum $\left|R_{\max }\right|$ angegeben, also die größte Abweichung eines Messwertes von seiner Vorhersage aus der Korrelationsfunktion.

$$
\begin{array}{r}
\mathrm{RMSE}=\sqrt{\frac{1}{n} \sum_{i}^{n} R_{i}^{2}} \\
\mathrm{MAE}=\frac{1}{n} \sum_{i}^{n}\left|R_{i}\right|
\end{array}
$$

Es lässt sich in Tab. 5.1 erkennen, dass die Anpassungsgüte beider Funktionen sehr ähnlich ist. Ebenfalls getestet wurde ein Zwei-Parameter-Ansatz gemäß Gl. 5.14.

$$
\tilde{v}=\lambda \omega+\kappa
$$

Allerdings führte diese flexiblere Form nur zu einer geringfügigen Reduzierung von RMSE und MAE, während deutliche Anzeichen einer Überanpassung auftraten. Dieser Ansatz wurde daher verworfen. Durch die Kenngrößen wird weiterhin bestätigt, was bereits durch visuelle Inspektion der Datenpunkte in Abb. 5.3 zu vermuteten war: Separate Anpassungen für die einzelnen Alkoholklassen beschreiben die Korrelation erheblich besser als eine Anpassung für alle Klassen gemeinsam. So sind MAE und RMSE für die einzelnen Klassen nur etwa halb so groß wie in der globalen Anpassung. Diese Aufteilung der Datenpunkte in die Alkoholklassen ist nicht willkürlich, sondern beruht auf klaren chemischen Unterschieden in der Molekülstruktur, für die nicht überraschend wäre, dass sie sich auf den Hydroxy-Oszillator auswirken. Dafür, dass es sich um einen systematischen Effekt handelt, spricht auch, dass sich $\kappa$ mit dem Substitutionsgrad monoton zu stärker negativen Werten verschiebt. Der von den primären Alkoholen abgesetzte Wert für Methanol legt nahe, diese Verbindung besser als Alkohol einer eigenen Klasse zu behandeln, und nicht als primären Alkohol „ehrenhalber“. Dieser Befund gilt unabhängig davon, welcher der Literaturwerte für Methanol gewählt wird.

Für die Begründung der unterschiedlichen Werte für $\kappa$ ergeben sich drei verschiedene Ansätze: unterschiedliche systematische Fehler bei der Bestimmung der experimentellen Werte, unterschiedliche systematische Fehler in der Elektronenstrukturmethode bei der Berechnung der harmonischen Wellenzahlen und/oder reale systematische Unterschiede in der Anharmonizität. Der erst genannte Erklärungsansatz kann ausgeschlossen werden, da experimentelle systematische Fehler, z. B. unzureichende Kalibrierung, nicht von einem molekularen Merkmal abhängen sollten, insbesondere nicht über mehrere gut übereinstimmende Detektionsmethoden hinweg. Es verbleiben die Möglichkeiten einer systematischen Verzerrung der berechneten harmonischen Wellenzahlen durch B3LYP- 
Tab. 5.1: Anpassungsergebnisse von zwei unterschiedlichen Korrelationsfunktionen an experimentelle Fundamentalübergänge $\tilde{v}$ in Relation zu auf B3LYP-D3(BJ)/may-cc-pVTZ-Niveau berechneten harmonischen Wellenzahlen $\omega$ für $\mathrm{OH}-\mathrm{Streckschwingungen} \mathrm{von} \mathrm{Alkoholen.}$

\begin{tabular}{|c|c|c|}
\hline tertiäre Alkohole & $\tilde{v}=\omega+\kappa$ & $\tilde{v}=\lambda \omega$ \\
\hline$\lambda$ & - & $0.9560(2)$ \\
\hline$\kappa / \mathrm{cm}^{-1}$ & $-167.6(6)$ & - \\
\hline $\mathrm{MAE} / \mathrm{cm}^{-1}$ & 1.4 & 1.4 \\
\hline $\mathrm{RMSE} / \mathrm{cm}^{-1}$ & 1.5 & 1.5 \\
\hline$\left|R_{\max }\right| / \mathrm{cm}^{-1}$ & 2.2 & 2.3 \\
\hline sekundäre Alkohole & $\tilde{v}=\omega+\kappa$ & $\tilde{v}=\lambda \omega$ \\
\hline$\lambda$ & - & $0.9567(1)$ \\
\hline$\kappa / \mathrm{cm}^{-1}$ & $-165.2(5)$ & - \\
\hline $\mathrm{MAE} / \mathrm{cm}^{-1}$ & 1.5 & 1.6 \\
\hline $\mathrm{RMSE} / \mathrm{cm}^{-1}$ & 1.9 & 1.9 \\
\hline$\left|R_{\max }\right| / \mathrm{cm}^{-1}$ & 3.7 & 3.6 \\
\hline primäre Alkohole & $\tilde{v}=\omega+\kappa$ & $\tilde{v}=\lambda \omega$ \\
\hline$\lambda$ & - & $0.9583(1)$ \\
\hline$\kappa / \mathrm{cm}^{-1}$ & $-159.4(5)$ & - \\
\hline $\mathrm{MAE} / \mathrm{cm}^{-1}$ & 1.7 & 1.7 \\
\hline $\mathrm{RMSE} / \mathrm{cm}^{-1}$ & 1.9 & 2.1 \\
\hline$\left|R_{\max }\right| / \mathrm{cm}^{-1}$ & 3.6 & 3.6 \\
\hline Methanol & $\tilde{v}=\omega+\kappa$ & $\tilde{v}=\lambda \omega$ \\
\hline$\lambda$ & - & 0.9610 \\
\hline$\kappa / \mathrm{cm}^{-1}$ & -149.4 & - \\
\hline alle Alkohole gemeinsam & $\tilde{v}=\omega+\kappa$ & $\tilde{v}=\lambda \omega$ \\
\hline$\lambda$ & - & $0.9573(2)$ \\
\hline$\kappa / \mathrm{cm}^{-1}$ & $-163.0(7)$ & \\
\hline $\mathrm{MAE} / \mathrm{cm}^{-1}$ & 3.5 & 3.9 \\
\hline $\mathrm{RMSE} / \mathrm{cm}^{-1}$ & 4.3 & 4.6 \\
\hline$\left|R_{\max }\right| / \mathrm{cm}^{-1}$ & 13.6 & 14.4 \\
\hline
\end{tabular}


D3(BJ)/may-cc-pVTZ und/oder ein realer systematischer Einfluss auf die Anharmonizität von Alkoholen. Beides wären wichtige Erkenntnisse. Die erste Möglichkeit könnte zukünftig durch den Vergleich mit weiteren harmonischen Rechnungen auf anderen Niveau überprüft werden - optimalerweise einer ab initio Methode, da die Möglichkeit eines gemeinsamen Defizits von Dichtefunktionalmethoden nicht ausgeschlossen werden kann. Schwache Indizien für die zweite Möglichkeit liefern experimentelle Werte für die diagonalen Anharmonizitätskonstanten aus Gasphasen- [249] und Überschallexpansionsmessungen [61]. Diese deuten an, dass die diagonale Anharmonizität tatsächlich sehr leicht mit dem Substitutionsgrad zu steigen scheint, allerdings im geringeren Maße als zur Erklärung der hier berechneten Differenzen in $\kappa$ notwendig. Zudem liegen Werte nur für sehr wenige Alkohole vor, aus denen bestenfalls nur sehr vorsichtig auf systematische Effekte geschlossen werden kann. Unbekannt bleibt auch der Einfluss der außerdiagonalen Anharmonizität, die in ihrer Summe experimentell weit schwieriger zugänglich ist. Daher wird zur Vereinfachung häufig angenommen, dass sich die außerdiagonalen Beiträge ungefähr gegenseitig aufheben, was bei den hier diskutierten geringen Differenzen aber nicht als sinnvolle Näherung erscheint. Auch hier wäre eine zukünftige theoretische Untersuchung dieses Trends sinnvoll, unter Verwendung einer quantenchemischen Methode, die die Anharmonizität angemessen berücksichtigt.

Insgesamt ist die Korrelation innerhalb der jeweiligen Alkoholklassen sehr hoch, so liegt der mittlere absolute Fehler jeweils nur zwischen 1.4 und $1.7 \mathrm{~cm}^{-1}$ und das größte absolute Residuum zwischen 2.2 und $3.7 \mathrm{~cm}^{-1}$. Je etwa $70 \%$ der Datenpunkte der primären und tertiären, sowie $60 \%$ der sekundären Alkohole weisen absolute Residuen kleiner $2 \mathrm{~cm}^{-1}$ auf. Ein Großteil der Abweichungen zwischen Experiment und Vorhersage wäre somit prinzipiell bereits mit der geschätzten Unsicherheit von etwa $2 \mathrm{~cm}^{-1}$, über die verschiedenen experimentellen Methoden der Literaturwerte hinweg, erklärbar.

Nach der Etablierung des Korrelationsmodells anhand des Trainingsdatensatzes aus Literaturwerten, wird dieses nun auf eine Reihe von Alkoholen - einem Testdatensatz - angewendet. Dabei gewonnene neuen Zuordnungen werden anschließend in Abschnitt 5.18 zur Erweiterung der Datenbasis verwendet und mögliche zukünftige Anwendungen und Erweiterungen des Modells werden diskutiert. 


\section{$5.5 n$-Octanol}

n-Octanol ist ein primärer Alkohol, der von Rice [250] und Cézard et al. [235] am FiletJet untersucht wurde. Dabei wurde eine einzelne Bande bei $3680 \mathrm{~cm}^{-1}$ beobachtet und der voll gestreckten $T_{6} t$-Konformation (Abb. 5.4) auf der Basis von MP2-Rechnungen zugeordnet. Allerdings wies bereits Rice [250] daraufhin, dass nach den damals neuen Erkenntnissen von Wassermann et al. für kürzere $n$-Alkanole [14] auch die $T_{5} G t$ Konformation in Frage kommt. Diese alternative Zuordnungsvariante kann hier auf Grundlage des Korrelationsmodells auch anhand der absoluten Wellenzahl erhärtet werden. Nach Korrektur der harmonischen Wellenzahlen um den für primäre Alkohole bestimmten Wert für $\kappa$ von $-159.4 \mathrm{~cm}^{-1}$ werden Vorhersagen für $T_{5} G t$ von $3679 \mathrm{~cm}^{-1}$ und für $T_{6} t$ von $3675 \mathrm{~cm}^{-1}$ erhalten. $T_{5} G t$ ist damit plausibler für die Zuordnung der Bande bei $3680 \mathrm{~cm}^{-1}$. Dies wird auch bezüglich der Intensität durch die berechnete etwas geringere Energie, höhere IR-Aktivität und den statistischen Vorteil des chiralen $T_{5} G t$-Konformers unterstützt, auch wenn im Jet bezüglich der C-C-Torsion vermutlich nur eine unvollständige Gleichgewichtseinstellung stattfindet.[190]

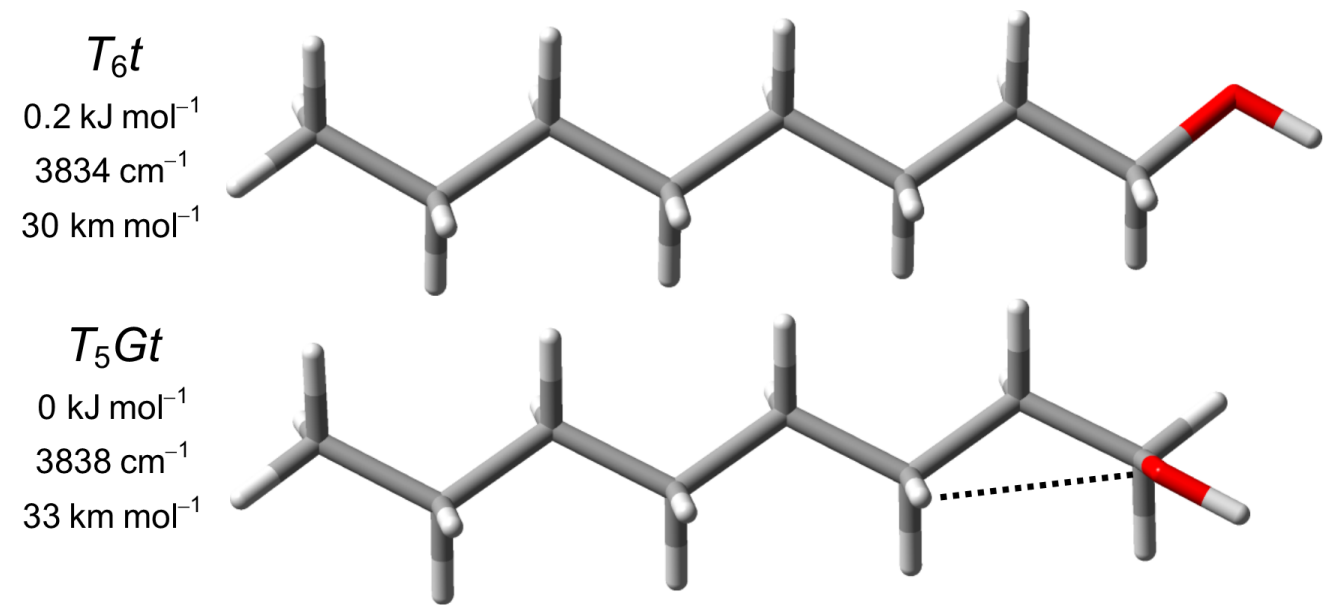

Abb. 5.4: Die beiden Konformere des $n$-Octanols auf B3LYP-D3(BJ)/may-cc-pVTZ-Niveau, die am ehesten für die Zuordnung der beobachteten Bande bei $3680 \mathrm{~cm}^{-1}$ in Frage kommen, mit relativen Energien, harmonischen $\mathrm{OH}-\mathrm{Streckschwingungswellenzahlen} \mathrm{und} \mathrm{IR-Aktivitäten.} \mathrm{Der} \mathrm{sta-}$ bilisierende $\mathrm{C}_{\gamma}-\mathrm{H} \cdots \mathrm{O}$-Kontakt für $T_{5} G t$ ist mit einer schwarz gestrichelten Linie gekennzeichnet. 


\subsection{Isopinocampheol}

Isopinocampheol ist ein chiraler, sekundärer Monoterpenalkohol, dessen Struktur in Abb. 5.5 gezeigt ist. Andere Bezeichnungen für das (-)-Enantiomer dieser Verbindung sind $(1 R, 2 R, 3 R, 5 S)$-3-Pinanol und $(1 R, 2 R, 3 R, 5 S)$-2,6,6-Trimethylbicyclo[3.1.1] heptan-3-ol.

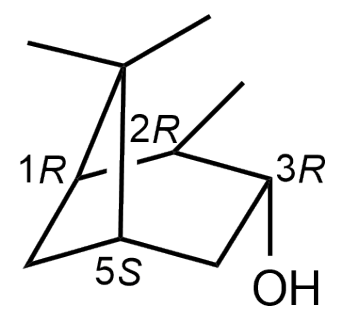

Abb. 5.5: Strukturformel von (-)-Isopinocampheol mit absoluter Konfiguration der Stereozentren.

Isopinocampheol kommt in der Natur in einigen Pflanzen vor, findet aber außerhalb der Forschung keine größere Verwendung. Isopinocampheol teilt mit dem in Kap. 6 untersuchten Verbindung $\alpha$-Pinen die Pinan-Grundstruktur und kann aus dieser durch Hydroborierung und anschließende Oxidation gewonnen werden,[251] was einer formalen Addition eines Wassermoleküls entspricht.

\subsubsection{Konformationsisomerie}

Das Bicyclo[3.1.1]heptan-Kohlenwasserstoffgerüst ist weitgehend, aber nicht vollständig starr. In Abhängigkeit von den Substituenten bevorzugt der Sechsring, der das doppelt methylierte quartäre Kohlenstoffatom einschließt, eine Halbsessel-, Sessel- oder Wannenkonformation.[252] In Übereinstimmung mit ${ }^{13} \mathrm{C}-\mathrm{NMR}$-spektroskopischen Untersuchungen [252-254] wird nach den hier durchgeführten Rechnungen das Kohlenstoffatom in 3-Position leicht in Richtung des quartären Kohlenstoffatoms ausgelenkt gefunden, was einer Übergangsform zwischen Halbsessel- und Wannenkonformation des Sechsringes entspricht. Weitere Minima von Isopinocampheol bezüglich der C-C-Torsionswinkel konnten quantenchemisch nicht gefunden werden. Die Bicyclo[3.1.1]heptan-Grundstruktur selbst ist achiral, die $C_{\mathrm{s}}$-Symmetrie wird aber durch die Methylgruppe in 2-Position gebrochen. Eine Optimierung des $C_{\mathrm{s}}$-symmetrischen, in 2-Position demethyliertes Derivats ergibt eine deutlichere Wannenkonformation, dagegen wird bei einer zusätzlichen symmetrischen Methylierung in 4-Position die Umwandlung in eine Sessel-Konformation erhalten.

Die in Isopinocampheol vorliegende gespannte Ringkonformation führt zu deutlichen Abweichungen von den in acyclischen Alkoholen bevorzugten gestaffelten Anordnungen bezüglich der $\mathrm{OC}_{\alpha} C_{\beta} \mathrm{H}$-Torsionswinkel, die für die Isopinocampheol-Konformere näher an ekliptischen Anordnungen sind.

Für die zu erwartende Isomerie bezüglich der $\mathrm{HOC}_{\alpha} \mathrm{H}$-Torsion sind dem Autor bisher keine Untersuchungen bekannt. Die Konformere werden bezüglich dieses Torsionswinkels als $g+, g-$ und $t$ bezeichnet. Durch die Chiralität von Isopinocampheol sind die $g+-$ und $g$--Konformere diastereomer. Das $g--$ Konformer wird etwas weniger stabil, geringfügig niederfrequenter und weniger IR-aktiv vorhergesagt als das $g+$-Konformer. Für das $t$-Konformer gilt dies jeweils noch im stärkeren Maße. Die drei Konformere und deren wichtigste Eigenschaften auf B3LYP-D3(BJ)/may-cc-pVTZ-Niveau sind in Abb. 5.6 


\section{Torsionsisomerie von Alkoholen}

gezeigt. Weitere Rechnungen mit anderen Methoden bestätigen diese energetische und spektrale Abfolge der Konformere (Tab. 5.2).
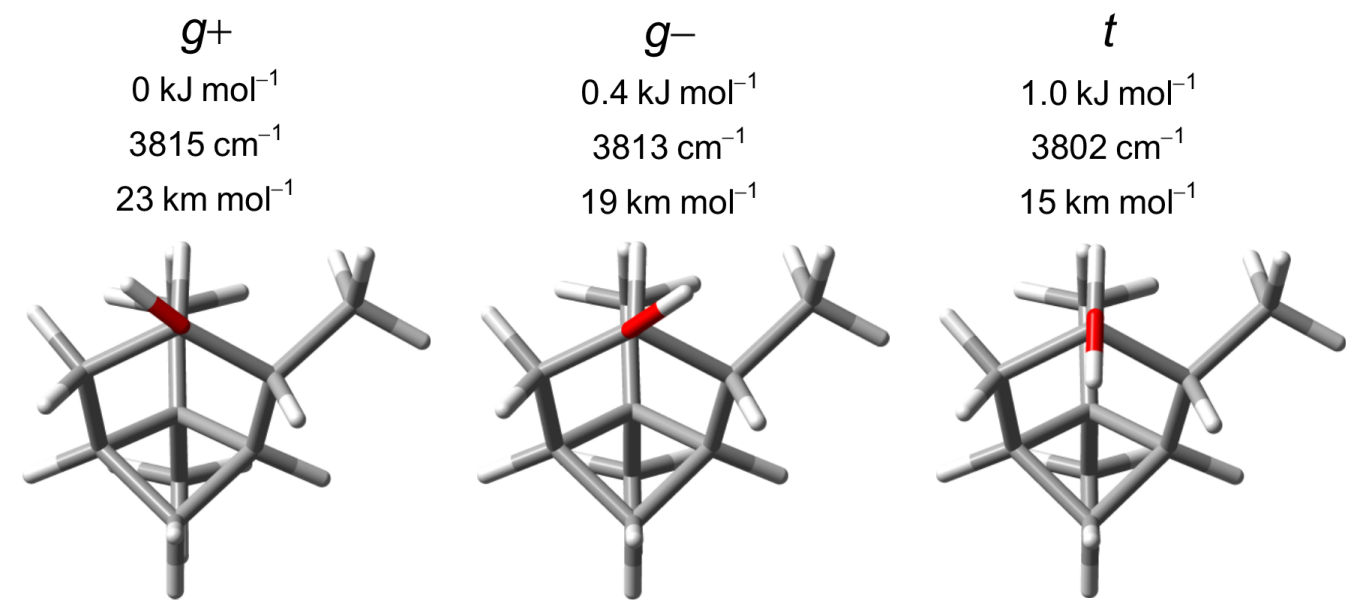

Abb. 5.6: Die drei Konformere des (-)-Isopinocampheols, klassifiziert nach der Einstellung des $\mathrm{HCOH}-$ Torsionswinkels, mit relativen Energien, OH-Streckschwingungswellenzahlen und IRAktivitäten auf B3LYP-D3(BJ)/may-cc-pVTZ-Niveau. Die Blickrichtung auf die Konformere erfolgt entlang der O-C-Bindung und der Pseudo-Spiegelebene.

Tab. 5.2: Differenzen der nullpunktskorrigierten Energien und harmonischen OH-Streckschwingungswellenzahlen der $g+$ und $g$--Konformere von (-)-Isopinocampheol-Monomer-Tabelle auf verschiedenen Rechenniveaus. Die $a b$ initio-Rechnungen wurden als Punktrechnungen unter Verwendung der Geometrien und Nullpunktsschwingungsenergien von B3LYP-D3(BJ)/aug-cc-pVTZ durchgeführt.

\begin{tabular}{lcc}
\hline Methode & $\begin{array}{c}E_{0}(g-)-E_{0}(g+) \\
/ \mathrm{kJ} \mathrm{mol}\end{array}$ & $\begin{array}{c}\omega_{\mathrm{OH}}(g+)-\omega_{\mathrm{OH}}(g-) \\
/ \mathrm{cm}^{-1}\end{array}$ \\
\hline M06-2X/def2-TZVP & 0.5 & 7 \\
$\omega$ B97X-D/def2-TZVP & 0.2 & 4 \\
B3LYP-D3(BJ)/def2-TZVP & 0.5 & 2 \\
B3LYP-D3(BJ)/may-cc-pVTZ & 0.4 & 2 \\
B3LYP-D3(BJ)/aug-cc-pVTZ & 0.4 & 2 \\
B2PLYP-D3(BJ)/may-cc-pVTZ & 0.6 & 2 \\
MP2/may-cc-pVTZ & 0.8 & - \\
MP2/aug-cc-pVQZ & 0.7 & - \\
CCSD(T)/may-cc-pVTZ & 0.7 & - \\
CCSD(T)/aug-cc-pVQZ* & 0.6 & - \\
\hline
\end{tabular}




\subsubsection{Schwingungsspektren}

Isopinocampheol schmilzt bei $57^{\circ} \mathrm{C}$ und siedet bei Normaldruck bei $219^{\circ} \mathrm{C}$.[255] Extrapolation der in Kap. 3.1.1 abgeleiteten Antoine-Gleichung lässt einen Sublimationsdruck von lediglich $4 \mathrm{~Pa}$ bei der verwendeten Sättigertemperatur von $24^{\circ} \mathrm{C}$ erwarten. Abb. 5.7 zeigt das FTIR-Spektrum einer Überschallexpansion von (-)-Isopinocampheol in Helium im OH-Streckschwingungsbereich. Dieses zeigt eine einzelne Bande im Monomerbereich mit Maximum bei $3648 \mathrm{~cm}^{-1}$. Bei der breiten Signatur im Bereich um $3500 \mathrm{~cm}^{-1}$ handelt es sich nicht um eine Absorptionsbande, sondern um ein Artefakt der Basislinie. Dieses ist auch in den Spektren von einigen anderen Substanzen vorhanden, fällt aber hier wegen der sehr geringen Absorbanz des Monomer-Signals besonders auf. Clusterbanden von Isopinocampheol sind wegen der geringen Konzentration nicht zu erkennen.

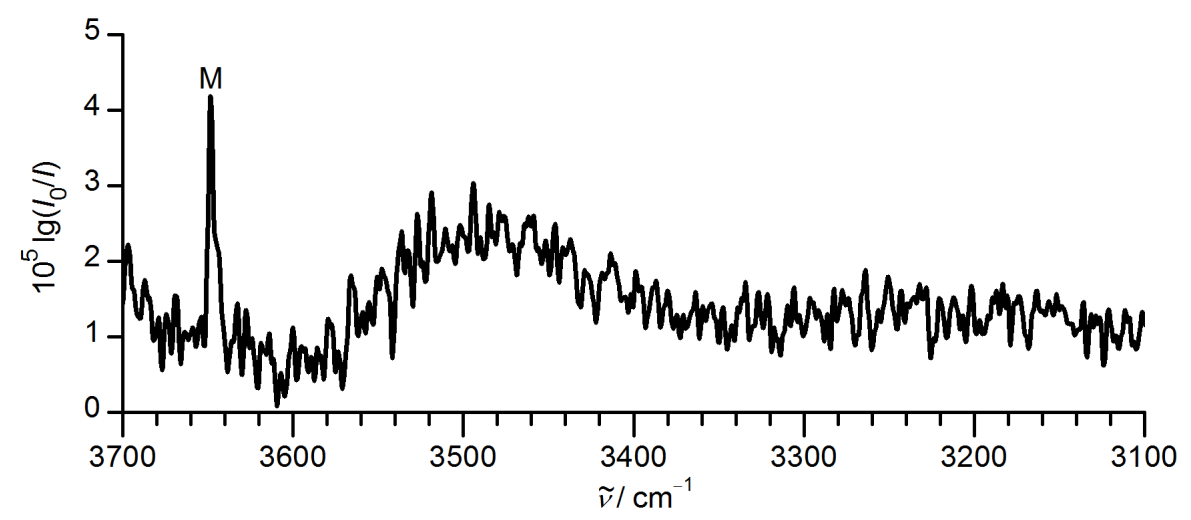

Abb. 5.7: Filet-Jet-Spektrum von (-)-Isopinocampheol im OH-Streckschwingungsbereich.

Ein näherer Blick auf die Monomerbande in Abb. 5.8 lässt zusätzlich eine Schulter bei etwa $3644 \mathrm{~cm}^{-1}$ erkennen. Durch Vergleich mit der Simulation lässt sich die Bande bei $3648 \mathrm{~cm}^{-1}$ dem $g+-$ Konformer zuordnen (Abweichung von der Vorhersage aus dem Korrelationsmodell: $-1 \mathrm{~cm}^{-1}$ ). Die Schulter bei $3644 \mathrm{~cm}^{-1}$ wird vermutlich vom $g-$ Konformer verursacht, allerdings sind für eine sichere Zuordnung die Auflösung und das Signal-zu-Rausch-Verhältnis nicht ausreichend. Die Position würde geringfügig stärker von der Vorhersage $\left(-3 \mathrm{~cm}^{-1}\right)$ abweichen. Mittels der für Monomere empfindlicheren und besser auflösenden Ramanspektroskopie könnte eventuell künftig diese Vermutung bestätigt oder widerlegt werden. Das $t$-Konformer ist wegen seiner hohen Energie und geringen IR-Aktivität unterhalb der Detektionsschwelle. Das Gasphasenspektrum bei Zimmertemperatur zeigt ein Maximum an der gleichen Position wie das Jet-Spektrum, die breite Bandenstruktur fällt zur niederfrequenten Seite etwas flacher ab. Dies wäre vereinbar mit der Vorhersage, dass die weniger stabilen und schwächer IR-aktiven Konformere bei niedrigeren Wellenzahlen als $g+$ absorbieren. 


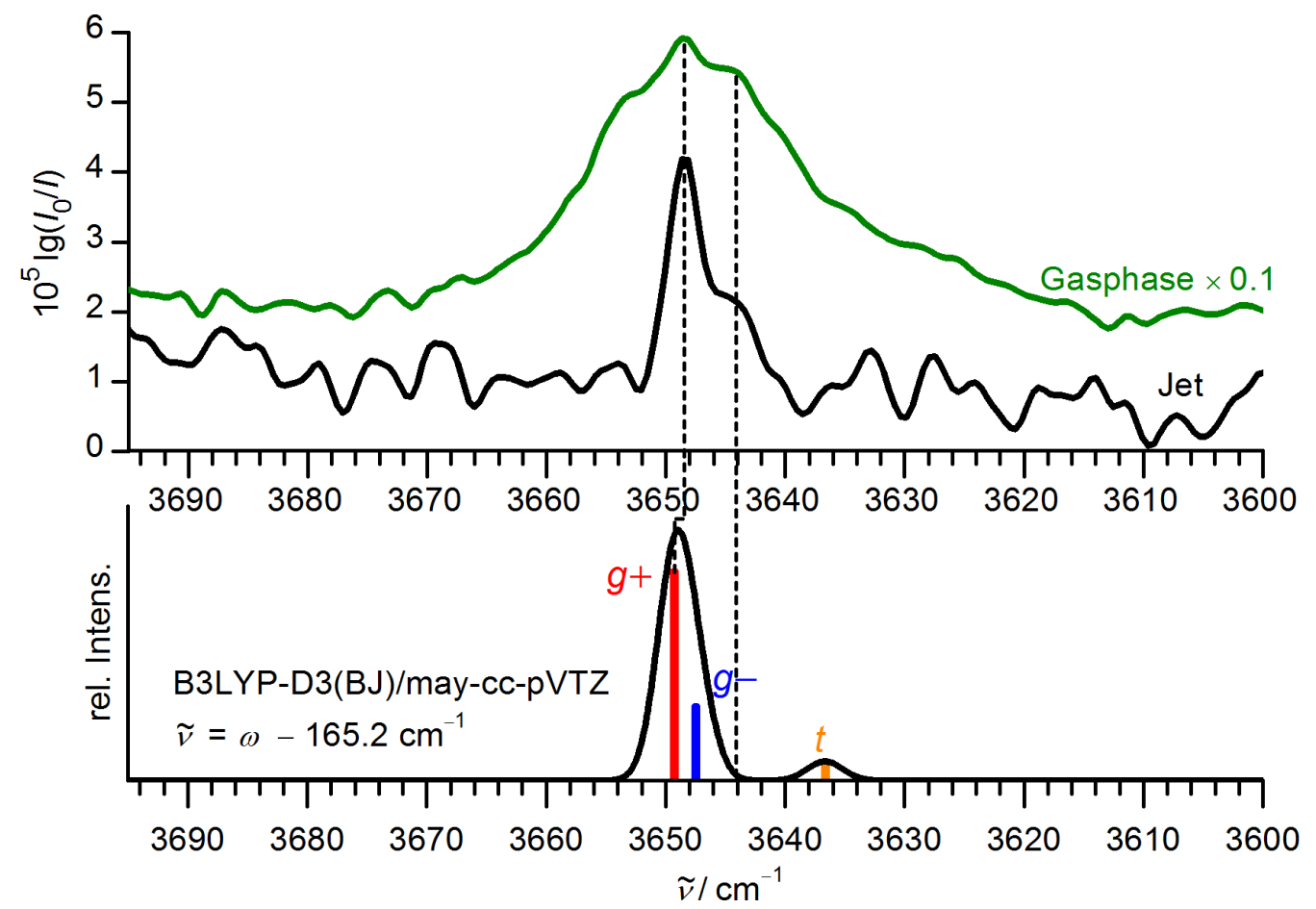

Abb. 5.8: Detailansicht des Filet-Jet-Spektrums von (-)-Isopinocampheol im Monomerbereich im Jet und in der Gasphase bei Zimmertemperatur. Simulationsparameter sind einheitliche Verschiebung der harmonischen Wellenzahlen um $\kappa=-165.2 \mathrm{~cm}^{-1}$ (aus dem Korrelationsmodell), eine Konformationstemperatur von $64 \mathrm{~K}$ und Gauss-Profile mit FWHM $=3.5 \mathrm{~cm}^{-1}$. 


\subsection{Borneol}

Borneol ist strukturell eng verwandt mit Isopinocampheol (Abb. 5.9) und ebenfalls ein chiraler, sekundärer und bicyclischer Monoterpenalkohol. Eine historische Bezeichnung für (+)-Borneol ist Borneo-Campher, was auf sein Vorkommen gemeinsam mit seinem Oxidationsprodukt Campher u. a. in Bäumen auf der Insel Borneo zurückgeht.[256] Die systematische Bezeichnung für (-)-Borneol lautet $(1 S, 2 R, 4 S)-1,7,7$ Trimethylbicyclo[2.2.1]heptan-2-ol. Durch die im Vergleich zu Isopinocampheol unterschiedliche Überbrückung ist die Bicyclo[2.2.1]heptan-Grundstruktur noch starrer: Der Sechsring ist in einer Wannenkonformation fixiert, die beiden Fünfringe in Briefumschlagkonformationen. Nach Berechnung auf B3LYP-D3(BJ)/may-cc-pVTZ-Niveau ist Borneol etwa $75 \mathrm{~kJ} \mathrm{~mol}^{-1}$ stabiler als sein Konstitutionsisomer Isopinocampheol, was auf einen erheblichen Abbau der Ringspannung hindeutet.
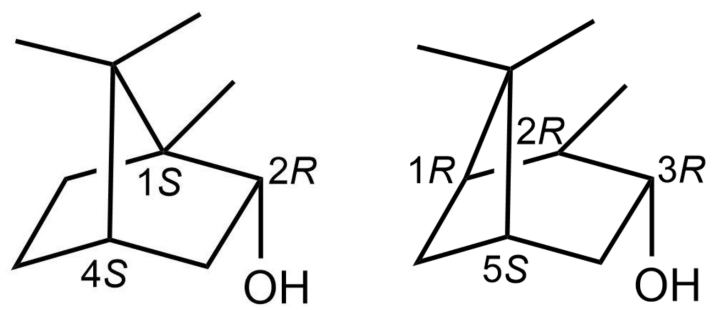

Abb. 5.9: Strukturformeln von (-)-Borneol (links) und (-)-Isopinocampheol im Vergleich mit absoluter Konfiguration der Stereozentren.

\subsubsection{Konformationsisomerie}

Im Gegensatz zu (-)-Isopinocampheol wird für (-)-Borneol die $g-$-Konformation als stabilste auf B3LYP-D3(BJ)/may-cc-pVTZ-Niveau vorhergesagt (Abb. 5.10), die Abfolgen der $\mathrm{OH}-$ Streckschwingungswellenzahlen und IR-Aktivitäten der drei Konformere bleiben dagegen erhalten. Die OH-Streckschwingungswellenzahlen sind aber um 7-21 cm ${ }^{-1}$
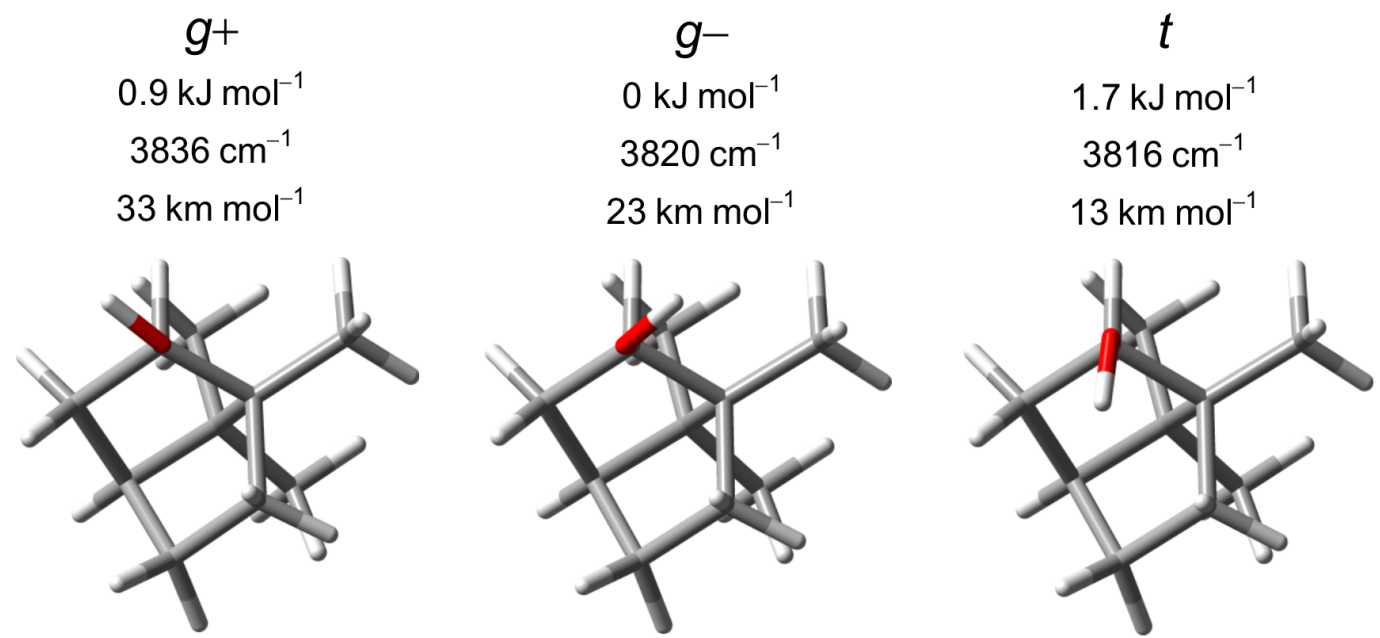

Abb. 5.10: Die drei Konformere des (-)-Borneol, klassifiziert nach der Einstellung des $\mathrm{HCOH}-$ Torsionswinkels, mit relativen Energien, OH-Streckschwingungswellenzahlen und IR-Aktivitäten auf B3LYP-D3(BJ)/may-cc-pVTZ-Niveau. Die Blickrichtung auf die Konformere erfolgt entlang der O-C-Bindung. 


\section{Torsionsisomerie von Alkoholen}

zu höheren Wellenzahlen verschoben. Weitere Rechnungen auf anderen Niveaus stiutzen diese Energie- und Frequenzabfolge der Konformere (Tab. 5.3).

Tab. 5.3: Differenzen der nullpunktskorrigierten Energien und harmonischen OH-Streckschwingungswellenzahlen der $g+$ und $g$--Konformere von (-)-Borneol verschiedenen Rechenniveaus. Die $a b$ initio-Rechnungen wurden als Punktrechnungen unter Verwendung der Geometrien und Nullpunktsschwingungsenergien von B3LYP-D3(BJ)/aug-cc-pVTZ durchgeführt.

\begin{tabular}{lcc}
\hline Methode & $\begin{array}{c}E_{0}(g-)-E_{0}(g+) \\
/ \mathrm{kJ} \mathrm{mol}\end{array}$ & $\begin{array}{c}\omega_{\mathrm{OH}}(g+)-\omega_{\mathrm{OH}}(g-) \\
/ \mathrm{cm}^{-1}\end{array}$ \\
\hline M06-2X/def2-TZVP & -0.8 & 16 \\
$\omega$ B97X-D/def2-TZVP & -1.6 & 19 \\
B3LYP-D3(BJ)/def2-TZVP & -1.1 & 16 \\
B3LYP-D3(BJ)/may-cc-pVTZ & -0.9 & 16 \\
B3LYP-D3(BJ)/aug-cc-pVTZ & -0.9 & 16 \\
B2PLYP-D3(BJ)/may-cc-pVTZ & -0.7 & 14 \\
MP2/may-cc-pVTZ & -0.2 & - \\
MP2/aug-cc-pVQZ & -0.3 & - \\
CCSD(T)/may-cc-pVTZ & -0.5 & - \\
CCSD(T)/aug-cc-pVQZ* & -0.6 & - \\
\hline
\end{tabular}

\subsubsection{Schwingungsspektren}

Die Normalsiedetemperatur $\left(212^{\circ} \mathrm{C}\right.$ [257] $)$ und der interpolierte Dampfdruck bei $24{ }^{\circ} \mathrm{C}$ (3 Pa) von Borneol sind vergleichbar mit den Werten von Isopinocampheol $\left(219^{\circ} \mathrm{C}, 4 \mathrm{~Pa}\right.$ [255]). Dagegen ist der Schmelzpunkt von Borneol mit $207^{\circ} \mathrm{C}$ [64] deutlich höher als der von Isopinocampheol mit $57^{\circ} \mathrm{C}$ [255], was auf deutliche Unterschiede in der Packung im Kristall hindeutet. Die Kristallstrukturen der beiden Verbindungen sind bisher nicht bekannt.

Borneol wurde bereits 2007 am Popcorn-Jet von Rice [250] untersucht. Abb. 5.11 zeigt die damals aufgenommenen Spektren im OH-Streckschwingungsbereich, zusammen mit einem hier neu aufgenommen am Filet-Jet. In allen vier Spektren wird eine Bande im Monomer-Bereich bei $3653 \mathrm{~cm}^{-1}$ gefunden. Im Gegensatz zum Filet-Jet-Spektrum werden in den Popcorn-Jet-Spektren auch Clusterbanden beobachtet, ermöglicht durch die höheren Konzentrationen in der geheizten Probenkammer. Auf Grundlage der steigenden Tiefverschiebung und Konzentrationsabhängigkeit wurde von Rice die Bande bei $3542 \mathrm{~cm}^{-1}$ als Dimer und die bei $3483 \mathrm{~cm}^{-1}$ als Trimer eingeschätzt. Rice verweist auf die im Vergleich zu den meisten anderen Alkoholen ungewöhnlich niedrigen Verschiebungen dieser beiden Banden relativ zum Monomer. Dies könnte auf eine adaptive Aggregation mit Wechsel von $g-$ auf das höherfrequente $g+$ im Dimer hindeuten, wie es für das gemischte Dimer von Borneol mit $\alpha$-Pinen in Kap. 6 gefunden wird. Quantenchemische Rechnungen für Dimere aus zwei Borneol-Molekülen wurden aber bisher nicht durchgeführt. Im Filet-Jet-Spektrum ist zudem die gleiche Instabilität der Basislinie wie bei Isopinocampheol im Bereich um $3500 \mathrm{~cm}^{-1}$ zu erkennen. Der Vergleich mit den höher konzentrierten Popcorn-Spektren zeigt, dass es sich hierbei eindeutig nicht um Cluster 


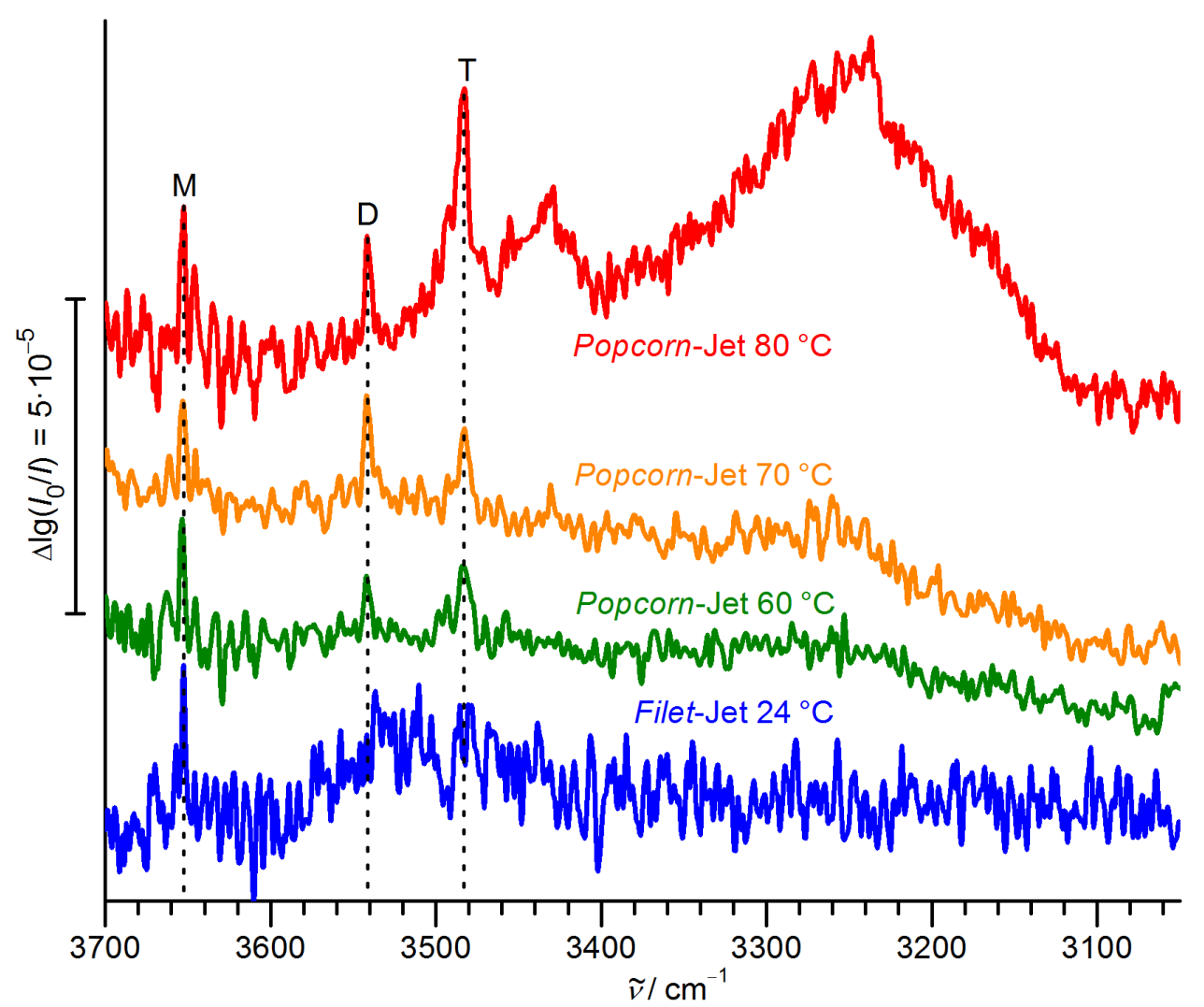

Abb. 5.11: Übersicht von Popcorn- und Filet-Jet-Spektren von (-)-Borneol im OHStreckschwingungsbereich. Angegeben sind jeweils die Substanztemperaturen. Das Spektrum bei $60{ }^{\circ} \mathrm{C}$ wurde bereits in Ref. [250] veröffentlicht, die Spektren bei 70 und $80^{\circ} \mathrm{C}$ stammen aus Archivmaterial der selben Messreihe. Die tentativen Zuordnungen zu Clustergrößen wurden aus Ref. [250] übernommen.

handelt. Abb. 5.12 zeigt eine Detailansicht der Spektren im Monomerbereich, ergänzt um ein Gasphasenspektrum bei Zimmertemperatur und einer Simulation auf B3LYP-D3(BJ)/ may-cc-pVTZ-Niveau. Die Bande bei $3653 \mathrm{~cm}^{-1}$ kann demnach dem Konformer $g-$ von (-)-Borneol zugeordnet werden. Dies entspricht einer Abweichung von $-2 \mathrm{~cm}^{-1}$ von der vom Korrelationsmodell vorhergesagten Position. Das Signal-zu-Rausch-Verhältnis ist in den Spektren der Überschallexpansionen nicht ausreichend um $g+\mathrm{zu}$ beobachten, allerdings gibt das Gasphasen-Spektrum einen klaren Hinweis darauf, dass die vorhergesagte Position von $\approx 3670 \mathrm{~cm}^{-1}$ plausibel ist. Die breite Bande der überlagerten $\mathrm{OH}$ Streckschwingungsübergänge ist in der Gasphase nahezu symmetrisch, wobei ein Maximum an der gleichen Position wie in der Überschallexpansion gefunden wird, sowie ein weiteres, gleich intensives bei $3668 \mathrm{~cm}^{-1}$. Dies stimmt mit der quantenchemischen Vorhersage überein, dass eine geringere Population von $g+$ durch die berechnete höhere IR-Aktivität bei hohen Temperaturen kompensiert werden kann. Das $t$-Konformer wird energetisch sehr hochliegend, wenig IR-aktiv und spektral sehr nahe bei $g$ - vorhergesagt, dementsprechend finden sich in keinem der Spektren Hinweise darauf.

Die Konformationsisomerie von Borneol wurde auch zuvor bereits mehrfach quantenchemisch untersucht.[258-260] Die Energieabfolge der drei Konformere stimmt in allen Rechnungen mit der hier gefundenen überein, mit der Ausnahme von solchen auf CBSQ-Niveau,[260] mit denen unrealistisch große Energieunterschiede vorhersagt wurden. 


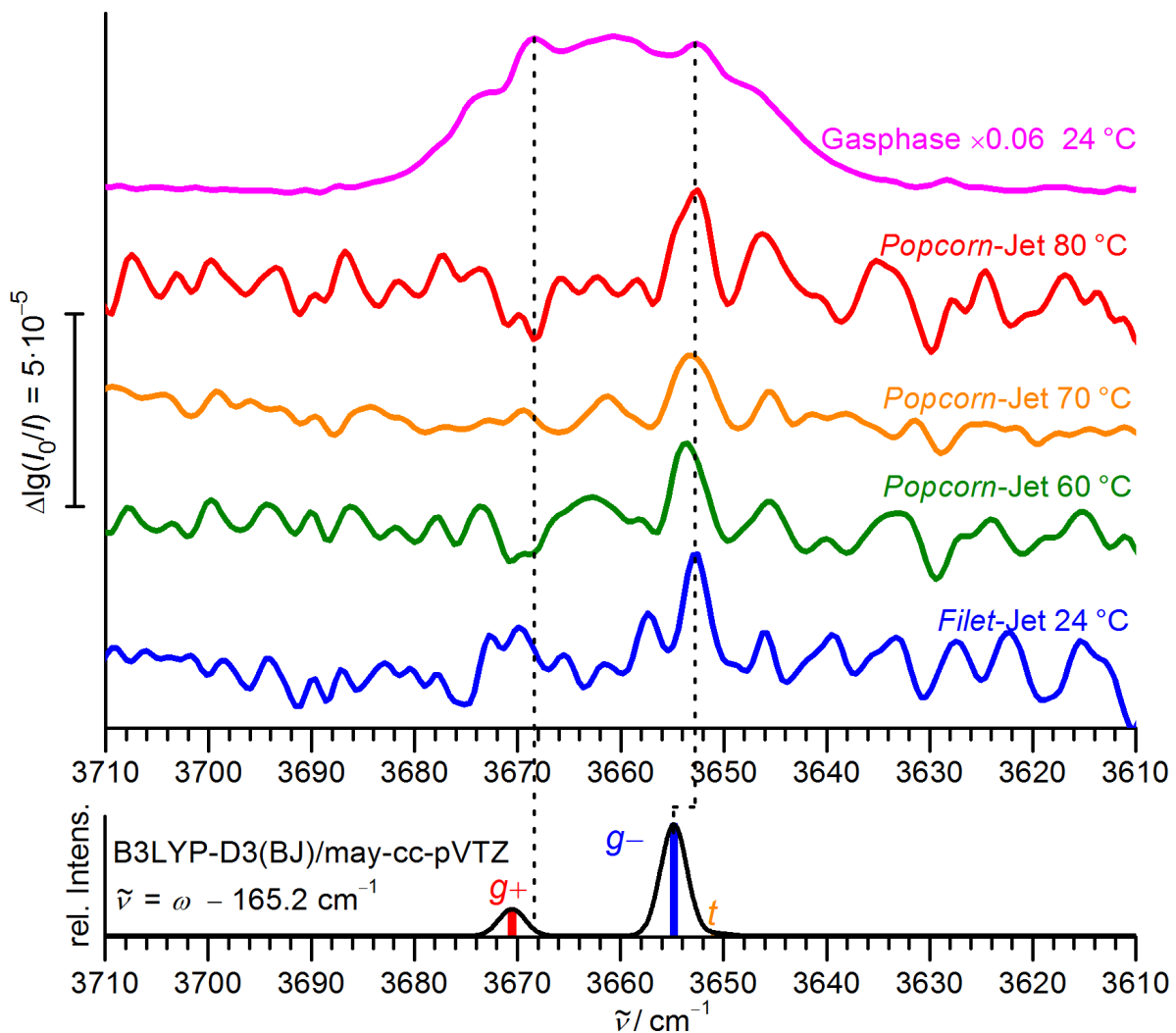

Abb. 5.12: Detailansicht von Popcorn- und Filet-Jet-Spektren von (-)-Borneol im Monomerbereich im Jet und in der Gasphase bei Zimmertemperatur. Angegeben sind jeweils die Substanztemperaturen. Das Spektrum bei $60^{\circ} \mathrm{C}$ wurde bereits in Ref. [250] veröffentlicht, die Spektren bei 70 und $80^{\circ} \mathrm{C}$ stammen aus Archivmaterial der selben Messreihe. Simulationsparameter sind einheitliche Verschiebung der harmonischen Wellenzahlen um $\kappa=-165.2 \mathrm{~cm}^{-1}$, eine Konformationstemperatur von $64 \mathrm{~K}$ und Gauss-Profile mit FWHM $=3 \mathrm{~cm}^{-1}$.

In Ref. [260] wurde mit Photoelektronen-Circulardichroismus gefunden, dass in einer Helium-Überschallexpansion die beste Übereinstimmung zwischen experimentellen und simulierten Spektren erreicht wird, wenn die gleiche Population für alle drei Konformere angenommen wird. Dies steht im klaren Widerspruch zu dem hier präsentierten FTIRspektroskopischen Befund und den übereinstimmenden quantenchemischen Rechnungen auf verschiedenen Niveaus, gemäß denen wegen der relativ großen Energieunterschiede unter Jet-Bedingungen $g$ - dominieren sollte. 


\section{$5.8 \alpha$-Fenchol - Teil 1}

$\alpha$-Fenchol, auch endo-Fenchylalkohol genannt, ist, wie die zuvor diskutierten Verbindungen Isopinocampheol und Borneol, ein chiraler, sekundärer und bicyclischer Monoterpenalkohol. $\alpha$-Fenchol und sein Oxidationsprodukt Fenchon finden sich als Naturstoffe in vielen Pflanzen, besonders im namensgebenden Fenchel.[75, 261] $(+)-\alpha$-Fenchol hat Bedeutung in der Parfümindustrie,[261] hat aber konzentriert einen ,sehr durchdringenden und belästigenden Geruch" (Zitat von Otto Wallach in der Erstbeschreibung 1891 [75]). Der systematische Name des (-)-Enantiomers lautet ( $1 S, 2 S, 4 R)-1,3,3$-Trimethylbicyclo[2.2.1] heptan-2-ol. Von seinem Konstitutionsisomer Borneol unterscheidet es sich nur in der Position von zwei Methylgruppen, die sich bei $\alpha$-Fenchol in 3-Position, bei Borneol dagegen in 7-Position befinden (Abb. 5.13). Nach Berechnung auf B3LYP-D3(BJ)/ may-cc-pVTZ-Niveau sind $\alpha$-Fenchol und Borneol vergleichbar stabile Konstitutionsisomere, im Gegensatz zum deutlich höherenergetischen Isopinocampheol. Auch wenn experimentell das rechtsdrehende Enantiomer verwendet wurde, wird hier, für die bessere Vergleichbarkeit mit den anderen beiden Terpenalkoholen, $(-)-\alpha$-Fenchol betrachtet.
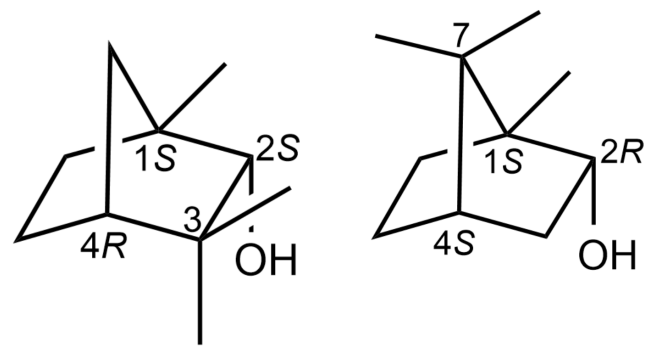

Abb. 5.13: Strukturformeln von (-)- $\alpha$-Fenchol (links) und (-)-Borneol im Vergleich mit absoluter Konfiguration der Stereozentren.

\subsubsection{Konformationsisomerie}

$\alpha$-Fenchol weist das gleiche starre und asymmetrische Grundgerüst wie Borneol auf, daher sind ebenfalls drei Konformere bezüglich der C-O-Torsion zu erwarten. Diese sind in Abb. 5.14 mit ihren wichtigsten spektralen Eigenschaften gezeigt. Die energetische und spektrale Reihenfolge entspricht der von Isopinocampheol, allerdings werden die $\mathrm{OH}$ Streckschwingungen, ähnlich wie Borneol, bei deutlich höheren Wellenzahlen vorhergesagt.

\subsubsection{Schwingungsspektren}

$\alpha$-Fenchol liegt bei Zimmertemperatur als ,prachtvoll aussehende, bei 40 bis $41^{\circ}$ schmelzende weise Krystallmasse“ (Zitat Otto Wallach 1891 [75]) vor. Die Normalsiedetemperatur beträgt $201{ }^{\circ} \mathrm{C}$ und der extrapolierte Sublimationsdruck bei $24^{\circ} \mathrm{C}$ ist mit $21 \mathrm{~Pa}$ deutlicher höher als der von Borneol (3 Pa) und Isopinocampheol (4 Pa) (Details in Kap. 3.1.1). Dies erlaubt auch ohne Heizung am Filet-Jet die Beobachtung von Dimeren, wie die Übersichtsspektren für den OH-Streckschwingungsbereich in Abb. 5.15 zeigen. Bei $3499 \mathrm{~cm}^{-1}$ findet sich eine intensive, sowie bei $3494 \mathrm{~cm}^{-1}$ und $3485 \mathrm{~cm}^{-1}$ zwei weniger intensive Banden. Aufgrund ihrer spektralen Lage und ihrer stärkeren Skalierung mit 


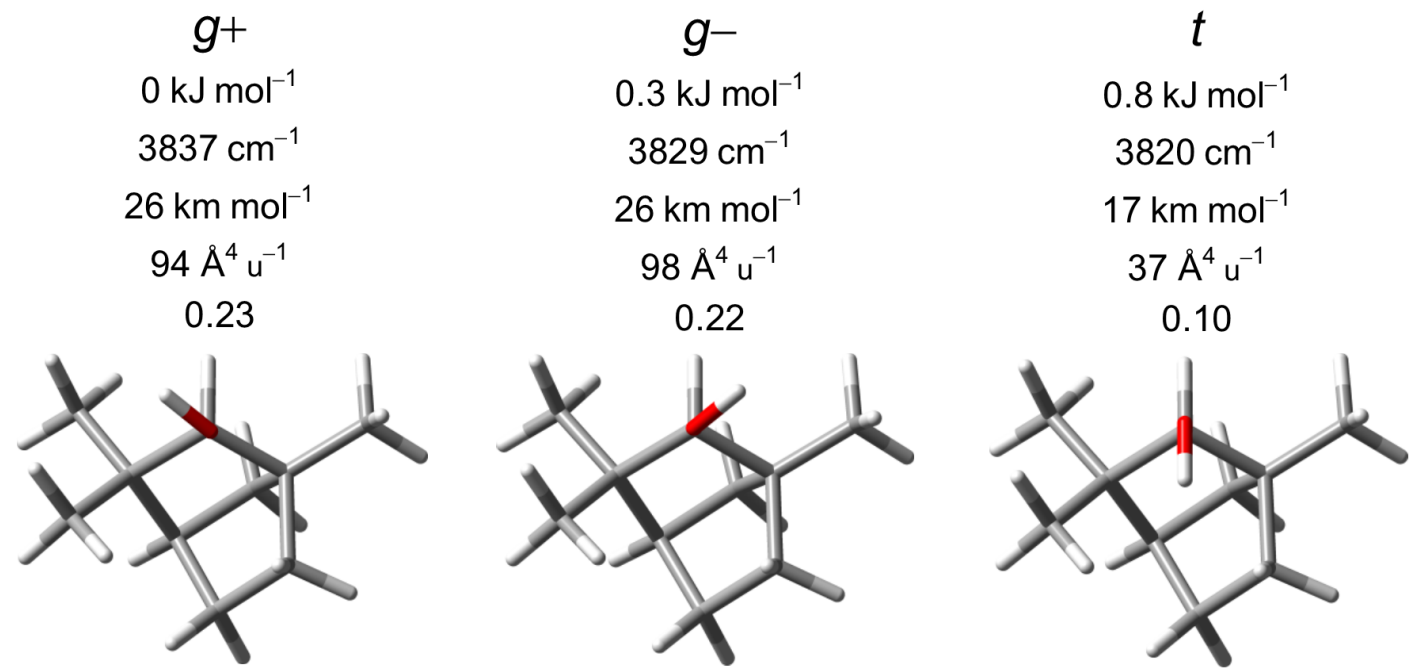

Abb. 5.14: Die drei Konformere des (-)- $\alpha$-Fenchols, klassifiziert nach der Einstellung des $\mathrm{HCOH}-T o r s i o n s w i n k e l s$, mit relativen nullpunktskorrigierten Energien, OHStreckschwingungswellenzahlen, IR-Aktivitäten, Raman-Aktivitäten und Depolarisationsverhältnissen auf B3LYP-D3(BJ)/may-cc-pVTZ-Niveau. Die Blickrichtung auf die Konformere erfolgt entlang der $\mathrm{O}-\mathrm{C}$-Bindung.

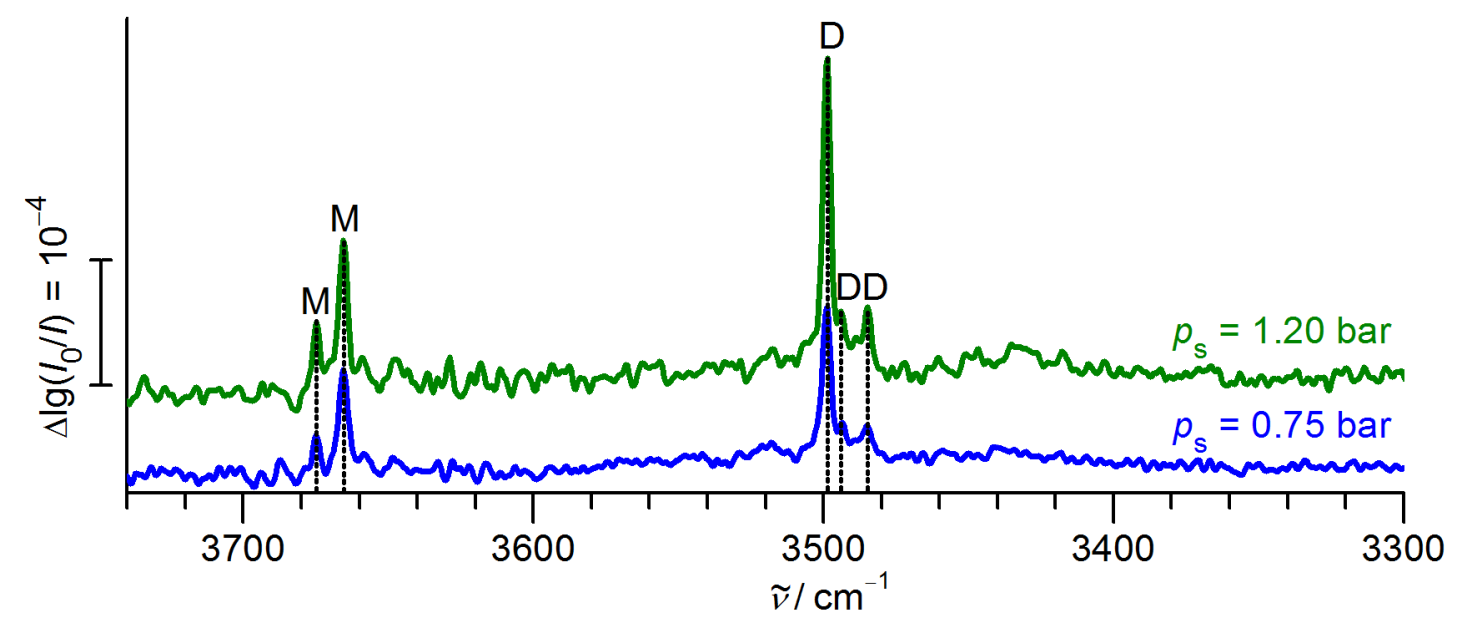

Abb. 5.15: Übersicht der Filet-Jet-Spektren von (+)- $\alpha$-Fenchol im OH-Streckschwingungsbereich bei zwei verschiedenen Stagnationsdrücken mit tentativen Bandenzuordnungen.

dem Stagnationsdruck sollte es sich um drei Dimer-Donorbanden handeln. Diese werden ausführlich in Kap. 7 behandelt. Bei $3675 \mathrm{~cm}^{-1}$ und $3665.5 \mathrm{~cm}^{-1}$ werden zwei Banden beobachtet, die Monomeren zugeordnet werden können. Eine Detailansicht des Monomerbereichs, ergänzt um ein Gasphasenspektrum und eine Simulation, bietet die obere Hälfte von Abb. 5.16. Die beiden Monomerbanden wären in ihren Positionen mit den Vorhersagen für $g+$ und $g$ - aus dem Korrelationsmodel innerhalb von $2-3 \mathrm{~cm}^{-1}$ kompatibel, allerdings ist das Intensitätsverhältnis umgekehrt. Da der berechnete Energievorteil von $g+$ nur $0.3 \mathrm{~kJ} \mathrm{~mol}^{-1}$ beträgt, wäre eine qualitativ falsche Vorhersage für die Energieabfolge von B3LYP-D3(BJ)/may-cc-pVTZ als Erklärung für die Diskrepanz am naheliegendsten. Das Verhältnis der beiden Banden verändert sich nicht bei Beimischung von 1\% Argon 


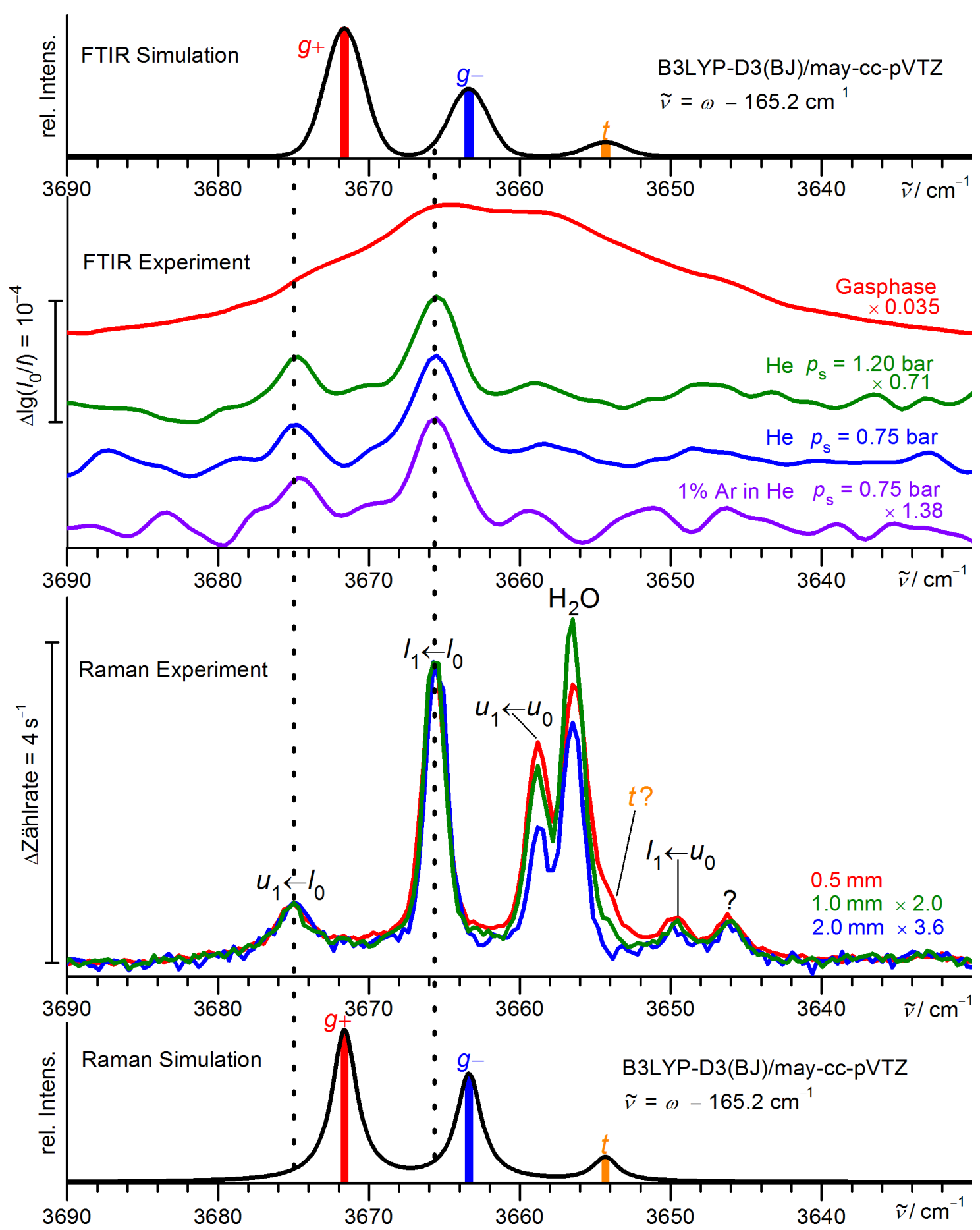

Abb. 5.16: Obere Hälfte: Filet-Jet-Spektren von $\alpha$-Fenchol im Monomerbereich im Jet bei verschiedenen Stagnationsdrücken und mit Argon-Beimischung ${ }^{\mathrm{b}}$ und in der Gasphase bei Zimmertemperatur, im Vergleich mit einer Simulation. Untere Hälfte: Curry-Jet-Spektren von $\alpha$-Fenchol im Monomerbereich bei verschiedenen Detektionsabständen mit tentativen Bandenzuordnungen (für Erläuterungen der Kürzel siehe Fließtext auf S. 133). Die Spektren wurden mit den angegebenen Faktoren auf die gleiche Maximalintensität der Bande bei $3665.5 \mathrm{~cm}^{-1}$ skaliert. Simulationsparameter sind einheitliche Verschiebung der harmonischen Wellenzahlen um $\kappa=-165.2 \mathrm{~cm}^{-1}$, eine Konformationstemperatur von $64 \mathrm{~K}$ für das IR-Spektrum und $100 \mathrm{~K}$ für das Raman-Spektrum, Gauss-Profile mit FWHM $=3 \mathrm{~cm}^{-1}$ für das IR-Spektrum und Lorentz-Profile mit FWHM $=2 \mathrm{~cm}^{-1}$ für das Raman-Spektrum. 


\section{Torsionsisomerie von Alkoholen}

in Helium ${ }^{\mathrm{a}}$, was in der Tat auf einen sehr kleinen Energieunterschied hindeutet. Alternativ müssten bezüglich der relativen harmonischen $\mathrm{OH}$-Streckschwingungswellenzahlen oder der IR-Intensitäten grob falsche Vorhersagen vorliegen, um die Diskrepanz erklären zu können. Dies erscheint deutlich unwahrscheinlicher, insbesondere in Hinblick auf die guten Vorhersagen der Methode für Isopinocampheol und Borneol.

Ungewöhnlich ist das IR-Gasphasenspektrum bei Zimmertemperatur. Der Schwerpunkt der breiten Bande ist im Vergleich zu den beiden Signalen in der Überschallexpansion deutlich zu niedrigeren Wellenzahlen verschoben. Im Bereich um $3654 \mathrm{~cm}^{-1}$ wäre zwar nach dem Korrelationsmodell noch die Absorption des hochenergetischen $t$ Konformers zu erwarten, welches bei Zimmertemperatur etwas stärker populiert sein sollte. Allerdings kann dieses auch wegen seiner vorhergesagten geringen IR-Aktivität nicht für eine solch drastische Verschiebung des Bandenschwerpunktes verantwortlich sein.

$\alpha$-Fenchol wurde kürzlich auch von zwei Arbeitsgruppen, denen um Huet [262] und Pate [263], unabhängig voneinander rotationsspektroskopisch untersucht. Diese haben in kälteren Expansionen mit Neon als Trägergas nur das $g+$-Konformer zugeordnet. Dies würde dafür sprechen, dass die quantenchemische Vorhersage von $g+$ als stabileres Konformer korrekt ist.

Um den Widerspruch zum FTIR-spektroskopischen Befund weiter zu untersuchen, wurden zunächst die drei Konformere auch auf anderen Rechenniveaus verglichen. Die wichtigsten Ergebnisse für die Unterschiede von $g+$ und $g-$ sind in Tab. 5.4 zusammengestellt. Es lässt sich erkennen, dass der leichte Energievorteil von $g+$ stabil über sämtliche getesteten Rechenniveaus vorhergesagt wird, allerdings mit tendenziell höherem Niveau fast verschwindet. Auch die $\mathrm{OH}-$ Streckschwingungswellenzahl von $g+$ ist auf allen ge-

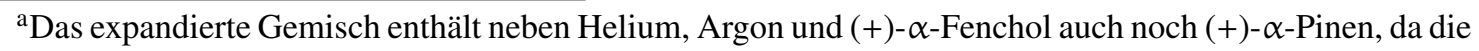
Messung im Kontext der Chiralitätserkennung durchgeführt wurde (Kap. 6). Die Präsenz von $\alpha$-Pinen sollte aber entweder keinen, oder einen ebenfalls leicht kühlenden Einfluss auf das Konformerengleichgewicht des Monomers haben.

Tab. 5.4: Differenzen der nullpunktskorrigierten Energien und harmonische OH-Streckschwingungswellenzahlen der $g+$ und $g-$-Konformere von $(-)-\alpha$-Fenchol auf verschiedenen Rechenniveaus. Die ab initio-Rechnungen wurden als Punktrechnungen unter Verwendung der Geometrien und Nullpunktsschwingungsenergien von B3LYP-D3(BJ)/aug-cc-pVTZ durchgeführt.

\begin{tabular}{lcc}
\hline Methode & $\begin{array}{c}E_{0}(g-)-E_{0}(g+) \\
/ \mathrm{kJ} \mathrm{mol}\end{array}$ & $\begin{array}{c}\omega_{\mathrm{OH}}(g+)-\omega_{\mathrm{OH}}(g-) \\
/ \mathrm{cm}^{-1}\end{array}$ \\
\hline M06-2X/def2-TZVP & 0.35 & 4 \\
$\omega$ B97X-D/def2-TZVP & 0.50 & 8 \\
B3LYP-D3(BJ)/def2-TZVP & 0.55 & 8 \\
B3LYP-D3(BJ)/may-cc-pVTZ & 0.33 & 8 \\
B3LYP-D3(BJ)/aug-cc-pVTZ & 0.33 & 8 \\
B2PLYP-D3(BJ)/may-cc-pVTZ & 0.22 & 8 \\
MP2/may-cc-pVTZ & 0.12 & - \\
MP2/aug-cc-pVQZ & 0.01 & - \\
CCSD(T)/may-cc-pVTZ & 0.17 & - \\
CCSD(T)/aug-cc-pVQZ* & 0.06 & - \\
\hline
\end{tabular}


testeten Niveaus höher als von $g-$. Die fast identischen IR-Aktivitäten von $g+$ und $g-$, sowie die höhere Energie, geringere $\mathrm{OH}-$ Streckschwingungswellenzahl und geringere IRAktivität von $t$ finden sich ebenfalls auf allen Niveaus wieder (Werte nicht aufgeführt).

Diese Energieabfolge, mit ebenfalls geringen Abständen zwischen $g+$ und $g-$, wurde auch auf MP2-Niveau mit verschiedenen Basissätzen von Neeman und Huet [262] gefunden. Nur auf MP2/aug-cc-pVTZ-Niveau wurde eine Umkehr der Energieabfolge festgestellt, allerdings beinhalten diese Rechnungen keine Schwingungsnullpunktskorrektur. Eine solche Umkehr der energetischen Präferenz durch die Korrektur wird auch in der vorliegenden Arbeiten mit ab initio-Punktrechnungen gefunden, da die elektronische Differenz so gering sind, dass die um $0.15-0.20 \mathrm{~kJ} \mathrm{~mol}^{-1}$ (abhängig vom DFT-Funktional) höhere Schwingungsnullpunktsenergie von $g-$ den Ausschlag gibt. Eine qualitative Fehlvorhersage von B3LYP-D3(BJ)/may-cc-pVTZ bezüglich der relativen Energien, Wellenzahlen oder IR-Intensitäten scheint daher als Erklärung des Widerspruches auszuscheiden.

Zur weiteren Untersuchung wurden Raman-Spektren am Curry-Jet bei verschiedenen Düsenabständen aufgenommen, um die Auflösung und Empfindlichkeit zu erhöhen, sowie um die Konformationstemperatur einfacher variieren zu können. Die Ergebnisse sind im unteren Teil von Abb. 5.16 zusammengestellt. Alle drei Spektren wurden auf die gleiche maximale Intensität der intensiven Bande bei $3665.5 \mathrm{~cm}^{-1}$ skaliert, um die relative Intensitätsentwicklung analysieren zu können. Die aus den IR-Spektren bekannten Banden bei 3675 und $3665.5 \mathrm{~cm}^{-1}$ sind auch in den Raman-Spektren vorhanden, dabei ist die niederfrequente der beiden noch dominanter. Die quantenchemische Vorhersage lautet, dass auch die Raman-Aktivitäten der beiden Konformeren sehr ähnlich sind. Durch die tendenziell höhere Konformationstemperatur in der Expansion des Curry-Jets wäre daher eher stattdessen ein Angleichen der Intensitäten zu erwarten gewesen. Ferner zeigt das Intensitätsverhältnis der beiden Banden keinerlei Abhängigkeit vom Abstand zwischen Düse und Detektionsbereich. Falls aber der deutliche Intensitätsunterschied zwischen beiden Banden auf eine Energiedifferenz zwischen zwei Konformeren zurückzuführen sein sollte, sollte dieser empfindlich auf die Expansionsbedingungen reagieren. Dies wird nicht beobachtet. Weitere Banden werden im Raman-Spektrum bei niedrigeren Wellenzahlen gefunden. Ursachen hierfür sind in der vermutlich höheren Konformationstemperatur, dem besseren Signal-zu-Rauschverhältnis und eventuell in unterschiedlichen Verhältnissen in den Raman- und IR-Aktivitäten der Übergänge zu vermuten. Die Bande bei $3659 \mathrm{~cm}^{-1}$ zeigt eine deutliche, monotone Abhängigkeit vom Detektionsabstand. Bei der benachbarten Bande bei $3656.5 \mathrm{~cm}^{-1}$ handelt es sich um die symmetrische Streckschwingung einer Wasserverunreinigung [238], die in ihrer relativen Intensität schwankt, da das Wasser nicht (nur) aus der Probe stammt. Zwei weitere schwache Banden befinden sich bei 3649.5 und $3646 \mathrm{~cm}^{-1}$, deren Skalierungen mit dem Detektionsabstand wegen ihrer geringen Intensitäten schwieriger zu beurteilen sind. Tendenziell scheinen diese Banden aber ebenfalls mit steigender Konformationstemperatur an relativer Intensität zu gewinnen. Diese drei niederfrequenten Banden und ihre Temperaturabhängigkeit erklärt die Verschiebung des Schwerpunktes im IR-Gasphasenspektrum bei Zimmertemperatur. Ein weiterer mit dem Detektionsabstand variierender Spektralbereich ist die niederfrequente Flanke der Wasserbande um $3654 \mathrm{~cm}^{-1}$. In diesem Bereich könnte eine weitere Bande von $\alpha$-Fenchol verdeckt sein - gemäß dem Korrelationsmodell wäre dort das $t$ Konformer zu erwarten. Um diese Möglichkeit näher zu untersuchen, wurden unter sonst 
identischen Bedingungen Expansionen von (formal) reinem Helium durchgeführt, in denen die Wasserverunreinigung ebenfalls detektierbar ist. Diese Spektren sind in Abb. 5.17 gezeigt.

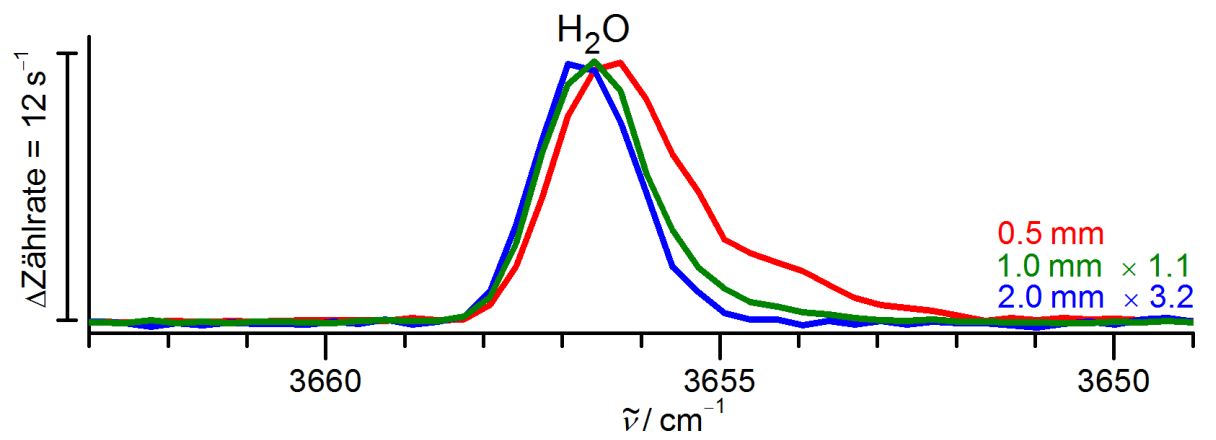

Abb. 5.17: Raman-Spektren der symmetrischen Streckschwingung von Wasser in Abhängigkeit vom Detektionsabstand.

Es ist zu erkennen, dass sich die Wasserbande mit geringerem Detektionsabstand geringfügig zu niedrigeren Wellenzahlen verschiebt und vor allem eine niederfrequente Flanke entwickelt. Dies ist auf die unterschiedliche Rotationsanregung des Wassermoleküls und damit Veränderungen im Q-Zweig zurückzuführen. Dieser Effekt kann auch die in den $\alpha$-Fenchol-Spektren beobachtete Flanke größtenteils erklären. Weitergehende Versuche mit Differenzspektren ergaben keine eindeutige Ergebnisse - es kann weder ausgeschlossen noch bestätigt werden, dass die Wasserbande möglicherweise eine weitere schwache Bande von $\alpha$-Fenchol verdeckt.

Es wird klar, dass, im Gegensatz zu Isopinocampheol und Borneol, das Spektrum von $\alpha$-Fenchol schon allein aufgrund der Zahl der beobachteten Banden nicht durch Rechnungen in harmonischer Näherung erklärt werden kann. Ein anharmonischer Effekt, der zu einer Erhöhung der Zahl an Banden führen könnte, wäre eine spektrale Tunnelaufspaltung. Ein spektrales Indiz hierfür ist ein auffälliges Muster, welches zwischen vier der beobachteten Banden existiert: Jeweils zwei Bandenpaare sind in doppelter Weise durch gleiche Wellenzahldifferenzen $\left(9.5\right.$ und $16.0 \mathrm{~cm}^{-1}$ ) voneinander getrennt. Dies ist in Abb. 5.18 illustriert. Eine mögliche Interpretation dieser vier Banden ist sie vier der sechs Übergangsmöglichkeiten in einem Vier-Niveau-System zuzuordnen. Die sich wiederholenden Abstände zwischen Übergängen entsprechen dann nach der Methode der Kombinationsdifferenzen den Abständen zwischen Zuständen, die an Übergängen mit jeweils gleichem Ausgangs- oder Endniveau beteiligt sind. Die Bande höchster Frequenz ist demnach dem Übergang zwischen dem untersten und dem obersten der vier Niveaus zuzuordnen. Die mit der niedrigsten Frequenz entspricht dem Übergang zwischen den beiden mittleren Niveaus. Die beiden Übergänge mittlerer Frequenz finden zwischen dem untersten und dem zweithöchsten, sowie zwischen dem zweituntersten und dem höchsten Niveau statt. Eine Zuordnung der jeweiligen Bande zu diesen beiden Alternativen bietet die Temperaturabhängigkeit: Die Bande bei $3659.0 \mathrm{~cm}^{-1}$ (wie auch die bei $3649.5 \mathrm{~cm}^{-1}$ ) gewinnt mit steigender Expansionstemperatur an relativer Intensität, da deren Ursprungsniveau thermisch populiert wird. Die zwei verbleibenden Übergangsmöglichkeiten zwischen dem untersten und zweituntersten, sowie zwischen dem zweithöchsten und höchsten Niveau würden bei einer solchen Zuordnung weit außerhalb des untersuchten Spektralbereichs bei 9.5 und $16.0 \mathrm{~cm}^{-1}$ liegen. Übergänge mit derart kleinen Stokes-Shift sind aufgrund der Nähe zur 


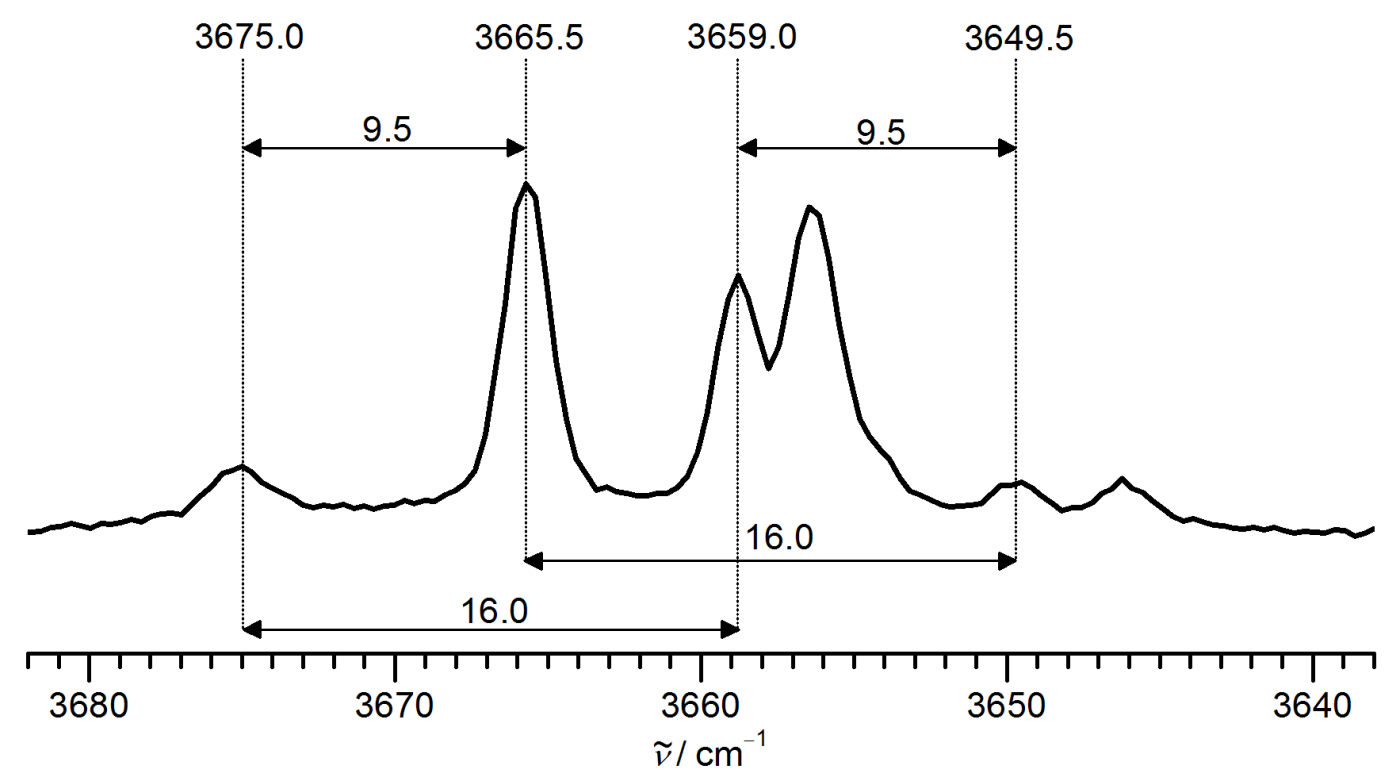

Abb. 5.18: Spektrales Muster mit sich wiederholenden Abständen im OHStreckschwingungsbereich von $\alpha$-Fenchol. Das gezeigte Spektrum entspricht dem bei $0.5 \mathrm{~mm}$ Detektionsabstand in Abb. 5.16.

Rayleigh-Linie mit dem Curry-Jet nicht zugänglich. Das sich aus diesen Überlegungen ergebende Energieniveauschema ist in Abb. 5.19 dargestellt.

Eine Erklärung für die vier Zustände und die dazwischen erlaubten Übergänge würde die Annahme einer Delokalisation des Hydroxyprotons zwischen den $g+-$ und der $g--$ Konformeren von $(-)-\alpha$-Fenchol bieten. In symmetrischen Alkoholen, bei denen $g+$ und $g$ - enantiomer zueinander sind, führt die jeweilige Tunneldynamik im Grund- und angeregten Zustand der OH-Streckschwingung ebenso zu vier Zuständen. Diese werden phänomenologisch nach ihrer Energieabfolge als $l$ (lower) und $u$ (upper) bezeichnet, getrennt nach der jeweiligen Quantenzahl der OH-Streckschwingung (0 bzw. 1), die als Index angegeben ist. Im asymmetrischen Fall von $\alpha$-Fenchol sind auch ohne Tunnelaufspaltung bereits vier Zustände vorhanden, allerdings ist normalerweise kein Übergang zwischen unterschiedlichen Konformeren erlaubt, da deren Wellenfunktionen keine Überlappung aufweisen (schneller ,senkrechter“ Übergang bei konstanten Kernabständen). Eine Delokalisation des Protons durch Tunneln zwischen beiden Konformeren, sei es im Grundzu-

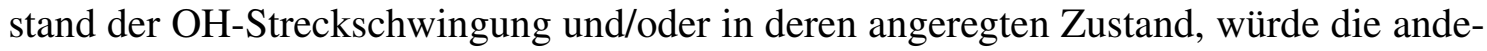
ren Übergänge ermöglichen. Die Wellenfunktionen der delokalisierten Zustände können näherungsweise als Linearkombinationen der lokalisierten $g+-$ und $g$--Zustände konstruiert werden, wobei wegen der Asymmetrie die Koeffizienten nicht identisch sein müssen.[264] Die Annahme einer annähernden aber nicht vollständigen Symmetrie könnte auch eine Erklärung für die beobachteten relativen Intensitäten sein: die Übergänge $l_{1} \leftarrow l_{0}$ und $u_{1} \leftarrow u_{0}$, bei denen die (Pseudo-)Symmetrie der Wellenfunktionen erhalten bleibt, sind deutlich intensiver als die Übergänge $u_{1} \leftarrow l_{0}$ und $l_{1} \leftarrow u_{0}$, bei denen dies nicht der Fall ist. [265] Eine alternative Sichtweise ist die Einstufung der weniger intensiven Banden als Kombinationsübergänge, da mit der Anregung der $\mathrm{OH}-\mathrm{Streckschwingung}$ auch die $\mathrm{OH}$-Torsion an- oder abgeregt wird. Dass das Verhältnis der Intensitäten der beiden hochfrequenten Übergänge $l_{1} \leftarrow l_{0}$ und $u_{1} \leftarrow l_{0}$ bei Infrarot-Detektion größer ist als bei Raman-Detektion, könnte auf eine begrenzte symmetriebedingte Komplementarität 


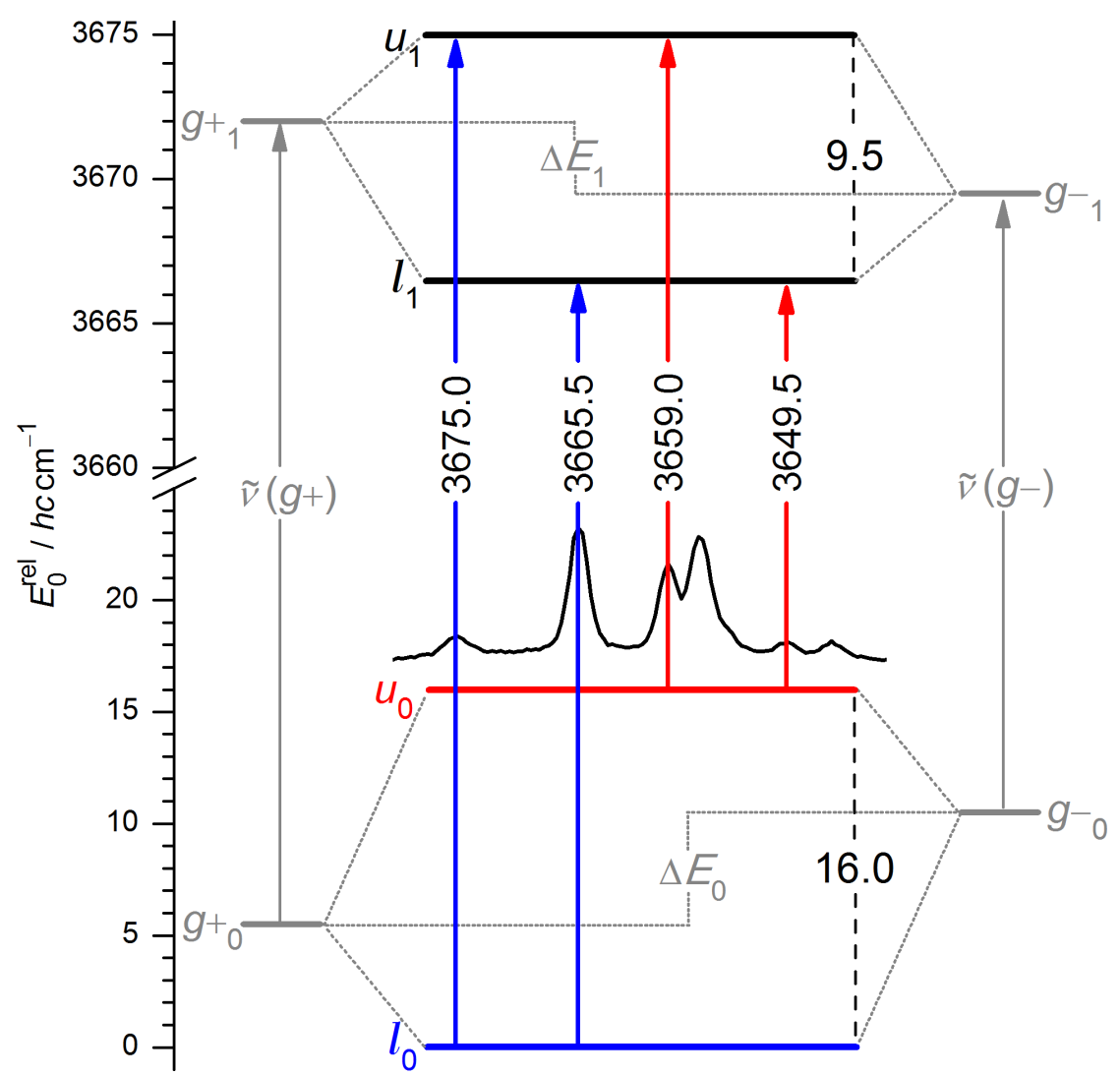

Abb. 5.19: Energieniveauschema für $\mathrm{OH}-$ Streckschwingungsübergänge im gaucheDoppelminimumpotential von $(-)-\alpha$-Fenchol. Der Grundzustand $l_{0}$ und daraus stattfindende Übergänge sind blau dargestellt, der erste angeregte Zustand $u_{0}$ und daraus stattfindende heiße Übergänge sind rot markiert. Nicht beobachtbare Modellgrößen für die Erklärung der Zustände sind in grau gehalten, die dafür verwendeten Rechenergebnisse, $\Delta E_{0}=\left(g-{ }_{0}\right)-\left(g+_{0}\right)=h c 5 \mathrm{~cm}^{-1}$ und $\omega(g+)-\omega(g-)=8 \mathrm{~cm}^{-1} \approx \tilde{v}(g+)-\tilde{v}(g-)$, wurden auf CCSD(T)/aug-cc-pVQZ*//B3LYPD3(BJ)/aug-cc-pVTZ-Niveau berechnet. Der Index 0 bzw. 1 gibt die Quantenzahl der $\mathrm{OH}-$ Streckschwingung an.

der Methoden hinweisen, oder alternativ auch in unterschiedlich hohen $g+$ und $g-$ Anteilen der Wellenfunktionen (und damit auch abweichenden IR- und Raman-Aktivitäten der Übergänge) begründet sein.

Ein Indiz, welches eher gegen eine solche Tunnel-Interpretation der Übergänge sprechen würde, sind die Schätzwerte aus dem Korrelationsmodell für die Wellenzahlen der Übergänge zwischen den lokalisierten Konformeren. Unabhängig vom Energieunterschied zwischen $g+$ und $g-$ im Schwingungsgrundzustand und der Größe der Tunnelaufspaltung sollten die Schwerpunkte der Übergänge gemäß Gleichung 5.15 zusammenfallen:

$$
\begin{aligned}
\frac{1}{4}\left[\tilde{v}\left(u_{1} \leftarrow l_{0}\right)+\tilde{v}\left(l_{1} \leftarrow l_{0}\right)+\tilde{v}\left(u_{1}\right.\right. & \left.\left.\leftarrow u_{0}\right)+\tilde{v}\left(l_{1} \leftarrow u_{0}\right)\right] \\
& \stackrel{!}{=} \frac{1}{2}[\tilde{v}(g-)+\tilde{v}(g+)]
\end{aligned}
$$


Werden für den linken Term der Gleichung die entsprechend zugeordneten Bandenpositionen eingesetzt, so wird $3662.3 \mathrm{~cm}^{-1}$ erhalten, während der Schätzwert aus dem Korrelationsmodell für den Term auf der rechten Seite $3667.5 \mathrm{~cm}^{-1}$ beträgt, also $5.2 \mathrm{~cm}^{-1}$ größer. Auch die experimentellen Wellenzahlen der zugeordneten Isopinocampheolund Borneol-Konformere wurden ebenfalls überschätzt, allerdings im geringeren Maße (um 1-2 $\mathrm{cm}^{-1}$ ). Das größte Residuum im Trainingsset des Korrelationsmodells beträgt $+3.7 \mathrm{~cm}^{-1}$ (Neomenthol). Es ist unklar ob diese größere Diskrepanz aus einer falschen Zuordnung dieser Banden, aus einer Schwäche in der Berechnung der harmonischen Wellenzahlen von B3LYP für dieses System, oder einer ungewöhnlichen lokalen Anharmonizität von $\alpha$-Fenchol resultiert.

Eine Tunnelaufspaltung (und dazu in dieser erheblichen Größe) erscheint auch angesichts der Molekülstruktur von $\alpha$-Fenchol zunächst als eine höchst unwahrscheinliche Hypothese. Denn Tunnelaufspaltungen finden in aller Regel, mit nur wenigen bekannten Ausnahmen, durch Interaktion von symmetrieäquivalenten Zuständen statt. Für $\alpha$ Fenchol kann bestenfalls eine lokale $C_{\mathrm{s}}$-Symmetrie im Übergangszustand zwischen $g+$ und $g-$ angenommen werden. Im Folgenden wird anhand verschiedener Indizien gezeigt, dass dennoch einiges für diese Hypothese spricht.

Für den Großteil der symmetrischen molekularen Systeme wird gefunden, dass das Einbringen schon kleiner Asymmetrien in der Regel Tunnelaufspaltungen löscht. Dazu zählen asymmetrische Substitutionen des Moleküls [166, 266-271], einschließlich Isotopensubstitutionen [12, 167, 272-278], interne Rotation von Molekülgruppen [12, 166, 276] und auch asymmetrische Umgebungen wie eine Edelgasmatrix [168, 265, 272], die Kristallstruktur [279] oder die flüssige Phase [166]. In der besonders schwach wechselwirkenden Umgebung von Helium-Nanotröpfchen wird keine Löschung, aber eine Verringerung von Tunnelaufspaltungen, sowie Veränderungen von relativen Bandenintensitäten beobachtet.[280, 281] Als Ursache hierfür wird, neben Einflüssen auf die Barrierenhöhe und die Tunnelmasse, auch ein symmetriebrechender Einfluss von Heliumatomen außerhalb des suprafluiden Zustands diskutiert.[280]

Nur in wenigen Fällen wurde bisher auch zwischen nicht entarteten Zuständen eine Tunnelaufspaltung gefunden. Ein Beispiel hierfür ist asymmetrisch am Ring deuteriertes Porphycen, welches abhängig von der Schwingungsanregung Tunnelaufspaltungen aufweist, die mit der symmetrischen Stammverbindung vergleichbar sind (etwa $4 \mathrm{~cm}^{-1}$ im Schwingungsgrundzustand).[282] Eine solche Isotopensubstitution an der Peripherie des relativ großen Moleküls führt zu einer berechneten Asymmetrie von 1.5-5.6 cm ${ }^{-1}$ (abhängig von der Position) durch Veränderung der Nullpunktsschwingungsenergie. Als Faustregel wurde abgeleitet, dass Asymmetrien, die größer als die Tunnelaufspaltung im symmetrischen Fall sind, zu weitreichender Lokalisierung führen.[264, 272]. Eine solche Lokalisierung findet im jeweils asymmetrisch ${ }^{18} \mathrm{O}$-substituierten Tropolon [273], 3Isopropyltropolon [274] und 2-Methyl-9-hydroxyphenalenon [272] statt, während der jeweils elektronisch angeregte Zustand wegen einer geringeren Barriere delokalisiert bleibt. Die Dimere von Chlor- [283] und Bromwasserstoff [284] bleiben bei asymmetrischer Isotopensubstitution von einem der beiden Halogenkerne hochgradig delokalisiert, durch den Symmetriebruch werden aber weitere Übergänge schwach erlaubt [280, 283]. Allerdings ist diese Interpretation nicht unumstritten.[285] Das ChlorwasserstoffDimer stellt insofern einen Grenzfall dar, dass die relative Energie des oberen Tunnelzustandes bereits mit der Barrierenhöhe vergleichbar ist.[286] Bei asymmetrischer H/D- 


\section{Torsionsisomerie von Alkoholen}

Isotopensubstitution wird für $\mathrm{HCl} \cdots \mathrm{DCl}$ daher auch eine nur unvollständige Lokalisierung angenommen.[287] Die entsprechende Substitution im Fluorwasserstoff-Dimer lokalisiert dagegen den Grundzustand. Eine Mischung wird allerdings wegen einer zufälligen Resonanz zwischen zwei sowohl schwingungs- wie auch rotationsangeregten $\mathrm{Zu}$ ständen beobachtet.[275] Die Barriere im Dimer von Iodwasserstoff ist soweit abgesenkt, dass der Grundzustand besser als einzelne symmetrische Struktur zu beschreiben ist. Aber auch hier wird vorhergesagt, dass Monodeuterierung wieder zu einer deutlich asymmetrischen Struktur führt.[288]

Nur ein einziges Beispiel für ein molekulares System mit Asymmetrie bereits in der Elektronenstruktur (innerhalb der Born-Oppenheimer-Näherung) und Tunnelaufspaltung im Grundzustand wurde in der Literatur gefunden: Benzoesäure-Dimere in dotierten Kristallen.[279] Während isolierte Benzoesäure-Dimere ein symmetrisches Tunnelpotential aufweisen, wird dieses im Kristall durch die Wechselwirkung mit weiteren Dimeren soweit gestört, dass die Tunnelaufspaltung gelöscht wird. Diese Asymmetrie kann aber in der Nachbarschaft von geeigneten Dotanden ausreichend weit gesenkt werden, dass die Tunnelaufspaltung beobachtbar wird. Allerdings befindet sich auch hier die Asymmetrie fern in der Peripherie. Die anspruchsvolle quantenmechanische Behandlung der Abhängigkeit der Tunnelaufspaltung von der Asymmetrie eines Doppelminimumpotentials ist Gegenstand anhaltender Diskussionen.[289-291]

In Hinblick auf diese Beispiele erscheint eine Tunnelaufspaltung auch für $\alpha$-Fenchol nur dann möglich, sofern 1.) die lokalisierten $g+-$ und $g$--Zustände energetisch nur um wenige $h c \mathrm{~cm}^{-1}$ getrennt sind und 2.) die dazwischen liegende, energetisch niedrige Barriere durch die 3.) räumlich geringe Verschiebung von wenig Masse durchtunnelt werden kann. Diese drei Kriterien werden im Folgenden für $\alpha$-Fenchol geprüft.

Die Energiedifferenz zwischen $g+$ und $g-$ als erstes Kriterium wurde bereits zuvor diskutiert. Auf dem höchsten einzuschätzenden Niveau (CCSD(T)/aug-cc-pVQZ*//B3LYPD3(BJ)/aug-cc-pVTZ) wurde gefunden, dass die Zustände tatsächlich fast isoenergetisch sind $\left(\Delta E_{0}=0.06 \mathrm{~kJ} \mathrm{~mol}^{-1}=h c 5 \mathrm{~cm}^{-1}\right)$. Allerdings ist dieser Wert nicht zuletzt wegen des deutlichen Einflusses der Schwingungsnullpunktsenergie mit einiger Unsicherheit behaftet.

Für die Beurteilung des zweiten Kriteriums wird hier als Tunnelkoordinate der Minimum-Energiepfad für die HOCH-Torsion evaluiert. Dieser Ansatz ermöglichte zuvor gute Vorhersagen für Tunnelaufspaltungen von Alkoholen.[292] Dieser Pfad ist eventuell nicht komplett identisch mit den Torsions-Normalschwingungen der Konformere, denn für $g$ - wird (zumindest in harmonischer Näherung) eine Kopplung der HOCH-Torsion an eine Gerüstschwingung vorhergesagt, die zu symmetrischen und antisymmetrischen Kombinationen mit vergleichbaren HOCH-Torsionscharakter führt. Durch diese Kopplung weisen auch Kohlenstoffkerne größere Auslenkungen auf, die damit verbundene hohe effektive Tunnelmasse disqualifiziert diese Normalschwingung als Tunnelkoordinate. Bei Deuterierung der Hydroxygruppe wird diese Kopplung aufgehoben. Ein relaxierter Scan des HOCH-Torsionswinkel ist in Abb. 5.20 dargestellt.

Die Energie des Übergangszustandes wird elektronisch zu $2.5 \mathrm{~kJ} \mathrm{~mol}^{-1}$ auf B3LYPD3(BJ)/may-cc-pVTZ gefunden (alle Angaben relativ zum globalen Minimum $g+$ ). Dieser verringert sich bei voller Schwingungsnullpunktskorrektur (d. h. unter Berücksichtigung aller Moden mit Ausnahme der imaginären des Übergangszustandes) auf $1.4 \mathrm{~kJ} \mathrm{~mol}^{-1}=h c 120 \mathrm{~cm}^{-1}$. Die CCSD(T)-Basissatz-Extrapolation für den Übergangszu- 
stand scheiterte, da die MP2/aug-cc-pVQZ-Punktrechnung, auch bei geringer Variation der Struktur, aus unbekannter Ursache stets einen unrealistischen Wert ergab. Auf dem höchsten ohne Extrapolation zugänglichen Niveau (CCSD(T)/may-cc-pVTZ//B3LYPD3(BJ)/aug-cc-pVTZ) beträgt die Barrierenhöhe vergleichbare $1.5 \mathrm{~kJ} \mathrm{~mol}^{-1}$. Die Übergangszustände zu $t$ liegen deutlich höher, ausgehend von $g$ - elektronisch bei $6.3 \mathrm{~kJ} \mathrm{~mol}^{-1}$ und ausgehend von $g+$ bei $4.6 \mathrm{~kJ} \mathrm{~mol}^{-1}$ (B3LYP-D3(BJ)/may-cc-pVTZ, nicht gezeigt im Diagramm). Das Potential kann für die $g$-Konformere daher gut als Doppelminimum genähert werden.

Die für den Tunnelprozess relevante Barrierenhöhe im adiabatischen Kanal wird durch Addition der HOCH-Torsionsschwingungsnullpunktsenergie auf die vollschwingungsnullpunktskorrigierte Barriere erhalten (dies ist äquivalent $\mathrm{zu}$ einer Nullpunktskorrektur für die elektronische Barriere ohne Berücksichtigung der Torsionsmode). Die entsprechende Schwingungswellenzahl (B3LYP-D3(BJ)/may-cc-pVTZ) beträgt für $g+240 \mathrm{~cm}^{-1}$, für $g-$ wird der Mittelwert der beiden gekoppelten Schwingungen (250 / $260 \mathrm{~cm}^{-1}$ ) verwendet. Dies führt zu einer Barriere von $h c 240 \mathrm{~cm}^{-1}$ ausgehend von $g+$ und $h c 220 \mathrm{~cm}^{-1}$ ausgehend von $g-$ (B3LYP-D3(BJ)/may-cc-pVTZ). Im Folgenden wird vereinfacht der Mittelwert von $h c 230 \mathrm{~cm}^{-1}=2.75 \mathrm{~kJ} \mathrm{~mol}^{-1}$ verwendet. Um die Höhe dieses Wertes einordnen zu können, wird im nächsten Abschnitt ein einfaches Modell für die Korrelation zwischen Barrierenhöhen und Tunnelaufspaltungen von Alkoholen entwickelt.

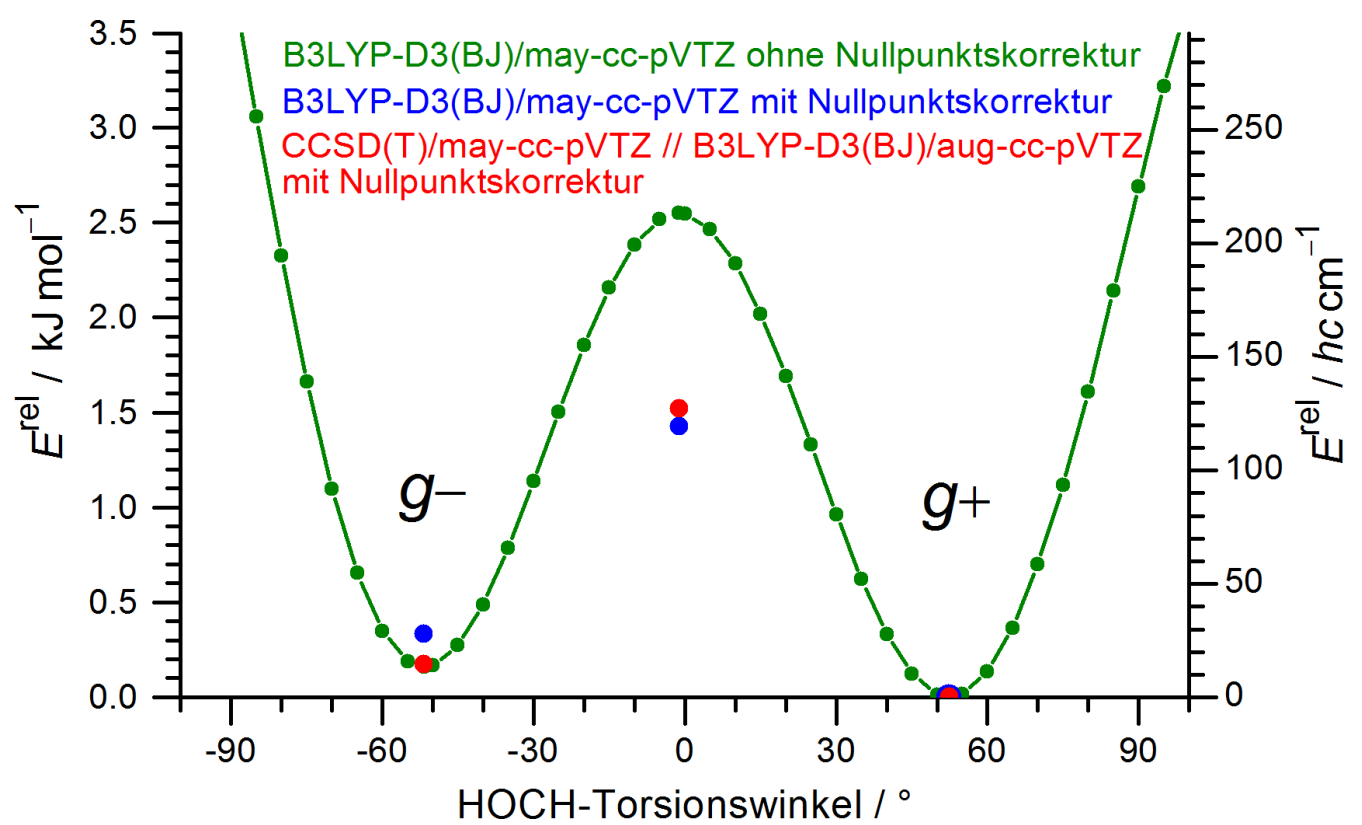

Abb. 5.20: Relaxierter Scan der elektronischen Energie auf B3LYP-D3(BJ)/may-cc-pVTZ-Niveau bezüglich des $\mathrm{HOCH}$-Torsionswinkel von $(-)$ - $\alpha$-Fenchol in $5^{\circ}$-Schritten, ergänzt um die volloptimierten stationären Punkte. Als weitere Datenpunkte sind die Energien nach Schwingungsnullpunktskorrektur und mit $a b$ initio Punktrechnungen angegeben. Alle Werte sind relativ zu $g+$ auf dem jeweiligen Theorieniveau. 


\subsection{Barrierenhöhemodell für Tunnelaufspaltungen in Alkoholen}

In diesem Abschnitt wird gezeigt, dass die Tunnelaufspaltungen vieler Alkohole hauptsächlich von Unterschieden in der Höhe der Barriere, und weniger von anderen Parametern, abhängen. Dies lässt sich mit einer in ihrer Form physikalisch begründbaren und bezüglich der Werte empirisch angepassten Beziehung nutzen, um mit kostengünstigen quantenchemischen Routinerechnungen Tunnelaufspaltungen vorherzusagen. Ferner wird gezeigt, dass auch der Isotopeneffekt auf die Tunnelaufspaltung bei Wechsel des Isotops des Hydroxywasserstoffs mit einer einfachen Beziehung beschrieben werden kann. Dies lässt darauf schließen, dass die effektive Tunnelmasse für die im Folgenden betrachteten Alkohole ähnlich ist (mit einer Ausnahme).

Als Startpunkt wurde eine Literaturrecherche für experimentell bekannte GrundzustandTunnelaufspaltungen von Alkoholen mit Kohlenwasserstoffrest durchgeführt. Diese Aufspaltungen sind aus rotationsspektroskopischen Messungen für eine ganze Reihe von Alkoholen teils sehr genau bekannt (werden hier aber aus Gründen der Übersichtlichkeit mit nur zwei signifikanten Stellen angegeben). Für viele Alkohole liegen Daten sowohl für eine protiierte wie auch eine deuterierte Hydroxygruppe vor. Diese Werte werden mit den Barrierenhöhen des adiabatischen Kanals für die Torsionsbewegung auf B3LYP-D3(BJ)/ may-cc-pVTZ-Niveau korreliert. Auch für zwei weitere Alkohole, neben dem zuvor diskutierten $\alpha$-Fenchol, ergibt sich dabei die Schwierigkeit, dass zwei Normalschwingungen deutlichen OH- bzw. OD-Torsionscharakter aufweisen. Dies betrifft Propargylalkohol (OH und $\mathrm{OD})$ und 1-Ethenylcyclopropan-1-ol $(\mathrm{OH})$. Auch in diesen Fällen wurde die Wellenzahl beider Schwingungen gemittelt. Die Aufspaltung der Torsionsschwingung, und damit die zusätzliche Unsicherheit, ist aber gering gegenüber der Barrierenhöhe. Für das asymmetrische $\alpha$-Fenchol wurde die Barrierenhöhe für $g-$ und $g+$ gemittelt. Die Barrierenhöhen sind den Tunnelaufspaltungen in Tab. 5.5 gegenübergestellt.

Die Tunnelaufspaltung nimmt tendenziell mit steigender Barrierenhöhe ab. Dies deutet daraufhin, dass Unterschiede in der Barrierenhöhe die Abweichungen in der Potentialform und der effektiven Tunnelmasse dominieren. Werden die Moleküle Benzylalkohol, Methanol, tert-Butylalkohol und Ethanol nicht berücksichtigt, ist die Korrelation zwischen Tunnelaufspaltung und Barrierenhöhe sogar monoton. Diese Ausnahmen lassen sich jeweils anhand der Molekülstruktur begründen. In Benzylalkohol ist die C-OTorsion stark an eine C-C-Torsion gekoppelt. Daher ist die effektive Tunnelmasse wahrscheinlich deutlich höher, sodass die Tunnelaufspaltung sehr viel kleiner ausfällt als es anhand der der relativ geringen Barrierenhöhe zu erwarten wäre. Dies äußert sich auch in einem, im Vergleich zu den anderen Alkoholen, stark abweichenden Isotopeneffekt (vide infra). Benzylalkohol wird in Kap. 5.16 im Detail diskutiert. Für Methanol und tert-Butylalkohol ist aus der Struktur der Moleküle ersichtlich, dass die Tunnelaufspaltung nicht in einem Doppelminimumpotential, sondern in einem dreizähligen periodischen Torsionspotential mit identischen Barrieren stattfindet. Auch für Ethanol ist die Beschreibung als einfaches Doppelminimumpotential unzureichend. Im Gegensatz zu den anderen hier betrachteten Alkoholen, liegt für Ethanol das $t$-Konformer energetisch tiefer als die $g$-Konformere und die $g \leftrightarrow t$-Barriere ist geringer als die für $g \leftrightarrow g$. Daher kann der Tunnelpfad durch die doppelten $g \leftrightarrow t$-Barrieren nicht vernachlässigt werden. Nach nu- 
Tab. 5.5: Gegenüberstellung von berechneten Barrierenhöhen $V_{0}$ im adiabatischen Kanal (ohne Nullpunktsenergie der OH- bzw. (OD)-Torsionsschwingung) zwischen $g$-Konformeren von verschiedenen Alkoholen auf B3LYP-D3(BJ)/may-cc-pVTZ-Niveau und experimentelle Tunnelaufspaltungen im Grundzustand $\Delta_{0}$, jeweils mit protiierter und (deuterierter) Hydroxygruppe.

\begin{tabular}{lcc}
\hline Alkohol (Konformation) & $V_{0} \mathrm{OH}(\mathrm{OD}) / \mathrm{hc} \mathrm{cm} \mathrm{cm}^{-1}$ & $\Delta_{0} \mathrm{OH}(\mathrm{OD}) / \mathrm{hc} \mathrm{cm}{ }^{-1}$ \\
\hline Propargylalkohol & $220(200)$ & $22(7.1)[293]$ \\
$\alpha$-Fenchol & $230(220)$ & $\leq 16 ?(-)$ \\
Benzylalkohol & $320(310)$ & $0.016(0.0045)[294]$ \\
Methanol & $370(360)$ & $9.1(2.6)[295,296]$ \\
1-Methylcyclohexanol (eq) & $370(350)$ & $-(0.61)[297]$ \\
1-Methylcyclohexanol (ax) & $390(370)$ & $-(0.52)[297]$ \\
tert-Butylalkohol & $420(410)$ & $3.8(-)[298]$ \\
Ethanol & $420(410)$ & $3.2(0.57)[12]$ \\
Cyclohexanol (eq) & $420(410)$ & $1.7(-)[299]$ \\
2-Propanol & $460(450)$ & $1.6(0.15)[300]$ \\
Allylalkohol (syn) & $590(580)$ & $0.47(0.031)[301]$ \\
Cyclopropanol & $720(710)$ & $0.14(0.0055)[302]$ \\
1-Ethenylcyclopropan-1-ol (syn) & $820(800)$ & $0.076(0.0024)[303]$ \\
\hline
\end{tabular}

merischen Berechnungen in Ref. [292] haben die (nominellen) $g$-Wellenfunktionen auch signifikante Amplituden im Winkelbereich des $t$-Potentialtopfes, im Gegensatz zu z. B. 2-Propanol. ${ }^{\mathrm{b}}$ Die Tunnelaufspaltungen in Methanol, tert-Butylalkohol und Ethanol sind durch diese zusätzlichen relevanten Tunnelpfade daher höher, als es allein aus der berechneten Barrierenhöhe, im Vergleich zu den anderen Alkoholen, zu erwarten wäre.

Für die anderen Datenpunkte ist es vielversprechend den Zusammenhang zwischen Barrierenhöhe und Tunnelaufspaltung näher zu analysieren, um damit die zu erwartende Tunnelaufspaltung für weitere Alkohole, darunter auch $\alpha$-Fenchol, zu interpolieren. Hierfür wird das Konzept von Berger et al. [34] aufgegriffen, die Tunnelaufspaltung in Abhängigkeit von der Skalierung eines Potentials zu beschreiben. Die Autoren entwickelten diese Methode, um extrem kleine Tunnelaufspaltungen im Zusammenhang mit der Paritätsverletzung zu extrapolieren, die aus numerischen Gründen nicht direkt berechenbar waren. Hierzu wird ein Doppelminimumpotential $V(x)$ mit einem Faktor $f$ skaliert. Dabei steigt die Barrierenhöhe $V_{0}$ linear mit $f$, während die harmonische Schwingungsfrequenz $\omega$ in den Minima sich wegen der Abhängigkeit $\omega \propto \sqrt{k}$ von der Kraftkonstanten $k$, also der Krümmung am Potentialminimum, mit $\sqrt{f}$ ändert. Dies ist in Abb. 5.21 illustriert.

Für die Berechnung der Tunnelaufspaltung in einem solchen symmetrischen Doppelminimumpotential (mit ansonsten beliebiger Form) wird die Näherung nach WentzelKramers-Brillouin (WKB) in der Formulierung von Garg [304] verwendet (Gl. 5.165.18).

\footnotetext{
${ }^{\mathrm{b}}$ Allerdings zeigen die Wellenfunktionen einige Anomalien hinsichtlich Symmetrie und Knotenzahlen, sie sollten daher mit einer gewissen Vorsicht betrachtet werden.
} 


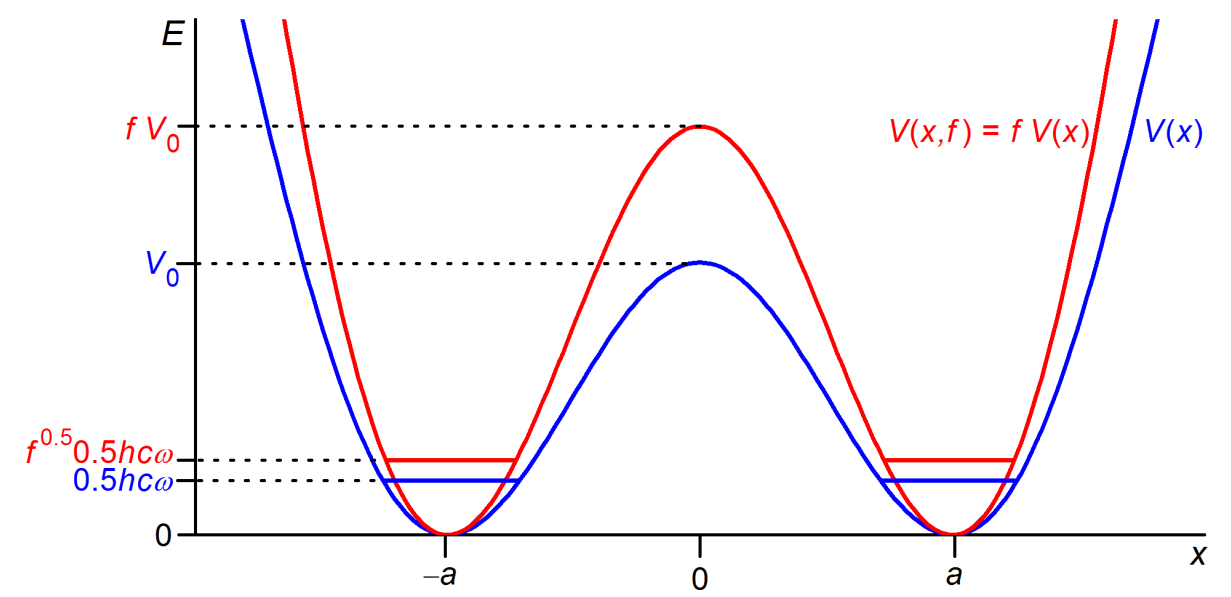

Abb. 5.21: Skalierung eines Doppelminimumpotentials $V(x)$ mit einem Faktor $f$ führt zu einer Erhöhung der Barrierenhöhe $V_{0}$ ebenfalls um $f$ und einer Skalierung der harmonischen Schwingungsfrequenz $\omega$ in den Minima um $f^{0.5}$.

$$
\begin{aligned}
\Delta_{0} & =2 \hbar \omega \sqrt{\frac{m \omega a^{2}}{\pi \hbar}} \mathrm{e}^{A} \mathrm{e}^{-S_{0} / \hbar} \\
S_{0} & =\int_{-a}^{a} \sqrt{2 m V(x)} \mathrm{d} x \\
A & =\int_{0}^{a}\left[\frac{m \omega}{\sqrt{2 m V(x)}}-\frac{1}{a-x}\right] \mathrm{d} x
\end{aligned}
$$

Dabei ist $\Delta_{0}$ die Tunnelaufspaltung des Grundzustandes, $\hbar$ das reduzierte Plancksche Wirkungsquantum, $\omega$ die harmonische Kreiswellenzahl der Schwingung in den Minima des Potentials $V(x), m$ die effektive Tunnelmasse, $a$ und $-a$ die Positionen der beiden Minima auf der Tunnelkoordinate $x, \pi$ die Kreiszahl und e die Eulersche Zahl. Gesucht wird die Abhängigkeit $\Delta_{0}(f)$, wobei $\Delta_{0}(f=1)$ das unskalierte Referenzpotential darstellt. Mit den Skalierungen gemäß Gl. 5.19 und 5.20 werden die folgenden Abhängigkeiten der Integrale $S_{0}$ und $A$ von $f$ erhalten (Gl. 5.21 und 5.22):

$$
\begin{aligned}
\omega(f) & =\sqrt{f} \omega \\
V(f, x) & =f V(x) \\
S_{0}(f) & =\int_{-a}^{a} \sqrt{2 m f V(x)} \mathrm{d} x=\sqrt{f} S_{0} \\
A(f) & =\int_{0}^{a}\left[\frac{m \sqrt{f} \omega}{\sqrt{2 m f V(x)}}-\frac{1}{a-x}\right] \mathrm{d} x=A
\end{aligned}
$$


Ersetzen durch die in Gl. 5.16 und Umformen liefert:

$$
\begin{aligned}
\Delta_{0}(f) & =2 \hbar \sqrt{f} \omega \sqrt{\frac{m \sqrt{f} \omega a^{2}}{\pi \hbar}} \mathrm{e}^{A} \mathrm{e}^{-\sqrt{f} S_{0} / \hbar} \\
\Delta_{0}(f) & =2 \hbar(\sqrt{f})^{1.5} \omega \sqrt{\frac{m \omega a^{2}}{\pi \hbar}} \mathrm{e}^{A} \mathrm{e}^{-\sqrt{f} S_{0} / \hbar} \\
\ln \left[\Delta_{0}(f) /\left(h c \mathrm{~cm}^{-1}\right)\right] & =1.5 \ln \sqrt{f}+\ln \left(2 \hbar \omega \sqrt{\frac{m \omega a^{2}}{\pi \hbar}} \mathrm{e}^{A}\right)-\sqrt{f} \frac{S_{0}}{\hbar} \\
\lg \left[\Delta_{0}(f) /\left(h c \mathrm{~cm}^{-1}\right)\right] & =1.5 \lg \sqrt{f}+\lg \left(2 \hbar \omega \sqrt{\frac{m \omega a^{2}}{\pi \hbar}} \mathrm{e}^{A}\right)-\sqrt{f} \frac{S_{0}}{\hbar \ln 10}
\end{aligned}
$$

G1. 5.26 gibt die Abhängigkeit des dekadischen Logarithmus der Tunnelaufspaltung von der Wurzel des Skalierungsfaktors an. Wird das unskalierte Potential so gewählt, dass dessen Barriere $V_{0}(f=1)=h c 1 \mathrm{~cm}^{-1}$ beträgt, so kann $f$ durch die berechneten Barrieren $V_{0} /\left(h c \mathrm{~cm}^{-1}\right)$ in Tab. 5.5 ersetzt werden. Die Korrelation zwischen den experimentell ermittelten Werten für $\lg \left[\Delta_{0} /\left(h c \mathrm{~cm}^{-1}\right)\right]$ und den berechneten Werten für $\sqrt{V_{0} /\left(h c \mathrm{~cm}^{-1}\right)}$ sollte daher gut durch eine Funktion der Form nach Gl. 5.26 beschrieben werden können. Abb. 5.22 zeigt, dass dies der Fall ist. Für die Vergleichbarkeit zwischen den folgenden Diagrammen werden in den Anpassungen nur die Alkohole berücksichtigt, für die

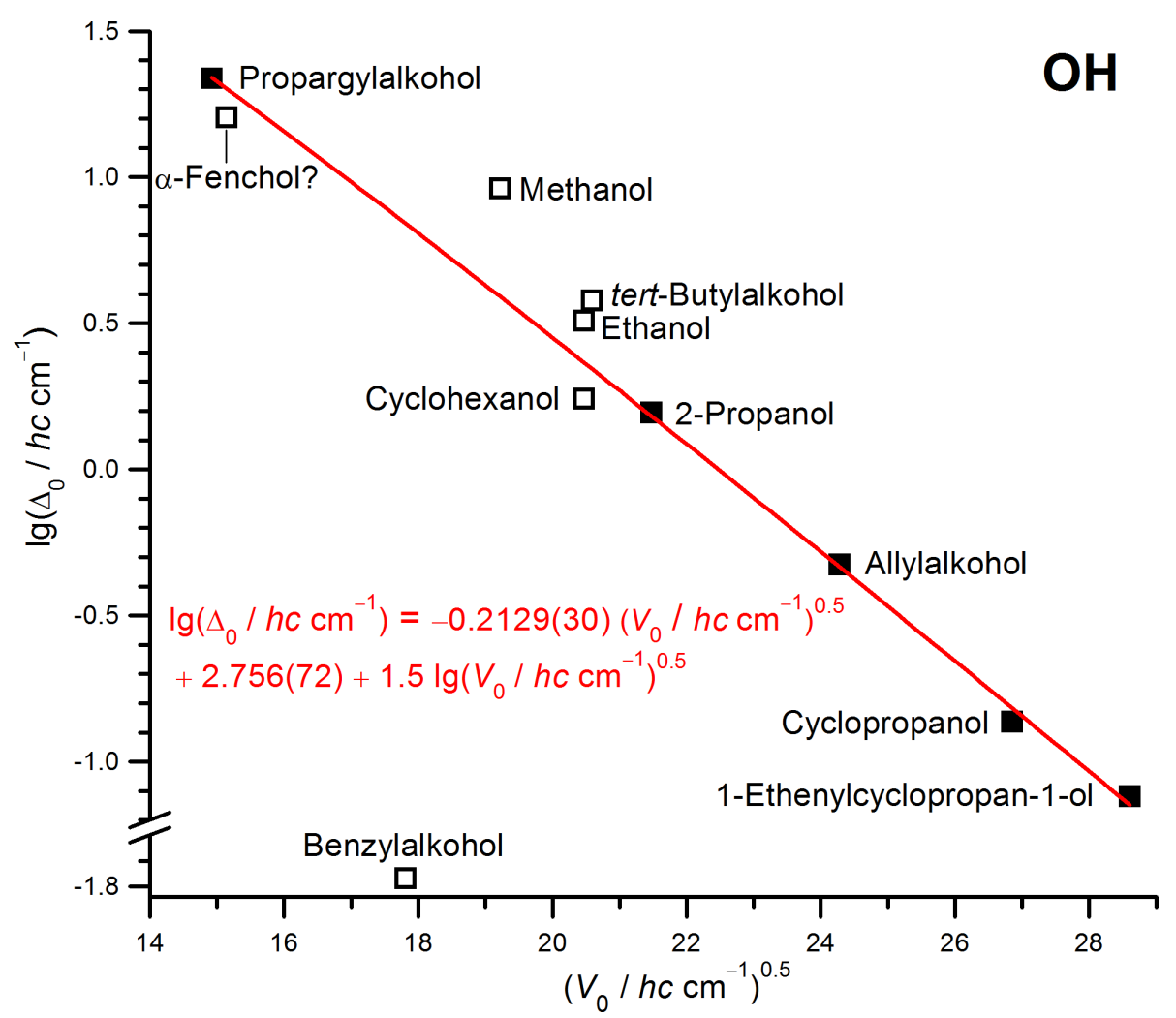

Abb. 5.22: Korrelation zwischen der dekadisch logarithmierten Tunnelaufspaltung und der Wurzel der Barrierenhöhe zwischen $g$-Konformeren von verschiedenen protiierten Alkoholen (Werte in Tab. 5.5). Für die Anpassung wurden nur die Datenpunkte einbezogen, die mit ausgefüllten Quadraten dargestellt sind. 


\section{Torsionsisomerie von Alkoholen}

sowohl Werte mit protiierter wie auch deuterierter Hydroxygruppe vorliegen (gekennzeichnet durch ausgefüllte Quadrate). Die Nicht-Berücksichtigung von Methanol, tertButylalkohol, Ethanol und Benzylalkohol wegen Nicht-Erfüllung der Grundannahmen des Modells (Doppelminimumpotential, vergleichbare Tunnelmasse) wurde bereits diskutiert. Die Tunnelaufspaltung von Cyclohexanol wird durch Interpolation um etwa $\frac{1}{3}$ überschätzt (vorhergesagt: $\Delta_{0}=h c 2.3 \mathrm{~cm}^{-1}$, gemessen: $h c 1.7 \mathrm{~cm}^{-1}$ [299]).

Eine Extrapolation auf die besonders kleine Tunnelaufspaltung von Phenol-OH liefert mit einer Vorhersage von $h c 0.0031 \mathrm{~cm}^{-1}$ (basierend auf einer berechneten adiabatischen Barriere von $h c 1270 \mathrm{~cm}^{-1}$ ) durchaus eine gute Übereinstimmung mit dem beobachteten Wert von $h c 0.0021 \mathrm{~cm}^{-1}$ [204]. Allerdings ist dies vermutlich eher eine Fehlerkompensation aus zwei Einflüssen der abweichenden Barrierenform. In Phenol liegt zwar auch ein Doppelminimumpotential vor, dieses ist allerdings periodisch, sodass zwei äquivalente Tunnelpfade zur Aufspaltung beitragen. Als dem entgegenlaufender Einfluss ist die Barriere mit $180^{\circ}$ breiter als für Alkohole mit $\approx 120^{\circ}$.

Dass dieser Zusammenhang auch gut für die deuterierten Isotopologen gilt, zeigt Abb. 5.23. Durch die Deuterierung verringern sich die berechneten Barrieren im adiabatischen Kanal für alle Alkohole nur um jeweils etwa $10-20 \mathrm{hc} \mathrm{cm}^{-1}$, sodass die Veränderung der Tunnelaufspaltung durch die Änderung der Tunnelmasse dominiert sein sollte. Die Tunnelaufspaltungen für die Konformere von deuteriertem 1-Methylcyclohexanol, mit der Hydroxygruppe in äquatorialer bzw. axialer Position, werden sehr gut interpoliert.

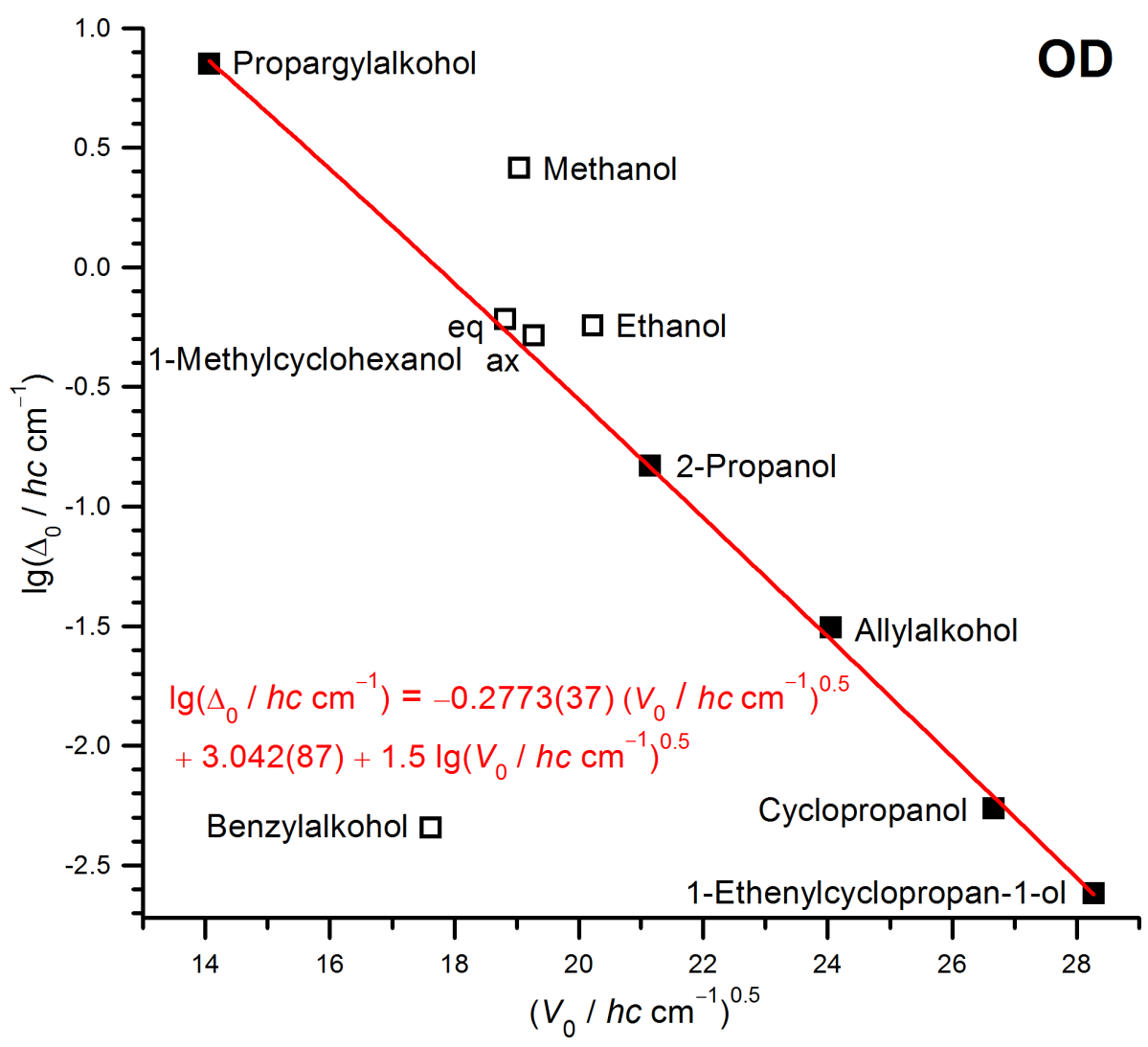

Abb. 5.23: Korrelation zwischen der dekadisch logarithmierten Tunnelaufspaltung und der Wurzel der $g \leftrightarrow g$-Barrierenhöhe von verschiedenen deuterierten Alkoholen (Werte in Tab. 5.5). Für die Anpassung wurden nur die Datenpunkte einbezogen, die mit ausgefüllten Quadraten dargestellt sind. 
Von Berger et al. [34] wurde abweichend von der hier verwendeten Form kein konstanter Vorfaktor von 1.5 vor dem logarithmischen Term der Anpassungsfunktion verwendet, sondern dieser als dritter Anpassungsparameter behandelt. Von den Autoren wurde auch angemerkt, dass die Daten fast ebenso gut durch einen rein linearen Zusammenhang beschrieben werden können. Dies ist auch für die hier betrachteten Daten der Fall. Die lineare Form entspricht einem näherungsweise konstanten oder vernachlässigbar kleinen Term 1.5 lg $\sqrt{f}$ in Gl. 5.26. Ein solch linearer Zusammenhang wurde auch bereits für die Beschreibung der Korrelation zwischen berechneten Barrierenhöhen und ebenfalls berechneten Tunnelaufspaltungen für Dimere von Carbonsäuren eingesetzt.[305] In diesem Fall wurde eine größere Streuung der Datenpunkte um die Anpassung gefunden als hier für Alkohole. Die Autoren nennen als Ursache hierfür nicht berücksichtigten Unterschiede in $\omega$, Länge und Form des Tunnelpfads, sowie Einflüsse der Carbonsäurereste auf die effektive Tunnelmasse im intermolekularen Tunnelprozess.[305]

Die logarithmierten Tunnelaufspaltungen sowohl der protiierten wie auch der deuterierten Alkohole korrelieren nahezu linear mit den Wurzeln der jeweiligen berechneten Barrieren. Diese Barrieren ändern sich zudem nur wenig durch Effekte auf die Schwingungsnullspunktsenergie. Dies lässt erwarten, dass auch zwischen den logarithmierten Tunnelaufspaltungen beider Isotopologe eine näherungsweise lineare Korrelation besteht. Abb. 5.24 zeigt, dass dies sehr gut zutrifft. Diese Beschreibung des Isotopeneffekts eignet sich ebenfalls für die Vorhersage von Tunnelaufspaltungen von Alkoholen, sofern ein experimenteller Wert für eines der beiden Isotopologen vorliegt. Quantenchemische Rech-

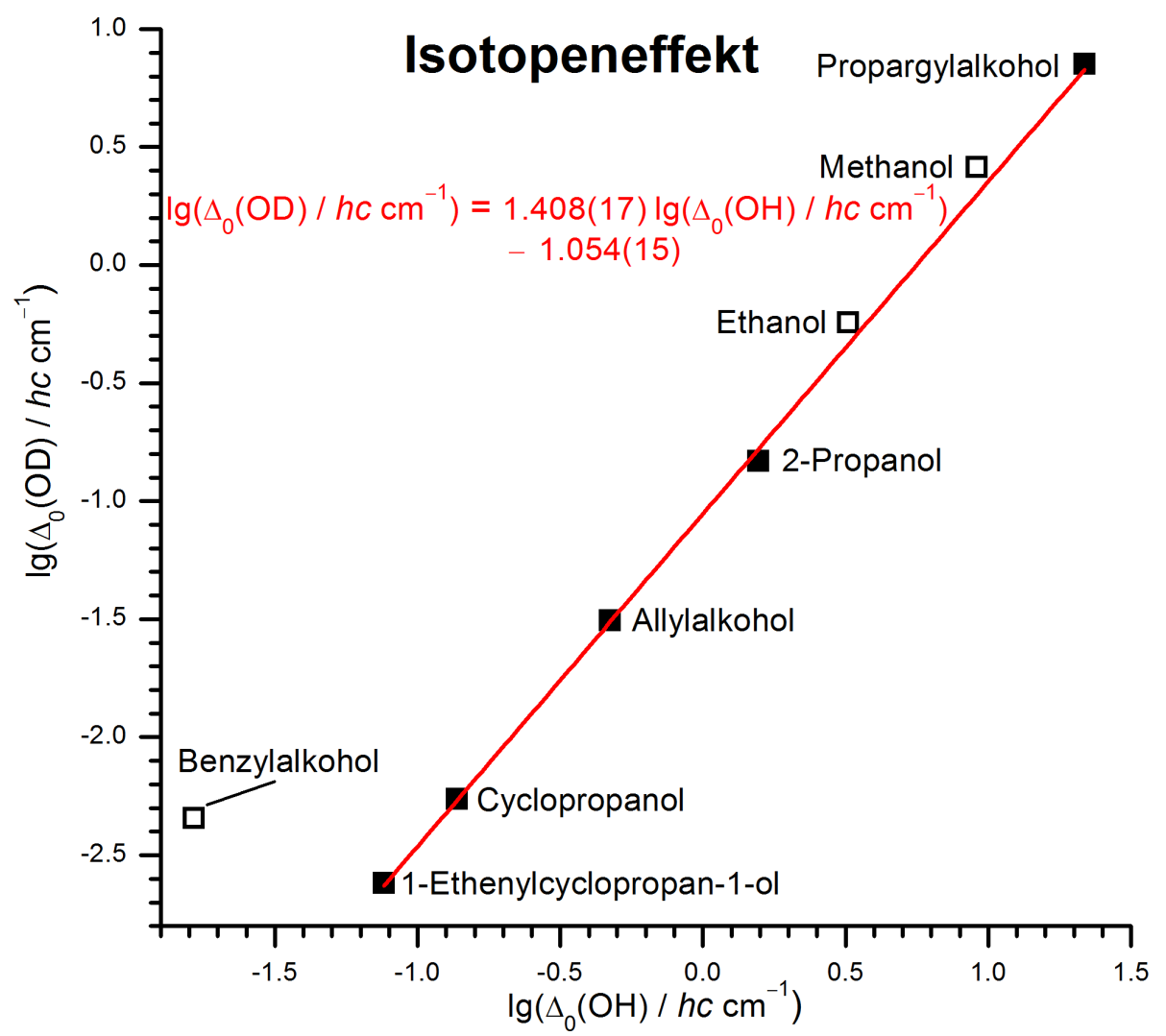

Abb. 5.24: Korrelation zwischen den dekadisch logarithmierten Tunnelaufspaltungen von protiierten und deuterierten Alkoholen (Werte in Tab. 5.5). Für die lineare Anpassung wurden die gleichen Alkohole wie in Abb. 5.22 und 5.23 verwendet, dargestellt mit ausgefüllten Quadraten. 


\section{Torsionsisomerie von Alkoholen}

nungen sind in diesem Fall nicht notwendig. Auch Methanol und Ethanol fügen sich in diese Korrelation gut ein. Dies könnte ein Hinweis darauf sein, dass ein Zusammenhang unabhängig von der Form des Potentials existiert.

Zur physikalischen Interpretation wird im Folgenden die Abhängigkeit der Tunnelaufspaltung von der Tunnelmasse betrachtet, wenn letztere mit einem Faktor $z$ skaliert wird. In Gl. 5.27 bis 5.30 sind die Abhängigkeiten der einzelnen Größen von $z$ angegeben:

$$
\begin{aligned}
m(z) & =z m \\
\omega(z, f) & =\frac{\sqrt{f} \omega}{\sqrt{z}} \\
S_{0}(z, f) & =\int_{-a}^{a} \sqrt{2 z m f V(x)} \mathrm{d} x=\sqrt{z} \sqrt{f} S_{0} \\
A(z, f) & =\int_{0}^{a}\left[\frac{z m \sqrt{f} \omega}{\sqrt{z} \sqrt{2 z m f V(x)}}-\frac{1}{a-x}\right] \mathrm{d} x=A
\end{aligned}
$$

Durch Ersetzen in Gl. 5.16 ergeben sich dann für die Tunnelaufspaltung Gl. 5.31 und 5.32:

$$
\begin{aligned}
\Delta_{0}(z, f)= & z^{-0.25}(\sqrt{f})^{1.5} 2 \hbar \omega \sqrt{\frac{m \omega a^{2}}{\pi \hbar}} \mathrm{e}^{A} \mathrm{e}^{-\sqrt{z} \sqrt{f} S_{0} / \hbar} \\
\lg \left[\Delta_{0}(z, f) /\left(h c \mathrm{~cm}^{-1}\right)\right]= & -0.25 \lg z+1.5 \lg \sqrt{f}+\lg \left(2 \hbar \omega \sqrt{\frac{m \omega a^{2}}{\pi \hbar}} \mathrm{e}^{A}\right) \\
& -\sqrt{z} \sqrt{f} \frac{S_{0}}{\hbar \ln 10}
\end{aligned}
$$

Um den in Abb. 5.24 gefundenen Zusammenhang zu beschreiben, müssen die Terme $\lg \left[\Delta_{0}(z, f) /\left(h c \mathrm{~cm}^{-1}\right)\right]$ und $\lg \left[\Delta_{0}(z=1, f) /\left(h c \mathrm{~cm}^{-1}\right)\right]$ verknüpft werden. Zur mathematischen Vereinfachung lässt sich der logarithmische Term $1.5 \lg \sqrt{f}$ als konstant oder vernachlässigbar annehmen, wie die gefundene annähernde Linearität andeutet. Gl. 5.32 und 5.26 lassen sich dann jeweils nach $\sqrt{f} \frac{S_{0}}{\hbar \ln 10}$ auflösen und gleichsetzen. Dies beinhaltet als weitere Näherung, dass sich die Barrieren durch die Deuterierung nur unwesentlich ändern - so wie es quantenchemisch gefunden wurde. Nach weiteren Umformungen ergibt sich Gl. 5.33:

$$
\begin{aligned}
\lg \left[\Delta_{0}(z) /\left(h c \mathrm{~cm}^{-1}\right)\right]= & \sqrt{z} \lg \left[\Delta_{0}(z=1) /\left(h c \mathrm{~cm}^{-1}\right)\right]-0.25 \lg z \\
& -(\sqrt{z}-1) \cdot\left[\lg \left(2 \hbar \omega \sqrt{\frac{m \omega a^{2}}{\pi \hbar}} \mathrm{e}^{A}\right)+1.5 \lg \sqrt{f}\right]
\end{aligned}
$$

Mit den Annahmen $1.5 \lg \sqrt{f} \approx$ const. und $z \approx$ const. für die betrachteten Alkohole entspricht Gl. 5.33 einem linearen Zusammenhang mit der Steigung $\sqrt{z}$. Mit dem aus den experimentellen Daten bestimmten Wert der Steigung von 1.408(17) ergibt sich $z=$ 
1.98(5). Dieser Wert entspricht dem bekannten Verhältnis der Massen von Deuteron zu Proton: 2.00.

Die erneut starke Abweichung des Datenpunktes von Benzylalkohol kann auf eine deutlich größere effektive Tunnelmasse zurückgeführt werden. Diese ändert sich bei Austausch des Wasserstoffisotops relativ gesehen weniger stark als bei den anderen Alkoholen. So weist Benzylalkohol-OD eine ähnlich niedrige Tunnelfrequenz wie Cyclopropanol-OD und 1-Ethenylcyclopropan-1-ol-OD auf. Während sich der Tunnelprozess der beiden letztgenannten bei Protierung um einen Faktor 25 bzw. 31 beschleunigt, geschieht dies für Benzylalkohol dagegen nur um etwa einen Faktor 4.

Für die bisher fehlenden experimentellen Werte in Tab. 5.5 lassen sich auf Basis der vorgestellten Korrelationen die in Tab. 5.6 gelisteten Vorhersagen aufstellen. Für 1-Methylcyclohexanol-OH existieren bereits Vorhersagen gemäß Meyers flexiblem Modell aus Ref. [297]. Während für die axiale Form sehr ähnliche Werte erhalten werden im Vergleich zu Ref. [297], sind diese für die äquatoriale Form signifikant höher, sodass ein experimenteller Test in Zukunft interessant sein könnte, um die Vorhersagekraft der Modelle zu vergleichen.

Tab. 5.6: Vorhersagen für Tunnelaufspaltungen von Alkoholen im Grundzustand, gemäß Interpolation aufgrund der berechneten Barrierenhöhe (Abb. 5.22 und 5.23); des Isotopeneffekts anderer Alkohole (Abb. 5.24); und gemäß Meyers flexiblen Modell aus Ref. [297] auf Basis der Tunnelaufspaltung des Isotopologen.

\begin{tabular}{lc}
\hline Alkohol (Konformation) & $\Delta_{0} / h c \mathrm{~cm}^{-1}$ \\
& (Barrierenhöhe; Isotopeneffekt; Ref. [297]) \\
\hline Cyclohexanol-OD (eq) & $0.25 ; 0.19 ;-$ \\
1-Methylcyclohexanol-OH (eq) & $3.9 ; 3.9 ; 3.436$ \\
1-Methylcyclohexanol-OH (ax) & $3.3 ; 3.5 ; 3.419$ \\
tert-Butylalkohol-OD & $-; 0.57 ;-$ \\
\hline
\end{tabular}




\section{$5.10 \alpha$-Fenchol - Teil 2}

Wird die in Abb. 5.22 erhaltene Anpassung auf die gemittelte Barriere von $\alpha$-Fenchol angewendet, so wird für die Tunnelaufspaltung eine Vorhersage von $h c 20 \mathrm{~cm}^{-1}$ erhalten, vergleichbar mit der beobachteten Aufspaltung von $h c 16 \mathrm{~cm}^{-1}$. Die Genauigkeit dieses interpolierten Wertes sollte nicht überbewertet werden, insbesondere wegen des Einflusses der Asymmetrie. Diese Interpolation zeigt aber, dass eine Tunnelaufspaltung in dieser Größenordnung kompatibel wäre mit der berechneten Barrierenhöhe. Damit die Delokalisation durch die Asymmetrie nicht weitgehend verloren geht, sollte letztere nicht wesentlich größer als die Tunnelaufspaltung im symmetrischen Fall sein. Wird die experimentelle Aufspaltung als Obergrenze verwendet, so ergibt sich ein kompatibler Bereich für die Asymmetrie von $\pm h c 16 \mathrm{~cm}^{-1}= \pm 0.19 \mathrm{~kJ} \mathrm{~mol}^{-1}$. Dies ist kein unplausibles Szenario für den Energieunterschied zwischen $g+$ und $g-$ von $\alpha$-Fenchol, die DFT-Rechnungen liegen geringfügig bis mäßig außerhalb dieser Spanne, die ab initio-Punktrechnungen alle innerhalb.

Über die harmonische OH-Streckschwingungswellenzahl des Übergangszustandes lässt sich auch sehr grob die Barriere und die mögliche Tunnelaufspaltung im schwingungsangeregten Zustand abschätzen. Die berechnete Wellenzahl des Übergangszustandes von $\alpha$-Fenchol ist um $49 \mathrm{~cm}^{-1}$ höher als die von $g$ - und um $41 \mathrm{~cm}^{-1}$ höher als die von $g+$. Die mittlere Barriere steigt demnach um $h c 45 \mathrm{~cm}^{-1}$. ${ }^{\mathrm{c}}$ Bei einer dann erhaltenen Barrierenhöhe von etwa $h c 270 \mathrm{~cm}^{-1}$ besteht nach Interpolation gemäß dem Barrierenhöhemodell eine Erwartung für die Tunnelaufspaltung von $h c 11 \mathrm{~cm}^{-1}$. Dieser Wert liegt im Bereich der zugeordneten Aufspaltung von $h c 9.5 \mathrm{~cm}^{-1}$. Durch die unterschiedlichen Wellenzahlen von $g$ - und $g+$ ändert sich aber auch die Asymmetrie bei Schwingungsanregung. Nach der Rechnung auf dem höchsten Niveau liegt $g+$ um $h c 5 \mathrm{~cm}^{-1}$ unterhalb von $g-$, nach Schwingungsanregung dagegen $h c 3 \mathrm{~cm}^{-1}$ oberhalb. In diesem Szenario würde sich der Betrag der Asymmetrie also nur wenig ändern, sodass auch im schwingungsangeregten Zustand eine Delokalisierung durchaus möglich erscheint. Für definitive Aussagen hierzu sind die Genauigkeit der hier verwendeten Rechnungen und einfachen Modelle aber unzureichend.

Voraussetzung dafür, dass die Tunnelaufspaltung durch Vergleich mit anderen Alkoholen über die Barrierenhöhe abgeschätzt werden kann, ist zudem eine ähnliche effektive Tunnelmasse. Diese hängt davon ab, um welche Distanzen die Atomkerne massengewichtet tunneln. Um dies zu untersuchen wurde die gekrümmte Torsionsbewegung hierzu in zwei als linear genäherte Teilschritte aufgeteilt: $g-\leftrightarrow \ddot{U Z Z}$ und ÜZ $\leftrightarrow g+$ (ÜZ: Übergangszustand). Die Strukturen auf B3LYP-D3(BJ)/may-cc-pVTZ-Niveau wurden durch Translation und Rotation derart überlagert, dass der RMSE für die massengewichteten Atomkernpositionen minimiert wurde (mittels Chemcraft 1.8). Für die beiden Schritte wurden jeweils die folgenden Tunneldistanzen gefunden: Hydroxyproton $\approx 0.7 \AA$, Sauerstoffkern $\approx 0.07 \AA$, alle anderen Protonen $<0.05 \AA$ und Kohlenstoffkerne $<0.02 \AA$. Dies ist Abb. 5.25 grafisch dargestellt. Die Atome des Übergangszustandes sind schwarz eingefärbt und für die Übersichtlichkeit wurden ihren Radien jeweils um $0.02 \AA$ verringert. Dadurch werden sie nur sichtbar, wenn sich die jeweilige Kernposition um mehr als diesen Betrag ändert, ansonsten werden sie von den größeren Atomen der Minimumstruktur voll-

\footnotetext{
${ }^{\mathrm{c}}$ Eine entsprechende Betrachtung für Methanol sagt eine Erhöhung um $h c 36 \mathrm{~cm}^{-1}$ voraus, vergleichbar
} mit einem experimentell abgeleiteten Wert von $h c 41 \mathrm{~cm}^{-1}$ [306]. 
ständig verdeckt. Es ist zu erkennen, dass Tunneldistanzen $>0.02 \AA$ auf das Hydroxyproton, den Sauerstoffkern und im geringen Maße $(<0.05 \AA)$ auf einige weitere Protonen in der näheren Umgebung der Hydroxygruppe beschränkt sind. Die Struktur von $\alpha$-Fenchol kann damit als starr genug eingeschätzt werden, dass Änderungen der Kernpositionen auf diesem Umwandlungspfad in guter Näherung auf die Hydroxygruppe beschränkt sind.

Zum Vergleich wurden dieses Verfahren auch für die Alkohole angewendet, deren Barrierenhöhen als Referenzen verwendet werden (Propargylalkohol, 2-Propanol, Allylalkohol, Cyclopropanol, 1-Ethenylcyclopropan-1-ol). Dabei wurden Tunneldistanzen in ähnlicher Größe für die jeweiligen Kernsorten gefunden. Dies entspricht dem Befund aus Kapitel 5.9, nach dem sich Unterschiede in den Tunnelaufspaltungen in guter Näherung allein mit unterschiedlich hohen Barrieren begründen lassen.

Zusammengefasst scheint die Erfüllung aller drei Kriterien für eine Tunnelaufspaltung (niedrige energetische Asymmetrie, geringe Barrierenhöhe, niedrige effektive Tunnelmasse) durchaus plausibel zu sein. Ein zukünftiger Beweis muss hier aber auf experimenteller Ebene erfolgen. Am vielversprechendsten erscheint die Untersuchung von $\alpha$ Fenchol am Curry-Jet in weiteren Spektralbereichen. Bei Verringerung des Detektionsabstandes könnten auch für andere Schwingungsübergänge heiße Banden erscheinen bzw. sich intensivieren, die um die vermutete Grundzustandsaufspaltung von $16 \mathrm{~cm}^{-1} \mathrm{zu}$ niedrigeren Wellenzahlen verschoben sind. Solche Übergänge setzen allerdings voraus, dass sich das Torsionspotential auch durch andere Schwingungsanregungen signifikant ändert.

Möglich wäre aber auch das Szenario, dass das Hydroxyproton von $\alpha$-Fenchol nur im angeregten Zustand der $\mathrm{OH}-$ Streckschwingung delokalisiert sein könnte, was zur Erklärung des beobachteten Bandenmusters ausreichend wäre. Dieses Szenario wäre schwieriger nachzuweisen oder zu widerlegen.

Von Interesse ist auch, ob eine Delokalisation im OH-Streckschwingungsgrundzustand auch mit den bereits erwähnten rotationsspektroskopischen Ergebnissen in Einklang zu bringen wäre, gemäß denen $\alpha$-Fenchol nur im $g+$-Zustand beobachtet wurde. Die Gruppe um Pate basiert ihre Zuordnung auf die gute Übereinstimmung der experimentellen Rotationskonstanten mit den berechneten für $g+$ und erklärt die Abwesenheit weiterer Konformere mit der starken Kühlung in der eingesetzten Neon-Expansion in Verbindung mit der geringen Barriere.[263] Die g--Alternative wurde dabei nicht näher betrachtet. Die Grup-
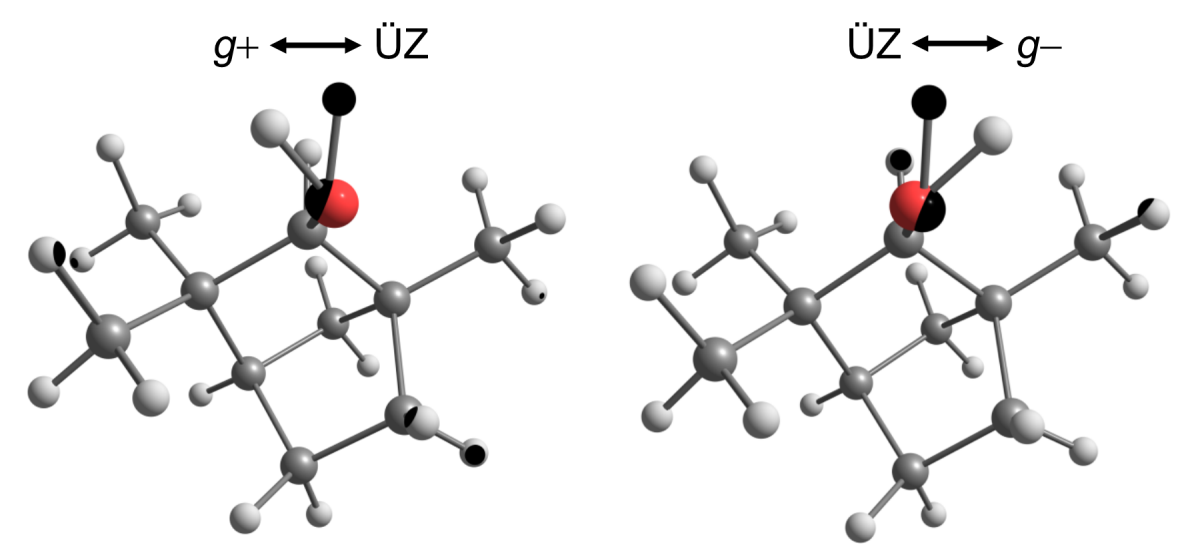

Abb. 5.25: Überlagerungen der (-)- $\alpha$-Fenchol-Konformere $g+$ und $g-$ jeweils mit dem Übergangszustand. Atome des Übergangszustandes sind schwarz dargestellt und werden aufgrund ihrer kleiner gewählten Radien bei Abweichungen $<0.02 \AA$ vollständig verdeckt. 


\section{Torsionsisomerie von Alkoholen}

pe um Huet verweist dagegen darauf, dass es nicht möglich ist anhand der Rotationskonstanten und der Dipolmomentkomponenten zwischen $g+$ und $g-$ zu unterscheiden. Dies ist darin begründet, dass die Konformere sich nur durch eine Verlagerung eines leichten Wasserstoffatoms relativ zu dem massereichen starren Gerüst von $\alpha$-Fenchol unterscheiden. Dieser Einfluss liegt im Bereich typischer Unsicherheiten beim Vergleich zwischen Experiment und Theorie, die u. a. in der Nicht-Berücksichtigung von Nullpunktsschwingungen begründet sind. Die Zuordnung zu $g+$ stützt sich wesentlich auf die Untersuchung von $\alpha$-Fenchol-OD und der daraus abgeleiteten Substitutionsstruktur sowie den Quadrupol-Konstanten des Deuterons. Für $\alpha$-Fenchol-OD stellt sich die Frage, inwieweit dessen Struktur der von $\alpha$-Fenchol-OH entspricht, denn die Annahme einer isotopenunabhängigen Struktur ist Grundlage des Substitutionsverfahrens [307]. Isotopeneffekte auf die Nullpunktsschwingungen und besonders Large Amplitude Motions, wie die Tunnelbewegung, können hierbei zu Anomalien führen.[308]

Die höhere Masse des Deuterons reduziert die Tunnelwahrscheinlichkeit deutlich. Für ein symmetrisches Potential mit der berechneten mittleren Barrierenhöhe von $\alpha$-FencholOD wäre gemäß der Interpolation nach Abb. 5.23 nur noch eine Tunnelaufspaltung von $h c 4-5 \mathrm{~cm}^{-1}$ zu erwarten, auch eine Abschätzung gemäß dem Isotopeneffekt in Abb. 5.24 führt zu einem vergleichbaren Wert. Die Wahrscheinlichkeit ist daher für $\alpha$-Fenchol-OD deutlich höher, dass die Struktur durch die Asymmetrie weitgehend lokalisiert wird. Aber auch die Asymmetrie selbst kann durch Schwingungsnullspunktsenergieeffekte verändert werden. Die von Neeman und Huet gefundene Substitutionsstruktur gleicht bezüglich der O-H-Bindungslänge stärker der von $g+$, ist aber auch für $g-$ nicht völlig abwegig. Dies wäre grundsätzlich auch mit einer delokalisierten $\alpha$-Fenchol-OH-Struktur kompatibel, mit einem größeren Anteil von $g+$ in der Wellenfunktion des delokalisierten Grundzustandes. Schließlich schlagen Neeman und Huet noch eine weitere Hypothese für die Nicht-Beobachtung von $g$ - vor: der zugehörige Potentialtopf könnte überhaupt keinen stabilen Zustand aufweisen. Allerdings erscheint die hierfür genannte Begründung auf Grundlage der imaginären Frequenz des Übergangszustandes nicht nachvollziehbar. Zusammengefasst bestätigen die rotationsspektroskopischen Ergebnisse die bereits quantenchemisch gefundene Präferenz für $g+$, erlauben aber keine Aussage darüber, ob die Lokalisierung in $g+$ vollständig oder unvollständig erfolgt. Beides wäre mit den schwingungsspektroskopischen Daten vereinbar, sofern stattdessen (oder auch zusätzlich) im OH-Streckschwingungsangeregten Zustand eine Tunnelaufspaltung existiert.

Unklar bleibt schließlich noch die Zuordnung der schwachen niederfrequenten Bande bei $3646 \mathrm{~cm}^{-1}$. Zwei naheliegende Möglichkeiten können aber ausgeschlossen werden. Die erste Möglichkeit wäre eine Dimer-Akzeptorbande. Das in den Filet-Jet-Spektren intensive Dimer-Donor-Signal bei $3499 \mathrm{~cm}^{-1}$ wird auch in den Curry-Jet-Spektren beobachtet, allerdings dort deutlich schwächer (Abb. 5.26). Das Verhältnis von Dimer zu Monomer nimmt mit steigendem Detektionsabstand zu. Im Monomer-Bereich findet sich keine Bande mit der selben Abhängigkeit. Die der Donorbande zugeordnete Struktur (Kap. 7) hat ein berechnetes Verhältnis der Raman-Streuquerschnitte von Donor zu Akzeptor von etwa 4:1, sodass die Akzeptorbande noch deutlich weniger intensiv zu erwarten ist. Eine Anwendung des Korrelationsmodells auf die Akzeptorwellenzahl lässt eine Position um $3650 \mathrm{~cm}^{-1}$ vermuten. Die Akzeptorbande könnte die gegenläufige Abstandsabhängigkeit des dort zugeordneten heißen Überganges etwas verfälschen. Eine Zuordnung zur 
unerklärten Bande bei $3646 \mathrm{~cm}^{-1}$ ist wegen der unterschiedlichen Abhängigkeiten vom Detektionsabstand auszuschließen.

Eine zweite Möglichkeit wäre eine Verunreinigung mit $\beta$-Fenchol, welches rotationsspektroskopisch in kommerziellen Proben von $\alpha$-Fenchol nachgewiesen wurde.[262, 263] $\beta$-Fenchol, auch exo-Fenchylalkohol genannt, ist ein Epimer von $\alpha$-Fenchol, bei dem die Konfiguration am $\alpha$-Kohlenstoffatom invertiert ist. Die Konfigurationen der anderen Stereozentren sind unverändert, sodass $\beta$ - und $\alpha$-Fenchol sich diastereomer zueinander verhalten. Die Diastereomerenreinheit der eingesetzten Probe wird vom Hersteller im Analysenzertifikat mit $98.5 \%$ angegeben, sodass potentiell bis zu $1.5 \% \beta$-Fenchol vorhanden sein könnte. Die berechneten Eigenschaften der drei zu erwartenden Konformere von $\beta$-Fenchol sind in Abb. 5.27 gezeigt.

Im Gegensatz zu $\alpha$-Fenchol bestehen für $\beta$-Fenchol größere Energieunterschiede zwischen den Konformeren, sodass bei diesem Molekül keine Tunnelaufspaltung und damit

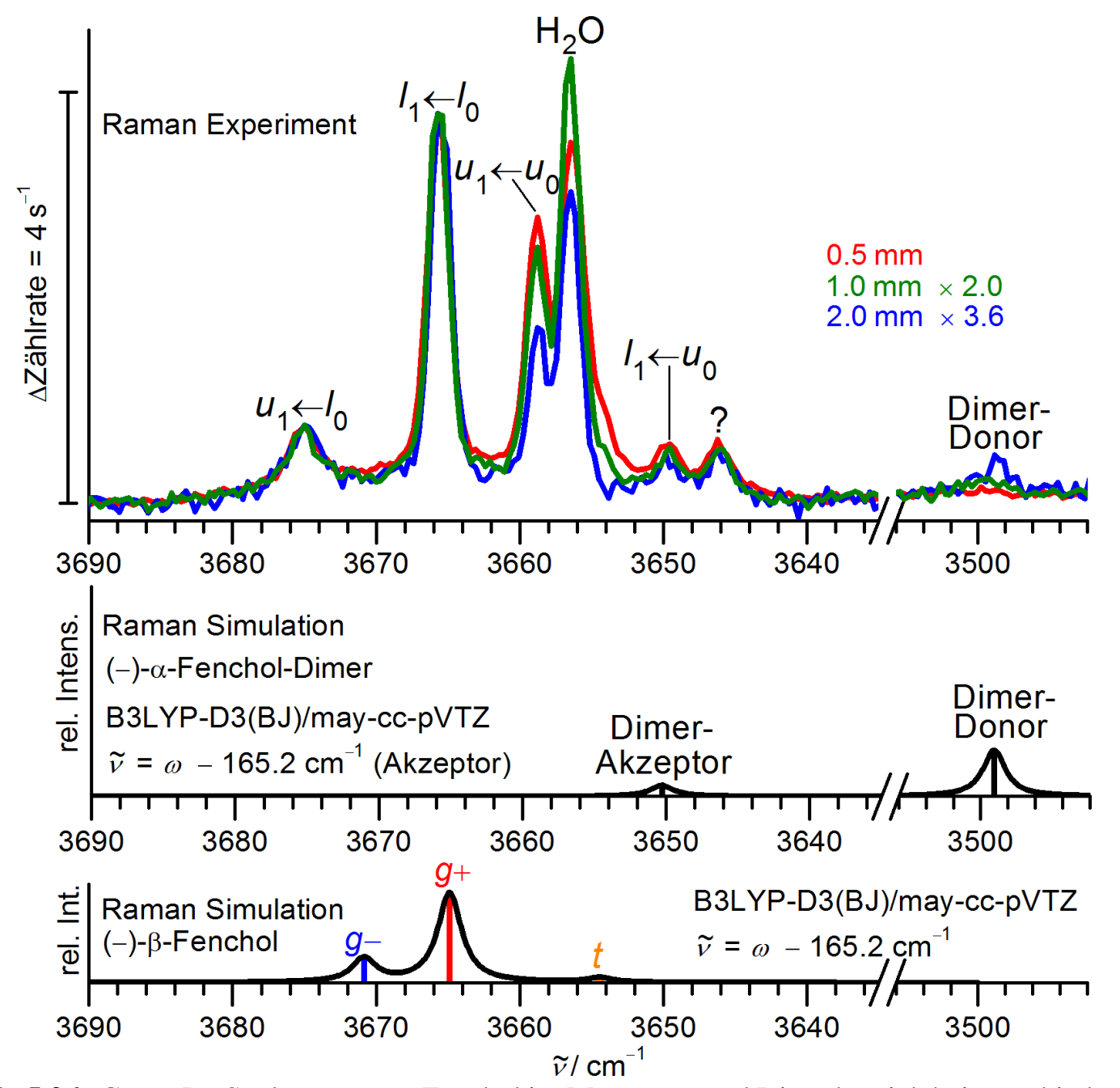

Abb. 5.26: Curry-Jet-Spektren von $\alpha$-Fenchol im Monomer- und Dimerbereich bei verschiedenen Detektionsabständen mit tentativen Bandenzuordnungen. Die Spektren wurden mit den angegebenen Faktoren auf die gleiche Maximalintensität der Bande bei $3665.5 \mathrm{~cm}^{-1}$ skaliert. Simulationsparameter sind einheitliche Verschiebung der harmonischen Monomer- und Dimer-AkzeptorWellenzahlen um $\kappa=-165.2 \mathrm{~cm}^{-1}$, der Dimer-Donor-Wellenzahl auf $3499 \mathrm{~cm}^{-1}$ eine Konformationstemperatur von $100 \mathrm{~K}$ und Lorentz-Profile mit FWHM $=2 \mathrm{~cm}^{-1}$. 


$g+$
$0 \mathrm{~kJ} \mathrm{~mol}^{-1}$
$3830 \mathrm{~cm}^{-1}$
$96 \AA^{4} \mathrm{u}^{-1}$

0.22

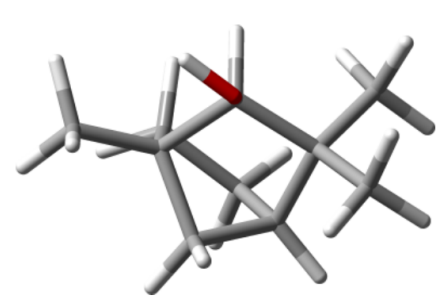

g-

$1.0 \mathrm{~kJ} \mathrm{~mol}^{-1}$

$3836 \mathrm{~cm}^{-1}$

$87 \AA^{4} u^{-1}$

0.21

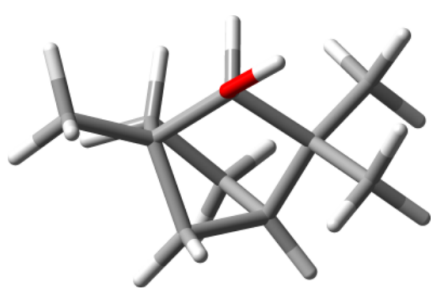

$t$

$1.7 \mathrm{~kJ} \mathrm{~mol}^{-1}$

$3820 \mathrm{~cm}^{-1}$

$37 \AA^{4} u^{-1}$

0.12

Abb. 5.27: Die drei Konformere des (-)- $\beta$-Fenchols, klassifiziert nach der Einstellung des $\mathrm{HCOH}-$ Torsionswinkels, mit relativen nullpunktskorrigierten Energien, $\mathrm{OH}-$ Streckschwingungswellenzahlen, Raman-Aktivitäten und Depolarisationsverhältnissen auf B3LYP-D3(BJ)/may-cc-pVTZ-Niveau. Die Blickrichtung auf die Konformere erfolgt entlang der $\mathrm{O}-\mathrm{C}-$ Bindung.

eine bessere Gültigkeit des Korrelationsmodells zu erwarten ist. Das hierzu simulierte Spektrum ist im unteren Teil von Abb. 5.26 dargestellt. Die dominante Bande des $g+-$ Konformers von $\beta$-Fenchol ist bei etwa $3665 \mathrm{~cm}^{-1}$ zu erwarten. Falls $\beta$-Fenchol vorhanden ist, so wird diese Bande daher wohl von der intensiven Bande von $\alpha$-Fenchol an dieser Position verdeckt. Im niederfrequenten Bereich unter $3650 \mathrm{~cm}^{-1}$ ist auch für $\beta$-Fenchol keine Bande zu erwarten, sodass eine Verunreinigung mit dieser Substanz auch nicht als Erklärung für die Bande bei $3646 \mathrm{~cm}^{-1}$ dienen kann.

Schließlich ist auch eine Zuordnung zu dem $t$-Konformer von $\alpha$-Fenchol denkbar, hierfür müsste aber eine größere Abweichung von $-8 \mathrm{~cm}^{-1}$ von dem vom Korrelationsmodell vorhergesagten Wert vorliegen. Die Zuordnung dieser Bande bleibt daher auf Grundlage der vorliegenden Daten unklar.

Zusammengefasst zeigt $\alpha$-Fenchol unerwartet komplexe Schwingungsspektren im $\mathrm{OH}-$ Streckschwingungsbereich, die durch harmonische Rechnungen nicht beschrieben werden können. Einige Indizien für eine Tunnelaufspaltung, trotz der Asymmetrie des Moleküls, wurden zusammengetragen. Weitere Messungen zur Absicherung dieser Hypothese sind notwendig. Vorgeschlagen sei hierfür die Untersuchung von $\alpha$-Fenchol-OH in anderen Spektralbereichen und von $\alpha$-Fenchol-OD im OD-Streckschwingungsbereich. Möglich wäre auch das Einbringen von $\alpha$-Fenchol in Matrizen oder in Helium-Nanotröpfchen, um die Asymmetrie weiter zu erhöhen und so womöglich die lokalisierten Übergänge beobachten zu können (allerdings mit zusätzlichen Bandenverschiebungen durch die Umgebungseinflüsse). Schließlich wäre auch die rotationsspektroskopische Untersuchung in wärmeren Expansionen interessant, in denen auch der oberere Tunnelzustand (oder alternativ das zweite Konformer) populiert ist. 


\subsection{Pinakolylalkohol}

Pinakolylalkohol, auch 3,3-Dimethyl-2-butanol oder tert-Butylmethylcarbinol genannt, ist ein chiraler, sekundärer Alkohol, dessen Struktur in Abb. 5.28 gezeigt ist. Pinakolylalkohol ist ein direktes Edukt für den Nervenkampfstoff Soman und steht daher nach Liste 2 der Chemiewaffenkonvention unter besonderer Regulierung und Beobachtung. Auch bei der Hydrolyse von Soman wird die Verbindung wieder frei. Da Pinakolylalkohol sonst kaum Anwendung findet und auch nicht natürlich vorkommt [309], ist dessen Detektion von besonderem Interesse, da der Nachweis ein wichtiger Indikator für die Herstellung oder den Einsatz von Soman ist [310, 311].

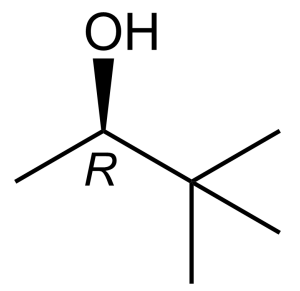

Abb. 5.28: Strukturformel von (-)-(R)-Pinakolylalkohol.

Da Pinakolylalkohol kommerziell nur als racemisches Gemisch verfügbar ist, ist es für die Chiralitätserkennung in dieser Arbeit weniger geeignet. Allerdings stellt Pinakolylalkohol grob einen offenkettigen Ausschnitt aus der Struktur der bicyclischen Monoterpenalkohole dar. Das mit den (-)-Enantiomeren der Monoterpenalkohole strukturell am ehesten vergleichbare Enantiomer von Pinakolylalkohol ist ebenfalls linksdrehend und hat $(R)$ Konfiguration [312]. Für Pinakolylalkohol sind ebenso drei Konformere zu erwarten, die in Abb. 5.29 mit ihren wichtigsten berechneten spektralen Eigenschaften gezeigt sind.
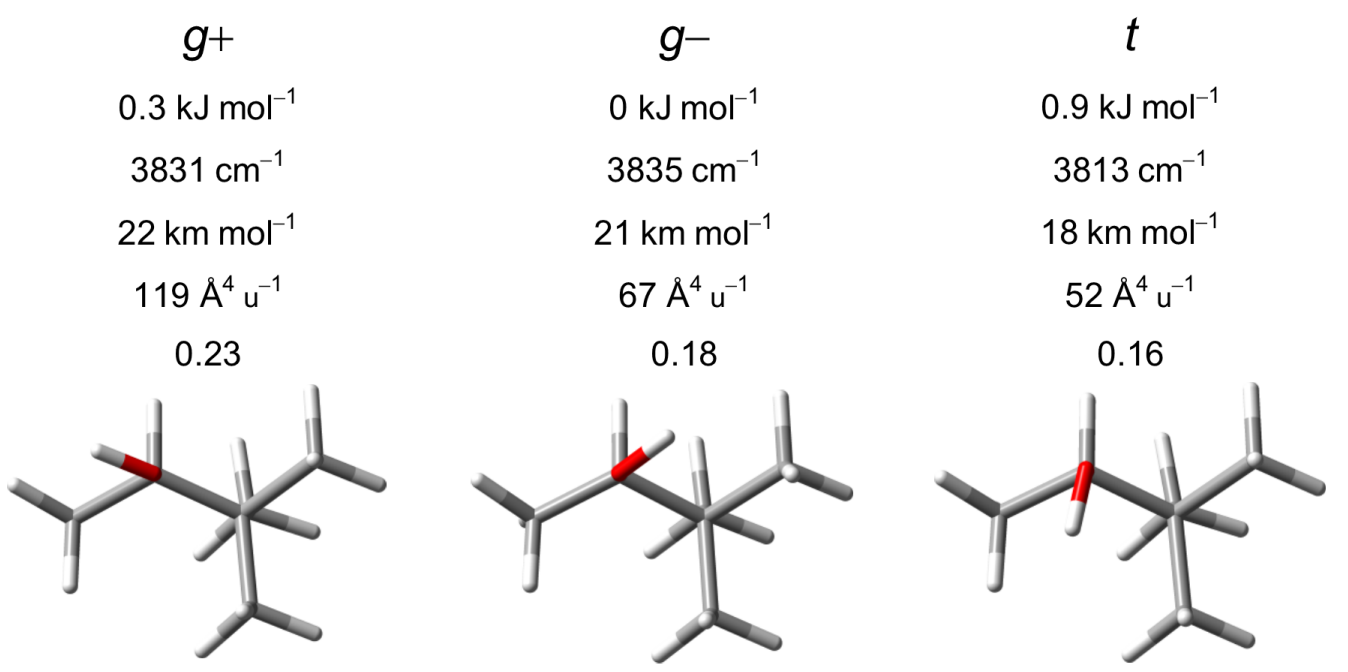

Abb. 5.29: Die drei Konformere des (-)-Pinakolylalkohols, klassifiziert nach der Einstellung des $\mathrm{HC}-\mathrm{OH}-$ Torsionswinkels, mit relativen Energien, harmonischen $\mathrm{OH}-$ Streckschwingungswellenzahlen, IR- und Raman-Aktivitäten sowie Depolarisationsverhältnissen auf B3LYP-D3(BJ)/may-cc-pVTZ-Niveau. Die Blickrichtung auf die Konformere erfolgt entlang der O-C-Bindung. 


\section{Torsionsisomerie von Alkoholen}

Eine Übersicht über den OH-Streckschwingungsbereich bieten die Filet-Jet-Spektren in Abb. 5.30. Diese zeigen eine Monomerbande bei $3666 \mathrm{~cm}^{-1}$ und eine Dimerbande bei $3514 \mathrm{~cm}^{-1}$. Eine Detailansicht der Spektren im Monomerbereich ist in der oberen Hälfte in Abb. 5.30 dargestellt.

Die experimentelle Monomerbande ist in den Filet-Jet-Spektren auffällig breit (FWHM $=8 \mathrm{~cm}^{-1}$ ), sowohl im Vergleich zur Dimerbande, wie auch zu den Banden einzelner Konformere anderer hier untersuchter monomerer Alkohole. Dies wäre mit der in der Simulation vorhergesagten Überlagerung von $g-$ und $g+$ vereinbar, wobei der Schwerpunkt der Bande vom Korrelationsmodell um etwa $2 \mathrm{~cm}^{-1}$ überschätzt werden würde. In den FiletJet-Spektren gibt es keinen Hinweis auf das $t$-Konformer, im Gasphasen-Spektrum zeigt sich aber eine Schulter bei $3645 \mathrm{~cm}^{-1}$, die gut mit der vorhergesagten Position vereinbar ist.

Um die breite Monomerbande aufzulösen wurde ein Curry-Jet-Spektrum aufgenommen, welches im unteren Teil von Abb. 5.31 mit einer Simulation verglichen ist. Gemäß der quantenchemischen Vorhersage sollte Ramanspektroskopisch $g+$ leicht intensiver erscheinen, da dessen fast doppelt so hohe Raman-Aktivität den Energienachteil überkompensieren sollte. Dagegen werden hier aber nicht zwei ähnlich intensive, sondern eine einzelne sehr intensive Bande bei $3666 \mathrm{~cm}^{-1}$ (wie mit FTIR-Detektion) sowie zwei unvollständig aufgelöste, schwache Satelliten bei $3669 \mathrm{~cm}^{-1}$ und $3663 \mathrm{~cm}^{-1}$ beobachtet. Eine mögliche Interpretation wäre, dass die Wellenzahldifferenz zwischen $g+$ und $g$ - überschätzt wird und deren Übergänge stattdessen bei $3666 \mathrm{~cm}^{-1}$ koinzidieren. Als Erklärung für die Satelliten könnten dann heiße Übergange nach thermischer Anregung einer niederfrequenten Mode, eine Dimer-Akzeptorschwingung und/oder einer Verunreinigung in Frage kommen.

Alternativ könnte auch die Energiedifferenz beider Konformere deutlich größer als berechnet sein, sodass einer der Satelliten dem instabileren Konformer zugeordnet werden könnte. Punktrechnungen auf CCSD(T)/aug-cc-pVQZ*//B3LYP-D3(BJ)/may-cc-pVTZNiveau sprechen aber gegen dieses Szenario. Diese sagen stattdessen eine Verringerung der Energiedifferenz von $0.26 \mathrm{~kJ} \mathrm{~mol}^{-1}$ auf nur noch $0.06 \mathrm{~kJ} \mathrm{~mol}^{-1}$ voraus. Die relative Energie des $t$-Konformers steigt von 0.7 auf $1.2 \mathrm{~kJ} \mathrm{~mol}^{-1}$. Dies würde erklären, warum

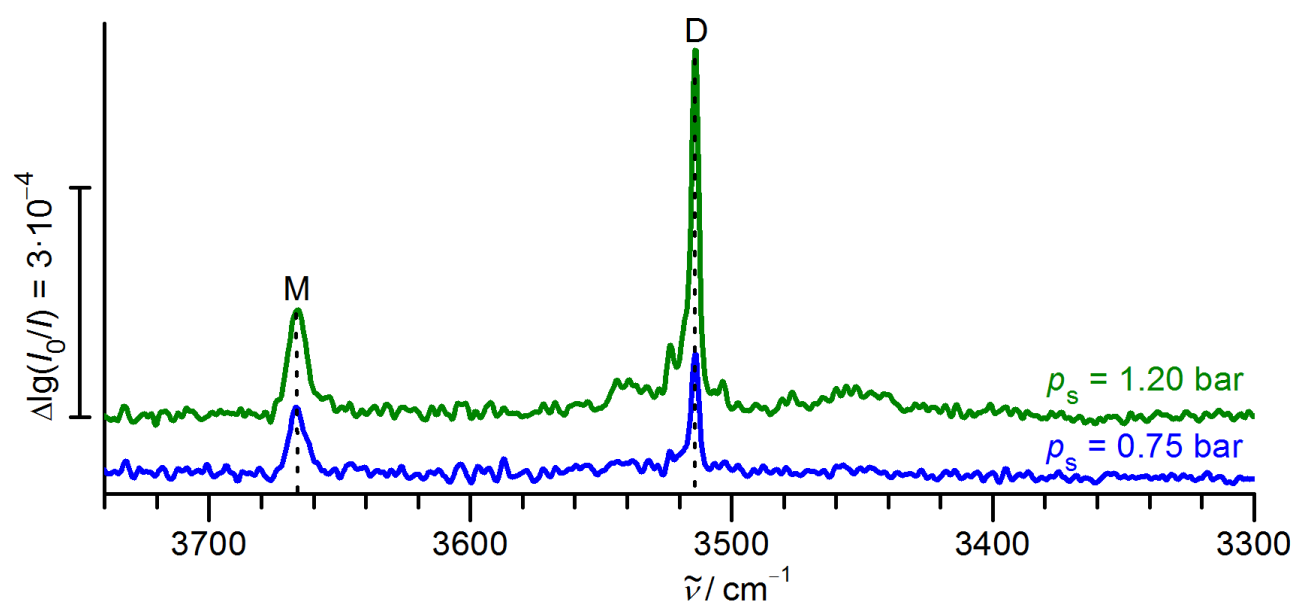

Abb. 5.30: Übersicht der Filet-Jet-Spektren von Pinakolylalkohol im OH-Streckschwingungsbereich bei zwei verschiedenen Stagnationsdrücken mit tentativen Bandenzuordnungen. 


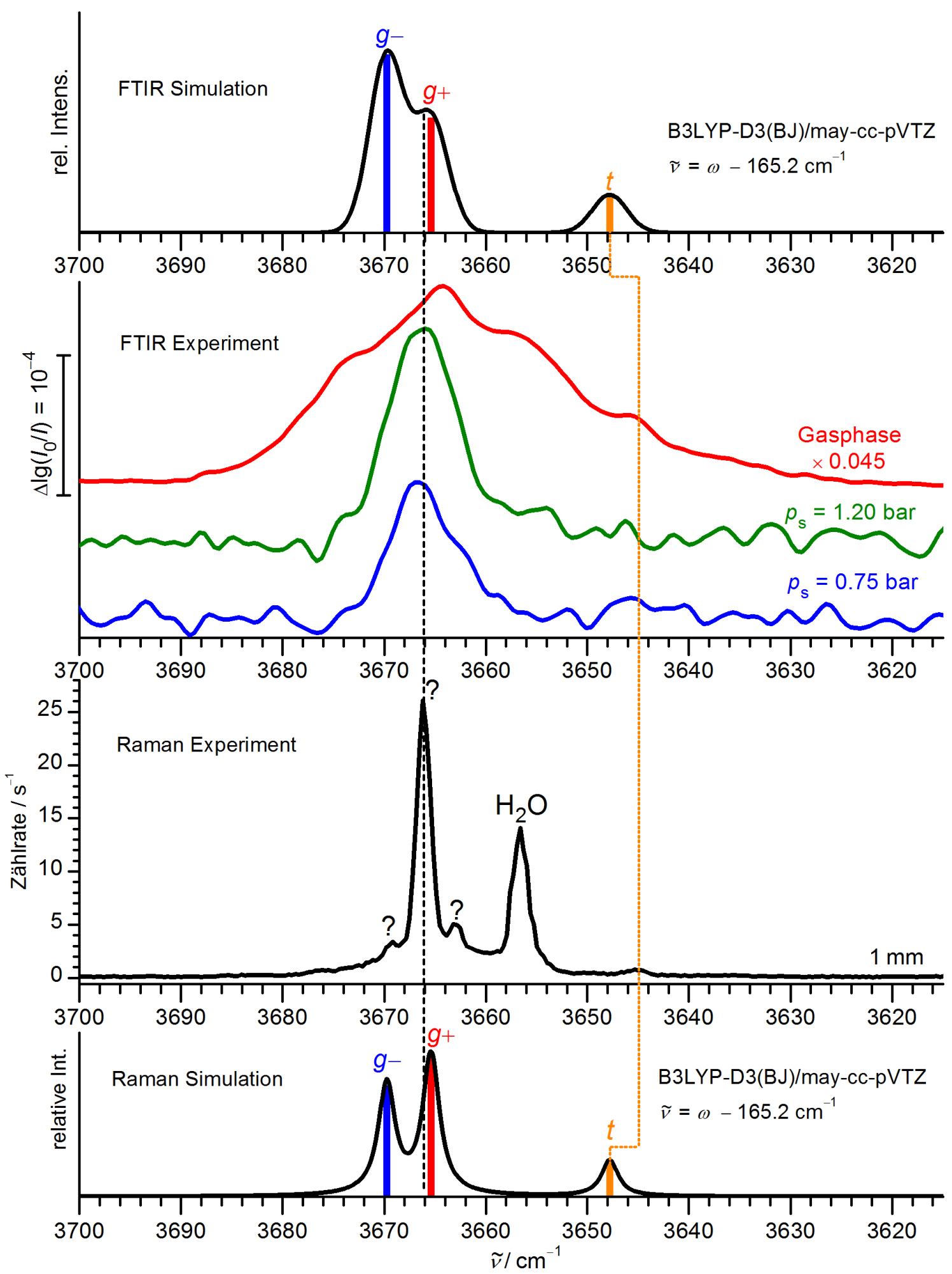

Abb. 5.31: Detailansicht von Filet-Jet-Spektren von Pinakolylalkohol im Monomerbereich bei verschiedenen Stagnationsdrücken und in der Gasphase bei Zimmertemperatur, sowie Curry-JetSpektrum von Pinakolylalkohol bei $1 \mathrm{~mm}$ Detektionsabstand. Dem gegenübergestellt sind Simulationen. Simulationsparameter sind einheitliche Verschiebung der harmonischen Wellenzahlen um $\kappa=-165.2 \mathrm{~cm}^{-1}$, eine Konformationstemperatur von $64 \mathrm{~K}$ für das IR- und $100 \mathrm{~K}$ für das RamanSpektrum, sowie Gauss-Profile mit FWHM $=4 \mathrm{~cm}^{-1}$ für das IR- und Lorentz-Profile mit FWHM $=2 \mathrm{~cm}^{-1}$ für das Raman-Spektrum. 


\section{Torsionsisomerie von Alkoholen}

nur eine sehr schwache Bande bei $3645 \mathrm{~cm}^{-1}$ beobachtet wird, in gleicher Position wie die Schulter im IR-Gasphasenspektrum, die $t$ zugeordnet wird.

Eine weitere, stärker spekulative Interpretation des Bandenmusters wäre eine Delokalisation des Hydroxyprotons auch bei Pinakolylalkohol. Die mittlere Barriere im adiabatischen Kanal des Schwingungsgrundzustandes ist mit $h c 350 \mathrm{~cm}^{-1}$ höher als für $\alpha$-Fenchol ( $h c 230 \mathrm{~cm}^{-1}$ ), würde im symmetrischen Fall gemäß dem Barrierenhöhemodell aber noch eine Tunnelaufspaltung von etwa $h c 5 \mathrm{~cm}^{-1}$ erwarten lassen. Dies lässt weniger Spielraum für eine tolerierte Asymmetrie, die auf dem höchsten Niveau aber ebenso groß mit $0.06 \mathrm{~kJ} \mathrm{~mol}^{-1}=h c 5 \mathrm{~cm}^{-1}$ berechnet wird. Durch die um $4 \mathrm{~cm}^{-1}$ größer vorhergesagte $\mathrm{OH}-$ Streckschwingung von $g-$ würde sich die Asymmetrie im angeregten Zustand sogar auf $h c 1 \mathrm{~cm}^{-1}$ verringern. Grob abgeschätzt, basierend auf der OH-Streckschwingung des Übergangszustandes von $3872 \mathrm{~cm}^{-1}$, erhöht sich die mittlere Barriere im schwingungsangeregten Zustand auf $390 \mathrm{~cm}^{-1}$ mit einer verringerten potentiellen Tunnelaufspaltung von $h c 3 \mathrm{~cm}^{-1}$. Eine teilweise Delokalisation wäre daher im Grundzustand und/oder im angeregten Zustand nicht völlig abwegig. Dies würde weitere Übergänge erlauben. Eine mögliche Interpretation wäre dann, dass die Aufspaltung im Grund- und angeregten Zustand fast identisch ist (so wie es auch für Ethanol vermutet wird [22]), sodass die intensiven $l_{1} \leftarrow l_{0}$ und $u_{1} \leftarrow u_{0}$ Übergänge in der Bande bei $3666 \mathrm{~cm}^{-1}$ zusammenfallen. Die Satelliten würden in diesem Szenario dann den schwächer erlaubten $u_{1} \leftarrow l_{0}\left(3669 \mathrm{~cm}^{-1}\right)$ und $l_{1} \leftarrow u_{0}\left(3666 \mathrm{~cm}^{-1}\right)$ Übergängen entsprechen. Dies ist in Abb. 5.32 dargestellt. Der Schwerpunkt der vier Banden bei $3666 \mathrm{~cm}^{-1}$ würde gut dem erwarteten Schwerpunkt der lokalisierten $g$ - und $g+$-Übergänge nach dem Korrelationsmodell bei $3668 \mathrm{~cm}^{-1}$ entsprechen (etwas überschätzt, wie bei den Terpenalkoholen).

Zwischen diesen vorgeschlagenen Szenarien kann auf Grundlage der vorhandenen Daten nicht entschieden werden, hier wären weitere Messungen mit systematisch variierten Expansionsbedingungen notwendig. Die Aufklärung erscheint wegen den kleineren Aufspaltungen schwieriger als für $\alpha$-Fenchol, da hierdurch die thermischen Effekte geringer ausfallen und die Banden nur unvollständig aufgelöst werden können. 

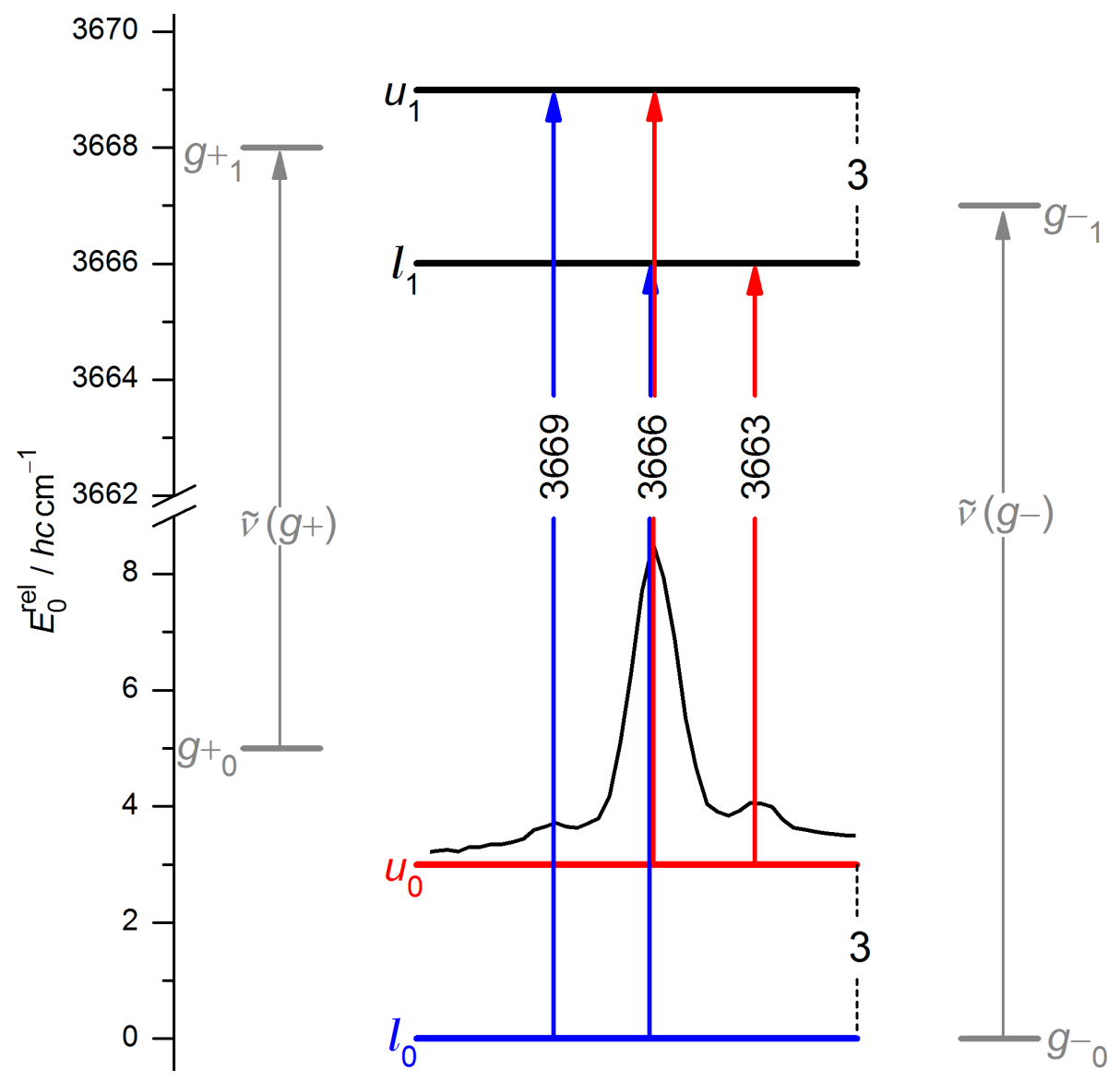

Abb. 5.32: Spekulatives Energieniveauschema für OH-Streckschwingungsübergänge im $g$ Doppelminimumpotential von (-)-Pinakolylalkohol. Der Grundzustand $l_{0}$ und daraus stattfindende Übergänge sind blau dargestellt, der erste angeregte Zustand $u_{0}$ und daraus stattfindende heiße Übergänge sind rot markiert. Vorhersagen für die lokalisierten $g$-Zustände sind in grau gehalten, die dafür verwendeten Rechenergebnisse, $\left(g+_{0}\right)-(g-0)=h c 5 \mathrm{~cm}^{-1}$ und $\omega(g-)-\omega(g+)=$ $4 \mathrm{~cm}^{-1} \approx \tilde{v}(g+)-\tilde{v}(g-)$, wurden auf CCSD(T)/aug-cc-pVQZ*//B3LYP-D3(BJ)/may-cc-pVTZNiveau berechnet. Der Index 0 bzw. 1 gibt die Quantenzahl der OH-Streckschwingung an. 


\subsection{2 tert-Butyldimethylcarbinol}

tert-Butyldimethylcarbinol (systematisch: 2,3,3-Trimethylbutan-2-ol) ist ein tertiärer Alkohol, der sich von Ethanol ableitet, wenn jedes an Kohlenstoff gebundene Wasserstoffatom durch eine Methylgruppe ersetzt wird. Entsprechend ist auch für tertButyldimethylcarbinol eine $t$ - und einer entartete $g$-Konformation $\mathrm{zu}$ erwarten (Abb. 5.33).
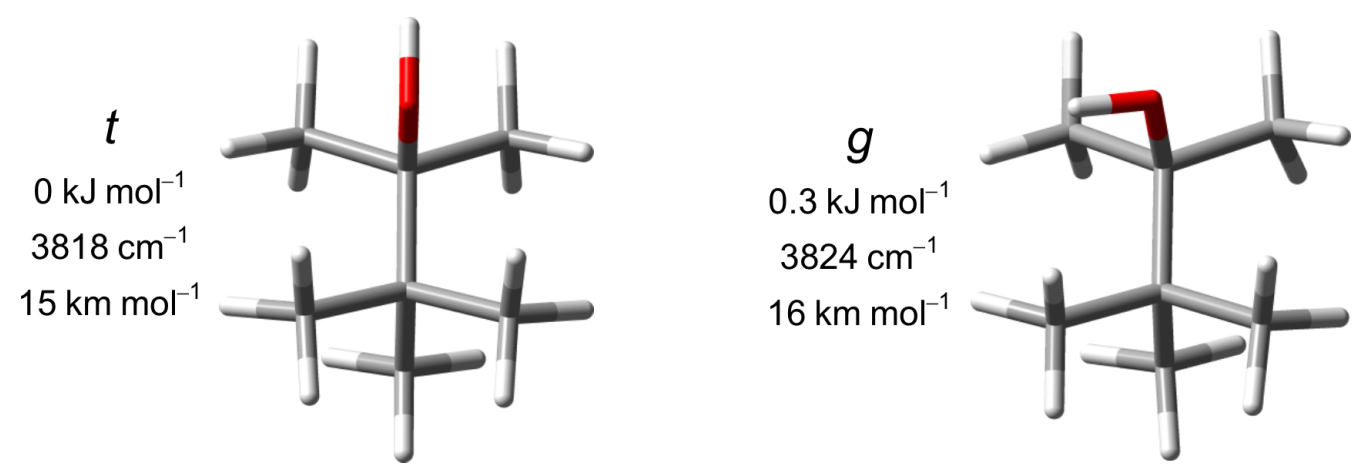

Abb. 5.33: Die beiden Konformere des tert-Butyldimethylcarbinol auf B3LYP-D3(BJ)/may-ccpVTZ-Niveau mit relativen Energien, harmonischen $\mathrm{OH}-$ Streckschwingungswellenzahlen und IRAktivitäten.

Alternativ lässt sich die Verbindung auch formal durch Methylierung am $\alpha$ Kohlenstoffatom aus Pinakolylalkohol erhalten. tert-Butyldimethylcarbinol wurde von Häber et al. [68, 127] und Zimmermann [313] am Ragout-Jet (dem Vorläufer des Filet-Jets) untersucht. Dabei wurde im Monomerbereich eine einzelne Bande bei $3650 \mathrm{~cm}^{-1}$ beobachtet (Abb. 5.34). Diese wurde später von Cézard et al. [235] dem $g$-Konformer zugeordnet, offenbar auf Grundlage der relativen Energie aus AMBERKraftfeldrechnungen. Auf B3LYP-D3(BJ)/may-cc-pVTZ-Niveau ist dagegen $t$ um $0.3 \mathrm{~kJ} \mathrm{~mol}^{-1}$, auf CCSD(T)/aug-cc-pVQZ*//B3LYP-D3(BJ)/may-cc-pVTZ-Niveau um $0.1 \mathrm{~kJ} \mathrm{~mol}^{-1}$ stabiler. Die Korrektur der auf B3LYP-D3(BJ)/may-cc-pVTZ berechneten Wellenzahlen mit dem Korrelationsmodell um $\kappa=-167.6 \mathrm{~cm}^{-1}$ für tertiäre Alkohole lassen die Absorption des $g$-Konformers bei $3656 \mathrm{~cm}^{-1}$ und die des $t$-Konformers bei $3650 \mathrm{~cm}^{-1}$ erwarten. Die Zuordnung des $t$-Konformers zu der experimentellen Bande bei $3650 \mathrm{~cm}^{-1}$ wäre daher gemäß dem Korrelationsmodell deutlich plausibler. Bezüglich der vorhergesagten Intensität lässt sich hier keine Präferenz erhalten: das $t$-Konformer wird zwar geringfügig stabiler berechnet, allerdings würde dies durch den statistischen Vorteil des $g$-Konformers in etwa kompensiert werden. Es stellt sich daher die Frage, warum nur eine einzelne Bande beobachtet wird. Auch das Zimmertemperatur-Gasphasenspektrum bietet keinen Hinweis auf ein zweites Konformer: dieses hat nur ein einzelnes Maximum an der gleichen Position wie im Jet und eine symmetrische Form. Die naheliegendste Erklärung wäre, dass verschiedene Übergänge innerhalb der Auflösung zusammenfallen, wie auch bei Pinakolylalkohol vermutet. Diese Abweichung von der harmonischen Vorhersage könnte im Torsionspotential begründet sein, bei dem eine ähnliche Situation wie bei Ethanol vorhergesagt wird: $t$ ist etwas stabiler als $g$ und die $g \leftrightarrow t$-Barriere ist niedriger als die $g \leftrightarrow g$-Barriere. Im Vergleich zu Ethanol ist die $g \leftrightarrow t$-Barriere zudem niedriger ( $h c 290 \mathrm{~cm}^{-1}$ gegenüber $h c 360 \mathrm{~cm}^{-1}$ ), sodass eine deutliche Tunnelaufspaltung zu erwarten ist, möglicherweise wegen des geringen Energieunterschiedes auch mit Ein- 


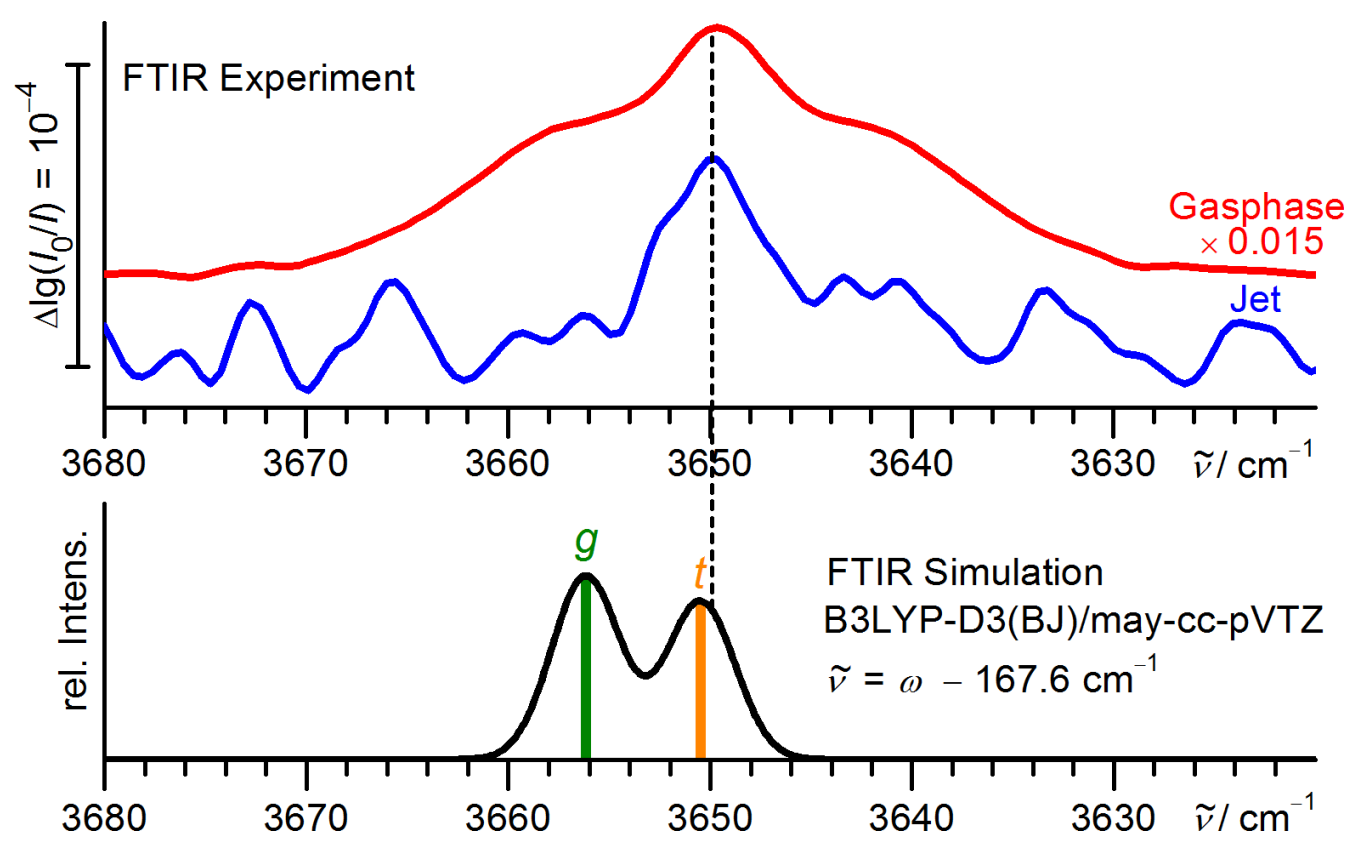

Abb. 5.34: Vergleich zwischen einem Ragout-Jet-Spektrum, einem Gasphasen-Spektrum bei Zimmertemperatur und eine Simulation für tert-Butyldimethylcarbinol. Die experimentellen Spektren wurden von Häber $[68,127]$ übernommen. Simulationsparameter sind einheitliche Verschiebung der harmonischen Wellenzahlen um $\kappa=-167.6 \mathrm{~cm}^{-1}$, eine Konformationstemperatur von $64 \mathrm{~K}$ und Gauss-Profile mit FWHM $=4 \mathrm{~cm}^{-1}$.

fluss auf die $t$-Wellenfunktion. Wegen dieser Unklarheiten wurde die Literaturzuordnung von $g$ bei $3650 \mathrm{~cm}^{-1}$ nicht in das Trainingsset des Korrelationsmodells übernommen. Möglicherweise könnten zukünftige Messungen am Curry-Jet weitere Übergänge oder eine Aufspaltung der im IR beobachteten Bande nachweisen. 


\subsection{3 tert-Butylcarbinol}

tert-Butylcarbinol, auch 2,2-Dimethylpropan-1-ol oder Neopentylalkohol genannt, ist ein primärer Alkohol, der sich von Ethanol ableitet, wenn die drei Wasserstoffatome der Methylgruppe durch weitere Methylgruppen ersetzt werden. Wie für Ethanol und tertButyldimethylcarbinol sind entsprechend ein $t$ - und ein zweifach entartetes $g$-Konformer zu erwarten (Abb. 5.35).

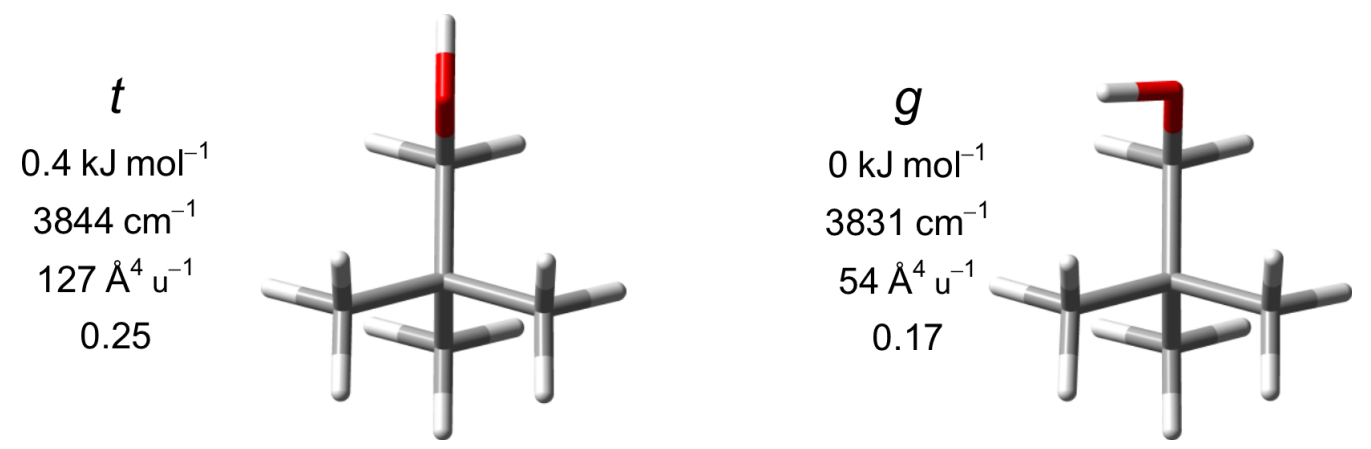

Abb. 5.35: Die beiden Konformere des tert-Butylcarbinol auf B3LYP-D3(BJ)/may-cc-pVTZNiveau mit relativen Energien, harmonischen $\mathrm{OH}-$ Streckschwingungswellenzahlen, RamanAktivitäten und Depolarisationsverhältnissen.

Die Verbindung wurde bereits intensiv durch Xue [236] am Filet- und Curry-Jet studiert. In den Raman-Spektren wurden drei intensive Banden bei 3692, 3676 und $3668 \mathrm{~cm}^{-1}$ beobachtet (Abb. 5.36). Durch Konzentrationsvariation konnten die weniger intensiven Signale um $3659 \mathrm{~cm}^{-1}$ als Dimer-Akzeptorbanden zugeordnet werden. Die Diskrepanz zwischen der Zahl von drei Banden und zwei erwarten diastereomeren

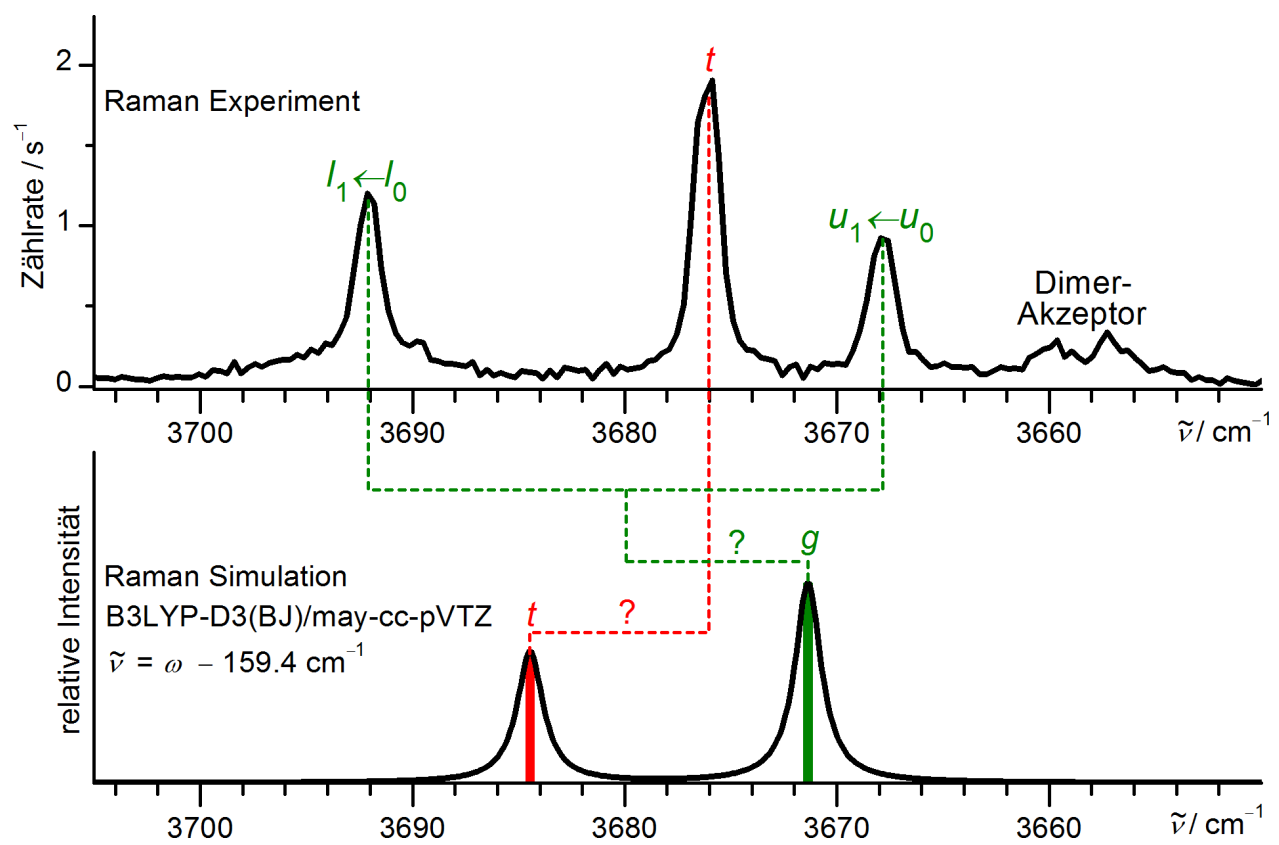

Abb. 5.36: Curry-Jet-Spektrum von tert-Butylcarbinol, übernommen zusammen mit der Interpretation von Xue [236]. Im Vergleich dazu ist eine Simulation mit dem Korrelationsmodell dargestellt. Simulationsparameter sind einheitliche Verschiebung der harmonischen Wellenzahlen um $\kappa=-159.4 \mathrm{~cm}^{-1}$, eine Konformationstemperatur von $100 \mathrm{~K}$ und Lorentz-Profile mit FWHM = $1.5 \mathrm{~cm}^{-1}$. 
Konformeren deutet klar auf eine Tunnelaufspaltung hin. Durch Vergleich mit Spektren der deuterierten Verbindung wurde die Bande bei $3676 \mathrm{~cm}^{-1}$ dem Übergang des $t$-Konformers, die Bande bei $3692 \mathrm{~cm}^{-1}$ dem $l_{1} \leftarrow l_{0}$-Übergang und die Bande bei $3668 \mathrm{~cm}^{-1}$ dem $u_{1} \leftarrow u_{0}$-Übergang zugeordnet. Dies würde eine Verringerung der Tunnelaufspaltung um $h c 24 \mathrm{~cm}^{-1}$ zwischen dem Schwingungsgrund- und dem angeregten Zustand bedeuten. Die absoluten Größen der Aufspaltungen konnten wegen der nichtbeobachteten weiteren Tunnelübergänge in diesem Fall nicht bestimmt werden, da diese im symmetrischen Fall praktisch keine Intensität aufweisen. Der lokalisierte $g$-Übergang entspricht dann gemäß dem Modell dem Mittelwert der beiden beobachteten Tunnelübergänge und ist bei $3680 \mathrm{~cm}^{-1} \mathrm{zu}$ erwarten.

In dieser Arbeit durchgeführte Rechnungen wecken aber einige Zweifel an dieser Interpretation von Xue. Wie bei Ethanol wird für tert-Butylcarbinol eine energetisch hohe $g \leftrightarrow g$-Barriere und eine niedrige $g \leftrightarrow t$-Barriere gefunden. Letztere hat bei tertButylcarbinol im adiabatischen Kanal eine Höhe von $h c 250 \mathrm{~cm}^{-1}$, was erheblich niedriger ist als bei Ethanol $\left(h c 360 \mathrm{~cm}^{-1}\right)$ und daher in der Tat eine große Tunnelaufspaltung erwarten lässt. Der nach der Interpretation von Xue lokalisierte $g$-Übergang bei $3680 \mathrm{~cm}^{-1}$ hätte eine größere Wellenzahl als der $t$-Übergang bei $3676 \mathrm{~cm}^{-1}$. Dies wäre für primäre Alkohole höchst ungewöhnlich, da üblicherweise gefunden wird, dass stattdessen der $t$-Übergang eine deutlich höhere Wellenzahl aufweist. Strukturell am ehesten vergleichbar mit tert-Butylcarbinol sind die $G t$ - und $G g$ het-Konformationen von $n$-Propanol $\left(\Delta \tilde{\nu}=13 \mathrm{~cm}^{-1}\right), G t$ und $G g$ von Cyclohexylmethanol $\left(\Delta \tilde{v}=12 \mathrm{~cm}^{-1}\right)$, sowie $G t$ und $G g$ von Isopropylmethanol $\left(\Delta \tilde{v}=12 \mathrm{~cm}^{-1}\right)$ (Werte aus Tab. A.7 im Anhang auf S. 309). Eine Differenz in dieser Größe $\left(\omega=13 \mathrm{~cm}^{-1}\right)$ wird auch für die lokalisierten $g$ und $t$ Zustände von tert-Butylcarbinol auf B3LYP-D3(BJ)/may-cc-pVTZ-Niveau vorhergesagt. Die Anwendung des Korrelationsmodells lässt den $t$-Übergang im Bereich um $3684 \mathrm{~cm}^{-1}$ und den $g$-Übergang um $3671 \mathrm{~cm}^{-1}$ erwarten. Diese Vorhersagen weichen erheblich von experimentellen Zuordnungen bei $3676 \mathrm{~cm}^{-1}(t)$ und $3680 \mathrm{~cm}^{-1}(\mathrm{~g}) \mathrm{ab}$ (siehe Abb. 5.35). Interessanterweise wäre die vorhergesagte $t$-Wellenzahl auch mit keiner der beiden anderen beobachteten Banden besser kompatibel. Xue ging auf der Basis von MP2-Rechnungen davon aus, dass das $t$-Konformer energetisch erheblich niedriger liegt $\left(1.1 \mathrm{~kJ} \mathrm{~mol}^{-1}\right)$ und daher von einer Tunneldynamik zwischen den $g$-Konformeren nicht beeinflusst wird. Auf CCSD(T)/aug-cc-pVQZ*//B3LYP-D3(BJ)/may-cc-pVTZ-Niveau beträgt der Energievorteil von $t$ nur noch $0.2 \mathrm{~kJ} \mathrm{~mol}^{-1}$, auf B3LYP-D3(BJ)/may-cc-pVTZ-Niveau kehrt er sich sogar um $\left(-0.4 \mathrm{~kJ} \mathrm{~mol}^{-1}\right)$. Eine Überschätzung der Stabilität von $t$ durch MP2 und eine Unterschätzung durch B3LYP-D3(BJ)/may-cc-pVTZ würde dem Befund für Ethanol entsprechen (Kap. 4.2). Auf Basis der sehr niedrigen Barriere, der geringen Energiedifferenz zwischen den Konformeren und der inkompatiblen Vorhersage der $\mathrm{OH}-$ Streckschwingungswellenzahl lässt sich daher vermuten, dass auch die $t$-Wellenfunktion nicht lokalisiert ist und daher bei tert-Butylcarbinol ein besonders komplizierter Fall von Tunnelaufspaltungen zwischen drei Zuständen vorliegt. Da für tert-Butylcarbinol bereits umfangreiche experimentelle Daten vorliegen, wäre eine weitere Untersuchung dieser Diskrepanzen vor allem aus theoretischer Sicht interessant. Hierbei würde es sich anbieten die Schrödingergleichung für das 1D-Torsionspotential direkt zu lösen. Herausforderungen sind hier eine angemessene Berücksichtigung der Schwingungsnullpunktsenergie im adiabatischen Kanal sowie die Änderungen des Potentials bei Anregung der $\mathrm{OH}-$ Streckschwingung. 


\subsection{Tri-tert-butylcarbinol}

Tri-tert-butylcarbinol (systematisch: 3-tert-Butyl-2,2,4,4-tetramethyl-pentan-3-ol) ist ein tertiärer Alkohol mit mehreren ungewöhnlichen Eigenschaften. Die Struktur (Abb. 5.37) leitet sich von tert-Butylalkohol ab, wenn alle Wasserstoffatome der Methylgruppen durch weitere Methylgruppen ersetzt werden.
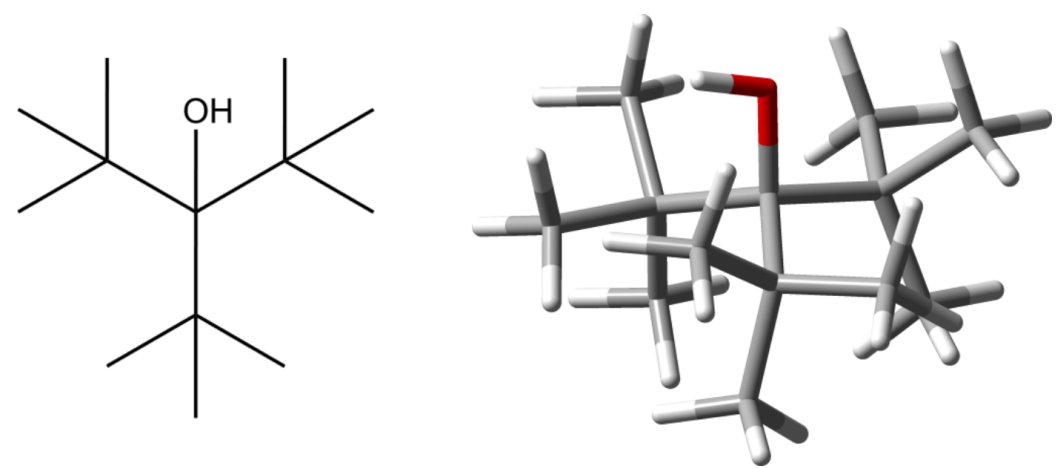

Abb. 5.37: Strukturformel und optimierte Struktur von Tri-tert-butylcarbinol auf B3LYPD3(BJ)/may-cc-pVTZ-Niveau.

Durch den sterischen Anspruch der eng gepackten Methylgruppen ist für die $\mathrm{OC}_{\alpha} \mathrm{C}_{\beta} \mathrm{C}_{\gamma}$-Torsionswinkel eine gestaffelte Anordnung sehr ungünstig und alle drei tertButylgruppen weichen davon durch Torsion in die gleiche Richtung ab, sodass sie wie Zahnräder ineinander - Methylgruppe in Lücke - greifen. Die Symmetrie des AlkoholatIons und des $\mathrm{HOC}_{\alpha} C_{\beta}$-Torsionspotentials des Alkohols wird hierdurch von $C_{3 \mathrm{v}}$ auf $C_{3}$ reduziert. Die beiden möglichen Torsionrichtungen der tert-Butylgruppen führen so zu zwei zueinander enantiomeren Konformeren. Tri-tert-butylcarbinol stellt damit ein seltenes Beispiel für einen chiralen Alkohol dar, der keine diastereomeren Konformere bezüglich der HOCC-Torsion aufweist. Ein weiterer wahrscheinlicher Kandidat hierfür ist der „,molekulare Propeller“ [314] Triphenylmethanol, allerdings erweist sich dessen hohe Normalsiedetemperatur von $370^{\circ} \mathrm{C}$ als Hindernis für Untersuchungen in der Gasphase. Die Normalsiedetemperatur von Tri-tert-butylcarbinol $\left(204^{\circ} \mathrm{C}\right)$ ist dagegen vergleichbar mit den in dieser Arbeit untersuchten Monoterpenalkoholen.

Da eine ungerade Zahl an Zahnrädern zyklisch ineinander greift, können benachbarte tert-Butylgruppen nicht ohne Hindernisse in gegenläufige Richtungen rotieren. Eine NMR-Studie in Lösung [315] fand für diese Libration durch die gestaffelte Anordnung eine Barriere von etwa $38 \mathrm{~kJ} \mathrm{~mol}^{-1}$. Diese ist vergleichbar mit der Barriere für die Ringinversion von Cyclohexan [316], sodass zu erwarten ist, dass Tri-tert-butylcarbinol bei Zimmertemperatur transient chiral ist, sich unter Jet-Bedingungen dagegen wie ein racemisches Gemisch eines permanent chiralen Alkohols verhält.

Die auf B3LYP-D3(BJ)/may-cc-pVTZ-Niveau berechnete harmonische OH-Streckschwingungswellenzahl ist mit $3830 \mathrm{~cm}^{-1}$ deutlich höher als für alle anderen hier betrachteten tertiären Alkohole $\left(3818-3800 \mathrm{~cm}^{-1}\right)$ und sogar höher als für alle sekundären Alkohole (3829-3799 $\left.\mathrm{cm}^{-1}\right)$. Als Ursache hierfür kommen zwei Effekte in Frage: Eine Versteifung des Potentials durch sterische Wechselwirkung des Hydroxyprotonen mit benachbarten Methylprotonen (es liegen vier $\mathrm{OH}$ - HCH-Abstände $<2.5 \AA$ vor) und/oder eine starke Abschwächung von induktiven Effekten durch die Schwäche der $\mathrm{C}_{\alpha}-\mathrm{C}_{\beta}$-Bindungen. Diese sind durch die sterischen Konflikte mit berechneten Werten 
von um die $1.63 \AA$ deutlich verlängert (tert-Butylalkohol: $1.53 \AA$ ). Die berechnete adiabatische Barriere von $h c 510 \mathrm{~cm}^{-1}$ lässt nur eine geringe Tunnelaufspaltung erwarten (tertButylalkohol: $V_{0}=h c 420 \mathrm{~cm}^{-1}, \Delta_{0}=h c 3.8 \mathrm{~cm}^{-1}$ [298])

Neben seinen ungewöhnlichen Eigenschaften als Monomer, erhielt Tri-tertbutylcarbinol auch Aufmerksamkeit für ein ebenso herausstechendes Merkmal seiner Aggregate: Die Verbindung gilt als einziger Alkohol, der ohne die Ausbildung von Wasserstoffbrücken aggregiert [317], da diese weder in Lösung [318, 319] noch im Festkörper [317, 318] nachgewiesen werden konnten.

Während angesichts der Struktur des Alkohols kooperative Wasserstoffbrücken in Trimeren oder größeren Aggregaten tatsächlich sterisch unmöglich erscheinen, erscheint dieser Befund für isolierte Wasserstoffbrücken in Dimeren weniger eindeutig. Dies wurde hier dadurch bestätigt, dass je zwei homo- und heterochirale Dimere (mit jeweils unterschiedlicher Koordination der diastereomeren Elektronenpaare) mit einer Wasserstoffbrücke optimiert werden konnten. Wegen direkter sterischer Hinderung und/oder den schwachen induktiven Effekten fallen die Verschiebungen der $\mathrm{OH}$ Streckschwingungswellenzahl des Donors und die Steigerungen der IR-Intensität geringer aus als für das Dimer von tert-Butylalkohol. Allerdings ist die Dissoziationsenergie von weiteren Dimer-Konformeren ohne eine Wasserstoffbrücke weiterhin erheblich geringer (18-22 gegenüber 34-37 kJ mol ${ }^{-1}$ auf B3LYP-D3(BJ)/def2-TZVP-Niveau). Tritert-butylcarbinol wäre mit dieser quantenchemischen Vorhersage daher ein Kandidat für einen Alkohol, der auf mikroskopischer Ebene in isolierten Dimeren völlig anders aggregieren könnte als in kondensierter Phase. Zusammen mit seiner besonderen Form von Chiralität und der ungewöhnlichen $\mathrm{OH}-$ Streckschwingungswellenzahl als Test für das Korrelationsmodell, wäre der Alkohol ein interessantes Objekt für eine zukünftige experimentelle Untersuchung. Tri-tert-butylcarbinol ist kommerziell verfügbar. 


\subsection{2-Butanol}

2-Butanol ist einer der kleinsten chiralen sekundären Alkohole, die Struktur des $(+)-(S)-$ Enantiomers ist in Abb. 5.38 dargestellt, welches im Folgenden betrachtet wird. Die Verbindung hat Bedeutung als Lösungsmittel und Zwischenprodukt für chemische Synthesen.

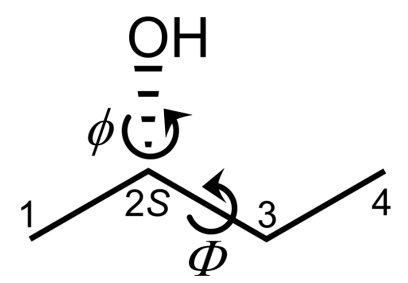

Abb. 5.38: Strukturformel von (+)-(S)-2-Butanol mit Nummerierung der Kohlenstoffatome und mit für die Konformation relevante Torsionswinkel: $\mathrm{OC}_{2} \mathrm{C}_{3} \mathrm{C}_{4}(\Phi)$ und $\mathrm{HOC}_{2} \mathrm{C}_{3}(\phi)$.

\subsubsection{Konformationsisomerie}

Im Gegensatz zu den zuvor diskutierten Alkoholen (mit Ausnahme von $n$-Octanol), können sich unterscheidbare Konformere für 2-Butanol sowohl bezüglich der Torsion um die $\mathrm{O}-\mathrm{C}_{2-}$, wie auch um die $\mathrm{C}_{2}-\mathrm{C}_{3}$-Bindung ergeben. Dies wird hier durch die $\mathrm{OC}_{2} \mathrm{C}_{3} \mathrm{C}_{4}{ }^{-}$ $(\Phi)$ und $\mathrm{HOC}_{2} \mathrm{C}_{3}$-Torsionswinkel $(\phi)$ beschrieben. Die gestaffelten gauche-, gauche+ und trans-Anordnungen werden für $\Phi$ in Großbuchstaben $(G-, G+, T)$, für $\phi$ hingegen in Kleinbuchstaben $(g-, g+, t)$ angegeben. Nicht näher festgelegte Einstellungen werden mit $X$ bzw. $x$ benannt. Diese Anordnungen sind in Abb. 5.39 in Form von Newman-Projektionen dargestellt. Diese Nomenklatur folgt der von Wassermann verwendeten [57, 190] für $n$-Propanol, aus dem sich 2-Butanol durch formale Methylierung am $\alpha$-Kohlenstoffatom ergibt, wodurch Vergleiche der beiden Systeme erleichtert werden. Für die zuvor diskutierten sekundären Alkohole wurde davon abweichend der $\mathrm{HOCH}-$ Torsionswinkel angeben, für (S)-2-Butanol gilt dabei: Die Einstellung $g+$ für $\phi\left(\mathrm{HOC}_{2} \mathrm{C}_{3}\right)$ entspricht $t$ für $\tau\left(\mathrm{HOC}_{2} \mathrm{H}\right)$.
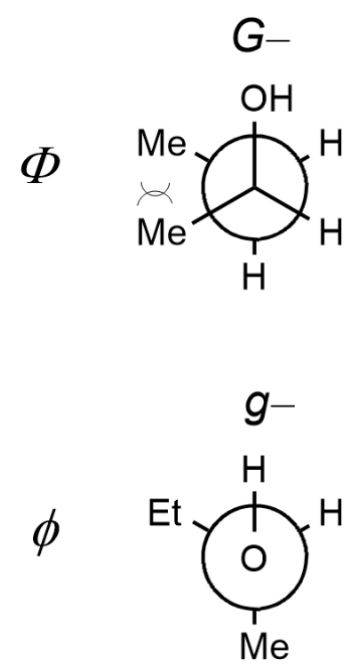

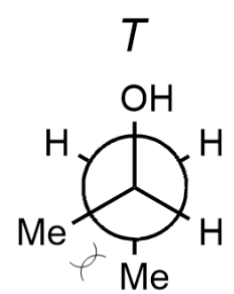<smiles>CCC1CCCC(C)C1</smiles><smiles>CC1C2CC3(O)CC1C(C)C3C2C</smiles><smiles>CCC1CCCCC(C)C1O</smiles>

$\mathrm{H}$

Abb. 5.39: Newman-Projektionen bezüglich der $\mathrm{C}_{2}-\mathrm{C}_{3}$ - (oben) und $\mathrm{O}-\mathrm{C}_{2}$-Bindung für unterschiedliche Einstellungen der $\mathrm{OC}_{2} \mathrm{C}_{3} \mathrm{C}_{4}{ }^{-}(\Phi)$ und $\mathrm{HO}_{2} \mathrm{C}_{3} \mathrm{C}$-Torsionswinkel $(\phi)$. Ungünstige gauche-Wechselwirkungen zwischen Methylgruppen sind gekennzeichnet. 
Um den gesamten relevanten Konformationsraum einschließlich der Übergangszustände zu erfassen, wurde ein zweidimensional relaxierter Scan der elektronischen Energie auf B3LYP-D3(BJ)/may-cc-pVTZ-Niveau bezüglich $\Phi$ und $\phi$ in $10^{\circ}$-Schritten durchgeführt (Abb. 5.40). Dieser zeigt neun Minima an jeweils den Positionen, an denen beide Torsionsanordnungen näherungsweise gestaffelt vorliegen. Diese entsprechen den stabilen Konformeren von $(S)$-2-Butanol, deren Strukturen und wichtigste spektrale Eigenschaften in Abb. 5.41 aufgeführt sind. 18 Übergangszustände liegen vor wenn eine Anordnung gestaffelt und die andere ekliptisch eingestellt ist. Auch das strukturell eng verwandte $n$-Propanol weist ebenfalls neun Konformere auf, von diesen bilden aber acht insgesamt vier Enantiomerenpaare, sodass nur fünf Konformere spektral unterscheidbar sind. Diese Symmetrie wird durch die formale Methylierung am $\alpha$-Kohlenstoffatom gebrochen, sodass alle neun Konformere des 2-Butanols energetisch und spektral unterscheidbar werden. Während die Konformere des $n$-Propanols auf B3LYP-D3(BJ)/may-ccpVTZ-Niveau in einem engen Energiebereich von nur $0.3 \mathrm{~kJ} \mathrm{~mol}^{-1}$ vorhergesagt werden, kommt es für 2-Butanol zu größeren Unterschieden. Bereits im 2D-Scan lässt sich erken-

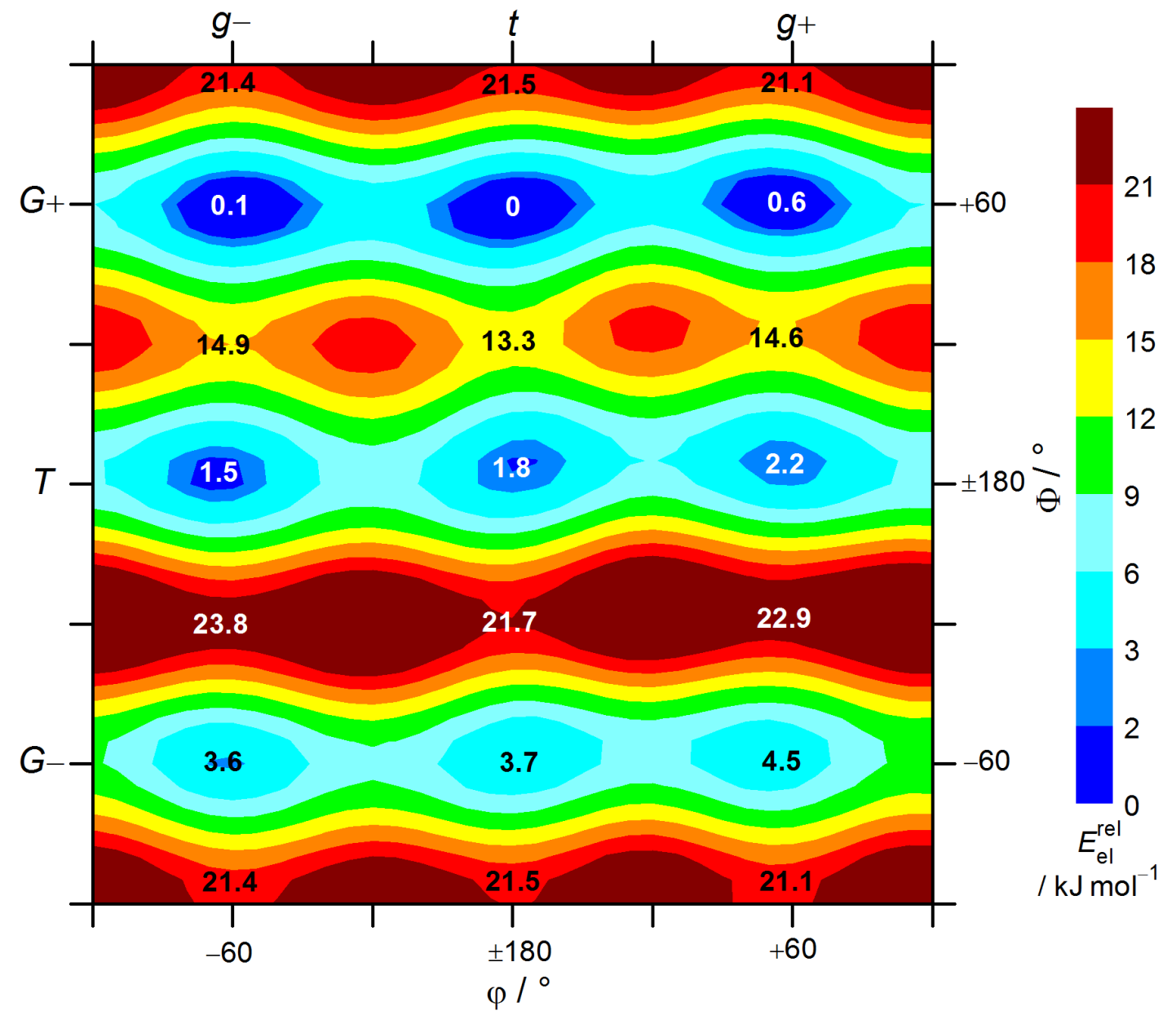

Abb. 5.40: Zweidimensionaler relaxierter Scan der elektronischen Potentialfläche auf B3LYPD3(BJ)/may-cc-pVTZ-Niveau bezüglich der $\mathrm{OC}_{2} \mathrm{C}_{3} \mathrm{C}_{4}-(\Phi)$ und $\mathrm{HO}_{2} \mathrm{C}_{3} \mathrm{C}$-Torsionswinkel $(\phi)$ von $(S)$-2-Butanol in $10^{\circ}$-Schritten (1369 Datenpunkte). Die Zahlenwerte im Diagramm entsprechen den relativen Energien der volloptimierten Minima sowie der Übergangszustände bezüglich $\Phi$ mit zusätzlicher Nullpunktsenergiekorrektur. 


\section{Torsionsisomerie von Alkoholen}

nen, dass für die relative Energie dieser neun Konformere eine klare Hierarchie bezüglich $\Phi$ vorliegt: es gilt $E(G+x)<E(T x)<E(G-x)$. Die hohe Stabilität von $G+x$ wird vermutlich vor allem durch die Abwesenheit einer ungünstigen gauche-Wechselwirkungen der beiden Methylgruppen bestimmt, wie sie in $T x$ und $G-x$ vorliegt (siehe Abb. 5.39). Im hochenergetischen $G-x$ findet zusätzlich eine gauche-Wechselwirkung zwischen der Hydroxygruppe und einer Methylgruppe statt, die scheinbar ebenfalls destabilisierend wirkt. Eine alternative Interpretation wäre, dass die Position der Hydroxygruppe in $G-x$ eine begrenzte Aufweitung des CCCC-Torsionwinkels behindert, welche in $T x$ die gauche-Wechselwirkung der Methylgruppen verringert. So weicht in $T x$ der CCCCTorsionwinkel um 4-6 $6^{\circ}$ von der idealen gestaffelten Anordnung ab (in Abb. 5.41 anhand des daran gekoppelten OCCC-Torsionwinkels mit bloßem Auge erkennbar), während er bei $G-x$ nur unwesentlich $\left( \pm 2^{\circ}\right)$ um den erwarteten Wert von $60^{\circ}$ streut.

Die Abfolge bezüglich $\phi$ ist weniger eindeutig ausgeprägt: es gilt $E(X g-) \approx E(X t)<$ $E(X g+)$ für $X=$ const. Dies entspricht dem Befund für andere sekundäre Alkohole bezüglich der HOCH-Torsion. Indizien für einen stabilisierenden Charakter eines $\mathrm{C}_{\gamma}-\mathrm{H} \cdots \mathrm{O}-$ Kontaktes, wie er für die geringe Energie des $G t$-Konformers von $n$-Propanol angeführt wird [190], sind in 2-Butanol widersprüchlich. Hiermit könnte die leicht höhere Stabilität des globalen Minimums $G+t$ gegenüber $G+g-$ erklärt werden, dagegen haben $G-t$
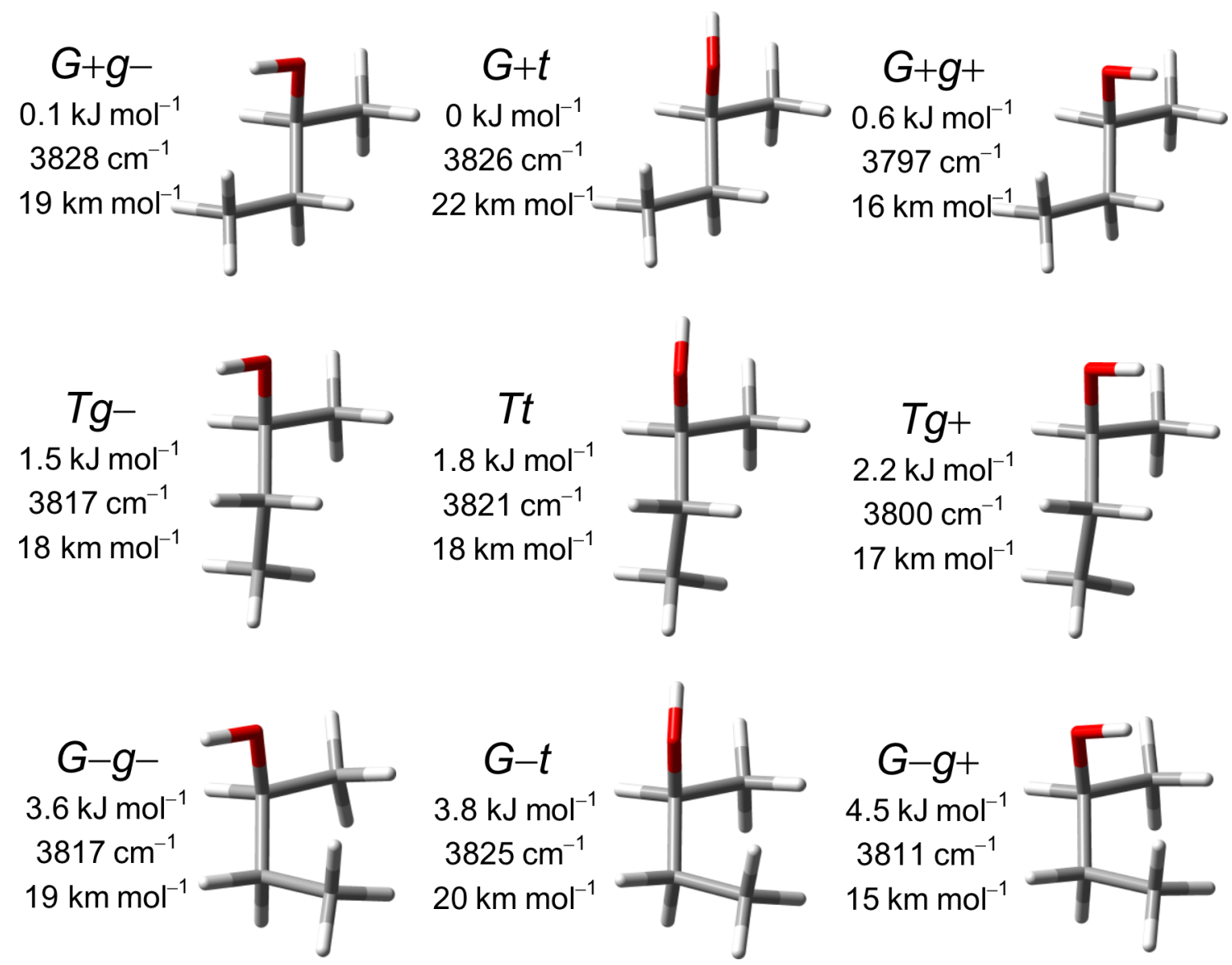

Abb. 5.41: Die neun Konformere von $(+)-(S)$-2-Butanol, klassifiziert nach den Einstellungen der $\mathrm{OC}_{2} \mathrm{C}_{3} \mathrm{C}_{4^{-}}$(Großbuchstaben) und $\mathrm{HO}_{2} \mathrm{C}_{3} \mathrm{C}$-Torsionswinkel (Kleinbuchstaben), mit relativen Energien, OH-Streckschwingungswellenzahlen und IR-Aktivitäten auf B3LYP-D3(BJ)/may-ccpVTZ-Niveau. 
und $G-g-$ die umgekehrte Energieabfolge. Auch für $T t$ und $T g-$ wird eine Energieaufspaltung berechnet, ohne dass in der unmittelbaren Umgebung der Hydroxygruppe eine Änderung auftritt, da sich dort das $\gamma$-Kohlenstoffatom in $T$-Position zur Hydroxygruppe befindet.

Die geringe molekulare Größe von 2-Butanol erlaubt auch Vergleiche mit den deutlich aufwändigeren Methoden B3LYP-D3(BJ)/aug-cc-pVQZ und B2PLYP-D3(BJ)/augcc-pVTZ. Die erhaltenen Werte für Energie- und Wellenzahldifferenzen sind auf B3LYPD3(BJ)/aug-cc-pVQZ-Niveau, wie bereits für Ethanol-Monomere und -Dimere, praktisch identisch zu denen bei Verwendung des erheblich kleineren Basissatzes may-cc-pVTZ (daher nicht aufgeführt). Auch mit B2PLYP-D3(BJ)/aug-cc-pVTZ wird die selbe Energieabfolge der neun Konformere gefunden, allerdings mit tendenziell größeren Energieabständen. Das höchste in der Literatur verfügbare Rechenniveau für die 2-ButanolKonformere ist MP2/6-311++G**, allerdings ohne Nullpunktsenergiekorrektur [320]. Die B3LYP- und B2PLYP-Ergebnisse legen nahe, dass eine Nullpunktsenergiekorrektur auf die Energieabstände bezüglich $\phi$ nur geringen Einfluss hat, aber bezüglich $\Phi$ die relative Energie von $T x$ und noch stärker $G-x$ erhöht. Auch auf MP2-Niveau ist die Energieabfolge der neun Konformere identisch, mit der Ausnahme einer um zwei Positionen geringeren Stabilität von $T g+$, welches hinter $G-g-$ und $G-t$ zurückfällt. Eine mögliche Ursache hierfür ist die fehlende Nullpunktsenergiekorrektur, die wie erwähnt $G-x$ besonders destabilisiert. Die Ergebnisse der drei Methoden sind in Tab. 5.7 in Matrixform zusammengestellt.

Die Übergangsbarrieren zwischen den Konformeren bezüglich $\phi$ sind generell niedrig (im Bereich von 3-6 kJ mol${ }^{-1}$ ), wobei die höheren Werte die Umwandlungen $G+g+$ $\leftrightarrow G+g-$ und $G-g+\leftrightarrow G-g-$ betreffen, da es in diesen Fällen offenbar zu einer ungünstigen sterischen Wechselwirkung zwischen dem Hydroxywasserstoffatom und einem $\mathrm{C}_{\gamma}$-Wasserstoffatom kommt. Dies ist für die effektive Umwandlung aber weitgehend unerheblich, da es sich um ein zyklisches Potential handelt und ein günstigerer Relaxationspfad über $X t$ offensteht. Die Barrieren bezüglich $\Phi$ sind deutlich höher, die $G-x$-Konformere müssen auf dem günstigsten Relaxationspfad eine Barriere von etwa

Tab. 5.7: Relative Energien der neun Konformere von $(S)$-2-Butanol in $\mathrm{kJ} \mathrm{mol}^{-1}$ auf B3LYPD3(BJ)/may-cc-pVTZ-, B2PLYP-D3(BJ)/aug-cc-pVTZ- und MP2/6-311++G**-Niveau, letzteres ohne Nullpunktsenergiekorrektur [320].

$\begin{array}{cccc} & \boldsymbol{g}- & \boldsymbol{t} & \boldsymbol{g}+ \\ \boldsymbol{G}+ & 0.1 & 0 & 0.6 \\ & 0.7 & 0 & 1.1 \\ & 1.2 & 0 & 1.7 \\ & & & \\ \boldsymbol{T} & 1.5 & 1.8 & 2.2 \\ & 2.1 & 2.3 & 3.1 \\ & 1.5 & 2.3 & 3.2 \\ & & & \\ \boldsymbol{G}- & 3.6 & 3.8 & 4.5 \\ & 4.3 & 4.3 & 5.8 \\ & 2.8 & 3.1 & 5.7\end{array}$


$18 \mathrm{~kJ} \mathrm{~mol}^{-1}$ überwinden, die $T x$-Konformere von etwa $11 \mathrm{~kJ} \mathrm{~mol}^{-1}$. Werte auf MP2/6$311++\mathrm{G}^{* *}$-Niveau ohne Nullpunktsenergiekorrektur [320] sind vergleichbar zu den hier berechneten.

Die berechneten harmonischen $\mathrm{OH}-$ Streckschwingungswellenzahlen der Konformere von 2-Butanol lassen sich systematisch aus denen von $n$-Propanol ableiten. Letztere wurden detailliert von Wassermann analysiert, und beobachtete und berechnete Unterschiede über das empirische 3-Parameter-Modell strukturellen Eigenschaften zugeordnet.[14, 57] Ausgehend von dieser Grundlage erlaubt die Erweiterung von Xue [236] auf sekundäre Alkohole hier auch die Rationalisierung für 2-Butanol. In Abb. 5.42 sind die berechneten Wellenzahlen der Konformere von $n$-Propanol und 2-Butanol gegenübergestellt. Jeweils in einer gemeinsamen Farbe sind diejenigen Konformere gekennzeichnet, die aus dem jeweiligen $n$-Propanol-Konformer durch Methylierung am $\alpha$-Kohlenstoffatom erhalten lassen. Es lässt sich erkennen, dass entweder fast keine Änderung der Wellenzahl stattfindet

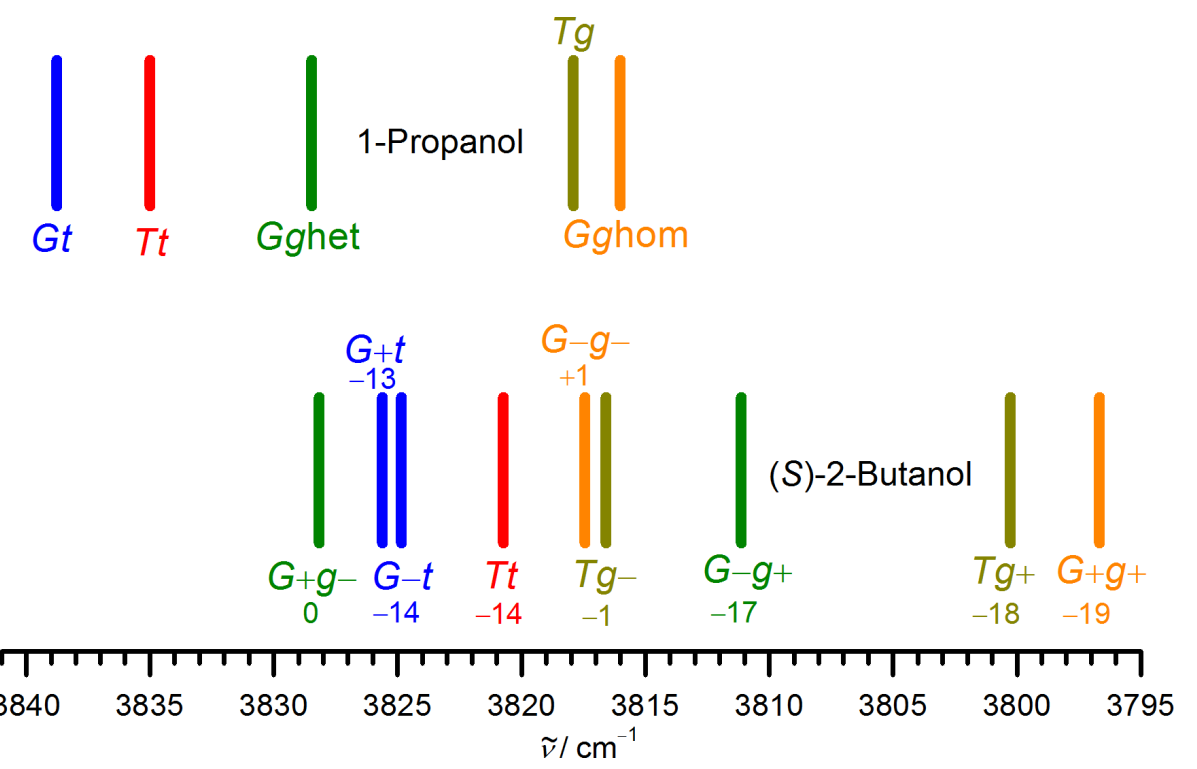

Abb. 5.42: Gegenüberstellung der harmonischen OH-Streckschwingungswellenzahlen der Konformere von $n$-Propanol und ( $S$ )-2-Butanol auf B3LYP-D3(BJ)/may-cc-pVTZ-Niveau. Konformere, die sich durch formale Methylierung am $\alpha$-Kohlenstoffatom von $n$-Propanol voneinander ableiten lassen, sind in einer einheitlichen Farbe gekennzeichnet. Die dadurch verursachten Verschiebungen der $\mathrm{OH}-$ Streckschwingungswellenzahlen sind in $\mathrm{cm}^{-1}$ mitangegeben.

oder eine deutliche Tiefverschiebung von 14 bzw. $17-19 \mathrm{~cm}^{-1}$. Diese drei Fälle werden vom 3-Parameter-Modell von Xue (welches in der Variante für sekundäre Alkohole tatsächlich bereits fünf Parameter enthält) erfolgreich vorhergesagt. Nach dem Modell ist eine Verschiebung von etwa $-15 \mathrm{~cm}^{-1}$ durch das Einführen einer $g$-Anordnung bezüglich eines $\mathrm{HOC}_{\alpha} \mathrm{C}_{\beta}$-Torsionswinkels zu erwarten. Bei der Methylierung der $X t$-Konformere von $n$-Propanol erfolgt dies zwangsläufig, sodass die dadurch abgeleiteten Konformere von 2-Butanol alle um etwa diesen Wert verschoben sind. Für die $X g$-Konformere gibt es dagegen zwei Alternativen: entweder die Methylierung erfolgt in $t$-Position bezüglich der Hydroxygruppe, sodass sich die Wellenzahl nicht ändert, oder die Methylierung erfolgt $g$, womit sich die Wellenzahl um $17-19 \mathrm{~cm}^{-1}$ verringert. Dieses Konzept ist in Abb. 5.43 illustriert. Dass die Verschiebung für $X g$-Konformere Fall etwas größer ausfällt als für die $X t$-Konformere wird im Modell nach Xue durch einen weiteren Korrekturterm 
von $-5 \mathrm{~cm}^{-1}$ berücksichtigt. Dieser ist anzuwenden wenn zwei $\mathrm{HOC}_{\alpha} \mathrm{C}_{\beta}$-Torsionswinkel in $g$-Stellung vorliegen - so wie es für die $\mathrm{Xg}+$-Konformere von 2-Butanol der Fall ist. Als strukturelle Begründung für diesen empirischen Befund wird von Xue die Abwesenheit einer $\mathrm{O}-\mathrm{H} \cdots \mathrm{H}-\mathrm{C}_{\alpha}$-Abstoßung angeführt. Kleinere zusätzliche Abweichungen könnten vorliegen, weil hier B3LYP-D3(BJ)/may-cc-pVTZ verwendet wird, während das 3P-Modell auf B3LYP/6-311+G* basiert.

Das 3P-Modell wird hier zum ersten Mal zur Rationalisierung von Verschiebungen zwischen primären und sekundären Alkoholen angewendet. Die Variante von Wassermann sieht dagegen nur Vergleiche zwischen primären $n$-Alkanolen, und die Variante von Xue nur zwischen sekundären Alkoholen vor. Wie im Korrelationsmodell festgestellt wurde, laufen aber die experimentellen und harmonischen B3LYP-Schwingungswellenzahlen nicht mehr parallel wenn der Substitutionsgrad am $\alpha$-Kohlenstoffatom verändert wird. Daher ist zu erwarten, dass die experimentellen Wellenzahlen der 2-Butanol-Konformere zusätzlich noch um die Differenz von $\kappa$, also um weitere $-6 \mathrm{~cm}^{-1}$, zu tieferen Werten verschoben sind.

Nach der Etablierung der quantenchemischen Befunde, lässt sich nun prüfen inwieweit diese mit experimentellen Ergebnissen im Einklang stehen. King und Howard haben das Relaxationsverhalten der Konformere von 2-Butanol in Überschallexpansionen mit Argon und Helium als Trägergas studiert.[320, 321] In der Argon-Expansion konnte nur $G-g-, T g-$ und $G+t$ nachgewiesen werden. Dies stimmt mit den quantenchemischen Vorhersagen überein, gemäß denen diese Konformere die jeweiligen energetisch tiefsten Minima bezüglich $\phi$ für die drei Einstellungen von $\Phi$ sind. Dies zeigt auch, dass mit

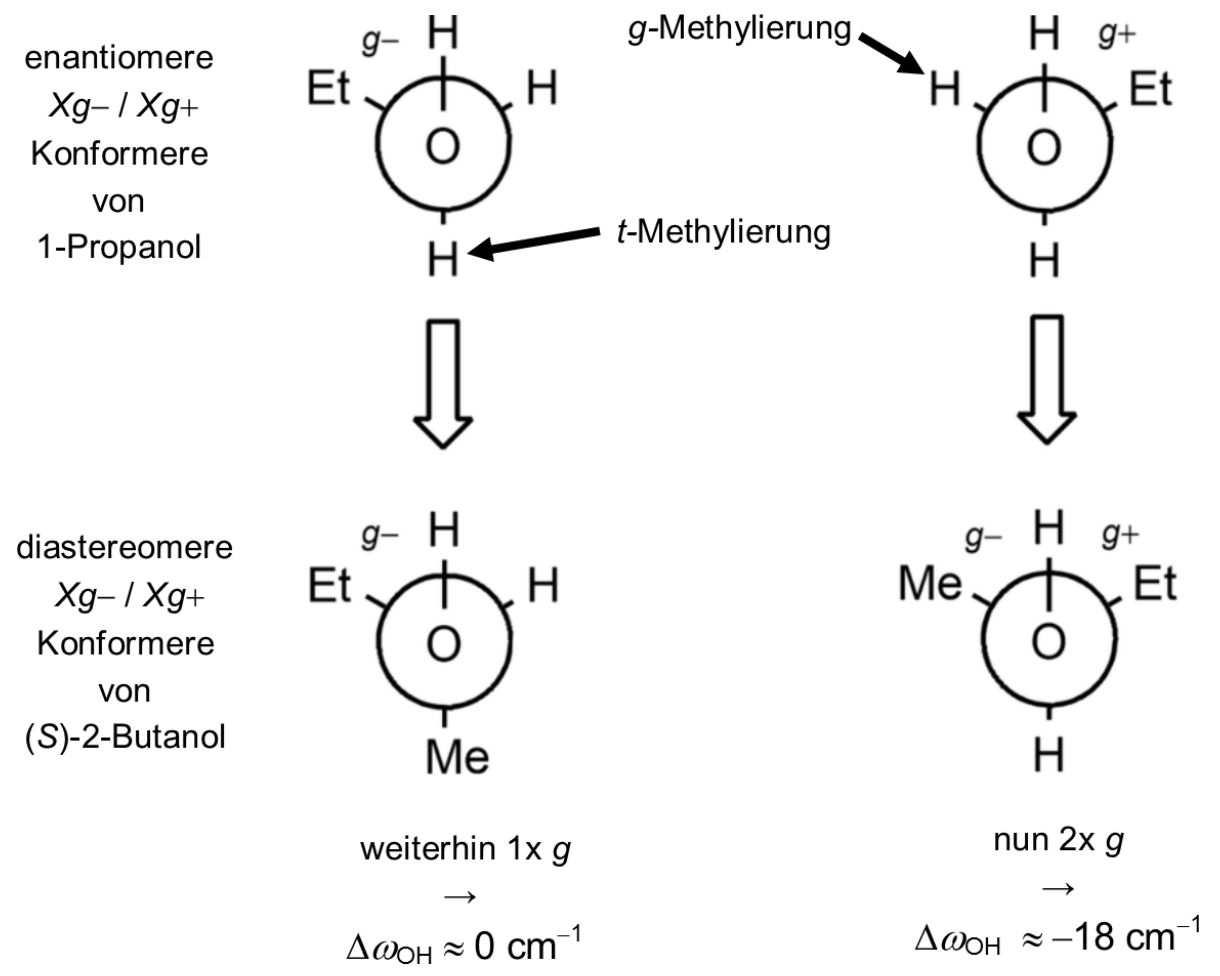

Abb. 5.43: Erhalt der Konformere von $(S)$-2-Butanol durch selektive formale Methylierung von Konformeren von $n$-Propanol am Beispiel von $X g$. Die Zahl der $g$-Anordnungen bezüglich der $\mathrm{HOC}_{\alpha} \mathrm{C}_{\beta}$-Torsionswinkel bestimmt maßgeblich die $\mathrm{OH}$-Streckschwingungswellenzahl (siehe Fließtext). 


\section{Torsionsisomerie von Alkoholen}

Argon zwar effektiv die Konformere bezüglich $\phi$ relaxiert werden können, aber für $\Phi$ keine oder nur eine unvollständige Relaxation stattfindet. Auch dies entspricht der Erwartung aus den sehr unterschiedlichen Barrierenhöhen. Die beobachteten Intensitäten (unter qualitativer Berücksichtigung von berechneten Dipolmomentkomponenten) folgen der Beziehung $I(G-g-)<I(T g-)<I(G+t)$, welche mit der Erwartung aus der berechneten Energieabfolge bezüglich $\Phi$ übereinstimmt. In der wärmeren Helium-Expansion konnten zusätzlich $G+t, G+g+$ und $T g+$ beobachtet werden, in den Intensitätsabfolgen $I(G+g+)$ $<I(G+g-)<I(G+t)$ und $I(T g+)<I(T g-)$. Auch diese entspricht der Erwartung aus den berechneten Energien. Allerdings wurde $T t$ nicht beobachtet, obwohl es stabiler als das nachgewiesene $T g+$ vorhergesagt gesagt wird. Diese scheinbare Abwesenheit wird von den Autoren aber durch die sehr geringe berechnete Dipolmomentkomponente für die untersuchten Übergänge erklärt. $G-t$ und $G-g+$ sind trotz gehinderter Relaxation bezüglich $\Phi$ offenbar zu gering populiert um nachgewiesen zu werden. Die berechnete Energieabfolge der Konformere steht damit im Einklang mit den rotationsspektroskopischen Befunden.

\subsubsection{Schwingungsspektren}

2-Butanol liegt bei Zimmertemperatur als Flüssigkeit vor und siedet bei $100{ }^{\circ} \mathrm{C}$. Durch den hohen Dampfdruck von etwa $2.3 \mathrm{kPa}$ bei $24^{\circ} \mathrm{C}$ [65] lassen sich auch ohne Heizung größere Aggregate nachweisen. FTIR-Spektren von 2-Butanol in Überschallexpansionen mit Helium als Trägergas wurden bereits 2001 von Borho am Ragout-Jet aufgenommen [322], auf die hier zurückgegriffen wird (Abb. 5.44). Die Spektren im OHStreckschwingungsbereich sind sehr ähnlich zu anderen kleinen aliphatischen Alkoholen mit Konformationsvielfalt strukturiert, wie z. B. Ethanol. Aufgrund dieser Analogie wur-

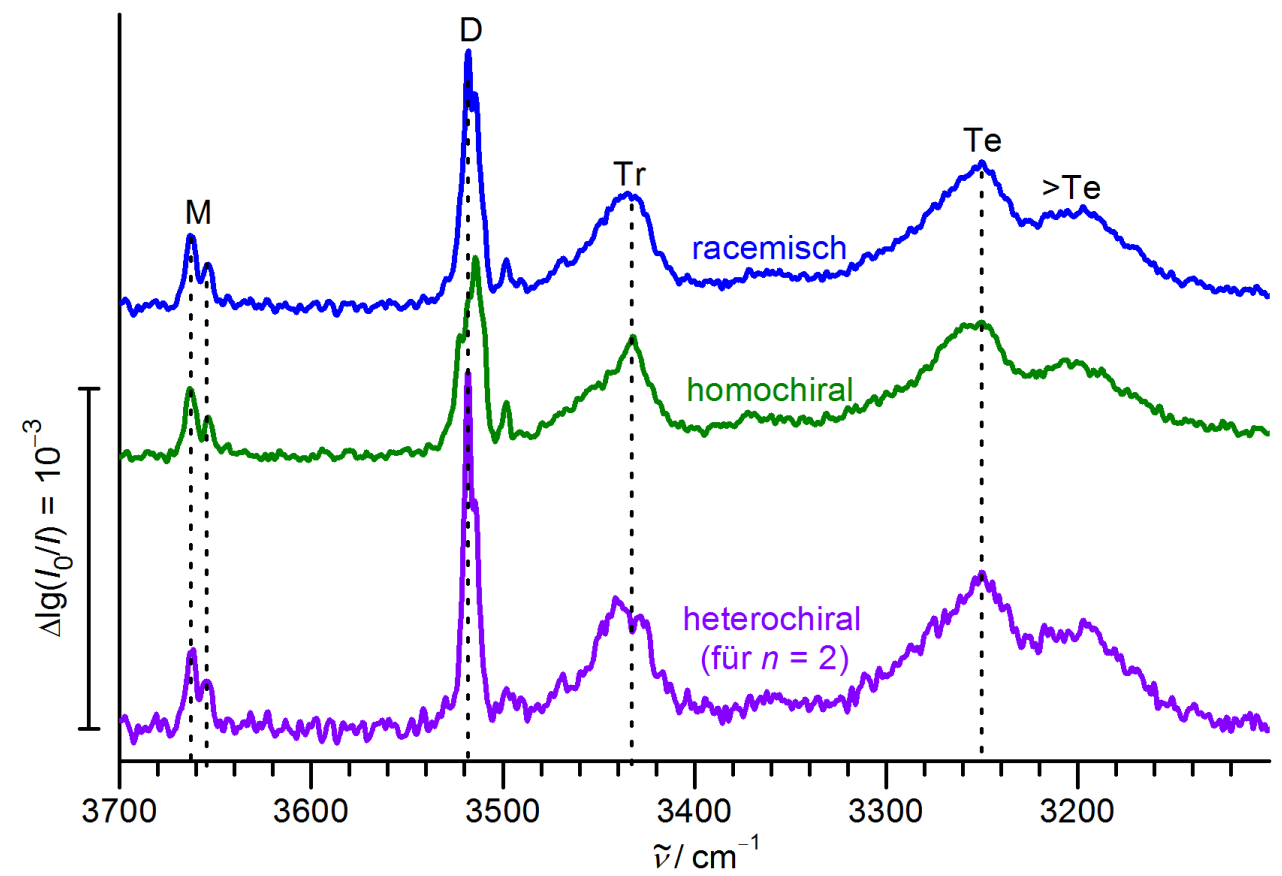

Abb. 5.44: Übersicht der Ragout-Jet-Spektren von rac-2-Butanol, (+)-(S)-2-Butanol im OHStreckschwingungsbereich mit tentativen Bandenzuordnungen, übernommen aus Ref. [322]. Das artifizielle Spektrum der heterochiralen Dimere wurde gemäß Gl. 2.2 auf S. 10 berechnet. 
den von Borho die Spektren in Bereiche für Monomere, Dimere, Trimere, Tetramere und größere Cluster unterteilt, die für Abb. 5.44 übernommen wurden. Im Monomerbereich sind zwei Banden bei 3663 und $3654 \mathrm{~cm}^{-1}$ zu erkennen. Der Bereich um $3520 \mathrm{~cm}^{-1}$ unterscheidet sich etwas zwischen den dort zugeordneten homochiralen und heterochiralen Dimeren. Entweder liegt eine etwas größere Vielfalt an homochiralen Dimeren vor oder diese unterscheiden sich zumindest spektral stärker als die heterochiralen Dimere. Auch der Trimerbereich um $3430 \mathrm{~cm}^{-1}$ zeigt gewisse Unterschiede, während dies für den Bereich der Tetramere (um $3250 \mathrm{~cm}^{-1}$ ) und noch größeren Cluster (um $3200 \mathrm{~cm}^{-1}$ ) kaum noch der Fall ist. Da die Chiralitätsdiskriminierung spektral eher moderat ausfällt, von Überlagerungen geprägt ist und quantenchemisch wohl nur sehr aufwändig zu modellieren ist, erscheint eine tiefer gehende Analyse wenig lohnenswert. Wie die zuvor diskutierte unvollständige Relaxation auch mit Einsatz von Argon zeigt, können die höher energetischen Konformere des 2-Butanols nicht vernachlässigt werden. Dies lässt sowohl für die homo- wie auch heterochiralen Dimere eine hohe Konformationsvielfalt in jeweils $9^{2}=81$ Klassen erwarten, für die durch die Inäquivalenz der freien Elektronenpaare und die Torsion um die Wasserstoffbrücke noch weitere Isomerie zu erwarten ist.

Weniger von spektralen Überlappungen betroffen ist die Rotationsspektroskopie, sodass einzelne Konformere besser identifiziert werden können, sofern eine gute Vorhersage für die Rotationskonstanten zur Verfügung steht. Es ist daher King und Howard gelungen ein heterochirales Dimer nachzuweisen.[323] Betrachtet wurden dabei nur homo- und hetereochirale Dimere, die aus zwei Molekülen der stabilsten $G+t$-Konformation (bzw. deren Entsprechung $G-t$ für $(R)$-2-Butanol) zusammengesetzt sind. Interessanterweise konnte dagegen kein entsprechendes homochirales Dimer nachgewiesen werden.

Eine Detailansicht des Monomer-Bereichs der Ragout-Jet-Spektren, ergänzt um ein Gasphasen-Spektrum, ist in Abb. 5.45 dargestellt. Geringe Unterschiede zwischen den Spektren des enantiomerenreinen und racemischen 2-Butanols erscheinen insignifikant. Wegen der hohen Konzentration könnten Akzeptor-Banden von Dimeren beitragen, die prinzipiell abhängig von der relativen Chiralität des Donors sein könnten. In Kapitel 6 diskutierte Spektren von Expansionen mit Beimischung von $\alpha$-Pinen, in denen die Bildung von 2-Butanol-Dimeren deutlich unterdrückt ist, zeigen aber hierzu keine signifikanten Unterschiede im Monomerbereich.

Diese Jet-Spektren konnten von Borho mit B3LYP/6-31+G*-Rechnungen für die Monomere nicht interpretiert werden.[322] In der Tat wird auch mit B3LYP-D3(BJ)/maycc-pVTZ bei Annahme einer einfachen Boltzmann-Verteilung nur für eine der beiden Banden eine Erklärung gefunden (untere Simulation in Abb. 5.45). Für die Ursache dieser Diskrepanz wurde von Borho die mangelnde Zuverlässigkeit der berechneten Wellenzahldifferenzen vermutet. Als Hauptursache hierfür kann mit dem heutigen Kenntnisstand stattdessen die angenommene Population der Konformere identifiziert werden, denn durch die hohen Barrieren bezüglich $\Phi$ kann sich unter Überschallexpansionen keine Gleichgewichtsverteilung mit niedriger Konformationstemperatur einstellen, wie King und Howard gezeigt haben. Dadurch sind auch hochenergetische Konformere noch substantiell populiert. Um dies zu modellieren, wird im Folgenden angenommen, dass die Abkühlung sehr schnell zu Beginn der Expansion stattfindet und damit die Verteilung bezüglich $\Phi$ bei Zimmertemperatur erhalten bleibt. Berechnete relative freien Enthalpien (Tab. A.10 im Anhang auf S. 311) lassen auf eine Population von etwa 56\% $G+x, 31 \%$ 


\section{Torsionsisomerie von Alkoholen}

Tx und 13\% $G-x$ bei Zimmertemperatur schließen. 2-Butanol verhält sich mit dieser Annahme nach Beginn der Expansion wie eine Mischung aus drei separaten Molekülen, die sich nicht mehr ineinander umwandeln können, aber jeweils ein internes Gleichgewicht bezüglich $\phi$ mit tiefer Konformationstemperatur ausbilden. Die Simulation einer solchen Überlagerung ist ebenfalls in Abb. 5.45 gezeigt, die in guter Übereinstimmung mit den beiden beobachteten Banden steht. Die absolute Position der beiden Banden wird vom Korrelationsmodell um etwa $2 \mathrm{~cm}^{-1}$ unterschätzt und/oder die Population der höherfrequenten Konformere ist tatsächlich etwas höher als modelliert.

Die vorhergesagte schwache Bande um $3632 \mathrm{~cm}^{-1}$, die von $G+g+$ und $T g+$ verursacht werden soll, wird in den Jet-Spektren nicht beobachtet. Dies kann dem begrenzten Signal-zu-Rausch-Verhältnis der Spektren und/oder einer Überschätzung der Stabilität dieser Konformere auf B3LYP-Niveau geschuldet sein. Letzteres würden die B2PLYPund MP2-Rechnungen nahelegen, wie auch die Ergebnisse für entsprechende Konformere anderer sekundären Alkohole in diesem Kapitel. $G+g+$ und $T g+$ wurden von King und Howard in einer Helium-Expansion nachgewiesen, allerdings nur mit geringer Intensi-

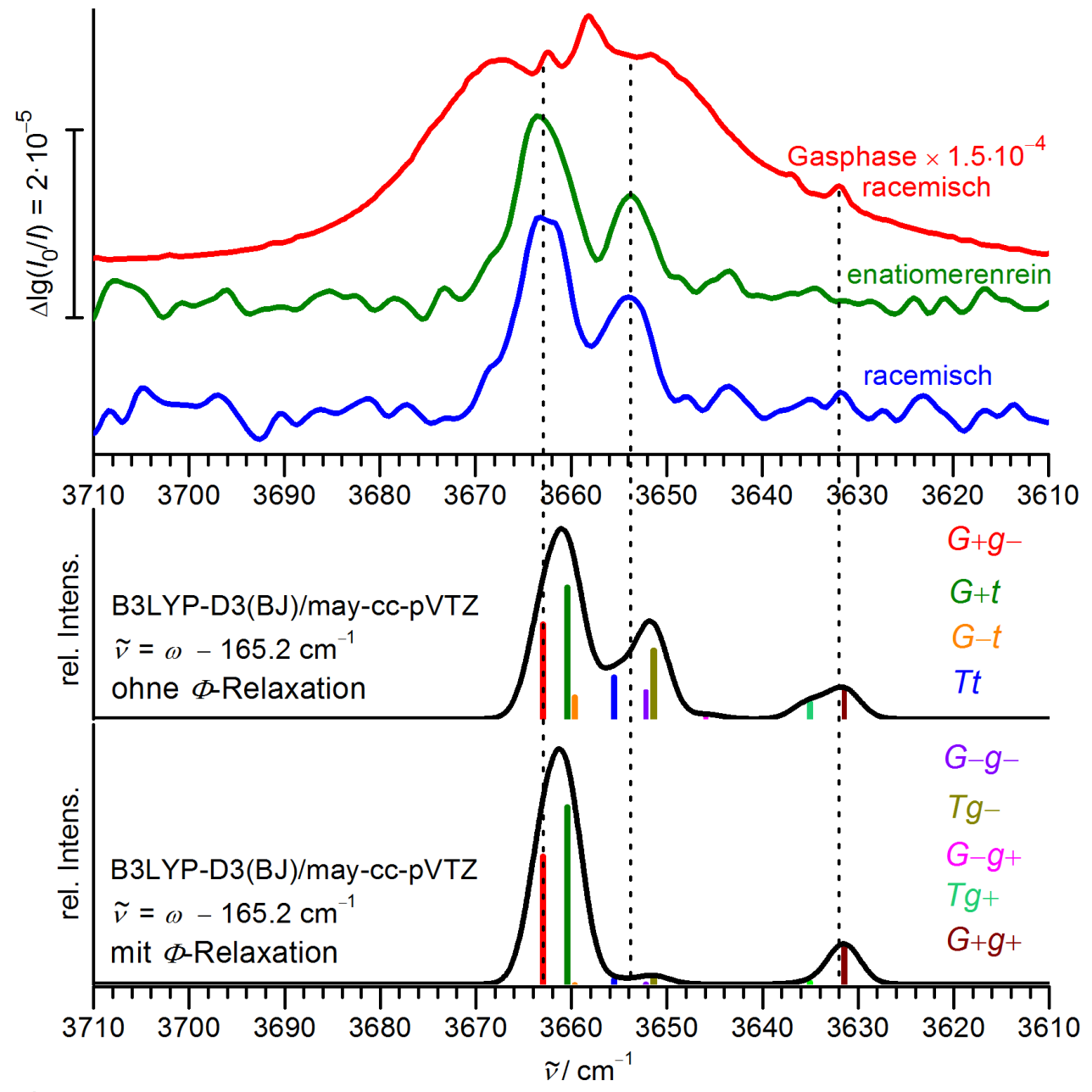

Abb. 5.45: Detailansicht von Filet-Jet- und Gasphasenspektren von 2-Butanol im Monomerbereich der OH-Streckschwingung, übernommen aus Ref. [322]. Simulationsparameter sind einheitliche Verschiebung der harmonischen Wellenzahlen um $\kappa=-165.2 \mathrm{~cm}^{-1}$, eine Konformationstemperatur von $64 \mathrm{~K}$ (mit Einschränkung in der oberen Simulation, siehe Fließtext) und Gauss-Profile mit FWHM $=4 \mathrm{~cm}^{-1}$. 
tät. Dass die vorhergesagte spektrale Position dieser Konformere plausibel ist, zeigt der Vergleich mit dem Gasphasen-Spektrum (Abb. 5.45). Dieses läuft zu niedrigeren Wellenzahlen flacher aus und besitzt sogar zwei kleine Nebenmaxima an den vorhergesagten Positionen. In Gasphasen-Obertonspektren von 2-Butanol wird die Separation in diese zwei Spektralbereiche deutlicher als im Fundamentalbereich. Daraus wurde eine zu erwartende Bandenposition für die Fundamentalübergänge bei $3628(2) \mathrm{cm}^{-1}$ abgeleitet [324], welche nahe an der hier vorhergesagten Position von $3632 \mathrm{~cm}^{-1}$ liegt.

Zusammenfassend geben die quantenchemische Vorhersagen ein schlüssiges Bild für die Beschreibung der Energieabfolge und spektralen Positionen der neun 2-ButanolKonformere. Konkrete Zuordnungen von einzelnen Konformeren können aber wegen der spektralen Überlagerungen nicht getroffen werden. Zukünftige Messungen am Curry-Jet könnten dies durch die Beobachtung der Q-Zweige ermöglichen, wie es für $n$-Propanol gelang [57]. Durch die höhere Sensitivität könnte vermutlich auch die vorhergesagte Bande um $3632 \mathrm{~cm}^{-1}$ bestätigt oder widerlegt werden. Gemäß den Resultaten von King und Howard erscheint die Beimischung von Argon aussichtsreich um die konformationelle Vielfalt zu verringern, allerdings mit der möglichen Komplikation der Bildung von Komplexen aus 2-Butanol und Argon [325]. Neon-Beimischung wäre hier eine teuere, aber komplikationsärmere Alternative. Schließlich empfehlen sich niedriger konzentrierte Expansionen, denn neben Akzeptorbanden könnte prinzipiell auch Solvent-Assisted Conformational Isomerization (SACI) [326] den analysierten Spektralbereich beeinflussen. 


\subsection{Benzylalkohol}

Benzylalkohol ist ein primärer Alkohol, dessen Konstitution in Abb. 5.46 dargestellt ist. Die Verbindung findet breite Anwendung u. a. als Lösungs- und Konservierungsmittel und kommt auch in der Natur in vielen Pflanzen vor. Benzylalkohol lässt sich von Methanol ableiten, wenn ein Wasserstoffatom durch eine Phenylgruppe ersetzt wird, demgemäß findet auch die Bezeichnung Phenylmethanol Verwendung. Die Eignung der Phenylgruppe als Akzeptor für inter- und intramolekulare $\mathrm{OH} \cdots \pi$-Wasserstoffbrücken [220] macht Benzylalkohol zu einem wichtigen Prototyp für diese Wechselwirkung. Wie Ethanol ist Benzylalkohol eine achirale Verbindung, die transient chirale Konformationen ausbildet. Neben der Untersuchung im Kontext von Chiralitätssynchronisation und -induktion wurde Benzylalkohol für die vorliegende Arbeit auch als Vergleichssystem für das im Anschluss behandelte permanent chirale 1-Phenylethanol ausgewählt.

\subsubsection{Konformationsisomerie}

Wie das im vorherigen Abschnitt diskutierte 2-Butanol weist Benzylalkohol verschiedene Konformere bezüglich der Torsion um eine O-C- und eine C-C-Bindung auf. NewmanProjektionen für die wichtigsten Einstellungen der beiden relevanten Torsionswinkel sind in Abb. 5.47 gezeigt.

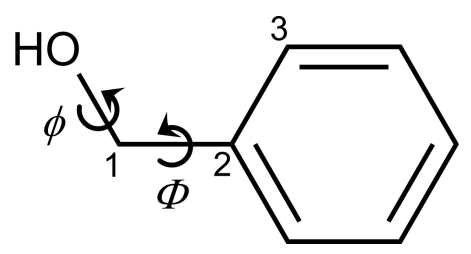

Abb. 5.46: Strukturformel von Benzylalkohol mit Benennung der für die Konformation relevanten Kohlenstoffatome und Torsionswinkel: $\mathrm{OC}_{1} \mathrm{C}_{2} \mathrm{C}_{3}(\Phi)$ und $\mathrm{HOC}_{1} \mathrm{C}_{2}(\phi)$.
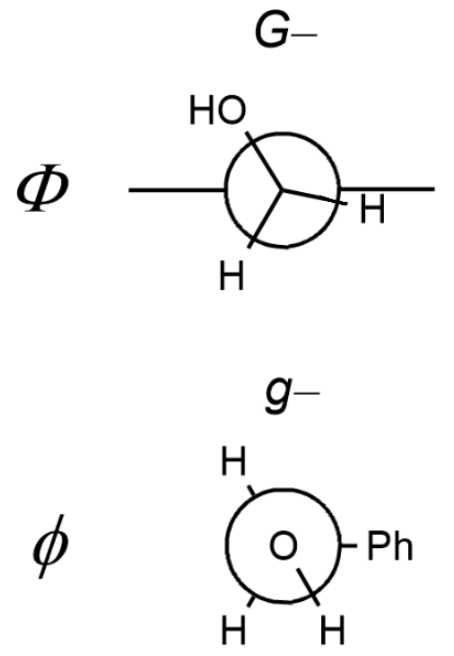
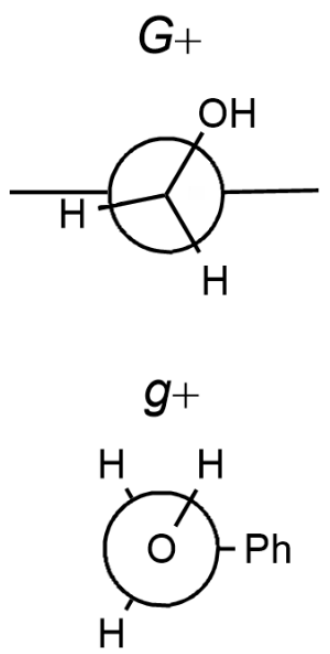
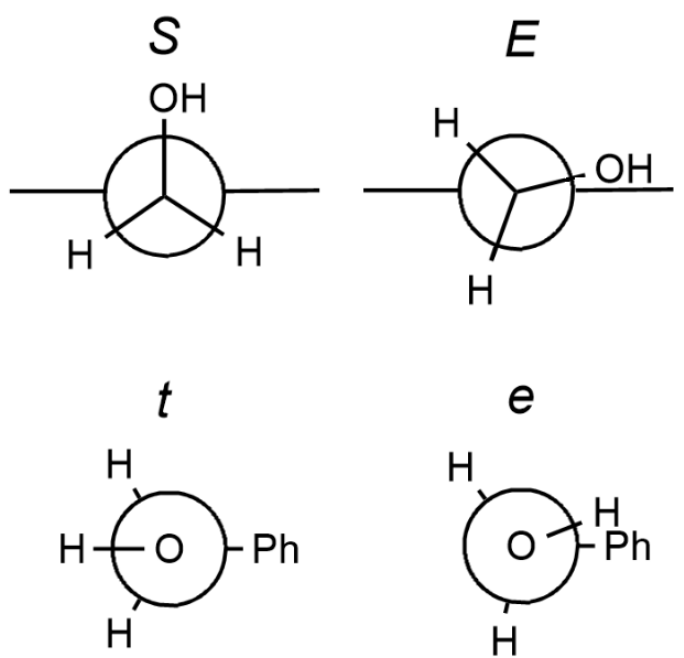

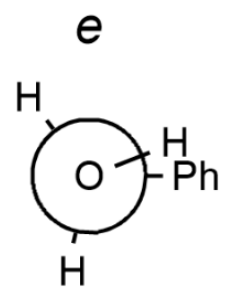

Abb. 5.47: Newman-Projektionen bezüglich der $\mathrm{C}_{1}-\mathrm{C}_{2}$ - (oben) und $\mathrm{O}-\mathrm{C}_{1}$-Bindung für unterschiedliche Einstellungen der $\mathrm{OC}_{1} \mathrm{C}_{2} \mathrm{C}_{3}-(\Phi)$ und $\mathrm{HOC}_{1} \mathrm{C}_{2}$-Torsionswinkel $(\phi) . G / g$ steht für gauche, $S$ für senkrechte, $t$ für trans und $E / e$ für ekliptische Anordnungen. 
Im Vergleich zu 2-Butanol ist Benzylalkohol durch generell niedrige Barrieren flexibler und es kommt zu einer starken Kopplung der beiden Torsionswinkel, d. h. die Geometrien und relativen Energien der stabilen Konformere lassen sich nicht mehr durch eine unabhängige Betrachtung der beiden Winkel vorhersagen. Deswegen war lange Zeit unklar bzw. umstritten, welche Anordnung, insbesondere bezüglich $\Phi$, in der stabilsten Konformation von Benzylalkohol vorliegt. Die Charakterisierung aller Minima und Übergangszustände gelang qualitativ richtig (nach heutigem Kenntnisstand) erstmals Glaser und Nichols [327]. Deren quantenchemische Suche und Rationalisierung der stationären Punkte, sowie Vergleiche mit widersprüchlichen experimentellen Daten, gleicht einem Kriminalroman. Während Glaser und Nichols dies nur durch die Optimierung von spezifischen Strukturideen bewerkstelligten, ist es systematischer und anschaulicher für diese Fragestellung einen relaxierten zweidimensionalen Scan beider Torsionswinkel auszuwerten. Entsprechende Scans auf verschiedenen Niveaus wurden zuvor bereits mehrfach veröffentlicht [328-331], aber nicht im Detail diskutiert. Aus Gründen des Rechenaufwandes wurde die in Abb. 5.48 dargestellte Potentialfläche auf B3LYP-D3(BJ)/def2-TZVPNiveau berechnet, und deren Minima und Übergangszustände anschließend auf B3LYPD3(BJ)/may-cc-pVTZ-Niveau nachoptimiert. Die Ergebnisse ändern sich zwischen beiden Niveaus nur graduell, nicht qualitativ. Konkrete Energie- und Geometrieangaben beziehen sich stets auf das höhere Niveau.

Hilfreich ist es zunächst die Symmetrie des Moleküls zu betrachten, welche auch eine Symmetrie der Potentialfläche nach sich zieht. Die zweizählige Symmetrie des Phenylringes führt dazu, dass eine Torsion um die $\mathrm{C}_{1}-\mathrm{C}_{2}$-Bindung $(\Phi)$ um $\pm 180^{\circ}$ stets in einer identischen Anordnung resultiert. Dies ist im Diagramm daran zu erkennen, dass sich alle Merkmale bei vertikaler Verschiebung um diesen Betrag wiederholen. Des Weiteren muss, wegen des nicht-permanent chiralen Charakters von Benzylalkohol, bei Inversion der Vorzeichen beider Torsionswinkel (und Relaxation der übrigen Geometrieparameter) stets eine zur Ausgangsstruktur isoenergetische Anordnung resultieren. Diese kann enantiomer oder identisch zur Ausgangsstruktur sein, abhängig davon, ob letztere transient chiral oder achiral ist. Dies äußert sich im Diagramm dadurch, dass dessen Mittelpunkt als Inversionszentrum für alle Merkmale agiert. Alternativ kann anstatt der Punktspiegelung auch gedanklich eine Drehung des Diagramms um $180^{\circ}$ in der Papierebene durchgeführt werden, diese Symmetrieoperationen sind für eine zweidimensionale Fläche äquivalent. Diese beiden Symmetrieelemente führen dazu, dass bereits ein Quadrant des Diagramms die vollständige Information enthält. Für die einfachere Nachvollziehbarkeit von Entartungen und Umwandlungspfaden ist es aber anschaulicher die volle $360^{\circ} \times 360^{\circ}$-Fläche zu betrachten.

Das globale Minimum von Benzylalkohol ist die $g$-Konformation (Abb. 5.49), bei der beide Torsionswinkel eine gauche-Einstellung mit gleichen Vorzeichen aufweisen $(G-g-/ G+g+)$. Diese Präferenz des Moleküls ist seit etwa zwei Jahrzehnten sowohl quantenchemisch [294, 327, 331-333] wie experimentell durch Messungen in Überschallexpansionen [220, 294, 332, 333] etabliert. Neben den beiden zueinander enantiomeren Varianten $g+$ und $g-$ ist die Konformation noch zusätzlich bezüglich der Drehung des Phenylringes entartet, sodass eine insgesamt vierfache Entartung resultiert. Der mit B3LYPD3(BJ)/may-cc-pVTZ berechnete Wert für $g-$ von $\Phi=-55.6^{\circ}$ steht in guter Übereinstimmung mit dem rotationsspektroskopischen Ergebnis von -54.5(7) ${ }^{\circ}$ [294]. Auf B3LYPD3(BJ)/def2-TZVP-Niveau wird dagegen $-39^{\circ}$ erhalten. Diese Unterschätzung wurde 


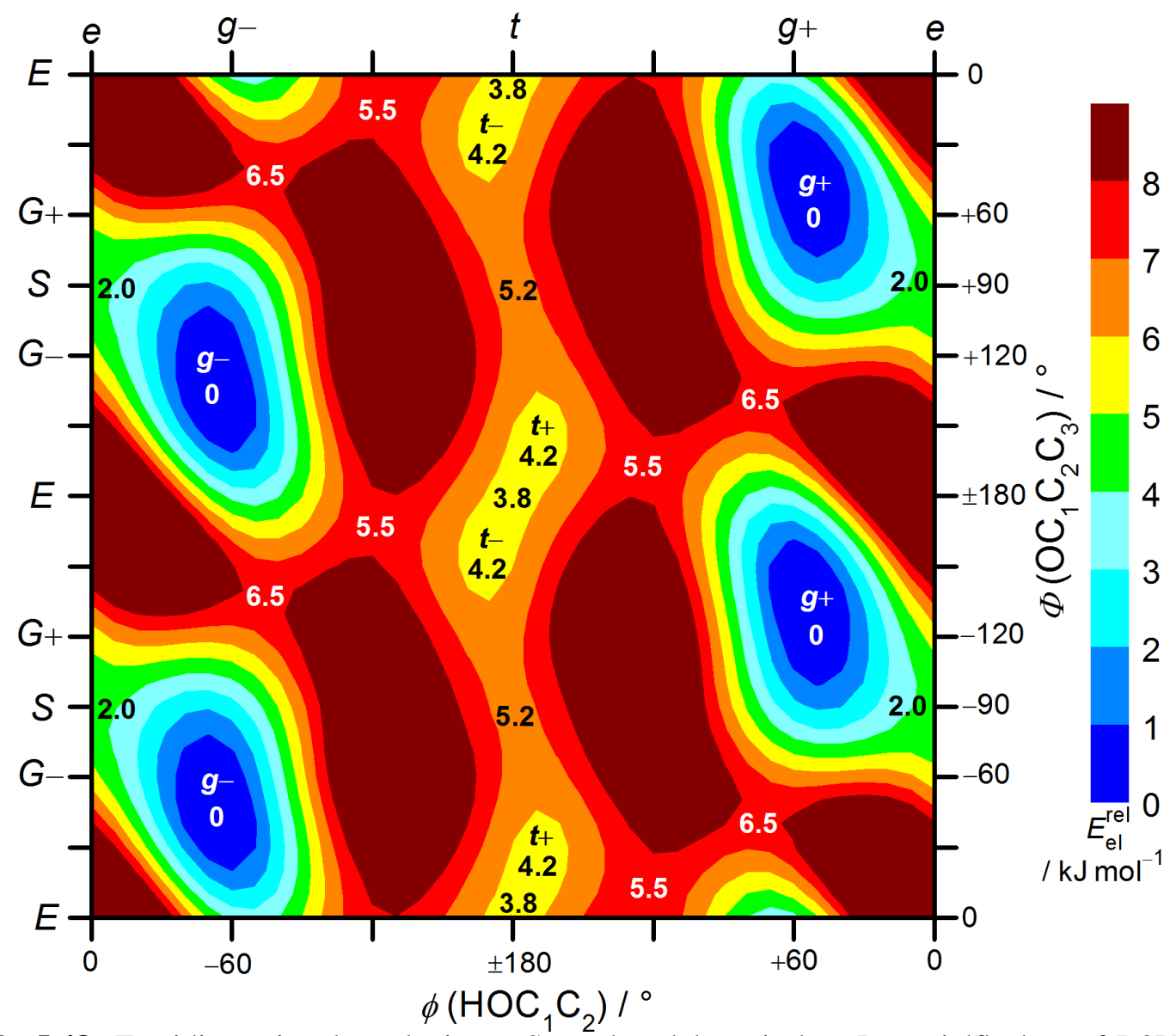

Abb. 5.48: Zweidimensionaler relaxierter Scan der elektronischen Potentialfläche auf B3LYPD3(BJ)/def2-TZVP-Niveau bezüglich der $\mathrm{OC}_{1} \mathrm{C}_{2} \mathrm{C}_{3}-(\Phi)$ und $\mathrm{HOC}_{1} \mathrm{C}_{2}$-Torsionswinkel $(\phi)$ von Benzylalkohol in $10^{\circ}$-Schritten (1369 Datenpunkte). Die Zahlenwerte im Diagramm entsprechen den Energien der volloptimierten Minima (mit Benennung) sowie der Übergangszustände (ohne Benennung) auf B3LYP-D3(BJ)/may-cc-pVTZ-Niveau mit zusätzlicher Nullpunktsenergiekorrektur relativ zum globalen Minimum.

zuvor auch mit anderen Basissätzen ohne diffuse Funktionen für MP2 und B3LYP gefunden (siehe Supporting Information von Ref. [334]). Thiobenzylalkohol (Benzylmercaptan) besitzt eine analoge Konformation mit $\Phi=-74^{\circ}$.[278]

Der experimentelle Wert für $\phi$ von $-64.6(46)^{\circ}$ für Benzylalkohol [294] wird mit B3LYP-D3(BJ) sowohl mit may-cc-pVTZ wie auch def2-TZVP Basis betragsmäßig um etwa $12^{\circ}$ unterschätzt. Werte auf MP2/6-311++G**- [294] und DF-LMP2/cc-pVQZNiveau [331] sind für beide Torsionswinkel vergleichbar mit denen von B3LYP-D3(BJ)/ may-cc-pVTZ, weisen also auch eine ähnliche Diskrepanz zum experimentellen Wert von $\phi$ auf. Abweichungen können durch den quantenchemisch nicht berücksichtigten Einfluss von Nullpunktsschwingungen entstehen.

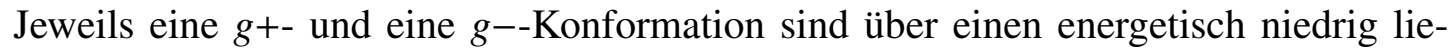
genden, $C_{\mathrm{s}}$-symmetrischen Übergangszustand voneinander getrennt, bei dem die $\mathrm{C}-\mathrm{O}$ und O-H-Bindungen in einer Spiegelebene senkrecht zur Ebene des Phenylringes liegen (S e-Anordnung). Dieser Umwandlungspfad ist in Abb. 5.49 gezeigt. Dass diese Geometrie einen Übergangszustand für Benzylalkohol darstellt, ist in der Reihe Benzylmethan [335], Benzylamin [336], Benzylalkohol und Benzylfluorid [337] eine Besonderheit, da 

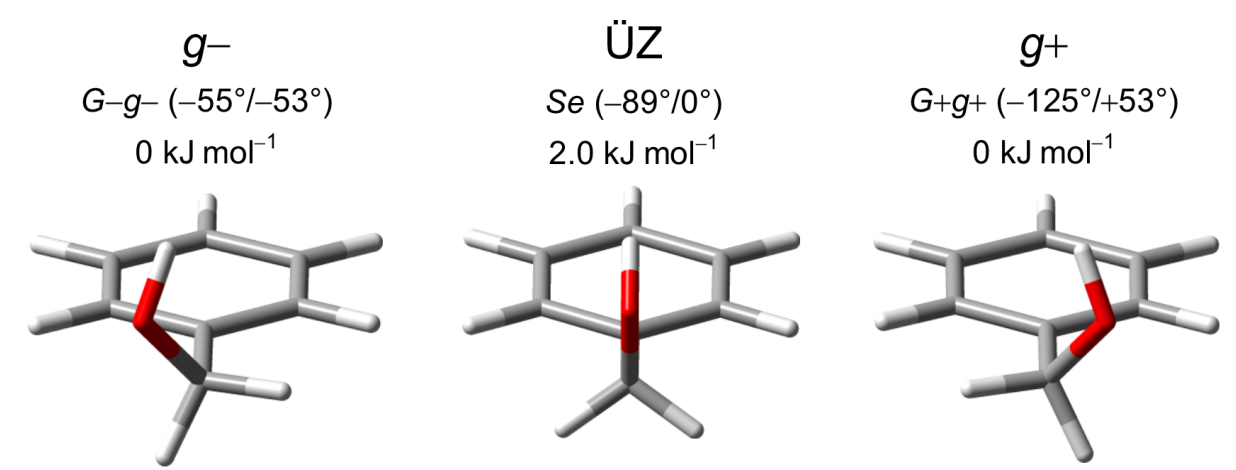

Abb. 5.49: Geometrien und nullpunktskorrigierte Energien auf B3LYP-D3(BJ)/may-cc-pVTZNiveau für die direkte Umwandlung zwischen $g-$ und $g+$. Der von $\Phi=-90^{\circ}$ leicht abweichende Wert des Übergangszustandes beruht auf einer nicht-exakten Planarität des Phenylringes.

die $S e$-Konformation für die anderen drei Moleküle stattdessen dem globalen Minimum entspricht (bei Benzylamin aber praktisch isoenergetisch zu $g$ ). Der Übergangszustand ist energetisch niedrig genug, dass eine geringe Tunnelaufspaltung von etwa $0.02 \mathrm{~cm}^{-1}$ beobachtet wird [294], die mit den in der vorliegenden Arbeit verwendeten schwingungsspektroskopischen Methoden nicht aufgelöst werden kann. Dieser Tunnelprozess in Benzylalkohol und dessen Derivaten wurde in den letzten Jahren detailliert untersucht.[266-269, 278, 331] Durch die Kopplung der beiden Torsionsfreiheitsgrade (diagonale Bewegung im Diagramm) beinhaltet der Tunnelprozess nicht nur eine Bewegung des Hydroxyprotons, sondern auch der anderen Atome der Seitenkette. Wie anhand des Isotopeneffektes in Abschnitt 5.9 gezeigt wurde, erscheint die effektive Tunnelmasse deutlich höher als für Tunnelprozesse zwischen $g$-Konformeren anderer Alkohole. Analog dazu zeigt auch die Tunnelaufspaltung in Thiobenzylalkohol eine deutliche Abhängigkeit von der Masse des Schwefel- und des $\alpha$-Kohlenstoffatoms.[278] Die Barrierenhöhe für Benzylalkohol beträgt auf B3LYP-D3(BJ)/may-cc-pVTZ-Niveau mit voller Schwingungsnullpunktskorrektur $2.0 \mathrm{~kJ} \mathrm{~mol}^{-1}$.

Durch selektive Fluorierung des Ringes kann der Tunnelprozess beeinflusst werden.[266-269, 331, 338] Bei Fluorierung in para-Position bleibt die Symmetrie des Moleküls erhalten, der Tunnelprozess wird etwas verlangsamt.[331, 338] Asymmetrische Fluorierung des Ringes in ortho- oder meta-Position [266-269, 331] führt dagegen zur Aufspaltung der vierfach entarteten $g$-Konformation in zwei zueinander diastereomere Enantiomerenpaare. Dieser Energieunterschied führt zu einer Löschung der Tunnelaufspaltung, genau wie bei einer asymmetrischen ${ }^{13} \mathrm{C}$-Isotopensubstitution in Thiobenzylalkohol.[278]

Es bestehen noch weitere Pfade auf der Potentialfläche, um $g$-Konformere ineinander umzuwandeln. Die zweite Möglichkeit ist die direkte Umwandlung zwischen identischen $g+$ bzw. $g$--Minima durch Rotation des Phenylringes um $180^{\circ}$, während $\phi$ nur wenig verändert wird (annähernd vertikale Bewegung im Diagramm). Dieser Pfad ist in Abb. 5.50 dargestellt. Diese direkte Umwandlung ist mit einer relativ hohen Barriere $\left(6.5 \mathrm{~kJ} \mathrm{~mol}^{-1}\right.$ nach Schwingungsnullpunktskorrektur) verbunden, wozu eine ungünstige sterischen Wechselwirkung zwischen dem Hydroxywasserstoffatom und einem Wasserstoffatom in ortho-Position des Phenylringes beitragen könnte.

Als dritte Möglichkeit kann auch das Hydroxywasserstoffatom vom Phenylring zunächst weggedreht werden um diesen ungünstigen Kontakt zu vermeiden, was über eine 


\section{Torsionsisomerie von Alkoholen}
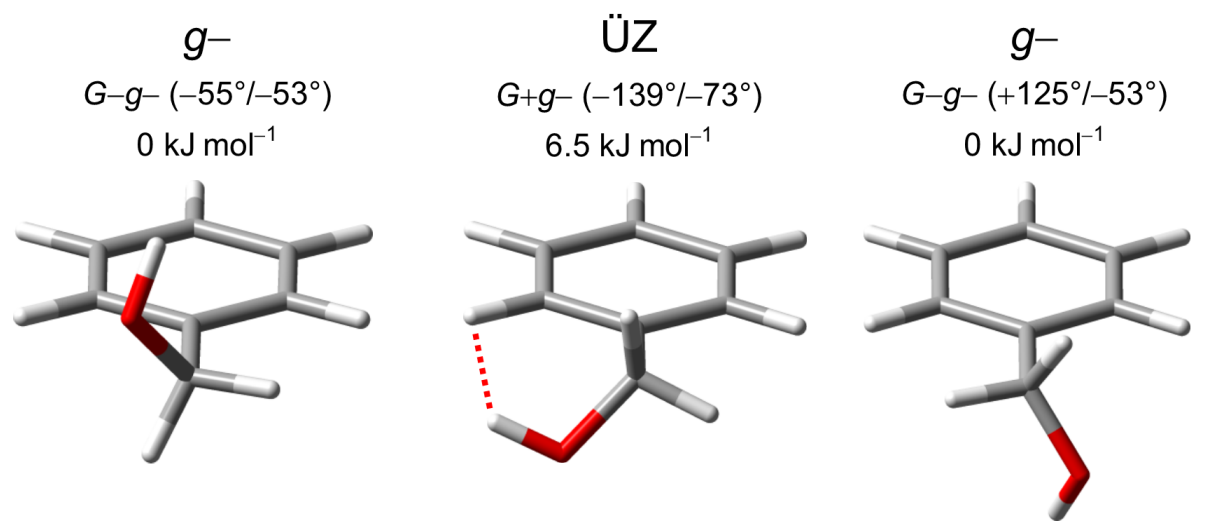

Abb. 5.50: Geometrien und nullpunktskorrigierte Energien auf B3LYP-D3(BJ)/may-cc-pVTZNiveau für die direkte Umwandlung zwischen $g$ - und seiner identischen Entsprechung. Im Übergangszustand ist ein vermutlich sterisch ungünstiger $\mathrm{OH} \cdots \mathrm{HC}$-Kontakt von $2.3 \AA$ markiert.

etwas niedrigere Barriere $\left(5.5 \mathrm{~kJ} \mathrm{~mol}^{-1}\right) \mathrm{zu}$ der hochenergetischen $t$-Konformation von Benzylalkohol führt. Dieser Pfad ist in Abb. 5.51 gezeigt. Eine Fortsetzung dieser Bewe-
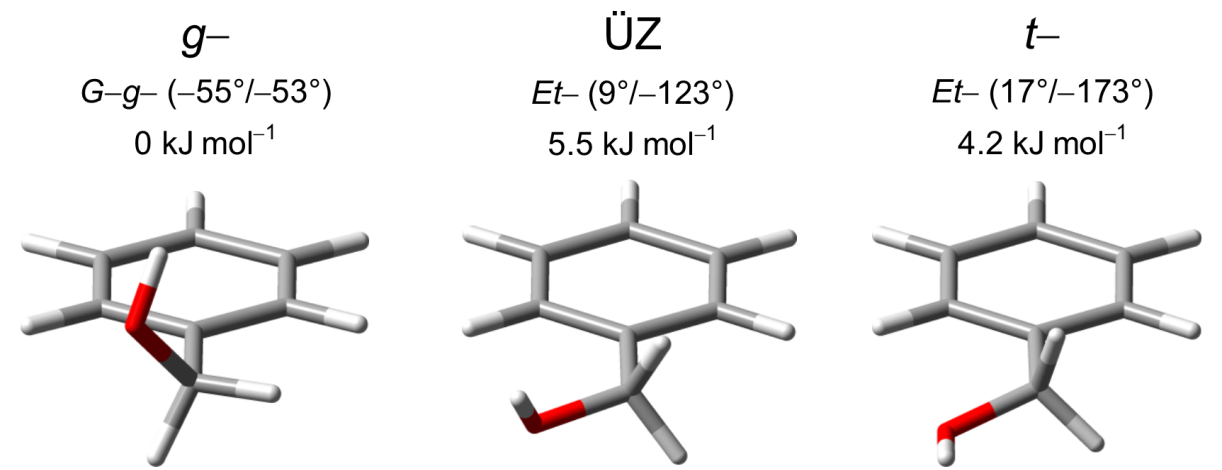

Abb. 5.51: Geometrien und nullpunktskorrigierte Energien auf B3LYP-D3(BJ)/may-cc-pVTZNiveau für die direkte Umwandlung zwischen $g-$ und $t-$.

gung führt schließlich zu einer $g$-Konformation enantiomer zum Ausgangszustand. Für diesen Pfad sind Veränderungen von $\phi$ und $\Phi$ stärker miteinander gekoppelt, sodass sich statt eines horizontalen ein geschwungener Verlauf im Diagramm in Abb. 5.48 ergibt. Dieser Pfad führt für in meta-Position fluorierte Derivate ebenfalls zu einer winzigen Tunnelaufspaltung.[266, 269, 331] Dass eine solche nicht auch für die Stammverbindung Benzylalkohol beobachtet wird, liegt vermutlich daran, dass durch die Fluorierung der Betrag von $\Phi$ verringert, also das Sauerstoffatom näher an die Ringebene geführt wird, sodass die Barriere in den Derivaten schmaler wird.[269] Eine Übersicht über die Änderungen in der Geometrie und den Tunnelaufspaltungen von Benzylalkohol durch selektive Fluorierung bietet Referenz [269].

Auch die hochenergetische $E t$-Konformation (kurz: $t$ ) von Benzylalkohol wird auf der elektronischen Potentialfläche asymmetrisch gefunden, sodass ebenfalls eine $t+-$ und eine $t-$-Variante existieren, die jeweils wiederum zweifach entartet sind. Allerdings sind $t+$ und $t$ - nur durch eine schmale und energetisch äußerst niedrige elektronische Barriere von $0.1 \mathrm{~kJ} \mathrm{~mol}^{-1}$ voneinander getrennt, die nach Nullpunktkorrektur sogar negativ, d. h. zu einem lokalen Minimum bezüglich der Gesamtenergie, wird. Es erscheint daher sinnvoller, $t$ als nur doppelt entartete, achirale Konformation zu behandeln. Dies ist in Abb. 
5.52 illustriert. Mit dem Hydroxywasserstoff in $t$-Position kann die Rotation des Phenyl-
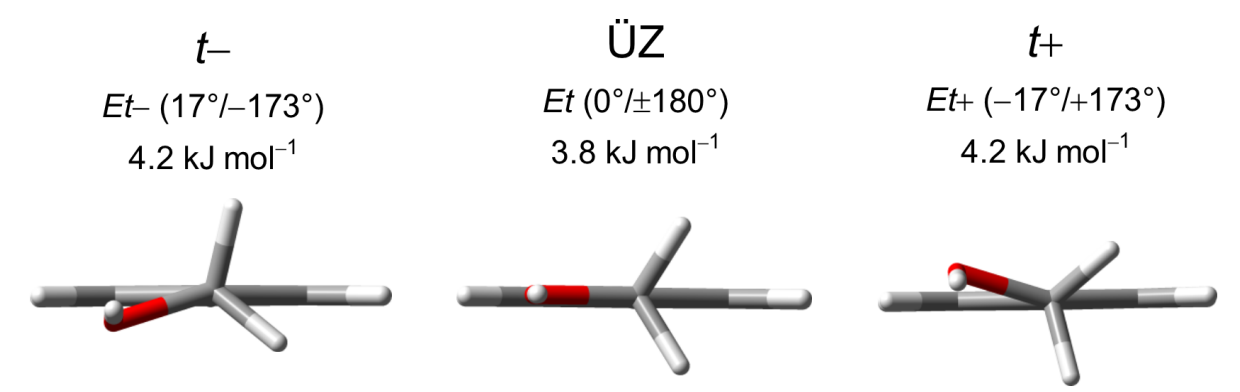

Abb. 5.52: Geometrien und nullpunktskorrigierte Energien auf B3LYP-D3(BJ)/may-cc-pVTZNiveau für die direkte Umwandlung zwischen $t-$ und $t+$. Der Übergangszustand liegt auf der elektronischen Potentialfläche nur sehr geringfügig oberhalb der $t-/ t+-$ Minima, dies kehrt sich nach Nullpunktskorrektur um.

ringes über eine Barriere von nur $1.4 \mathrm{~kJ} \mathrm{~mol}^{-1}$ erfolgen. Über einen solchen zusätzlichen Schritt (gezeigt in Abb. 5.53), der in den dritten Umwandlungspfad (Abb. 5.51) eingeschoben wird, lässt sich $g$ auf eine weitere Art in eine identische Entsprechung umwandeln. Der höchste dafür zu überwindende Übergangszustand von $5.5 \mathrm{~kJ} \mathrm{~mol}^{-1}$ ist in dieser vierten Möglichkeit energetisch niedriger als der in der direkten, vertikalen Umwandlung $\left(6.5 \mathrm{~kJ} \mathrm{~mol}^{-1}\right)$.
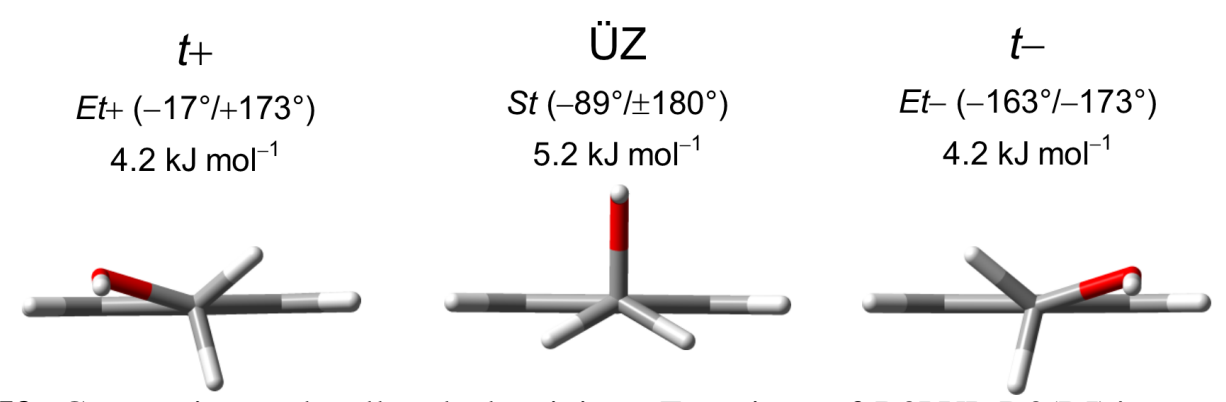

Abb. 5.53: Geometrien und nullpunktskorrigierte Energien auf B3LYP-D3(BJ)/may-cc-pVTZNiveau für die alternative Umwandlung zwischen $t-$ und $t+$. Der von $\Phi=-90^{\circ}$ leicht abweichende Wert des Übergangszustandes beruht auf einer nicht-exakten Planarität des Phenylringes.

In der Literatur wird kontrovers diskutiert, wie der Energievorteil und die Geometrie von $g \mathrm{zu}$ begründen ist. Die geometrische Ausrichtung der Hydroxygruppe zumindest grob in Richtung der $\pi$-Elektronenwolke und die im Vergleich zu anderen primären Alkoholen ungewöhnlich niedrige OH-Streckschwingungswellenzahl (siehe auch Abb. 5.62 auf Seite 189) werden von den meisten Autoren als Hinweise auf eine schwache intramolekulare $\mathrm{OH} \cdots \pi$-Wasserstoffbrücke gedeutet.[219, 220, 329, 330, 332, 337, 339] Die Bedeutung der Wasserstoffbrücke für die Stabilität von $g$ wird aber unterschiedlich bewertet. Zum Teil wird sie als nur untergeordneter Faktor eingeschätzt, mit Dipol-Dipol-Wechselwirkung zwischen den $\mathrm{C}_{1}-\mathrm{H}$ - und $\mathrm{C}_{3}-\mathrm{H}$-Bindungen als Haupterklärung [337]. Andere Autoren negieren die Präsenz einer $\mathrm{OH} \cdots \pi$-Wasserstoffbrücke grundsätzlich.[327, 334, 340] Alternative Erklärungen umfassen die Abstoßung zwischen den freien Elektronenpaaren des Sauerstoffs und den $\pi$-Elektronen [334], $\pi \rightarrow \sigma_{\mathrm{C}-\mathrm{O}}^{*}$-Donationen [340] oder das Zusammenspiel von mehreren Beiträgen: Abstoßung zwischen den freien Elektronenpaaren des Sauerstoffs und den $\pi$-Elektronen, attraktive $\mathrm{OH} \cdots \pi$-Wechselwirkung (abgegrenzt 


\section{Torsionsisomerie von Alkoholen}

zu einer Wasserstoffbrücke) und Dipol-Dipol-Wechselwirkung zwischen den $\mathrm{C}_{1}-\mathrm{C}_{2}$ - und $\mathrm{O}-\mathrm{H}$-Bindungen [327].

Auch anhand dieser anhaltenden Diskussion lässt sich erkennen, dass Benzylalkohol trotz seiner einfachen Konstitution einen komplexen Konformationsraum mit weiterhin offenen Fragen aufweist.

\subsubsection{Schwingungsspektren}

Die schwingungsspektroskopischen Kenngrößen der $g$ - und $t$-Konformere von Benzylalkohol sind in Abb. 5.54 gegenübergestellt. Die berechnete harmonische OH-Streckschwingungswellenzahl von $t$ ist wegen der komplizierten Potentialfläche in dessen Umgebung mit einer größeren Unsicherheit behafte. So ist sie für den (vermeintlichen) Übergangszustand $E t$ um $3 \mathrm{~cm}^{-1}$ höher als für das entartete elektronische Minimum $t$.
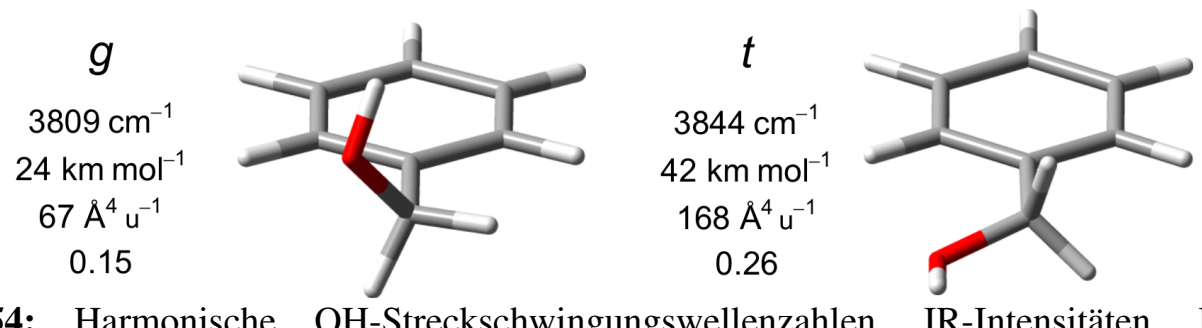

Abb. 5.54: Harmonische OH-Streckschwingungswellenzahlen, IR-Intensitäten, RamanAktivitäten und Depolarisationsverhältnisse der Konformere von Benzylalkohol auf B3LYPD3(BJ)/may-cc-pVTZ-Niveau.

Benzylalkohol liegt bei Zimmertemperatur als Flüssigkeit vor und hat eine Normalsiedetemperatur von $206{ }^{\circ} \mathrm{C}$. Der extrapolierte Dampfdruck bei $24^{\circ} \mathrm{C}$ (siehe Tab. 3.1 auf S. 21) beträgt nur etwa $8 \mathrm{~Pa}$. Für die Beobachtung von Aggregation (die in Kapitel 8 behandelt wird) ist ein geheizter Aufbau sinnvoll, Spektren wurden daher am Popcorn- und Curry-Jet aufgenommen. Abb. 5.55 zeigt den Monomerbereich im Vergleich mit Simulationen. Das $g$-Konformer kann der intensiven Bande bei $3647.5 \mathrm{~cm}^{-1}$ zugeordnet werden. Die Vorhersage aus dem Korrelationsmodell überschätzt diese Wellenzahl um $2 \mathrm{~cm}^{-1}$, wobei hier zu erwähnen ist, dass bereits ein Literaturwert von $3648 \mathrm{~cm}^{-1}$ [333] Bestandteil des Trainingssets des Korrelationsmodells war. Das $t$-Konformer ist an einer Position um $3685 \mathrm{~cm}^{-1}$ zu erwarten. Dies wäre, zusammen mit Methanol, wohl mit die höchste bekannte OH-Streckschwingungswellenzahl für Alkohole (siehe auch Abb. 5.3 auf Seite 113). Doch kann weder an dieser noch an einer anderen Position eine Bande gefunden werden, die dem $t$-Konformer zugeordnet werden könnte. Die Bande bei $3656.5 \mathrm{~cm}^{-1}$ in den Raman-Spektren stammt von der symmetrischen Streckschwingung einer Wasserverunreinigung.[238] Auch bei einer erhöhten Düsentemperatur von $200{ }^{\circ} \mathrm{C}$ kann, bis auf eine leichte Verbreiterung der Bande des $g$-Konformers, keine Änderung der Spektren festgestellt werden. Unter ähnlichen Bedingungen konnte kürzlich das cis-Konformer von Ameisensäure, mit einer relativen Energie von etwa $16 \mathrm{~kJ} \mathrm{~mol}^{-1}$ gegenüber dem stabileren trans-Konformer, nachgewiesen werden.[342] Dass diese Strategie nicht auch für das $t$-Konformer von Benzylalkohol gelingt, dürfte der sehr niedrigen Barriere von etwa 1$2 \mathrm{~kJ} \mathrm{~mol}^{-1}$ und einer dadurch sehr schnellen Relaxation in $g$, auch unter Jet-Bedingungen, geschuldet sein. Das cis-Konformer von Ameisensäure besitzt dagegen nach schneller Abkühlung noch eine Lebensdauer in der Größenordnung von Minuten und kann damit 
auch nach Deposition in einer Matrix noch beobachtet werden.[343] Bei der in den Simulationen in Abb. 5.55 angenommenen Konformationstemperatur von $100 \mathrm{~K}$ wäre ein t: g-Intensitätsverhältnis von 1:170 (IR) bzw. 1:130 (Raman) zu erwarten - offenbar zu gering für eine Detektion.

Die Suche nach der $t$-Konformation von Benzylalkohol war bisher auch in der Gasphase bei Zimmertemperatur erfolglos, den Obertonbereich eingeschlossen,[332] Auch in gebundener Form in der Kristallstruktur liegt eine nur geringfügig deformierte $g$ Konformation mit $\Phi=-44^{\circ}$ und $\phi=-60^{\circ}$ vor, basierend auf Auswertung der kristallographischen Daten aus Referenz [344]. ${ }^{\mathrm{d}}$ In verdünnter Lösung in Tetrachlormethan wurde die Asymmetrie der OH-Streckschwingungsbande von Benzylalkohol als Überlagerung von zwei Konformeren interpretiert, wobei die intensivere, niederfrequente Komponente

${ }^{\mathrm{d}}$ In der Publikation ist aber offenbar die Position des Hydroxywasserstoffatoms im EinzelmolekülAusschnitt der Kristallstruktur falsch dargestellt.

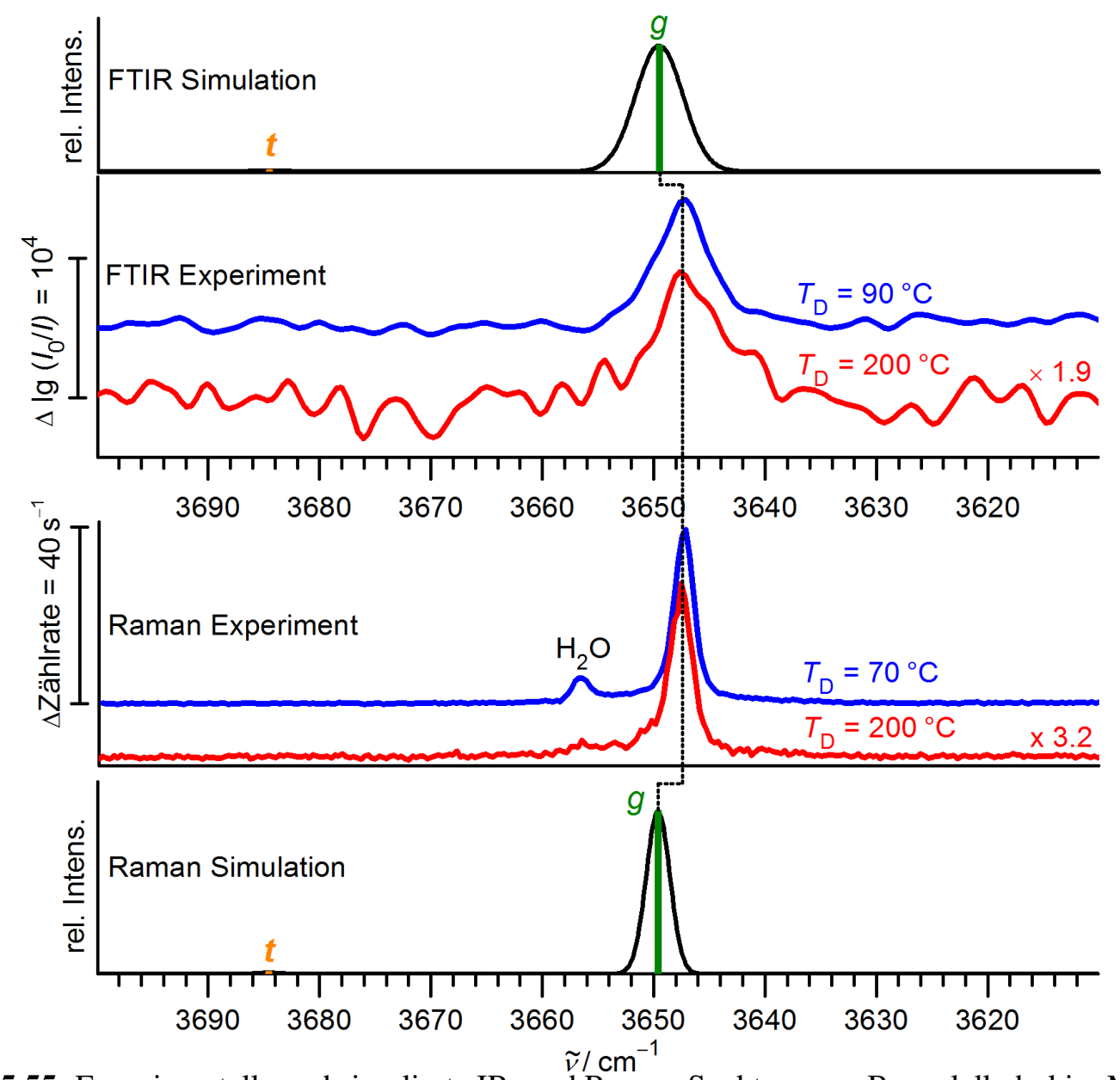

Abb. 5.55: Experimentelle und simulierte IR- und Raman-Spektren von Benzylalkohol im Monomerbereich. Die IR-Spektren wurden aus Ref. [341] (90 ${ }^{\circ} \mathrm{C}$ Düsentemperatur) bzw. Ref. [333] $\left(200^{\circ} \mathrm{C}\right.$ Düsentemperatur) übernommen. Die Bande bei $3656.5 \mathrm{~cm}^{-1}$ in den Raman-Spektren stammt von der symmetrischen Streckschwingung einer Wasserverunreinigung.[238] Simulationsparameter sind einheitliche Verschiebung der harmonischen Wellenzahlen um $\kappa=-159.4 \mathrm{~cm}^{-1}$, eine Konformationstemperatur von $100 \mathrm{~K}$ und Gauss-Profile mit $\mathrm{FWHM}=5 \mathrm{~cm}^{-1}$ für die IR- und $2.5 \mathrm{~cm}^{-1}$ für die Raman-Banden. 
einer intramolekular gebundenen Hydroxygruppe und die höherfrequente Komponente einer freien Hydroxygruppe zugeordnet wird.[329, 345-348] Zumindest in Lösung gibt es daher Hinweise auf das $t$-Konformer. Durch das Einbringen von elektronenziehenden Substitutenten in meta-Position kann das $t$-Konformer stabilisiert werden.[346, 348] Dies wird durch eigene Rechnungen gestützt, gemäß denen der energetische Nachteil von $t$ in beispielsweise 3-Nitrobenzylalkohol von 4 auf $1 \mathrm{~kJ} \mathrm{~mol}^{-1}$ sinkt und sich in 3,5-Dinitrobenzylalkohol sogar in einen Vorteil von $1 \mathrm{~kJ} \mathrm{~mol}^{-1}$ verwandelt. Interessanterweise geht diese drastische Änderung in der relativen Energie fast ohne Einfluss auf die berechneten $\mathrm{OH}-\mathrm{Streckschwingungswellenzahlen} \mathrm{einher.} \mathrm{Dies} \mathrm{wurde} \mathrm{der} \mathrm{Methylengrup-}$ pe zugeschrieben, die die Elektronendichte des Ringes von der Polarität der Hydroxygruppe entkoppelt.[346]

Zusammengefasst tritt Benzylalkohol unter Jet-Bedingungen, im Gegensatz zu Ethanol, effektiv nur in zwei zueinander enantiomeren Konformeren auf ( $g-$ und $g+)$. Diese können auch noch bei geringer Temperatur, unterstützt von einem Tunnelprozess, ineinander umwandeln - anders als beispielsweise die unter Jet-Bedingungen effektiv permanent chiralen Konformere von Ethylenglycol [27]. Zusammen mit der Phenylgruppe - als potentieller Akzeptor für Wasserstoffbrücken und polarisierbares Strukturelement für starke Dispersionswechselwirkung - bietet Benzylalkohol damit gute Voraussetzungen für die Untersuchung von Chiralitätssynchronisation und -induktion, für die in Kapitel 8 Beispiele behandelt werden. 


\subsection{1-Phenylethanol}

1-Phenylethanol, auch sec-Phenethanol, sec-Phenylethylalkohol oder $\alpha$-Methylbenzylalkohol genannt, ist ein chiraler sekundärer Alkohol, dessen Konstitution in Abb. 5.46 gezeigt ist. Wie die Namen der Verbindung es nahelegen, lässt sie sich aus dem zuvor besprochenen Benzylalkohol durch Methylierung oder aus Ethanol durch Phenylierung, jeweils am $\alpha$-Kohlenstoffatom, ableiten. 1-Phenylethanol kommt in der Natur als Bestandteil vieler pflanzlicher Aromen vor und weist einen blumigen Geruch auf. Einen olfaktorischen Unterschied zwischen dem (-)- $(S)$-Enantiomer und der racemischen Mischung konnte der Autor der vorliegenden Arbeit nicht feststellen.

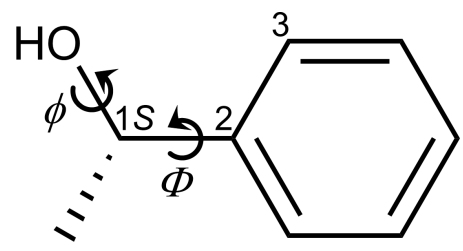

Abb. 5.56: Strukturformel von (-)-(S)-Phenylethanol mit Nummerierung der Kohlenstoffatome und für die Konformation relevante Torsionswinkel: $\mathrm{OC}_{1} \mathrm{C}_{2} \mathrm{C}_{3}(\Phi)$ und $\mathrm{HOC}_{1} \mathrm{C}_{2}(\phi)$.

\subsubsection{Konformationsisomerie}

Die Konformationsisomerie von 1-Phenylethanol wurde bereits zuvor in unterschiedlicher Detailtiefe untersucht [237, 330, 348-352], wobei besonders die Arbeit von Shin-ya et al. [351] hervorzuheben ist. In Erweiterung zu diesen früheren Arbeiten werden hier auf der elektronischen Potentialfläche zwei weitere Minima und die Übergangszustände charakterisiert.

Der Konformationsraum lässt sich analog zu Benzylalkohol durch zwei Torsionswinkel beschreiben (unter Nichtberücksichtigung der Methyltorsion), deren wichtigsten Einstellungen in Abb. 5.57 dargestellt sind.
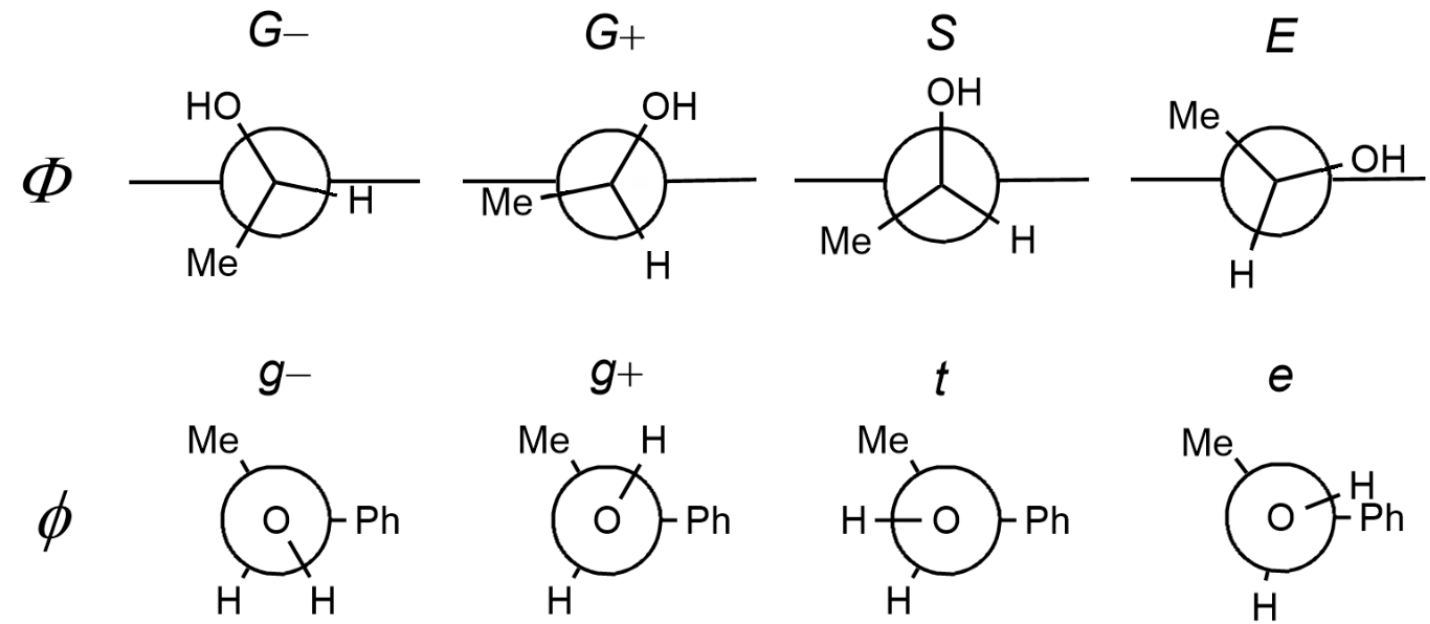

Abb. 5.57: Newman-Projektionen bezüglich der $\mathrm{C}_{1}-\mathrm{C}_{2}$ - (oben) und der $\mathrm{O}-\mathrm{C}_{1}$-Bindung für unterschiedliche Einstellungen der $\mathrm{OC}_{1} \mathrm{C}_{2} \mathrm{C}_{3^{-}}(\Phi)$ und $\mathrm{HOC}_{1} \mathrm{C}_{2}$-Torsionswinkel $(\phi)$ für $(S)$-1Phenylethanol. $G / g$ steht für gauche, $S$ für senkrechte, $t$ für trans und $E / e$ für ekliptische Anordnungen. 


\section{Torsionsisomerie von Alkoholen}

Mit identischen Parametern wie für Benzylalkohol wurde die Potentialfläche für beide Torsionswinkel (mit Relaxation der übrigen Parameter) auf B3LYP-D3(BJ)/def2-TZVPNiveau gescannt und alle Minima und Übergangszustände auf B3LYP-D3(BJ)/may-ccpVTZ-Niveau nachoptimiert. Das Ergebnis ist in Abb. 5.58 dargestellt.

Die Potentialfläche zeigt sowohl wichtige Gemeinsamkeiten wie auch größere Unterschiede zu der von Benzylalkohol (Abb. 5.48 auf S. 174). Die Symmetrie bezüglich der Rotation der Phenylgruppe (Veränderung von $\Phi$ um $\pm 180^{\circ}$ ) bleibt erhalten, die Punktsymmetrie bezüglich des Zentrums des Diagramms geht dagegen verloren. Letzteres ist in der permanenten Chiralität von 1-Phenylethanol begründet. Bei Inversion der beiden betrachteten Torsionswinkel können die übrigen Geometrieparameter des Moleküls nicht mehr in eine enantiomere bzw. identische Anordnung relaxieren. Hierfür müsste die Konfiguration am asymmetrisch-substituierten Kohlenstoffatom invertiert werden, was durch einfache Drehung um Bindungen nicht möglich ist. Durch die Punktspiegelung werden stattdessen die Potentialflächen von $(S)$ - und $(R)$-1-Phenylethanol ineinander überführt.

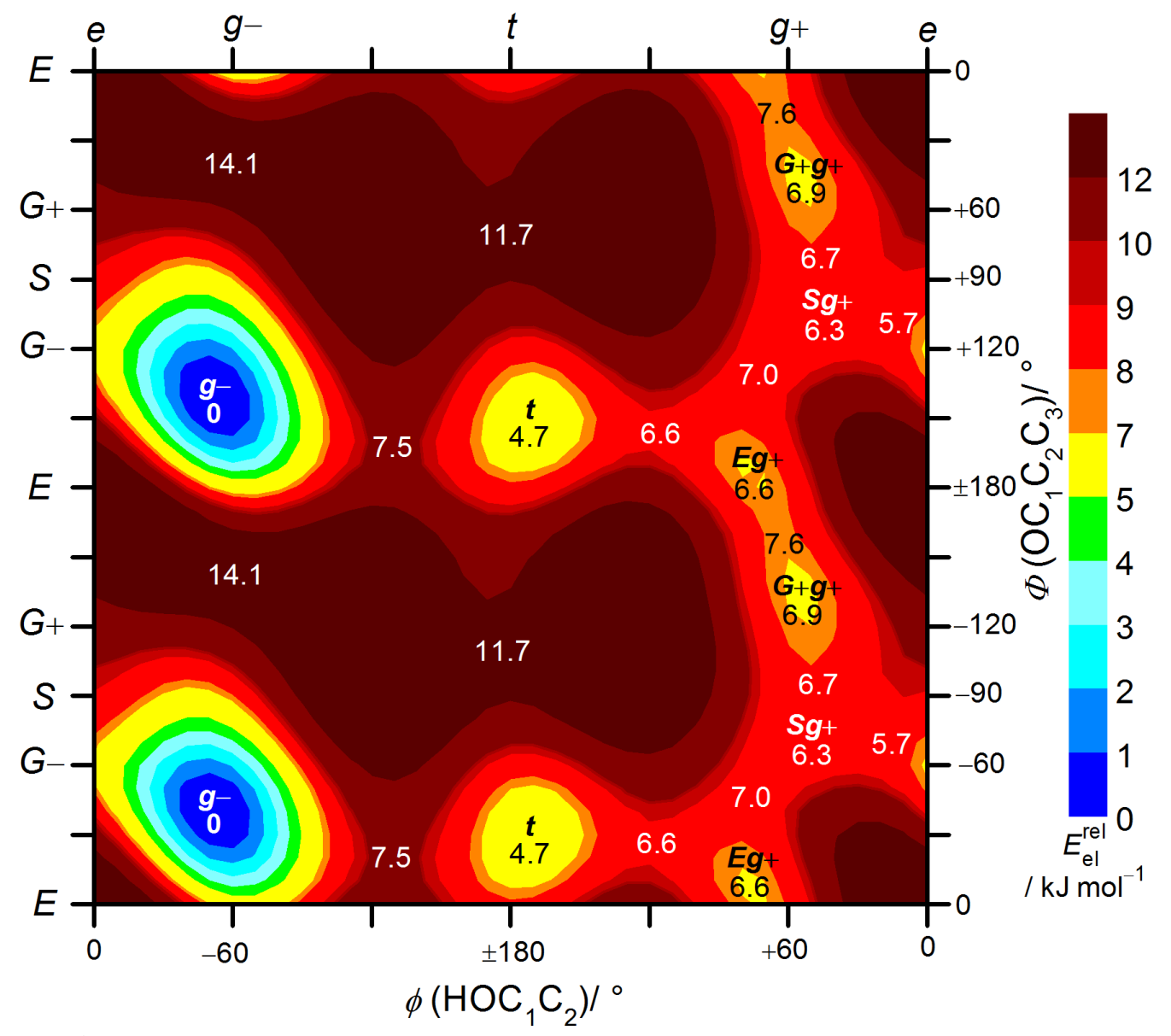

Abb. 5.58: Zweidimensionaler relaxierter Scan der elektronischen Potentialfläche auf B3LYPD3(BJ)/def2-TZVP-Niveau bezüglich der $\mathrm{OC}_{1} \mathrm{C}_{2} \mathrm{C}_{3}-(\Phi)$ und $\mathrm{HOC}_{1} \mathrm{C}_{2}$-Torsionswinkel $(\phi)$ von (S)-1-Phenylethanol in $10^{\circ}$-Schritten (1369 Datenpunkte). Die Zahlenwerte im Diagramm entsprechen den Energien der volloptimierten Minima (mit Benennung) sowie der Übergangszustände (ohne Benennung) auf B3LYP-D3(BJ)/may-cc-pVTZ-Niveau mit zusätzlicher Nullpunktsenergiekorrektur relativ zum globalen Minimum. 
Auf der elektronischen Potentialfläche finden sich fünf zueinander diastereomere Konformere, die jeweils zweifach entartet sind. Deren Strukturen sind in Abb. 5.59 gezeigt.

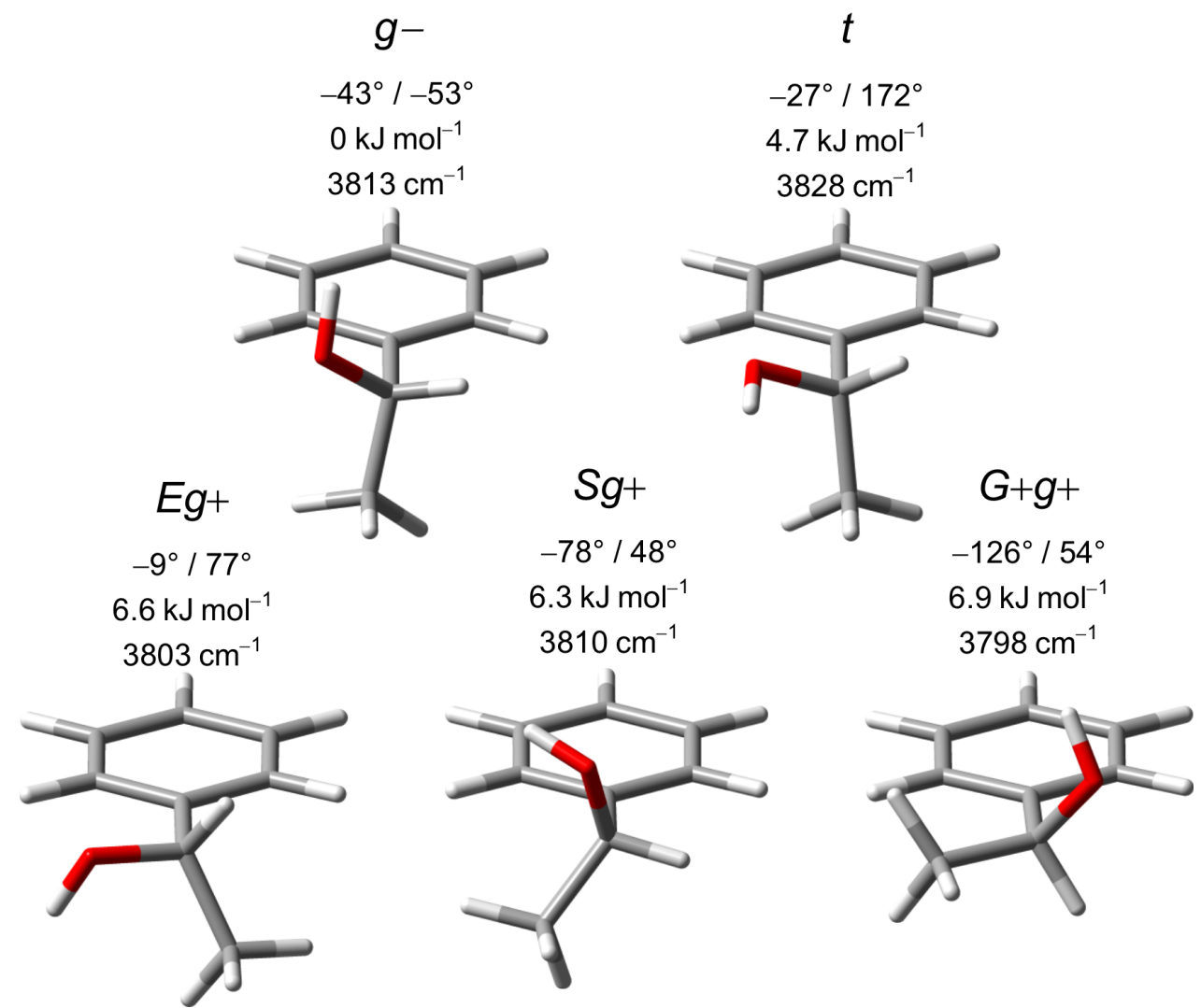

Abb. 5.59: Geometrien mit Angabe von $\Phi$ und $\phi$, nullpunktskorrigierte Energien und harmonische OH-Streckschwingungswellenzahlen der Konformere von $(S)$-1-Phenylethanol auf B3LYPD3(BJ)/may-cc-pVTZ-Niveau.

Das klare globale Minimum ist $G-g-$ (kurz: $g-$ ). Dessen hohe Stabilität gegenüber den anderen Konformeren kann durch die Stellung der beiden Substituenten am $\alpha$ Kohlenstoffatom begründet werden. Die Methylgruppe entspricht in ihrer weitgehend senkrechten Ausrichtung zur Ringebene der Präferenz in Ethylbenzol [335]. Ein idealer Wert von $90^{\circ}$ für den $\mathrm{C}_{\mathrm{Me}} \mathrm{C}_{1} \mathrm{C}_{2} \mathrm{C}_{3}$-Torsionswinkel würde wegen der tetraedrischen Symmetrie am sp ${ }^{3}$-hybridisierten $\alpha$-Kohlenstoffatom aber in $\Phi=-30^{\circ}$ resultieren, während die Präferenz der Hydroxygruppe in Benzylalkohol bei $-56^{\circ}$ gefunden wurde. Der für 1-Phenylethanol berechnete Wert von $-43^{\circ}$ stellt daher einen Kompromiss aus den bevorzugten Einstellungen der beiden Substituenten dar. Aus einem Abstract für einen Vortrag von 2003 [350] geht hervor, dass 1-Phenylethanol von Utzat et al. mit Benzylalkohol rotationsspektroskopisch verglichen wurde, die Ergebnisse für 1-Phenylethanol wurden aber in Folge nicht publiziert. Angegeben wird, dass nur eine Konformation gefunden wurde mit $\Phi \approx-30^{\circ}$ und ohne Anzeichen für eine Tunnelaufspaltung, was mit $g-$ im Rahmen der Unsicherheiten kompatibel ist.

Bei Vergleich der Potentialtöpfe von $g-$ in Abb. 5.58 mit dem von Benzylalkohol in Abb. 5.48 auf S. 174 fällt auf, dass die Methylgruppe nicht nur eine verschobene Präferenz bezüglich $\Phi$, sondern auch eine Verringerung der Flexibilität hinsichtlich dieser 


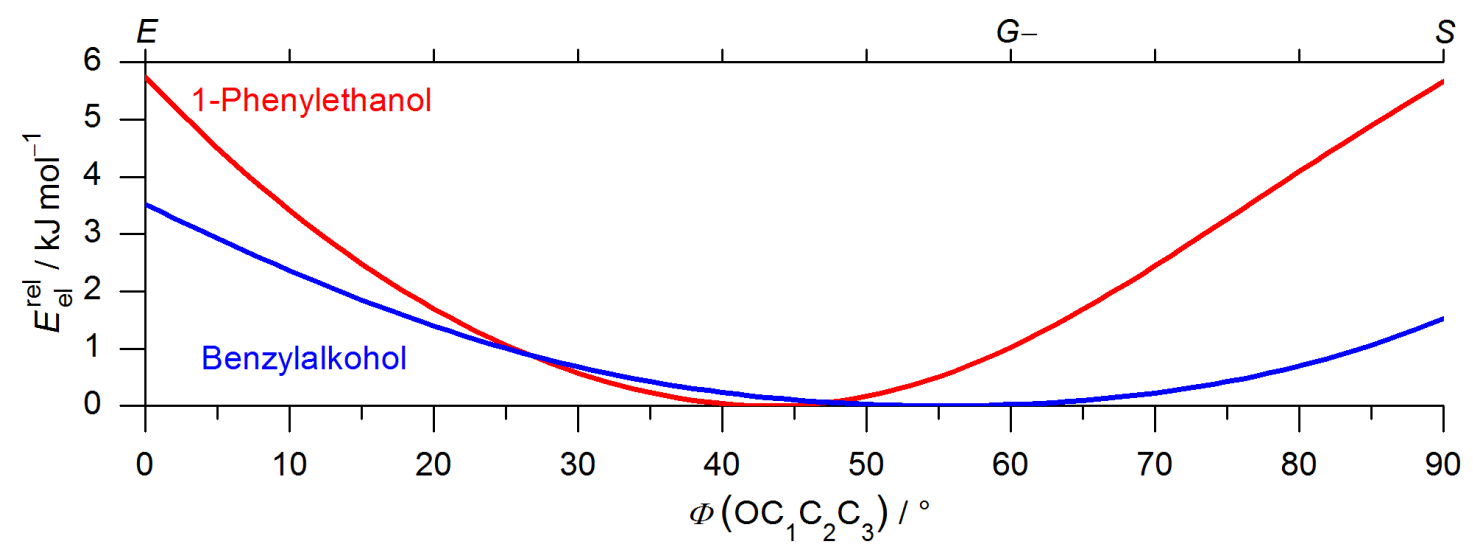

Abb. 5.60: Relaxierter Scan der elektronischen Energie auf B3LYP-D3(BJ)/may-cc-pVTZ-Niveau in Abhängigkeit des $\mathrm{OC}_{1} \mathrm{C}_{2} \mathrm{C}_{3}$-Torsionswinkels $(\Phi)$ für $(S)$-1-Phenylethanol und Benzylalkohol in $1^{\circ}$-Schritten (jeweils 91 Datenpunkte, interpoliert).

Koordinate bewirkt. Dies ist eindimensional in Abb. 5.60 näher analysiert. Benzylalkohol ist damit flexibler für größere Verformungen, was Implikationen für die Präferenz der beiden Molekülen in Dimerstrukturen hat, wie in Kap. 8 gezeigt werden wird.

Das Et-Konformer (kurz: $t$ ) entspricht in seiner relativen Energie weitgehend seinem Pendant in Benzylalkohol. Durch die verringerte Wechselwirkung zwischen dem Hydroxywasserstoffatom und dem Phenylring kann die Methylgruppe eine nahezu senkrechte Position zur Ringebene einnehmen, sodass für $\Phi$ fast $-30^{\circ}$ resultiert.

Das $g+-$ Konformer ist nicht länger isoenergetisch zu $g$ - wie bei Benzylalkohol, sondern wird durch die Einführung der Methylgruppe energetisch sehr deutlich um etwa 6$7 \mathrm{~kJ} \mathrm{~mol}^{-1}$ in seiner relativen Energie angehoben. Ferner wird $g+$ in drei separate Minima auf der elektronischen Potentialfläche aufgespalten: $E g+, S g+$ und $G+g+$. Von diesen drei Konformeren ist bisher nur $G+g+$ in der Literatur beschrieben, nachdem es als Minimum in vergleichbaren Scans der Potentialfläche auf HF/6-31G**- [351] und B3LYP/6$311++G^{* *-N i v e a u}[352]$ identifiziert wurde. Ein genauer Blick auf diese Potentialflächen zeigt aber, dass sich auch dort zwei weitere Konformere als Minima andeuteten. Nachoptimierungen der Strukturen auf HF/6-31G** und B3LYP/6-311++G**-Niveau bestätigten dies. Die Minima wurden wegen ihrer geringen Ausprägung von den Autoren entweder übersehen oder ignoriert. Die hohe Energie der drei $g+-$ Konformere kann durch ungünstige Kompromisse zwischen der Stellung der Hydroxy- und der Methylgruppe erklärt werden. So ist beispielsweise in $G+g+$ die Hydroxygruppe günstig zum Phenylring ausgerichtet, die Methylgruppe ist dagegen sterisch sehr ungünstig ekliptisch zur Ringebene platziert. Wegen ihrer hohen Energien und der nach Schwingungsnullspunktskorrektur praktisch verschwindenden Barrieren (siehe Abb. 5.58), sind die drei g+-Konformere zumindest bei tiefen Temperaturen ohne Bedeutung. Während für Benzylalkohol gefunden wurde, dass der Phenylring nahezu frei rotierbar ist, mit der Hydroxygruppe in $t$-Stellung, ist dies für 1-Phenylethanol bei der $g$ +-Einstellung der Fall.

Zusammengefasst weist 1-Phenylethanol wegen seiner Chiralität und seinen zwei flexiblen Torsionswinkeln eine komplexe Potentialfläche mit fünf diastereomeren Konformeren auf. Durch die hohen Energieunterschiede und geringen Umwandlungsbarrieren kann 1-Phenylethanol unter Jet-Bedingungen aber dennoch als effektiv monokonformationell betrachtet werden, womit es einen Sonderstatus unter den chiralen Alkoholen ein- 
nimmt. Dies erlaubt es bei der Untersuchung von Chiralitätserkennung die intramolekulare Isomerie weitgehend zu ignorieren. Auch andere chirale Moleküle mit Hydroxyfunktion, wie z. B. Methyllactat [353], haben eine dominante Konformation, allerdings ist dies häufig durch eine relativ starke intramolekulare Wasserstoffbrücke bedingt. Diese wird bei der Aggregation häufig durch eine intermolekulare Wasserstoffbrücke ersetzt [341, 354], womit der energetische Vorteil der Konformation verloren geht und damit auch andere Anordnungen berücksichtigt werden müssen. Daneben kann der Bruch einer starken intermolekularen Wasserstoffbrïcke aber auch kinetisch gehindert oder thermodynamisch ungünstig sein.[5] So wurde für die Mischung aus Methyllactat und Limonen experimentell kein Hinweis auf ein gemischtes Dimer gefunden.[237]

Besonders interessant ist die große chemische Ähnlichkeit zwischen dem permanent chiralen 1-Phenylethanol und dem transient chiralen Benzylalkohol. Effektiv wird das flexible Molekül Benzylalkohol in einer seiner beiden zueinander enantiomeren Anordnungen durch die Methylgruppe als konformationellen Anker fixiert. Dies lässt sich im Kontext von Chiralitätserkennung, -synchronisation und -induktion gezielt nutzen, wie in Kapitel 8 gezeigt werden wird.

Die Struktur von 1-Phenylethanol ist auch als Bestandteil größerer Moleküle von Bedeutung. Fluorierung [355, 356] oder Chlorierung [357] in para-Position ändert wenig an der Konformationsisomerie, dagegen führt Fluorierung in ortho- [356, 358] oder metaPosition [356] zu einer Aufspaltung der zuvor doppelt entarteten Konformere, da die Rotation der Phenylgruppe um $180^{\circ}$ nicht mehr in identischen Strukturen resultiert. Gleiches gilt für eine Ringerweiterung von Phenyl zu Naphthyl [239]. Das Einbringen von Heteroatomen in $\beta$-Position an der Methylgruppe führt zu einer Änderung der Konformationspräferenz zugunsten von intramolekularen Wasserstoffbrücken mit diesen. Beispiele hierfür sind 1-Phenyl-2,2,2-trifluorethanol [359, 360], Mandelsäure und ihre Ester [361, 362] sowie Phenylpropanolamine wie Ephedrin [362].

1-Phenylethanol ist auch eng mit 1-Indanol verwandt. In 1-Indanol ist die Methylgruppe über eine Methylenbrücke mit dem Phenylring in ortho-Position verknüpft, sodass ein Fünfring entsteht. Die Torsion um die $\mathrm{C}_{1}-\mathrm{C}_{2}$-Bindung ist dadurch eingeschränkt und transformiert nun zwischen zwei Konformationen des Fünfringes. Die stabilste Konformation von $(S)$-1-Indanol, 1eq [70, 363], ist bezüglich $\Phi\left(-142^{\circ}\right)$ und $\phi\left(-54^{\circ}\right)$ ähnlich zu $G+g+$ von $(S)-1-P h e n y l e t h a n o l\left(-126^{\circ} /-53^{\circ}\right)$, sowie auch in der Stellung der Methylbzw. Methylengruppe. Dies äußert sich auch in analogen sehr niedrig berechneten harmonischen OH-Streckschwingungswellenzahlen (3793 bzw. $3798 \mathrm{~cm}^{-1}$ ). Die verringerte Flexibilität der C-C-Torsion von 1-Indanol macht weitere Konformere energetisch kompetitiver als bei 1-Phenylethanol, sodass dessen intramolekulare Isomerie auch in den Dimeren präsent ist.[70, 363]

\subsubsection{Schwingungsspektren}

1-Phenylethanol liegt bei Zimmertemperatur als Flüssigkeit vor und hat eine Normalsiedetemperatur von $204{ }^{\circ} \mathrm{C}$. Der extrapolierte Dampfdruck bei $24^{\circ} \mathrm{C}$ beträgt $40 \mathrm{~Pa}$. Dies ist deutlich höher als für Benzylalkohol ( $8 \mathrm{~Pa}$ ) und etwas höher als für $\alpha$-Fenchol (21 Pa). Unter geeigneten Bedingungen ist 1-Phenylethanol daher auch ungeheizt am Filet-Jet untersuchbar. Archivmaterial zeigt, dass bereits Borho dies bei der Erprobung des FiletJets im Jahre 2004 versucht hat, allerdings war die Optimierung noch nicht weit genug 


\section{Torsionsisomerie von Alkoholen}

um im OH-Streckschwingungsbereich ein ausreichendes Signal-zu-Rausch-Verhältnis zu erzielen. Sechs Jahre später gelang es Falkowski [237] ein intensives Signal im Monomerbereich und zwei schwächere Banden im Dimerbereich zu beobachten. Durch weitere technische Verbesserungen in der Zwischenzeit (u. a. intensivere Lichtquelle, Teflonbeschichtung des Reservoirs [364]) sowie Optimierung der Messparameter (höchstmögliche Konzentration bei sommerlichen Temperaturen, hoher Stagnationsdruck) konnte das Signal-zu-Rausch-Verhältnis in der vorliegenden Arbeit nochmals deutlich gesteigert werden. Zusätzlich wurden Raman-Spektren am Curry-Jet mit geheizter Substanz aufgenommen. Aggregate von 1-Phenylethanol mit Hinblick auf Chiralitätserkennung werden in Kap. 8.4 behandelt, der Monomerbereich ist in Abb. 5.61 gezeigt. Eine einzelne intensive Bande wird bei $3647.5 \mathrm{~cm}^{-1}$ gefunden, dies stimmt mit den Befunden von Falkowski (3648 $\mathrm{cm}^{-1}$ [237]) und aus der Doppelresonanzstudie von Le Barbu et al. $\left(3647 \mathrm{~cm}^{-1}\right.$ [349]) überein. Dies gilt auch vollumfänglich für die Vorhersage aus dem Korrelationsmodell $\left(3647.5 \mathrm{~cm}^{-1}\right)$, wobei der Wert von Le Barbu bereits als einer von

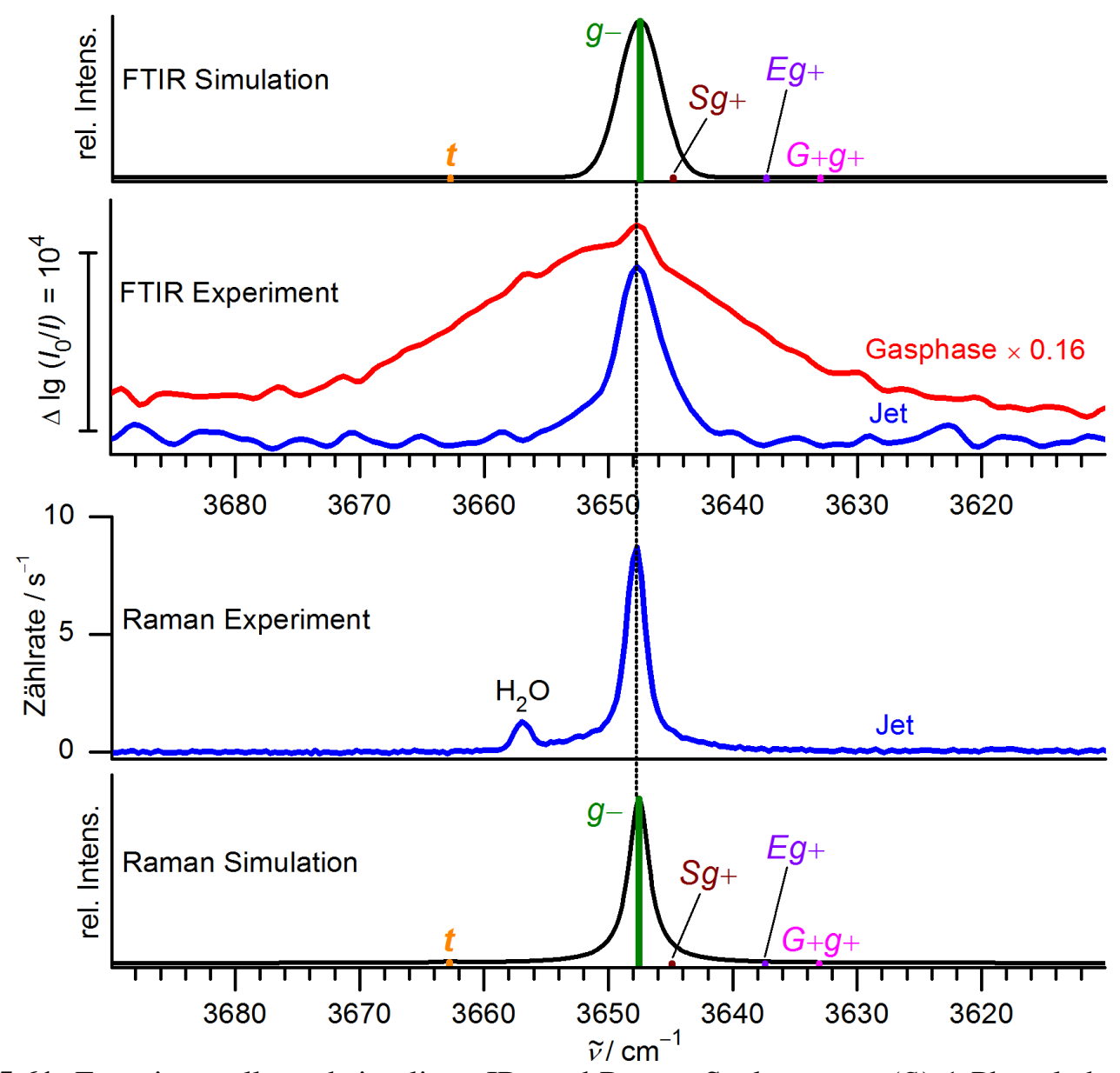

Abb. 5.61: Experimentelle und simulierte IR- und Raman-Spektren von $(S)$-1-Phenylethanol im Monomerbereich. Die Bande bei $3656.5 \mathrm{~cm}^{-1}$ im Raman-Spektrum stammt von der symmetrischen Streckschwingung einer Wasserverunreinigung.[238] Simulationsparameter sind einheitliche Verschiebung der harmonischen Wellenzahlen um $\kappa=-165.2 \mathrm{~cm}^{-1}$, eine Konformationstemperatur von $64 \mathrm{~K}$ für das Filet-Jet-Sektrum und $100 \mathrm{~K}$ für das Curry-Jet-Spektrum, GaussProfile mit FWHM $=4 \mathrm{~cm}^{-1}$ für die IR-Banden sowie Lorentz-Profile mit FWHM $=2 \mathrm{~cm}^{-1}$ für die Raman-Banden. 
fünfzehn Datenpunkten im Trainingsset für sekundäre Alkohole berücksichtigt wurde. Die experimentelle Bandenposition stimmt innerhalb der Messgenauigkeit mit der von Benzylalkohol überein. Die berechnete harmonische Wellenzahl von 1-Phenylethanol ist dagegen um rund $4 \mathrm{~cm}^{-1}$ größer, ein Beispiel der systematischen Diskrepanz zwischen primären und sekundären Alkoholen, die im Korrelationsmodell berücksichtigt wird.

Hinweise auf die anderen vier Konformere sind in den Jet-Spektren nicht vorhanden, was der Erwartung aus deren hohen Energien und geringen Relaxationsbarrieren entspricht. Das Gasphasen-FTIR-Spektrum bei Zimmertemperatur hat sein Maximum an der gleichen Position wie die Jetspektren, läuft aber zu höheren Wellenzahlen etwas flacher aus. Es ist nicht klar, ob dies aus einer besonders hohen Asymmetrie der Zweige der Rotationsschwingungsübergänge resultiert oder auf die Population eines höherfrequenten Konformers oder auf einen sonstigen heißen Übergang hindeutet. Das $t$-Konformer wäre dafür tendenziell zu hochfrequent vorhergesagt. In verdünnter TetrachlormethanLösung wurde ebenfalls eine leichte Asymmetrie der Bande beobachtet und die $\mathrm{OH}$ Streckschwingung des $t$-Konformers $11 \mathrm{~cm}^{-1}$ oberhalb von $g-$ im Intensitätsverhältnis $0.16: 1$ zugeordnet.[351] In einer anderen Studie wurde die leichte Asymmetrie dagegen nicht mit einem zweiten Konformer assoziiert.[348] 


\subsection{Fazit und Aktualisierung des Korrelationsmodells}

In der vorgehenden Abschnitten wurde das Korrelationsmodell an einer Reihe von Alkoholen erprobt. Dabei wurde, wie zuvor für das Trainingsset, gefunden, dass die Mehrheit der beobachteten Banden mit Vorhersagen in etwa einem Bereich von $2 \mathrm{~cm}^{-1}$ kompatibel sind. Nicht immer konnten dabei aber einzelne Konformere wegen spektralen Überlappungen oder Tunnelaufspaltungen zugeordnet werden. Bezüglich relativer Energien von Konformeren erscheint B3LYP-D3(BJ)/may-cc-pVTZ weitgehend robust für zumindest qualitativ korrekte Abfolgen, gemessen am Experiment und aufwändigeren quantenchemischen Methoden. Eine beobachtete Diskrepanz zum Experiment veranlasste z. B. überhaupt erst die nähere Untersuchung von $\alpha$-Fenchol. Nur bei tert-Butylcarbinol wurde mit CCSD(T)/aug-cc-pVQZ*-Punktrechnungen eine abweichende Energieabfolge erhalten. Durch die Tunnelaufspaltung in diesem System ist eine experimentelle Bestimmung der Abfolge nicht ohne weiteres möglich. Die Stabilität der $t$-Konformere bezüglich des HOCH-Torsionswinkels von sekundären Alkoholen scheint etwas überschätzt zu werden. Insgesamt hat sich für die Vorhersage der Konformationsisomerie und der zugehörigen $\mathrm{OH}-$ Streckschwingungsspektren B3LYP-D3(BJ)/may-cc-pVTZ in harmonischer Näherung als effiziente Methode erwiesen, wenn sie um eine empirische Anharmonizitätskorrektur nach dem Korrelationsmodell und ggf. bei engen Energieabständen um zusätzliche Punktrechnungen ergänzt wird.

Die Grenzen des Modells sind besonders beim Auftreten von spektralen Tunnelaufspaltungen deutlich geworden. Aber auch eine größere Diskrepanz zwischen Vorhersage und Experiment kann als Hinweis auf größere anharmonische Effekte genutzt werden, die interessante Objekte für weitere Untersuchungen darstellen. Wie die Stichprobe der näher betrachteten Alkoholen anzudeuten scheint, könnten Tunnelaufspaltungen weiter verbreitet sein als bisher angenommen. Der Fall des $\alpha$-Fenchols weist daraufhin, dass diese auch bei asymmetrischen Alkoholen nicht von vornherein auszuschließen sind. Empfehlenswert ist es daher stets auch die Übergangszustände mit zu betrachten. Wie gezeigt wurde, reicht in vielen Fällen bereits ein einfacher Vergleich der Barrierenhöhe mit anderen Systemen aus, um die Höhe einer möglichen Tunnelaufspaltung zumindest grob abzuschätzen.

Die in Tab. 5.8 auf S. 190 gelisteten Konformere konnten entweder neu zugeordnet bzw. deren spektrale Position konnte durch Beobachtung der scharfen Q-Zweige präzisiert werden. Mit diesen Daten wurde das Korrelationsmodell aktualisiert ( $n$-Octanol wird aus zuvor diskutierten Gründen dabei nicht berücksichtigt). Die Neuanpassung der Korrelationsfunktionen verändert $\kappa$ unwesentlich für primäre Alkohole von -159.5(5) auf $-159.4(5) \mathrm{cm}^{-1}$ und für sekundäre Alkohole von $-165.2(5)$ auf $-165.5(5) \mathrm{cm}^{-1}$. Die aktualisierte Gesamtübersicht aller Datenpunkte sowie die aktualisierten statistischen Auswertungen sind im Anhang in Abb. A.2 und Tab. A.12 auf S. 312 und 313 zu finden.

Eine Aufschlüsselung der Datenpunkte auf der Konformeren-Ebene erfolgt in den Abb. 5.62 und 5.63. Besonders für primäre Alkohole wird deutlich, dass sich die Konformere nach gemeinsamen Strukturmerkmalen gruppieren, wie sie teilweise im 3-ParameterModell zur Erklärung der Wellenzahlen benutzt werden. Das 3-Parameter-Modell könnte anhand der hier zusammengetragenen Daten künftig aktualisiert und mit weiteren Strukturmerkmalen parametrisiert werden. 

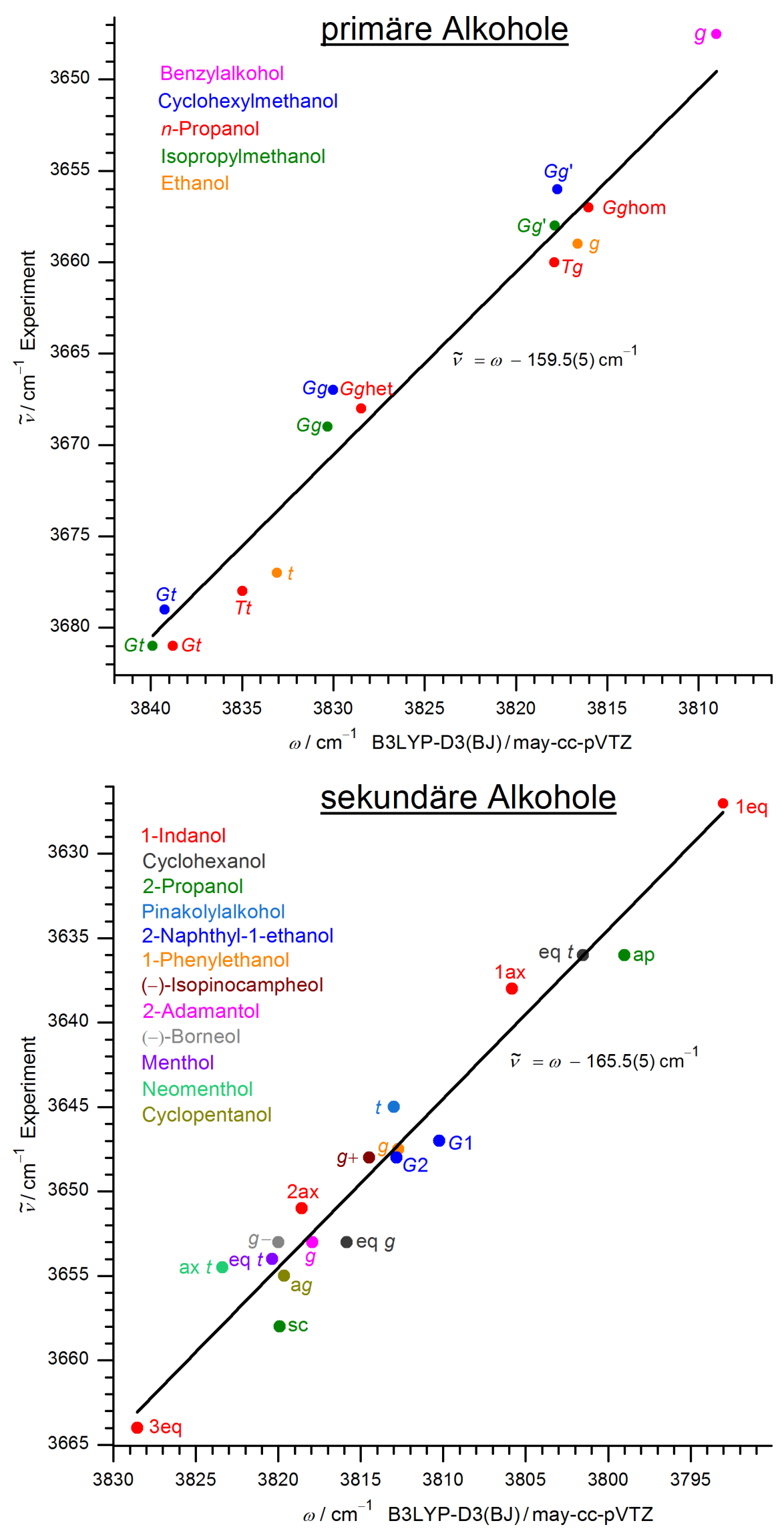

Abb. 5.62: Korrelation von experimentellen $\mathrm{OH}-\mathrm{Streckschwingungswellenzahlen} \mathrm{von} \mathrm{primären}$ und sekundären Alkoholen mit berechneten in harmonischer Näherung mit B3LYP-D3(BJ)/maycc-pVTZ. 


\section{Torsionsisomerie von Alkoholen}

Tab. 5.8: Neu zugeordnete bzw. in der Bandenposition präzisierte Alkohol-Konformere mit harmonischer OH-Streckschwingungswellenzahl auf B3LYP-D3(BJ)/may-cc-pVTZ-Niveau und experimenteller Bandenposition.

\begin{tabular}{llll}
\hline Alkohol & Konformer & $\omega_{\mathrm{OH}} / \mathrm{cm}^{-1}$ & $\tilde{v}_{\mathrm{OH}} / \mathrm{cm}^{-1}$ \\
\hline n-Octanol & $T_{5} G t$ & 3838.3 & $3680(1)$ \\
(-)-Isopinocampheol & $g+$ & 3814.5 & $3648(1)$ \\
(-)-Borneol & $g-$ & 3820.0 & $3653(1)$ \\
Pinakolylalkohol & $t$ & 3813.0 & $3645.0(5)$ \\
Benzylalkohol & $g$ & 3809.0 & $3647.5(5)$ \\
1-Phenylethanol & $g$ & 3812.7 & $3647.5(5)$ \\
\hline
\end{tabular}

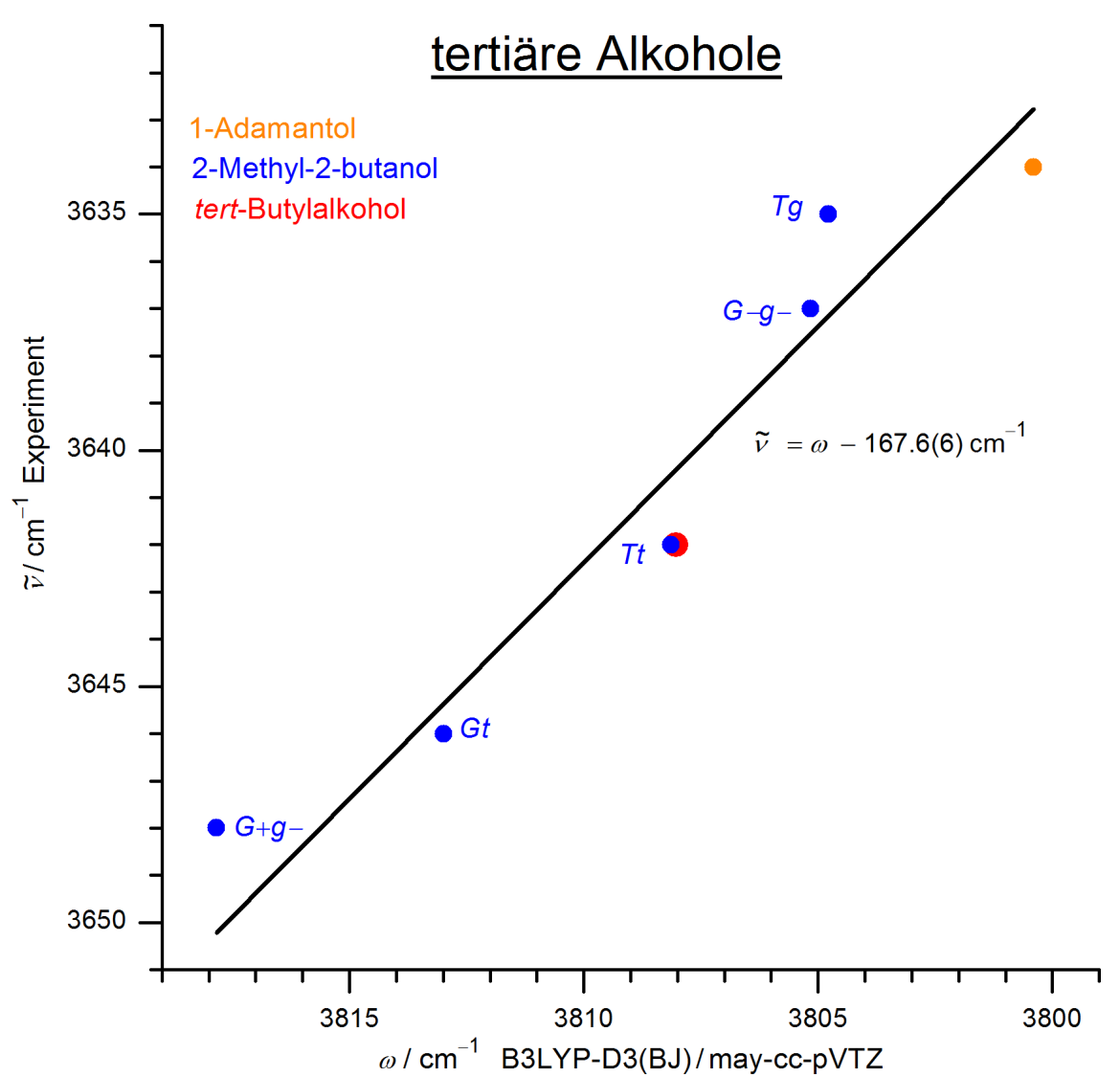

Abb. 5.63: Korrelation von experimentellen $\mathrm{OH}-\mathrm{Streckschwingungswellenzahlen} \mathrm{von} \mathrm{tertiären}$ Alkoholen mit berechneten in harmonischer Näherung mit B3LYP-D3(BJ)/may-cc-pVTZ. 


\subsection{Anwendungen des Korrelationsmodells}

Das Korrelationsmodell wurde in erster Linie als Hilfe zur Interpretation von Spektren von Alkoholen in Überschallexpansionen entwickelt und in den vorherigen Abschnitten auch erfolgreich angewendet. Auch für die Interpretation von Spektren von Zimmertemperatur-Gasphasen kann es hilfreich sein. Für gelöste Alkohole ist es dagegen wegen der Lösungsmittelshifts nur sehr eingeschränkt anwendbar. Nachträgliche empirische Korrekturen der bestehenden Werte oder Neuberechnung der quantenchemischen Werte mit Lösungsmittelkorrekturen wären aber zumindest denkbar.

Kurz sei hier die konkrete Verwendung des Korrelationsmodells erläutert. Die quantenchemischen Rechnungen wurden mit Gaussian 09 Revision E.01 durchgeführt, einem weit verbreiteten, aber leider nicht frei verfügbaren Softwarepaket. Ältere Versionen sollten wegen eines Fehlers in der Implementierung der Dispersionskorrektur nicht verwendet werden. Testrechnungen mit der derzeit aktuellen Version Gaussian 16 C.01 deuten darauf hin, dass diese identische Rechenergebnisse liefert. Die zu verwendene Befehlszeile lautet:

\# b3lyp empiricaldispersion=gd3bj may-cc-pvtz int=ultrafine fopt=verytight freq

Die quantenchemisch erhaltene harmonische $\mathrm{OH}-$ Streckschwingungswellenzahl ist um die in Abb. 5.62 und 5.63 angegebenen Korrekturwerte zu verringern. Berücksichtigt werden sollten bei der Zuordnung auch die relative Energie der Konformere. Das Vorliegen einer Tunnelaufspaltung sollte ebenso stets geprüft werden.

Daneben bieten sich die zusammengetragenen Werte auch als Benchmarking-Datensatz für quantenchemische Methoden an. Dies gilt besonders für anharmonische Methoden, die ihre höheren Kosten mit einer besseren Korrelation rechtfertigen können sollten. Folgende Kriterien könnten hierfür herangezogen werden:

1. Eine gute Vorhersage von Unterschieden in der Anharmonizität innerhalb der jeweiligen Klassen, die im harmonischen Korrelationsmodell vernachlässigt werden, sollte zu einer besseren Korrelation führen.

2. Eine ähnlich gute Korrelation für alle Alkohole gemeinsam, wie innerhalb der einzelnen Klassen, ist erstrebenswert. Eine gute Vorhersage der vermuteten systematischen Änderung der Anharmonizität zwischen den Klassen erlaubt die Vereinigung zu einem gemeinsamen Datensatz.

3. Die Vorhersage von absoluten Wellenzahlen sollte mit möglichst geringer, bestenfalls keiner, nachträglichen empirischen Korrektur gelingen $(\kappa \rightarrow 0$ bzw. $\lambda \rightarrow 1)$.

4. Eine besondere Herausforderung stellt die Vorhersage von Übergängen in Alkoholen mit spektraler Tunnelaufspaltung dar, da hierfür eine Betrachtung der lokalen Anharmonizität in der Umgebung der Minimumsstruktur nicht mehr ausreichend ist.

Es ist geplant, die Daten auch im Rahmen des von der Deutschen Forschungsgemeinschaft geförderten Graduiertenkollegs „GRK 2455 Bewertende Experimente für die numerische 


\section{Torsionsisomerie von Alkoholen}

Quantenchemie“ zugänglich zu machen. Hierzu zählen die experimentellen und harmonischen B3LYP-D3(BJ)/may-cc-pVTZ Wellenzahlen, die statistischen Auswertungen des Korrelationsmodells als Vergleich für Bewertungen, sowie die Strukturkoordinaten der Alkohol-Konformere, sodass diese mit weiteren Methoden nur noch nachoptimiert werden müssen.

\subsection{Ausblick}

Da für das Korrelationsmodell bisher nur Alkohole mit Kohlenwasserstoffresten und ohne (stärkere) intramolekulare Wasserstoffbrücken berücksichtigt wurden, ist der Raum für Erweiterungen groß. Ob sich auch Moleküle mit Heteroatomen und/oder intramolekularen Wasserstoffbrücken gut in die Korrelation einfügen, hängt davon $a b$, ob a) die zusätzlichen Strukturelemente zu keinen signifikanten Änderungen in der Anharmonizität führen und b) ob die Auswirkungen auf die harmonische Wellenzahl ähnlich gut von B3LYP-D3(BJ)/may-cc-pVTZ beschrieben werden können. Analysen von Poblotzki [69] $\mathrm{zu}$ intermolekularen Wasserstoffbrücken mit der verwandten Methode B3LYP-D3(BJ, $\mathrm{abc}$ )/def2-TZVP zeigen hier eher ein wenig vielversprechendes Bild mit größerer Streuung und systematischen Abweichungen in Abhängigkeit von der Stärke und Art des Akzeptors. Eigene Testrechnungen zu Mono-, Di- und Trifluorethanol und den ChlorAnaloga [365] deuten daraufhin, dass sich diese alle mit absoluten Residuen $<4 \mathrm{~cm}^{-1}$ in die bestehende Korrelation für primäre Alkohole einfügen würden. Dabei werden aber offenbar systematisch die Wellenzahlen der Fluorethanole unter- und die der Chlorethanole überschätzt. Auch Erweiterungen auf Hydroxyverbindungen jenseits von Alkoholen wären denkbar. Ein guter Startpunkt hierfür könnten die von Bouteiller et al. [227] zusammengetragenen Daten für freie Hydroxyoszillatoren von Jet-gekühlten Biomolekülen sein.

Angeregt sei auch eine tiefer gehende Untersuchung der Tunneldynamik in Alkoholen, die in der Vergangenheit meist eher unerwartet festgestellt wurde und daher selten im Mittelpunkt des Interesses stand. Die hier gefundenen empirischen Zusammenhänge lassen es möglich erscheinen bereits im Vorfeld mit kostengünstigen Routinerechnungen $\mathrm{zu}$ evaluieren, ob für eine Verbindung eine für die jeweilige Spektroskopiemethode auflösbare Tunnelaufspaltung zu erwarten wäre. Die vielversprechendsten könnten anschlieBend experimentell geprüft werden. Zudem sind die Spektren der Alkohole $\alpha$-Fenchol, Pinakolylalkohol, tert-Butyldimethylcarbinol, tert-Butylcarbinol und 3-Pentanol [236] bisher nur unvollständig verstanden.

Die schwingungsspektroskopische Charakterisierung von Propargylalkohol, mit seiner besonders hohen Tunnelaufspaltung von $22 \mathrm{~cm}^{-1}$ [293], steht in einer Überschallexpansion noch aus. Daneben sind auch dessen Derivate vielversprechend. Die hohe relative Energie der $t$-Konformere, die beispielsweise für die Stammverbindung hier zu fast $7 \mathrm{~kJ} \mathrm{~mol}^{-1}$ berechnet wird, sollte sowohl die Spektren, wie auch die Modellierung als Doppelminimumpotential vereinfachen. Verschiedene symmetrische Derivate sind kommerziell verfügbar und versprechen ebenfalls große Tunnelaufspaltungen. So wird mit dem Barrierenhöhemodell für 1,1-Dimethylpropargylalkohol (2-Methyl-3-butin-2-ol) eine Tunnelaufspaltung von $h c 16 \mathrm{~cm}^{-1}$ vorhergesagt und für 3-Methylpropargylalkohol 
(2-Butin-1-ol) ein Rekordwert von $h c 47 \mathrm{~cm}^{-1}$. In einer rotationsspektroskopischen Studie zur letztgenannten Verbindung wurde nur ein einzelnes Konformer mit unklarer Position des Hydroxyprotons gefunden.[366] Dies wäre mit einer großen Tunnelaufspaltung grundsätzlich kompatibel, wenn angenommen wird, dass bei starker Kühlung in der verwendeten Neon-Expansion nur der Grundzustand der Tunnelaufspaltung thermisch populiert ist. Ein besonderes Merkmal dieses Moleküls ist zudem die fast freie Methyltorsion, die auch ggf. mit der Hydroxytorsion koppeln könnte. Eine dazu verwandte Verbindung ist 3-Phenylpropargylalkohol (3-Phenyl-2-propin-1-ol), welche Benzylalkohol mit einem zusätzlichen Ethinyl-Linker zwischen der Phenyl- und der Hydroxymethylgruppe entspricht. Die Kopplung zwischen der Phenyl- und Hydroxytorsion bleibt dabei laut Testrechnungen erhalten, ist aber durch die größere Distanz abgeschwächt. Dies senkt die beiden unterschiedlichen Barrieren und verspricht schnelles Schweratomtunneln zwischen den vier isoenergetischen $g$-Minima.

Besonders interessant sind auch Tunnelaufspaltungen in asymmetrischen Potentialen, für die es in der Literatur bisher nur sehr wenige experimentelle Beispiele gibt, insbesondere für den experimentell und quantenchemisch leichter zugänglichen Grundzustand. Die Substitution von Alkoholen mit geringer Barriere, wie Propargylalkohol oder bestimmter sekundärer Alkohole wie $\alpha$-Fenchol, ist hier vielversprechend, erfordert aber vermutlich synthetischen Aufwand. So wird bei einfacher Deuterierung eines Methylenwasserstoffes bei Propargylalkohol eine Asymmetrie von $h c 10 \mathrm{~cm}^{-1}$ berechnet, welche geringer wäre als die Tunnelaufspaltung des symmetrischen Moleküls $\left(h c 22 \mathrm{~cm}^{-1}\right)$. Die Erwartung wäre daher, dass die Lokalisierung nur unvollständig stattfindet. Der Symmetriebruch macht es zudem wahrscheinlicher, dass vier, statt nur zwei Übergänge, zwischen Tunnelniveaus ausreichend spektrale Aktivität erhalten, sodass schwingungsspektroskopisch nicht nur die Differenz, sondern auch absolute Werte für Tunnelaufspaltungen bestimmt werden können. Alternativ bietet die Rotationsspektroskopie Zugang zu Tunnelaufspaltungen im Grundzustand, aus der sich dann die Tunnelaufspaltung im angeregten Zustand verschiedener Schwingungen ableiten lässt.[168]

Nach ausführlicher Charakterisierung der monomeren Alkohole in diesem Kapitel, werden diese im Folgenden in Komplexen mit Chiralitätserkennung, -synchronisation und -induktion eingesetzt. 



\section{Chiralitätsdiskriminierung von Alkoholen mit $\alpha$-Pinen}

\subsection{Vorwort}

In diesem Kapitel wird die Chiralitätsdiskriminierung zwischen fünf enantiomerenreinen Alkoholen und den Enantiomeren von $\alpha$-Pinen untersucht. Diese manifestiert sich bei Wechsel der relativen Chiralität in einer Verschiebung der spektroskopischen Signatur der $\mathrm{OH}-$ Streckschwingung, welche mit Vorhersagen verschiedener quantenchemischen Methoden verglichen wird.

Die Messungen und B3LYP-D3(BJ)-Rechnungen für die Komplexe von $\alpha$-Pinen mit $\alpha$ Fenchol wurden zusammen mit Helen Preiß im Rahmen eines PC-Forschungspraktikums, für die mit den weiteren Alkoholen zusammen mit Caroline Stelbrink im Rahmen ihrer Bachelorarbeit (Ref. [367]), durchgeführt. Die in diesem Kapitel präsentierten Ergebnisse (mit Ausnahme der Komplexe mit 2-Butanol) wurde bereits in gekürzter Form in Ref. [4] publiziert.

\subsection{Einleitung}

$\alpha$-Pinen, systematisch 2,6,6-Trimethylbicyclo[3.1.1]hept-2-en, ist ein chirales bicyclisches Monoterpen (Abb. 6.1), welches in der Natur in erheblicher Menge [368] von Pflanzen gebildet und emittiert wird. So wird die jährliche Freisetzung allein für ein ver-
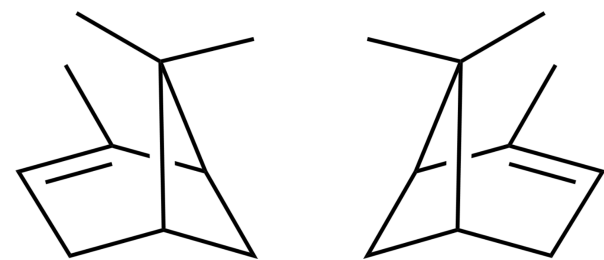

Abb. 6.1: Die Strukturformeln von (-)- und (+)- $\alpha$-Pinen.

gleichsweise kleines Land wie Finnland auf etwa 30 Kilotonnen pro Jahr geschätzt.[369] Die Produkte aus der schrittweisen Oxidation in Luft $[368,370]$ haben als Kondensationskeime Einfluss auf die Wolkenbildung und damit das Klima [368]. Die enantiomere Zusammensetzung der Emission variiert deutlich zwischen verschiedenen Spezies, geographischen Lagen, den Tages- und Jahreszeiten, und sogar zwischen einzelnen Individuen einer Spezies.[371] Durch die Omnipräsenz von $\alpha$-Pinen ist es nicht überraschend, dass die Enantiomere von so unterschiedlichen Tieren, wie Asiatischen Elefanten [372], Südafrikanischen Seebären [373], Honigbienen [374] und Menschen [6], unterschieden werden können. Nach Wahrnehmung des Autors dieser Arbeit haben beide Enantiomere 


\section{Chiralitätsdiskriminierung von Alkoholen mit $\alpha$-Pinen}

einen an Kiefern und Wälder erinnernden Grundgeruch, aber mit klar unterscheidbaren Noten. Während (+)- $\alpha$-Pinen einen intensiv süßlichen Eindruck vermittelt, riecht (-)- $\alpha$-Pinen schwächer und muffig. Auch mikrobiologisch haben die Enantiomere unterschiedliche Wirkungen.[375, 376] Die Zugänglichkeit von $\alpha$-Pinen und anderen Terpenen in guter Enantiomerenreinheit aus biologischen Quellen (dem sog. Chiral Pool) macht sie interessant für die asymmetrische Katalyse [377], für deren Weiterentwicklung das Verständnis der molekularen Prinzipien von Chiralitätsdiskriminierung und -induktion entscheidend ist.

Die Untersuchung von gemischten Dimeren aus chiralen Alkoholen und Terpenen am Filet-Jet wurde bereits 2004 von Nicole Borho vorgeschlagen, die für andere chirale Substanzklassen bereits intensiv die Selbsterkennung studiert hatte.[37] Chiralitätsdiskriminierung mit Terpenen in der Gasphase [378, 379], darunter auch $\alpha$-Pinen [379], wurde zuvor auch mit anderen experimentellen Methoden studiert. Dabei wurden mit den Enantiomeren von 2-Naphthyl-1-ethanol Unterschiede im Fluoreszensanregungsspektrum und in der Fluoreszenzlebensdauer gefunden.[379] Am Filet-Jet folgten 2010/11 Arbeiten zur Diskriminierung von chiralen Hydroxyverbindungen mit Limonen [237, 380] und Carvon [381] (Strukturen in Abb. 6.2). Besonders die Untersuchung mit Limonen kann als direkter Vorläufer zu der in diesem Kapitel vorgestellten Studie angesehen werden, da Limonen und $\alpha$-Pinen beide ungesättigte cyclische Monoterpene und darüber hinaus Konstitutionsisomere sind.
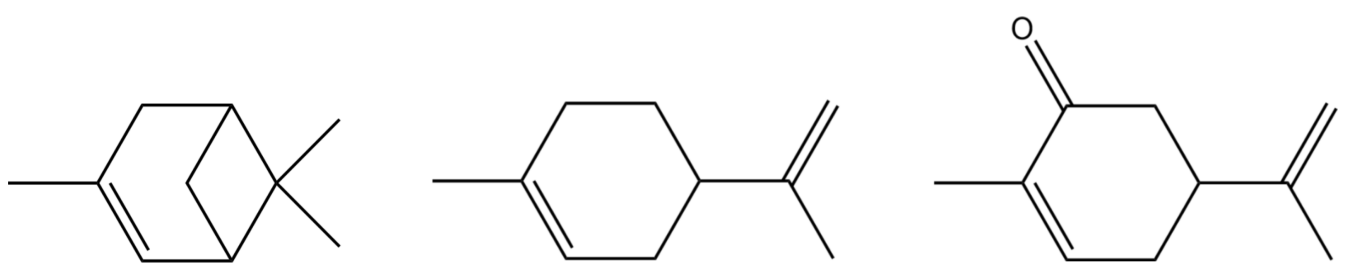

Abb. 6.2: Die Strukturformeln von $\alpha$-Pinen, Limonen und Carvon (von links nach rechts) in zweidimensionaler Darstellung.

In diesen Arbeiten konnten mit enantiomerenreinem 2-Butanol, 1,2-Propandiol, Glycidol, 1-Phenylethanol und 1-Amino-2-propanol spektrale Unterschiede in unterschiedlicher Ausprägung bei Komplexbildung mit den Limonen-Enantiomeren beobachtet werden. Allerdings konnten diese experimentellen Befunden nicht quantenchemisch gedeutet werden, sodass eine Erklärung auf molekularer Ebene für die jeweilige spektrale Chiralitätsdiskriminierung (oder deren Fehlen) offen blieb. Gleiches gilt auch für die Diskriminierung mit Carvon. Rückblickend können hierfür die große Konformationsvielfalt, in Kombination mit den damals verfügbaren quantenchemischen Methoden, als wesentliche Schwierigkeiten identifiziert werden.

So besitzt Limonen sechs Konformere, die sich aus der Ringinversion und der Torsion der Isopropenylgruppe relativ zum Ring ergeben. Auch die koordinierenden Hydroxyverbindungen besitzen teils reichhaltige intramolekulare Konformationsisomerie. Hinzu kommen eine Vielzahl an möglichen intermolekularen Anordnungen. Neben der Torsion um die Wasserstoffbrücke als konformationeller Freiheitsgrad kann Regioselektivität bezüglich der zwei Doppelbindungen, die zusätzlich von jeweils zwei unterschiedlichen Seiten koordiniert werden können, auftreten. Diese Konformationsvielfalt konnte quantenchemisch nur unvollständig abgedeckt werden. Mit der Ausnahme des Komplexes mit 
1,2-Propandiol, wurde dabei stets nur eine einzelne $\mathrm{OH} \cdots \pi$-Wasserstoffbrücke als gerichteter Kontakt gefunden. Die Diskriminierung muss daher über weitere schwächere Kontakte zwischen den Substituenten, maßgeblich vermittelt über die Dispersionswechselwirkung, erfolgen. Da die beobachteten spektralen Unterschiede nur einige $\mathrm{cm}^{-1}$ betragen, stellt dies hohe Anforderungen an die theoretische Beschreibung der Komplexe. Als quantenchemische Methoden kamen B97-D, B3LYP und MP2, jeweils mit 6-311+G(d)-Basis, zum Einsatz. Dass mit B3LYP ohne Dispersionskorrektur die experimentellen Ergebnisse nicht erklärt werden konnten, überrascht zumindest im Rückblick nicht. Dies galt aber auch für das kostengünstige B97-D Funktional, welches eine solche Korrektur der ersten Generation beinhaltet. MP2 kann die Dispersionswechselwirkung über die Elektronenkorrelation grundsätzlich beschreiben, besitzt aber auch diesbezüglich bekannte Defizite [382-384]. Wegen der nicht unerheblichen molekularen Ausdehnung der Dimere und deren großen Konformationsvielfalt war zudem die Größe des einsetzbaren Basissatzes eingeschränkt, was vermutlich vor allem für MP2 negativ zum Tragen kam.

In dieser Arbeit wurde versucht diese Probleme zu umgehen. Hinsichtlich der Konformationsvielfalt führt die Struktur von $\alpha$-Pinen gegenüber Limonen zu weitreichenden Vereinfachungen. Die bi- statt monocyclische Grundstruktur führt zu einem starren Gerüst ohne intramolekulare Konformationsisomerie.[385] Durch den zusätzlichen Ringschluss verbleibt zudem nur eine einzelne Doppelbindung, die zusätzlich von einer Seite sterisch weitgehend durch eine Methylgruppe abgeschirmt ist. Dies begrenzt die Konformationsisomerie intermolekular auf die Torsion um die Wasserstoffbrücke und intramolekular auf die Isomerie der koordinierenden Wasserstoffbrückendonoren. Letztere wurde für die hier eingesetzten Alkohole im Vorkapitel ausführlich betrachtet. Quantenchemisch werden hier drei verschiedene DFT-Funktionale eingesetzt und evaluiert, die um Dispersionskorrekturen erweitert wurden (B3LYP-D3(BJ) und $\omega$ B97X-D) oder für deren Parametrisierung bereits Komplexe mit hohem Dispersionsanteil berücksichtigt wurden (M06-2X).

\subsection{Schwingungsspektren}

In Abb. 6.3 sind Filet-Jet-Spektren der beiden Enantiomere von $\alpha$-Pinen in Kombination mit jeweils einem enantiomerenreinen Alkohol: (-)-Borneol, (+)- $\alpha$-Fenchol, (-)Isopinocampheol oder (-)-1-Phenylethanol, zusammengestellt. In allen Spektren wird eine dominante Bande beobachtet, mit Ausnahme für die Kombination aus (+)- $\alpha$-Pinen und (+)- $\alpha$-Fenchol. In diesem Fall werden zwei Banden ähnlicher Intensität beobachtet, die im Folgenden mit $a$ und $b$ bezeichnet werden. Spektren mit 2-Butanol sind dagegen komplexer und werden separat in einem späterem Abschnitt gezeigt und diskutiert.

Während mit Isopinocampheol und 1-Phenylethanol nur unerhebliche spektrale Verschiebungen zwischen den diastereomeren Enantiomerenpaarungen zu erkennen sind (in beiden Fällen mit etwa $1 \mathrm{~cm}^{-1}$ am Rande der Messgenauigkeit), fallen diese mit Borneol $\left(11 \mathrm{~cm}^{-1}\right)$ und $\alpha$-Fenchol $\left(2\right.$ und $13 \mathrm{~cm}^{-1}$, bezogen auf die Banden $a$ und $\left.b\right)$ deutlicher aus. Die spektrale Chiralitätsdiskriminierung der beiden letztgenannten Systeme wäre auch bei Einsatz von racemischen Mischungen noch als getrennte Banden erkennbar, da die Verschiebungen mehr als doppelt so groß sind wie die vollen Halbwertsbreiten $\left(3 \mathrm{~cm}^{-1}\right)$ der Banden. Die absoluten Positionen der Banden, die gemischten Dimerem zugeordnet werden, sind im Anhang in Tab. A.13 auf S. 314 dokumentiert. Wegen der geringen Dampf- 
drücke der Alkohole wurden alle Spektren in Abb. 6.3 jeweils mit einem fünffachen Überschuss des leichter flüchtigen $\alpha$-Pinen aufgenommen, um die absoluten Signalintensitäten zu steigern. Spektren bei weiteren Bedingungen sind im Anhang in Abb. A.3-A.6 auf S. 314-316 zusammengestellt, deren Interpretation wie folgt zusammengefasst ist:

- Die Spektren der Reinsubstanzen zeigen, dass die hier diskutierten Signale tatsächlich erst bei Mischung der jeweiligen Substanzen auftreten.

- Weitere schwache Banden, die tiefverschoben zu den intensiven Signalen auftreten, skalieren stärker mit der $\alpha$-Pinen-Konzentration und sind daher wahrscheinlich gemischten Trimeren mit zwei Molekülen $\alpha$-Pinen zuzuordnen. Analoge Banden wurden auch zuvor bei verwandten achiralen Systemen gefunden und entsprechend interpretiert.[386]

- Spektren der Mischungen aus racemischem $\alpha$-Pinen und (+)- $\alpha$-Fenchol entsprechen in guter Näherung Mittelungen der Spektren, für deren Aufnahme die beiden

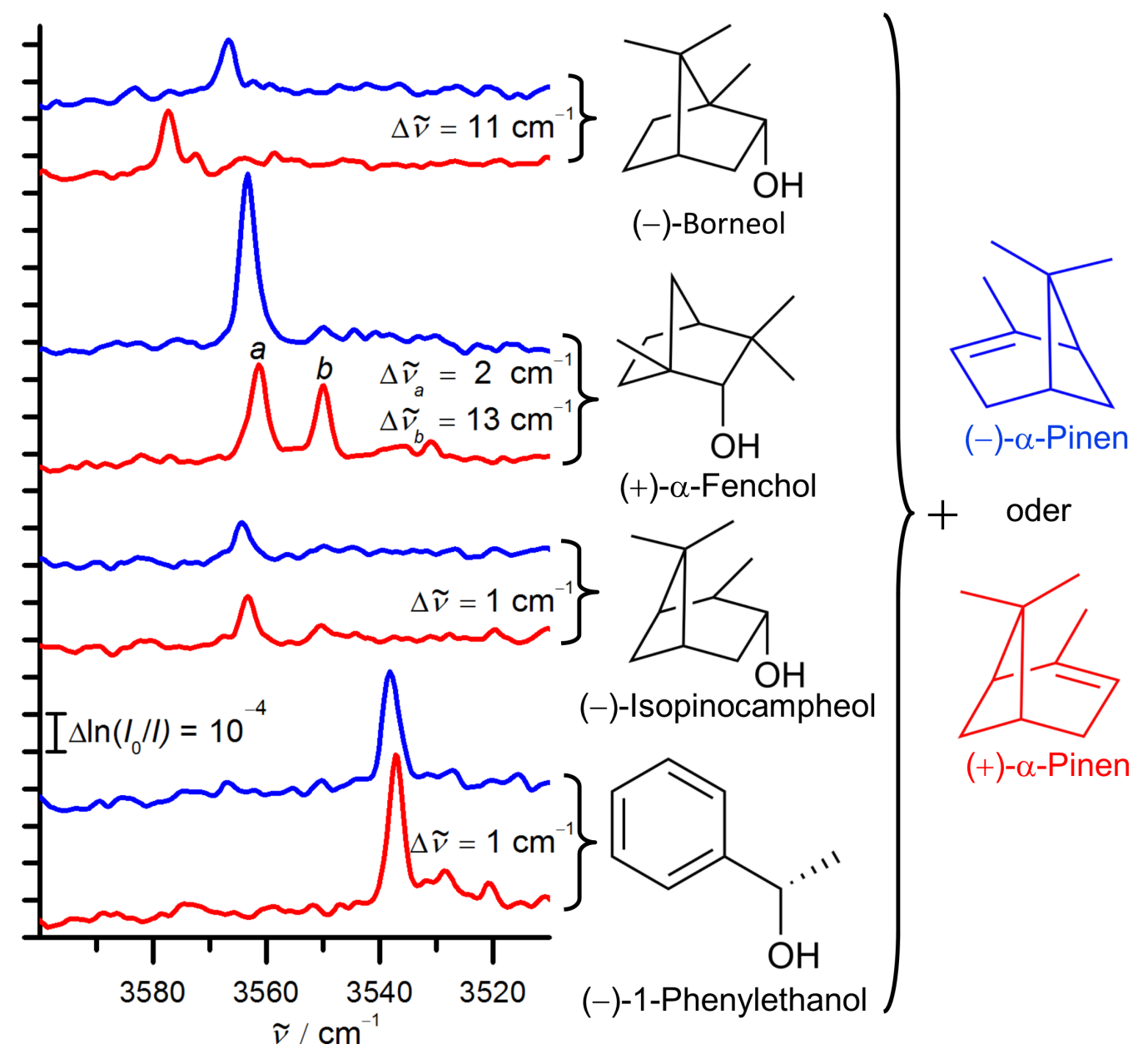

Abb. 6.3: Filet-Jet-Spektren im OH-Streckschwingungsbereich von Koexpansionen aus Helium, enantiomerenreinen Alkoholen und entweder (-)- $\alpha$-Pinen (blau) oder (+)- $\alpha$-Pinen (rot). Beträge für Verschiebungen zwischen den intensivsten Banden bei Wechsel des $\alpha$-Pinen-Enantiomers sind angegeben. Mit Anpassungen übernommen aus Ref. [4] mit Erlaubnis von John Wiley and Sons. 
$\alpha$-Pinen-Enantiomere getrennt eingesetzt wurden. Dies weist auf eine weitgehend statistische Bildung der Dimere unterschiedlicher relativer Chiralität hin.

- Durch Zumischung von $1 \%$ Argon in die Expansion von $(+)-\alpha$-Fenchol und (+)$\alpha$-Pinen wurde versucht die Energieabfolge der Dimer-Konformere $a$ und $b \mathrm{zu}$ bestimmen. Dies misslang, da nicht nur Intensitätsänderungen, sondern auch Verbreiterungen und leichte Verschiebungen der Signale beobachtet wurden. Offenbar lagert auch bei dieser relativ geringen Beimischung bereits Argon an die ausgedehnten, und damit relativ leicht polarisierbaren, Dimere an. Eine Expansion mit Neon-Beimischung am Gratin-Jet [132] könnte hierfür zukünftig aussagekräftiger sein.

\subsection{Quantenchemische Rechnungen}

Die spektroskopischen Ergebnisse deuten daraufhin, dass die Dimere stets sehr weitgehend in ihr globales Minimum relaxieren, mit Ausnahme der Kombination von (+)- $\alpha-$ Pinen und $(+)-\alpha$-Fenchol, bei der zwei Konformere signifikant populiert zu sein scheinen. Für die Komplexe mit Borneol und $\alpha$-Fenchol wurde signifikante spektrale Chiralitätsdiskriminierung festgestellt, mit Isopinocampheol und 1-Phenylethanol dagegen nicht. Diese klaren experimentellen Befunde eignen sich gut für das Benchmarking von quantenchemischen Methoden, auch in harmonischer Näherung. Enantiomere besitzen die gleichen inhärenten Akzeptor- bzw. Donorstärken für eine Wasserstoffbrücke, die beobachteten spektralen Unterschiede sind daher allein Resultat aus kleinen strukturellen Änderungen durch die unterschiedlichen sekundären Wechselwirkungen. Die Nichtberücksichtigung von anharmonischen Effekten und weitere Defizite der Theorie sollten sich daher beim Vergleich der beiden Kombinationen der Enantiomere weitgehend kompensieren. Eine Ausnahme hiervon stellen Einflüsse dar, die explizit von der relativen Chiralität abhängen (Chiraliätsinduzierte Spinselektivität (CISS) [1, 2]). Systematische Diskrepanzen zwischen Experiment und der hier eingesetzten Theorie ohne explizite Berücksichtigung von Spin-Bahn-Kopplung können daher obere Grenzen für das Ausmaß solcher Effekte aufzeigen. In dieser Arbeit werden die DFT-Funktionale B3LYP-D3(BJ), $\omega$ B97X-D und M06-2X verwendet, jeweils mit dem def2-TZVP Basissatz. Strukturen aus B3LYP-D3(BJ)/def2-TZVP wurden zusätzlich mit dem größeren may-cc-pVTZ Basissatz nachoptimiert. Die semi-empirische AM1-Methode wird als Kontrollreferenz verwendet, von der zu erwarten ist, dass sie die grundsätzliche Wechselwirkung, aber nicht die subtilen Unterschiede zwischen den Enantiomeren erfassen kann. Zum Auffinden der globalen Minima für die Zuordnung der Banden wurde eine umfassende systematische Suche durchgeführt, die für die vier Methoden auf Basis der selben Startstrukturen, aber ansonsten unabhängig voneinander erfolgte (d. h. ohne Voroptimierungen o. ä.).

Ein wesentlicher Freiheitsgrad der Systeme ist die Regioselektivität bezüglich der Koordination der diastereotopen Seiten des $\pi$-Systems von $\alpha$-Pinen. Mit B3LYP-D3(BJ) wurden beide Seiten intensiv untersucht, dabei wurde gefunden, dass die stabilsten Komplexe mit der Seite, auf der sich die doppelten Methylgruppen befinden, stets energetisch um mindestens $3 \mathrm{~kJ} \mathrm{~mol}^{-1}$ höher lagen als die mit der Seite ohne Methylgruppen. Dies ist auf einen sterischen Konflikt zurückzuführen, der in Abb. 6.4 illustriert ist. Auch für die 


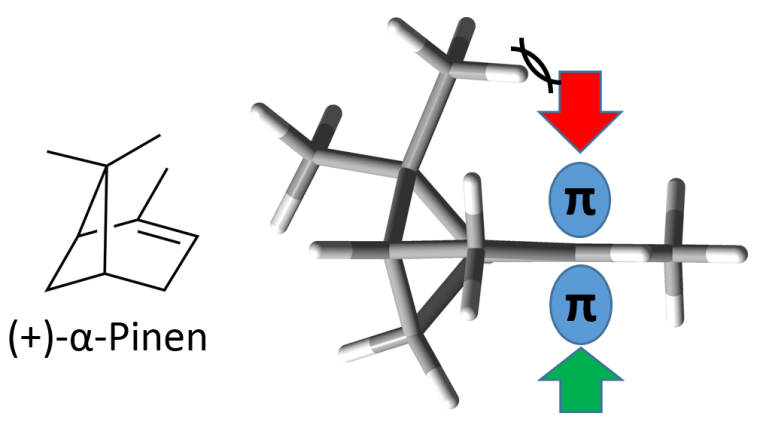

Abb. 6.4: Optimierte Struktur von (+)- $\alpha$-Pinen mit Zugänglichkeit der $\pi$-Orbitale.

Reaktionen von $\alpha$-Pinen mit Ozon [387] und Wasserstoff [388] wurde eine solche Regioselektivität beobachtet. Für Komplexe mit der schwieriger zugänglichen Seite werden wegen der ungünstigen Geometrie der gebildeten Wasserstoffbrücke auch deutlich höhere $\mathrm{OH}-$ Streckschwingungswellenzahlen vorhergesagt, für die es keine experimentellen Hinweise gibt. Es ist unklar, ob Komplexe mit Koordination dieser Seite bereits initial nicht signifikant gebildet werden und/oder ob effektive Relaxationsspfade in die stabileren Isomere existieren. Hierfür müsste die Wasserstoffbrücke zunächst wieder gebrochen und der Donor auf die andere Molekülseite von $\alpha$-Pinen wandern, wofür intuitiv eine hohe und breite Umwandlungsbarriere zu erwarten wäre. Allerdings deuten Ergebnisse für Dimere und Trimere von tert-Butylalkohol mit den ebenfalls diasteretopen Seiten von Norbornen auf eine weitgehende Einstellung eines Gleichgewichts und nicht auf eine statistische Bildung hin.[386] Möglicherweise kann auch ein komplexerer Umwandlungsmechanismus, unter Beteiligung eines dritten Moleküls vorliegen, wie er auch für andere Komplexe erwogen wurde.[174, 389, 390] Für die anderen quantenchemischen Methoden wurde die sterisch gehinderte Seite daher weniger intensiv mit jeweils nur etwa zwei Startstrukturen pro Kombination aus $\alpha$-Pinen-Enantiomer und Alkohol-Konformer berücksichtigt, wobei stets eine vergleichbare Benachteiligung gefunden wurde.

Diese deutliche Regioselektivät reduziert die Komplexität auf zwei verbleibende relevante Isomerisierung-Freiheitsgrade: die intramolekulare Konformationsisomerie der Alkohole und die intermolekulare Torsion um die Wasserstoffbrücke. Die Konformere der eingesetzten Alkohole wurden in der Komplexbildung vollständig in den Startstrukturen berücksichtigt. Die Torsion um die Wasserstoffbrücke wird näherungsweise über den $(\mathrm{Me}) \mathrm{C}=\mathrm{C} \cdots \mathrm{O}-\mathrm{C}$-Diederwinkel beschrieben, für den jeweils 12 Startstrukturen in $30^{\circ}$ Intervallen erstellt wurden, wobei einige Strukturen bezüglich anderer Winkel leicht modifiziert wurden, um offensichtliche sterische Konflikte zu vermeiden. Mit vier Alkoholen mit jeweils drei bis fünf Konformeren, zwei $\alpha$-Pinen-Enantiomeren, zwölf plus zwei Strukturen bezüglich der Torsion um die Wasserstoffbrücken und vier quantenchemischen Methoden wurden insgesamt eine vierstellige Zahl an Strukturoptimierungen durchgeführt, um die jeweiligen globalen Minima aufzufinden. Für alle optimierten Strukturen mit relativen elektronischen Energien innerhalb von $3 \mathrm{~kJ} \mathrm{~mol}^{-1}$ wurden Normalmodenanalysen durchgeführt, um diese durch Abwesenheit von imaginären Frequenzen als Minima zu bestätigen und um die Energien um die Schwingungsnullpunktsbeiträge zu korrigieren (mit Ausnahme von AM1, bei der die Schwingungsnullpunktsenergie, zusammen mit thermischen Beiträgen, bereits Teil der Parametrisierung ist [89]). Die berechneten spektralen Verschiebungen zwischen den globalen Minima der beiden Enantiomerenkombinationen lassen sich mit den experimentellen Verschiebungen vergleichen. Für die Kom- 
bination aus $(+)$ - $\alpha$-Pinen mit $(+)$ - $\alpha$-Fenchol wurde neben dem globalen Minimum auch die zweitstabilste Struktur berücksichtigt. Da die experimentelle Energieabfolge zwischen den beiden Konformeren $a$ und $b$ experimentell nicht bekannt ist, wird zugunsten der Theorie die Struktur mit der höheren berechneten $\mathrm{OH}-$ Streckschwingungswellenzahl der Bande $a$ und die mit der niedrigeren der Bande $b$ zugeordnet. Die absoluten Abweichungen aus den fünf berechneten und experimentellen Verschiebungen (unter Berücksichtigung der Vorzeichen) bei Wechsel des $\alpha$-Pinen-Enantiomers wurden gemittelt, und der erhaltene mittlere absolute Fehler (MAE) für die Bewertung der Performance der Methoden herangezogen. Die Ergebnisse für die verschiedenen Alkohole und Methoden sind in Abb. 6.5 zusammengestellt.

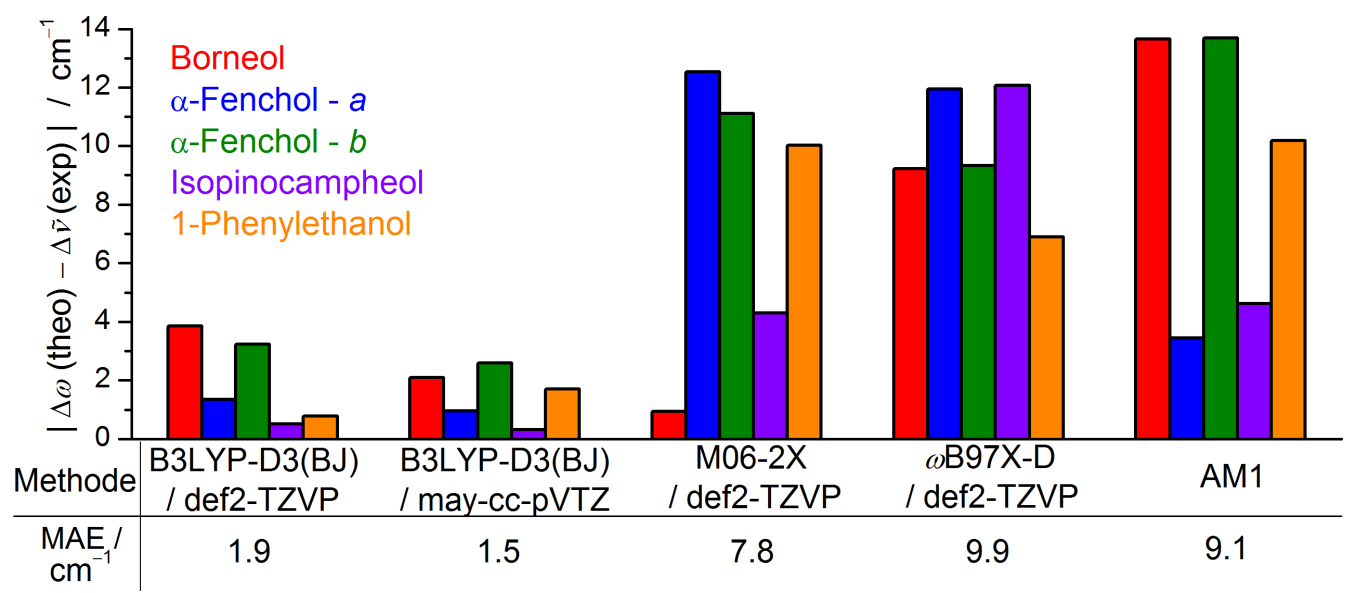

Abb. 6.5: Absolute Abweichungen zwischen berechneten und experimentellen Verschiebungen der OH-Streckschwingungswellenzahlen bei Wechsel der relativen Chiralität in gemischten Dimeren zwischen Alkoholen und $\alpha$-Pinen, für verschiedene quantenchemische Methoden. Mit Anpassungen übernommen aus Ref. [4] mit Erlaubnis von John Wiley and Sons.

B3LYP-D3(BJ)/def2-TZVP erreicht einen mittleren absoluten Fehler von $1.9 \mathrm{~cm}^{-1}$ und sagt für alle System die spektrale Chiralitätsdiskriminierung (oder deren Abwesenheit) qualitativ korrekt voraus. Nachoptimierung der globalen Minima mit may-cc-pVTZ reduziert den MAE weiter auf $1.5 \mathrm{~cm}^{-1}$ mit einer größten Einzelabweichung von $2.6 \mathrm{~cm}^{-1}$, welche somit bereits nur noch unwesentlich höher als die experimentelle Auflösung $\left(2 \mathrm{~cm}^{-1}\right)$ ist. Die Robustheit der Vorhersagen über vier verschiedene Systeme mit fünf spektralen Shifts deutet daraufhin, dass die gute Performance nicht nur auf zufälliger, sondern auf systematischer Fehlerkompensation (z. $\dot{B}$. hinsichtlich der Anharmonizität) beruht. Im deutlichen Kontrast hierzu zeigen die anderen beiden getesteten DFTMethoden keine Vorhersagekraft, mit MAEs von $9.9 \mathrm{~cm}^{-1}$ (M06-2X) bzw. $7.8 \mathrm{~cm}^{-1}$ $(\omega \mathrm{B} 97 \mathrm{X}-\mathrm{D})$. Diese spektroskopischen Vorhersagen sind nicht besser als die der AM1Methode $\left(\right.$ MAE $\left.=9.1 \mathrm{~cm}^{-1}\right)$, deren Strukturvorhersagen deutlich von denen der anderen Methoden abweichen. Die lokalen Minima der drei DFT-Methoden sind untereinander qualitativ ähnlicher, unterscheiden sich aber hinsichtlich relativer Energien und $\mathrm{OH}$ Streckschwingungswellenzahlen. Alle globalen Minimumstrukturen (sowie die zweitstabilste Struktur für (+)- $\alpha$-Pinen mit $(+)-\alpha$-Fenchol) sind für alle Methoden mit den wichtigsten Kennzahlen im Anhang in Abb. A.7 bis A.10 auf S. 317-320 zusammengestellt. Hier im Haupttext werden im Folgenden deren wichtigsten Unterschiede herausgestellt und diskutiert. 


\section{Chiralitätsdiskriminierung von Alkoholen mit $\alpha$-Pinen}

In den B3LYP-D3(BJ)-Strukturen ist interessanterweise in sämtlichen globalen Minima stets das jeweilige $g+-$ Konformer der Alkohole bezüglich des HOCH-Torsionswinkels präsent, wenn das jeweilige (-)-Enantiomer betrachtet wird. Dies gilt auch für Borneol, für welches $g+$ im freien Alkohol nur das zweitstabilste Konformer darstellt. Dieser Fall von adaptativer Aggregation kann damit begründet werden, dass in den $g$--Konformeren aller drei hier untersuchten (-)-Terpenalkohole das Hydroxyproton stets in Richtung einer Methylgruppe zeigt und daher sterisch weniger gut zugänglich ist (siehe beispielsweise Abb. 5.10 auf S. 123 für Borneol). In den anderen Alkoholen ist die analoge g+Einstellung dieses Torsionswinkels zusätzlich bereits im freien Alkohol bevorzugt.

Dass der Wechsel der Händigkeit des $\alpha$-Pinens keinen Konformationswechsel im Alkohol induziert, unterstreicht die Subtilität der Änderung in der Wechselwirkung und erleichtert die quantenchemische Beschreibung, da somit beim Vergleich der Diastereomere bessere Fehlerkompensation zu erwarten ist. Die anderen beiden DFT-Methoden geben für die monomeren Alkohole die gleiche Energieabfolge der Konformere wie B3LYPD3(BJ) an, in den globalen Minima der gemischten Dimeren treten dagegen teilweise andere Alkohol-Konformere auf. AM1 hat sowohl für die freien, wie auch die gebundenen Alkohole, eine starke Präferenz für das jeweilige $t$-Konformer. Um zu untersuchen, ob diese abweichenden intramolekularen Konformationspräferenzen der entscheidende Faktor für die schlechte Performance dieser Methoden ist, wurde die Auswahl testweise auf die jeweils stabilsten Dimere mit dem $g+$-Konformer, also der Vorhersage von B3LYPD3(BJ), eingeschränkt. Dies Beschränkung verringert den mittleren absoluten Fehler für M06-2X und $\omega$ B97X-D etwas (auf 5.5 bzw. $5.7 \mathrm{~cm}^{-1}$, mehr Details in Abb. A.11 auf S. $321 \mathrm{im}$ Anhang). Einige der so ausgewählten Strukturen unterscheiden sich allerdings auch zusätzlich bezüglich der Torsion um die Wasserstoffbrücke qualitativ von den globalen Minima von B3LYP-D3(BJ). Eine noch stärkere Einschränkung durch direkte Nachoptimierung der globalen Minima von B3LYP-D3(BJ) verringert die MAEs weiter, auf $4.7 \mathrm{~cm}^{-1}$ (M06-2X), $2.7 \mathrm{~cm}^{-1}$ ( $\omega$ B97X-D) und $6.7 \mathrm{~cm}^{-1}$ (AM1) (Abb. A.12 auf S. $321 \mathrm{im}$ Anhang). Dabei wird aber weiterhin nicht die Übereinstimmung von B3LYP-D3(BJ) erreicht. Die Defizite der anderen Methoden beschränken sich daher offenbar nicht nur auf die Vorhersage der relativen Energien der Minima. Im Fall von M06-2X sind hierfür teils starke Unterschiede bezüglich der vorhergesagten Verschiebung verantwortlich. So sind die globalen Minima der Dimere mit 1-Phenylethanol für B3LYP-D3(BJ) und M06-2X strukturell zwar sehr ähnlich, dagegen ist die zugehörige Verschiebung aber sehr verschieden $\left(+0.8 \mathrm{~cm}^{-1}\right.$ gegenüber $-11.0 \mathrm{~cm}^{-1}$, Experiment: $\left.-1 \mathrm{~cm}^{-1}\right)$. Um zu prüfen, inwieweit die schlechte Performance von M06-2X auf die bekannte Sensitivität der Methode auf das Integrationsgitter [391, 392] zurückzuführen ist, wurden mit M06-2X und verfeinerten Parametern [Int (Grid=SuperFine) und CPHF (Grid=UltraFine)] sowohl die globalen Minima von M06-2X [Int (Grid=UltraFine)] wie auch B3LYP-D3(BJ) nachoptimiert. Dies führt zu mäßigen Änderungen in den Wellenzahlunterschieden, aber nicht zu einer Annäherung an die experimentellen Werten (Details in Abb. A.13 auf S. 322 im Anhang).

Die kartesischen Koordinaten der neun zugeordneten Strukturen auf B3LYPD3(BJ)/def2-TZVP-Niveau stehen für zukünftige Tests von Frequenzvorhersagen weiterer quantenchemischen Methoden in den Supporting Information von Ref. [4] zur Verfügung. 
Nach der Feststellung, dass B3LYP-D3(BJ)/may-cc-pVTZ die Verschiebungen offenbar sehr gut beschreiben kann, lohnt ein Blick auf die Strukturen, um molekulare Unterschiede mit dem Ausmaß der spektralen Chiralitätsdiskriminierung zu korrelieren. Alle zugeordneten Strukturen auf B3LYP-D3(BJ)/may-cc-pVTZ sind in Abb. 6.6 zusammengestellt. Abweichend von den experimentell verwendeten Enantiomeren, werden zur einfacheren Vergleichbarkeit alle Strukturen mit $(+)-\alpha$-Pinen und variiertem AlkoholEnantiomers dargestellt. Durch diese Betrachtungsweise lässt sich die Torsion um die Wasserstoffbrücke in Abhängigkeit von der Struktur des Alkohols verdeutlichen. Dabei lassen sich für die Komplexe der eng verwandten Terpenalkohole gewisse Beziehungen zwischen den Dimer-Strukturen und den $\mathrm{OH}-$ Streckschwingungswellenzahlen erkennen. So sind die Dimere aus den (+)-Enantiomeren von Borneol und Isopinocampheol mit $(+)-\alpha$-Pinen strukturell ähnlich und haben auch vergleichbare vorhergesagte $\mathrm{OH}-$ Streckschwingungswellenzahlen $\left(\Delta \omega=+2 \mathrm{~cm}^{-1}\right)$, in Übereinstimmung mit dem Experiment $\left(\Delta \tilde{v}=+3 \mathrm{~cm}^{-1}\right)$. Gleiches gilt für die Dimere aus den (-)-Enantiomeren von $\alpha$ Fenchol und Isopinocampheol mit $(+)-\alpha$-Pinen $\left(\Delta \omega=+1 \mathrm{~cm}^{-1}, \Delta \tilde{v}=+2 \mathrm{~cm}^{-1}\right)$. Dies ist insofern bemerkenswert, als die Wellenzahl des $g+$-Konformers von (-)-Isopinocampheol in freier Form über $20 \mathrm{~cm}^{-1}$ niedriger vorhergesagt wird als für seine Entsprechungen in (-)-Borneol und (-)- $\alpha$-Fenchol. Auch 1-Phenylethanol weist im freier Form eine ähnlich niedrige $\mathrm{OH}-\mathrm{Streckschwingungswellenzahl} \mathrm{wie} \mathrm{Isopinocampheol} \mathrm{auf.} \mathrm{Diese} \mathrm{ist} \mathrm{dagegen}$ im Komplex mit $\alpha$-Pinen noch tiefer verschoben im Vergleich zu den Terpenalkoholen. Möglicherweise wird die Wasserstoffbrücke durch eine höhere Dispersionswechselwirkung mit dem gut polarisierbaren und kompakteren Phenylring [384] komprimiert, wozu auch die höher berechnete Dissoziationsenergie passen würde (etwa $30 \mathrm{~kJ} \mathrm{~mol}^{-1}$ gegenüber 24-27 kJ mol ${ }^{-1}$ für die Komplexe der Terpenalkohole).

Dass signifikante spektrale Verschiebungen bei Wechsel der relativen Chiralität mit Borneol und $\alpha$-Fenchol, jedoch nicht mit Isopinocampheol, beobachtet werden, beruht offenbar darauf, dass bei diesen in jeweils einer Enantiomeren-Kombination die Lage des globalen Minimums auf der Torsionskoordinate im Vergleich zu Isopinocampheol verschoben ist. Tatsächlich wurden jeweils lokale Minima gefunden, die sowohl bezüglich des Torsionswinkels, wie auch der OH-Streckschwingungswellenzahl, weitgehend den globalen Minima der anderen Terpenalkoholen entsprechen. Es scheint daher eine übergeordnete Beziehung zwischen intermolekularer Torsion und $\mathrm{OH}$ Streckschwingungswellenzahl zu existieren. 

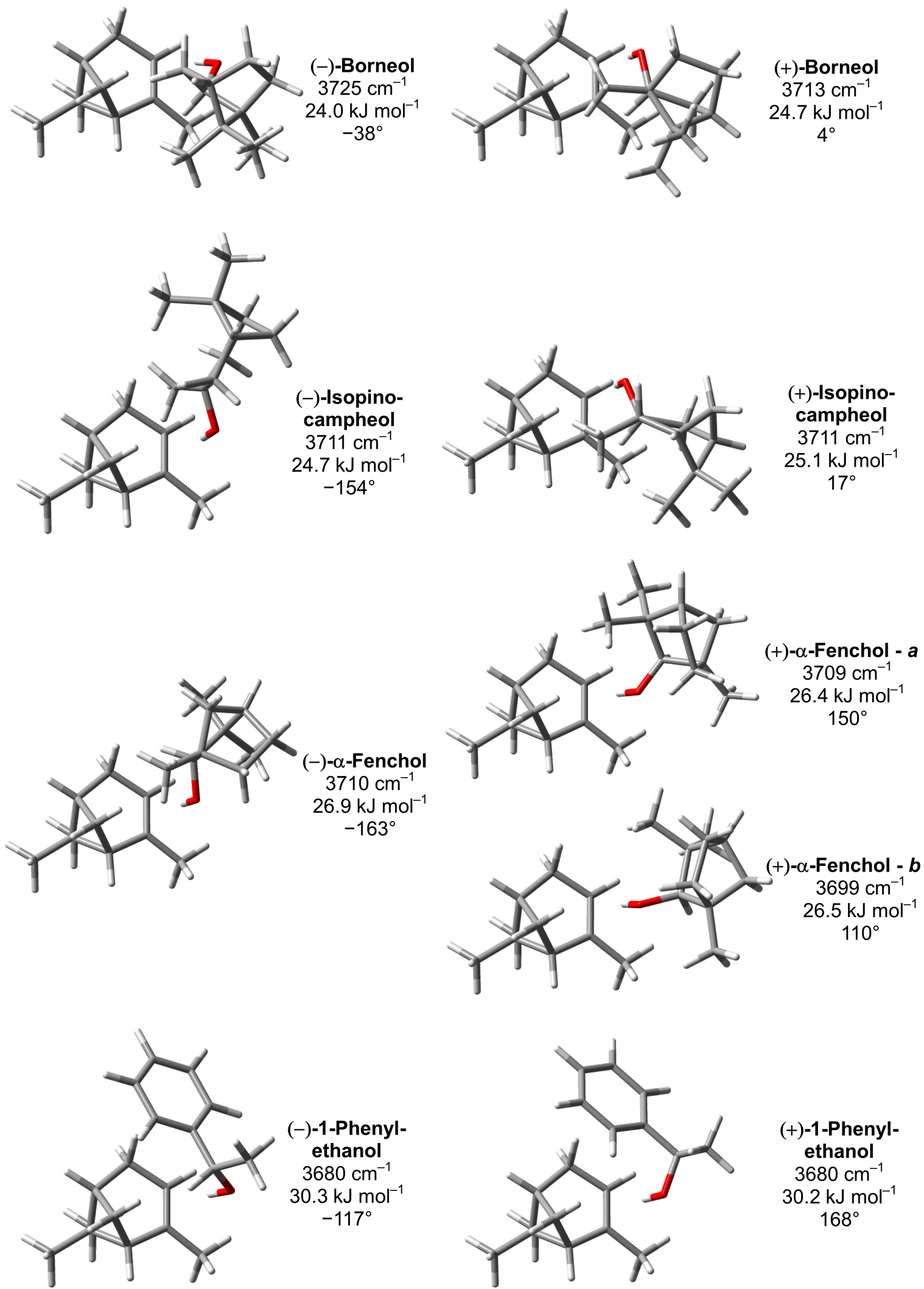

(+)-1-Phenylethanol $3680 \mathrm{~cm}^{-1}$ $30.2 \mathrm{~kJ} \mathrm{~mol}^{-1}$ $168^{\circ}$

Abb. 6.6: Strukturen von zugeordneten Dimeren aus (+)- $\alpha$-Pinen und verschiedenen Alkoholen. Angeben sind die OH-Streckschwingungswellenzahlen, Dissoziationsenergien (relativ zu den jeweils stabilsten Monomeren) und $\mathrm{MeC}=\mathrm{C} \cdots \mathrm{O}-\mathrm{C}-$ Diederwinkel auf B3LYP-D3(BJ)/may-ccpVTZ-Niveau. 
Das Auftreten der Banden $a$ und $b$ im Spektrum der Mischung von $(+)-\alpha$-Fenchol und $(+)-\alpha$-Pinen wird quantenchemisch durch zwei fast isoenergetische Minima im intermolekularen Torsionspotential erklärt. Relaxierte Scans dieser Koordinate sind in Abb. 6.7 gezeigt. Während für die Kombination aus $(-)$ - $\alpha$-Fenchol und $(+)-\alpha$-Pinen ein einzelnes deutliches Minimum besteht, ist für $(+)-\alpha$-Fenchol und $(+)-\alpha$-Pinen stattdessen ein Doppelminimum ausgeprägt. Auch sonst sind die beiden Potentialkurven hinsichtlich der Position von Minima und Maxima sehr verschieden, was zeigt, dass $(+)$ - $\alpha$-Pinen empfindlich auf die Unterschiede zwischen (+)- und (-)- $\alpha$-Fenchol reagiert. Weitere lokale Minima sind erkennbar, diese sind jedoch energetisch recht hoch $\left(>1 \mathrm{~kJ} \mathrm{~mol}^{-1}\right)$ und von niedrigen Barrieren umgeben. Dies steht in Übereinstimmung mit der beobachteten Relaxation in das jeweilige globale (Doppel-)Minimum. In Bereichen ohne Datenpunkte kommt es aufgrund von sterischen Konflikten während der Optimierung zur Umwandlung in andere intramolekulare Konformere von $\alpha$-Fenchol, die ebenfalls über niedrige Barrieren zugänglich sind. Während der Struktursuche wurden auch lokale Minima gefunden, bei denen der HOCH-Torsionswinkel von $\alpha$-Fenchol ekliptisch vorliegt - dies unterstreicht die ungewöhnlich niedrige Barriere zwischen den $g$-- und $g+-$ Konformeren, die in Kap. 5.8 diskutiert wurde. Auch M06-2X und $\omega$ B97X-D sagen das Doppelminimum, sowie die zugehörige spektrale Aufspaltung, vorher. Allerdings wurde mit beiden Methoden eine noch stabilere Struktur mit dem $t$-Konformer von $\alpha$-Fenchol gefunden, deren relative spektrale Position nicht mit dem Experiment kompatibel ist.

Auf B3LYP-D3(BJ)/may-cc-pVTZ-Niveau liegt Konformer $a$ elektronisch nur $0.02 \mathrm{~kJ} \mathrm{~mol}^{-1}$ oberhalb von Konformer $b$, was sich durch die Schwingungsnullpunktskorrektur auf $0.15 \mathrm{~kJ} \mathrm{~mol}^{-1}$ erhöht. Die Barrierenhöhe beträgt relativ zum höher ener-

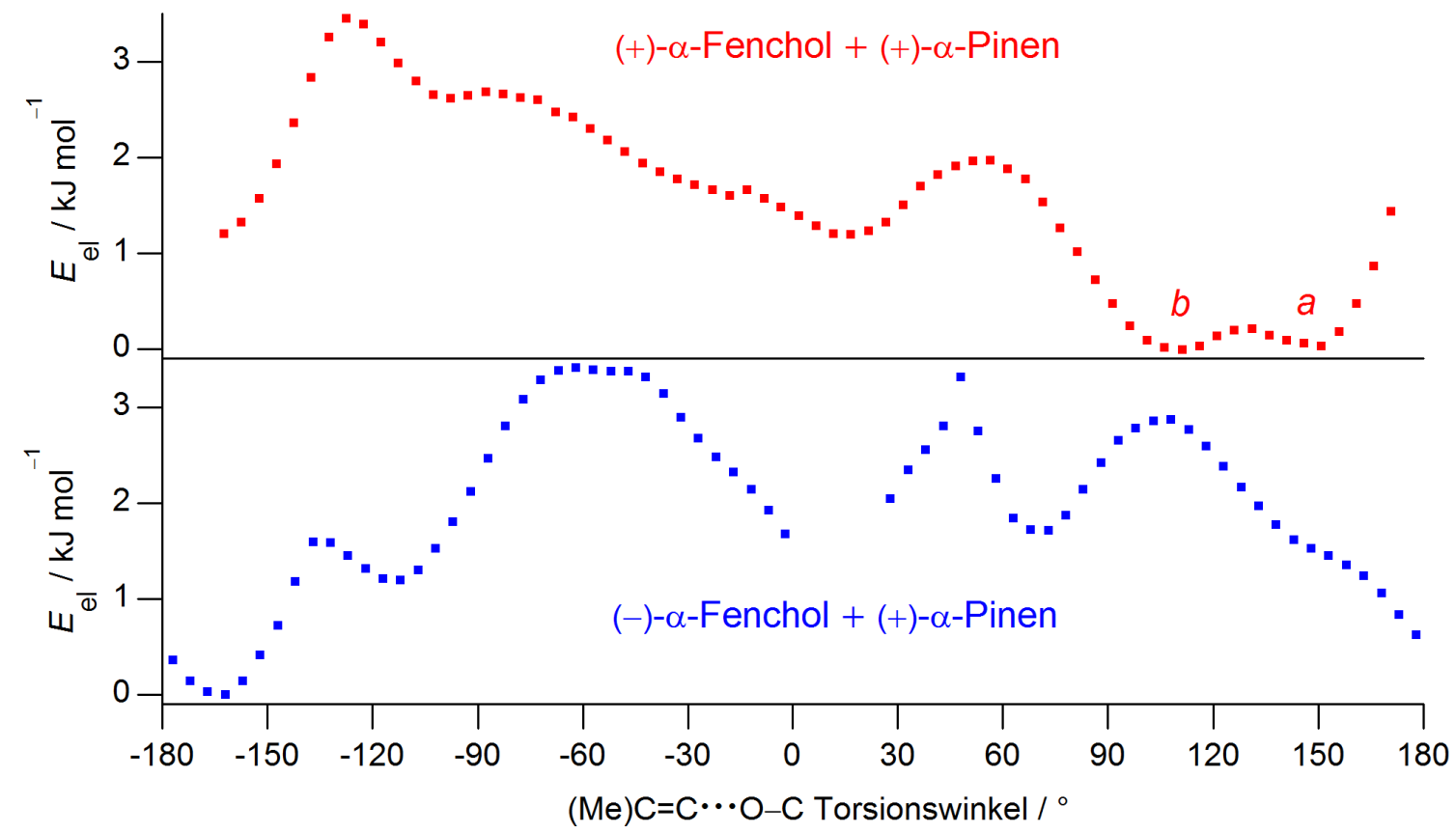

Abb. 6.7: Relaxierte Scans des $\mathrm{MeC}=\mathrm{C} \cdots \mathrm{O}-\mathrm{C}$-Torsionswinkels für gemischte Dimere aus $\alpha$ Fenchol ( $g+-$ Konformation bezogen das (-)-Enantiomer) und $\alpha$-Pinen auf B3LYP-D3(BJ)/maycc-pVTZ-Niveau. In Bereichen ohne Datenpunkte kommt es aufgrund von sterischen Konflikten während der Optimierung zur Umwandlung in andere intramolekulare Konformere von $\alpha$ Fenchol. 


\section{Chiralitätsdiskriminierung von Alkoholen mit $\alpha$-Pinen}

getischen Konformer $a$ elektronisch $0.18 \mathrm{~kJ} \mathrm{~mol}^{-1}$, wird allerdings durch Schwingungsnullpunktskorrektur negativ mit $-0.02 \mathrm{~kJ} \mathrm{~mol}^{-1}$. Wegen der Abwesenheit einer effektiven Barriere wäre daher eine alternative Interpretation, dass es sich nicht um getrennte Konformere, sondern um Zustände der sehr niederfrequenten intermolekularen Torsion (berechnet zu $\omega=18$ bzw. $6 \mathrm{~cm}^{-1}$ für $a$ und $b$ ) eines einzelnen Konformers handelt. Dennoch stehen die für beide (elektronische) Minima berechneten Positionen der $\mathrm{OH}-$ Streckschwingungsübergänge in guter Übereinstimmung mit den beobachteten. Die Bedeutung von Isomeren bezüglich der Torsionbewegung um eine Wasserstoffbrücke ohne effektive Barrieren nach Schwingungsnullpunktskorrektur wurde bereits in Kap. 4.3.7 anhand der Ethanol-Dimere diskutiert. Der Befund hier stützt die dortige Vermutung, dass diese bei der Interpretation von Spektren nicht vernachlässigt werden sollten.

Neben den spektralen Aspekten spielt die chirodiastaltische Energie eine zentrale Rolle in der Chiralitätserkennung. Durch den permanent chiralen Charakter der Verbindungen ist diese, mangels Gleichgewichtseinstellung, mit den hier verwendeten Methoden nicht experimentell zugänglich. Quantenchemische Vorhersagen sind in Abb. 6.8 gezeigt. Wegen der sehr unterschiedlichen Strukturen von $\alpha$-Pinen und den Alkoholen ist unklar, welche Kombinationen als homo- und welche als heterochiral einzustufen sind. Die zu erwartende Richtung eines möglichen nicht berücksichtigten Einflusses durch Chiraliätsinduzierte Spinselektivität ist daher unbekannt. Zur Klassifizierung werden hier stattdessen die optischen Aktivitäten der Verbindungen betrachtet, die gleiche oder entgegengesetzte Vorzeichen aufweisen können. Die absoluten chirodiastaltischen Energien fallen relativ gering aus mit Werten von $1 \mathrm{~kJ} \mathrm{~mol}^{-1}$ oder weniger. Werden die Vorhersagen von B3LYP-D3(BJ)/may-cc-pVTZ zugrunde gelegt, besteht keine klare Korrelation mit der spektralen Chiralitätsdiskriminierung.

Interessant ist diesbezüglich die Frage, ob sich alternativ eine Bestimmung der chirodiastaltischen Energien über die Messung der Dissoziationsenergien nicht als besserer

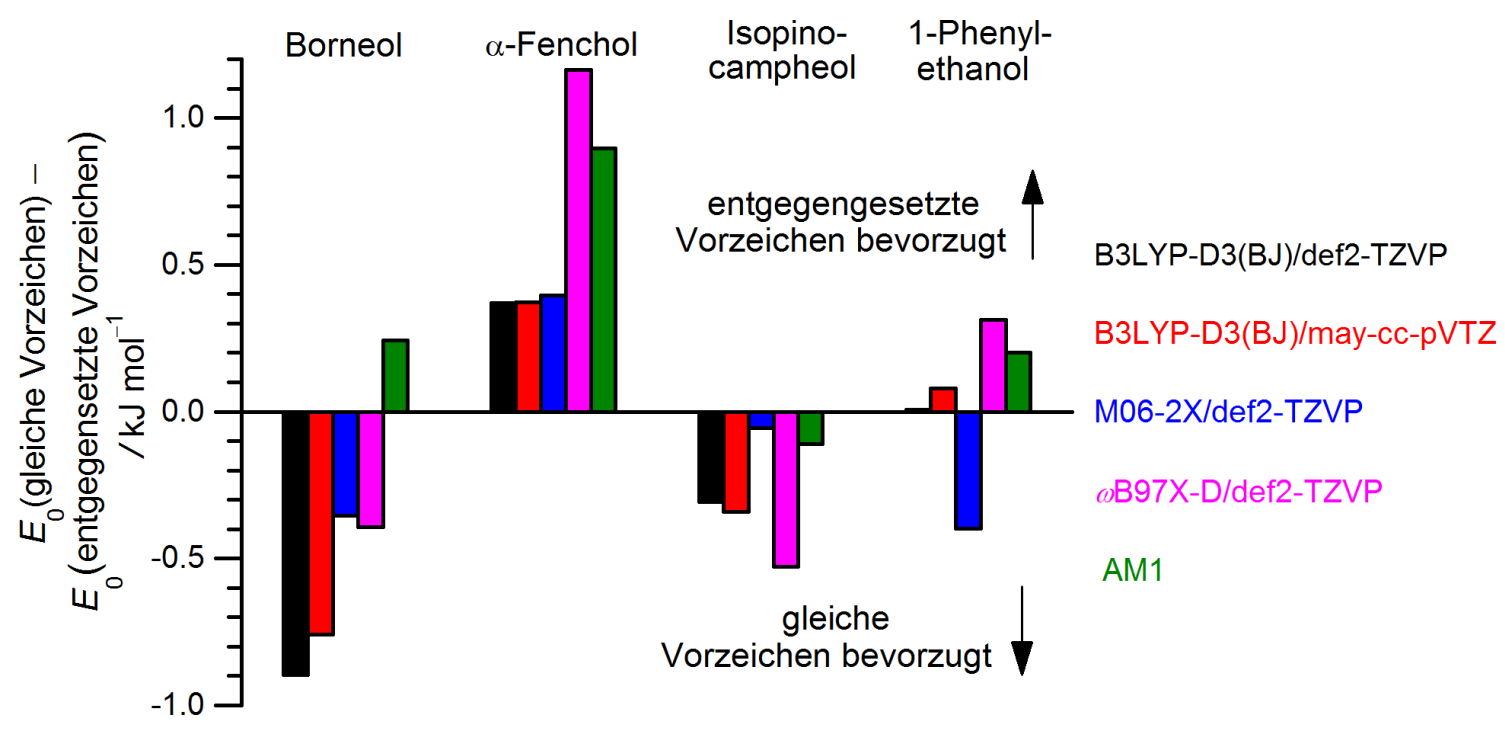

Abb. 6.8: Vorhergesagte Energiedifferenzen zwischen den globalen Minima der Komplexe aus den untersuchten Molekülen mit optischen Aktivitäten gleicher und entgegengesetzter Vorzeichen gemäß verschiedenen quantenchemischen Methoden. Mit Anpassungen übernommen aus Ref. [4] mit Erlaubnis von John Wiley and Sons. 
Benchmark für die hier evaluierten quantenchemische Methoden eignen würde. Für deren Beantwortung muss die Genauigkeit bestehender experimenteller Methoden mit den Unterschieden in den Vorhersagen verglichen werden. Hierzu wird hypothetisch angenommen, dass B3LYP-D3(BJ)/may-cc-pVTZ auch die chirodiastaltische Energien gut vorhersagt und dementsprechend als Referenz verwendet. Die resultierenden Abweichungen der anderen Methoden sind in Abb. 6.9 zusammengestellt.

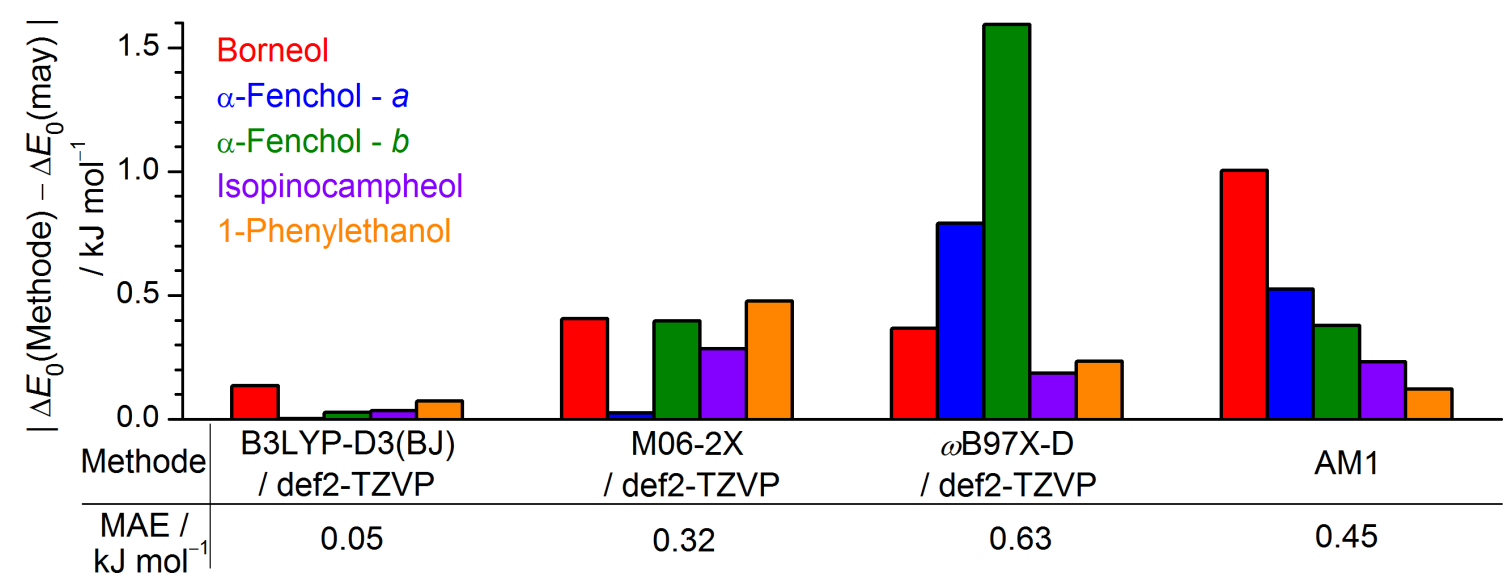

Abb. 6.9: Absolute Abweichungen zwischen auf B3LYP-D3(BJ)/may-cc-pVTZ-Niveau und anderen Methoden berechneten Energieunterschieden bei Chiralitätswechsel in gemischten Dimeren von Alkoholen und $\alpha$-Pinen. Mit Anpassungen übernommen aus Ref. [4] mit Erlaubnis von John Wiley and Sons.

Demnach müsste eine experimentelle Methode eine Genauigkeit von mindestens $\pm 0.1 \mathrm{~kJ} \mathrm{~mol}^{-1}$ für Dissoziationsenergien (und damit $\pm 0.2 \mathrm{~kJ} \mathrm{~mol}^{-1}$ für chirodiastaltische Energien) erreichen, um die diese System gut beschreibende Methode B3LYPD3(BJ)/def2-TZVP von der ungeeigneten M06-2X/def2-TZVP zu unterscheiden. Dies erscheint mit den bekannten Möglichkeiten zur Zeit nicht konsistent erreichbar, insbesondere da in den Komplexen mit den Terpenalkoholen ein UV-Chromophor fehlt, welches für viele der Methoden Voraussetzung ist.[393, 394] Die Genauigkeit des hier verwendeten schwingungsspektroskopische Ansatzes von $\pm 1 \mathrm{~cm}^{-1}$ für Bandenpositionen (und damit $\pm 2 \mathrm{~cm}^{-1}$ für spektrale Unterschiede) ist dagegen ausreichend, um die Methoden zu diskriminieren - sofern im gewählten System ausreichende Fehlerkompensation, z. B. bezüglich anharmonischer Effekte, stattfindet. Eine analoge Auswertung für die spektralen Verschiebungen mit B3LYP-D3(BJ)/may-cc-pVTZ als Referenz ist in Abb. A.14 auf S. $322 \mathrm{im}$ Anhang dokumentiert. Dabei wird gefunden, dass sich die mittleren absoluten Fehler der anderen Methoden sich nur im Bereich von $\pm 1 \mathrm{~cm}^{-1}$ durch den Wechsel der Referenz ändern.

Eine interessante molekulare Eigenschaft von $\alpha$-Pinen ist, dass es durch Substitution der doppelten Methylgruppen am Vierring durch Wasserstoffatome zu einem prochiralen Alken wird. Die beiden enantiotopen Seiten entsprechen denen der $\alpha$-Pinen-Enantiomere, wo sie jeweils einzeln zugänglich sind (Abb. 6.10). Da die beiden Methylgruppen sich auf der anderen Molekülseite befinden, sollte sich deren An- oder Abwesenheit nur geringfügig auf die Wasserstoffbrücke im Dimer auswirken. Rechnungen auf B3LYPD3(BJ)/may-cc-pVTZ sagen voraus, dass die Substitution der Methylgruppen die absoluten $\mathrm{OH}-$ Streckschwingungswellenzahlen in den neun zugeordneten Strukturen ohne 


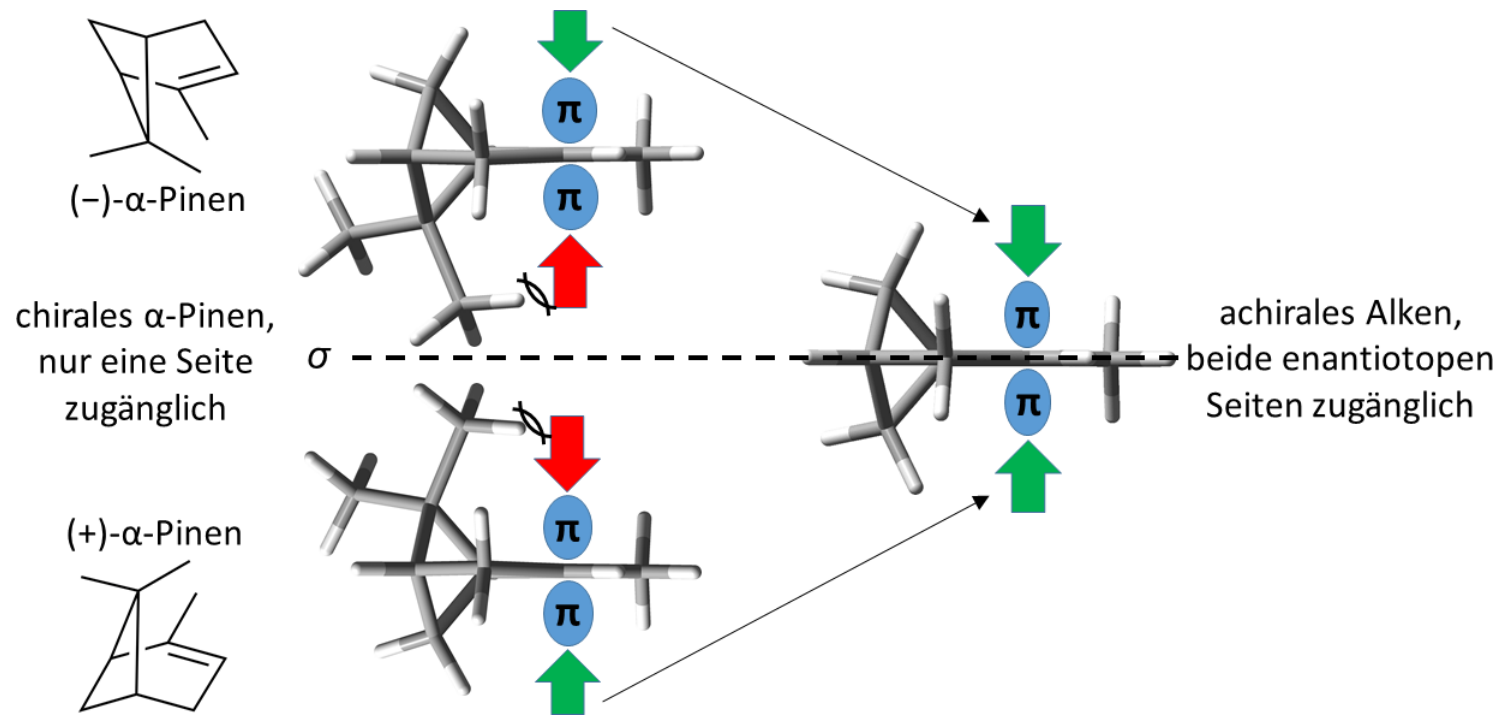

Abb. 6.10: Vereinigung der einzeln zugänglichen enantiotopen Seiten der beiden $\alpha$-PinenEnantiomere im prochiralen 2-Methylbicyclo[3.1.1]hept-2-en durch Substitution zweier Methylgruppen durch Wasserstoffatome.

klaren Trend nur um -2.0 bis $+1.3 \mathrm{~cm}^{-1}$ ändern (Mittelwert $-0.5 \mathrm{~cm}^{-1}$ ). Die spektralen Unterschiede zwischen den Diastereomeren-Paaren verändern sich noch weniger $(-1.4$ bis $+0.7 \mathrm{~cm}^{-1}$, Mittelwert $-0.2 \mathrm{~cm}^{-1}$ ). Die vorhergesagten Dissoziationsenergien verringern sich dagegen im Vorzeichen einheitlich um -0.1 bis $-0.6 \mathrm{~kJ} \mathrm{~mol}^{-1}$, vermutlich wegen des fehlenden langreichweitigen dispersiven Einflusses der Methylgruppen. In diesen vorhergesagten nur geringfügigen Änderungen werden aber möglicherweise nicht angemessen explizit chiralitätsabhängige Effekte berücksichtigt, wie sie im Rahmen der chiralitätsinduzierten Spinselektivität diskutiert werden (Kap. 2.4). Durch einen künftigen experimentellen Vergleich der Wechselwirkung von chiralen Alkoholen mit $\alpha$-Pinen und seinem prochiralen Pendant 2-Methylbicyclo[3.1.1]hept-2-en könnte somit weiter geprüft werden, ob solche Effekte einen signifikanten Einfluss haben oder nicht.

Neben den absoluten Bandenpositionen und spektralen Aufspaltungen der Komplexe mit 2-Methylbicyclo[3.1.1]hept-2-en sind dabei auch die relativen Bandenintensitäten interessant. Diese könnten Aufschluss darüber geben, ob die Bildung der Dimere mit den beiden Seiten statistisch oder als Gleichgewichtsverteilung erfolgt. Falls letzteres zutrifft, könnten aus den relativen Bandenintensitäten chirodiastaltische Energien experimentell abgeschätzt werden. 2-Methylbicyclo[3.1.1] hept-2-en müsste hierfür allerdings zunächst synthetisiert werden. Dieses Konzept wird im Ausblick dieser Arbeit (Kap. 10) auf weitere Systeme erweitert.

\subsection{Diskriminierung mit 2-Butanol}

2-Butanol besitzt einige Eigenschaften, die die Verbindung für die Chiralitätserkennung in der Gasphase interessant machen. Als einfachster chiraler aliphatischer Alkohol besitzt sie Prototyp-Charakter, weist einen hohen Dampfdruck auf und beide Enantiomere sind kommerziell verfügbar. Daher war 2-Butanol bereits Bestandteil einer größeren Zahl von entsprechenden Studien.[237, 323, 357, 395-404] Allerdings werden die Spek- 
tren und quantenchemischen Rechnungen durch die intramolekulare Konformationsvielfalt von 2-Butanol verkompliziert (Kap. 5.15). Dies gilt insbesondere wegen der hohen Umwandlungsbarrieren, die die Relaxation in die stabilsten Anordnungen unter Überschallexpansionsbedingungen einschränken. Bei der Diskriminierung von 2-Butanol mit $\alpha$-Pinen werden daher komplexe und von Überlagerungen geprägte Spektren im $\mathrm{OH}$ Streckschwingungsbereich erhalten, die in Abb. 6.11 gegenübergestellt sind. Im Bereich von 3530-3490 $\mathrm{cm}^{-1}$ sind die in Kap. 5.15 diskutierten Banden der homochiralen Dimere aus zwei Molekülen (+)-2-Butanol zu erkennen. Diese werden allerdings offenbar leicht durch gemischte und vermutlich größere Cluster überlagert. Intensive neue Signale treten im Bereich von 3580-3530 $\mathrm{cm}^{-1}$ auf. Die relativen Banden-Intensitäten in diesem Bereich sind unempfindlich gegen Veränderungen des Stagnationsdruckes und des Mischungsverhältnisses (weitere Spektren in Abb. A.15 auf S. 323 im Anhang), daher werden diese Banden Clustern gleicher Zusammensetzung und Größe zugeordnet. Im gleichen Spektralbereich wurden die Banden der zuvor diskutierten gemischten Dimere von $\alpha$-Pinen und anderen Alkoholen gefunden, was eine analoge Zuordnung nahe legt. Während in der Kombination der Verbindungen mit entgegengesetzten optischen Aktivitäten eine dominante Bande neben weiteren schwächeren Signalen zu erkennen ist, verteilt sich in der Kombination mit gleichgerichteten optischen Aktivitäten die Intensität gleichmäßiger auf mehrere Banden. Die Positionen der Banden, die gemischten Dimeren zugeordnet werden, sind im Anhang in Tab. A.13 auf S. 314 dokumentiert. Die Spektren, die unter Verwendung von racemischem 2-Butanol erhalten wurden, entsprechen im Bereich der gemischten Dimere sehr gut Mittelungen der Spektren mit Einsatz der reinen Enantiomere, was auch hier auf statistische Bildung hinweist.

Um die Spektren auf molekularer Ebene zu interpretieren, wurde die Potentialfläche der gemischten Dimere gemäß der zuvor für die anderen Alkohole erläuterten Strategie auf B3LYP-D3(BJ)/def2-TZVP-Niveau evaluiert und alle gefundenen Minima mit B3LYP-D3(BJ)/may-cc-pVTZ nachoptimiert. Die jeweils zwei stabilsten DimerKonformere mit gleichen bzw. entgegensetzten Vorzeichen sind in Abb. 6.12 dargestellt.

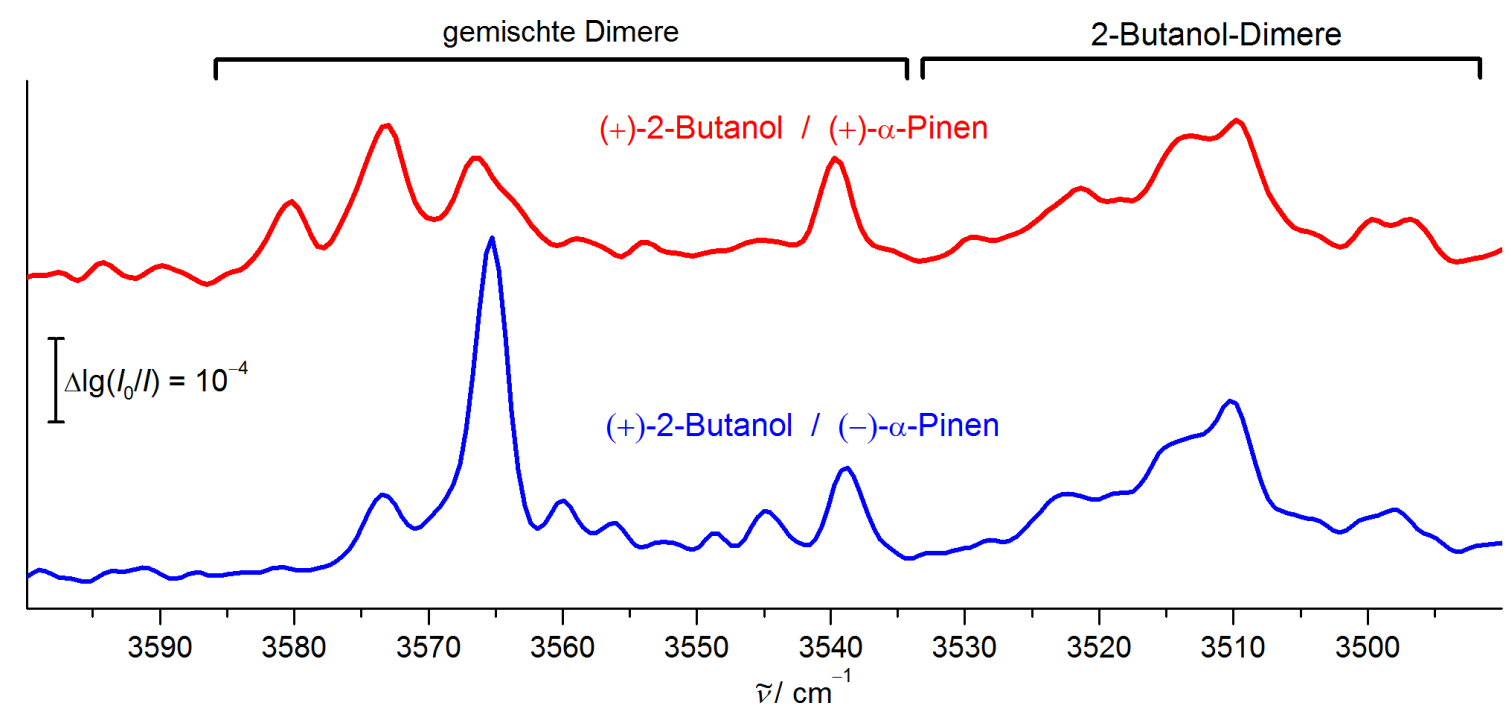

Abb. 6.11: Filet-Jet-Spektren im OH-Streckschwingungsbereich für Expansionen einer etwa äquimolaren Mischung von (+)-2-Butanol mit (+)- bzw. (-)- $\alpha$-Pinen. Spektralbereiche für Banden von Dimeren verschiedener Zusammensetzung sind markiert. 


\section{Chiralitätsdiskriminierung von Alkoholen mit $\alpha$-Pinen}

Das $G+t$-Konformer von (+)-2-Butanol, welches für den freien Alkohol die stabilste Anordnung darstellt, ist auch an beiden globalen Minima der Dimere mit $\alpha$-Pinen beteiligt. Das globale Minimum mit gleichen Vorzeichen wird dabei um $0.4 \mathrm{~kJ} \mathrm{~mol}^{-1}$ stabiler gefunden als seine Entsprechung mit entgegengesetzten Vorzeichen. Wird für den Vergleich mit den anderen zuvor diskutierten Alkoholen der HOCH-Torsionswinkel des (-)Enantiomers von 2-Butanol betrachtet, so nimmt dieser in den globalen Minima erneut eine $g+$-Einstellung ein. Das im freien Alkohol zweitstabilste $G+g--$ Konformers ist dagegen im Komplex destabilisiert. Dies kann vermutlich auf die eingeschränkte Zugänglichkeit des Hydroxywasserstoffatoms zurückgeführt werden, welche in der Präsenz der benachbarten Ethylgruppe begründet ist (siehe Abb. 5.41 auf 164). Ein vergleichbarer Effekt wurde zuvor auch für die analogen Konformere der Terpenalkohole gefunden.

Werden vereinfacht die intensivsten beobachteten Banden den globalen Minima zugeordnet, so entspricht der experimentelle Shift von $8 \mathrm{~cm}^{-1}$ auch für dieses System sehr gut dem berechneten von $9 \mathrm{~cm}^{-1}$. Allerdings werden mit 2-Butanol, anders als für die zuvor diskutierten Alkohole, auch Banden nicht mehr vernachlässigbarer Intensität für weitere Dimere beobachtet. Daher wurden Modellierungen der Spektren auf Basis aller gefundenen 34 (gleiche Vorzeichen) bzw. 31 (entgegengesetzte Vorzeichen) lokalen Minima durchgeführt. Dabei wurden folgende Annahmen/Vereinfachungen gemacht:

- Die Anharmonizität der OH-Streckschwingung aller gemischten Dimere wird als vergleichbar angenommen und wird durch eine einheitliche empirische Subtraktion von den harmonischen Wellenzahlen berücksichtigt.

- Der OCCC-Torsionwinkel von 2-Butanol wird in der Verteilung bei Zimmertemperatur als eingefroren betrachtet, entsprechend der erfolgreichen Modellierung der

(+)-2-Butanol / (+)- $\alpha$-Pinen

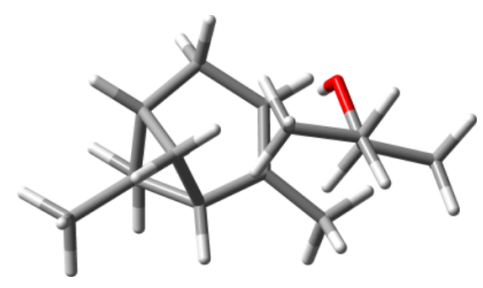

$\mathrm{G}+t$

$21.6 \mathrm{~kJ} \mathrm{~mol}^{-1}$

$3715 \mathrm{~cm}^{-1}$
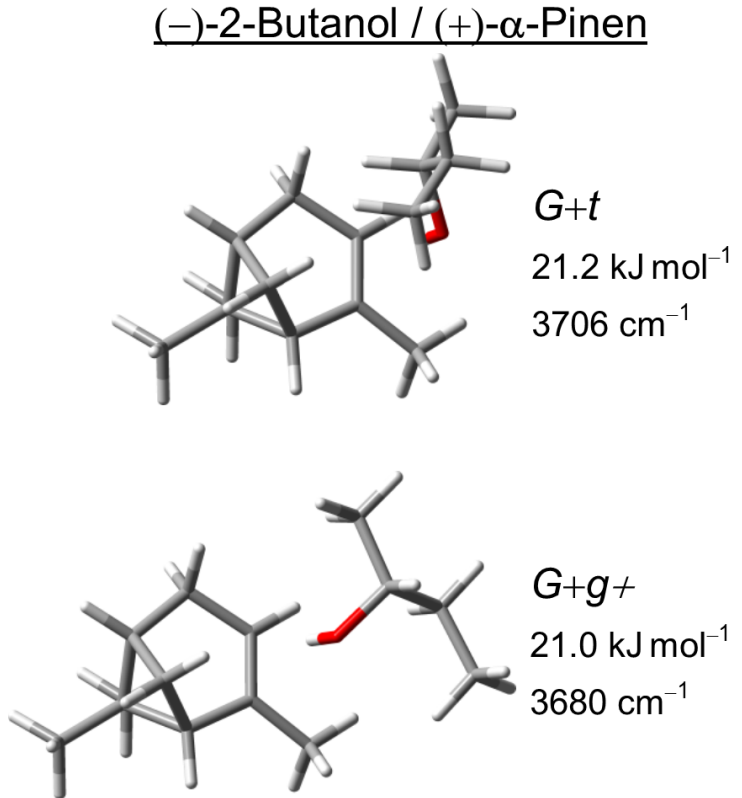

$\mathrm{G}+\mathrm{g}+$ $21.0 \mathrm{~kJ} \mathrm{~mol}^{-1}$ $3680 \mathrm{~cm}^{-1}$

\footnotetext{
Abb. 6.12: Die jeweils zwei stabilsten Konformere für die Dimere aus (+)- $\alpha$-Pinen und (+)- bzw. (-)-2-Butanol auf B3LYP-D3(BJ)/may-cc-pVTZ-Niveau. Angeben sind jeweils das beteiligte 2Butanol-Konformer (die Vorzeichen der Torsionswinkel (Kap. 5.15) sind bezogen auf das (+)Enantiomer), die Dissoziationsenergie (bezogen auf die stabilsten Monomer-Konformere), sowie die harmonische $\mathrm{OH}-$ Streckschwingungswellenzahl.
} 
Spektren des freien Alkohols in Kap. 5.15. Die Dimerisierung verläuft diesbezüglich statistisch. Für alle anderen intra- und intermolekularen Konformationsfreiheitsgerade wird eine Gleichgewichtseinstellung bei $64 \mathrm{~K}$ angenommen, entsprechend dem Befund für Ethanol-Monomere und -Dimere in Kap. 4.3.4.

- Die Modellierung aller Übergänge erfolgt mit Gauß-Profilen einheitlicher Halbwertsbreite.

Modellierungen auf Basis dieser Voraussetzungen sind in Abb. 6.13 ober- bzw. unterhalb der experimentellen Spektren gezeigt. Der Vergleich zeigt, dass es plausibel ist, dass die jeweiligen globalen Minima (die jeweils intensivsten dunkelgrünen Signale in den Strichspektren) tatsächlich zu den intensivsten Banden beitragen.

Die jeweils zweitstabilsten Dimere werden mit dem $G+g+$-Konformer von (+)-2Butanol gebildet (Abb. 6.12), die wegen ihrer besonders niedrigen OH-Streckschwingungswellenzahlen spektral abgesetzt erscheinen sollten (die intensivsten braunen Signale in den Strichspektren in Abb. 6.13) und daher eindeutiger zugeordnet werden können. Tatsächlich findet sich jeweils nahe an den erwarteten Position für beide Enantiomerenkombinationen eine Bande passender relativer Intensität. Die Verschiebung von $1 \mathrm{~cm}^{-1}$ bei Wechsel der relativen Chiralität entspricht gut der berechneten von $3 \mathrm{~cm}^{-1}$. Im freien Alkohol ist das $G+g+$-Konformer nur die drittstabilste Konformation und konnte dort schwingungsspektroskopisch in den Jet-Spektren nicht nachgewiesen werden (siehe Abb. 5.45 auf S. 170). Die vermutete niederfrequente Position wird hier durch entsprechende niederfrequente Banden im Dimer gestützt. Auch in Kombination mit beiden LimonenEnantiomeren [237] und bei der Selbstdiskriminierung von 2-Butanol finden sich entsprechend abgesetzte Banden, für die analoge Zuordnungen vermutet werden können.

Mit einfachen sterischen Betrachtungen kann nachvollzogen werden, warum mit dem $G+t$-Konformer eine spektrale Chiralitätsdiskriminierung vorhergesagt wird, mit dem $G+g+$-Konformer dagegen nicht. Ein Vergleich der beide Dimere von $G+g+$ mit den unterschiedlichen $\alpha$-Pinen-Enantiomeren in Abb. 6.12 zeigt, dass die Strukturen im Wesentlichen durch Inversion der Konfiguration von 2-Butanol durch gedachte Vertauschung von Methyl- und Ethylgruppe ineinander überführt werden können. Dabei treten nur geringe Änderungen in der lokalen Umgebung der Hydroxygruppe und damit deren Streckschwingungswellenzahl auf. Dagegen führt für die Dimere mit $G+t$ eine solche Vertauschung jeweils zu einem sterischen Konflikt mit $\alpha$-Pinen, welcher im Komplex eine Torsion um die Wasserstoffbrücke und damit die spektrale Chiralitätsdiskriminierung bewirkt.

Wird die gesamte Modellierung mit dem Experiment verglichen, so stehen für die Kombination mit entgegengesetzten Vorzeichen sowohl die relativen Bandenpositionen wie auch die Intensitäten in guter Übereinstimmung mit dem Experiment. Für die Kombination mit gleichen Vorzeichen werden die Bandenpositionen ebenfalls gut wiedergegeben, allerdings liegen hier größere Diskrepanzen bei den relativen Intensitäten vor. Die Simulationen für beide Kombinationen mit unterschiedlicher relativer Chiralität ähneln sich insgesamt stark und können damit die prägnanten experimentellen Intensitätsunterschiede nicht wiedergeben.

Eine mögliche Ursache für das Versagen des Modells hinsichtlich dieses Aspektes könnte die getroffene Annahme bezüglich des bereits bei Zimmertemperatur eingefro- 


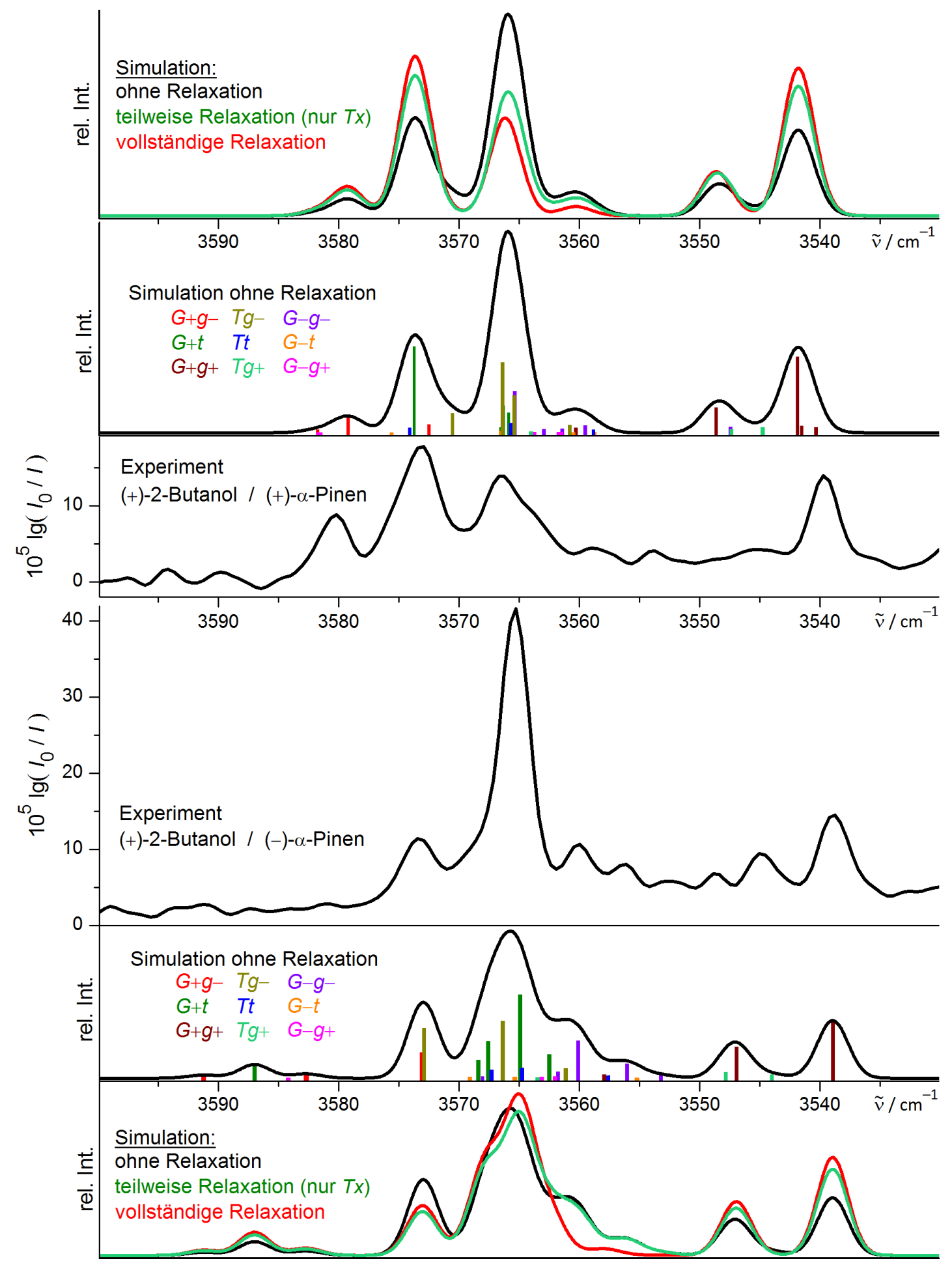

Abb. 6.13: Simulierte und experimentelle Spektren von Dimeren aus (+)-2-Butanol und (+)- $\alpha-$ Pinen (obere Hälfte) bzw. (-)- $\alpha$-Pinen (untere Hälfte). Die Filet-Jet-Spektren im Zentrum der Abbildung wurden mit verdünnten 1:1-Mischungen der beiden Verbindungen aufgenommen. Darüber bzw. darunter befinden sich Simulationen auf Basis von B3LYP-D3(BJ)/may-cc-pVTZ mit der Annahme, dass der OCCC-Torsionwinkel von 2-Butanol seine berechnete Verteilung bei Zimmertemperatur beibehält. Weitere Modellierungen in grün und rot weichen diese Einschränkung schrittweise auf. Sonstige Simulationsparameter sind einheitliche Verschiebung der harmonischen Wellenzahlen um $-141 \mathrm{~cm}^{-1}$, eine Konformationstemperatur von $64 \mathrm{~K}$ und Gauss-Profile mit einer Halbwertsbreite von $3 \mathrm{~cm}^{-1}$. 
renen OCCC-Torsionwinkel von 2-Butanol sein. Um dies zu untersuchen, wurde die Modellierung mit zwei modifizierten Annahmen wiederholt. Die erste lässt eine Gleichgewichtseinstellung bei niedriger Temperatur zwischen den $T x$ - und den $G+x$-Konformeren von (+)-2-Butanol zu, da hierfür im freien Alkohol geringere Barrieren gefunden wurden als für die Relaxation der $G-x$-Konformere (siehe Abb. 5.40 auf S. 163). In der zweiten Variante wird eine Gleichgewichtseinstellung sämtlicher Konformere bei niedriger Konformationstemperatur modelliert. Eine solche einheitliche Konformationstemperatur trotz deutlich unterschiedlicher Barrieren ist in diesem Fall als Vereinfachung zu werten, um qualitativ die resultierenden spektralen Auswirkungen zu untersuchen. Diese drei Modellierungen sind in im oberen bzw. unteren Teil von Abb. 6.13 verglichen. Für die Dimere mit gleichen Vorzeichen wird durch die zusätzliche Relaxation eine deutliche Annäherung an die experimentellen relativen Bandenintensitäten gefunden. Diese liegt darin begründet, dass die ohne Relaxation am intensivsten modellierte Bande bei $3566 \mathrm{~cm}^{-1}$ maßgeblich auf eine Metastabilität von höherenergetischen Konformere begründet ist. Für die Kombination mit entgegengesetzten Vorzeichen bleibt eine Bande in (zufällig) gleicher Postion auch bei zugelassener Relaxation dagegen die intensivste und gewinnt gegenüber der bei $3573 \mathrm{~cm}^{-1}$ sogar an relativer Intensität. Die experimentellen Spektren beider Kombinationen werden daher tendenziell besser beschrieben, wenn in der Modellierung zumindest eine teilweise Relaxation für $T x$-Konformere angenommen wird.

Verschiedene Erklärungsansätze können diskutiert werden, warum 2-Butanol im Komplex mit $\alpha$-Pinen einfacher relaxieren könnte, als im freien Molekül:

- Barrierenhöhe: Die Barrieren könnten erniedrigt sein, allerdings erscheint ein gröBerer Effekt durch die eher schwache Wechselwirkung der Ethylgruppe von 2Butanol mit $\alpha$-Pinen eher unwahrscheinlich. Eine Testrechnung für einen einzelnen Umwandlungspfad ergab für die direkte Isomerisierung von $T g-\mathrm{zu} \mathrm{G+g-}$ eine leicht erniedrigte Barriere von $11 \mathrm{~kJ} \mathrm{~mol}^{-1}$ im Komplex, im Vergleich $\mathrm{zu}$ $14 \mathrm{~kJ} \mathrm{~mol}^{-1}$ im freien Alkohol.

- Triebkraft: Gegen eine erhöhte Triebkraft für die Relaxation spricht, dass die berechneten Energieabstände zwischen den 2-Butanol-Konformeren in den DimerKonformeren zumindest hinsichtlich des OCCC-Torsionswinkels ähnlich bleiben.

- Solvent-assisted conformational isomerization: Bei der Bildung von Dimeren wird die Bindungsenergie frei, die zusätzlich für die Isomerisierung zur Verfügung steht. Wird ein Teil nicht direkt in einem Drei-Teilchen-Stoß wieder abgeführt, dissoziert der Komplex wieder.[20] Die hier berechneten Bindungsenergien (relativ zu den jeweils beteiligten Konformeren) sind mit einem Betrag von etwa $21 \mathrm{~kJ} \mathrm{~mol}^{-1}$ größer als die Isomerisierungsbarrieren von etwa $12 \mathrm{~kJ} \mathrm{~mol}^{-1}$ für $T x$ und etwa $17 \mathrm{~kJ} \mathrm{~mol}^{-1}$ für $G-x$. Auch bei einem aus kalten Monomeren gebildetes Dimer wäre damit prinzipiell genug Energie vorhanden, um diese Barrieren zu überwinden. Dieser Relaxationseffekt ähnelt den bereits diskutierten mit polarisierbaren Trägergasbeimischungen wie Argon, und wurde mit verschiedenen Bindungspartnern als Solventassisted conformational isomerization systematisch studiert.[24, 326] Im Vergleich zu den Spektren der reinen 2-Butanol Expansionen wird aber keine signifikante Veränderung im Monomer-Bereich beobachtet. Dies wäre zu erwarten, falls relaxierte Monomeren aus nur temporär gebildeten Komplexen wieder freigesetzt werden. 
Eine erste mögliche Ursache hierfür wäre, dass der Effekt auch ohne $\alpha$-Pinen durch die Bildung von 2-Butanol Dimeren auftritt. Dies würde aber dem Befund in Abb. 5.45 auf S. 170 widersprechen, wonach das Monomer-Spektrum besser durch die Metastabilität, statt Relaxation, hochenergetischer Konformere modelliert werden kann. Ein zweiter Erklärungsansatz wäre, dass die Isomerisierung in diesen Komplexen deutlich langsamer verläuft als die Dissoziation, und daher hauptsächlich 2Butanol Molekülen betrifft, die bereits teilweise abgekühlt und damit im Komplex gefangen sind. Die aus der internen Relaxation von $T x$-Konformeren freigesetzte Energie ist mit etwa 1.5 bis $3 \mathrm{~kJ} \mathrm{~mol}^{-1}$ relativ gering gegenüber der Dissoziationsenergie.

Sicherlich ist das System aus 2-Butanol und $\alpha$-Pinen mit seiner vielfältigen intra- und intermolekularen Konformationsisomerie zu komplex, um solche vielschichtigen Relaxationseffekte eindeutig beurteilen zu können. Eine Vereinfachung könnte in Zukunft mit der Untersuchung des Komplexes aus $n$-Propanol und Ethylen erreicht werden. Dieses System ist der Prototyp für eine Koordination eines Alkens mit einem Alkohol, der $\mathrm{C}-\mathrm{C}-$ Torsionsisomerie mit hohen Barrieren aufweist. In diesem System wären die unterscheidbaren Alkohol-Konformere auf fünf reduziert und sehr wahrscheinlich wäre auch die Isomerie bezüglich der Torsion um die Wasserstoffbrücke stark vereinfacht. Durch Substitution von Ethylen könnte über induktive und dispersive Effekte die Bindungsenergie variiert und somit deren Einfluss systematisch untersucht werden.

\subsection{Fazit}

Zusammengefasst kann festgestellt werden, dass die molekularen Ursachen der für einige Alkohole beobachteten Chiralitätsdiskriminierung mit $\alpha$-Pinen weitgehend mit B3LYPD3(BJ) verstanden werden können. Die von der Methode vorhergesagten Unterschiede in den $\mathrm{OH}-$ Streckschwingungswellenzahlen stehen in sehr guter Übereinstimmung mit beobachteten spektralen Bandenverschiebungen. Für ein signifikantes Defizit der Methode durch die Nichtberücksichtigung von Chiralitätsinduzierter Spinselektivität gibt es damit zumindest für diese Systeme keine Hinweise. Auch für den besonders komplizierten Fall der Diskriminierung mit 2-Butanol können für die wichtigsten Signale plausible Vorschläge gemacht werden, auch wenn Unklarheiten bezüglich der Population der Konformere und damit der Bandenintensitäten verbleiben. Der Vergleich über fünf verschiedene Systeme hinweg wurde genutzt, um die Wahrscheinlichkeit zu reduzieren, dass es sich dabei nur um zufällige Übereinstimmungen handelt. Die drei anderen getesteten Methoden ( $\omega$ B97X-D, M06-2X und AM1) konnten dagegen die spektralen Unterschiede nur für einzelne, aber nicht konsistent für mehrere Systeme vorhersagen. 


\section{Selbstdiskriminierung von $\alpha$-Fenchol}

\subsection{Einleitung}

Bei der Untersuchung des Fenchol-Monomers in Kap. 5.8 und dessen Komplex mit $\alpha$ Pinen in Kap. 6 wurden weitere Banden beobachtet, die aufgrund ihrer spektralen Position und Konzentrationsabhängkeit Dimeren von $\alpha$-Fenchol zugeordnet werden. Diese werden in diesem Kapitel näher analysiert. Die quantenchemische Betrachtung von Dimeren aus bicyclischen Monoterpen-Alkoholen ist nicht nur wegen deren molekularer Größe anspruchsvoll, sondern auch vor allem wegen der auftretenden intra- und intermolekularen Konformationsvielfalt und der hohen Bedeutung der Dispersionswechselwirkung.

\subsection{Quantenchemische Rechnungen}

$\alpha$-Fenchol besitzt, ähnlich wie Ethanol (Kap. 4), drei Konformere bezüglich der HOCHTorsion: $g-, g+$ und $t$ (Kap. 5.8). Die, im Gegensatz zu Ethanol, permanente Chiralität des Alkohols führt zu einer größeren Zahl an möglichen Dimer-Strukturen, die als homooder heterochiral eingeteilt werden können. Eine Umwandlung zwischen diesen beiden Klassen ist nicht möglich. Die beiden freien Elektronenpaare sind diastereotop, sodass deren jeweilige Koordination zu weiteren diastereomeren Strukturen führt (statt teilweise zu enantiomeren bei Ethanol). Zusätzlich wechselwirken die wesentlich größeren Kohlenwasserstoffreste stärker miteinander, was bezüglich der Torsion um die Wasserstoffbrücke zu weiteren Minima führt. Unter Berücksichtigung dieser konformationellen Freiheitsgrade wurden auf B3LYP-D3(BJ)/def2-TZVP-Niveau insgesamt 51 homochirale und 39 heterochirale Minima innerhalb einer Energiespanne von $11 \mathrm{~kJ} \mathrm{~mol}^{-1}$ gefunden, was eine erhebliche Erweiterung zu den insgesamt 20 gefundenen Ethanol-Dimeren innerhalb von $3 \mathrm{~kJ} \mathrm{~mol}^{-1}$ darstellt. Strukturen innerhalb eines Bereiches von $2 \mathrm{~kJ} \mathrm{~mol}^{-1}$ relativ zum homo- bzw. heterochiralen globalen Minimum wurden auf B3LYP-D3(BJ)/may-ccpVTZ-Niveau nachoptimiert, die nachfolgend für die Modellierung der Spektren verwendet werden. Diese Konformere sind in Tab. 7.1 gelistet. Der Donor ist dabei stets als $(-)$ - $\alpha$-Fenchol festgelegt. Sowohl das homo- wie auch das heterochirale globale Minimum (Abb. 7.1) werden aus einem $g+$-Konformer als Donor und einem $t$-Konformer als Akzeptor gebildet. Die Präferenz für das $g+-$ Konformer in der Donor-Rolle wurde bereits in Kap. 6 im Komplex mit den beiden $\alpha$-Pinen-Enantiomeren gefunden. In der Akzeptor-Rolle agiert das $t$-Konformer auch im Komplex mit Wasser [405]. Für die globalen Minima der drei Systeme, in denen $\alpha$-Fenchol als Akzeptor agiert, wurde zudem eine übereinstimmende Präferenz für eines der beiden Elektronenpaare gefunden.

Herausstechend ist die besondere Stabilität des homochiralen globalen Minimums. Diese Struktur ist um $1.5 \mathrm{~kJ} \mathrm{~mol}^{-1}$ stabiler als das zweite homochirale Konformer (Tab. 7.1) und sogar um $1.7 \mathrm{~kJ} \mathrm{~mol}^{-1}$ gegenüber dem heterochiralen globalen Minimum. Zu- 


\section{Selbstdiskriminierung von $\alpha$-Fenchol}

sammen mit der vorhergesagten Differenz in der OH-Streckschwingung des Donors von $19 \mathrm{~cm}^{-1}$ ist dies die größte Chiralitätserkennung für in dieser Arbeit untersuchten Systeme.

Ein interessantes strukturelles Detail des homochiralen globalen Minimums ist, dass das Gerüst ohne die beiden Hydroxyprotonen nahezu $C_{2}$-symmetrisch ist. Möglicherweise könnten daher Donor- und Akzeptor (fast) nur durch Bewegung der beiden Hydroxyprotonen ihre Rolle vertauschen, analog zu den intermolekularen Dynamiken der Wasser[406] und Halogenwasserstoff-Dimere [275, 284, 286, 288] sowie zu den intramolekula-

Tab. 7.1: Schwingungsnullpunktskorrigierte Energien von $\alpha$-Fenchol-Dimeren auf B3LYP$\mathrm{D} 3(\mathrm{BJ}) /$ may-cc-pVTZ-Niveau innerhalb von $2 \mathrm{~kJ} \mathrm{~mol}^{-1}$, jeweils relativ zum homochiralen bzw. heterochiralen globalen Minimum. Das heterochirale globale Minimum liegt energetisch $1.7 \mathrm{~kJ} \mathrm{~mol}^{-1}$ oberhalb des homochiralen. Die HOCH-Torsionwinkel und damit die beteiligten Monomer-Konformere sind qualitativ $(g-, g+, t)$ in der Reihenfolge Donor-Akzeptor angegeben. Der Donor ist stets $(-)-\alpha$-Fenchol.

\begin{tabular}{cc|cc}
\hline hom-Konformer & $E_{0}^{\mathrm{rel}} / \mathrm{kJ} \mathrm{mol}^{-1}$ & het-Konformer & $E_{0}^{\mathrm{rel}} / \mathrm{kJ} \mathrm{mol}^{-1}$ \\
\hline$g+t$ & 0 & $g+t$ & 0 \\
$g-t$ & 1.5 & $g-t$ & 0.3 \\
$g+g-$ & 1.8 & $g+t$ & 0.4 \\
$g+g+$ & 1.9 & $t t$ & 0.9 \\
& & $g-g-$ & 1.0 \\
& & $g-g+$ & 1.2 \\
& & $g+g-$ & 1.5 \\
& & $g+t$ & 1.5 \\
& & $g+g-$ & 1.8 \\
\hline
\end{tabular}
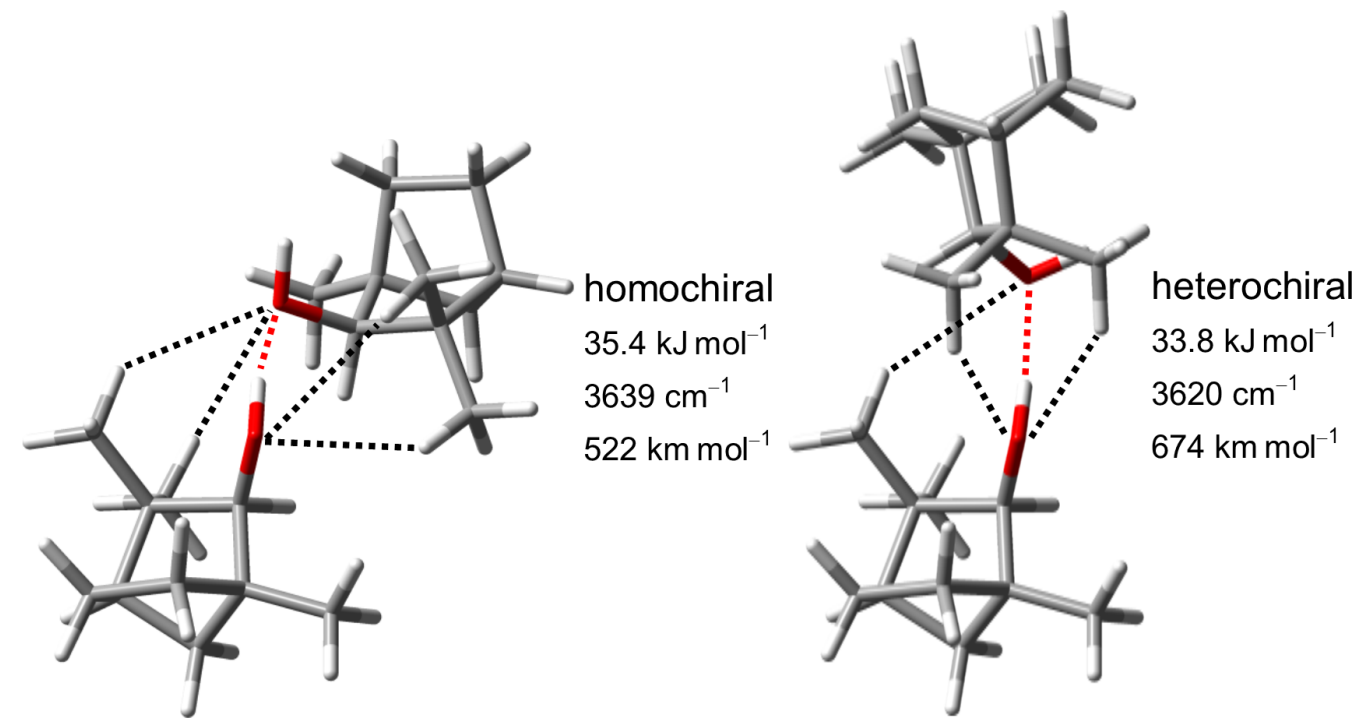

Abb. 7.1: Strukturen, Dissoziationsenergien (relativ zu vollständig relaxierten Monomeren), Donor-OH-Streckschwingungswellenzahlen und zugehörige IR-Aktivitäten für die homo- und heterochiralen globalen Minima des $\alpha$-Fenchol-Dimers. OH ‥O-Wasserstoffbrücken sind mit roten, intermolekulare $\mathrm{CH} \cdots \mathrm{O}$-Kontakte mit Abständen $<3.0 \AA$ A mit schwarzen gestrichelten Linien hervorgehoben. 
ren Entsprechungen in Diolen [407-410]. Abhängig davon, inwieweit dabei weitere Atome ihre Lager ändern, wäre hierfür eine (mit hochauflösenden Methoden) nachweisbare Tunnelaufspaltung denkbar. Fehlende Symmetrie verhindert dagegen beispielsweise im Methanol-Dimer die Tunneldynamik.[138] Für den zugehörigen $C_{2}$-symmetrischen Übergangszustand des $\alpha$-Fenchol-Dimers wird eine voll-schwingungsnullpunktskorrigierte relative Energie von $10 \mathrm{~kJ} \mathrm{~mol}^{-1}$ berechnet. Tatsächlich zeigen unvollständige rotationsspektroskopische Analysen des Dimers eine ungewöhnliche Aufspaltung.[411, 412] Da das Dimer insgesamt sechs Methylgruppen aufweist, wäre auch ein Tunnelprozess im Zusammenhang mit deren Torsion denkbar. Allerdings zeigt das Monomer keine solche Aufspaltung.[262]

\subsection{Schwingungsspektren}

Die Vorhersage einer besonders stabilen homochiralen Dimer-Konformation stimmt insofern mit dem Spektrum der Expansion von (weitgehend) enantiomerenreinem (+)- $\alpha$ Fenchol in Abb. 7.2 überein, dass dieses im Dimerbereich von einer einzelnen Bande bei $3499 \mathrm{~cm}^{-1}$ dominiert wird.

Aufgrund des Fehlens einer besonders stabilen heterochiralen Struktur sollte sich im Spektrum der heterochiralen Dimere die Intensität stärker auf mehrere Banden aufteilen. Allerdings ist weder (-)- $\alpha$-Fenchol noch die racemische Mischung kommerziell verfügbar, sodass diese Vorhersage nicht ohne synthetischen Aufwand überprüft werden kann. $(-)-\alpha$-Fenchol könnte hierfür durch Reduktion von (+)-Fenchon hergestellt werden [413416].

Für die weiteren schwachen Banden im Spektrum der Expansion von (+)- $\alpha$-Fenchol ist uneindeutig, ob es sich um höherenergetische homochirale Konformere, oder um andere Dimere unter Beteiligung von (enantiomeren) Verunreinigungen handelt. Gegen einen größeren Cluster spricht die gleiche Skalierung der Banden mit dem Stagnationsdruck der Expansion. Zugabe von Wasser zur Probe führte zu keiner Verstärkung der Banden. Ein gemischtes Dimer aus $\alpha$ - und $\beta$-Fenchol ist dagegen nicht völlig auszuschließen.

Der Vergleich mit den spektralen Vorhersagen in Abb. 7.2 lässt vermuten, dass für die niederfrequente Bande bei $3485 \mathrm{~cm}^{-1}$ eher ein heterochirales, als ein homochirales, Dimer verantwortlich sein könnte. Das Analysenzertifikat der verwendeten Probe gibt für die eingesetzte Probe eine Diastereomerenreinheit von 98.5\% und einen spezifischen Drehwinkel von $+10.3^{\circ} \frac{\mathrm{ml}}{\mathrm{gdm}}$ in Ethanol an. Gemäß den Betrachtungen in Kap. 3.5 lässt sich daraus ein Enantiomerenüberschuss in einer breiten Spannweite zwischen etwa 77 und 99\% abschätzen, je nachdem welcher Literaturwert als Referenz verwendet wird (und unter der Annahme, dass diastereomere Verunreinigungen nicht ebenfalls optisch aktiv sind). Dies entspricht einem möglichen Anteil von heterochiralen Clustern bei statistischer Bildung von bis zu $20 \%$.

Eine Beobachtung in dieser Arbeit spricht allerdings dagegen, dass ein signifikanter Anteil von (-)- $\alpha$-Fenchol in der Probe vorliegt. Im Spektrum der Kombination von (+)$\alpha$-Fenchol mit (-)- $\alpha$-Pinen deutet sich die Bande $b$ nur schwach an, die aus einer wesentlichen Verunreinigung mit (-)- $\alpha$-Fenchol zu erwarten wäre (Abb. 6.3 auf S. 198). Dies gilt, obwohl für das eingesetzte (-)- $\alpha$-Pinen nur ein begrenzter Enantiomerenüberschuss von $87 \%$ vom Hersteller angegeben wurde, sodass auch der dazu enantiomere Komplex 


\section{Selbstdiskriminierung von $\alpha$-Fenchol}

aus $(+)$ - $\alpha$-Fenchol und (+)- $\alpha$-Pinen zur Bande beiträgt. Im Gegentest mit racemischen $\alpha$ Pinen ist die Bande dagegen sehr deutlich vorhanden (Abb. A.4 auf S. 315 im Anhang). Die Zuordnung der Nebenbanden im Spektrum von $(+)-\alpha$-Fenchol bleibt damit unklar.

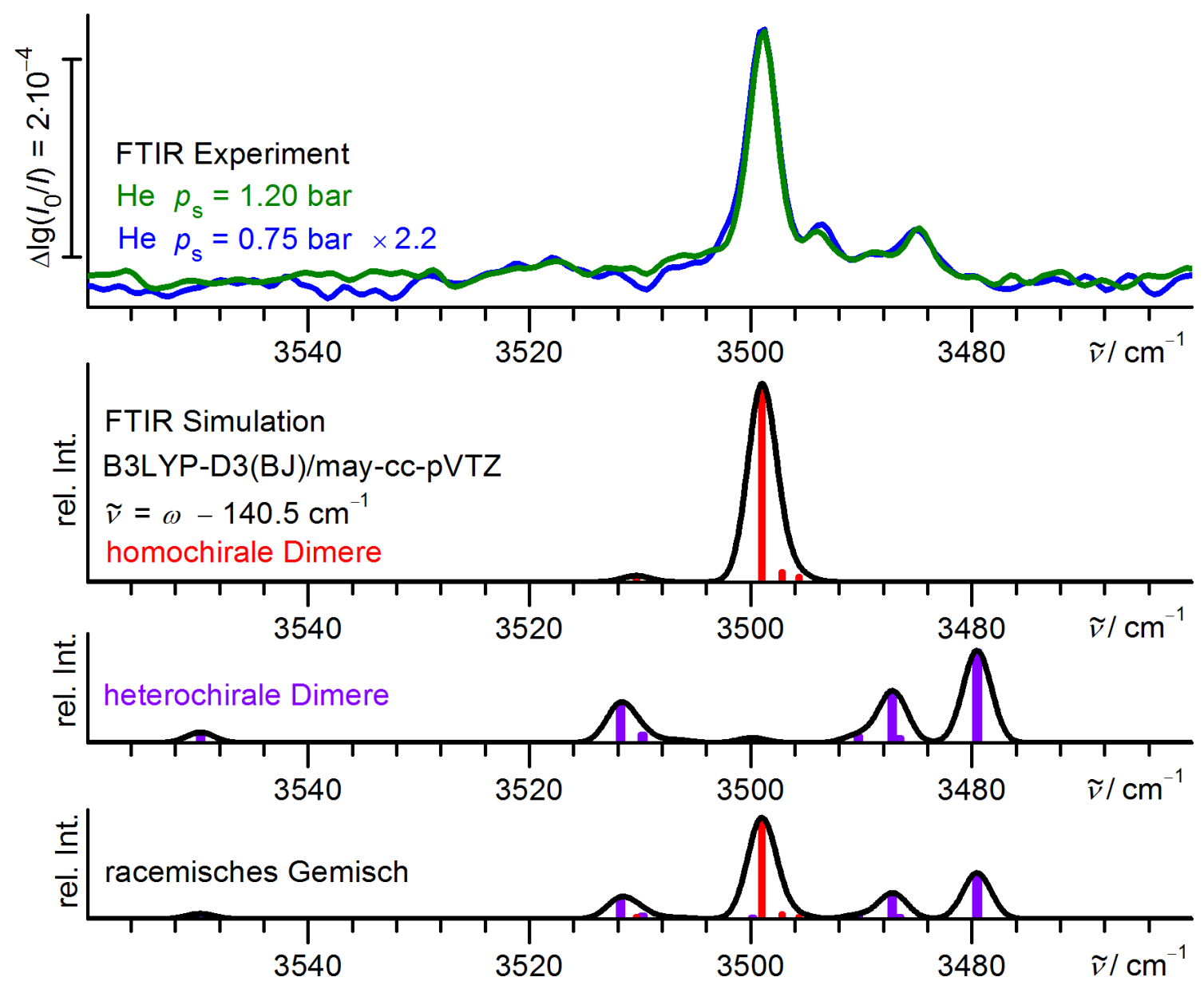

Abb. 7.2: Experimentelle Filet-Jet-Spektren von (+)- $\alpha$-Fenchol bei zwei unterschiedlichen Stagnationsdrücken im Vergleich mit Simulationen auf B3LYP-D3(BJ)/may-cc-pVTZ-Niveau für homochirale und heterochirale Dimere sowie dem racemischen Gemisch. Simulationsparameter sind einheitliche Verschiebung der harmonischen Wellenzahlen um $-140.5 \mathrm{~cm}^{-1}$, eine Konformationstemperatur von $64 \mathrm{~K}$, Gauss-Profile mit einer Halbwertsbreite von $3 \mathrm{~cm}^{-1}$ sowie die Annahme, dass die Gesamtkonzentration der gebildeten Dimere unabhängig von der enantiomeren Zusammensetzung ist. Berücksichtigt wurden nur Strukturen innerhalb von $2 \mathrm{~kJ} \mathrm{~mol}^{-1}$. 


\section{Komplementarität von Chiralitätssynchronisation und Chiralitätsdiskriminierung in Dimeren von Benzylalkohol und 1-Phenylethanol}

\subsection{Vorwort}

Die FTIR-Messungen zu 1-Phenylethanol in diesem Kapitel wurden zusammen mit Caroline Stelbrink als Teil ihrer Bachelorarbeit [367] aufgenommen, die Raman-Spektren zu Benzylalkohol und 1-Phenylethanol zusammen mit Enno Meyer im Rahmen eines PCForschungspraktikums.

\subsection{Einleitung}

Ein transient chirales Molekül kann sich bei Dimerisierung der Chiralität eines Wechselwirkungspartners anpassen. Abhängig davon, ob der Partner ebenfalls transient chiral oder permanent chiral ist, wird dies als Chiralitätssynchronisation bzw. Chiralitätsinduktion bezeichnet. Bei ausreichend niedrigen Barrieren kann sich ein Gleichgewicht zwischen homo- und heterochiralen Anordnungen ausbilden, dessen Lage Aufschluss geben kann über die chirodiastaltische Energie als Triebkraft dieser Anpassung. Bei der Untersuchung solcher Gleichgewichte kann als Schwierigkeit auftreten, dass die spektralen Signaturen nicht immer eindeutig zugeordnet werden können. Denn die häufig subtilen strukturellen Unterschiede zwischen homo- und heterochiralen Strukturen manifestieren sich in ebenso geringen spektralen und energetischen Differenzen. Deren korrekte theoretische Vorhersage stellt auch für moderne quantenchemische Methoden eine Herausforderung dar, wie in Kap. 4.3 für Ethanol-Dimere und in Kap. 6 für gemischte Dimere von Alkoholen mit $\alpha$-Pinen deutlich wurde.

Bei der Chiralitätsdiskriminierung zwischen permanent chiralen Molekülen ist dagegen keine Anpassung möglich, sodass in der Regel eine weitgehend statistische Bildung homo- und heterochiraler Komplexe resultiert. Über die Wahl der eingesetzten Enantiomere können daher die Anteile der gebildeten Dimere gesteuert werden. Die resultierenden spektralen Intensitätsänderungen geben Aufschluss über die Zuordnung von Banden zu homo- und heterochiralen Komplexen. Dagegen werden keine Informationen über die relative Stabilität der nebeneinander vorliegenden Diastereomere unterschiedlicher relativer Chiralität erhalten. 


\section{Komplementarität in Dimeren von Benzylalkohol und 1-Phenylethanol}

Durch Betrachtung der Chiralitätsdiskriminierung auf der einen, und der Chiralitätssynchronisation und/oder -induktion auf der anderen Seite, können zueinander komplementäre Informationen erhalten und kombiniert werden. Voraussetzung hierfür ist allerdings, dass die betrachteten Wechselwirkungen zwischen den beteiligten Molekülen tatsächlich vergleichbar sind. Die Überführung eines transient chiralen in ein permanent chirales Molekül erfordert eine Modifikation der chemischen Struktur, woraus sich aber stets auch weitere Implikationen ergeben, die beim Vergleich berücksichtigt werden müssen.

Parallel zu der vorliegenden Arbeit wurde ein solcher Vergleich für die Dimerisierung von 1,2-Ethandiol (Ethylenglycol) und trans-1,2-Cyclohexandiol durch Hartwig et al. [27] erarbeitet. Die (bei Zimmertemperatur) transiente Chiralität von 1,2-Ethandiol bezüglich des OCCO-Torsionswinkels wird in trans-1,2-Cyclohexandiol durch den Ringschluss fixiert. Da die Monomere in den Dimerstrukturen relativ stark deformiert werden, kommt die unterschiedliche Flexibilität der beiden Moleküle zum Tragen. Die Erweiterung um je eine $\mathrm{C}_{4} \mathrm{H}_{6}$-Einheit in beiden Molekülen erhöht zudem, über dispersive und induktive Effekte, die Wechselwirkung zwischen den Monomeren. Dies führt zu begrenzten Änderungen in der Energieabfolge der Dimerkonformere, laut den Rechnungen bleibt aber die sehr hohe chirodiastaltische Energie von etwa $7 \mathrm{~kJ} \mathrm{~mol}^{-1}$ bestehen. Als Ergebnis dieser Studie wurde erhalten, dass die Dimerisierung von 1,2-Ethandiol in einer Überschallexpansion nicht wie vorher angenommen [174] als Synchronisation, sondern als Diskriminierung mit weitgehend statistischer Bildung zu beschreiben ist. Der Grund hierfür sind die hohen Racemisierungbarrieren, sowohl im Monomer $\left(20 \mathrm{~kJ} \mathrm{~mol}^{-1}\right)$, wie auch im Dimer $\left(14 \mathrm{~kJ} \mathrm{~mol}^{-1}\right)$ [174]. Diese können bei Zimmertemperatur, jedoch nicht unter Jet-Bedingungen überwunden werden, sodass 1,2-Ethandiol sich unter letzteren effektiv wie ein racemisches Gemisch einer permanent chiralen Verbindung verhält. Mit verbesserten Aufnahmebedingungen und dem Vergleich mit trans-1,2-Cyclohexandiol konnte die zuvor gefundene [174] scheinbare Abwesenheit von homochiralen Strukturen aufgeklärt werden. Diese beruhte nicht auf Relaxation in das heterochirale globale Minimum über andere Mechanismen (z. B. transiente Trimere [174]), sondern auf einer stärkeren Verteilung der Intensität auf Banden von mehreren homochiralen Konformeren. Durch die weitgehend statistische Bildung konnten keine experimentellen Informationen über die chirodiastaltische Energie gewonnen werden, auch wenn die theoretische Vorhersage indirekt durch die gute Performance von B3LYP-D3(abc,BJ)/def2-QZVP für andere Messgrößen gestützt wird.

Das in diesem Kapitel behandelte Molekül-Paar Benzylalkohol/1-Phenylethanol scheint für den Vergleich von Chiralitätssynchronisation bzw. -induktion mit Chiralitätsdiskriminierung gute Voraussetzungen aufzuweisen. So ist die berechnete Racemisierungbarriere im monomeren Benzylalkohol mit $2 \mathrm{~kJ} \mathrm{~mol}^{-1}$ um einen Faktor 10 geringer als in 1,2-Ethandiol. Die permanente Chiralität wird durch die Einführung einer einzelnen Methylgruppe erreicht, sodass die Wechselwirkung in den Dimeren nur begrenzt beeinflusst wird. Im Gegensatz zu Ethanol (Kap. 4), 2-Butanol (Kap. 5.15) und $\alpha$-Fenchol (Kap. 7 und 5.8) sind in Benzylalkohol und 1-Phenylethanol keine weiteren Konformere energetisch konkurrenzfähig, sodass die Konformationsvielfalt der Selbstdiskriminierung durch inter- statt intramolekulare Isomerie geprägt ist. Ähnlich wie für die Diole wird ein en- 
ger Kontakt über mehrere Wasserstoffbrücken, aber zusätzlich auch über substantielle Dispersionswechselwirkung zwischen den gut polarisierbaren und kompakten Phenylringen [384], hergestellt. Durch unterschiedliche Ausrichtung der Moleküle zueinander werden dabei verschiedene Strukturmotive erhalten. Die dabei auftretenden Konkurrenzen zwischen jeweils: Wasserstoffbrücken und Dispersionswechselwirkung, $\mathrm{OH} \cdots \mathrm{O}$ - und $\mathrm{OH} \cdots \pi$-Wasserstoffbrücken, sowie kooperativen und isolierten Anordnungen von Wasserstoffbrücken, stellen besonders hohe Anforderungen an quantenchemische Methoden.

\subsection{Chiralitätssynchronisation in Benzylalkohol-Dimeren}

\subsubsection{Einleitung}

Aufbauend auf der Charakterisierung des Monomers in Kap. 5.16, werden in diesem Abschnitt Dimere von Benzylalkohol untersucht, ergänzt um einen Ausblick auf gröBere Cluster. Einen ersten Einblick in die Aggregation von Benzylalkohol bietet dessen Kristallstruktur. In dieser bilden sich über kooperative $\mathrm{OH} \cdots \mathrm{O}$-Wasserstoffbrücken und $\left(\mathrm{sp}^{3}\right) \mathrm{C}-\mathrm{H} \cdots \pi$-Kontakte unendliche Ketten aus [344], wobei alle Moleküle homochiral synchronisiert sind. Für Thiobenzylalkohol wird dagegen ein heterochirales dimeres Motiv mit zwei nicht-kooperativen $\mathrm{SH} \cdots \pi$-Kontakten gefunden,[344] dessen Einheiten untereinander über $\left(\mathrm{sp}^{3}\right) \mathrm{C}-\mathrm{H} \cdots \pi$-Kontakte verknüpft sind.

Dimere von Benzylalkohol wurde bereits 1940 in verdünnter Tetrachlormethan-Lösung untersucht.[345] Diesen wurde im OH-Streckschwingungsbereich zwei Banden zugeordnet, die jeweils einem gebundenen und einem freien Hydroxyoszillator zugeschrieben wurden.

Ein Benzylalkohol-Dimer in einer Überschallexpansion wurden vermutlich das erste Mal von Guchhait et al. [218, 219] 1999 beobachtet, dessen Bande bei $3585 \mathrm{~cm}^{-1}$ aber noch fälschlich einem Benzylalkohol-Monomer zugeordnet wurde. Dies wurde ein Jahr später von Mons et al. [220] revidiert und die Bande stattdessen einem Hydroxyoszillator in einer intermolekularen $\mathrm{OH} \cdots \pi$-Wasserstoffbrücke zugeschrieben. Eine zweite Bande wurde bei $3515 \mathrm{~cm}^{-1}$ festgestellt, die gemäß der Konformerenselektivität der eingesetzten Doppelresonanzmethode von der selben Struktur verursacht wird. Die deutlich tiefere Wellenzahl dieser zweiten Bande deutet auf eine stärkere $\mathrm{OH} \cdots \mathrm{O}$-Wasserstoffbrücke hin. Auf HF/6-31G*-Niveau wurden von Mons et al. vier Dimer-Konformere gefunden, die jeweils diese beiden Arten von Wasserstoffbrücken (O $\pi$-Motiv) aufweisen. Die in der Publikation abgebildeten zwei stabilsten Strukturen sind jeweils heterochiral synchronisiert.

Dieses Thema wurde 2013 in größerer Tiefe von Oswald in seiner Bachelorarbeit [417] mittels FTIR-Spektroskopie am Popcorn-Jet aufgegriffen. Die Ergebnisse wurden in Folge zusammen mit Altnöder und Suhm publiziert [333], und fanden auch Eingang in die Dissertation von Altnöder [70]. Basierend auf ausgedehnten Rechnungen mit MP2 und verschiedenen dispersionskorrigierten Dichtefunktionalmethoden wurden die beiden benannten Banden der auf fast allen Niveaus stabilsten Struktur - einem homochiralen $\mathrm{O} \pi-$ Dimer - zugeordnet. Auffallend ist dabei aber, auch im Vergleich zu den Rechnungen von Mons et al., dass keine der in der Publikation und Dissertation diskutierten Konformere (in einer Spanne von etwa $6 \mathrm{~kJ} \mathrm{~mol}^{-1}$ ) ein heterochirales $\mathrm{O} \pi$-Motiv aufweist. Die ande- 


\section{Komplementarität in Dimeren von Benzylalkohol und 1-Phenylethanol}

ren diskutierten Motive treten dagegen als Paare verschiedener relativer Chiralität und ähnlicher Energie auf. In der Bachelorarbeit von Oswald wurde hingegen noch eine solche heterochirale $\mathrm{O} \pi$-Struktur (wenn auch dort noch nicht als solche klassifiziert) mit allen drei eingesetzten dispersionskorrigierten Dichtefunktionalmethoden als zweitstabilste Struktur gefunden, teils nur wenige Zehntel $\mathrm{kJ} \mathrm{mol}^{-1}$ oberhalb des homochiralen globalen Minimums. Diese heterochirale Struktur entspricht qualitativ der stabilsten Struktur von Mons et al. In der darauf aufbauenden Publikation und der Doktorarbeit von Altnöder wird sie aber überraschenderweise nicht mehr erwähnt. Durchsicht von Archivmaterial zeigt, dass die Struktur auf den MP2- und B2PLYP-D-Niveaus, auf die sich die beiden nachfolgenden Arbeiten wesentlich stützen, nicht mehr nachoptimiert wurde. Der Grund hierfür ist unklar. Die beiden Strukturen wurden einerseits spektral ähnlich genug vorhergesagt, dass eine eindeutige Zuordnung zu den Banden nur auf Grundlage der vorhergesagten Verschiebungen relativ zum Monomer nicht möglich ist, andererseits aber auch nicht derart ähnlich, dass eine Koinzidenz in beiden Banden naheliegend wäre.

Eine dritte, neu beobachtete Bande bei $3598 \mathrm{~cm}^{-1}$ wurde von Oswald und Altnöder der antisymmetrischen Schwingung eines symmetrischen Dimers mit zwei isolierten (nichtkooperativen) $\mathrm{OH} \cdots \pi$-Wasserstoffbrücken ( $\pi \pi$-Motiv) zugeschrieben. Dabei blieb die relative Chiralität der beiden Konstituenten offen, da sich für dieses Motiv die energetischen und spektralen Vorhersagen der homo- und heterochiralen Varianten noch stärker ähneln. Eine Reihe weiterer neu beobachteter Banden wurde auf Trimere und noch gröBere Cluster zurückgeführt.

Hinsichtlich der Chiralitätssynchronisation in Benzylalkohol-Dimeren lassen sich daher die folgenden offenen Fragen formulieren:

- Wie robust ist die Zuordnung der beiden intensivsten Banden zu einer homochiralen $\mathrm{O} \pi$-Struktur, angesichts der von Altnöder vernachlässigten heterochiralen Alternative?

- Wo befinden sich die Banden der O $\pi$-Struktur der anderen relativen Chiralität, wenn diese laut den Berechnungen von Oswald energetisch konkurrenzfähig sein soll?

- Wird im konkurrierenden $\pi \pi$-Motiv des Benzylalkohol-Dimers bevorzugt die gleiche oder die entgegengesetzte Chiralitätssynchronisation im Vergleich zum $\mathrm{O} \pi$ Motiv realisiert?

Diese Fragen werden in diesem Kapitel weitgehend beantwortet. Hierzu werden im Signal-zu-Rausch-Verhältnis verbesserte FTIR-Spektren, dazu teilweise komplementäre Raman-Spektren, und weitere Rechnungen, auch für Übergangszustände, herangezogen. Aufschlussreich ist auch der Vergleich mit den Dimeren des permanent chiralen 1Phenylethanol sowie mit gemischten Dimeren aus 1-Phenylethanol und Benzylalkohol.

Gemischte Dimere von Benzylalkohol wurden auch mit Wasser [219, 220], Ammoniak [418], Cyclohexylmethanol [333], und Methyllactat [341] untersucht. Mit den beiden erst genannten Molekülen wird ebenfalls bevorzugt ein $\mathrm{O} \pi$ bzw. $\mathrm{N} \pi$-Motiv ausgebildet. Mit Cyclohexylmethanol wurde stattdessen wahrscheinlich eine Struktur mit nur einer einzelnen Wasserstoffbrücke beobachtet, in der Benzylalkohol als Donor agiert. Bei Methyllactat insertiert Benzylalkohol hingegen vermutlich in dessen intramolekulare Wasserstoffbrücke für ein kooperatives Motiv mit zwei $\mathrm{OH}$...O-Wasserstoffbrücken. 


\subsubsection{Quantenchemische Rechnungen}

Die quantenchemische Suche nach Dimeren erfolgte auf B3LYP-D3(BJ)/def2-TZVPNiveau, deren Ergebnisse anschließend mit dem größeren Basissatz may-cc-pVTZ nachoptimiert wurden. Die wichtigsten Strukturen wurden auch mit den Funktionalen B2PLYP-D3(BJ), $\omega$ B97X-D und M06-2X - ebenfalls mit may-cc-pVTZ Basissatz nachoptimiert. Eine Gesamtübersicht über die erhaltenen relativen Energien und spektralen Verschiebungen findet sich im Anhang auf S. 324 in Tab. A.14 und A.15. Zusätzliche Nachoptimierungen auf B3LYP-D3(BJ)/aug-cc-pVTZ-Niveau ergaben erneut praktisch identische Ergebnisse wie mit may-cc-pVTZ hinsichtlich energetischer und spektraler Unterschiede zwischen den Strukturen.

Startpunkt für die Suche waren die Strukturen von Oswald und Altnöder, einschließlich von solchen im Archiv, die in den veröffentlichten Arbeiten nicht erwähnt werden. Diese wurden durch eigene Strukturideen ergänzt, sodass insgesamt 17 DimerKonformere erhalten wurden. Für die Klassifizierung wird die Nomenklatur von Altnöder [333] weitgehend übernommen und erweitert. Liegen beide Moleküle in der stabileren $g$ Konformation vor, so werden diese gemäß ihrer relativer Chiralität mit der Vorsilbe hom oder het versehen. Ist dagegen ein achirales $t$-Konformer enthalten, so werden die Dimere mit $g t$ oder $t g$, in der Reihenfolge der nachfolgend angegebenen WasserstoffbrückenAkzeptoren, benannt. Angegeben werden die Akzeptoren von Wasserstoffbrücken mit $\mathrm{O}$, $\pi$ und für freie Hydroxygruppen mit V (,Vakuum-Akzeptor"). Die beiden freien Elektronenpaare (E) des Sauerstoffatoms im $g$-Konformer sind diastereotop und lassen sich bezüglich des Torsionswinkels $\mathrm{EOC}_{\alpha} \mathrm{C}_{\beta}$ als gauche $\left(\mathrm{O}^{g}\right)$ bzw. trans $\left(\mathrm{O}^{t}\right)$ klassifizieren. Für die relative Stellung der Phenylringe werden Anordnungen gefunden, die grob den beiden stabilsten Benzol-Dimer-Konformeren [382] ähneln: parallel-versetzt oder T-förmig. Dimere mit letztgenannter Anordnung werden entsprechend mit einem T gekennzeichnet, ansonsten wird eine eher parallel-versetzte Anordnung impliziert. Falls eine von $C_{1}$ abweichende Punktgruppe vorliegt, wird diese in Tab. 8.1 mitangegeben, in der alle Dimere gelistet sind. Diese Nomenklatur ist nicht völlig ausreichend um auch für Dimere mit Beteiligung des $t$-Konformer komplett eindeutig zu sein, da in diesem die Elektronenpaare des Sauerstoffatoms enantiotop sind und damit bei deren Koordination durch ein transient chirales $g$-Konformer ebenfalls diastereomere Strukturen resultieren. Wegen der geringen Bedeutung des $t$-Konformers wird aber hierfür die Nomenklatur nicht weiter verkompliziert.

Die fünf stabilsten Dimer-Konformere, sowie das heterochirale Pendant zur fünft stabilsten Struktur, sind in Abb. 8.1 dargestellt. Dabei wird der Donor der $\mathrm{OH} \cdots \pi$ Wasserstoffbrücke stets in $g$--Konformation und gleich ausrichtet dargestellt, sodass die Unterschiede in der Wechselwirkung mit dem zweiten Molekül direkt ersichtlich sind. Fast alle Strukturmotive wurden in einer homo- und einer heterochiralen Variante gefunden, deren enge strukturelle Verwandtschaft in der Gegenüberstellung in Abb. 8.1 deutlich wird. Die berechnete chirodiastaltische Energie fällt innerhalb der einzelnen Motive im Betrag zum Teil größer $\left(\mathrm{O}^{t} \pi\right.$ : $\left.1.6 \mathrm{~kJ} \mathrm{~mol}^{-1}\right)$, zum Teil kleiner $\left(\mathrm{O}^{g} \pi: 0.2 \mathrm{~kJ} \mathrm{~mol}^{-1}\right)$ aus, und ist auch im Vorzeichen variabel. Auch die aus der Chiralitätssynchronisation resultierenden spektralen Unterschiede sind uneinheitlich in Größe und Vorzeichen.

Das stabilste Strukturmotiv $\mathrm{O}^{g} \pi$ weist je eine $\mathrm{OH} \cdots \mathrm{O}-$ und eine $\mathrm{OH} \cdots \pi$ Wasserstoffbrücke auf, die kooperativ zusammenwirken. Die heterochirale Variante hat 
Tab. 8.1: Schwingungsnullpunktskorrigierte relative Energien von Benzylalkohol-Dimeren auf B3LYP-D3(BJ)/may-cc-pVTZ-Niveau. Für die Nomenklatur siehe Fließtext.

\begin{tabular}{cc|cc}
\hline Konformer & $E_{0}^{\mathrm{rel}} / \mathrm{kJ} \mathrm{mol}^{-1}$ & Konformer & $E_{0}^{\mathrm{rel}} / \mathrm{kJ} \mathrm{mol}^{-1}$ \\
\hline $\operatorname{homO}^{g} \pi$ & 0 & $g t \mathrm{OV}$ & 5.2 \\
$\operatorname{hetO}^{g} \pi$ & 0.2 & homO $^{g} \mathrm{~V} \mathrm{~T}$ & 5.3 \\
$\operatorname{het} \pi \pi C_{\mathrm{i}}$ & 1.6 & $\operatorname{hetO}^{t} \pi \mathrm{T}$ & 5.5 \\
$\operatorname{hom} \pi \pi C_{2}$ & 2.0 & gt OV & 7.5 \\
$\operatorname{homO}^{t} \pi$ & 3.2 & $\operatorname{tg} \mathrm{O}^{g} \mathrm{~V}$ & 8.4 \\
$\operatorname{hetO}^{g} \mathrm{~V} \mathrm{~T}$ & 4.3 & gt $\mathrm{O} \pi$ & 10.5 \\
$\operatorname{homO}^{g} \mathrm{~V}$ & 4.4 & gt $\mathrm{O} \pi$ & 12.1 \\
$\operatorname{hetO}^{g} \mathrm{~V}$ & 4.5 & tg O & 20.8 \\
$\operatorname{hetO}^{t} \pi$ & 4.8 & & \\
\hline
\end{tabular}
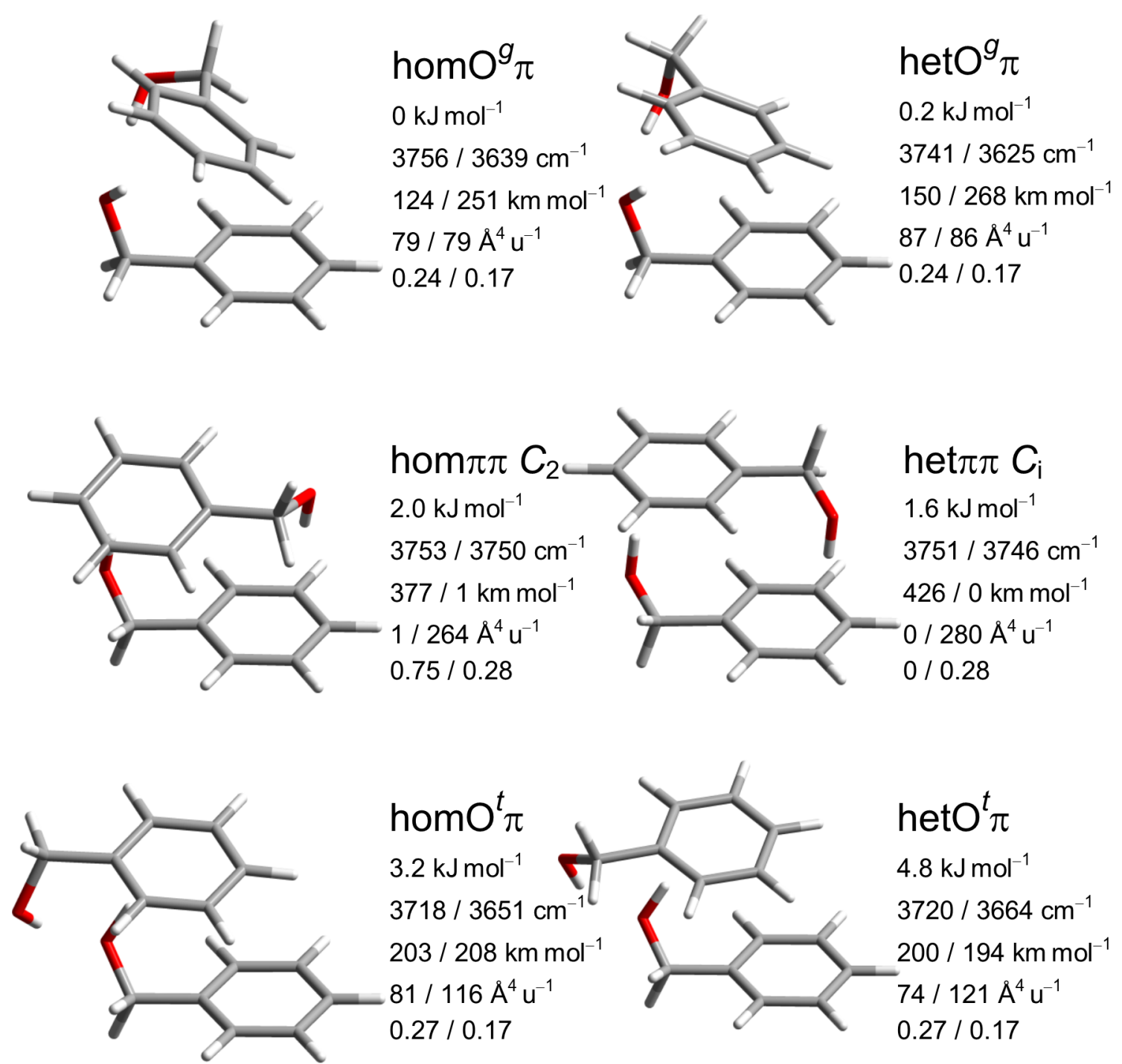

Abb. 8.1: Nullpunktskorrigierte relative Energien, harmonische OH-Streckschwingungswellenzahlen, IR-Intensitäten, Raman-Aktivitäten und Depolarisationsverhältnisse der wichtigsten Benzylalkohol-Dimere auf B3LYP-D3(BJ)/may-cc-pVTZ-Niveau. 
dabei etwas größere Tiefverschiebungen, IR- und Raman-Aktivitäten - was auf etwas stärkere Wasserstoffbrücken hindeutet - aber dennoch eine geringfügig kleinere Dissoziationsenergie. Auch das stabilste Dimer von Propargylalkohol, bei dem die Phenylgruppe durch eine Ethinylgruppe ersetzt ist, kann analog als homO ${ }^{g} \pi$ klassifiziert werden.[419] Das drittstabilste Strukturmotiv $\mathrm{O}^{t} \pi$ unterscheidet sich von $\mathrm{O}^{g} \pi$ durch die Koordination des anderen Elektronenpaares am Sauerstoffatom. Im Vergleich zu $\mathrm{O}^{g} \pi$ sind die $\mathrm{OH} \cdots \pi-$ Streckschwingungen von $\mathrm{O}^{t} \pi$ niederfrequenter und stärker IR-aktiv, umgekehrtes gilt für die $\mathrm{OH}$...O-Streckschwingungen. Dieses Strukturmotiv scheint daher eher einen Kompromiss zu Gunsten der $\mathrm{OH} \cdots \pi$-Wasserstoffbrücke darzustellen. Für das $\mathrm{O}^{t} \pi$-Motiv von Benzylalkohol ist unter Jet-Bedingungen keine nachweisbare Population zu erwarten, da seine Energie $\left(>3 \mathrm{~kJ} \mathrm{~mol}^{-1}\right.$ ) beträgt, was einem Boltzmann-Faktor $<3 \%$ bei $100 \mathrm{~K}$ entspricht. Es wird hier dennoch diskutiert, da sich daran wichtige Umwandlungspfade veranschaulichen lassen und es für 1-Phenylethanol relevanter wird.

Energetisch zwischen den beiden $\mathrm{O} \pi$-Motiven liegt das $\pi \pi$-Motiv mit zwei isolierten, nicht-kooperativen $\mathrm{OH} \cdots \pi$-Wasserstoffbrücken. Die beiden Moleküle sind in diesem Motiv symmetrieäquivalent, und lassen sich im homochiralen Fall über eine zweizählige Drehachse, im heterochiralen Fall durch eine Punktspiegelung ineinander überführen. Da damit auch die Hydroxyoszillatoren äquivalent sind, koppeln deren Streckschwingungen zu einer Raman-aktiven symmetrischen und einer IR-aktiven antisymmetrischen Schwingung mit einer geringen Davydov-Aufspaltung von $4-5 \mathrm{~cm}^{-1}$. Das Alternativverbot bezüglich der IR- und Raman-Aktivitäten gilt dabei nur im heterochiralen Fall streng, ist

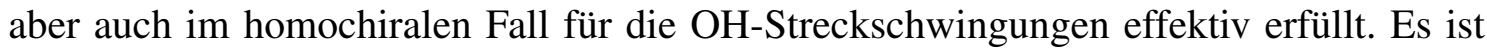
zu erwarten, dass die hohen spektralen Aktivitäten der Schwingungen die vergleichsweise hohen relativen Energien (1.6 bzw. $\left.2.0 \mathrm{~kJ} \mathrm{~mol}^{-1}\right)$ und die statistischen Nachteile (hom $\pi \pi$ wegen der Rotationssymmetriezahl, het $\pi \pi$ wegen der Achiralität) der Strukturen im Spektrum nur teilweise kompensieren können. het $\pi \pi$ entspricht qualitativ der in der Kristallstruktur von Thiobenzylalkohol realisierten Anordnung [344].

Die Konkurrenz zwischen symmetrischen Motiven mit isolierten Wasserstoffbrücken und asymmetrischen Motiven mit kooperativen Wasserstoffbrücken findet sich auch in Dimeren des permanent chiralen Glycidols [420] und des prochiralen Glycolaldehyds [421]. In diesen Molekülen ist die Phenylgruppe durch eine Epoxy- bzw. Formylgruppe substituiert. Vermutlich wegen der höheren Akzeptorstärke des jeweils enthaltenen Sauerstoffatoms sind in diesen Systemen die symmetrischen Strukturen das jeweilige globale Minimum.

In den verschiedenen Varianten des OV-Motives von Benzylalkohol-Dimeren wird nur eine $\mathrm{OH}$... O-Wasserstoffbrücke ausgebildet, in Entsprechung zu Dimeren von anderen Alkoholen, die keine zusätzliche Akzeptorfunktion aufweisen. Die Wasserstoffbrücke wird in diesem Motiv aber durch einen $\left(\mathrm{sp}^{3}\right) \mathrm{C}-\mathrm{H} \cdots \pi$-Kontakt unterstützt (wie in der Kristallstruktur) bzw. bei T-förmiger Anordnung der Phenylringe durch einen $\left(\mathrm{sp}^{2}\right) \mathrm{C}-\mathrm{H} \cdots \pi$-Kontakt. Ohne die geometrische Restriktion einer zweiten Wasserstoffbrücke, kann in diesem Motiv die $\mathrm{OH}$...O-Wasserstoffbrücke optimiert werden, deren $\mathrm{OH}-$ Streckschwingung daher in diesen Strukturen besonders große Tiefverschiebungen und IR-Aktivitäten erreicht. Allerdings lassen die hohen relativen Energien jenseits von $4 \mathrm{~kJ} \mathrm{~mol}^{-1}$ nicht erwarten, dass OV-Strukturen in einer Überschallexpansion ausgebildet werden. Die bereits im Monomer hochenergetische und experimentell bisher nicht nachgewiesene $t$-Konformation von Benzylalkohol wird auch im Dimer nicht konkur- 


\section{Komplementarität in Dimeren von Benzylalkohol und 1-Phenylethanol}

renzfähiger gefunden.

Um die Population der verschiedenen Dimere unter Jet-Bedingungen verstehen zu können, sind neben den Energieunterschieden auch die Barrieren zwischen den Konformeren aufschlussreich. Abb. 8.2 zeigt ein Energiediagramm mit den Strukturen der drei stabilsten Strukturmotive, sowie die Barrieren für Umwandlungen zwischen diesen. Die Barrierenhöhen beziehen sich dabei stets auf die Relaxation der jeweils höherenergetischen Struktur, für die Barriere der umgekehrten Umwandlung muss entsprechend die Energiedifferenz der beteiligten Dimere addiert werden. Die Barrierenhöhen werden anhand des Richtwertes von $5 \mathrm{~kJ} \mathrm{~mol}^{-1}$ nach dem Befund aus Ref. [23] für Umwandlungen unter Jet-Bedingungen als leicht oder schwierig überwindbar eingeteilt. Weitere nicht auszuschließende Umwandlungspfade, bei denen sich die relative Chiralität und das Bindungsmotiv konzertiert verändern, konnten wegen ihrer hohen Komplexität nicht berücksichtigt werden.

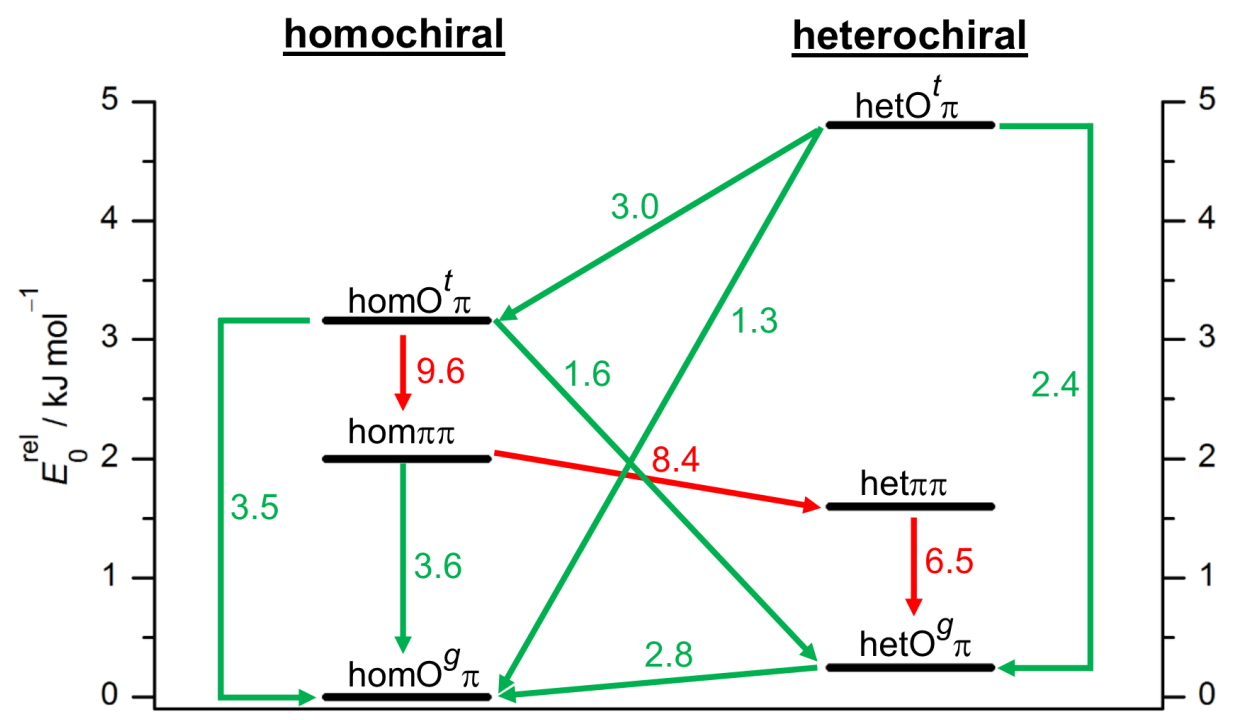

Abb. 8.2: Schwingungsnullpunktskorrigierte relative Energien und Barrierenhöhen in $\mathrm{kJ} \mathrm{mol}^{-1}$ der wichtigsten Benzylalkohol-Dimere auf B3LYP-D3(BJ)/may-cc-pVTZ-Niveau. Die Barrieren beziehen sich auf Relaxation des instabileren Konformers und sind farblich gekennzeichnet, ob sie unterhalb (grün) oder oberhalb (rot) des Richtwertes von $5 \mathrm{~kJ} \mathrm{~mol}^{-1}$ [23] liegen.

Die Umwandlung zwischen den $\mathrm{O}^{t} \pi$ - und den $\mathrm{O}^{g} \pi$-Motiven kann direkt durch Wechsel des koordinierten Elektronenpaares erfolgen, was mit einer nur geringen Barriere von 2.4 bzw. $3.5 \mathrm{~kJ} \mathrm{~mol}^{-1}$, ausgehend von $\mathrm{O}^{t} \pi$, verbunden ist.

Wie in Kap. 5.16 gefunden wurde, kann die Racemisierung zwischen den beiden enantiomeren $g$-Konformeren im isolierten Benzylalkohol-Molekül über eine Barriere von nur $2.0 \mathrm{~kJ} \mathrm{~mol}^{-1}$ erfolgen, welche zusätzlich noch durch einen Tunnelprozess unterstützt wird. In den Dimeren sind in der Regel zwei unterschiedliche Überführungen zwischen homo- und heterochiralen Strukturen möglich, da die beiden Moleküle meist inäquivalent sind. Wird in den $\mathrm{O}^{t} \pi$ - und $\mathrm{O}^{g} \pi$-Motiven der Donor der $\mathrm{OH} \cdots \mathrm{O}$-Wasserstoffbrücke umgewandelt, wird direkt die Variante des selben Motivs mit entgegengesetzter relativer Chiralität erhalten. Dies geschieht, ausgehend von der höherenergetischen Struktur, über Barrieren von 3.0 bzw. $2.8 \mathrm{~kJ} \mathrm{~mol}^{-1}$, die somit nur geringfügig höher sind als im isolierten Monomer. Eine Umwandlung des Donor-Moleküls der $\mathrm{OH} \cdots \pi$-Wasserstoffbrücke 
überführt dagegen zusätzlich den Charakter des koordinierten Elektronenpaares zwischen $\mathrm{O}^{g}$ und $\mathrm{O}^{t}$, womit auch ein Übergang zwischen den entsprechenden Motiven stattfindet. Die berechneten Barrieren hierfür $\left(1.3\right.$ und $\left.1.6 \mathrm{~kJ} \mathrm{~mol}^{-1}\right)$ sind sogar geringer als im Monomer. Im $\pi \pi$-Motiv sind dagegen beide Moleküle symmetrieäquivalent, sodass es unerheblich ist, welches der beiden seine transiente Chiralität wechselt. Die Barriere wird hier wesentlich höher gefunden $\left(8.4 \mathrm{~kJ} \mathrm{~mol}^{-1}\right)$. Dies kann damit begründet werden, dass die Umwandlung zwischen den enantiomeren Benzylalkohol-Konformationen über einen $\mathrm{OC}_{1} \mathrm{C}_{2} \mathrm{C}_{3}$-Torsionswinkel von $90^{\circ} \mathrm{im}$ Übergangszustand erfolgt (siehe Abb. 5.49 auf Seite 175). In den $\pi \pi$-Dimeren liegt der Betrag dieses Winkels aber davon besonders weit entfernt ( $17^{\circ}$ für het $\pi \pi$ bzw. $31^{\circ}$ für hom $\pi \pi$, Vergleichswert des Monomers: $55^{\circ}$ ). Diese geringen Werte, bei denen sich das Sauerstoffatom der Ebene des Phenylringes annähert, erlauben im $\pi \pi$-Motiv eine besonders große Annäherung zwischen beiden Molekülen. Für die Racemisierung muss diese kompakte Struktur aufgebrochen werden, wodurch der Übergangszustand destabilisiert wird. Ebenfalls evaluiert wurde ein alternativer Racemisierungspfad mit der gegenläufigen Torsionsrichtung, bei der die Hydroxygruppe einen längeren Weg über die Rückseite des Phenylringes nimmt. Für einen solchen Seitenwechsel wurde aber bereits im Monomer eine hohe Barriere gefunden, im Dimer wird diese noch zusätzlich durch den notwendigen Bruch einer Wasserstoffbrücke gesteigert und ist damit energetisch sehr hochliegend.

Bei der Umwandlung zwischen den $\pi \pi$ - und den $\mathrm{O} \pi$-Motiven muss eine Wasserstoffbrücke einen Akzeptorwechsel zwischen dem $\pi$-System des Phenylringes und einem freien Elektronenpaar des Sauerstoffatoms vollziehen. Hierzu müssen die beiden Moleküle in Gänze gegeneinander rotiert werden, da in den $\pi \pi$-Motiven die Hydroxygruppen an gegenüberliegenden Enden des Dimers liegen. Dieser Pfad kann näherungsweise über den $\mathrm{C}_{1} \mathrm{C}_{2} \mathrm{C}_{1}^{\prime} \mathrm{C}_{2}^{\prime}$-Torsionswinkel beschrieben werden, dessen Scan zum Auffinden von geeigneten Startstrukturen für die Optimierung der Übergangszustände genutzt wurde. Ausgehend von hom $\pi \pi$ sind die beiden Richtungen der intermolekularen Torsion inäquivalent: die eine führt zu homO ${ }^{g} \pi$, die andere $\mathrm{zu}$ homO ${ }^{t} \pi$. Die Barriere für den Übergang zu homO ${ }^{g} \pi$ ist dabei wesentlich niedriger $\left(3.6 \mathrm{~kJ} \mathrm{~mol}^{-1}\right)$ als die $\mathrm{zu} \mathrm{homO}^{t} \pi$ $\left(10.7 \mathrm{~kJ} \mathrm{~mol}^{-1}\right)$. Dies liegt darin begründet, dass die Distanz zwischen dem $\pi$-System und dem $\mathrm{O}^{g}$-Elektronenpaar sehr viel geringer ist als die zu dem $\mathrm{O}^{t}$-Elektronenpaar. Im zweiten Fall muss die Wasserstoffbrücke daher im Übergangszustand komplett gebrochen werden. Für het $\pi \pi$ sind aufgrund der Inversionssymmetrie dagegen beide Richtungen der Torsion äquivalent und führen zu hetO ${ }^{g} \pi$. Obwohl der Wechsel zu einem $\mathrm{O}^{g}$-Elektronenpaar erfolgt, ist die Barriere relativ hoch $\left(6.5 \mathrm{~kJ} \mathrm{~mol}^{-1}\right)$. Die geometrische Begründung hierfür ist weniger eindeutig. Möglicherweise ist die große notwendige Veränderung des $\mathrm{OC}_{1} \mathrm{C}_{2} \mathrm{C}_{3}$-Torsionswinkels des Donors für den Akzeptorwechsel verantwortlich, die im heterochiralen Fall von 17 auf $65^{\circ}$ erfolgt, im homochiralen Fall dagegen nur von 31 auf $44^{\circ}$. Ein direkter Übergangspfad von het $\pi \pi \mathrm{zu}$ hetO ${ }^{t} \pi$ erscheint nicht möglich, die Umwandlung erfolgt stattdessen stets über hetO ${ }^{g} \pi$ als Zwischenschritt.

Zusammengefasst wurden damit für alle wichtigsten Dimer-Strukturen Relaxationspfade in ein energetisch tieferes Minimum mit niedriger Barrierenhöhe gefunden - mit Ausnahme von het $\pi \pi$, welches damit bei tiefen Temperaturen kinetisch isoliert erscheint.

Trimere von Benzylalkohol sind im Hinblick auf das in Kap. 4.9.2 diskutierte Gleichgewicht zwischen cyclischen Strukturen mit allen drei Alkohol-Resten auf der gleichen 


\section{Komplementarität in Dimeren von Benzylalkohol und 1-Phenylethanol}

(иии) oder alternativ auf unterschiedlichen (uиd) Seiten des Ringes aus Hydroxygruppen interessant. Die Phenylgruppen können starke Dispersionswechselwirkungen untereinander ausbilden und damit potentiell das $и и и$-Motiv stabilisieren. Archivmaterial zeigt, dass bereits Oswald einige Trimer-Strukturen optimiert hat, die aber keinen Eingang in die Veröffentlichungen gefunden haben. Diese wurden hier auf B3LYP-D3(BJ)/def2TZVP-Niveau nachoptimiert und um einige weitere Strukturideen ergänzt. Dabei wurde zumindest für den homochiralen Fall gefunden, dass das uud-Motiv keine stabile Struktur darstellt und sich bei der Optimierung stets in $и и и$ umwandelt. Heterochiral wurde eine uud-Konformation als lokales Minimum gefunden, dieses ist aber sehr hochenergetisch (12 $\mathrm{kJ} \mathrm{mol}^{-1}$ oberhalb des homochiralen $u и u$-Konformers). Dies steht im Kontrast zum umgekehrten Befund für Alkohole mit aliphatischen Resten (Methanol, Ethanol) in Kap. 4.9.2. Allerdings wurde für Benzylalkohol noch eine deutlich stabilere (um $4.7 \mathrm{~kJ} \mathrm{~mol}^{-1}$ ) heterochirale Struktur gefunden, bei der der Ringschluss nicht mit dem Sauerstoffatom des dritten Moleküls, sondern mit dessen $\pi$-System erfolgt. Auf diese Weise wird die Ringspannung des Dreiringes vermieden. Auch bei der verwandten Verbindung 1-Indanol wurde von Altnöder eine klare Präferenz für OO $\pi$-Strukturen gefunden - im Einklang mit dem Experiment.[70, 363] Die zwei stabilsten bisher gefundenen BenzylalkoholTrimere sind in Abb. 8.3 dargestellt. Die Betrachtung der umfangreichen konformationellen Möglichkeiten für Trimere ist hier allerdings fernab von erschöpfend erfolgt, für eine zukünftige Fortsetzung erscheint der Einsatz von automatisierten Methoden zur Konformerensuche sinnvoll.

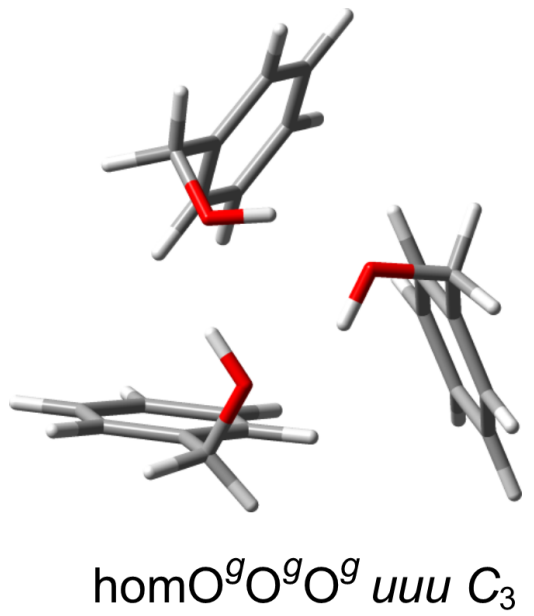

$4.7 \mathrm{~kJ} \mathrm{~mol}^{-1}$

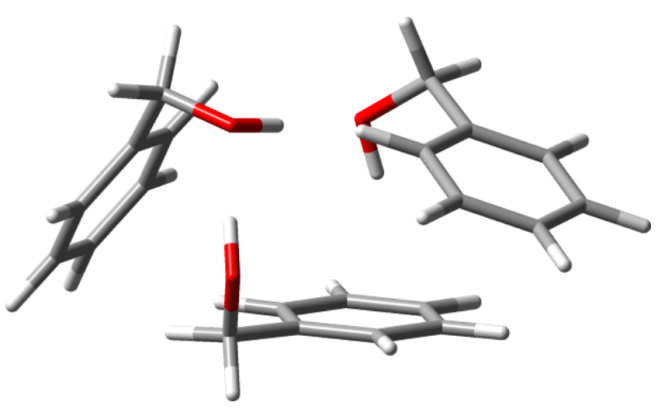

$\operatorname{hetO}{ }^{g} \mathrm{O}^{g} \pi$

$0 \mathrm{~kJ} \mathrm{~mol}^{-1}$

Abb. 8.3: Die zwei stabilsten bisher gefundenen Benzylalkohol-Trimere mit relativen nullpunktskorrigierten Energien auf B3LYP-D3(BJ)/def2-TZVP-Niveau.

\subsubsection{Schwingungsspektren}

Der obere Teil von Abb. 8.4 zeigt eine Übersicht des OH-Streckschwingungsbereiches im 2019 von Sennert [341] neu aufgenommenen Spektrum von Benzylalkohol am verbesserten Popcorn-Jet. Diesem ist das Spektrum von Oswald von 2013 [70, 333, 417], aufgenommen mit der älteren Variante des Popcorn-Jets, gegenübergestellt. Die Clustergrößenverteilung ist in beiden FTIR-Spektren sehr ähnlich, da der Stagnationsdruck und 


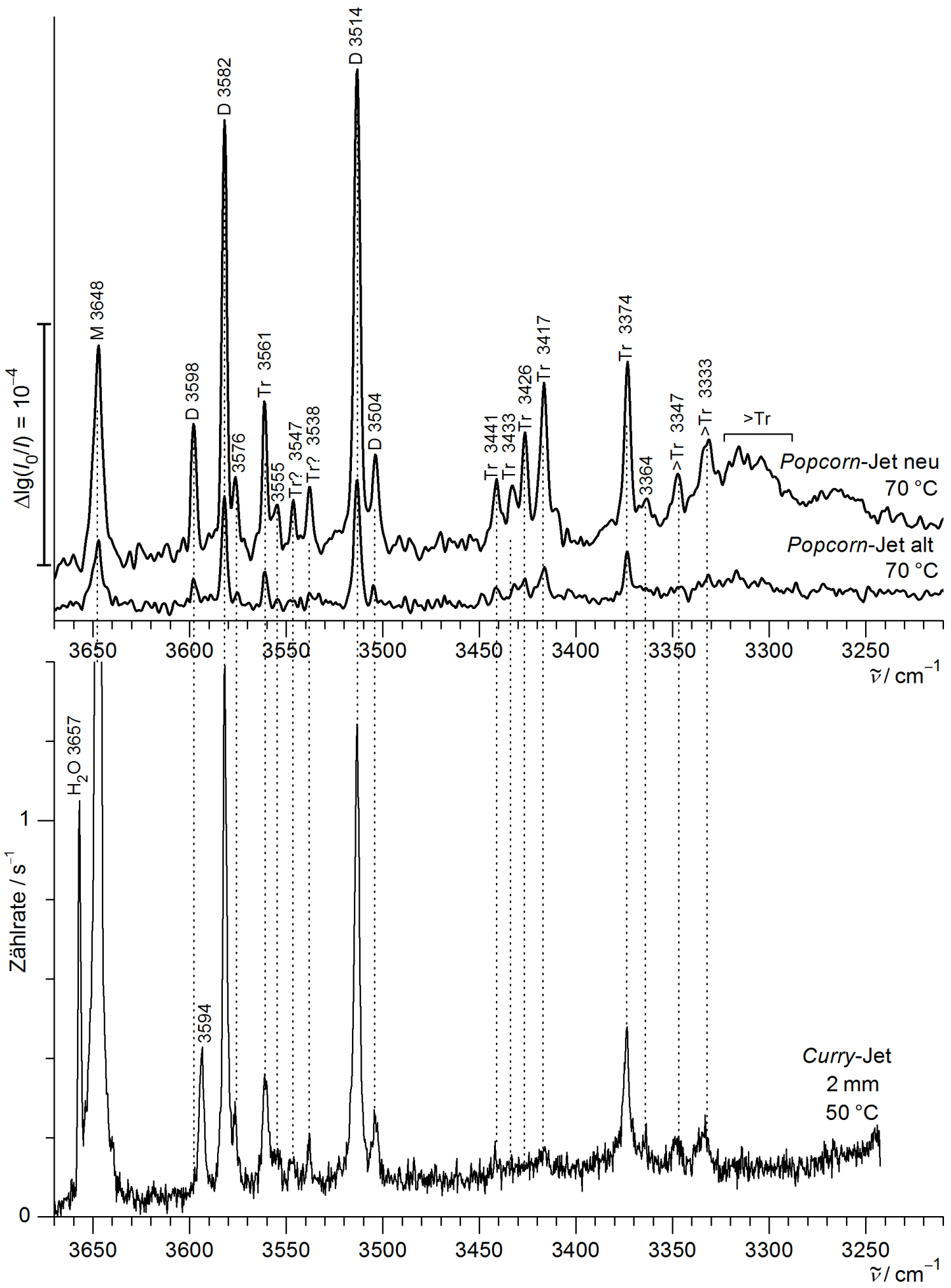

Abb. 8.4: Vergleich zwischen neuem [341] und altem Popcorn-Jet-Spektrum [70, 333, 417] sowie neuem Curry-Jet-Spektrum von Benzylalkohol im OH-Streckschwingungsbereich. Die Banden sind mit ihren spektralen Position in $\mathrm{cm}^{-1}$ sowie der zugeordneten Clustergröße gemäß Ref. [70, 333, 417] (soweit vorhanden) gekennzeichnet. M kennzeichnet Monomere, D Dimere, Tr Trimere und $>\operatorname{Tr}$ Cluster größer als Trimere. 


\section{Komplementarität in Dimeren von Benzylalkohol und 1-Phenylethanol}

die Temperatur der Probenkammer (und damit der Dampfdruck) gleich gewählt wurden. Die Intensität der Signale (und damit wegen der ähnlichen Wiederholungszahl auch das Signal-zu-Rausch-Verhältnis) konnte um etwa um einen Faktor vier gesteigert werden. Dieser erhebliche Fortschritt ist auf Änderungen an der Düsengeometrie, an der Synchronisation zwischen Expansion und FTIR-Datenaufnahme und vor allem auf der Optimierung des Gasflusses durch Lange [422] und Poblotzki [69] zurückzuführen. Die Banden sind mit von Altnöder und Oswald zugeordneten Clustergrößen gekennzeichnet, die aus Konzentrationsvariation und Einsatz der Hochtemperaturdüse ermittelt wurden.[70, 333, 417] Im unteren Teil der Abbildung ist ein hier neu aufgenommenes, komplementäres Raman-Spektrum dargestellt. Archivmaterial zeigt, dass bereits Altnöder 2013 Benzylalkohol am Curry-Jet untersucht hat. Da dabei aber keine Heizung der Substanz erfolgte, ist in diesen älteren Spektren ausschließlich die Monomerbande bei $3648 \mathrm{~cm}^{-1} \mathrm{zu}$ erkennen. Benzylalkohol wurde kürzlich auch von Lange ebenfalls ungeheizt am Filet-Jet untersucht. Neben der Monomerbande sind auch dort die Banden des stabilsten Dimers bei 3582 und $3514 \mathrm{~cm}^{-1}$ erkennbar, allerdings mit deutlich schlechterem Signal-zu-RauschVerhältnis als in den Popcorn-Jet Spektren. Sowohl die neuen FTIR-, wie auch RamanMessungen, bei erhöhter Temperatur geben Einblick in zusätzliche Details der Aggregation von Benzylalkohol. Bisher nicht beschriebene Banden befinden sich bei 3594, 3576, 3555 und $3364 \mathrm{~cm}^{-1}$.

Die Bande bei $3504 \mathrm{~cm}^{-1}$ wurde auf Grundlage ihrer Konzentrationsabhängigkeit von Oswald als Benzylalkohol-Dimer eingeordnet.[417] In der nachfolgenden Publikation [333] und Doktorarbeit von Altnöder [70] wurde aber eine Interpretation als gemischtes Benzylalkohol-Cyclohexylmethanol-Dimer als Folge einer Verunreinigung wahrscheinlicher angesehen, da Cyclohexylmethanol direkt davor gemessen wurde und das gemischte Dimer der beiden Alkohole an genau dieser Position gefunden wurde.[70, 333, 417] Da die Bande aber hier erneut beobachtet wird, bestätigt dies die ursprüngliche Interpretation von Oswald als Bande einer reinen Benzylalkohol-Spezies.

Die FTIR- und Raman-Spektren sind insgesamt ähnlich, allerdings gibt es auch einige markante Unterschiede. So ist die Bande im IR-Spektrum bei $3598 \mathrm{~cm}^{-1}$ nicht im RamanSpektrum erkennbar, dafür tritt dort eine neue Bande bei $3594 \mathrm{~cm}^{-1}$ auf. Dies beweist die von Altnöder und Oswald vermutete Zuordnung der Bande bei $3598 \mathrm{~cm}^{-1}$ zu einer symmetrischen $\pi \pi$-Struktur, da deren symmetrische und antisymmetrische Schwingungen jeweils (fast) ausschließlich IR- bzw. Raman-aktiv sind und um eine Davydov-Aufspaltung von $4 \mathrm{~cm}^{-1}$ getrennt sind. Bemerkenswert ist, dass keine der beiden Banden aufgespalten oder deutlich verbreitert erscheint. Dies spricht dafür, dass im Wesentlichen entweder hom- oder het $\pi \pi$ vorliegt, oder alternativ diese beiden Strukturen nahezu perfekt in beiden Banden koinzidieren.

Die im IR-Spektrum intensive Trimer-Bandenformation zwischen 3445 und $3415 \mathrm{~cm}^{-1}$ deutet sich nur sehr schwach im Raman-Spektrum an. Hierfür kommen zwei Ursachen in Frage. Zum einem könnten diese Banden stattdessen von höheren Oligomeren verursacht werden, die in der Expansion des Curry-Jets kaum gebildet werden, da die Substanztemperatur mit $50^{\circ} \mathrm{C}$ (und damit die Konzentration) geringer ist gegenüber dem Popcorn-Jet $\left(70^{\circ} \mathrm{C}\right)$. Allerdings sind auch die sonstigen Expansionsbedingungen zwischen Curry- und Popcorn-Jet durch die Verwendung unterschiedlicher Düsentemperaturen und -geometrien, Detektionsabstände sowie Stagnations- und Hintergrunddrücke der Expansion nur begrenzt vergleichbar. Gegen einen größeren Unterschied in der Clustergrößen- 
verteilung spricht aber, dass die sonstigen Trimerbanden in beiden Spektren sehr ähnliche Intensitäten relativ zu den Dimerbanden aufweisen (unter der Annahme, dass sich die Dimer-zu-Trimer-Verhältnisse der IR- und Raman-Aktivitäten nicht deutlich systematisch verändern). Auch die Banden bei 3347 und $3333 \mathrm{~cm}^{-1}$, die größeren Cluster zugeordnet wurden, sind in beiden Spektren mit ähnlicher relativer Intensität zu den anderen Banden erkennbar. Es wurden auch hier nicht gezeigte Raman-Spektren bei höherer Substanztemperatur aufgenommen, diese weisen ebenso keinen Anstieg bzw. Erscheinen der fraglichen Banden auf (dafür aber zunehmend thermische Verbreiterungen und Probleme mit der Basislinie, da auch die Düsentemperatur erhöht wurde). Daher erscheint ein reiner Konzentrationseffekt als Ursache für die geringe Intensität dieser Banden unwahrscheinlich. Die breiten Banden unterhalb von $3330 \mathrm{~cm}^{-1}$, die vermutlich von noch größeren Clustern stammen, sind im Raman-Spektrum überhaupt nicht erkennbar.

Die alternative Erklärung für die intensitätsschwach ausfallenden Banden im RamanSpektrum wäre, dass es sich um Banden (weitgehend) symmetrischer Tri- oder Tetramere handelt. In diesem Fall wären allerdings weitere, stark Raman-aktive Banden zu erwarten. Diese könnten aber durch Kooperativität und Davydov-Aufspaltungen unterhalb von $3240 \mathrm{~cm}^{-1}$, und damit außerhalb des Messbereiches, verschoben sein. Der Vergleich mit den (annähernd) symmetrischen Tetrameren von Methanol und Ethanol (Kap. 4.8) zeigt, dass dies durchaus plausibel wäre. Deren stark IR-aktive Schwingungen absorbieren ebenfalls im Bereich um $3300 \mathrm{~cm}^{-1}$, während die stark Raman-aktiven Schwingungen im Bereich um $3200 \mathrm{~cm}^{-1}$ streuen. Auch die IR-aktiven Banden der (annähernd) symmetrischen Trimere vom Methanol (Kap. 4.4.2) und Ethanol (Kap. 4.4.2) liegen um $3450 \mathrm{~cm}^{-1}$ und damit im passenden Bereich der Benzylalkohol-Trimer-Bandenformation, allerdings fehlen im dann erwarteten Bereich um $3400 \mathrm{~cm}^{-1}$ bei Benzylalkohol stark Raman-, aber wenig IR-aktive Banden der symmetrischen Schwingung(en). Weitere Banden, die tentativ Benzylalkohol-Trimeren zugeordnet wurden (3561, 3547, 3538 und $3374 \mathrm{~cm}^{-1}$ ) sind dagegen ähnlich stark IR- und Raman-aktiv und deuten daher auf deutlich asymmetrische Strukturen hin. Für eine nähere Beurteilung größerer Cluster wären weiteren Rechnungen und Messungen notwendig, daher fokussiert sich die weitere Analyse der Spektren auf den Dimer-Bereich von 3600-3500 $\mathrm{cm}^{-1}$.

Den experimentellen Spektren sind Simulationen auf B3LYP-D3(BJ)/may-cc-pVTZNiveau in Abb. 8.5 gegenübergestellt, bei denen die harmonischen Wellenzahlen einheitlich derart verschoben wurden, dass die vorhergesagte und experimentelle Position des $g$ Monomers übereinstimmt. Effektiv verglichen werden also experimentelle und vorhergesagte harmonische Verschiebungen relativ zum Monomer. Die experimentelle DavydovAufspaltung von $4 \mathrm{~cm}^{-1}$ zwischen den Banden bei 3598 und $3594 \mathrm{~cm}^{-1}$ würde sowohl gut mit den Vorhersagen für hom $\pi \pi\left(4.0 \mathrm{~cm}^{-1}\right)$, wie auch für het $\pi \pi\left(4.6 \mathrm{~cm}^{-1}\right)$, übereinstimmen. Verschiedene Indizien weisen aber daraufhin, dass die Bande maßgeblich von het $\pi \pi$ verursacht wird. Von allen in dieser Arbeit (Tab. 8.2) und von Oswald und Altnöder getesteten Methoden wird het $\pi \pi$ als niederenergetischer gefunden, mit der Ausnahme von M06-2X. Die spektrale Sichtbarkeit von het $\pi \pi$ würde zudem weiter durch die etwas höher vorhergesagten IR- und Raman-Aktivitäten verstärkt. Zudem wurde für het-, aber nicht hom $\pi \pi$, eine erhöhte Barriere für die Umwandlung in das globale Minimum gefunden, was die Population weiter erhöhen kann. Im folgenden Abschnitt wird zudem gezeigt, dass auch der Vergleich mit 1-Phenylethanol die (überwiegend) heterochirale Zuordnung unterstützt. hom $\pi \pi$ ist zu schwach populiert, um nachgewiesen zu 


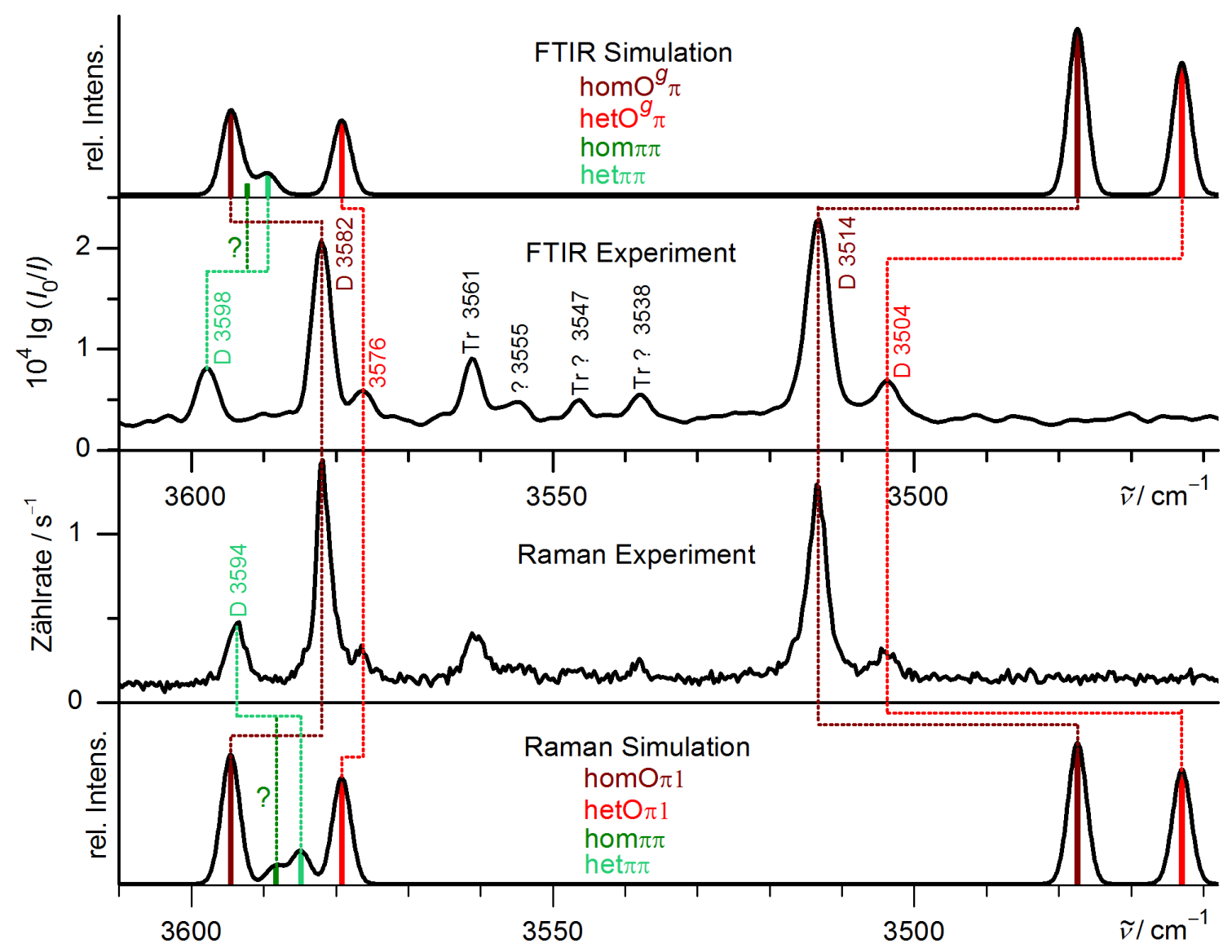

Abb. 8.5: Simulierte und experimentelle IR- [341] und Raman-Spektren von Benzylalkohol im Dimer-Bereich. Simulationsparameter auf Basis von B3LYP-D3(BJ)/may-cc-pVTZ sind einheitliche Verschiebung der harmonischen Wellenzahlen um die Differenz von experimenteller und harmonischer $g$-Monomerwellenzahl $\left(-161.5 \mathrm{~cm}^{-1}\right)$, eine Konformationstemperatur von $100 \mathrm{~K}$ und Gauss-Profile mit einer vollen Halbwertsbreite von $3 \mathrm{~cm}^{-1}$.

werden, und/oder koinzidiert spektral fast perfekt mit het $\pi \pi$. Die Tiefverschiebungen der OH-Streckschwingungswellenzahlen durch die Ausbildung der isolierten $\mathrm{OH} \cdots \pi$ Wasserstoffbrücken wird von B3LYP-D3(BJ) in harmonischer Näherung leicht überschätzt (Tab. 8.2), dies steht im Einklang mit den Befunden für die in Kap. 6 diskutierten Alkohol- $\alpha$-Pinen-Komplexen. Ein Nachweis von hom $\pi \pi$ wäre durch Rotationsspektroskopie denkbar, während het $\pi \pi$ für diese Spektroskopiemethode wegen des fehlenden Dipolmoments unsichtbar ist. Beide Konformere wären grundsätzlich über konformerenselektive Doppelresonanz-Methoden beobachtbar, sofern sich ihre UV-Spektren stärker unterscheiden sollten.

Entgegen den Vorhersagen von B3LYP-D3(BJ) und B2PLYP-D3(BJ), nach denen sich hom- und hetO ${ }^{g} \pi$ energetisch nur um wenige Zehntel $\mathrm{kJ} \mathrm{mol}^{-1}$ unterscheiden (Tab. 8.3), wird nur ein intensives Bandenpaar (bei $3582 / 3514 \mathrm{~cm}^{-1}$ ) für das $\mathrm{O}^{g} \pi$-Motiv im Experiment gefunden. Die Barriere zwischen hom- und het ${ }^{g} \pi$ wurde als gering gefunden $\left(2.8 \mathrm{~kJ} \mathrm{~mol}^{-1}\right.$, B3LYP-D3(BJ/may-cc-pVTZ) sodass sich auch unter Jet-Bedingungen ein Gleichgewicht einstellen sollte. Die wahrscheinlichste Erklärung für die Diskrepanz scheint zu sein, dass die Energiedifferenz von diesen Methoden unterschätzt wird. Eine Koinzidenz in beiden Banden erscheint dagegen unwahrscheinlicher. Mit allen hier (Tab. 
Tab. 8.2: Differenzen (hom - het) der nullpunktskorrigierten Energien und Verschiebungen der $\mathrm{OH}$-Streckschwingungswellenzahlen (relativ zum $g$-Monomer) der hom $\pi \pi$ - und het $\pi \pi$-Strukturen auf verschiedenen Rechenniveaus im Vergleich zum Experiment.

\begin{tabular}{lccc}
\hline Methode & $\begin{array}{c}\Delta E_{0} \\
/ \mathrm{kJ} \mathrm{mol}^{-1}\end{array}$ & $\begin{array}{c}-\Delta \tilde{v}(\omega) \text { hom } \\
/ \mathrm{cm}^{-1}\end{array}$ & $\begin{array}{c}-\Delta \tilde{v}(\omega) \text { het } \\
/ \mathrm{cm}^{-1}\end{array}$ \\
\hline Experiment & $?$ & \multicolumn{2}{c}{$50 / 54$} \\
B3LYP-D3(BJ)/may-cc-pVTZ & 0.4 & $56 / 59$ & $58 / 63$ \\
B2PLYP-D3(BJ)/may-cc-pVTZ & 0.3 & $55 / 59$ & $56 / 60$ \\
$\omega B$ B7X-D/may-cc-pVTZ & 1.4 & $45 / 49$ & $47 / 51$ \\
M06-2X/may-cc-pVTZ & -0.5 & $31 / 35$ & $30 / 35$ \\
\hline
\end{tabular}

Tab. 8.3: Differenzen (hom - het) der nullpunktskorrigierten Energien und OH-Streckschwingungswellenzahlen der homO ${ }^{g} \pi$ - und hetO ${ }^{g} \pi$-Strukturen auf verschiedenen Rechenniveaus im Vergleich zur experimentellen Zuordnung.

\begin{tabular}{lccc}
\hline Methode & $\begin{array}{c}\Delta E_{0} \\
/ \mathrm{kJ} \mathrm{mol}^{-1}\end{array}$ & $\begin{array}{c}\Delta \tilde{v}(\omega) \mathrm{OH} \cdots \pi \\
/ \mathrm{cm}^{-1}\end{array}$ & $\begin{array}{c}\Delta \tilde{v}(\omega) \mathrm{OH} \cdots \mathrm{O} \\
/ \mathrm{cm}^{-1}\end{array}$ \\
\hline Experiment & $<0$ & 6 & 10 \\
B3LYP-D3(BJ)/may-cc-pVTZ & -0.2 & 15 & 14 \\
B2PLYP-D3(BJ)/may-cc-pVTZ & -0.1 & 16 & 14 \\
$\omega$ B97X-D/may-cc-pVTZ & -1.7 & 18 & 8 \\
M06-2X/may-cc-pVTZ & -2.8 & 7 & 6 \\
\hline
\end{tabular}

8.3) und von Oswald [417] geprüften Methoden wird $\mathrm{homO}^{g} \pi$ als stabiler und in beiden $\mathrm{OH}-$ Streckschwingungen höherfrequenter als hetO ${ }^{g} \pi$ vorhergesagt. Daher wird die hochfrequente und intensive Bande bei $3582 \mathrm{~cm}^{-1}$ homO $^{g} \pi$ zugeordnet. Gemäß der ähnlichen Intensität und dem konformerenselektiven Befund von Mons et al. [220] gehört die Bande bei $3514 \mathrm{~cm}^{-1}$ zur selben Struktur. Dies entspricht der Zuordnung von Oswald und Altnöder. Die beiden Banden von hetO ${ }^{g} \pi$ sind gegenüber homO ${ }^{g} \pi$ jeweils niederfrequenter und weniger intensiv zu erwarten. Bezüglich der OH $\cdots$ O-Streckschwingung kommt demnach nur die Bande bei $3504 \mathrm{~cm}^{-1}$ in Frage, eine solche Zuordnung wurde auch bereits von Oswald [417] erwogen. Die Wellenzahldifferenz bei Wechsel der relativen Chiralität von $10 \mathrm{~cm}^{-1}$ würde akzeptabel zu den theoretischen Vorhersagen passen (Tab. 8.3). Die Überschätzung der Verschiebung zum Monomer beträgt für B3LYP-D3(BJ)/may-ccpVTZ dann sowohl für hom- wie hetO ${ }^{g} \pi$ jeweils etwa $28 \%$. Dies ist weniger als für das $g+g+$-Dimer von Ethanol (37\%), allerdings ist die Vergleichbarkeit wegen der in $\mathrm{O}^{g} \pi$ kooperativen und gespannten Wasserstoffbrücke eingeschränkt.

Da Oswald keine passende Bande für die $\mathrm{OH} \cdots \pi$-Streckschwingung von hetO ${ }^{g} \pi$ beobachtet hat, wurde eine Koinzidenz mit der Bande bei $3582 \mathrm{~cm}^{-1}$ diskutiert.[417] Als Alternative kann hier nun die neu entdeckte Bande bei $3576 \mathrm{~cm}^{-1}$ vorgeschlagen werden. Der spektrale Unterschied des $\mathrm{OH} \cdots \pi$-Streckschwingungsüberganges zwischen hom- und hetO $^{g} \pi$ wäre in diesem Fall von allen Methoden, mit Ausnahme von M06-2X, überschätzt (Tab. 8.3). Eine weitere Möglichkeit wäre eine Verdeckung durch die Trimer- 


\section{Komplementarität in Dimeren von Benzylalkohol und 1-Phenylethanol}

bande bei $3561 \mathrm{~cm}^{-1}$, was einer spektralen Verschiebung von $21 \mathrm{~cm}^{-1}$, und damit einer ähnlich großen Diskrepanz zur Theorie entsprechen würde. Der kommende Vergleich mit 1-Phenylethanol stützt aber die Zuordnung zu $3576 \mathrm{~cm}^{-1}$. Sowohl für homO ${ }^{g} \pi$ (deutlich) wie auch hetO ${ }^{g} \pi$ (geringfügig) würde B3LYP-D3(BJ)/may-cc-pVTZ dann die Verschiebung zum Monomer unterschätzen, entgegen der Überschätzung für isolierte $\mathrm{OH} \cdots \pi$ Wasserstoffbrücken. Dieser Befund wird ebenfalls bei 1-Phenylethanol bestätigt werden.

Die Überschätzung der Verschiebung der isolierten, wenig gespannten $\mathrm{OH} \cdots \pi$ Wasserstoffbrücken im $\pi \pi$-Motiv und die Unterschätzung für die kooperativen, gespannten Kontakte im $O^{g} \pi$-Motiv führen in Kombination zu einer qualitativ falschen Vorhersage für die Abfolge der Banden durch B3LYP-D3(BJ) (Abb. 8.5), die zum Teil auch bei B2PLYP-D3(BJ) besteht (Tab. 8.4). M06-2X/may-cc-pVTZ und $\omega$ B97X-D/may-ccpVTZ sagen die Bandenabfolge dagegen korrekt vorher. Dies ist aber wegen der strapazierten Annahme ähnlicher Anharmonizität bei Vergleich unterschiedlicher Wasserstoffbrückenmotiven kein zwangsläufiges Qualitätskriterium. Gravierender ist dagegen, dass $\omega$ B97X-D/may-cc-pVTZ, und ganz besonders M06-2X/may-cc-pVTZ, jeweils eine Struktur des $\pi \pi$-Motivs als globales Minimum vorhersagen. Dies steht im Widerspruch zu den geringen Intensitäten der zugehörigen Banden. Auch für Komplexe von Methanol mit verschieden substituierten Anisolen wurde für M06-2X eine mit dem Experiment inkompatible $\mathrm{OH} \cdots \pi$-Präferenz gefunden, während B3LYP-D3 eine gute Beschreibung der Balance mit der OH*.O-Alternative gelingt.[207] Im völligen Kontrast zu den hier durchgeführten M06-2X/may-cc-pVTZ Rechnungen wurde von Altnöder [70] mit M06-2X/QZVP gefunden, dass beide $\pi \pi$-Konformere stattdessen um mehr als $8 \mathrm{~kJ} \mathrm{~mol}^{-1}$ instabiler als hom $O^{g} \pi$ sein sollen. Solche wiederholt erratischen Ergebnisse der M06-2X Methode wurden von Altnöder auch für weitere Systeme angemerkt.

Da sowohl für hom- wie auch für hetO ${ }^{g} \pi$ jeweils ein Bandenpaar für die zwei Wasserstoffbrücken beobachtet wird, können die relativen Intensitäten mit den Vorhersagen für die IR-Aktivitäten und Raman-Streuquerschnitte verglichen werden. Integration des IR-Spektrums liefert für das intensivere Bandenpaar ein Verhältnis von 1.5 und für das weniger intensive von 1.8, jeweils zugunsten der jeweils stärker verschobenen Bande der $\mathrm{OH}$-..O-Wasserstoffbrücke. Dem gegenüber stehen die einheitlichen theoretischen Vorhersagen, dass $\operatorname{homO}^{g} \pi$ das höhere Verhältnis aufweisen soll (Tab. 8.5). Gemessen an dieser Tendenz wäre eine Zuordnung umgekehrt zur bisher diskutierten naheliegender.

Tab. 8.4: Differenzen der nullpunktskorrigierten Energien und der $\mathrm{OH} \cdots \pi$-Streckschwingungswellenzahlen der homO ${ }^{g} \pi$ - und het $\pi \pi$-Strukturen (letztere getrennt in Bezug auf die antisymmetrische und symmetrische Streckschwingung) auf verschiedenen Rechenniveaus im Vergleich zur experimentellen Zuordnung.

\begin{tabular}{lccc}
\hline Methode & $\begin{array}{c}\Delta E_{0} \\
/ \mathrm{kJ} \text { mol }\end{array}$ & $\begin{array}{c}\Delta \tilde{v}(\omega) \text { antis. } \\
/ \mathrm{cm}^{-1}\end{array}$ & $\begin{array}{c}\Delta \tilde{v}(\omega) \text { symm. } \\
/ \mathrm{cm}^{-1}\end{array}$ \\
\hline Experiment & $>0$ & 16 & 12 \\
B3LYP-D3(BJ)/may-cc-pVTZ & 1.6 & -5 & -10 \\
B2PLYP-D3(BJ)/may-cc-pVTZ & 2.2 & 1 & -4 \\
$\omega$ B97X-D/may-cc-pVTZ & -0.04 & 7 & 3 \\
M06-2X/may-cc-pVTZ & -2.2 & 29 & 24 \\
\hline
\end{tabular}


Tab. 8.5: Intensitätsverhältnisse der Fundamentalübergänge der $\mathrm{OH} \cdots \mathrm{O}-\mathrm{zu}$ den $\mathrm{OH} \cdots \pi$ Oszillatoren bei IR- und Raman-Detektion gemäß Experiment und Theorie. Für die theoretischen Raman-Streuquerschnitte wurde die Wellenzahlabhängigkeit der Detektionsempfindlichkeit am Curry-Jet (siehe Kap. 3.7) durch Verwendung der experimentellen Wellenzahlen berücksichtigt. Für B2PLYP wurden keine Raman-Streuquerschnitte berechnet, da diese nur mit sehr erheblichem Rechenaufwand numerisch zugänglich sind.

\begin{tabular}{lcccc}
\hline Methode & \multicolumn{2}{c}{ homO $^{g} \pi$} & \multicolumn{2}{c}{ hetO $^{g} \pi$} \\
& IR & Raman & IR & Raman \\
\hline Experiment & 1.5 & 1.2 & 1.8 & 1.5 \\
B3LYP-D3(BJ)/may-cc-pVTZ & 2.0 & 1.1 & 1.8 & 1.1 \\
B2PLYP-D3(BJ)/may-cc-pVTZ & 2.1 & - & 1.8 & - \\
$\omega$ B97X-D/may-cc-pVTZ & 2.2 & 1.1 & 1.8 & 1.1 \\
M06-2X/may-cc-pVTZ & 1.4 & 1.2 & 1.3 & 1.3 \\
\hline
\end{tabular}

Wahrscheinlicher erscheinen eher allerdings experimentelle und/oder quantenchemische Unsicherheiten bei der Bestimmung dieses Verhältnisses als Erklärung für diese Diskrepanz. So ist das Intensitätsverhältnis der beiden schwächeren Banden wegen ihrer geringen Intensität und der teilweisen spektralen Überlappung mit den stärkeren Banden mit einer größeren Unsicherheit behaftet. Nicht ausgeschlossen werden können auch unerkannte spektrale Überlagerungen mit weiteren Banden - für 1-Phenylethanol werden im nächsten Abschnitt Koinzidenzen mit dem $\mathrm{O}^{t} \pi$-Motiv der jeweils anderen relativen Chiralität festgestellt. Bei 1-Phenylethanol werden noch größere Abweichungen für relative Bandenstärken zwischen Theorie und Experiment gefunden. In qualitativer Übereinstimmung mit den theoretischen Vorhersagen nähert sich das Intensitätsverhältnis innerhalb der Bandenpaare im Raman-Spektrum von Benzylalkohol an.

Auf Basis der experimentellen Bandenintensitäten sowie den berechneten chirodiastaltischen Energien und IR-Aktivitäten bzw. Raman-Streuquerschnitten lassen sich nach Gl. 8.1 Konformationstemperaturen bei Annahme einer Boltzmann-Verteilung abschätzen.[132, 423, 424] Gemessen an deren Realitätsnähe lässt sich beurteilen, ob eine Methode eine plausible Vorhersage für den Energieunterschied zwischen $\operatorname{homO}^{g} \pi$ und hetO ${ }^{g} \pi$ macht.

$$
T_{\mathrm{K}}=\frac{E_{j}-E_{i}}{R}\left[\ln \left(\frac{A_{i}}{A_{j}} \cdot \frac{B_{j}}{B_{i}} \cdot \frac{g_{j}}{g_{i}} \cdot \frac{\sigma_{i}}{\sigma_{j}}\right)\right]^{-1}
$$

Diese Gleichung wird aus Umstellung von G1. 3.5 auf S. 31 erhalten, die enthaltenen Größen sind dort erklärt. Für jede Methode werden vier Konformationstemperaturen bestimmten, je zwei aus den IR- und Raman-Spektren und je zwei aus den Bandenintensitätsverhältnissen der $\mathrm{OH} \cdots \pi$ - und $\mathrm{OH} \cdots \mathrm{O}$-Wasserstoffbrïcken. Dabei wird davon ausgegangen, dass bei Vergleich der Banden für Wasserstoffbrücken mit einheitlicher Akzeptorart $(\pi / \mathrm{O})$ eine bessere Fehlerkompensation stattfindet als bei Kreuzvergleichen. Die Übersicht der erhaltenen Werte ist in Tab. 8.6 dargestellt.

Unabhängig von der Betrachtung des $\mathrm{OH} \cdots \pi$ - oder des $\mathrm{OH} \cdots \mathrm{O}$-Bandenpaares werden stets ähnliche Werte erhalten, was die Zuordnung dieser Banden weiter stützt. Auch sind die Konformationstemperaturen in den Expansionen des Popcorn- und Cur- 
Tab. 8.6: Konformationstemperaturen für das Verhältnis von homO $^{g} \pi$ und hetO ${ }^{g} \pi$ gemäß Gl. 8.1 für verschiedene quantenchemische Methoden, jeweils mit may-cc-pVTZ Basissatz. Die Werte wurden auf Basis der IR- oder Raman-Spektren, sowie der $\mathrm{OH} \cdots \pi$ - oder $\mathrm{OH} \cdots \mathrm{O}$-Bandenpaaren bestimmt bestimmt. Werte oberhalb von $20 \mathrm{~K}$ sind auf Vielfache von $10 \mathrm{~K}$ gerundet.

\begin{tabular}{lcccc}
\hline Methode & $\begin{array}{c}T_{\mathrm{K}} / \mathrm{K} \\
(\mathrm{IR}, \pi)\end{array}$ & $\begin{array}{c}T_{\mathrm{K}} / \mathrm{K} \\
(\mathrm{IR}, \mathrm{O})\end{array}$ & $\begin{array}{c}T_{\mathrm{K}} / \mathrm{K} \\
(\text { Raman, } \pi)\end{array}$ & $\begin{array}{c}T_{\mathrm{K}} / \mathrm{K} \\
(\text { Raman, O) }\end{array}$ \\
\hline B3LYP-D3(BJ) & 15 & 18 & 14 & 16 \\
B2PLYP-D3(BJ) & 8 & 10 & - & - \\
$\omega B$ 97X-D & 100 & 130 & 100 & 110 \\
M06-2X & 170 & 200 & 160 & 170 \\
\hline
\end{tabular}

ry-Jets offenbar ebenfalls sehr ähnlich. Die Verhältnisse der IR-Aktivitäten und RamanStreuquerschnitte zwischen homO ${ }^{g} \pi$ und hetO ${ }^{g} \pi$ variieren nur wenig zwischen den Methoden. Größere Unterschiede existieren dagegen bezüglich der vorhergesagten chirodiastaltischen Energie (siehe Tab. 8.3), sodass die berechnete Konformationstemperatur maßgeblich von dieser abhängt. Alle erhaltenen Temperaturen sind positiv, was zeigt, dass die Methoden die chirodiastaltische Energie qualitativ richtig mit einem negativen Vorzeichen hervorsagen. Als realistischer Bereich wird hier 60-150 K eingeschätzt. Von den getesteten Methoden liegt nur $\omega$ B97X-D in diesem Bereich mit einer vorhergesagten chirodiastaltischen Energie von -1.7 kJ mol. B3LYP-D3(BJ) und B2PLYP-D3(BJ) unterschätzen dagegen die homochirale Präferenz recht deutlich, M06-2X überschätzt sie leicht. Eine Unterschätzung wäre grundsätzlich kompatibel mit einem möglicherweise relevanten und auf dem Theorieniveau nicht berücksichtigten Effekt der Chiralitätsinduzierten Spinselektivität (Kap. 2.4). Wird umgekehrt das Intervall der Konformationstemperaturen von $60-150 \mathrm{~K}$ in Gl. 8.1 eingesetzt, wird über die spektralen Aktivitäten aller vier Methoden hinweg eine realistische Spannweite von -0.8 bis $-2.6 \mathrm{~kJ} \mathrm{~mol}^{-1}$ für die chirodiastaltische Energie erhalten.

Auf die gleiche Weise lassen sich auch Konformationstemperaturen zwischen homO ${ }^{g} \pi$ und het $\pi \pi$ bestimmen. Hierfür kann entweder die $\mathrm{OH} \cdots \pi$ - oder die $\mathrm{OH} \cdots \mathrm{O}$-Bande von homO $^{g} \pi$ genutzt werden, was zu leicht unterschiedlichen Werten führt. Eine bessere Fehlerkompensation ist für den Vergleich mit der $\mathrm{OH} \cdots \pi$-Bande zu erwarten, da die Wechselwirkung ähnlicher zu het $\pi \pi$ ist. Die Annahme für diese Berechnung ist allerdings, dass eine möglicher Beitrag von hom $\pi \pi$ vernachlässigbar ist. Dieser Einfluss, sowie die quantenchemisch gefundene erhöhte Barriere, führen zu tendenziell zu hohen Konformationstemperaturen. Die Ergebnisse sind in Tab. 8.7 zusammengestellt. Die mit B3LYPD3(BJ) und B2PLYP-D3(BJ) erhaltenen Konformationstemperaturen sind unter diesen Einschränkungen realistisch. Die aus $\omega$ B97X-D und M06-2X erhaltenen Werte sind dagegen negativ und unphysikalisch. Für Curry- und Popcorn-Jet werden erneut ähnliche Werte erhalten.

Die Angabe von Konformationstemperaturen für das Verhältnis von hetO ${ }^{g} \pi$ und het $\pi \pi$ erweist sich als nicht sinnvoll, da der Term $\left[\ln \left(\frac{A_{i}}{A_{j}} \cdot \frac{B_{j}}{B_{i}} \cdot \frac{g_{j}}{g_{i}} \cdot \frac{\sigma_{i}}{\sigma_{j}}\right)\right]^{-1}$ in diesem Fall sehr groß ist und schon kleine Fehler in der berechneten Energiedifferenz zu massiven Ausschlägen in der Konformationstemperatur führen. Dies ist ein bekanntes Problem dieser Auswertungsmethode.[132] Thermodynamisch ist dies darin begründet, dass sehr hohe (oder 
Tab. 8.7: Konformationstemperaturen für das Verhältnis von homO ${ }^{g} \pi$ und het $\pi \pi$ gemäß Gl. 8.1 für verschiedene quantenchemische Methoden, jeweils mit may-cc-pVTZ Basissatz. Die Werte wurden auf Basis der IR- oder Raman-Spektren, sowie der $\mathrm{OH} \cdots \pi$ - oder $\mathrm{OH} \cdots \mathrm{O}$-Bande von homO $^{g} \pi$ bestimmt. Werte mit Beträgen oberhalb von $20 \mathrm{~K}$ sind auf Vielfache von $10 \mathrm{~K}$ gerundet.

\begin{tabular}{lcccc}
\hline Methode & $\begin{array}{c}T_{\mathrm{K}} / \mathrm{K} \\
(\mathrm{IR}, \pi)\end{array}$ & $\begin{array}{c}T_{\mathrm{K}} / \mathrm{K} \\
(\mathrm{IR}, \mathrm{O})\end{array}$ & $\begin{array}{c}T_{\mathrm{K}} / \mathrm{K} \\
(\text { Raman, } \pi)\end{array}$ & $\begin{array}{c}T_{\mathrm{K}} / \mathrm{K} \\
(\text { Raman, O) }\end{array}$ \\
\hline B3LYP-D3(BJ) & 110 & 140 & 110 & 100 \\
B2PLYP-D3(BJ) & 150 & 190 & - & - \\
$\omega$ B97X-D & -2 & -3 & -2 & -2 \\
M06-2X & -170 & -170 & -150 & -150 \\
\hline
\end{tabular}

stark negative) Konformationstemperaturen benötigt werden, um bei einem berechneten signifikanten Energieunterschied fast gleiche beobachtete Populationen (unter Berücksichtigung von Entartungen) erklären zu können. Das beobachtete Intensitätsverhältnis ist in Kombination mit den berechneten spektralen Aktivitäten konsistent mit einem sehr kleinen Energievorteil im Bereich von wenigen Zehntel $\mathrm{kJ} \mathrm{mol}^{-1}$ zugunsten von het $\pi \pi$. Dies ist mit keiner der Vorhersagen der vier getesteten Methoden konsistent, die het $\pi \pi$ entweder als energetisch deutlich höher (B3LYP-D3(BJ). B2PLYP-D3(BJ)) oder als deutlich niedriger $(\omega \mathrm{B} 97 \mathrm{X}-\mathrm{D}, \mathrm{M} 06-2 \mathrm{X})$ vorhersagen gegenüber hetO ${ }^{g} \pi$ (Tab. A.14 auf S. 324). Unter Berücksichtigung, dass die Banden von het $\pi \pi$ durch eine kinetische Stabilisierung oder einen Beitrag von hom $\pi \pi$ tendenziell zu intensiv erscheinen, sind die Vorhersagen von B3LYP-D3(BJ) und B2PLYP-D3(BJ) plausibler.

In den Spektren lassen sich keine Hinweise darauf finden, dass eventuell auch eine Struktur des hochenergetisch vorhergesagten OV-Motivs realisiert werden könnte. Hierfür fehlen sowohl die noch etwas stärker verschoben vorhergesagten Banden der $\mathrm{OH} \cdots \mathrm{O}-$ Wasserstoffbrücken, wie auch die zugehörigen Akzeptorbanden der freien Hydroxygruppen in spektraler Nähe des Monomers.

Die Positionen aller beobachteten Banden und deren vorgeschlagenen Zuordnungen sind in im Anhang in Tab. A.16 auf S. 324 dokumentiert.

Zusammengefasst erweist sich die Beschreibung der Benzylalkohol-Dimere quantenchemisch anspruchsvoll, alle vier getesteten Methoden zeigen unterschiedliche Defizite für relative Energien, Bandenpositionen und spektrale Sichtbarkeit. Daher wird im nächsten Abschnitt ein Vergleich mit den Dimeren von 1-Phenylethanol durchgeführt. In diesem System können Banden homo- und heterochiraler Cluster klarer identifiziert und mögliche systematische Defizite der Theorie herausgestellt werden. 


\subsection{Chiralitätsdiskriminierung in 1-Phenylethanol-Dimeren}

\subsubsection{Einleitung}

1-Phenylethanol kann aus Benzylalkohol durch Methylierung am $\alpha$-Kohlenstoffatom erhalten werden. Die Implikationen auf die Konformere des Monomers wurden in Kap. 5.17 diskutiert.

Dimere von enantiomerenreinem (-)-(S)-1-Phenylethanol wurden 2007 von Shin-ya et al. quantenchemisch, in Lösung und in der flüssigen Phase untersucht.[351] Auf B3LYP/6-31+G(d,p)-Niveau wurden dabei vier Dimerstrukturen gefunden, die Kombinationen aus dem $g--$ und dem $t$-Konformer (siehe Kap. 5.17) entsprechen: $g-g-$, $g-t, t g-$ und $t t$. Diese Strukturen weisen je nur eine einzelne intermolekulare $\mathrm{OH} \cdots \mathrm{O}-$ Wasserstoffbrücke aus. Intermolekulare Isomerie wurde quantenchemisch nicht erwogen, obwohl experimentell Hinweise auf intermolekulare $\mathrm{OH} \cdots \pi$-Wasserstoffbrücken gefunden wurden. Aufgrund der Beteiligung des hochenergetischen $t$-Konformers, der geringen Kompaktheit (was vermutlich zum Teil dem bekannten Defizit von B3LYP ohne Dispersionskorrektur geschuldet ist) und der fehlenden Absättigung der zweiten Hydroxygruppe sind diese Strukturen in einer Überschallexpansion nicht zu erwarten.

Enantiomerenreines 1-Phenylethanol wurde 2010 von Falkowski am Filet-Jet untersucht.[237] Dabei wurden zwei Banden bei 3575 und $3516 \mathrm{~cm}^{-1}$ einem oder zwei Dimeren zugeordnet, aber nicht näher analysiert. Gemischte Dimere mit anderen Molekülen wie Limonen [237], 2-Butanol [398-400, 425, 426], 2-Butylamin [399, 426], Wasser [349, 398, 425-427] und $\alpha$-Pinen (Kap. 6) wurden intensiver ausgewertet. Auch eng verwandte Derivate von 1-Phenylethanol, wie 1-Phenyl-1-propanol [426-429], 2Naphthyl-1-ethanol [239, 379, 430], 1-Indanol [240, 427, 428] und verschiedene halogenierte 1-Phenylethanole [356, 357, 359, 403] waren Bestandteile von weiteren untersuchten gemischten Dimeren. Die Chiralitätsdiskriminierung bei der Dimerisierung aus zwei identischen/spiegelbildlichen Derivaten wurde im Fall von 1-Indanol [70, 363] intensiv untersucht und wird an späterer Stelle mit der von 1-Phenylethanol verglichen. Das von Borho [37] am Filet-Jet gemessene 3-Buten-2-ol entspricht einem Ausschnitt von 1-Phenylethanol (mit einer Ethenyl- statt einer Phenylgruppe) und zeigt Analogien im Cluster-Spektrum, dieses wurde aber bisher nicht näher ausgewertet. Die Kristallstruktur von 1-Phenylethanol ist nicht bekannt.

\subsubsection{Quantenchemische Rechnungen}

Die Struktursuche für Dimere von 1-Phenylethanol erfolgte analog zu der für Benzylalkohol. Eine Übersicht über die 30 gefundenen 1-Phenylethanol-Dimere auf B3LYPD3(BJ)/may-cc-pVTZ-Niveau bietet Tab. 8.8. Ergebnisse für die wichtigsten Strukturen mit weiteren Methoden sind im Anhang auf S. 325 in Tab. A.17 und A.18 zusammengefasst. Die Nomenklatur erfolgt analog zu den Benzylalkohol-Dimeren. Aufbauend auf der Betrachtung des Monomers in Kap. 5.17 wird das stabilste 1-Phenylethanol Monomer-Konformer als $g$ bezeichnet (entspricht $G-g$ - für (-)-(S)-1-Phenylethanol) und das trans-Konformer als $t$. Die weniger stabilen gauche-Konformere werden als $g^{\prime}$ zusammengefasst (diese entsprechen $X g+$ für $(-)-(S)-1-P h e n y l e t h a n o l)$. Werden voran- 
gestellt keine Konformere angegeben, so wird impliziert, dass beide Moleküle in der stabilsten Konformation $g$ vorliegen. In einem energetischen Bereich von etwa $3 \mathrm{~kJ} \mathrm{~mol}^{-1}$ wurden ausschließlich $g g$-Dimere gefunden. Für die anderen vorhergesagten, hochenergetischen Monomer-Konformere ist damit auch als Bestandteil eines Dimers kein Nachweis zu erwarten.

Die jeweils drei stabilsten homo- und heterochiralen Dimere und deren wichtigste schwingungsspektroskopische Eigenschaften sind in Abb. 8.6 dargestellt. Ein Vergleich mit den Benzylalkohol-Dimeren in Abb. 8.1 auf Seite 224 zeigt, dass die strukturellen Analogien sehr weitgehend sind. Diese sind darin begründet, dass sich in der relevanten $g$-Konformation die Methylgruppe auf der gegenüberliegenden Molekülseite in Bezug auf die Hydroxygruppe befindet und daher nicht unmittelbar sterisch die Aggregation beeinflusst. Mittelbar führt die Methylgruppe aber über Veränderung des intramolekularen Torsionspotentials, sowie durch den induktiven Effekt und zusätzliche Dispersionswechselwirkung, zu energetischen Verschiebungen zwischen den Konformeren, welche an späterer Stelle in Abschnitt 8.6 im Detail diskutiert werden. Das $\alpha$ Kohlenstoffatom wird ferner durch die Methylierung zu einem stereogenen Zentrum, sodass sich die Chiralitätssynchronisation in Benzylalkohol zu einer Chiralitätsdiskriminierung in 1-Phenylethanol wandelt. Unterschieden werden muss zwischen den Vorzeichen der $\mathrm{HOC}_{1} \mathrm{C}_{2}$ - und $\mathrm{OC}_{1} \mathrm{C}_{2} \mathrm{C}_{3}$-Torsionswinkel (über die in den Benzylalkohol-Dimeren die relative transiente Chiralität bestimmt wurde) und der relativen permanenten Chiralität der 1-Phenylethanol-Moleküle. Die Ergebnisse dieser beiden Betrachtungsweisen stimmen allerdings für die stabileren 1-Phenylethanol-Dimeren überein, die aus zwei $g$ Konformeren bestehen. Die Methylgruppe legt als konformationeller Anker das Vorzei-

Tab. 8.8: Nullpunktskorrigierte Energien von 1-Phenylethanol-Dimeren auf B3LYP-D3(BJ)/maycc-pVTZ-Niveau, jeweils relativ zum homochiralen bzw. heterochiralen globalen Minimum. Das heterochirale globale Minimum liegt $0.9 \mathrm{~kJ} \mathrm{~mol}^{-1}$ oberhalb des homochiralen. Für die Nomenklatur siehe Fließtext.

\begin{tabular}{|c|c|c|c|}
\hline hom-Konformer & $E_{0}^{\mathrm{rel}} / \mathrm{kJ} \mathrm{mol}^{-1}$ & het-Konformer & $E_{0}^{\mathrm{rel}} / \mathrm{kJ} \mathrm{mol}^{-1}$ \\
\hline $\mathrm{O}^{g} \pi$ & 0 & $\mathrm{O}^{g} \pi$ & 0 \\
\hline$\pi \pi C_{2}$ & 1.0 & $\pi \pi C_{\mathrm{i}}$ & 1.1 \\
\hline $\mathrm{O}^{t} \pi$ & 1.9 & $\mathrm{O}^{t} \pi$ & 1.8 \\
\hline $\mathrm{O}^{g} \mathrm{~V} \mathrm{~T}$ & 2.6 & $\mathrm{O}^{g} \mathrm{~V} \mathrm{~T}$ & 2.3 \\
\hline $\mathrm{O}^{t} \mathrm{~V} \mathrm{~T}$ & 4.4 & $\mathrm{gg}^{\prime} \mathrm{O}^{g} \pi$ & 3.3 \\
\hline $\mathrm{gg}^{\prime} \mathrm{O}^{g} \pi$ & 6.0 & $\mathrm{O}^{t} \mathrm{~V} \mathrm{~T}$ & 3.9 \\
\hline $\mathrm{g}^{\prime} \mathrm{g} \mathrm{O}^{g} \pi$ & 6.7 & $\mathrm{~g}^{\prime} \mathrm{g} \mathrm{O}^{t} \pi$ & 6.7 \\
\hline $\mathrm{O}^{g} \mathrm{~V}$ & 7.0 & $g^{\prime} g \pi \pi$ & 7.7 \\
\hline$g^{\prime} g \pi \pi$ & 7.8 & gg' $\mathrm{O}^{t} \pi$ & 7.9 \\
\hline $\operatorname{gg}^{\prime} \mathrm{O}^{t} \pi$ & 8.9 & gt $\mathrm{O} \pi$ & 8.4 \\
\hline gt $\mathrm{O} \pi$ & 9.0 & $\mathrm{gt} \mathrm{O} \pi$ & 10.3 \\
\hline $\mathrm{g}^{\prime} \mathrm{g} \mathrm{O}^{t} \pi$ & 9.2 & $\mathrm{~g}^{\prime} \mathrm{g}^{\prime} \mathrm{O}^{g} \pi$ & 10.9 \\
\hline $\mathrm{gt} \mathrm{O} \pi$ & 10.9 & $g^{\prime} g^{\prime} \pi \pi$ & 14.0 \\
\hline $\mathrm{g}^{\prime} \mathrm{g}^{\prime} \mathrm{O}^{g} \pi$ & 11.2 & $\mathrm{~g}^{\prime} \mathrm{g}^{\prime} \mathrm{O}^{t} \pi$ & 15.4 \\
\hline $\mathrm{g}^{\prime} \mathrm{g}^{\prime} \pi \pi C_{2}$ & 14.2 & & \\
\hline $\mathrm{g}^{\prime} \mathrm{g}^{\prime} \mathrm{O}^{t} \pi$ & 14.7 & & \\
\hline
\end{tabular}



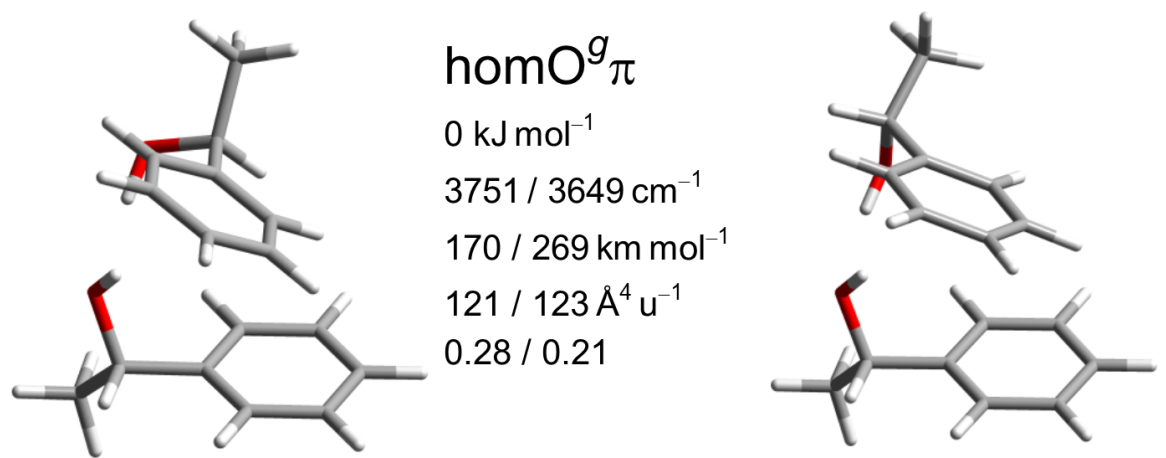

hetO $^{g} \pi$

$0.9 \mathrm{~kJ} \mathrm{~mol}^{-1}$

$3742 / 3636 \mathrm{~cm}^{-1}$

178 / $297 \mathrm{~km} \mathrm{~mol}^{-1}$

$122 / 132 \AA^{4} u^{-1}$

$0.28 / 0.21$

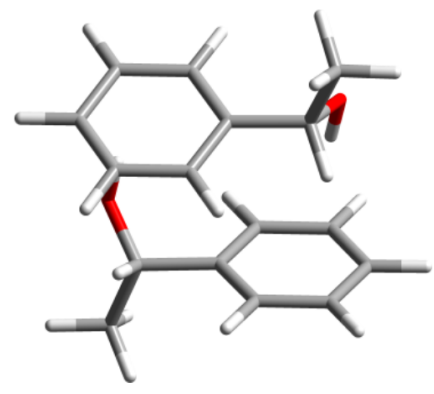

hom $\pi \pi C_{2}$

$1.0 \mathrm{~kJ} \mathrm{~mol}^{-1}$

$3761 / 3757 \mathrm{~cm}^{-1}$

$386 / 2 \mathrm{~km} \mathrm{~mol}^{-1}$

$1 / 343 \AA^{4} u^{-1}$

$0.75 / 0.28$

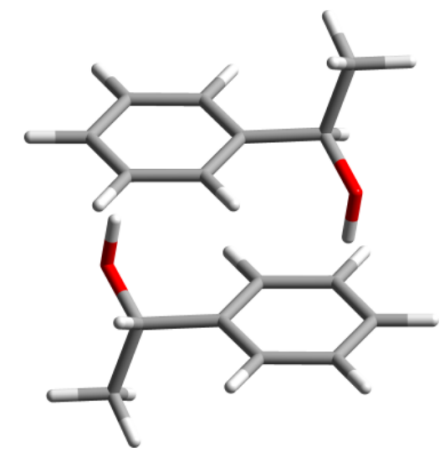

het $\pi \pi C_{\mathrm{i}}$

$2.0 \mathrm{~kJ} \mathrm{~mol}^{-1}$

$3754 / 3750 \mathrm{~cm}^{-1}$

$478 / 0 \mathrm{~km} \mathrm{~mol}^{-1}$

$0 / 400 \AA^{4} u^{-1}$

$0 / 0.30$
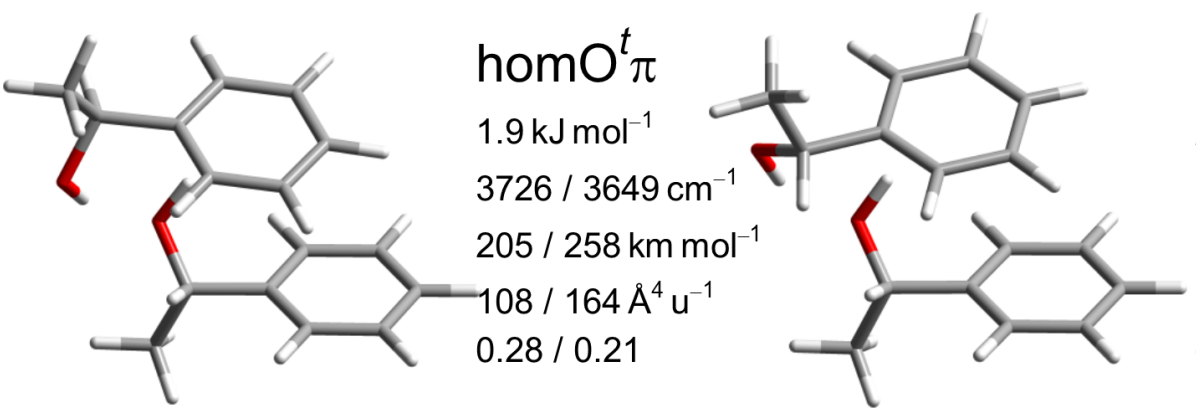

$\operatorname{hetO}^{t} \pi$

$2.7 \mathrm{~kJ} \mathrm{~mol}^{-1}$

$3729 / 3654 \mathrm{~cm}^{-1}$

$198 / 259 \mathrm{~km} \mathrm{~mol}^{-1}$

$106 / 164 \AA^{4} u^{-1}$

$0.28 / 0.21$

Abb. 8.6: Nullpunktskorrigierte relative Energien, harmonische OH-Streckschwingungswellenzahlen, IR-Intensitäten, Raman-Aktivitäten und Depolarisationsverhältnisse von 1-PhenylethanolDimeren auf B3LYP-D3(BJ)/may-cc-pVTZ-Niveau.

chen der Torsionswinkel effektiv fest $(G-g-$ für $(-)-1-P h e n y l e t h a n o l ~ u n d ~ G+g+$ für $(+)$ 1-Phenylethanol), wie in Kap. 5.17 beschrieben wurde.

Während hetO ${ }^{g} \pi$ in Benzylalkohol durch die mögliche Relaxation in homO ${ }^{g} \pi$ nur schwach populiert ist, kann die Konzentration dieses Konformers in 1-Phenylethanol über die Enantiomerenzusammensetzung kontrolliert werden. Die auf diese Weise bestimmten Bandenpositionen können mit denen von homO ${ }^{g} \pi$ und den theoretischen Vorhersagen verglichen werden, um so Rückschlüsse auf die Plausibilität der Zuordnung in Benzylalkohol zu erhalten. Entsprechendes gilt auch für die getrennte spektrale Charakterisierung von hom $\pi \pi$ und het $\pi \pi$. Für alle drei hier näher betrachteten Motive wird von den Methoden fast einheitlich eine homochirale Präferenz vorhergesagt. Dies lässt sich aber wegen der nicht möglichen Gleichgewichtseinstellung bei 1-Phenylethanol mit den hier verwendeten Methoden experimentell nicht prüfen. Hierfür müssten die Bindungsenergien der Komplexe mit anderen Methoden [393, 394] direkt bestimmt und verglichen werden.

Im Vergleich zu Benzylalkohol werden die Varianten des $\mathrm{O}^{t} \pi$-Motivs energetisch abgesenkt, sodass diese auch nachweisbar populiert sein könnten. Interessant ist, dass die 
vorhergesagten Energieabstände zwischen den Strukturmotiven innerhalb der homo- und heterochiralen Cluster sehr ähnlich sind (zumindest auf B3LYP-D3(BJ)-Niveau), sodass auch vergleichbare Intensitäten zu erwarten wären. Dies gilt aber nur bei Vernachlässigung von kinetischen Aspekten. Bezüglich der berechneten Umwandlungsbarrieren (Abb. 8.7) ergibt sich ein analoges Bild zu Benzylalkohol. Trotz der verhinderten Umwandlung zwischen homo- und heterochiralen Strukturen bleibt mindestens ein günstiger Relaxationspfad für jede Struktur offen - aber erneut mit Ausnahme von het $\pi \pi$, welches auch bei 1-Phenylethanol eine erhöhte Barriere für die Relaxation in das (heterochirale) globale Minimum aufweist.

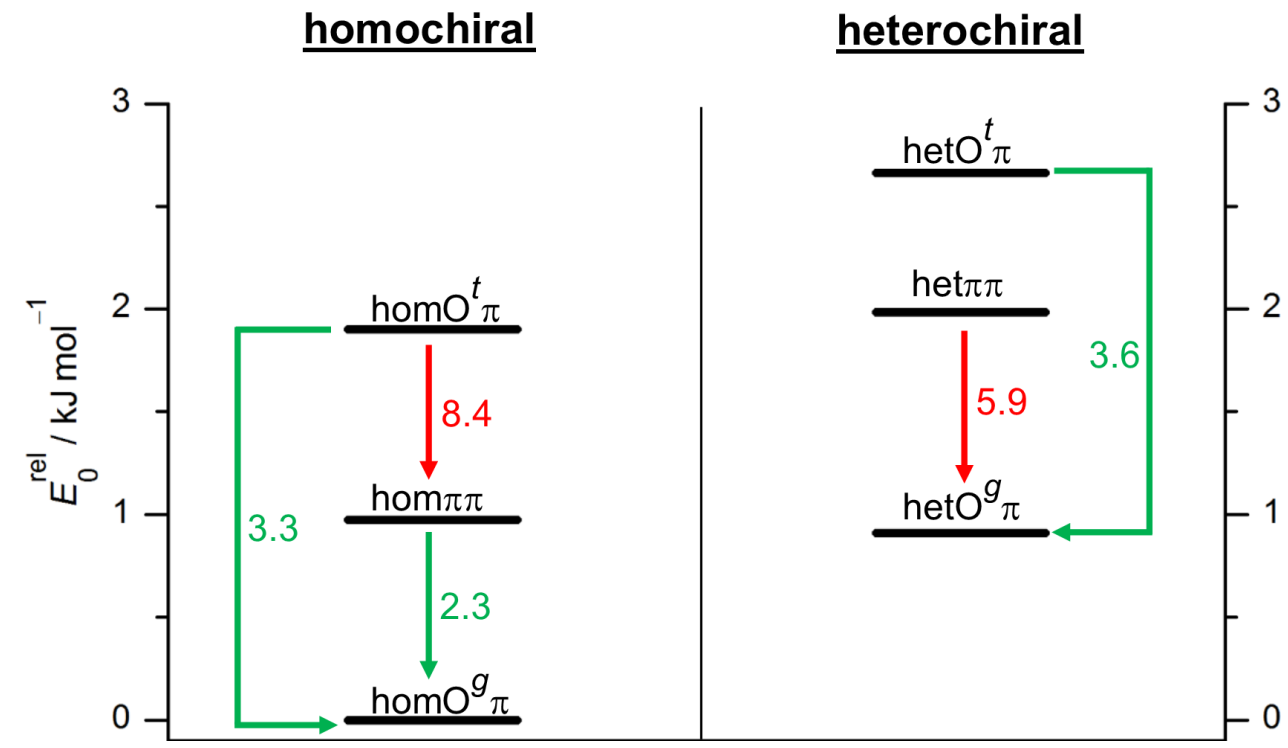

Abb. 8.7: Schwingungsnullpunktskorrigierte relative Energien und Barrierenhöhen in $\mathrm{kJ} \mathrm{mol}^{-1}$ von 1-Phenylethanol-Dimeren auf B3LYP-D3(BJ)/may-cc-pVTZ-Niveau. Die Barrieren beziehen sich auf Relaxation des instabileren Konformers und sind farblich gekennzeichnet, ob sie unterhalb (grün) oder oberhalb (rot) des Richtwertes von $5 \mathrm{~kJ} \mathrm{~mol}^{-1}$ [23] liegen.

\subsubsection{Schwingungsspektren}

Abb. 8.8 gibt einen Überblick über den OH-Streckschwingungsbereich von 3670$3240 \mathrm{~cm}^{-1}$ für Expansionen von 1-Phenylethanol in Helium. Die IR-Spektren wurden am Filet-Jet aufgenommen, die Raman-Spektren am Curry-Jet. Im Vergleich ist davon auszugehen, dass die Expansionen am Filet-Jet verdünnter und stärker gekühlt sind. 1Phenylethanol wurde sowohl enantiomerenrein, wie auch racemisch expandiert. Da die Diastereo- und Enantiomerenreinheiten (bzw. der racemische Charakter) der eingesetzten Proben sehr hoch sind (siehe Kap. 3.5), entspricht das Spektrum der enantiomerenreinen Substanz in guter Näherung dem der homochiralen Cluster. Die künstlichen Spektren der heterochiralen Cluster können in diesem Fall über einfache Linearkombinationen gebildet werden. Für die Dimere erfolgte dies über $I_{\text {het }}^{\mathrm{D}}(\tilde{v})=2 I_{\text {rac }}(\tilde{v})-I_{\text {hom }}(\tilde{v})$ und für die Trimere nach $I_{\text {het }}^{\operatorname{Tr}}(\tilde{v})=\frac{4}{3} I_{\operatorname{rac}}(\tilde{v})-\frac{1}{3} I_{\text {hom }}(\tilde{v})($ gemäß Gl. 2.2 auf S. 10).

In der IR-detektierten Expansion der enantiomerenreinen Substanz werden vier Banden beobachtet. Dazu zählen die bereits in Kap. 5.17 diskutierte Monomerbande bei 
8 Komplementarität in Dimeren von Benzylalkohol und 1-Phenylethanol

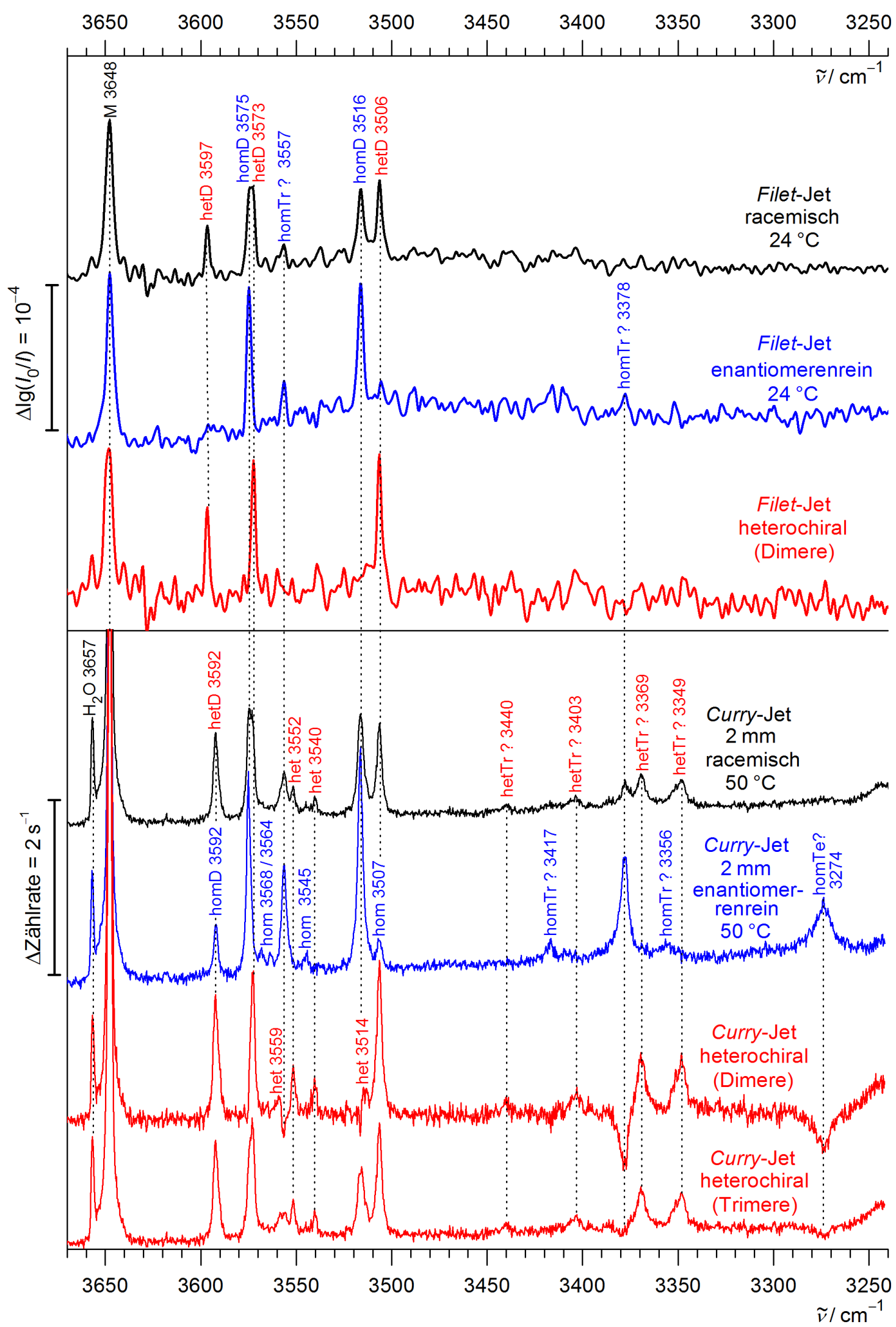

Abb. 8.8: Experimentelle IR- und Raman-Spektren von 1-Phenylethanol im OHStreckschwingungsbereich. Die Banden sind mit der relativen Chiralität, teilweise mit der vermuteten Clustergröße und mit ihrer Position in $\mathrm{cm}^{-1}$ gekennzeichnet. M kennzeichnet Monomere, D Dimere, Tr Trimere und Te Tetramere. 
$3648 \mathrm{~cm}^{-1}$ und zwei intensive Banden bei 3575 und $3516 \mathrm{~cm}^{-1}$, die auch bereits von Falkowski [237] beobachtet und Dimeren zugeordnet wurden. Diese Zuordnung wird hier weiter durch die Beobachtung gestützt, dass sich Bandenintensitäten in der Expansion der racemischen Substanz halbieren, wie es bei statistischer Bildung zu erwarten ist. Die weitgehende Analogie zu Benzylalkohol legt eine analoge Zuordnung zu homO ${ }^{g} \pi$ nahe. Eine bisher nicht beschriebene, weniger intensive Bande wird bei $3557 \mathrm{~cm}^{-1}$ gefunden, deren Intensität sich ebenfalls zu halbieren scheint. Allerdings ist sie in der Expansion der racemischen Substanz praktisch auf das Rauschlevel abgefallen, sodass diese Bewertung unsicher ist. In der höher konzentrierten Raman-detektierten Expansion zeigt die Bande dagegen eher eine Konzentrationsabhängigkeit, die einem Trimer entspricht. Auch bei Benzylalkohol wurde eine Bande an ähnlicher Position $\left(3561 \mathrm{~cm}^{-1}\right)$ gefunden, die als Trimer bewertet wurde. Sofern das Trimer nicht (näherungsweise) $C_{3}$-symmetrisch ist (die hohe Wellenzahl spricht eher für eine $\mathrm{OH} \cdots \pi$-Wasserstoffbrücke und steht damit dem entgegen), so wären aber noch weitere Banden der Struktur zu erwarten. Die im RamanSpektrum intensive Trimer-Bande bei $3378 \mathrm{~cm}^{-1}$ könnte sich auch im IR-Spektrum andeuten.

Aus der breiten Bande bei $3574 \mathrm{~cm}^{-1}$ im Spektrum der racemischen Mischung wird durch die Subtraktion des homochiralen Spektrums eine neue schmale Bande bei $3573 \mathrm{~cm}^{-1}$ erhalten. Diese ist somit, wie eine zweite intensive Bande bei $3506 \mathrm{~cm}^{-1}$, leicht tiefverschoben zu einer homochiralen Entsprechung. Die Konzentrationsabhängigkeit dieser Banden kann an dieser Stelle nicht herangezogen werden, allerdings lässt der Vergleich mit Benzylalkohol und den quantenchemischen Rechnungen auf hetO ${ }^{g} \pi$ schließen. Im IR-Spektrum der heterochiralen Cluster wird eine hochfrequente Bande bei $3597 \mathrm{~cm}^{-1}$ gefunden, die bei Raman-Detektion nicht beobachtet wird. Diese lässt sich daher klar der antisymmetrischen $\mathrm{OH}-$ Streckschwingung von het $\pi \pi$ zuordnen.

Wegen der höheren Konzentration, der wahrscheinlich höheren Konformationstemperatur, sowie wegen teilweise komplementärer Auswahlregeln, lassen sich in den RamanSpektren eine Reihe weiterer Banden beobachten. So werden die Banden der symmetrischen Streckschwingungen von hom $\pi \pi$ und het $\pi \pi$ übereinstimmend bei $3592 \mathrm{~cm}^{-1}$ gefunden, allerdings für den heterochiralen Komplex in deutlich höherer Intensität. Weitere schwache Banden im hochfrequenten Teil des Spektralbereichs liegen für homochirale Cluster bei 3568, 3564, 3545 und $3507 \mathrm{~cm}^{-1}$, und für heterochirale Cluster bei 3559 , 3552, 3540 und $3514 \mathrm{~cm}^{-1}$. Gemessen an den Positionen könnte es sich um Banden hochenergetischer Dimere, mutmaßlich des $\mathrm{O}^{t} \pi$-Motivs, und/oder Banden von kooperativ verstärkten $\mathrm{OH} \cdots \pi$-Wasserstoffbrücken von Trimeren handeln. Stärker verschoben, im erwarteten Bereich von $\mathrm{OH}$... O-Wasserstoffbrücken von Trimeren und Tetrameren, finden sich ebenso weitere Banden: homochiral bei 3417, 3378, 3356 und $3274 \mathrm{~cm}^{-1}$, sowie heterochiral bei $3440,3403,3369$ und $3349 \mathrm{~cm}^{-1}$. Die intensive Bande im homochiralen Spektrum bei $3378 \mathrm{~cm}^{-1}$ hat eine spektrale Entsprechung bei Benzylalkohol bei $3374 \mathrm{~cm}^{-1}$ (Abb. 8.4 auf S. 229). Dies könnte ein Indiz dafür sein, dass auch Benzylalkohol-Trimere vornehmlich homochiral synchronisiert sein könnten - wie die Dimere und die Kristallstruktur. Die Bande im Spektrum der homochiralen Cluster bei $3274 \mathrm{~cm}^{-1}$ ist eher als Tetramer denn als Trimer zu werten - einerseits wegen ihrer hohen Tiefverschiebung und Halbwertsbreite, anderseits auf Grund des Umstandes, dass sie im Spektrum racemischer Cluster nicht beobachtet wird (im Gegensatz zur Bande bei $3378 \mathrm{~cm}^{-1}$ ). Ob der Anstieg der Basislinie am niederfrequenten Ende des untersuchten 


\section{Komplementarität in Dimeren von Benzylalkohol und 1-Phenylethanol}

Spektralbereichs als abgeschnittene Bandenformation oder Artefakt zu werten ist, bleibt unklar.

Zusammengefasst zeigen sich im Bereich größerer Cluster sogar noch größere Unterschiede zwischen homo- und heterochiralen Kombinationen als im Dimerbereich, was eine zukünftige Untersuchung lohnenswert erscheinen lässt. Wie für Benzylalkohol bieten sich weitere Raman-Messungen bei niedrigeren Wellenzahlen an, um den $\mathrm{OH}-$ Streckbereich komplett zu erfassen. Ein Vergleich von Raman- und IR-Detektion für größere Cluster ist wegen der niedrigen Konzentration am Filet-Jet an dieser Stelle nicht möglich. Der Befund bei Benzylalkohol lässt aber auch für 1-Phenylethanol große Komplementarität zum Raman-Spektrum erwarten, die in Zukunft mit höher konzentrierten Messungen am Popcorn-, Gratin- oder Risotto-Jet erfasst werden könnte.

Eine Detailansicht des Dimerbereiches, experimentell und simuliert auf B3LYPD3(BJ)/may-cc-pVTZ-Niveau, ist in Abb. 8.9 gezeigt. Die Zuordnung der Banden bei 3575 und $3516 \mathrm{~cm}^{-1} \mathrm{zu}$ homO $^{g} \pi$, sowie der Banden bei 3573 und $3516 \mathrm{~cm}^{-1} \mathrm{zu}$ hetO$^{g} \pi$, ist naheliegend. Wie bei Benzylalkohol werden dabei die Verschiebungen der Banden der $\mathrm{OH}$-... O-Wasserstoffbrücken überschätzt, bei gleichzeitig guter Vorhersage der Aufspaltung zwischen den homo- und heterochiralen Varianten. Dies gilt auch für die Unterschätzung der Verschiebungen der zugehörigen kooperativ eingebundenen $\mathrm{OH} \cdots \pi$ Oszillatoren. Dabei wird, erneut wie bei Benzylalkohol, die Position für homO ${ }^{g} \pi$ deutlicher unterschätzt. Die Analogie der beiden Systeme setzt sich in der Überschätzung der Verschiebungen der Banden des $\pi \pi$-Motivs fort, sodass auch hier, im Rahmen der harmonischen Näherung, eine falsche Abfolge von het $\pi \pi$ und homO ${ }^{g} \pi$ vorhergesagt wird. Die beobachtete Davydov-Aufspaltung von het $\pi \pi\left(5 \mathrm{~cm}^{-1}\right)$ wird auch hier gut vorhergesagt $\left(4.7 \mathrm{~cm}^{-1}\right)$. Die symmetrischen Streckschwingungen von het $\pi \pi$ und hom $\pi \pi$ koinzidieren, was von keiner der quantenchemischen Methoden antizipiert wird, am ehesten noch von B2PLYP-D3(BJ) $\left(\Delta \omega=4 \mathrm{~cm}^{-1}\right)$. Die aus der Chiralitätsdiskriminierung resultierenden Verschiebungen von Banden der verschiedenen Motive sind in Tab. 8.9 zusammengefasst.

Interessanterweise wird die antisymmetrische Streckschwingung von hom $\pi \pi$ nicht im IR-Spektrum beobachtet, während die Banden von het $\pi \pi$ mit beiden Detektionsmethoden deutlich intensiver erscheinen, als gemäß den Erwartungen aus den angenommenen Boltzmann-Verteilungen auf B3LYP-D3(BJ)-Niveau. Am Curry-Jet wird dagegen hom $\pi \pi$ beobachtet, was auf eine höhere Konformationstemperatur und vor allem das

Tab. 8.9: Differenzen (hom - het) der OH-Streckschwingungswellenzahlen in $\mathrm{cm}^{-1}$ bei Wechsel der relativen Chiralität in 1-Phenylethanol Dimere-Motiven im Vergleich zwischen Experiment und harmonischer Theorie.

\begin{tabular}{lccc}
\hline Methode & $\begin{array}{c}\pi \pi \\
\text { symm. }\end{array}$ & $\begin{array}{c}\mathrm{O}^{g} \pi \\
\mathrm{OH} \cdots \pi\end{array}$ & $\begin{array}{c}\mathrm{O}^{g} \pi \\
\mathrm{OH} \cdots \mathrm{O}\end{array}$ \\
\hline Experiment & 0 & 2 & 10 \\
B3LYP-D3(BJ)/may-cc-pVTZ & 7 & 8 & 13 \\
B2PLYP-D3(BJ)/may-cc-pVTZ & 4 & 9 & 12 \\
$\omega$ B97X-D/may-cc-pVTZ & 9 & 13 & 10 \\
M06-2X/may-cc-pVTZ & -11 & 4 & 9 \\
\hline
\end{tabular}




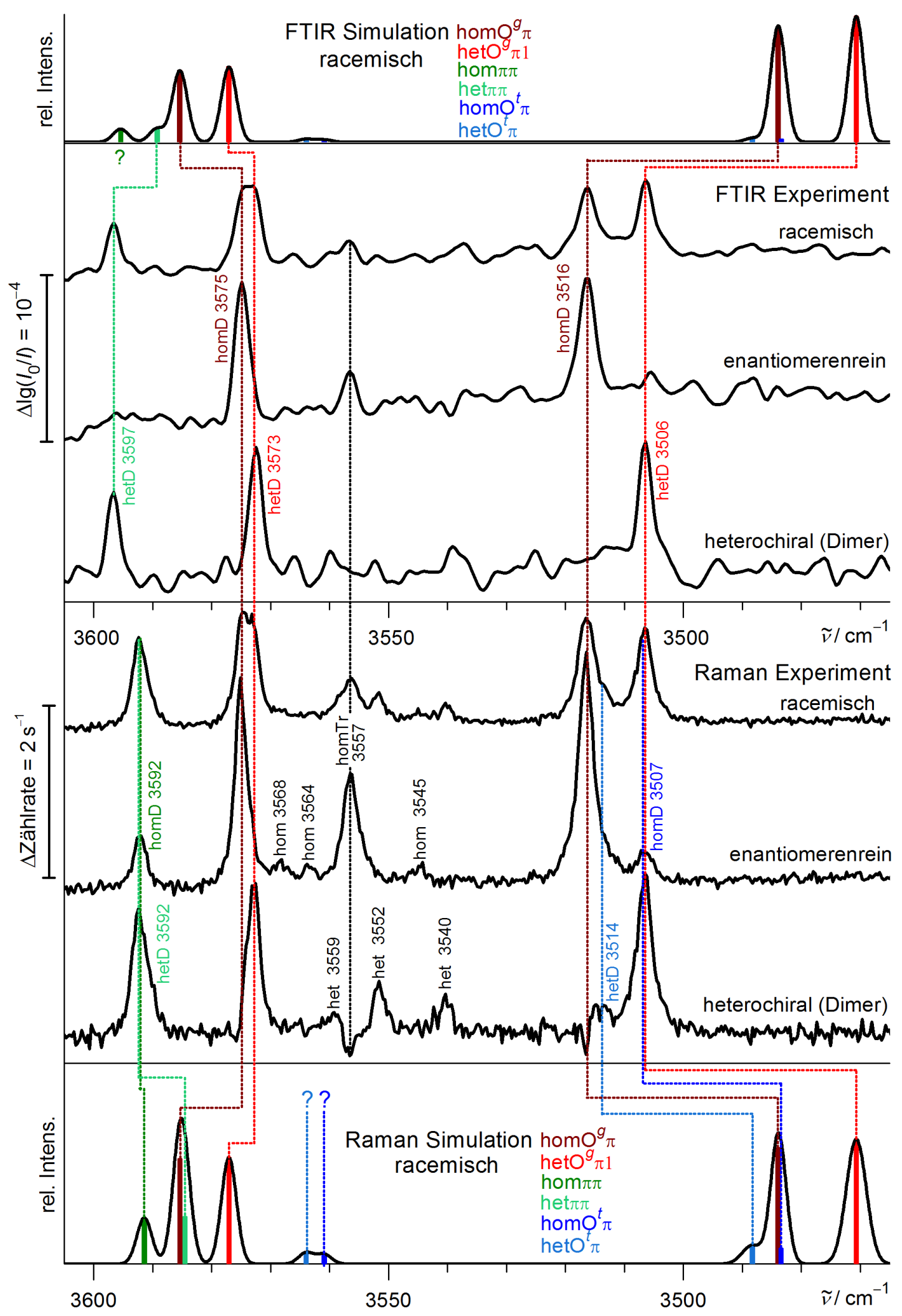

Abb. 8.9: Simulierte und experimentelle IR- und Raman-Spektren von 1-Phenylethanol im DimerBereich. Simulationsparameter für die racemische Mischung auf Basis von B3LYP-D3(BJ)/maycc-pVTZ sind einheitliche Verschiebung der harmonischen Wellenzahlen um die Differenz von experimenteller und harmonischer $g$-Monomerwellenzahl $\left(-165.2 \mathrm{~cm}^{-1}\right)$, zwei getrennte Gleichgewichte (hom/het) mit Konformationstemperaturen von $64 \mathrm{~K}$ (IR) bzw. $100 \mathrm{~K}$ (Raman) und GaussProfile mit einer vollen Halbwertsbreite von $3 \mathrm{~cm}^{-1}$. 
Tab. 8.10: Konformationstemperaturen für die Verhältnisse von homO ${ }^{g} \pi \mathrm{zu}$ hom $\pi \pi$ (obere Hälfte), sowie von hetO ${ }^{g} \pi$ und het $\pi \pi$ (untere Hälfte), gemäß Gl. 8.1 für verschiedene quantenchemische Methoden, jeweils mit may-cc-pVTZ Basissatz. Die Werte wurden auf Basis der IR- oder Raman-Spektren, sowie der $\mathrm{OH} \cdots \pi$ - oder $\mathrm{OH} \cdots \mathrm{O}-\mathrm{Bande}$ von hom- bzw. hetO ${ }^{g} \pi$ bestimmt. Die Temperaturen sind auf Vielfache von $10 \mathrm{~K}$ gerundet.

\begin{tabular}{lcccc}
\hline Methode & $\begin{array}{c}T_{\mathrm{K}} / \mathrm{K} \\
(\mathrm{IR}, \pi)\end{array}$ & $\begin{array}{c}T_{\mathrm{K}} / \mathrm{K} \\
(\mathrm{IR}, \mathrm{O})\end{array}$ & $\begin{array}{c}T_{\mathrm{K}} / \mathrm{K} \\
(\text { Raman, } \pi)\end{array}$ & $\begin{array}{c}T_{\mathrm{K}} / \mathrm{K} \\
(\text { Raman, O) }\end{array}$ \\
\hline B3LYP-D3(BJ) & $<70$ & $<90$ & 70 & 60 \\
B2PLYP-D3(BJ) & $<70$ & $<100$ & - & - \\
$\omega$ B97X-D & $<60$ & $<90$ & 60 & 50 \\
M06-2X & -150 & $>-150$ & -130 & -130 \\
\hline B3LYP-D3(BJ) & 180 & 320 & 280 & 190 \\
B2PLYP-D3(BJ) & 180 & 290 & - & - \\
$\omega$ B97X-D & -240 & -490 & -560 & -490 \\
M06-2X & -800 & -960 & -750 & -760 \\
\hline
\end{tabular}

weit bessere Signal-zu-Rausch-Verhältnis zurückgeführt werden kann. Analog zu Benzylalkohol lassen sich Konformationstemperaturen für das Verhältnis zwischen dem $\mathrm{O}^{g} \pi$ und dem $\pi \pi$-Motiv bestimmen, nun allerdings getrennt für die homo- und heterochiralen Kombinationen. Mit dem erzielten Signal-zu-Rausch-Verhältnis kann die Intensität der erwarteten IR-aktiven Bande von hom $\pi \pi$ nicht mehr als $20 \%$ betragen, im Vergleich zu den Banden von homO ${ }^{g} \pi$. Die aus diesem Verhältnis bestimmte Konformationstemperatur stellt somit eine Obergrenze dar. Die Ergebnisse für die Konformationstemperaturen sind in Tab. 8.10 zusammengestellt. Die erhaltenen Temperaturen für das Verhältnis der homochiralen Konformere sind realistisch auf B3LYP-D3(BJ), B2PLYP-D3(BJ) und $\omega$ B97X-D-Niveau. Für M06-2X werden dagegen unphysikalische stark negative Temperaturen erhalten, was bedeutet, dass das Experiment der Vorhersage der Methode (hom $\pi \pi$ als globales Minimum) widerspricht. Für die heterochiralen Konformere sind die Konformationstemperaturen für B3LYP-D3(BJ) und B2PLYP-D3(BJ) relativ hoch, aber nicht oberhalb der eingesetzten Düsentemperaturen als plausible Obergrenzen (298 K für die IR-Spektren, $333 \mathrm{~K}$ für die Raman-Spektren), abgesehen von einer einzelnen geringfügigen Überschreitung. Dies ist qualitativ kompatibel mit der quantenchemisch gefundenen erhöhten Barriere. Die Konformationstemperaturen für $\omega$ B97X-D und M06-2X sind dagegen stark negativ, was deren Vorhersagen für het $\pi \pi$ als heterochirales globales Minimum widerlegt.

Im Vergleich zu Benzylalkohol wird bei 1-Phenylethanol laut Vorhersage das $\mathrm{O}^{t} \pi$ Motiv energetisch abgesenkt, sodass zumindest in der wärmeren Expansion des Curry-Jets eine nachweisbare Population möglich erscheint. Als plausible Positionen für deren $\mathrm{OH}$. . O-Fundamentalen erscheinen die Banden bei $3514 \mathrm{~cm}^{-1}$ (heterochiral) und $3507 \mathrm{~cm}^{-1}$ (homochiral). Die erstgenannte Bande hat durch eine nicht perfekte Subtraktion des Spektrums der homochiralen Cluster eine unregelmäßige Form und wird vermutlich in ihrer Intensität als zu gering wiedergeben, da die Konzentration von 1Phenylethanol in der enantiomerenreinen Expansion geringfügig höher erscheint. Die 
Bande bei $3507 \mathrm{~cm}^{-1}$ im Spektrum der homochiralen Cluster koinzidiert mit der intensiven Bande von hetO ${ }^{g} \pi$, sodass auch diese Struktur als alternative Erklärung für die Bande in Folge einer Enantiomerenverunreinigung diskutiert werden kann. Dagegen spricht aber die vom Hersteller angegebene hohe Enantiomerenreinheit der eingesetzten Substanz, die statistisch einen Anteil von heterochiralen Dimeren von nur $0.4 \%$ erwarten lässt. Zudem wäre in diesem Fall die zweite Bande von hetO ${ }^{g} \pi$ in ähnlicher Intensität bei $3573 \mathrm{~cm}^{-1}$ zu erwarten, was nicht der Fall ist. Für die Zuordnung der $\mathrm{OH} \cdots \pi$-Fundamentalen von hom- und hetO ${ }^{t} \pi$ gibt es jeweils mehrere plausible Kandidaten im Bereich zwischen $3570-3540 \mathrm{~cm}^{-1}$, für die wegen der größeren Unsicherheiten in den berechneten Verschiebungen und Intensitäten keine nähere Zuordnung getroffen werden kann.

Die getroffenen Zuordnungen für Benzylalkohol und 1-Phenylethanol lassen sich durch den direkten Vergleich der beiden Systeme weiter erhärten. In Abb. 8.10 ist eine Gegenüberstellung der Raman-Spektren und der zugehörigen Simulationen gezeigt. Das $\mathrm{O}^{t} \pi$-Motiv wird hierfür vernachlässigt. Durch die formale doppelte Methylierung des Benzylalkohol-Dimers zum 1-Phenylethanol-Dimer werden die $\mathrm{OH} \cdots \mathrm{O}$ Wasserstoffbrücken des $\mathrm{O}^{g} \pi$-Motivs leicht zu höheren Wellenzahlen verschoben, wäh-

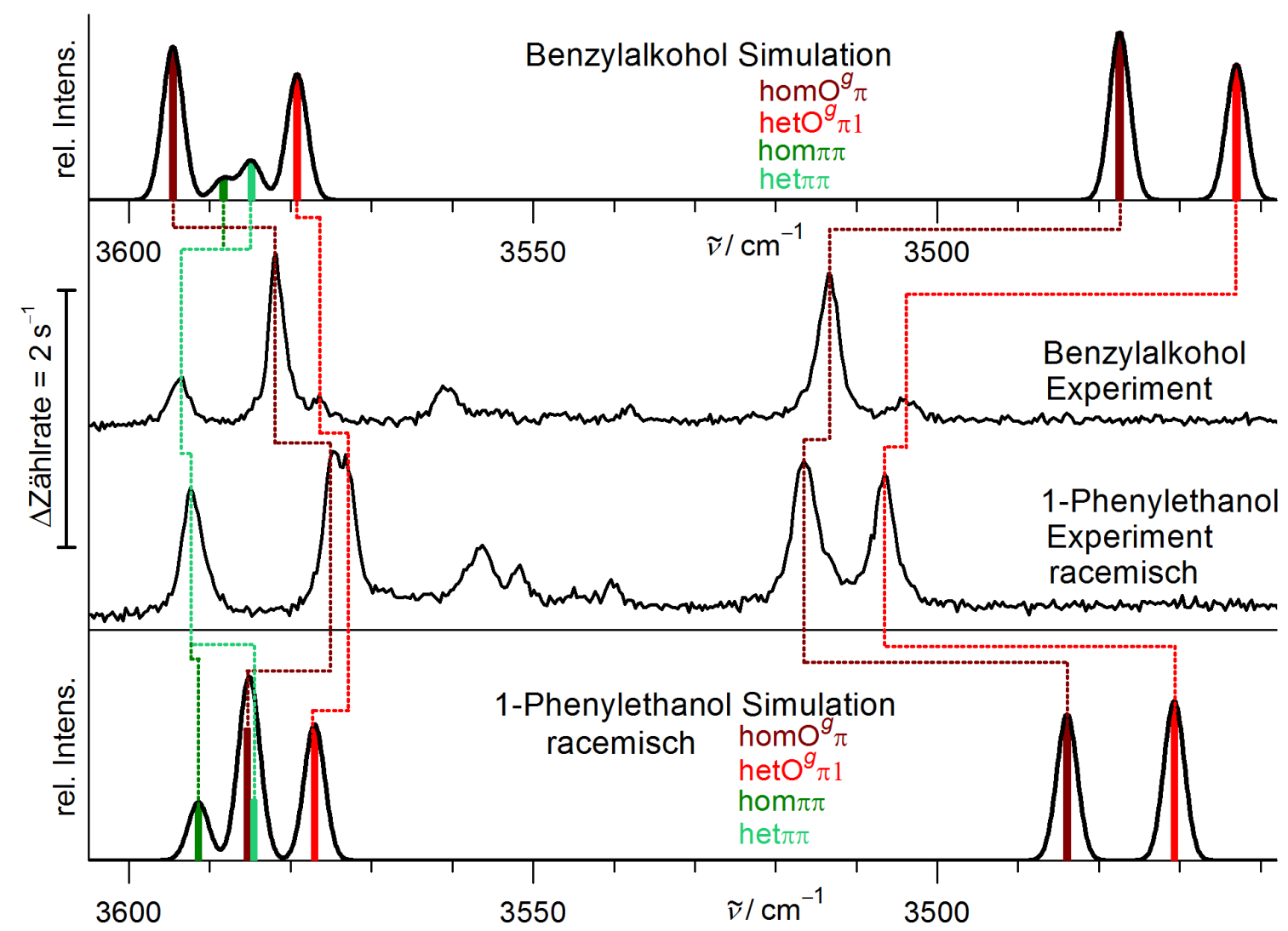

Abb. 8.10: Simulierte und experimentelle Raman-Spektren von Benzylalkohol und racemischem 1-Phenylethanol im Dimer-Bereich im Vergleich. Simulationsparameter auf Basis von B3LYP$\mathrm{D} 3(\mathrm{BJ}) /$ may-cc-pVTZ sind einheitliche Verschiebung der harmonischen Wellenzahlen um die Differenz von experimenteller und harmonischer $g$-Monomerwellenzahl $\left(-161.5 \mathrm{~cm}^{-1}\right.$ für Benzylalkohol und $-165.2 \mathrm{~cm}^{-1}$ für 1-Phenylethanol), eine Konformationstemperatur von $100 \mathrm{~K}$ und Gauss-Profile mit einer vollen Halbwertsbreite von $3 \mathrm{~cm}^{-1}$. 


\section{Komplementarität in Dimeren von Benzylalkohol und 1-Phenylethanol}

rend das Umgekehrte für die $\mathrm{OH} \cdots \pi$-Wasserstoffbrücken gilt. Beide Banden von het $\pi \pi$ werden geringfügig zu niedrigeren Wellenzahlen verschoben. Alle diese Effekte werden von B3LYP-D3(BJ) mindestens qualitativ korrekt vorhergesagt, wie Tab. 8.11 zeigt, wenn statt absoluter Wellenzahlen die Verschiebungen relativ zum jeweiligen Monomer betrachtet werden. Hierdurch werden die unterschiedlichen Anharmonizitäten (oder alternativ: das Defizit von B3LYP-D3(BJ)) berücksichtigt, da es sich bei Benzylalkohol um einen primären, bei 1-Phenylethanol dagegen um einen sekundären Alkohol handelt (siehe Diskussion in Kap. 5.4). Während die experimentellen Monomerübergänge im Rahmen der Auflösung koinzidieren, beträgt die Differenz der harmonischen Wellenzahlen $3.7 \mathrm{~cm}^{-1}$. Ohne Berücksichtigung dieses Umstandes würden deutlich schlechtere Vorhersagen für die Verschiebungen zwischen den Dimeren beider Alkohole erhalten werden. Eine ähnliche nachträgliche Korrektur auf die experimentelle $t / g$-Wellenzahldifferenz von Ethanol hatte sich in Kap. 4.3 für Ethanol-Dimere als hilfreich erwiesen.

Auch für 1-Phenylethanol lassen sich die Intensitätsverhältnisse der Banden der $\mathrm{OH} \cdots \mathrm{O}-$ zu den $\mathrm{OH} \cdots \pi$-Wasserstoffbrücken für das $\mathrm{O}^{g} \pi$-Motiv zwischen Experiment und Theorie vergleichen. B3LYP-D3(BJ) und $\omega$ B97X-D sagen hier voraus, dass bei IR-Detektion die Übergänge der $\mathrm{OH}$...O-Oszillatoren intensiver erscheinen sollen, aber bei RamanDetektion eine Angleichung stattfinden soll - analog zu Benzylalkohol. Doch experimentell wird das Gegenteil gefunden, dies entspricht dem Ergebnis von M06-2X (Tab. 8.12). Für diese Diskrepanz, sowohl zwischen Experiment und Theorie, wie auch zwischen Benzylalkohol und 1-Phenylethanol, kommen auch hier sowohl experimentelle wie theoretische Schwächen in Frage. So ist in den Filet-Jet das Signal-zu-Rausch-Verhältnis mäßig und in der höher konzentrierten und wärmeren Expansion des Curry-Jet könnten unerkannte Überlagerungen mit Banden weiterer Strukturen vorliegen.

Zusammengefasst scheinen auch 1-Phenylethanol-Dimere durch B3LYP-D3(BJ)/maycc-pVTZ ausreichend beschrieben zu werden, mit einigen Defiziten, wie für die Intensitätsverteilung zwischen den beiden Übergängen. Eine Übersicht über alle beobachteten Banden und deren vorgeschlagenen Zuordnungen bietet im Anhang Tab. A.19 auf S. 326.

Die Absicherung der Zuordnungen über die sehr weitgehende Analogie zwischen Benzylalkohol und 1-Phenylethanol erlaubt es nun, die eingangs formulierten offenen Fragen für Benzylalkohol-Dimere wie folgt zu beantworten:

Tab. 8.11: Experimentelle und vorhergesagte (B3LYP-D3(BJ)/may-cc-pVTZ) Veränderungen in den Verschiebungen relativ zum jeweiligen Monomer für verschiedene Banden von Dimeren beim Wechsel von Benzylalkohol zu 1-Phenylethanol.

\begin{tabular}{lcc}
\hline Bande & $\Delta \tilde{v}(\exp ) / \mathrm{cm}^{-1}$ & $\Delta \tilde{\nu}($ theo $) / \mathrm{cm}^{-1}$ \\
\hline $\operatorname{homO}^{g} \pi(\mathrm{OH} \cdots \mathrm{O})$ & 2 & 6.5 \\
$\operatorname{hetO}^{g} \pi(\mathrm{OH} \cdots \mathrm{O})$ & 2 & 7.7 \\
$\operatorname{homO}^{g} \pi(\mathrm{OH} \cdots \pi)$ & -7 & -9.2 \\
$\operatorname{hetO}^{g} \pi(\mathrm{OH} \cdots \pi)$ & -3 & -2.1 \\
$\operatorname{het}^{\prime} \pi$ (antisymmetrisch) & -1 & -0.2 \\
het $\pi \pi$ (symmetrisch) & -2 & -0.3 \\
\hline
\end{tabular}


Tab. 8.12: Intensitätsverhältnisse der Banden der $\mathrm{OH} \cdots \mathrm{O}-\mathrm{zu}$ den $\mathrm{OH} \cdots \pi$-Oszillatoren bei IRund Raman-Detektion gemäß Experiment und Theorie.

\begin{tabular}{lcccc}
\hline Methode & \multicolumn{2}{c}{ homO $^{g} \pi$} & \multicolumn{2}{c}{ hetO $^{g} \pi$} \\
& IR & Raman & IR & Raman \\
\hline Experiment & 1.1 & 1.5 & 1.2 & 1.4 \\
B3LYP-D3(BJ)/may-cc-pVTZ & 1.6 & 1.1 & 1.7 & 1.1 \\
B2PLYP-D3(BJ)/may-cc-pVTZ & 1.5 & - & 1.6 & - \\
$\omega B$ B7X-D/may-cc-pVTZ & 1.7 & 1.1 & 1.7 & 1.3 \\
M06-2X/may-cc-pVTZ & 1.1 & 1.3 & 1.3 & 1.4 \\
\hline
\end{tabular}

- Die Zuordnung von Oswald und Altnöder der beiden intensivsten Banden zu $\operatorname{homO}^{g} \pi$ wird mit den neuen Daten bestätigt.

- Auch die heterochirale Variante des Motivs konnte nun zugeordnet und dessen relative Energie in einen Bereich von 0.8 bis $2.6 \mathrm{~kJ} \mathrm{~mol}^{-1}$ eingegrenzt werden.

- Im $\pi \pi$-Motiv wird sehr wahrscheinlich stattdessen bevorzugt die heterochirale Variante ausgebildet. Hierzu können sowohl energetische, wie auch kinetische Aspekte beitragen.

Abschließend sei auf einige Gemeinsamkeiten und Unterschiede zur Chiralitätsdiskriminierung im verwandten System 1-Indanol [70, 363] hingewiesen. Auch dort bilden die Dimere $\mathrm{O}^{g} \pi$ - und $\pi \pi$-Motive aus, während das $\mathrm{O}^{t} \pi$-Motiv unter den diskutierten konkurrenzfähigen Konformeren nicht erwähnt wird. Die Zuordnungen sind weniger eindeutig, da die intramolekulare äquatorial/axial-Isomerie des Fünfringes von 1-Indanol sich auch auf die Dimere überträgt. Das $\pi \pi$-Motiv konnte nur in der homochiralen Kombination nachgewiesen werden, während es hier für 1-Phenylethanol in beiden Kombinationen beobachtet wurde - allerdings deutlich intensiver im heterochiralen Fall. Weiterhin fällt die Davydov-Aufspaltung bei 1-Indanol hom $\pi \pi$ mit $16-19 \mathrm{~cm}^{-1}$ (der Wert wurde leicht unterschiedlich mit verschiedenen Detektionsmethoden bestimmt) deutlich größer aus als bei 1-Phenylethanol het $\pi \pi\left(5 \mathrm{~cm}^{-1}\right)$. Die spektralen Intensitäten der asymmetrischen und symmetrischen Streckschwingungen sind bei 1-Indanol hom $\pi \pi$ nur begrenzt komplementär, da beide Übergänge sowohl mit IR- wie Raman-Detektion nachweisbar sind. Die Rechnungen auf B3LYP-D3/def2-TZVP-Niveau sagen im Widerspruch dazu eine Situation mit praktisch vollständiger Komplementarität, äquivalent zu 1-Phenylethanol, voraus. Eine mögliche alternative Interpretation wäre die Präsenz von mehreren leicht unterschiedlichen hom $\pi \pi$-Strukturen. Zudem treten die isolierten $\mathrm{OH} \cdots \pi$-Wasserstoffbrücken in hom $\pi \pi$ bei tieferen Wellenzahlen auf als die kooperativen in $\mathrm{O}^{g} \pi-$ möglicherweise eine Folge der Beteiligung von unterschiedlichen Monomer-Konformeren. Für die $\mathrm{O}^{g} \pi$-Strukturen wurde gefunden, dass, wie bei 1-Phenylethanol, die Bande der $\mathrm{OH} \cdots \pi$ Wasserstoffbrücke im homochiralen Fall etwas hochfrequenter auftritt. Die Bande der $\mathrm{OH}$... O-Wasserstoffbrücke wird homochiral dagegen deutlich niederfrequenter als heterochiral gefunden. Als globales Minimum des Systems wird zudem die heterochirale Variante des Motivs vorhergesagt. Zusammengefasst ist die Analogie zwischen 1Phenylethanol und Benzylalkohol, trotz des Wechsels zwischen permanenter und transienter Chiralität, sehr viel weitgehender als die zwischen 1-Phenylethanol und 1-Indanol. 


\subsection{Chiralitätsinduktion in gemischten 1-Phenylethanol-Benzylalkohol-Dimeren}

\subsubsection{Einleitung}

Gemischte Dimere sind ein wichtiger Zwischenschritt beim Übergang zwischen Benzylalkohol- und 1-Phenylethanol-Dimeren, die helfen Änderungen in den Energieabständen zwischen den Motiven bei chemischer Substitution verstehen zu können. In den vorherigen Abschnitten wurde in der Synchronisation in Benzylalkohol-Dimeren für das stabilste $\mathrm{O}^{g} \pi$-Motiv eine homochirale Präferenz bestätigt, die laut den Rechnungen energetisch noch stärker in der Diskriminierung in 1-Phenylethanol-Dimeren ausgeprägt sein soll. Die naheliegende Erwartung für ein gemischtes Dimer wäre daher, dass 1-Phenylethanol seine permanente Chiralität ebenfalls homochiral in Benzylalkohol induzieren kann. Dies wird in diesem Kapitel untersucht und dabei auftretende experimentelle Herausforderungen diskutiert. Dagegen wurde für das $\pi \pi$-Motiv bei Benzylalkohol eine heterochirale und bei 1-Phenylethanol eine homochirale Präferenz vorausgesagt, sodass die Erwartung für das gemischte Dimer unklar ist. Sowohl die neu auftretende DonorAkzeptor-Isomerie in den $\mathrm{O} \pi$-Motivem, wie auch der zwangsläufige Symmetriebruch im $\pi \pi$-Motiv fügen der theoretischen Beschreibung weitere Herausforderungen hinzu.

\subsubsection{Quantenchemische Rechnungen}

Aufbauend auf den Ergebnissen der beiden vorherigen Abschnitte, wurden für gemischte Dimere nur die $\mathrm{O}^{g} \pi$-, $\pi \pi$ - und $\mathrm{O}^{t} \pi$-Motive, unter Beteiligung der jeweils stabilsten Monomer-Konformere beider Moleküle, auf B3LYP-D3(BJ)/may-cc-pVTZ-Niveau berücksichtigt. In den $\mathrm{O} \pi$-Motiven ergibt sich dabei eine neue Form von Isomerie: eines der beiden ungleichen Moleküle tritt als Donor der $\mathrm{OH} \cdots \mathrm{O}-$ und Akzeptor der $\mathrm{OH} \cdots \pi$ Wasserstoffbrücke auf, während das andere Molekül in den umgekehrten Rollen agiert. Zur Unterscheidung wird den Strukturbezeichnungen ein Kürzel BP oder PB angehängt, wobei B für Benzylalkohol und P für 1-Phenylethanol steht. Die Reihenfolge entspricht der Angabe der adressierten Wasserstoffbrücken-Akzeptoren in der Bezeichnung $\mathrm{O} \pi$. Als Beispiel liegt in homO $^{g} \pi$-PB eine homochirale Induktion bezüglich der Torsionswinkel vor, mit 1-Phenylethanol als Donor der OH. . O- und Benzylalkohol als Donor der $\mathrm{OH} \cdots \pi$-Wasserstoffbrücke. In homO ${ }^{g} \pi$-BP sind die Rollen entsprechend vertauscht. Dimere aus zwei Einheiten Benzylalkohol werden mit der Endung BB, und solche aus zwei Molekülen 1-Phenylethanol mit PP gekennzeichnet.

Die sechs stabilsten gemischten Dimere und deren schwingungsspektroskopischen Eigenschaften sind in Abb. 8.11 dargestellt. Das globale Minimum ist homO ${ }^{g} \pi-\mathrm{PB}$, mit einem deutlichen energetischen Vorteil von $1.3 \mathrm{~kJ} \mathrm{~mol}^{-1}$ vor dessen heterochiraler Variante hetO ${ }^{g} \pi$-PB. Diese berechnete homochirale Präferenz ist sowohl höher als die für BB $\left(0.2 \mathrm{~kJ} \mathrm{~mol}^{-1}\right)$, wie auch die für PP $\left(0.9 \mathrm{~kJ} \mathrm{~mol}^{-1}\right)$. Es folgen hetO ${ }^{g} \pi-\mathrm{BP}$ und homO ${ }^{g} \pi-\mathrm{BP}$ mit relativen Energien von $1.47 \mathrm{~kJ} \mathrm{~mol}^{-1}$ und $1.52 \mathrm{~kJ} \mathrm{~mol}^{-1}$. Bei Vertauschung der DonorAkzeptor-Rollen wird also stattdessen ein minimaler heterochiraler Vorteil gefunden.

1-Phenylethanol nimmt im $\mathrm{O}^{g} \pi$-Motiv bevorzugt die Rolle des Donors der $\mathrm{OH} \cdots \mathrm{O}-$ Wasserstoffbrücke ein, für das $\mathrm{O}^{t} \pi$-Motiv wird dagegen eine umgekehrte Rollenvertei- 

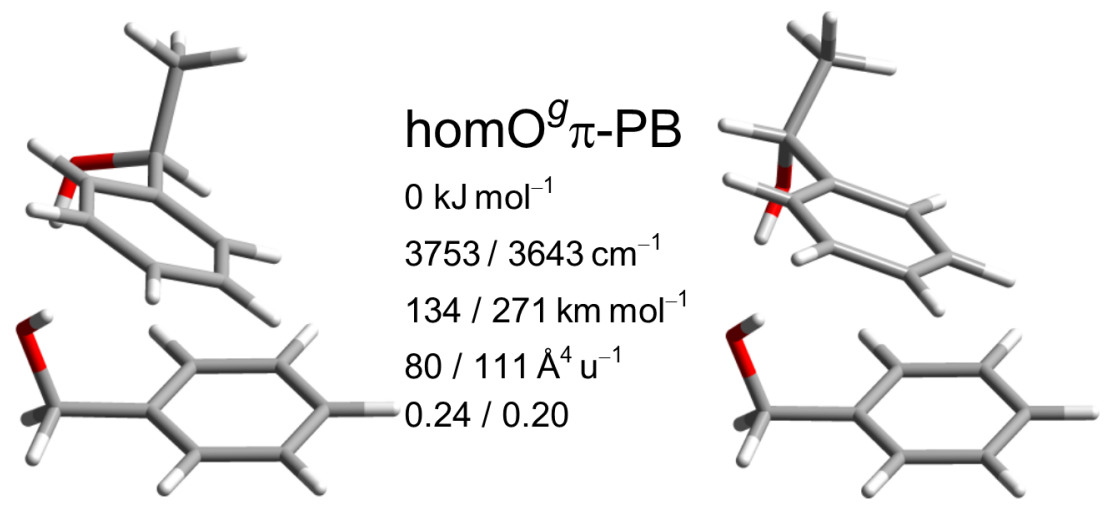

\section{hetO $^{g} \pi-\mathrm{PB}$}

$1.3 \mathrm{~kJ} \mathrm{~mol}^{-1}$

$3742 / 3635 \mathrm{~cm}^{-1}$

$148 / 286 \mathrm{~km} \mathrm{~mol}^{-1}$

$83 / 121 \AA^{4} u^{-1}$

$0.24 / 0.20$
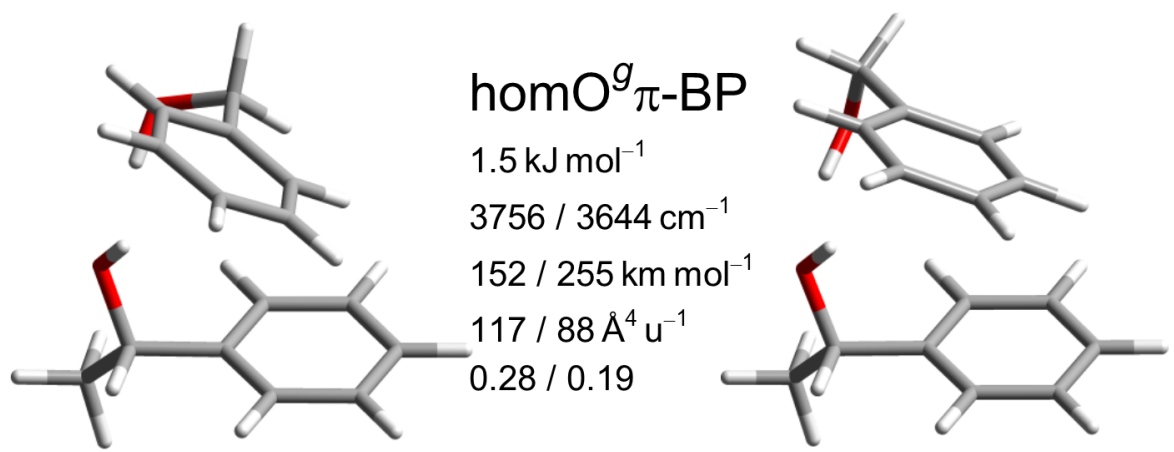

$\operatorname{hetO}^{g} \pi-\mathrm{BP}$

$1.5 \mathrm{~kJ} \mathrm{~mol}^{-1}$

$3741 / 3627 \mathrm{~cm}^{-1}$

$178 / 277 \mathrm{~km} \mathrm{~mol}^{-1}$

$126 / 93 \AA^{4} u^{-1}$

$0.28 / 0.19$
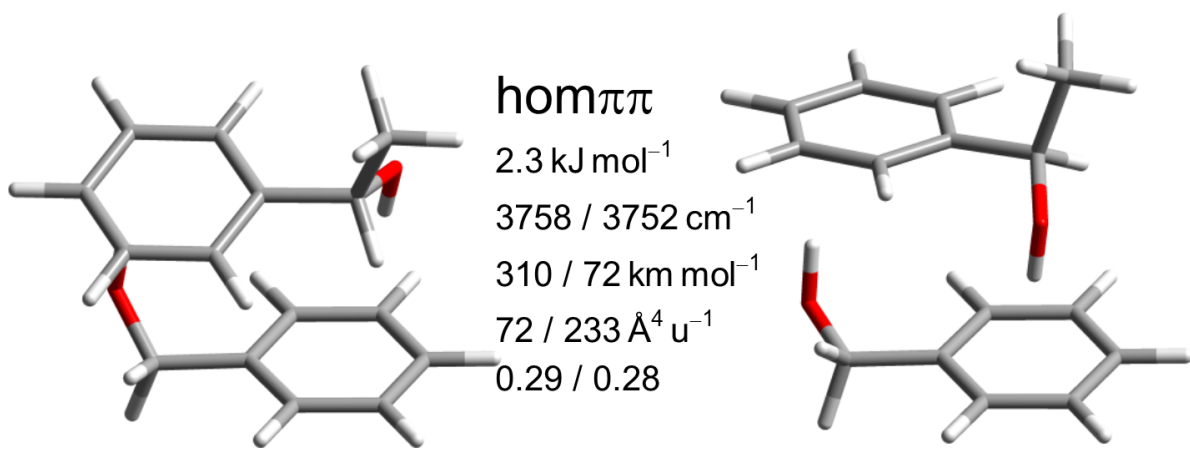

het $\pi \pi$

$2.2 \mathrm{~kJ} \mathrm{~mol}^{-1}$ $3759 / 3748 \mathrm{~cm}^{-1}$ $309 / 131 \mathrm{~km} \mathrm{~mol}^{-1}$ $98 / 223 \AA^{4} u^{-1}$ $0.29 / 0.29$

Abb. 8.11: Nullpunktskorrigierte relative Energien, harmonische OH-Streckschwingungswellenzahlen, IR-Intensitäten, Raman-Aktivitäten und Depolarisationsverhältnisse von gemischten 1Phenylethanol-Benzylalkohol-Dimeren auf B3LYP-D3(BJ)/may-cc-pVTZ-Niveau.

lung vorhergesagt. Die Ursache für diesen interessanten Befund wird im nächsten Unterkapitel analysiert.

het $\pi \pi$ wird mit einer relativen Energie von $2.2 \mathrm{~kJ} \mathrm{~mol}^{-1}$ um $0.1 \mathrm{~kJ} \mathrm{~mol}^{-1}$ stabiler gefunden als hom $\pi \pi$. Diese minimale heterochirale Präferenz entspricht der von BB $\left(0.4 \mathrm{~kJ} \mathrm{~mol}^{-1}\right)$, aber nicht der von PP $\left(-1.0 \mathrm{~kJ} \mathrm{~mol}^{-1}\right)$. Problematisch ist die Anwendung der harmonischen Näherung auf die $\pi \pi$-Strukturen. Durch den Bruch der Symmetrie verlieren diese nicht nur ihren statistischen Nachteil, sondern auch die Kopplung zwischen den $\mathrm{OH}-$ Streckschwingungen wird beeinträchtigt. Wie bereits diskutiert, stimmen die Fundamentalübergänge der Benzylalkohol- und 1-Phenylethanol-Monomere experimentell, aber nicht quantenchemisch in harmonischer Näherung überein. Daher ist zu vermuten, dass auch die Kopplung in harmonischer Näherung unterschätzt wird, da die Schwingungsfrequenzen tatsächlich ähnlicher sind als berechnet. Die (pseudo-)antisymmetrische Streckschwingung erhält in hom $\pi \pi$ etwa ein Drittel, im stärker asymmetrischen het $\pi \pi$ so- 


\section{Komplementarität in Dimeren von Benzylalkohol und 1-Phenylethanol}

gar fast die Hälfte der Raman-Aktivität der (pseudo-)symmetrischen Schwingung. Ähnliches gilt umgekehrt für die IR-Aktivitäten.

Die vier Strukturen des $\mathrm{O}^{t} \pi$-Motivs haben relative Energien zwischen 2.4 und $4.8 \mathrm{~kJ} \mathrm{~mol}^{-1}$ und werden daher in den folgenden Spektren-Modellierungen nicht berücksichtigt.

\subsubsection{Schwingungsspektren}

Die schwingungsspektroskopische Charakterisierung von gemischten 1-PhenylethanolBenzylalkohol-Dimeren weist zwei experimentelle Herausforderungen auf: spektrale Überlagerungen zwischen Banden und unterschiedliche (sowie niedrige) Dampfdrücke der beiden Verbindungen. Die zusätzliche PB/BP-Isomerie geht laut den Rechnungen nur mit geringen spektralen Unterschieden und damit mit zu erwartenden Überlappungen einher. Für homO ${ }^{g} \pi$-PB ist eine Zuordnung zumindest auf Grundlage seiner energetischen Dominanz zu erhoffen. Daneben lässt es sich experimentell nicht vermeiden, dass ebenfalls BB- und PP-Dimere gebildet werden, von denen ebenfalls zu erwarten ist, dass deren Banden mit denen der gemischten Dimeren spektral überlagern. Dies ist eine Folge der Grundidee dieses Kapitels: die Ausnutzung der großen chemischen Ähnlichkeit der beiden Substanzen.

Der Anteil der gemischten Dimere in der Expansion gegenüber denen der unerwünschten BB- und PP-Dimere wird entscheidend vom Partial- bzw. Dampfdruckverhältnis der beiden Substanzen bestimmt. Dessen Abhängigkeit von der Temperatur ist in Abb. 8.12 dargestellt. Bei Annahme statistischer Bildung wird der größte Anteil an gemischten Dimeren bei einem Verhältnis von 1:1 der Monomere erhalten, und zwar 50\% gegenüber 25\% BB und 25\% PP. Die Dampfdrücke nähern sich aber erst bei hoher Temperatur diesem Optimalfall an, wie auch in den sehr ähnlichen Normalsiedetemperaturen (206 und $204^{\circ} \mathrm{C}$ ) zum Ausdruck kommt. Bei derart hohen Temperaturen wäre zusätzliche Verdünnung notwendig, um die Bildung höherer Oligomere zu begrenzen. Bei $24^{\circ} \mathrm{C}$ (der in der Praxis höchstmöglichen Filet-Jet Temperatur) ist ein Überschuss 1-Phenylethanol von etwa Faktor 5, bei der als Messtemperatur am Curry-Jet optimal gefundenen Temperatur von $50{ }^{\circ} \mathrm{C}$ von etwa $2.5 \mathrm{zu}$ erwarten. Diese Verhältnisse lassen Anteile von $28 \%$ und

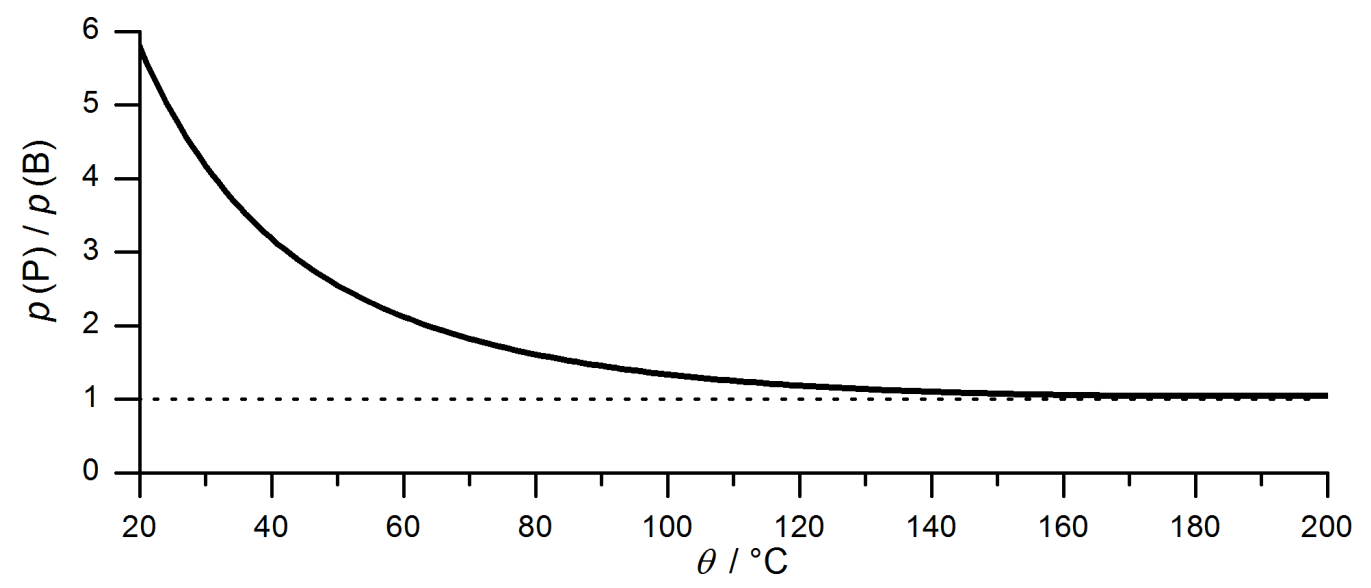

Abb. 8.12: Verhältnis der Dampfdrücke von 1-Phenylethanol (P) zu Benzylalkohol (B) in Abhängigkeit der Temperatur gemäß der teilweise extrapolierten Dampfdruckdaten in Tab. 3.1 auf $\mathrm{S}$. 21. 
$41 \%$ an gemischten Dimeren erwarten. Die absolute Konzentration sinkt zudem noch weit stärker mit der Temperatur. Durch Kontrolle der Temperatur, bzw. optimalerweise zweier unabhängiger Temperaturen, können daher die Dampfdrücke beider Substanzen und damit die gemischte Dimerkonzentration optimiert werden. Die bestehenden Apparaturen in der Abteilung Suhm bieten hierfür unterschiedliche Voraussetzungen:

Filet- und Curry-Jet sind mit jeweils zwei getrennt temperierbaren Glassättigern ausgestattet, die die Zimmertemperatur nicht überschreiten können, da sonst die Substanz in den ungeheizten Leitungen kondensieren würde. Durch das nachträgliche Zusammenführen der beiden Bestandteile im Reservoir werden die erreichbaren Partialdrücke weiter verringert, sodass hier keine ausreichenden Konzentrationen für die Untersuchung von gemischten Dimeren zu erwarten sind. Neben einem baugleichen Glassättiger weist der Popcorn-Jet eine geheizte Probenkammer auf. In dieser wurden zuvor Mischungen von Benzylalkohol und Cyclohexylmethanol untersucht, wobei aber dabei ausgenutzt wurde, dass die Dampfdrücke dieser beiden Substanzen ähnlicher und deren Cluster-Spektren unterschiedlicher sind.[70, 417] Derzeit wird eine Modifikation mit zwei geheizten Probenkammern erprobt, sodass die Dampfdrücke zweier schwerflüchtiger Substanzen getrennt eingestellt werden können (,double pick-up“) [69].

Ein alternativer Ansatz wird im nächsten Kapitel 9 in Form des in Erprobung befindlichen Risotto-Konzepts vorgestellt, bei dem ein Tropfen einer Flüssigkeitsmischung vollständig verdampft werden soll, sodass das Stoffmengenverhältnis in der Gasphase dem der flüssigen Mischung entspricht. Alternativ erlaubt der Risotto-Jet auch eine gewisse Entkopplung von Konzentration und Temperatur. Auf diese Weise lassen sich auch bei sehr hoher Temperatur bis $200^{\circ} \mathrm{C}$, bei der die Dampfdrücke angeglichen sind, ausreichend verdünnte Expansionen untersuchen.

Auch der ebenfalls in Erprobung befindliche Gratin-Jet erlaubt ebenfalls die unabhängige Einstellung von Partialdrücken zweier Substanzen durch vollständige Verdampfung. Allerdings ist dies derzeit noch auf Partialdrücke unterhalb des Dampfdruckes bei Zimmertemperatur beschränkt, da noch nicht die gesamte Apparatur beheizt ist.

Auch der Curry-Jet wurde mit einem beheizten Metallsättiger ergänzt [94]. Dieser kann aber, anders die Probenkammer des Popcorn-Jet, vermutlich nicht komplikationsfrei mit einem der Glassättiger kombiniert werden. Da das Trägergas diese hintereinander und nicht parallel durchläuft, würde sich die Gasphase der einen Substanz in der flüssigen Phase der anderen lösen. Ein alternativer Ansatz am Curry-Jet ist die Kontrolle des Dampfdruckverhältnisses bei einheitlicher Temperatur über die Zusammensetzung einer flüssigen Mischung, was in dieser Arbeit erstmals am geheizten Metallsättiger erprobt wurde. Wegen der großen chemischen Ähnlichkeit von 1-Phenylethanol und Benzylalkohol kann die Mischung näherungsweise als ideal vermutet werden, sodass gemäß dem Raoultschen Gesetz der Dampfdruck jeder Substanz proportional zum Stoffmengenanteil in der flüssigen Phase wäre. Anders als beim Popcorn- und Risotto-Jet kann das Mischungsverhältnis hier unkompliziert verändert werden, sofern zumindest eine Komponente flüssig ist. Hierzu wird über die Einfüllöffnung des Sättigers die zu erhöhende Komponente zugegeben. Da das sich im Sättiger noch befindliche Volumen nicht verfolgt werden kann, ist eine exakte Einstellung eines Mischungsverhältnisses auf diese Weise nicht möglich. Dies wäre aber auch nur begrenzt sinnvoll, denn ein Nachteil dieser Methode ist, dass das Mischungsverhältnis zeitlich nicht konstant ist [431], sofern nicht zufällig (oder gezielt) ein azeotropes Gemisch eingesetzt wird. 


\section{Komplementarität in Dimeren von Benzylalkohol und 1-Phenylethanol}

Am Ende einer Messung von enantiomerenreinem 1-Phenylethanol enthielt der Metallsättiger noch eine geringe Restmenge (die Signalintensitäten in den Spektren fielen bereits ab). Zu dieser wurden je $7 \mathrm{ml} \mathrm{B}$ und $7 \mathrm{ml}$ enantiomerenreines $\mathrm{P}$ zugegeben, sodass wegen ähnlicher Dichten und molarer Massen näherungsweise eine äquimolare Mischung erhalten wurde. Diese Mischung wurde über einen Zeitraum von 60 min expandiert und dann um weitere $5 \mathrm{ml} \mathrm{B}$ ergänzt und erneut gemessen. Der Vergleich der jeweils ersten und letzten 3 min Messblöcke deutet auf nur wenig Konzentrationsänderung während der Messreihen hin.

Es wurden die zwei in schwarz und grau dargestellten Spektren im oberen Teil von Abb. 8.13 erhalten. Im Vergleich mit den Spektren der Reinsubstanzen unter gleichen Expansionsbedingungen in grün $(\mathrm{P})$ und rot $(\mathrm{B})$ treten neue Signale auf. So befindet sich ein neues Maximum in beiden Spektren bei $3579 \mathrm{~cm}^{-1}$, welches somit zwischen den bekannten Bandenpositionen der Reinsubstanzen bei 3582 und $3575 \mathrm{~cm}^{-1}$ auftritt. Zur näheren Analyse wurden Differenzspektren gebildet. Die Skalierungsfaktoren der Spektren der Reinsubstanzen wurden hierfür größtmöglich gewählt, ohne dass dabei Signale unterhalb der Basislinie im Differenzspektrum resultieren. Die Differenzspektren stellen somit die Raman-Streuung dar, die nicht durch eine Überlagerung der Spektren der Reinsubstanzen erklärt werden kann. Da die Skalierungsfaktoren auch kleiner gewählt werden könnten, stellt dies eine Untergrenze für die Streuung der gemischten Komplexe dar. Die verwendeten Skalierungsfaktoren sind angegeben und auf die Spektren der Reinsubstanzen angewandt, sodass die Subtraktion visuell nachvollzogen werden kann. Es ist zu erkennen, dass die Anpassung an Bandenflanken in den Spektren der Mischungen die iterative Skalierung der Reinspektren erleichtert. Bei der näherungsweise äquimolaren Mischung ist die abgezogene Streuung von reinen P-Dimeren nach der Erwartung aus den unterschiedlichen Dampfdrücken deutlich höher als die der B-Dimere. Bei der flüssigen Mischung mit B-Überschuss sind die Intensitäten von BB und PP ähnlicher, wobei aber zu berücksichtigen ist, dass die Raman-Streuquerschnitte der P-Dimere etwa 50\% höher vorhergesagt werden als die der B-Dimere.

Die beiden erhaltenen Differenzspektren im mittleren Teil der Abbildung sind sich sehr ähnlich. Bei der Mischung ähnlicher Gasphasen-Konzentration von 1-Phenylethanol und Benzylalkohol wurde ein etwas intensiveres Spektrum der gemischten Cluster erhalten und die Differenzbildung gelang einfacher. Neben der bereits vor der Differenzbildung identifizierbaren neuen Bande bei $3579 \mathrm{~cm}^{-1}$ werden nun weitere Bandenmaxima bei 3592 und $3513 \mathrm{~cm}^{-1}$ erkennbar. Die breiteren Banden im Bereich um $3550 \mathrm{~cm}^{-1}$ sind nur bedingt aussagekräftig, da Trimere bei Verdünnung unterschiedlich mit der Konzentration skalieren und die Differenzbildung auf Grundlage der Dimerbanden durchgeführt wurde. Für die Simulation im unteren Teil der Abbildung wurden die weitgehend lokalisierten harmonischen Wellenzahlen der $\mathrm{OH}$-Streckschwingungen in den $\mathrm{O}^{g} \pi$-Strukturen getrennt für P und B um die Differenz der experimentellen und harmonischen MonomerWellenzahl korrigiert, die stärker gekoppelten der $\pi \pi$-Strukturen dagegen um den Mittelwert von B und P. Die Peaks bei 3579 und $3513 \mathrm{~cm}^{-1}$ können dem stabilsten gemischten Dimer homO ${ }^{g} \pi$-PB zugeordnet werden. Neben den hohen Intensitäten sprechen dafür auch die Bandenpositionen. In Tab. 8.13 sind experimentelle und berechnete Verschiebungen miteinandner verglichen. Die beobachteten Bandenpositionen stimmen mit den Vorhersagen für homO ${ }^{g} \pi$-PB überein, nach denen sich die Bande der $\mathrm{OH} \cdots \pi$ Wasserstoffbrücke spektral zwischen den Banden von BB und PP befinden, während die 


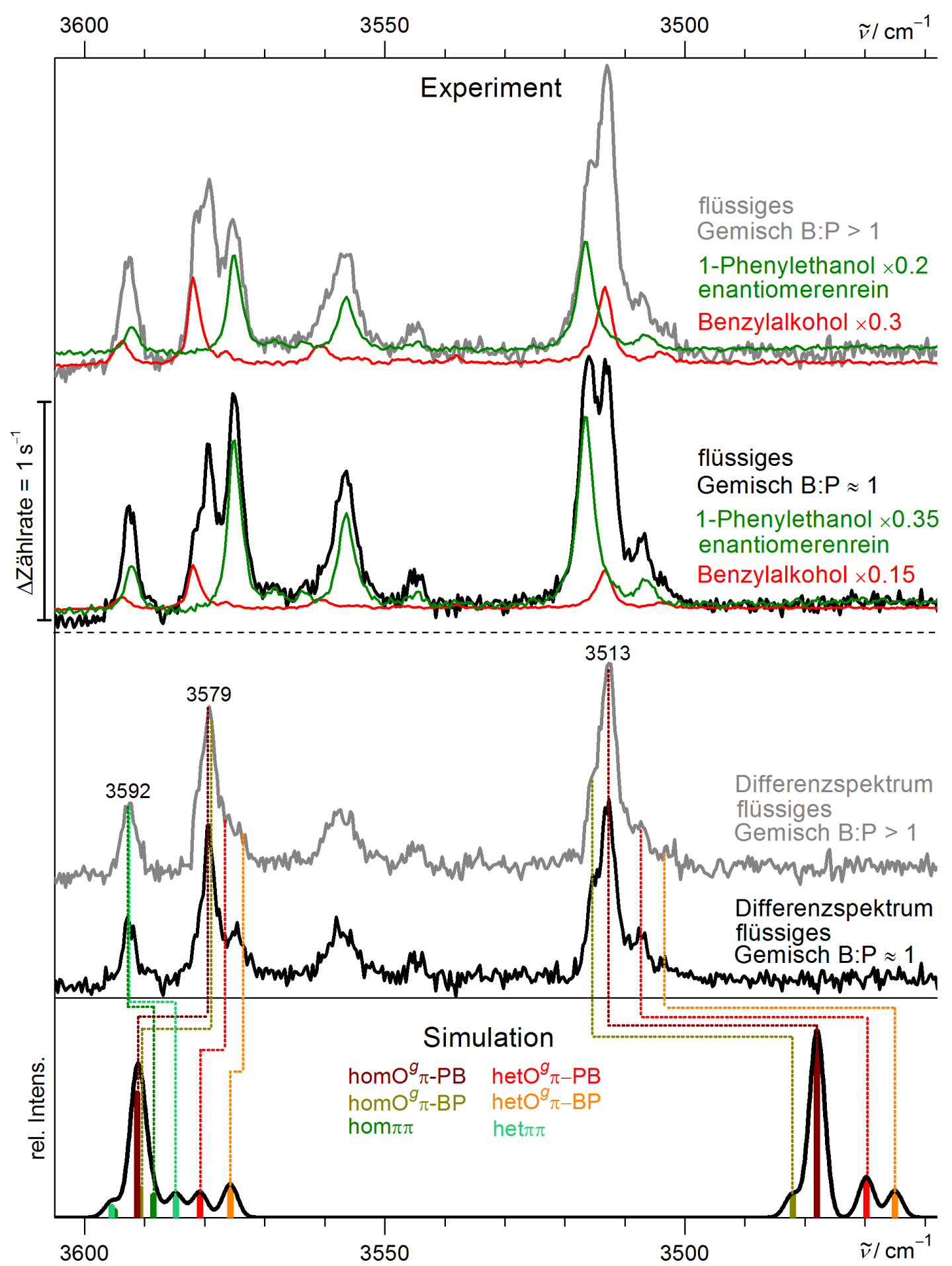

Abb. 8.13: Oberer Teil: Experimentelle Raman-Spektren von zwei Mischungen aus 1Phenylethanol und Benzylalkohol und skalierte Spektren der beiden Reinsubstanzen bei $50{ }^{\circ} \mathrm{C}$ Sättigertemperatur und $2 \mathrm{~mm}$ Detektionsabstand. Mittlerer Teil: Differenzspektren aus den Spektren der Mischungen und den skalierten Spektren der Reinsubstanzen. Unterer Teil: Simulation des Spektrums der gemischten Dimere auf Basis von B3LYP-D3(BJ)/may-cc-pVTZ. Die harmonischen Wellenzahlen wurden um die Differenz der experimentellen und harmonischen Wellenzahl des jeweiligen Monomers verschoben $\left(-165.2 \mathrm{~cm}^{-1}\right.$ für 1-Phenylethanol und $-161.5 \mathrm{~cm}^{-1}$ für Benzylalkohol). Für die stärker gekoppelten $\pi \pi$-Strukturen wurde davon abweichend der Mittelwert benutzt. Es wurden eine Konformationstemperatur von $100 \mathrm{~K}$ und Gauss-Profile mit einer vollen Halbwertsbreite von $3 \mathrm{~cm}^{-1}$ verwendet. 


\section{Komplementarität in Dimeren von Benzylalkohol und 1-Phenylethanol}

Bande der $\mathrm{OH} \cdots \mathrm{O}-$ Wasserstoffbrücke fast mit der von BB koinzidieren soll. Allerdings werden homO ${ }^{g} \pi$-PB und homO ${ }^{g} \pi$-BP bezüglich der Bandenpositionen sehr ähnlich vorhergesagt und überlappen vermutlich. Die Schulter bei $3515 \mathrm{~cm}^{-1}$ könnte auf die $\mathrm{OH} \cdots \mathrm{O}-$ Streckschwingung von homO ${ }^{g} \pi$-BP hinweisen. Darauf, dass vorwiegend homO ${ }^{g} \pi-\mathrm{PB}$ gebildet wird, deutet nicht nur die deutlich geringer berechnete Energie, sondern auch das Verhältnis der vorhergesagten Raman-Aktivitäten hin. Die höhere Intensität der Bande bei $3513 \mathrm{~cm}^{-1}$ gegenüber der bei $3579 \mathrm{~cm}^{-1}$ stimmt mit der Vorhersage überein, dass die Bande der Wasserstoffbrücke, in der 1-Phenylethanol als Donor agiert, stets die höhere Raman-Aktivität aufweisen sollte (siehe Abb. 8.11). Falls stattdessen homO ${ }^{g} \pi$-BP die dominante Struktur wäre, wäre die umgekehrte Intensitätsverteilung zu erwarten. Dies bleibt aber ein schwaches Indiz, da wegen der Differenzbildung die experimentelle Intensitätsverteilung nur begrenzt verlässlich ist und auch für die theoretische Vorhersage der relative Streuquerschnitte zuvor Schwächen festgestellt wurden. Ein experimenteller Beweis wäre durch ${ }^{18} \mathrm{O}$-Isotopensubstitution von entweder 1-Phenylethanol oder Benzylalkohol möglich. Banden von Wasserstoffbrücken, in denen das isotopensubstituierte Molekül als Donor agiert, sollten sich dann wegen der erhöhten reduzierte Masse der Schwingung zu niedrigeren Wellenzahlen verschieben. Mit dieser Methode gelang zuvor der experimentelle Beweis von Präferenzen für Donor-Akzeptor-Isomerien in anderen gemischten Dimeren.[131, 432]

Die Banden bei 3579 und $3513 \mathrm{~cm}^{-1}$ besitzen jeweils einen niederfrequenten Ausläufer. Diese können hetO $^{g} \pi$-PB und hetO ${ }^{g} \pi$-BP zugeordnet werden, wenn davon ausgegangen wird, dass die spektrale Aufspaltung zwischen den homo- und heterochiralen Strukturen ähnlich überschätzt wird, wie es bei BB und PP festgestellt wurde.

Schließlich kann die Bande bei $3592 \mathrm{~cm}^{-1}$ het $\pi \pi$ und/oder hom $\pi \pi$ zugeordnet werden. Im Vergleich zu den analogen Banden bei BB und PP wird keine wesentliche Aufspaltung oder Verbreiterung beobachtet. Bereits bei PP wurde festgestellt, dass die symmetrischen Streckschwingungen von het $\pi \pi$ und hom $\pi \pi$ entgegen den Vorhersagen koinzidieren. Ebenso gibt es keine Evidenz dafür, dass die (pseudo-)antisymmetrische Streckschwingung durch den Symmetriebruch wie vorhergesagt signifikante Raman-Aktivität erhalten würde. Wie bereits zuvor diskutiert wurde, könnte die Ursache hierfür die Unzulänglichkeit der harmonischen Näherung sein, wodurch das Ausmaß der verbleibenden Kopplung unterschätzt wird. Für alle Banden der verschiedenen Strukturmotive ergeben sich ähnliche Über- bzw. Unterschätzungen der Bandenpositionen wie bei BB und PP.

Zusammengefasst konnte in diesem Abschnitt gezeigt werden, dass auch mit dem ein-

Tab. 8.13: Experimentelle und vorhergesagte (B3LYP-D3(BJ)/may-cc-pVTZ) Verschiebungen der Bandenpositionen von homO ${ }^{g} \pi$-PB relativ zu homO ${ }^{g} \pi-\mathrm{BB}$ und homO ${ }^{g} \pi$-PP. Die vorhergesagten Bandenpositionen, auf denen die Shifts beruhen, sind jeweils um die Differenz zwischen experimenteller und harmonischer Wellenzahl des jeweiligen Monomers korrigiert.

\begin{tabular}{lcc}
\hline & $\Delta \tilde{v}(\exp ) / \mathrm{cm}^{-1}$ & $\Delta \tilde{v}($ theo $) / \mathrm{cm}^{-1}$ \\
\hline OH $\cdots \pi$ (relativ zu BB) & -3 & -3.4 \\
OH $\cdots \pi$ (relativ zu PP) & 4 & 5.8 \\
OH $\cdots$ O (relativ zu BB) & -1 & 0.6 \\
OH $\cdots$ O (relativ zu PP) & -3 & -6.0 \\
\hline
\end{tabular}


zelnen Metallsättiger des Curry-Jets gemischte Dimere zweier schwerflüchtiger Verbindungen beobachtet werden können und dabei das Mischungsverhältnis variiert werden kann. Die erhaltenen Differenzspektren sind zumindest für das $\mathrm{O}^{g} \pi$-Motiv in guter Übereinstimmung mit den Berechnungen und stützen die Vorhersage, dass in gemischten 1-Phenylethanol-Benzylalkohol-Clustern eine homochirale Induktion stattfindet, mit wahrscheinlich 1-Phenylethanol als Donor der $\mathrm{OH} \cdots \mathrm{O}$-Wasserstoffbrücke. Im folgenden Unterkapitel wird quantenchemisch ergründet, warum diese Rollenverteilung eingenommen wird und warum die homochirale Präferenz im gemischten Dimer besonders stark ausfällt.

\subsection{Substitutionseffekte und chirodiastaltische Energien}

In den vorherigen Abschnitten wurden Dimere aus zwei Molekülen Benzylalkohol, zwei Molekülen 1-Phenylethanol und gemischte Dimere beider Substanzen analysiert. Diese zeigten weitgehende Gemeinsamkeiten, aber auch einige mehr oder weniger subtile Unterschiede. Auf B3LYP-D3(BJ)/may-cc-pVTZ-Niveau gelang die Vorhersage einer mit dem Experiment stets zumindest qualitativ kompatiblen Energieabfolge der Strukturen, sofern auch kinetische Aspekte mitberücksichtigt wurden. Dabei galt stets die Abfolge $E_{0}\left(\mathrm{O}^{g} \pi\right)<E_{0}(\pi \pi)<E_{0}\left(\mathrm{O}^{t} \pi\right)$, wobei sich aber das Ausmaß der Energieabstände veränderte. So erschien $\mathrm{O}^{t} \pi$ mit relativen Energien unter $2 \mathrm{~kJ} \mathrm{~mol}^{-1}$ für PP relevanter als für BB mit dortigen relativen Energien jenseits von $3 \mathrm{~kJ} \mathrm{~mol}^{-1}$. Im $\mathrm{O}^{g} \pi$-Motiv wurde stets eine homochirale Präferenz gefunden, allerdings in variierender Ausprägung. Für das $\pi \pi$-Motiv wurde dagegen ein Wechsel von heterochiraler Präferenz bei BB und PB auf eine homochirale Bevorzugung in PP vorhergesagt. Derartige Substitutionseffekte der Methylgruppe auf die Dimere und damit die chirodiastaltische Energien werden in diesem Unterkapitel quantenchemisch näher analysiert.

Der Vergleich zwischen Dimeren unterschiedlicher Zusammensetzung ist über die Betrachtung der schwingungsnullpunktskorrigierten Bindungsenergien (jeweils relativ zu den stabilsten Monomer-Konformationen) möglich. Einen Überblick gibt Abb. 8.14. Das Diagramm ist folgendermaßen zu lesen: die beiden inneren Spalten beinhalten die BBDimere, nach außen gehend wird in beide Richtungen schrittweise Benzylalkohol gegen 1-Phenylethanol ausgetauscht, was einer formalen Methylierung entspricht. Zu erkennen ist dabei für die gemischten Dimere die Aufspaltung der $\mathrm{O}^{g} \pi$ - und $\mathrm{O}^{t} \pi$-Motive in die BP- und PB-Varianten. In den beiden äußeren Spalten befinden sich schließlich die PPDimere. Bei Verfolgung der analogen Strukturen von innen nach außen ergeben sich keine klaren Trends. In einigen Fällen sinkt die Bindungsenergie, in anderen steigt sie. Wie ist dies zu erklären?

Einen ersten Hinweis gibt ein auffälliges Muster: der Effekt einer doppelten Methylierung (d.h. von BB zu PP) entspricht in guter Näherung stets der Summe der beiden möglichen einfachen Methylierungen bei den $\mathrm{O}^{g} \pi$ - und $\mathrm{O}^{t} \pi$-Motiven. Dies spricht für lokalisierte Effekte. Zur näheren Analyse kann der Dimerisierungsprozess gedanklich und rechnerisch in folgende Einzelschritte zerlegt werden: die beiden isolierten Monomere werden zunächst derart deformiert, wie sie im Dimer vorliegen, dies erfordert Energie. Anschließend werden die beiden deformierten Monomere zum Dimer kombiniert, wobei die Wechselwirkungsenergie frei wird. Begleitet werden diese Schritte von einer Ände- 


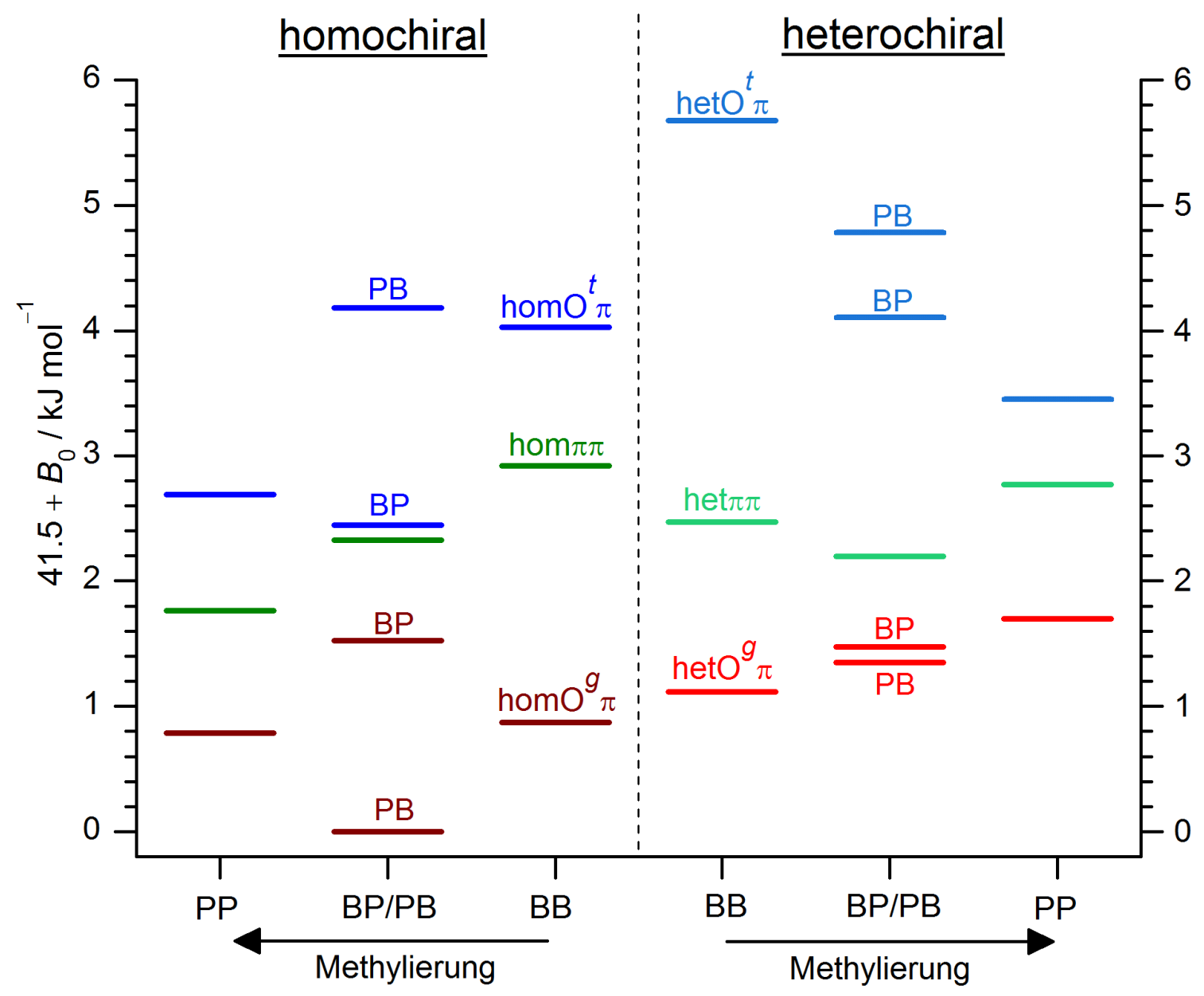

Abb. 8.14: Schwingungsnullpunktskorrigierte Bindungsenergien $B_{0}$ von Dimeren von Benzylalkohol (BB), 1-Phenylethanol (PP) und gemischt aus beiden Molekülen (BP/PB). Ausgehend von den beiden inneren Spalten findet eine schrittweise Substitution von Benzylalkohol durch 1-Phenylethanol, eine formale Methylierung, statt.

rung der Schwingungsnullpunktsenergie. Als Summe dieser Beiträge ergibt sich schließlich die schwingungsnullpunktskorrigierte Bindungsenergie, wie sie im Diagramm aufgetragen ist. Im Detail ausgeführt ist dies im oberen Teil von Tab. 8.14 für homO ${ }^{g} \pi$. 
Tab. 8.14: Elektronische Deformationsenergien der Monomere in Rolle des OH...O-Donors $\Delta E_{\mathrm{el}}($ Def : $\mathrm{OH} \cdots \mathrm{O})$ bzw. OH $\cdots \pi$-Donors $\Delta E_{\mathrm{el}}($ Def : OH $\cdots \pi)$, elektronische Wechselwirkungsenergien $\Delta E_{\mathrm{el}}$ (Wechselwirkung) der deformierten Monomere, Änderungen der Schwingungsnullpunktsenergie bei der Dimerisierung $\Delta E_{0}$ (Schwingungsnullp.) und die sich jeweils als Summe ergebenden Bindungsenergien $B_{0}$ für Dimere von Benzylalkohol (BB), 1-Phenylethanol (PP) und gemischte Dimere $(\mathrm{PB} / \mathrm{BP})$ der $\mathrm{O}^{g} \pi$ - und $\mathrm{O}^{t} \pi$-Motive. Alle Werte sind in $\mathrm{kJ} \mathrm{mol}^{-1}$ angegeben und auf B3LYP-D3(BJ)/may-cc-pVTZ-Niveau berechnet.

\begin{tabular}{lcccc}
\hline & homO $^{g} \pi-\mathrm{BB}$ & homO $^{g} \pi-\mathrm{PB}$ & homO $^{g} \pi-\mathrm{BP}$ & homO $^{g} \pi-\mathrm{PP}$ \\
$\Delta E_{\mathrm{el}}$ (Def : OH $\cdots$ O) & 1.0 & 0.8 & 0.9 & 0.8 \\
$\Delta E_{\mathrm{el}}$ (Def : OH $\left.\cdots \pi\right)$ & 0.7 & 0.7 & 2.2 & 2.2 \\
$\Delta E_{\mathrm{el}}$ (Wechselwirkung) & -48.8 & -49.2 & -49.5 & -49.9 \\
$\Delta E_{0}$ (Schwingungsnullp. $)$ & 6.5 & 6.3 & 6.4 & 6.2 \\
\hline$B_{0}$ & -40.7 & -41.5 & -40.0 & -40.7 \\
& & & & \\
\hline & & & & \\
$\Delta E_{\mathrm{el}}($ Def : OH $\cdots \mathrm{O})$ & 0.9 & 1.7 & 1.1 & 1.6 \\
$\Delta E_{\mathrm{el}}$ (Def : OH $\left.\cdots \pi\right)$ & 0.5 & 0.5 & 1.7 & 1.6 \\
$\Delta E_{\mathrm{el}}$ (Wechselwirkung) & -48.3 & -48.6 & -49.2 & -49.2 \\
$\Delta E_{0}$ (Schwingungsnullp. $)$ & 6.5 & 6.2 & 6.4 & 6.1 \\
\hline$B_{0}$ & -40.4 & -40.2 & -40.1 & -39.8
\end{tabular}

\begin{tabular}{lrrrr}
$\Delta E_{\mathrm{el}}($ Def : OH $\cdots \mathrm{O})$ & 0.6 & 1.2 & 0.7 & 1.3 \\
$\Delta E_{\mathrm{el}}($ Def : OH $\cdots \pi)$ & 1.6 & 1.7 & 1.3 & 1.3 \\
$\Delta E_{\mathrm{el}}$ (Wechselwirkung) & -46.0 & -46.2 & -47.2 & -47.4 \\
$\Delta E_{0}$ (Schwingungsnullp. $)$ & 6.2 & 5.9 & 6.2 & 5.9 \\
\hline$B_{0}$ & -37.5 & -37.3 & -39.1 & -38.8
\end{tabular}

\begin{tabular}{lcccc}
\hline & hetO $^{t} \pi-\mathrm{BB}$ & hetO $^{t} \pi-\mathrm{PB}$ & hetO $^{t} \pi-\mathrm{BP}$ & hetO $^{t} \pi-\mathrm{PP}$ \\
$\Delta E_{\mathrm{el}}($ Def $: \mathrm{OH} \cdots \mathrm{O})$ & 0.8 & 0.6 & 0.9 & 0.9 \\
$\Delta E_{\mathrm{el}}($ Def $:$ OH $\cdots \pi)$ & 1.9 & 2.0 & 1.5 & 1.6 \\
$\Delta E_{\mathrm{el}}$ (Wechselwirkung) & -44.4 & -44.9 & -45.5 & -46.2 \\
$\Delta E_{0}$ (Schwingungsnullp. $)$ & 5.9 & 5.5 & 5.7 & 5.7 \\
\hline$B_{0}$ & -35.9 & -36.7 & -37.4 & -38.1 \\
\hline
\end{tabular}




\section{Komplementarität in Dimeren von Benzylalkohol und 1-Phenylethanol}

Es ist zu erkennen, dass die Deformation des Donors der $\mathrm{OH} \cdots \mathrm{O}$-Wasserstoffbrücke in homO $^{g} \pi$ stets einen vergleichbaren Energiebetrag benötigt $\left(0.8-1.0 \mathrm{~kJ} \mathrm{~mol}^{-1}\right)$, unabhängig davon ob diese Rolle von Benzylalkohol oder 1-Phenylethanol ausgeübt wird. Dagegen ist die Deformationsenergie für den Donor der $\mathrm{OH} \cdots \pi$-Wasserstoffbrücke deutlich höher, 2.2 statt $0.7 \mathrm{~kJ} \mathrm{~mol}^{-1}$, wenn 1-Phenylethanol sich in dieser Position befindet. Bezüglich der Wechselwirkungsenergie der deformierten Monomere ist ein Energiegewinn festzustellen bei einfacher Methylierung von -0.4 bzw. $-0.7 \mathrm{~kJ} \mathrm{~mol}^{-1}$ und bei doppelter Methylierung etwa die Summe daraus von $-1.1 \mathrm{~kJ} \mathrm{~mol}^{-1}$. Dass der Wechselwirkungsgewinn in $\mathrm{homO}^{g} \pi$-BP (und auch in allen anderen $\mathrm{O} \pi$-BP Strukturen) größer ausfällt, dürfte darauf zurückzuführen sein, dass der $+\mathrm{I}$-Effekt der Methylgruppe vor allem die Akzeptorstärke der Hydroxygruppe verstärkt.[131] In O $\pi$-PB agiert die Hydroxygruppe von 1-Phenylethanol dagegen als Donor und das von der Methylgruppe weiter entfernte $\pi$ System als Akzeptor. Überlagert wird dies von der zusätzlichen langreichweitigen Dispersionswechselwirkung der Methylgruppe. Die höhere elektronische Wechselwirkung wird weiterhin im geringen Maße durch einen tendenziell etwas kleineren Anstieg der Schwingungsnullpunktsenergie unterstützt, je höher der 1-Phenylethanol-Anteil ist. In Summe ist die Erklärung für die geringere Gesamtbindungsenergie von homO $^{g} \pi$-BP gegenüber homO $^{g} \pi$-PB daher nicht etwa eine schwächere Wechselwirkung zwischen den Monomeren, sondern eine höhere notwendige Deformationsenergie damit 1-Phenylethanol als Donor der $\mathrm{OH} \cdots \pi$-Wasserstoffbrücke agieren kann. Diese höhere Deformationsenergie ist wiederum in der in Kap. 5.17 analysierten geringen intramolekularen Flexibilität von 1Phenylethanol begründet. Eine näherer Betrachtung der Struktur von homO ${ }^{g} \pi$-BB zeigt, dass sich die beiden Moleküle im Dimer vor allem im $\Phi\left(\mathrm{OC}_{1} \mathrm{C}_{2} \mathrm{C}_{3}\right)$-Torsionswinkel unterscheiden. So beträgt der Wert von $\Phi$ des Donors der $\mathrm{OH} \cdots \mathrm{O}$-Wasserstoffbrücke $44^{\circ}$, der des Donors der $\mathrm{OH} \cdots \pi$-Wasserstoffbrücke dagegen $70^{\circ}$. Letzter Wert liegt erheblich weiter entfernt vom Optimum im isolierten 1-Phenylethanol-Monomer $\left(43^{\circ}\right)$, was die höhere Deformationsenergie erklärt. Bezüglich des zweiten flexiblen Torsionswinkels $\phi\left(\mathrm{HOC}_{1} \mathrm{C}_{2}\right)$ zeigen die verschiedenen Dimere deutlich weniger Varianz zwischen den Strukturmotiven.

Die realisierten Werte sind für die weiteren BB-Dimere in Abb. 8.15 zusammengestellt und mit relaxierten Scans des Torsionswinkels für die isolierten Monomere von Benzylalkohol und 1-Phenylethanol verglichen. Es ist zu erkennen, dass beide Moleküle in dem markierten Bereich von 14-57 ähnlich flexibel sind. Diese Grenzen entsprechen einer maximal um $0.7 \mathrm{~kJ} \mathrm{~mol}^{-1}$ höheren Deformationsenergie von 1-Phenylethanol gegenüber Benzylalkohol, was dem zuvor festgestellten mittleren Gewinn an Wechselwirkungsenergie und Schwingungsnullpunktsenergie bei Methylierung entspricht. Innerhalb dieses Bereiches überkompensiert der zusätzliche intermolekulare Energiegewinn die notwendige intramolekulare Deformationsenergie. Unterhalb und besonders oberhalb dieser Grenzen steigt dagegen die Differenz der Deformationsenergien stark an. Die Winkel der Benzylalkohol-Dimere liegen teilweise innerhalb, teilweise außerhalb dieses Bereiches. Durch die starke Wechselwirkung über zwei Wasserstoffbrücken und erhebliche Dispersionskräfte sind die Dimere weitgehend starr, daher verschiebt sich durch einfache Methylierung der Torsionswinkel nur um wenige Grad in Richtung des Optimums von 1-Phenylethanol, während der Wert des verbleibenden Benzylalkohol-Moleküls fast unverändert bleibt. Diese lokalisierte Strukturänderung erklärt, warum die energetische Auswirkung der doppelten Methylierung nahezu der Summe der einfachen Methylierung 


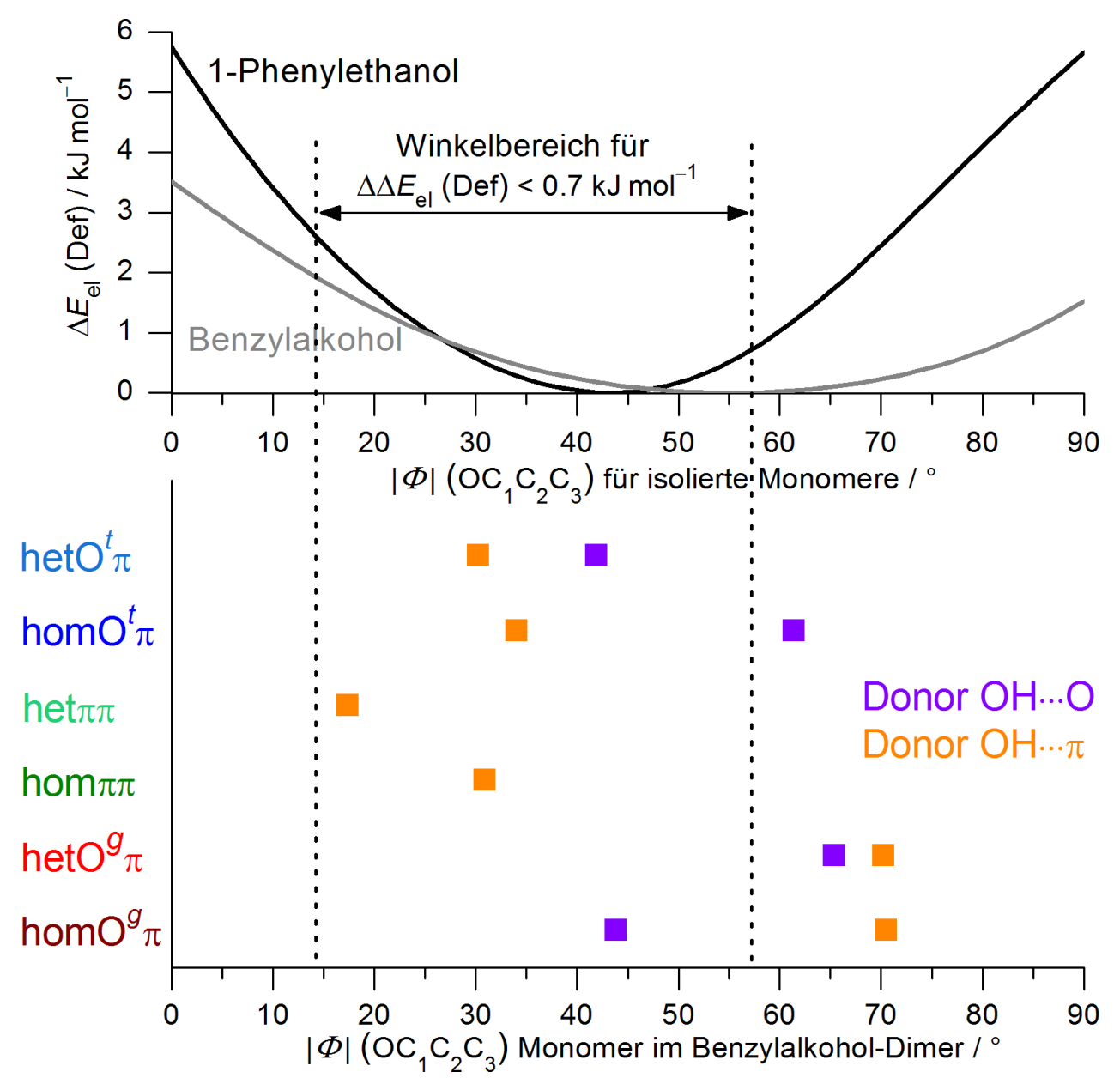

Abb. 8.15: Relaxierte Scans des $\Phi\left(\mathrm{OC}_{1} \mathrm{C}_{2} \mathrm{C}_{3}\right)$-Torsionswinkels von 1-Phenylethanol und Benzylalkohol im Vergleich mit den realisierten Werten in Benzylalkohol-Dimeren. Markiert ist der Winkelbereich von $16-55^{\circ}$, in dem die elektronische Deformationsenergie von 1-Phenylethanol höchstens $0.5 \mathrm{~kJ} \mathrm{~mol}^{-1}$ höher ist als die von Benzylalkohol. Methylierung des betreffenden Moleküls in Benzylalkohol-Dimeren in diesem Bereich führt zu einer Erhöhung der Bindungsenergie, ansonsten zu einer Erniedrigung (siehe Abb. 8.14)

entspricht. Mit diesem einfachen geometrischen Kriterium, ob der Winkel sich im Bereich von $14-57^{\circ}$ befindet oder nicht, lässt sich qualitativ korrekt vorhersagen, welche Strukturen durch Methylierung eine Erhöhung, und welche eine Erniedrigung in der Bindungsenergie erfahren.

So kann beispielsweise nachvollzogen werden, warum die Bindungsenergie von homO $^{g} \pi$ ambivalent von einer einfachen Methylierung beeinflusst wird, während ihr Betrag in hetO ${ }^{g} \pi$ dagegen bei Methylierung in beiden Positionen erniedrigt wird. Mit 1Phenylethanol in der richtigen Position in gemischten Dimer für homO ${ }^{g} \pi$ kann die Bindungsenergie gesteigert werden, ohne dass dafür zusätzliche Deformationsenergie aufgewendet werden muss, während dies für hetO ${ }^{g} \pi$ nicht möglich ist. Dies erklärt die höhere chirodiastaltische Energie im gemischten Dimer im Vergleich zu BB. Die (zweite) Methylierung am Donor der $\mathrm{OH} \cdots \pi$-Wasserstoffbrücke ist für homO ${ }^{g} \pi$ dagegen besonders ungünstig, sodass sich die Energien von homO ${ }^{g} \pi$ und hetO ${ }^{g} \pi$ bei PP wieder annähern. Grundsätzlich ist aber hetO ${ }^{g} \pi$ gegenüber homO $^{g} \pi$ vor allem durch eine geringere Wech- 


\section{Komplementarität in Dimeren von Benzylalkohol und 1-Phenylethanol}

selwirkungsenergie benachteiligt, während Schwingungsnullspunktsenergieeffekte kaum eine Rolle spielen und Unterschiede in der Deformationsenergie nur im gemischten Dimer relevant sind.

Auch die erhöhte Bedeutung des $\mathrm{O}^{t} \pi$-Motivs in PP ergibt sich aus dieser Betrachtung, insbesondere für die bei BB besonders instabile het ${ }^{t} \pi$-Variante. Wie der Vergleich zwischen den $\mathrm{O}^{g} \pi$ - und $\mathrm{O}^{t} \pi$-Motiven in Tab. 8.14 zeigt, ist $\mathrm{O}^{t} \pi$ generell durch geringe Wechselwirkungsenergien destabilisiert, und in BB zusätzlich durch relativ hohe Deformationsenergien. Dies wird durch die etwas geringeren Schwingungsnullpunktsenergien nur wenig abgemildert. Allerdings profitiert dieses Motiv besonders stark von der doppelten Methylierung, da die enthaltenen Monomere Geometrien nahe am Optimum von 1-Phenylethanol aufweisen. Aus diesem Grund ist für $\mathrm{O}^{t} \pi$ im gemischten Dimer auch BP stabiler als $\mathrm{PB}$, wenn also 1-Phenylethanol als Akzeptor der $\mathrm{OH} \cdots \mathrm{O}$-Wasserstoffbrücke agiert. Dies entspricht der Erwartung bei Dimerisierung zwischen unterschiedlich substituierten Alkoholen [131]. Im $\mathrm{O}^{g} \pi$-Motiv wird dagegen durch die besonders große notwendige strukturelle Deformation die umgekehrte Rollenverteilung für den Gewinn der hohen Wechselwirkungsenergie erzwungen.

Eine entsprechende Zerlegung der Energiebeiträge ist auch für das $\pi \pi$-Motiv in Tab. 8.15 gezeigt. hom $\pi \pi$ hat im Vergleich zum $\mathrm{O}^{g} \pi$-Motiv eine verringerte Wechselwirkungsenergie, die sich bei Methylierung kaum ändert. Auch die Deformationsenergien sind relativ hoch, die sich aber bei Methylierung etwas verringern, da die in hom $\pi \pi$ realisierten Werte für $\Phi$ (31-34 ${ }^{\circ}$, je nach Zusammensetzung) näher am Optimum für 1-Phenylethanol $\left(43^{\circ}\right)$ als für Benzylalkohol $\left(55^{\circ}\right)$ liegen. Dabei sinkt zudem die Schwingungsnullpunkt-

Tab. 8.15: Elektronische Deformationsenergien vom Benzylalkohol $\Delta E_{\mathrm{el}}$ (Def : B) bzw. 1Phenylethanol $\Delta E_{\text {el }}\left(\right.$ Def : P), elektronische Wechselwirkungsenergien $\Delta E_{\text {el }}$ (Wechselwirkung) der deformierten Monomere, Änderungen der Schwingungsnullpunktsenergie bei der Dimerisierung $\Delta E_{0}$ (Schwingungsnullp.) und die sich jeweils als Summe ergebenden Gesamtbindungsenergien $B_{0}$ für Dimere von Benzylalkohol (BB), 1-Phenylethanol (PP) und gemischte Dimere (BP) des $\pi \pi$-Motivs. Alle Werte sind in $\mathrm{kJ} \mathrm{mol}^{-1}$ angegeben und auf B3LYP-D3(BJ)/may-cc-pVTZ-Niveau berechnet.

\begin{tabular}{lccc}
\hline & hom $\pi \pi-\mathrm{BB}$ & hom $\pi \pi-\mathrm{BP}$ & hom $\pi \pi-\mathrm{PP}$ \\
$\Delta E_{\mathrm{el}}($ Def : B $)$ & $2 \times 1.4$ & 1.5 & - \\
$\Delta E_{\mathrm{el}}($ Def : P $)$ & - & 1.2 & $2 \times 1.2$ \\
$\Delta E_{\mathrm{el}}$ (Wechselwirkung $)$ & -45.8 & -46.0 & -45.9 \\
$\Delta E_{0}$ (Schwingungsnullp. $)$ & 4.3 & 4.1 & 3.8 \\
\hline$B_{0}$ & -38.7 & -39.2 & -39.8 \\
\hline & het $\pi \pi-\mathrm{BB}$ & het $\pi \pi-\mathrm{BP}$ & het $\pi \pi-\mathrm{PP}$ \\
$\Delta E_{\mathrm{el}}($ Def : B $)$ & $2 \times 2.4$ & 3.0 & - \\
$\Delta E_{\mathrm{el}}$ (Def : P $)$ & - & 1.5 & $2 \times 2.0$ \\
$\Delta E_{\mathrm{el}}$ (Wechselwirkung) & -47.7 & -47.6 & -46.5 \\
$\Delta E_{0}$ (Schwingungsnullp. $)$ & 3.9 & 3.8 & 3.7 \\
\hline$B_{0}$ & -39.1 & -39.3 & -38.8 \\
\hline
\end{tabular}


senergie, die deutlich niedriger ist als bei $\mathrm{O}^{g} \pi$ und den elektronischen Nachteil teilweise kompensiert.

het $\pi \pi$ hat eine vom Betrag höhere Wechselwirkungs- und geringere Schwingungsnullpunktsenergie, aber auch eine größere Deformationsenergie als hom $\pi \pi$. Abweichend von den anderen Strukturen erfährt het $\pi \pi$ starke geometrische Veränderung durch einfache Methylierung. Beträgt der Wert von $\Phi$ im symmetrischen het $\pi \pi$-BB für beide Moleküle $17^{\circ}$, so verändert er sich im gemischten Dimer für 1-Phenylethanol zu $29^{\circ}$, während der Torsionwinkel im verbleibenden, flexibleren Benzylalkohol nur noch $10^{\circ}$ beträgt. Hieraus wird im einfachen Modell mit dem relevanten Winkelbereich von $14-57^{\circ}$ wiederum verständlich, warum die erste Methylierung mit einer Erhöhung der Bindungsenergie, die zweite dagegen mit einer Erniedrigung verbunden ist. Allerdings gilt dies hier aus anderen Gründen, denn für $\pi \pi$ geht die Methylierung nicht mit einem Gewinn an Wechselwirkungsenergie, sondern mit Abbau von Deformations- und Schwingungsnullpunktsenergie einher. Im wieder symmetrischen, doppelt methylierten het $\pi \pi$-PP beträgt der Wert für beide Moleküle schließlich 23․ Derartige Konformationsänderungen zu kleinen Werten von $\Phi$ bei Aggregation wurden bereits auch bei Wechselwirkungen von Benzylalkohol und 1-Phenylethanol mit ausgedehnten $\pi$-Systemen diskutiert.[330, 334]

Auffällig ist die verringerte Wechselwirkungsenergie in het $\pi \pi$-PP gegenüber BB und BP. Der direkte Vergleich zwischen BB und PP zeigt, dass PP einen etwas größeren Abstand zwischen beiden Molekülen aufweist als BB. Offenbar reicht der Wechselwirkungsgewinn bei weiterer Annäherung nicht aus, um die dafür notwendige höhere Deformationsenergie von 1-Phenylethanol aufzubringen. Die geringe Flexibilität von 1Phenylethanol ist daher dafür verantwortlich, dass für PP die chirodiastaltische Energie im $\pi \pi$-Motiv ihr Vorzeichen wechselt.

Zusammengefasst kann durch eine einfache Betrachtung des geometrischen Parameters $\Phi$ qualitativ vorhergesagt werden, ob die Einführung einer Methylgruppe zu einer Erhöhung oder Erniedrigung der Bindungsenergie führt. Die dahinter stehenden Substitutionseffekte sind vielfältig und reichen von der Beeinflussung der intramolekularen Flexibilität des Moleküls über induktive Effekte auf die Wasserstoffbrücken und Beeinflussung der Dispersionswechselwirkung bis hin zu Veränderungen der Schwingungsnullpunktsenergie. In Summe kann so durch selektive chemische Substitution die subtile Balance zwischen homo- und heterochiralen Anordnungen, sowie zwischen verschiedenen Strukturmotiven einschließlich Donor-Akzeptor-Isomerie, gesteuert werden.

\subsection{Ausblick}

Bereits in den einzelnen Unterkapiteln wurde auf die vielversprechend erscheinende Untersuchung von Tri- und womöglich Tetrameren von Benzylakohol und 1-Phenylethanol hingewiesen. Anders als die Cluster von Ethanol (Kap. 4.4.2 und 4.8.2) und 2-Butanol (Kap. 5.15.2) zeigen diese weitgehend aufgelöste Banden, sodass die Chance besteht diese einzelnen Konformeren zuzuordnen. Genutzt werden kann hierfür sowohl die Komplementarität zwischen IR- und Raman-Detektion, wie auch die zwischen Chiralitätssynchronisation und -diskriminierung. Durch das Vorhandensein von UV-Chromophoren können auch ergänzend größen- und konformerenselektive Doppelresonanzmethoden 


\section{Komplementarität in Dimeren von Benzylalkohol und 1-Phenylethanol}

genutzt werden. Größere Cluster stellen aber weitere Herausforderungen bezüglich der längeren Rechenzeit und des größeren Konformationsraumes dar. Auch die Energiezerlegung zum Verständnis der Dimer-Resultate kann noch verfeinert werden, mit z. B. einer Quantifizierung der Dispersionswechselwirkung [27].

Das Molekülpaar Benzylalkohol/1-Phenylethanol besitzt wegen seiner sehr weitgehenden Analogie Potential für die Untersuchung von gemischten Dimeren mit anderen permanent chiralen Molekülen. Bei der Diskriminierung mit 1-Phenylethanol lassen sich homound heterochirale Komplexe getrennt identifizieren, während deren relative Stabilität in der Induktion mit Benzylalkohol beurteilt werden kann. Allerdings müssen dabei auch die Substitutionseffekte der Methylgruppe beachtet werden, die besonders zum Tragen kommen, wenn die Moleküle in den Dimeren stark deformiert werden.

Ein Beispiel mit geringer Deformation ist die Kombination mit $\alpha$-Pinen, die für 1Phenylethanol in Kap. 6 behandelt wurde. Für die Substitution mit Benzylalkohol wird hier berechnet, dass die spektrale Aufspaltung von 1 auf $4 \mathrm{~cm}^{-1}$ erhöht wird (bei Verschiebung der absoluten Bandenpositionen um -2 bzw. $-5 \mathrm{~cm}^{-1}$ ), während sich die Energiedifferenz von -0.1 auf $+0.2 \mathrm{~kJ} \mathrm{~mol}^{-1}$ ändern soll. Auch wenn die Substitutionseffekte absolut gesehen klein sind, ist die Chiralitätserkennung in diesem Fall vermutlich zu subtil um das Vorzeichen der chirodiastaltischen Energie mit Benzylalkohol eindeutig aufzuklären.

Ein Beispiel mit stärkerer Deformation des Alkohols ist das gemischte Dimer von Benzylalkohol mit Methyllactat, welches von Sennert [341] untersucht wurde. Hier wird eine ausgeprägte Induktion mit einer chirodiastaltischen Energie mit einem Betrag von $2.5 \mathrm{~kJ} \mathrm{~mol}^{-1}$ (nach eigener Rechnung auf B3LYP-D3(BJ)/may-cc-pVTZ-Niveau) vorhergesagt. In Übereinstimmung hierzu wurden experimentell nur Hinweise auf eine einzelne Struktur im Dimerbereich gefunden. Da Benzylalkohol in der instabileren Kombination besonders stark deformiert ist, weitet sich die Energiedifferenz auf $3.2 \mathrm{~kJ} \mathrm{~mol}^{-1}$ bei Substitution mit 1-Phenylethanol aus. Durch die Kombination von enantiomerenreinem Methyllactat mit den beiden Enantiomeren von 1-Phenylethanol könnte die Zuordnung weiter unterstützt werden. Entspricht die beobachtete Struktur der zugeordneten, sollte in der Kombination von (-)-(S)-Methyllactat mit (-)-(S)-1-Phenylethanol durch den Substitutionseffekt sich die Bande laut harmonischer Vorhersage um $+14 \mathrm{~cm}^{-1}$ verschieben. Wird dagegen die ungünstig vorhergesagte Kombination mit (+)-(R)-1-Phenylethanol erzwungen, sollte diese dagegen bei einer um $-7 \mathrm{~cm}^{-1}$ niedrigeren Wellenzahl erscheinen. Eingeschränkt werden diese Vorhersagen aber durch weitere mögliche Dimer-Konformere, sowie durch die starke Kopplung der beiden vorhandenen OH-Streckschwingungen, deren Beeinflussung bei Substitution möglicherweise in harmonischer Näherung nicht gut beschrieben wird. 


\section{Umbau des RESS- zum Risotto-Jet}

\section{1 Übersicht}

In diesem Kapitel werden zunächst der RESS-Jet und daran unternommene Messungen vorgestellt. Ursprüngliche Zielsetzung war es, das Aggregationsverhalten und die Konformationspräferenzen von langkettigen Alkoholen zu untersuchen. Wenn eine erhitzte und komprimierte Lösung des Alkohols in einem leichtflüchtigen apolaren Lösungsmittel ins Vakuum expandiert wird, kann dieser Phasenübergang potentiell Änderungen im Bindungsmotiv bewirken. So sind Anordnungen denkbar, in denen die Dispersionswechselwirkung zwischen oder auch innerhalb [149] der Alkylketten optimiert ist. In Konkurrenz dazu können Strukturen treten, bei denen Wasserstoffbrücken zwischen den Hydroxygruppen das Motiv bestimmten. Während die Clustergenerierung in den vorherigen Kapiteln durch Aggregation von gasförmigen Monomeren erfolgte, ist es Ziel dieses alternativen Ansatzes, dies durch die Zerstäubung und der teilweisen Verdampfung der kondensierten Phase zu erreichen. Da dieser Prozess aber keine diesbezüglich auswertbaren Resultate ergab, wurde der RESS-Jet anschließend zum Risotto-Jet umgebaut. In diesem wird der flüssige Analyt in einem zusätzlichen Schritt vor der Expansion zunächst verdampft und mit Helium als Trägergas vermischt. Dies stellt die Rückkehr zum bewährten Ansatz der Clustererzeugung dar, aber unter Beibehaltung bestimmter Vorteile der Flüssigkeitsinjektion.

\subsection{Messungen am RESS -Jet}

\subsubsection{Aufbau des RESS -Jets}

RESS ist die Abkürzung für „Rapid Expansion of Supercritical Solutions“, also die rasche Expansion von überkritischen Lösungen. Der RESS-Jet wurde ursprünglich von Dana Hermsdorf in der Gruppe von Ruth Signorell aufgebaut und zur Bildung und Untersuchung von Nanopartikeln bei Expansion einer Lösung von organischen Molekülen in überkritischem Kohlenstoffdioxid genutzt.[17] Die Apparatur wurde später von Sascha Abraham in die hier beschriebene Form gebracht und zur Charakterisierung von Lösungsmittel-Expansionen (Distickstoffmonoxid, $n$-Pentan und tert-Butylalkohol) eingesetzt.[433] Der Aufbau ist in Abb. 9.1 schematisch dargestellt.

In der folgenden Beschreibung der Apparatur liegt der Fokus auf Bestandteilen, die sich auch nach dem späteren Umbau in den Risotto-Jet noch in Verwendung befinden (Beschreibung in Gegenwartsform). Nicht übernommene Bauteile (HPLC-Pumpen, Extraktor, Reservoir, Heizkabel und Lochdüse) werden hingegen in Vergangenheitsform beschrieben, für weitere Details zu diesen sei auf Ref. [433] verwiesen. 
Für den RESS-Jet wird der flüssige Analyt zunächst in ein gläsernes Vorratsgefäß gefüllt, welches über einen Teflonschlauch mit der Ansaugöffnung einer Hochdruckflüssigkeitspumpe verbunden war, welche von den Chemie-Werkstätten nach Vorbild der High Pressure Liquid Chromatography (HPLC) entwickelt wurde. Nach Druckaufbau in der HPLC-Pumpe durchläuft der Analyt den Vorerhitzer (eine beheizte Kapillare in Spiralform), dessen Zweck es ist, den Druckanstieg durch Erwärmung in empfindlicheren Teilen der Apparatur zu begrenzen. Der gesamte weitere Weg des Analyten bis zur Düse war auf die selbe oder leicht höhere Temperaturen beheizt. Dies wurde, mit Ausnahme des Heizschlauches, für alle Teile durch Umwicklung mit Heizkabeln realisiert. Optional konnte ein Lösungsmittel den Extraktor durchlaufen, in dem eine Substanz gelöst werden konnte, die im Lösungsmittel vor der Kompression und Erwärmung nicht löslich gewesen wäre. Dies wurde in der Vergangenheit bei Verwendung von überkritischem Kohlenstoffdioxid als Lösungsmittel genutzt.[17] Der Analyt gelangte in das Reservoir einem Hohlzylinder mit bis zu $98 \mathrm{ml}$ nutzbarem Volumen. Das Reservoir war nach unten durch einen beweglichen Kolben begrenzt, dessen Position durch Druckaufbau an dessen Ober- oder Unterseite veränderlich war. An der Unterseite geschah dies durch eine zweite HPLC-Pumpe, die mit $n$-Heptan als Hydraulikflüssigkeit arbeitete. Nach Befüllen des Reservoirs wurde die erste HPLC-Pumpe abgestellt, der Zieldruck wurde nun stattdessen über das Druckgleichgewicht am Kolben mit der zweiten HPLC-Pumpe gehalten. Wurde etwas Analyt entnommen, so sank der Druck kurzzeitig ab, wurde aber durch Verschieben des Kolbens nach oben über Kompression der verbleibenden Substanz wieder hergestellt. Über einen flexiblen Heizschlauch und eine anschließende Kapillare gelangt der Analyt zum pneumatischen Ventil (PV in Abb. 9.1). Die Kapillare stellt dabei den Übergang zwischen den Abschnitten der Apparatur her, die sich in Atmosphäre bzw. im Vakuum des Expansionsrohres befinden. Dieser Übergangsbereich ist im unteren Teil von Abb. 9.2 dargestellt. Das pneumatische Ventil Typ 6 NC 710.3364 der Firma SITEC ist

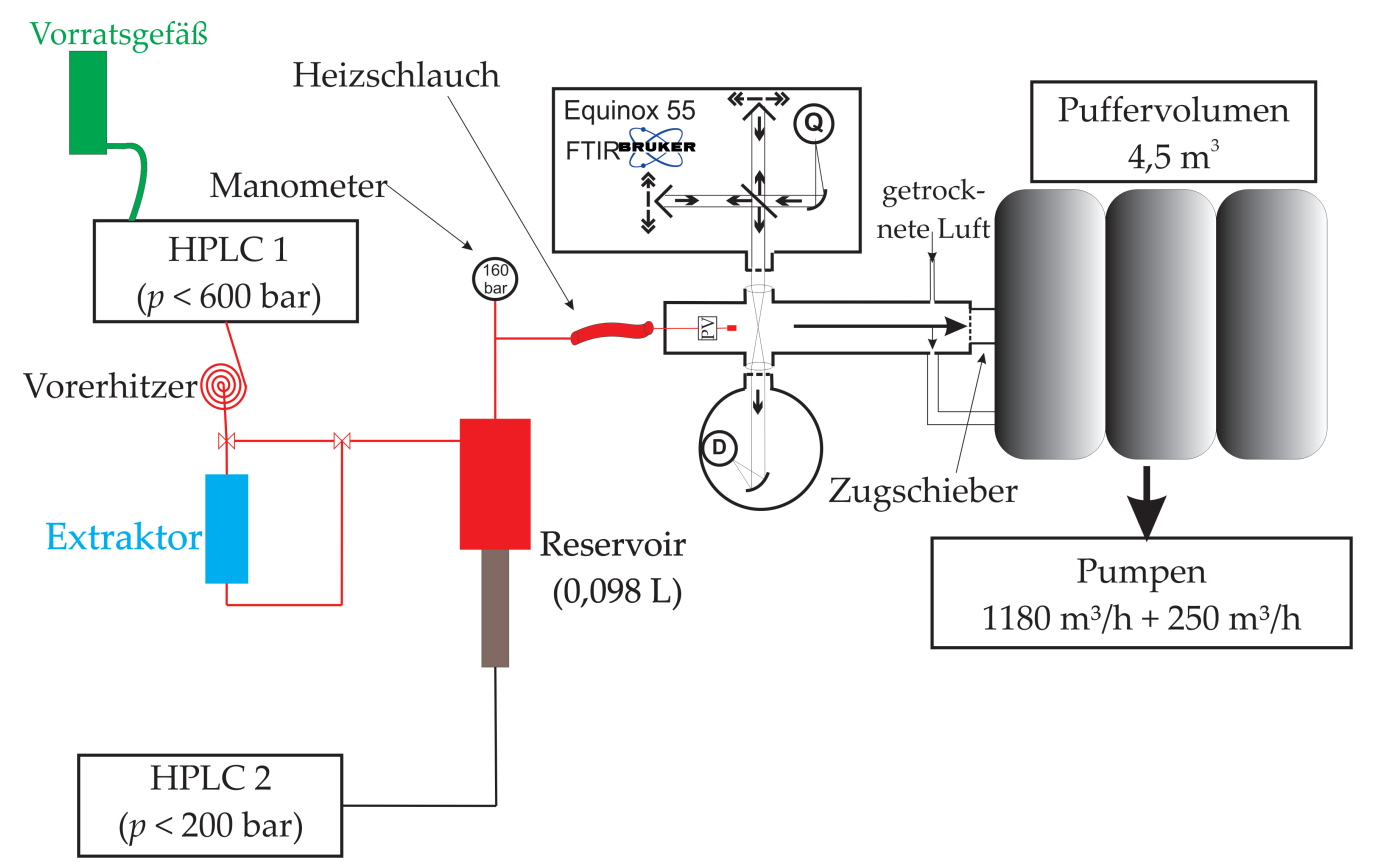

Abb. 9.1: Schematischer Aufbau des RESS-Jets, Abbildung übernommen aus Ref. [433], lizenziert nach CC BY-NC-ND 3.0. 
für Drücke bis 1000 bar und Medientemperaturen bis $200^{\circ} \mathrm{C}$ ausgelegt. Es ist im Normalzustand geschlossen und kann durch das Anlegen von 6 bar Stickstoff an der Ventilmechanik geöffnet werden, wobei eine Nadel zurückgezogen wird, die den Durchlass freigibt. Das Ventil ist von einem gasdichten Gehäuse umschlossen, welches es in Position hält und verhindert, dass das eingesetzte Stickstoffgas das umgebende Vakuum beeinträchtigt. Montiert auf den Ausgang des Ventils war eine Lochdüse mit $160 \mu \mathrm{m}$ Durchmesser, aus der die Expansion des Analyten in das Vakuum erfolgte. Zwischen Ventilnadel und Düse befand sich noch ein durch die Lochdüse evakuiertes Totvolumen von etwa $0.36 \mathrm{ml}$. Der im Vakuum befindliche Abschnitt der Kapillare, der vordere Abschnitt des Ventils mit der Öffnungsnadel und die Düse wurden über Umwicklung mit einem einzelnen Heizkabel beheizt, wobei eine etwas höhere Temperatur der Düse über eine engere Wickelung erzielt wurde.

Das Expansionsrohr (dargestellt im oberen Bereich von Abb. 9.2) ist über einen Zugschieber und einen Bypass mit einem Puffervolumen von $4.5 \mathrm{~m}^{3}$ verbunden. In diesem wird das Gas verteilt, sodass der Hintergrunddruck während der gepulsten Expansion nicht zu stark ansteigt. Das Puffervolumen wird von zwei hintereinander angeordneten Pumpen evakuiert, einer Okta 1000A Wälzkolbenpumpe von Pfeiffer Vacuum mit einer Saugleistung von $1180 \mathrm{~m}^{3} \mathrm{~h}^{-1}$ und einer Leybold Heraeus E225 Sperrschieberpumpe mit $250 \mathrm{~m}^{3} \mathrm{~h}^{-1}$. Zwischen beiden Pumpen befindet sich eine mit Flüssigstickstoff gekühlte Kühlfalle. Der Druck wird über zwei Pfeiffer Vacuum TPR 280 PTR26950A Drucksonden (platziert im Puffervolumen unmittelbar vor der Wälzkolbenpumpe sowie zwischen den Pumpen) über die Wärmeleitfähigkeit des Gases gemessen. Der bei längerer Evakuierung erreichbare tiefste Druck liegt bei etwa $2 \mu \mathrm{bar}$, im Messbetrieb mit einer typischen Wartezeit von 1-2 Minuten zwischen den Wiederholungen des Experiments schwankt er zwischen etwa $10 \mu$ bar vor und 20-200 $\mu$ bar nach einer Expansion, abhängig von der expandierten Substanzmenge. Dieser Werte gelten für Luft, für Helium wären sie laut Ka-
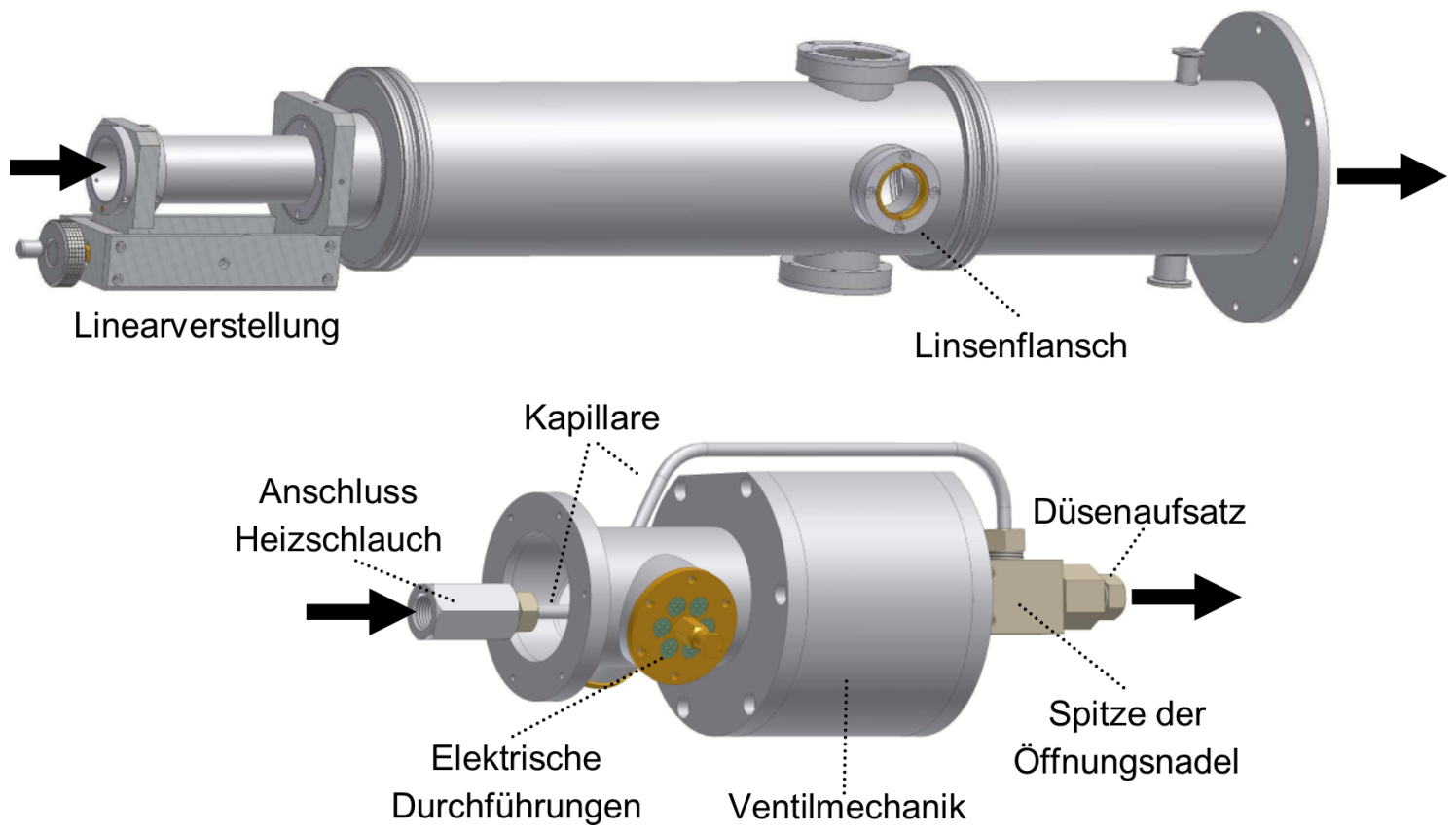

Abb. 9.2: Technische Zeichnungen des Expansionsrohrs (oben) [434] und dessen inneren Bereiches (unten) [435]. Die Pfeile geben die Fließ- bzw. Expansionsrichtung des Analyten an. 
librierung des Herstellers 20\% niedriger, für schwere Gase wie die hier expandierten Substanzen dagegen tendenziell höher. Neben dem erwähnten Hauptzugang (in Abb. 9.2 oben links), durch den der Heizschlauch, die Kabel der Heizungen und die Stickstoffleitung gehen, sowie der Verbindung zum Puffervolumen über den Zugschieber und den Bypass, besitzt das Expansionsrohr fünf weitere kleinere Zugänge. Einer davon befindet sich sich oberhalb des Bypasses nahe am Zugschieber, von den vier restlichen sind zwei horizontal und zwei vertikal oberhalb der Düse in der Mitte des Expansionsrohres angeordnet. Die beiden horizontalen Zugänge beinhalten Linsen, die anderen drei wurden in dieser Ausbaustufe für ein Sichtfenster, eine zusätzliche MKS Baratron 722A11MGA2FK Drucksonde und für das Einleiten von Luft zur gezielten Erhöhung des Hintergrunddruckes genutzt. Durch eine Linearverstellung kann der gesamte im Expansionsrohr befindliche Apparaturteil millimetergenau relativ zur Optik vor- und zurück verschoben werden, um unterschiedliche Abschnitte der Expansion zu detektieren.

Diese Detektion erfolgt über ein Bruker Equinox 55 FTIR-Spektrometer, welches ursprünglich am Filet-Jet eingesetzt wurde [37], dort aber später gegen ein Bruker IFS 66v/S ersetzt wurde [436] (dieses Modell wird auch am Popcorn-Jet verwendet). Während das Equinox 55 eher für den Routine-Laboreinsatz ausgelegt ist, ist das IFS 66v/S ein spezielleres Forschungsgerät. Nachteile des Equinox 55 sind im Vergleich eine geringere Gesamtintensität und höhere Divergenz der Strahlung, ein geringes maximales Auflösungsvermögen [37] sowie die fehlende Möglichkeit der Evakuierung zur besseren Reduktion von atmosphärischer Absorption durch Wasser und Kohlenstoffdioxid [436]. Ein Vorteil ist die größere Robustheit des Cube-Corner-Interferometers gegenüber dem gasgelagerten Linearscanner des IFS 66v/S. Als Strahlungsquelle stehen wahlweise ein wassergekühlter $100 \mathrm{~W}$ Globar (MIR) oder eine $20 \mathrm{~W}$ Wolframlampe (NIR) zur Verfügung. Wegen der geringen Leistung der verbauten Wolframlampe (20 gegenüber $150 \mathrm{~W}$ beim Filet-Jet) bietet der Globar auch im relativ hochfrequenten $\mathrm{OH}-$ Streckschwingungsbereich eine höhere Lichtintensität. Nach dem Durchlaufen des Interferometers mit einem KBr-Strahlteiler (optional: $\mathrm{CaF}_{2}$ ) wird der IR-Strahl über ein $\mathrm{KBr}$-Fenster aus dem Spektrometer hinaus und über eine in einem Flansch platzierte $\mathrm{KBr}$-Sammellinse mit $105 \mathrm{~mm}$ Brennweite in das Expansionsrohr hineingeführt. Auf der anderen Seite wird das Licht über eine baugleiche Linse wieder parallelisiert. Der Durchmesser des Expansionsrohrs beträgt generell $150 \mathrm{~mm}$, ist durch die Linsenflansche an dieser Stelle aber auf einen Linsenabstand von $200 \mathrm{~mm}$ verbreitert. Durch ein weiteres Fenster tritt das Licht in die Detektorkammer ein, in der es über einen goldbeschichteten Parabolspiegel und durch einen Filter (4100$2500 \mathrm{~cm}^{-1}$ ) auf einen mit Flüssigstickstoff gekühlten InSb-Detektor fokussiert wird. Das Spektrometer, die Detektorkammer sowie die Übergänge zum Expansionsrohr werden mit Druckluft gespült, welche zuvor über Ölfilter und einen Lufttrockner weit möglichst von Öl, Wasser und Kohlenstoffdioxid befreit wird. Zusätzlich werden die Linsen auf $50{ }^{\circ} \mathrm{C}$ beheizt, um Kondensation vorzubeugen.

Die Synchronisation der gepulsten Expansion mit den Scans des Spektrometers erfolgt über einen DG535 Delay-Generator (Stanford Research Systems). Nach der Aufnahme von 20 Hintergrundscans sendet das Spektrometer am Ende des Hintergrundscans ein TTL-Signal, welches vom Delay-Generator verzögert und in der Länge moduliert an eine TTL-Box weiterleitet wird. Letztere öffnet für die gewählte Dauer ein Magnetventil für die Stickstoffversorgung des pneumatischen Ventils, welches in Folge selbst öffnet. Die Synchronisation des RESS-Jets war derart, dass nach den 20 Hintergrundscans und dem 
Vorscan (als Kontrolle und Backup-Hintergrund) ein einzelner Probescan während der Expansion stattfand, auf den zwei weitere Nachscans nach Schließen des Ventils folgten. Dies ist fast identisch mit dem Ablauf am Filet- und Popcorn-Jet, mit den Ausnahmen, dass dort ein doppelter statt ein einzelner Vorscan erfolgt sowie dass das TTl-Signal zu Beginn der beiden Vorscans gesendet wird. Die Interferogramme von typischerweise fünf bis einigen hundert Wiederholungen werden gemittelt, fouriertransformiert und die erhaltenen Hintergrund- mit den Probe-Einkanalspektren zu Extinktionsspektren verrechnet.

\subsection{2 n-Pentan}

Bevor Expansionen von Alkohol-Lösungen untersucht werden, ist es sinnvoll, zunächst das reine Lösungsmittel als Referenz zu charakterisieren. $n$-Pentan erscheint als Lösungsmittel für die Expansion von langkettigen Alkoholen aus verschiedenen Gründen gut geeignet. Es liegt mit einer Normalsiedetemperatur von $36^{\circ} \mathrm{C}$ bei Zimmertemperatur und Atmosphärendruck flüssig vor, sodass Lösungen definierter Konzentration durch einfaches Mischen vor dem Einfüllen in die Apparatur hergestellt werden können. Von längerkettigen Alkoholen ist dabei anzunehmen, dass sie mit Alkanen vollständig mischbar sind. $n$-Pentan ist dabei aber deutlich flüchtiger als langkettige Alkohole (das hier untersuchte $n$-Dodecanol hat eine Normalsiedetemperatur von $260^{\circ} \mathrm{C}$ ), sodass es bei Einsatz einer hohen Düsentemperatur relativ leicht vom Alkohol im Vakuum verdampft werden können sollte. $n$-Pentan weist im OH-Streckschwingungsbereich keine Fundamentalübergänge auf, sodass dort Signale des Alkohols nur von intensitätsschwachen Kombinationsübergängen oder Obertönen überlagert sind. Im Bereich des verwendeten Filters lässt sich gleichzeitig auch der CH-Streckschwingungsbereich untersuchen, in dem sowohl $n$ Pentan wie auch Alkohole hohe IR-Aktivität aufweisen. Aus den unterschiedlichen Formen der dort überlagerten Banden lässt sich das Konzentrationsverhältnis grob abschätzen.

$n$-Pentan wurde bereits von Abraham am RESS-Jet im Fingerprintbereich zwischen 1400 und $700 \mathrm{~cm}^{-1}$ untersucht, dabei konnten die beobachteten Signale den beiden wichtigsten Konformeren zugeordnet werden. Allerdings wurde dabei fast keine Abhängigkeit von den Expansionsbedingungen festgestellt, sodass keine Aussage über das Ausmaß der Verdampfung getroffen werden konnte.[433]

Um das Experiment zu optimieren und Streueffekte in den tolerablen Bereich zu bringen, wurden einerseits spektroskopische Parameter variiert, diese umfassen das Strahlteiler-Material, die Strahlungsquelle, die Blendengröße, den Detektorvorwiderstand, die Spiegelgeschwindigkeit, den Scan-Modus, die Auflösung, die Faltungsgrenzen, den Abstand zwischen Düse und Detektionsbereich, sowie die Synchronisation von Expansion und Scan. Andererseits auch Einstellungen, die die Expansion selbst beeinflussen, dies sind der Expansionsdruck, die Analyttemperatur, die Düsentemperatur, die Düsenöffnungszeit, und der Hintergrunddruck. Das Ziel dabei war, die nachfolgend beschriebenen Streueffekte zu minieren. Exemplarisch sind in Abb. 9.3 einzelne Spektren einer Messreihe an $n$-Pentan gezeigt, in der der Abstand zwischen Düse und Detektionsbereich variiert wurde. Streueffekte machen sich dabei auf vier Arten in den Spektren bemerkbar:

1. Die Basislinie der Extinktion ist über den gesamten Spektralbereich zu höheren Werten verschoben, da durch die Streuung während des Probescans weniger Strah- 
lung den Detektor erreicht als während des Hintergrundscans. Die dekadische Extinktion erreicht in den dargestellten Spektren bei $4000 \mathrm{~cm}^{-1}$ einen Wert von bis zu $\lg \frac{I_{0}}{I}=0.12$, was gleichbedeutend ist mit $I=0.75 I_{0}$. Es wird also bis zu einem Viertel der Strahlung in diesem Spektralbereich durch Flüssigkeitströpfchen gestreut.

2. Die Basislinienverschiebung ist nicht einheitlich, sondern steigt zu höheren Wellenzahlen an. Dies erfolgt aber nicht in der Abhängigkeit $\propto \lambda^{4}$, wie es für die Rayleigh-Streuung bei Tröpfchen zu erwarten wäre, die deutlich kleiner sind als die eingesetzten Wellenlängen. Dies deutet darauf hin, dass auch Tröpfchen in ähnlichen Größen wie die Wellenlängen vorliegen und Mie-Streuung verursachen (4000-2600 $\mathrm{cm}^{-1}$ entsprechen 2.5-3.8 $\mu \mathrm{m}$ ).

3. Die starke Änderung des Brechungsindex im Bereich einer Absorptionsbande führt zu einer starken Wellenlängen-Abhängigkeit der Streuung. Durch den ChristiansenEffekt [437, 438] sind Absorptionsbanden asymmetrisch verzerrt. Während die hochfrequenten Flanken nach unten gezogen sind, verlaufen die niederfrequenten ungewöhnlich flach aus. Dies verändert auch die Position der (scheinbaren) Absorptionsmaxima. Dieser Effekt ist auch bekannt bei IR-Spektroskopie von Substanzen verteilt in einer Matrix (z. B. KBr-Presslingen) [244] und von Stäuben [439]. Ursa-
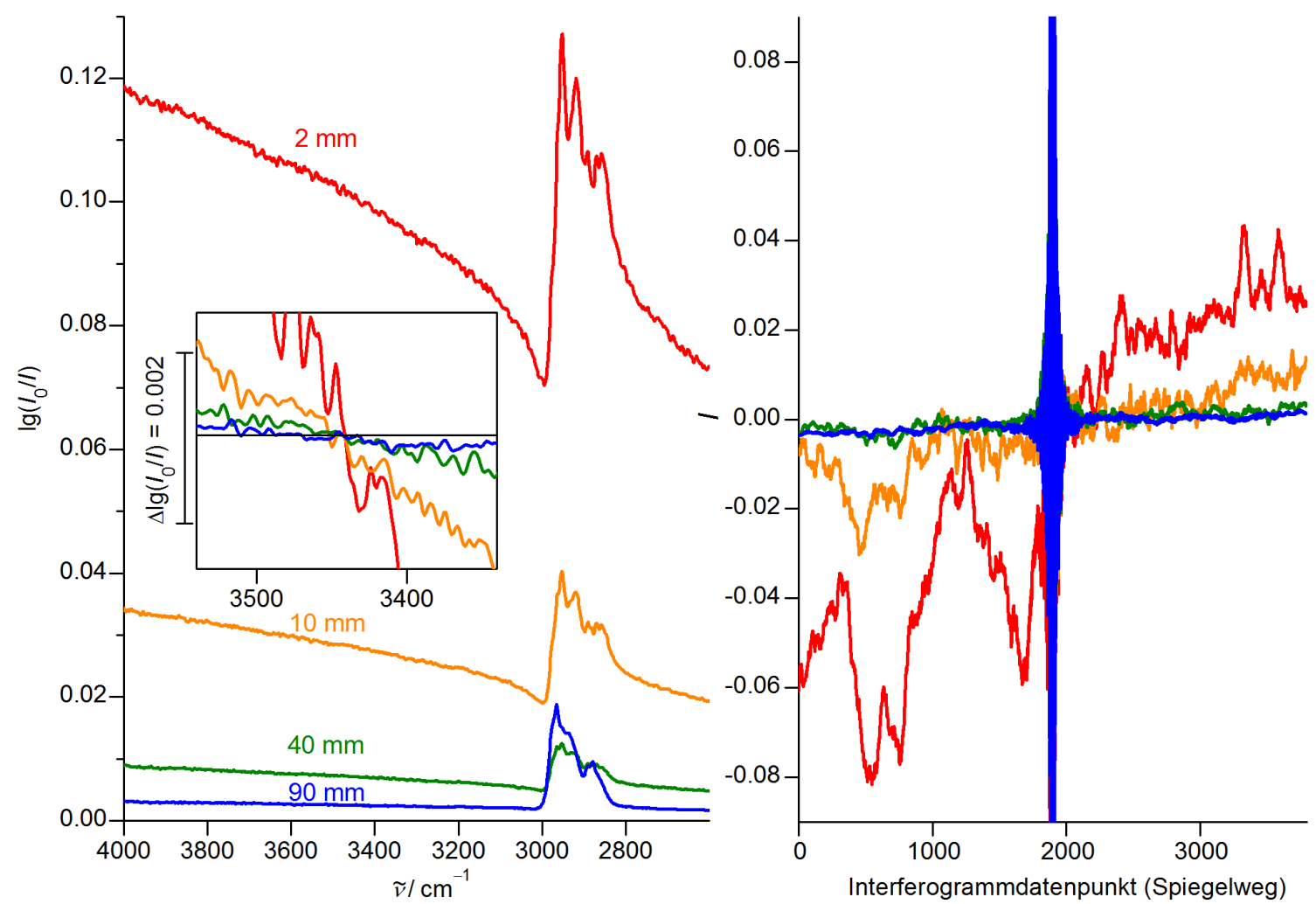

Abb. 9.3: Linke Seite: RESS -Jet-Spektren von $n$-Pentan-Expansionen bei verschiedenen Detektionsabständen (verwendet wird hier die Distanz bis zum Beginn des Signalabfalls durch Blockierung des IR-Strahls, für die Distanz bis zum halben Signalabfall sind $3 \mathrm{~mm}$ zu addieren). Expansionsparameter sind $120^{\circ} \mathrm{C}$ Zuleitungs- und $190^{\circ} \mathrm{C}$ Düsentemperatur sowie 20 bar Flüssigkeitsdruck, je 10 Wiederholungen. Insert: Vergleich der Basislinienneigung und des Rauschniveaus bei vertikaler Verschiebung auf die gleiche Extinktion bei $3440 \mathrm{~cm}^{-1}$. Rechte Seite: zugehörige Interferogramme (gleiche Farbcodierung). 
che ist auch dort eine grobkörnige Probe in ähnlicher Größe wie die Wellenlänge der Strahlung.

4. Ein statistisches Rauschen überlagert das Spektrum.

Deutlich ist erkennbar, wie die Streueffekte bei steigendem Detektionsabstand abnehmen. Die Änderungen in der Steigung der Basislinie und des Rauschens sind im Insert in Abb. 9.3 besser beurteilbar, in dem die Spektren auf die gleiche Extinktion bei einer Wellenzahl vertikal verschoben sind. Die Abnahme der Streueffekte verläuft überproportional gegenüber der Abnahme der Intensität der $\mathrm{CH}$-Absorptionsbande, was darauf hindeutet, dass ersteres nicht nur durch die Divergenz der Expansion, sondern auch durch Verdampfung der Tröpfchen erfolgt. Zudem durchläuft die Intensität der $\mathrm{CH}$-Absorptionsbande bei etwa $40 \mathrm{~mm}$ ein Minimum. Der danach folgende Wiederanstieg kann als Auftreffen auf die Machscheibe der Expansion gedeutet werden, wodurch die Substanz abgebremst und damit wieder komprimiert wird. Diese Interpretation wird durch eine Verschiebung des Intensitätsminimums zu kleineren Abständen bei Erhöhung des Hintergrunddruckes gestützt. So wird das Minimum beispielsweise bei $25 \mathrm{~mm}$ gefunden, wenn das Expansionsrohr bei geschlossenem Zugschieber nur durch den Bypass evakuiert wird. Die Streueffekte sind in den zugehörigen Interferogrammen (rechte Seite von Abb. 9.3) auch nach Mittelung von 10 Wiederholungen deutlich erkennbar. Dies ist eine Folge von wiederholten Abschattungen durch einzelne Tröpfchen, die den IR-Strahl passieren.[433] Neben dem Detektionsabstand (und damit der Flugzeit) wurden Temperatur und Substanzdruck als entscheidende Größen für die Reduzierung von Streueffekten identifiziert.

Bisher wurde, wie erwähnt, die Kapillare, der vordere Teil des Ventils und die Düse über ein gemeinsames Heizkabel erwärmt, wodurch die Temperatur der Düse durch eine engere Wickelung nur wenig höher war als die der anderen Bauteile. Durch die begrenzte Temperaturtoleranz des Ventils (bis $200^{\circ} \mathrm{C}$ ) limitierte dies die Höchsttemperatur der Düse. Durch den Einbau einer zusätzlichen, unabhängigen Heizung der Düse konnte die dort erreichbare Temperatur deutlich erhöht werden. Dabei wurde gefunden, dass die Streueffekte entscheidend von der Düsentemperatur, aber nur in geringem Maße von der Temperatur der Kapillare und des Ventils abhängen. Ebenfalls nehmen die Streueffekte stark mit einem geringeren Substanzdruck ab. Dieser ist nach unten durch den Dampfdruck der unterkritischen Substanz limitiert. Eine Absenkung bis auf den Dampfdruck führt zu einer deutlichen Reduzierung der Signalintensität im Spektrum, vermutlich wegen einer Reduzierung der Flüssigkeitsdichte durch Dampfblasen. Auch diesbezüglich erwies sich eine Reduzierung der Substanztemperatur bei gleichzeitig hoher Düsentemperatur als Fortschritt, da damit auch der Druck weiter abgesenkt werden kann.

Als wichtigster spektroskopischer Parameter wurde die Spiegelgeschwindigkeit, wie von Abraham gefunden [433], bestätigt. Auch wenn dabei auf das Gain Ranging ${ }^{\text {a ver- }}$ zicht wird, lassen sich stark fluktuierende Expansionen erheblich besser bei möglichst hoher Spiegelgeschwindigkeit untersuchen. Die Messungen am RESS-Jet wurden daher mit einer Modulationsfrequenz des HeNe-Lasers von $280 \mathrm{kHz}^{\mathrm{b}}$, statt mit $80 \mathrm{kHz}$ wie am Filet-

\footnotetext{
${ }^{\mathrm{a}}$ Gain Ranging ist ein Verstärkungsmechanismus für die Flanken des Interferogramms zur besseren Ausnutzung der Bandbreite des Analog-Digital-Wandlers. Dieser funktioniert aber nur bis Spiegelgeschwindigkeiten von $80 \mathrm{kHz}$.

${ }^{\mathrm{b}}$ Die Modulationsfrequenz des HeNe-Lasers $f_{\mathrm{HeNe}}$, ist mit der Spiegelgeschwindigkeit $v$ über $v=2 \cdot f_{\mathrm{HeNe}}$. $15800 \mathrm{~cm}^{-1}$ verknüpft.[433]
} 
und Popcorn-Jet, durchgeführt. Nachdem die Bedingungen soweit optimiert wurden, dass auch schwache Kombinationsbanden von $n$-Pentan im OH-Streckschwingungsbereich beobachtet werden konnten, wurde im nächsten Schritt $n$-Dodecanol zugemischt.

\subsection{3 $n$-Dodecanol in $n$-Pentan}

$n$-Dodecanol ist ein langkettiger Alkohol mit einer linearen Alkylkette aus 12 Kohlenstoffatomen, einer Normalsiedetemperatur von $260^{\circ} \mathrm{C}$ und einer Schmelztemperatur von $23^{\circ} \mathrm{C}$. Da letztere im Bereich der Zimmertemperatur liegt, wurde $n$-Dodecanol für eine mögliche Anwendung als Latentwärmespeicher untersucht, gemischt mit langkettigen Alkanen [440] oder Ketonen [441].

IR-Spektren von $n$-Dodecanol in verschiedenen Aggregatzuständen sind in Abb. 9.4 zusammengestellt. Im oberen Teil der Abbildung sind Referenzspektren der Substanz im gasförmigen, flüssigen und festen Aggregatzustand vergleichend dargestellt. Diese unterscheiden sich vor allem im $\mathrm{OH}$ - und im geringeren Maße auch im $\mathrm{CH}$-Streckschwingungsbereich. Im gasförmigen Zustand liegt eine $\mathrm{OH}-$ Streckschwingungsbande mit einem Maximum bei $3670 \mathrm{~cm}^{-1}$, im flüssigen bei $3330 \mathrm{~cm}^{-1}$ und im festen bei $3290 \mathrm{~cm}^{-1}$ vor.

Darunter sind Jet-Spektren, aufgenommen am Falafel- [70] und Popcorn-Jet, dargestellt. Auch hier sind breite $\mathrm{OH}-\mathrm{Streckschwingungsbanden} \mathrm{vorhanden,} \mathrm{die} \mathrm{scheinbar} \mathrm{aber}$ zweigeteilt sind, wobei der schwache hochfrequente Bereich bei $3500-3550 \mathrm{~cm}^{-1} \mathrm{zu}$ finden ist. Dies entspricht in etwa dem Bereich, in dem für kürzere n-Alkanole Dimere zugeordnet wurden [14].

Am RESS-Jet wurde zunächst eine Lösung von 10 Molprozent $n$-Dodecanol in $n$ Pentan untersucht. Repräsentative Spektren bei 110 und $20 \mathrm{~mm}$ Düsenabstand sind im unteren Teil von Abb. 9.4 gezeigt, also jenseits sowie vor der Machscheibe. Streueffekte sind mit Zumischung von $n$-Dodecanol deutlich stärker als mit reinem $n$-Pentan. Bei großem Düsenabstand wird im Probescan, d. h. während das Ventil noch geöffnet ist, eine $\mathrm{CH}$-Streckschwingungsbande beobachtet, die der von $n$-Pentan ähnelt. Es sich aber auch schwächere Beiträge von $n$-Dodecanol zu erkennen, in etwa gemäß der Erwartung aus dem Mischungsverhältnis. Im direkten Vergleich mit einem unter gleichen Bedingungen aufgenommen $n$-Pentan-Spektrum lässt sich auch eine schwache sehr breite Bande im OH-Streckschwingungsbereich feststellen. Im ersten Nachscan, etwa 80 ms später und nach Schließung des Ventils, weist das Profil der $\mathrm{CH}-$ Streckschwingungsbande dagegen praktisch reinen $n$-Dodecanol-Charakter auf, am ehestens vergleichbar mit dem Spektrum von flüssigem $n$-Dodecanol. Breite und intensivere OH-Streckschwingungsbanden sind erkennbar, allerdings verzerrt durch den Christiansen-Effekt. Offenbar ist bereits kurz nach Schließen des Ventils das leichtflüchtige Lösungsmittel weitgehend verdampft und im Puffervolumen verteilt, während weiterhin $n$-Dodecanol Tröpfchen im Mikrometerbereich detektiert werden.

Wird die Expansion näher an der Düse detektiert, wird ein sehr ungewöhnliches Spektrum erhalten. Die CH-Bande kann als Überlagerung aus einem positiven $n$-Pentan- und einem negativen $n$-Dodecanol-Beitrag interpretiert werden. Dies bedeutet, dass im Hintergrundscan vor der Expansion mehr $n$-Dodecanol detektiert wird als während der Expansion. Im ersten Nachscan wird in Summe eine schwach positive Bande mit $n$-DodecanolCharakter erhalten. Dieses Spektrum ist allerdings von einer Oszillation mit einer Periode 

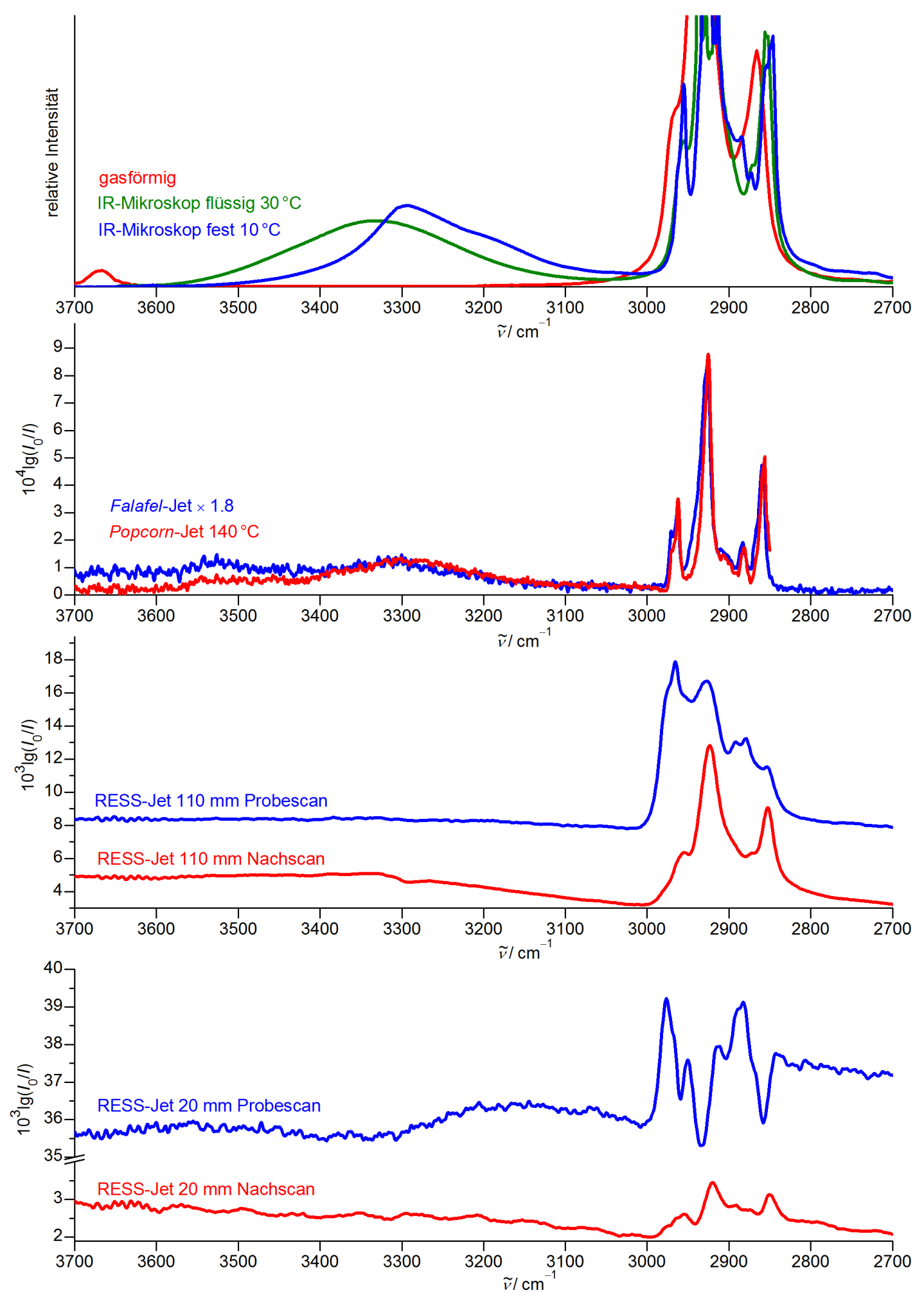

Abb. 9.4: Spektren von $n$-Dodecanol in verschiedenen Aggregatzuständen. Oben: Gasphase [442] und flüssig/fest (gemessen am IR-Mikroskop [441] von Alexandra Domanskaya). Oben Mitte: Falafel-Jet-Spektrum von Altnöder [70] und Popcorn-Spektrum von Juhyon Lee (Archivmaterial). Unten Mitte und Unten: RESS-Jet-Spektren einer Lösung von zehn Molprozent $n$-Dodenanol in $n$-Pentan bei zwei verschiedenen Detektionsabständen, sowie während und nach der Ventilöffnung (Probe-/Nachscan). Expansionsparameter waren $120^{\circ} \mathrm{C}$ Zuleitungs- und $190^{\circ} \mathrm{C}$ Düsentemperatur sowie 20 bar Flüssigkeitsdruck, je 100 Scans. 
von etwa $70 \mathrm{~cm}^{-1}$ unbekannter Ursache überlagert. In den zugehörigen Interferogrammen wurden dagegen keine Auffälligkeiten beobachtet. Störsignale durch die Düsenschließung können ausgeschlossen werden, da die Störung auch in späteren Nachscans (bei Erweiterung des Messablaufs), aber nicht bei einem größeren Detektionsabstand oder bei der Expansion von reinem $n$-Pentan auftritt. Es handelt sich dabei offenbar um ein weiteres spektrales Streuartefakt von $n$-Dodecanol-Tröpfchen.

Für die Persistenz von $n$-Dodecanol zwischen den Wiederholungen (für die hier gezeigte Spektren durchgeführt mit einer Frequenz von etwa $1 \mathrm{~min}^{-1}$ ) kommen verschiedene Möglichkeiten in Betracht:

1. n-Dodecanol kondensiert an den Wänden des Expansionsrohrs und auch auf den Linsen.

2. In der Gasphase bleibt $n$-Dodecanol als Aerosol im Expansionsrohr zurück.

3. n-Dodecanol expandiert auch nach dem Schließen des Ventils fortlaufend weiter aus dem Totvolumen zwischen Ventilnadel und Düse.

Die erste Möglichkeit erscheint unwahrscheinlich, da das im Hintergrundscan detektierte $n$-Dodecanol offenbar durch die nachfolgende Expansion verdrängt wird. Gegen die zweite Möglichkeit spricht, dass sich das Aerosol im $4.5 \mathrm{~m}^{3}$ großen Puffervolumen stark verdünnen müsste und konstant abgepumpt wird. $n$-Dodecanol bleibt nach einem Puls etwa 10 Minuten lang nachweisbar im Vergleich zu dem ersten Hintergrundspektrum der Messreihe. Auch Altnöder merkte am Falafel-Jet Probleme mit negativen $n$-DodecanolBanden an.[70] Eine dort gefundene Lösung war, die Helium-Expansion bereits vor dem Hintergrundscan zu starten, um das $n$-Dodecanol aus dem Detektionsbereich auszuspülen, während neues $n$-Dodecanol anschließend durch Laserverdampfung in den Heliumstrom eingebracht wird.

Weitere Messungen wurden mit verringerten Konzentrationen von $n$-Dodecanol durchgeführt. Dabei nehmen die Streueffekte im gleichen Maße ab wie die Banden von $n$ Dodecanol. Konzentrationseffekte auf Bandenpositionen, die sich nicht durch veränderte Überlagerungen von $n$-Dodecanol- und $n$-Pentan-Banden erklären lassen, wurden nicht festgestellt. Dies kann so interpretiert werden, dass durch Verdünnung nur die Menge, aber nicht die Eigenschaften der $n$-Dodecanol-Aggregate verändert wird. Hinweise auf kleinere Cluster oder Monomere wurden nicht gefunden.

Zusammengefasst kann festgestellt werden, dass sich $n$-Pentan als leichtflüchtiges Lösungsmittel zwar offenbar gut vom schwerflüchtigen Analyten $n$-Dodecanol trennen, letzteres sich aber nicht ausreichend zerstäuben bzw. verdampfen lässt. Streueffekte konnten nicht ausreichend reduziert werden, um Effekte auf $\mathrm{OH}-$ Streckschwingungsbanden beim Übergang aus dem gelösten in den Clusterzustand zu analysieren. Neue Probleme traten durch die Persistenz von $n$-Dodecanol auf. Daher wurde im nächsten Schritt zum Vergleich Methanol als besonders leichtflüchtiger Alkohol untersucht.

\subsubsection{Methanol}

Vor den Messungen mit Methanol wurden Optimierungen an der Heizungssteuerung vorgenommen, wodurch Düsentemperaturen bis $350{ }^{\circ} \mathrm{C}$ erreichbar wurden. In Kombination 
mit der hohen Flüchtigkeit von Methanol wurde es so möglich, Streueffekte bei der Expansion fast vollständig zu vermeiden. Durch Variation des Düsenabstandes konnte so die schrittweise Verdampfung von Methanol entlang der Expansion beobachtet werden. Zwei repräsentative Spektren sind in Abb. 9.5 dargestellt.

In $20 \mathrm{~mm}$ Abstand zur Düse ist die OH-Bande wasserstoffverbrückter Moleküle ähnlich intensiv wie die $\mathrm{CH}$-Bande, Anzeichen für Monomere sind nicht erkennbar. Bei $110 \mathrm{~mm}$ Abstand ist dagegen die OH-Bande wasserstoffverbrückter Moleküle schon deutlich schwächer als die $\mathrm{CH}$-Bande und ähnlich intensiv wie die $\mathrm{OH}-\mathrm{Bande}$ der Monomere. Da die IR-Aktivität freier OH-Oszillatoren deutlich geringer ist, als die solcher in Wasserstoffbrücken (in Kap. 4 wurde ein Verstärkungs-Faktor 40 zwischen dem MethanolMonomer und Clustern mittlerer Größe berechnet), kann geschlossen werden, dass der überwiegende Teil der Methanol-Moleküle während dieser Flugstrecke von $90 \mathrm{~mm}$ verdampft. Die Größe der Methanol-Tröpfchen ist offenbar unterhalb des Mikrometerbereiches, sodass Streueffekte praktisch nicht mehr auftreten, allerdings auch noch nicht klein genug, dass eine spektrale Größenauflösung gelingt. Bei Absenkung der Temperatur und besonders bei Erhöhung des Druckes traten dagegen wieder starke Streuartefakte auf.

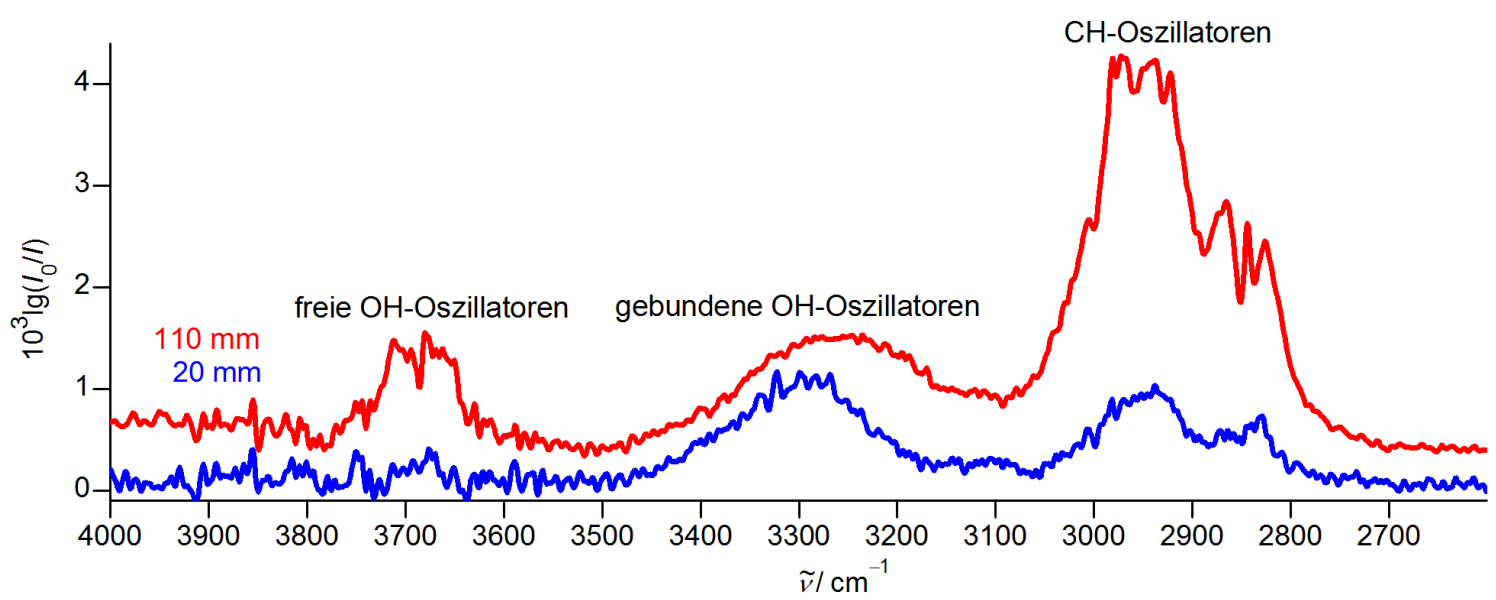

Abb. 9.5: RESS-Jet-Spektren von Methanol bei zwei verschiedenen Detektionsabständen. Expansionsparameter waren $120^{\circ} \mathrm{C}$ Zuleitungs- und $350^{\circ} \mathrm{C}$ Düsentemperatur sowie 20 bar Flüssigkeitsdruck, je 10 Wiederholungen.

\subsection{Umbau zum Risotto-Jet}

\subsubsection{Prinzip}

Auch wenn einige Fortschritte in der Zerstäubung und Verdampfung von Flüssigkeitsexpansionen erzielt wurden, blieb dabei die Beobachtung von auf molekularer Ebene auswertbaren Effekten aus. Daher wurde beschlossen, den RESS-Jet in den Risotto-Jet umzubauen. Risotto steht für "Rapid injection of solutions into a turbulent flow oven“, also die rasche Einspritzung von Lösungen in einen Ofen mit turbulenter Strömung. In diesem Ofen, der im Folgenden Mischkammer genannt wird, soll ein injizierter Flüssigkeitstropfen vollständig verdampft, mit Helium vermischt und durch eine Schlitzdüse ins Vakuum expandiert werden. In diesem Grundprinzip, bei dem der Analyt zunächst verdampft und anschließend in einer Überschallexpansion wieder aggregiert wird, ähnelt 
der Risotto-Jet wieder stärker den anderen in dieser Arbeit eingesetzten Apparaturen. Im Detail lassen sich aus diesem Prinzip bzw. aus dessen derzeitiger technischer Umsetzung, einige Vor- und Nachteile erwarten:

Vorteile:

- Die im Vakuum befindliche Mischkammer kann geheizt werden, sodass auch schwerflüchtige Substanzen verdampft werden können (anders als am Filet-Jet). Im Gegensatz zum Curry- und Gratin-Jet, und ähnlich zum Popcorn- und Falafel-Jet, erfolgt die Verdampfung erst kurz vor der Düse. Die Notwendigkeit der Beheizung größerer Apparaturteile entfällt damit.

- Eine vollständige Verdampfung einer binären Mischung sollte prinzipiell die Einstellung eines Partialdruckverhältnisses erlauben, welches unabhängig vom Dampfdruckverhältnis der beiden Komponenten ist. Dieses Problem wurde für das konkrete Beispiel der Mischung von Benzylalkohol/1-Phenylethanol bereits in Kap. 8.5 diskutiert. Ein weiterer Ansatz für die Einstellung eines beliebigen Partialdruckverhältnisses befindet sich derzeit in der Form des Double-Pickup am Popcorn-Jet in Erprobung.[69]

- Da der Analyt stets nur in der für jede einzelne Wiederholung nötigen Menge in die Kammer eingebracht wird, kann die volle thermische Beanspruchung vermutlich auf Millisekunden bis Sekunden begrenzt werden, im Gegensatz zu einer Verweildauer von Minuten bis Stunden beim Popcorn- und Gratin-Jet. Dies könnte sich als Vorteil bei der Untersuchung von thermolabilen Substanzen erweisen. Ein anderer Ansatz hierfür war die Laserdesorption beim Falafel-Jet, mit der aber keine ausreichenden Konzentrationen erzielt werden konnten.[70].

- Der Analyt kann über ein Vorratsgefäß außerhalb der Apparatur nachgefüllt werden, ein regelmäßiger Zugang zum Vakuumteil, wie am Popcorn-Jet, ist daher nicht notwendig.

- Da der Partialdruck des Analyten in der Umsetzung mit offener Mischkammer nur kurzzeitig aufgebaut wird und zeitlich nicht konstant ist, lässt sich die untersuchte Konzentration sehr einfach durch Veränderung der doppelten Synchronisation von FTIR-Scan, Substanz-Injektion und Trägergas-Puls anpassen, ohne hierfür eine Wiedereinstellung eines veränderten Temperaturgleichgewichts abwarten zu müssen. Auch über diese Entkoppelung von Temperatur und Konzentration wäre eine begrenzte Kontrolle des Dampfdruck- und damit Partialdruckverhältnisses einer binären Mischung denkbar.

Nachteile:

- Schwerflüchtige Substanzen müssen bei Laborbedingungen flüssig sein oder gelöst werden. Die zusätzliche Anwesenheit eines Lösungsmittels kann dabei die Expansion beeinflussen. Alternativ kann aber auch gerade der Vergleich zwischen einer Lösung in flüssiger Phase und einer Mischung gleicher Zusammensetzung in einer Überschallexpansion für die Untersuchung von Lösungsmitteleffekten interessant 
sein. Leichter flüchtige Substanzen können, unabhängig vom Aggregatzustand bei Laborbedingungen, dem Helium beigemischt werden - sei es über eine Vormischung in einer Druckgasflasche, oder über einen Sättiger in einer möglichen zukünftigen Ausbaustufe.

- Das vor dem Ventil befindliche Zuleitungssystem muss zunächst mit dem Analyten vollständig befüllt werden. Dies erfordert zumindest in der derzeitigen Umsetzung eine um mehrere Größenordnungen höhere Substanzmenge, als sie für aussagekräftige Spektren expandiert werden muss. Dies macht den Wechsel des Analyten (oder einer Veränderung der Konzentration in einer Mischung) aufwändig, da hierfür das gesamte Zuleitungssystem gespült werden muss. Zudem sind hierdurch teure Analyten (wie der Großteil der enantiomerenreinen Verbindungen) momentan nicht $\mathrm{zu}$ akzeptablen Kosten untersuchbar.

- Die notwendige doppelte Synchronisation zwischen Substanzinjektion, Heliumpuls und FTIR-Scan ist komplexer und muss wahrscheinlich für jeden Analyten nachjustiert werden.

Die Apparatur, die dem Risotto-Jet vermutlich am nächsten kam, ist die in Ref. [443] beschriebene aus dem Arbeitskreis von Michel Herman. Auch dort wurde das expandierte Gasgemisch in einer geheizten Mischkammer generiert, in die der flüssige Analyt injiziert, vollständig verdampft und mit dem Trägergas vermischt wurde. Die Motivation war aber dort nicht die Untersuchung von schwerflüchtigen Substanzen oder Mischungen, sondern die Generierung einer hohen und gut kontrollierbaren Konzentration. Dies war für flüssige Substanzen über die konventionelle Anreicherung in einem Sättiger bei hohem Trägergas-Durchfluss nicht in dem Maße möglich, wie er für eine kontinuierliche Expansion gekoppelt mit hochauflösender FTIR-Spektroskopie mit langen Scanzeiten benötigt wird.[443] Die Injektion der Flüssigkeit war zwar prinzipiell gepulst, lässt sich bei hoher Frequenz oder Pulslänge aber auch quasi-kontinuierlich gestalten. Dieser Ansatz war für Methanol erfolgreich, wurde nach begrenzten Ergebnissen für andere Substanzen aber offenbar nicht weiterverfolgt. Die Aggregation bei höheren Dampfdrücken limitiert einerseits die Konzentration von Monomeren, lieferte andererseits aber nicht genug Signalstärke für die Untersuchung von Clustern.[18] Dem letztgenannten Problem wird hier durch den Fokus auf OH-Streckschwingungen Rechnung getragen, für die bei Wasserstoffbrückenbildung eine starke Steigerung der IR-Aktivität stattfindet.

Jüngst wurde gezeigt, dass sich auch mit dem kommerziellen Controlled Evaporation Mixer der Firma Bronkhorst, welches nach dem gleichen Grundprinzip funktioniert, geeignete Gasgemische für FTIR-Untersuchungen von schwerflüchtigen flüssigen Substanzen, wie Methoxyphenole, in Überschallexpansionen erzeugen lassen.[444]

\subsubsection{Vorversuche}

Mitentscheidend für die Eigenschaften der Expansion (Temperatur, Clustergrößenverteilung etc.) sind die Partialdrücke des Trägergases und des Analyten. Erfahrungen an den anderen Apparaturen des Arbeitskreises Suhm [55, 105] zeigen, dass ein Trägergasdruck von etwa 0.7-1.5 bar und ein Analytpartialdruck im Bereich von 1-10 mbar (also etwa 0.1-1\% Stoffmengenanteil) für die Signaloptimierung von kleinen Clustern gut geeignet 
sind. Soll ein Flüssigkeitstropfen vollständig verdampft werden, müssen also die Volumina der Mischkammer und des Flüssigkeitstropfens in einem passenden Verhältnis stehen. Ist das Verhältnis zu groß, so ist der Analyt in der Expansion zu gering konzentriert. Ist das Verhältnis dagegen zu klein, kann unter Umständen der Analyt nicht vollständig verdampfen und es besteht die Gefahr von Streuartefakten durch Flüssigkeitstropfen. Letzteres entsprach der Situation am RESS-Jet mit seinem sehr kleinen Totvolumen zwischen Ventil und Düse.

Um die notwendige Dimensionierung der Mischkammer grob abschätzen zu können, wurde zunächst die expandierte Flüssigkeitsmenge des RESS-Jets näher bestimmt. Hierzu wurde die Düse vom Ventil abmontiert und die ungeheizte Flüssigkeit unter atmosphärischen Bedingungen aufgefangen und nach 30 Pulsen ausgewogen. Bei einer Öffnungszeit von $70 \mathrm{~ms}$ und einem Flüssigkeitsdruck von 15 bar wurden Volumina von $0.77 \mathrm{ml} / \mathrm{Puls}$ für Methanol, $0.72 \mathrm{ml} / \mathrm{Puls}$ für Aceton und $0.56 \mathrm{ml} / \mathrm{Puls}$ für Ethylenglycol erhalten. Wird berücksichtigt, dass auch die Lochdüse den Durchsatz zusätzlich beeinflusst, erscheinen diese Werte kompatibel mit der von Abraham abgeschätzten Menge von etwa $2 \mathrm{ml} / \mathrm{Puls}$ bei $120 \mathrm{~ms}$ Öffnungszeit und 100 bar für eine nicht näher angegebene Flüssigkeit [433]. Diese Größenordnung entspricht auch den eigenen Erfahrungen für die notwendige Nachfüllhäufigkeit des Reservoirs am RESS -Jet. Eine alternative Bestimmung über den Druckanstieg bei Verdampfung im geschlossenen Expansionsrohr erwies sich wegen Undichtigkeiten und vermutlich auch unvollständiger Verdampfung wegen Erreichen des Sättigungsdampfdruckes als weniger geeignet.

Soll nun durch vollständige Verdampfung von beispielsweise $0.6 \mathrm{ml}$ Ethylenglycol in einer $200^{\circ} \mathrm{C}$ heißen Mischkammer ein Druck von etwa 1 mbar aufgebaut werden, so wird überschlagsmäßig mit $\rho=1 \frac{\mathrm{g}}{\mathrm{ml}}$ und $M=62 \frac{\mathrm{g}}{\mathrm{mol}}$ gemäß dem idealen Gasgesetz ein Mischkammervolumen von etwa $400 \mathrm{~L}$ benötigt. Dies ist zum einen erheblich größer als das zur Verfügung stehende Expansionsrohr. Zum anderen zeigt der Popcorn-Jet, dass bereits mit deutlich kleineren Substanzmengen die Konzentration über einen Zeitraum von etwa $100 \mathrm{~ms}$ aufrecht erhalten werden kann für die Synchronisation mit einem Spektrometerscan dieser Dauer bei $2 \mathrm{~cm}^{-1}$ Auflösung. Am Popcorn-Jet wird der Dampfdruck in einer nur etwa $20 \mathrm{ml}^{\mathrm{c}}$ großen Probenkammer [445] aufgebaut, in der ähnlich zur Mischkammer des Risotto-Jets die Mischung mit dem Trägergas stattfindet. Die erste Voraussetzung für eine Verwirklichung des Risotto-Jets war daher zunächst eine Reduzierung des injizierten Flüssigkeitsvolumens um mehrere Größenordnungen, für die einige Vorversuche durchgeführt wurden.

Gemäß dem Gesetz von Hagen-Poiseuille (Gl. 9.1) hängt das Volumen $\Delta V$ eines homogenen Newton'schen Fluids, welches in einer Zeit $\Delta t$ laminar durch eine Kapillare fließt, sowohl vom Innenradius $r$ und der Länge $l$ der Kapillare, wie auch vom Druckunterschied $\Delta p$ und der dynamischen Viskosität des Fluids $\eta \mathrm{ab}$.

$$
\Delta V=\frac{\Delta t \pi r^{4} \Delta p}{8 \eta l}
$$

Dass trotz der deutlich höheren Viskosität von Ethylenglycol bei Zimmertemperatur $(20 \mathrm{mPa} \cdot \mathrm{s})$ im Vergleich zu Methanol $(0.5 \mathrm{mPa} \cdot \mathrm{s})$ und Aceton $(0.3 \mathrm{mPa} \cdot \mathrm{s})$ nur ein we-

\footnotetext{
${ }^{\mathrm{c}}$ Von diesem Rauminhalt sind zudem noch die Volumina der Molsiebkügelchen und der Glaswolle abzuziehen.
} 
nig kleinerer Durchsatz festgestellt wurde, ist vermutlich auf den schnellen Druckabfall bei Entspannung einer wenig kompressiblen Flüssigkeit zurückzuführen (beobachtbar am Manometer der Apparatur). Auch sind gerade niederviskose Fluide für Turbulenzen und damit höhere Strömungswiderstände anfällig. Dieser nivellierende Effekt erleichtert die Handhabung des Risotto-Jets, da sich die injizierte Flüssigkeitsmenge nicht, wie die Viskosität der Analyten, um Größenordnungen bei Wechsel der Substanz verändert.

Der verwendete Flüssigkeitsdruck und die Öffnungszeit des eingesetzten Ventils lassen sich technisch bedingt nicht weiter absenken. Eine Verkleinerung der Injektionsmenge lässt sich dagegen am effektivsten über die Wahl des Radius erreichen, welcher in G1. 9.1 in vierter Potenz eingeht. Aus diesem Grund wurde die bestehende Kapillare von $1.6 \mathrm{~mm}$ Innendurchmesser kurz nach dem Übergang in den Vakuumbereich zerteilt und das Verbindungsstück zum Ventil durch eine weitere Kapillare von $0.18 \mathrm{~mm}$ Innendurchmesser ersetzt. Dies würde gemäß Gl. 9.1 ohne den nivellierenden Effekt eine Reduzierung des Durchsatzes um etwa den Faktor 6000 erwarten lassen. Ebenfalls zur Verfügung stehen Kapillaren mit $0.25 \mathrm{~mm}$ Innendurchmesser (Faktor 1700) und $0.13 \mathrm{~mm}$ Innendurchmesser (Faktor 23000), mit denen die Injektionsmenge bei Bedarf weiter nach unten oder wieder nach oben angepasst werden kann. Solch geringe Substanzmengen lassen sich nicht mehr durch einfaches Auffangen vor dem Ventil bestimmen. Zum einen, da kleine Mengen leichtflüchtiger Substanzen, wie Methanol und Aceton, in kürzester Zeit verdampfen. Zum anderen benetzt das schwerflüchtige Ethylenglycol zunächst die Oberflächen und perlt erst bei Bildung größerer Tropfen wenig reproduzierbar ab. Die für dieses Problem gefundene Methode nutzt zusammengerollte Streifen von Filterpapier, welche direkt vor die Öffnungsnadel in das Ventil eingeführt wurden und dort das Ethylenglycol unmittelbar aufsaugen. Durch Auswiegen wurden Massenzunahmen zwischen 1.6 und $2.2 \mathrm{mg}$ pro Puls festgestellt, auch bei Verwendung des selben Streifens für mehrere Pulse. Dies entspricht einer Verringerung des Injektionsvolumens um etwa einen Faktor 300. Die Abweichung vom berechneten Faktor 6000 ist auch hier vermutlich auf den stärkeren Druckabfall bei höherem Durchsatz zurückzuführen.

\subsubsection{Mischkammer}

Nach diesen Vorversuchen wurde eine Mischkammer aus Bronze konstruiert, die $100 \mathrm{~mm}$ lang, $120 \mathrm{~mm}$ breit und $34 \mathrm{~mm}$ hoch ist (Außenmaße). Unter Berücksichtigung der relativ hohen Wandstärken (für die Integration der Heizungen) ergibt sich ein Innenvolumen von etwa $250 \mathrm{ml}$. Die Mischkammer besteht aus einer oberen und unteren Hälfte, was ein Öffnen für die Reinigung oder eine Modifikation des Innenraums erlaubt. Technische Zeichnungen der Mischkammerhälften und der angrenzenden Apparaturteile sind in Abb. 9.6 gezeigt. Beim Zusammenführen von Deckel und Boden wird durch Aussparungen an der Vorderseite eine Schlitzdüse mit $90 \mathrm{~mm}$ Breite und $0.2 \mathrm{~mm}$ Höhe gebildet. Gegenüber der zuvor beim RESS-Jet verwendeten Lochdüse weist diese eine Reihe von Vorteilen auf, die u. a. von Herman et al. diskutiert wurden.[18] So wird auf kurzen und mittleren Abständen die Kühlung und Clusterbildung effektiver.[18] Insbesondere wird der Absorptionsweg deutlich erhöht, wenn der IR-Strahl entlang der Schlitzdüse geführt wird. Dies wird bei den besonders langen Schlitzdüsen am Filet- $(600 \mathrm{~mm})$ und Gratin-Jet $(700 \mathrm{~mm})$ ausgenutzt. Da sich die Expansion effektiv nur in einer statt zwei Dimensionen aufweitet, 
sinkt die Teilchendichte langsamer mit dem Abstand ab, was der Detektion mit den relativ breiten FTIR-Strahlprofilen (im Vergleich zu Lasern) entgegenkommt.[18]

Eine Fokussierung des Strahlprofils mittels Sammellinsen führt einerseits zu höherer Intensität in der Ebene der Schlitzdüse und damit im Expansionsbereich. Anderseits entsteht dabei aber auch ein dreieckförmiger, nicht-detektierter Bereich nahe der Schlitzdüse, in dem gerade die höchsten Teilchendichten vorliegen. Eine Neuerung des Risotto-Jets ist es daher, dass die Geometrie der Schlitzdüse dem Strahlprofil angepasst ist. Aus dieser Überlegung ist die Schlitzdüse nicht linear $\left(180^{\circ}\right)$, sondern leicht angewinkelt $\left(162^{\circ}\right)$ konstruiert.

Das Strahlprofil im mittleren Infrarot bei $5 \mathrm{~mm}$ Spektrometer-Blende wurde durch das Einbringen einer Irisblende bestimmt, deren schrittweises Schließen zu Signalabfall am Detektor führt. Der Beginn des Signalabfalls ist nicht völlig scharf, lässt aber dennoch eine gute Bestimmung des Strahldurchmessers an unterschiedlichen Positionen zu. Dabei wurde im Fokus ein Durchmesser von $6 \mathrm{~mm}$ gefunden, der sich jeweils $60 \mathrm{~mm}$ rechts und links davon zu $25 \mathrm{~mm}$ aufweitet. Im sichtbaren Bereich wurden unter Verwendung der Wolframlampe, Millimeterpapier und einer Abdunkelung des Labors vergleichbare Werte erhalten. Unter Annahme eines doppelten Kegelstumpfes als Strahlprofil lässt sich aus diesen Daten trigonometrisch die genannte Abwinkelung der Düse bestimmen. Ein äquivalentes Düsendesign wurde auch erfolgreich für den Popcorn-Jet adaptiert [69] und führte dort, in Verbindung mit weiteren Optimierungen des Gasflusses [422], zu erheblichen Verbesserungen, die in Kap. 8 genutzt wurden.

Die Kontaktflächen zwischen Deckel und Boden der Mischkammer bilden eine Labyrinth-Dichtung, die ohne temperaturanfällige Dichtungsmaterialien auskommt. Deren begrenzte Vakuumdichtigkeit ist hier kein Nachteil, da die Kammer bereits über die Schlitzdüse permanent offen steht. Entscheidend ist nur, dass das Gas die Kammer vor-

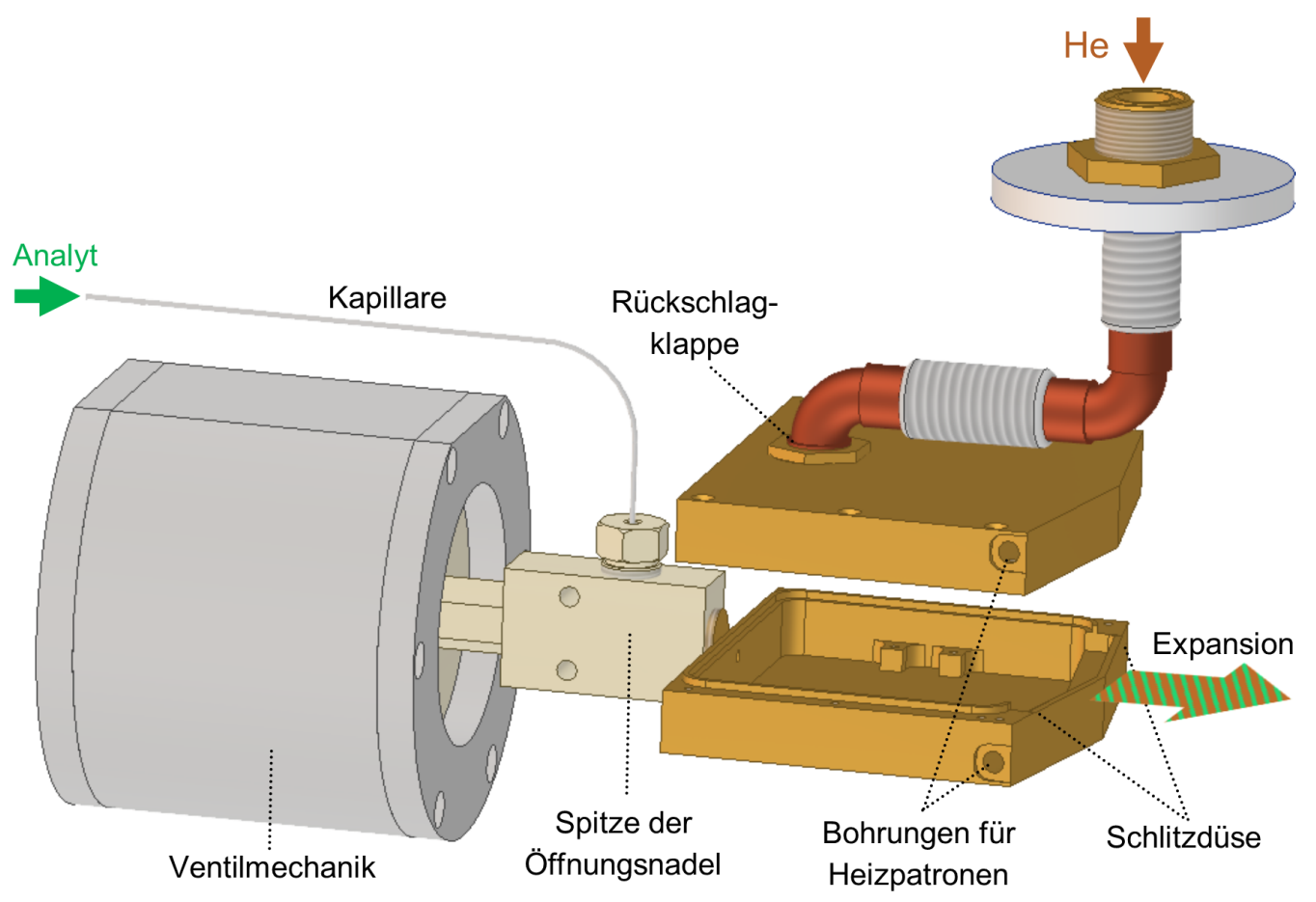

Abb. 9.6: Technische Zeichnungen der Mischkammer (mit angehobenem Deckel), des Ventils und des letzten Abschnittes der Helium-Zufuhr des Risotto-Jets, adaptiert aus [446]. 
wiegend über die Schlitzdüse verlässt. Die Mischkammer weist in der hinteren Wand einen abgewinkelten Zugang auf, durch den die injizierte Flüssigkeit in Richtung Boden gelenkt wird. Hierdurch wird eine direkte Flugbahn zwischen Ventilnadel und Schlitzdüse verhindert, sodass Flüssigkeitstropfen nicht ohne Kontakt mit den heißen Oberflächen der Kammer in den Detektionsbereich gelangen können. Durch eine weitere Öffnung im Deckel kann über einen Wellschlauch Trägergas zugeführt werden. Der Wellschlauch als Verbindung zwischen beweglicher Kammer und dem oberen Zugang des stationären Expansionsrohrs erlaubt eine begrenzte Flexibilität, um weiterhin den Abstand zwischen Schlitzdüse und IR-Strahl um einige Zentimeter variieren zu können. Im Übergang zwischen Kammer und Wellschlauch befindet sich eine Rückschlagklappe, die nur öffnet, wenn sich im Wellschlauch ein höherer Druck als in der Kammer befindet. Dies verhindert, dass verdampfter Analyt in ungeheizte Teile der Apparatur gelangen kann. Die Klappe öffnet in Richtung des pneumatischen Ventils (statt in Richtung der Schlitzdüse), um die Durchmischung des Trägergases und des verdampften Analyten zu fördern. Zusätzlich befinden sich noch mehrere bisher ungenutzte Befestigungsanker in der Kammer, die zukünftig z. B. ein Gitter oder poröses Material halten könnten, welches bei Bedarf den Strömungswiderstand und die Durchmischung weiter steigern könnte.

Die Heizung der Mischkammer erfolgt über drei unabhängige Heizungen unter Verwendung von Heizpatronen der HS Heizelemente GmbH mit Leistungen von $160 \mathrm{~W}$ je Patrone. Die Heizpatronen sind über Bohrungen in die Wände und den Boden der Mischkammer integriert. Je zwei Heizpatronen befinden sich direkt ober- und unterhalb der vollen Länge der Schlitzdüse (zwei der Bohrungen sind in Abb. 9.6 erkennbar) und bilden zusammen die erste Heizung. Die zweite Heizung umfasst zwei Heizpatronen im hinteren Boden der Mischkammer, also in dem Bereich in dem der Analyt und das Trägergas die Mischkammer zugeführt werden. Schließlich sind zwei weitere Heizpatronen in Messingplatten integriert, die seitlich an den vordersten Ventilteil angebracht sind (nicht dargestellt in Abb. 9.6). Jede Heizung verfügt über einen eigenen Temperaturfühler (Thermoelement Typ K), welche ebenfalls über Bohrungen integriert sind. Gegenüber der vorherigen Umwicklung durch Heizbänder am RESS-Jet ist durch diese Umsetzung eine schnellere Aufheizung sowie eine bessere Temperaturverteilung und -kontrolle zu erwarten. Bei Kontrollmessungen an Luft wurden mit einem zusätzlichen externen Temperaturfühler eine sehr einheitliche Temperaturverteilung insbesondere entlang der Schlitzdüse bestätigt. Das Ventil ist weiterhin auf maximal $200^{\circ} \mathrm{C}$ limitiert, allerdings sind höhere Temperaturen in der Mischkammer und insbesondere an der Schlitzdüse durch einen Temperaturgradienten erreichbar, wenn die vorderste Heizung über Konduktion die gesamte Heizleistung übernimmt. So wird beispielsweise bei einer Düsentemperatur von $300^{\circ} \mathrm{C}$ eine Gleichgewichtstemperatur von $240^{\circ} \mathrm{C}$ am Temperaturfühler im hinteren Boden und von $165^{\circ} \mathrm{C}$ am Ventil erhalten. An der Schlitzdüse sind ohne Überhitzung des Ventils stabil $350^{\circ} \mathrm{C}$, kurzzeitig auch $400{ }^{\circ} \mathrm{C}$ einsetzbar.

\subsubsection{Zuleitungssystem}

Daneben wurden auch einige Änderungen am Zuleitungssystem durchgeführt. Ein vereinfachtes Schema des komplettierten Risotto-Jets ist in Abb. 9.7 dargestellt.

Alle Heizungen vor dem Ventil wurden entweder demontiert oder stillgelegt. Die Heizung des Zuleitungssystems war prinzipiell auch bereits am RESS-Jet nur dann zwin- 
gend notwendig, wenn z. B. Kohlenstoffdioxid im überkritischen Zustand gehalten werden musste, um einen Phasenübergang mit möglicher Entmischung auszuschließen. In der Praxis ließ sich aber das Reservoir wegen der Elastizität der Dichtungen für den beweglichen Kolben nur im geheizten Zustand zuverlässig verwenden. Dieses störungsanfällige und wartungsaufwändige Bauteil wurde überbrückt, was auch die notwendige Flüssigkeitsmenge in der Apparatur von fast 200 auf etwa $75 \mathrm{ml}$ reduzierte. Der Druck wird nun stattdessen über die erste HPLC-Pumpe zwischen den Pulsen durch Fördern neuer Substanz aus dem Vorratsbehälter wiederhergestellt, statt durch Kompression des Volumens über die zweite HPLC-Pumpe. Hierdurch entfällt auch die regelmäßig notwendige manuelle Neubefüllung des Reservoirs. Vergleichsmessungen zeigten keine Auswirkungen auf die Spektren, obwohl durch das geringere Volumen der Druck prinzipiell schneller abfällt. Anders als beim RESS-Jet wird beim Risotto-Jet generell kaum eine Abhängigkeit vom Druck der Flüssigkeit gefunden.

Nach Förderproblemen mit Ethylenglycol, mutmaßlich wegen dessen hoher Viskosität, wurde die von der Mechanik-Werkstatt gebaute HPLC-Pumpe durch ein älteres kommerzielles Modell der KNAUER GmbH ersetzt. Dies verhindert die Probleme nicht vollständig, reduziert sie aber. Dieses einfache mechanische Modell wird mit Druckluft betrieben. Modernere elektrische Modelle wurden dagegen als ungeeignet gefunden, da die dort verwendete Software einen Druckabfall als Störung einstuft und daher eine Abschaltung der Pumpe veranlasst.

Zusätzlich wurden die $\mathrm{KBr}$-Linsen durch $\mathrm{CaF}_{2}$-Linsen gleicher Brennweite ausgetauscht. Dies behebt Probleme mit ungewöhnlichen Absorptionsbanden im Einkanalspektrum der KBr-Linsen, die auf eine Kontamination mit einem Alkohol hindeuten.

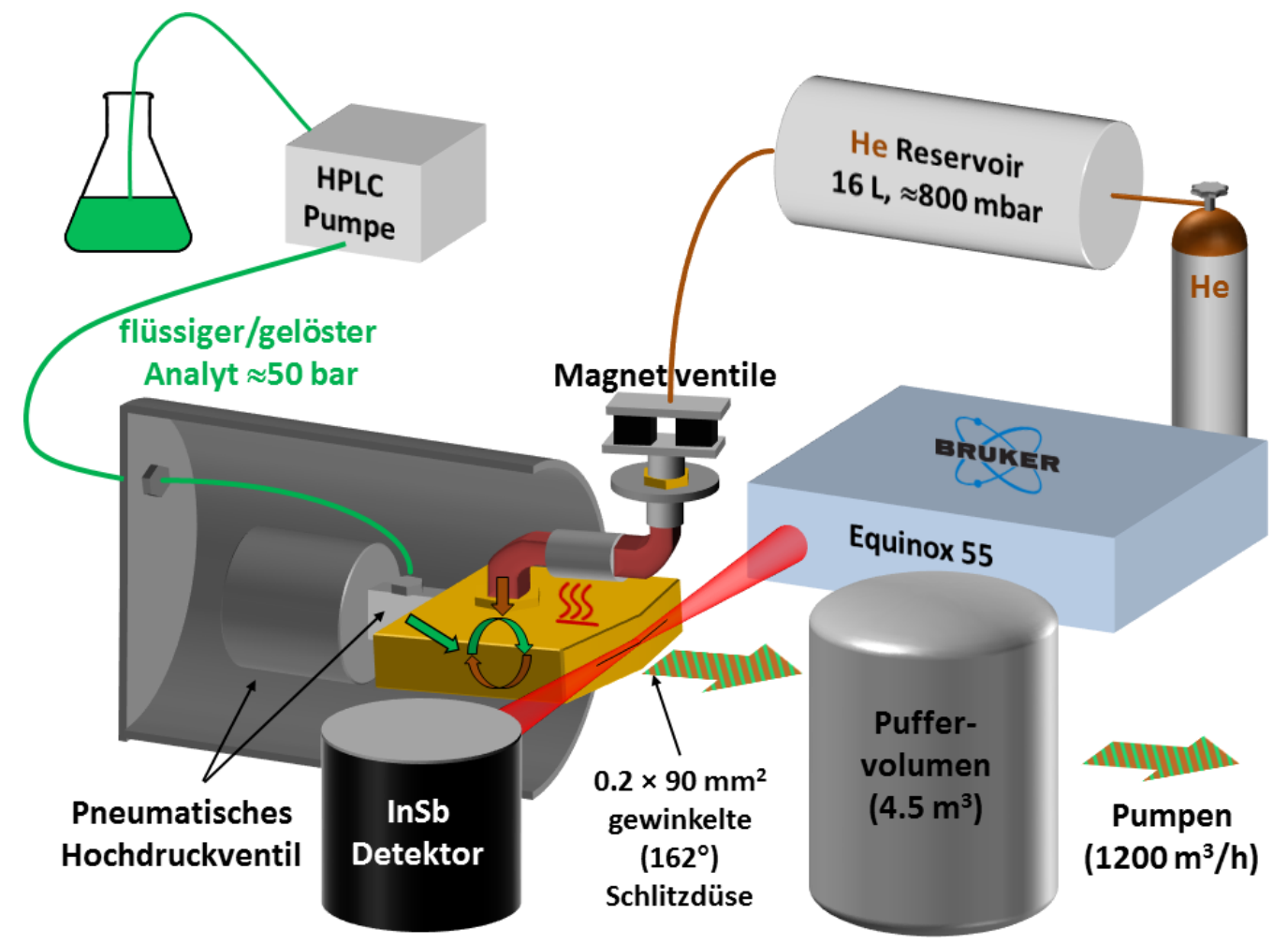

Abb. 9.7: Vereinfachtes Schema des Risotto-Jets. Adaptiert von einer ähnlichen Darstellung des Popcorn-Jets [69], freundlicherweise zur Verfügung gestellt von Anja Poblotzki. 
Dieses Problem konnte zurïckverfolgt werden auf Messungen mit tert-Butylalkohol im Jahr 2012 durch Abraham [433]. $\mathrm{CaF}_{2}$ sollte diesbezüglich unempfindlicher sein, wie auch bezüglich Aufnahme von atmosphärischem Wasser. Die Heizung und Spülung der Linsen mit Trockenluft wurde aber beibehalten. Die geringere spektrale Durchlässigkeit von $\mathrm{CaF}_{2}$ im niederfrequenten Bereich ist für Untersuchungen im $\mathrm{OH}$ Streckschwingungsbereich unerheblich.

Bevor im nächsten Schritt das System für die Trägergaszufuhr installiert wurde, wurde zunächst die Mischkammer mit Methanol getestet. Dieser Aufbau ähnelt grundsätzlich der des RESS-Jets, allerdings mit einem erheblich erweiterten Totvolumen zwischen Ventil und Düse. Abhängig von den Temperaturen, der injizierten Menge und der Synchronisation zwischen Injektion und Scan wurde ein Spektrum des Methanol-Monomers mit oder ohne einer zusätzlichen breiten Clusterbande erhalten. Ein wesentlicher Unterschied zu den früheren Messungen ist aber, dass mit der neuen Düse zu keinem Zeitpunkt die in Abschnitt 9.2.2 beschriebenen Streueffekte beobachtet wurden, die zuvor nur unter Extrembedingungen (sehr hohe Düsentemperatur, niedriger Druck) vermieden werden konnten. Dies zeigt, dass die Verdampfung in der Mischkammer zu Monomeren (oder zumindest sehr kleinen Tröpfchen) effektiv erfolgt. Kleinere Cluster im größenaufgelösten Bereich wurden auch mit der neuen Düse nicht beobachtet, wenn kein Trägergas eingesetzt wird.

In der neugebauten Trägergaszufuhr gelangt Helium aus einer Druckgasflasche über ein Magnetventil (Asco 238913-006) in ein Reservoir mit 16 L Volumen. Das Magnetventil wird über eine Steuerung betätigt, die einen gewählten Solldruck (Stagnationsdruck) mit dem von einer Drucksonde (Omega PXM219, Messbereich 0-1.6 bar absolut) gemessenen abgleicht. Ein Sicherheitsventil (Drukon UAC-01-0,5 bar) öffnet im Fall von Fehlbedienung bei einem Überdruck von 0.5 bar gegenüber Atmosphäre. Das Reservoir steht über einen Wellschlauch und zwei parallel angeordnete Magnetventile (Parker Lucifer 22J3301E) mit dem Vakuumteil der Apparatur (Abb. 9.6) in Verbindung. Hierfür wird der Zugang des Expansionsrohres oberhalb des Strahlengangs benutzt, welcher beim RESS-Jet noch ein Sichtfenster enthielt. Für Magnetventile diesen Typs wurde eine effektive Nennweite von $5.8 \mathrm{~mm}$ bestimmt [37], was einer kreisförmigen Querschnittsfläche von rund $26 \mathrm{~mm}^{2}$ entspricht. Damit in der Mischkammer während des Heliumpulses ein Druck ähnlich zum Reservoir aufgebaut werden kann, sollte die Gesamtquerschnittsfläche der Wellschlauchverbindung und der Magnetventile größer sein als die der Schlitzdüse $\left(90 \mathrm{~mm} \times 0.2 \mathrm{~mm}=18 \mathrm{~mm}^{2}\right)$ [37]. Um dies sicherzustellen, werden daher zwei parallel angeordnete Magnetventile verwendet. Die Expansion des Trägergases ist über die Magnetventile mit der Analytinjektion und dem Spektrometerscan mittels des DelayGenerators synchronisiert. Die Magnetventile können über die Steuerung auch manuell geöffnet werden, um das Reservoir komplett zu evakuieren.

\subsubsection{Synchronisation}

Vielfältige Möglichkeiten, aber auch Herausforderungen, bestehen bei der doppelten Synchronisation von Analytinjektion, Heliumpuls und den Spektrometerscans. Das Ende der Hintergrundscans wird hierfür als zeitlicher Nullpunkt definiert, da das Spektrometer zu diesem Zeitpunkt ein TTL-Signal an den Delay-Generator sendet, welches für die Analytinjektion und Heliumpuls unterschiedlich lange verzögert und in der Dauer modifiziert 
Tab. 9.1: Scan- $D_{\mathrm{S}}$ und Pausendauern $D_{\mathrm{P}}$ in Abhängigkeit von spektroskopischen Parametern. DSFR steht für den Double-Sided-Fast-Return-Modus und SSFR für den Single-Sided-Fast-ReturnModus. Einheitlich wurde eine Phasenauflösung von $16 \mathrm{~cm}^{-1}$ verwendet. Alle Werte wurden aus den OPUS-Dateien entsprechender Messungen entnommen, für eine Diskussion der technischen Zusammenhänge sei auf Ref. [433] verwiesen.

\begin{tabular}{ccccc}
\hline $\begin{array}{c}f_{\mathrm{HeNe}} \\
/ \mathrm{kHz}\end{array}$ & $\begin{array}{c}\text { Auflösung } \\
/ \mathrm{cm}^{-1}\end{array}$ & Modus & $\begin{array}{c}D_{\mathrm{S}} \\
/ \mathrm{ms}\end{array}$ & $\begin{array}{c}D_{\mathrm{P}} \\
/ \mathrm{ms}\end{array}$ \\
\hline 280 & 5.0 & DSFR & 20 & 61 \\
280 & 3.5 & DSFR & 29 & 69 \\
80 & 5.0 & SSFR & 47 & 84 \\
80 & 3.5 & SSFR & 63 & 92 \\
80 & 2.0 & SSFR & 100 & 117 \\
\hline
\end{tabular}

wird. Diese am Delay-Generator modifizierbaren Werte werden im Folgenden mit $V(\mathrm{~A})$, $D(\mathrm{~A}), V(\mathrm{He})$ und $D(\mathrm{He})$ angegeben, wobei $V$ für Verzögerung und $D$ für Dauer steht. Die einstellbaren digitalen Verzögerungen werden noch zusätzlich durch Signallaufzeiten, Reaktionszeiten der Ventile und die Laufzeiten der Substanzen zwischen den Ventilen und dem Detektionsbereich verlängert. Dabei wurde gefunden, dass der Analyt etwa ab $[V(\mathrm{~A})+80 \mathrm{~ms}]$ nach Ende der Hintergrundscans detektiert werden kann. Für den Beginn der Auswirkungen einer zusätzlichen Helium-Beimischung auf das Spektrum müssen zur digitalen Verzögerung dagegen nur etwa $20 \mathrm{~ms}$ addiert werden. Die Synchronisation mit dem Spektrometerscan ist insbesondere abhängig von der gewählten Spiegelgeschwindigkeit, der Auflösung und dem Aufnahmemodus (Single- oder Double-Sided ${ }^{\mathrm{d}}$ etc.), da diese Parameter die Dauer der Scans $D_{\mathrm{S}}$ und der Pausen $D_{\mathrm{P}}$ zwischen diesen beeinflussen. Tab. 9.1 listet Werte für in dieser Arbeit am Risotto-Jet verwendete Aufnahmebedingungen.

Für die Charakterisierung der Messabläufe und der zeitlichen Konzentrationsentwicklung in der Expansion wurde zunächst die vorher für den RESS -Jet genutzte hohe Spiegelgeschwindigkeit $(280 \mathrm{kHz})$ und die niedrige Auflösung $\left(5 \mathrm{~cm}^{-1}\right)$ in Verbindung mit dem Double-Sided-Fast-Return-Modus beibehalten. Diese Kombination bietet mit $D_{\mathrm{S}}=20 \mathrm{~ms}$ und $D_{\mathrm{P}}=61 \mathrm{~ms}$ eine hohe Zeitauflösung bei akzeptablem Signal-zu-Rausch-Verhältnis. Durch eine Messreihe mit schrittweise erhöhter Verzögerung der Analytinjektion und ggf. des Heliumpulses lässt sich damit ein langer Zeitraum des Experiments mit guter Zeitauflösung in einem Raster erfassen. Der Messablauf wurde hierfür derart modifiziert, dass nach dem Vorscan nun acht, statt zuvor drei, jeweils einzeln mit den Hintergrundscans verrechnete Scans gemessen werden. Die bei den Filet-, Popcorn- und RESS-Jets genutzte Unterteilung in einen einzelnen Probescan (während der Expansion) und zwei Nachscans (nach der Expansion) ist für den Risotto-Jet bis zur Etablierung einer festen Messroutine nicht sinnvoll, Die gemischte Expansion weist häufig eine geeignete Konzentration erst in einem späteren Zeitfenster auf, welches besser mit einem der späteren kurzen Scans synchronisiert werden kann. Welcher dies ist, ist stark abhängig von den Aufnahmeparametern. Daher werden die Probescans durchnummeriert und nicht in Vorund Nachscans unterteilt. Die Start- und Endzeitpunkte bestimmter Ereignisse im De-

\footnotetext{
${ }^{\mathrm{d}}$ Im Double-Sided-Modus wird ein symmetrisches Interferogramm gemessen, dessen beide Arme je einem vollständigen Scan entsprechen. Im Single-Sided-Modus ist dagegen ein Arm des Interferogramms stark verkürzt und wird nur für die Phaseninformation genutzt.
} 
tektionsbereich lassen sich allgemein gemäß den Formeln in Tab. 9.2 abschätzen. Die Schwankung in der Synchronisation zwischen einzelnen Pulsen beträgt etwa $\pm 10 \mathrm{~ms}$ und die zeitliche Unschärfe der Messpunkte durch die endliche Dauer der genutzten Scans nochmals etwa die gleiche Größe. Auf Basis dieser Formeln lässt sich bei Wechsel der spektroskopischen Parameter die Synchronisation grob einstellen. Eine darauffolgende empirische Optimierung ist im Bereich von $\pm 20 \mathrm{~ms}$ aber anhand der konkreten Spektren notwendig. Insbesondere muss während des relevanten Probescans eine zeitliche Überlappung mit den Störsignalen durch Ventilöffnungen und -schließungen vermieden werden.

Tab. 9.2: Abgeschätze Start- und Endzeitpunkte von Ereignissen für die Synchronisation von Spektrometer-Scans, Analytinjektion und Heliumpuls. $D_{\mathrm{S}}$ ist die Dauer eines Scans, $D_{\mathrm{P}}$ die Pause zwischen Scans, $V$ die zusätzliche digitale Verzögerung und $D$ die Dauer der Signale für die Analytinjektion A bzw. den Heliumpuls He.

\begin{tabular}{lll}
\hline Ereignis & $t($ Start $) / \mathrm{ms}$ & $t($ Ende $) / \mathrm{ms}$ \\
\hline 20 Hintergrundscans & $-\left(20 \cdot D_{\mathrm{S}}+19 \cdot D_{\mathrm{P}}\right)$ & 0 \\
Vorscan & $D_{\mathrm{P}}$ & $D_{\mathrm{P}}+D_{\mathrm{S}}$ \\
Probescan Nr. $N$ & $N \cdot D_{\mathrm{S}}+(N+1) \cdot D_{\mathrm{P}}$ & $(N+1) \cdot\left(D_{\mathrm{S}}+D_{\mathrm{P}}\right)$ \\
Substanzinjektion & $80+V_{\mathrm{A}}$ & $80+V_{\mathrm{A}}+D_{\mathrm{A}}$ \\
Heliumpuls & $20+V_{\mathrm{He}}$ & $20+V_{\mathrm{He}}+D_{\mathrm{He}}$ \\
\hline
\end{tabular}

Eine grundlegende Frage bei der Synchronisation von Analytinjektion und Heliumpuls ist, ob besser zunächst der Analyt injiziert und verdampft werden sollte, um anschlieBend vom Heliumpuls aufgenommen zu werden (ähnlich zum Ablauf beim Popcorn-Jet) oder ob stattdessen eine Injektion in den bereits laufenden Heliumpuls eine geeignete Alternative wäre (wie beim Falafel-Jet erprobt [70]). Für den Risotto-Jet wurde nur die erstgenannte Abfolge als durchführbar gefunden, denn ab moderaten Heliumdrücken von etwa 500 mbar (Wert im Reservoir zu Beginn des Pulses) wird der Analyt nicht mehr am erwarteten Zeitpunkt kurz nach der Injektion detektiert, sondern erst deutlich später nach Abschluss des Heliumpulses. Dies bedeutet, dass der Analyt vom Helium blockiert wird. Während Gase zwar grundsätzlich vollständig mischbar sind, erfolgt dies ab bestimmten Teilchendichten nur noch langsam, da die mittlere freie Weglänge durch die zunehmende Anzahl an Stößen sinkt. Dieses Phänomenen der effektiven Nichtmischung von zwei Gasströmen wurde auch bei der versuchten Kreuzung von zwei Überschallexpansionen beobachtet.[447] Dass umgekehrt der verdampfte Analyt aber vom Helium tatsächlich aufgenommen und nicht (nur) geschoben wird, belegt die Verschmälerung von MonomerBanden und das Auftreten von Banden kleiner Cluster. Die erhaltenen Spektren sind vergleichbar mit denen von einer Expansion eines vorgemischten Gases, wie beispielsweise am Filet-Jet. Dieser unterschiedliche Vermischungsgrad in Abhängigkeit von der Reihenfolge ist vermutlich durch die Dichteabnahme des Analyten begründet, wenn dieser in der Mischkammer zunächst verdampft und verteilt wird. Als optimal wurde ein Heliumdruck von 800 mbar gefunden. Bei tieferen Werten sinkt der Clusteranteil und Banden beginnen sich zu verbreitern, für größere Werte wurden keine spektralen Auswirkungen gefunden, während aber der Heliumverbrauch weiter ansteigt. 


\subsubsection{Heliumverbrauch}

Angesichts steigender Heliumpreise [448] ist der Verbrauch ein zunehmend relevanter werdendes Kriterium für das Jet-Design, welches u. a. mit als Motivation zur Konstruktion des Gratin-Jets [132] mit Gas-Recycling beitragen hat. Als Richtwert für den Risotto-Jet wird im 16 L Reservoir ein Druckabfall von etwa 70 mbar bei einem Stagnationsdruck von 800 mbar und einer Pulsdauer von $140 \mathrm{~ms}$ beobachtet (gemessen bei abgeschalteter Nachfüllung). Überschlagsmäßig abgeschätzt mit dem idealen Gasgesetz lassen sich mit einer Helium-Druckgasflasche mit 50 L Volumen und 200 bar Druck grob 9000 Pulse dieser Art durchführen. Diese abgeschätzte Größenordnung entspricht auch der eigenen Erfahrung, so wurde bei der gesamten Erprobung des Risotto-Jets nur etwas mehr als eine Gasflasche verbraucht. Für die Messungen am Filet-Jet in dieser Arbeit wurde hingegen zum Teil die gleiche Menge an einem einzelnen Tag benötigt.

\subsubsection{Charakterisierung des Konzentrationsverlaufes}

Die zeitliche Entwicklung der Konzentration des Analyten Ethylenglycol in der Expansion kann anhand des Integrals der CH-Schwingungen $\left(3050-2700 \mathrm{~cm}^{-1}\right)$ verfolgt werden, deren IR-Aktivität weitgehend unabhängig vom Aggregatzustand ist. Ein Integral von $100 \mathrm{~cm}^{-1}$ entspricht in diesem Fall einer maximalen dekadischen Absorbanz von etwa $2 \cdot 10^{-3}$. Für die Zeitauflösung wurden die acht je $20 \mathrm{~ms}$ dauernden Probescans in einem Raster von $10 \mathrm{~ms}$ verschoben und je 5 Wiederholungen gemittelt. Dies ist in Abb. 9.8 für drei Mischkammertemperaturen von 100,150 und $200^{\circ} \mathrm{C}$ gezeigt (die Düse ist jeweils noch $5^{\circ} \mathrm{C}$ höher geheizt).

Im oberen Teil der Abbildung ist der Verlauf mit einer Ethylenglycol-Injektion mit einem Druck von 90 bar und einer Dauer von 60 ms, ohne zusätzlichen Heliumpuls, dargestellt. Der zeitliche Nullpunkt wurde in dieser Darstellung auf denjenigen Messpunkt festgelegt, bei dem Ethylenglycol als erstes beobachtet wird. Bei $150^{\circ} \mathrm{C}$ und noch stärker bei $200^{\circ} \mathrm{C}$ zeigt sich ein intensives Signal ungefähr in der Dauer der Ventilöffnung. Auch für die weiteren 550 ms des Messfensters wird Ethylenglycol bei diesen Bedingungen in sehr geringer Konzentration beobachtet. Es ist aus diesen Daten nicht eindeutig, ob die Flüssigkeit bereits kurz nach Ende der Injektion vollständig verdampft wird und das Gas anschließend nur noch langsam aus der Schlitzdüse diffundiert - oder ob alternativ ein länger bestehendes dynamisches Gleichgewicht aus Verdampfung und Diffusion bei niedrigem Druck vorliegt. Bei $100{ }^{\circ} \mathrm{C}$ ist Ethylenglycol dagegen nur an wenigen Messpunkten im Spektrum nachweisbar. Im mittleren Teil der Abbildung wurde jeweils ein Heliumpuls derart synchronisiert, dass sein Beginn mit dem Zeitpunkt der höchsten beobachteten Ethylenglycol-Konzentration bei $t=20 \mathrm{~ms}$ zusammenfällt. Hierdurch wird bei 150 und $200^{\circ} \mathrm{C}$ die beobachtete Konzentration stark erhöht, die anschließend während des laufenden Heliumpulses abfällt, bis schließlich kein Analyt mehr nachweisbar ist.

Bei $100^{\circ} \mathrm{C}$ ist Ethylenglycol auch mit Heliumpuls weiterhin meist unter der Nachweisgrenze. Dies erscheint zunächst überraschend, da die optimale Temperatur der Probenkammer für die Untersuchung von Ethylenglycol-Dimeren am Popcorn-Jet bei nur etwa 60-70 ${ }^{\circ} \mathrm{C}$ liegt [27]. Ein wesentlicher Unterschied zwischen den beiden Apparaturen ist allerdings, dass die Probenkammer des Popcorn-Jets durch ein Rückschlagventil vom Vakuum getrennt ist, welches erst bei Druckaufbau durch den Heliumpuls öffnet. Hierdurch 


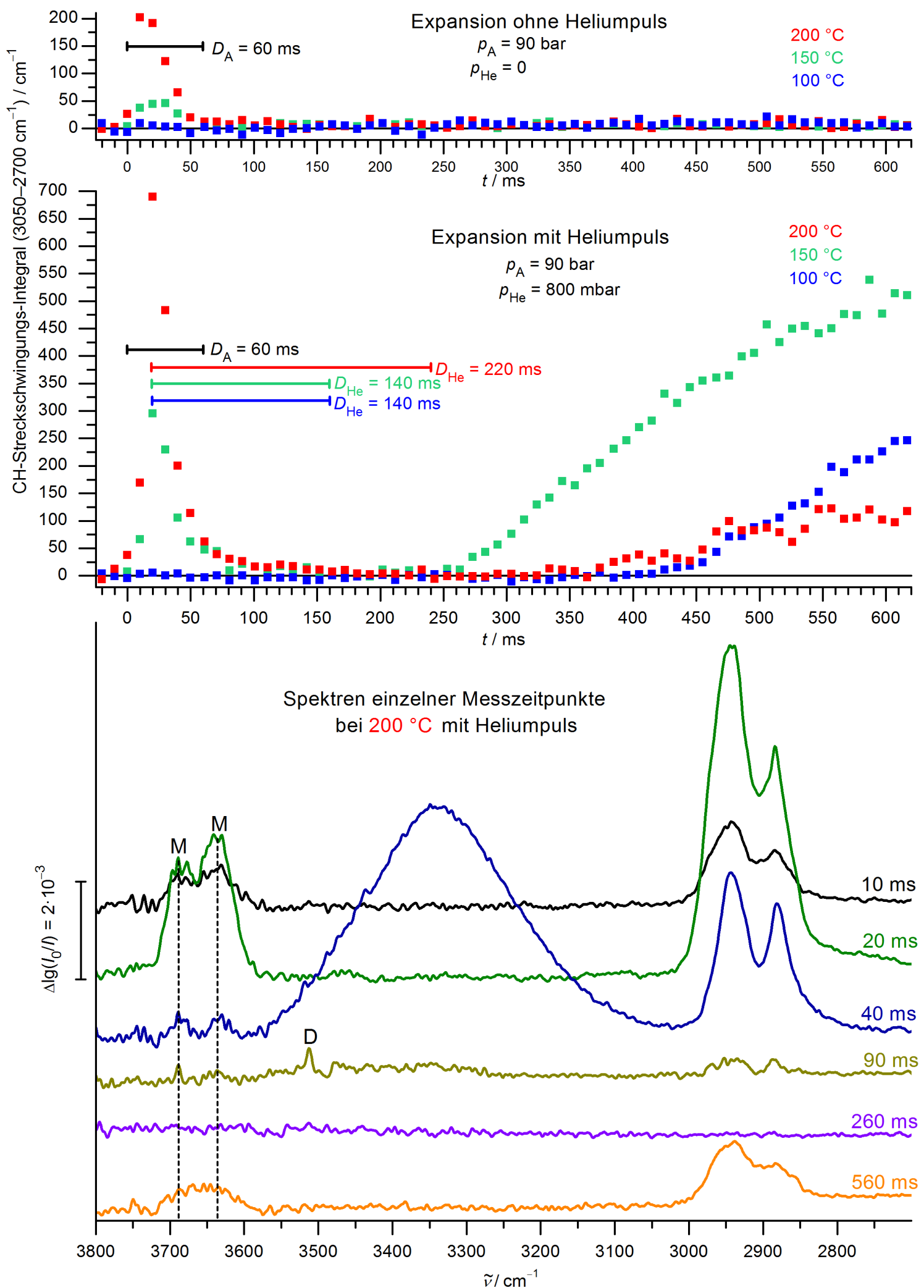

Abb. 9.8: Zeitliche Entwicklung der Konzentration von Ethylenglycol bei verschiedenen Mischkammertemperaturen ohne (oben) und mit (Mitte) zusätzlichem Heliumpuls. Zusätzlich angegeben als Balken sind die zeitliche Lage von Analytinjektion und Heliumpuls. Im unteren Teil der Abbildung sind die zugehörigen Spektren für einige Messzeitpunkte der Expansion bei $200{ }^{\circ} \mathrm{C}$ und mit Heliumpuls gezeigt. Gemittelt wurden je 5 Wiederholungen. 
kann sich während der Wartezeit von etwa einer Minute zwischen den Wiederholungen der Dampfdruck des Analyten aufbauen. Beim Risotto-Jet steht dagegen die Mischkammer über die Schlitzdüse zum Vakuum permanent offen, sodass der volle Dampfdruck wegen des fortwährenden Verlustes in das Vakuum nicht erreicht werden kann. Diese Interpretation wird durch die Beobachtung einer intensiven Nachexpansion einige Zeit nach Ende des Heliumpulses gestützt. Offenbar blockiert der Heliumpuls einen Teil des Analyten im hinteren Teil der Mischkammer (und/oder im Kanal zwischen Ventilnadel und Mischkammer) und wirkt wie ein dynamisches Rückschlagventil. Der sich dort aufbauende höhere Dampfdruck kann erst dann entweichen, sobald nach Schließen der Magnetventile der blockierende Heliumdruck ausreichend stark gefallen ist. Zu dieser Deutung passt, dass die Nachexpansion bei $150^{\circ} \mathrm{C}$ früher und intensiver erfolgt als bei $100^{\circ} \mathrm{C}$. Die Nachexpansion bei $200^{\circ} \mathrm{C}$ ist damit nicht direkt vergleichbar, da hier ein längerer Heliumpuls verwendet wurde. Dieses Phänomenen könnte zukünftig gezielt für sehr schwerflüchtige Substanzen genutzt werden, indem ein erster Heliumpuls als dynamisches Rückschlagventil den Aufbau des vollen Dampfdruckes fördert, während ein zweiter dann mit der intensiven Nachexpansion vermischt wird, um eine höher konzentrierte Expansion zu generieren.

Der untere Teil von Abb. 9.8 zeigt am Beispiel der Expansion bei $200^{\circ} \mathrm{C}$ mit Heliumpuls die Auswirkungen auf den $\mathrm{OH}-$ Streckschwingungsbereich. Die spektrale Informationen liefert Hinweise auf den Aggregationsgrad von Ethylenglycol während des Messablaufs. Das oberste Spektrum wurde bei $10 \mathrm{~ms}$ noch vor Eintreffen des Heliumpulses aufgenommen. Es sind die beiden (hier überlappenden) Banden des stabilsten MonomerKonformers (M) von Ethylenglycol bei 3689 und $3636 \mathrm{~cm}^{-1}$ [174] zu erkennen. Bei $20 \mathrm{~ms}$ ist dieses Spektrum in der Intensität deutlich verstärkt, zeigt aber weiterhin keine Zeichen von Aggregation. Offenbar wird die Gasphase in der Mischkammer zunächst nur vom Heliumpuls komprimiert und aus der Schlitzdüse gedrückt. Bei 40 ms ist die Konzentration schon wieder etwas abgefallen, allerdings ist nun der Großteil der Moleküle aggregiert. Es ist unklar, ob dieses Spektrum aus der Expansion von noch unverdampften Flüssigkeitströpfchen oder alternativ von re-aggregierten Monomeren resultiert. Störsignale durch Streuung wurden zu keinem Zeitpunkt beobachtet. Im weiteren Verlauf des Heliumpulses sinkt die Konzentration weiter, dabei wird auch eine Verschmälerung der Bandenformen, wie auch die zunehmende Verlagerung zu kleineren Clustern beobachtet. Dies belegt, dass Analyt und Helium zumindest teilweise vermischen. In dem zeitlichen Fenster zwischen etwa 90 und 210 ms ist die stark IR-aktive Bande des besonders stabilen heterochiralen Ethylenglycol-Dimers bei $3513 \mathrm{~cm}^{-1}$ [174] erkennbar und intensiver als Banden größerer Cluster. Dieser Bereich ist daher optimal für die Untersuchung von Dimeren und wurde im Folgenden für Messungen mit besserer Auflösung und höherem Signal-zu-Rausch-Verhältnis genutzt. Die Konzentration fällt kontinuierlich weiter ab bis schließlich Ethylenglycol nicht mehr nachweisbar ist - im Gegensatz zur entsprechenden Expansion ohne Heliumpuls, wo es durchgehend nachweisbar bleibt. Nach Ende des Heliumpulses, teilweise mit deutlicher Verzögerung, erfolgt die intensive Nachexpansion von heißen Monomeren, von der noch unklar ist, wie lange sie anhält. Die hohen Konzentrationen über mindestens einige hundert Millisekunden zeigen aber, dass offenbar nur ein kleiner Teil des injizierten Analyten mit dem Heliumpuls aufgenommen und zur Clusterbildung zur Verfügung steht. 


\subsubsection{Optimierung der Aufnahmebedingungen}

Messreihen bei unterschiedlichen spektroskopischen Einstellungen mit je 135 Wiederholungen im identifizierten optimalen Konzentrations- bzw. Zeitfenster für Dimere sind in Abb. 9.9 miteinander verglichen. Zum Vergleich sind im unteren Teil Spektren dargestellt, die an der bisherigen Variante des Popcorn-Jets mit Doppelschlitz-Düse und mit der neuen V-Düse aufgenommen wurden. Diese wurden auf die gleiche Intensität für die intensive Dimerbande bei $3513 \mathrm{~cm}^{-1}$ skaliert (Faktor 2.22 für die ältere und 0.62 für die neue Variante). Zugeordnete Banden für Ethylenglycol sind mit M, M', D, und >D gekennzeichnet (für Details zu Ethylenglycol-Clustern siehe Ref. [27]).

Beim Wechsel von 280 auf $80 \mathrm{kHz}$ Spiegelgeschwindigkeit findet durch das dann verfügbare Gain Ranging eine deutliche Verbesserung des Signal-zu-Rausch-Verhältnisses statt, trotz des wegen der längeren Scandauer notwendigen Wechsel von DSFR auf SSFR. Dies ist am besten erkennbar beim Vergleich der rot und blau dargestellten Spektren, bei denen alle weiteren Parameter konstant gehalten wurden. Bei Verbesserung der Auflösung von 3.5 auf $2.0 \mathrm{~cm}^{-1}$ (und hierfür notwendiger Verkleinerung der Blende der Strahlungsquelle von 5.0 auf $3.5 \mathrm{~mm}$ ) treten im Bereich zwischen 3550 und $3950 \mathrm{~cm}^{-1}$ Störsignale auf, die weiter unten diskutiert werden. Als bisher beste Aufnahmebedingungen erwiesen sich daher $80 \mathrm{kHz}$ Spiegelgeschwindigkeit und $3.5 \mathrm{~cm}^{-1}$ Auflösung. Das Signal-zu-Rausch-Verhältnis ist dabei ähnlich bis leicht besser im Vergleich zur früheren Variante des Popcorn-Jets mit Doppel-Schlitz-Düse. Dabei wurden für das Popcorn-JetSpektrum etwas weniger Wiederholungen (125 gegenüber 135) durchgeführt, diese beinhalten aber jeweils durch den dort verwendeten Double-Sided-Modus nominell doppelt so viele Scans. Nicht erreicht wird dagegen das Signal-zu-Rausch-Verhältnis der (durch die Vorversuche der vorliegenden Arbeit inspirierten) verbesserten Variante des Popcorn-Jets mit V-Düse, trotz der im Spektrum gemittelten niedrigeren Zahl an Wiederholungen (86), wenn auch nominell wieder mit doppelt so vielen Scans. Inwieweit dieser Nachteil in der schlechteren Optik, noch ungenügender Optimierung und/oder im Grundprinzip der Generierung der Expansion begründet ist, ist noch unklar. Für die Nutzung eines langen (177 ms) Double-Sided-Scans mit mittlerer Spiegelgeschwindigkeit $(80 \mathrm{kHz})$ und hoher Auflösung $\left(2.0 \mathrm{~cm}^{-1}\right)$ verändert sich die Konzentration am Risotto-Jet in der derzeitigen Verwirklichung zu schnell.

Obwohl die verwendeten Temperaturen am Risotto-Jet $\left(200^{\circ} \mathrm{C}\right.$ Mischkammer und Ventil, $205^{\circ}$ Düse) sehr unterschiedlich zu denen am Popcorn-Jet ( 85 bzw. $70{ }^{\circ} \mathrm{C}$ Probenkammer, 105 bzw. $90^{\circ} \mathrm{C}$ Düse) sind, wurden sehr ähnliche Spektren erhalten. Der größte Unterschied betrifft den Aggregationsgrad. Bei gleich skalierter Intensität der Dimerbanden, sind die Monomer-OH- und $\mathrm{CH}-\mathrm{Streckschwingungsbanden} \mathrm{(letztere} \mathrm{als} \mathrm{Maß}$ für die Gesamtzahl an detektierten Molekülen) in den Risotto-Jet Spektren etwas intensiver. Dies kann auf die höheren Temperaturen zurückgeführt werden und wird im nächsten Unterkapitel am Beispiel Methanol näher betrachtet.

Zunächst werden aber die Störsignale im grünen Spektrum in Abb. 9.9 diskutiert, die bislang Messungen oberhalb von $3550 \mathrm{~cm}^{-1}$ bei höheren Auflösungen beeinträchtigen. Diese Störsignale stimmen mit den Positionen von Rotationsschwingungsübergängen von atmosphärischen Wasser im Einkanalspektrum überein. Dabei handelt es sich aber nicht um einen Konzentrationsdrift (wie in Kap. 3.1.2 diskutiert), sondern um ein anderes, noch nicht völlig verstandenes Phänomenen, durch das offenbar der Probescan derart gestört 


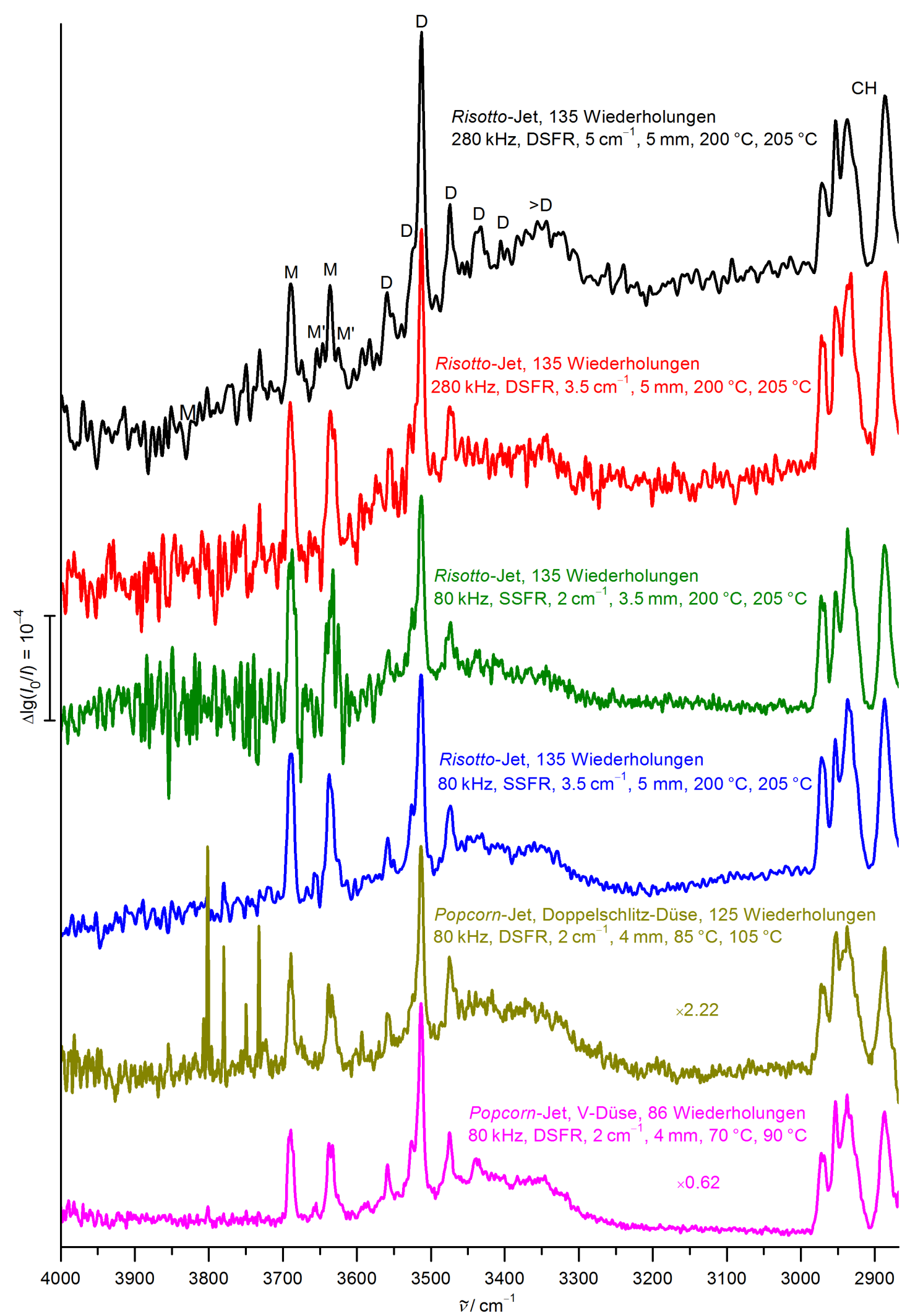

Abb. 9.9: Spektren von Ethylenglycol am Risotto- und Popcorn-Jet aufgenommen mit verschiedenen Parametern (Spiegelgeschwindigkeit, Aufnahmemodus, Auflösung, Blendendurchmesser, Substanztemperatur, Düsentemperatur). Popcorn-Jet-Spektren wurden von Manuel Lange [422] gemessen und mit den angegeben Faktoren auf die gleiche Intensität der Dimer-Bande bei $3513 \mathrm{~cm}^{-1}$ skaliert. 
wird, dass sich die Postionen der Wasserbanden leicht verschieben und im Extinktionsspektrum daher keine ausreichende Kompensation mehr stattfindet. Vergleichbare Störsignale wurden von Borho [37] bei der Erprobung des Filet-Jets beschrieben, sowie später erneut durch Heger [54], und auf eine Reflexion des IR-Strahls an den Blenden der Schlitzdüse zurückgeführt. Eine Vergrößerung des Abstandes zwischen Schlitzdüse und IR-Strahl führt beim Risotto-Jet allerdings zu keiner Verbesserung (eine Verringerung allerdings zu einer Verschlechterung), sodass es sich offenbar um unterschiedliche Ursachen mit vergleichbaren Auswirkungen handelt. Stattdessen wird hier ein Zusammenhang mit dem Heliumpuls vermutet.

Auch das Öffnen und Schließen des Hochdruckventils führt zu charakteristischen Störsignalen, die sowohl im Spektrum wie auch im Interferogramm erkennbar sind. Diese sind allerdings nur von kurzer Dauer und lassen sich durch geeignete Synchronisation zeitlich außerhalb der relevanten Scans platzieren. Die von der Öffnung der Magnetventile für den Heliumpuls erzeugte Störung klingt dagegen nur langsam über einige hundert Millisekunden ab und ist im Interferogramm als Oszillation erkennbar.

Dies ist die Begründung für die bei der Charakterisierung der Expansion (Abschnitt 9.3.7) gewählte Form der Synchronisation, bei der der Heliumpuls so abgestimmt ist, dass er die höchstmögliche Menge an Analyt aufnimmt. Der Probescan erfolgt dabei erst dann, wenn die Analytkonzentration während des Heliumpulses auf das gewünschte Maß abgesunken ist. Dies erlaubt die höchstmögliche Zeitdifferenz zwischen dem Öffnen der Magnetventile und dem Probescan.

Ohne diese langanhaltende Störung wäre es dagegen ökonomischer, mit dem Heliumpuls zu warten, bis durch einfache Diffusion ins Vakuum die Analytkonzentration in der Mischkammer auf das gewünschte Maß abgefallen ist. In diesem Fall müsste der Heliumpuls nur wenig länger gewählt werden als der Probescan. Mit diesem Vorgehen würde nicht nur der Heliumverbrauch niedriger ausfallen, sondern auch die Wartezeit zwischen den Wiederholungen des Experiments, da weniger Gas zur Wiederherstellung des Hintergrunddrucks abgepumpt werden muss. Aus diesen Gründen war dies die ursprünglich erprobte Form der Synchronisation, dabei wurden allerdings die Störsignale auch bei niedrigeren Auflösungen beobachtet.

Abb. 9.10 zeigt zwei Messungen für Methanol, die mit den alternativen Synchronisations-Strategien optimiert wurden und ansonsten identische Parameter verwenden. Durch die Umstellung der Strategie konnte die Störung bei niedriger Auflösung umgangen werden, allerdings funktioniert dies aus unbekanntem Grund nicht bei hoher Auflösung. Hinsichtlich der Übertragung der Störung zwischen Heliumsystem und Spektrometer konnte eine elektrische Kopplung durch Verwendung unabhängiger Spannungsversorgungen ausgeschlossen werden (auf diese Weise gelang es allerdings, Störeinflüsse durch den Lufttrockner zu eliminieren). Als Alternative kommt eine mechanische Übertragung durch Schwingungen in Betracht. Möglicherweise wirkt es sich ungünstig aus, dass das Helium-Reservoir an dem Labortisch befestigt ist, auf dem auch das Spektrometer steht. Diese Vermutung benötigt aber weitere Untersuchungen.

Zusätzlich könnte geprüft werden, ob eine geringere Auflösung nicht sogar die bessere Alternative wäre, da sie sich auch außerhalb des gestörten Bereiches eher positiv auf das Signal-zu-Rausch-Verhältnis auszuwirken scheint und zumindest bei Ethylenglycol scheinbar ohne Informationsverlust einhergeht. Die kürzere Scandauer würde zudem eher eine Umstellung auf den Double-Sided-Modus ermöglichen. Für eine solche Be- 
wertung sollte aber zunächst ein störungsfreies Vergleichsspektrum bei hoher Auflösung zur Verfügung stehen. Allerdings sind die Ethylenglycol-Dimerbanden auch in den höher aufgelösten Popcorn-Spektren mit Halbwertsbreiten von $6-7 \mathrm{~cm}^{-1}$ ungewöhnlich breit und profitieren daher nicht von einer hohen Auflösung. Für die in dieser Arbeit untersuchten Dimerbanden anderer Systeme wurden dagegen niedrigere Halbwertsbreiten von 3-4 $\mathrm{cm}^{-1}$ gefunden. Eine Ursache hierfür können die kooperativen WasserstoffbrückenNetzwerke in den Ethylenglycol-Dimeren sein. Strukturell verwandte Motive liegen auch beispielsweise in den Methanol-Tri- und Tetrameren vor, die ebenfalls breite Banden aufweisen (Kap. 4.4). Denkbar als Ursache hierfür wäre eine Lebensdauerverbreiterung durch schnelle Umverteilung der Anregungsenergie in den stark gekoppelten $\mathrm{OH}-$ Streckschwingungsmoden.

\subsubsection{Variation der Expansionstemperatur am Beispiel Methanol}

Wie bereits im vorherigen Abschnitt erwähnt, wurde der Risotto-Jet nicht nur mit dem schwerflüchtigen Ethylenglycol, sondern auch mit dem leichtflüchtigen Methanol erprobt. Letzteres kann auch ohne Heizung und somit über einen größeren Temperaturbereich untersucht werden. Bei niedriger Temperatur konnten Methanol-Spektren gut reproduziert werden, die bereits in Abb. 4.18 auf S. 73 und Abb. 4.29 auf S. 91 diskutiert wurden. Durch die geringere verwendete Auflösung von $5 \mathrm{~cm}^{-1}$ gehen aber einige Details verloren, wie die Aufspaltung der intensivsten Trimerbande. Mit steigender Temperatur der Expansion (kontrollierbar über die Düsentemperatur und den Heliumdruck) sinkt der Aggregationsgrad, die absolute Menge an Clustern kann aber durch eine höhere Gesamtkonzentration an Methanol aufrechterhalten werden. Für solche Experimente ist der Risotto-Jet hervorragend geeignet, da über geringe Änderungen an den Verzögerungszeiten die Konzentration über einen großen Bereich variiert werden kann. Die Konzentrationen für die Spektren in Abb. 9.11 wurden so gewählt, dass die niederfrequente Bande großer Cluster (Tetramere und größer) stets eine vergleichbare Intensität hat. Neben der Erhöhung der Düsentemperatur, kann die Expansionstemperatur auch durch Absenkung des Heliumdruckes erhöht werden. Dies wurde für Methanol bereits durch Larsen et al. am

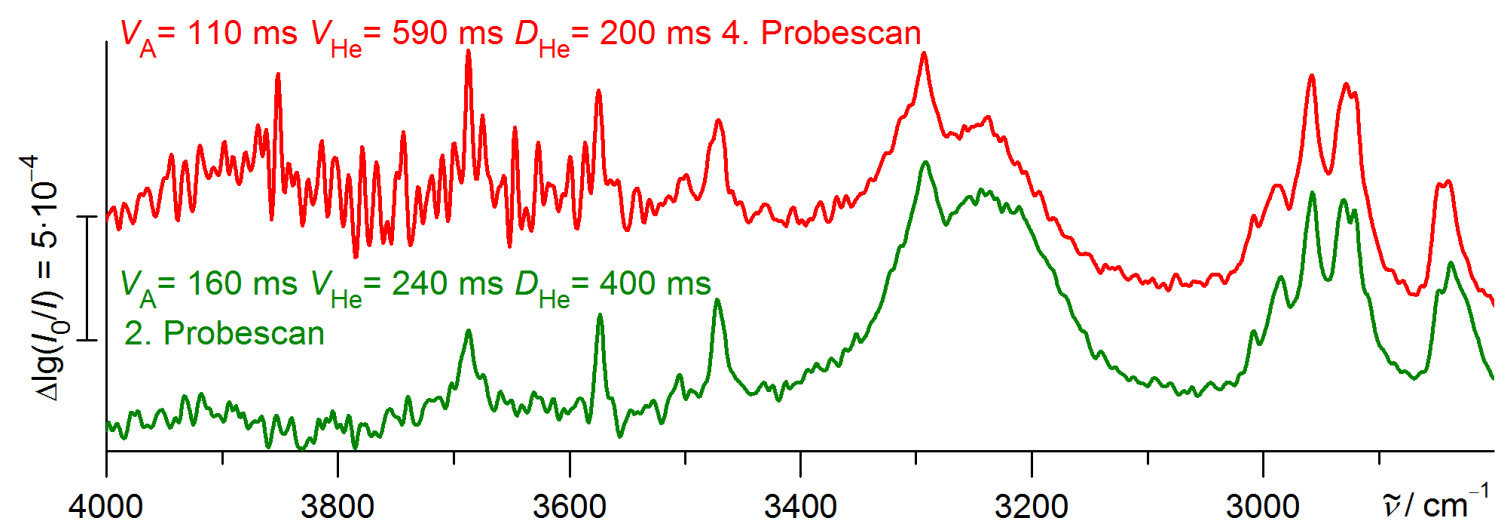

Abb. 9.10: Vergleich von Methanol-Spektren mit unterschiedlichen Strategien für die Synchronisation (siehe Fließtext), mit und ohne Störung. Angegeben sind die unterschiedlichen Synchronisationsparameter. Sonstige Parameter sind identisch: $p_{\mathrm{A}}=65$ bar, $D_{\mathrm{A}}=60 \mathrm{~ms}, p_{\mathrm{He}}=1200 \mathrm{mbar}$, Düsentemperatur $60^{\circ} \mathrm{C}$, Mischkammertemperatur $57^{\circ} \mathrm{C}$, Spiegelgeschwindigkeit $80 \mathrm{kHz}, 5 \mathrm{~cm}^{-1}$ Auflösung, Scanmodus SSFR, 10 Wiederholungen. 
Filet-Jet gezeigt.[130] Durch die Kombination aus Heizung und niedrigem Heliumdruck können schließlich besonders warme Expansionen erhalten werden, die aber weiterhin intensive Banden, auch von kleinen Clustern, aufweisen. In dieser Temperaturreihe wurde die Gesamtkonzentration insgesamt um etwa einen Faktor 10 erhöht (gemessen an der Intensität der CH-Streckschwingungen), um ähnliche Intensitäten der Clusterbanden aufrechtzuerhalten.

Die Variation der Expansionsbedingungen hat unterschiedliche Auswirkungen auf die Banden der Cluster verschiedener Größe. Die Bande des Methanol-Dimers bleibt als intensives Signal erhalten, erfährt aber eine Verbreiterung zu höheren Wellenzahlen, vermutlich weil die Anregung niederfrequenter Moden die Wasserstoffbrücke schwächt.[130] Das intensive Signal der beiden überlagernden stark IR-aktiven Übergänge des Methanol-Trimers (siehe Kap. 4.4.2) wird durch einen breiten, zu höheren Wellenzahlen verschobenen Sockel ersetzt. Verloren geht ebenso das relativ scharfe Signal der stark IR-aktiven Bande des Tetramers, welche aus der breiten Bande der größeren Cluster bei tiefen Temperaturen heraussticht (siehe auch Kap. 4.8). Die verbleibende breite Bande erfährt eine zunehmende Verschiebung zu höheren Wellenzahlen. Die Position des Maximums dieser Bande hat im Spektrum der wärmsten Expansion mit etwa $3340 \mathrm{~cm}^{-1}$ einen Wert vergleichbar mit dem von flüssigem Methanol bei Zimmertemperatur [449] erreicht.

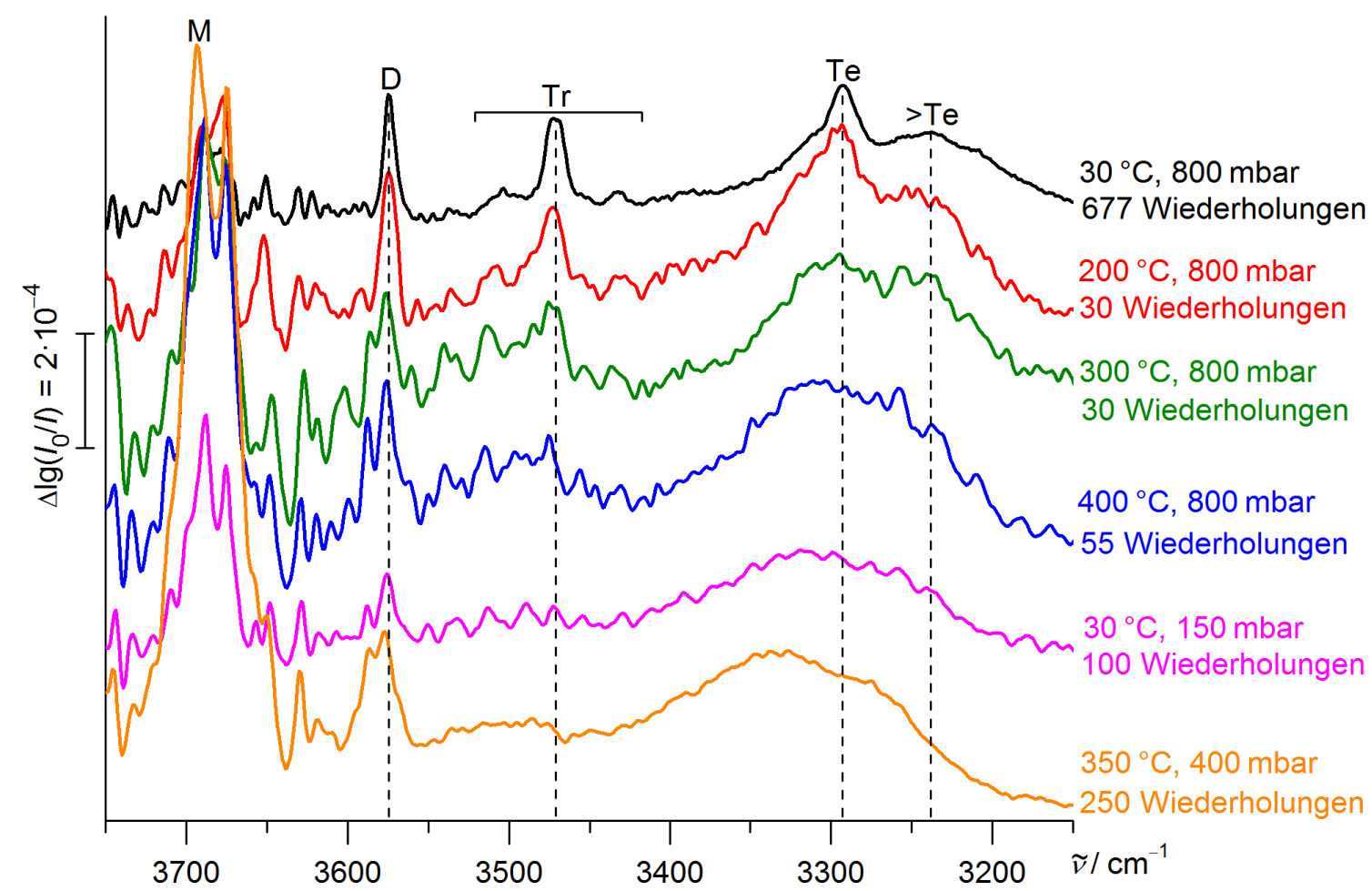

Abb. 9.11: Spektrale Entwicklung von Methanol-Clustern bei Erhöhung der Expansionstemperatur (qualitativ von oben nach unten). Die Konzentration wurde über Variation der Synchronisation stets so gewählt, dass ein ähnlich intensives Signal für größere Cluster erhalten wird. Die Bandenpositionen sind für die kältesten Bedingungen mit $\mathrm{M}$ für Monomer, $\mathrm{D}$ für Dimer, Tr für Trimer, Te für Tetramer und $>$ Te größer als Tetramer gekennzeichnet. Der Aufnahmemodus ist einheitlich 80 $\mathrm{kHz}$ Spiegelgeschwindigkeit, $5 \mathrm{~cm}^{-1}$ Auflösung, SSFR. Da diese Spektren noch mit der ursprünglichen Synchronisationsstrategie aufgenommen wurden, ist der Bereich oberhalb von $3550 \mathrm{~cm}^{-1}$ durch Störsignale überlagert. 
Im Unterschied zu Spektren der Flüssigkeit und zu den RESS-Jet-Spektren der MethanolTröpfchen (Abb. 9.5) ist aber weiterhin ein intensives Signal des Dimers präsent. Eine mögliche Interpretation dieser spektralen Effekte ist, dass die gespannten cyclischen Trimere, und möglicherweise auch die weniger gespannten Tetramere, bei thermischer Anregung in offene, lineare Strukturen aufbrechen. Für die bereits bei tiefen Temperaturen offenkettigen Dimere ist eine solche Isomerisierung nicht möglich, sodass die spektralen Effekte begrenzter sind. Auf diese Weise wurden ähnliche spektrale Effekte bei analogen Wasser-Clustern interpretiert.[450]

\subsection{Fazit und Ausblick}

In diesem Kapitel wurde gezeigt, dass sich auch durch die Injektion von Flüssigkeit in eine geheizte Mischkammer Gasgemische für Expansionen erzeugen lassen, die sowohl für leicht- (Methanol), wie auch schwerflüchtige Verbindungen (Ethylenglycol) vergleichbare spektrale Informationen zugänglich machen wie mit anderen Ansätzen für die Herstellung des Gasgemisches. In einer Weiterführung dieser Arbeit sollte es darum gehen, auf der einen Seite bekannte Schwächen zu verringern, und auf der anderen Seite potentielle Vorteile dieses Prinzips näher zu erproben und an geeigneten Systemen zur Geltung zu bringen. Hierfür werden im Folgenden einige Vorschläge gemacht.

Zunächst sollten auch die Verwendung höherer Auflösungen durch Vermeidung der diskutieren Störsignale ermöglicht werden. Sofern wie vermutet die Magnetventile des Heliumpulses hierfür verantwortlich sind, sollte dies prinzipiell erreichbar sein, da das selbe Spektrometer bereits in der Frühphase des Filet-Jets [37] mit den gleichen Magnetventilen erfolgreich kombiniert wurde.

Messungen bei höherer Auflösung würden aufgrund der längeren Scandauer auch von Maßnahmen für eine sich zeitlich langsamer verändernde Expansion profitieren. Hierdurch könnten auch Double-Sided-Scans wie am Popcorn-Jet möglich werden. Denkbar wäre sogar, dass mehr als ein Probescan verwertbare Informationen liefert, um ein molekulares System in einer einzigen Messreihe bei mehreren Konzentrationen zu charakterisieren. Dies wurde mit sehr kurzen Scans bereits erprobt, allerdings ist dies durch die dabei notwendige hohe Spiegelgeschwindigkeit und geringe Auflösung nicht konkurrenzfähig zu zwei nacheinander durchgeführten Messreihen. Mögliche Ansätze hierzu wären eine Verringerung der Querschnittsfläche der Schlitzdüse oder ein Strömungswiderstand im Inneren, wie ein Gitter oder Glaswolle. Allerdings würde durch den geringeren Gasfluss wohl auch die Intensität der Signale beeinträchtigt werden. Diesbezüglich neutraler wäre eine Vergrößerung der Mischkammer. Die bestehende nutzt die Breite des Expansionsrohrs bereits gut aus, hätte aber noch Spielraum in der Höhe und vor allem in der Länge, da das Ventil im Expansionsrohr durch die Linearverstellung weiter zurückgefahren werden kann.

Als ein mögliches Problem hat sich die Bildung der Schlitzdüse aus Aussparungen bei Zusammensetzung der zwei Hälften der Mischkammer erwiesen. Nach zwischenzeitlicher Öffnung und Wiederzusammenbau konnten Spektren erst nach größeren Veränderungen an der Synchronisation wieder (weitgehend) reproduziert werden. Diese Änderungen deuteten daraufhin, dass die Fläche der Schlitzdüse etwas größer geworden ist. Möglicherweise hat das genaue Anzugsdrehmoment der die beiden Hälften verbindenden 
Schrauben signifikanten Einfluss im Mikrometerbereich. Bei Handhabung der Mischkammer sollte daher auf eine reproduzierbare Vorgehensweise geachtet werden.

Hinsichtlich des Zuleitungssystems sollte eine Alternative für die eingesetzte kommerzielle HPLC-Pumpe gefunden werden. Ähnlich, wenn auch nicht im gleichen Ausmaß wie die vorher eingesetzte HPLC-Pumpe aus Werkstatt-Konstruktion, zeigt diese Probleme bei Verwendung von Ethylenglycol. Sofern dies auf die Viskosität zurückzuführen ist, dürfte dieses Problem auch bei anderen schwerflüchtigen Substanzen zu erwarten sein. Die Ansaugöffnung im Vorratsgefäß sollte mit einem Filter versehen werden, um einer Verstopfung der feinen Kapillaren mit Staub oder anderen Verunreinigungen vorzubeugen. Das Zuleitungssystem sollte zudem stark in seinem Volumen verringert werden, um auch höherpreisige Analyten untersuchen zu können. So besitzt allein der Heizschlauch ein Innenvolumen von knapp 19 ml [433] und sollte durch eine dünnere Kapillare ersetzt werden. Hier könnte auf bestehende Erfahrungen mit HPLC-Systemen an der Fakultät zurückgegriffen werden.

Das Potential des Risotto-Jets ist sicherlich auch durch sein veraltetes Spektrometer eingeschränkt, wobei allerdings zunächst die Limits bei der Generierung der Expansion erforscht werden sollten. Eine begrenzte Verbesserungsmöglichkeit wäre die Installation einer $150 \mathrm{~W}$ Wolframlampe wie am Filet-Jet, hierfür könnte vermutlich die Spannungsversorgung des Globars weiterverwendet werden. Auch für den Popcorn-Jet könnte dies nach dem Erfolg der neuen V-Düse ein sinnvolles Upgrade sein.

Da die Nutzbarkeit von sehr hohen Drücken nicht mehr maßgeblich für den Risotto-Jet ist, ist das für die Injektion der Flüssigkeit eingesetzte Hochdruckventil überdimensioniert und limitiert die einsetzbaren Temperaturen. Kompakte und temperaturbeständige Einspritzdüsen finden in Dieselmotoren Verwendung.

Vor größeren baulichen Investitionen sollte aber zunächst evaluiert werden, inwieweit eine Hauptmotivation für den Risotto-Jet tatsächlich umgesetzt werden kann: die Untersuchung von binären Analyten-Gemischen in einem Verhältnis unabhängig von den individuellen Dampfdrücken. Bisherige Untersuchungen an Einzelsubstanzen deuten eher daraufhin, dass das Ziel der schnellen vollständigen Verdampfung des injizierten Tropfens noch nicht erreicht ist und nur ein kleiner Teil durch den Heliumpuls aufgenommen wird. Inwiefern dies zu Abweichungen in der Zusammensetzung zwischen flüssiger und gasförmiger Phase führt, wäre zu erproben. Ein geeignetes Testsystem sollte aus zwei schwer-, aber doch unterschiedlich flüchtigen Komponenten bestehen, die sich im $\mathrm{CH}-$ Streckschwingungsbereich deutlich genug unterscheiden, um durch Integration der Banden das Stoffmengenverhältnis bestimmen zu können. Mögliche Veränderungen, um eine vollständige Verdampfung zu begünstigen, wären der Einsatz der noch dünneren Kapillare, ein größeres Volumen der Mischkammer, höhere Temperaturen und Maßnahmen, die die Verweildauer des Analyten in der Mischkammer erhöhen. Für letzteres könnte auch ein zweiter Heliumpuls, wie diskutiert, als dynamisches Rückschlagventil genutzt werden. Alternativ wäre auch ein mechanisches Rückschlagventil wie beim Popcorn-Jet denkbar. Umgekehrt könnte eine sequentielle Verdampfung aber sogar für eine Kombination aus einem schwerflüchtigen, eventuell festen Analyten, und einem leichtflüchtigen Lösungsmittel wünschenswert sein, wie sie am RESS-Jet für die Mischung aus $n$-Dodecanol und $n$-Pentan beobachtet wurde. Sollte sich eine vollständige Verdampfung ohne Trennung des Gemisches als nicht möglich erweisen, könnte auch eine begrenzte Steuerung des Partialdruckverhältnisses über die Mischkammertemperatur erzielt 
werden, da die Gesamtkonzentration über die Synchronisation verringert werden kann. So könnte vermutlich aus einer 1:1-Mischung der flüssigen Phase auch eine annähernde 1:1-Gasphasen-Mischung von Benzylalkohol und 1-Phenylethanol bei $200^{\circ} \mathrm{C}$ erzeugt werden, da dann die Dampfdrücke gemäß der Interpolation in Abb. 8.12 auf S. 252 fast übereinstimmen.

Eine weitere offene Frage ist die Eignung für die Untersuchung von schwerflüchtigen thermolabilen Substanzen. Mit dem derzeitigen Messablauf vergeht zwischen Beginn der Injektion und der Detektion eine Zeitspanne von etwa $100 \mathrm{~ms}$, in der der Analyt der vollen Temperaturbelastung ausgesetzt ist. Allerdings befindet sich die Substanz auch bereits vor der Injektion nicht durchgehend auf Zimmertemperatur, da der vorderste Abschnitt der zuführenden Kapillare über Wärmeleitung zwangsläufig miterwärmt wird. Die aus den Vorversuchen abgeschätzte Menge von $2 \mu$ würde bei laminarer Strömung aus den vordersten $8 \mathrm{~cm}$ der Kapillare mit $0.18 \mathrm{~mm}$ Innendurchmesser entnommen werden. Auch eine Temperatur oberhalb der Zimmertemperatur und unterhalb des Wertes der Mischkammer, würde die Zersetzungsgeschwindigkeit vermutlich schon deutlich verringern. Ein Aufenthalt der Substanz in diesem Bereich von etwa 1-2 Minuten vor dem Experiment könnte daher tolerabel sein.

Die hier durchgeführten grundlegenden Arbeiten für das Risotto-Prinzip zeigen Potential, es bestehen aber auch noch größere offene Fragen. 


\section{Fazit und Ausblick: Chiralitätserkennung mit prochiralen Molekülen}

In den vorangegangenen Kapiteln wurde eine Vielfalt an molekularen Systemen untersucht und hinsichtlich von Phänomenen wie Konformationsvielfalt, adaptive Aggregation und Chiralitätserkennung charakterisiert. Dabei wurden subtile energetische und spektrale Differenzen, sowohl zwischen Konformeren von Alkoholen, wie auch zwischen Komplexen mit unterschiedlicher relativer Händigkeit, beobachtet. Für die Vorhersage von $\mathrm{OH}-$ Streckschwingungsübergängen und Tunnelaufspaltungen in monomeren Alkoholen wurden Modelle präsentiert. Es wurde gefunden, dass diese Effekte insgesamt gut und zu akzeptablen Rechenkosten mit der DFT-Methode B3LYP-D3(BJ)/may-ccpVTZ in doppelt-harmonischer Näherung in Kombination mit empirischen Korrekturen beschrieben werden können. Auch mit B2PLYP-D3(BJ) werden ähnliche Resultate erhalten, allerdings sind die Einsatzmöglichkeiten dieser Methode aufgrund der hohen Rechenkosten eingeschränkt. Die Abwesenheit von größeren Abweichungen legt nahe, dass diese Methoden CISS gut beschreiben können oder/und dass der Einfluss von CISS auf Chiralitätserkennung nicht so substantiell ist, wie behauptet wurde. Verbleibende Defizite könnten auch in der doppelt-harmonischen Näherung und in der semi-empirischen Behandlung von Austausch und Korrelation (einschließlich Dispersion) im DFT-Ansatz begründet sein, ohne hierfür eine (weitgehend) fehlende wichtige Komponente in der molekularen Erkennung, wie CISS, annehmen zu müssen.

Für weitere Untersuchungen werden im folgenden einige Systeme vorgeschlagen. Prochirale Moleküle sind hinsichtlich dieser Fragestellung interessant, da sie achiral sind, aber dennoch Chiralitätserkennung zeigen. Dies könnte es erlauben, zwei Beiträge zur Chiralitätserkennung weitgehend zu separieren: einerseits Unterschiede in der räumlichen Passform, andererseits Effekte, die explizit von der Chiralität beider Moleküle abhängen, wie CISS.

Ein erster Ansatz ist es, das etablierte Konzept der intermolekularen Erkennung in Carbonylwaagen [132, 210, 212, 423, 424] mit Chiralitätserkennung zu verbinden. In bisher untersuchten Carbonylwaagen tragen die $\alpha$-Kohlenstoffatome von Ketonen unterschiedliche Substituenten (z. B. in Phenylmethylketon), sodass die beiden freien Elektronenpaare diastereotop sind. Deren Koordination mit Wasserstoffbrücken-Donoren führt zu Unterschieden in der Energie und in der OH-Streckschwingungswellenzahl. Diese werden als Benchmark für die Beschreibung von zwischenmolekularen Wechselwirkungen durch quantenchemische Methoden genutzt. Die hierfür bisher eingesetzten Ketone besitzen Konformationen (oder niedrig liegende Übergangszustände) mit einer Spiegelebene in der Ebene der Carbonylgruppe (definiert durch die weitgehend planare $\mathrm{C}-\mathrm{CO}-\mathrm{C}$ - 
Einheit). Da damit in dieser Spiegelebene auch die freien Elektronenpaare liegen, ist keine Erkennung diesbezüglich mit den Enantiomeren von chiralen Donoren zu erwarten. Auch völlig asymmetrische Ketone wurden unter Beteiligung des Autors der vorliegenden Arbeit an den Beispielen Campher und Fenchon bereits untersucht. Diese zeigen deutliche spektrale Unterschiede bei der Chiralitätsdiskriminierung mit dem Wasserstoffbrückendonor $\alpha$-Fenchol (unveröffentlicht).

Ein „Twist“ hierzu, sowohl im übertragenen wie auch im buchstäblichen Sinne, sind prochirale Ketone, die eine Spiegelebene nicht innerhalb, sondern senkrecht zu der Carbonylebene aufweisen. Hierdurch sind die beiden freien Elektronenpaare des Sauerstoffatoms nicht mehr diastereotop, sondern enantiotop. Für achirale Donoren ist deren Koordination dadurch gleichwertig, die sich diesbezüglich bildenden Konformere des Komplexes sind enantiomer und damit spektroskopisch nicht unterscheidbar. Für chirale Donoren hingegen ist eine Chiralitätserkennung mit den enantiotopen Seiten des Ketons zu erwarten. Bezüglich der Koordination der beiden Seiten kann sich ein Gleichgewicht einstellen, dessen Lage Rückschlüsse auf die chirodiastaltische Energie zulässt.

Da die enantiotopen Elektronenpaare die gleichen inhärenten Akzeptorstärken aufweisen, sind spektrale und energetische Unterschiede allein auf die Auswirkungen der unterschiedlichen sekundären Wechselwirkungen zurückzuführen, die die Chiralitätserkennung vermitteln. Für deren Beschreibung durch Elektronenstrukturmethoden sollte das Experiment daher einen besonders feinen Benchmark darstellen. Im Vergleich zu bisher untersuchten Carbonylwaagen, ist für andere Aspekte, wie die Stärke der primären Wasserstoffbrücke oder die Anharmonizität, tendenziell noch weitergehende Fehlerkompensation zu erwarten. Die erwartete geringe chirodiastaltische Energie sollte in der Regel im angestrebten Bereich für Carbonylwaagen von $<2 \mathrm{~kJ} \mathrm{~mol}^{-1}$ [423] liegen, sodass beide Konformere des Komplexes ausreichend für einen Nachweis populiert sein können. Ein Nachteil sind die geringeren spektralen Unterschiede, die Zuordnungen weniger eindeutig machen.[212]

Eine naheliegende Grundstruktur eines Ketons mit der angestrebten Symmetrie folgt dem Schema in Abb. 10.1, allerdings besteht in dieser offenen Form vermutlich eine verkomplizierende Konformationsvielfalt bezüglich der C-C-Torsion.

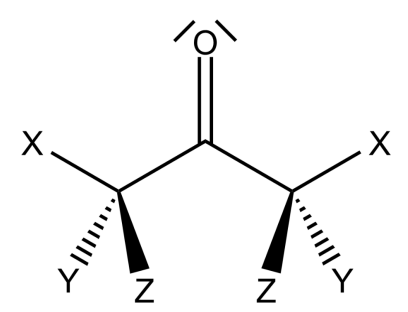

Abb. 10.1: Allgemeine Strukturformel eines meso-Ketons.

Diese Flexibilität kann durch Ringschluss eingeschränkt werden. Cyclohexanon als cyclisches Keton hat eine sehr deutliche Präferenz für eine einzelne $C_{\mathrm{s}}$-symmetrische Konformation (Abb. 10.2) [451-455], die auch im Komplex mit Wasser beibehalten wird.[455] Testrechnungen von Cyclohexanon für die Chiralitätssynchronisation mit Benzylalkohol und die Chiralitätsinduktion mit 1-Phenylethanol ergaben auf B3LYP-D3(BJ)/may-cc-pVTZ-Niveau vielversprechende Energieunterschiede von 0.7 bzw. $0.6 \mathrm{~kJ} \mathrm{~mol}^{-1}$ und Differenzen in der OH-Streckschwingungswellenzahl von $20 \mathrm{bzw}$. 
$12 \mathrm{~cm}^{-1}$ zwischen den Konformeren. Dieses Thema wird derzeit von Moritz Niessner in einer Bachelorarbeit untersucht. Erste Ergebnisse erscheinen vielversprechend.

Eine noch stärkere Fixierung kann durch einen zweiten Ringschluss erfolgen, Abb. 10.3 listet hierfür einige Beispielmoleküle.

Interessant könnte dabei der direkte Vergleich mit solchen chiralen Ketonen sein, bei denen eine der beiden Seiten sehr selektiv koordiniert werden kann. Die sterische Abschirmung einer Seite, wie es in analog für das chirale Alken $\alpha$-Pinen verwirklicht ist (Kap. 6), scheint für Ketone allerdings kaum umsetzbar. Eine elegante Alternative hierzu wären $C_{2}$-symmetrische Derivate mit zwei homotopen Seiten, wie im Beispiel-Molekül in Abb. 10.4. Dies würde den Vergleich zwischen Komplexen ermöglichen, die sehr ähnliche räumliche Beiträge zur Erkennung aufweisen. Dagegen wären zusätzliche Effekte, die eine (induzierte) Chiralität des Ketons voraussetzen, wie CISS, weit stärker mit dem intrinsisch chiralen $C_{2}$-symmetrischen Keton zu erwarten.

Auch bei weiteren Sauerstoff-Verbindungen, wie Oxiranen (Epoxiden), treten enantiotope freie Elektronenpaare auf. Abb. 10.5 zeigt das prochirale 2,2-Dimethyloxiran, sowie die Enantiomere von trans-2,3-Dimethyloxiran als chirale Vergleichssubstanzen. 2,2-Dimethyloxiran sowie das racemische Gemisch von trans-2,3-Dimethyloxiran sind
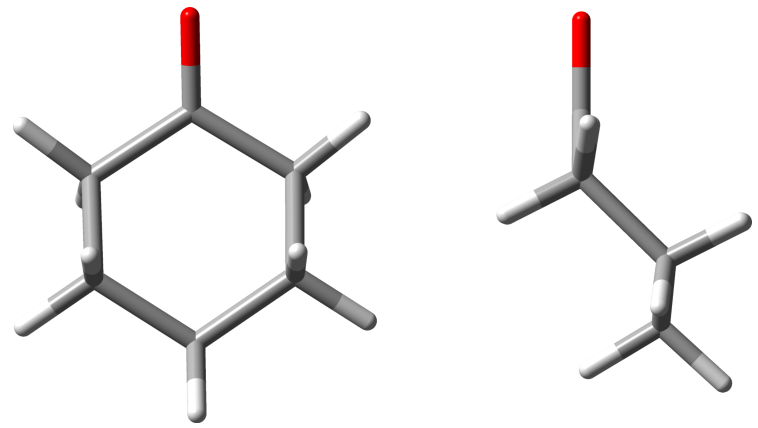

Abb. 10.2: Optimierte Konformation von Cyclohexanon mit Blickrichtung entlang (links) und senkrecht zur Spiegelebene (rechts) auf B3LYP-D3(BJ)/def2-TZVP-Niveau.
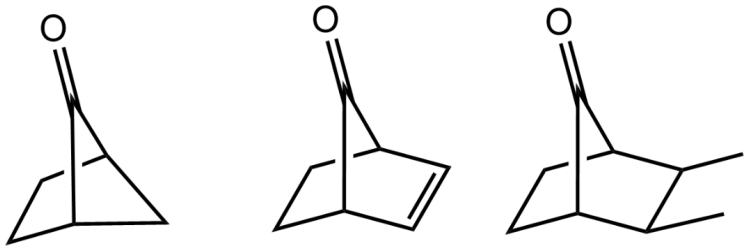

Abb. 10.3: Beispiele von bicyclischen Ketonen mit einer Spiegelebene senkrecht zur Ebene der Carbonylgruppe.

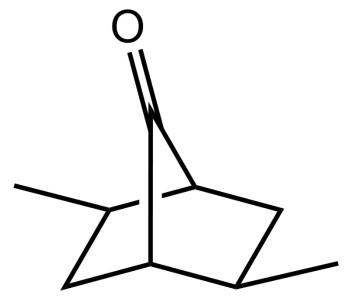

Abb. 10.4: Beispiel eines chiralen $C_{2}$-symmetrischen bicyclischen Ketons mit zwei identischen Seiten. Diese entsprechen der vorderen Seite der dritten Struktur in Abb. 10.3. 
kommerziell verfügbar. Bei dieser Substanzklasse kann auf Vorarbeiten zur Charakteri-
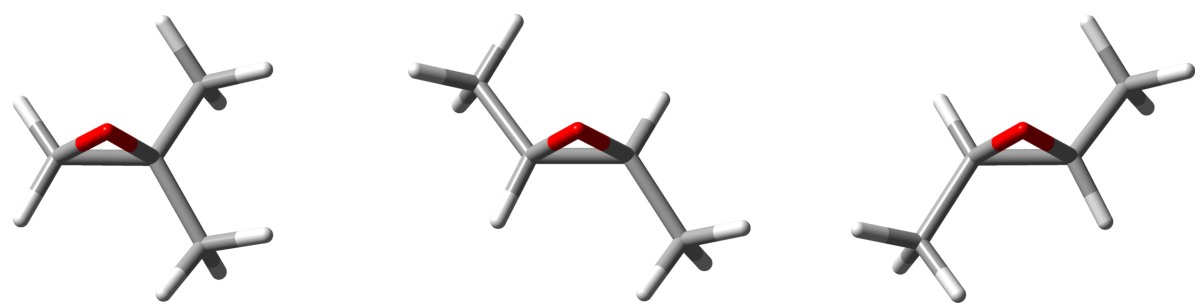

Abb. 10.5: Von links nach rechts: Strukturen von 2,2-Dimethyloxiran, (2S,3S)-2,3Dimethyloxiran und $(2 R, 3 R)$-2,3-Dimethyloxiran.

sierung der Oxirane [456-458] und deren Chiralitätsinduktion auf andere Moleküle wie Wasserstoffperoxid [459], Ethanol [460, 461] und 2-Fluorethanol [462] zurückgegriffen werden. Auch bestimmte andere cyclische Ether, wie das bicyclische Monoterpen Eucalyptol (1,8-Cineol) [463, 464], besitzen zwei enantiotope Elektronenpaare.

Auch viele Alkohole sind prochiral. Die Selbstsynchronisation für Cluster des Prototypen Methanol wurde bereits in Kap. 4 betrachtet. Auch das $t$-Konformer von Ethanol ist prochiral. Für die quantenchemische Untersuchung der Konformationsvielfalt der Ethanol-Dimere wurde in Kap. 4.3.3 willkürlich die Koordination des gleichen freien Elektronenpaares festgelegt und die Konformation des Donors variiert. Alternativ kann aber auch das koordinierte freie Elektronenpaar bei festgehaltenen Konformationen verändert werden. In dieser alternativen Beschreibung wird verständlich, dass auch die Diastereomerie der als $g-t$ - und $g+t$ bezeichneten Dimere von Ethanol als Ergebnis von Chiralitätssynchronisation beschrieben werden kann.

Das System der Ethanol-Dimere wird allerdings durch Donor-Akzeptor-Isomerie zwischen den Konformeren verkompliziert. Ähnliches gilt auch für gemischte Dimere von Alkoholen. So ist Methanol ein schlechter Akzeptor für Wasserstoffbrücken und tritt daher im Dimer mit Ethanol nur als Donor auf.[131] Ein besserer geeigneter Akzeptor mit gleicher Symmetrie wäre tert-Butylalkohol.

Aber auch Methanol kann beispielsweise in Insertionskomplexen in die Akzeptor-Rolle gebracht werden (zusätzlich zur Donor-Rolle). Ein Beispiel für die Chiralitätssynchronisation sind die zwei jüngst in einer Stickstoff-Matrix nachgewiesenen gemischten Dimere mit Propargylalkohol.[465] Weitere Beispiele sind die von Borho et al. [432] untersuchten Komplexe von Methanol mit den prochiralen $\alpha$-Hydroxyestern Methylglykolat und Methyl- $\alpha$-hydroxyisobutyrat, sowie mit dem permanent chiralen Methyllactat. Die Prochiralität von Methanol wurde in dieser Publikation angesprochen, aber nur unvollständig berücksichtigt. Nach eigenen Rechnungen auf B3LYP-D3(BJ)/may-cc-pVTZ-Niveau (und bestätigt auf dem in der Publikation verwendeten B3LYP/6-31+G*-Niveau) wurde das jeweils zweitstabilste Dimer-Konformer übersehen. Abb. 10.6 zeigt beispielhaft die zwei stabilsten Konformere von Methanol mit Methylglykolat. Da die Energiedifferenz allerdings mit jeweils etwa $2 \mathrm{~kJ} \mathrm{~mol}^{-1}$ groß ausfällt, ändert dies nichts an der Interpretation der Spektren, in denen nur jeweils ein Komplex nachgewiesen wurde.

Auch in der Kombination von Methanol mit dem transient chiralen Benzylalkohol und dem permanent chiralen 1-Phenylethanol ist eine Chiralitätssynchronisation bzw. -induktion in einem Insertionskomplex zu erwarten. Abb. 10.7 zeigt die zugehörigen Strukturen für das System Methanol-Benzylalkohol. 
Im Vergleich zu Carbonylwaagen bieten Insertionskomplexe einige Vorteile als Benchmark für quantenchemische Methoden. Wie auch in Kap. 8 für Dimere von Benzylalkohol und 1-Phenylethanol gezeigt, werden durch die Ausbildung von zwei unterschiedlichen Wasserstoffbrücken im OH-Streckschwingungsbereich im Idealfall zwei Bandenpaare erhalten. Mit den theoretischen Vorhersagen können dann mindestens zwei spektrale Aufspaltungen verglichen werden. Auch Konformationstemperaturen bzw. Energieunterschiede können auf mehrere Arten abgeschätzt werden. Dies erlaubt zusätzlich auch Rückschlüsse auf die Verlässlichkeit von berechneten relativen IR-Aktivitäten und Raman-Streuquerschnitten.

Interessanterweise ist die gleiche Erkennung auch mit Wasser anstelle von Methanol zu erwarten, obwohl das freie Wassermolekül zwei homotope freie Elektronenpaare aufweist. Dies ist darin begründet, dass die Symmetrie des Wassermoleküls im Insertionskomplex verringert wird, da eines der beiden Wasserstoffatome als Donor einer Wasserstoffbrücke gebunden wird. Hierdurch ist auch zu erwarten, dass die beiden Konformere nicht mehr durch eine einfache barrierefreie Torsion um die Wasserstoffbrücke ineinander umgewandelt werden können (wie in Komplexen, in denen Wasser nur entweder als Do-

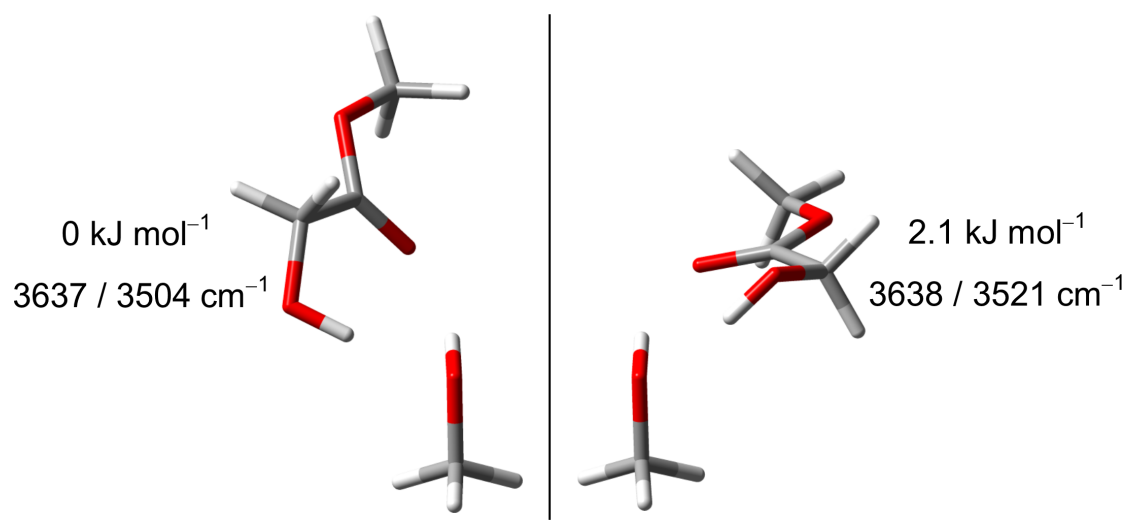

Abb. 10.6: Die zwei stabilsten Konformere des gemischten Dimers aus Methanol und Methylglykolat, welche sich aus der Chiralitätssynchronisation der beiden prochiralen Moleküle ergeben. Die Chiralität von Methylglykolat ist in beiden Komplexen gleich. Angegeben sind die relativen schwingungsnullpunktskorrigierten Energien und die harmonischen $\mathrm{OH}-$ Streckschwingungswellenzahlen auf B3LYP-D3(BJ)/may-cc-pVTZ-Niveau. Die auf der linken Seite dargestellte Struktur entspricht der von Borho et al. [432] zugeordneten.

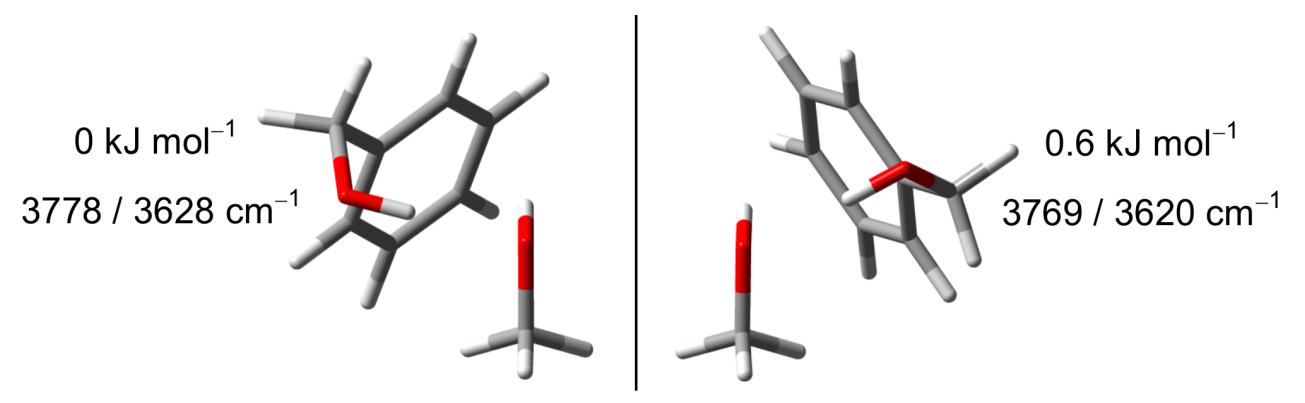

Abb. 10.7: Die zwei stabilsten Konformere des gemischten Dimers aus Methanol und Benzylalkohol. Die Chiralität von Benzylalkohol ist in beiden Komplexen gleich. Angegeben sind die relativen schwingungsnullpunktskorrigierten Energien und die harmonischen $\mathrm{OH}-$ Streckschwingungswellenzahlen auf B3LYP-D3(BJ)/may-cc-pVTZ-Niveau. Additionskomplexe mit nur einer intermolekularen Wasserstoffbrücke sind deutlich weniger stabil. 
nor oder Akzeptor agiert). Zwei Studien zu dem gemischten Dimer aus Benzylalkohol und Wasser [219, 220] konnten nur eine einzelne - vermutlich die gleiche - Struktur nachweisen, ordnen sie aber interessanterweise unterschiedlichen Konformeren zu, die sich durch die hier diskutierte Isomerie unterscheiden. Auch Isomerie in Wasser-Clustern lässt sich teilweise auf die gleiche Weise beschreiben, da in diesen die Wassermoleküle ebenfalls gleichzeitig als Donor und Akzeptor agieren. Dies zeigt wie universal die in dieser Arbeit diskutierten Prinzipien von Chiralitätserkennung angewendet werden können. 


\section{Danksagung}

Viele Personen haben zum Gelingen dieser Arbeit beigetragen.

Als allererstes gilt Prof. Dr. Martin A. Suhm besonderer Dank für die hervorragende Betreuung, seine Diskussionsbereitschaft, sowie seine Begeisterungsfähigkeit. Auch für die Möglichkeit, dieser Arbeit nach anfänglichen Schwierigkeiten einen neuen Schwerpunkt zu geben, bedanke ich mich herzlich.

Prof. Dr. Ricardo A. Mata danke ich ebenfalls für die Betreuung und nützliche Hinweise. Prof. Dr. Konrad Koszinowski, Dr. Sebastian Kruss, Dr. Oliver Bünermann und Dr. Tim Schäfer danke ich für ihre Bereitschaft der Prüfungskommission anzugehören.

Besonderer Dank geht an die Mitarbeiter der Mechanik- und Elektronik-Werkstätten, ohne die die experimentelle Arbeit, insbesondere in Kap. 9, unmöglich gewesen wäre. Stellvertretend seien hier Reinhard Hildebrandt und Andreas Knorr genannt.

Für administrative und technische Hilfe möchte ich mich bei Petra Lawecki, Dr. Markus Hold, Clemens Heymann, Dr. Ulrich Schmitt, Dr. Nils Lüttschwager, Werner Noack und Norbert Neisen bedanken.

Caroline Stelbrink, Enno Meyer, Helen Preiß, Moritz Niessner und Johann Benedikt Meyer danke ich für ihre engagierte Zusammenarbeit in verschiedenen studentischen Projekten und Abschlussarbeiten.

Danken möchte ich auch der gesamten Suhm Group, insbesondere Beppo Hartwig, Charlotte Zimmermann, Manuel Lange, Arman Nejad, Dr. Sönke Oswald, Dr. Anja Poblotzki, Caroline Stelbrink, Taija Fischer und Maxim Gawrilow, die einzelne Abschnitte oder ganze Kapitel dieser Arbeit vorab gelesen haben.

Für die wissenschaftliche Kooperation bei den Systemen $\alpha$-Fenchol/Campher und $\alpha$ Fenchol/Fenchon möchte ich mich bei Mariyam Fatima, María Mar Quesada-Moreno, Prof. Dr. Melanie Schnell und Xaiza Aniban bedanken.

Meiner Familie und meinen Freunden danke ich für die langjährige Unterstützung. 



\section{A Anhang}

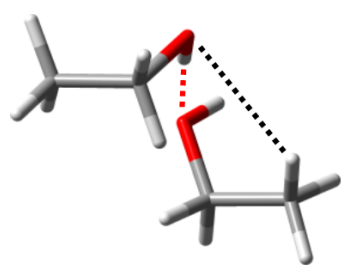

$g-g+2 \quad 0.92$

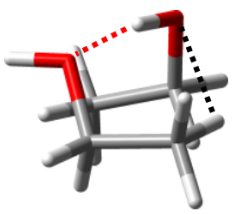

$g+t 2 \quad 1.60$

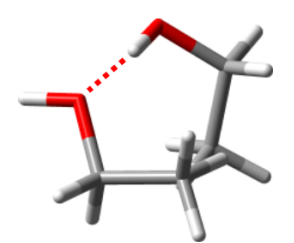

$g+t 31.81$

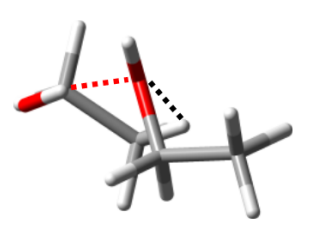

$g+g-1.40$

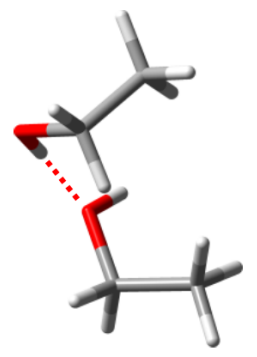

$g+g+2 \quad 1.69$

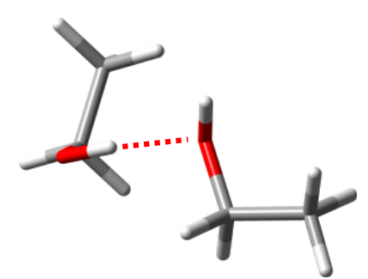

$g-g-1.87$

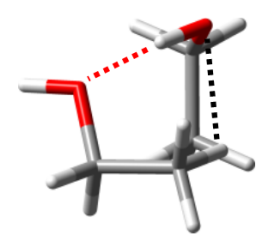

g-t2 1.46
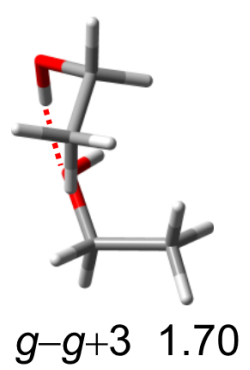

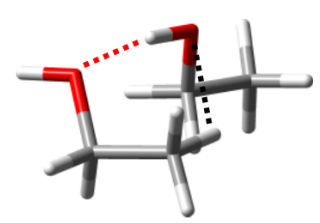

tt2 1.55

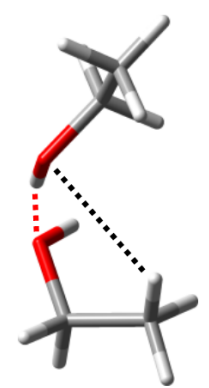

$\operatorname{tg}+21.77$

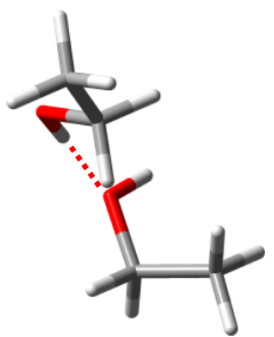

$\operatorname{tg}+3 \quad 1.89$

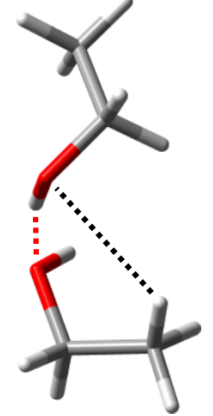

$g+g+3 \quad 1.90$

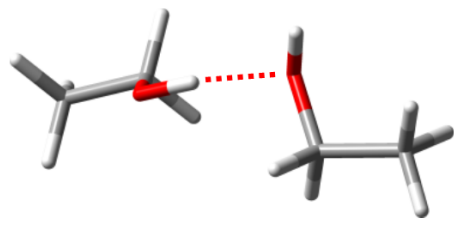

$\operatorname{tg}-1.95$

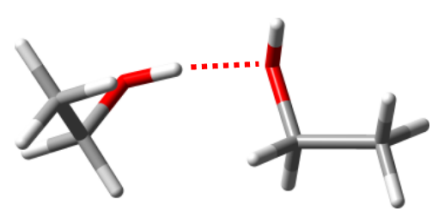

$g+g-2 \quad 2.81$

Abb. A.1: Strukturen der 14 weniger stabilen Ethanol-Dimer-Konformere auf B3LYPD3(BJ)/may-cc-pVTZ-Niveau mit nullpunktskorrigierten Energien relativ zum globalen Minimum $g+g+$ in $\mathrm{kJ} \mathrm{mol}^{-1}$. OH $\cdots \mathrm{O}$-Wasserstoffbrücken sind mit roten, $\mathrm{CH} \cdots \mathrm{O}$-Kontakte mit $\mathrm{Ab}$ ständen $<3.0 \AA$ A mit schwarzen gestrichelten Linien hervorgehoben. 
Tab. A.1: Nullpunktskorrigierte Energien und harmonische OH-Streckschwingungswellenzahlen des Donors der 20 Ethanol-Dimere relativ zu $g+g+\left(\omega_{\mathrm{OH}}=3641.4 \mathrm{~cm}^{-1}\right)$, sowie zugehörige IR-Aktivitäten, Raman-Aktivitäten und Depolarisationsverhältnisse auf B3LYP-D3(BJ)/may-ccpVTZ-Niveau.

\begin{tabular}{lccccc}
\hline Konformer & $\begin{array}{c}E_{0}^{\mathrm{rel}} \\
/ \mathrm{kJ} \mathrm{mol}^{-1}\end{array}$ & $\begin{array}{c}\omega_{\mathrm{OH}}^{\mathrm{rel}} \\
/ \mathrm{cm}^{-1}\end{array}$ & $\begin{array}{c}I \\
/ \mathrm{km} \mathrm{mol}^{-1}\end{array}$ & $\begin{array}{c}A \\
/ \AA^{4} \mathrm{u}^{-1}\end{array}$ & $P$ \\
\hline$g+g+$ & 0 & 0 & 447 & 146 & 0.23 \\
$g-t$ & 0.11 & 9.6 & 445 & 145 & 0.22 \\
$t t$ & 0.31 & 13.7 & 540 & 200 & 0.25 \\
$g+t$ & 0.32 & 6.4 & 469 & 153 & 0.23 \\
$t g+$ & 0.52 & 4.7 & 569 & 213 & 0.27 \\
$g-g+$ & 0.53 & -3.3 & 492 & 162 & 0.24 \\
$g-g+2$ & 0.92 & 14.8 & 373 & 121 & 0.21 \\
$g+g-$ & 1.40 & 16.0 & 492 & 173 & 0.24 \\
$g-t 2$ & 1.46 & 22.9 & 409 & 132 & 0.22 \\
$t t 2$ & 1.55 & 27.6 & 445 & 160 & 0.24 \\
$g+t 2$ & 1.60 & 17.1 & 369 & 115 & 0.20 \\
$g+g+2$ & 1.69 & 33.9 & 370 & 119 & 0.21 \\
$g-g+3$ & 1.70 & 22.0 & 427 & 135 & 0.21 \\
$t g+2$ & 1.77 & 19.4 & 478 & 173 & 0.25 \\
$g+t 3$ & 1.81 & 13.1 & 446 & 146 & 0.22 \\
$g-g-$ & 1.87 & 10.9 & 541 & 191 & 0.24 \\
$t g+3$ & 1.89 & 32.3 & 477 & 174 & 0.25 \\
$g+g+3$ & 1.90 & 12.6 & 405 & 129 & 0.22 \\
$t g-$ & 1.95 & 14.9 & 654 & 262 & 0.27 \\
$g+g-2$ & 2.81 & 27.8 & 420 & 143 & 0.21 \\
\hline & & & & &
\end{tabular}


Tab. A.2: Nullpunktskorrigierte Energien und harmonische $\mathrm{OH}-\mathrm{Streckschwingungswellenzahlen}$ des Donors der 20 Ethanol-OD-Dimere relativ zu $g+g+\left(\omega_{\mathrm{OH}}=2652.4 \mathrm{~cm}^{-1}\right)$, sowie zugehörige IR-Aktivitäten, Raman-Aktivitäten und Depolarisationsverhältnisse auf B3LYP-D3(BJ)/may-ccpVTZ-Niveau.

\begin{tabular}{lcrccc}
\hline Konformer & $\begin{array}{c}E_{0}^{\mathrm{rel}} \\
/ \mathrm{kJ} \mathrm{mol}^{-1}\end{array}$ & $\begin{array}{c}\omega_{\mathrm{OH}}^{\mathrm{rel}} \\
/ \mathrm{cm}^{-1}\end{array}$ & $\begin{array}{c}I \\
/ \mathrm{km} \mathrm{mol}^{-1}\end{array}$ & $\begin{array}{c}A \\
/ \AA^{4} \mathrm{u}^{-1}\end{array}$ & $P$ \\
\hline$g+g+$ & 0.00 & 0.0 & 255 & 82 & 0.22 \\
$g-t$ & 0.10 & 6.3 & 251 & 83 & 0.21 \\
$t t$ & 0.29 & 10.0 & 299 & 104 & 0.24 \\
$g+t$ & 0.32 & 4.5 & 268 & 87 & 0.22 \\
$t g+$ & 0.52 & 4.1 & 318 & 109 & 0.25 \\
$g-g+$ & 0.53 & -2.4 & 278 & 90 & 0.22 \\
$g-g+2$ & 0.95 & 9.9 & 208 & 71 & 0.20 \\
$g+g-$ & 1.45 & 11.1 & 274 & 100 & 0.23 \\
$g-t 2$ & 1.51 & 16.1 & 232 & 76 & 0.21 \\
$t t 2$ & 1.56 & 19.4 & 242 & 85 & 0.22 \\
$g+t 2$ & 1.63 & 11.5 & 205 & 68 & 0.19 \\
$g-g+3$ & 1.80 & 15.2 & 236 & 80 & 0.21 \\
$t g+2$ & 1.81 & 13.9 & 265 & 91 & 0.23 \\
$g+g+2$ & 1.82 & 23.5 & 205 & 71 & 0.20 \\
$g+t 3$ & 1.88 & 9.2 & 248 & 85 & 0.21 \\
$g-g-$ & 1.90 & 7.3 & 297 & 108 & 0.23 \\
$g+g+3$ & 1.96 & 8.4 & 227 & 74 & 0.20 \\
$t g-$ & 1.98 & 11.0 & 354 & 136 & 0.26 \\
$t g+3$ & 1.98 & 22.9 & 256 & 93 & 0.23 \\
$g+g-2$ & 2.90 & 19.1 & 228 & 85 & 0.21 \\
\hline & & & & &
\end{tabular}

Tab. A.3: Verwendete Skalierungsfaktoren für die interne Nachkalibrierung der Wellenzahlinformation älterer Raman-Spektren von Ethanol auf die $t$-Monomerbande (Ausnahme: EtOD). Der Wert 0.999713 entspricht dem Kehrwert des Brechungsindex von Luft.

\begin{tabular}{cclc}
\hline Abb. & Ref. & Bedingungen & Skalierungsfaktor \\
\hline $4.3,4.9$ & {$[21]$} & He $1 \mathrm{~mm}$ & 0.999142 \\
$4.3,4.8$ & {$[21]$} & $\mathrm{He} 3 \mathrm{~mm}$ & 0.999194 \\
$4.3,4.10$ & {$[22]$} & $\mathrm{He}+8 \%$ Ar $2 \mathrm{~mm}$ & 0.999540 \\
$4.3,4.10$ & {$[21]$} & $\mathrm{He}+7.5 \% \mathrm{~N}_{2} 3 \mathrm{~mm}$ & 0.999191 \\
$4.3,4.11$ & {$[22]$} & $\mathrm{Ne} 3 \mathrm{~mm}$ & 0.999705 \\
4.12 & {$[22]$} & He $1 \mathrm{~mm}$ EtOD & 0.999713 \\
\hline
\end{tabular}




\section{A Anhang}

Tab. A.4: Relative nullpunktskorrigierte Energien, Rotationskonstanten und DipolmomentKomponenten der drei stabilsten Ethanol-Trimere auf B3LYP-D3(BJ)/may-cc-pVTZ-Niveau.

\begin{tabular}{cccc}
\hline & $u u d / g-t g+$ & $u u d / t g-g+$ & $u u d / g+g-g+$ \\
\hline$E_{0}^{\mathrm{rel}} / \mathrm{kJ} \mathrm{mol}^{-1}$ & 0 & 0.2 & 0.3 \\
$A / \mathrm{GHz}$ & 1.273 & 0.982 & 1.260 \\
$B / \mathrm{GHz}$ & 0.810 & 0.980 & 0.818 \\
$C / \mathrm{GHz}$ & 0.579 & 0.575 & 0.583 \\
$\left|\mu_{\mathrm{A}}\right| / \mathrm{D}$ & 0.75 & 0.71 & 0.71 \\
$\left|\mu_{\mathrm{B}}\right| / \mathrm{D}$ & 0.34 & 0.24 & 0.25 \\
$\mid \mu_{\mathrm{C}} / \mathrm{D}$ & 0.59 & 0.66 & 0.71 \\
\hline
\end{tabular}

Tab. A.5: Relative nullpunktskorrigierte Energien, Rotationskonstanten und DipolmomentKomponenten der zweit- bis viertstabilsten Ethanol-Tetramere auf B3LYP-D3(BJ)/may-cc-pVTZNiveau. Die stabilste Struktur besitzt kein Dipolmoment.

\begin{tabular}{cccc}
\hline & $u d u d / g-g+g-t$ & $u d u d / g-g+t t$ & $u d u d / g+t g-g+$ \\
\hline$E_{0}^{\mathrm{rel}} / \mathrm{kJ} \mathrm{mol}^{-1}$ & 0.5 & 0.7 & 0.8 \\
$A / \mathrm{GHz}$ & 0.581 & 0.546 & 0.614 \\
$B / \mathrm{GHz}$ & 0.499 & 0.525 & 0.483 \\
$C / \mathrm{GHz}$ & 0.488 & 0.469 & 0.470 \\
$\left|\mu_{\mathrm{A}}\right| / \mathrm{D}$ & 0.25 & 0.13 & 0.48 \\
$\left|\mu_{\mathrm{B}}\right| / \mathrm{D}$ & 0.07 & 0.34 & 0.00 \\
$\mid \mu_{\mathrm{C}} / / \mathrm{D}$ & 0.06 & 0.08 & 0.04 \\
\hline
\end{tabular}

Tab. A.6: Berechnete (B3LYP-D3(BJ)/may-cc-pVTZ) und experimentelle OHStreckschwingungswellenzahlen von Methanol.

\begin{tabular}{lll}
\hline Alkohol & $\omega_{\mathrm{OH}} / \mathrm{cm}^{-1}$ & $\tilde{v}_{\mathrm{OH}} / \mathrm{cm}^{-1}$ \\
\hline Methanol & 3835.4 & $3686[130] 3685$ [466] 3683.4 [364] 3684 [105] \\
& & $3683 / 3684$ [184] 3681.5 [183] 3684 [182] \\
\hline
\end{tabular}


Tab. A.7: Berechnete (B3LYP-D3(BJ)/may-cc-pVTZ) und experimentelle OHStreckschwingungswellenzahlen von primären Alkoholen, alphabetisch sortiert. Experimentelle Werte ohne Quellenangabe wurden in der vorliegenden Arbeit ermittelt. Ältere Curry-Jet-Werte sind mit einem * markiert und wurden um $1 \mathrm{~cm}^{-1}$ verringert.

\begin{tabular}{llll}
\hline Alkohol & Konformer & $\omega_{\mathrm{OH}} / \mathrm{cm}^{-1}$ & $\tilde{v}_{\mathrm{OH}} / \mathrm{cm}^{-1}$ \\
\hline Benzylalkohol & $g$ & 3809.0 & $3648[333] 3649[220]$ \\
& & & $3585[218,219] 3650[332] 3647.5$ \\
Cyclohexylmethanol & $G t$ & 3839.2 & $3679^{*}[70,333]$ \\
Cyclohexylmethanol & $G g$ & 3830.0 & $3667^{*}[70,333]$ \\
Cyclohexylmethanol & $G g^{\prime}$ & 3817.9 & $3656^{*}[70,333]$ \\
Ethanol & $g$ & 3816.6 & $3659^{*}[22] 3659.3[61,105]$ \\
& & & $3667[144]$ \\
Ethanol & $t$ & 3833.1 & $3677^{*}[22] 3676.6[61,105]$ \\
& & & $3682[144]$ \\
Isopropylmethanol & $G t$ & 3839.9 & $3681^{*}[70,333]$ \\
Isopropylmethanol & $G g$ & 3830.3 & $3669^{*}[70,333]$ \\
Isopropylmethanol & $G g^{\prime}$ & 3817.9 & $3658^{*}[70,333]$ \\
n-Propanol & $G t$ & 3838.8 & $3681^{*}[190]$ \\
$n$-Propanol & $T t$ & 3835.0 & $3678^{*}[190]$ \\
n-Propanol & $G g h e t$ & 3828.5 & $3668^{*}[190]$ \\
n-Propanol & $T g$ & 3817.9 & $3660^{*}[190]$ \\
n-Propanol & $G g h o m$ & 3816.0 & $3657^{*}[190]$ \\
\hline
\end{tabular}




\section{A Anhang}

Tab. A.8: Berechnete (B3LYP-D3(BJ)/may-cc-pVTZ) und experimentelle OHStreckschwingungswellenzahlen von sekundären Alkoholen, alphabetisch sortiert. Experimentelle Werte ohne Quellenangabe wurden in der vorliegenden Arbeit ermittelt. Ältere Curry-Jet-Werte sind mit einem * markiert und wurden um $1 \mathrm{~cm}^{-1}$ verringert.

\begin{tabular}{llll}
\hline Alkohol & Konformer & $\omega_{\mathrm{OH}} / \mathrm{cm}^{-1}$ & $\tilde{v}_{\mathrm{OH}} / \mathrm{cm}^{-1}$ \\
\hline 2-Adamantol & $g$ & 3817.9 & $3653[235]$ \\
(-)-Borneol & $g-$ & 3820.0 & 3653 \\
Cyclohexanol & eq $g$ & 3815.9 & $3653[150]$ \\
Cyclohexanol & eq $t$ & 3801.5 & $3636[150]$ \\
Cyclopentanol & ag & 3819.6 & $3655^{*}[236] 3655[127,236]$ \\
& Briefumschlag & & \\
1-Indanol & OH an der Klappe & & \\
& 1eq & 3793.0 & $3627^{*}[70,363] 3627$ [70, 240, 363] \\
1-Indanol & & 3828.6 & $3664^{*}[70,363]$ \\
1-Indanol & 3 eq & 3805.8 & $3638^{*}[70] 3637 *[363] 3636[70]$ \\
1-Indanol & $1 \mathrm{ax}$ & 3818.6 & $3651^{*}[70,363] 3650[70,363]$ \\
& 2ax & & $3652[70,363]$ \\
(-)-Isopinocampheol & $g+$ & 3814.5 & 3648 \\
Menthol & & 3820.4 & $3654[216]$ \\
2-Napththyl-1-ethanol & $G 1$ & 3810.2 & $3647[239]$ \\
2-Napththyl-1-ethanol & $G 2$ & 3812.8 & $3648[239]$ \\
Neomenthol & ax $t$ & 3823.4 & $3654.5[216]$ \\
1-Phenylethanol & $g$ & 3812.7 & $3647[349] 3648[237] 3647.5$ \\
Pinakolylalkohol & $t$ & 3813.0 & 3645 \\
2-Propanol & sc & 3819.9 & $3658[468]$ \\
2-Propanol & ap & 3799.0 & $3636[468]$ \\
\hline
\end{tabular}


Tab. A.9: Berechnete (B3LYP-D3(BJ)/may-cc-pVTZ) und experimentelle OHStreckschwingungswellenzahlen von tertiären Alkoholen, alphabetisch sortiert. Ältere Curry-Jet-Werte sind mit einem * markiert und wurden um $1 \mathrm{~cm}^{-1}$ verringert.

\begin{tabular}{llll}
\hline Alkohol & Konformer & $\omega_{\mathrm{OH}} / \mathrm{cm}^{-1}$ & $\tilde{v}_{\mathrm{OH}} / \mathrm{cm}^{-1}$ \\
\hline 1-Adamantol & - & 3800.4 & $3634[235]$ \\
tert-Butylalkohol & - & 3808.3 & $3642[126]$ \\
2-Methyl-2-butanol & $T g$ & 3804.8 & $3635^{*}[236]$ \\
2-Methyl-2-butanol & $G-g-$ & 3805.2 & $3637^{*}[236]$ \\
2-Methyl-2-butanol & $T t$ & 3808.1 & $3642^{*}[236]$ \\
2-Methyl-2-butanol & $G t$ & 3813.0 & $3646^{*}[236]$ \\
2-Methyl-2-butanol & $G+g-$ & 3817.8 & $3648^{*}[236]$ \\
\hline
\end{tabular}

Tab. A.10: Relative freie Enthalpien der neun Konformere von $(S)$-2-Butanol und deren Boltzmann-Populationen bei $25^{\circ} \mathrm{C}$ und 1 atm auf B3LYP-D3(BJ)/may-cc-pVTZ-Niveau.

\begin{tabular}{lcc}
\hline Konformer & $G / \mathrm{kJ} \mathrm{mol}^{-1}$ & $P$ \\
\hline$G+g-$ & 0.2 & $19 \%$ \\
$G+t$ & 0 & $21 \%$ \\
$G+g+$ & 0.6 & $16 \%$ \\
$T g-$ & 1.4 & $12 \%$ \\
$T t$ & 1.7 & $11 \%$ \\
$T g+$ & 2.0 & $9 \%$ \\
$G-g-$ & 3.8 & $4 \%$ \\
$G-t$ & 3.8 & $4 \%$ \\
$G-g+$ & 4.4 & $4 \%$ \\
\hline
\end{tabular}

Tab. A.11: IR-Intensitäten, Raman-Aktivitäten und Depolarisationsverhältnisse auf B3LYPD3(BJ)/may-cc-pVTZ-Niveau für die fünf Konformere von $(S)$-1-Phenylethanol.

\begin{tabular}{lccc}
\hline Konformer & $\begin{array}{c}I \\
/ \mathrm{km} \mathrm{mol}^{-1}\end{array}$ & $\begin{array}{c}A \\
/ \AA^{4} \mathrm{u}^{-1}\end{array}$ & $P$ \\
\hline$g-$ & 23 & 86 & 0.19 \\
$t$ & 26 & 147 & 0.25 \\
$E g+$ & 21 & 58 & 0.17 \\
$S g+$ & 19 & 48 & 0.11 \\
$G+g+$ & 20 & 62 & 0.14 \\
\hline
\end{tabular}


A Anhang

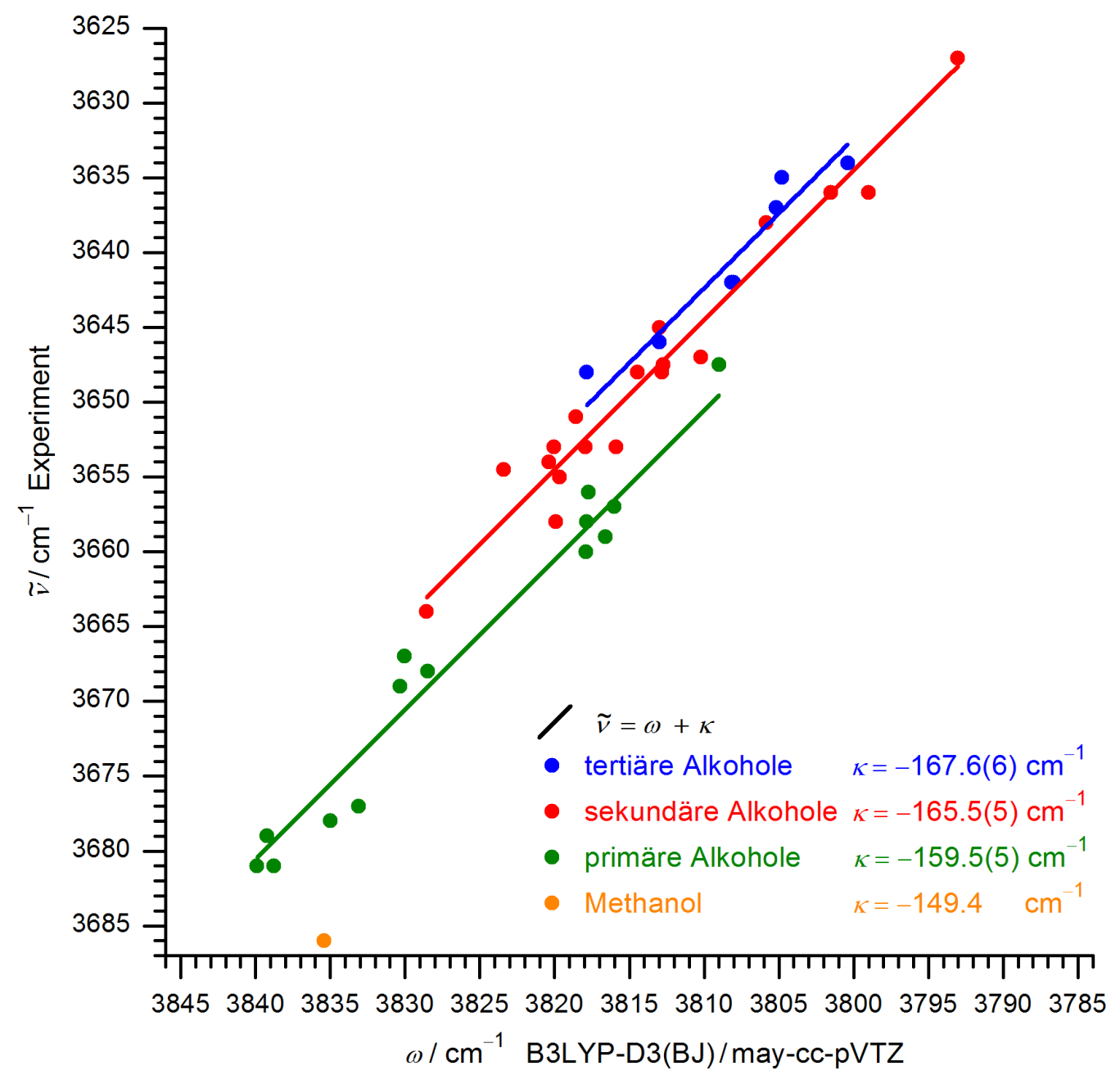

Abb. A.2: Aktualisierte Korrelation von experimentellen OH-Streckschwingungswellenzahlen von Alkoholen mit berechneten in harmonischer Näherung mit B3LYP-D3(BJ)/may-cc-pVTZ. Die Ausgleichsgeraden entsprechen der Gleichung $\tilde{v}=\omega+\kappa$ für die verschiedenen Alkoholklassen. 
Tab. A.12: Aktualisierte Anpassungsergebnisse von zwei unterschiedlichen Korrelationsfunktionen an experimentelle Fundamentalübergänge $\tilde{v}$ in Relation zu auf B3LYP-D3(BJ)/may-cc-pVTZNiveau berechneten harmonischen Wellenzahlen $\omega$ für OH-Streckschwingungen von Alkoholen. MAE ist der Mean Absolute Error, RMSE der Root Mean Square Error und $\left|R_{\max }\right|$ das größte absolute Residuum.

\begin{tabular}{lcc} 
tertiäre Alkohole & $\tilde{v}=\omega+\kappa$ & $\tilde{v}=\lambda \omega$ \\
\hline$\lambda$ & - & $0.9560(2)$ \\
$\kappa / \mathrm{cm}^{-1}$ & $-167.6(6)$ & - \\
$\mathrm{MAE} / \mathrm{cm}^{-1}$ & 1.4 & 1.4 \\
$\mathrm{RMSE} / \mathrm{cm}^{-1}$ & 1.5 & 1.5 \\
$\left|R_{\max }\right| / \mathrm{cm}^{-1}$ & 2.2 & 2.3
\end{tabular}

\begin{tabular}{lcc} 
sekundäre Alkohole & $\tilde{v}=\omega+\kappa$ & $\tilde{v}=\lambda \omega$ \\
\hline$\lambda$ & - & $0.9566(1)$ \\
$\kappa / \mathrm{cm}^{-1}$ & $-165.5(5)$ & - \\
$\mathrm{MAE} / \mathrm{cm}^{-1}$ & 1.6 & 1.6 \\
$\mathrm{RMSE} / \mathrm{cm}^{-1}$ & 1.9 & 1.9 \\
$\left|R_{\max }\right| / \mathrm{cm}^{-1}$ & 3.6 & 3.8
\end{tabular}

\begin{tabular}{lcc} 
primäre Alkohole & $\tilde{v}=\omega+\kappa$ & $\tilde{v}=\lambda \omega$ \\
\hline$\lambda$ & - & $0.9583(2)$ \\
$\kappa / \mathrm{cm}^{-1}$ & $-159.5(5)$ & - \\
$\mathrm{MAE} / \mathrm{cm}^{-1}$ & 1.7 & 1.7 \\
$\mathrm{RMSE} / \mathrm{cm}^{-1}$ & 1.9 & 2.1 \\
$\mid R_{\max } / / \mathrm{cm}^{-1}$ & 3.6 & 3.6 \\
Methanol & & \\
\hline$\lambda$ & $\tilde{v}=\omega+\kappa$ & $\tilde{v}=\lambda \omega$ \\
$\kappa / \mathrm{cm}^{-1}$ & -149.4 & 0.9610
\end{tabular}

\begin{tabular}{lcc} 
alle Alkohole gemeinsam & $\tilde{v}=\omega+\kappa$ & $\tilde{v}=\lambda \omega$ \\
\hline$\lambda$ & - & $0.9572(2)$ \\
$\kappa / \mathrm{cm}^{-1}$ & $-163.4(7)$ & - \\
$\mathrm{MAE} / \mathrm{cm}^{-1}$ & 3.5 & 3.8 \\
$\mathrm{RMSE} / \mathrm{cm}^{-1}$ & 4.3 & 4.6 \\
$\mid R_{\max } / \mathrm{cm}^{-1}$ & 14.0 & 14.7 \\
\hline
\end{tabular}


Tab. A.13: Experimentelle Bandenpositionen für $\mathrm{OH}-$ Streckschwingungs-Fundamentalübergänge von Dimeren aus enantiomerenreinen Alkoholen und $\alpha$-Pinen.

\begin{tabular}{lll}
\hline Alkohol & $\alpha$-Pinen Enantiomer & $\tilde{v} / \mathrm{cm}^{-1}$ \\
\hline (-)-Borneol & $(-)-\alpha$-Pinen & 3567 \\
$(-)$-Borneol & $(+)-\alpha$-Pinen & 3577 \\
\hline$(+)-\alpha$-Fenchol & $(-)-\alpha$-Pinen & 3563 \\
$(+)-\alpha$-Fenchol & $(+)-\alpha$-Pinen & 3561,3550 \\
\hline (-)-Isopinocampheol & $(-)-\alpha$-Pinen & 3564 \\
$(-)-$ Isopinocampheol & $(+)-\alpha$-Pinen & 3563 \\
\hline$(-)-1-P h e n y l e t h a n o l$ & $(-)-\alpha$-Pinen & 3538 \\
$(-)-1-P h e n y l e t h a n o l$ & $(+)-\alpha$-Pinen & 3537 \\
\hline$(+)-2-B u t a n o l$ & $(-)-\alpha$-Pinen & $3580,3573,3566,3540$ \\
$(+)-2-B u t a n o l$ & $(+)-\alpha$-Pinen & $3573,3565,3560,3545,3539$ \\
\hline
\end{tabular}

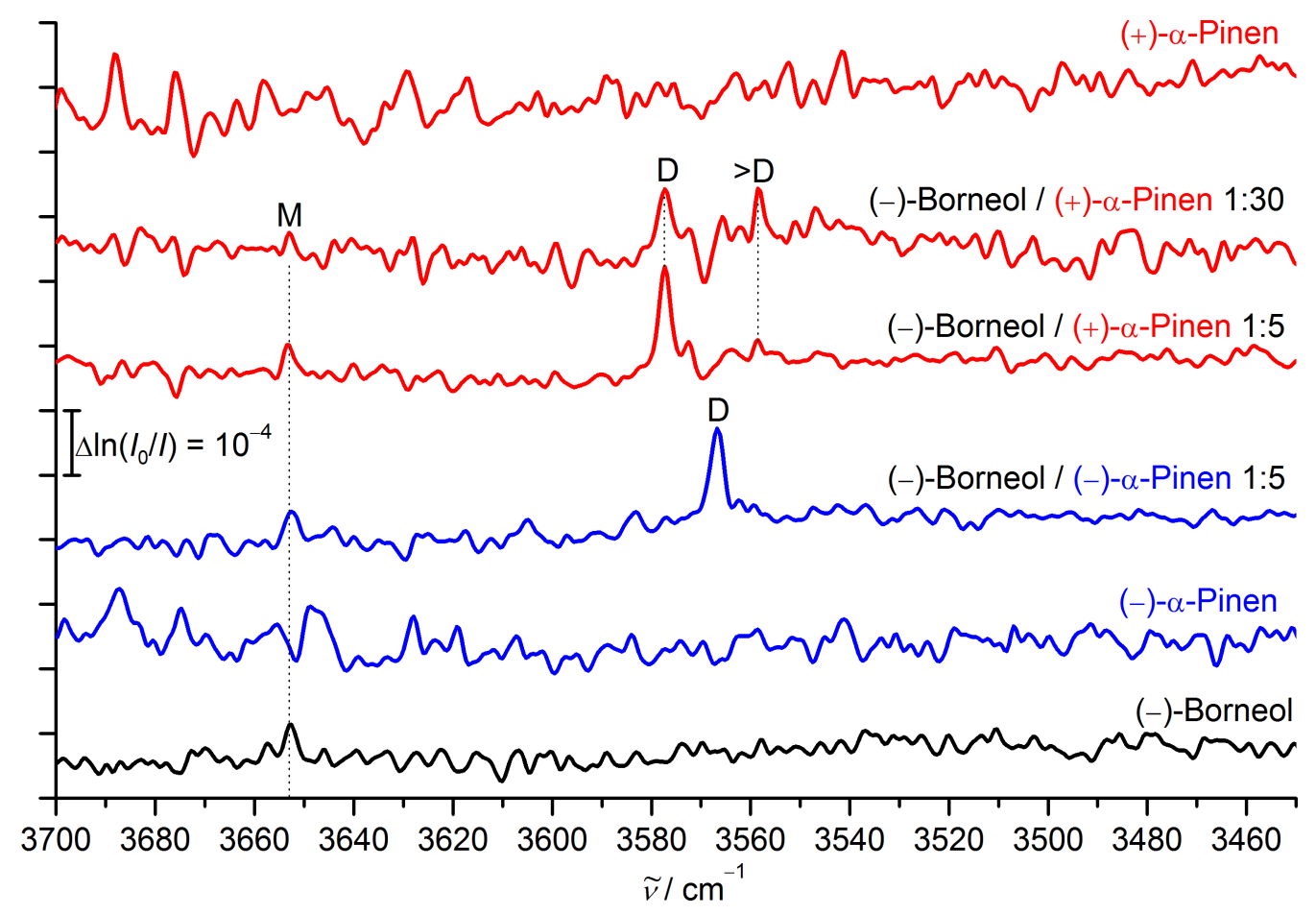

Abb. A.3: Filet-Jet-Spektren von (-)-Borneol / $\alpha$-Pinen bei verschiedenen Mischungsverhältnissen mit zugeordneten Clustergrößen: Monomer (M), Dimer (D) und größer als Dimer ( $>$ D). Das verstärkte Rauschen in den Kontrollspektren der reinen $\alpha$-Pinen-Enantiomere ist auf eine geringere Wiederholungszahl zurückzuführen. Mit Anpassungen übernommen aus Ref. [4] mit Erlaubnis von John Wiley and Sons. 


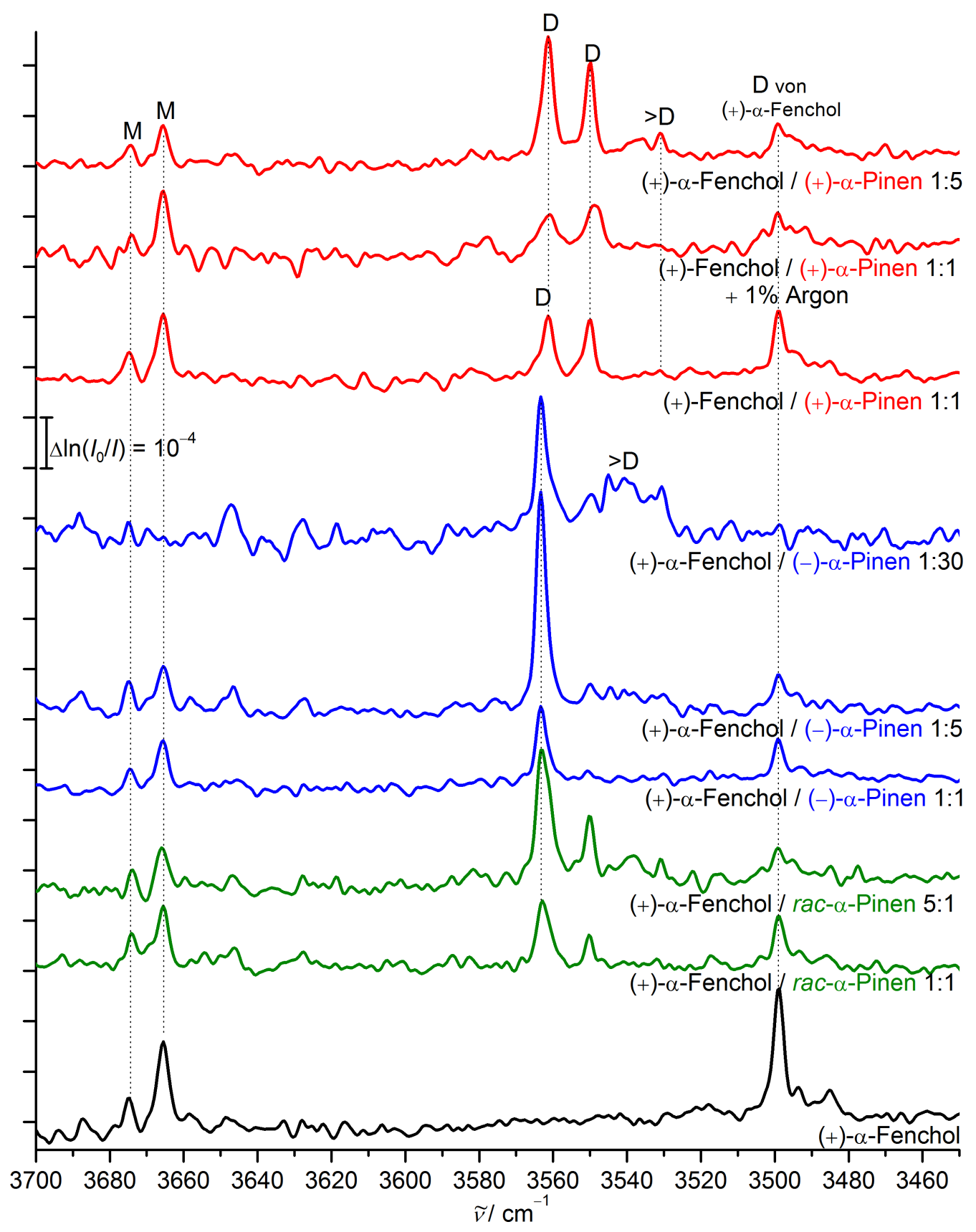

Abb. A.4: Filet-Jet-Spektren von (+)-Fenchol / $\alpha$-Pinen bei verschiedenen Mischungsverhältnissen mit zugeordneten Clustergrößen: Monomer (M), Dimer (D) und größer als Dimer ( $>$ D). Mit Anpassungen übernommen aus Ref. [4] mit Erlaubnis von John Wiley and Sons. 


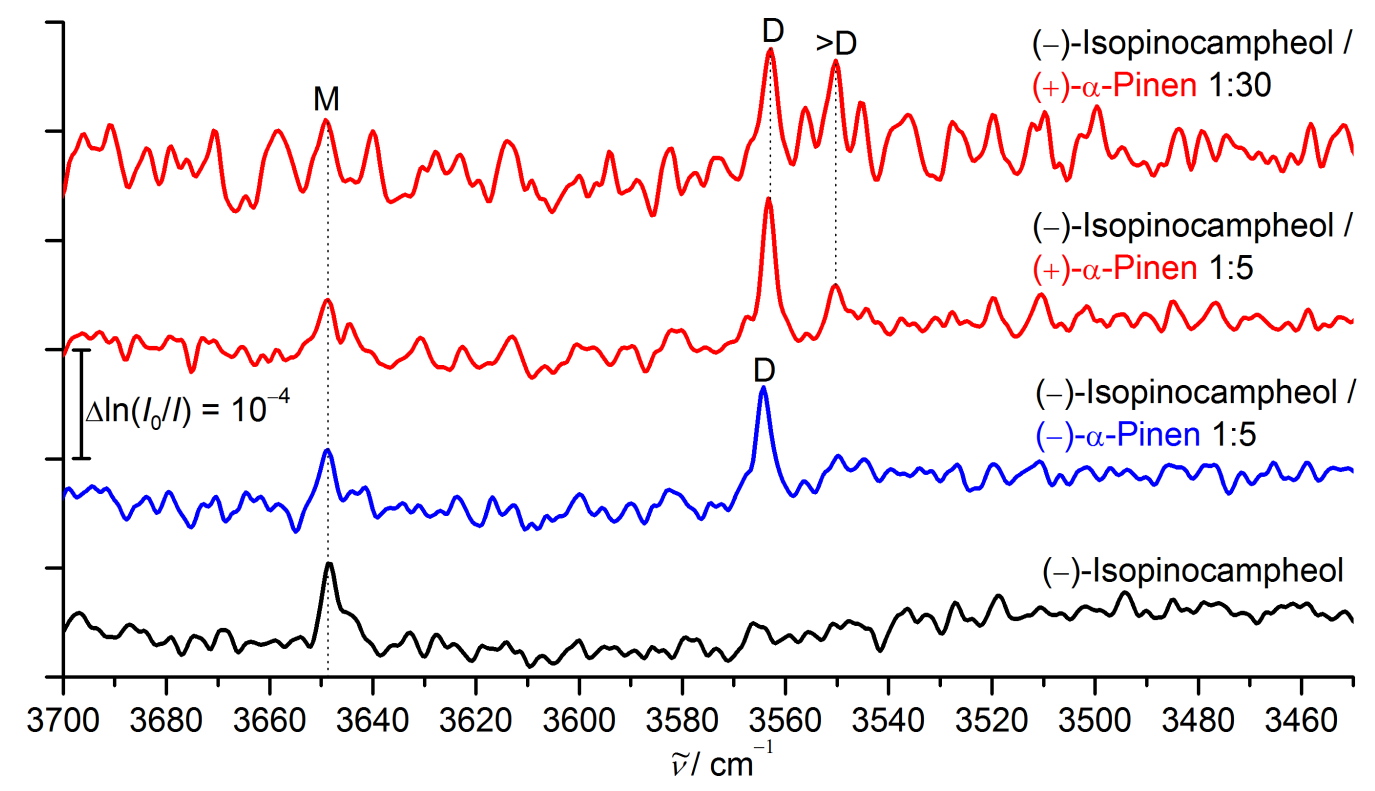

Abb. A.5: Filet-Jet-Spektren von (-)-Isopinocampheol / $\alpha$-Pinen bei verschiedenen Mischungsverhältnissen mit zugeordneten Clustergrößen: Monomer (M), Dimer (D) und größer als Dimer (>D). Mit Anpassungen übernommen aus Ref. [4] mit Erlaubnis von John Wiley and Sons.

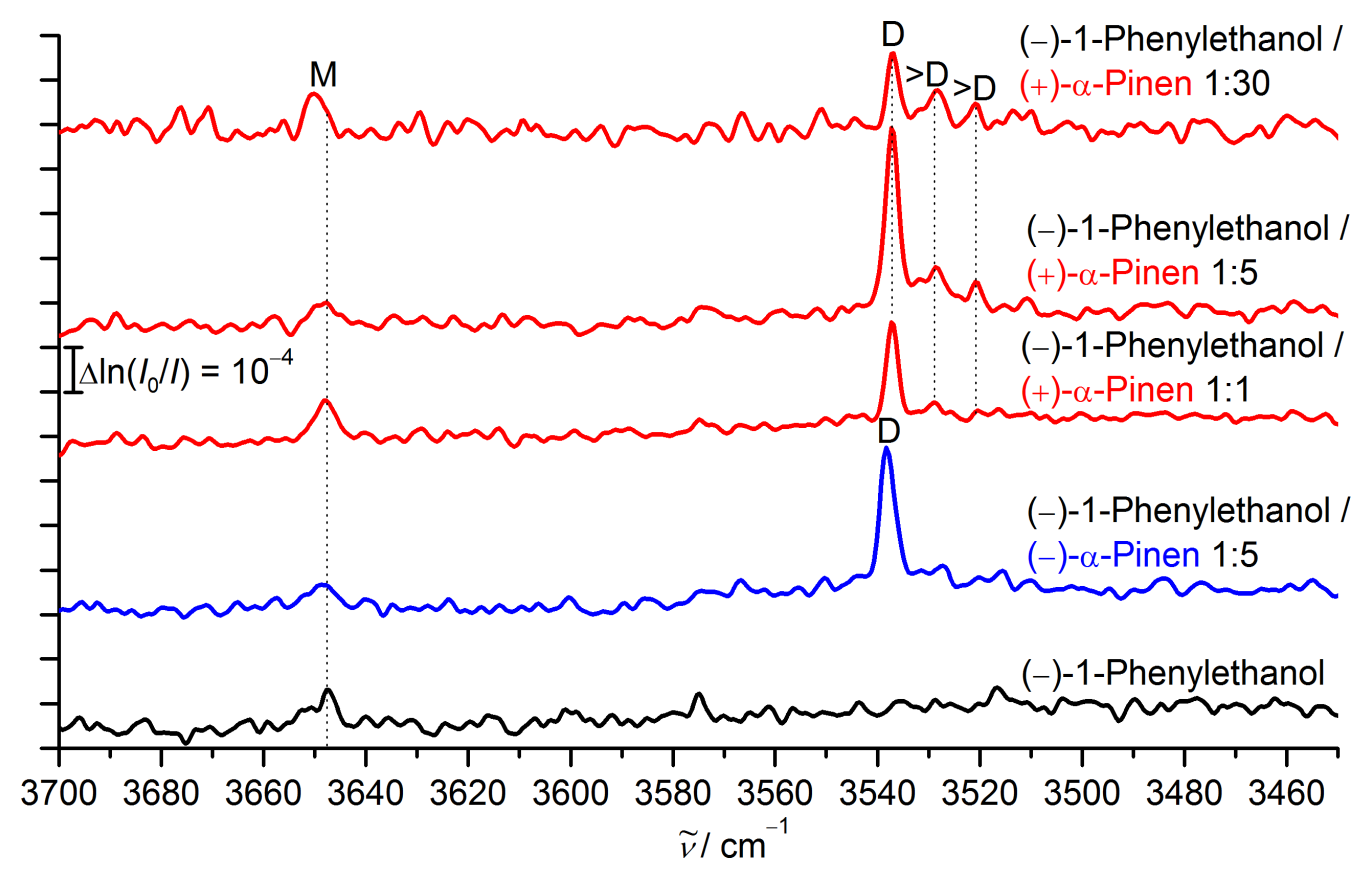

Abb. A.6: Filet-Jet-Spektren von (-)-1-Phenylethanol / $\alpha$-Pinen bei verschiedenen Mischungsverhältnissen mit zugeordneten Clustergrößen: Monomer (M), Dimer (D) und größer als Dimer (>D). Mit Anpassungen übernommen aus Ref. [4] mit Erlaubnis von John Wiley and Sons. 


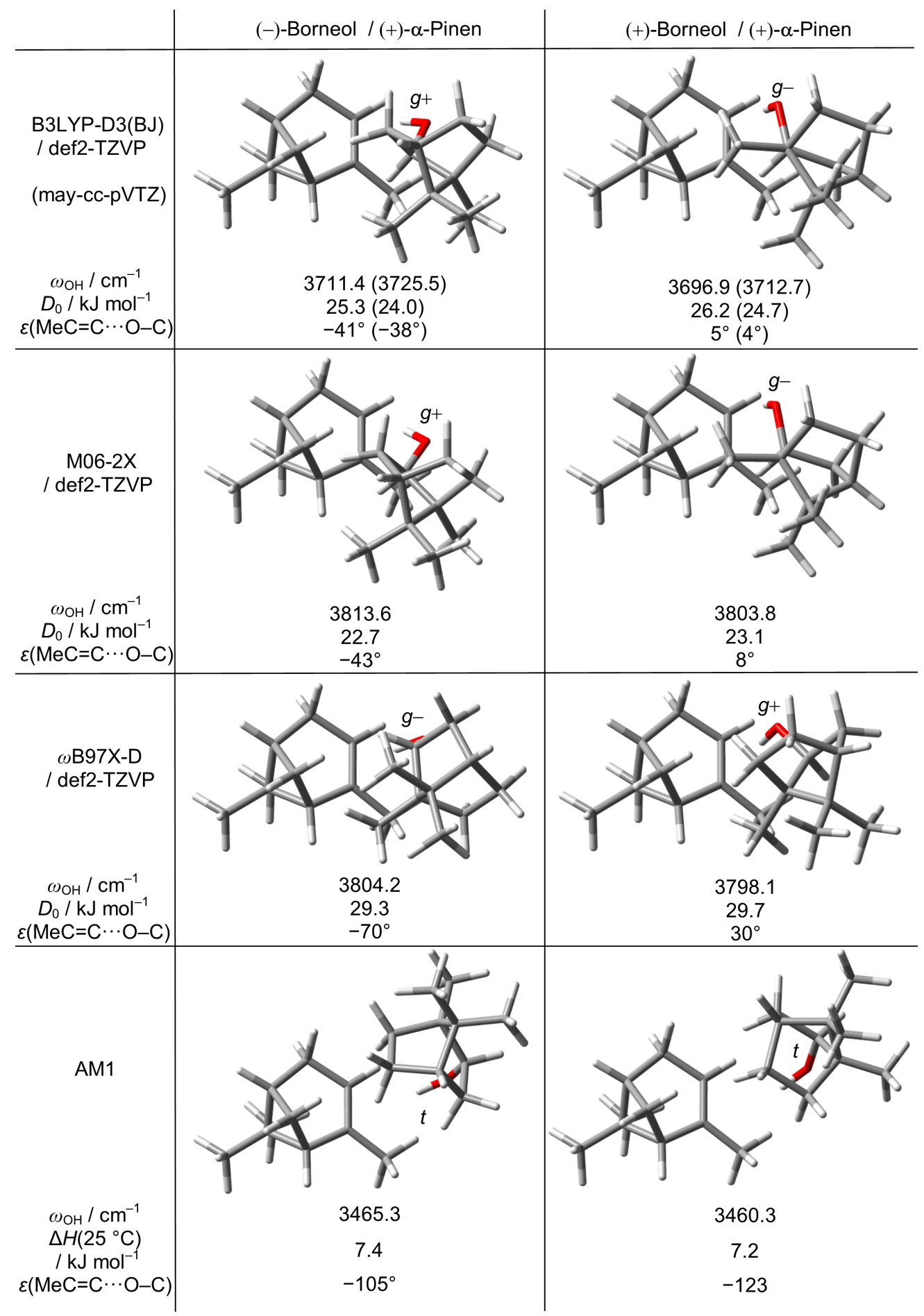

Abb. A.7: Strukturen, OH-Streckschwingungswellenzahlen und Dissoziationsenergien (relativ zu den jeweils stabilsten Monomeren), $\mathrm{MeC}=\mathrm{C} \cdots \mathrm{O}-\mathrm{C}-$ und $\mathrm{H}-\mathrm{C}-\mathrm{O}-\mathrm{H}$-Diederwinkel (letztere vereinfacht als $g-, g+, t$ ) für die globalen Minima von Borneol / $(+)-\alpha$-Pinen Dimeren gemäß verschiedenen quantenchemischen Methoden. B3LYP-D3(BJ)/may-cc-pVTZ Strukturen sind nahezu identisch mit denen aus B3LYP-D3(BJ)/def2-TZVP, die zugehörigen Werte sind in Klammern angegeben. Für bessere Vergleichbarkeit sind alle Komplexe mit dem (+)-Enantiomer von $\alpha$-Pinen mit identischer Ausrichtung gezeigt. Die teilweise davon abweichenden experimentellen Kombinationen mit einheitlichem Alkohol-Enantiomer können durch Spiegelung erhalten werden, wobei alle Diederwinkel ihr Vorzeichen ändert. Mit Anpassungen übernommen aus Ref. [4] mit Erlaubnis von John Wiley and Sons. 


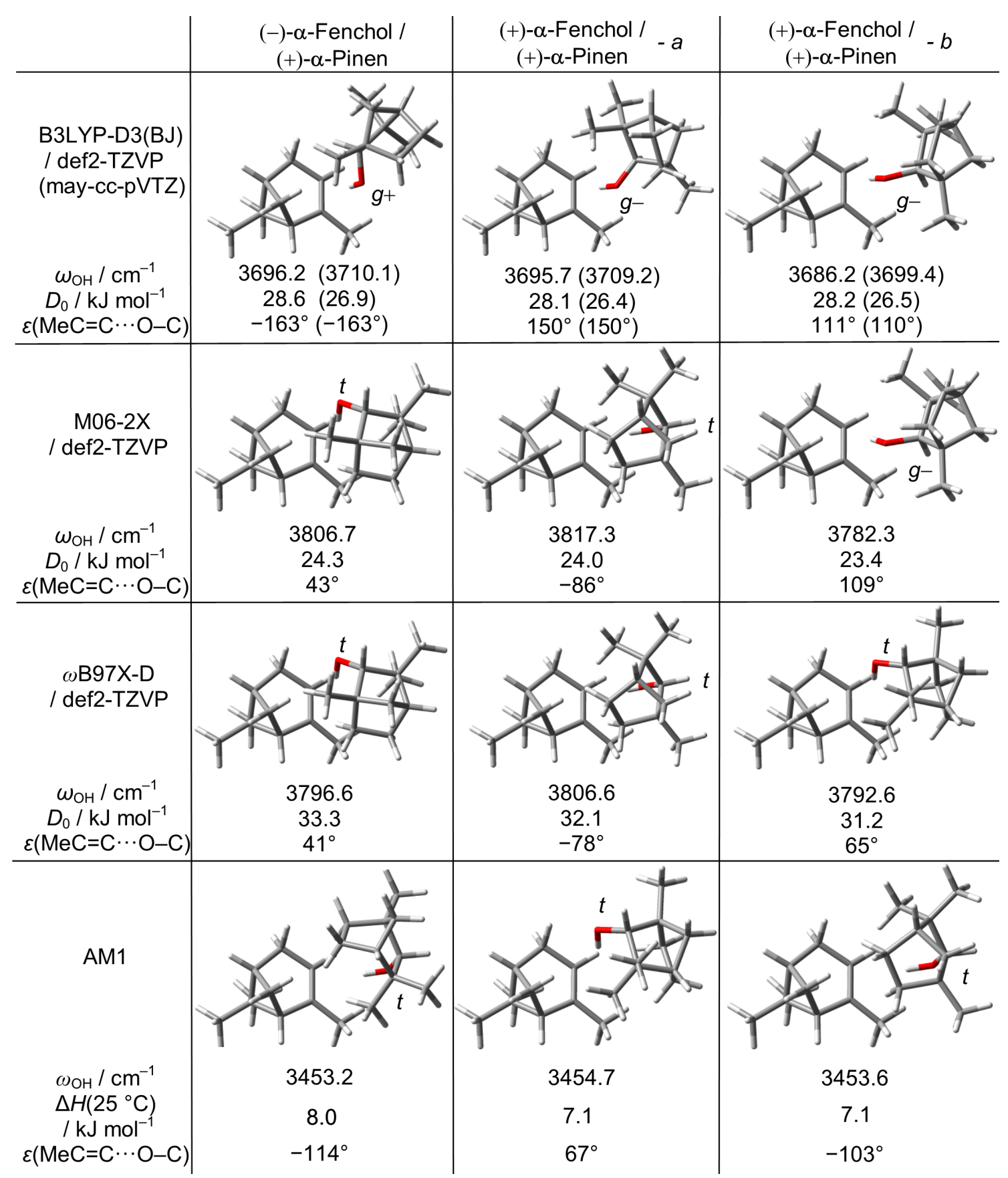

Abb. A.8: Strukturen, $\mathrm{OH}-$ Streckschwingungswellenzahlen und Dissoziationsenergien (relativ zu den jeweils stabilsten Monomeren), $\mathrm{MeC}=\mathrm{C} \cdots \mathrm{O}-\mathrm{C}-$ und $\mathrm{H}-\mathrm{C}-\mathrm{O}-\mathrm{H}-\mathrm{Diederwinkel}$ (letztere vereinfacht als $g-, g+, t$ ) für die globalen Minima von $\alpha$-Fenchol $/(+)$ - $\alpha$-Pinen Dimeren gemäß verschiedenen quantenchemischen Methoden. B3LYP-D3(BJ)/may-cc-pVTZ Strukturen sind nahezu identisch mit denen aus B3LYP-D3(BJ)/def2-TZVP, die zugehörigen Werte sind in Klammern angegeben. Für bessere Vergleichbarkeit sind alle Komplexe mit dem (+)-Enantiomer von $\alpha$-Pinen mit identischer Ausrichtung gezeigt. Die teilweise davon abweichenden experimentellen Kombinationen mit einheitlichem Alkohol-Enantiomer können durch Spiegelung erhalten werden, wobei alle Diederwinkel ihr Vorzeichen ändert. Mit Anpassungen übernommen aus Ref. [4] mit Erlaubnis von John Wiley and Sons. 


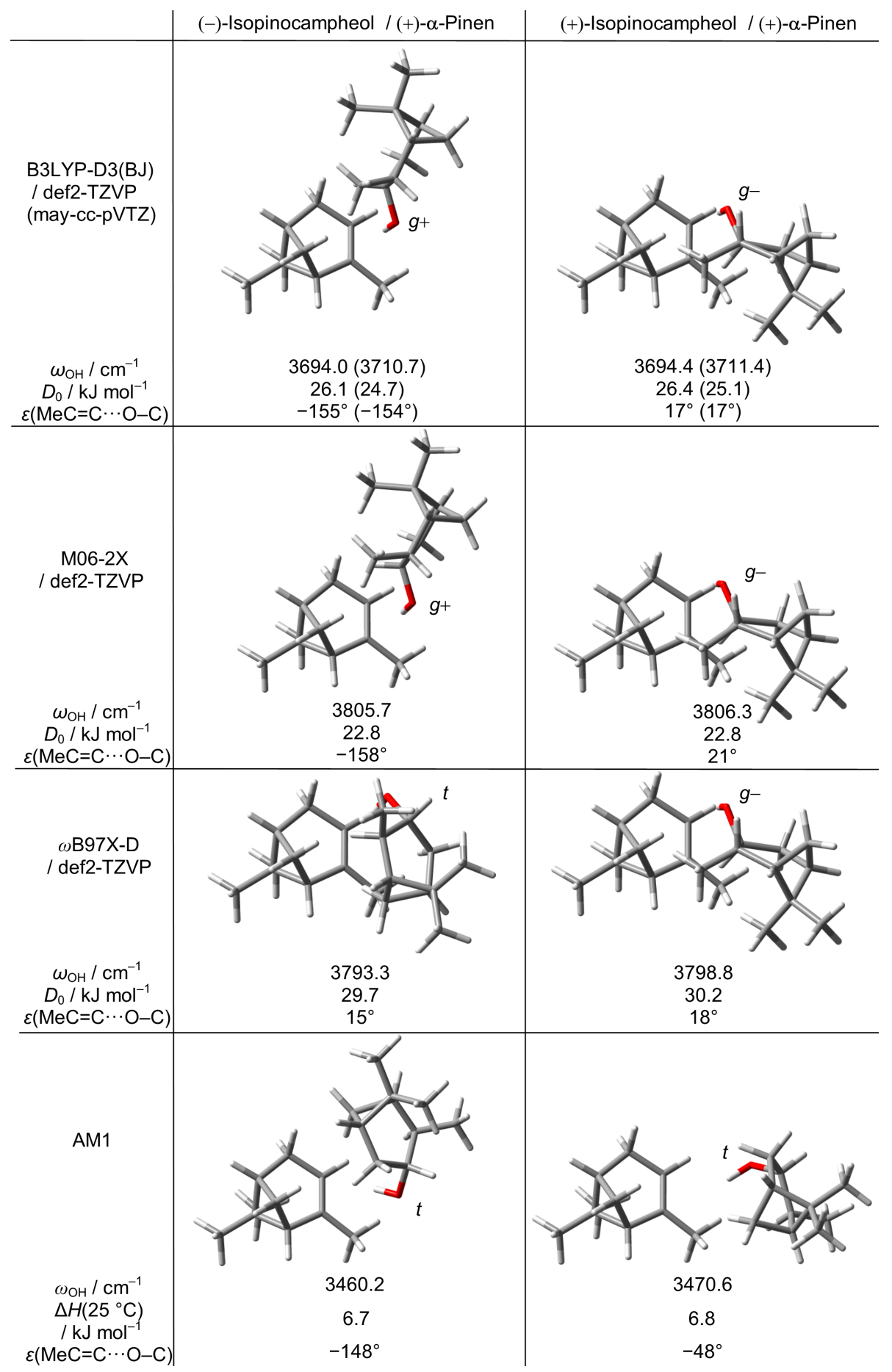

Abb. A.9: Strukturen, $\mathrm{OH}-$ Streckschwingungswellenzahlen und Dissoziationsenergien (relativ zu den jeweils stabilsten Monomeren), $\mathrm{MeC}=\mathrm{C} \cdots \mathrm{O}-\mathrm{C}-$ und $\mathrm{H}-\mathrm{C}-\mathrm{O}-\mathrm{H}-\mathrm{Diederwinkel} \mathrm{(letztere} \mathrm{ver-}$ einfacht als $g-, g+, t$ ) für die globalen Minima von Isopinocampheol / (+)- $\alpha$-Pinen Dimeren gemäß verschiedenen quantenchemischen Methoden. B3LYP-D3(BJ)/may-cc-pVTZ Strukturen sind nahezu identisch mit denen aus B3LYP-D3(BJ)/def2-TZVP, die zugehörigen Werte sind in Klammern angegeben. Für bessere Vergleichbarkeit sind alle Komplexe mit dem (+)-Enantiomer von $\alpha$-Pinen mit identischer Ausrichtung gezeigt. Die teilweise davon abweichenden experimentellen Kombinationen mit einheitlichem Alkohol-Enantiomer können durch Spiegelung erhalten werden, wobei alle Diederwinkel ihr Vorzeichen ändert. Mit Anpassungen übernommen aus Ref. [4] mit Erlaubnis von John Wiley and Sons. 


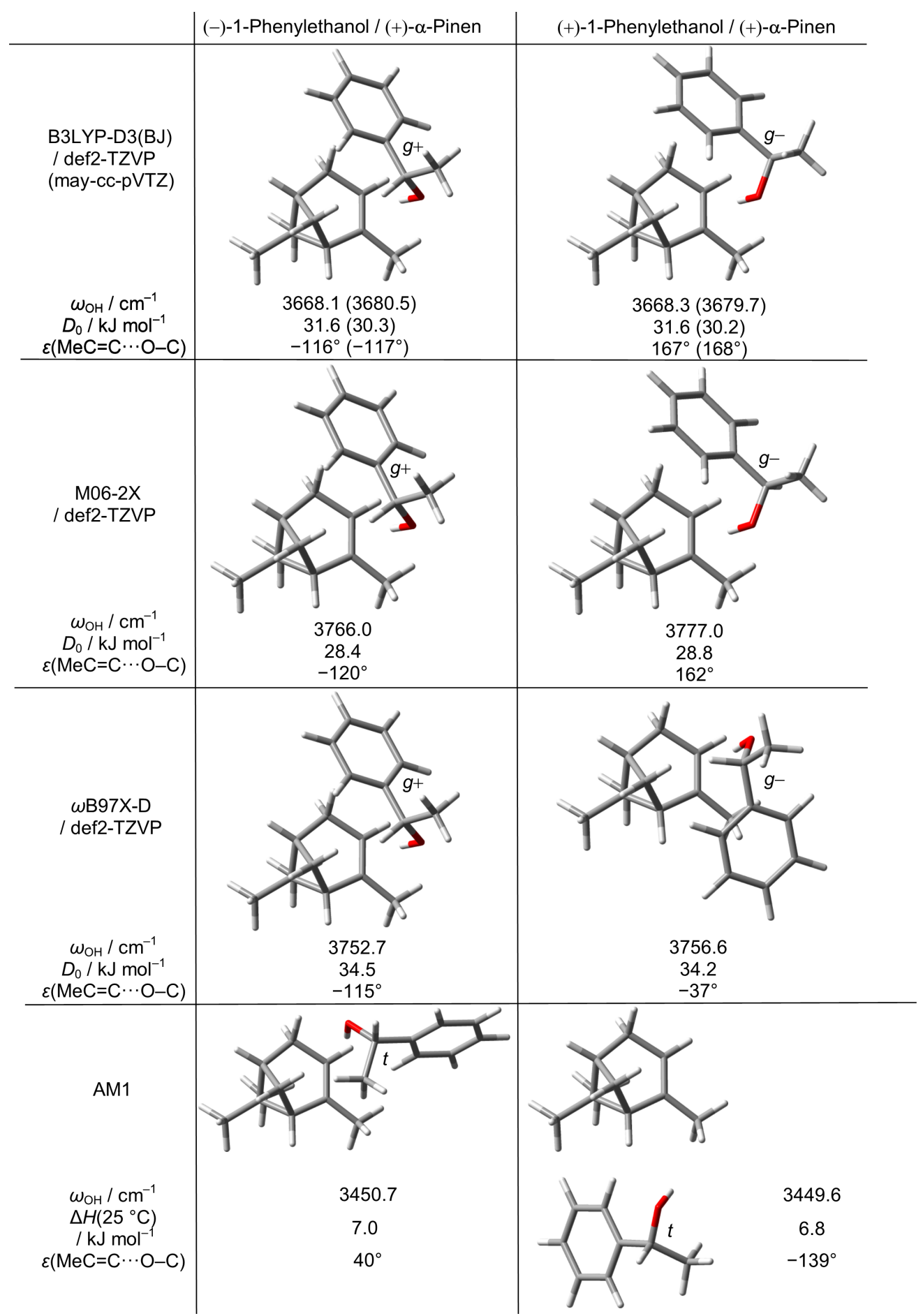

Abb. A.10: Strukturen, OH-Streckschwingungswellenzahlen und Dissoziationsenergien (relativ zu den jeweils stabilsten Monomeren), $\mathrm{MeC}=\mathrm{C} \cdots \mathrm{O}-\mathrm{C}-$ und $\mathrm{H}-\mathrm{C}-\mathrm{O}-\mathrm{H}-$ Diederwinkel (letztere vereinfacht als $g-, g+, t)$ für die globalen Minima von 1-Phenylethanol / (+)- $\alpha$-Pinen Dimeren gemäß verschiedenen quantenchemischen Methoden. B3LYP-D3(BJ)/may-cc-pVTZ Strukturen sind nahezu identisch mit denen aus B3LYP-D3(BJ)/def2-TZVP, die zugehörigen Werte sind in Klammern angegeben. Für bessere Vergleichbarkeit sind alle Komplexe mit dem (+)-Enantiomer von $\alpha$-Pinen mit identischer Ausrichtung gezeigt. Die teilweise davon abweichenden experimentellen Kombinationen mit einheitlichem Alkohol-Enantiomer können durch Spiegelung erhalten werden, wobei alle Diederwinkel ihr Vorzeichen ändert. Mit Anpassungen übernommen aus Ref. [4] mit Erlaubnis von John Wiley and Sons. 


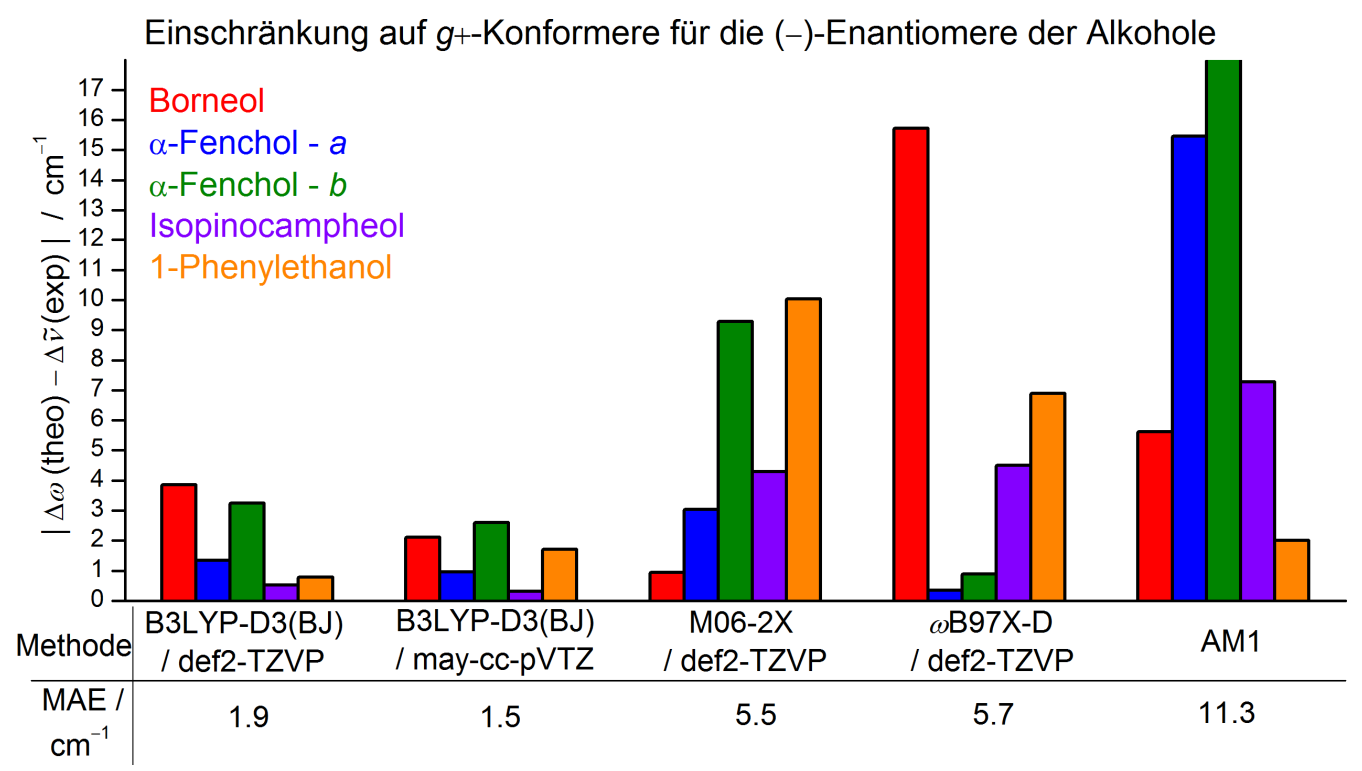

Abb. A.11: Absolute Abweichungen zwischen berechneten und experimentellen Verschiebungen der OH-Streckschwingungswellenzahlen bei Wechsel der relativen Chiralität in gemischten Dimeren zwischen Alkoholen und $\alpha$-Pinen. Die Auswahl wurde dabei eingeschränkt auf die stabilsten Dimere mit g+-Konformer der (-)-Alkohole, wie es von B3LYP-D3(BJ) vorhergesagt wird. Mit Anpassungen übernommen aus Ref. [4] mit Erlaubnis von John Wiley and Sons.

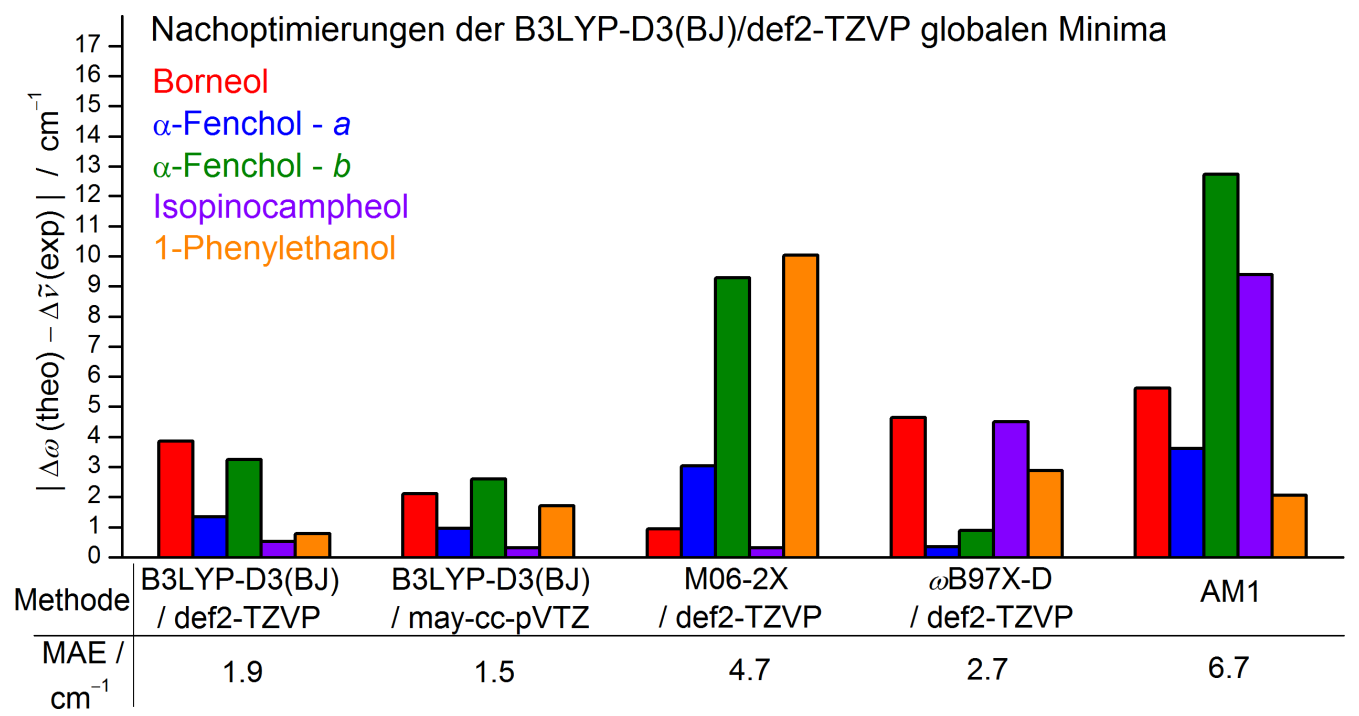

Abb. A.12: Absolute Abweichungen zwischen berechneten und experimentellen Verschiebungen der OH-Streckschwingungswellenzahlen bei Wechsel der relativen Chiralität in gemischten Dimeren zwischen Alkoholen und $\alpha$-Pinen. In diesem Fall wurden nur direkte Nachoptimierungen der B3LYP-D3(BJ)/def2-TZVP Minima berücksichtigt. Mit Anpassungen übernommen aus Ref. [4] mit Erlaubnis von John Wiley and Sons. 


\section{A Anhang}

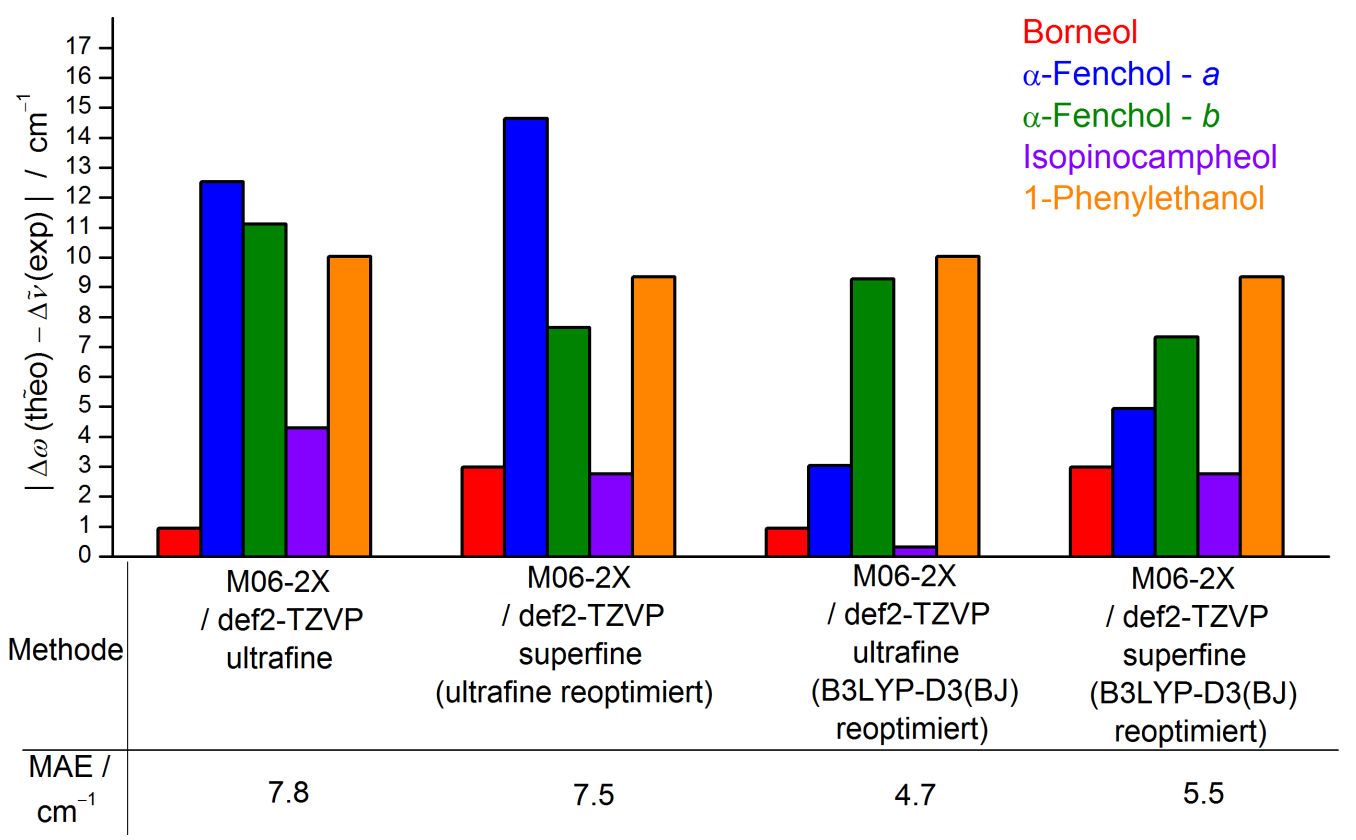

Abb. A.13: Absolute Abweichungen zwischen berechneten und experimentellen Verschiebungen der OH-Streckschwingungswellenzahlen bei Wechsel der relativen Chiralität in gemischten Dimeren zwischen Alkoholen und $\alpha$-Pinen. Vergleich zwischen unterschiedlich feinen Integrationsgittern für M06-2X/def2-TZVP. Mit Anpassungen übernommen aus Ref. [4] mit Erlaubnis von John Wiley and Sons.

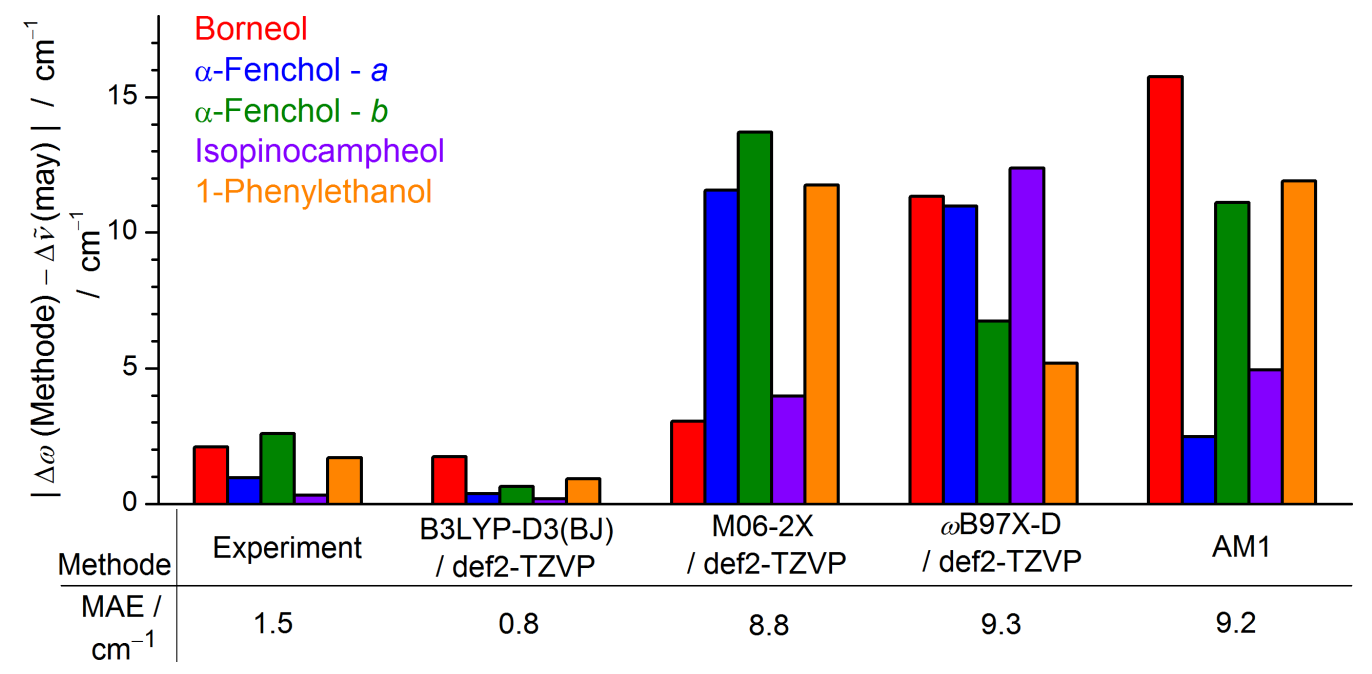

Abb. A.14: Absolute Abweichungen zwischen verschiedenen Methoden mit B3LYP-D3(BJ)/maycc-pVTZ als Referenz für Verschiebungen der OH-Streckschwingungswellenzahlen bei Wechsel der relativen Chiralität in gemischten Dimeren zwischen Alkoholen und $\alpha$-Pinen. Mit Anpassungen übernommen aus Ref. [4] mit Erlaubnis von John Wiley and Sons. 


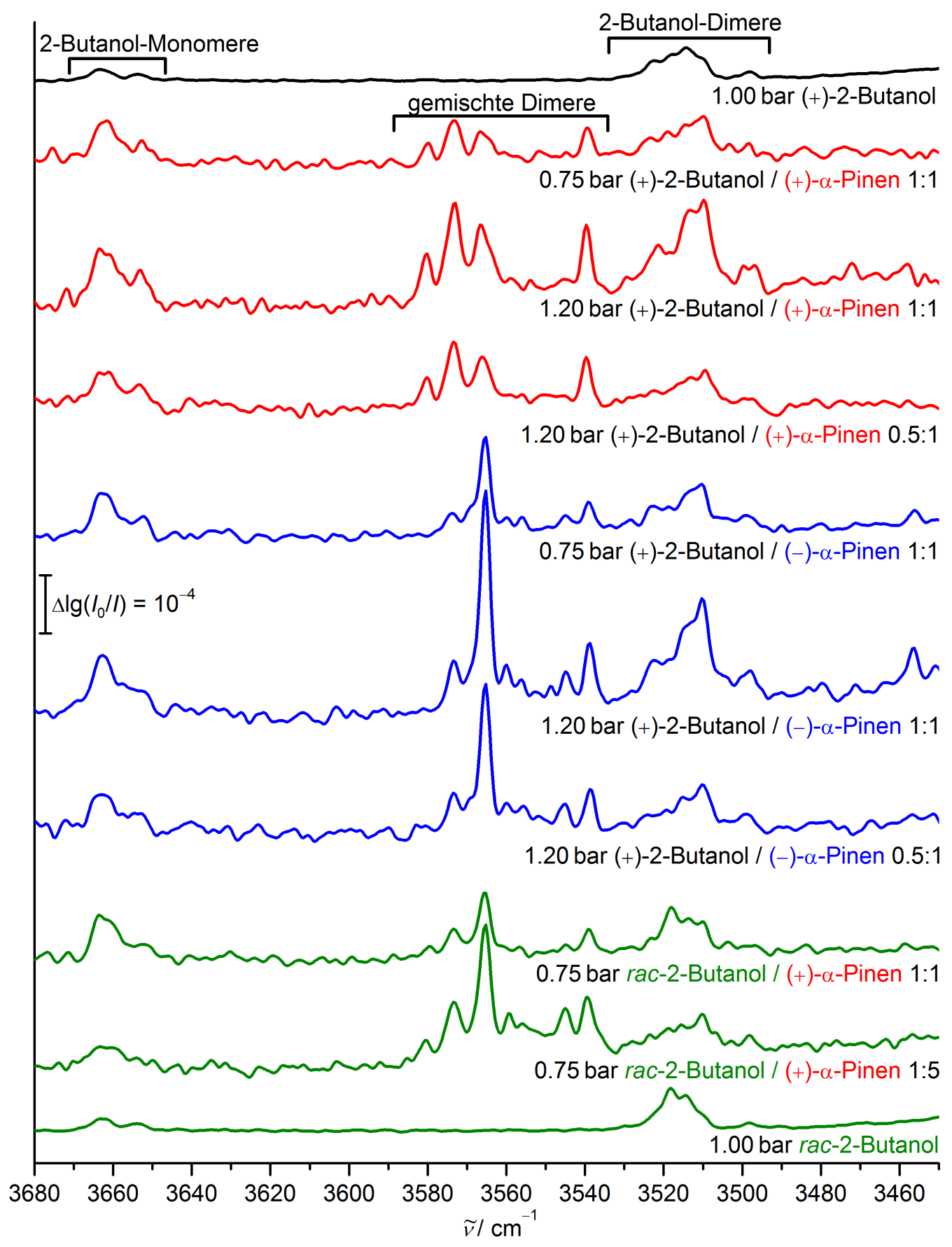

Abb. A.15: Filet-Jet-Spektren von 2-Butanol / $\alpha$-Pinen bei verschiedenen EnantiomerenKombinationen, Mischungsverhältnissen und Stagnationsdrücken mit Zuordnungen der Spektralbereiche. Ragout-Jet-Spektren von reinem 2-Butanol wurden aus Ref. [322] übernommen. 
Tab. A.14: Schwingungsnullpunktskorrigierte Energien von Konformeren von BenzylalkoholDimeren, jeweils relativ zur Struktur $\mathrm{homO}^{g} \pi$ in $\mathrm{kJ} \mathrm{mol}^{-1}$, gemäß verschiedenen DFTFunktionalen, jeweils mit may-cc-pVTZ Basissatz.

\begin{tabular}{lrcc|ccc}
\hline & \multicolumn{3}{c}{ homochiral } & \multicolumn{3}{c}{ heterochiral } \\
Methode & $\pi \pi$ & $\mathrm{O}^{g} \pi$ & $\mathrm{O}^{t} \pi$ & $\pi \pi$ & $\mathrm{O}^{g} \pi$ & $\mathrm{O}^{t} \pi$ \\
\hline B3LYP-D3(BJ) & 2.0 & 0 & 3.2 & 1.6 & 0.2 & 4.8 \\
B2PLYP-D3(BJ) & 2.5 & 0 & 3.3 & 2.2 & 0.1 & 5.0 \\
$\omega$ B97X-D & 1.3 & 0 & 3.2 & -0.04 & 1.7 & 4.3 \\
M06-2X & -2.7 & 0 & 3.7 & -2.2 & 2.8 & 3.8 \\
\hline
\end{tabular}

Tab. A.15: Harmonische OH-Streckschwingungswellenzahl des $g$-Konformers von Benzylalkohol und Tiefverschiebungen von Benzylalkohol-Dimeren relativ hierzu in $\mathrm{cm}^{-1}$ gemäß verschiedenen DFT-Funktionalen, jeweils mit may-cc-pVTZ Basissatz.

\begin{tabular}{lc|ccc|ccc}
\hline & & \multicolumn{3}{|c}{ homochiral } & \multicolumn{3}{c}{ heterochiral } \\
Methode & $g$ & $\pi \pi$ & $\mathrm{O}^{g} \pi$ & $\mathrm{O}^{t} \pi$ & $\pi \pi$ & $\mathrm{O}^{g} \pi$ & $\mathrm{O}^{t} \pi$ \\
\hline B3LYP-D3(BJ) & 3809 & $56 / 59$ & $53 / 170$ & $91 / 158$ & $58 / 63$ & $68 / 184$ & $89 / 145$ \\
B2PLYP-D3(BJ) & 3827 & $55 / 59$ & $57 / 163$ & $95 / 154$ & $56 / 60$ & $72 / 177$ & $92 / 172$ \\
$\omega$ B97X-D & 3895 & $45 / 49$ & $54 / 169$ & $93 / 143$ & $47 / 51$ & $72 / 176$ & $89 / 139$ \\
M06-2X & 3875 & $31 / 35$ & $59 / 128$ & $81 / 113$ & $30 / 35$ & $66 / 133$ & $84 / 114$ \\
\hline
\end{tabular}

Tab. A.16: Experimentelle Bandenpositionen und Zuordnungen im Benzylalkohol-System.

\begin{tabular}{cl}
\hline$\tilde{v} / \mathrm{cm}^{-1}$ & Zuordnung \\
\hline 3648 & Monomer $g$ \\
3598 & Dimer het $\pi \pi(+$ hom $\pi \pi ?)$ antisymmetrisch \\
3594 & Dimer het $\pi \pi(+$ hom $\pi \pi ?)$ symmetrisch \\
3582 & Dimer homO $^{g} \pi(\mathrm{OH} \cdots \pi)$ \\
3576 & Dimer hetO $^{g} \pi(\mathrm{OH} \cdots \pi)$ \\
3561 & Trimer \\
3555 & $?$ \\
3547 & Trimer? \\
3538 & Trimer? \\
3514 & Dimer homO \\
3504 & Dimer hetO ${ }^{g} \pi(\mathrm{OH} \cdots \mathrm{O} \cdots)$ \\
3441 & Trimer \\
3433 & Trimer \\
3426 & Trimer \\
3517 & Trimer \\
3374 & Trimer \\
3364 & $?$ \\
3347 & $>$ Trimer \\
3333 & $>$ Trimer \\
$3330-3250$ & $>$ Trimer \\
\hline &
\end{tabular}


Tab. A.17: Schwingungsnullpunktskorrigierte Energien von Konformeren von 1-PhenylethanolDimeren, jeweils relativ zur Struktur homO ${ }^{g} \pi$ in $\mathrm{kJ} \mathrm{mol}^{-1}$, gemäß verschiedenen DFTFunktionalen, jeweils mit may-cc-pVTZ Basissatz.

\begin{tabular}{lrcc|rcc}
\hline & \multicolumn{3}{c}{ homochiral } & \multicolumn{3}{c}{ heterochiral } \\
Methode & $\pi \pi$ & $\mathrm{O}^{g} \pi$ & $\mathrm{O}^{t} \pi$ & $\pi \pi$ & $\mathrm{O}^{g} \pi$ & $\mathrm{O}^{t} \pi$ \\
\hline B3LYP-D3(BJ) & 1.0 & 0 & 1.9 & 2.0 & 0.9 & 2.7 \\
B2PLYP-D3(BJ) & 1.1 & 0 & 1.9 & 1.9 & 0.9 & 2.6 \\
$\omega$ B97X-D & 0.9 & 0 & 2.7 & 1.2 & 2.5 & 2.9 \\
M06-2X & -2.0 & 0 & 2.2 & -1.7 & 2.1 & 2.0 \\
\hline
\end{tabular}

Tab. A.18: Harmonische OH-Streckschwingungswellenzahl des $g$-Konformers von 1Phenylethanol und Tiefverschiebungen von 1-Phenylethanol-Dimeren relativ hierzu in $\mathrm{cm}^{-1}$ gemäß verschiedenen DFT-Funktionalen, jeweils mit may-cc-pVTZ Basissatz.

\begin{tabular}{lc|ccc|ccc}
\hline & & \multicolumn{3}{|c}{ homochiral } & \multicolumn{3}{c}{ heterochiral } \\
Methode & $g$ & $\pi \pi$ & $\mathrm{O}^{g} \pi$ & $\mathrm{O}^{t} \pi$ & $\pi \pi$ & $\mathrm{O}^{g} \pi$ & $\mathrm{O}^{t} \pi$ \\
\hline B3LYP-D3(BJ) & 3813 & $52 / 56$ & $62 / 164$ & $87 / 164$ & $58 / 63$ & $70 / 177$ & $84 / 159$ \\
B2PLYP-D3(BJ) & 3827 & $53 / 57$ & $68 / 156$ & $92 / 159$ & $56 / 61$ & $76 / 168$ & $89 / 153$ \\
$\omega$ B97X-D & 3897 & $42 / 46$ & $61 / 164$ & $90 / 152$ & $51 / 55$ & $74 / 174$ & $87 / 151$ \\
M06-2X & 3877 & $41 / 45$ & $59 / 121$ & $81 / 119$ & $30 / 34$ & $63 / 130$ & $76 / 120$ \\
\hline
\end{tabular}


Tab. A.19: Experimentelle Bandenpositionen und Zuordnungen im 1-Phenylethanol-System.

\begin{tabular}{ll}
\hline$\tilde{v} / \mathrm{cm}^{-1}$ & Zuordnung \\
\hline 3648 & Monomer $g$ \\
3597 & Dimer het $\pi \pi$ antisymmetrisch \\
3592 & Dimer het $\pi \pi$ symmetrisch \\
3592 & Dimer hom $\pi \pi$ symmetrisch \\
3575 & Dimer homO $^{g} \pi(\mathrm{OH} \cdots \pi)$ \\
3573 & Dimer hetO ${ }^{g} \pi(\mathrm{OH} \cdots \pi)$ \\
3568 & ? hom \\
3564 & ? hom \\
3559 & ? het \\
3557 & Trimer? hom \\
3552 & ? het \\
3545 & ? hom \\
3540 & ? het \\
3516 & Dimer homO ${ }^{g} \pi(\mathrm{OH} \cdots \mathrm{O})$ \\
3514 & Dimer hetO $\pi(\mathrm{OH} \cdots \mathrm{O})$ ? \\
3507 & Dimer homO ${ }^{t} \pi(\mathrm{OH} \cdots \mathrm{O}) ?$ \\
3506 & Dimer hetO ${ }^{g} \pi(\mathrm{OH} \cdots \mathrm{O})$ \\
3440 & Trimer? het \\
3417 & Trimer? hom \\
3403 & Trimer? het \\
3378 & Trimer? hom \\
3369 & Trimer? het \\
3356 & Trimer? hom \\
3349 & Trimer? het \\
3274 & Tetramer? hom \\
\hline &
\end{tabular}




\section{Über den Autor}

\section{Persönliche Daten}

$\begin{array}{ll}\text { Vorname } & \text { Robert } \\ \text { Nachname } & \text { Medel } \\ \text { Geburtsort } & \text { Leipzig, Deutschland }\end{array}$

\section{Ausbildung}

2007

Abitur, Bismarckschule Hannover

10/2007-08/2010 Bachelor of Science in Chemie ,mit Auszeichnung“, Georg-AugustUniversität Göttingen, Bachelorarbeit: „Intramolekulare Dynamik an rigiden Farbstoffsonden"

10/2010-03/2014 Master of Science in Chemie, Georg-August-Universität Göttingen, Masterarbeit: „Infrarotspektroskopie von Alkohol-AlkenKomplexen: konkurrierende schwache Wechselwirkungen“"

07/2014-06/2020 Promotionsstudium, Georg-August University School of Science (GAUSS), Grundprogramm Chemie, Georg-August-Universität Göttingen

\section{Publikationen}

Folgende Publikationen sind während der Promotion entstanden:

- R. Medel, M. Heger, M. A. Suhm, Molecular Docking via Olefinic OH $\cdots \pi$ Interactions: A Bulky Alkene Model System and Its Cooperativity, J. Phys. Chem. A 2015, 119, 1723-1730.

- R. Medel, C. Stelbrink, M. A. Suhm, Vibrational Signatures of Chirality Recognition Between $\alpha$-Pinene and Alcohols for Theory Benchmarking, Angew. Chem. Int. Ed. 2019, 58, 8177-8181.

- B. Hartwig, M. Lange, A. Poblotzki, R. Medel, A. Zehnacker, M. A. Suhm, The Reduced Cohesion of Homoconfigurational 1,2-Diols, Phys. Chem. Chem. Phys. 2020, 22, 1122-1136.

Die eigenständige Veröffentlichung weiterer Ergebnisse dieser Arbeit ist geplant.

Göttingen, 09.06.2020 


\section{Betreute studentische Arbeiten}

Folgende studentische Forschungsprojekte wurden betreut:

- Helen Preiß, The Molecular Nose - Chirality Recognition between Bicyclic Monoterpenes, IPC-Forschungspraktikum 2017.

- Caroline Stelbrink, Wettstreit der molekularen Nasen: Chiralitätserkennung zwischen $\alpha$-Pinen und Alkoholen, Bachelorarbeit 2018.

- Enno Meyer, The dimers of 1-phenylethanol and benzyl alcohol examined by Raman spectroscopy, IPC-Forschungspraktikum 2019.

- Lukas Hasecke, Jonas Heitland, Johann Benedikt Meyer, Moritz Niessner, Spektroskopie des molekularen Händedrucks, Forschungsorientierten Lehren und Lernen (FoLL XVI) 2019, Betreuung zusammen mit Manuel Lange, Caroline Stelbrink, Ricardo A. Mata und Martin A. Suhm.

- Moritz Niessner, Chiralitätserkennung in Carbonylwaagen: Wasserstoffbrückenkomplexe von Alkoholen mit dem prochiralen Keton Cyclohexanon, Bachelorarbeit 2020, Betreuung zusammen mit Charlotte Zimmermann.

- Johann Richard Springborn, Modellierung und Raman-spektroskopische Untersuchung der Tunneldynamik bei Alkoholen, Bachelorarbeit 2020. 


\section{Spektrenverzeichnis}

Gelistet sind die Dateinamen der in dieser Arbeit abgebildeten Spektren, wie sie im Archiv aufzufinden sind. Getrennt nach Apparatur sind die Spektren in der gleichen vertikalen Reihenfolge angegeben, wie sie in der Abbildung dargestellt sind. Für Filet-JetSpektren ist zusätzlich der ggf. verwendete Skalierungsfaktor für die Differenzbildung mit dem Spektrum der atmosphärischen Wasserbanden angegeben (siehe Abb. 3.3 auf S. 25).

Tab. A.20: Curry-Jet-Spektren

\begin{tabular}{|c|c|}
\hline Abb. & Dateiname \\
\hline 4.3 & $1 \mathrm{~mm} \_0 \% \_\mathrm{N} 2$ \\
\hline 4.3 & 3mm_0\%_N2 \\
\hline 4.3 & 080827_EtOH_m15C_8ArHe_1_6_0_72bar_659nmMC2051_300s_2mm_75mum_smooth3 \\
\hline 4.3 & $3 \mathrm{~mm} \_7.5 \%$ N2 \\
\hline 4.3 & 080813_EtOH_m23_5C_Ne_1_6_0_85bar_659nmMC2051_500s_3mm_75mum_smooth3 \\
\hline 4.8 & $3 \mathrm{~mm} \_0 \% \_\mathrm{N} 2$ \\
\hline 4.9 & $1 \mathrm{~mm} \_0 \%$ N2 \\
\hline 4.10 & 080827_EtOH_m15C_8ArHe_1_6_0_72bar_659nmMC2051_300s_2mm_75mum_smooth3 \\
\hline 4.10 & $3 \mathrm{~mm} \_7.5 \%$ N2 \\
\hline 4.11 & 080813_EtOH_m23_5C_Ne_1_6_0_85bar_659nmMC2051_500s_3mm_75mum_smooth3 \\
\hline 4.12 & 090306_EtOD_m23_5C_He_1_5_0p8bar_621nmMC2051_300s_1 $1 \mathrm{~mm}$ 77 $75 \mathrm{mum}$ smooth3 \\
\hline 4.14 & $3 \mathrm{~mm} \_0 \% \_\mathrm{N} 2$ \\
\hline 4.18 & 5.2_MeOH_Raman_Jet_500mm \\
\hline 4.19 & 060919_EtOH_m8gD150d1_654nm_acton_600 \\
\hline 4.29 & 5.2_MeOH_Raman_Jet_500mm \\
\hline 4.30 & 060919_EtOH_m8gD150d1_654nm_acton_600 \\
\hline 5.16 & 20181017_d+f_Fenchol_22,5C_He_0,7bar_0.5mm_D26C_75microm_M652_17x6min \\
\hline 5.16 & 20181017 b+e_Fenchol_22,5C_He_0,7bar_1mm_D26C_75microm_M652_10x6min \\
\hline 5.16 & 20181017_c+g_Fenchol_22,5C_He_0,7bar_2mm_D26C_75microm_M652_3x6min \\
\hline 5.17 & 20181022 d_HedurchschwarzenSaettiger_0,7bar_0,5mm_D26C_75microm_M652_4x6min \\
\hline 5.17 & 20181022_b_HedurchschwarzenSaettiger_0,7bar_1mm_D26C_75microm_M652_4x6min \\
\hline 5.17 & 20181022_c_HedurchschwarzenSaettiger_0,7bar_2mm_D26C_75microm_M652_4x6min \\
\hline 5.18 & 20181017_d+f_Fenchol_22,5C_He_0,7bar_0.5mm_D26C_75microm_M652_17x6min \\
\hline 5.19 & 20181017_d+f_Fenchol_22,5C_He_0,7bar_0.5mm_D26C_75 microm_M652_17x6min \\
\hline 5.26 & 20181017_d+f_Fenchol_22,5C_He_0,7bar_0.5mm_D26C_75 microm_M652_17x6min \\
\hline 5.26 & 20181017_b+e_Fenchol_22,5C_He_0,7bar_1mm_D26C_75 microm_M652_10x6min \\
\hline 5.26 & 20181017_c+g_Fenchol_22,5C_He_0,7bar_2mm_D26C_75microm_M652_3x6min \\
\hline 5.31 & 20181018_c+d_Pinakolylalkohol_6C_He_0,7bar_1mm_D26C_75microm_M652_4x6min \\
\hline 5.32 & 20181018 cc+d_Pinakolylalkohol_6C_He_0,7bar_1mm_D26C_75microm_M652_4x6min \\
\hline 5.36 & 071129_2DmPrOH_9g_1mm_659_500mbar \\
\hline 5.55 & 20181001_a_BzOH_60C_He_0,7bar_1mm_D70C_75microm_M652_2min \\
\hline 5.55 & 20181002_h_BzOH_70C_He_0,7bar_1mm_D200C_75microm_M652_2min \\
\hline 5.61 & 20181015_g_S(-)-PhEtOH_51C_He_1,0bar_2mm_D60C_75microm_M652_20x3min \\
\hline 8.4 & 20181016_h_BzOH_50C_He_1,0bar_2mm_D60C_75microm_M652_20x3min_calib_mit_i00 \\
\hline 8.5 & 20181016_h_BzOH_50C_He_1,0bar_2mm_D60C_75microm_M652_20x3min_calib_mit_i00 \\
\hline 8.8 & 20181010_g+h_PhEtOH_49C_He_1,0bar_2mm_D60C_75microm_M652_22x3min_calib_mit_f \\
\hline 8.8 & 20181015_g_S(-)-PhEtOH_51C_He_1,0bar_2mm_D60C_75microm_M652_20x3min_recalib \\
\hline 8.8 & 20181010_heterochiral_PhEtOH_49C_He_1,0bar_2mm_D60C_75microm_M652_22 $33 \mathrm{~min}$ recalib \\
\hline 8.8 & 20181015_heterochiral-Trimer_51C_He_1,0bar_2mm_D60C_75microm_M652_20x3min_recalib_interp \\
\hline 8.9 & 20181010 g + h_PhEtOH_49C_He_1,0bar_2mm_D60C 75 microm_M652_22 33min_calib_mit_f \\
\hline 8.9 & 20181015_g_S(-)-PhEtOH_51C_He_1,0bar_2mm_D60C_75microm_M652_20x3min_recalib \\
\hline 8.9 & 20181010_heterochiral_PhEtOH_49C_He_1,0bar_2mm_D60C_75 microm_M652_22 $33 \mathrm{~min}$ recalib \\
\hline 8.10 & 20181016_h_BzOH_50C_He_1,0bar_2mm_D60C_75microm_M652_20x3min_calib_mit_00 \\
\hline 8.10 & 20181010_g+h_PhEtOH_49C_He_1,0bar_2mm_D60C_75microm_M652_22x3min_calib_mit_f \\
\hline 8.13 & 20181016_d_S(-)-PhEtOH+mehrBzOH_50C_He_1,0bar_2mm_D60C_75 microm_M652_20x3min_calib \\
\hline 8.13 & 20181016_c_S(-)-PhEtOH+BzOH_50C_He_1,0bar_2mm_D60C_75microm_M652_20x3min_calib \\
\hline 8.13 & BzOH-Ueberschuss_0.3_0.2_mit_interpolation \\
\hline 8.13 & 1-PeOH-Ueberschuss_0.15_0.35_mit_interpolation \\
\hline
\end{tabular}


Tab. A.21: Filet-Jet-Spektren mit Skalierungsfaktor für die Wasserkorrektur WK.

\begin{tabular}{|c|c|c|}
\hline Abb. & WK & Dateiname \\
\hline 3.2 & - & 20180409-a-(-)-Borneol-24C-leer-0,75barp_single. 0 \\
\hline 3.2 & - & 20180409-d-(-)-Borneol-24C-leer-0,75barp_single.7 \\
\hline 3.3 & - & 180315-Notch2-InSbSWneu-2,2kO-F13a-CaF2+W150-3,5mm-80kHz \\
\hline 3.3 & - & Zusammengemittelt_(-)IPC(+)P(1_5).285 \\
\hline 3.3 & 0.03 & Zusammengemittelt_(-)IPC(+)P(1_5)WK.285 \\
\hline 4.3 & - & 151120_a+151208_a+151214_a_He0.75bar.500 \\
\hline 4.3 & - & $151210-\mathrm{a}-\mathrm{EtOH}-\mathrm{He} \_\mathrm{N} 2 \_0,75 \mathrm{barpm} .200$ \\
\hline 4.3 & - & $151221-\mathrm{a}+151223-\mathrm{c}-\mathrm{EtOH}-\mathrm{He}-\mathrm{Ar}-0,75 \mathrm{barpm} .650$ \\
\hline 4.3 & - & $160603-\mathrm{b}-\mathrm{EtOH}-0.1 \%-\mathrm{O} 2-2.5 \%-\mathrm{He}-0,75$ barpm. 325 \\
\hline 4.5 & - & $151120 \_a+151208 \_a+151214 \_a \_H e 0.75 b a r .500$ \\
\hline 4.6 & - & $151120 \_a+151208 \_a+151214 \_a-H e 0.75 b a r .500$ \\
\hline 4.7 & - & $151120 \_a+151208 \_a+151214 \_a \_H e 0.75 b a r .500$ \\
\hline 4.10 & - & $151210-\mathrm{a}-\mathrm{EtOH}-\mathrm{He} \_\mathrm{N} 2 \_0,75 \mathrm{barpm} .200$ \\
\hline 4.10 & - & $151221-\mathrm{a}+151223-\mathrm{c}-\mathrm{EtOH}-\mathrm{He}-\mathrm{Ar}-0,75 \mathrm{barpm} .650$ \\
\hline 4.10 & - & $160603-\mathrm{b}-\mathrm{EtOH}-0.1 \%-\mathrm{O} 2-2.5 \%-\mathrm{He}-0,75$ barpm. 325 \\
\hline 4.19 & - & 151120_a+151208_a+151214_a_He0.75bar.500 \\
\hline 4.22 & - & 151120_a+151208_a+151214_a_He0.75bar.500 \\
\hline 4.30 & - & 151120_a+151208_a+151214_a_He0.75bar.500 \\
\hline 5.7 & 0.015 & Zusammengemittelt_IsopinocampheolWK.287 \\
\hline 5.8 & -0.1148 & 20180426-jGP-(-)-Isopinocampheol-24C-1,00barWK \\
\hline 5.8 & 0.015 & Zusammengemittelt_IsopinocampheolWK.287 \\
\hline 5.11 & - & Zusammengemittelt_Borneol_s.Messdaten_hellgruen.139 \\
\hline 5.12 & 0.1875 & 20180409-fGP-(-)-Borneol-24C-leer-0,75barWK \\
\hline 5.12 & - & Zusammengemittelt_Borneol_s.Messdaten_hellgruen.139 \\
\hline 5.15 & 0.0405 & Fenchol 1,20 bar. 107 \\
\hline 5.15 & 0.025 & Fenchol 0,75 bar. 307 \\
\hline 5.16 & 0.55 & 20170308-e-(+)-alpha-Fenchol-25C-leer-1,20bar-GP_WK \\
\hline 5.16 & 0.0405 & Fenchol 1,20bar. 107 \\
\hline 5.16 & 0.025 & Fenchol 0,75 bar. 307 \\
\hline 5.16 & - & $(+)-$ Pinen+Fenchol 1 zu $1+$ Argon 0,75 bar. 388 \\
\hline 5.30 & - & $20181115-\mathrm{h}+\mathrm{i}+\mathrm{j}-$ Pinakolylalkohol-6C -+ Gas $-0,75$ barpm .95 \\
\hline 5.30 & - & $20181115-\mathrm{k}+\mathrm{l}+\mathrm{m}+\mathrm{n}+\mathrm{o}-$ Pinakolylalkohol $-6 \mathrm{C}-+$ mehrGas $-0,75 \mathrm{barpm} .175$ \\
\hline 5.31 & - & 20181115-oGP-Pinakolylalkohol-6C-+mehrGas-0,75bar \\
\hline 5.31 & - & $20181115-\mathrm{h}+\mathrm{i}+\mathrm{j}-$ Pinakolylalkohol-6C -+ Gas $-0,75$ barpm .95 \\
\hline 5.31 & - & $20181115-\mathrm{k}+\mathrm{l}+\mathrm{m}+\mathrm{n}+\mathrm{o}-$ Pinakolylalkohol $-6 \mathrm{C}-+$ mehrGas $-0,75 \mathrm{barpm} .175$ \\
\hline 5.61 & -0.1823 & 20180417-gGP-(-)-1-Phenylethanol-24C-0,75barWK \\
\hline 5.61 & 0.0154 & Zusammengemittelt_(-)-1-Phenylethanol_homochiralWK.171 \\
\hline 6.3 & 0.0163 & Zusammengemittelt_(-)B(-)P(1_5)_1,20barWK.259 \\
\hline 6.3 & 0.0132 & Zusammengemittelt_(-) $\mathrm{B}(+) \mathrm{P}\left(1 \_5\right) \_1,20 \mathrm{barWK} .357$ \\
\hline 6.3 & - & $(-)-$ Pinen+Fenchol 5 zu 10,75 bar. 389 \\
\hline 6.3 & - & (+)-Pinen+Fenchol 5 zu $1 \quad 0,75$ bar.391 \\
\hline 6.3 & - & Zusammengemittelt_(-)IPC(-)P(1_5).283 \\
\hline 6.3 & 0.03 & Zusammengemittelt_(-)IPC(+)P(1_5)WK.285 \\
\hline 6.3 & 0.023 & Zusammengemittelt_(-)Pe(-)P_s.Messdaten_grasgruenWK.208 \\
\hline 6.3 & 0.307 & Zusammengemittelt_(-) $\mathrm{Pe}(+) \mathrm{P} \_s-$ Messdaten_tuerkisWK.640 \\
\hline 6.11 & 0.0313 & Zusammengemittelt_(+)-2Bu(-)P(1_1)x3-1,20barWK.55 \\
\hline 6.11 & - & Zusammengemittelt_(+)-2Bu(+) $\mathrm{P}\left(1 \_1\right) \times 3-1,20 \mathrm{bar} .34$ \\
\hline 6.13 & 0.0313 & Zusammengemittelt_(+)-2Bu(-)P(1_1)×3-1,20barWK.55 \\
\hline 6.13 & - & Zusammengemittelt_(+)-2Bu(+)P(1_1) $\times 3-1,20$ bar.34 \\
\hline 7.2 & 0.0405 & Fenchol 1,20bar.107 \\
\hline 7.2 & 0.025 & Fenchol 0,75 bar. 307 \\
\hline 8.8 & - & Zusammengemittelt_rac-1-Phenylethanol.481 \\
\hline 8.8 & 0.0154 & Zusammengemittelt_(-)-1-Phenylethanol_homochiralWK.171 \\
\hline 8.8 & - & Zusammengemittelt_(-)-1-Phenylethanol_heterochiralWK.0 \\
\hline 8.9 & - & Zusammengemittelt_rac-1-Phenylethanol.481 \\
\hline 8.9 & 0.0154 & Zusammengemittelt_(-)-1-Phenylethanol_homochiralWK.171 \\
\hline 8.9 & - & Zusammengemittelt_(-)-1-Phenylethanol_heterochiralWK.0 \\
\hline A. 3 & - & (+)-alpha-Pinen 0,75 bar .55 \\
\hline A. 3 & 0.0436 & Zusammengemittel__(-)B(+)P(1_30)_1,20barWK.134 \\
\hline A. 3 & 0.0132 & Zusammengemittelt_(-)B(+)P(1_5)_1,20barWK.357 \\
\hline A. 3 & 0.0163 & Zusammengemittelt_(-)B(-)P(1_5)_1,20barWK.259 \\
\hline A. 3 & - & $20170309-a+b+c-(-)$-alpha-Pinen-24C-leer-0,75barpm. 110 \\
\hline A. 3 & - & Zusammengemittelt_Borneol_s.Messdaten_hellgruen.139 \\
\hline A. 4 & - & (+)-Pinen+Fenchol 5 zu 10,75 bar .391 \\
\hline A. 4 & - & (+)-Pinen+Fenchol 1 zu $1+$ Argon 0,75 bar. 388 \\
\hline A. 4 & - & (+)-Pinen+Fenchol 1 zu 1 0,75bar.391 \\
\hline A. 4 & - & (-)-Pinen+Fenchol 30 zu 1 0,75bar.98 \\
\hline A. 4 & - & (-)-Pinen+Fenchol 5 zu 10,75 bar. 389 \\
\hline A. 4 & - & (-)-Pinen+Fenchol 1 zu 1 0,75bar.393 \\
\hline A. 4 & - & rac-Pinen+Fenchol 5 zu 1 0,75bar. 199 \\
\hline A. 4 & - & rac-Pinen+Fenchol 1 zu 1 0,75bar. 191 \\
\hline A. 4 & 0.025 & Fenchol 0,75 bar.307 \\
\hline A. 5 & 0.03 & Zusammengemittelt_(-)IPC(+)P(1_30)WK.153 \\
\hline A. 5 & 0.03 & Zusammengemittelt_(-)IPC(+)P(1_5)WK.285 \\
\hline A. 5 & - & Zusammengemittelt_(-)IPC(-)P(1_5).283 \\
\hline A. 5 & 0.015 & Zusammengemittelt_IsopinocampheolWK.287 \\
\hline A. 6 & 0.04 & Zusammengemittelt_(-)Pe_(+)P_ps0,75_(30_1)WK.88 \\
\hline A. 6 & 0.045 & Zusammengemittelt_(-)Pe_(+)P_ps0,75_(5_1)WK.263 \\
\hline A. 6 & 0.0365 & Zusammengemittelt_(-)Pe_(+)P_ps0,75_(1_1)WK.289 \\
\hline A. 6 & 0.023 & Zusammengemittelt_(-)Pe(-)P_s.Messdaten_grasgruenWK.208 \\
\hline A. 6 & 0.0154 & Zusammengemittelt_(-)-1-Phenylethanol_homochiralWK.171 \\
\hline A. 15 & -0.0313 & Zusammengemittelt_(+)-2Bu(+)P(1_1) x3-0,75barWK.119 \\
\hline A. 15 & - & Zusammengemittelt_(+)-2Bu(+)P(1_1) $\times 3-1,20$ bar.34 \\
\hline A. 15 & - & Zusammengemittelt_(+)-2Bu(+)P(0,5_1) $\times 3-1,20$ bar. 100 \\
\hline A. 15 & -0.0156 & Zusammengemittelt_(+)-2Bu(-)P(1_1) $33-0,75$ barWK.175 \\
\hline A. 15 & 0.0313 & Zusammengemittelt_(+)-2Bu(-)P(1_1) x3-1,20barWK.55 \\
\hline A. 15 & - & Zusammengemittelt_(+)-2Bu(-)P(0,5_1) $\times 3-1,20$ bar.99 \\
\hline A. 15 & - & Zusammengemittelt_rac-2Bu(+) $\mathrm{P}\left(1 \_1\right) \times 3.117$ \\
\hline A. 15 & - & Zusammengemittelt_rac-2Bu(+) $\mathrm{P}\left(1 \_5\right) \times 3.80$ \\
\hline
\end{tabular}


Tab. A.22: Ragout-Jet-Spektren

\begin{tabular}{ll}
\hline Abb. & Dateiname \\
\hline 4.18 & 5.2_MeOH_FTIR_Jet \\
4.22 & 5.2_MeOH_FTIR_Jet \\
4.29 & 5.2_MeOH_FTIR_Jet \\
5.34 & TBDMC10.000 \\
5.34 & $400012 \mathrm{AG} .000$ \\
5.44 & rsbu18mm.400 \\
5.44 & ssbu18mm.400 \\
5.44 & het_n=2 \\
5.45 & 2buohgas \\
5.45 & ssbu18mm.400 \\
5.45 & rsbu18mm.400 \\
A.15 & ssbu18mm.400 \\
A.15 & rsbu18mm.400 \\
\hline
\end{tabular}

Tab. A.23: Popcorn-Jet-Spektren

\begin{tabular}{ll}
\hline Abb. & Dateiname \\
\hline 5.11 & Borneol 80 C.779 \\
5.11 & Borneol 70 C.547 \\
5.11 & Borneol 60 C.690 \\
5.12 & Borneol 80 C.779 \\
5.12 & Borneol 70 C.547 \\
5.12 & Borneol 60 C.690 \\
5.55 & BA_mittelwert70C_190515c-i_190516d-j.313 \\
5.55 & BzOH_OH_He_85C_HT200C_d,e,g,i_pm.51 \\
8.4 & BA_mittelwert70C_190515c \\
8.4 & BzOH_OH_He_70C_c-h_pm.283 \\
8.5 & BA_mittelwert70C_190515c \\
9.4 & DDnol_140C_OH_15bar_cpm.50 \\
9.9 & Ethylenglycol_OH_He_85C_alt.125 \\
9.9 & EG_70_90_86Scans_neu.86 \\
\hline
\end{tabular}

Tab. A.24: RESS-Jet-Spektren

\begin{tabular}{ll}
\hline Abb. & Dateiname \\
\hline 9.3 & 141118A_20_120_190_2_70_OZ_2mmpm.10 \\
9.3 & 141118C_20_120_190_10_70_OZ_10mmpm.10 \\
9.3 & 141118G_20_120_190_40_70_OZ_40mmpm.10 \\
9.3 & 141118J_20_120_190_90_70_OZ_90mmpm.10 \\
9.4 & 150120A_20_120_190_110_70_OZ_D1P9pm.100 \\
9.4 & 150120A_20_120_190_110_70_OZ_D1P9n1m.100 \\
9.4 & 150119A_20_120_190_20_70_OZ_D1P9pm.100 \\
9.4 & 150119A_20_120_190_20_70_OZ_D1P9n1m.100 \\
9.5 & 151023A_20_120_350_110_70_OZ_MeOH_5n+10+n+30+npm.10 \\
9.5 & 151021E_20_120_350_20_70_OZ_MeOH_5n+10+n+30+npm.10 \\
\hline
\end{tabular}

Tab. A.25: Falafel-Jet-Spektren

\begin{tabular}{ll}
\hline Abb. & Dateiname \\
\hline 9.4 & C12OH_CH_He_140917_120C_02-04_pvm.75
\end{tabular}


Tab. A.26: Risotto-Jet-Spektren

\begin{tabular}{|c|c|}
\hline Abb. & Dateiname \\
\hline 9.8 & 1_180824X_EG_90bar_A=70_60_205_200_200_800mbar_C=A+80_220pm.5 \\
\hline 9.8 & 2_180824W_EG_90bar_A=60_60_205_200_200_800mbar_C $=\mathrm{A}+80 \_220 \mathrm{pm} .5$ \\
\hline 9.8 & 3_180824U_EG_90bar_A=40_60_205_200_200_800mbar_C=A+80_220pm.5 \\
\hline 9.8 & 4_180824X_EG_90bar_A=70_60_205_200_200_800mbar_C=A+80_220n1m.5 \\
\hline 9.8 & 5_180824W_EG_90bar_A=60_60_205_200_200_800mbar_C=A+80_220n3m.5 \\
\hline 9.8 & 6_180824Z_EG_90bar_A=90_60_205_200_200_800mbar_C=A+80_220n7m.5 \\
\hline 9.9 & $280 \mathrm{kHz} \_5$ res_5mm_DS_180302+180306.135 \\
\hline 9.9 & $\begin{array}{l}\text { 280kHz_3.5res_5mm_DS_180907B+C+D+E+F+C+H+H+I+J+K+L+M+N+O+P+P } \\
\text { EG_90bar_A }=60 \_60 \_205 \_200 \_200 \_800 \mathrm{mbar}-\mathrm{C}=\mathrm{A}+80 \_160 \mathrm{pm} .135\end{array}$ \\
\hline 9.9 & $80 \mathrm{kHz} \_2$ res_3.5mm_SS $180830 \mathrm{~F}+\mathrm{G}+\mathrm{L}+\mathrm{M}+\mathrm{N}+\mathrm{O} \_$EG_90bar_A $=120 \_60 \_205 \_200 \_200 \_800 \mathrm{mbar} \_\mathrm{C}=\mathrm{A}+80 \_220 \mathrm{pm} .135$ \\
\hline 9.9 & $\begin{array}{l}\text { 80kHz_3.5res_5mm_SS_180907P+Q+R+S+T+U+V+W+X+Y+Z+ZA+ZB+ZC_EG } \\
\text { _90bar_A }=140 \_60 \_205 \_200 \_200 \_800 \mathrm{mbar} \_\mathrm{C}=\mathrm{A}+80 \_240 \mathrm{n} 1 \mathrm{~m} .135\end{array}$ \\
\hline 9.10 & 170413G_MeOH_65bar_A=110_60_60_57_53_He_1200mbar_590_200n3m.10 \\
\hline 9.10 & 170421H_MeOH_65bar_A=160_60_60_57_53_He_1200mbar_240_400n1m.10 \\
\hline 9.11 & 0629ABCDG0713ABFJKLSTUVWX0714BCDEFGHJKLM_MeOH_65bar_A=190_60_30_29_27_He_800mbar_280_300n1m.677 \\
\hline 9.11 & 170609C+D_MeOH_65bar_A=185_60_200_157_101_He_800mbar_275_400n1m.30 \\
\hline 9.11 & 170609H+I_MeOH_65bar_A $=200 \_60 \_300 \_237 \_156 \_$He_800mbar_290_400n $1 \mathrm{~m} .30$ \\
\hline 9.11 & $170609 \mathrm{~W}+\mathrm{X}+\mathrm{Y} \_\mathrm{MeOH} \_65 \mathrm{bar} \_\mathrm{A}=188 \_100 \_400 \_309 \_200 \_\mathrm{He} \_800 \mathrm{mbar} \_278 \_400 \mathrm{n} 1 \mathrm{~m} .55$ \\
\hline 9.11 & 170628I_MeOH_65bar_A=185_100_30_29_27_He_150mbar_275_400n1m.100 \\
\hline 9.11 & $170705 \mathrm{O}+\mathrm{P}+0706 \mathrm{~A}+\mathrm{C}+\mathrm{D}+\mathrm{E}+\mathrm{H}+\mathrm{I}+\mathrm{K}+\mathrm{L}+\mathrm{N} \_\mathrm{MeOH} \_65 \mathrm{bar} \_\mathrm{A}=190 \_150 \_350 \_281 \_195 \_\mathrm{He} \_400 \mathrm{mbar} \_280 \_200 \mathrm{n} 1 \mathrm{~m} .250$ \\
\hline
\end{tabular}




\section{Literaturverzeichnis}

[1] K. Michaeli, N. Kantor-Uriel, R. Naaman, D. H. Waldeck, The electron's spin and molecular chirality - how are they related and how do they affect life processes?, Chem. Soc. Rev. 2016, 45, 6478-6487.

[2] A. Kumar, E. Capua, M. K. Kesharwani, J. M. L. Martin, E. Sitbon, D. H. Waldeck, R. Naaman, Chirality-induced spin polarization places symmetry constraints on biomolecular interactions, Proc. Natl. Acad. Sci. 2017, 114, 2474-2478.

[3] M. S. Zöllner, S. Varela, E. Medina, V. Mujica, C. Herrmann, Chiral-Induced Spin Selectivity: A Symmetry Analysis of Electronic Transmission, ChemRxiv Prepr. 2019.

[4] R. Medel, C. Stelbrink, M. A. Suhm, Vibrational Signatures of Chirality Recognition Between $\alpha$-Pinene and Alcohols for Theory Benchmarking, Angew. Chem. Int. Ed. 2019, 58, 8177-8181.

[5] A. Zehnacker, M. A. Suhm, Chirality Recognition between Neutral Molecules in the Gas Phase, Angew. Chem. Int. Ed. 2008, 47, 6970-6992.

[6] M. Laska, Olfactory Discrimination Ability of Human Subjects for Ten Pairs of Enantiomers, Chem. Senses 1999, 24, 161-170.

[7] T. Yamamoto, E. Tokunaga, S. Nakamura, N. Shibata, T. Toru, Synthesis and Configurational Stability of $(S)$ - and $(R)$-Deuteriothalidomides, Chem Pharm Bull 2010, 58, 110-112.

[8] V. Jacques, A. W. Czarnik, T. M. Judge, L. H. T. Van der Ploeg, S. H. DeWitt, Differentiation of antiinflammatory and antitumorigenic properties of stabilized enantiomers of thalidomide analogs, Proc. Natl. Acad. Sci. 2015, 112, E1471E1479.

[9] T. Mori, T. Ito, S. Liu, H. Ando, S. Sakamoto, Y. Yamaguchi, E. Tokunaga, N. Shibata, H. Handa, T. Hakoshima, Structural basis of thalidomide enantiomer binding to cereblon, Sci. Rep. 2018, 8.

[10] A. Kohen, J. P. Klinman, Enzyme Catalysis: Beyond Classical Paradigms, Acc. Chem. Res. 1998, 31, 397-404.

[11] G. P. Moss, P. A. S. Smith, D. Tavernier, Glossary of class names of organic compounds and reactivity intermediates based on structure (IUPAC Recommendations 1995), Pure Appl. Chem. 1995, 67, 1307-1375.

[12] R. K. Kakar, C. R. Quade, Microwave rotational spectrum and internal rotation in gauche ethyl alcohol, J. Chem. Phys. 1980, 72, 4300-4307.

[13] B. T. Lutz, J. H. van der Maas, The sensorial potentials of the $\mathrm{OH}$ stretching mode, J. Mol. Struct. 1997, 436-437, 213-231.

[14] T. N. Wassermann, P. Zielke, J. J. Lee, C. Cézard, M. A. Suhm, Structural Preferences, Argon Nanocoating, and Dimerization of $n$-Alkanols as Revealed by $\mathrm{OH}$ Stretching Spectroscopy in Supersonic Jets, J. Phys. Chem. A 2007, 111, 74377448.

[15] G. Seyfang, M. Quack, Trendbericht Physikalische Chemie 2017: Atomare und molekulare Tunnelprozesse, Nachrichten Aus Chem. 2018, 66, 307-315.

[16] R. A. Mata, M. A. Suhm, Benchmarking Quantum Chemical Methods: Are We Heading in the Right Direction?, Angew. Chem. Int. Ed. 2017, 56, 11011-11018. 
[17] D. Hermsdorf, Schnelle Expansion von überkritischen Lösungen zur Herstellung von organischen Nanopartikeln, Dissertation, Cuvillier Verlag, Göttingen, 2006.

[18] M. Herman, R. Georges, M. Hepp, D. Hurtmans, High resolution Fourier transform spectroscopy of jet-cooled molecules, Int. Rev. Phys. Chem. 2000, 19, 277325.

[19] J. M. Hayes, G. J. Small, Supersonic Jets, Rotational Cooling, and Analytical Chemistry, Anal. Chem. 1983, 55, 565A-574A.

[20] U. Erlekam, M. Frankowski, G. von Helden, G. Meijer, Cold collisions catalyse conformational conversion, Phys. Chem. Chem. Phys. 2007, 9, 3786.

[21] S. Oswald, Weak Hydrogen Bonds to Molecular Nitrogen and Oxygen as Experimental Benchmarks for Quantum Chemistry, Dissertation, Georg-AugustUniversität Göttingen, 2019.

[22] T. N. Wassermann, M. A. Suhm, Ethanol Monomers and Dimers Revisited: A Raman Study of Conformational Preferences and Argon Nanocoating Effects, $J$. Phys. Chem. A 2010, 114, 8223-8233.

[23] R. S. Ruoff, T. D. Klots, T. Emilsson, H. S. Gutowsky, Relaxation of conformers and isomers in seeded supersonic jets of inert gases, J. Chem. Phys. 1990, 93, 3142-3150.

[24] W. Y. Sohn, K.-J. Cho, S. Y. Lee, S. S. Kang, Y. D. Park, H. Kang, Solvent-assisted conformational isomerization (SACI) of meta-substituted phenols: Tuning relative stability, isomerization barrier, and IVR rate, Chem. Phys. Lett. 2012, 525-526, 37-43.

[25] R. Campargue, Progress in overexpanded supersonic jets and skimmed molecular beams in free-jet zones of silence, J. Phys. Chem. 1984, 88, 4466-4474.

[26] R. S. Cahn, C. Ingold, V. Prelog, Specification of Molecular Chirality, Angew. Chem. Int. Ed. Engl. 1966, 5, 385-415.

[27] B. Hartwig, M. Lange, A. Poblotzki, R. Medel, A. Zehnacker, M. A. Suhm, The reduced cohesion of homoconfigurational 1,2-diols, Phys. Chem. Chem. Phys. 2020, 22, 1122-1136.

[28] T. Benincori, G. Celentano, T. Pilati, A. Ponti, S. Rizzo, F. Sannicolò, Configurationally Stable Molecular Propellers: First Resolution of Residual Enantiomers, Angew. Chem. Int. Ed. 2006, 45, 6193-6196.

[29] M. Quack, Struktur und Dynamik chiraler Moleküle, Angew. Chem. 1989, 101, 588-604.

[30] B. Wiesmann, Chirale Moleküle: Zur Erklärung der Stabilität optischer Isomere aus fundamentalen Wechselwirkungen, Dissertation, Universität Regensburg, 2000.

[31] R. Bentley, Ogston and the development of prochirality theory, Nature 1978, 276, 673-676.

[32] L. D. Barron, Chirality, magnetism and light, Nature 2000, 405, 895-896.

[33] R. Berger, J. Stohner, Parity violation, Wiley Interdiscip. Rev. Comput. Mol. Sci. 2019, 9, e1396.

[34] R. Berger, M. Gottselig, M. Quack, M. Willeke, Parity Violation Dominates the Dynamics of Chirality in Dichlorodisulfane, Angew. Chem. Int. Ed. 2001, 40, 4195-4198.

[35] H.-Q. Wang, W.-N. Cheng, N. Chen, L.-Y. Zhu, X.-G. Hu, Energetics of pairwise interaction between glycidol enantiomers in (dimethylformamide+water) mixtures rich in water at $T=298.15 \mathrm{~K}, J$. Chem. Thermodyn. 2015, 87, 8-12. 
[36] D. P. Craig, D. P. Mellor in Topics in Current Chemistry: Bonding and Structure, Vol. 63, Springer-Verlag, Berlin/Heidelberg, 1976.

[37] N. Borho, Chirale Erkennung in Molekülclustern: maßgeschneiderte Aggregation von $\alpha$-Hydroxyestern, Dissertation, Cuvillier Verlag, Göttingen, 2005.

[38] D. E. Koshland, The Key-Lock Theory and the Induced Fit Theory, Angew. Chem. Int. Ed. Engl. 1995, 33, 2375-2378.

[39] V. A. Davankov, The nature of chiral recognition: Is it a three-point interaction?, Chirality 1997, 9, 99-102.

[40] D. P. Craig, E. A. Power, T. Thirunamachandran, The Interaction of Optically Active Molecules, Proc. R. Soc. Math. Phys. Eng. Sci. 1971, 322, 165-179.

[41] D. P. Craig, T. Thirunamachandran, Molecular quantum electrodynamics: an introduction to radiation-molecule interactions, Academic Press, London ; Orlando, 1984.

[42] R. Kuroda, S. F. Mason, C. D. Rodger, R. H. Seal, Chiral discriminations: An extended transition monopole model, Mol. Phys. 1981, 42, 33-50.

[43] S. F. Mason, Molecular Handedness and the Origins of Chiral Discrimination, Int. Rev. Phys. Chem. 1983, 3, 217-241.

[44] K. Ray, Asymmetric Scattering of Polarized Electrons by Organized Organic Films of Chiral Molecules, Science 1999, 283, 814-816.

[45] R. Naaman, D. H. Waldeck, Chiral-Induced Spin Selectivity Effect, J. Phys. Chem. Lett. 2012, 3, 2178-2187.

[46] R. S. Rowland, R. Taylor, Intermolecular Nonbonded Contact Distances in Organic Crystal Structures: Comparison with Distances Expected from van der Waals Radii, J Phys Chem 1996, 100, 7384-7391.

[47] A. Otero-de-la-Roza, J. E. Hein, E. R. Johnson, Reevaluating the Stability and Prevalence of Conglomerates: Implications for Preferential Crystallization, Cryst. Growth Des. 2016, 16, 6055-6059.

[48] J. Jacques, A. Collet, S. H. Wilen, Enantiomers, racemates, and resolutions, Reissue with corrections, Krieger, Malabar, Florida, 1994.

[49] C. P. Brock, W. B. Schweizer, J. D. Dunitz, On the validity of Wallach's rule: on the density and stability of racemic crystals compared with their chiral counterparts, J. Am. Chem. Soc. 1991, 113, 9811-9820.

[50] K. B. Wiberg, P. H. Vaccaro, J. R. Cheeseman, Conformational Effects on Optical Rotation. 3-Substituted 1-Butenes, J. Am. Chem. Soc. 2003, 125, 1888-1896.

[51] T. Tsunetsugu, J. Furukawa, T. Fueno, Optical rotatory properties of synthetic polymers. I. Optical inversion of poly(propylene oxide), J. Polym. Sci. [A1] 1971, 9, 3529-3540.

[52] M. Geyer, R. Gutierrez, V. Mujica, G. Cuniberti, Chirality-Induced Spin Selectivity in a Coarse-Grained Tight-Binding Model for Helicene, J. Phys. Chem. C 2019, 123, 27230-27241.

[53] G. Herzberg, Infrared and Raman spectra of polyatomic molecules, Reprint ed. with corr, Krieger, Malabar, Florida, 1991.

[54] M. Heger, Diagonal and Off-Diagonal Anharmonicity in Hydrogen-Bonded Systems, Dissertation, Georg-August-Universität-Göttingen, 2016.

[55] M. A. Suhm, F. Kollipost, Femtisecond single-mole infrared spectroscopy of molecular clusters, Phys. Chem. Chem. Phys. 2013, 15, 10702.

[56] P. R. Griffiths, J. A. de Haseth, Fourier transform infrared spectrometry, 2nd edition, John Wiley \& Sons, Inc., Hoboken, New Jersey, 2007. 
[57] T. N. Wassermann, Umgebungseinflüsse auf die $C-C$ - und $C-O$-Torsionsdynamik in Molekülen und Molekülaggregaten: Schwingungsspektroskopie bei tiefen Temperaturen, Dissertation, Georg-August-Universität Göttingen, 2010.

[58] D. A. Long, The Raman effect: a unified treatment of the theory of Raman scattering by molecules, John Wiley \& Sons, Chichester, New York, 2002.

[59] S. Oswald, E. Meyer, M. A. Suhm, Dinitrogen as a Sensor for Metastable Carboxylic Acid Dimers and a Weak Hydrogen Bond Benchmarking Tool, J. Phys. Chem. A 2018, 122, 2933-2946.

[60] V. Barone, Anharmonic vibrational properties by a fully automated second-order perturbative approach, J. Chem. Phys. 2005, 122, 014108.

[61] F. Kollipost, K. Papendorf, Y.-F. Lee, Y.-P. Lee, M. A. Suhm, Alcohol dimers - how much diagonal OH anharmonicity?, Phys. Chem. Chem. Phys. 2014, 16, $15948-15956$.

[62] J. Řezáč, P. Hobza, Describing Noncovalent Interactions beyond the Common Approximations: How Accurate Is the "Gold Standard," $\operatorname{CCSD(T)}$ at the Complete Basis Set Limit?, J. Chem. Theory Comput. 2013, 9, 2151-2155.

[63] J. A. Widegren, T. J. Bruno, Vapor Pressure Measurements on Low-Volatility Terpenoid Compounds by the Concatenated Gas Saturation Method, Environ. Sci. Technol. 2010, 44, 388-393.

[64] V. Štejfa, M. Fulem, K. Růžička, C. Červinka, Thermodynamic study of selected monoterpenes II, J. Chem. Thermodyn. 2014, 79, 272-279.

[65] J. Dykyj, H. Landolt, R. Börnstein, W. Martienssen, Numerical data and functional relationships in science and technology: new series. Group 4 Vol. 20 Subvol. b: Physical chemistry Vapor pressure of chemicals Vapor pressure and Antoine constants for oxygen containing organic compounds, (Eds.: K. R. Hall, O. Madelung), Springer, Berlin, 2000.

[66] J. Dykyj, H. Landolt, R. Börnstein, W. Martienssen, O. Madelung, Numerical data and functional relationships in science and technology: new series. Group 4 Vol. 20 Subvol. a: Physical chemistry Vapor pressure of chemicals Vapor pressure and Antoine constants for hydrocarbons, and sulfur, selenium, tellurium, and halogen containing organic compounds, (Ed.: K. R. Hall), Springer, Berlin, 1999.

[67] R. Medel, Infrarotspektroskopie von Alkohol-Alken-Komplexen: konkurrierende schwache Wechselwirkungen, Masterarbeit, Georg-August-Universität Göttingen, 2014.

[68] T. Häber, Ragout-Jet-FTIR-Spektroskopie: Eine neuartige Methode zur Untersuchung der Dyamik von Oligomeren und nanometer-großen Molekülclustern, Dissertation, Georg-August-Universität Göttingen, 2000.

[69] A. Poblotzki, Intermolecular energy scales based on aromatic ethers and alcohols, Dissertation, Georg-August-Universität Göttingen, 2019.

[70] J. Altnöder, Untersuchungen zum Einfluss von London-Dispersionswechselwirkungen auf die Molekülaggregation, Dissertation, Georg-August-Universität Göttingen, 2015.

[71] T. Forsting, M. Suhm, "Curry-Jet SETUP”, DOI: 10.6084/m9.figshare. 6395840.v1, 2019.

[72] S. Bocklitz, Conformational spectroscopy of flexible chain molecules near the folding limit, Dissertation, Georg-August-Universität Göttingen, 2018.

[73] R. Hänsel, O. Sticher, E. Steinegger, Pharmakognosie - Phytopharmazie, 6. Aufl, Springer, Berlin, 1999. 
[74] E. Breitmaier, G. Jung, Organische Chemie: Grundlagen, Stoffklassen, Reaktionen, Konzepte, Molekülstruktur, 5. überarb. Aufl, Thieme, Stuttgart, 2005.

[75] O. Wallach, Zur Kenntniss der Terpene und der ätherischen Oele. Ueber neue Verbindungen der Campherreihe und ein neues Terpen, Justus Liebigs Ann. Chem. 1891, 263, 129-156.

[76] J. Bertram, J. Helle, Ueber Isofenchylalkohol, J. Für Prakt. Chem. 1900, 61, $293-$ 306.

[77] J. A. Gardner, G. B. Cockburn, XXVII.-Researches on the terpenes. II. On the oxidation of fenchene, J Chem Soc Trans 1898, 73, 275-280.

[78] A. Blumann, O. Zeitschel, Beitrag zur Oxydation des Fenchyl-alkohols, Berichte Dtsch. Chem. Ges. 1909, 42, 2698-2702.

[79] M. J. Frisch, G. W. Trucks, H. B. Schlegel, G. E. Scuseria, M. A. Robb, J. R. Cheeseman, G. Scalmani, V. Barone, B. Mennucci, G. A. Petersson, H. Nakatsuji, M. Caricato, X. Li, H. P. Hratchian, A. F. Izmaylov, J. Bloino, G. Zheng, J. L. Sonnenberg, M. Hada, M. Ehara, K. Toyota, K. Fukuda, J. Hasegawa, M. Ishida, T. Nakajima, Y. Honda, O. Kitao, H. Nakai, T. Vreven, J. A. J. Montgomery, J. E. Peralta, F. Ogliaro, M. Bearpark, J. J. Heyd, E. Brothers, K. N. Kudin, V. N. Staroverov, R. Kobayashi, J. Normand, K. Raghavachari, A. Rendell, J. C. Burant, S. S. Iyengar, J. Tomasi, M. Cossi, N. Rega, J. M. Millam, M. Klene, J. E. Knox, J. B. Cross, V. Bakken, C. Adamo, J. Jaramillo, R. Gomperts, R. E. Stratmann, O. Yazyev, A. J. Austin, R. Cammi, C. Pomelli, J. W. Ochterski, R. L. Martin, K. Morokuma, V. G. Zakrzewski, G. A. Voth, P. Salvador, J. J. Dannenberg, S. Dapprich, A. D. Daniels, Ö. Farkas, J. B. Foresman, J. V. Ortiz, J. Cioslowski, D. J. Fox, Gaussian 09, Revision E.01, Gaussian, Inc., Wallingford CT, 2009.

[80] A. D. Becke, Density-functional exchange-energy approximation with correct asymptotic behavior, Phys. Rev. A 1988, 38, 3098-3100.

[81] C. Lee, W. Yang, R. G. Parr, Development of the Colle-Salvetti correlation-energy formula into a functional of the electron density, Phys. Rev. B 1988, 37, 785-789.

[82] A. D. Becke, Density-functional thermochemistry. III. The role of exact exchange, J. Chem. Phys. 1993, 98, 5648-5652.

[83] P. J. Stephens, F. J. Devlin, C. F. Chabalowski, M. J. Frisch, Ab Initio Calculation of Vibrational Absorption and Circular Dichroism Spectra Using Density Functional Force Fields, J. Phys. Chem. 1994, 98, 11623-11627.

[84] J.-D. Chai, M. Head-Gordon, Long-range corrected hybrid density functionals with damped atom-atom dispersion corrections, Phys. Chem. Chem. Phys. 2008, $10,6615$.

[85] Y. Zhao, D. G. Truhlar, The M06 suite of density functionals for main group thermochemistry, thermochemical kinetics, noncovalent interactions, excited states, and transition elements: two new functionals and systematic testing of four M06class functionals and 12 other functionals, Theor. Chem. Acc. 2008, 120, 215241.

[86] S. Grimme, Semiempirical hybrid density functional with perturbative secondorder correlation, J. Chem. Phys. 2006, 124, 034108.

[87] A. Austin, G. A. Petersson, M. J. Frisch, F. J. Dobek, G. Scalmani, K. Throssell, A Density Functional with Spherical Atom Dispersion Terms, J. Chem. Theory Comput. 2012, 8, 4989-5007.

[88] L. Goerigk in A Comprehensive Overview of the DFT-D3 London-Dispersion Correction, in: Non-Covalent Interactions in Quantum Chemistry and Physics, Elsevier, 2017, 195-219. 
[89] M. J. S. Dewar, E. G. Zoebisch, E. F. Healy, J. J. P. Stewart, Development and use of quantum mechanical molecular models. 76. AM1: a new general purpose quantum mechanical molecular model, J. Am. Chem. Soc. 1985, 107, 3902-3909.

[90] F. Weigend, R. Ahlrichs, Balanced basis sets of split valence, triple zeta valence and quadruple zeta valence quality for $\mathrm{H}$ to $\mathrm{Rn}$ : Design and assessment of accuracy, Phys. Chem. Chem. Phys. 2005, 7, 3297.

[91] D. Rappoport, F. Furche, Property-optimized Gaussian basis sets for molecular response calculations, J. Chem. Phys. 2010, 133, 134105.

[92] E. Papajak, J. Zheng, X. Xu, H. R. Leverentz, D. G. Truhlar, Perspectives on Basis Sets Beautiful: Seasonal Plantings of Diffuse Basis Functions, J. Chem. Theory Comput. 2011, 7, 3027-3034.

[93] M. Pitoňák, T. Janowski, P. Neogrády, P. Pulay, P. Hobza, Convergence of the CCSD(T) Correction Term for the Stacked Complex Methyl Adenine-Methyl Thymine: Comparison with Lower-Cost Alternatives, J. Chem. Theory Comput. 2009, 5, 1761-1766.

[94] N. O. B. Lüttschwager, Raman Spectroscopy of Conformational Rearrangements at Low Temperatures Folding and Stretching of Alkanes in Supersonic Jets, Springer International Publishing, Cham, 2014.

[95] P. Zielke, Ramanstreuung am Überschallstrahl: Wasserstoffbrückendynamik aus neuer Perspektive, Dissertation, Georg-August-Universität Göttingen, 2007.

[96] M. P. Balanay, D. H. Kim, H. Fan, Revisiting the Formation of Cyclic Clusters in Liquid Ethanol, J. Chem. Phys. 2016, 144, 154302.

[97] M. Šoltésová, L. Benda, M. Peksa, J. Czernek, J. Lang, Determination of Size of Molecular Clusters of Ethanol by Means of NMR Diffusometry and Hydrodynamic Calculations, J. Phys. Chem. B 2014, 118, 6864-6874.

[98] W. O. George, T. Has, M. Fokhray Hossain, B. F. Jones, R. Lewis, Hydrogenbonded forms of ethanol-IR spectra and ab initio computations, J. Chem. Soc. Faraday Trans. 1998, 94, 2701-2708.

[99] N. Nishi, S. Takahashi, M. Matsumoto, A. Tanaka, K. Muraya, T. Takamuku, T. Yamaguchi, Hydrogen-Bonded Cluster Formation and Hydrophobic Solute Association in Aqueous Solutions of Ethanol, J. Phys. Chem. 1995, 99, 462-468.

[100] J. Pearson, K. Sastry, E. Herbst, F. C. De Lucia, The Millimeter- and Submillimeter-Wave Spectrum of Gauche-Ethyl Alcohol, J. Mol. Spectrosc. 1996, 175, 246-261.

[101] J. R. Durig, W. E. Bucy, C. J. Wurrey, L. A. Carreira, Raman spectra of gases. XVI. Torsional transitions in ethanol and ethanethiol, J. Phys. Chem. 1975, 79, 988-993.

[102] H. L. Fang, R. L. Swofford, Molecular conformers in gas-phase ethanol: A temperature study of vibrational overtones, Chem. Phys. Lett. 1984, 105, 5-11.

[103] J. Durig, R. Larsen, Torsional vibrations and barriers to internal rotation for ethanol and 2,2,2-trifluoroethanol, J. Mol. Struct. 1990, 238, 195-222.

[104] J. C. Pearson, K. V. L. N. Sastry, E. Herbst, F. C. De Lucia, Gauche Ethyl Alcohol: Laboratory Assignments and Interstellar Identification, Astrophys. J. 1997, 480, 420-431.

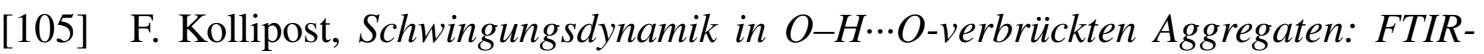
Spektroskopie vom Nah- bis zum Ferninfraroten, Dissertation, Georg-AugustUniversität Göttingen, 2015.

[106] V. Dyczmons, Dimers of Ethanol, J. Phys. Chem. A 2004, 108, 2080-2086. 
[107] J. D. Weibel, C. F. Jackels, R. L. Swofford, Experimental and ab initio investigation of the O-H overtone vibration in ethanol, J. Chem. Phys. 2002, 117, 42454254.

[108] C. Emmeluth, V. Dyczmons, T. Kinzel, P. Botschwina, M. A. Suhm, M. Yáñez, Combined jet relaxation and quantum chemical study of the pairing preferences of ethanol, Phys. Chem. Chem. Phys. 2005, 7, 991-997.

[109] B. Hartwig, Aggregation und Isomerisierung eines realistischen Peptidbindungsmodells, Bachelorarbeit, Georg-August-Universität Göttingen, 2016.

[110] D. Loru, I. Peña, M. Sanz, Ethanol dimer: Observation of three new conformers by broadband rotational spectroscopy, J. Mol. Spectrosc. 2017, 335, 93-101.

[111] A. Malloum, J. J. Fifen, J. Conradie, Exploration of the potential energy surface of the ethanol hexamer, J. Chem. Phys. 2019, 150, 124308.

[112] P.-G. Jönsson, Hydrogen bond studies. CXIII. The crystal structure of ethanol at $87 \mathrm{~K}$, Acta Crystallogr. B 1976, 32, 232-235.

[113] D. R. Allan, S. J. Clark, Comparison of the high-pressure and low-temperature structures of ethanol and acetic acid, Phys. Rev. B 1999, 60, 6328-6334.

[114] D. R. Allan, S. Parsons, S. J. Teat, High-pressure single-crystal X-ray diffraction facilities on station 9.8 at the SRS Daresbury Laboratory - hydrogen location in the high-pressure structure of ethanol, J. Synchrotron Radiat. 2001, 8, 10-17.

[115] C. L. Bull, S. A. Barnett, D. R. Allan, W. G. Marshall, Hydrogen bonding in ethanol - a high pressure neutron diffraction study, High Press. Res. 2019, 39, 179-184.

[116] M. Ehbrecht, F. Huisken, Vibrational Spectroscopy of Ethanol Molecules and Complexes Selectively Prepared in the Gas Phase and Adsorbed on Large Argon Clusters, J. Phys. Chem. A 1997, 101, 7768-7777.

[117] L. González, O. Mó, M. Yáñez, Density functional theory study on ethanol dimers and cyclic ethanol trimers, J. Chem. Phys. 1999, 111, 3855-3861.

[118] S. Coussan, M. E. Alikhani, J. P. Perchard, W. Q. Zheng, Infrared-Induced Isomerization of Ethanol Dimers Trapped in Argon and Nitrogen Matrices: Monochromatic Irradiation Experiments and DFT Calculations, J. Phys. Chem. A 2000, 104, 5475-5483.

[119] R. A. Provencal, R. N. Casaes, K. Roth, J. B. Paul, C. N. Chapo, R. J. Saykally, G. S. Tschumper, H. F. Schaefer, Hydrogen Bonding in Alcohol Clusters: A Comparative Study by Infrared Cavity Ringdown Laser Absorption Spectroscopy, J. Phys. Chem. A 2000, 104, 1423-1429.

[120] M. L. Senent, Y. G. Smeyers, R. Domínguez-Gómez in New Trends in Quantum Systems in Chemistry and Physics, Vol. 6, Kluwer Academic Publishers, Dordrecht, 2002, 359-374.

[121] J. P. I. Hearn, R. V. Cobley, B. J. Howard, High-resolution spectroscopy of induced chiral dimers: A study of the dimers of ethanol by Fourier transform microwave spectroscopy, J. Chem. Phys. 2005, 123, 134324.

[122] S. Patel, C. L. Brooks, Structure, thermodynamics, and liquid-vapor equilibrium of ethanol from molecular-dynamics simulations using nonadditive interactions, J. Chem. Phys. 2005, 123, 164502.

[123] A. Vargas-Caamal, F. Ortiz-Chi, D. Moreno, A. Restrepo, G. Merino, J. L. Cabellos, The rich and complex potential energy surface of the ethanol dimer, Theor. Chem. Acc. 2015, 134: 16, 1-9. 
[124] M. Umer, W. A. Kopp, K. Leonhard, Efficient yet accurate approximations for $a b$ initio calculations of alcohol cluster thermochemistry, J. Chem. Phys. 2015, 143, 214306.

[125] I. A. Finneran, P. B. Carroll, G. J. Mead, G. A. Blake, Hydrogen bond competition in the ethanol-methanol dimer, Phys. Chem. Chem. Phys. 2016, 18, 2256522572.

[126] S. Oswald, M. Wallrabe, M. A. Suhm, Cooperativity in Alcohol-Nitrogen Complexes: Understanding Cryomatrices through Slit Jet Expansions, J. Phys. Chem. A 2017, 121, 3411-3422.

[127] T. Häber, U. Schmitt, M. A. Suhm, FTIR-spectroscopy of molecular clusters in pulsed supersonic slit-jet expansions, Phys. Chem. Chem. Phys. 1999, 1, 55735582.

[128] C. Emmeluth, Donor-/Akzeptorisomerie in wasserstoffbrückengebundenen Dimeren, Dissertation, Georg-August-Universität Göttingen, 2005.

[129] P. Zielke, M. A. Suhm, Concerted proton motion in hydrogen-bonded trimers: A spontaneous Raman scattering perspective, Phys. Chem. Chem. Phys. 2006, 8, 2826-2830.

[130] R. W. Larsen, P. Zielke, M. A. Suhm, Hydrogen-bonded OH stretching modes of methanol clusters: A combined IR and Raman isotopomer study, J. Chem. Phys. 2007, 126, 194307.

[131] C. Emmeluth, V. Dyczmons, M. A. Suhm, Tuning the Hydrogen Bond Donor/Acceptor Isomerism in Jet-Cooled Mixed Dimers of Aliphatic Alcohols, J. Phys. Chem. A 2006, 110, 2906-2915.

[132] T. L. Fischer, Schwingungsspektroskopie von zwischenmolekularen Waagen, Masterarbeit, Georg-August-Universität Göttingen, 2019.

[133] T. Scharge, T. Häber, M. A. Suhm, Quantitative chirality synchronization in trifluoroethanol dimers, Phys. Chem. Chem. Phys. 2006, 8, 4664-4667.

[134] T. Scharge, C. Cézard, P. Zielke, A. Schütz, C. Emmeluth, M. A. Suhm, A peptide co-solvent under scrutiny: self-aggregation of 2,2,2-trifluoroethanol, Phys. Chem. Chem. Phys. 2007, 9, 4472.

[135] J. Thomas, Y. Xu, Chirality Synchronization in Trifluoroethanol Dimer Revisited: The Missing Heterochiral Dimer, J. Phys. Chem. Lett. 2014, 5, 1850-1855.

[136] D. Loru, I. Peña, M. E. Sanz, The role of secondary interactions on the preferred conformers of the fenchone-ethanol complex, Phys. Chem. Chem. Phys. 2019, 21, 2938-2945.

[137] A. Mukhopadhyay, W. T. Cole, R. J. Saykally, The water dimer I: Experimental characterization, Chem. Phys. Lett. 2015, 633, 13-26.

[138] F. Lovas, S. Belov, M. Tretyakov, W. Stahl, R. Suenram, The $a$-Type $K=0$ Microwave Spectrum of the Methanol Dimer, J. Mol. Spectrosc. 1995, 170, 478492.

[139] G. R. Desiraju, T. Steiner, The weak hydrogen bond: in structural chemistry and biology, 1. Auflage, Oxford University Press, Oxford, 2001.

[140] S. L. Boyd, R. J. Boyd, A Density Functional Study of Methanol Clusters, J. Chem. Theory Comput. 2007, 3, 54-61.

[141] P. Golub, I. Doroshenko, V. Pogorelov, Quantum-chemical modeling of energy parameters and vibrational spectra of chain and cyclic clusters of monohydric alcohols, Phys. Lett. A 2014, 378, 1937-1944.

[142] F. N. Keutsch, J. D. Cruzan, R. J. Saykally, The Water Trimer, Chem. Rev. 2003, 103, 2533-2578. 
[143] B. D. Kay, A. Castleman Jr, Molecular beam electric deflection study of the hydrogen-bonded water, methanol, and ethanol clusters $\left(\mathrm{H}_{2} \mathrm{O}\right)_{N},\left(\mathrm{CH}_{3} \mathrm{OH}\right)_{N}$, and $\left(\mathrm{C}_{2} \mathrm{H}_{5} \mathrm{OH}\right)_{N}$, J. Phys. Chem. 1985, 89, 4867-4868.

[144] Y. J. Hu, H. B. Fu, E. R. Bernstein, Infrared plus vacuum ultraviolet spectroscopy of neutral and ionic ethanol monomers and clusters, J. Chem. Phys. 2006, 125, 154305.

[145] M. Weimann, M. Fárník, M. A. Suhm, M. Alikhani, J. Sadlej, Cooperative and anticooperative mixed trimers of $\mathrm{HCl}$ and methanol, J. Mol. Struct. 2006, 790, $18-26$.

[146] N. A. Seifert, A. L. Steber, J. L. Neill, C. Pérez, D. P. Zaleski, B. H. Pate, A. Lesarri, The interplay of hydrogen bonding and dispersion in phenol dimer and trimer: structures from broadband rotational spectroscopy, Phys. Chem. Chem. Phys. 2013, 15, 11468.

[147] J. Thomas, N. A. Seifert, W. Jäger, Y. Xu, A Direct Link from the Gas to the Condensed Phase: A Rotational Spectroscopic Study of 2,2,2-Trifluoroethanol Trimers, Angew. Chem. Int. Ed. 2017, 56, 6289-6293.

[148] N. Borho, M. A. Suhm, Self-organization of lactates in the gas phase, Org. Biomol. Chem. 2003, 1, 4351.

[149] N. O. B. Lüttschwager, T. N. Wassermann, R. A. Mata, M. A. Suhm, The Last Globally Stable Extended Alkane, Angew. Chem. Int. Ed. 2013, 52, 463-466.

[150] B. Hartwig, Ramanspektroskopie zur Konformation und Aggregation von Cyclohexanderivaten, Masterarbeit, Georg-August-Universität Göttingen, 2018.

[151] R. Ludwig, F. Weinhold, T. C. Farrar, Quantum cluster equilibrium theory of liquids: molecular clusters and thermodynamics of liquid ethanol, Mol. Phys. 1999, 97, 465-477.

[152] M. Huelsekopf, R. Ludwig, Temperature dependence of hydrogen bonding in alcohols, J. Mol. Liq. 2000, 85, 105-125.

[153] G. Matisz, A.-M. Kelterer, W. M. F. Fabian, S. Kunsági-Máté, Application of the Quantum Cluster Equilibrium (QCE) Model for the Liquid Phase of Primary Alcohols Using B3LYP and B3LYP-D DFT Methods, J. Phys. Chem. B 2011, 115, 3936-3941.

[154] S. M. Mejía, E. Flórez, F. Mondragón, An orbital and electron density analysis of weak interactions in ethanol-water, methanol-water, ethanol and methanol small clusters, J. Chem. Phys. 2012, 136, 144306.

[155] A. K. Sum, S. I. Sandler, Ab Initio Calculations of Cooperativity Effects on Clusters of Methanol, Ethanol, 1-Propanol, and Methanethiol, J. Phys. Chem. A 2000, 104, 1121-1129.

[156] F. Huisken, M. Kaloudis, M. Koch, O. Werhahn, Experimental study of the O-H ring vibrations of the methanol trimer, J. Chem. Phys. 1996, 105, 8965-8968.

[157] U. Buck, J.-G. Siebers, R. J. Wheatley, Structure and vibrational spectra of methanol clusters from a new potential model, J. Chem. Phys. 1998, 108, 20-32.

[158] R. A. Provencal, J. B. Paul, K. Roth, C. Chapo, R. N. Casaes, R. J. Saykally, G. S. Tschumper, H. F. Schaefer, Infrared cavity ringdown spectroscopy of methanol clusters: Single donor hydrogen bonding, J. Chem. Phys. 1999, 110, 4258-4267.

[159] G. S. Tschumper, J. M. Gonzales, H. F. Schaefer, Assignment of the infrared spectra of the methanol trimer, J. Chem. Phys. 1999, 111, 3027-3034.

[160] U. Buck, F. Huisken, Infrared Spectroscopy of Size-Selected Water and Methanol Clusters, Chem. Rev. 2000, 100, 3863-3890. 
[161] M. Mandado, A. M. Graña, R. A. Mosquera, On the structures of the methanol trimer and their cooperative effects, Chem. Phys. Lett. 2003, 381, 22-29.

[162] M. A. Suhm, D. J. Nesbitt, Potential surfaces and dynamic of weakly bound trimers: perspectives from high resolution IR spectroscopy, Chem. Soc. Rev. 1995, 24,45 .

[163] M. I. Sulaiman, S. Yang, A. M. Ellis, Infrared Spectroscopy of Methanol and Methanol/Water Clusters in Helium Nanodroplets: The OH Stretching Region, J. Phys. Chem. A 2017, 121, 771-776.

[164] M. V. Vener, J. Sauer, Vibrational spectra of the methanol tetramer in the OH stretch region. Two cyclic isomers and concerted proton tunneling, J. Chem. Phys. 2001, 114, 2623-2628.

[165] M. Mandziuk, On the tunneling splitting in a cyclic water trimer, Chem. Phys. Lett. 2016, 661, 263-268.

[166] J. R. De la Vega, Role of symmetry in the tunneling of the proton in doubleminimum potentials, Acc. Chem. Res. 1982, 15, 185-191.

[167] C. F. Su, C. Quade, Microwave Spectra of gauche $\mathrm{CH}_{2} \mathrm{DCH}_{2} \mathrm{OH}$ Including Excited States of the -OH Torsion, J. Mol. Spectrosc. 1998, 188, 1-8.

[168] T. N. Wassermann, D. Luckhaus, S. Coussan, M. A. Suhm, Proton tunneling estimates for malonaldehyde vibrations from supersonic jet and matrix quenching experiments, Phys. Chem. Chem. Phys. 2006, 8, 2344-2348.

[169] S. M. Mejía, J. F. Espinal, F. Mondragón, Cooperative effects on the structure and stability of (ethanol) ${ }_{3}$-water, (methanol) $)_{3}$-water heterotetramers and (ethanol) ${ }_{4}$, (methanol) 4 tetramers, J. Mol. Struct. Theochem 2009, 901, 186-193.

[170] J. David, D. Guerra, A. Restrepo, Structural Characterization of the (Methanol) 4 Potential Energy Surface, J. Phys. Chem. A 2009, 113, 10167-10173.

[171] J. F. Pérez, C. Z. Hadad, A. Restrepo, Structural studies of the water tetramer, Int. J. Quantum Chem. 2008, 108, 1653-1659.

[172] M. Mella, K. D. M. Harris, Pathways for hydrogen bond switching in a tetrameric methanol cluster, Phys. Chem. Chem. Phys. 2009, 11, 11340.

[173] D. L. Jadhav, N. Karthick, P. Kannan, R. Shanmugam, A. Elangovan, G. Arivazhagan, Molecular interaction forces in acetone + ethanol binary liquid solutions: FTIR and theoretical studies, J. Mol. Struct. 2017, 1130, 497-502.

[174] F. Kollipost, K. E. Otto, M. A. Suhm, A Symmetric Recognition Motif between Vicinal Diols: The Fourfold Grip in Ethylene Glycol Dimer, Angew. Chem. Int. Ed. 2016, 55, 4591-4595.

[175] D. Sun, Y.-H. Li, F.-J. Liu, H.-J. Hao, R.-B. Huang, L.-S. Zheng, Characterization of an unprecedented (ethanol) 4 cluster in a novel photoluminescent silver(I) coordination polymer, Inorg. Chem. Commun. 2011, 14, 1871-1875.

[176] Y. Nishimura, Y.-P. Lee, S. Irle, H. A. Witek, Critical interpretation of $\mathrm{CH}-$ and $\mathrm{OH}-$ stretching regions for infrared spectra of methanol clusters $\left(\mathrm{CH}_{3} \mathrm{OH}\right)_{n}(n=$ 2-5) using self-consistent-charge density functional tight-binding molecular dynamics simulations, J. Chem. Phys. 2014, 141, 094303.

[177] C. Perez, M. T. Muckle, D. P. Zaleski, N. A. Seifert, B. Temelso, G. C. Shields, Z. Kisiel, B. H. Pate, Structures of Cage, Prism, and Book Isomers of Water Hexamer from Broadband Rotational Spectroscopy, Science 2012, 336, 897-901.

[178] K. Nauta, Formation of Cyclic Water Hexamer in Liquid Helium: The Smallest Piece of Ice, Science 2000, 287, 293-295. 
[179] C. Steinbach, M. Fárník, I. Ettischer, J. Siebers, U. Buck, Isomeric transitions in size-selected methanol hexamers probed by OH-stretch spectroscopy, Phys. Chem. Chem. Phys. 2006, 8, 2752-2758.

[180] K. Hull, R. M. Soliday, I. Sumner, P. L. Raston, Comment on "Revisiting the formation of cyclic clusters in liquid ethanol" [J. Chem. Phys. 144, 154302 (2016)], J. Chem. Phys. 2019, 150, 057101.

[181] A. Malloum, J. J. Fifen, J. Conradie, Exploration of the Potential Energy Surfaces of Small Ethanol Clusters, Phys. Chem. Chem. Phys. 2020, 22, 13201-13213.

[182] Y. J. Hu, H. B. Fu, E. R. Bernstein, Infrared plus vacuum ultraviolet spectroscopy of neutral and ionic methanol monomers and clusters: New experimental results, J. Chem. Phys. 2006, 125, 154306.

[183] H. B. Fu, Y. J. Hu, E. R. Bernstein, IR+Vacuum ultraviolet (118 nm) nonresonant ionization spectroscopy of methanol monomers and clusters: Neutral cluster distribution and size-specific detection of the $\mathrm{OH}$ stretch vibrations, J. Chem. Phys. 2006, 124, 024302.

[184] H.-L. Han, C. Camacho, H. A. Witek, Y.-P. Lee, Infrared absorption of methanol clusters $\left(\mathrm{CH}_{3} \mathrm{OH}\right)_{n}$ with $n=2-6$ recorded with a time-of-flight mass spectrometer using infrared depletion and vacuum-ultraviolet ionization, J. Chem. Phys. 2011, $134,144309$.

[185] U. Buck, I. Ettischer, Vibrational predissociation spectra of size selected methanol clusters: New experimental results, J. Chem. Phys. 1998, 108, 33-38.

[186] M. Behrens, R. Fröchtenicht, M. Hartmann, J.-G. Siebers, U. Buck, F. C. Hagemeister, Vibrational spectroscopy of methanol and acetonitrile clusters in cold helium droplets, J. Chem. Phys. 1999, 111, 2436-2443.

[187] D. Zimmermann, T. Häber, H. Schaal, M. A. Suhm, Hydrogen bonded rings, chains and lassos: the case of $t$-butyl alcohol clusters, Mol. Phys. 2001, 99, 413425.

[188] W. Klopper, M. Quack, M. A. Suhm, Explicitly correlated coupled cluster calculations of the dissociation energies and barriers to concerted hydrogen exchange of $(\mathrm{HF})_{n}$ oligomers $(n=2,3,4,5)$, Mol. Phys. 1998, 94, 105-119.

[189] W. Li, L. Evangelisti, Q. Gou, W. Caminati, R. Meyer, The Barrier to Proton Transfer in the Dimer of Formic Acid: A Pure Rotational Study, Angew. Chem. Int. Ed. 2019, 58, 859-865.

[190] T. N. Wassermann, M. A. Suhm, P. Roubin, S. Coussan, Isomerization around $\mathrm{C}-\mathrm{C}$ and $\mathrm{C}-\mathrm{O}$ bonds in 1-propanol: Collisional relaxation in supersonic jets and selective IR photo-isomerization in cryogenic matrices, J. Mol. Struct. 2012, 1025, 20-32.

[191] C. Červinka, M. Fulem, State-of-the-Art Calculations of Sublimation Enthalpies for Selected Molecular Crystals and Their Computational Uncertainty, J. Chem. Theory Comput. 2017, 13, 2840-2850.

[192] G. Nichols, S. Kweskin, M. Frericks, S. Reiter, G. Wang, J. Orf, B. Carvallo, D. Hillesheim, J. Chickos, Evaluation of the Vaporization, Fusion, and Sublimation Enthalpies of the 1-Alkanols: The Vaporization Enthalpy of 1-, 6-, 7-, and 9-Heptadecanol, 1-Octadecanol, 1-Eicosanol, 1-Docosanol, 1-Hexacosanol, and Cholesterol at $T=298.15 \mathrm{~K}$ by Correlation Gas Chromatography, J. Chem. Eng. Data 2006, 51, 475-482.

[193] J. R. Dixon, W. O. George, M. Fokhray Hossain, R. Lewis, J. M. Price, Hydrogenbonded forms of methanol IR spectra and ab initio calculations, J. Chem. Soc. Faraday Trans. 1997, 93, 3611-3618. 
[194] P.-J. Hsu, K.-L. Ho, S.-H. Lin, J.-L. Kuo, Exploration of hydrogen bond networks and potential energy surfaces of methanol clusters using a two-stage clustering algorithm, Phys. Chem. Chem. Phys. 2017, 19, 544-556.

[195] S. Coussan, A. Loutellier, J. P. Perchard, S. Racine, A. Peremans, A. Tadjeddine, W. Q. Zheng, Infrared laser induced isomerization of methanol polymers trapped in nitrogen matrix. I. Trimers, J. Chem. Phys. 1997, 107, 6526-6540.

[196] M. T. Kirchner, D. Das, R. Boese, Cocrystallization with Acetylene: Molecular Complex with Methanol, Cryst. Growth Des. 2008, 8, 763-765.

[197] T.-J. Lin, C.-R. Hsing, C.-M. Wei, J.-L. Kuo, Structure prediction of the solid forms of methanol: an ab initio random structure searching approach, Phys. Chem. Chem. Phys. 2016, 18, 2736-2746.

[198] D. R. Moberg, D. Becker, C. W. Dierking, F. Zurheide, B. Bandow, U. Buck, A. Hudait, V. Molinero, F. Paesani, T. Zeuch, The end of ice I, Proc. Natl. Acad. Sci. 2019, 201914254.

[199] I. Dauster, M. A. Suhm, U. Buck, T. Zeuch, Experimental and theoretical study of the microsolvation of sodium atoms in methanol clusters: differences and similarities to sodium-water and sodium-ammonia, Phys. Chem. Chem. Phys. 2008, $10,83-95$.

[200] R. M. Forck, I. Dauster, U. Buck, T. Zeuch, Sodium Microsolvation in Ethanol: Common Features of $\mathrm{Na}(\mathrm{HO}-\mathrm{R})_{n}\left(\mathrm{R}=\mathrm{H}, \mathrm{CH}_{3}, \mathrm{C}_{2} \mathrm{H}_{5}\right)$ Clusters, J. Phys. Chem. A 2011, 115, 6068-6076.

[201] R. M. Forck, C. C. Pradzynski, S. Wolff, M. Ončák, P. Slavíček, T. Zeuch, Size resolved infrared spectroscopy of $\mathrm{Na}\left(\mathrm{CH}_{3} \mathrm{OH}\right)_{n}(n=4-7)$ clusters in the OH stretching region: unravelling the interaction of methanol clusters with a sodium atom and the emergence of the solvated electron, Phys. Chem. Chem. Phys. 2012, 14, 3004.

[202] D. Troya, Reactivity Consequences of Conformational Isomerism in 1-Propanol, J. Phys. Chem. A 2019, 123, 1044-1050.

[203] H. Kolbe, Ueber den natürlichen Zusammenhang der organischen mit den unorganischen Verbindungen, die wissenschaftliche Grundlage zu einer naturgemässen Classification der organischen chemischen Körper, Ann. Chem. Pharm. 1860, 113, 293-332.

[204] L. Kolesniková, A. Daly, J. Alonso, B. Tercero, J. Cernicharo, The millimeter wave tunneling-rotational spectrum of phenol, J. Mol. Spectrosc. 2013, 289, 1320.

[205] M. A. Suhm in Advances in Chemical Physics, Vol. 142, (Ed.: S. A. Rice), John Wiley \& Sons, Inc., Hoboken, NJ, USA, 2008, 1-57.

[206] M. Heger, J. Altnöder, A. Poblotzki, M. A. Suhm, To $\pi$ or not to $\pi$ - how does methanol dock onto anisole?, Phys. Chem. Chem. Phys. 2015, 17, 13045-13052.

[207] H. C. Gottschalk, J. Altnöder, M. Heger, M. A. Suhm, Control over the HydrogenBond Docking Site in Anisole by Ring Methylation, Angew. Chem. Int. Ed. 2016, 55, 1921-1924.

[208] C. Medcraft, S. Zinn, M. Schnell, A. Poblotzki, J. Altnöder, M. Heger, M. A. Suhm, D. Bernhard, A. Stamm, F. Dietrich, M. Gerhards, Aromatic embedding wins over classical hydrogen bonding - a multi-spectroscopic approach for the diphenyl ether-methanol complex, Phys. Chem. Chem. Phys. 2016, 18, 2597525983.

[209] A. Poblotzki, J. Altnöder, M. A. Suhm, Subtle solvation behaviour of a biofuel additive: the methanol complex with 2,5-dimethylfuran, Phys. Chem. Chem. Phys. 2016, 18, 27265-27271. 
[210] A. Poblotzki, H. C. Gottschalk, M. A. Suhm, Tipping the Scales: Spectroscopic Tools for Intermolecular Energy Balances, J. Phys. Chem. Lett. 2017, 8, 56565665.

[211] D. Bernhard, M. Fatima, A. Poblotzki, A. L. Steber, C. Pérez, M. A. Suhm, M. Schnell, M. Gerhards, Dispersion-controlled docking preference: multispectroscopic study on complexes of dibenzofuran with alcohols and water, Phys. Chem. Chem. Phys. 2019, 21, 16032-16046.

[212] C. Zimmermann, H. C. Gottschalk, M. A. Suhm, Three-dimensional docking of alcohols to ketones: an experimental benchmark based on acetophenone solvation energy balances, Phys. Chem. Chem. Phys. 2020, 22, 2870-2877.

[213] P. E. Hansen, J. Spanget-Larsen, NMR and IR Investigations of Strong Intramolecular Hydrogen Bonds, Molecules 2017, 22, 552.

[214] Y. Yu, W. Fan, Y. Wang, X. Zhou, J. Sun, S. Liu, Probe of Alcohol Structures in the Gas and Liquid States Using C-H Stretching Raman Spectroscopy, Sensors 2018, 18, 2061.

[215] Z. Kisiel, O. Dorosh, A. Maeda, I. R. Medvedev, F. C. D. Lucia, E. Herbst, B. J. Drouin, J. C. Pearson, S. T. Shipman, Determination of precise relative energies of conformers of n-propanol by rotational spectroscopyw, 2010, 13.

[216] M. Albrecht, J. Will, M. A. Suhm, Chiralitätserkennung bei Menthol und Neomenthol: bevorzugte Bildung homokonfigurierter Aggregate, Angew. Chem. 2010, 122, 6339-6342.

[217] D. Schmitz, V. A. Shubert, T. Betz, M. Schnell, Exploring the conformational landscape of menthol, menthone, and isomenthone: a microwave study, Front. Chem. 2015, 3.

[218] N. Guchhait, T. Ebata, N. Mikami, Discrimination of Rotamers of Aryl Alcohol Homologues by Infrared-Ultraviolet Double-Resonance Spectroscopy in a Supersonic Jet, J. Am. Chem. Soc. 1999, 121, 5705-5711.

[219] N. Guchhait, T. Ebata, N. Mikami, Structures of hydrogen-bonded clusters of benzyl alcohol with water investigated by infrared-ultraviolet double resonance spectroscopy in supersonic jet, J. Chem. Phys. 1999, 111, 8438-8447.

[220] M. Mons, E. G. Robertson, J. P. Simons, Intra- and Intermolecular $\pi$-Type Hydrogen Bonding in Aryl Alcohols: UV and IR-UV Ion Dip Spectroscopy, J. Phys. Chem. A 2000, 104, 1430-1437.

[221] A. P. Scott, L. Radom, Harmonic Vibrational Frequencies: An Evaluation of Hartree-Fock, Møller-Plesset, Quadratic Configuration Interaction, Density Functional Theory, and Semiempirical Scale Factors, J. Phys. Chem. 1996, 100, 16502-16513.

[222] I. M. Alecu, J. Zheng, Y. Zhao, D. G. Truhlar, Computational Thermochemistry: Scale Factor Databases and Scale Factors for Vibrational Frequencies Obtained from Electronic Model Chemistries, J. Chem. Theory Comput. 2010, 6, 28722887.

[223] M. P. Andersson, P. Uvdal, New Scale Factors for Harmonic Vibrational Frequencies Using the B3LYP Density Functional Method with the Triple- $\zeta$ Basis Set 6-311+G(d,p), J. Phys. Chem. A 2005, 109, 2937-2941.

[224] P. Sinha, S. E. Boesch, C. Gu, R. A. Wheeler, A. K. Wilson, Harmonic Vibrational Frequencies: Scaling Factors for HF, B3LYP, and MP2 Methods in Combination with Correlation Consistent Basis Sets, J. Phys. Chem. A 2004, 108, 9213-9217.

[225] J. P. Merrick, D. Moran, L. Radom, An Evaluation of Harmonic Vibrational Frequency Scale Factors, J. Phys. Chem. A 2007, 111, 11683-11700. 
[226] G. Rauhut, P. Pulay, Transferable Scaling Factors for Density Functional Derived Vibrational Force Fields, J. Phys. Chem. 1995, 99, 3093-3100.

[227] Y. Bouteiller, J.-C. Gillet, G. Grégoire, J. P. Schermann, Transferable Specific Scaling Factors for Interpretation of Infrared Spectra of Biomolecules from Density Functional Theory, J. Phys. Chem. A 2008, 112, 11656-11660.

[228] H. Yoshida, K. Takeda, J. Okamura, A. Ehara, H. Matsuura, A New Approach to Vibrational Analysis of Large Molecules by Density Functional Theory: Wavenumber-Linear Scaling Method, J. Phys. Chem. A 2002, 106, 3580-3586.

[229] M. Sibaev, D. L. Crittenden, Quadratic Corrections to Harmonic Vibrational Frequencies Outperform Linear Models, J. Phys. Chem. A 2015, 119, 13107-13112.

[230] H. Yoshida, A. Ehara, H. Matsuura, Density functional vibrational analysis using wavenumber-linear scale factors, Chem. Phys. Lett. 2000, 325, 477-483.

[231] J. Spanget-Larsen, B. K. Hansen, P. E. Hansen, OH stretching frequencies in systems with intramolecular hydrogen bonds: Harmonic and anharmonic analyses, Chem. Phys. 2011, 389, 107-115.

[232] J. Baker, A. A. Jarzecki, P. Pulay, Direct Scaling of Primitive Valence Force Constants: An Alternative Approach to Scaled Quantum Mechanical Force Fields, $J$. Phys. Chem. A 1998, 102, 1412-1424.

[233] C. Legler, N. Brown, R. Dunbar, M. Harness, K. Nguyen, O. Oyewole, W. Collier, Scaled Quantum Mechanical scale factors for vibrational calculations using alternate polarized and augmented basis sets with the B3LYP density functional calculation model, Spectrochim. Acta. A. Mol. Biomol. Spectrosc. 2015, 145, 1524.

[234] E. Kohls, M. Stein, Vibrational Scaling Factors for Rh(I) Carbonyl Compounds in Homogeneous Catalysis, Contrib. Sect. Nat. Math. Biotech. Sci. 2017, 38, 43.

[235] C. Cézard, C. A. Rice, M. A. Suhm, OH-Stretching Red Shifts in Bulky Hydrogen-Bonded Alcohols: Jet Spectroscopy and Modeling, J. Phys. Chem. A 2006, 110, 9839-9848.

[236] Z. Xue, Einflüsse von Konstitution und Konformation auf die $\mathrm{O}-\mathrm{H}$ Streckschwingungen gesättigter einwertiger $C_{5}$-Alkohole, Diplomarbeit, GeorgAugust-Universität Göttingen, 2007.

[237] D. Falkowski, Warum duften (R)-Limonen und sein Spiegelbild unterschiedlich?, Bachelorarbeit, Georg-August-Universität Göttingen, 2010.

[238] K. E. Otto, Z. Xue, P. Zielke, M. A. Suhm, The Raman spectrum of isolated water clusters, Phys. Chem. Chem. Phys. 2014, 16, 9849.

[239] K. Le Barbu-Debus, N. Seurre, F. Lahmani, A. Zehnacker-Rentien, Formation of hydrogen-bonded bridges in jet-cooled complexes of a chiral chromophore as studied by IR/UV double resonance spectroscopy. ( \pm )2-Naphthyl-1ethanol/(methanol) ${ }_{n=1,2}$ complexes, Phys Chem Chem Phys 2002, 4, 4866-4876.

[240] A. Bouchet, J. Altnöder, M. Broquier, A. Zehnacker, IR-UV spectroscopy of jetcooled 1-indanol: Restriction of the conformational space by hydration, J. Mol. Struct. 2014, 1076, 344-351.

[241] G. V. Hartland, B. F. Henson, V. A. Venturo, P. M. Felker, Ionization-loss stimulated Raman spectroscopy of jet-cooled hydrogen-bonded complexes containing phenols, J. Phys. Chem. 1992, 96, 1164-1173.

[242] Y. Shen, C.-F. Chen, Helicenes: Synthesis and Applications, Chem. Rev. 2012, $112,1463-1535$. 
[243] J. van der Maas, E. Lutz, Structural information from $\mathrm{OH}$ stretching frequencies monohydric saturated alcohols, Spectrochim. Acta Part Mol. Spectrosc. 1974, 30, 2005-2019.

[244] H. Günzler, H.-U. Gremlich, IR-Spektroskopie Eine Einführung, 4. Auflage, Wiley-VCH, Weinheim, 2012.

[245] J. Caballero, J. Van Der Maas, Molecular mechanics calculation on the hydroxyl stretching frequency in saturated alcohols, J. Mol. Struct. 1985, 127, 57-75.

[246] H. Günzler, H.-U. Gremlich, IR spectroscopy: an introduction, Wiley-VCH, Weinheim, 2002.

[247] C. Willmott, K. Matsuura, Advantages of the mean absolute error (MAE) over the root mean square error (RMSE) in assessing average model performance, Clim. Res. 2005, 30, 79-82.

[248] T. Chai, R. R. Draxler, Root mean square error (RMSE) or mean absolute error (MAE)? - Arguments against avoiding RMSE in the literature, Geosci. Model Dev. 2014, 7, 1247-1250.

[249] J. Phillips, J. Orlando, G. Tyndall, V. Vaida, Integrated intensities of OH vibrational overtones in alcohols, Chem. Phys. Lett. 1998, 296, 377-383.

[250] C. A. Rice, Jet-FTIR spectroscopy of biomolecular model systems, Dissertation, Georg-August-Universität-Göttingen, 2007.

[251] G. Zweifel, H. C. Brown, Hydroboration of Terpenes. II. The Hydroboration of $\alpha$ - and $\beta$-Pinene-The Absolute Configuration of the Dialkylborane from the Hydroboration of $\alpha$-Pinene, J. Am. Chem. Soc. 1964, 86, 393-397.

[252] E. F. Weigand, H.-J. Schneider, ${ }^{13}$ C-NMR-spektroskopische und stereochemische Untersuchungen. XXIII.-Konformationen, ${ }^{13} \mathrm{C}$-NMR-Verschiebungen- und ${ }^{13} \mathrm{C}-{ }^{1} \mathrm{H}-$ Kopplungen bei Pinanverbindungen, Org. Magn. Reson. 1979, 12, 637644.

[253] C. M. Holden, D. Whittaker, Conformational studies of monoterpenes. IIconformational studies of bicyclo[3.1.1] heptane derivatives by ${ }^{13} \mathrm{C}$ NMR, Org. Magn. Reson. 1975, 7, 125-127.

[254] J. Texter, E. S. Stevens, Conformational analysis of pinanols, J. Org. Chem. 1979, 44, 3222-3225.

[255] Z. G. Isaeva, B. A. Arbuzov, Reduction of 2,3-epoxypinane and 3,4-epoxycarane, Bull. Acad. Sci. USSR Div. Chem. Sci. 1959, 8, 1013-1021.

[256] A. Gossauer, Struktur und Reaktivität der Biomoleküle: eine Einführung in die organische Chemie, 1. Auflage, Verl. Helvetica Chimica Acta AG, Zürich, 2006.

[257] C. Boutekedjiret, F. Bentahar, R. Belabbes, J. M. Bessiere, Extraction of rosemary essential oil by steam distillation and hydrodistillation, Flavour Fragr. J. 2003, 18, 481-484.

[258] F. J. Devlin, P. J. Stephens, P. Besse, Conformational Rigidification via Derivatization Facilitates the Determination of Absolute Configuration Using Chiroptical Spectroscopy: A Case Study of the Chiral Alcohol endo-Borneol, J. Org. Chem. 2005, 70, 2980-2993.

[259] F. Gangemi, R. Gangemi, G. Longhi, S. Abbate, Experimental and ab-initio calculated vcd spectra of the first OH-stretching overtone of $(1 R)-(-)$ and $(1 S)-(+)-$ endo-BORNEOL, Phys. Chem. Chem. Phys. 2009, 11, 2683.

[260] G. A. Garcia, H. Soldi-Lose, L. Nahon, I. Powis, Photoelectron Circular Dichroism Spectroscopy in an Orbitally Congested System: The Terpene Endoborneol, J. Phys. Chem. A 2010, 114, 847-853. 
[261] E. Breitmaier, Terpene: Aromen, Düfte, Pharmaka, Pheromone, 2. Auflage, Wiley-VCH, Weinheim, 2005.

[262] E. M. Neeman, T. R. Huet, Identification of the maze in the conformational landscape of fenchol, Phys. Chem. Chem. Phys. 2018, 20, 24708-24715.

[263] P. L. Polavarapu, Chiral analysis: advances in spectroscopy, chromatography and emerging methods, Second edition, Elservier, Amsterdam Oxford Cambridge, 2018.

[264] H. Sekiya in Atom Tunneling Phenomena in Physics, Chemistry and Biology, (Ed.: T. Miyazaki), Springer Berlin Heidelberg, Berlin, Heidelberg, 2004, 201-231.

[265] N. O. Lüttschwager, T. N. Wassermann, S. Coussan, M. A. Suhm, Vibrational tuning of the Hydrogen transfer in malonaldehyde - a combined FTIR and Raman jet study, Mol. Phys. 2013, 111, 2211-2227.

[266] S. Tang, Z. Xia, A. Maris, W. Caminati, Tunnelling splittings in the rotational spectrum of 3-fluoro-benzylalcohol, Chem. Phys. Lett. 2010, 498, 52-55.

[267] L. Evangelisti, L. B. Favero, W. Caminati, Rotational spectrum of 2-fluorobenzyl alcohol, J. Mol. Struct. 2010, 978, 279-281.

[268] L. Evangelisti, G. Feng, Q. Gou, W. Caminati, Rotational spectrum of 2,5difluorobenzyl alcohol, J. Mol. Struct. 2012, 1023, 15-17.

[269] L. Evangelisti, Q. Gou, G. Feng, W. Caminati, Effects of ring fluorination on the transient atropisomerism of benzyl alcohol: the rotational spectrum of 3,4difluorobenzyl alcohol, Mol. Phys. 2013, 111, 1994-1998.

[270] A. M. Pejlovas, W. Lin, S. G. Kukolich, Microwave spectra and structure of the cyclopropanecarboxylic acid-formic acid dimer, J. Chem. Phys. 2015, 143, 124311.

[271] J. Thomas, M. J. Carrillo, A. Serrato, F. Xie, W. Jäger, Y. Xu, W. Lin, Microwave spectrum of the complex of 3,3,3-trifluoro-2-(trifluoromethyl)propanoic acid and formic acid, Mol. Phys. 2019, 117, 1193-1199.

[272] R. Rossetti, R. Rayford, R. C. Haddon, L. E. Brus, Proton localization in an asymmetric double-minimum potential: 2-methyl-9-hydroxyphenalenone, J. Am. Chem. Soc. 1981, 103, 4303-4307.

[273] R. L. Redington, T. E. Redington, M. A. Hunter, R. W. Field, $\tilde{A}^{1} B_{2}-\tilde{X}^{1} A_{1} 26^{v}{ }_{0}$ transitions of ${ }^{18} \mathrm{O}$-enriched tropolone, J. Chem. Phys. 1990, 92, 6456-6462.

[274] H. Sekiya, H. Takesue, Y. Nishimura, Z.-H. Li, A. Mori, H. Takeshita, Electronic spectra of jet-cooled isopropyltropolones in the $S_{1}-S_{0}$ region. Proton tunneling in the $S_{1}$ state, J. Chem. Phys. 1990, 92, 2790-2796.

[275] J. T. Farrell, M. A. Suhm, D. J. Nesbitt, Breaking symmetry with hydrogen bonds: Vibrational predissociation and isomerization dynamics in HF-DF and DF-HF isotopomers, J. Chem. Phys. 1996, 104, 9313-9331.

[276] A. Lesarri, S. T. Shipman, J. L. Neill, G. G. Brown, R. D. Suenram, L. Kang, W. Caminati, B. H. Pate, Interplay of Phenol and Isopropyl Isomerism in Propofol from Broadband Chirped-Pulse Microwave Spectroscopy, J. Am. Chem. Soc. 2010, 132, 13417-13424.

[277] S. Albert, P. Lerch, R. Prentner, M. Quack, Tunneling and Tunneling Switching Dynamics in Phenol and Its Isotopomers from High-Resolution FTIR Spectroscopy with Synchrotron Radiation, Angew. Chem. Int. Ed. 2013, 52, 346-349.

[278] R. T. Saragi, M. Juanes, W. Caminati, A. Lesarri, L. Enríquez, M. Jaraíz, Rotational Spectrum, Tunneling Motions, and Intramolecular Potential Barriers in Benzyl Mercaptan, J. Phys. Chem. A 2019, 123, 8435-8440.

[279] C. Rambaud, H. Trommsdorff, Cooperative proton transfer and tunneling in dye doped benzoic acid crystals, Chem. Phys. Lett. 1999, 306, 124-132. 
[280] M. Ortlieb, Ö. Birer, M. Letzner, G. W. Schwaab, M. Havenith, Observation of Rovibrational Transitions of $\mathrm{HCl},(\mathrm{HCl})_{2}$, and $\mathrm{H}_{2} \mathrm{O}-\mathrm{HCl}$ in Liquid Helium Nanodroplets, J. Phys. Chem. A 2007, 111, 12192-12199.

[281] P. L. Raston, T. Liang, G. E. Douberly, Infrared spectroscopy and tunneling dynamics of the vinyl radical in ${ }^{4}$ He nanodroplets, J. Chem. Phys. 2013, 138, 174302.

[282] E. T. Mengesha, A. Zehnacker-Rentien, J. Sepioł, M. Kijak, J. Waluk, Spectroscopic Study of Jet-Cooled Deuterated Porphycenes: Unusual Isotopic Effects on Proton Tunneling, J. Phys. Chem. B 2015, 119, 2193-2203.

[283] M. D. Schuder, C. M. Lovejoy, R. Lascola, D. J. Nesbitt, High resolution, jetcooled infrared spectroscopy of $(\mathrm{HCl})_{2}$ : Analysis of $v_{1}$ and $v_{2} \mathrm{HCl}$ stretching fundamentals, interconversion tunneling, and mode-specific predissociation lifetimes, J. Chem. Phys. 1993, 99, 4346-4362.

[284] J. Castillo-Chará, A. L. McIntosh, Z. Wang, R. R. Lucchese, J. W. Bevan, Nearinfrared spectra and rovibrational dynamics on a four-dimensional ab initio potential energy surface of (HBr $)_{2}$, J. Chem. Phys. 2004, 120, 10426-10441.

[285] K. Imura, T. Kasai, H. Ohoyama, R. Naaman, Focusing of $\mathrm{DCl}$ and $\mathrm{HCl}$ dimers by an electrostatic hexapole field: The role of the tunneling motion, J. Chem. Phys. 1999, 110, 355-358.

[286] M. Fárník, S. Davis, D. J. Nesbitt, High-resolution IR studies of hydrogen bonded clusters: Large amplitude dynamics in $(\mathrm{HCl})_{n}$, Faraday Discuss. 2001, 118, 6378.

[287] M. D. Schuder, D. J. Nesbitt, High resolution near infrared spectroscopy of $\mathrm{HCl}-\mathrm{DCl}$ and $\mathrm{DCl}-\mathrm{HCl}$ : Relative binding energies, isomer interconversion rates, and mode specific vibrational predissociation, J. Chem. Phys. 1994, 100, 72507267.

[288] Z. Wang, B. A. McElmurry, R. R. Lucchese, J. W. Bevan, L. H. Coudert, Paired hydrogen bonds in the hydrogen halide homodimer $(\mathrm{HI})_{2}$, J. Chem. Phys. 2011, $134,064317$.

[289] D.-Y. Song, Tunneling and energy splitting in an asymmetric double-well potential, Ann. Phys. 2008, 323, 2991-2999.

[290] D.-Y. Song, Localization or tunneling in asymmetric double-well potentials, Ann. Phys. 2015, 362, 609-620.

[291] S. M. H. Halataei, A. J. Leggett, Tunnel Splitting in Asymmetric Double Well Potentials: An Improved WKB Calculation, 2017.

[292] U. Kuenzer, T. S. Hofer, A periodic Numerov approach applied to the torsional tunneling splitting in hydrogen peroxide, aliphatic alcohols and phenol, Chem. Phys. Lett. 2019, 728, 195-200.

[293] J. Pearson, B. Drouin, The ground state torsion-rotation spectrum of propargyl alcohol ( $\left.\mathrm{HCCCH}_{2} \mathrm{OH}\right)$, J. Mol. Spectrosc. 2005, 234, 149-156.

[294] K. A. Utzat, R. K. Bohn, J. A. Montgomery, H. H. Michels, W. Caminati, Rotational Spectrum, Tunneling Motions, and Potential Barriers of Benzyl Alcohol, $J$. Phys. Chem. A 2010, 114, 6913-6916.

[295] L.-H. Xu, X. Wang, T. J. Cronin, D. S. Perry, G. T. Fraser, A. S. Pine, SubDoppler Infrared Spectra and Torsion-Rotation Energy Manifold of Methanol in the CH-Stretch Fundamental Region, J. Mol. Spectrosc. 1997, 185, 158-172.

[296] I. Mukhopadhyay, Y. Duan, K. Takagi, High resolution spectroscopy of $\mathrm{CH}_{3} \mathrm{OD}$ involving the ground and first excited torsional states in the vibrational ground state, Spectrochim. Acta. A. Mol. Biomol. Spectrosc. 1998, 54, 1325-1335. 
[297] W. Li, L. Spada, L. Evangelisti, W. Caminati, Conformational Equilibrium and Potential Energy Functions of the O-H Internal Rotation in the Axial and Equatorial Species of 1-Methylcyclohexanol, J. Phys. Chem. A 2016, 120, 4338-4342.

[298] E. Cohen, B. Drouin, E. Valenzuela, R. Woods, W. Caminati, A. Maris, S. Melandri, The rotational spectrum of tertiary-butyl alcohol, J. Mol. Spectrosc. 2010, $260,77-83$.

[299] M. Juanes, W. Li, L. Spada, L. Evangelisti, A. Lesarri, W. Caminati, Internal dynamics of cyclohexanol and the cyclohexanol-water adduct, Phys. Chem. Chem. Phys. 2019, 21, 3676-3682.

[300] E. Hirota, Y. Kawashima, Internal Rotation of the Hydroxyl Group in Isopropanol and the Chirality of the Gauche Form: Fourier Transform Microwave Spectroscopy of $\left(\mathrm{CH}_{3}\right)_{2} \mathrm{CHOD}$, J. Mol. Spectrosc. 2001, 207, 243-253.

[301] S. Melandri, P. G. Favero, W. Caminati, Detection of the syn conformer of allyl alcohol by free jet microwave spectroscopy, Chem. Phys. Lett. 1994, 223, 541545.

[302] J. N. Macdonald, D. Norbury, J. Sheridan, Microwave spectrum, dipole moment and internal rotation potential function of gauche-cyclopropanol, J. Chem. Soc. Faraday Trans. 2 1978, 74, 1365.

[303] A. Leonov, K.-M. Marstokk, A. de Meijere, H. Møllendal, Microwave Spectrum, Conformational Equilibrium, Intramolecular Hydrogen Bonding, Tunneling, and Quantum Chemical Calculations for 1-Ethenylcyclopropan-1-ol, J. Phys. Chem. A 2000, 104, 4421-4428.

[304] A. Garg, Tunnel splittings for one-dimensional potential wells revisited, Am. J. Phys. 2000, 68, 430-437.

[305] C. S. Tautermann, A. F. Voegele, K. R. Liedl, The ground-state tunneling splitting of various carboxylic acid dimers, J. Chem. Phys. 2004, 120, 631-637.

[306] D. Rueda, O. V. Boyarkin, T. R. Rizzo, I. Mukhopadhyay, D. S. Perry, Torsion-rotation analysis of $\mathrm{OH}$ stretch overtone-torsion combination bands in methanol, J. Chem. Phys. 2002, 116, 91.

[307] J. Kraitchman, Determination of Molecular Structure from Microwave Spectroscopic Data, Am. J. Phys. 1953, 21, 17-24.

[308] S. Herbers, D. A. Obenchain, P. Kraus, D. Wachsmuth, J.-U. Grabow, Blurring out hydrogen: The dynamical structure of teflic acid, J. Chem. Phys. 2018, 148, 194307.

[309] T. Mozga, Z. Prokop, R. Chaloupková, J. Damborský, Chiral aliphatic hydroxy compounds in nature: A review of biological functions and practical applications, Collect. Czechoslov. Chem. Commun. 2009, 74, 1195-1278.

[310] R. L. F. Albo, C. A. Valdez, R. N. Leif, H. A. Mulcahy, C. Koester, Derivatization of pinacolyl alcohol with phenyldimethylchlorosilane for enhanced detection by gas chromatography-mass spectrometry, Anal. Bioanal. Chem. 2014, 406, 52315234.

[311] C. A. Valdez, R. N. Leif, S. Hok, B. R. Hart, Analysis of chemical warfare agents by gas chromatography-mass spectrometry: methods for their direct detection and derivatization approaches for the analysis of their degradation products, Rev. Anal. Chem. 2018, 37, 1-26.

[312] J. Jacobus, Z. Majerski, K. Mislow, P. v. R. Schleyer, Absolute configuration of pinacolyl alcohol, J. Am. Chem. Soc. 1969, 91, 1998-2000. 
[313] D. Zimmermann, Überschallstrahlmessungen und quantenchemische Rechungen zur Aggregation sterisch anspruchsvoller Alkohole, Diplomarbeit, Georg-AugustUniversität Göttingen, 2000.

[314] N. Prusinowska, W. Bendzińska-Berus, M. Jelecki, U. Rychlewska, M. Kwit, Triphenylacetic Acid Amides: Molecular Propellers with Induced Chirality: Molecular Propellers with Induced Chirality, Eur. J. Org. Chem. 2015, 2015, 738-749.

[315] J. E. Anderson, Chemical shifts of the protons within a methyl group in a saturated hydrocarbon. Slow methyl group rotation, Chem. Commun. 1996, 93.

[316] F. R. Jensen, D. S. Noyce, C. H. Sederholm, A. J. Berlin, The energy barrier for the chair-chair interconversion of cyclohexane, J. Am. Chem. Soc. 1960, 82, $1256-1257$.

[317] I. Majerz, I. Natkaniec, Experimental and theoretical IR, R, and INS spectra of 2,2,4,4-tetramethyl-3-t-butyl-pentane-3-ol, J. Mol. Struct. 2006, 788, 93-101.

[318] Z. Malarski, Solid Rotator Phases in 2,2,4,4-Tetramethyl-3-t-butyl-pentane-3-ol (t-Bu 3 COH), Mol. Cryst. Liq. Cryst. 1974, 25, 259-272.

[319] E. M. Arnett, L. E. Small, R. T. McIver, J. S. Miller, Ionization and fragmentation of tri-tert-butylcarbinol. Evidence for a transient tert-butyl carbanion in dimethyl sulfoxide?, J. Org. Chem. 1978, 43, 815-817.

[320] A. K. King, B. J. Howard, An investigation into the relaxation of the conformers of butan-2-ol in a supersonic expansion, J. Mol. Spectrosc. 2009, 257, 205-212.

[321] A. K. King, B. J. Howard, A High-Resolution Microwave Study of the Conformations of Butan-2-ol in a Supersonic Expansion, J. Mol. Spectrosc. 2001, 205, $38-42$.

[322] N. Borho, Spektroskopische Untersuchungen zum Schlüssel-Schloss-Prinzip in der Gasphase, Diplomarbeit, Georg-August-Universität Göttingen, 2001.

[323] A. K. King, B. J. Howard, A microwave study of the hetero-chiral dimer of butan2-ol, Chem. Phys. Lett. 2001, 348, 343-349.

[324] S. Xu, Y. Liu, G. Sha, C. Zhang, J. Xie, The O-H Stretching $\Delta v=3$, 4, and 5 Vibrational Overtones and Conformational Study of 2-Butanol, J. Phys. Chem. A 2000, 104, 8671-8676.

[325] A. K. King, B. J. Howard, A High-Resolution Microwave Study of the Butan-2-ol Argon Complex, J. Mol. Spectrosc. 2002, 214, 97-102.

[326] W. Y. Sohn, M. Kim, S.-S. Kim, Y. D. Park, H. Kang, Solvent-assisted conformational isomerization and the conformationally-pure REMPI spectrum of 3aminophenol, Phys. Chem. Chem. Phys. 2011, 13, 7037.

[327] R. Glaser, G. R. Nichols, Conformational Preferences and Pathways for Enantiomerization and Diastereomerization of Benzyl Alcohol. Data Mining and ab Initio Quantum-Mechanical Study, J. Org. Chem. 2000, 65, 755-766.

[328] T. Schaefer, R. Sebastian, J. Peeling, G. H. Penner, K. Koh, Conformational properties of benzyl alcohol in dilute solution, Can. J. Chem. 1989, 67, 1015-1021.

[329] M. H. Langoor, J. H. van der Maas, On the competitive intramolecular H-bonding in alcohols with two accepting sites, J. Mol. Struct. 1997, 403, 213-229.

[330] V. V. Varfolomeeva, A. V. Terent'ev, Adsorption of benzyl alcohol and 1phenylethanol on graphitized thermal carbon black, Russ. J. Phys. Chem. A 2010, 84, 1592-1597.

[331] T. V. Alves, L. Simón-Carballido, F. R. Ornellas, A. Fernández-Ramos, Hindered rotor tunneling splittings: an application of the two-dimensional non-separable method to benzyl alcohol and two of its fluorine derivatives, Phys. Chem. Chem. Phys. 2016, 18, 8945-8953. 
[332] B. J. Miller, H. G. Kjaergaard, K. Hattori, S.-i. Ishiuchi, M. Fujii, The most stable conformer of benzyl alcohol, Chem. Phys. Lett. 2008, 466, 21-26.

[333] J. Altnöder, S. Oswald, M. A. Suhm, Phenyl- vs Cyclohexyl-Substitution in Methanol: Implications for the $\mathrm{OH}$ Conformation and for Dispersion-Affected Aggregation from Vibrational Spectra in Supersonic Jets, J. Phys. Chem. A 2014, $118,3266-3279$.

[334] V. V. Varfolomeeva, A. V. Terentev, Weak intramolecular and intermolecular hydrogen bonding of benzyl alcohol, 2-phenylethanol and 2-phenylethylamine in the adsorption on graphitized thermal carbon black, Phys. Chem. Chem. Phys. 2015, 17, 24282-24293.

[335] B. Maté, R. D. Suenram, C. Lugez, Microwave studies of three alkylbenzenes: Ethyl, $n$-propyl, and $n$-butylbenzene, J. Chem. Phys. 2000, 113, 192-199.

[336] S. Melandri, A. Maris, P. G. Favero, W. Caminati, Intramolecular Hydrogen Bonds and Conformational Properties of Benzylamine, ChemPhysChem 2001, 2, 172-177.

[337] K. Utzat, A. A. Restrepo, R. K. Bohn, H. H. Michels, Conformational studies of benzyl alcohol and benzyl fluoride, Int. J. Quantum Chem. 2004, 100, 964-972.

[338] R. G. Bird, A. E. Nikolaev, D. W. Pratt, Microwave and UV Excitation Spectra of 4-Fluorobenzyl Alcohol at High Resolution. $S_{0}$ and $S_{1}$ Structures and Tunneling Motions along the Low Frequency $-\mathrm{CH}_{2} \mathrm{OH}$ Torsional Coordinate in Both Electronic States, J. Phys. Chem. A 2011, 115, 11369-11377.

[339] H. S. Im, E. R. Bernstein, H. V. Secor, J. I. Seeman, Supersonic jet studies of benzyl alcohols: minimum energy conformations and torsional motion, J. Am. Chem. Soc. 1991, 113, 4422-4431.

[340] J. Osío Barcina, I. Fernández, M. del Rosario Colorado Heras, 7-Arylnorbornanes: Model Compounds for the Study of $\mathrm{CH} \cdots \pi$ and $\mathrm{OH} \cdots \pi$ Interactions, Eur. $J$. Org. Chem. 2012, 2012, 940-947.

[341] E. Sennert, Methyllaktat als multifunktionaler chiraler Induktor, Bachelorarbeit, Georg-August-Universität Göttingen, 2019.

[342] K. A. E. Meyer, M. A. Suhm, Stretching of cis-formic acid: warm-up and cooldown as molecular work-out, Chem. Sci. 2019, 10, 6285-6294.

[343] L. Khriachtchev, M. Pettersson, R. B. Gerber, Tribute to Markku O. Räsänen, J. Phys. Chem. A 2015, 119, 2187-2190.

[344] S. K. Nayak, R. Sathishkumar, T. N. G. Row, Directing role of functional groups in selective generation of $\mathrm{C}-\mathrm{H} \cdots \pi$ interactions: In situ cryo-crystallographic studies on benzyl derivatives, CrystEngComm 2010, 12, 3112.

[345] J. J. Fox, A. E. Martin, Infra-red absorption of the hydroxyl group in relation to inter- and intramolecular hydrogen bonds, Trans. Faraday Soc. 1940, 36, 897.

[346] P. J. Krueger, B. F. Hawkins, Infrared Absorption Band Shape Studies: Fundamental OH Stretching Vibration of Benzyl Alcohol Conformers in Dilute Carbon Tetrachloride Solution, Can. J. Chem. 1973, 51, 3250-3262.

[347] T. Visser, J. Van Der Maas, Structural information from $\mathrm{OH}$ stretching vibrations-XVII. On the different conformers in benzylalcohols and anthracylmethanols, Spectrochim. Acta Part Mol. Spectrosc. 1986, 42, 599-602.

[348] E. Bogdan, G. Compain, L. Mtashobya, J.-Y. Le Questel, F. Besseau, N. Galland, B. Linclau, J. Graton, Influence of Fluorination on the Conformational Properties and Hydrogen-Bond Acidity of Benzyl Alcohol Derivatives, Chem. - Eur. J. 2015, $21,11462-11474$. 
[349] K. Le Barbu, F. Lahmani, M. Mons, M. Broquier, A. Zehnacker, IR-UV investigation of the structure of the 1-phenylethanol chromophore and its hydrated complexes, Phys. Chem. Chem. Phys. 2001, 3, 4684-4688.

[350] K. Utzat, R. K. Bohn, E. Chu, Microwave spectral studies of benzyl alcohol and sec-phenethanol, Ohio State University, Abstracts of OSU International Symposium on Molecular Spectroscopy 2000-2009, 2003.

[351] K. Shin-ya, H. Sugeta, S. Shin, Y. Hamada, Y. Katsumoto, K. Ohno, Absolute Configuration and Conformation Analysis of 1-Phenylethanol by Matrix-Isolation Infrared and Vibrational Circular Dichroism Spectroscopy Combined with Density Functional Theory Calculation, J. Phys. Chem. A 2007, 111, 8598-8605.

[352] J. Kapitán, C. Johannessen, P. Bouř, L. Hecht, L. D. Barron, Vibrational Raman optical activity of 1-phenylethanol and 1-phenylethylamine: Revisiting old friends, Chirality 2009, 21, E4-E12.

[353] A. Borba, A. Gómez-Zavaglia, L. Lapinski, R. Fausto, Matrix isolation FTIR spectroscopic and theoretical study of methyl lactate, Vib. Spectrosc. 2004, 36, $79-88$.

[354] T. B. Adler, N. Borho, M. Reiher, M. A. Suhm, Chirality-Induced Switch in Hydrogen-Bond Topology: Tetrameric Methyl Lactate Clusters in the Gas Phase, Angew. Chem. 2006, 118, 3518-3523.

[355] B. Velino, M. Satta, W. Caminati, Conformational preferences of chiral molecules: free-jet rotational spectrum of 1-(4-fluorophenyl)-1-ethanol, Phys. Scr. 2008, $78,058106$.

[356] M. Speranza, F. Rondino, A. Giardini, A. Paladini, A. R. Hortal, S. Piccirillo, M. Satta, Conformational Landscape of Supersonically Expanded 1(Fluorophenyl)ethanols and Their Monohydrated Clusters, ChemPhysChem 2009, 10, 1859-1867.

[357] F. Rondino, M. Satta, S. Piccirillo, A. Ciavardini, A. Giardini, M. Speranza, L. Avaldi, A. Paladini, Chlorine Para-Substitution of 1-Phenylethanol: Resonant Photoionization Spectroscopy and Quantum Chemical Calculations of Hydrated and Diastereomeric Complexes, J. Phys. Chem. A 2016, 120, 5023-5031.

[358] S. Tang, W. Caminati, Free jet rotational spectrum of the most stable conformer of 1-(2-fluorophenyl)-1-ethanol, J. Mol. Spectrosc. 2010, 260, 120-123.

[359] A. Giardini, F. Rondino, G. Cattenacci, A. Paladini, S. Piccirillo, M. Satta, M. Speranza, Van der Waals interactions in a monosolvated chiral fluorinated molecule: R2PI vibroelectronic spectra of $(R)$-1-phenyl-2,2,2-trifluoroethanol clustered with water, Chem. Phys. Lett. 2007, 435, 230-235.

[360] C. D. Carlson, N. A. Seifert, M. Heger, F. Xie, J. Thomas, Y. Xu, Conformational dynamics of 1-phenyl-2,2,2-trifluoroethanol by rotational spectroscopy and ab initio calculations, J. Mol. Spectrosc. 2018, 351, 62-67.

[361] M. Albrecht, A. Borba, K. Le Barbu-Debus, B. Dittrich, R. Fausto, S. Grimme, A. Mahjoub, M. Nedić, U. Schmitt, L. Schrader, M. A. Suhm, A. Zehnacker-Rentien, J. Zischang, Chirality influence on the aggregation of methyl mandelate, New J. Chem. 2010, 34, 1266.

[362] M. Nedić, Adaptive Aggregation and Chirality Recognition of Neuroactive Substances and their Building Blocks: A Vibrational Spectroscopic and Microscopic Study, Dissertation, Georg-August-Universität Göttingen, 2012.

[363] J. Altnöder, A. Bouchet, J. J. Lee, K. E. Otto, M. A. Suhm, A. Zehnacker-Rentien, Chirality-dependent balance between hydrogen bonding and London dispersion in isolated ( \pm )-1-indanol clusters, Phys. Chem. Chem. Phys. 2013, 15, 10167. 
[364] M. Heger, J. Andersen, M. A. Suhm, R. Wugt Larsen, The donor OH stretching-libration dynamics of hydrogen-bonded methanol dimers in cryogenic matrices, Phys. Chem. Chem. Phys. 2016, 18, 3739-3745.

[365] T. Scharge, T. N. Wassermann, M. A. Suhm, Weak Hydrogen Bonds Make a Difference: Dimers of Jet-Cooled Halogenated Ethanols, Z. Für Phys. Chem. 2008, 222, 1407-1452.

[366] R. Subramanian, S. E. Novick, R. K. Bohn, Torsional analysis of 2-butynol, J. Mol. Spectrosc. 2003, 222, 57-62.

[367] C. Stelbrink, Wettstreit der molekularen Nasen: Chiralitätserkennung zwischen $\alpha$ Pinen und Alkoholen, Bachelorarbeit, Georg-August-Universität Göttingen, 2018.

[368] M. Kanakidou, J. H. Seinfeld, S. N. Pandis, I. Barnes, F. J. Dentener, M. C. Facchini, R. V. Dingenen, B. Ervens, A. Nenes, C. J. Nielsen, E. Swietlicki, J. P. Putaud, Y. Balkanski, S. Fuzzi, J. Horth, G. K. Moortgat, R. Winterhalter, C. E. L. Myhre, K. Tsigaridis, E. Vignati, E. G. Stephanou, J. Wilson, Organic aerosol and global climate modelling: a review, Atmos Chem Phys 2005, 71.

[369] V. Tarvainen, H. Hakola, J. Rinne, H. Hellén, S. Haapanala, Towards a comprehensive emission inventory of terpenoids from boreal ecosystems, Tellus B 2007, 59, 526-534.

[370] M. Sax, R. Zenobi, U. Baltensperger, M. Kalberer, Time Resolved Infrared Spectroscopic Analysis of Aerosol Formed by Photo-Oxidation of 1,3,5Trimethylbenzene and $\alpha$-Pinene, Aerosol Sci. Technol. 2005, 39, 822-830.

[371] W. Song, Laboratory and field measurements of enantiomeric and nonenantiomeric biogenic VOCs and anthropogenic BTEX compounds, Dissertation, Johannes Gutenberg-Universität Mainz, 2012.

[372] A. Rizvanovic, M. Amundin, M. Laska, Olfactory Discrimination Ability of Asian Elephants (Elephas maximus) for Structurally Related Odorants, Chem. Senses 2013, 38, 107-118.

[373] S. Kim, M. Amundin, M. Laska, Olfactory discrimination ability of South African fur seals (Arctocephalus pusillus) for enantiomers, J. Comp. Physiol. A 2013, 199, 535-544.

[374] M. Laska, C. G. Galizia, Enantioselectivity of odor perception in honeybees (Apis mellifera carnica)., Behav. Neurosci. 2001, 115, 632-639.

[375] A. C. R. da Silva, P. M. Lopes, M. M. B. de Azevedo, D. C. M. Costa, C. S. Alviano, D. S. Alviano, Biological Activities of $\alpha$-Pinene and $\beta$-Pinene Enantiomers, Molecules 2012, 17, 6305-6316.

[376] K. Ložienè, J. Švedienè, A. Paškevičius, V. Raudonienè, O. Sytar, A. Kosyan, Influence of plant origin natural $\alpha$-pinene with different enantiomeric composition on bacteria, yeasts and fungi, Fitoterapia 2018, 127, 20-24.

[377] M. S. I. El Alami, M. A. El Amrani, F. Agbossou-Niedercorn, I. Suisse, A. Mortreux, Chiral Ligands Derived from Monoterpenes: Application in the Synthesis of Optically Pure Secondary Alcohols via Asymmetric Catalysis, Chem. - Eur. J. 2015, 21, 1398-1413.

[378] A. R. Al-Rabaa, K. L. Barbu, F. Lahmani, A. Zehnacker-Rentien, Laser induced fluorescence of jet-cooled complexes between chiral molecules: a photophysical method for chiral discrimination, J. Photochem. Photobiol. Chem. 1997, 6.

[379] F. Lahmani, K. Le Barbu, A. Zehnacker-Rentien, Photophysical Studies on Molecular Chirality: Ground and Excited State Enantioselective Interactions between 2-Naphthyl-1-ethanol and Natural Bicyclic Compounds, J. Phys. Chem. A 1999, 103, 1991-1996. 
[380] S. Nehls, Chiralitätserkennung am Beispiel des Limonens: Einfache Modelle für den Geruchssinn, Bachelorarbeit, Georg-August-Universität Göttingen, 2011.

[381] M. Hendrich, Chiralitätserkennung bei Carvon: Ein einfaches Modellsystem für den Geruchssinn, Bachelorarbeit, Georg-August-Universität Göttingen, 2011.

[382] M. O. Sinnokrot, E. F. Valeev, C. D. Sherrill, Estimates of the ab initio Limit for $\pi-\pi$ Interactions: The Benzene Dimer, J. Am. Chem. Soc. 2002, 124, 1088710893.

[383] A. Tkatchenko, R. A. DiStasio, M. Head-Gordon, M. Scheffler, Dispersioncorrected Moller-Plesset second-order perturbation theory, J. Chem. Phys. 2009, $131,094106$.

[384] T. Janowski, P. Pulay, A Benchmark Comparison of $\sigma / \sigma$ and $\pi / \pi$ Dispersion: the Dimers of Naphthalene and Decalin, and Coronene and Perhydrocoronene, J. Am. Chem. Soc. 2012, 134, 17520-17525.

[385] E. M. Neeman, J. R. Avilés Moreno, T. R. Huet, The gas phase structure of $\alpha$ pinene, a main biogenic volatile organic compound, J. Chem. Phys. 2017, 147, 214305.

[386] R. Medel, M. Heger, M. A. Suhm, Molecular Docking via Olefinic OH $\cdots \pi$ Interactions: A Bulky Alkene Model System and Its Cooperativity, J. Phys. Chem. A 2015, 119, 1723-1730.

[387] R. W. Kugel, B. S. Ault, Infrared Matrix Isolation and Theoretical Studies of Reactions of Ozone with Bicyclic Alkenes: $\alpha$-Pinene, Norbornene, and Norbornadiene, J. Phys. Chem. A 2015, 119, 312-322.

[388] S.-H. Ko, T.-C. Chou, T.-J. Yang, Selective Hydrogenation of (-)- $\alpha$-Pinene over Nickel Catalysts Prepared by Electroless Deposition, Ind. Eng. Chem. Res. 1995, 34, 457-467.

[389] J. Zischang, J. J. Lee, M. A. Suhm, Communication: Where does the first water molecule go in imidazole?, J. Chem. Phys. 2011, 135, 061102.

[390] A. Mahjoub, K. Le Barbu-Debus, A. Zehnacker, Structural Rearrangement in the Formation of Jet-Cooled Complexes of Chiral $(S)$-1,2,3,4-Tetrahydro-3isoquinolinemethanol with Methyl Lactate: Chirality Effect in Conformer Selection, J. Phys. Chem. A 2013, 117, 2952-2960.

[391] E. R. Johnson, A. D. Becke, C. D. Sherrill, G. A. DiLabio, Oscillations in metageneralized-gradient approximation potential energy surfaces for dispersionbound complexes, J. Chem. Phys. 2009, 131, 034111.

[392] N. Mardirossian, M. Head-Gordon, How Accurate Are the Minnesota Density Functionals for Noncovalent Interactions, Isomerization Energies, Thermochemistry, and Barrier Heights Involving Molecules Composed of Main-Group Elements?, J. Chem. Theory Comput. 2016, 12, 4303-4325.

[393] J. A. Frey, C. Holzer, W. Klopper, S. Leutwyler, Experimental and Theoretical Determination of Dissociation Energies of Dispersion-Dominated Aromatic Molecular Complexes, Chem. Rev. 2016, 116, 5614-5641.

[394] S. Haldar, R. Gnanasekaran, P. Hobza, A comparison of ab initio quantummechanical and experimental $D_{0}$ binding energies of eleven H-bonded and eleven dispersion-bound complexes, Phys. Chem. Chem. Phys. 2015, 17, 26645-26652.

[395] M. Pierini, A. Troiani, M. Speranza, S. Piccirillo, C. Bosman, D. Toja, A. Giardini-Guidoni, Gas-Phase Enantiodifferentiation of Chiral Molecules: Chiral Recognition of 1-Phenyl-1-propanol/2-Butanol Clusters by Resonance Enhanced Multiphoton Ionization Spectroscopy, Angew. Chem. Int. Ed. Engl. 1997, 36, $1729-1731$. 
[396] K. Le Barbu, V. Brenner, P. Millié, F. Lahmani, A. Zehnacker-Rentien, An Experimental and Theoretical Study of Jet-Cooled Complexes of Chiral Molecules: The Role of Dispersive Forces in Chiral Discrimination, J. Phys. Chem. A 1998, $102,128-137$.

[397] A. Latini, D. Toja, A. Giardini-Guidoni, A. Palleschi, S. Piccirillo, M. Speranza, Spectroscopic enantiodifferentiation of chiral molecules in the gas phase, Chirality 1999, 11, 376-380.

[398] M. Mons, F. Piuzzi, I. Dimicoli, A. Zehnacker, F. Lahmani, Binding energy of hydrogen-bonded complexes of the chiral molecule 1-phenylethanol, as studied by 2C-R2PI: comparison between diastereoisomeric complexes with butan-2-ol and the singly hydrated complex, Phys. Chem. Chem. Phys. 2000, 2, 5065-5070.

[399] A. Giardini Guidoni, S. Piccirillo, D. Scuderi, M. Satta, T. Di Palma, M. Speranza, A. Filippi, A. Paladini, R2PI Study of intermolecular hydrogen bond in solventfree chiral complexes, Chirality 2001, 13, 727-730.

[400] K. Le Barbu, A. Zehnacker, F. Lahmani, M. Mons, F. Piuzzi, I. Dimicoli, Spectroscopic studies of enantiomeric discrimination in jet-cooled chiral complexes, Chirality 2001, 13, 715-721.

[401] A. Giardini, F. Rondino, A. Paladini, M. Speranza, M. Satta, S. Piccirillo, Enantioselective HF Loss Promoted by Resonant Two-Photon Ionization of Supersonically Expanded (R)-1-Phenyl-2,2,2-trifluoroethanol Clusters, J. Phys. Chem. A 2009, 113, 15127-15135.

[402] F. Rondino, A. Paladini, A. Ciavardini, A. Casavola, D. Catone, M. Satta, H. D. Barth, A. Giardini, M. Speranza, S. Piccirillo, Chiral recognition between 1-(4fluorophenyl)ethanol and 2-butanol: higher binding energy of homochiral complexes in the gas phase, Phys Chem Chem Phys 2011, 13, 818-824.

[403] A. Ciavardini, F. Rondino, A. Paladini, M. Speranza, S. Fornarini, M. Satta, S. Piccirillo, The effect of fluorine substitution on chiral recognition: interplay of $\mathrm{CH} \cdots \pi, \mathrm{OH} \cdots \pi$ and $\mathrm{CH} \cdots \mathrm{F}$ interactions in gas-phase complexes of 1-aryl-1ethanol with butan-2-ol, Phys. Chem. Chem. Phys. 2013, 15, 19360.

[404] F. Rondino, A. Ciavardini, M. Satta, A. Paladini, C. Fraschetti, A. Filippi, B. Botta, A. Calcaterra, M. Speranza, A. Giardini, S. Piccirillo, Ultraviolet and infrared spectroscopy of neutral and ionic non-covalent diastereomeric complexes in the gas phase, Rendiconti Lincei 2013, 24, 259-267.

[405] T. Huet, E. Neeman, Isotopic substitutions unveiled the identification of the most stable conformer of fenchol and of its water complex, Proc. 73rd Int. Symp. Mol. Spectrosc. 2018, DOI 10.15278/isms .2018. RK08.

[406] Y. Watanabe, T. Taketsugu, D. J. Wales, An ab initio study of tunneling splittings in the water dimer, J. Chem. Phys. 2004, 120, 5993-5999.

[407] D. Christen, L. Coudert, J. Larsson, D. Cremer, The Rotational-Torsional Spectrum of the $\mathrm{g}^{\prime} \mathrm{Gg}$ Conformer of Ethylene Glycol: Elucidation of an Unusual Tunneling Path, J. Mol. Spectrosc. 2001, 205, 185-196.

[408] J. Paul, I. Hearn, B. J. Howard, Chiral recognition in a single molecule: a study of homo and heterochiral butan-2,3-diol by Fourier transform microwave spectroscopy, Mol. Phys. 2007, 105, 825-839.

[409] M. Olschewski, J. Lindner, P. Vöhringer, A Hydrogen-Bond Flip-Flop through a Bjerrum-Type Defect, Angew. Chem. Int. Ed. 2013, 52, 2602-2605.

[410] L. Evangelisti, Q. Gou, L. Spada, G. Feng, W. Caminati, Conformational analysis of 1,4-butanediol: A microwave spectroscopy study, Chem. Phys. Lett. 2013, 556, 55-58.

[411] M. Fatima, Persönliche Mitteilung an Robert Medel, 2018. 
[412] L. Evangelisti, Persönliche Mitteilung an Robert Medel, 2020.

[413] H. C. Brown, H. R. Deck, Selective Reductions. VIII. The Stereochemistry of Reduction of Cyclic and Bicyclic Ketones by the Alkoxy-Substituted Lithium Aluminum Hydrides, J. Am. Chem. Soc. 1965, 87, 5620-5625.

[414] A. Coulombeau, A. Rassat, Metal-ammonia reduction of some bicyclo[2,2,1]heptan-2-ones, Chem Commun Lond. 1968, 0, 1587-1589.

[415] R. J. Taillefer, Base-Catalyzed Protium-Deuterium Exchange in Bicyclo[2.2.1.] heptanones, Dissertation, McMaster University Hamilton, 1973.

[416] V. Rautenstrauch, Endo-selectivity and kinetic control in alkali metal- $\mathrm{NH}_{3}-\mathrm{NH}_{4}^{+}$ reductions of bicyclo[2.2.1] heptan-2-ones, J Chem Soc Chem Commun 1986, $1558-1560$.

[417] S. Oswald, Aromatische und aliphatische Sechsringe in Wasserstoffbrückenkonkurrenz, Bachelorarbeit, Georg-August-Universität Göttingen, 2013.

[418] N. Guchhait, Benzyl alcohol-ammonia (1:1) cluster structure investigated by combined IR-UV double resonance spectroscopy in jet and ab initio calculation, $J$. Chem. Sci. 2001, 113, 235-244.

[419] D. Mani, E. Arunan, Rotational spectra of propargyl alcohol dimer: A dimer bound with three different types of hydrogen bonds, J. Chem. Phys. 2014, 141, 164311.

[420] N. Borho, M. A. Suhm, Glycidol dimer: anatomy of a molecular handshake, Phys. Chem. Chem. Phys. 2002, 4, 2721-2732.

[421] J. Altnöder, J. J. Lee, K. E. Otto, M. A. Suhm, Molecular Recognition in Glycolaldehyde, the Simplest Sugar: Two Isolated Hydrogen Bonds Win Over One Cooperative Pair, ChemistryOpen 2012, 1, 269-275.

[422] M. Lange, IR-Studien zur Aggregation und Komplexierung von 1,2-Diolen, Masterarbeit, Georg-August-Universität Göttingen, 2018.

[423] C. Zimmermann, FTIR-Spektroskopie an Intermolekularen Carbonyl-Waagen, Masterarbeit, Georg-August-Universität Göttingen, 2018.

[424] J. Stachowiak, Alkohol-Keton-Dimere als zwischenmolekulares Waagensystem, Bachelorarbeit, Georg-August-Universität Göttingen, 2018.

[425] A. Giardini Guidoni, S. Piccirillo, D. Scuderi, M. Satta, T. M. Di Palma, M. Speranza, Chirality and intermolecular forces: studies using R2PI experiments in supersonic beams, Phys. Chem. Chem. Phys. 2000, 2, 4139-4142.

[426] A. Filippi, A. Giardini, A. Latini, S. Piccirillo, D. Scuderi, M. Speranza, Chiral discrimination of monofunctional alcohols and amines in the gas phase, Int. J. Mass Spectrom. 2001, 210-211, 483-488.

[427] D. Scuderi, A. Paladini, M. Satta, D. Catone, A. Filippi, S. Piccirillo, A. Laganà, M. Speranza, A. Guidoni, Gas-phase complexes: noncovalent interactions and stereospecificity, Int. J. Mass Spectrom. 2003, 223-224, 159-168.

[428] D. Scuderi, A. Paladini, M. Satta, D. Catone, F. Rondino, A. Filippi, S. Piccirillo, M. Speranza, A. G. Guidoni, Solvent free interactions in contact pairs of molecules of biological interest: Laser spectroscopic and electrospray mass spectrometric studies, Int. J. Photoenergy 2004, 6, 17-21.

[429] A. Giardini Guidoni, A. Paladini, F. Rondino, S. Piccirillo, M. Satta, M. Speranza, Chiral clusters in a supersonic beam: R2PI-TOF spectroscopy of diastereomeric carboxylic esters/(R)-(+)-1-phenyl-1-propanol complexes, Org. Biomol. Chem. 2005, 3, 3984.

[430] N. Seurre, K. Le Barbu-Debus, F. Lahmani, N. Borho, M. A. Suhm, A. Zehnacker, Chiral Recognition in Jet-Cooled Complexes, Aust. J. Chem. 2004, 57, 1149. 
[431] M. Weimann, M. Fárník, M. A. Suhm, A first glimpse at the acidic proton vibrations in $\mathrm{HCl}-$ water clusters via supersonic jet FTIR spectroscopy, Phys Chem Chem Phys 2002, 4, 3933-3937.

[432] N. Borho, M. A. Suhm, K. Le Barbu-Debus, A. Zehnacker, Intra- vs. intermolecular hydrogen bonding: dimers of alpha-hydroxyesters with methanol, Phys. Chem. Chem. Phys. 2006, 8, 4449.

[433] S. Abraham, Schwingungsspektroskopie nah- und überkritischer Lösungsmittel, Dissertation, Georg-August-Universität Göttingen, 2013.

[434] R. Hildebrandt, Technische Zeichnung: Montage Expansionsrohr mit Düse, Chemie-Werkstätten Mechanik, Georg-August-Universität Göttingen, 2010.

[435] R. Hildebrandt, Technische Zeichnung: Microventil mit Gehäuse, ChemieWerkstätten Mechanik, Georg-August-Universität Göttingen, 2010.

[436] S. Hesse, Schwache Wechselwirkungen zwischen organischen Molekülen: strukturelle Vielfalt und ihre schwingungsspektroskopischen Auswirkungen, Dissertation, Cuvillier Verlag, Göttingen, 2010.

[437] C. Christiansen, Untersuchungen über die optischen Eigenschaften von fein vertheilten Körpern, Ann. Phys. 1884, 259, 298-306.

[438] C. Christiansen, Untersuchungen über die optischen Eigenschaften von fein vertheilten Körpern, Ann. Phys. 1885, 260, 439-446.

[439] O. Reinhold, Anwendung der FTIR-Spektroskopie für die quantitative Cristobalitanalyse im Arbeitsschutzbereich, Dissertation, Ruhr-Universität Bochum, 2009.

[440] S. Bocklitz, Charakterisierung von Phasenwechselmaterialien als thermische Speicher, Bachelorarbeit, Georg-August-Universität Göttingen, 2011.

[441] A. Poblotzki, Charakterisierung von Phasenwechselmaterialien, Bachelorarbeit, Georg-August-Universität Göttingen, 2012.

[442] P. Linstrom, NIST Chemistry WebBook, NIST Standard Reference Database 69, 1997.

[443] M. Hepp, F. Herregodts, M. Herman, Fourier transform infrared jet spectroscopy using a heated slit source, Chem. Phys. Lett. 1998, 294, 528-532.

[444] P. Asselin, J. Bruckhuisen, A. Roucou, M. Goubet, M.-A. Martin-Drumel, A. Jabri, Y. Belkhodja, P. Soulard, R. Georges, A. Cuisset, Jet-cooled rovibrational spectroscopy of methoxyphenols using two complementary FTIR and QCL based spectrometers, J. Chem. Phys. 2019, 151, 194302.

[445] M. Albrecht, Biologisch relevante Wasserstoffbrückensysteme im Überschallstrahl: Steuerung der Aggregation durch Substitution, Dissertation, Sierke, Göttingen, 2009.

[446] R. Hildebrandt, Technische Zeichnung: Microventil mit Gehäuse Umbau 2016, Chemie-Werkstätten Mechanik, Georg-August-Universität Göttingen, 2016.

[447] K. A. E. Meyer, M. A. Suhm, Formic acid aggregation in 2D supersonic expansions probed by FTIR imaging, J. Chem. Phys. 2017, 147, 144305.

[448] W. P. Halperin, The impact of helium shortages on basic research, Nat. Phys. 2014, 10, 467-470.

[449] M. Falk, E. Whalley, Infrared Spectra of Methanol and Deuterated Methanols in Gas, Liquid, and Solid Phases, J. Chem. Phys. 1961, 34, 1554-1568.

[450] J. Zischang, M. A. Suhm, The OH stretching spectrum of warm water clusters, $J$. Chem. Phys. 2014, 140, 064312. 
[451] C. Romers, An electron-diffraction investigation into the vapours of cyclohexanone and 2-methylcyclohexanone: Second communication on the molecular structures of ketones, Recl. Trav. Chim. Pays-Bas 1956, 75, 956-964.

[452] Y. Ohnishi, K. Kozima, Microwave Spectrum of Cyclohexanone, Bull. Chem. Soc. Jpn. 1968, 41, 1323-1325.

[453] J. Dillen, H. Geise, The molecular structure of cyclohexanone determined by gasphase electron diffraction, including microwave data, J. Mol. Struct. 1980, 69, 137-144.

[454] J. L. Alonso, Microwave spectrum of cyclohexanone, J. Mol. Struct. 1981, 73, 63-69.

[455] J. Gao, N. A. Seifert, W. Jäger, A microwave spectroscopic and ab initio study of keto-enol tautomerism and isomerism in the cyclohexanone-water complex, Phys. Chem. Chem. Phys. 2019, 21, 12872-12880.

[456] M. R. Emptage, Microwave Spectrum of trans-2,3-Epoxybutane, J. Chem. Phys. 1967, 47, 1293-1299.

[457] H. Hartwig, H. Dreizler, The Rotational Spectrum of 2,2-Dimethyloxirane, Z. Für Naturforschung A 1992, 47, DOI 10.1515/zna-1992-1010.

[458] H. Hartwig, H. Dreizler, The structure of 2,2-dimethyloxirane obtained by microwave spectroscopy A comparison of oxiranes and thiiranes, J. Mol. Struct. 1997, 8.

[459] S. Portmann, A. Inauen, H. P. Lüthi, S. Leutwyler, Chiral discrimination in hydrogen-bonded complexes, J. Chem. Phys. 2000, 113, 9577-9585.

[460] N. Borho, Y. Xu, Lock-and-Key Principle on a Microscopic Scale: The Case of the Propylene Oxide...Ethanol Complex, Angew. Chem. Int. Ed. 2007, 46, 22762279.

[461] N. Borho, Y. Xu, Molecular recognition in $1: 1$ hydrogen-bonded complexes of oxirane and trans-2,3-dimethyloxirane with ethanol: a rotational spectroscopic and abinitio study, Phys. Chem. Chem. Phys. 2007, 9, 4514-4520.

[462] N. Borho, Y. Xu, Tailoring the Key in a Molecular Lock-and-Key Model System: The Propylene Oxide...2-Fluoroethanol Complex, J. Am. Chem. Soc. 2008, 130, 5916-5921.

[463] C. Medcraft, M. Schnell, A Comparative Study of Two Bicyclic Ethers, Eucalyptol and 1,4-Cineole, by Broadband Rotational Spectroscopy, Z Phys Chem 2016, 230, 1-14.

[464] P. Raveendran, D. Zimmermann, T. Häber, M. A. Suhm, Exploring a hydrogenbond terminus: spectroscopy of eucalyptol-alcohol clusters, Phys. Chem. Chem. Phys. 2000, 2, 3555-3563.

[465] J. Saini, K. S. Viswanathan, From the propargyl alcohol-water complex to the propargyl alcohol dimer: where does the propargyl alcohol-methanol complex fit in?, New J. Chem. 2019, 43, 3969-3980.

[466] R. Hunt, W. Shelton, F. A. Flaherty, W. Cook, Torsion-Rotation Energy Levels and the Hindering Potential Barrier for the Excited Vibrational State of the $\mathrm{OH}-$ Stretch Fundamental Band $v_{1}$ of Methanol, J. Mol. Spectrosc. 1998, 192, 277293.

[467] K. Le Barbu-Debus, F. Lahmani, A. Zehnacker-Rentien, N. Guchhait, S. S. Panja, T. Chakraborty, Fluorescence spectroscopy of jet-cooled chiral ( \pm )-indan-1-ol and its cluster with ( \pm )-methyl- and ethyl-lactate, J. Chem. Phys. 2006, 125, 174305.

[468] H. Schaal, T. Häber, M. A. Suhm, Hydrogen Bonding in 2-Propanol. The Effect of Fluorination, J. Phys. Chem. A 2000, 104, 265-274. 